\title{
Beyond Chemical Accuracy for Alkane Thermochemistry: the DHthermo approach
}

Hanwei Lia ${ }^{\mathrm{a}}$, Bernardino Tirria ${ }^{\mathrm{a}}$, Eric Brémond ${ }^{\mathrm{b}}$, Juan Carlos Sancho-García ${ }^{\mathrm{c}}$ and Carlo Adamo $^{\mathrm{a}, \mathrm{d}^{*}}$

Chimie ParisTech, PSL Research University, CNRS, Institute of Chemistry for Health and Life Sciences, F-75005 Paris, France, Université de Paris, ITODYS, CNRS, F-75006 Paris, France; Departamento de Química Física, Universidad de Alicante, E-03080 Alicante, Spain ; Institut Universitaire de France, 103 Boulevard Saint Michel, F-75005 Paris, France

\section{SUPPLEMENTARY INFORMATION}




\section{Table of Contents}

Table S1. Optimized exponents of the DH-SVPD basis set. These exponents replace the corresponding exponents of the original Def2-SVPD basis set, which also reported for comparison. All other exponents are kept as in the original basis set....

Table S2. Example input for Gaussian code, reporting the optimized DH-SVPD basis set for $\mathrm{C}$ and $\mathrm{H}$ atoms .S4

Table S3. Relative energies ( $\mathrm{kcal} / \mathrm{mol}$ ) for the considered isodesmic reactions, obtained with the DH-SVPD basis set.

Table S4. Relative energies ( $\mathrm{kcal} / \mathrm{mol})$ for the considered isodesmic reactions, obtained with the CC-PVTZ basis set.

Table S5. Relative energies ( $\mathrm{kcal} / \mathrm{mol})$ for the considered isodesmic reactions, obtained with the CC-PVQZ basis set

Table S6. Relative energies $(\Delta \mathrm{E})$, including also ZPE correction $(\Delta \mathrm{E}+\mathrm{ZPE})$ and enthalpies $(\Delta \mathrm{H})$ at $298 \mathrm{~K}$ for the isodesmic reactions of Scheme 1, computed with the DH-SVPD basis set

Table S7. Relative energies $(\Delta \mathrm{E})$, including also $\mathrm{ZPE}$ correction $(\Delta \mathrm{E}+\mathrm{ZPE})$ and enthalpies $(\Delta \mathrm{H})$ at $298 \mathrm{~K}$ for the isodesmic reactions of Scheme 1, computed with the cc-pVTZ basis set

Coordinates of the molecules of 11 Alkane isodesmic reactions and the zero-point energy, thermal Enthalpies, thermal Free Energies....

Total energy of the molecules of 11 Alkane isodesmic reactions S94

Total energy and geometries of BSR36 dataset. 
Table S1. Optimized exponents of the DH-SVPD basis set. These exponents replace the corresponding exponents of the original Def2-SVPD basis set, which are also reported for comparison. All other exponents are kept as in the original basis set.

\begin{tabular}{ccccccc}
\hline atom & function & def2-SVPD & DH-SVPD & function & def2-SVPD & DH-SVPD \\
\hline $\mathrm{H}$ & $\mathrm{s}$ & 0.12194962000 & 0.4617867850 & $\mathrm{p}$ & 0.11704099050 & 0.07913402419 \\
$\mathrm{C}$ & $\mathrm{p}$ & 0.15268613795 & 0.1508036550 & $\mathrm{~d}$ & 0.11713185140 & 0.3229294790
\end{tabular}


Table S2. Example input for Gaussian code, reporting the optimized DH-SVPD basis set for $\mathrm{C}$ and $\mathrm{H}$ atoms

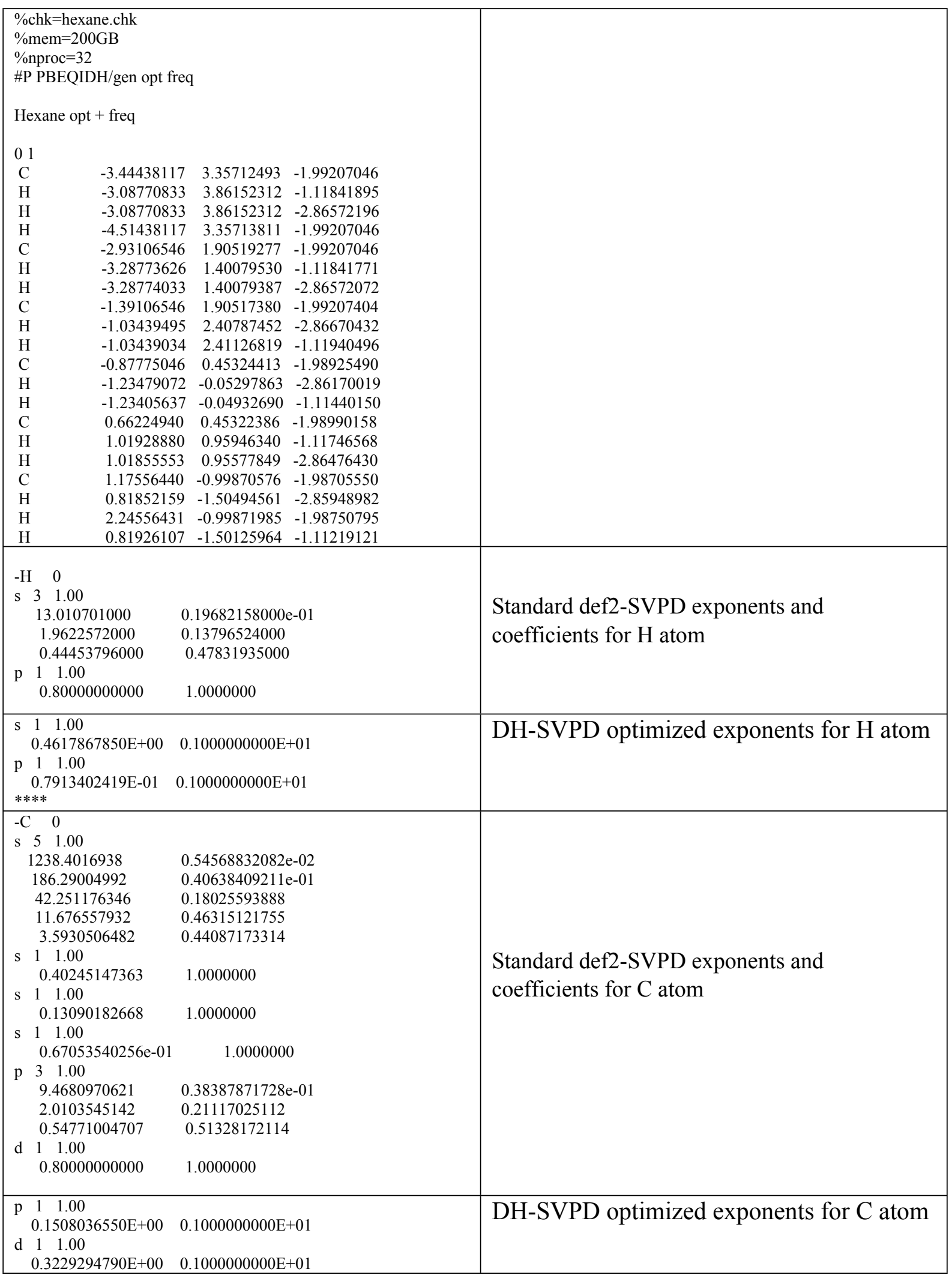


Table S3. Relative energies $(\mathrm{kcal} / \mathrm{mol})$ for the considered isodesmic reactions, obtained with the DH-SVPD basis set. In parenthesis are reported the relative errors with respect to the experimental data of reference 18

\begin{tabular}{|c|c|c|c|c|c|c|c|c|c|c|c|c|c|c|c|}
\hline \multirow[t]{2}{*}{$\begin{array}{l}1 . \\
2 . \\
3 .\end{array}$} & \multicolumn{3}{|c|}{$\begin{array}{l}\text { 2ethane } \rightarrow \text { propane }+ \text { methane } \\
\text { 3ethane } \rightarrow \text { butane }+2 \text { methane } \\
\text { 3ethane } \rightarrow \text { isobutane }+2 \text { methane }\end{array}$} & \multicolumn{4}{|c|}{$\begin{array}{ll}\text { 4. } & \text { 3ethane } \rightarrow \text { pentane }+3 \text { methane } \\
\text { 5. } & \text { 4ethane } \rightarrow \text { neopentane }+3 \text { methane } \\
\text { 6. } & \text { 5ethane } \rightarrow \text { hexane }+4 \text { methane }\end{array}$} & \multicolumn{5}{|c|}{$\begin{array}{ll}\text { 7. } & \text { 5ethane } \rightarrow 22 \text {-dimethylbutane }+4 \text { methane } \\
\text { 8. } & \text { 6ethane } \rightarrow \text { heptane }+5 \text { methane } \\
\text { 9. } & \text { 6ethane } \rightarrow 223 \text { trimethylbutane }+5 \text { methane }\end{array}$} & \multicolumn{3}{|c|}{$\begin{array}{l}\text { 10. 7ethane } \rightarrow \text { octane }+6 \text { methane } \\
\text { 11. } 7 \text { 7ethane } \rightarrow 2233 \text { tetramethylbutane }+6 \mathrm{~m} \\
\text { ethane }\end{array}$} \\
\hline & B3LYP & B3LYP-D3 & B2PLYP & $\begin{array}{c}\text { B2PLYP- } \\
\text { D3 }\end{array}$ & PBE0 & PBE0-DH & PBE-QIDH & M06 & M06L & wB97XD & TPSSh & $\begin{array}{l}\text { CAM- } \\
\text { B3LYP }\end{array}$ & $\begin{array}{c}\text { PBE0-DH- } \\
\text { D3(BJ) }\end{array}$ & \begin{tabular}{|c|} 
PBE-QIDH- \\
D3(BJ) \\
\end{tabular} & $\begin{array}{c}\text { DSD- } \\
\text { PBEP86 }\end{array}$ \\
\hline 1 & $\begin{array}{c}-2.22 \\
(+0.95)\end{array}$ & $\begin{array}{c}-2.74 \\
(+0.43)\end{array}$ & $\begin{array}{c}-2.70 \\
(+0.47)\end{array}$ & $\begin{array}{c}-2.98 \\
(+0.19)\end{array}$ & $\begin{array}{c}-2.42 \\
(+0.75)\end{array}$ & $\begin{array}{c}-2.64 \\
(+0.53)\end{array}$ & $\begin{array}{c}-2.91 \\
(+0.26)\end{array}$ & $\begin{array}{l}-2.71 \\
(+0.46)\end{array}$ & $\begin{array}{c}-2.38 \\
(+0.79)\end{array}$ & $\begin{array}{c}-2.79 \\
(+0.38)\end{array}$ & $\begin{array}{c}-2.04 \\
(+1.13)\end{array}$ & $\begin{array}{c}-2.52 \\
(+0.65)\end{array}$ & $\begin{array}{l}-2.96 \\
(+0.21)\end{array}$ & $\begin{array}{c}-3.00 \\
(+0.17)\end{array}$ & $\begin{array}{c}-3.13 \\
(+0.04)\end{array}$ \\
\hline 2 & $\begin{array}{c}-4.48 \\
(+2.27)\end{array}$ & $\begin{array}{c}-5.61 \\
(+1.14)\end{array}$ & $\begin{array}{l}-5.51 \\
+1.24)\end{array}$ & $\begin{array}{c}-6.13 \\
(+0.62)\end{array}$ & $\begin{array}{c}-4.91 \\
(+1.84)\end{array}$ & $\begin{array}{c}-5.39 \\
(+1.36)\end{array}$ & $\begin{array}{c}-5.99 \\
(+0.77)\end{array}$ & $\begin{array}{l}-5.58 \\
(+1.17)\end{array}$ & $\begin{array}{c}-4.86 \\
(+1.89)\end{array}$ & $\begin{array}{c}-5.73 \\
(+1.02)\end{array}$ & $\begin{array}{c}-4.13 \\
(+2.62)\end{array}$ & $\begin{array}{c}-5.09 \\
(+1.66)\end{array}$ & $\begin{array}{c}-6.10 \\
(+0.65)\end{array}$ & $\begin{array}{c}-6.20 \\
(+0.55)\end{array}$ & $\begin{array}{c}-6.45 \\
(+0.30)\end{array}$ \\
\hline 3 & $\begin{array}{c}-5.51 \\
(+2.95)\end{array}$ & $\begin{array}{l}-7.12 \\
(+1.34)\end{array}$ & $\begin{array}{c}-7.01 \\
(+1.45)\end{array}$ & $\begin{array}{c}-7.88 \\
(+0.58)\end{array}$ & $\begin{array}{c}-6.13 \\
(+2.33)\end{array}$ & $\begin{array}{c}-6.83 \\
(+1.63)\end{array}$ & $\begin{array}{c}-7.70 \\
(+0.76)\end{array}$ & $\begin{array}{c}-7.44 \\
(+1.02)\end{array}$ & $\begin{array}{c}-6.20 \\
(+2.26)\end{array}$ & $\begin{array}{c}-7.22 \\
(+1.24)\end{array}$ & $\begin{array}{c}-5.08 \\
(+3.38)\end{array}$ & $\begin{array}{c}-6.42 \\
(+2.04)\end{array}$ & $\begin{array}{c}-7.81 \\
(+0.65)\end{array}$ & $\begin{array}{c}-7.96 \\
(+0.50)\end{array}$ & $\begin{array}{l}-8.39 \\
(0.07)\end{array}$ \\
\hline 4 & $\begin{array}{c}-6.71 \\
(+3.45)\end{array}$ & $\begin{array}{c}-8.50 \\
(+1.66)\end{array}$ & $\begin{array}{c}-8.33 \\
(+1.83)\end{array}$ & $\begin{array}{c}-9.31 \\
(+0.85)\end{array}$ & $\begin{array}{c}-7.37 \\
(+2.79)\end{array}$ & $\begin{array}{c}-8.13 \\
(+2.03)\end{array}$ & $\begin{array}{c}-9.07 \\
(+1.09)\end{array}$ & $\begin{array}{l}-8.46 \\
(+1.70)\end{array}$ & $\begin{array}{c}-7.50 \\
(+2.66)\end{array}$ & $\begin{array}{c}-8.71 \\
(+1.45)\end{array}$ & $\begin{array}{c}-6.20 \\
(+3.96)\end{array}$ & $\begin{array}{c}-7.64 \\
(+2.52)\end{array}$ & $\begin{array}{c}-9.24 \\
(+0.92)\end{array}$ & $\begin{array}{c}-9.43 \\
(+0.73)\end{array}$ & $\begin{array}{l}-9.81 \\
(0.35)\end{array}$ \\
\hline 5 & $\begin{array}{c}-8.61 \\
(+6.58)\end{array}$ & $\begin{array}{c}-11.90 \\
(+3.29)\end{array}$ & $\begin{array}{l}-11.77 \\
(+3.42)\end{array}$ & $\begin{array}{c}-13.54 \\
(+1.65)\end{array}$ & $\begin{array}{c}-9.92 \\
(+5.27)\end{array}$ & $\begin{array}{c}-11.44 \\
(+3.75)\end{array}$ & $\begin{array}{c}-13.29 \\
(+1.90)\end{array}$ & $\begin{array}{c}-13.05 \\
(+2.14)\end{array}$ & $\begin{array}{l}-10.54 \\
(+4.65)\end{array}$ & $\begin{array}{c}-12.22 \\
(+2.97)\end{array}$ & $\begin{array}{c}-8.07 \\
(+7.12)\end{array}$ & $\begin{array}{l}-10.43 \\
(+4.76)\end{array}$ & $\begin{array}{c}-13.44 \\
(+1.75)\end{array}$ & $\begin{array}{c}-13.83 \\
(+1.36)\end{array}$ & $\begin{array}{c}-14.68 \\
(0.51)\end{array}$ \\
\hline 6 & $\begin{array}{c}-8.99 \\
(+4.60)\end{array}$ & $\begin{array}{l}-11.44 \\
(+2.15)\end{array}$ & $\begin{array}{l}-11.20 \\
(+2.39)\end{array}$ & $\begin{array}{l}-12.55 \\
(+1.04)\end{array}$ & $\begin{array}{c}-9.88 \\
(+3.71)\end{array}$ & $\begin{array}{l}-10.92 \\
(+2.67)\end{array}$ & $\begin{array}{l}-12.21 \\
(+1.38)\end{array}$ & $\begin{array}{l}-11.36 \\
(+2.23)\end{array}$ & $\begin{array}{l}-10.00 \\
(+3.59)\end{array}$ & $\begin{array}{l}-11.73 \\
(+1.86)\end{array}$ & $\begin{array}{c}-8.32 \\
(+5.27)\end{array}$ & $\begin{array}{l}-10.24 \\
(+3.35)\end{array}$ & $\begin{array}{c}-12.44 \\
(+1.15)\end{array}$ & $\begin{array}{l}-12.72 \\
(+0.87)\end{array}$ & $\begin{array}{l}-13.22 \\
(0.37)\end{array}$ \\
\hline 7 & $\begin{array}{c}-9.02 \\
(+7.76)\end{array}$ & $\begin{array}{c}-13.62 \\
(+3.16)\end{array}$ & $\begin{array}{c}-13.14 \\
(+3.64)\end{array}$ & $\begin{array}{l}-15.63 \\
(+1.15)\end{array}$ & $\begin{array}{c}-10.86 \\
(+5.92)\end{array}$ & $\begin{array}{c}-12.83 \\
(+3.95)\end{array}$ & $\begin{array}{l}-15.23 \\
(+1.55)\end{array}$ & $\begin{array}{c}-15.26 \\
(+1.52)\end{array}$ & $\begin{array}{l}-12.70 \\
(+4.08)\end{array}$ & $\begin{array}{l}-14.47 \\
(+2.31)\end{array}$ & $\begin{array}{c}-8.54 \\
(+8.24)\end{array}$ & $\begin{array}{l}-11.35 \\
(+5.43)\end{array}$ & $\begin{array}{c}-15.66 \\
(+1.12)\end{array}$ & $\begin{array}{c}-16.05 \\
(+0.73)\end{array}$ & $\begin{array}{l}-17.11 \\
(-0.33)\end{array}$ \\
\hline 8 & $\begin{array}{c}-11.29 \\
(+5.73)\end{array}$ & $\begin{array}{l}-14.40 \\
(+2.62)\end{array}$ & $\begin{array}{l}-14.09 \\
(+2.93)\end{array}$ & $\begin{array}{l}-15.80 \\
(+1.22)\end{array}$ & $\begin{array}{l}-12.39 \\
(+4.63)\end{array}$ & $\begin{array}{c}-13.73 \\
(+3.29)\end{array}$ & $\begin{array}{c}-15.36 \\
(+1.66)\end{array}$ & $\begin{array}{l}-14.29 \\
(+2.73)\end{array}$ & $\begin{array}{l}-12.71 \\
(+4.31)\end{array}$ & $\begin{array}{l}-14.77 \\
(+2.25)\end{array}$ & $\begin{array}{c}-10.44 \\
(+6.58)\end{array}$ & $\begin{array}{l}-12.85 \\
(+4.17)\end{array}$ & $\begin{array}{l}-15.65 \\
(+1.37)\end{array}$ & $\begin{array}{c}-16.02 \\
(+1.00)\end{array}$ & $\begin{array}{c}-16.64 \\
(+0.38)\end{array}$ \\
\hline 9 & $\begin{array}{c}-9.24 \\
(+10.37)\end{array}$ & $\begin{array}{l}-15.68 \\
(+3.93)\end{array}$ & $\begin{array}{l}-14.86 \\
(+4.75)\end{array}$ & $\begin{array}{l}-18.34 \\
(+1.27)\end{array}$ & $\begin{array}{l}-11.84 \\
(+7.77)\end{array}$ & $\begin{array}{l}-14.51 \\
(+5.10)\end{array}$ & $\begin{array}{l}-17.77 \\
(+1.84)\end{array}$ & $\begin{array}{l}-18.01 \\
(+1.60)\end{array}$ & $\begin{array}{l}-15.07 \\
(+4.54)\end{array}$ & $\begin{array}{l}-16.71 \\
(+2.90)\end{array}$ & $\begin{array}{c}-8.89 \\
(+10.72)\end{array}$ & $\begin{array}{l}-12.34 \\
(+7.27)\end{array}$ & $\begin{array}{l}-18.53 \\
(+1.08)\end{array}$ & $\begin{array}{l}-18.96 \\
(+0.65)\end{array}$ & $\begin{array}{l}-20.40 \\
(-0.79)\end{array}$ \\
\hline 10 & $\begin{array}{c}-13.58 \\
(+6.87)\end{array}$ & $\begin{array}{c}-17.36 \\
(+3.09)\end{array}$ & $\begin{array}{c}-16.98 \\
(+3.47)\end{array}$ & $\begin{array}{l}-19.06 \\
(+1.39)\end{array}$ & $\begin{array}{l}-14.92 \\
(+5.53)\end{array}$ & $\begin{array}{c}-16.54 \\
(+3.91)\end{array}$ & $\begin{array}{c}-18.52 \\
(+1.93)\end{array}$ & $\begin{array}{c}-17.22 \\
(+3.23)\end{array}$ & $\begin{array}{l}-15.26 \\
(+5.19)\end{array}$ & $\begin{array}{l}-17.81 \\
(+2.64)\end{array}$ & $\begin{array}{c}-12.57 \\
(+7.88)\end{array}$ & $\begin{array}{l}-15.46 \\
(+4.99)\end{array}$ & $\begin{array}{c}-18.87 \\
(+1.58)\end{array}$ & $\begin{array}{c}-19.33 \\
(+1.12)\end{array}$ & $\begin{array}{c}-20.08 \\
(+0.37)\end{array}$ \\
\hline 11 & $\begin{array}{c}-8.23 \\
(+14.90)\end{array}$ & $\begin{array}{c}-17.32 \\
(+5.81)\end{array}$ & $\begin{array}{l}-16.22 \\
(+6.91)\end{array}$ & $\begin{array}{l}-21.16 \\
(+1.97)\end{array}$ & $\begin{array}{c}-12.06 \\
(+11.07)\end{array}$ & $\begin{array}{c}-15.85 \\
(+7.28)\end{array}$ & $\begin{array}{c}-20.49 \\
(+2.64)\end{array}$ & $\begin{array}{l}-20.94 \\
(+2.19)\end{array}$ & $\begin{array}{l}-17.43 \\
(+5.70)\end{array}$ & $\begin{array}{l}-19.16 \\
(+3.97)\end{array}$ & $\begin{array}{c}-8.46 \\
(+14.67)\end{array}$ & $\begin{array}{c}-12.63 \\
(+10.50)\end{array}$ & $\begin{array}{c}-21.45 \\
(+1.68)\end{array}$ & $\begin{array}{c}-22.15 \\
(+0.98)\end{array}$ & $\begin{array}{l}-24.14 \\
(-1.01)\end{array}$ \\
\hline MAD & 6.04 & 2.60 & 2.96 & 1.08 & 4.69 & 3.23 & 1.43 & 1.82 & 3.60 & 2.09 & 6.51 & 4.30 & 1.10 & 0.79 & 0.41 \\
\hline
\end{tabular}


Table S4. Relative energies $(\mathrm{kcal} / \mathrm{mol})$ for the considered isodesmic reactions, obtained with the CC-PVTZ basis set. In parenthesis are reported the relative errors with respect to the experimental data of reference 18 .

\begin{tabular}{|c|c|c|c|c|c|c|c|c|c|c|c|c|c|c|c|}
\hline & B3LYP & $\begin{array}{c}\text { B3LYP- } \\
\text { D3 }\end{array}$ & B2PLYP & $\begin{array}{c}\text { B2PLYP } \\
\text {-D3 } \\
\end{array}$ & PBE0 & $\begin{array}{c}\text { PBE0- } \\
\text { DH }\end{array}$ & $\begin{array}{l}\text { PBE- } \\
\text { QIDH }\end{array}$ & M06 & M06L & wB97XD & TPSSh & $\begin{array}{l}\text { CAM- } \\
\text { B3LYP }\end{array}$ & $\begin{array}{c}\text { PBE0-DH- } \\
\text { D3(BJ) }\end{array}$ & $\begin{array}{l}\text { PBEQIDH } \\
\text {-D3(BJ) }\end{array}$ & $\begin{array}{c}\text { DSD- } \\
\text { PBEP86 } \\
\end{array}$ \\
\hline 1 & $\begin{array}{l}-1.91 \\
(1.26)\end{array}$ & $\begin{array}{c}-2.44 \\
(+0.73)\end{array}$ & $\begin{array}{c}-2.38 \\
(+0.79)\end{array}$ & $\begin{array}{c}-2.67 \\
(+0.50)\end{array}$ & $\begin{array}{c}-2.18 \\
(+0.99)\end{array}$ & $\begin{array}{c}-2.39 \\
(+0.78)\end{array}$ & $\begin{array}{c}-2.63 \\
(+0.54)\end{array}$ & $\begin{array}{c}-2.46 \\
(+0.71)\end{array}$ & $\begin{array}{c}-2.10 \\
(+1.07)\end{array}$ & $\begin{array}{c}-2.51 \\
(+0.66)\end{array}$ & $\begin{array}{c}-1.80 \\
(+1.37)\end{array}$ & $\begin{array}{c}-2.23 \\
(+0.94)\end{array}$ & $\begin{array}{c}-2.71 \\
(+0.46)\end{array}$ & $\begin{array}{c}-2.72 \\
(+0.45)\end{array}$ & $\begin{array}{c}-2.81 \\
(+0.36)\end{array}$ \\
\hline 2 & $\begin{array}{l}-3.88 \\
(2.87)\end{array}$ & $\begin{array}{c}-5.02 \\
(+1.73)\end{array}$ & $\begin{array}{c}-4.87 \\
(+1.88)\end{array}$ & $\begin{array}{l}-5.50 \\
(+1.25)\end{array}$ & $\begin{array}{c}-4.45 \\
(+2.30)\end{array}$ & $\begin{array}{c}-4.90 \\
(+1.85)\end{array}$ & $\begin{array}{c}-5.42 \\
(+1.33)\end{array}$ & $\begin{array}{c}-5.21 \\
(+1.54)\end{array}$ & $\begin{array}{c}-4.41 \\
(+2.34)\end{array}$ & $\begin{array}{c}-5.24 \\
(+1.51)\end{array}$ & $\begin{array}{c}-3.69 \\
(+3.06)\end{array}$ & $\begin{array}{c}-4.54 \\
(+2.21)\end{array}$ & $\begin{array}{c}-5.61 \\
(+1.14)\end{array}$ & $\begin{array}{c}-5.64 \\
(+1.11)\end{array}$ & $\begin{array}{c}-5.79 \\
(+0.96)\end{array}$ \\
\hline 3 & $\begin{array}{l}-4.66 \\
(3.80)\end{array}$ & $\begin{array}{c}-6.29 \\
(+2.17)\end{array}$ & $\begin{array}{c}-6.16 \\
(+2.30)\end{array}$ & $\begin{array}{c}-7.04 \\
(+1.42)\end{array}$ & $\begin{array}{c}-5.49 \\
(+2.97)\end{array}$ & $\begin{array}{c}-6.18 \\
(+2.28)\end{array}$ & $\begin{array}{c}-6.97 \\
(+1.49)\end{array}$ & $\begin{array}{c}-6.83 \\
(+1.63)\end{array}$ & $\begin{array}{c}-5.56 \\
(+2.90)\end{array}$ & $\begin{array}{c}-6.54 \\
(+1.92)\end{array}$ & $\begin{array}{c}-4.48 \\
(+3.98)\end{array}$ & $\begin{array}{c}-5.64 \\
(+2.82)\end{array}$ & $\begin{array}{c}-7.17 \\
(+1.29)\end{array}$ & $\begin{array}{c}-7.23 \\
(+1.23)\end{array}$ & $\begin{array}{c}-7.53 \\
(+0.93)\end{array}$ \\
\hline 4 & $\begin{array}{l}-5.80 \\
(4.36)\end{array}$ & $\begin{array}{c}-7.60 \\
(+2.56)\end{array}$ & $\begin{array}{c}-7.34 \\
(+2.82)\end{array}$ & $\begin{array}{c}-8.33 \\
(+1.83)\end{array}$ & $\begin{array}{c}-6.66 \\
(+3.50)\end{array}$ & $\begin{array}{c}-7.37 \\
(+2.79)\end{array}$ & $\begin{array}{c}-8.18 \\
(+1.98)\end{array}$ & $\begin{array}{c}-7.84 \\
(+2.32)\end{array}$ & $\begin{array}{c}-6.74 \\
(+3.42)\end{array}$ & $\begin{array}{c}-7.95 \\
(+2.21)\end{array}$ & $\begin{array}{c}-5.52 \\
(+4.64)\end{array}$ & $\begin{array}{c}-6.80 \\
(+3.36)\end{array}$ & $\begin{array}{c}-8.48 \\
(+1.68)\end{array}$ & $\begin{array}{c}-8.54 \\
(+1.62)\end{array}$ & $\begin{array}{c}-8.75 \\
(+1.41)\end{array}$ \\
\hline 5 & $\begin{array}{l}-7.26 \\
(7.93)\end{array}$ & $\begin{array}{l}-10.57 \\
(+4.62)\end{array}$ & $\begin{array}{l}-10.41 \\
(+4.78)\end{array}$ & $\begin{array}{l}-12.20 \\
(+2.99)\end{array}$ & $\begin{array}{c}-8.97 \\
(+6.22)\end{array}$ & $\begin{array}{l}-10.45 \\
(+4.74)\end{array}$ & $\begin{array}{l}-12.12 \\
(+3.07)\end{array}$ & $\begin{array}{c}-12.42 \\
(+2.77)\end{array}$ & $\begin{array}{c}-9.76 \\
(+5.43)\end{array}$ & $\begin{array}{l}-11.19 \\
(+4.00)\end{array}$ & $\begin{array}{c}-7.17 \\
(+8.02)\end{array}$ & $\begin{array}{c}-9.21 \\
(+5.98)\end{array}$ & $\begin{array}{l}-12.47 \\
(+2.72)\end{array}$ & $\begin{array}{c}-12.66 \\
(+2.53)\end{array}$ & $\begin{array}{l}-13.30 \\
(+1.89)\end{array}$ \\
\hline 6 & $\begin{array}{l}-7.75 \\
(5.84)\end{array}$ & $\begin{array}{l}-10.21 \\
(+3.38)\end{array}$ & $\begin{array}{c}-9.84 \\
(+3.75)\end{array}$ & $\begin{array}{c}-11.19 \\
(+2.40)\end{array}$ & $\begin{array}{c}-8.91 \\
(+4.68)\end{array}$ & $\begin{array}{c}-9.88 \\
(+3.71)\end{array}$ & $\begin{array}{l}-10.98 \\
(+2.61)\end{array}$ & $\begin{array}{l}-10.58 \\
(+3.01)\end{array}$ & $\begin{array}{c}-9.04 \\
(+4.55)\end{array}$ & $\begin{array}{l}-10.70 \\
(+2.89)\end{array}$ & $\begin{array}{c}-7.40 \\
(+6.19)\end{array}$ & $\begin{array}{c}-9.09 \\
(+4.50)\end{array}$ & $\begin{array}{l}-11.40 \\
(+2.19)\end{array}$ & $\begin{array}{c}-11.48 \\
(+2.11)\end{array}$ & $\begin{array}{l}-11.77 \\
(+1.82)\end{array}$ \\
\hline 7 & $\begin{array}{l}-7.04 \\
(9.74)\end{array}$ & $\begin{array}{l}-11.66 \\
(+5.12)\end{array}$ & $\begin{array}{l}-11.13 \\
(+5.65)\end{array}$ & $\begin{array}{l}-13.63 \\
(+3.15)\end{array}$ & $\begin{array}{c}-9.39 \\
(+7.39)\end{array}$ & $\begin{array}{l}-11.28 \\
(+5.50)\end{array}$ & $\begin{array}{l}-13.44 \\
(+3.34)\end{array}$ & $\begin{array}{l}-14.18 \\
(+2.60)\end{array}$ & $\begin{array}{l}-11.48 \\
(+5.30)\end{array}$ & $\begin{array}{l}-12.88 \\
(+3.90)\end{array}$ & $\begin{array}{c}-7.17 \\
(+9.61)\end{array}$ & $\begin{array}{c}-9.51 \\
(+7.27)\end{array}$ & $\begin{array}{l}-14.13 \\
(+2.65)\end{array}$ & $\begin{array}{l}-14.26 \\
(+2.52)\end{array}$ & $\begin{array}{l}-15.04 \\
(+1.74)\end{array}$ \\
\hline 8 & $\begin{array}{l}-9.71 \\
(7.31)\end{array}$ & $\begin{array}{l}-12.85 \\
(+4.17)\end{array}$ & $\begin{array}{l}-12.34 \\
(+4.68)\end{array}$ & $\begin{array}{l}-14.07 \\
(+2.95)\end{array}$ & $\begin{array}{l}-11.17 \\
(+5.85)\end{array}$ & $\begin{array}{l}-12.39 \\
(+4.63)\end{array}$ & $\begin{array}{l}-13.78 \\
(+3.24)\end{array}$ & $\begin{array}{l}-13.25 \\
(+3.77)\end{array}$ & $\begin{array}{l}-11.40 \\
(+5.62)\end{array}$ & $\begin{array}{l}-13.47 \\
(+3.55)\end{array}$ & $\begin{array}{c}-9.27 \\
(+7.75)\end{array}$ & $\begin{array}{l}-11.39 \\
(+5.63)\end{array}$ & $\begin{array}{l}-14.33 \\
(+2.69)\end{array}$ & $\begin{array}{c}-14.44 \\
(+2.58)\end{array}$ & $\begin{array}{l}-14.78 \\
(+2.24)\end{array}$ \\
\hline 9 & $\begin{array}{c}-6.33 \\
(13.28)\end{array}$ & $\begin{array}{l}-12.89 \\
(+6.72)\end{array}$ & $\begin{array}{l}-12.00 \\
(+7.61)\end{array}$ & $\begin{array}{l}-15.50 \\
(+4.11)\end{array}$ & $\begin{array}{c}-9.68 \\
(+9.93)\end{array}$ & $\begin{array}{l}-12.30 \\
(+7.31)\end{array}$ & $\begin{array}{l}-15.24 \\
(+4.37)\end{array}$ & $\begin{array}{l}-16.36 \\
(+3.25)\end{array}$ & $\begin{array}{l}-13.15 \\
(+6.46)\end{array}$ & $\begin{array}{l}-14.48 \\
(+5.13)\end{array}$ & $\begin{array}{c}-6.91 \\
(+12.70)\end{array}$ & $\begin{array}{c}-9.69 \\
(+9.92)\end{array}$ & $\begin{array}{l}-16.35 \\
(+3.26)\end{array}$ & $\begin{array}{c}-16.43 \\
(+3.18)\end{array}$ & $\begin{array}{l}-17.49 \\
(+2.12)\end{array}$ \\
\hline 10 & $\begin{array}{c}-11.68 \\
(8.77)\end{array}$ & $\begin{array}{l}-15.48 \\
(+4.97)\end{array}$ & $\begin{array}{c}-14.86 \\
(+5.59)\end{array}$ & $\begin{array}{l}-16.96 \\
(+3.49)\end{array}$ & $\begin{array}{l}-13.43 \\
(+7.02)\end{array}$ & $\begin{array}{l}-14.91 \\
(+5.54)\end{array}$ & $\begin{array}{l}-16.59 \\
(+3.86)\end{array}$ & $\begin{array}{l}-15.99 \\
(+4.46)\end{array}$ & $\begin{array}{l}-13.73 \\
(+6.72)\end{array}$ & $\begin{array}{c}-16.24 \\
(+4.21)\end{array}$ & $\begin{array}{l}-11.15 \\
(+9.30)\end{array}$ & $\begin{array}{l}-13.69 \\
(+6.76)\end{array}$ & $\begin{array}{l}-17.27 \\
(+3.18)\end{array}$ & $\begin{array}{c}-17.40 \\
(+3.05)\end{array}$ & $\begin{array}{l}-17.81 \\
(+2.64)\end{array}$ \\
\hline 11 & $\begin{array}{c}-4.51 \\
(18.62)\end{array}$ & $\begin{array}{l}-13.65 \\
(+9.48)\end{array}$ & $\begin{array}{c}-12.48 \\
(+10.65)\end{array}$ & $\begin{array}{c}-17.42 \\
(+5.71)\end{array}$ & $\begin{array}{c}-9.26 \\
(+13.87)\end{array}$ & $\begin{array}{c}-13.01 \\
(+10.12)\end{array}$ & $\begin{array}{l}-17.19 \\
(+5.94)\end{array}$ & $\begin{array}{l}-18.83 \\
(+4.30)\end{array}$ & $\begin{array}{l}-15.12 \\
(+8.01)\end{array}$ & $\begin{array}{c}-16.27 \\
(+6.86)\end{array}$ & $\begin{array}{c}-5.94 \\
(+17.19)\end{array}$ & $\begin{array}{c}-9.13 \\
(+14.0 \\
0)\end{array}$ & $\begin{array}{l}-18.62 \\
(+4.51)\end{array}$ & $\begin{array}{l}-18.83 \\
(+4.30)\end{array}$ & $\begin{array}{l}-20.31 \\
(+2.82)\end{array}$ \\
\hline MAD & 7.62 & 4.15 & 4.59 & 2.71 & 5.88 & 4.48 & 2.89 & 2.76 & 4.71 & 3.35 & 7.62 & 5.76 & 2.34 & 2.24 & 1.72 \\
\hline
\end{tabular}


Table S5. Relative energies ( $\mathrm{kcal} / \mathrm{mol})$ for the considered isodesmic reactions, obtained with the cc-PVQZ basis set. In parenthesis are reported the relative errors with respect to the experimental data of reference 18.

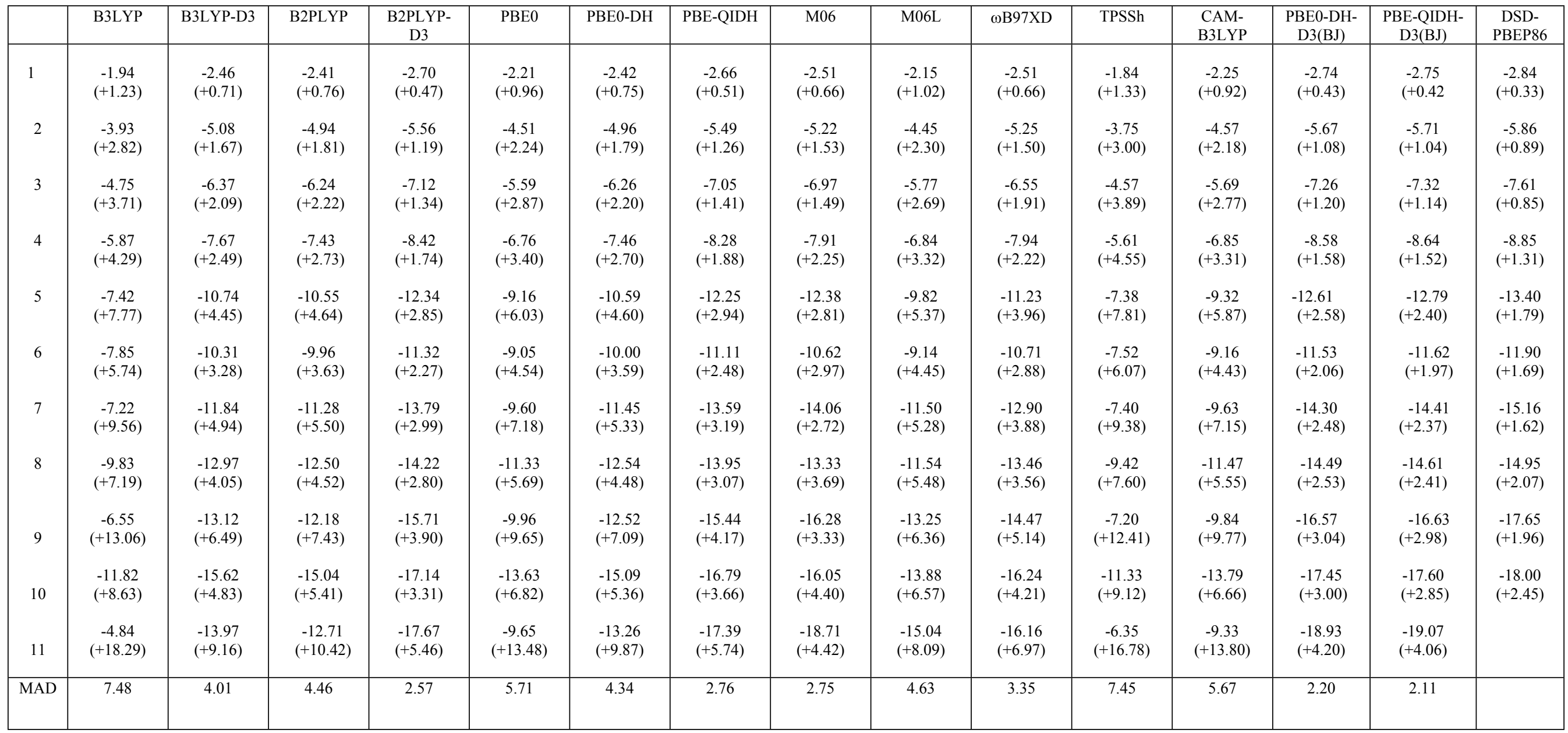


Table S6. Relative energies $(\Delta \mathrm{E})$, including also ZPE correction $(\Delta \mathrm{E}+\mathrm{ZPE})$ and enthalpies $(\Delta \mathrm{H})$ at $298 \mathrm{~K}$ for the isodesmic reactions of Scheme 1, computed with the DH-SVPD basis set. All the values were computed using the DH-SVPD basis set.

\begin{tabular}{|c|c|c|c|c|c|c|c|c|c|c|c|c|}
\hline \multirow[t]{2}{*}{ Reactions } & \multicolumn{3}{|c|}{ PBEQIDH-D3(BJ) } & \multicolumn{3}{|c|}{ PBE0DH-D3(BJ) } & \multicolumn{3}{|c|}{ B2PLYP-D3 } & \multicolumn{3}{|c|}{ B3LYP-D3 } \\
\hline & $\Delta(\mathrm{E}+\mathrm{ZPVE})$ & $\Delta \mathrm{E}$ & $\Delta \mathrm{H}$ & $\Delta(\mathrm{E}+\mathrm{ZPVE})$ & $\Delta \mathrm{E}$ & $\Delta \mathrm{H}$ & $\Delta(\mathrm{E}+\mathrm{ZPVE})$ & $\Delta \mathrm{E}$ & $\Delta \mathrm{H}$ & $\Delta(\mathrm{E}+\mathrm{ZPVE})$ & $\Delta \mathrm{E}$ & $\Delta \mathrm{H}$ \\
\hline 1 & -3.03 & -2.24 & -2.72 & -2.94 & -2.16 & -2.68 & -2.96 & -2.23 & -2.71 & -2.73 & -2.03 & -2.48 \\
\hline 2 & -6.25 & -4.52 & -5.50 & -6.07 & -4.36 & -5.41 & -6.10 & -4.50 & -5.45 & -5.59 & -4.07 & -4.95 \\
\hline 3 & -8.00 & -5.98 & -7.32 & -7.78 & -5.78 & -7.17 & -7.84 & -5.99 & -7.26 & -7.09 & -5.35 & -6.52 \\
\hline 4 & -9.49 & -6.83 & -8.29 & -9.20 & -6.57 & -8.11 & -9.27 & -6.78 & -8.20 & -8.46 & -6.09 & -7.40 \\
\hline 5 & -13.87 & -10.43 & -12.79 & -13.38 & -10.00 & -12.41 & -13.49 & -10.39 & -12.56 & -11.85 & -8.97 & -10.95 \\
\hline 6 & -12.79 & -9.15 & -11.11 & -12.39 & -8.79 & -10.84 & -12.49 & -9.09 & -10.97 & -11.39 & -8.15 & -9.89 \\
\hline 7 & -16.10 & -11.74 & -14.49 & -15.60 & -11.31 & -14.17 & -15.57 & -11.60 & -14.19 & -13.56 & -9.87 & -12.22 \\
\hline 8 & -16.10 & -11.49 & -13.94 & -15.59 & -11.03 & -13.59 & -15.73 & -11.41 & -13.76 & -14.33 & -10.22 & -12.39 \\
\hline 9 & -19.03 & -13.55 & -17.05 & -18.45 & -13.06 & -16.64 & -18.26 & -13.28 & -16.53 & -15.61 & -10.92 & -13.88 \\
\hline 10 & -19.42 & -13.82 & -16.77 & -18.80 & -13.27 & -16.34 & -18.98 & -13.73 & -16.55 & -17.28 & -12.29 & -14.89 \\
\hline 11 & -22.23 & -15.66 & -20.05 & -21.37 & -14.97 & -19.40 & -21.08 & -15.19 & -19.19 & -17.25 & -11.75 & -15.37 \\
\hline
\end{tabular}




\begin{tabular}{|c|c|c|c|c|c|c|c|c|c|c|c|c|}
\hline \multirow[t]{2}{*}{ Reactions } & \multicolumn{3}{|c|}{ PBE-QIDH } & \multicolumn{3}{|c|}{ B2PLYP } & \multicolumn{3}{|c|}{ PBE0-DH } & \multicolumn{3}{|c|}{ DSD-PBEP86 } \\
\hline & $\Delta(\mathrm{E}+\mathrm{ZPVE})$ & $\Delta \mathrm{E}$ & $\Delta \mathrm{H}$ & $\Delta(\mathrm{E}+\mathrm{ZPVE})$ & $\Delta \mathrm{E}$ & $\Delta \mathrm{H}$ & $\Delta(\mathrm{E}+\mathrm{ZPVE})$ & $\Delta \mathrm{E}$ & $\Delta \mathrm{H}$ & $\Delta(\mathrm{E}+\mathrm{ZPVE})$ & $\Delta \mathrm{E}$ & $\Delta \mathrm{H}$ \\
\hline 1 & -2.898 & -2.105 & -2.637 & -2.686 & -1.931 & -2.425 & -2.625 & -1.837 & -2.363 & -3.13 & -2.37 & -2.87 \\
\hline 2 & -5.961 & -4.233 & -5.297 & -5.484 & -3.840 & -4.821 & -5.369 & -3.653 & -4.704 & -6.45 & -4.79 & -5.78 \\
\hline 3 & -7.665 & -5.637 & -7.060 & -6.980 & -5.060 & -6.372 & -6.800 & -4.785 & -6.190 & -8.39 & -6.45 & -7.78 \\
\hline 4 & -9.037 & -6.368 & -7.940 & -8.297 & -5.745 & -7.204 & -8.100 & -5.450 & -7.002 & -9.81 & -7.24 & -8.71 \\
\hline 5 & -13.237 & -9.795 & -12.268 & -11.724 & -8.488 & -10.750 & -11.392 & -7.974 & -10.413 & -14.68 & -11.40 & -13.71 \\
\hline 6 & -12.163 & -8.519 & -10.616 & -11.156 & -7.672 & -9.615 & -10.881 & -7.264 & -9.332 & -13.22 & -9.71 & -11.68 \\
\hline 7 & -15.169 & -10.798 & -13.743 & -13.090 & -8.981 & -11.666 & -12.783 & -8.449 & -11.350 & -17.11 & -12.96 & -15.68 \\
\hline 8 & -15.303 & -10.681 & -13.300 & -14.030 & -9.610 & -12.027 & -13.673 & -9.087 & -11.669 & -16.64 & -12.19 & -14.64 \\
\hline 9 & -17.703 & -12.205 & -15.901 & -14.798 & -9.603 & -12.972 & -14.450 & -8.974 & -12.618 & -20.40 & -15.21 & -18.62 \\
\hline 10 & -18.450 & -12.841 & -15.987 & -16.916 & -11.549 & -14.460 & -16.473 & -10.910 & -14.008 & -20.08 & -14.68 & -17.61 \\
\hline 11 & -20.409 & -13.836 & -18.442 & -16.151 & -10.007 & -14.158 & -15.790 & -9.275 & -13.796 & -24.14 & -17.95 & -22.18 \\
\hline
\end{tabular}




\begin{tabular}{|c|c|c|c|c|c|c|c|c|c|c|c|c|c|c|c|}
\hline \multirow[t]{2}{*}{ Reactions } & \multicolumn{3}{|c|}{ PBE0 } & \multicolumn{3}{|c|}{ B3LYP } & \multicolumn{3}{|c|}{ M06 } & \multicolumn{3}{|c|}{ TPSSH } & \multicolumn{3}{|c|}{ M06-L } \\
\hline & $\Delta(\mathrm{E}+\mathrm{ZPVE})$ & $\Delta \mathrm{E}$ & $\Delta \mathrm{H}$ & $\Delta \mathrm{E}+\mathrm{ZPVE}$ & $\Delta \mathrm{E}$ & $\Delta \mathrm{H}$ & $\Delta(\mathrm{E}+\mathrm{ZPVE})$ & $\Delta \mathrm{E}$ & $\Delta \mathrm{H}$ & $\Delta(\mathrm{E}+\mathrm{ZPVE})$ & $\Delta \mathrm{E}$ & $\Delta \mathrm{H}$ & $\Delta(\mathrm{E}+\mathrm{ZPVE})$ & $\Delta \mathrm{E}$ & $\Delta \mathrm{H}$ \\
\hline 1 & -2.410 & -1.649 & -2.147 & -2.211 & -1.480 & -1.949 & -2.703 & -1.980 & -2.448 & -2.032 & -1.302 & -1.758 & -2.372 & -1.571 & -2.104 \\
\hline 2 & -4.889 & -3.232 & -4.226 & -4.457 & -2.865 & -3.796 & -5.555 & -3.965 & -4.898 & -4.117 & -2.518 & -3.433 & -4.840 & -3.193 & -4.174 \\
\hline 3 & -6.108 & -4.164 & -5.495 & -5.490 & -3.636 & -4.880 & -7.406 & -5.553 & -6.822 & -5.063 & -3.183 & -4.418 & -6.178 & -4.190 & -5.564 \\
\hline 4 & -7.337 & -4.774 & -6.239 & -6.684 & -4.210 & -5.595 & -8.422 & -5.895 & -7.321 & -6.175 & -3.709 & -5.057 & -7.475 & -4.754 & -6.346 \\
\hline 5 & -9.881 & -6.585 & -8.893 & -8.572 & -5.462 & -7.592 & -13.001 & -10.070 & -12.121 & -8.035 & -4.810 & -6.983 & -10.494 & -7.122 & -9.506 \\
\hline 6 & -9.839 & -6.335 & -8.299 & -8.953 & -5.576 & -7.418 & -11.311 & -7.850 & -9.752 & -8.286 & -4.913 & -6.711 & -9.965 & -6.338 & -8.387 \\
\hline 7 & -10.818 & -6.640 & -9.377 & -8.980 & -5.029 & -7.553 & -15.198 & -11.355 & -13.821 & -8.510 & -4.412 & -6.993 & -12.649 & -8.254 & -11.171 \\
\hline 8 & -12.342 & -7.906 & -10.341 & -11.241 & -6.952 & -9.252 & -14.232 & -9.815 & -12.211 & -10.394 & -6.126 & -8.351 & -12.655 & -7.922 & -10.587 \\
\hline 9 & -11.792 & -6.473 & -9.921 & -9.200 & -4.140 & -7.315 & -17.939 & -13.144 & -16.245 & -8.852 & -3.628 & -6.891 & -15.015 & -9.552 & -13.213 \\
\hline 10 & -14.857 & -9.477 & -12.398 & -13.531 & -8.329 & -11.086 & -17.148 & -11.784 & -14.660 & -12.518 & -7.337 & -10.009 & -15.204 & -9.512 & -12.672 \\
\hline 11 & -12.011 & -5.671 & -9.968 & -8.199 & -2.279 & -6.162 & -20.859 & -15.227 & -19.013 & -8.425 & -2.162 & -6.256 & -17.358 & -10.789 & -15.384 \\
\hline
\end{tabular}




\begin{tabular}{|c|c|c|c|c|c|c|}
\hline \multirow{2}{*}{ Reactions } & \multicolumn{3}{|c|}{ CAM-B3LYP } & \multicolumn{3}{c|}{ wB97X-D } \\
\hline & $\Delta$ (E+ZPVE) & $\Delta \mathrm{E}$ & $\Delta \mathrm{H}$ & $\Delta \mathrm{E}+\mathrm{ZPVE}$ & $\Delta \mathrm{E}$ & $\Delta \mathrm{H}$ \\
\hline 1 & -2.511 & -1.756 & -2.250 & -2.780 & -2.004 & -2.520 \\
\hline 2 & -5.07 & -3.428 & -4.411 & -5.71 & -4.036 & -5.040 \\
\hline 3 & -6.39 & -4.477 & -5.786 & -7.19 & -5.285 & -6.611 \\
\hline 4 & -7.61 & -5.061 & -6.525 & -8.68 & -6.079 & -7.591 \\
\hline 5 & -10.39 & -7.180 & -9.423 & -12.17 & -8.937 & -11.235 \\
\hline 6 & -10.20 & -6.714 & -8.665 & -11.68 & -8.142 & -10.138 \\
\hline 7 & -11.30 & -7.242 & -9.888 & -14.41 & -10.157 & -12.964 \\
\hline 8 & -12.80 & -8.379 & -10.804 & -14.71 & -10.225 & -12.716 \\
\hline 9 & -12.29 & -7.164 & -10.474 & -16.64 & -11.584 & -14.930 \\
\hline 10 & -15.40 & -10.044 & -12.954 & -17.74 & -12.307 & -15.283 \\
\hline 11 & -12.58 & -6.520 & -10.602 & -19.08 & -12.909 & -17.163 \\
\hline
\end{tabular}


Table S7. Relative energies $(\Delta \mathrm{E})$, including also ZPE correction $(\Delta \mathrm{E}+\mathrm{ZPE})$ and enthalpies $(\Delta \mathrm{H})$ at $298 \mathrm{~K}$ for the isodesmic reactions of Scheme 1 , computed with the cc-PVTZ basis set.

\begin{tabular}{|c|c|c|c|c|c|c|c|c|}
\hline \multicolumn{3}{|c|}{ B2PLYP } & \multicolumn{3}{c|}{ PBE-QIDH } & \multicolumn{3}{c|}{ PBE-QIDH-D3(BJ) } \\
\hline$\Delta(\mathrm{E}+\mathrm{ZPVE})$ & $\Delta \mathrm{E}$ & $\Delta \mathrm{H}$ & $\Delta(\mathrm{E}+\mathrm{ZPVE})$ & $\Delta \mathrm{E}$ & $\Delta \mathrm{H}$ & $\Delta(\mathrm{E}+\mathrm{ZPVE})$ & $\Delta \mathrm{E}$ & $\Delta \mathrm{H}$ \\
\hline & & & & & & & \\
\hline-2.374 & -1.613 & -2.103 & -2.62 & -1.82 & -2.35 & -2.71 & -1.909 & -2.440 \\
\hline-4.855 & -3.208 & -4.178 & -5.40 & -3.67 & -4.72 & -5.62 & -3.883 & -4.938 \\
\hline-6.139 & -4.183 & -5.509 & -6.94 & -4.88 & -6.31 & -7.21 & -5.145 & -6.577 \\
\hline-7.310 & -4.759 & -6.198 & -8.15 & -5.47 & -7.03 & -8.50 & -5.829 & -7.386 \\
\hline-10.372 & -6.990 & -9.358 & -12.07 & -8.50 & -11.07 & -12.61 & -9.040 & -11.609 \\
\hline-9.797 & -6.321 & -8.230 & -10.93 & -7.28 & -9.35 & -11.44 & -7.790 & -9.862 \\
\hline-11.082 & -6.896 & -9.617 & -13.39 & -8.96 & -11.92 & -14.20 & -9.781 \\
\hline-12.295 & -7.882 & -10.255 & -13.73 & -9.10 & -11.68 & -14.38 & -12.734 \\
\hline-11.948 & -6.662 & -10.026 & -15.18 & -9.62 & -13.28 & -16.37 & -10.809 & -14.454 \\
\hline-14.800 & -9.445 & -12.300 & -16.53 & -10.91 & -14.01 & -17.33 & -11.719 & -14.818 \\
\hline-12.435 & -6.107 & -10.317 & -17.12 & -10.48 & -15.06 & -18.75 & -12.144 & -16.700 \\
\hline
\end{tabular}




\begin{tabular}{|c|c|c|c|c|c|c|c|c|}
\hline \multicolumn{3}{|c|}{ B2PLYP-D3 } & \multicolumn{2}{c|}{ PBE0 } & \multicolumn{2}{c|}{ B3LYP } \\
\hline$\Delta(\mathrm{E}+\mathrm{ZPVE})$ & $\Delta \mathrm{E}$ & $\Delta \mathrm{H}$ & $\Delta(\mathrm{E}+\mathrm{ZPVE})$ & $\Delta \mathrm{E}$ & $\Delta \mathrm{H}$ & $\Delta(\mathrm{E}+\mathrm{ZPVE})$ & $\Delta \mathrm{E}$ & $\Delta \mathrm{H}$ \\
\hline & & & & & & & \\
\hline-2.657 & -1.918 & -2.395 & -2.169 & -1.422 & -1.91 & -1.906 & -1.185 & -1.639 \\
\hline-5.479 & -3.873 & -4.818 & -4.43 & -2.805 & -3.77 & -3.86 & -2.298 & -3.194 \\
\hline-7.012 & -5.123 & -6.410 & -5.47 & -3.553 & -4.87 & -4.65 & -2.800 & -4.025 \\
\hline-8.296 & -5.804 & -7.204 & -6.63 & -4.127 & -5.55 & -5.78 & -3.352 & -4.678 \\
\hline-12.149 & -8.902 & -11.188 & -8.94 & -5.620 & -7.96 & -7.23 & -4.044 & -6.230 \\
\hline-11.149 & -7.749 & -9.608 & -8.88 & -5.456 & -7.35 & -7.72 & -4.414 & -6.169 \\
\hline-13.575 & -9.527 & -12.158 & -9.35 & -5.218 & -7.92 & -7.01 & -3.052 & -5.562 \\
\hline-14.018 & -9.697 & -12.017 & -11.13 & -6.783 & -9.14 & -9.68 & -5.472 & -7.666 \\
\hline-15.443 & -10.332 & -13.597 & -9.64 & -4.412 & -7.75 & -6.30 & -1.310 & -4.404 \\
\hline-16.891 & -11.646 & -14.425 & -13.38 & -8.111 & -10.93 & -11.63 & -6.530 & -9.156 \\
\hline-17.354 & -11.308 & -15.362 & -9.22 & -2.975 & -7.13 & -4.49 & -1.527 & -2.334 \\
\hline
\end{tabular}




\begin{tabular}{|c|c|c|c|c|c|c|c|c|}
\hline \multicolumn{3}{|c|}{ B3LYP-D3 } & \multicolumn{3}{c|}{ PBE0-DH-D3(BJ) } & \multicolumn{3}{c|}{ PBE0-DH } \\
\hline$\Delta(\mathrm{E}+\mathrm{ZPVE})$ & $\Delta \mathrm{E}$ & $\Delta \mathrm{H}$ & $\Delta(\mathrm{E}+\mathrm{ZPVE})$ & $\Delta \mathrm{E}$ & $\Delta \mathrm{H}$ & $\Delta(\mathrm{E}+\mathrm{ZPVE})$ & $\Delta \mathrm{E}$ & $\Delta \mathrm{H}$ \\
\hline & & & & & & & \\
\hline-2.430 & -1.746 & -2.176 & -2.701 & -1.924 & -2.433 & -2.377 & -1.594 & -2.108 \\
\hline-5.00 & -3.511 & -4.359 & -5.59 & -3.901 & -4.916 & -4.88 & -3.183 & -4.206 \\
\hline-6.27 & -4.532 & -5.685 & -7.15 & -5.151 & -6.523 & -6.15 & -4.141 & -5.527 \\
\hline-7.57 & -5.250 & -6.503 & -8.45 & -5.846 & -7.340 & -7.34 & -4.719 & -6.227 \\
\hline-10.53 & -7.576 & -9.613 & -12.42 & -8.978 & -11.418 & -10.41 & -6.934 & -9.401 \\
\hline-10.17 & -7.002 & -8.662 & -11.36 & -7.805 & -9.792 & -9.84 & -6.263 & -8.269 \\
\hline-11.62 & -7.920 & -10.252 & -14.07 & -9.808 & -12.623 & -11.24 & -6.922 & -9.780 \\
\hline-12.79 & -8.756 & -10.829 & -14.27 & -9.766 & -12.246 & -12.34 & -7.807 & -10.312 \\
\hline-12.84 & -8.104 & -11.012 & -16.28 & -10.895 & -14.376 & -12.25 & -6.781 & -10.326 \\
\hline-15.42 & -10.513 & -12.992 & -17.20 & -11.728 & -14.701 & -14.85 & -9.350 & -12.354 \\
\hline-13.60 & -7.950 & -11.602 & -18.54 & -12.168 & -16.498 & -12.96 & -6.429 & -10.878 \\
\hline
\end{tabular}




\begin{tabular}{|c|c|c|c|c|c|c|c|c|}
\hline \multicolumn{3}{|c|}{$\mathrm{M} 06-\mathrm{L}$} & \multicolumn{3}{c|}{$\mathrm{M} 06$} & \multicolumn{3}{c|}{$\omega \mathrm{B} 97 \mathrm{XD}$} \\
\hline$\Delta(\mathrm{E}+\mathrm{ZPVE})$ & $\Delta \mathrm{E}$ & $\Delta \mathrm{H}$ & $\Delta(\mathrm{E}+\mathrm{ZPVE})$ & $\Delta \mathrm{E}$ & $\Delta \mathrm{H}$ & $\Delta(\mathrm{E}+\mathrm{ZPVE})$ & $\Delta \mathrm{E}$ & $\Delta \mathrm{H}$ \\
\hline & & & & & & & \\
\hline-2.090 & -1.350 & -1.819 & -2.448 & -1.780 & -2.201 & -2.501 & -94.559 & -2.243 \\
\hline-4.391 & -2.803 & -3.714 & -5.19 & -3.651 & -4.540 & -5.22 & -98.160 & -4.551 \\
\hline-5.540 & -3.624 & -4.920 & -6.80 & -5.057 & -6.240 & -6.51 & -135.332 & -5.916 \\
\hline-6.714 & -4.192 & -5.586 & -7.81 & -5.465 & -6.739 & -7.92 & -192.046 & -6.835 \\
\hline-9.717 & -6.366 & -8.722 & -12.37 & -9.392 & -11.485 & -11.14 & -194.493 & -10.190 \\
\hline-9.003 & -5.589 & -7.427 & -10.54 & -7.280 & -9.017 & -10.66 & -249.792 & -9.125 \\
\hline-11.436 & -7.180 & -9.931 & -14.12 & -10.398 & -12.762 & -12.83 & -251.210 & -11.390 \\
\hline-11.360 & -6.981 & -9.300 & -13.20 & -9.102 & -11.234 & -13.41 & -307.547 & -11.422 \\
\hline-13.095 & -7.935 & -11.248 & -16.30 & -11.703 & -14.597 & -14.43 & -307.884 & -12.640 \\
\hline-13.672 & -8.376 & -11.141 & -15.93 & -10.920 & -13.497 & -16.18 & -365.300 & -13.717 \\
\hline-15.056 & -8.755 & -13.001 & -18.75 & -13.379 & -16.903 & -16.21 & -364.460 & -14.220 \\
\hline
\end{tabular}




\begin{tabular}{|c|c|c|c|c|c|c|c|c|}
\hline \multicolumn{3}{|c|}{ TPSSh } & \multicolumn{3}{|c|}{ CAM-B3LYP } & \multicolumn{3}{c|}{ DSD-PBEP86 } \\
\hline$\Delta(\mathrm{E}+\mathrm{ZPVE})$ & $\Delta \mathrm{E}$ & $\Delta \mathrm{H}$ & $\Delta(\mathrm{E}+\mathrm{ZPVE})$ & $\Delta \mathrm{E}$ & $\Delta \mathrm{H}$ & $\Delta(\mathrm{E}+\mathrm{ZPVE})$ & $\Delta \mathrm{E}$ & $\Delta \mathrm{H}$ \\
\hline & & & & & & & \\
\hline-1.797 & -1.067 & -1.515 & -2.225 & -1.482 & -1.959 & -2.809 & -2.039 & -2.538 \\
\hline-3.68 & -2.085 & -2.981 & -4.52 & -2.908 & -3.852 & -5.788 & -4.121 & -5.109 \\
\hline-4.46 & -2.563 & -3.794 & -5.62 & -3.715 & -5.000 & -7.531 & -5.548 & -6.899 \\
\hline-5.50 & -3.056 & -4.356 & -6.77 & -4.272 & -5.674 & -8.754 & -6.177 & -7.632 \\
\hline-7.15 & -3.837 & -6.075 & -9.17 & -5.894 & -8.181 & -13.297 & -9.868 & -12.279 \\
\hline-7.37 & -4.030 & -5.774 & -9.05 & -5.644 & -7.504 & -11.768 & -8.247 & -10.195 \\
\hline-7.14 & -3.031 & -5.600 & -9.48 & -5.418 & -8.039 & -15.036 & -10.805 & -13.567 \\
\hline-9.23 & -5.004 & -7.159 & -11.34 & -7.014 & -9.327 & -14.783 & -10.319 & -12.736 \\
\hline-6.88 & -1.666 & -4.862 & -9.65 & -4.540 & -7.762 & -17.491 & -12.188 & -15.593 \\
\hline-11.11 & -5.976 & -8.578 & -13.63 & -8.385 & -11.154 & -17.808 & -12.393 & -15.289 \\
\hline-5.91 & 0.387 & -3.647 & -9.09 & -2.998 & -7.020 & -20.315 & -14.016 & -18.255 \\
\hline
\end{tabular}


Coordinates of the molecules of 11 Alkane isodesmic reactions, zero-point energy, thermal Enthalpies, thermal Free Energies

Basis set: DH-SVPD

Functional: M06-L

Ethane:

Standard orientation:

\begin{tabular}{|c|c|c|c|c|}
\hline \multirow{2}{*}{$\begin{array}{l}\text { Center } \\
\text { Number }\end{array}$} & Atomic & Atomic & \multicolumn{2}{|c|}{ Coordinates (Angstroms) } \\
\hline & Number & Type & $\mathrm{X}$ & Z \\
\hline 1 & 6 & -0.000000 & -0.000000 & 0.758047 \\
\hline 2 & 1 & -0.507299 & 0.878735 & 1.162409 \\
\hline 3 & 1 & 1.014656 & -0.000034 & 1.162409 \\
\hline 4 & 1 & -0.507357 & -0.878701 & 1.162409 \\
\hline 5 & 6 & 0.000000 & -0.000000 & -0.758047 \\
\hline 6 & 1 & 0.507299 & 0.878735 & -1.162409 \\
\hline 7 & 1 & 0.507357 & -0.878701 & -1.162409 \\
\hline 8 & 1 & -1.014656 & -0.000034 & -1.162409 \\
\hline \multicolumn{3}{|c|}{ Zero-point correction $=$} & \multicolumn{2}{|c|}{0.075475 (Hartree/Particle) } \\
\hline \multicolumn{3}{|c|}{ Thermal correction to Energy= } & \multicolumn{2}{|c|}{0.078959} \\
\hline \multicolumn{3}{|c|}{ Thermal correction to Enthalpy= } & \multicolumn{2}{|c|}{0.079903} \\
\hline \multicolumn{4}{|c|}{ Thermal correction to Gibbs Free Energy= } & 0.054064 \\
\hline \multicolumn{4}{|c|}{ Sum of electronic and zero-point Energies $=$} & -79.686694 \\
\hline \multicolumn{4}{|c|}{ Sum of electronic and thermal Energies $=$} & -79.683210 \\
\hline \multicolumn{4}{|c|}{ Sum of electronic and thermal Enthalpies $=$} & -79.682266 \\
\hline \multicolumn{4}{|c|}{ Sum of electronic and thermal Free Energies $=$} & -79.708104 \\
\hline
\end{tabular}

Methane:

Standard orientation:

\begin{tabular}{|c|c|c|c|c|}
\hline \multirow{2}{*}{$\begin{array}{l}\text { Center } \\
\text { Number }\end{array}$} & \multirow{2}{*}{$\begin{array}{l}\text { Atomic } \\
\text { Numbe }\end{array}$} & \multirow{2}{*}{$\begin{array}{r}\text { Atomic } \\
\text { Type }\end{array}$} & \multicolumn{2}{|c|}{ Coordinates (Angstroms) } \\
\hline & & & $\mathrm{X}$ & $\mathrm{Z}$ \\
\hline 1 & 6 & 0.000000 & 0.000000 & 0.000000 \\
\hline 2 & 1 & 0.628413 & 0.628413 & 0.628413 \\
\hline 3 & 1 & -0.628413 & -0.628413 & 0.628413 \\
\hline 4 & 1 & -0.628413 & 0.628413 & -0.628413 \\
\hline 5 & 1 & 0.628413 & -0.628413 & -0.628413 \\
\hline \multicolumn{3}{|c|}{ Zero-point correction $=$} & \multicolumn{2}{|c|}{0.045228 (Hartree/Particle) } \\
\hline \multicolumn{3}{|c|}{ Thermal correction to Energy= } & \multicolumn{2}{|c|}{0.048098} \\
\hline \multicolumn{3}{|c|}{ Thermal correction to Enthalpy= } & \multicolumn{2}{|c|}{0.049043} \\
\hline \multicolumn{4}{|c|}{ Thermal correction to Gibbs Free Energy= } & 0.027916 \\
\hline \multicolumn{4}{|c|}{ Sum of electronic and zero-point Energies= } & -40.437083 \\
\hline \multicolumn{4}{|c|}{ Sum of electronic and thermal Energies $=$} & -40.434212 \\
\hline
\end{tabular}


Sum of electronic and thermal Enthalpies=

$-40.433268$

Sum of electronic and thermal Free Energies=

Propane: Standard orientation:

\begin{tabular}{cccrrr} 
Center & \multicolumn{2}{c}{ Atomic } & \multicolumn{2}{c}{ Atomic } & \multicolumn{3}{c}{ Coordinates (Angstroms) } \\
Number & Number & Type & \multicolumn{1}{c}{ X } & Y & Z \\
\hline 1 & 6 & 0 & -1.259942 & -0.259079 & 0.000000 \\
\hline 2 & 1 & 0 & -2.168285 & 0.349722 & -0.000039 \\
3 & 1 & 0 & -1.296952 & -0.907759 & 0.881611 \\
4 & 1 & 0 & -1.296919 & -0.907821 & -0.881567 \\
5 & 6 & 0 & 0.000000 & 0.589828 & -0.000000 \\
6 & 1 & 0 & -0.000000 & 1.250859 & 0.875696 \\
7 & 1 & 0 & 0.000001 & 1.250838 & -0.875711 \\
8 & 6 & 0 & 1.259942 & -0.259079 & 0.000000 \\
9 & 1 & 0 & 1.296960 & -0.907746 & 0.881621 \\
10 & 1 & 0 & 1.296908 & -0.907834 & -0.881558 \\
11 & 1 & 0 & 2.168286 & 0.349720 & -0.000057
\end{tabular}

Zero-point correction=

Thermal correction to Energy=

0.103361 (Hartree/Particle)

Thermal correction to Enthalpy=

0.107910

Thermal correction to Gibbs Free Energy=

0.108854

Sum of electronic and zero-point Energies=

0.077704

$-118.859035$

Sum of electronic and thermal Energies=

$-118.854486$

Sum of electronic and thermal Enthalpies=

$-118.853542$

Sum of electronic and thermal Free Energies=

$-118.884692$

Butane:

Standard orientation:

\begin{tabular}{cccccc} 
Center & \multicolumn{2}{c}{ Atomic } & Atomic & \multicolumn{3}{c}{ Coordinates (Angstroms) } \\
Number & Number & Type & X & Y & Z \\
\hline-1 & 6 & 0 & 1.939639 & -0.117625 & 0.000051 \\
2 & 1 & 0 & 2.082570 & -0.751122 & -0.881692 \\
3 & 1 & 0 & 2.082481 & -0.751060 & 0.881852 \\
4 & 1 & 0 & 2.736557 & 0.630932 & 0.000065 \\
5 & 6 & 0 & 0.559249 & 0.514881 & -0.000057 \\
6 & 1 & 0 & 0.446420 & 1.168088 & 0.876254 \\
7 & 1 & 0 & 0.446500 & 1.168004 & -0.876441 \\
8 & 6 & 0 & -0.559249 & -0.514881 & -0.000057 \\
9 & 1 & 0 & -0.446500 & -1.168002 & -0.876442 \\
10 & 1 & 0 & -0.446420 & -1.168090 & 0.876252 \\
11 & 6 & 0 & -1.939639 & 0.117625 & 0.000051 \\
12 & 1 & 0 & -2.082567 & 0.751130 & -0.881686 \\
13 & 1 & 0 & -2.736557 & -0.630932 & 0.000055 \\
14 & 1 & 0 & -2.082484 & 0.751051 & 0.881857 \\
& & & & &
\end{tabular}


Zero-point correction=

Thermal correction to Energy=

Thermal correction to Enthalpy=

Thermal correction to Gibbs Free Energy=

Sum of electronic and zero-point Energies=

Sum of electronic and thermal Energies=

Sum of electronic and thermal Enthalpies=

Sum of electronic and thermal Free Energies=

Isobutane:

Standard orientation:
0.131823 (Hartree/Particle)

0.137649

0.138593
0.103762

$-158.087419$

$-158.081594$

$-158.080649$

$-158.115480$

\begin{tabular}{|c|c|c|c|c|}
\hline \multirow{2}{*}{$\begin{array}{l}\text { Center } \\
\text { Number }\end{array}$} & Atomic & \multirow{2}{*}{$\begin{array}{l}\text { Atomic } \\
\text { Type }\end{array}$} & \multicolumn{2}{|c|}{ Coordinates (Angstroms } \\
\hline & Number & & $\mathrm{X}$ & $\mathrm{Z}$ \\
\hline 1 & 6 & -1.435649 & 0.165481 & -0.095239 \\
\hline 2 & 1 & -1.871508 & 1.106526 & 0.254201 \\
\hline 3 & 1 & -1.476817 & 0.169543 & -1.191233 \\
\hline 4 & 1 & -2.074445 & -0.651031 & 0.255307 \\
\hline 5 & 6 & -0.000005 & -0.000006 & 0.379663 \\
\hline 6 & 1 & 0.000001 & 0.000016 & 1.481514 \\
\hline 7 & 6 & 0.861137 & 1.160544 & -0.095243 \\
\hline 8 & 1 & 1.893820 & 1.067979 & 0.254962 \\
\hline 9 & 1 & 0.885966 & 1.193651 & -1.191235 \\
\hline 10 & 1 & 0.472900 & 2.122074 & 0.254579 \\
\hline 11 & 6 & 0.574511 & -1.326033 & -0.095241 \\
\hline 12 & 1 & 1.601375 & -1.470504 & 0.254506 \\
\hline 13 & 1 & -0.021946 & -2.174097 & 0.254996 \\
\hline 14 & 1 & 0.590689 & -1.364071 & -1.191232 \\
\hline
\end{tabular}

Zero-point correction=

Thermal correction to Energy=

Thermal correction to Enthalpy=

Thermal correction to Gibbs Free Energy=

Sum of electronic and zero-point Energies=

Sum of electronic and thermal Energies $=$

Sum of electronic and thermal Enthalpies=

Sum of electronic and thermal Free Energies=

Pentane:
0.131404 (Hartree/Particle)

$$
0.137113
$$

0.138057

$$
\begin{gathered}
0.103797 \\
-158.090369 \\
-158.084659 \\
-158.083715 \\
-158.117975
\end{gathered}
$$

Standard orientation:

\begin{tabular}{cccccc} 
Center & \multicolumn{2}{c}{ Atomic } & \multicolumn{2}{c}{ Atomic } & \multicolumn{3}{c}{ Coordinates } \\
Number & Number & Type & X & Y & Z \\
- \hdashline 1 & 6 & 0 & 2.528279 & 0.325603 & -0.000001 \\
2 & 1 & 0 & 2.565371 & 0.973995 & -0.881643 \\
3 & 1 & 0 & 2.565361 & 0.974030 & 0.881615 \\
4 & 1 & 0 & 3.436884 & -0.282310 & 0.000016 \\
5 & 6 & 0 & 1.270277 & -0.524445 & 0.000008 \\
6 & 1 & 0 & 1.266609 & -1.186725 & -0.876465
\end{tabular}




$\begin{array}{cccccc}7 & 1 & 0 & 1.266607 & -1.186700 & 0.876500 \\ 8 & 6 & 0 & 0.000000 & 0.309074 & -0.000005 \\ 9 & 1 & 0 & -0.000006 & 0.973545 & 0.876932 \\ 10 & 1 & 0 & 0.000006 & 0.973535 & -0.876950 \\ 11 & 6 & 0 & -1.270277 & -0.524445 & -0.000009 \\ 12 & 1 & 0 & -1.266605 & -1.186720 & 0.876468 \\ 13 & 1 & 0 & -1.266611 & -1.186704 & -0.876497 \\ 14 & 6 & 0 & -2.528279 & 0.325603 & 0.000006 \\ 15 & 1 & 0 & -3.436884 & -0.282310 & 0.000002 \\ 16 & 1 & 0 & -2.565363 & 0.974003 & 0.881642 \\ 17 & 1 & 0 & -2.565369 & 0.974022 & -0.881616\end{array}$

Zero-point correction=

Thermal correction to Energy=

Thermal correction to Enthalpy=

Thermal correction to Gibbs Free Energy=

Sum of electronic and zero-point Energies=

Sum of electronic and thermal Energies=

Sum of electronic and thermal Enthalpies=

Sum of electronic and thermal Free Energies=

Neopentane:
0.160176 (Hartree/Particle)

$$
\begin{aligned}
& 0.167343 \\
& 0.168287
\end{aligned}
$$

$$
\begin{gathered}
0.129680 \\
-197.315826 \\
-197.308659 \\
-197.307714 \\
-197.346321
\end{gathered}
$$

\begin{tabular}{|c|c|c|c|c|}
\hline \multirow{2}{*}{$\begin{array}{l}\text { Center } \\
\text { Number }\end{array}$} & Atomic & Atomic & \multicolumn{2}{|c|}{ Coordinates (Angstroms) } \\
\hline & Numbe & Type & $X$ & $\mathrm{Z}$ \\
\hline 1 & 6 & -1.434305 & 0.518973 & 0.003540 \\
\hline 2 & 1 & -2.069456 & -0.079810 & 0.665286 \\
\hline 3 & 1 & -1.477753 & 1.557629 & 0.348770 \\
\hline 4 & 1 & -1.870149 & 0.482307 & -1.000729 \\
\hline 5 & 6 & -0.000006 & 0.000007 & -0.000028 \\
\hline 6 & 6 & 0.568485 & 0.067168 & 1.413773 \\
\hline 7 & 1 & 1.599893 & -0.300568 & 1.442700 \\
\hline 8 & 1 & 0.569934 & 1.095728 & 1.790516 \\
\hline 9 & 1 & -0.022673 & -0.541468 & 2.106551 \\
\hline 10 & 6 & 0.016442 & -1.445547 & -0.486446 \\
\hline 11 & 1 & -0.387031 & -1.525372 & -1.501682 \\
\hline 12 & 1 & 1.035825 & -1.846223 & -0.499700 \\
\hline 13 & 1 & -0.586609 & -2.088165 & 0.164123 \\
\hline 14 & 6 & 0.849370 & 0.859399 & -0.930861 \\
\hline 15 & 1 & 0.464290 & 0.830964 & -1.955935 \\
\hline 16 & 1 & 0.856684 & 1.905382 & -0.605716 \\
\hline 17 & 1 & 1.887132 & 0.509591 & -0.954056 \\
\hline Zero-poin & at correct & $n^{-1}$ & 0.15953 & 2 (Hartree/Particle) \\
\hline Thermal & correctior & to Energy= & 0.16 & 6348 \\
\hline Thermal & correctior & to Enthalpy= & 0.1 & 67292 \\
\hline Thermal & correctior & to Gibbs Free & ergy $=$ & 0.130562 \\
\hline Sum of e & lectronic & and zero-point & nergies $=$ & -197.323123 \\
\hline
\end{tabular}

Standard orientation: 
Sum of electronic and thermal Energies $=$

$-197.316308$

Sum of electronic and thermal Enthalpies=

Sum of electronic and thermal Free Energies=

Hexane:

Standard orientation:

\begin{tabular}{cccccc} 
Center & \multicolumn{2}{c}{ Atomic } & \multicolumn{2}{c}{ Atomic } & \multicolumn{3}{c}{ Coordinates } \\
Number & Number & Type & X & Y & $Z$ \\
-1 & 6 & 0 & -3.185715 & 0.207211 & 0.000020 \\
2 & 1 & 0 & -3.268791 & 0.851373 & 0.881653 \\
3 & 1 & 0 & -3.268784 & 0.851486 & -0.881532 \\
4 & 1 & 0 & -4.048834 & -0.463739 & -0.000027 \\
5 & 6 & 0 & -1.870384 & -0.550954 & -0.000025 \\
6 & 1 & 0 & -1.819310 & -1.211545 & 0.876333 \\
7 & 1 & 0 & -1.819327 & -1.211468 & -0.876442 \\
8 & 6 & 0 & -0.662432 & 0.370768 & 0.000003 \\
9 & 1 & 0 & -0.710523 & 1.033168 & -0.877014 \\
10 & 1 & 0 & -0.710537 & 1.033138 & 0.877041 \\
11 & 6 & 0 & 0.662432 & -0.370768 & 0.000003 \\
12 & 1 & 0 & 0.710522 & -1.033170 & -0.877012 \\
13 & 1 & 0 & 0.710537 & -1.033136 & 0.877043 \\
14 & 6 & 0 & 1.870384 & 0.550954 & -0.000025 \\
15 & 1 & 0 & 1.819310 & 1.211544 & 0.876333 \\
16 & 1 & 0 & 1.819327 & 1.211469 & -0.876441 \\
17 & 6 & 0 & 3.185715 & -0.207211 & 0.000020 \\
18 & 1 & 0 & 3.268789 & -0.851478 & -0.881538 \\
19 & 1 & 0 & 4.048834 & 0.463739 & -0.000016 \\
20 & 1 & 0 & 3.268786 & -0.851382 & 0.881647
\end{tabular}

Zero-point correction=

Thermal correction to Energy=

Thermal correction to Enthalpy=

Thermal correction to Gibbs Free Energy=

Sum of electronic and zero-point Energies=

Sum of electronic and thermal Energies $=$

Sum of electronic and thermal Enthalpies=

Sum of electronic and thermal Free Energies=

2,2-dimethylbutane
0.188533 (Hartree/Particle)

$$
\begin{gathered}
0.197064 \\
0.198008
\end{gathered}
$$

\subsection{6}

$-236.544267$

$-236.535735$

$-236.534791$

$-236.577153$

\begin{tabular}{|c|c|c|c|c|}
\hline \multirow{2}{*}{$\begin{array}{l}\text { Center } \\
\text { Number }\end{array}$} & \multirow{2}{*}{$\begin{array}{l}\text { Atomic } \\
\text { Number }\end{array}$} & \multirow{2}{*}{$\begin{array}{l}\text { Atomic } \\
\text { Type }\end{array}$} & \multicolumn{2}{|c|}{ Coordinates (Angstroms) } \\
\hline & & & $\mathrm{X}$ & $\mathrm{Z}$ \\
\hline 1 & 6 & -1.609030 & 0.969744 & 0.000868 \\
\hline 2 & 1 & -1.589875 & 1.614629 & 0.886214 \\
\hline 3 & 1 & -1.590008 & 1.615974 & -0.883499 \\
\hline 4 & 1 & -2.562939 & 0.431767 & 0.000532 \\
\hline 5 & 6 & -0.430452 & -0.000147 & 0.000016 \\
\hline 6 & 6 & 0.864115 & 0.826044 & 0.000526 \\
\hline
\end{tabular}

Standard orientation: 


$\begin{array}{cccccc}7 & 1 & 0 & 0.843255 & 1.488539 & -0.876455 \\ 8 & 1 & 0 & 0.843335 & 1.487353 & 0.878408 \\ 9 & 6 & 0 & 2.163406 & 0.038521 & -0.000017 \\ 10 & 1 & 0 & 2.254939 & -0.600339 & -0.883554 \\ 11 & 1 & 0 & 3.025911 & 0.710495 & 0.000078 \\ 12 & 1 & 0 & 2.255232 & -0.600977 & 0.883023 \\ 13 & 6 & 0 & -0.513133 & -0.877882 & 1.245857 \\ 14 & 1 & 0 & 0.271978 & -1.639828 & 1.263351 \\ 15 & 1 & 0 & -0.421075 & -0.277995 & 2.158501 \\ 16 & 1 & 0 & -1.474699 & -1.400674 & 1.286955 \\ 17 & 6 & 0 & -0.513422 & -0.875921 & -1.247195 \\ 18 & 1 & 0 & -1.474984 & -1.398684 & -1.288837 \\ 19 & 1 & 0 & -0.421660 & -0.274580 & -2.158908 \\ 20 & 1 & 0 & 0.271688 & -1.637832 & -1.266147\end{array}$

Zero-point correction= 0.187923 (Hartree/Particle)

Thermal correction to Energy= 0.196165

Thermal correction to Enthalpy= 0.197110

Thermal correction to Gibbs Free Energy=

Sum of electronic and zero-point Energies=

0.156409

Sum of electronic and thermal Energies $=$ $-236.550462$

$-236.542220$

Sum of electronic and thermal Enthalpies= $-236.541276$

Sum of electronic and thermal Free Energies $=\quad-236.581976$ Heptane

Standard orientation:

\begin{tabular}{|c|c|c|c|c|}
\hline \multirow{2}{*}{$\begin{array}{l}\text { Center } \\
\text { Number }\end{array}$} & Atomic & \multirow{2}{*}{$\begin{array}{l}\text { Atomic } \\
\text { Type }\end{array}$} & \multicolumn{2}{|c|}{ Coordinates (Angstroms } \\
\hline & Numbe & & $\mathrm{X}$ & Z \\
\hline 1 & 6 & -3.772213 & -0.367911 & -0.001015 \\
\hline 2 & 1 & -3.771596 & -0.984713 & -0.875345 \\
\hline 3 & 1 & -3.772831 & -0.986636 & 0.871956 \\
\hline 4 & 1 & -4.645865 & 0.249854 & -0.000952 \\
\hline 5 & 6 & -2.514809 & 0.521207 & 0.000852 \\
\hline 6 & 1 & -2.514191 & 1.139931 & -0.872120 \\
\hline 7 & 1 & -2.515428 & 1.138012 & 0.875182 \\
\hline 8 & 6 & -1.257404 & -0.367912 & 0.000766 \\
\hline 9 & 1 & -1.257044 & -0.985252 & 0.874718 \\
\hline 10 & 1 & -1.257765 & -0.986102 & -0.872585 \\
\hline 11 & 6 & -0.000000 & 0.521208 & -0.000186 \\
\hline 12 & 1 & 0.000136 & 1.139713 & 0.872941 \\
\hline 13 & 1 & -0.000138 & 1.138231 & -0.874361 \\
\hline 14 & 6 & 1.257405 & -0.367912 & 0.000371 \\
\hline 15 & 1 & 1.257258 & -0.986431 & -0.872747 \\
\hline 16 & 1 & 1.257552 & -0.984922 & 0.874555 \\
\hline 17 & 6 & 2.514809 & 0.521208 & -0.000608 \\
\hline 18 & 1 & 2.514953 & 1.139729 & 0.872508 \\
\hline 19 & 1 & 2.514664 & 1.138216 & -0.874794 \\
\hline 20 & 6 & 3.772214 & -0.367911 & -0.000047 \\
\hline 21 & 1 & 3.772072 & -0.986427 & -0.873167 \\
\hline
\end{tabular}




$\begin{array}{rrrrr}1 & 0 & 4.645865 & 0.249854 & -0.000720 \\ 1 & 0 & 3.772356 & -0.984924 & 0.874135\end{array}$

Zero-point correction $=$

Thermal correction to Energy=

0.216854 (Hartree/Particle)

Thermal correction to Enthalpy=

0.226758

0.227702

Thermal correction to Gibbs Free Energy=

0.181541

Sum of electronic and zero-point Energies=

$-275.772761$

Sum of electronic and thermal Energies $=$

$-275.762857$

Sum of electronic and thermal Enthalpies=

$-275.761913$

Sum of electronic and thermal Free Energies=

$-275.808074$

2,2,3-trimethylbutane

Standard orientation:

\begin{tabular}{|c|c|c|c|c|}
\hline \multirow{2}{*}{$\begin{array}{l}\text { Center } \\
\text { Number }\end{array}$} & Atomic & \multirow{2}{*}{$\begin{array}{l}\text { Atomic } \\
\text { Type }\end{array}$} & \multicolumn{2}{|c|}{ Coordinates (Angstroms } \\
\hline & Numbe & & $\mathrm{X}$ & Z \\
\hline 1 & 6 & -1.302560 & -1.319488 & -0.393350 \\
\hline 2 & 1 & -1.144802 & -1.542994 & -1.455066 \\
\hline 3 & 1 & -0.922511 & -2.163361 & 0.189284 \\
\hline 4 & 1 & -2.382767 & -1.267742 & -0.222476 \\
\hline 5 & 6 & -0.637726 & -0.000289 & -0.000871 \\
\hline 6 & 6 & 0.845738 & -0.001701 & -0.453684 \\
\hline 7 & 1 & 0.815222 & -0.009304 & -1.557423 \\
\hline 8 & 6 & 1.616179 & 1.241829 & -0.023555 \\
\hline 9 & 1 & 1.779049 & 1.249638 & 1.059777 \\
\hline 10 & 1 & 2.603255 & 1.259683 & -0.494829 \\
\hline 11 & 1 & 1.112094 & 2.173558 & -0.292955 \\
\hline 12 & 6 & 1.624104 & -1.237814 & -0.016130 \\
\hline 13 & 1 & 1.631593 & -1.341725 & 1.074903 \\
\hline 14 & 1 & 1.224199 & -2.162645 & -0.438099 \\
\hline 15 & 1 & 2.667792 & -1.160769 & -0.335358 \\
\hline 16 & 6 & -0.777418 & 0.191932 & 1.508059 \\
\hline 17 & 1 & -0.421330 & 1.175253 & 1.830080 \\
\hline 18 & 1 & -1.829866 & 0.115147 & 1.801308 \\
\hline 19 & 1 & -0.227638 & -0.568414 & 2.072462 \\
\hline 20 & 6 & -1.389222 & 1.125731 & -0.711249 \\
\hline 21 & 1 & -1.325581 & 1.017071 & -1.799913 \\
\hline 22 & 1 & -2.449441 & 1.110502 & -0.437823 \\
\hline 23 & 1 & -1.003835 & 2.114898 & -0.449186 \\
\hline
\end{tabular}

Zero-point correction=

Thermal correction to Energy=

0.216252 (Hartree/Particle)

Thermal correction to Enthalpy=

0.225635

0.226579

Thermal correction to Gibbs Free Energy=

0.183491

Sum of electronic and zero-point Energies=

$-275.778668$

Sum of electronic and thermal Energies=

$-275.769285$

Sum of electronic and thermal Enthalpies=

$-275.768341$

Sum of electronic and thermal Free Energies=

$-275.811430$

Octane 
Standard orientation:

\begin{tabular}{|c|c|c|c|c|c|}
\hline \multirow{2}{*}{$\begin{array}{l}\text { Center } \\
\text { Number }\end{array}$} & Atomic & \multirow{2}{*}{\multicolumn{2}{|c|}{$\begin{array}{l}\text { Atomic } \\
\text { Type }\end{array}$}} & \multicolumn{2}{|c|}{ Coordinates (Angstroms } \\
\hline & Numbe & & & $\mathrm{X}$ & $\mathrm{Z}$ \\
\hline 1 & 6 & 0 & -4.445653 & 0.256371 & 0.000044 \\
\hline 2 & 1 & 0 & -4.508254 & 0.902914 & 0.881704 \\
\hline 3 & 1 & 0 & -4.508260 & 0.903081 & -0.881494 \\
\hline 4 & 1 & 0 & -5.329566 & -0.386931 & -0.000014 \\
\hline 5 & 6 & 0 & -3.154828 & -0.542750 & -0.000038 \\
\hline 6 & 1 & 0 & -3.124360 & -1.204755 & 0.876310 \\
\hline 7 & 1 & 0 & -3.124391 & -1.204625 & -0.876485 \\
\hline 8 & 6 & 0 & -1.918519 & 0.340396 & 0.000005 \\
\hline 9 & 1 & 0 & -1.945643 & 1.004272 & -0.876942 \\
\hline 10 & 1 & 0 & -1.945651 & 1.004202 & 0.877004 \\
\hline 11 & 6 & 0 & -0.617328 & -0.441969 & -0.000015 \\
\hline 12 & 1 & 0 & -0.590629 & -1.105369 & -0.877138 \\
\hline 13 & 1 & 0 & -0.590606 & -1.105369 & 0.877105 \\
\hline 14 & 6 & 0 & 0.617328 & 0.441969 & -0.000029 \\
\hline 15 & 1 & 0 & 0.590606 & 1.105398 & 0.877070 \\
\hline 16 & 1 & 0 & 0.590629 & 1.105340 & -0.877173 \\
\hline 17 & 6 & 0 & 1.918519 & -0.340396 & 0.000014 \\
\hline 18 & 1 & 0 & 1.945650 & -1.004282 & -0.876925 \\
\hline 19 & 1 & 0 & 1.945644 & -1.004193 & 0.877020 \\
\hline 20 & 6 & 0 & 3.154828 & 0.542750 & -0.000026 \\
\hline 21 & 1 & 0 & 3.124366 & 1.204741 & 0.876333 \\
\hline 22 & 1 & 0 & 3.124384 & 1.204639 & -0.876462 \\
\hline 23 & 6 & 0 & 4.445653 & -0.256371 & 0.000034 \\
\hline 24 & 1 & 0 & 4.508266 & -0.903050 & -0.881526 \\
\hline 25 & 1 & 0 & 5.329566 & 0.386931 & 0.000005 \\
\hline 26 & 1 & 0 & 4.508248 & -0.902945 & 0.881673 \\
\hline
\end{tabular}

Zero-point correction=

Thermal correction to Energy=

0.245190 (Hartree/Particle)

Thermal correction to Enthalpy=

0.256473

0.257417

Thermal correction to Gibbs Free Energy=

Sum of electronic and zero-point Energies $=$

0.207444

$-315.001245$

Sum of electronic and thermal Energies $=\quad-314.989962$

Sum of electronic and thermal Enthalpies $=\quad-314.989018$

Sum of electronic and thermal Free Energies $=\quad-315.038991$

2,2,3,3-tetramethylbutane

Standard orientation:

\begin{tabular}{|c|c|c|c|c|}
\hline \multirow{2}{*}{$\begin{array}{l}\text { Center } \\
\text { Number }\end{array}$} & Atomic & \multirow{2}{*}{$\begin{array}{l}\text { Atomic } \\
\text { Type }\end{array}$} & \multicolumn{2}{|c|}{ Coordinates (Angstroms) } \\
\hline & Number & & $\mathrm{X}$ & Z \\
\hline 1 & 6 & -1.342446 & -0.190964 & -1.413090 \\
\hline 2 & 1 & -0.928620 & 0.528494 & -2.126733 \\
\hline 3 & 1 & -1.151728 & -1.196547 & -1.798566 \\
\hline 4 & 1 & -2.428100 & -0.048631 & -1.405001 \\
\hline
\end{tabular}




$\begin{array}{cccccc}5 & 6 & 0 & -0.785134 & 0.000265 & 0.000096 \\ 6 & 6 & 0 & 0.785091 & 0.000214 & -0.000224 \\ 7 & 6 & 0 & 1.342159 & -0.124579 & 1.420463 \\ 8 & 1 & 0 & 0.927835 & 0.627244 & 2.099570 \\ 9 & 1 & 0 & 1.151691 & -1.111157 & 1.852456 \\ 10 & 1 & 0 & 2.427738 & 0.017679 & 1.405892 \\ 11 & 6 & 0 & -1.341855 & 1.319702 & 0.541684 \\ 12 & 1 & 0 & -0.925462 & 1.578922 & 1.520280 \\ 13 & 1 & 0 & -1.153471 & 2.155999 & -0.137478 \\ 14 & 1 & 0 & -2.427094 & 1.240873 & 0.663906 \\ 15 & 6 & 0 & -1.341191 & -1.128542 & 0.872262 \\ 16 & 1 & 0 & -2.426812 & -1.193604 & 0.745293 \\ 17 & 1 & 0 & -0.926421 & -2.105784 & 0.605422 \\ 18 & 1 & 0 & -1.150097 & -0.959854 & 1.935879 \\ 19 & 6 & 0 & 1.342245 & 1.292692 & -0.603000 \\ 20 & 1 & 0 & 2.427802 & 1.208834 & -0.719122 \\ 21 & 1 & 0 & 0.927804 & 1.504675 & -1.593648 \\ 22 & 1 & 0 & 1.151958 & 2.160278 & 0.035156 \\ 23 & 6 & 0 & 1.341091 & -1.168440 & -0.818279 \\ 24 & 1 & 0 & 2.426644 & -1.227872 & -0.687929 \\ 25 & 1 & 0 & 0.925942 & -2.131956 & -0.506149 \\ 26 & 1 & 0 & 1.150632 & -1.049687 & -1.888698\end{array}$

Zero-point correction $=$

Thermal correction to Energy=

0.244762 (Hartree/Particle)

Thermal correction to Enthalpy=

0.255023

0.255967

Thermal correction to Gibbs Free Energy=

0.211677

Sum of electronic and zero-point Energies=

$-315.007160$

Sum of electronic and thermal Energies=

$-314.996899$

Sum of electronic and thermal Enthalpies=

$-314.995955$

Sum of electronic and thermal Free Energies=

$-315.040246$

Basis set: DH-SVPD

Functional: TPSSH

Ethane:

Standard orientation:

\begin{tabular}{cccccc} 
Center & \multicolumn{2}{c}{ Atomic } & \multicolumn{2}{c}{ Atomic } & \multicolumn{2}{c}{ Coordinates (Angstrom } \\
Number & Number & Type & \multicolumn{1}{c}{ X } & Y & Z \\
- \hdashline 1 & 6 & 0 & -0.000000 & -0.000000 & 0.765784 \\
2 & 1 & 0 & -0.510308 & 0.883999 & 1.163626 \\
3 & 1 & 0 & 1.020719 & -0.000060 & 1.163626 \\
4 & 1 & 0 & -0.510412 & -0.883939 & 1.163626 \\
5 & 6 & 0 & 0.000000 & -0.000000 & -0.765784 \\
6 & 1 & 0 & 0.510308 & 0.883999 & -1.163626 \\
7 & 1 & 0 & 0.510412 & -0.883939 & -1.163626 \\
8 & 1 & 0 & -1.020719 & -0.000060 & -1.163626
\end{tabular}

Zero-point correction $=$

0.074774 (Hartree/Particle)

Thermal correction to Energy=

0.078272 
Thermal correction to Enthalpy=

0.079216

Thermal correction to Gibbs Free Energy=

0.053334

Sum of electronic and zero-point Energies=

$-79.712113$

Sum of electronic and thermal Energies $=$

$-79.708615$

Sum of electronic and thermal Enthalpies=

$-79.707671$

Sum of electronic and thermal Free Energies=

$-79.733553$

Methane:

Standard orientation:

\begin{tabular}{|c|c|c|c|c|}
\hline \multirow{2}{*}{$\begin{array}{l}\text { Center } \\
\text { Number }\end{array}$} & Atomic & \multirow{2}{*}{$\begin{array}{r}\text { Atomic } \\
\text { Type }\end{array}$} & \multicolumn{2}{|c|}{ Coordinates (Angstro } \\
\hline & Numbe & & $\mathrm{X}$ & Z \\
\hline 1 & 6 & 0.000000 & 0.000000 & 0.000000 \\
\hline 2 & 1 & 0.630717 & 0.630717 & 0.630717 \\
\hline 3 & 1 & -0.630717 & -0.630717 & 0.630717 \\
\hline 4 & 1 & -0.630717 & 0.630717 & -0.630717 \\
\hline 5 & 1 & 0.630717 & -0.630717 & -0.630717 \\
\hline
\end{tabular}

Zero-point correction=

Thermal correction to Energy=

0.044974 (Hartree/Particle)

Thermal correction to Enthalpy=

0.047841

Thermal correction to Gibbs Free Energy $=\quad 0.027653$

Sum of electronic and zero-point Energies=

$-40.451412$

Sum of electronic and thermal Energies=

$-40.448546$

Sum of electronic and thermal Enthalpies=

$-40.447601$

Sum of electronic and thermal Free Energies $=\quad-40.468734$

Propane:

Standard orientation:

\begin{tabular}{|c|c|c|c|c|}
\hline \multirow{2}{*}{$\begin{array}{l}\text { Center } \\
\text { Number }\end{array}$} & Atomic & \multirow{2}{*}{$\begin{array}{l}\text { Atomic } \\
\text { Type }\end{array}$} & \multicolumn{2}{|c|}{ Coordinates (Angstroms) } \\
\hline & Number & & $\mathrm{X}$ & $\mathrm{Z}$ \\
\hline 1 & 6 & -1.275617 & -0.260482 & 0.000000 \\
\hline 2 & 1 & -2.175224 & 0.364946 & -0.000008 \\
\hline 3 & 1 & -1.318075 & -0.907001 & 0.884658 \\
\hline 4 & 1 & -1.318070 & -0.907016 & -0.884648 \\
\hline 5 & 6 & 0.000000 & 0.588375 & -0.000000 \\
\hline 6 & 1 & -0.000000 & 1.246844 & 0.878826 \\
\hline 7 & 1 & 0.000001 & 1.246832 & -0.878834 \\
\hline 8 & 6 & 1.275618 & -0.260482 & 0.000000 \\
\hline 9 & 1 & 1.318103 & -0.906957 & 0.884689 \\
\hline 10 & 1 & 1.318038 & -0.907060 & -0.884617 \\
\hline 11 & 1 & 2.175226 & 0.364944 & -0.000070 \\
\hline \multicolumn{3}{|c|}{ Zero-point correction $=$} & \multicolumn{2}{|c|}{0.103411 (Hartree/Particle) } \\
\hline \multicolumn{3}{|c|}{ Thermal correction to Energy= } & \multicolumn{2}{|c|}{0.107976} \\
\hline \multicolumn{3}{|c|}{ Thermal correction to Enthalpy= } & \multicolumn{2}{|c|}{0.108921} \\
\hline \multicolumn{4}{|c|}{ Thermal correction to Gibbs Free Energy= } & 0.077710 \\
\hline \multicolumn{5}{|c|}{ Sum of electronic and zero-point Energies $=$} \\
\hline \multicolumn{5}{|c|}{ Sum of electronic and thermal Energies $=$} \\
\hline
\end{tabular}


Sum of electronic and thermal Enthalpies=

$-118.970543$

Sum of electronic and thermal Free Energies=

Butane:

Standard orientation:

\begin{tabular}{cccccc} 
Center & \multicolumn{2}{c}{ Atomic } & \multicolumn{2}{c}{ Atomic } & \multicolumn{2}{c}{ Coordinates } \\
Number & Number & Type & X & Y & $Z$ \\
\hline 1 & 6 & 0 & 1.960329 & -0.120907 & 0.000036 \\
2 & 1 & 0 & 2.105500 & -0.752166 & -0.884656 \\
3 & 1 & 0 & 2.105446 & -0.752087 & 0.884793 \\
4 & 1 & 0 & 2.749222 & 0.639314 & 0.000025 \\
5 & 6 & 0 & 0.566962 & 0.516092 & -0.000040 \\
6 & 1 & 0 & 0.460934 & 1.167176 & 0.879201 \\
7 & 1 & 0 & 0.460990 & 1.167113 & -0.879336 \\
8 & 6 & 0 & -0.566962 & -0.516092 & -0.000041 \\
9 & 1 & 0 & -0.460990 & -1.167112 & -0.879337 \\
10 & 1 & 0 & -0.460934 & -1.167178 & 0.879200 \\
11 & 6 & 0 & -1.960329 & 0.120907 & 0.000036 \\
12 & 1 & 0 & -2.105494 & 0.752180 & -0.884648 \\
13 & 1 & 0 & -2.749222 & -0.639314 & 0.000009 \\
14 & 1 & 0 & -2.105451 & 0.752074 & 0.884802
\end{tabular}

Zero-point correction=

0.131826 (Hartree/Particle)

Thermal correction to Energy=

0.137676

Thermal correction to Enthalpy=

0.138621

Thermal correction to Gibbs Free Energy=

Sum of electronic and zero-point Energies $=$

0.103709

Sum of electronic and thermal Energies $=$

$-158.240076$

$-158.234227$

Sum of electronic and thermal Enthalpies=

$-158.233282$

Sum of electronic and thermal Free Energies= $-158.268194$

Isobutane:

Standard orientation:

\begin{tabular}{|c|c|c|c|c|}
\hline \multirow{2}{*}{$\begin{array}{l}\text { Center } \\
\text { Number }\end{array}$} & Atomic & \multirow{2}{*}{$\begin{array}{r}\text { Atomic } \\
\text { Type }\end{array}$} & \multicolumn{2}{|c|}{ Coordinates (Angstroms } \\
\hline & Numbe & & $\mathrm{X}$ & Z \\
\hline 1 & 6 & -1.178797 & -0.863070 & -0.096032 \\
\hline 2 & 1 & -2.133732 & -0.463782 & 0.264438 \\
\hline 3 & 1 & -1.222759 & -0.895276 & -1.192144 \\
\hline 4 & 1 & -1.086574 & -1.893994 & 0.264456 \\
\hline 5 & 6 & -0.000006 & 0.000004 & 0.373843 \\
\hline 6 & 1 & -0.000010 & 0.000013 & 1.475313 \\
\hline 7 & 6 & -0.158050 & 1.452388 & -0.096038 \\
\hline 8 & 1 & 0.665016 & 2.079842 & 0.264706 \\
\hline 9 & 1 & -0.163600 & 1.506542 & -1.192146 \\
\hline 10 & 1 & -1.097135 & 1.887881 & 0.264149 \\
\hline 11 & 6 & 1.336848 & -0.589319 & -0.096029 \\
\hline 12 & 1 & 2.183524 & 0.006056 & 0.264433 \\
\hline 13 & 1 & 1.468577 & -1.615944 & 0.264472 \\
\hline
\end{tabular}


Zero-point correction $=$

Thermal correction to Energy=

Thermal correction to Enthalpy=

Thermal correction to Gibbs Free Energy=

Sum of electronic and zero-point Energies=

Sum of electronic and thermal Energies $=$

Sum of electronic and thermal Enthalpies=

Sum of electronic and thermal Free Energies=

Pentane:

Standard orientation:
0.131378 (Hartree/Particle)

0.137167

0.138111

0.103662

$-158.241584$

$-158.235795$

$-158.234851$

$-158.269300$

\begin{tabular}{|c|c|c|c|c|}
\hline \multirow{2}{*}{$\begin{array}{l}\text { Center } \\
\text { Number }\end{array}$} & Atomic & \multirow{2}{*}{$\begin{array}{l}\text { Atomic } \\
\text { Type }\end{array}$} & \multicolumn{2}{|c|}{ Coordinates (Angstrom } \\
\hline & Number & & $\mathrm{X}$ & Z \\
\hline 1 & 6 & 2.556962 & 0.325935 & -0.000005 \\
\hline 2 & 1 & 2.599195 & 0.972313 & -0.884724 \\
\hline 3 & 1 & 2.599188 & 0.972365 & 0.884677 \\
\hline 4 & 1 & 3.457142 & -0.298564 & 0.000016 \\
\hline 5 & 6 & 1.282935 & -0.525198 & 0.000015 \\
\hline 6 & 1 & 1.282860 & -1.184601 & -0.879261 \\
\hline 7 & 1 & 1.282859 & -1.184557 & 0.879325 \\
\hline 8 & 6 & 0.000000 & 0.314449 & -0.000003 \\
\hline 9 & 1 & -0.000011 & 0.975281 & 0.879610 \\
\hline 10 & 1 & 0.000011 & 0.975272 & -0.879622 \\
\hline 11 & 6 & -1.282935 & -0.525198 & -0.000016 \\
\hline 12 & 1 & -1.282857 & -1.184601 & 0.879261 \\
\hline 13 & 1 & -1.282862 & -1.184557 & -0.879324 \\
\hline 14 & 6 & -2.556962 & 0.325935 & 0.000009 \\
\hline 15 & 1 & -3.457142 & -0.298565 & -0.000076 \\
\hline 16 & 1 & -2.599222 & 0.972264 & 0.884761 \\
\hline 17 & 1 & -2.599161 & 0.972414 & -0.884640 \\
\hline
\end{tabular}

Zero-point correction $=$

Thermal correction to Energy=

0.160243 (Hartree/Particle)

Thermal correction to Enthalpy=

0.167417

Thermal correction to Gibbs Free Energy=

0.168361

Sum of electronic and zero-point Energies=

0.129765

Sum of electronic and thermal Energies=

$-197.504057$

$-197.496884$

Sum of electronic and thermal Enthalpies=

$-197.495940$

Sum of electronic and thermal Free Energies= $-197.534536$

Neopentane:

Standard orientation:

\begin{tabular}{cccccc}
$\begin{array}{l}\text { Center } \\
\text { Number }\end{array}$ & Atomic & \multicolumn{2}{c}{ Atomic } & \multicolumn{2}{c}{ Coordinates (Angstroms) } \\
- & Tyme & X & Y & Z \\
\hline 1 & 6 & 0 & -0.503128 & 1.170994 & -0.863151 \\
2 & 1 & 0 & -1.265341 & 0.835040 & -1.576266
\end{tabular}




$\begin{array}{cccccc}3 & 1 & 0 & -0.947912 & 1.956264 & -0.240330 \\ 4 & 1 & 0 & 0.318031 & 1.619630 & -1.434798 \\ 5 & 6 & 0 & -0.000003 & -0.000011 & 0.000017 \\ 6 & 6 & 0 & -1.179078 & -0.602208 & 0.785241 \\ 7 & 1 & 0 & -0.848559 & -1.440458 & 1.410115 \\ 8 & 1 & 0 & -1.637794 & 0.146608 & 1.441904 \\ 9 & 1 & 0 & -1.955068 & -0.974477 & 0.105804 \\ 10 & 6 & 0 & 0.616210 & -1.079260 & -0.908239 \\ 11 & 1 & 0 & 1.460517 & -0.676821 & -1.480580 \\ 12 & 1 & 0 & 0.983458 & -1.927298 & -0.318062 \\ 13 & 1 & 0 & -0.122875 & -1.461317 & -1.622480 \\ 14 & 6 & 0 & 1.066005 & 0.510472 & 0.986159 \\ 15 & 1 & 0 & 1.919524 & 0.945217 & 0.452431 \\ 16 & 1 & 0 & 0.653513 & 1.282459 & 1.646647 \\ 17 & 1 & 0 & 1.442473 & -0.304773 & 1.615452\end{array}$

Zero-point correction=

Thermal correction to Energy=

Thermal correction to Enthalpy=

Thermal correction to Gibbs Free Energy=

Sum of electronic and zero-point Energies=

Sum of electronic and thermal Energies $=$

Sum of electronic and thermal Enthalpies=

Sum of electronic and thermal Free Energies=
0.159036 (Hartree/Particle)

0.166104

0.167048

0.129772

$-197.507020$

$-197.499953$

$-197.499009$

$-197.536284$

Hexane:

Standard orientation:

\begin{tabular}{|c|c|c|c|c|}
\hline \multirow{2}{*}{$\begin{array}{l}\text { Center } \\
\text { Number }\end{array}$} & Atomic & \multirow{2}{*}{$\begin{array}{l}\text { Atomic } \\
\text { Type }\end{array}$} & \multicolumn{2}{|c|}{ Coordinates (Angstroms) } \\
\hline & Numbe & & $\mathrm{X}$ & Z \\
\hline 1 & 6 & -3.220319 & 0.209165 & 0.000051 \\
\hline 2 & 1 & -3.307276 & 0.851006 & 0.884845 \\
\hline 3 & 1 & -3.307288 & 0.851255 & -0.884561 \\
\hline 4 & 1 & -4.075069 & -0.476228 & -0.000042 \\
\hline 5 & 6 & -1.890271 & -0.551364 & -0.000064 \\
\hline 6 & 1 & -1.844285 & -1.209388 & 0.879114 \\
\hline 7 & 1 & -1.844330 & -1.209210 & -0.879378 \\
\hline 8 & 6 & -0.668464 & 0.375167 & -0.000007 \\
\hline 9 & 1 & -0.714879 & 1.034164 & -0.879618 \\
\hline 10 & 1 & -0.714925 & 1.034129 & 0.879627 \\
\hline 11 & 6 & 0.668464 & -0.375167 & 0.000020 \\
\hline 12 & 1 & 0.714894 & -1.034201 & -0.879563 \\
\hline 13 & 1 & 0.714910 & -1.034092 & 0.879683 \\
\hline 14 & 6 & 1.890271 & 0.551364 & -0.000050 \\
\hline 15 & 1 & 1.844297 & 1.209383 & 0.879133 \\
\hline 16 & 1 & 1.844319 & 1.209215 & -0.879359 \\
\hline 17 & 6 & 3.220319 & -0.209165 & 0.000039 \\
\hline 18 & 1 & 3.307289 & -0.851222 & -0.884597 \\
\hline 19 & 1 & 4.075069 & 0.476228 & -0.000026 \\
\hline 20 & 1 & 3.307275 & -0.851039 & 0.884809 \\
\hline
\end{tabular}


Zero-point correction=

Thermal correction to Energy=

Thermal correction to Enthalpy=

Thermal correction to Gibbs Free Energy=

Sum of electronic and zero-point Energies=

Sum of electronic and thermal Energies=

Sum of electronic and thermal Enthalpies=

Sum of electronic and thermal Free Energies=

2,2-dimethylbutane

Standard orientation:
0.188599 (Hartree/Particle)

0.197130

0.198074

$$
\begin{aligned}
& 0.155787 \\
& -236.768122 \\
& -236.759591 \\
& -236.758646 \\
& -236.800934
\end{aligned}
$$

\begin{tabular}{|c|c|c|c|c|}
\hline \multirow{2}{*}{$\begin{array}{l}\text { Center } \\
\text { Number }\end{array}$} & Atomic & \multirow{2}{*}{$\begin{array}{r}\text { Atomic } \\
\text { Type }\end{array}$} & \multicolumn{2}{|c|}{ Coordinates (Angstrom } \\
\hline & Numbe & & $X$ & $\mathrm{Z}$ \\
\hline 1 & 6 & -1.620430 & 0.983303 & 0.001742 \\
\hline 2 & 1 & -1.596205 & 1.627077 & 0.889119 \\
\hline 3 & 1 & -1.596389 & 1.629959 & -0.883543 \\
\hline 4 & 1 & -2.576688 & 0.446953 & 0.000966 \\
\hline 5 & 6 & -0.435428 & -0.000497 & 0.000016 \\
\hline 6 & 6 & 0.873782 & 0.828988 & 0.000964 \\
\hline 7 & 1 & 0.856791 & 1.489977 & -0.877346 \\
\hline 8 & 1 & 0.856872 & 1.487899 & 0.880839 \\
\hline 9 & 6 & 2.186060 & 0.036262 & 0.000021 \\
\hline 10 & 1 & 2.276995 & -0.601290 & -0.885929 \\
\hline 11 & 1 & 3.042806 & 0.718976 & 0.000352 \\
\hline 12 & 1 & 2.277376 & -0.602652 & 0.884943 \\
\hline 13 & 6 & -0.521865 & -0.886622 & 1.256262 \\
\hline 14 & 1 & 0.273856 & -1.639065 & 1.277422 \\
\hline 15 & 1 & -0.442669 & -0.283961 & 2.169340 \\
\hline 16 & 1 & -1.480003 & -1.418628 & 1.287567 \\
\hline 17 & 6 & -0.522421 & -0.882780 & -1.258905 \\
\hline 18 & 1 & -1.480423 & -1.414973 & -1.291233 \\
\hline 19 & 1 & -0.443999 & -0.277262 & -2.170155 \\
\hline 20 & 1 & 0.273493 & -1.634934 & -1.282938 \\
\hline
\end{tabular}

Zero-point correction=

Thermal correction to Energy=

Thermal correction to Enthalpy=

Thermal correction to Gibbs Free Energy=

Sum of electronic and zero-point Energies=

Sum of electronic and thermal Energies=

Sum of electronic and thermal Enthalpies=

0.187443 (Hartree/Particle)

0.195883

\begin{tabular}{|c|c|c|c|}
\hline Cent & Atomic & Atomic & Coordinates (Angstrom \\
\hline Number & Number & Type & $\mathrm{X}$ \\
\hline
\end{tabular}

0.196827

Sum of electronic and thermal Free Energies $=\quad-236.800030$

Heptane:

Standard orientation: 


$\begin{array}{cccccc}1 & 6 & 0 & -3.838311 & -0.355592 & -0.000008 \\ 2 & 1 & 0 & -3.880427 & -1.001983 & -0.884649 \\ 3 & 1 & 0 & -3.880418 & -1.002051 & 0.884583 \\ 4 & 1 & 0 & -4.738411 & 0.268726 & 0.000021 \\ 5 & 6 & 0 & -2.564602 & 0.495812 & 0.000017 \\ 6 & 1 & 0 & -2.564380 & 1.155279 & -0.879185 \\ 7 & 1 & 0 & -2.564380 & 1.155228 & 0.879256 \\ 8 & 6 & 0 & -1.281391 & -0.343482 & -0.000005 \\ 9 & 1 & 0 & -1.281679 & -1.004120 & 0.879545 \\ 10 & 1 & 0 & -1.281690 & -1.004096 & -0.879572 \\ 11 & 6 & 0 & 0.000000 & 0.498097 & -0.000003 \\ 12 & 1 & 0 & -0.000003 & 1.158303 & 0.879637 \\ 13 & 1 & 0 & 0.000003 & 1.158292 & -0.879651 \\ 14 & 6 & 0 & 1.281391 & -0.343482 & 0.000007 \\ 15 & 1 & 0 & 1.281682 & -1.004124 & -0.879539 \\ 16 & 1 & 0 & 1.281688 & -1.004091 & 0.879579 \\ 17 & 6 & 0 & 2.564602 & 0.495812 & -0.000014 \\ 18 & 1 & 0 & 2.564381 & 1.155275 & 0.879191 \\ 19 & 1 & 0 & 2.564380 & 1.155232 & -0.879250 \\ 20 & 6 & 0 & 3.838311 & -0.355592 & 0.000006 \\ 21 & 1 & 0 & 3.880421 & -1.002039 & -0.884594 \\ 22 & 1 & 0 & 4.738411 & 0.268726 & -0.000011 \\ 23 & 1 & 0 & 3.880424 & -1.001995 & 0.884639\end{array}$

Zero-point correction=

Thermal correction to Energy=

0.216972 (Hartree/Particle)

0.226881

0.227825

Thermal correction to Gibbs Free Energy=

Sum of electronic and zero-point Energies=

0.181815

$-276.032182$

$-276.022273$

Sum of electronic and thermal Energies $=$

Sum of electronic and thermal Enthalpies=

Sum of electronic and thermal Free Energies=

$-276.021329$

$-276.067339$

2,2,3-trimethylbutane:

Standard orientation:

\begin{tabular}{|c|c|c|c|c|}
\hline \multirow{2}{*}{$\begin{array}{l}\text { Center } \\
\text { Number }\end{array}$} & Atomic & \multirow{2}{*}{$\begin{array}{l}\text { Atomic } \\
\text { Type }\end{array}$} & \multicolumn{2}{|c|}{ Coordinates (Angstroms } \\
\hline & Number & & $\mathrm{X}$ & Z \\
\hline 1 & 6 & -1.335392 & -1.291179 & -0.482006 \\
\hline 2 & 1 & -1.195397 & -1.435372 & -1.560689 \\
\hline 3 & 1 & -0.951794 & -2.178463 & 0.031517 \\
\hline 4 & 1 & -2.413103 & -1.239244 & -0.288480 \\
\hline 5 & 6 & -0.643760 & 0.000093 & -0.000052 \\
\hline 6 & 6 & 0.854539 & -0.001034 & -0.452772 \\
\hline 7 & 1 & 0.827572 & -0.004150 & -1.555170 \\
\hline 8 & 6 & 1.632953 & 1.252765 & -0.019347 \\
\hline 9 & 1 & 1.752004 & 1.291593 & 1.069687 \\
\hline 10 & 1 & 2.637689 & 1.239954 & -0.456332 \\
\hline 11 & 1 & 1.147866 & 2.179663 & -0.340078 \\
\hline 12 & 6 & 1.635542 & -1.252189 & -0.016218 \\
\hline
\end{tabular}




$\begin{array}{rrrrrr}13 & 1 & 0 & 1.676095 & -1.339783 & 1.076036 \\ 14 & 1 & 0 & 1.201973 & -2.174363 & -0.413963 \\ 15 & 1 & 0 & 2.668917 & -1.193093 & -0.375922 \\ 16 & 6 & 0 & -0.787332 & 0.099282 & 1.530510 \\ 17 & 1 & 0 & -0.377122 & 1.038912 & 1.916093 \\ 18 & 1 & 0 & -1.846015 & 0.061389 & 1.812960 \\ 19 & 1 & 0 & -0.281222 & -0.727998 & 2.040474 \\ 20 & 6 & 0 & -1.377825 & 1.192301 & -0.646663 \\ 21 & 1 & 0 & -1.279812 & 1.169170 & -1.739081 \\ 22 & 1 & 0 & -2.446573 & 1.157529 & -0.405311 \\ 23 & 1 & 0 & -0.993419 & 2.154024 & -0.292454\end{array}$

Zero-point correction=

Thermal correction to Energy=

Thermal correction to Enthalpy=

Thermal correction to Gibbs Free Energy=

Sum of electronic and zero-point Energies=

Sum of electronic and thermal Energies=

Sum of electronic and thermal Enthalpies=

Sum of electronic and thermal Free Energies=

Octane:

Standard orientation:

\begin{tabular}{|c|c|c|c|c|}
\hline \multirow{2}{*}{$\begin{array}{l}\text { Center } \\
\text { Number }\end{array}$} & Atomic & \multirow{2}{*}{$\begin{array}{l}\text { Atomic } \\
\text { Type }\end{array}$} & \multicolumn{2}{|c|}{ Coordinates (Angstroms) } \\
\hline & Numbe & & $X$ & Z \\
\hline 1 & 6 & -4.492767 & 0.257532 & 0.000015 \\
\hline 2 & 1 & -4.560175 & 0.901786 & 0.884659 \\
\hline 3 & 1 & -4.560173 & 0.901865 & -0.884572 \\
\hline 4 & 1 & -5.367738 & -0.401545 & -0.000016 \\
\hline 5 & 6 & -3.186673 & -0.543290 & -0.000019 \\
\hline 6 & 1 & -3.160568 & -1.202307 & 0.879184 \\
\hline 7 & 1 & -3.160575 & -1.202248 & -0.879266 \\
\hline 8 & 6 & -1.937252 & 0.345524 & 0.000004 \\
\hline 9 & 1 & -1.963343 & 1.005678 & -0.879546 \\
\hline 10 & 1 & -1.963350 & 1.005647 & 0.879578 \\
\hline 11 & 6 & -0.624000 & -0.445299 & -0.000001 \\
\hline 12 & 1 & -0.598199 & -1.105152 & -0.879589 \\
\hline 13 & 1 & -0.598191 & -1.105146 & 0.879590 \\
\hline 14 & 6 & 0.624000 & 0.445299 & -0.000010 \\
\hline 15 & 1 & 0.598192 & 1.105163 & 0.879569 \\
\hline 16 & 1 & 0.598198 & 1.105134 & -0.879610 \\
\hline 17 & 6 & 1.937252 & -0.345524 & 0.000008 \\
\hline 18 & 1 & 1.963347 & -1.005682 & -0.879539 \\
\hline 19 & 1 & 1.963346 & -1.005643 & 0.879585 \\
\hline 20 & 6 & 3.186673 & 0.543290 & -0.000011 \\
\hline 21 & 1 & 3.160570 & 1.202299 & 0.879198 \\
\hline 22 & 1 & 3.160573 & 1.202257 & -0.879252 \\
\hline 23 & 6 & 4.492767 & -0.257532 & 0.000010 \\
\hline 24 & 1 & 4.560175 & -0.901847 & -0.884590 \\
\hline
\end{tabular}




$\begin{array}{rrrrrr}25 & 1 & 0 & 5.367738 & 0.401545 & -0.000005 \\ 26 & 1 & 0 & 4.560173 & -0.901804 & 0.884641\end{array}$

Zero-point correction $=$

Thermal correction to Energy=

Thermal correction to Enthalpy=

Thermal correction to Gibbs Free Energy=

Sum of electronic and zero-point Energies=

Sum of electronic and thermal Energies $=$

Sum of electronic and thermal Enthalpies=

Sum of electronic and thermal Free Energies= 2233tetramethylbutane:

Standard orientation:

\begin{tabular}{|c|c|c|c|c|}
\hline \multirow{2}{*}{$\begin{array}{l}\text { Center } \\
\text { Number }\end{array}$} & Atomic & \multirow{2}{*}{$\begin{array}{l}\text { Atomic } \\
\text { Type }\end{array}$} & \multicolumn{2}{|c|}{ Coordinates (Angstroms } \\
\hline & Numbe & & $X$ & Z \\
\hline 1 & 6 & -1.350938 & -0.990346 & -1.043944 \\
\hline 2 & 1 & -1.143321 & -0.663780 & -2.067998 \\
\hline 3 & 1 & -0.939083 & -1.997804 & -0.917493 \\
\hline 4 & 1 & -2.439303 & -1.065078 & -0.936624 \\
\hline 5 & 6 & -0.792365 & 0.000024 & 0.000023 \\
\hline 6 & 6 & 0.792277 & -0.000024 & -0.000019 \\
\hline 7 & 6 & 1.350830 & 1.183981 & 0.817818 \\
\hline 8 & 1 & 1.142914 & 2.145461 & 0.337479 \\
\hline 9 & 1 & 0.939286 & 1.214127 & 1.832913 \\
\hline 10 & 1 & 2.439238 & 1.089671 & 0.907885 \\
\hline 11 & 6 & -1.350745 & 1.399378 & -0.335638 \\
\hline 12 & 1 & -1.143429 & 2.122588 & 0.459578 \\
\hline 13 & 1 & -0.938313 & 1.793875 & -1.270984 \\
\hline 14 & 1 & -2.439049 & 1.343907 & -0.454598 \\
\hline 15 & 6 & -1.350765 & -0.408956 & 1.379694 \\
\hline 16 & 1 & -2.439148 & -0.278768 & 1.390908 \\
\hline 17 & 1 & -1.142928 & -1.459084 & 1.608722 \\
\hline 18 & 1 & -0.938814 & 0.204254 & 2.188950 \\
\hline 19 & 6 & 1.350743 & 0.116249 & -1.434320 \\
\hline 20 & 1 & 2.439093 & 0.242003 & -1.397720 \\
\hline 21 & 1 & 1.143331 & -0.780684 & -2.026696 \\
\hline 22 & 1 & 0.938719 & 0.979952 & -1.968135 \\
\hline 23 & 6 & 1.350833 & -1.300290 & 0.616393 \\
\hline 24 & 1 & 2.439250 & -1.331088 & 0.489690 \\
\hline 25 & 1 & 1.142980 & -1.365236 & 1.689240 \\
\hline 26 & 1 & 0.939351 & -2.194410 & 0.134843 \\
\hline
\end{tabular}

Zero-point correction=

Thermal correction to Energy=

Thermal correction to Enthalpy=

Thermal correction to Gibbs Free Energy=

Sum of electronic and zero-point Energies=

Sum of electronic and thermal Energies=
0.243593 (Hartree/Particle)

0.254333

0.255277

$$
\begin{aligned}
& 0.209939 \\
& -315.289745 \\
& -315.279005
\end{aligned}
$$


Sum of electronic and thermal Enthalpies=

Sum of electronic and thermal Free Energies=

Basis set: DH-SVPD

Functional: B3LYP

Ethane:

Standard orientation:

\begin{tabular}{cccccc} 
Center & \multicolumn{2}{c}{ Atomic } & \multicolumn{2}{c}{ Atomic } & \multicolumn{2}{c}{ Coordinates (Angstrom } \\
Number & Number & Type & X & Y & Z \\
- \hdashline 1 & 6 & 0 & -0.000000 & 0.000000 & 0.765779 \\
2 & 1 & 0 & -0.509353 & 0.882281 & 1.165178 \\
3 & 1 & 0 & 1.018754 & -0.000028 & 1.165178 \\
4 & 1 & 0 & -0.509401 & -0.882253 & 1.165178 \\
5 & 6 & 0 & 0.000000 & 0.000000 & -0.765779 \\
6 & 1 & 0 & 0.509353 & 0.882281 & -1.165178 \\
7 & 1 & 0 & 0.509401 & -0.882253 & -1.165178 \\
8 & 1 & 0 & -1.018754 & -0.000028 & -1.165178
\end{tabular}

Zero-point correction=

Thermal correction to Energy=

Thermal correction to Enthalpy=

Thermal correction to Gibbs Free Energy=

Sum of electronic and zero-point Energies=

Sum of electronic and thermal Energies=

Sum of electronic and thermal Enthalpies=

Sum of electronic and thermal Free Energies=

Methane:

$$
\begin{array}{cc}
0.074688 & \text { (Hartree/Particle) } \\
& 0.078188 \\
& 0.079132 \\
\text { rgy }= & 0.053246 \\
\text { gies }= & -79.707602 \\
\text { s }= & -79.704102 \\
\text { pies }= & -79.703158 \\
\text { Energies }= & -79.729043
\end{array}
$$

\begin{tabular}{|c|c|c|c|c|}
\hline \multirow{2}{*}{$\begin{array}{l}\text { Center } \\
\text { Number }\end{array}$} & \multirow{2}{*}{$\begin{array}{l}\text { Atomic } \\
\text { Number }\end{array}$} & \multirow{2}{*}{$\begin{array}{l}\text { Atomic } \\
\text { Type }\end{array}$} & \multicolumn{2}{|c|}{ Coordinates (Angstro } \\
\hline & & & $\mathrm{X}$ & Z \\
\hline 1 & 6 & 0.000000 & 0.000000 & 0.000000 \\
\hline 2 & 1 & 0.629976 & 0.629976 & 0.629976 \\
\hline 3 & 1 & -0.629976 & -0.629976 & 0.629976 \\
\hline 4 & 1 & -0.629976 & 0.629976 & -0.629976 \\
\hline 5 & 1 & 0.629976 & -0.629976 & -0.629976 \\
\hline
\end{tabular}

Standard orientation:

Zero-point correction=

Thermal correction to Energy=

Thermal correction to Enthalpy=

Thermal correction to Gibbs Free Energy=

Sum of electronic and zero-point Energies=

Sum of electronic and thermal Energies $=$

Sum of electronic and thermal Enthalpies=

Sum of electronic and thermal Free Energies=

Propane:

$$
\begin{array}{cc}
0.044837 \text { (Hartree/Particle) } & 0.047706 \\
& 0.048650 \\
\text { gy }= & 0.027519 \\
\text { gies }= & -40.450415 \\
\text { s }= & -40.447547 \\
\text { pies }= & -40.446603 \\
\text { ergies }= & -40.467734
\end{array}
$$

Standard orientation: 


\begin{tabular}{cccccc}
$\begin{array}{c}\text { Center } \\
\text { Number }\end{array}$ & \multicolumn{2}{c}{ Atomic } & \multicolumn{2}{c}{ Atomic } & \multicolumn{3}{c}{ Coordinates (Angstroms) } \\
- & Number & Type & X & Y & Z \\
---------------- \\
1 & 6 & 0 & -1.276691 & -0.259758 & 0.000000 \\
2 & 1 & 0 & -2.175332 & 0.364785 & -0.000005 \\
3 & 1 & 0 & -1.321971 & -0.906212 & 0.883015 \\
4 & 1 & 0 & -1.321968 & -0.906222 & -0.883007 \\
5 & 6 & 0 & -0.000000 & 0.586814 & 0.000000 \\
6 & 1 & 0 & -0.000001 & 1.245760 & 0.877121 \\
7 & 1 & 0 & 0.000001 & 1.245748 & -0.877130 \\
8 & 6 & 0 & 1.276692 & -0.259757 & 0.000000 \\
9 & 1 & 0 & 1.322003 & -0.906162 & 0.883050 \\
10 & 1 & 0 & 1.321932 & -0.906273 & -0.882972 \\
11 & 1 & 0 & 2.175334 & 0.364783 & -0.000075
\end{tabular}

Zero-point correction=

Thermal correction to Energy=

Thermal correction to Enthalpy=

Thermal correction to Gibbs Free Energy=

Sum of electronic and zero-point Energies=

Sum of electronic and thermal Energies=

Sum of electronic and thermal Enthalpies=

Sum of electronic and thermal Free Energies=

Butane:
0.103374 (Hartree/Particle)

0.107923

0.108868

$-118.968312$

$-118.963763$

$-118.962819$

$-118.994000$

Standard orientation:

\begin{tabular}{|c|c|c|c|c|}
\hline \multirow{2}{*}{$\begin{array}{l}\text { Center } \\
\text { Number }\end{array}$} & Atomic & \multirow{2}{*}{$\begin{array}{l}\text { Atomic } \\
\text { Type }\end{array}$} & \multicolumn{2}{|c|}{ Coordinates (Angstrom } \\
\hline & Numbe & & $X$ & Z \\
\hline 1 & 6 & 1.961530 & -0.120448 & 0.000042 \\
\hline 2 & 1 & 2.109448 & -0.751229 & -0.882986 \\
\hline 3 & 1 & 2.109391 & -0.751122 & 0.883155 \\
\hline 4 & 1 & 2.749879 & 0.638422 & 0.000020 \\
\hline 5 & 6 & 0.567818 & 0.514670 & -0.000047 \\
\hline 6 & 1 & 0.461990 & 1.166266 & 0.877516 \\
\hline 7 & 1 & 0.462057 & 1.166190 & -0.877674 \\
\hline 8 & 6 & -0.567818 & -0.514670 & -0.000047 \\
\hline 9 & 1 & -0.462056 & -1.166189 & -0.877675 \\
\hline 10 & 1 & -0.461990 & -1.166267 & 0.877516 \\
\hline 11 & 6 & -1.961530 & 0.120448 & 0.000042 \\
\hline 12 & 1 & -2.109441 & 0.751244 & -0.882976 \\
\hline 13 & 1 & -2.749879 & -0.638422 & 0.000001 \\
\hline 14 & 1 & -2.109397 & 0.751107 & 0.883164 \\
\hline
\end{tabular}

Zero-point correction=

Thermal correction to Energy=

Thermal correction to Enthalpy=

Thermal correction to Gibbs Free Energy=

Sum of electronic and zero-point Energies=

Sum of electronic and thermal Energies=
0.131852 (Hartree/Particle)

0.137669

0.138613

0.103769

$-158.229079$

$-158.223262$ 
Sum of electronic and thermal Enthalpies=

Sum of electronic and thermal Free Energies= Isobutane:

Standard orientation:
$-158.222318$

$-158.257162$

\begin{tabular}{|c|c|c|c|c|}
\hline \multirow{2}{*}{$\begin{array}{l}\text { Center } \\
\text { Number }\end{array}$} & Atomic & \multirow{2}{*}{$\begin{array}{r}\text { Atomic } \\
\text { Type }\end{array}$} & \multicolumn{2}{|c|}{ Coordinates (Angstrom } \\
\hline & Numbe & & $\mathrm{X}$ & Z \\
\hline 1 & 6 & -0.067571 & -1.460063 & -0.095468 \\
\hline 2 & 1 & -0.975791 & -1.953687 & 0.264689 \\
\hline 3 & 1 & -0.070437 & -1.518343 & -1.190130 \\
\hline 4 & 1 & 0.791356 & -2.035353 & 0.264423 \\
\hline 5 & 6 & -0.000004 & 0.000011 & 0.371512 \\
\hline 6 & 1 & -0.000011 & 0.000030 & 1.472411 \\
\hline 7 & 6 & -1.230671 & 0.788547 & -0.095473 \\
\hline 8 & 1 & -1.203987 & 1.821925 & 0.264604 \\
\hline 9 & 1 & -1.279790 & 0.820095 & -1.190136 \\
\hline 10 & 1 & -2.158325 & 0.332380 & 0.264526 \\
\hline 11 & 6 & 1.298244 & 0.671511 & -0.095470 \\
\hline 12 & 1 & 1.367074 & 1.702964 & 0.264525 \\
\hline 13 & 1 & 2.179800 & 0.131664 & 0.264612 \\
\hline 14 & 1 & 1.350126 & 0.698288 & -1.190131 \\
\hline
\end{tabular}

Zero-point correction=

Thermal correction to Energy=

Thermal correction to Enthalpy=

Thermal correction to Gibbs Free Energy=

Sum of electronic and zero-point Energies=

Sum of electronic and thermal Energies=

Sum of electronic and thermal Enthalpies=

Sum of electronic and thermal Free Energies=

Pentane:
0.131435 (Hartree/Particle)

0.137171

0.138116

0.103763

$-158.230725$

$-158.224989$

$-158.224045$

$-158.258397$

Standard orientation:

\begin{tabular}{|c|c|c|c|c|}
\hline \multirow{2}{*}{$\begin{array}{l}\text { Center } \\
\text { Number }\end{array}$} & Atomic & \multirow{2}{*}{$\begin{array}{r}\text { Atomic } \\
\text { Type }\end{array}$} & \multicolumn{2}{|c|}{ Coordinates (Angstroms } \\
\hline & & & $X$ & $\mathrm{Z}$ \\
\hline 1 & 6 & 2.558826 & 0.325034 & -0.000006 \\
\hline 2 & 1 & 2.604169 & 0.971350 & -0.883072 \\
\hline 3 & 1 & 2.604159 & 0.971424 & 0.883007 \\
\hline 4 & 1 & 3.457951 & -0.298648 & 0.000024 \\
\hline 5 & 6 & 1.283749 & -0.523676 & 0.000023 \\
\hline 6 & 1 & 1.283913 & -1.183586 & -0.877549 \\
\hline 7 & 1 & 1.283910 & -1.183520 & 0.877643 \\
\hline 8 & 6 & 0.000000 & 0.313382 & -0.000008 \\
\hline 9 & 1 & -0.000015 & 0.974701 & 0.877933 \\
\hline 10 & 1 & 0.000016 & 0.974682 & -0.877962 \\
\hline 11 & 6 & -1.283749 & -0.523676 & -0.000024 \\
\hline 12 & 1 & -1.283905 & -1.183582 & 0.877552 \\
\hline 13 & 1 & -1.283917 & -1.183524 & -0.877640 \\
\hline
\end{tabular}




$\begin{array}{rrrrrc}14 & 6 & 0 & -2.558826 & 0.325034 & 0.000014 \\ 15 & 1 & 0 & -3.457951 & -0.298648 & -0.000095 \\ 16 & 1 & 0 & -2.604201 & 0.971288 & 0.883122 \\ 17 & 1 & 0 & -2.604126 & 0.971485 & -0.882957\end{array}$

$\begin{array}{lc}\text { Zero-point correction= } & 0.160298 \text { (Hartree/Particle) } \\ \text { Thermal correction to Energy= } & 0.167431 \\ \text { Thermal correction to Enthalpy= } & 0.168375 \\ \text { Thermal correction to Gibbs Free Energy= } & 0.129863 \\ \text { Sum of electronic and zero-point Energies }= & -197.489815 \\ \text { Sum of electronic and thermal Energies }= & -197.482683 \\ \text { Sum of electronic and thermal Enthalpies }= & -197.481739 \\ \text { Sum of electronic and thermal Free Energies }= & -197.520251\end{array}$

Neopentane:

Standard orientation:

\begin{tabular}{|c|c|c|c|c|}
\hline \multirow{2}{*}{$\begin{array}{l}\text { Center } \\
\text { Number }\end{array}$} & Atomic & \multirow{2}{*}{$\begin{array}{l}\text { Atomic } \\
\text { Type }\end{array}$} & \multicolumn{2}{|c|}{ Coordinates (Angstroms } \\
\hline & Numbe & & $X$ & Z \\
\hline 1 & 6 & 1.120096 & -1.056165 & 0.023982 \\
\hline 2 & 1 & 0.761095 & -2.003767 & 0.439122 \\
\hline 3 & 1 & 1.497149 & -1.255427 & -0.984630 \\
\hline 4 & 1 & 1.964741 & -0.722634 & 0.635951 \\
\hline 5 & 6 & -0.000002 & -0.000012 & -0.000020 \\
\hline 6 & 6 & -1.165369 & -0.507367 & -0.869014 \\
\hline 7 & 1 & -1.979181 & 0.224463 & -0.905266 \\
\hline 8 & 1 & -0.839158 & -0.694664 & -1.897445 \\
\hline 9 & 1 & -1.575251 & -1.442650 & -0.473531 \\
\hline 10 & 6 & -0.500489 & 0.250006 & 1.434430 \\
\hline 11 & 1 & 0.308213 & 0.612506 & 2.077676 \\
\hline 12 & 1 & -1.299600 & 0.998580 & 1.449372 \\
\hline 13 & 1 & -0.895531 & -0.668557 & 1.880923 \\
\hline 14 & 6 & 0.545757 & 1.313534 & -0.589398 \\
\hline 15 & 1 & 1.377669 & 1.699638 & 0.008943 \\
\hline 16 & 1 & 0.910022 & 1.166822 & -1.611630 \\
\hline 17 & 1 & -0.230125 & 2.085719 & -0.619370 \\
\hline
\end{tabular}

Zero-point correction=

Thermal correction to Energy=

Thermal correction to Enthalpy=

Thermal correction to Gibbs Free Energy=

Sum of electronic and zero-point Energies=

Sum of electronic and thermal Energies $=$

Sum of electronic and thermal Enthalpies=

Sum of electronic and thermal Free Energies=

Hexane:
0.159284 (Hartree/Particle)

$$
0.166241
$$

0.167186

\subsection{5}

$-197.492824$

$-197.485866$

$-197.484922$

$-197.521993$

Standard orientation:

Center Atomic Atomic Coordinates (Angstroms)




\begin{tabular}{cccccc} 
Number & Number & Type & $X$ & $Y$ & $Z$ \\
\hline 1 & 6 & 0 & -3.222397 & 0.208475 & 0.000065 \\
2 & 1 & 0 & -3.312283 & 0.850036 & 0.883227 \\
3 & 1 & 0 & -3.312291 & 0.850368 & -0.882857 \\
4 & 1 & 0 & -4.076313 & -0.475820 & -0.000062 \\
5 & 6 & 0 & -1.891662 & -0.549854 & -0.000083 \\
6 & 1 & 0 & -1.845983 & -1.208440 & 0.877376 \\
7 & 1 & 0 & -1.846040 & -1.208203 & -0.877722 \\
8 & 6 & 0 & -0.668684 & 0.373930 & -0.000007 \\
9 & 1 & 0 & -0.715250 & 1.033427 & -0.877919 \\
10 & 1 & 0 & -0.715314 & 1.033381 & 0.877937 \\
11 & 6 & 0 & 0.668684 & -0.373930 & 0.000028 \\
12 & 1 & 0 & 0.715270 & -1.033476 & -0.877847 \\
13 & 1 & 0 & 0.715294 & -1.033331 & 0.878008 \\
14 & 6 & 0 & 1.891662 & 0.549854 & -0.000065 \\
15 & 1 & 0 & 1.845998 & 1.208431 & 0.877401 \\
16 & 1 & 0 & 1.846025 & 1.208212 & -0.877696 \\
17 & 6 & 0 & 3.222397 & -0.208475 & 0.000049 \\
18 & 1 & 0 & 3.312296 & -0.850319 & -0.882908 \\
19 & 1 & 0 & 4.076313 & 0.475820 & -0.000034 \\
20 & 1 & 0 & 3.312279 & -0.850085 & 0.883177
\end{tabular}

Zero-point correction $=$

0.188710 (Hartree/Particle)

Thermal correction to Energy=

0.197185

Thermal correction to Enthalpy= 0.198130

Thermal correction to Gibbs Free Energy=

0.155963

Sum of electronic and zero-point Energies=

$-236.750618$

Sum of electronic and thermal Energies $=$

$-236.742143$

Sum of electronic and thermal Enthalpies=

$-236.741199$

Sum of electronic and thermal Free Energies $=\quad-236.783365$

22-dimethylbutane:

Standard orientation:

\begin{tabular}{|c|c|c|c|c|}
\hline \multirow{2}{*}{$\begin{array}{l}\text { Center } \\
\text { Number }\end{array}$} & Atomic & \multirow{2}{*}{$\begin{array}{r}\text { Atomic } \\
\text { Type }\end{array}$} & \multicolumn{2}{|c|}{ Coordinates (Angstroms) } \\
\hline & Numbe & & $X$ & $\mathrm{Z}$ \\
\hline 1 & 6 & -1.619196 & 0.986944 & 0.001380 \\
\hline 2 & 1 & -1.595981 & 1.631225 & 0.886557 \\
\hline 3 & 1 & -1.596137 & 1.633489 & -0.882150 \\
\hline 4 & 1 & -2.576552 & 0.455730 & 0.000781 \\
\hline 5 & 6 & -0.436334 & -0.000267 & 0.000011 \\
\hline 6 & 6 & 0.876728 & 0.825059 & 0.000642 \\
\hline 7 & 1 & 0.861240 & 1.486327 & -0.875768 \\
\hline 8 & 1 & 0.861315 & 1.484922 & 0.878115 \\
\hline 9 & 6 & 2.190925 & 0.035647 & 0.000010 \\
\hline 10 & 1 & 2.286700 & -0.601669 & -0.883805 \\
\hline 11 & 1 & 3.045657 & 0.718867 & 0.000089 \\
\hline 12 & 1 & 2.287064 & -0.602358 & 0.883283 \\
\hline 13 & 6 & -0.525940 & -0.886128 & 1.257028 \\
\hline
\end{tabular}




$\begin{array}{rrrrrr}14 & 1 & 0 & 0.263919 & -1.642200 & 1.280638 \\ 15 & 1 & 0 & -0.444028 & -0.286461 & 2.170121 \\ 16 & 1 & 0 & -1.484543 & -1.414095 & 1.291189 \\ 17 & 6 & 0 & -0.526498 & -0.883239 & -1.259006 \\ 18 & 1 & 0 & -1.484955 & -1.411434 & -1.293768 \\ 19 & 1 & 0 & -0.445379 & -0.281410 & -2.170745 \\ 20 & 1 & 0 & 0.263569 & -1.639022 & -1.284928\end{array}$

Zero-point correction $=$

Thermal correction to Energy=

Thermal correction to Enthalpy=

Thermal correction to Gibbs Free Energy=

Sum of electronic and zero-point Energies=

Sum of electronic and thermal Energies $=$

Sum of electronic and thermal Enthalpies=

Sum of electronic and thermal Free Energies=

Heptane:
0.187797 (Hartree/Particle)

0.196098

0.197042

0.156407

$-236.750660$

$-236.742359$

$-236.741415$

$-236.782050$

Standard orientation:

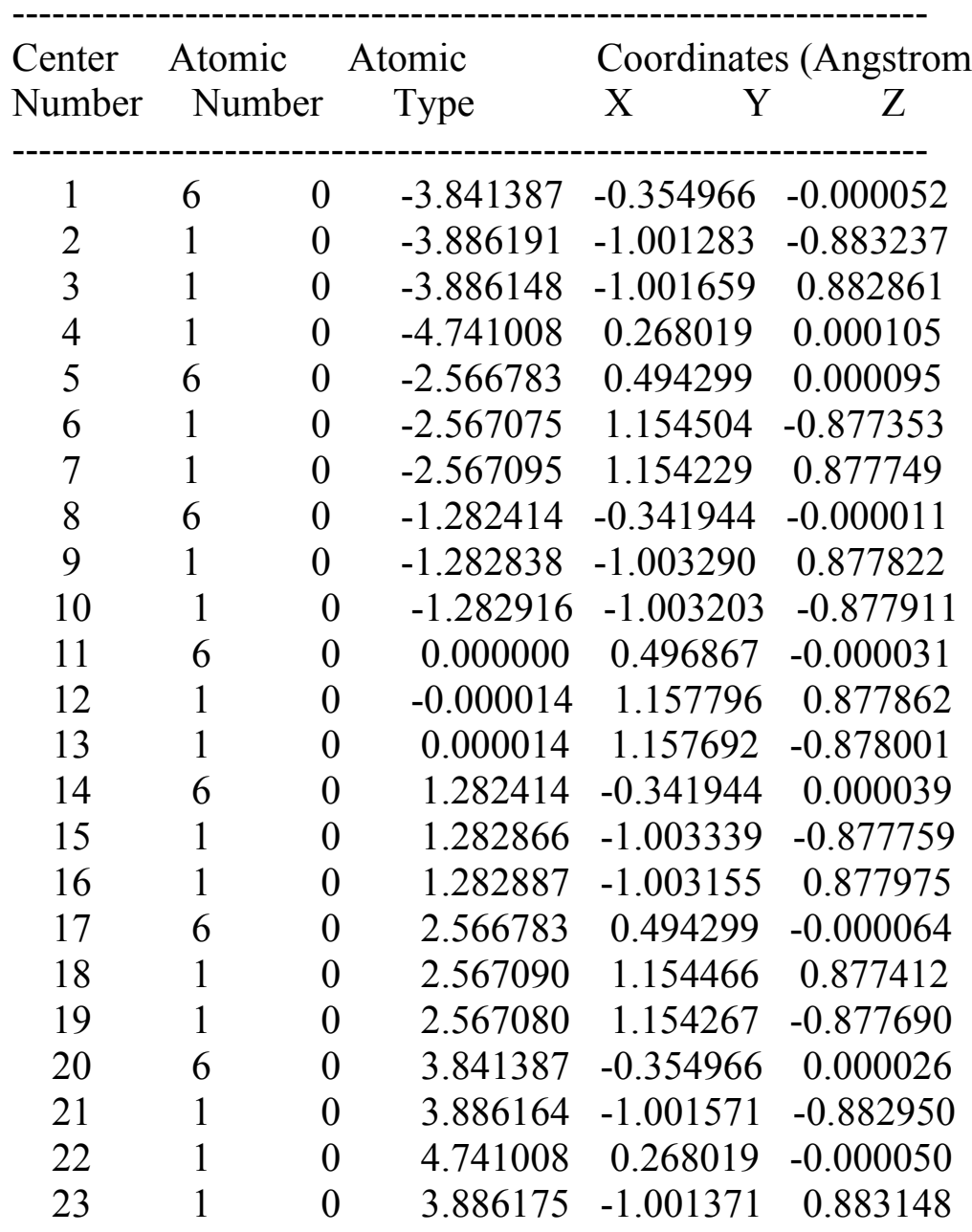

Zero-point correction=

Thermal correction to Energy=

Thermal correction to Enthalpy=

Thermal correction to Gibbs Free Energy=
0.217108 (Hartree/Particle)

0.226938

0.227882

0.182077 
Sum of electronic and zero-point Energies=

$-276.011451$

Sum of electronic and thermal Energies=

$-276.001621$

Sum of electronic and thermal Enthalpies=

Sum of electronic and thermal Free Energies=

223trimethylbutane

Standard orientation:

\begin{tabular}{|c|c|c|c|c|}
\hline \multirow{2}{*}{$\begin{array}{l}\text { Center } \\
\text { Number }\end{array}$} & Atomic & \multirow{2}{*}{$\begin{array}{l}\text { Atomic } \\
\text { Type }\end{array}$} & \multicolumn{2}{|c|}{ Coordinates (Angstrom } \\
\hline & Number & & $\mathrm{X}$ & $\mathrm{Z}$ \\
\hline 1 & 6 & -1.350002 & -1.263961 & -0.536175 \\
\hline 2 & 1 & -1.218234 & -1.362638 & -1.619448 \\
\hline 3 & 1 & -0.974610 & -2.176964 & -0.067637 \\
\hline 4 & 1 & -2.425121 & -1.215306 & -0.336478 \\
\hline 5 & 6 & -0.645616 & 0.000013 & -0.000207 \\
\hline 6 & 6 & 0.857096 & -0.000365 & -0.447862 \\
\hline 7 & 1 & 0.832120 & -0.001630 & -1.549294 \\
\hline 8 & 6 & 1.638850 & 1.252943 & -0.017459 \\
\hline 9 & 1 & 1.739245 & 1.310532 & 1.071222 \\
\hline 10 & 1 & 2.650847 & 1.225691 & -0.433003 \\
\hline 11 & 1 & 1.172992 & 2.179314 & -0.360987 \\
\hline 12 & 6 & 1.639937 & -1.252577 & -0.016189 \\
\hline 13 & 1 & 1.708200 & -1.330252 & 1.073847 \\
\hline 14 & 1 & 1.195102 & -2.176908 & -0.391255 \\
\hline 15 & 1 & 2.663736 & -1.206389 & -0.399979 \\
\hline 16 & 6 & -0.795243 & 0.038628 & 1.533413 \\
\hline 17 & 1 & -0.359428 & 0.945011 & 1.963387 \\
\hline 18 & 1 & -1.854300 & 0.023246 & 1.810947 \\
\hline 19 & 1 & -0.321249 & -0.823263 & 2.012062 \\
\hline 20 & 6 & -1.366519 & 1.225348 & -0.600279 \\
\hline 21 & 1 & -1.251146 & 1.259055 & -1.689313 \\
\hline 22 & 1 & -2.438277 & 1.182857 & -0.381837 \\
\hline 23 & 1 & -0.990896 & 2.167474 & - -0.193682 \\
\hline
\end{tabular}

Zero-point correction=

Thermal correction to Energy=

0.215878 (Hartree/Particle)

Thermal correction to Enthalpy=

0.225542

0.226486

Thermal correction to Gibbs Free Energy=

0.181767

Sum of electronic and zero-point Energies=

$-276.008199$

Sum of electronic and thermal Energies=

$-275.998535$

Sum of electronic and thermal Enthalpies=

$-275.997591$

Sum of electronic and thermal Free Energies $=\quad-276.042311$

Octane:

Standard orientation:

\begin{tabular}{|c|c|c|c|c|}
\hline Center & Atomic & Atomic & Coordinate & s (Angstroms) \\
\hline Number & Numbe & Type & $\mathrm{X}$ & $\mathrm{Z}$ \\
\hline 1 & 6 & -4.45 & 0.256818 & 0.000070 \\
\hline 2 & 1 & -4.566479 & 0.900879 & 0.883269 \\
\hline
\end{tabular}




$\begin{array}{rrrrrr}3 & 1 & 0 & -4.566449 & 0.901305 & -0.882822 \\ 4 & 1 & 0 & -5.370895 & -0.400926 & -0.000106 \\ 5 & 6 & 0 & -3.189448 & -0.541784 & -0.000097 \\ 6 & 1 & 0 & -3.163808 & -1.201578 & 0.877343 \\ 7 & 1 & 0 & -3.163847 & -1.201271 & -0.877768 \\ 8 & 6 & 0 & -1.938755 & 0.344049 & 0.000020 \\ 9 & 1 & 0 & -1.965009 & 1.004921 & -0.877800 \\ 10 & 1 & 0 & -1.965074 & 1.004797 & 0.877932 \\ 11 & 6 & 0 & -0.624552 & -0.443916 & 0.000015 \\ 12 & 1 & 0 & -0.598703 & -1.104510 & -0.877814 \\ 13 & 1 & 0 & -0.598665 & -1.104431 & 0.877902 \\ 14 & 6 & 0 & 0.624552 & 0.443916 & -0.000051 \\ 15 & 1 & 0 & 0.598675 & 1.104551 & 0.877747 \\ 16 & 1 & 0 & 0.598693 & 1.104390 & -0.877969 \\ 17 & 6 & 0 & 1.938755 & -0.344049 & 0.000034 \\ 18 & 1 & 0 & 1.965046 & -1.004946 & -0.877766 \\ 19 & 1 & 0 & 1.965037 & -1.004772 & 0.877966 \\ 20 & 6 & 0 & 3.189448 & 0.541784 & -0.000046 \\ 21 & 1 & 0 & 3.163822 & 1.201512 & 0.877443 \\ 22 & 1 & 0 & 3.163833 & 1.201338 & -0.877667 \\ 23 & 6 & 0 & 4.496361 & -0.256818 & 0.000041 \\ 24 & 1 & 0 & 4.566469 & -0.901181 & -0.882940 \\ 25 & 1 & 0 & 5.370895 & 0.400926 & -0.000021 \\ 26 & 1 & 0 & 4.566459 & -0.901004 & 0.883151\end{array}$

Zero-point correction=

Thermal correction to Energy=

0.245504 (Hartree/Particle)

0.256693

Thermal correction to Enthalpy=

0.257637

Thermal correction to Gibbs Free Energy $=\quad 0.208205$

Sum of electronic and zero-point Energies $=\quad-315.272287$

Sum of electronic and thermal Energies $=\quad-315.261099$

Sum of electronic and thermal Enthalpies $=\quad-315.260155$

Sum of electronic and thermal Free Energies $=\quad-315.309587$

2233tetramethylbutane

Standard orientation:

\begin{tabular}{cccccc} 
Center & \multicolumn{2}{c}{ Atomic } & \multicolumn{2}{c}{ Atomic } & \multicolumn{2}{c}{ Coordinates (Angstroms) } \\
Number & Number & Type & X & Y & $Z$ \\
- \hdashline 1 & 6 & 0 & -1.356824 & 1.047932 & 0.986655 \\
2 & 1 & 0 & -1.148778 & 0.785881 & 2.027039 \\
3 & 1 & 0 & -0.954076 & 2.048307 & 0.803949 \\
4 & 1 & 0 & -2.444250 & 1.112672 & 0.879566 \\
5 & 6 & 0 & -0.795250 & -0.000066 & 0.000037 \\
6 & 6 & 0 & 0.795306 & 0.000025 & -0.000037 \\
7 & 6 & 0 & 1.356926 & -1.217275 & -0.767962 \\
8 & 1 & 0 & 1.148579 & -2.159167 & -0.254305 \\
9 & 1 & 0 & 0.954514 & -1.290278 & -1.782373 \\
10 & 1 & 0 & 2.444392 & -1.129860 & -0.857006 \\
11 & 6 & 0 & -1.356650 & -1.378547 & 0.414260
\end{tabular}




$\begin{array}{rrrrrr}12 & 1 & 0 & -1.148106 & -2.148600 & -0.332693 \\ 13 & 1 & 0 & -0.954213 & -1.720279 & 1.372176 \\ 14 & 1 & 0 & -2.444137 & -1.318363 & 0.523370 \\ 15 & 6 & 0 & -1.356881 & 0.330368 & -1.400827 \\ 16 & 1 & 0 & -2.444336 & 0.205571 & -1.403127 \\ 17 & 1 & 0 & -1.148635 & 1.362312 & -1.694233 \\ 18 & 1 & 0 & -0.954482 & -0.328211 & -2.175848 \\ 19 & 6 & 0 & 1.356826 & -0.056335 & 1.438191 \\ 20 & 1 & 0 & 2.444316 & -0.176932 & 1.407158 \\ 21 & 1 & 0 & 1.148166 & 0.859363 & 1.997025 \\ 22 & 1 & 0 & 0.954516 & -0.898431 & 2.008559 \\ 23 & 6 & 0 & 1.356611 & 1.273862 & -0.670304 \\ 24 & 1 & 0 & 2.444117 & 1.307483 & -0.550428 \\ 25 & 1 & 0 & 1.147852 & 1.299951 & -1.742734 \\ 26 & 1 & 0 & 0.954173 & 2.188792 & -0.226170\end{array}$

Zero-point correction=

0.244360 (Hartree/Particle)

Thermal correction to Energy=

0.254897

Thermal correction to Enthalpy=

0.255841

Thermal correction to Gibbs Free Energy=

Sum of electronic and zero-point Energies $=$

0.210862

Sum of electronic and thermal Energies $=$

$-315.263790$

$-315.253252$

Sum of electronic and thermal Enthalpies=

$-315.252308$

Sum of electronic and thermal Free Energies=

$-315.297287$

Basis set: DH-SVPD

Functional: PBE0

Ethane:

Standard orientation:

\begin{tabular}{|c|c|c|c|c|}
\hline \multirow{2}{*}{$\begin{array}{l}\text { Center } \\
\text { Number }\end{array}$} & Atomic & \multirow{2}{*}{$\begin{array}{l}\text { Atomic } \\
\text { Type }\end{array}$} & \multicolumn{2}{|c|}{ Coordinates (Angstrom } \\
\hline & Number & & $\mathrm{X}$ & $\mathrm{Z}$ \\
\hline 1 & 6 & -0.000000 & -0.000000 & 0.761372 \\
\hline 2 & 1 & -0.509474 & 0.882493 & 1.162555 \\
\hline 3 & 1 & 1.018998 & -0.000028 & 1.162555 \\
\hline 4 & 1 & -0.509524 & -0.882464 & 1.162555 \\
\hline 5 & 6 & 0.000000 & -0.000000 & -0.761372 \\
\hline 6 & 1 & 0.509474 & 0.882493 & -1.162555 \\
\hline 7 & 1 & 0.509524 & -0.882464 & -1.162555 \\
\hline 8 & 1 & -1.018998 & -0.000028 & -1.162555 \\
\hline
\end{tabular}

Zero-point correction=

Thermal correction to Energy=

Thermal correction to Enthalpy=

Thermal correction to Gibbs Free Energy=

Sum of electronic and zero-point Energies=

Sum of electronic and thermal Energies $=$

Sum of electronic and thermal Enthalpies=

Sum of electronic and thermal Free Energies=

Methane:
0.075015 (Hartree/Particle)

$$
0.078510
$$$$
0.079454
$$

0.053585

$-79.596549$

$-79.593053$

$-79.592109$

$-79.617978$ 
Standard orientation:

\begin{tabular}{cccccc} 
Center & \multicolumn{2}{c}{ Atomic } & \multicolumn{2}{c}{ Atomic } & \multicolumn{2}{c}{ Coordinates (Angstroms) } \\
Number & Number & Type & X & Y & Z \\
-1 & 6 & 0 & 0.000000 & 0.000000 & 0.000000 \\
2 & 1 & 0 & 0.630509 & 0.630509 & 0.630509 \\
3 & 1 & 0 & -0.630509 & -0.630509 & 0.630509 \\
4 & 1 & 0 & -0.630509 & 0.630509 & -0.630509 \\
5 & 1 & 0 & 0.630509 & -0.630509 & -0.630509
\end{tabular}

Zero-point correction $=$

Thermal correction to Energy=

0.044974 (Hartree/Particle)

Thermal correction to Enthalpy=

0.047844

Thermal correction to Gibbs Free Energy=

0.048789

Sum of electronic and zero-point Energies=

0.027653

Sum of electronic and thermal Energies=

$-40.388594$

$-40.385723$

$\begin{array}{lr}\text { Sum of electronic and thermal Enthalpies }= & -40.384779 \\ \text { Sum of electronic and thermal Free Energies }= & -40.405915\end{array}$

Propane:

Standard orientation:

\begin{tabular}{|c|c|c|c|c|}
\hline \multirow{2}{*}{$\begin{array}{l}\text { Center } \\
\text { Number }\end{array}$} & Atomic & \multirow{2}{*}{$\begin{array}{l}\text { Atomic } \\
\text { Type }\end{array}$} & \multicolumn{2}{|c|}{ Coordinates (Angstroms } \\
\hline & Number & & $X$ & Z \\
\hline 1 & 6 & -1.268448 & -0.258548 & 0.000000 \\
\hline 2 & 1 & -2.170319 & 0.362796 & -0.000020 \\
\hline 3 & 1 & -1.312485 & -0.906352 & 0.883287 \\
\hline 4 & 1 & -1.312469 & -0.906385 & -0.883263 \\
\hline 5 & 6 & 0.000000 & 0.585137 & -0.000000 \\
\hline 6 & 1 & -0.000000 & 1.245826 & 0.877026 \\
\hline 7 & 1 & 0.000001 & 1.245811 & -0.877036 \\
\hline 8 & 6 & 1.268448 & -0.258548 & 0.000000 \\
\hline 9 & 1 & 1.312501 & -0.906326 & 0.883305 \\
\hline 10 & 1 & 1.312449 & -0.906410 & -0.883245 \\
\hline 11 & 1 & 2.170321 & 0.362793 & -0.000056 \\
\hline
\end{tabular}

Zero-point correction=

0.103844 (Hartree/Particle)

Thermal correction to Energy=

0.108383

Thermal correction to Enthalpy=

0.109327

Thermal correction to Gibbs Free Energy=

Sum of electronic and zero-point Energies=

0.078180

Sum of electronic and thermal Energies=

$-118.808344$

$-118.803805$

Sum of electronic and thermal Enthalpies=

$-118.802861$

Sum of electronic and thermal Free Energies=

$-118.834009$

Butane:

Standard orientation:

\begin{tabular}{lcccc} 
Center & Atomic & Atomic & & \multicolumn{2}{c}{ Coordinates (Angstroms) } \\
Number & Number & Type & X & Y
\end{tabular}




$\begin{array}{cccccc}1 & 6 & 0 & 1.949833 & -0.119275 & 0.000034 \\ 2 & 1 & 0 & 2.097461 & -0.751366 & -0.883307 \\ 3 & 1 & 0 & 2.097407 & -0.751308 & 0.883426 \\ 4 & 1 & 0 & 2.741040 & 0.637803 & 0.000033 \\ 5 & 6 & 0 & 0.564082 & 0.512680 & -0.000038 \\ 6 & 1 & 0 & 0.456198 & 1.165849 & 0.877444 \\ 7 & 1 & 0 & 0.456250 & 1.165793 & -0.877569 \\ 8 & 6 & 0 & -0.564082 & -0.512680 & -0.000038 \\ 9 & 1 & 0 & -0.456250 & -1.165791 & -0.877570 \\ 10 & 1 & 0 & -0.456198 & -1.165851 & 0.877443 \\ 11 & 6 & 0 & -1.949833 & 0.119275 & 0.000034 \\ 12 & 1 & 0 & -2.097457 & 0.751376 & -0.883301 \\ 13 & 1 & 0 & -2.741040 & -0.637803 & 0.000021 \\ 14 & 1 & 0 & -2.097410 & 0.751298 & 0.883432\end{array}$

Zero-point correction=

0.132455 (Hartree/Particle)

Thermal correction to Energy=

0.138258

Thermal correction to Enthalpy=

0.139203

Thermal correction to Gibbs Free Energy=

Sum of electronic and zero-point Energies=

0.104409

Sum of electronic and thermal Energies $=$

$-158.020251$

$-158.014448$

Sum of electronic and thermal Enthalpies=

$-158.013503$

Sum of electronic and thermal Free Energies=

$-158.048297$

Isobutane:

Standard orientation:

\begin{tabular}{|c|c|c|c|c|}
\hline \multirow{2}{*}{$\begin{array}{l}\text { Center } \\
\text { Number }\end{array}$} & Atomic & \multirow{2}{*}{$\begin{array}{l}\text { Atomic } \\
\text { Type }\end{array}$} & \multicolumn{2}{|c|}{ Coordinates (Angstrom } \\
\hline & Number & & $X$ & Z \\
\hline 1 & 6 & -0.771373 & 1.231000 & -0.095024 \\
\hline 2 & 1 & -0.306357 & 2.156155 & 0.263081 \\
\hline 3 & 1 & -0.800663 & 1.277847 & -1.190967 \\
\hline 4 & 1 & -1.806748 & 1.215859 & 0.262974 \\
\hline 5 & 6 & 0.000020 & 0.000007 & 0.371919 \\
\hline 6 & 1 & 0.000029 & 0.000010 & 1.473714 \\
\hline 7 & 6 & 1.451772 & 0.052515 & -0.095032 \\
\hline 8 & 1 & 2.020415 & -0.812846 & 0.263003 \\
\hline 9 & 1 & 1.506973 & 0.054557 & -1.190976 \\
\hline 10 & 1 & 1.956403 & 0.956683 & 0.263051 \\
\hline 11 & 6 & -0.680407 & -1.283520 & -0.095021 \\
\hline 12 & 1 & -0.149814 & -2.172664 & 0.263162 \\
\hline 13 & 1 & -1.714207 & -1.343217 & 0.262871 \\
\hline 14 & 1 & -0.706110 & -1.332397 & -1.190967 \\
\hline
\end{tabular}

Zero-point correction=

Thermal correction to Energy=

0.131999 (Hartree/Particle)

Thermal correction to Enthalpy=

0.137721

Thermal correction to Gibbs Free Energy=

0.104362

Sum of electronic and zero-point Energies=

$-158.022193$ 
Sum of electronic and thermal Energies $=$

Sum of electronic and thermal Enthalpies=

Sum of electronic and thermal Free Energies=

Pentane:

Standard orientation:

\begin{tabular}{|c|c|c|c|c|}
\hline \multirow{2}{*}{$\begin{array}{l}\text { Center } \\
\text { Number }\end{array}$} & Atomic & \multirow{2}{*}{$\begin{array}{r}\text { Atomic } \\
\text { Type }\end{array}$} & \multicolumn{2}{|c|}{ Coordinates (Angstrom } \\
\hline & Numbe & & $X$ & Z \\
\hline 1 & 6 & 2.542715 & 0.323870 & -0.000002 \\
\hline 2 & 1 & 2.586989 & 0.971447 & -0.883263 \\
\hline 3 & 1 & 2.586985 & 0.971468 & 0.883245 \\
\hline 4 & 1 & 3.444702 & -0.296860 & 0.000008 \\
\hline 5 & 6 & 1.276104 & -0.521855 & 0.000006 \\
\hline 6 & 1 & 1.275004 & -1.183428 & -0.877541 \\
\hline 7 & 1 & 1.275004 & -1.183412 & 0.877564 \\
\hline 8 & 6 & 0.000000 & 0.311389 & -0.000002 \\
\hline 9 & 1 & -0.000003 & 0.974528 & 0.877899 \\
\hline 10 & 1 & 0.000003 & 0.974524 & -0.877907 \\
\hline 11 & 6 & -1.276104 & -0.521855 & $5-0.000006$ \\
\hline 12 & 1 & -1.275003 & -1.183426 & $5 \quad 0.877542$ \\
\hline 13 & 1 & -1.275006 & -1.183414 & -0.877562 \\
\hline 14 & 6 & -2.542715 & 0.323870 & 0.000003 \\
\hline 15 & 1 & -3.444702 & -0.296860 & -0.000001 \\
\hline 16 & 1 & -2.586986 & 0.971450 & 0.883263 \\
\hline 17 & 1 & -2.586988 & 0.971465 & -0.883245 \\
\hline
\end{tabular}

Zero-point correction=

0.161054 (Hartree/Particle)

Thermal correction to Energy=

0.168175

Thermal correction to Enthalpy=

0.169119

Thermal correction to Gibbs Free Energy=

0.130649

Sum of electronic and zero-point Energies=

$-197.232106$

Sum of electronic and thermal Energies=

$-197.224985$

Sum of electronic and thermal Enthalpies=

$-197.224041$

Sum of electronic and thermal Free Energies=

$-197.262512$

Neopentane:

Standard orientation:

\begin{tabular}{|c|c|c|c|c|}
\hline \multirow{2}{*}{$\begin{array}{l}\text { Center } \\
\text { Number }\end{array}$} & Atomic & \multirow{2}{*}{$\begin{array}{r}\text { Atomic } \\
\text { Type }\end{array}$} & \multicolumn{2}{|c|}{ Coordinates (Angstroms } \\
\hline & Numbe & & $X$ & Z \\
\hline 1 & 6 & -0.662928 & 0.073560 & 1.377866 \\
\hline 2 & 1 & -1.466236 & -0.666587 & 1.470279 \\
\hline 3 & 1 & 0.062532 & -0.119364 & 2.176709 \\
\hline 4 & 1 & -1.099011 & 1.063625 & 1.554665 \\
\hline 5 & 6 & 0.000003 & 0.000009 & -0.000009 \\
\hline 6 & 6 & 0.594537 & -1.395479 & -0.206375 \\
\hline 7 & 1 & 1.077569 & -1.478576 & -1.186899 \\
\hline 8 & 1 & 1.347845 & -1.621387 & 0.557266 \\
\hline 9 & 1 & -0.180924 & -2.168187 & -0.149474 \\
\hline
\end{tabular}




$\begin{array}{rrrrrr}10 & 6 & 0 & -1.045015 & 0.274923 & -1.084342 \\ 11 & 1 & 0 & -1.489444 & 1.269526 & -0.962262 \\ 12 & 1 & 0 & -0.598702 & 0.228973 & -2.084513 \\ 13 & 1 & 0 & -1.856948 & -0.460606 & -1.046781 \\ 14 & 6 & 0 & 1.113406 & 1.046991 & -0.087144 \\ 15 & 1 & 0 & 0.717024 & 2.058845 & 0.056702 \\ 16 & 1 & 0 & 1.878209 & 0.875796 & 0.679294 \\ 17 & 1 & 0 & 1.608070 & 1.017920 & -1.064959\end{array}$

Zero-point correction=

Thermal correction to Energy=

Thermal correction to Enthalpy=

Thermal correction to Gibbs Free Energy=

Sum of electronic and zero-point Energies=

Sum of electronic and thermal Energies $=$

Sum of electronic and thermal Enthalpies=

Sum of electronic and thermal Free Energies=

Hexane:
0.159885 (Hartree/Particle)

0.166831

0.167775

\subsection{0}

$-197.236161$

$-197.229215$

$-197.228271$

$-197.265296$

Standard orientation:

\begin{tabular}{|c|c|c|c|c|}
\hline \multirow{2}{*}{$\begin{array}{l}\text { Center } \\
\text { Number }\end{array}$} & Atomic & \multirow{2}{*}{$\begin{array}{r}\text { Atomic } \\
\text { Type }\end{array}$} & \multicolumn{2}{|c|}{ Coordinates (Angstrom } \\
\hline & Numbe & & $\mathrm{X}$ & $\mathrm{Z}$ \\
\hline 1 & 6 & -3.203444 & 0.207338 & 0.000044 \\
\hline 2 & 1 & -3.292540 & 0.850318 & 0.883451 \\
\hline 3 & 1 & -3.292534 & 0.850565 & -0.883185 \\
\hline 4 & 1 & -4.060476 & -0.474372 & -0.000056 \\
\hline 5 & 6 & -1.880815 & -0.547936 & -0.000059 \\
\hline 6 & 1 & -1.833625 & -1.208248 & 0.877367 \\
\hline 7 & 1 & -1.833661 & -1.208079 & -0.877614 \\
\hline 8 & 6 & -0.665118 & 0.371704 & 0.000001 \\
\hline 9 & 1 & -0.712141 & 1.033136 & -0.877910 \\
\hline 10 & 1 & -0.712185 & 1.033087 & 0.877947 \\
\hline 11 & 6 & 0.665118 & -0.371704 & 0.000018 \\
\hline 12 & 1 & 0.712150 & -1.033160 & -0.877874 \\
\hline 13 & 1 & 0.712176 & -1.033062 & 0.877983 \\
\hline 14 & 6 & 1.880815 & 0.547936 & -0.000050 \\
\hline 15 & 1 & 1.833632 & 1.208246 & 0.877378 \\
\hline 16 & 1 & 1.833653 & 1.208081 & -0.877603 \\
\hline 17 & 6 & 3.203444 & -0.207338 & 0.000036 \\
\hline 18 & 1 & 3.292542 & -0.850534 & -0.883215 \\
\hline 19 & 1 & 4.060477 & 0.474371 & -0.000031 \\
\hline 20 & 1 & 3.292532 & -0.850350 & 0.883422 \\
\hline
\end{tabular}

Zero-point correction=

Thermal correction to Energy=

Thermal correction to Enthalpy=

Thermal correction to Gibbs Free Energy=

Sum of electronic and zero-point Energies=

Sum of electronic and thermal Energies=
0.189596 (Hartree/Particle)

$$
\begin{gathered}
0.198044 \\
0.198989
\end{gathered}
$$

0.156921

$-236.444048$

$-236.435599$ 
Sum of electronic and thermal Enthalpies=

Sum of electronic and thermal Free Energies=

22-dimethylbutane:

Standard orientation:

\begin{tabular}{|c|c|c|c|c|}
\hline \multirow{2}{*}{$\begin{array}{l}\text { Center } \\
\text { Number }\end{array}$} & Atomic & \multirow{2}{*}{$\begin{array}{l}\text { Atomic } \\
\text { Type }\end{array}$} & \multicolumn{2}{|c|}{ Coordinates (Angstrom } \\
\hline & Numbe & & $\mathrm{X}$ & Z \\
\hline 1 & 6 & -1.612735 & 0.976966 & 0.001419 \\
\hline 2 & 1 & -1.591268 & 1.621934 & 0.887603 \\
\hline 3 & 1 & -1.591447 & 1.624254 & -0.883079 \\
\hline 4 & 1 & -2.569364 & 0.442071 & 0.000811 \\
\hline 5 & 6 & -0.433566 & -0.000366 & 0.000014 \\
\hline 6 & 6 & 0.868761 & 0.824300 & 0.000684 \\
\hline 7 & 1 & 0.850310 & 1.486982 & -0.876240 \\
\hline 8 & 1 & 0.850393 & 1.485474 & 0.878751 \\
\hline 9 & 6 & 2.174140 & 0.037799 & 0.000004 \\
\hline 10 & 1 & 2.268530 & -0.600599 & -0.884636 \\
\hline 11 & 1 & 3.032405 & 0.717871 & 0.000122 \\
\hline 12 & 1 & 2.268897 & -0.601402 & 0.884020 \\
\hline 13 & 6 & -0.518117 & -0.881478 & 1.249638 \\
\hline 14 & 1 & 0.276030 & -1.634978 & 1.272167 \\
\hline 15 & 1 & -0.438245 & -0.280862 & 2.163652 \\
\hline 16 & 1 & -1.475281 & -1.414319 & 1.284157 \\
\hline 17 & 6 & -0.518693 & -0.878483 & -1.251688 \\
\hline 18 & 1 & -1.475712 & -1.411545 & -1.286846 \\
\hline 19 & 1 & -0.439640 & -0.275613 & -2.164285 \\
\hline 20 & 1 & 0.275657 & -1.631695 & -1.276622 \\
\hline
\end{tabular}

Zero-point correction=

Thermal correction to Energy=

0.188521 (Hartree/Particle)

Thermal correction to Enthalpy=

0.196813

Thermal correction to Gibbs Free Energy=

0.197757

Sum of electronic and zero-point Energies=

0.157148

Sum of electronic and thermal Energies $=$

$-236.445609$

$-236.437317$

$-236.436373$

Sum of electronic and thermal Enthalpies=

$-236.476981$

Heptane:

Standard orientation:

\begin{tabular}{|c|c|c|c|c|}
\hline \multirow{2}{*}{$\begin{array}{l}\text { Center } \\
\text { Number }\end{array}$} & Atomic & \multirow{2}{*}{$\begin{array}{l}\text { Atomic } \\
\text { Type }\end{array}$} & \multicolumn{2}{|c|}{ Coordinates (Angstroms } \\
\hline & Number & & $\mathrm{X}$ & $\mathrm{Z}$ \\
\hline 1 & 6 & -3.817626 & -0.353689 & -0.000010 \\
\hline 2 & 1 & -3.861421 & -1.001379 & -0.883295 \\
\hline 3 & 1 & -3.861410 & -1.001450 & 0.883225 \\
\hline 4 & 1 & -4.720007 & 0.266469 & 0.000021 \\
\hline 5 & 6 & -2.551390 & 0.492501 & 0.000018 \\
\hline 6 & 1 & -2.550361 & 1.154297 & -0.877460 \\
\hline 7 & 1 & -2.550362 & 1.154244 & 0.877536 \\
\hline
\end{tabular}




$\begin{array}{ccrrrr}8 & 6 & 0 & -1.274701 & -0.340042 & -0.000005 \\ 9 & 1 & 0 & -1.275226 & -1.003131 & 0.877866 \\ 10 & 1 & 0 & -1.275238 & -1.003104 & -0.877896 \\ 11 & 6 & 0 & 0.000000 & 0.494708 & -0.000002 \\ 12 & 1 & 0 & -0.000004 & 1.157310 & 0.878005 \\ 13 & 1 & 0 & 0.000004 & 1.157303 & -0.878015 \\ 14 & 6 & 0 & 1.274701 & -0.340042 & 0.000007 \\ 15 & 1 & 0 & 1.275227 & -1.003136 & -0.877860 \\ 16 & 1 & 0 & 1.275236 & -1.003100 & 0.877902 \\ 17 & 6 & 0 & 2.551390 & 0.492501 & -0.000016 \\ 18 & 1 & 0 & 2.550362 & 1.154295 & 0.877464 \\ 19 & 1 & 0 & 2.550361 & 1.154246 & -0.877533 \\ 20 & 6 & 0 & 3.817626 & -0.353689 & 0.000007 \\ 21 & 1 & 0 & 3.861414 & -1.001441 & -0.883234 \\ 22 & 1 & 0 & 4.720007 & 0.266469 & -0.000013 \\ 23 & 1 & 0 & 3.861417 & -1.001388 & 0.883286\end{array}$

Zero-point correction=

Thermal correction to Energy=

Thermal correction to Enthalpy=

Thermal correction to Gibbs Free Energy=

Sum of electronic and zero-point Energies=

Sum of electronic and thermal Energies=

Sum of electronic and thermal Enthalpies=

Sum of electronic and thermal Free Energies=

223trimethylbutane:

Standard orientation:

\begin{tabular}{|c|c|c|c|c|}
\hline \multirow{3}{*}{$\begin{array}{l}\text { Center } \\
\text { Number } \\
1\end{array}$} & \multirow{2}{*}{$\begin{array}{l}\text { Atomic } \\
\text { Number }\end{array}$} & \multirow{2}{*}{$\begin{array}{l}\text { Atomic } \\
\text { Type }\end{array}$} & \multicolumn{2}{|c|}{ Coordinates (Angstroms) } \\
\hline & & & $\mathrm{X}$ & $\mathrm{Z}$ \\
\hline & 6 & -1.329933 & -1.280432 & -0.487794 \\
\hline 2 & 1 & -1.190796 & -1.420376 & -1.566864 \\
\hline 3 & 1 & -0.950510 & -2.172608 & 0.019381 \\
\hline 4 & 1 & -2.407649 & -1.230675 & -0.296057 \\
\hline 5 & 6 & -0.640901 & -0.000006 & -0.000256 \\
\hline 6 & 6 & 0.850276 & -0.000762 & -0.449101 \\
\hline 7 & 1 & 0.822191 & -0.003825 & -1.551750 \\
\hline 8 & 6 & 1.624473 & 1.246208 & -0.019886 \\
\hline 9 & 1 & 1.742811 & 1.289253 & 1.068888 \\
\hline 10 & 1 & 2.630786 & 1.233442 & -0.452166 \\
\hline 11 & 1 & 1.144864 & 2.174622 & -0.342862 \\
\hline 12 & 6 & 1.627166 & -1.245076 & -0.016798 \\
\hline 13 & 1 & 1.674687 & -1.332110 & 1.074996 \\
\hline 14 & 1 & 1.194153 & -2.169313 & -0.409164 \\
\hline 15 & 1 & 2.659377 & -1.190218 & -0.379188 \\
\hline 16 & 6 & -0.783364 & 0.089036 & 1.522661 \\
\hline 17 & 1 & -0.370631 & 1.023417 & 1.917180 \\
\hline 18 & 1 & -1.841340 & 0.054101 & 1.806761 \\
\hline 19 & 1 & -0.282789 & -0.742902 & 2.029662 \\
\hline
\end{tabular}




$\begin{array}{llllll}20 & 6 & 0 & -1.369070 & 1.191117 & -0.635367 \\ 21 & 1 & 0 & -1.268876 & 1.180081 & -1.727499 \\ 22 & 1 & 0 & -2.438641 & 1.155627 & -0.399673 \\ 23 & 1 & 0 & -0.989521 & 2.150969 & -0.272401\end{array}$

$\begin{array}{lc}\text { Zero-point correction }= & 0.216743 \text { (Hartree/Particle) } \\ \text { Thermal correction to Energy= } & 0.226345 \\ \text { Thermal correction to Enthalpy= } & 0.227289 \\ \text { Thermal correction to Gibbs Free Energy= } & 0.183271 \\ \text { Sum of electronic and zero-point Energies }= & -275.655116 \\ \text { Sum of electronic and thermal Energies }= & -275.645514 \\ \text { Sum of electronic and thermal Enthalpies }= & -275.644569 \\ \text { Sum of electronic and thermal Free Energies }= & -275.688587 \\ \text { Octane: } & \end{array}$

Standard orientation:

\begin{tabular}{|c|c|c|c|c|}
\hline \multirow{2}{*}{$\begin{array}{l}\text { Center } \\
\text { Number }\end{array}$} & Atomic & \multirow{2}{*}{$\begin{array}{l}\text { Atomic } \\
\text { Type }\end{array}$} & \multicolumn{2}{|c|}{ Coordinates (Angstroms } \\
\hline & Numbe & & $\mathrm{X}$ & $\mathrm{Z}$ \\
\hline 1 & 6 & -4.468724 & 0.255668 & 0.000016 \\
\hline 2 & 1 & -4.538002 & 0.901144 & 0.883303 \\
\hline 3 & 1 & -4.537999 & 0.901226 & -0.883211 \\
\hline 4 & 1 & -5.345996 & -0.399532 & -0.000016 \\
\hline 5 & 6 & -3.170129 & -0.539951 & -0.000020 \\
\hline 6 & 1 & -3.142964 & -1.201268 & 0.877456 \\
\hline 7 & 1 & -3.142973 & -1.201205 & -0.877543 \\
\hline 8 & 6 & -1.927149 & 0.342121 & 0.000005 \\
\hline 9 & 1 & -1.953705 & 1.004718 & -0.877864 \\
\hline 10 & 1 & -1.953712 & 1.004683 & 0.877900 \\
\hline 11 & 6 & -0.620703 & -0.441877 & -0.000003 \\
\hline 12 & 1 & -0.594674 & -1.104143 & -0.877943 \\
\hline 13 & 1 & -0.594665 & -1.104142 & 0.877939 \\
\hline 14 & 6 & 0.620703 & 0.441877 & -0.000010 \\
\hline 15 & 1 & 0.594665 & 1.104157 & 0.877920 \\
\hline 16 & 1 & 0.594674 & 1.104128 & -0.877962 \\
\hline 17 & 6 & 1.927149 & -0.342121 & 0.000009 \\
\hline 18 & 1 & 1.953709 & -1.004722 & -0.877856 \\
\hline 19 & 1 & 1.953709 & -1.004678 & 0.877908 \\
\hline 20 & 6 & 3.170129 & 0.539951 & -0.000013 \\
\hline 21 & 1 & 3.142967 & 1.201261 & 0.877468 \\
\hline 22 & 1 & 3.142970 & 1.201212 & -0.877531 \\
\hline 23 & 6 & 4.468724 & -0.255669 & 0.000011 \\
\hline 24 & 1 & 4.538001 & -0.901210 & -0.883228 \\
\hline 25 & 1 & 5.345996 & 0.399532 & -0.000006 \\
\hline 26 & 1 & 4.537999 & -0.901160 & 0.883287 \\
\hline
\end{tabular}

Zero-point correction=

Thermal correction to Energy=

Thermal correction to Enthalpy=

Thermal correction to Gibbs Free Energy=
0.246687 (Hartree/Particle)

0.257851

0.258795

0.209432 
Sum of electronic and zero-point Energies=

$-314.867955$

Sum of electronic and thermal Energies=

Sum of electronic and thermal Enthalpies=

$-314.855846$

Sum of electronic and thermal Free Energies=

$-314.905210$

2233tetramethylbutane

Standard orientation:

\begin{tabular}{|c|c|c|c|c|}
\hline \multirow{2}{*}{$\begin{array}{l}\text { Center } \\
\text { Number }\end{array}$} & Atomic & \multirow{2}{*}{$\begin{array}{r}\text { Atomic } \\
\text { Type }\end{array}$} & \multicolumn{2}{|c|}{ Coordinates (Angstroms } \\
\hline & & & $X$ & Z \\
\hline 1 & 6 & -1.344561 & 0.589794 & -1.304021 \\
\hline 2 & 1 & -1.139274 & 1.661210 & -1.390373 \\
\hline 3 & 1 & -0.936131 & 0.094497 & -2.191472 \\
\hline 4 & 1 & -2.432600 & 0.463328 & -1.334331 \\
\hline 5 & 6 & -0.788692 & 0.000034 & -0.000018 \\
\hline 6 & 6 & 0.788838 & -0.000024 & -0.000074 \\
\hline 7 & 6 & 1.344613 & -0.310206 & 1.397133 \\
\hline 8 & 1 & 1.138467 & 0.494861 & 2.109141 \\
\hline 9 & 1 & 0.936595 & -1.239258 & 1.809584 \\
\hline 10 & 1 & 2.432749 & -0.428866 & 1.345755 \\
\hline 11 & 6 & -1.344536 & 0.834542 & 1.162698 \\
\hline 12 & 1 & -1.139722 & 0.373644 & 2.133863 \\
\hline 13 & 1 & -0.935837 & 1.850627 & 1.177508 \\
\hline 14 & 1 & -2.432523 & 0.924376 & 1.068046 \\
\hline 15 & 6 & -1.344632 & -1.424165 & 0.141381 \\
\hline 16 & 1 & -2.432717 & -1.387157 & 0.265665 \\
\hline 17 & 1 & -1.139017 & -2.034944 & -0.743047 \\
\hline 18 & 1 & -0.936541 & -1.944781 & 1.014381 \\
\hline 19 & 6 & 1.344746 & 1.365072 & -0.429957 \\
\hline 20 & 1 & 2.432702 & 1.380273 & -0.300144 \\
\hline 21 & 1 & 1.139906 & 1.578646 & -1.483492 \\
\hline 22 & 1 & 0.935769 & 2.186840 & 0.167507 \\
\hline 23 & 6 & 1.344428 & -1.055053 & -0.967232 \\
\hline 24 & 1 & 2.432414 & -0.950567 & -1.045388 \\
\hline 25 & 1 & 1.139224 & -2.074016 & -0.625051 \\
\hline 26 & 1 & 0.935307 & -0.948676 & -1.977614 \\
\hline
\end{tabular}

Zero-point correction $=$

0.245157 (Hartree/Particle)

Thermal correction to Energy=

0.255657

Thermal correction to Enthalpy=

0.256602

Thermal correction to Gibbs Free Energy=

Sum of electronic and zero-point Energies=

0.211759

Sum of electronic and thermal Energies=

$-314.863420$

$-314.852919$

Sum of electronic and thermal Enthalpies=

$-314.851975$

Sum of electronic and thermal Free Energies=

$-314.896817$

Basis set: DH-SVPD

Functional: M06

Ethane:

Standard orientation: 


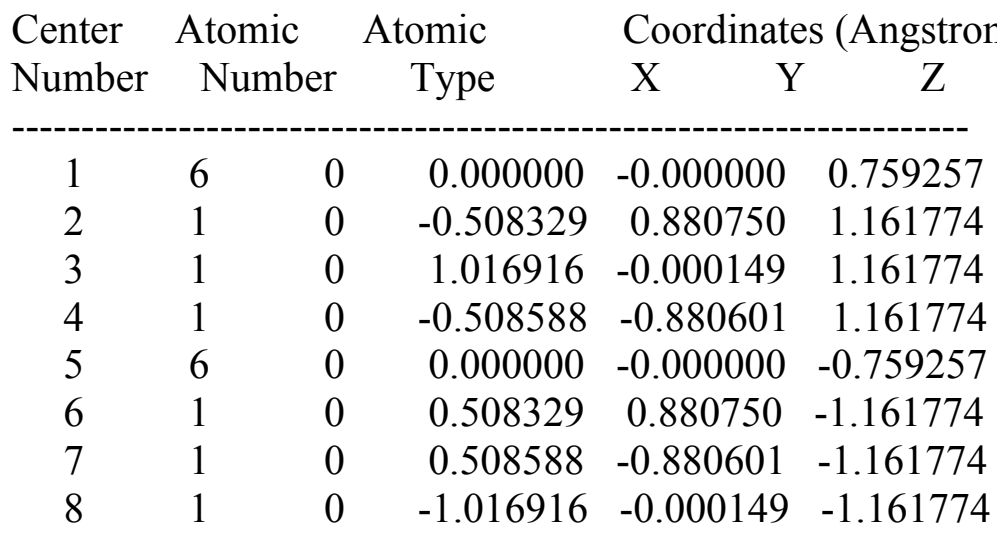

Zero-point correction $=$

Thermal correction to Energy=

Thermal correction to Enthalpy=

Thermal correction to Gibbs Free Energy=

Sum of electronic and zero-point Energies=

Sum of electronic and thermal Energies=

Sum of electronic and thermal Enthalpies=

Sum of electronic and thermal Free Energies=

Methane:

\section{Standard orientation:}

0.074668 (Hartree/Particle)

$$
0.078176
$$

0.079120

0.053241

$-79.630890$

$-79.627383$

$-79.626438$

$-79.652317$

\begin{tabular}{lrrrrr} 
Center & \multicolumn{2}{c}{ Atomic } & \multicolumn{2}{c}{ Atomic } & \multicolumn{2}{c}{ Coordinates (Angstroms) } \\
Number & Number & Type & X & Y & Z \\
\hline 1 & 6 & 0 & 0.000000 & 0.000000 & 0.000000 \\
2 & 1 & 0 & 0.629339 & 0.629339 & 0.629339 \\
3 & 1 & 0 & -0.629339 & -0.629339 & 0.629339 \\
4 & 1 & 0 & -0.629339 & 0.629339 & -0.629339 \\
5 & 1 & 0 & 0.629339 & -0.629339 & -0.629339
\end{tabular}

Zero-point correction $=$

Thermal correction to Energy=

Thermal correction to Enthalpy=

Thermal correction to Gibbs Free Energy=

Sum of electronic and zero-point Energies=

Sum of electronic and thermal Energies=

Sum of electronic and thermal Enthalpies=

Sum of electronic and thermal Free Energies=

Propane:

Standard orientation:
0.044823 (Hartree/Particle)

0.047695

0.048639

0.027506

$-40.407052$

$-40.404179$

$-40.403235$

$-40.424368$

\begin{tabular}{|c|c|c|c|c|}
\hline Center & Atomic & Atomic & Coordinate & es (Angstroms \\
\hline Number & Number & Type & $\mathrm{X}$ & Z \\
\hline 1 & 6 & -1.259942 & -0.259079 & 0.000000 \\
\hline 2 & 1 & -2.168285 & 0.349722 & -0.000039 \\
\hline 3 & 1 & -1.296952 & -0.907759 & 0.881611 \\
\hline 4 & 1 & -1.296919 & -0.907821 & -0.881567 \\
\hline
\end{tabular}




$\begin{array}{cccccc}5 & 6 & 0 & 0.000000 & 0.589828 & -0.000000 \\ 6 & 1 & 0 & -0.000000 & 1.250859 & 0.875696 \\ 7 & 1 & 0 & 0.000001 & 1.250838 & -0.875711 \\ 8 & 6 & 0 & 1.259942 & -0.259079 & 0.000000 \\ 9 & 1 & 0 & 1.296960 & -0.907746 & 0.881621 \\ 10 & 1 & 0 & 1.296908 & -0.907834 & -0.881558 \\ 11 & 1 & 0 & 2.168286 & 0.349720 & -0.000057\end{array}$

Zero-point correction=

Thermal correction to Energy=

Thermal correction to Enthalpy=

Thermal correction to Gibbs Free Energy=

Sum of electronic and zero-point Energies=

Sum of electronic and thermal Energies $=$

Sum of electronic and thermal Enthalpies=

Sum of electronic and thermal Free Energies=

Butane:
0.103361 (Hartree/Particle)

0.107910

0.108854

$-118.859035$

$-118.854486$

$-118.853542$

$-118.884692$

Standard orientation:

\begin{tabular}{|c|c|c|c|c|}
\hline \multirow{2}{*}{$\begin{array}{l}\text { Center } \\
\text { Number }\end{array}$} & Atomic & \multirow{2}{*}{$\begin{array}{l}\text { Atomic } \\
\text { Type }\end{array}$} & \multicolumn{2}{|c|}{ Coordinates (Angstroms } \\
\hline & Numbe & & $X$ & Z \\
\hline 1 & 6 & 1.939639 & -0.117625 & 0.000051 \\
\hline 2 & 1 & 2.082570 & -0.751122 & -0.881692 \\
\hline 3 & 1 & 2.082481 & -0.751060 & 0.881852 \\
\hline 4 & 1 & 2.736557 & 0.630932 & 0.000065 \\
\hline 5 & 6 & 0.559249 & 0.514881 & -0.000057 \\
\hline 6 & 1 & 0.446420 & 1.168088 & 0.876254 \\
\hline 7 & 1 & 0.446500 & 1.168004 & -0.876441 \\
\hline 8 & 6 & -0.559249 & -0.514881 & -0.000057 \\
\hline 9 & 1 & -0.446500 & -1.168002 & -0.876442 \\
\hline 10 & 1 & -0.446420 & -1.168090 & 0.876252 \\
\hline 11 & 6 & -1.939639 & 0.117625 & 0.000051 \\
\hline 12 & 1 & -2.082567 & 0.751130 & -0.881686 \\
\hline 13 & 1 & -2.736557 & -0.630932 & 0.000055 \\
\hline 14 & 1 & -2.082484 & 0.751051 & 0.881857 \\
\hline
\end{tabular}

Zero-point correction=

Thermal correction to Energy=

Thermal correction to Enthalpy=

Thermal correction to Gibbs Free Energy=

Sum of electronic and zero-point Energies=

Sum of electronic and thermal Energies=

Sum of electronic and thermal Enthalpies=

Sum of electronic and thermal Free Energies=

Isobutane:

Standard orientation:
0.131823 (Hartree/Particle)

0.137649

0.138593

$$
\begin{gathered}
0.103762 \\
-158.087419 \\
-158.081594 \\
-158.080649 \\
-158.115480
\end{gathered}
$$

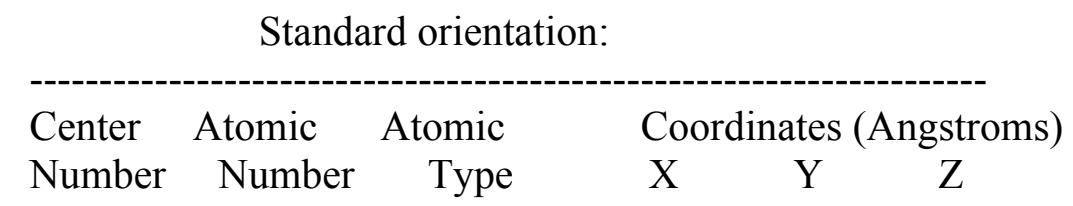




$\begin{array}{cccccc}1 & 6 & 0 & -1.435649 & 0.165481 & -0.095239 \\ 2 & 1 & 0 & -1.871508 & 1.106526 & 0.254201 \\ 3 & 1 & 0 & -1.476817 & 0.169543 & -1.191233 \\ 4 & 1 & 0 & -2.074445 & -0.651031 & 0.255307 \\ 5 & 6 & 0 & -0.000005 & -0.000006 & 0.379663 \\ 6 & 1 & 0 & 0.000001 & 0.000016 & 1.481514 \\ 7 & 6 & 0 & 0.861137 & 1.160544 & -0.095243 \\ 8 & 1 & 0 & 1.893820 & 1.067979 & 0.254962 \\ 9 & 1 & 0 & 0.885966 & 1.193651 & -1.191235 \\ 10 & 1 & 0 & 0.472900 & 2.122074 & 0.254579 \\ 11 & 6 & 0 & 0.574511 & -1.326033 & -0.095241 \\ 12 & 1 & 0 & 1.601375 & -1.470504 & 0.254506 \\ 13 & 1 & 0 & -0.021946 & -2.174097 & 0.254996 \\ 14 & 1 & 0 & 0.590689 & -1.364071 & -1.191232\end{array}$

Zero-point correction=

Thermal correction to Energy=

Thermal correction to Enthalpy=

Thermal correction to Gibbs Free Energy=

Sum of electronic and zero-point Energies=

Sum of electronic and thermal Energies $=$

Sum of electronic and thermal Enthalpies=

Sum of electronic and thermal Free Energies=
0.131404 (Hartree/Particle)

0.137113

0.138057

0.103797

$-158.090369$

$-158.084659$

$-158.083715$

$-158.117975$

Pentane:

Standard orientation:

\begin{tabular}{|c|c|c|c|c|}
\hline \multirow{2}{*}{$\begin{array}{l}\text { Center } \\
\text { Number }\end{array}$} & Atomic & \multirow{2}{*}{$\begin{array}{r}\text { Atomic } \\
\text { Type }\end{array}$} & \multicolumn{2}{|c|}{ Coordinates (Angstroms } \\
\hline & Numbe & & $\mathrm{X}$ & $\mathrm{Z}$ \\
\hline 1 & 6 & 2.528279 & 0.325603 & -0.000001 \\
\hline 2 & 1 & 2.565371 & 0.973995 & -0.881643 \\
\hline 3 & 1 & 2.565361 & 0.974030 & 0.881615 \\
\hline 4 & 1 & 3.436884 & -0.282310 & 0.000016 \\
\hline 5 & 6 & 1.270277 & -0.524445 & 0.000008 \\
\hline 6 & 1 & 1.266609 & -1.186725 & -0.876465 \\
\hline 7 & 1 & 1.266607 & -1.186700 & 0.876500 \\
\hline 8 & 6 & 0.000000 & 0.309074 & -0.000005 \\
\hline 9 & 1 & -0.000006 & 0.973545 & 0.876932 \\
\hline 10 & 1 & 0.000006 & 0.973535 & -0.876950 \\
\hline 11 & 6 & -1.270277 & -0.524445 & -0.000009 \\
\hline 12 & 1 & -1.266605 & -1.186720 & 0.876468 \\
\hline 13 & 1 & -1.266611 & -1.186704 & $4-0.876497$ \\
\hline 14 & 6 & -2.528279 & 0.325603 & 0.000006 \\
\hline 15 & 1 & -3.436884 & -0.282310 & 0.000002 \\
\hline 16 & 1 & -2.565363 & 0.974003 & 0.881642 \\
\hline 17 & 1 & -2.565369 & 0.974022 & -0.881616 \\
\hline
\end{tabular}

Zero-point correction=

Thermal correction to Energy=
0.160176 (Hartree/Particle)

0.167343 
Thermal correction to Enthalpy=

Thermal correction to Gibbs Free Energy=

Sum of electronic and zero-point Energies=

Sum of electronic and thermal Energies $=$

Sum of electronic and thermal Enthalpies=

Sum of electronic and thermal Free Energies=

Neopentane:
0.168287

0.129680

$-197.315826$

$-197.308659$

$-197.307714$

$-197.346321$

Standard orientation:

\begin{tabular}{|c|c|c|c|c|}
\hline \multirow{2}{*}{$\begin{array}{l}\text { Center } \\
\text { Number }\end{array}$} & Atomic & \multirow{2}{*}{$\begin{array}{l}\text { Atomic } \\
\text { Type }\end{array}$} & \multicolumn{2}{|c|}{ Coordinates (Angstroms } \\
\hline & Numbe & & $\mathrm{X}$ & $\mathrm{Z}$ \\
\hline 1 & 6 & -1.434305 & 0.518973 & 0.003540 \\
\hline 2 & 1 & -2.069456 & -0.079810 & 0.665286 \\
\hline 3 & 1 & -1.477753 & 1.557629 & 0.348770 \\
\hline 4 & 1 & -1.870149 & 0.482307 & -1.000729 \\
\hline 5 & 6 & -0.000006 & 0.000007 & -0.000028 \\
\hline 6 & 6 & 0.568485 & 0.067168 & 1.413773 \\
\hline 7 & 1 & 1.599893 & -0.300568 & 1.442700 \\
\hline 8 & 1 & 0.569934 & 1.095728 & 1.790516 \\
\hline 9 & 1 & -0.022673 & -0.541468 & 2.106551 \\
\hline 10 & 6 & 0.016442 & -1.445547 & -0.486446 \\
\hline 11 & 1 & -0.387031 & -1.525372 & -1.501682 \\
\hline 12 & 1 & 1.035825 & -1.846223 & -0.499700 \\
\hline 13 & 1 & -0.586609 & -2.088165 & 0.164123 \\
\hline 14 & 6 & 0.849370 & 0.859399 & -0.930861 \\
\hline 15 & 1 & 0.464290 & 0.830964 & -1.955935 \\
\hline 16 & 1 & 0.856684 & 1.905382 & -0.605716 \\
\hline 17 & 1 & 1.887132 & 0.509591 & -0.954056 \\
\hline
\end{tabular}

Zero-point correction=

Thermal correction to Energy=

Thermal correction to Enthalpy=

Thermal correction to Gibbs Free Energy=

Sum of electronic and zero-point Energies=

Sum of electronic and thermal Energies $=$

Sum of electronic and thermal Enthalpies=

Sum of electronic and thermal Free Energies=

Hexane:
0.159532 (Hartree/Particle)

$$
0.166348
$$

0.167292

$$
\begin{gathered}
0.130562 \\
-197.323123 \\
-197.316308 \\
-197.315364 \\
-197.352094
\end{gathered}
$$

Standard orientation:

\begin{tabular}{cccccc} 
Center & \multicolumn{2}{c}{ Atomic } & \multicolumn{2}{c}{ Atomic } & \multicolumn{3}{c}{ Coordinates } \\
Number & Number & Type & X & Y & Z \\
--------- & \\
1 & 6 & 0 & -3.185715 & 0.207211 & 0.000020 \\
2 & 1 & 0 & -3.268791 & 0.851373 & 0.881653 \\
3 & 1 & 0 & -3.268784 & 0.851486 & -0.881532 \\
4 & 1 & 0 & -4.048834 & -0.463739 & -0.000027 \\
5 & 6 & 0 & -1.870384 & -0.550954 & -0.000025 \\
6 & 1 & 0 & -1.819310 & -1.211545 & 0.876333
\end{tabular}




$\begin{array}{cccrrr}7 & 1 & 0 & -1.819327 & -1.211468 & -0.876442 \\ 8 & 6 & 0 & -0.662432 & 0.370768 & 0.000003 \\ 9 & 1 & 0 & -0.710523 & 1.033168 & -0.877014 \\ 10 & 1 & 0 & -0.710537 & 1.033138 & 0.877041 \\ 11 & 6 & 0 & 0.662432 & -0.370768 & 0.000003 \\ 12 & 1 & 0 & 0.710522 & -1.033170 & -0.877012 \\ 13 & 1 & 0 & 0.710537 & -1.033136 & 0.877043 \\ 14 & 6 & 0 & 1.870384 & 0.550954 & -0.000025 \\ 15 & 1 & 0 & 1.819310 & 1.211544 & 0.876333 \\ 16 & 1 & 0 & 1.819327 & 1.211469 & -0.876441 \\ 17 & 6 & 0 & 3.185715 & -0.207211 & 0.000020 \\ 18 & 1 & 0 & 3.268789 & -0.851478 & -0.881538 \\ 19 & 1 & 0 & 4.048834 & 0.463739 & -0.000016 \\ 20 & 1 & 0 & 3.268786 & -0.851382 & 0.881647\end{array}$

Zero-point correction=

Thermal correction to Energy=

Thermal correction to Enthalpy=

Thermal correction to Gibbs Free Energy=

Sum of electronic and zero-point Energies=

Sum of electronic and thermal Energies=

Sum of electronic and thermal Enthalpies=

Sum of electronic and thermal Free Energies=

22dimethylbutane

Standard orientation:

\begin{tabular}{|c|c|c|c|c|}
\hline \multirow{2}{*}{$\begin{array}{l}\text { Center } \\
\text { Number }\end{array}$} & Atomic & \multirow{2}{*}{$\begin{array}{r}\text { Atomic } \\
\text { Type }\end{array}$} & \multicolumn{2}{|c|}{ Coordinates (Angstroms } \\
\hline & Numbe & & $\mathrm{X}$ & Z \\
\hline 1 & 6 & -1.609030 & 0.969744 & 0.000868 \\
\hline 2 & 1 & -1.589875 & 1.614629 & 0.886214 \\
\hline 3 & 1 & -1.590008 & 1.615974 & -0.883499 \\
\hline 4 & 1 & -2.562939 & 0.431767 & 0.000532 \\
\hline 5 & 6 & -0.430452 & -0.000147 & 0.000016 \\
\hline 6 & 6 & 0.864115 & 0.826044 & 0.000526 \\
\hline 7 & 1 & 0.843255 & 1.488539 & -0.876455 \\
\hline 8 & 1 & 0.843335 & 1.487353 & 0.878408 \\
\hline 9 & 6 & 2.163406 & 0.038521 & -0.000017 \\
\hline 10 & 1 & 2.254939 & -0.600339 & -0.883554 \\
\hline 11 & 1 & 3.025911 & 0.710495 & 0.000078 \\
\hline 12 & 1 & 2.255232 & -0.600977 & 0.883023 \\
\hline 13 & 6 & -0.513133 & -0.877882 & 1.245857 \\
\hline 14 & 1 & 0.271978 & -1.639828 & 1.263351 \\
\hline 15 & 1 & -0.421075 & -0.277995 & 2.158501 \\
\hline 16 & 1 & -1.474699 & -1.400674 & 1.286955 \\
\hline 17 & 6 & -0.513422 & -0.875921 & -1.247195 \\
\hline 18 & 1 & -1.474984 & -1.398684 & -1.288837 \\
\hline 19 & 1 & -0.421660 & -0.274580 & -2.158908 \\
\hline 20 & 1 & 0.271688 & -1.637832 & -1.266147 \\
\hline
\end{tabular}


Zero-point correction=

Thermal correction to Energy=

Thermal correction to Enthalpy=

Thermal correction to Gibbs Free Energy=

Sum of electronic and zero-point Energies=

Sum of electronic and thermal Energies $=$

Sum of electronic and thermal Enthalpies=

Sum of electronic and thermal Free Energies= Heptane
0.187923 (Hartree/Particle)

0.196165

0.197110

$$
\begin{aligned}
& 0.156409 \\
& -236.550462 \\
& -236.542220 \\
& -236.541276 \\
& -236.581976
\end{aligned}
$$

Standard orientation:

\begin{tabular}{cccccc} 
Center & Atomic & \multicolumn{2}{c}{ Atomic } & \multicolumn{3}{c}{ Coordinates } \\
Number & Number & Type & X & Y & $Z$ \\
---------- & Z \\
1 & 6 & 0 & -3.797108 & -0.356172 & -0.000019 \\
2 & 1 & 0 & -3.833528 & -1.004695 & -0.881692 \\
3 & 1 & 0 & -3.833514 & -1.004822 & 0.881561 \\
4 & 1 & 0 & -4.706256 & 0.250924 & 0.000032 \\
5 & 6 & 0 & -2.539647 & 0.494513 & 0.000034 \\
6 & 1 & 0 & -2.536071 & 1.157085 & -0.876344 \\
7 & 1 & 0 & -2.536077 & 1.156984 & 0.876488 \\
8 & 6 & 0 & -1.268730 & -0.338092 & -0.000008 \\
9 & 1 & 0 & -1.269126 & -1.002466 & 0.876964 \\
10 & 1 & 0 & -1.269148 & -1.002416 & -0.877016 \\
11 & 6 & 0 & 0.000000 & 0.496051 & -0.000003 \\
12 & 1 & 0 & -0.000007 & 1.159760 & 0.877196 \\
13 & 1 & 0 & 0.000007 & 1.159743 & -0.877215 \\
14 & 6 & 0 & 1.268730 & -0.338091 & 0.000015 \\
15 & 1 & 0 & 1.269127 & -1.002476 & -0.876949 \\
16 & 1 & 0 & 1.269147 & -1.002407 & 0.877031 \\
17 & 6 & 0 & 2.539647 & 0.494513 & -0.000031 \\
18 & 1 & 0 & 2.536076 & 1.157081 & 0.876351 \\
19 & 1 & 0 & 2.536073 & 1.156989 & -0.876482 \\
20 & 6 & 0 & 3.797108 & -0.356172 & 0.000012 \\
21 & 1 & 0 & 3.833519 & -1.004806 & -0.881580 \\
22 & 1 & 0 & 4.706256 & 0.250924 & -0.000023 \\
23 & 1 & 0 & 3.833523 & -1.004711 & 0.881673
\end{tabular}

Zero-point correction=

Thermal correction to Energy=

Thermal correction to Enthalpy=

Thermal correction to Gibbs Free Energy=

Sum of electronic and zero-point Energies=

Sum of electronic and thermal Energies=

Sum of electronic and thermal Enthalpies=

Sum of electronic and thermal Free Energies=

223trimethylbutane

Standard orientation:
0.216854 (Hartree/Particle)

0.226758

0.227702

0.181541

$-275.772761$

$-275.762857$

$-275.761913$

$-275.808074$

Center Atomic Atomic Coordinates (Angstroms)




\begin{tabular}{cccccc} 
Number & Number & Type & X & $Y$ & $Z$ \\
\hline 1 & 6 & 0 & -1.302560 & -1.319488 & -0.393350 \\
2 & 1 & 0 & -1.144802 & -1.542994 & -1.455066 \\
3 & 1 & 0 & -0.922511 & -2.163361 & 0.189284 \\
4 & 1 & 0 & -2.382767 & -1.267742 & -0.222476 \\
5 & 6 & 0 & -0.637726 & -0.000289 & -0.000871 \\
6 & 6 & 0 & 0.845738 & -0.001701 & -0.453684 \\
7 & 1 & 0 & 0.815222 & -0.009304 & -1.557423 \\
8 & 6 & 0 & 1.616179 & 1.241829 & -0.023555 \\
9 & 1 & 0 & 1.779049 & 1.249638 & 1.059777 \\
10 & 1 & 0 & 2.603255 & 1.259683 & -0.494829 \\
11 & 1 & 0 & 1.112094 & 2.173558 & -0.292955 \\
12 & 6 & 0 & 1.624104 & -1.237814 & -0.016130 \\
13 & 1 & 0 & 1.631593 & -1.341725 & 1.074903 \\
14 & 1 & 0 & 1.224199 & -2.162645 & -0.438099 \\
15 & 1 & 0 & 2.667792 & -1.160769 & -0.335358 \\
16 & 6 & 0 & -0.777418 & 0.191932 & 1.508059 \\
17 & 1 & 0 & -0.421330 & 1.175253 & 1.830080 \\
18 & 1 & 0 & -1.829866 & 0.115147 & 1.801308 \\
19 & 1 & 0 & -0.227638 & -0.568414 & 2.072462 \\
20 & 6 & 0 & -1.389222 & 1.125731 & -0.711249 \\
21 & 1 & 0 & -1.325581 & 1.017071 & -1.799913 \\
22 & 1 & 0 & -2.449441 & 1.110502 & -0.437823 \\
23 & 1 & 0 & -1.003835 & 2.114898 & -0.449186
\end{tabular}

Zero-point correction=

Thermal correction to Energy=

Thermal correction to Enthalpy=

Thermal correction to Gibbs Free Energy=

Sum of electronic and zero-point Energies=

Sum of electronic and thermal Energies $=$

Sum of electronic and thermal Enthalpies=

0.216252 (Hartree/Particle)

0.225635

0.226579

0.183491

$-275.778668$

$-275.769285$

$-275.768341$

$-275.811430$

Octane

Standard orientation:

\begin{tabular}{|c|c|c|c|c|}
\hline \multirow{2}{*}{$\begin{array}{l}\text { Center } \\
\text { Number }\end{array}$} & Atomic & \multirow{2}{*}{$\begin{array}{r}\text { Atomic } \\
\text { Type }\end{array}$} & \multicolumn{2}{|c|}{ Coordinates (Angstrom } \\
\hline & Numbe & & $\mathrm{X}$ & Z \\
\hline 1 & 6 & -4.445653 & 0.256371 & 0.000044 \\
\hline 2 & 1 & -4.508254 & 0.902914 & 0.881704 \\
\hline 3 & 1 & -4.508260 & 0.903081 & -0.881494 \\
\hline 4 & 1 & -5.329566 & -0.386931 & -0.000014 \\
\hline 5 & 6 & -3.154828 & -0.542750 & -0.000038 \\
\hline 6 & 1 & -3.124360 & -1.204755 & 0.876310 \\
\hline 7 & 1 & -3.124391 & -1.204625 & -0.876485 \\
\hline 8 & 6 & -1.918519 & 0.340396 & 0.000005 \\
\hline 9 & 1 & -1.945643 & 1.004272 & -0.876942 \\
\hline 10 & 1 & -1.945651 & 1.004202 & 0.877004 \\
\hline
\end{tabular}




$\begin{array}{rrrrrr}11 & 6 & 0 & -0.617328 & -0.441969 & -0.000015 \\ 12 & 1 & 0 & -0.590629 & -1.105369 & -0.877138 \\ 13 & 1 & 0 & -0.590606 & -1.105369 & 0.877105 \\ 14 & 6 & 0 & 0.617328 & 0.441969 & -0.000029 \\ 15 & 1 & 0 & 0.590606 & 1.105398 & 0.877070 \\ 16 & 1 & 0 & 0.590629 & 1.105340 & -0.877173 \\ 17 & 6 & 0 & 1.918519 & -0.340396 & 0.000014 \\ 18 & 1 & 0 & 1.945650 & -1.004282 & -0.876925 \\ 19 & 1 & 0 & 1.945644 & -1.004193 & 0.877020 \\ 20 & 6 & 0 & 3.154828 & 0.542750 & -0.000026 \\ 21 & 1 & 0 & 3.124366 & 1.204741 & 0.876333 \\ 22 & 1 & 0 & 3.124384 & 1.204639 & -0.876462 \\ 23 & 6 & 0 & 4.445653 & -0.256371 & 0.000034 \\ 24 & 1 & 0 & 4.508266 & -0.903050 & -0.881526 \\ 25 & 1 & 0 & 5.329566 & 0.386931 & 0.000005 \\ 26 & 1 & 0 & 4.508248 & -0.902945 & 0.881673\end{array}$

Zero-point correction=

Thermal correction to Energy=

0.245190 (Hartree/Particle)

0.256473

Thermal correction to Enthalpy=

0.257417

Thermal correction to Gibbs Free Energy=

Sum of electronic and zero-point Energies=

0.207444

$-315.001245$

Sum of electronic and thermal Energies $=$

$-314.989962$

Sum of electronic and thermal Enthalpies $=$

$-314.989018$

Sum of electronic and thermal Free Energies $=\quad-315.038991$

2233tetramethylbutane

Standard orientation:

\begin{tabular}{cccccc} 
Center & \multicolumn{1}{c}{ Atomic } & \multicolumn{2}{c}{ Atomic } & \multicolumn{3}{c}{ Coordinates } \\
Number & Number & Type & X & Y & Z \\
- & Nutroms) \\
\hline 1 & 6 & 0 & -1.342446 & -0.190964 & -1.413090 \\
2 & 1 & 0 & -0.928620 & 0.528494 & -2.126733 \\
3 & 1 & 0 & -1.151728 & -1.196547 & -1.798566 \\
4 & 1 & 0 & -2.428100 & -0.048631 & -1.405001 \\
5 & 6 & 0 & -0.785134 & 0.000265 & 0.000096 \\
6 & 6 & 0 & 0.785091 & 0.000214 & -0.000224 \\
7 & 6 & 0 & 1.342159 & -0.124579 & 1.420463 \\
8 & 1 & 0 & 0.927835 & 0.627244 & 2.099570 \\
9 & 1 & 0 & 1.151691 & -1.111157 & 1.852456 \\
10 & 1 & 0 & 2.427738 & 0.017679 & 1.405892 \\
11 & 6 & 0 & -1.341855 & 1.319702 & 0.541684 \\
12 & 1 & 0 & -0.925462 & 1.578922 & 1.520280 \\
13 & 1 & 0 & -1.153471 & 2.155999 & -0.137478 \\
14 & 1 & 0 & -2.427094 & 1.240873 & 0.663906 \\
15 & 6 & 0 & -1.341191 & -1.128542 & 0.872262 \\
16 & 1 & 0 & -2.426812 & -1.193604 & 0.745293 \\
17 & 1 & 0 & -0.926421 & -2.105784 & 0.605422 \\
18 & 1 & 0 & -1.150097 & -0.959854 & 1.935879 \\
19 & 6 & 0 & 1.342245 & 1.292692 & -0.603000
\end{tabular}




$\begin{array}{llllll}20 & 1 & 0 & 2.427802 & 1.208834 & -0.719122 \\ 21 & 1 & 0 & 0.927804 & 1.504675 & -1.593648 \\ 22 & 1 & 0 & 1.151958 & 2.160278 & 0.035156 \\ 23 & 6 & 0 & 1.341091 & -1.168440 & -0.818279 \\ 24 & 1 & 0 & 2.426644 & -1.227872 & -0.687929 \\ 25 & 1 & 0 & 0.925942 & -2.131956 & -0.506149 \\ 26 & 1 & 0 & 1.150632 & -1.049687 & -1.888698\end{array}$

Zero-point correction=

Thermal correction to Energy=

Thermal correction to Enthalpy=

Thermal correction to Gibbs Free Energy=

Sum of electronic and zero-point Energies=

Sum of electronic and thermal Energies $=$

Sum of electronic and thermal Enthalpies=

Sum of electronic and thermal Free Energies=

Basis set: DH-SVPD

Functional: CAM-B3LYP

Ethane

Standard orientation:
0.244762 (Hartree/Particle)

0.255023

0.255967

0.211677

$-315.007160$

$-314.996899$

$-314.995955$

$-315.040246$

\begin{tabular}{|c|c|c|c|c|}
\hline \multirow{2}{*}{$\begin{array}{l}\text { Center } \\
\text { Number }\end{array}$} & \multirow{2}{*}{$\begin{array}{l}\text { Atomic } \\
\text { Number }\end{array}$} & \multirow{2}{*}{$\begin{array}{r}\text { Atomic } \\
\text { Type }\end{array}$} & \multicolumn{2}{|c|}{ Coordinates (Angstrom } \\
\hline & & & $\mathrm{X}$ & $\mathrm{Z}$ \\
\hline 1 & 6 & 0.000000 & -0.000000 & 0.762886 \\
\hline 2 & 1 & -0.508741 & 0.881194 & 1.161671 \\
\hline 3 & 1 & 1.017507 & -0.000014 & 1.161671 \\
\hline 4 & 1 & -0.508766 & -0.881179 & 1.161671 \\
\hline 5 & 6 & -0.000000 & -0.000000 & -0.762886 \\
\hline 6 & 1 & 0.508741 & 0.881194 & -1.161671 \\
\hline 7 & 1 & 0.508766 & -0.881179 & -1.161671 \\
\hline 8 & 1 & -1.017507 & -0.000014 & -1.161671 \\
\hline
\end{tabular}

Zero-point correction=

Thermal correction to Energy=

Thermal correction to Enthalpy=

0.075315 (Hartree/Particle)

Thermal correction to Gibbs Free Energy=

0.078806

0.079750

Sum of electronic and zero-point Energies=

0.053885

Sum of electronic and thermal Energies $=$

$-79.647437$

$-79.643947$

Sum of electronic and thermal Enthalpies $=$

$-79.643003$

Sum of electronic and thermal Free Energies $=\quad-79.668868$

Methane

Standard orientation:

\begin{tabular}{cccccc} 
Center & \multicolumn{2}{c}{ Atomic } & \multicolumn{2}{c}{ Atomic } & \multicolumn{2}{c}{ Coordinates (Angstroms) } \\
Number & Number & Type & X & Y & Z \\
-1 & 6 & 0 & 0.000000 & 0.000000 & 0.000000 \\
2 & 1 & 0 & 0.629392 & 0.629392 & 0.629392 \\
3 & 1 & 0 & -0.629392 & -0.629392 & 0.629392
\end{tabular}




$\begin{array}{rrrrrr}4 & 1 & 0 & -0.629392 & 0.629392 & -0.629392 \\ 5 & 1 & 0 & 0.629392 & -0.629392 & -0.629392\end{array}$

Zero-point correction $=$

Thermal correction to Energy=

Thermal correction to Enthalpy=

Thermal correction to Gibbs Free Energy=

Sum of electronic and zero-point Energies=

Sum of electronic and thermal Energies=

Sum of electronic and thermal Enthalpies=

Sum of electronic and thermal Free Energies=

Propane
0.045130 (Hartree/Particle)

0.047998

0.048942

0.027814

$-40.415961$

$-40.413093$

$-40.412149$

$-40.433277$

Standard orientation:

\begin{tabular}{|c|c|c|c|c|}
\hline Center & Atomic & Atomic & Coordinate & s (Angstrom \\
\hline Number & Numbe & Type & $X$ & Z \\
\hline 1 & 6 & -1.269532 & -0.259268 & 0.000000 \\
\hline 2 & 1 & -2.169320 & 0.361103 & -0.000014 \\
\hline 3 & 1 & -1.311573 & -0.905154 & 0.881967 \\
\hline 4 & 1 & -1.311562 & -0.905178 & -0.881949 \\
\hline 5 & 6 & 0.000000 & 0.586798 & -0.000000 \\
\hline 6 & 1 & -0.000000 & 1.244449 & 0.876260 \\
\hline 7 & 1 & 0.000001 & 1.244436 & -0.876269 \\
\hline 8 & 6 & 1.269532 & -0.259268 & 0.000000 \\
\hline 9 & 1 & 1.311586 & -0.905132 & 0.881982 \\
\hline 10 & 1 & 1.311545 & -0.905201 & -0.881934 \\
\hline 11 & 1 & 2.169321 & 0.361100 & -0.000045 \\
\hline
\end{tabular}

Zero-point correction $=$

Thermal correction to Energy=

Thermal correction to Enthalpy=

Thermal correction to Gibbs Free Energy=

Sum of electronic and zero-point Energies=

Sum of electronic and thermal Energies $=$

Sum of electronic and thermal Enthalpies=

Sum of electronic and thermal Free Energies=

Butane
0.104298 (Hartree/Particle)

0.108825

0.109770

Standard orientation:

\begin{tabular}{|c|c|c|c|c|}
\hline \multirow{2}{*}{$\begin{array}{l}\text { Center } \\
\text { Number }\end{array}$} & \multirow{2}{*}{$\begin{array}{l}\text { Atomic } \\
\text { Numbe }\end{array}$} & \multirow{2}{*}{$\begin{array}{r}\text { Atomic } \\
\text { Type }\end{array}$} & \multicolumn{2}{|c|}{ Coordinates (Angst } \\
\hline & & & $\mathbf{X}$ & $\mathbf{Z}$ \\
\hline 1 & 6 & 1.951443 & -0.119611 & 0.000029 \\
\hline 2 & 1 & 2.097078 & -0.750097 & -0.881956 \\
\hline 3 & 1 & 2.097043 & -0.750022 & 0.882072 \\
\hline 4 & 1 & 2.740455 & 0.636483 & 0.000011 \\
\hline 5 & 6 & 0.563984 & 0.513916 & -0.000032 \\
\hline 6 & 1 & 0.456585 & 1.164072 & 0.876685 \\
\hline 7 & 1 & 0.456629 & 1.164025 & -0.876791 \\
\hline
\end{tabular}




$\begin{array}{cccccc}8 & 6 & 0 & -0.563984 & -\mathbf{0 . 5 1 3 9 1 6} & -\mathbf{0 . 0 0 0 0 3 2} \\ 9 & 1 & 0 & -\mathbf{0 . 4 5 6 6 2 9} & -\mathbf{1 . 1 6 4 0 2 3} & \mathbf{- 0 . 8 7 6 7 9 2} \\ 10 & 1 & 0 & -\mathbf{0 . 4 5 6 5 8 5} & -\mathbf{1 . 1 6 4 0 7 4} & \mathbf{0 . 8 7 6 6 8 4} \\ 11 & 6 & 0 & -1.951443 & \mathbf{0 . 1 1 9 6 1 1} & \mathbf{0 . 0 0 0 0 2 9} \\ 12 & 1 & 0 & -2.097075 & \mathbf{0 . 7 5 0 1 0 4} & \mathbf{- 0 . 8 8 1 9 5 2} \\ 13 & 1 & 0 & -2.740455 & -\mathbf{0 . 6 3 6 4 8 3} & \mathbf{0 . 0 0 0 0 0 3} \\ 14 & 1 & 0 & -2.097046 & 0.750016 & \mathbf{0 . 8 8 2 0 7 7}\end{array}$

Zero-point correction $=$

Thermal correction to Energy=

Thermal correction to Enthalpy=

Thermal correction to Gibbs Free Energy=

Sum of electronic and zero-point Energies=

Sum of electronic and thermal Energies $=$

Sum of electronic and thermal Enthalpies=

Sum of electronic and thermal Free Energies=

Isobutane
0.133068 (Hartree/Particle)

$$
\mathbf{0 . 1 3 8 8 5 2}
$$

0.139796

\subsection{8}

$-158.118469$

$-158.112684$

$-158.111740$

$-158.146518$

Standard orientation:

\begin{tabular}{|c|c|c|c|c|}
\hline \multirow{3}{*}{$\begin{array}{l}\begin{array}{l}\text { Center } \\
\text { Number }\end{array} \\
-1\end{array}$} & \multirow{2}{*}{$\begin{array}{l}\text { Atomic } \\
\text { Number }\end{array}$} & \multirow{3}{*}{$\begin{array}{c}\begin{array}{c}\text { Atomic } \\
\text { Type }\end{array} \\
1.425044\end{array}$} & \multicolumn{2}{|c|}{ Coordinates (Angstr } \\
\hline & & & $\mathbf{X}$ & $\mathbf{Z}$ \\
\hline & 6 & & 0.288689 & -0.095360 \\
\hline 2 & 1 & 2.125035 & -0.471300 & 0.262101 \\
\hline 3 & 1 & 1.476937 & 0.299239 & -1.189103 \\
\hline 4 & 1 & 1.774115 & 1.261148 & 0.262166 \\
\hline 5 & 6 & 0.000006 & 0.000002 & 0.373093 \\
\hline 6 & 1 & -0.000001 & -0.000012 & 1.472467 \\
\hline 7 & 6 & -0.462507 & -1.378445 & -0.095362 \\
\hline 8 & 1 & -1.470732 & -1.604598 & 0.261976 \\
\hline 9 & 1 & -0.479166 & -1.428669 & -1.189103 \\
\hline 10 & 1 & 0.205035 & -2.167016 & 0.262271 \\
\hline 11 & 6 & -0.962537 & 1.089757 & $-\mathbf{0 . 0 9 5 3 5 9}$ \\
\hline 12 & 1 & -1.979305 & 0.905655 & 0.261915 \\
\hline 13 & 1 & -0.654562 & 2.075939 & 0.262338 \\
\hline 14 & 1 & -0.997391 & 1.129605 & -1.189098 \\
\hline
\end{tabular}

Zero-point correction $=$

Thermal correction to Energy=

Thermal correction to Enthalpy=

Thermal correction to Gibbs Free Energy=

Sum of electronic and zero-point Energies=

Sum of electronic and thermal Energies=

Sum of electronic and thermal Enthalpies=

Sum of electronic and thermal Free Energies=

Pentane
0.132636 (Hartree/Particle)
0.138334

0.139278

0.105002

$-158.120573$

$-158.114875$

$-158.113931$

$-\mathbf{1 5 8 . 1 4 8 2 0 7}$

Standard orientation:

\begin{tabular}{lccccc} 
Center & Atomic & Atomic & \multicolumn{2}{c}{ Coordinates (Angstroms) } \\
Number & Number & Type & $X$ & $Y$ & $Z$
\end{tabular}




\begin{tabular}{|c|c|c|c|c|c|}
\hline 1 & 6 & $\mathbf{0}$ & 2.544950 & 0.324987 & $-\mathbf{0 . 0 0 0 0 0 7}$ \\
\hline 2 & 1 & $\mathbf{0}$ & 2.586999 & 0.970753 & -0.882018 \\
\hline 3 & 1 & $\mathbf{0}$ & 2.586991 & 0.970825 & 0.881951 \\
\hline 4 & 1 & $\mathbf{0}$ & 3.445309 & -0.294351 & 0.000022 \\
\hline 5 & 6 & $\mathbf{0}$ & 1.277188 & -0.523223 & 0.000022 \\
\hline 6 & 1 & $\mathbf{0}$ & 1.276675 & -1.181971 & -0.876700 \\
\hline 7 & 1 & $\mathbf{0}$ & 1.276674 & -1.181907 & 0.876791 \\
\hline 8 & 6 & $\mathbf{0}$ & 0.000000 & 0.311439 & -0.000005 \\
\hline 9 & 1 & $\mathbf{0}$ & -0.000016 & 0.971754 & 0.877083 \\
\hline 10 & 1 & $\mathbf{0}$ & 0.000016 & 0.971741 & -0.877103 \\
\hline 11 & 6 & $\mathbf{0}$ & -1.277188 & -0.523223 & -0.000023 \\
\hline 12 & 1 & $\mathbf{0}$ & -1.276669 & -1.181969 & 0.876701 \\
\hline 13 & 1 & $\mathbf{0}$ & -1.276680 & -1.181909 & -0.876790 \\
\hline 14 & 6 & $\mathbf{0}$ & -2.544950 & 0.324987 & 0.000012 \\
\hline 15 & 1 & $\mathbf{0}$ & -3.445309 & -0.294351 & -0.000056 \\
\hline 16 & 1 & $\mathbf{0}$ & -2.587014 & 0.970721 & 0.882045 \\
\hline 17 & 1 & $\mathbf{0}$ & -2.586976 & $\mathbf{0 . 9 7 0 8 5 7}$ & -0.881924 \\
\hline
\end{tabular}

Zero-point correction $=$

Thermal correction to Energy=

0.161803 (Hartree/Particle)

Thermal correction to Enthalpy=

0.168891

Thermal correction to Gibbs Free Energy=

0.169836

Sum of electronic and zero-point Energies=

0.131415

$-197.353997$

$-197.346908$

Sum of electronic and thermal Energies $=$

$-197.345964$

Sum of electronic and thermal Free Energies $=\quad-197.384385$

Neopentane

Standard orientation:

\begin{tabular}{|c|c|c|c|c|c|}
\hline \multirow{3}{*}{$\begin{array}{l}\text { Center } \\
\text { Number } \\
1\end{array}$} & \multirow{2}{*}{\multicolumn{2}{|c|}{$\begin{array}{l}\text { Atomic } \\
\text { Number }\end{array}$}} & \multirow{3}{*}{$\begin{array}{c}\text { Atomic } \\
\text { Type }\end{array}$} & \multicolumn{2}{|c|}{ Coordinates (Angstr } \\
\hline & & & & $\mathbf{X}$ & $\mathbf{Z}$ \\
\hline & 6 & $\mathbf{0}$ & & -0.864823 & 0.674866 \\
\hline 2 & 1 & $\mathbf{0}$ & -2.050254 & -0.707179 & 0.216731 \\
\hline 3 & 1 & $\mathbf{0}$ & -0.827788 & -1.928201 & 0.589425 \\
\hline 4 & 1 & $\mathbf{0}$ & -1.155444 & -0.626050 & 1.738879 \\
\hline 5 & 6 & $\mathbf{0}$ & -0.000009 & -0.000001 & 0.000013 \\
\hline 6 & 6 & $\mathbf{0}$ & 0.088787 & -0.364295 & -1.485480 \\
\hline 7 & 1 & $\mathbf{0}$ & 0.846524 & 0.238623 & -1.994250 \\
\hline 8 & 1 & $\mathbf{0}$ & 0.355207 & -1.416951 & -1.617594 \\
\hline 9 & 1 & $\mathbf{0}$ & -0.866873 & -0.195521 & -1.990209 \\
\hline 10 & 6 & $\mathbf{0}$ & $-\mathbf{0 . 3 7 4 7 7 7}$ & 1.478774 & 0.141663 \\
\hline 11 & 1 & $\mathbf{0}$ & -0.445503 & 1.768088 & 1.194193 \\
\hline 12 & 1 & $\mathbf{0}$ & 0.372709 & 2.121637 & -0.332156 \\
\hline 13 & 1 & $\mathbf{0}$ & -1.340560 & 1.687027 & -0.327815 \\
\hline 14 & 6 & $\mathbf{0}$ & 1.355538 & -0.249652 & 0.668948 \\
\hline 15 & 1 & $\mathbf{0}$ & 1.322194 & 0.002723 & 1.732770 \\
\hline 16 & 1 & $\mathbf{0}$ & 1.649379 & -1.299843 & 0.583643 \\
\hline 17 & 1 & $\mathbf{0}$ & 2.140475 & 0.355631 & 0.206315 \\
\hline
\end{tabular}




$\begin{array}{lc}\text { Zero-point correction= } & \mathbf{0 . 1 6 0 7 5 1} \text { (Hartree/Particle) } \\ \text { Thermal correction to Energy= } & \mathbf{0 . 1 6 7 6 5 3} \\ \text { Thermal correction to Enthalpy }= & \mathbf{0 . 1 6 8 5 9 7} \\ \text { Thermal correction to Gibbs Free Energy= } & \mathbf{0 . 1 3 1 6 3 3} \\ \text { Sum of electronic and zero-point Energies }= & -197.358427 \\ \text { Sum of electronic and thermal Energies }= & -\mathbf{1 9 7 . 3 5 1 5 2 5} \\ \text { Sum of electronic and thermal Enthalpies }= & -\mathbf{1 9 7 . 3 5 0 5 8 1} \\ \text { Sum of electronic and thermal Free Energies }= & -\mathbf{- 1 9 7 . 3 8 7 5 4 5} \\ \text { Hexane } & \end{array}$

Standard orientation:

\begin{tabular}{|c|c|c|c|c|c|}
\hline \multirow{2}{*}{$\begin{array}{l}\text { Center } \\
\text { Number }\end{array}$} & \multicolumn{2}{|c|}{ Atomic } & \multirow{2}{*}{$\begin{array}{r}\text { Atomic } \\
\text { Type }\end{array}$} & \multicolumn{2}{|c|}{ Coordinates (Angstroms) } \\
\hline & & Number & & $\mathbf{X}$ & $\mathbf{Y}$ \\
\hline 1 & 6 & $\mathbf{0}$ & -3.205439 & 0.207950 & 0.000046 \\
\hline 2 & 1 & $\mathbf{0}$ & -3.292498 & 0.849148 & 0.882133 \\
\hline 3 & 1 & $\mathbf{0}$ & -3.292478 & 0.849439 & -0.881833 \\
\hline 4 & 1 & $\mathbf{0}$ & -4.060361 & -0.472751 & -0.000078 \\
\hline 5 & 6 & $\mathbf{0}$ & -1.881455 & -0.549356 & -0.000066 \\
\hline 6 & 1 & $\mathbf{0}$ & -1.834661 & -1.206701 & 0.876556 \\
\hline 7 & 1 & $\mathbf{0}$ & -1.834695 & -1.206506 & -0.876836 \\
\hline 8 & 6 & $\mathbf{0}$ & -0.665535 & 0.372467 & 0.000007 \\
\hline 9 & 1 & $\mathbf{0}$ & -0.712549 & 1.030926 & -0.877052 \\
\hline 10 & 1 & $\mathbf{0}$ & -0.712600 & 1.030863 & 0.877111 \\
\hline 11 & 6 & $\mathbf{0}$ & 0.665535 & -0.372467 & 0.000023 \\
\hline 12 & 1 & $\mathbf{0}$ & 0.712558 & -1.030951 & -0.877017 \\
\hline 13 & 1 & $\mathbf{0}$ & 0.712592 & -1.030838 & 0.877145 \\
\hline 14 & 6 & $\mathbf{0}$ & 1.881455 & 0.549356 & -0.000058 \\
\hline 15 & 1 & $\mathbf{0}$ & 1.834668 & 1.206698 & 0.876567 \\
\hline 16 & 1 & 0 & 1.834689 & 1.206509 & -0.876825 \\
\hline 17 & 6 & $\mathbf{0}$ & 3.205439 & -0.207950 & 0.000038 \\
\hline 18 & 1 & $\mathbf{0}$ & 3.292493 & -0.849396 & -0.881869 \\
\hline 19 & 1 & $\mathbf{0}$ & 4.060361 & 0.472751 & -0.000037 \\
\hline 20 & 1 & $\mathbf{0}$ & 3.292483 & -0.849190 & $\mathbf{0 . 8 8 2 0 9 7}$ \\
\hline
\end{tabular}

Zero-point correction $=$

Thermal correction to Energy=

Thermal correction to Enthalpy=

Thermal correction to Gibbs Free Energy=

Sum of electronic and zero-point Energies=

Sum of electronic and thermal Energies=

Sum of electronic and thermal Enthalpies=

Sum of electronic and thermal Free Energies=

22dimethylbutane
0.190502 (Hartree/Particle)

$$
0.198924
$$

0.199868

0.157815

$-236.589593$

$-236.581171$

$-236.580227$

$-236.622280$

Standard orientation:

\begin{tabular}{|c|c|c|c|}
\hline Center & Atomic & Atomic & Coordinates (Angstroms) \\
\hline Number & Number & Type & Y \\
\hline
\end{tabular}




$\begin{array}{cccccc}1 & 6 & 0 & -1.614487 & 0.977595 & 0.000392 \\ 2 & 1 & 0 & -1.592881 & 1.621575 & 0.884349 \\ 3 & 1 & 0 & -1.592894 & 1.622248 & -0.883075 \\ 4 & 1 & 0 & -2.568237 & 0.442410 & 0.000194 \\ 5 & 6 & 0 & -0.433683 & 0.000049 & 0.000006 \\ 6 & 6 & 0 & 0.869247 & 0.825400 & 0.000284 \\ 7 & 1 & 0 & 0.852173 & 1.484898 & -0.875852 \\ 8 & 1 & 0 & 0.852197 & 1.484283 & 0.876885 \\ 9 & 6 & 0 & 2.176752 & 0.037246 & 0.000005 \\ 10 & 1 & 0 & 2.269848 & -0.599649 & -0.882863 \\ 11 & 1 & 0 & 3.032435 & 0.716974 & 0.000150 \\ 12 & 1 & 0 & 2.269919 & -0.600119 & 0.882526 \\ 13 & 6 & 0 & -0.518960 & -0.881114 & 1.251411 \\ 14 & 1 & 0 & 0.271498 & -1.634835 & 1.272898 \\ 15 & 1 & 0 & -0.436347 & -0.280715 & 2.162576 \\ 16 & 1 & 0 & -1.475859 & -1.409595 & 1.286598 \\ 17 & 6 & 0 & -0.519020 & -0.880163 & -1.252069 \\ 18 & 1 & 0 & -1.475917 & -1.408624 & -1.287599 \\ 19 & 1 & 0 & -0.436465 & -0.279065 & -2.162778 \\ 20 & 1 & 0 & 0.271439 & -1.633865 & -1.274183\end{array}$

Zero-point correction=

Thermal correction to Energy=

Thermal correction to Enthalpy=

Thermal correction to Gibbs Free Energy=

Sum of electronic and zero-point Energies=

Sum of electronic and thermal Energies $=$

Sum of electronic and thermal Enthalpies=

Sum of electronic and thermal Free Energies= Heptane
0.189586 (Hartree/Particle)

$$
0.197816
$$

0.198760

$$
\begin{gathered}
0.158232 \\
-236.591351 \\
-236.583121 \\
-236.582177 \\
-236.622706
\end{gathered}
$$

\begin{tabular}{|c|c|c|c|c|}
\hline \multirow{2}{*}{$\begin{array}{l}\text { Center } \\
\text { Number }\end{array}$} & Atomic & \multirow{2}{*}{$\begin{array}{r}\text { Atomic } \\
\text { Type }\end{array}$} & \multicolumn{2}{|c|}{ Coordinates (Angstroms) } \\
\hline & Number & & $\mathrm{X}$ & Z \\
\hline 1 & 6 & -3.820039 & -0.355018 & -0.000014 \\
\hline 2 & 1 & -3.861676 & -1.000830 & -0.881994 \\
\hline 3 & 1 & -3.861666 & -1.000925 & 0.881897 \\
\hline 4 & 1 & -4.720519 & 0.263935 & 0.000026 \\
\hline 5 & 6 & -2.552786 & 0.493739 & 0.000025 \\
\hline 6 & 1 & -2.552131 & 1.152552 & -0.876668 \\
\hline 7 & 1 & -2.552135 & 1.152479 & 0.876772 \\
\hline 8 & 6 & -1.275331 & -0.340455 & -0.000005 \\
\hline 9 & 1 & -1.275812 & -1.000583 & 0.877045 \\
\hline 10 & 1 & -1.275829 & -1.000550 & -0.877081 \\
\hline 11 & 6 & 0.000000 & 0.496169 & -0.000003 \\
\hline 12 & 1 & -0.000005 & 1.155831 & 0.877132 \\
\hline 13 & 1 & 0.000005 & 1.155816 & -0.877149 \\
\hline 14 & 6 & 1.275331 & -0.340455 & 0.000011 \\
\hline 15 & 1 & 1.275815 & -1.000591 & -0.877034 \\
\hline
\end{tabular}

Standard orientation: 


$\begin{array}{rrrrrr}16 & 1 & 0 & 1.275826 & -1.000541 & 0.877092 \\ 17 & 6 & 0 & 2.552786 & 0.493739 & -0.000021 \\ 18 & 1 & 0 & 2.552134 & 1.152548 & 0.876674 \\ 19 & 1 & 0 & 2.552132 & 1.152483 & -0.876766 \\ 20 & 6 & 0 & 3.820039 & -0.355018 & 0.000009 \\ 21 & 1 & 0 & 3.861669 & -1.000912 & -0.881912 \\ 22 & 1 & 0 & 4.720519 & 0.263935 & -0.000017 \\ 23 & 1 & 0 & 3.861673 & -1.000843 & 0.881979\end{array}$

Zero-point correction=

Thermal correction to Energy=

Thermal correction to Enthalpy=

Thermal correction to Gibbs Free Energy=

Sum of electronic and zero-point Energies=

Sum of electronic and thermal Energies $=$

Sum of electronic and thermal Enthalpies=

Sum of electronic and thermal Free Energies=

223trimethylbutane
0.219200 (Hartree/Particle)

0.228974

0.229918

$-275.825210$

$-275.815436$

$-275.814491$

$-275.860205$

Standard orientation:

\begin{tabular}{|c|c|c|c|c|}
\hline \multirow{2}{*}{$\begin{array}{l}\text { Center } \\
\text { Number }\end{array}$} & Atomic & \multirow{2}{*}{$\begin{array}{l}\text { Atomic } \\
\text { Type }\end{array}$} & \multicolumn{2}{|c|}{ Coordinates (Angstrom } \\
\hline & Number & & $\mathrm{X}$ & Z \\
\hline 1 & 6 & -1.326811 & -1.291546 & -0.468664 \\
\hline 2 & 1 & -1.182784 & -1.449710 & -1.542174 \\
\hline 3 & 1 & -0.948210 & -2.171317 & 0.054965 \\
\hline 4 & 1 & -2.402904 & -1.239496 & -0.282338 \\
\hline 5 & 6 & -0.641209 & -0.000049 & -0.000783 \\
\hline 6 & 6 & 0.850598 & -0.000974 & -0.450288 \\
\hline 7 & 1 & 0.824464 & -0.004764 & -1.550359 \\
\hline 8 & 6 & 1.626439 & 1.247448 & -0.020006 \\
\hline 9 & 1 & 1.752447 & 1.283618 & 1.065669 \\
\hline 10 & 1 & 2.626697 & 1.238724 & -0.460438 \\
\hline 11 & 1 & 1.142415 & 2.174029 & -0.333048 \\
\hline 12 & 6 & 1.629990 & -1.245939 & -0.016191 \\
\hline 13 & 1 & 1.667696 & -1.337035 & 1.073228 \\
\hline 14 & 1 & 1.204417 & -2.167492 & -0.415825 \\
\hline 15 & 1 & 2.662525 & -1.184362 & -0.369440 \\
\hline 16 & 6 & -0.784601 & 0.111467 & 1.522399 \\
\hline 17 & 1 & -0.384095 & 1.054961 & 1.900473 \\
\hline 18 & 1 & -1.840042 & 0.067818 & 1.805739 \\
\hline 19 & 1 & -0.274492 & -0.704282 & 2.040804 \\
\hline 20 & 6 & -1.375860 & 1.179688 & -0.653481 \\
\hline 21 & 1 & -1.280950 & 1.149011 & -1.743331 \\
\hline 22 & 1 & -2.441694 & 1.145777 & -0.411774 \\
\hline 23 & 1 & -0.996762 & 2.143947 & -0.310068 \\
\hline
\end{tabular}

Zero-point correction=

Thermal correction to Energy=

Thermal correction to Enthalpy=
0.218074 (Hartree/Particle)

0.227566

0.228510 
Thermal correction to Gibbs Free Energy=

Sum of electronic and zero-point Energies=

Sum of electronic and thermal Energies $=$

Sum of electronic and thermal Enthalpies=

Sum of electronic and thermal Free Energies=

Octane
0.184838

$-275.824400$

$-275.814908$

$-275.813964$

$-275.857636$

Standard orientation:

\begin{tabular}{|c|c|c|c|c|}
\hline \multirow{2}{*}{$\begin{array}{l}\text { Center } \\
\text { Number }\end{array}$} & Atomic & \multirow{2}{*}{$\begin{array}{l}\text { Atomic } \\
\text { Type }\end{array}$} & \multicolumn{2}{|c|}{ Coordinates (Angstroms } \\
\hline & Numbe & & $\mathrm{X}$ & $\mathrm{Z}$ \\
\hline 1 & 6 & -4.471601 & 0.256498 & 0.000022 \\
\hline 2 & 1 & -4.538775 & 0.900166 & 0.882003 \\
\hline 3 & 1 & -4.538772 & 0.900274 & -0.881883 \\
\hline 4 & 1 & -5.346888 & -0.397602 & -0.000020 \\
\hline 5 & 6 & -3.171724 & -0.541380 & -0.000026 \\
\hline 6 & 1 & -3.144904 & -1.199731 & 0.876666 \\
\hline 7 & 1 & -3.144916 & -1.199649 & -0.876780 \\
\hline 8 & 6 & -1.928222 & 0.342631 & 0.000005 \\
\hline 9 & 1 & -1.954745 & 1.002263 & -0.877045 \\
\hline 10 & 1 & -1.954757 & 1.002220 & 0.877087 \\
\hline 11 & 6 & -0.620905 & -0.442922 & -0.000002 \\
\hline 12 & 1 & -0.594838 & -1.102255 & -0.877080 \\
\hline 13 & 1 & -0.594826 & -1.102248 & 0.877080 \\
\hline 14 & 6 & 0.620905 & 0.442922 & -0.000013 \\
\hline 15 & 1 & 0.594827 & 1.102271 & 0.877052 \\
\hline 16 & 1 & 0.594837 & 1.102232 & -0.877108 \\
\hline 17 & 6 & 1.928222 & -0.342631 & 0.000012 \\
\hline 18 & 1 & 1.954751 & -1.002270 & -0.877032 \\
\hline 19 & 1 & 1.954751 & -1.002213 & 0.877099 \\
\hline 20 & 6 & 3.171724 & 0.541380 & -0.000016 \\
\hline 21 & 1 & 3.144909 & 1.199720 & 0.876685 \\
\hline 22 & 1 & 3.144912 & 1.199660 & -0.876762 \\
\hline 23 & 6 & 4.471601 & -0.256498 & 0.000014 \\
\hline 24 & 1 & 4.538775 & -0.900251 & -0.881906 \\
\hline 25 & 1 & 5.346888 & 0.397602 & -0.000008 \\
\hline 26 & 1 & 4.538772 & -0.900189 & 0.881979 \\
\hline
\end{tabular}

Zero-point correction=

Thermal correction to Energy=

Thermal correction to Enthalpy=

Thermal correction to Gibbs Free Energy=

Sum of electronic and zero-point Energies $=$

Sum of electronic and thermal Energies $=$

Sum of electronic and thermal Enthalpies=

Sum of electronic and thermal Free Energies=

2233tetramethylbutane
0.247885 (Hartree/Particle)

0.259009

0.259953

$-315.060839$

$-315.049715$

$-315.048771$

$-315.098098$

Standard orientation:

Center Atomic Atomic Coordinates (Angstroms) 


\begin{tabular}{cccccc} 
Number & Number & Type & $X$ & $Y$ & $Z$ \\
\hline 1 & 6 & 0 & -1.346751 & 1.416791 & 0.212782 \\
2 & 1 & 0 & -1.144606 & 1.789079 & 1.219009 \\
3 & 1 & 0 & -0.936729 & 2.134876 & -0.501638 \\
4 & 1 & 0 & -2.431971 & 1.410647 & 0.079743 \\
5 & 6 & 0 & -0.789222 & 0.000035 & -0.000099 \\
6 & 6 & 0 & 0.789192 & -0.000114 & -0.000087 \\
7 & 6 & 0 & 1.346848 & -1.429957 & 0.088716 \\
8 & 1 & 0 & 1.145755 & -1.887840 & 1.059208 \\
9 & 1 & 0 & 0.936040 & -2.083548 & -0.684648 \\
10 & 1 & 0 & 2.431928 & -1.412309 & -0.044453 \\
11 & 6 & 0 & -1.347026 & -0.892467 & 1.120470 \\
12 & 1 & 0 & -1.146397 & -1.950204 & 0.939193 \\
13 & 1 & 0 & -0.935934 & -0.633752 & 2.099274 \\
14 & 1 & 0 & -2.432044 & -0.772863 & 1.182597 \\
15 & 6 & 0 & -1.347327 & -0.523972 & -1.333241 \\
16 & 1 & 0 & -2.432499 & -0.636232 & -1.260875 \\
17 & 1 & 0 & -1.145698 & 0.161370 & -2.158795 \\
18 & 1 & 0 & -0.937392 & -1.501626 & -1.598152 \\
19 & 6 & 0 & 1.346946 & 0.791623 & 1.193844 \\
20 & 1 & 0 & 2.431983 & 0.667108 & 1.245255 \\
21 & 1 & 0 & 1.146214 & 1.861116 & 1.105176 \\
22 & 1 & 0 & 0.935890 & 0.448767 & 2.146474 \\
23 & 6 & 0 & 1.347323 & 0.637994 & -1.282523 \\
24 & 1 & 0 & 2.432290 & 0.745098 & -1.199930 \\
25 & 1 & 0 & 1.147221 & 0.026367 & -2.164445 \\
26 & 1 & 0 & 0.936057 & 1.634354 & -1.462172 \\
----------------------------------------------------------
\end{tabular}

Zero-point correction=

Thermal correction to Energy=

0.246769 (Hartree/Particle)

Thermal correction to Enthalpy=

0.257144

0.258088

Thermal correction to Gibbs Free Energy=

0.213474

Sum of electronic and zero-point Energies=

$-315.056340$

Sum of electronic and thermal Energies $=$

$-315.045966$

$-315.045022$

Sum of electronic and thermal Free Energies $=\quad-315.089636$

Basis set DH-SVPD

Functonal: wB97X-D

Ethane

Standard orientation:

\begin{tabular}{|c|c|c|c|c|}
\hline Center & Atomic & Atomic & Coordinate & s (Angstror \\
\hline Number & Numbe & Type & $\mathrm{X}$ & $\mathrm{Z}$ \\
\hline 1 & 6 & 0.000000 & -0.000000 & 0.763403 \\
\hline 2 & 1 & 0.509618 & 0.882708 & 1.161814 \\
\hline 3 & 1 & 0.509639 & -0.882696 & 1.161814 \\
\hline 4 & 1 & -1.019256 & -0.000012 & 1.161814 \\
\hline
\end{tabular}




$\begin{array}{rrrrrr}5 & 6 & 0 & -0.000000 & -0.000000 & -0.763403 \\ 6 & 1 & 0 & 1.019256 & -0.000012 & -1.161814 \\ 7 & 1 & 0 & -0.509639 & -0.882696 & -1.161814 \\ 8 & 1 & 0 & -0.509618 & 0.882708 & -1.161814\end{array}$

Zero-point correction=

Thermal correction to Energy=

Thermal correction to Enthalpy=

Thermal correction to Gibbs Free Energy=

Sum of electronic and zero-point Energies=

Sum of electronic and thermal Energies $=$

Sum of electronic and thermal Enthalpies=

Sum of electronic and thermal Free Energies=

Methane
0.075266 (Hartree/Particle)

0.078752

0.079697

0.053839

$-79.678265$

$-79.674779$

$-79.673834$

$-79.699692$

Standard orientation:

\begin{tabular}{cccccc} 
Center & \multicolumn{2}{c}{ Atomic } & Atomic & \multicolumn{2}{c}{ Coordinates (Angstrom } \\
Number & Number & Type & X & Y & Z \\
-1 & 6 & 0 & 0.000000 & 0.000000 & 0.000000 \\
2 & 1 & 0 & 0.630259 & 0.630259 & 0.630259 \\
3 & 1 & 0 & -0.630259 & -0.630259 & 0.630259 \\
4 & 1 & 0 & -0.630259 & 0.630259 & -0.630259 \\
5 & 1 & 0 & 0.630259 & -0.630259 & -0.630259
\end{tabular}

Zero-point correction=

Thermal correction to Energy=

Thermal correction to Enthalpy=

Thermal correction to Gibbs Free Energy=

Sum of electronic and zero-point Energies=

Sum of electronic and thermal Energies=

Sum of electronic and thermal Enthalpies=

Sum of electronic and thermal Free Energies=

Propane
0.045080 (Hartree/Particle)

0.047949

0.048893

0.027761

$-40.433266$

$-40.430398$

$-40.429454$

$-40.450586$

Standard orientation:

\begin{tabular}{|c|c|c|c|c|}
\hline \multirow{2}{*}{$\begin{array}{l}\text { Center } \\
\text { Number }\end{array}$} & Atomic & \multirow{2}{*}{$\begin{array}{l}\text { Atomic } \\
\text { Type }\end{array}$} & \multicolumn{2}{|c|}{ Coordinates (Angstroms } \\
\hline & Numbe & & $\mathrm{X}$ & Z \\
\hline 1 & 6 & -1.270362 & -0.259701 & 0.000000 \\
\hline 2 & 1 & -2.171032 & 0.361952 & -0.000025 \\
\hline 3 & 1 & -1.311565 & -0.906245 & 0.883479 \\
\hline 4 & 1 & -1.311544 & -0.906285 & -0.883450 \\
\hline 5 & 6 & 0.000000 & 0.587632 & 0.000000 \\
\hline 6 & 1 & -0.000001 & 1.245899 & 0.877476 \\
\hline 7 & 1 & 0.000001 & 1.245883 & -0.877487 \\
\hline 8 & 6 & 1.270362 & -0.259701 & 0.000000 \\
\hline 9 & 1 & 1.311584 & -0.906215 & 0.883500 \\
\hline 10 & 1 & 1.311521 & -0.906315 & -0.883429 \\
\hline 11 & 1 & 2.171034 & 0.361949 & -0.000068 \\
\hline
\end{tabular}




$\begin{array}{lc}\text { Zero-point correction= } & 0.104215 \text { (Hartree/Particle) } \\ \text { Thermal correction to Energy= } & 0.108735 \\ \text { Thermal correction to Enthalpy= } & 0.109679 \\ \text { Thermal correction to Gibbs Free Energy= } & 0.078557 \\ \text { Sum of electronic and zero-point Energies }= & -118.927694 \\ \text { Sum of electronic and thermal Energies }= & -118.923174 \\ \text { Sum of electronic and thermal Enthalpies }= & -118.922230 \\ \text { Sum of electronic and thermal Free Energies }= & -118.953351 \\ \text { Butane } & \end{array}$

Standard orientation:

\begin{tabular}{cccccc} 
Center & \multicolumn{2}{c}{ Atomic } & \multicolumn{2}{c}{ Atomic } & \multicolumn{3}{c}{ Coordinates (Angstroms) } \\
Number & Number & Type & X & Y & $Z$ \\
-1 & 6 & 0 & 1.952513 & -0.119977 & 0.000037 \\
2 & 1 & 0 & 2.097002 & -0.751307 & -0.883497 \\
3 & 1 & 0 & 2.096950 & -0.751226 & 0.883637 \\
4 & 1 & 0 & 2.742372 & 0.637340 & 0.000025 \\
5 & 6 & 0 & 0.564298 & 0.514822 & -0.000042 \\
6 & 1 & 0 & 0.456946 & 1.165758 & 0.877892 \\
7 & 1 & 0 & 0.457003 & 1.165695 & -0.878029 \\
8 & 6 & 0 & -0.564298 & -0.514822 & -0.000042 \\
9 & 1 & 0 & -0.457003 & -1.165694 & -0.878030 \\
10 & 1 & 0 & -0.456947 & -1.165760 & 0.877891 \\
11 & 6 & 0 & -1.952513 & 0.119977 & 0.000037 \\
12 & 1 & 0 & -2.096999 & 0.751314 & -0.883492 \\
13 & 1 & 0 & -2.742372 & -0.637340 & 0.000016 \\
14 & 1 & 0 & -2.096952 & 0.751218 & 0.883642
\end{tabular}

Zero-point correction=

Thermal correction to Energy=

Thermal correction to Enthalpy=

Thermal correction to Gibbs Free Energy=

Sum of electronic and zero-point Energies=

Sum of electronic and thermal Energies $=$

Sum of electronic and thermal Enthalpies=

Sum of electronic and thermal Free Energies=
0.132975 (Hartree/Particle)

0.138763

0.139707
0.104911

$-158.177358$

$-158.171570$

$-158.170626$

$-158.205422$ Isobutane

Standard orientation:

\begin{tabular}{|c|c|c|c|c|}
\hline \multirow{2}{*}{$\begin{array}{l}\text { Center } \\
\text { Number }\end{array}$} & Atomic & \multirow{2}{*}{$\begin{array}{l}\text { Atomic } \\
\text { Type }\end{array}$} & \multicolumn{2}{|c|}{ Coordinates (Angstroms) } \\
\hline & Number & & $\mathrm{X}$ & Z \\
\hline 1 & 6 & -1.286046 & -0.681409 & -0.095543 \\
\hline 2 & 1 & -2.173676 & -0.150012 & 0.262981 \\
\hline 3 & 1 & -1.332256 & -0.705629 & -1.191038 \\
\hline 4 & 1 & -1.344657 & -1.714430 & 0.262564 \\
\hline 5 & 6 & 0.000013 & 0.000013 & 0.373704 \\
\hline 6 & 1 & 0.000035 & 0.000001 & 1.474157 \\
\hline
\end{tabular}




$\begin{array}{cccccc}7 & 6 & 0 & 0.052902 & 1.454452 & -0.095548 \\ 8 & 1 & 0 & 0.957279 & 1.957291 & 0.262321 \\ 9 & 1 & 0 & 0.054277 & 1.506549 & -1.191039 \\ 10 & 1 & 0 & -0.812085 & 2.021839 & 0.263173 \\ 11 & 6 & 0 & 1.233148 & -0.773040 & -0.095543 \\ 12 & 1 & 0 & 2.157084 & -0.307382 & 0.262673 \\ 13 & 1 & 0 & 1.216633 & -1.807504 & 0.262829 \\ 14 & 1 & 0 & 1.277266 & -0.800822 & -1.191038\end{array}$

Zero-point correction=

Thermal correction to Energy=

Thermal correction to Enthalpy=

Thermal correction to Gibbs Free Energy=

Sum of electronic and zero-point Energies=

Sum of electronic and thermal Energies $=$

Sum of electronic and thermal Enthalpies=

Sum of electronic and thermal Free Energies=

Pentane
0.132602 (Hartree/Particle)

0.138251

0.139195

0.105029

$-158.179722$

$-158.174073$

$-158.173129$

$-158.207295$

Standard orientation:

\begin{tabular}{cccccc} 
Center & Atomic & \multicolumn{2}{c}{ Atomic } & \multicolumn{3}{c}{ Coordinates } \\
Number & Number & Type & X & $Y$ & $Z$ \\
-1 & 6 & 0 & 2.546006 & 0.325458 & -0.000009 \\
2 & 1 & 0 & 2.586598 & 0.971892 & -0.883549 \\
3 & 1 & 0 & 2.586606 & 0.971924 & 0.883508 \\
4 & 1 & 0 & 3.447499 & -0.294794 & -0.000001 \\
5 & 6 & 0 & 1.277801 & -0.524162 & 0.000013 \\
6 & 1 & 0 & 1.277026 & -1.183549 & -0.878012 \\
7 & 1 & 0 & 1.277036 & -1.183516 & 0.878063 \\
8 & 6 & 0 & 0.000000 & 0.312287 & 0.000007 \\
9 & 1 & 0 & -0.000011 & 0.973398 & 0.878394 \\
10 & 1 & 0 & 0.000011 & 0.973410 & -0.878371 \\
11 & 6 & 0 & -1.277801 & -0.524162 & -0.000013 \\
12 & 1 & 0 & -1.277032 & -1.183555 & 0.878009 \\
13 & 1 & 0 & -1.277029 & -1.183511 & -0.878066 \\
14 & 6 & 0 & -2.546006 & 0.325458 & 0.000003 \\
15 & 1 & 0 & -3.447499 & -0.294794 & -0.000056 \\
16 & 1 & 0 & -2.586622 & 0.971855 & 0.883568 \\
17 & 1 & 0 & -2.586582 & 0.971961 & -0.883489
\end{tabular}

Zero-point correction=

Thermal correction to Energy=

Thermal correction to Enthalpy=

Thermal correction to Gibbs Free Energy=

Sum of electronic and zero-point Energies=

Sum of electronic and thermal Energies=

Sum of electronic and thermal Enthalpies=

Sum of electronic and thermal Free Energies=

Neopentane
0.161683 (Hartree/Particle)

$$
0.168759
$$

0.169704

$$
\begin{aligned}
& 0.131308 \\
& -197.427091 \\
& -197.420015 \\
& -197.419071 \\
& -197.457467
\end{aligned}
$$


Standard orientation:

\begin{tabular}{|c|c|c|c|c|c|}
\hline \multirow{2}{*}{$\begin{array}{l}\text { Center } \\
\text { Number }\end{array}$} & \multicolumn{2}{|l|}{ Atomic } & & \multicolumn{2}{|c|}{ Coordinates (Angstroms) } \\
\hline & Numbe & & & $\mathrm{X}$ & $\mathrm{Z}$ \\
\hline 1 & 6 & 0 & -0.154618 & -1.519361 & 0.142191 \\
\hline 2 & 1 & 0 & -0.149765 & -1.819033 & 1.195992 \\
\hline 3 & 1 & 0 & -1.096584 & -1.862642 & -0.299612 \\
\hline 4 & 1 & 0 & 0.663352 & -2.047359 & -0.360259 \\
\hline 5 & 6 & 0 & -0.000005 & -0.000003 & 0.000008 \\
\hline 6 & 6 & 0 & -1.162069 & 0.701255 & 0.714443 \\
\hline 7 & 1 & 0 & -1.077692 & 1.790228 & 0.628727 \\
\hline 8 & 1 & 0 & -2.125262 & 0.404767 & 0.284703 \\
\hline 9 & 1 & 0 & -1.178800 & 0.449210 & 1.780517 \\
\hline 10 & 6 & 0 & 1.327543 & 0.439967 & 0.629789 \\
\hline 11 & 1 & 0 & 2.177265 & -0.046295 & 0.138017 \\
\hline 12 & 1 & 0 & 1.464975 & 1.523408 & 0.542741 \\
\hline 13 & 1 & 0 & 1.363509 & 0.181944 & 1.693961 \\
\hline 14 & 6 & 0 & -0.010862 & 0.378133 & -1.486425 \\
\hline 15 & 1 & 0 & 0.810476 & -0.109473 & -2.023051 \\
\hline 16 & 1 & 0 & -0.949522 & 0.075040 & -1.963259 \\
\hline 17 & 1 & 0 & 0.098113 & 1.460258 & -1.618511 \\
\hline \multicolumn{4}{|c|}{ Zero-point correction $=$} & \multicolumn{2}{|c|}{0.160676 (Hartree/Particle) } \\
\hline \multicolumn{4}{|c|}{ Thermal correction to Energy= } & \multicolumn{2}{|c|}{0.167505} \\
\hline \multicolumn{4}{|c|}{ Thermal correction to Enthalpy= } & \multicolumn{2}{|c|}{0.168450} \\
\hline \multicolumn{5}{|c|}{ Thermal correction to Gibbs Free Energy= } & 0.131639 \\
\hline \multicolumn{5}{|c|}{ Sum of electronic and zero-point Energies= } & -197.432652 \\
\hline \multicolumn{5}{|c|}{ Sum of electronic and thermal Energies $=$} & -197.425822 \\
\hline \multicolumn{5}{|c|}{ Sum of electronic and thermal Enthalpies $=$} & -197.424878 \\
\hline \multicolumn{5}{|c|}{ Sum of electronic and thermal Free Energies $=$} & -197.461688 \\
\hline
\end{tabular}

Hexane

Standard orientation:

\begin{tabular}{|c|c|c|c|c|}
\hline \multirow{2}{*}{$\begin{array}{l}\text { Center } \\
\text { Number }\end{array}$} & Atomic & \multirow{2}{*}{$\begin{array}{r}\text { Atomic } \\
\text { Type }\end{array}$} & \multicolumn{2}{|c|}{ Coordinates (Angstroms } \\
\hline & Numbe & & $\mathrm{X}$ & Z \\
\hline 1 & 6 & -3.206681 & 0.208332 & 0.000048 \\
\hline 2 & 1 & -3.292335 & 0.850312 & 0.883655 \\
\hline 3 & 1 & -3.292327 & 0.850564 & -0.883379 \\
\hline 4 & 1 & -4.062675 & -0.473355 & -0.000055 \\
\hline 5 & 6 & -1.882122 & -0.550259 & -0.000058 \\
\hline 6 & 1 & -1.834982 & -1.208232 & 0.877846 \\
\hline 7 & 1 & -1.835021 & -1.208066 & -0.878088 \\
\hline 8 & 6 & -0.665777 & 0.373344 & -0.000002 \\
\hline 9 & 1 & -0.712473 & 1.032577 & -0.878389 \\
\hline 10 & 1 & -0.712507 & 1.032529 & 0.878419 \\
\hline 11 & 6 & 0.665777 & -0.373344 & 0.000011 \\
\hline 12 & 1 & 0.712477 & -1.032598 & -0.878361 \\
\hline 13 & 1 & 0.712503 & -1.032508 & 0.878447 \\
\hline
\end{tabular}




$\begin{array}{rrrrrr}14 & 6 & 0 & 1.882122 & 0.550259 & -0.000051 \\ 15 & 1 & 0 & 1.834985 & 1.208230 & 0.877855 \\ 16 & 1 & 0 & 1.835018 & 1.208068 & -0.878080 \\ 17 & 6 & 0 & 3.206681 & -0.208332 & 0.000042 \\ 18 & 1 & 0 & 3.292340 & -0.850530 & -0.883408 \\ 19 & 1 & 0 & 4.062675 & 0.473355 & -0.000020 \\ 20 & 1 & 0 & 3.292321 & -0.850346 & 0.883626\end{array}$

Zero-point correction=

Thermal correction to Energy=

Thermal correction to Enthalpy=

Thermal correction to Gibbs Free Energy=

Sum of electronic and zero-point Energies=

Sum of electronic and thermal Energies $=$

Sum of electronic and thermal Enthalpies=

Sum of electronic and thermal Free Energies=

22dimethylbutane
0.190372 (Hartree/Particle)

0.198793

0.199737

0.157651

$-236.676875$

$-236.668454$

$-236.667510$

$-236.709596$

Standard orientation:

\begin{tabular}{cccccc} 
Center & Atomic & \multicolumn{2}{c}{ Atomic } & \multicolumn{3}{c}{ Coordinates } \\
Number & Number & Type & X & Y & $Z$ \\
- & Nustroms) & Z \\
\hline 1 & 6 & 0 & -1.618258 & 0.976314 & 0.000695 \\
2 & 1 & 0 & -1.597625 & 1.620925 & 0.886370 \\
3 & 1 & 0 & -1.597746 & 1.621990 & -0.884207 \\
4 & 1 & 0 & -2.572260 & 0.438057 & 0.000436 \\
5 & 6 & 0 & -0.434029 & 0.000156 & 0.000012 \\
6 & 6 & 0 & 0.868680 & 0.828682 & 0.000375 \\
7 & 1 & 0 & 0.851887 & 1.489043 & -0.877170 \\
8 & 1 & 0 & 0.851938 & 1.488205 & 0.878555 \\
9 & 6 & 0 & 2.175849 & 0.038622 & -0.000013 \\
10 & 1 & 0 & 2.266546 & -0.599125 & -0.884589 \\
11 & 1 & 0 & 3.033519 & 0.717978 & 0.000107 \\
12 & 1 & 0 & 2.266720 & -0.599653 & 0.884165 \\
13 & 6 & 0 & -0.516284 & -0.882659 & 1.252307 \\
14 & 1 & 0 & 0.272876 & -1.640610 & 1.267886 \\
15 & 1 & 0 & -0.425796 & -0.282087 & 2.164609 \\
16 & 1 & 0 & -1.476238 & -1.408761 & 1.291913 \\
17 & 6 & 0 & -0.516557 & -0.881148 & -1.253337 \\
18 & 1 & 0 & -1.476520 & -1.407207 & -1.293342 \\
19 & 1 & 0 & -0.426298 & -0.279464 & -2.164927 \\
20 & 1 & 0 & 0.272589 & -1.639090 & -1.270047
\end{tabular}

Zero-point correction=

0.189236 (Hartree/Particle)

Thermal correction to Energy=

Thermal correction to Enthalpy=

0.197500

0.198444

Thermal correction to Gibbs Free Energy=

0.157689

Sum of electronic and zero-point Energies=

$-236.681222$

Sum of electronic and thermal Energies $=$

$-236.672958$

Sum of electronic and thermal Enthalpies=

$-236.672014$ 
Sum of electronic and thermal Free Energies=

Heptane

Standard orientation:

\begin{tabular}{|c|c|c|c|c|c|}
\hline \multirow{2}{*}{$\begin{array}{l}\text { Center } \\
\text { Number }\end{array}$} & \multicolumn{2}{|c|}{ Atomic } & \multirow{2}{*}{$\begin{array}{l}\text { Atomic } \\
\text { Type }\end{array}$} & \multicolumn{2}{|c|}{ Coordinates (Angstrom } \\
\hline & & & & $\mathrm{X}$ & Z \\
\hline 1 & 6 & 0 & -3.821161 & -0.355634 & -0.000008 \\
\hline 2 & 1 & 0 & -3.861260 & -1.002114 & -0.883490 \\
\hline 3 & 1 & 0 & -3.861257 & -1.002156 & 0.883445 \\
\hline 4 & 1 & 0 & -4.722824 & 0.264123 & 0.000009 \\
\hline 5 & 6 & 0 & -2.553578 & 0.494663 & 0.000011 \\
\hline 6 & 1 & 0 & -2.552664 & 1.154084 & -0.877977 \\
\hline 7 & 1 & 0 & -2.552667 & 1.154051 & 0.878024 \\
\hline 8 & 6 & 0 & -1.275567 & -0.341203 & -0.000001 \\
\hline 9 & 1 & 0 & -1.275641 & -1.002046 & 0.878384 \\
\hline 10 & 1 & 0 & -1.275647 & -1.002033 & -0.878395 \\
\hline 11 & 6 & 0 & 0.000000 & 0.497205 & -0.000001 \\
\hline 12 & 1 & 0 & -0.000002 & 1.157522 & 0.878516 \\
\hline 13 & 1 & 0 & 0.000002 & 1.157513 & -0.878525 \\
\hline 14 & 6 & 0 & 1.275567 & -0.341203 & 0.000006 \\
\hline 15 & 1 & 0 & 1.275641 & -1.002052 & -0.878375 \\
\hline 16 & 1 & 0 & 1.275647 & -1.002027 & 0.878405 \\
\hline 17 & 6 & 0 & 2.553578 & 0.494663 & -0.000010 \\
\hline 18 & 1 & 0 & 2.552666 & 1.154083 & 0.877980 \\
\hline 19 & 1 & 0 & 2.552665 & 1.154053 & -0.878022 \\
\hline 20 & 6 & 0 & 3.821161 & -0.355634 & 0.000003 \\
\hline 21 & 1 & 0 & 3.861258 & -1.002149 & -0.883454 \\
\hline 22 & 1 & 0 & 4.722824 & 0.264123 & -0.000008 \\
\hline 23 & 1 & 0 & 3.861259 & -1.002121 & 0.883481 \\
\hline
\end{tabular}

Zero-point correction $=$

Thermal correction to Energy=

Thermal correction to Enthalpy=

Thermal correction to Gibbs Free Energy=

Sum of electronic and zero-point Energies=

Sum of electronic and thermal Energies=

Sum of electronic and thermal Enthalpies=

Sum of electronic and thermal Free Energies=

223trimethylbutane
0.219045 (Hartree/Particle)

0.228809

0.229753

0.184027

$-275.926706$

$-275.916942$

$-275.915998$

$-275.961724$

Standard orientation:

\begin{tabular}{cccccc} 
Center & \multicolumn{2}{c}{ Atomic } & \multicolumn{2}{c}{ Atomic } & \multicolumn{2}{c}{ Coordinates (Angstroms) } \\
Number & Number & Type & X & Y & Z \\
- \hdashline 1 & 6 & 0 & -1.310606 & -1.324678 & -0.400296 \\
2 & 1 & 0 & -1.151548 & -1.544400 & -1.462541 \\
3 & 1 & 0 & -0.927861 & -2.168550 & 0.180594 \\
4 & 1 & 0 & -2.390877 & -1.273374 & -0.228576 \\
5 & 6 & 0 & -0.641809 & -0.000111 & -0.001205
\end{tabular}




$\begin{array}{cccccc}6 & 6 & 0 & 0.850601 & -0.001505 & -0.453958 \\ 7 & 1 & 0 & 0.825172 & -0.007658 & -1.555451 \\ 8 & 6 & 0 & 1.623876 & 1.249319 & -0.022559 \\ 9 & 1 & 0 & 1.776931 & 1.265548 & 1.061906 \\ 10 & 1 & 0 & 2.613496 & 1.261674 & -0.489770 \\ 11 & 1 & 0 & 1.118992 & 2.176807 & -0.305068 \\ 12 & 6 & 0 & 1.629969 & -1.246752 & -0.016890 \\ 13 & 1 & 0 & 1.637517 & -1.353566 & 1.073662 \\ 14 & 1 & 0 & 1.221431 & -2.166310 & -0.442765 \\ 15 & 1 & 0 & 2.672668 & -1.171863 & -0.341163 \\ 16 & 6 & 0 & -0.779785 & 0.187072 & 1.516343 \\ 17 & 1 & 0 & -0.413758 & 1.166412 & 1.839341 \\ 18 & 1 & 0 & -1.832012 & 0.116268 & 1.812083 \\ 19 & 1 & 0 & -0.231580 & -0.580032 & 2.072939 \\ 20 & 6 & 0 & -1.394208 & 1.136784 & -0.710730 \\ 21 & 1 & 0 & -1.319857 & 1.038843 & -1.799753 \\ 22 & 1 & 0 & -2.456611 & 1.116763 & -0.445616 \\ 23 & 1 & 0 & -1.010334 & 2.122669 & -0.434051\end{array}$

Zero-point correction=

Thermal correction to Energy=

Thermal correction to Enthalpy=

Thermal correction to Gibbs Free Energy=

Sum of electronic and zero-point Energies=

Sum of electronic and thermal Energies=

Sum of electronic and thermal Enthalpies=

Sum of electronic and thermal Free Energies=
0.218132 (Hartree/Particle)

0.227446

0.228390

0.185386

$-275.929785$

$-275.920471$

$-275.919527$

$-275.962530$

Octane

Standard orientation:

\begin{tabular}{|c|c|c|c|c|}
\hline \multirow{2}{*}{$\begin{array}{l}\text { Center } \\
\text { Number }\end{array}$} & Atomic & \multirow{2}{*}{$\begin{array}{l}\text { Atomic } \\
\text { Type }\end{array}$} & \multicolumn{2}{|c|}{ Coordinates (Angstroms) } \\
\hline & Number & & $\mathrm{X}$ & Z \\
\hline 1 & 6 & -4.472836 & 0.256945 & 0.000018 \\
\hline 2 & 1 & -4.538509 & 0.901348 & 0.883499 \\
\hline 3 & 1 & -4.538516 & 0.901410 & -0.883417 \\
\hline 4 & 1 & -5.349258 & -0.398018 & -0.000002 \\
\hline 5 & 6 & -3.172510 & -0.542354 & -0.000015 \\
\hline 6 & 1 & -3.145361 & -1.201307 & 0.877964 \\
\hline 7 & 1 & -3.145375 & -1.201259 & -0.878031 \\
\hline 8 & 6 & -1.928611 & 0.343460 & -0.000001 \\
\hline 9 & 1 & -1.954781 & 1.003822 & -0.878382 \\
\hline 10 & 1 & -1.954783 & 1.003802 & 0.878394 \\
\hline 11 & 6 & -0.620961 & -0.443787 & -0.000005 \\
\hline 12 & 1 & -0.594831 & -1.103781 & -0.878451 \\
\hline 13 & 1 & -0.594825 & -1.103775 & 0.878445 \\
\hline 14 & 6 & 0.620961 & 0.443787 & -0.000011 \\
\hline 15 & 1 & 0.594823 & 1.103791 & 0.878427 \\
\hline 16 & 1 & 0.594832 & 1.103765 & -0.878469 \\
\hline 17 & 6 & 1.928611 & -0.343460 & 0.000006 \\
\hline
\end{tabular}




$\begin{array}{rrrrrr}18 & 1 & 0 & 1.954784 & -1.003829 & -0.878368 \\ 19 & 1 & 0 & 1.954780 & -1.003795 & 0.878407 \\ 20 & 6 & 0 & 3.172510 & 0.542354 & -0.000008 \\ 21 & 1 & 0 & 3.145366 & 1.201300 & 0.877977 \\ 22 & 1 & 0 & 3.145371 & 1.201266 & -0.878018 \\ 23 & 6 & 0 & 4.472836 & -0.256945 & 0.000012 \\ 24 & 1 & 0 & 4.538516 & -0.901396 & -0.883434 \\ 25 & 1 & 0 & 5.349258 & 0.398018 & 0.000003 \\ 26 & 1 & 0 & 4.538509 & -0.901363 & 0.883482\end{array}$

Zero-point correction=

Thermal correction to Energy=

Thermal correction to Enthalpy=

Thermal correction to Gibbs Free Energy=

Sum of electronic and zero-point Energies=

Sum of electronic and thermal Energies $=$

Sum of electronic and thermal Enthalpies=

Sum of electronic and thermal Free Energies=

2233tetramethylbutane
0.247718 (Hartree/Particle)

0.258839

0.259783

$$
\begin{gathered}
0.210371 \\
-315.176535 \\
-315.165414 \\
-315.164470 \\
-315.213882
\end{gathered}
$$

Standard orientation:

\begin{tabular}{cccccc} 
Center & Atomic & \multicolumn{2}{c}{ Atomic } & \multicolumn{3}{c}{ Coordinates } \\
Number & Number & Type & X & Y & Z \\
---------- & \\
1 & 6 & 0 & -1.346572 & 0.035798 & -1.433814 \\
2 & 1 & 0 & -0.923344 & 0.856430 & -2.021744 \\
3 & 1 & 0 & -1.154857 & -0.898157 & -1.969599 \\
4 & 1 & 0 & -2.431689 & 0.180330 & -1.407357 \\
5 & 6 & 0 & -0.789763 & 0.000036 & -0.000025 \\
6 & 6 & 0 & 0.789862 & 0.000052 & -0.000063 \\
7 & 6 & 0 & 1.346163 & -0.366092 & 1.386816 \\
8 & 1 & 0 & 0.923769 & 0.258876 & 2.180057 \\
9 & 1 & 0 & 1.152403 & -1.412023 & 1.640945 \\
10 & 1 & 0 & 2.431528 & -0.221737 & 1.401854 \\
11 & 6 & 0 & -1.346344 & 1.223868 & 0.747964 \\
12 & 1 & 0 & -0.924059 & 1.321830 & 1.753103 \\
13 & 1 & 0 & -1.153252 & 2.154985 & 0.207747 \\
14 & 1 & 0 & -2.431658 & 1.129570 & 0.858799 \\
15 & 6 & 0 & -1.346066 & -1.259751 & 0.685915 \\
16 & 1 & 0 & -2.431390 & -1.308824 & 0.549025 \\
17 & 1 & 0 & -0.923562 & -2.179065 & 0.268056 \\
18 & 1 & 0 & -1.152723 & -1.257410 & 1.762348 \\
19 & 6 & 0 & 1.346459 & 1.384094 & -0.376374 \\
20 & 1 & 0 & 2.431632 & 1.324254 & -0.510133 \\
21 & 1 & 0 & 0.923352 & 1.759374 & -1.313573 \\
22 & 1 & 0 & 1.154244 & 2.126944 & 0.402923 \\
23 & 6 & 0 & 1.346385 & -1.017950 & -1.010483 \\
24 & 1 & 0 & 2.431565 & -1.103933 & -0.891861 \\
25 & 1 & 0 & 0.923221 & -2.017193 & -0.866733 \\
26 & 1 & 0 & 1.154079 & -0.714581 & -2.043469
\end{tabular}


Zero-point correction=

Thermal correction to Energy=

Thermal correction to Enthalpy=

Thermal correction to Gibbs Free Energy=

Sum of electronic and zero-point Energies=

Sum of electronic and thermal Energies=

Sum of electronic and thermal Enthalpies=

Sum of electronic and thermal Free Energies=

Basis set: DH-SVPD

Functional: B3LYP-D3

Ethane

Standard orientation:
0.246540 (Hartree/Particle)

0.256803

0.257747

0.213426

$-315.178673$

$-315.168409$

$-315.167465$

$-315.211786$

\begin{tabular}{|c|c|c|c|c|}
\hline \multirow{2}{*}{$\begin{array}{l}\text { Center } \\
\text { Number }\end{array}$} & Atomic & \multirow{2}{*}{$\begin{array}{r}\text { Atomic } \\
\text { Type }\end{array}$} & \multicolumn{2}{|c|}{ Coordinates (Angstron } \\
\hline & Numbe & & $\mathrm{X}$ & $\mathrm{Z}$ \\
\hline 1 & 6 & 0.000000 & -0.000000 & 0.766152 \\
\hline 2 & 1 & -0.509516 & 0.882570 & 1.165028 \\
\hline 3 & 1 & 1.019085 & -0.000031 & 1.165028 \\
\hline 4 & 1 & -0.509570 & -0.882538 & 1.165028 \\
\hline 5 & 6 & 0.000000 & -0.000000 & -0.766152 \\
\hline 6 & 1 & 0.509516 & 0.882570 & -1.165028 \\
\hline 7 & 1 & 0.509570 & -0.882538 & -1.165028 \\
\hline 8 & 1 & -1.019085 & -0.000031 & -1.165028 \\
\hline
\end{tabular}

Zero-point correction=

Thermal correction to Energy=

Thermal correction to Enthalpy=

Thermal correction to Gibbs Free Energy=

Sum of electronic and zero-point Energies=

Sum of electronic and thermal Energies $=$

Sum of electronic and thermal Enthalpies=

0.074710 (Hartree/Particle)

0.078199

0.079143

0.053277

$-79.709382$

$-79.705893$

$-79.704949$

$-79.730815$

Methane

Standard orientation:

\begin{tabular}{cccccc} 
Center & \multicolumn{2}{c}{ Atomic } & \multicolumn{2}{c}{ Atomic } & \multicolumn{2}{c}{ Coordinates (Angstrom } \\
Number & Number & Type & X & Y & Z \\
- & - & 0 & 0.000000 & 0.000000 & 0.000000 \\
2 & 1 & 0 & 0.630081 & 0.630081 & 0.630081 \\
3 & 1 & 0 & -0.630081 & -0.630081 & 0.630081 \\
4 & 1 & 0 & -0.630081 & 0.630081 & -0.630081 \\
5 & 1 & 0 & 0.630081 & -0.630081 & -0.630081
\end{tabular}

Zero-point correction=

Thermal correction to Energy=

Thermal correction to Enthalpy=

Thermal correction to Gibbs Free Energy=
0.044802 (Hartree/Particle)

0.047671

0.048615

0.027483 
Sum of electronic and zero-point Energies=

$-40.450606$

Sum of electronic and thermal Energies=

$-40.447737$

Sum of electronic and thermal Enthalpies=

$-40.446793$

Sum of electronic and thermal Free Energies=

$-40.467925$

Propane

Standard orientation:

\begin{tabular}{|c|c|c|c|c|}
\hline \multirow{2}{*}{$\begin{array}{l}\text { Center } \\
\text { Number }\end{array}$} & Atomic & \multirow{2}{*}{$\begin{array}{l}\text { Atomic } \\
\text { Type }\end{array}$} & \multicolumn{2}{|c|}{ Coordinates (Angstrom } \\
\hline & Number & & $\mathrm{X}$ & Z \\
\hline 1 & 6 & -1.274394 & -0.260512 & 0.000000 \\
\hline 2 & 1 & -2.175812 & 0.360062 & -0.000014 \\
\hline 3 & 1 & -1.315039 & -0.907296 & 0.883115 \\
\hline 4 & 1 & -1.315027 & -0.907319 & -0.883098 \\
\hline 5 & 6 & -0.000000 & 0.589825 & 0.000000 \\
\hline 6 & 1 & -0.000001 & 1.248161 & 0.877645 \\
\hline 7 & 1 & 0.000001 & 1.248146 & -0.877655 \\
\hline 8 & 6 & 1.274394 & -0.260512 & 0.000000 \\
\hline 9 & 1 & 1.315080 & -0.907232 & 0.883160 \\
\hline 10 & 1 & 1.314983 & -0.907383 & -0.883054 \\
\hline 11 & 1 & 2.175814 & 0.360060 & -0.000103 \\
\hline
\end{tabular}

Zero-point correction $=$

0.103515 (Hartree/Particle)

Thermal correction to Energy=

0.108020

Thermal correction to Enthalpy=

0.108964

Thermal correction to Gibbs Free Energy=

Sum of electronic and zero-point Energies=

0.077876

$-118.972509$

Sum of electronic and thermal Energies $=$

$-118.968004$

Sum of electronic and thermal Enthalpies=

$-118.967060$

Sum of electronic and thermal Free Energies $=\quad-118.998148$

Butane

Standard orientation:

\begin{tabular}{cccccc} 
Center & \multicolumn{2}{c}{ Atomic } & \multicolumn{2}{c}{ Atomic } & \multicolumn{2}{c}{ Coordinates } & (Angstroms) \\
Number & Number & Type & X & $Y$ & $Z$ \\
-1 & 6 & 0 & 1.959005 & -0.120114 & 0.000049 \\
2 & 1 & 0 & 2.103333 & -0.751704 & -0.883093 \\
3 & 1 & 0 & 2.103243 & -0.751630 & 0.883258 \\
4 & 1 & 0 & 2.749425 & 0.636620 & 0.000057 \\
5 & 6 & 0 & 0.566012 & 0.516356 & -0.000055 \\
6 & 1 & 0 & 0.458254 & 1.167255 & 0.877855 \\
7 & 1 & 0 & 0.458332 & 1.167170 & -0.878039 \\
8 & 6 & 0 & -0.566012 & -0.516356 & -0.000055 \\
9 & 1 & 0 & -0.458332 & -1.167168 & -0.878040 \\
10 & 1 & 0 & -0.458254 & -1.167256 & 0.877854 \\
11 & 6 & 0 & -1.959005 & 0.120114 & 0.000049 \\
12 & 1 & 0 & -2.103327 & 0.751719 & -0.883083 \\
13 & 1 & 0 & -2.749425 & -0.636620 & 0.000038 \\
14 & 1 & 0 & -2.103249 & 0.751615 & 0.883268
\end{tabular}




$\begin{array}{lc}\text { Zero-point correction= } & 0.132106 \text { (Hartree/Particle) } \\ \text { Thermal correction to Energy= } & 0.137850 \\ \text { Thermal correction to Enthalpy= } & 0.138794 \\ \text { Thermal correction to Gibbs Free Energy= } & 0.104125 \\ \text { Sum of electronic and zero-point Energies }= & -158.235842 \\ \text { Sum of electronic and thermal Energies }= & -158.230098 \\ \text { Sum of electronic and thermal Enthalpies }= & -158.229154 \\ \text { Sum of electronic and thermal Free Energies }= & -158.263823\end{array}$
Isobutane

Standard orientation:

\begin{tabular}{cccccc} 
Center & \multicolumn{2}{c}{ Atomic } & \multicolumn{2}{c}{ Atomic } & \multicolumn{3}{c}{ Coordinates (Angstroms) } \\
Number & Number & Type & X & Y & $Z$ \\
-1 & 6 & 0 & -1.110989 & -0.947302 & -0.095796 \\
2 & 1 & 0 & -2.092939 & -0.621022 & 0.261391 \\
3 & 1 & 0 & -1.149827 & -0.980961 & -1.190871 \\
4 & 1 & 0 & -0.944503 & -1.968312 & 0.262086 \\
5 & 6 & 0 & 0.000018 & -0.000017 & 0.375086 \\
6 & 1 & 0 & 0.000021 & 0.000005 & 1.476011 \\
7 & 6 & 0 & -0.264913 & 1.435779 & -0.095793 \\
8 & 1 & 0 & 0.508452 & 2.123123 & 0.261601 \\
9 & 1 & 0 & -0.274411 & 1.486299 & -1.190863 \\
10 & 1 & 0 & -1.232510 & 1.801955 & 0.261876 \\
11 & 6 & 0 & 1.375898 & -0.488472 & -0.095803 \\
12 & 1 & 0 & 2.176757 & 0.166792 & 0.261283 \\
13 & 1 & 0 & 1.584734 & -1.501629 & 0.262187 \\
14 & 1 & 0 & 1.424144 & -0.506176 & -1.190870
\end{tabular}

Zero-point correction=

Thermal correction to Energy=

Thermal correction to Enthalpy=

Thermal correction to Gibbs Free Energy=

Sum of electronic and zero-point Energies=

Sum of electronic and thermal Energies $=$

Sum of electronic and thermal Enthalpies=

Sum of electronic and thermal Free Energies=

Pentane

\subsection{2 (Hartree/Particle)}

$$
0.137395
$$

0.138339

$$
\begin{gathered}
0.104203 \\
-158.238235 \\
-158.232602 \\
-158.231658 \\
-158.265794
\end{gathered}
$$

Standard orientation:

\begin{tabular}{cccccc}
$\begin{array}{c}\text { Center } \\
\text { Number }\end{array}$ & \multicolumn{2}{c}{ Atomic } & \multicolumn{2}{c}{ Atomic } & \multicolumn{2}{c}{ Coordinates (Angstroms) } \\
- & Number & Type & X & Y & Z \\
\hline 1 & 6 & 0 & 2.554421 & 0.326741 & -0.000009 \\
2 & 1 & 0 & 2.594563 & 0.973421 & -0.883170 \\
3 & 1 & 0 & 2.594562 & 0.973473 & 0.883114 \\
4 & 1 & 0 & 3.456647 & -0.292471 & 0.000010 \\
5 & 6 & 0 & 1.282118 & -0.525853 & 0.000016 \\
6 & 1 & 0 & 1.281401 & -1.185322 & -0.877928
\end{tabular}




$\begin{array}{cccccc}7 & 1 & 0 & 1.281407 & -1.185279 & 0.877992 \\ 8 & 6 & 0 & 0.000000 & 0.312421 & 0.000003 \\ 9 & 1 & 0 & -0.000012 & 0.973588 & 0.878120 \\ 10 & 1 & 0 & 0.000012 & 0.973592 & -0.878110 \\ 11 & 6 & 0 & -1.282118 & -0.525853 & -0.000017 \\ 12 & 1 & 0 & -1.281405 & -1.185324 & 0.877927 \\ 13 & 1 & 0 & -1.281403 & -1.185277 & -0.877994 \\ 14 & 6 & 0 & -2.554421 & 0.326741 & 0.000006 \\ 15 & 1 & 0 & -3.456647 & -0.292471 & -0.000090 \\ 16 & 1 & 0 & -2.594598 & 0.973365 & 0.883205 \\ 17 & 1 & 0 & -2.594526 & 0.973529 & -0.883079\end{array}$

Zero-point correction=

Thermal correction to Energy=

Thermal correction to Enthalpy=

Thermal correction to Gibbs Free Energy=

Sum of electronic and zero-point Energies=

Sum of electronic and thermal Energies=

Sum of electronic and thermal Enthalpies=

Sum of electronic and thermal Free Energies=

Neopentane

Standard orientation:
0.160663 (Hartree/Particle)

$$
0.167697
$$

0.168641
0.130389

$-197.499198$

$-197.492164$

$-197.491220$

$-197.529473$

\begin{tabular}{|c|c|c|c|c|}
\hline \multirow{3}{*}{$\begin{array}{l}\text { Center } \\
\text { Number } \\
-1\end{array}$} & \multirow{2}{*}{$\begin{array}{l}\text { Atomic } \\
\text { Number }\end{array}$} & \multirow{2}{*}{$\begin{array}{l}\text { Atomic } \\
\text { Type }\end{array}$} & \multicolumn{2}{|c|}{ Coordinates (Angstroms) } \\
\hline & & & $\mathrm{X}$ & $\mathrm{Z}$ \\
\hline & 6 & -0.314848 & 1.326172 & 0.714463 \\
\hline 2 & 1 & -0.910388 & 1.155383 & 1.617403 \\
\hline 3 & 1 & 0.604621 & 1.841168 & 1.011952 \\
\hline 4 & 1 & -0.880450 & 2.000137 & 0.062542 \\
\hline 5 & 6 & -0.000046 & -0.000007 & -0.000006 \\
\hline 6 & 6 & 0.793230 & -0.917375 & 0.947356 \\
\hline 7 & 1 & 1.032255 & -1.870480 & 0.464029 \\
\hline 8 & 1 & 1.735872 & -0.449807 & 1.250580 \\
\hline 9 & 1 & 0.220649 & -1.136186 & 1.854781 \\
\hline 10 & 6 & -1.315080 & -0.691189 & -0.401491 \\
\hline 11 & 1 & -1.901560 & -0.059750 & -1.077113 \\
\hline 12 & 1 & -1.121195 & -1.639715 & -0.913194 \\
\hline 13 & 1 & -1.932047 & -0.904732 & 0.477648 \\
\hline 14 & 6 & 0.836699 & 0.282396 & -1.260329 \\
\hline 15 & 1 & 0.295734 & 0.934209 & -1.954328 \\
\hline 16 & 1 & 1.780390 & 0.775357 & -1.004233 \\
\hline 17 & 1 & 1.076389 & -0.645568 & -1.790022 \\
\hline \multicolumn{3}{|c|}{ Zero-point correction $=$} & \multicolumn{2}{|c|}{0.159853 (Hartree/Particle) } \\
\hline \multicolumn{3}{|c|}{ Thermal correction to Energy= } & \multicolumn{2}{|c|}{0.166631} \\
\hline \multicolumn{3}{|c|}{ Thermal correction to Enthalpy= } & \multicolumn{2}{|c|}{0.167575} \\
\hline \multicolumn{4}{|c|}{ Thermal correction to Gibbs Free Energy= } & 0.130872 \\
\hline \multicolumn{4}{|c|}{ Sum of electronic and zero-point Energies $=$} & -197.504597 \\
\hline \multicolumn{4}{|c|}{ Sum of electronic and thermal Energies $=$} & -197.497819 \\
\hline
\end{tabular}


Sum of electronic and thermal Enthalpies=

Sum of electronic and thermal Free Energies=

Hexane

Standard orientation:

\begin{tabular}{cccccc} 
Center & \multicolumn{2}{c}{ Atomic } & \multicolumn{2}{c}{ Atomic } & \multicolumn{3}{c}{ Coordinates } & (Angstroms) \\
Number & Number & Type & X & Y & $Z$ \\
-1 & 6 & 0 & -3.217433 & 0.208938 & 0.000052 \\
2 & 1 & 0 & -3.302809 & 0.851166 & 0.883289 \\
3 & 1 & 0 & -3.302816 & 0.851415 & -0.883003 \\
4 & 1 & 0 & -4.074052 & -0.471995 & -0.000042 \\
5 & 6 & 0 & -1.888418 & -0.552045 & -0.000063 \\
6 & 1 & 0 & -1.841150 & -1.210079 & 0.877777 \\
7 & 1 & 0 & -1.841193 & -1.209899 & -0.878039 \\
8 & 6 & 0 & -0.668090 & 0.373974 & -0.000007 \\
9 & 1 & 0 & -0.715028 & 1.033252 & -0.878098 \\
10 & 1 & 0 & -0.715066 & 1.033215 & 0.878110 \\
11 & 6 & 0 & 0.668090 & -0.373974 & 0.000015 \\
12 & 1 & 0 & 0.715037 & -1.033285 & -0.878051 \\
13 & 1 & 0 & 0.715057 & -1.033182 & 0.878158 \\
14 & 6 & 0 & 1.888418 & 0.552045 & -0.000051 \\
15 & 1 & 0 & 1.841158 & 1.210074 & 0.877793 \\
16 & 1 & 0 & 1.841186 & 1.209905 & -0.878023 \\
17 & 6 & 0 & 3.217433 & -0.208938 & 0.000042 \\
18 & 1 & 0 & 3.302820 & -0.851384 & -0.883035 \\
19 & 1 & 0 & 4.074052 & 0.471995 & -0.000023 \\
20 & 1 & 0 & 3.302805 & -0.851197 & 0.883257
\end{tabular}

Zero-point correction=

Thermal correction to Energy=

Thermal correction to Enthalpy=

Thermal correction to Gibbs Free Energy=

Sum of electronic and zero-point Energies=

Sum of electronic and thermal Energies $=$

Sum of electronic and thermal Enthalpies=

Sum of electronic and thermal Free Energies=

22dimethylbutane
$-197.496875$

$-197.533578$ 


$\begin{array}{cccccc}8 & 1 & 0 & 0.857451 & 1.488620 & 0.878226 \\ 9 & 6 & 0 & 2.186188 & 0.037558 & 0.000010 \\ 10 & 1 & 0 & 2.277806 & -0.600185 & -0.883793 \\ 11 & 1 & 0 & 3.043350 & 0.717554 & 0.000230 \\ 12 & 1 & 0 & 2.277860 & -0.600828 & 0.883343 \\ 13 & 6 & 0 & -0.520981 & -0.885100 & 1.256722 \\ 14 & 1 & 0 & 0.269379 & -1.640542 & 1.276039 \\ 15 & 1 & 0 & -0.435312 & -0.284036 & 2.168458 \\ 16 & 1 & 0 & -1.479493 & -1.412941 & 1.292975 \\ 17 & 6 & 0 & -0.521049 & -0.883912 & -1.257546 \\ 18 & 1 & 0 & -1.479563 & -1.411718 & -1.294239 \\ 19 & 1 & 0 & -0.435431 & -0.281981 & -2.168714 \\ 20 & 1 & 0 & 0.269310 & -1.639336 & -1.277626\end{array}$

Zero-point correction $=$

0.188474 (Hartree/Particle)

Thermal correction to Energy=

0.196580

Thermal correction to Enthalpy=

0.197524

Thermal correction to Gibbs Free Energy=

Sum of electronic and zero-point Energies $=$

0.157279

$-236.766098$

Sum of electronic and thermal Energies $=$

$-236.757992$

Sum of electronic and thermal Enthalpies=

$-236.757048$

Sum of electronic and thermal Free Energies $=\quad-236.797293$

Heptane

Standard orientation:

\begin{tabular}{|c|c|c|c|c|}
\hline \multirow{2}{*}{$\begin{array}{l}\text { Center } \\
\text { Number }\end{array}$} & Atomic & \multirow{2}{*}{$\begin{array}{l}\text { Atomic } \\
\text { Type }\end{array}$} & \multicolumn{2}{|c|}{ Coordinates (Angstrom } \\
\hline & Numbe & & $\mathrm{X}$ & Z \\
\hline 1 & 6 & -3.834899 & -0.357160 & -0.000035 \\
\hline 2 & 1 & -3.874468 & -1.003877 & -0.883292 \\
\hline 3 & 1 & -3.874418 & -1.004166 & 0.883014 \\
\hline 4 & 1 & -4.737641 & 0.261320 & 0.000094 \\
\hline 5 & 6 & -2.563103 & 0.495973 & 0.000070 \\
\hline 6 & 1 & -2.562446 & 1.155733 & -0.877764 \\
\hline 7 & 1 & -2.562450 & 1.155526 & 0.878057 \\
\hline 8 & 6 & -1.280368 & -0.341319 & -0.000016 \\
\hline 9 & 1 & -1.280436 & -1.002495 & 0.877991 \\
\hline 10 & 1 & -1.280485 & -1.002414 & -0.878083 \\
\hline 11 & 6 & -0.000000 & 0.498692 & -0.000019 \\
\hline 12 & 1 & -0.000013 & 1.159364 & 0.878074 \\
\hline 13 & 1 & 0.000013 & 1.159300 & -0.878160 \\
\hline 14 & 6 & 1.280368 & -0.341319 & 0.000029 \\
\hline 15 & 1 & 1.280450 & -1.002523 & -0.877957 \\
\hline 16 & 1 & 1.280471 & -1.002386 & 0.878118 \\
\hline 17 & 6 & 2.563103 & 0.495973 & -0.000050 \\
\hline 18 & 1 & 2.562451 & 1.155708 & 0.877801 \\
\hline 19 & 1 & 2.562445 & 1.155551 & -0.878020 \\
\hline 20 & 6 & 3.834899 & -0.357160 & 0.000022 \\
\hline 21 & 1 & 3.874440 & -1.004101 & -0.883073 \\
\hline 22 & 1 & 4.737641 & 0.261320 & -0.000036 \\
\hline
\end{tabular}


0.883232

Zero-point correction $=$

Thermal correction to Energy=

Thermal correction to Enthalpy=

Thermal correction to Gibbs Free Energy=

Sum of electronic and zero-point Energies=

Sum of electronic and thermal Energies=

Sum of electronic and thermal Enthalpies=

Sum of electronic and thermal Free Energies=

223trimethylbutane

Standard orientation:
0.217691 (Hartree/Particle)

0.227375

0.228319
0.182938
$-276.026109$
$-276.016424$
$-276.015480$
$-276.060862$

Zero-point correction $=$

Thermal correction to Energy=

Thermal correction to Enthalpy=

Thermal correction to Gibbs Free Energy=

Sum of electronic and zero-point Energies=

Sum of electronic and thermal Energies=

Sum of electronic and thermal Enthalpies=

Sum of electronic and thermal Free Energies=

Octane

\subsection{7 (Hartree/Particle)}

0.226123

0.227067

0.183789

$-276.028149$

$-276.018793$

$-276.017848$

$-276.061126$

Standard orientation: 


\begin{tabular}{|c|c|c|c|c|}
\hline \multirow{2}{*}{$\begin{array}{l}\text { Center } \\
\text { Number }\end{array}$} & Atomic & \multirow{2}{*}{$\begin{array}{l}\text { Atomic } \\
\text { Type }\end{array}$} & \multicolumn{2}{|c|}{ Coordinates (Angstroms } \\
\hline & Number & & $\mathrm{X}$ & $\mathrm{Z}$ \\
\hline 1 & 6 & -4.489133 & 0.257818 & 0.000042 \\
\hline 2 & 1 & -4.554439 & 0.902448 & 0.883309 \\
\hline 3 & 1 & -4.554383 & 0.902782 & -0.882988 \\
\hline 4 & 1 & -5.366563 & -0.396085 & -0.000111 \\
\hline 5 & 6 & -3.184385 & -0.543950 & -0.000069 \\
\hline 6 & 1 & -3.157407 & -1.203247 & 0.877759 \\
\hline 7 & 1 & -3.157414 & -1.203017 & -0.878070 \\
\hline 8 & 6 & -1.935920 & 0.343631 & 0.000029 \\
\hline 9 & 1 & -1.962198 & 1.004333 & -0.877962 \\
\hline 10 & 1 & -1.962235 & 1.004216 & 0.878107 \\
\hline 11 & 6 & -0.623251 & -0.444784 & 0.000010 \\
\hline 12 & 1 & -0.596954 & -1.105126 & -0.878011 \\
\hline 13 & 1 & -0.596916 & -1.105083 & 0.878062 \\
\hline 14 & 6 & 0.623251 & 0.444784 & -0.000037 \\
\hline 15 & 1 & 0.596927 & 1.105164 & 0.877956 \\
\hline 16 & 1 & 0.596943 & 1.105045 & -0.878118 \\
\hline 17 & 6 & 1.935920 & -0.343631 & 0.000028 \\
\hline 18 & 1 & 1.962218 & -1.004342 & -0.877956 \\
\hline 19 & 1 & 1.962215 & -1.004206 & 0.878114 \\
\hline 20 & 6 & 3.184385 & 0.543950 & -0.000038 \\
\hline 21 & 1 & 3.157408 & 1.203200 & 0.877824 \\
\hline 22 & 1 & 3.157413 & 1.203064 & -0.878004 \\
\hline 23 & 6 & 4.489133 & -0.257818 & 0.000028 \\
\hline 24 & 1 & 4.554413 & -0.902685 & -0.883070 \\
\hline 25 & 1 & 5.366564 & 0.396085 & -0.000022 \\
\hline 26 & 1 & 4.554409 & -0.902546 & 0.883227 \\
\hline
\end{tabular}

Zero-point correction=

Thermal correction to Energy=

Thermal correction to Enthalpy=

Thermal correction to Gibbs Free Energy=

Sum of electronic and zero-point Energies=

Sum of electronic and thermal Energies $=$

Sum of electronic and thermal Enthalpies=

Sum of electronic and thermal Free Energies=

2233tetramethylbutane
0.246193 (Hartree/Particle)

0.257215

0.258159

0.209227

$-315.289589$

$-315.278567$

$-315.277623$

$-315.326555$

Standard orientation:

\begin{tabular}{|c|c|c|c|c|}
\hline \multirow{2}{*}{$\begin{array}{l}\text { Center } \\
\text { Number }\end{array}$} & Atomic & \multirow{2}{*}{$\begin{array}{l}\text { Atomic } \\
\text { Type }\end{array}$} & \multicolumn{2}{|c|}{ Coordinates (Angstrom } \\
\hline & Numbe & & $\mathrm{X}$ & $\mathrm{Z}$ \\
\hline 1 & 6 & -1.352954 & 0.103347 & 1.435568 \\
\hline 2 & 1 & -1.157281 & -0.801329 & 2.016416 \\
\hline 3 & 1 & -0.933289 & 0.952913 & 1.981563 \\
\hline 4 & 1 & -2.438215 & 0.241304 & 1.403078 \\
\hline 5 & 6 & -0.793871 & -0.000038 & -0.000021 \\
\hline
\end{tabular}




$\begin{array}{cccccc}6 & 6 & 0 & 0.793898 & -0.000019 & 0.000061 \\ 7 & 6 & 0 & 1.352902 & -0.414414 & -1.378311 \\ 8 & 1 & 0 & 1.157531 & -1.466674 & -1.598938 \\ 9 & 1 & 0 & 0.932843 & 0.184927 & -2.190897 \\ 10 & 1 & 0 & 2.438110 & -0.273542 & -1.397273 \\ 11 & 6 & 0 & -1.352749 & -1.294999 & -0.628386 \\ 12 & 1 & 0 & -1.157050 & -1.345511 & -1.702289 \\ 13 & 1 & 0 & -0.932880 & -2.192582 & -0.165740 \\ 14 & 1 & 0 & -2.438006 & -1.336083 & -0.492673 \\ 15 & 6 & 0 & -1.352751 & 1.191596 & -0.807346 \\ 16 & 1 & 0 & -2.437959 & 1.094379 & -0.911059 \\ 17 & 1 & 0 & -1.157394 & 2.146902 & -0.314037 \\ 18 & 1 & 0 & -0.932612 & 1.239860 & -1.815876 \\ 19 & 6 & 0 & 1.352824 & -0.986476 & 1.048181 \\ 20 & 1 & 0 & 2.438072 & -1.073200 & 0.935875 \\ 21 & 1 & 0 & 1.157187 & -0.651398 & 2.069720 \\ 22 & 1 & 0 & 0.932919 & -1.989926 & 0.935354 \\ 23 & 6 & 0 & 1.352737 & 1.400961 & 0.330287 \\ 24 & 1 & 0 & 2.437983 & 1.347154 & 0.461573 \\ 25 & 1 & 0 & 1.157077 & 2.118113 & -0.470677 \\ 26 & 1 & 0 & 0.932749 & 1.804949 & 1.255682\end{array}$

Zero-point correction=

Thermal correction to Energy=

Thermal correction to Enthalpy=

Thermal correction to Gibbs Free Energy=

Sum of electronic and zero-point Energies=

Sum of electronic and thermal Energies=

Sum of electronic and thermal Enthalpies=

Sum of electronic and thermal Free Energies=

Basis set:DH-SVPD

Functional B2PLYP

Ethane

Standard orientation:

\begin{tabular}{|c|c|c|c|c|}
\hline \multirow{2}{*}{$\begin{array}{l}\text { Center } \\
\text { Number }\end{array}$} & Atomic & \multirow{2}{*}{$\begin{array}{l}\text { Atomic } \\
\text { Type }\end{array}$} & \multicolumn{2}{|c|}{ Coordinates (Angstroms) } \\
\hline & Number & & $X$ & Z \\
\hline 1 & 6 & -0.000000 & 0.000000 & 0.764911 \\
\hline 2 & 1 & -0.509111 & 0.881857 & 1.162560 \\
\hline 3 & 1 & 1.018266 & -0.000026 & 1.162560 \\
\hline 4 & 1 & -0.509156 & -0.881831 & 1.162560 \\
\hline 5 & 6 & 0.000000 & 0.000000 & -0.764911 \\
\hline 6 & 1 & 0.509111 & 0.881857 & -1.162560 \\
\hline 7 & 1 & 0.509156 & -0.881831 & -1.162560 \\
\hline 8 & 1 & -1.018266 & -0.000026 & -1.162560 \\
\hline \multicolumn{3}{|c|}{ Zero-point correction $=$} & \multicolumn{2}{|c|}{0.075472 (Hartree/Particle) } \\
\hline \multicolumn{3}{|c|}{ Thermal correction to Energy= } & \multicolumn{2}{|c|}{0.078957} \\
\hline \multicolumn{3}{|c|}{ Thermal correction to Enthalpy= } & \multicolumn{2}{|c|}{0.079901} \\
\hline
\end{tabular}


Thermal correction to Gibbs Free Energy=

0.054043

Sum of electronic and zero-point Energies=

$-79.600286$

Sum of electronic and thermal Energies $=$

$-79.596801$

Sum of electronic and thermal Enthalpies=

$-79.595857$

Sum of electronic and thermal Free Energies=

$-79.621715$

Methane

Standard orientation:

\begin{tabular}{|c|c|c|c|c|}
\hline \multirow{2}{*}{$\begin{array}{l}\text { Center } \\
\text { Number }\end{array}$} & Atomic & \multirow{2}{*}{$\begin{array}{l}\text { Atomic } \\
\text { Type }\end{array}$} & \multicolumn{2}{|c|}{ Coordinates (Angstrom } \\
\hline & Numbe & & $X$ & Z \\
\hline 1 & 6 & 0.000000 & 0.000000 & 0.000000 \\
\hline 2 & 1 & 0.629479 & 0.629479 & 0.629479 \\
\hline 3 & 1 & -0.629479 & -0.629479 & 0.629479 \\
\hline 4 & 1 & -0.629479 & 0.629479 & -0.629479 \\
\hline 5 & 1 & 0.629479 & -0.629479 & -0.629479 \\
\hline
\end{tabular}

Zero-point correction=

Thermal correction to Energy=

Thermal correction to Enthalpy=

Thermal correction to Gibbs Free Energy=
Sum of electronic and zero-point Energies

Sum of electronic and thermal Energies $=$

Sum of electronic and thermal Enthalpies=

Sum of electronic and thermal Free Energies=

Propane

$$
\begin{array}{cc}
0.045320 & \text { (Hartree/Particle) } \\
0.048186 \\
0.049130 \\
\text { rgy }= & 0.028004 \\
\text { gies }= & -40.390731 \\
\text { s }= & -40.387865 \\
\text { pies }= & -40.386921 \\
\text { Energies }= & -40.408047
\end{array}
$$

Standard orientation:

\begin{tabular}{cccccc} 
Center & \multicolumn{2}{c}{ Atomic } & \multicolumn{2}{c}{ Atomic } & \multicolumn{2}{c}{ Coordinates (Angstroms) } \\
Number & Number & Type & X & Y & Z \\
- \hdashline 1 & 6 & 0 & -1.272028 & -0.260418 & 0.000000 \\
2 & 1 & 0 & -2.172420 & 0.359978 & -0.000016 \\
3 & 1 & 0 & -1.312194 & -0.905969 & 0.882765 \\
4 & 1 & 0 & -1.312181 & -0.905996 & -0.882745 \\
5 & 6 & 0 & 0.000000 & 0.589372 & -0.000000 \\
6 & 1 & 0 & -0.000001 & 1.246387 & 0.877286 \\
7 & 1 & 0 & 0.000001 & 1.246373 & -0.877295 \\
8 & 6 & 0 & 1.272028 & -0.260418 & 0.000000 \\
9 & 1 & 0 & 1.312219 & -0.905930 & 0.882792 \\
10 & 1 & 0 & 1.312153 & -0.906035 & -0.882718 \\
11 & 1 & 0 & 2.172421 & 0.359976 & -0.000071
\end{tabular}

Zero-point correction=

Thermal correction to Energy=

Thermal correction to Enthalpy=

Thermal correction to Gibbs Free Energy=

Sum of electronic and zero-point Energies=

Sum of electronic and thermal Energies $=$

Sum of electronic and thermal Enthalpies=
0.104421 (Hartree/Particle)

0.108941
0.109885

0.078761

$-118.814122$

$-118.809602$

$-118.808657$ 
Sum of electronic and thermal Free Energies=

Butane

Standard orientation:

\begin{tabular}{cccccc} 
Center & Atomic & \multicolumn{2}{c}{ Atomic } & \multicolumn{3}{c}{ Coordinates (Angstrom } \\
Number & \multicolumn{2}{c}{ Number } & Type & X & $Y$ \\
----------------- \\
1 & 6 & 0 & 1.955834 & -0.120185 & 0.000039 \\
2 & 1 & 0 & 2.099713 & -0.750597 & -0.882774 \\
3 & 1 & 0 & 2.099652 & -0.750523 & 0.882914 \\
4 & 1 & 0 & 2.745133 & 0.636447 & 0.000034 \\
5 & 6 & 0 & 0.564874 & 0.515998 & -0.000044 \\
6 & 1 & 0 & 0.457433 & 1.165454 & 0.877825 \\
7 & 1 & 0 & 0.457493 & 1.165387 & -0.877969 \\
8 & 6 & 0 & -0.564874 & -0.515998 & -0.000044 \\
9 & 1 & 0 & -0.457493 & -1.165386 & -0.877970 \\
10 & 1 & 0 & -0.457433 & -1.165455 & 0.877824 \\
11 & 6 & 0 & -1.955834 & 0.120185 & 0.000039 \\
12 & 1 & 0 & -2.099709 & 0.750608 & -0.882767 \\
13 & 1 & 0 & -2.745133 & -0.636447 & 0.000021 \\
14 & 1 & 0 & -2.099656 & 0.750513 & 0.882921
\end{tabular}

Zero-point correction=

0.133156 (Hartree/Particle)

Thermal correction to Energy=

0.138935

Thermal correction to Enthalpy=

0.139879

Thermal correction to Gibbs Free Energy=

Sum of electronic and zero-point Energies=

0.105117

$-158.028135$

Sum of electronic and thermal Energies=

$-158.022356$

Sum of electronic and thermal Enthalpies=

$-158.021412$

Sum of electronic and thermal Free Energies $=\quad-158.056174$

Isobutane

Standard orientation:

\begin{tabular}{cccccc} 
Center & \multicolumn{2}{c}{ Atomic } & \multicolumn{2}{c}{ Atomic } & \multicolumn{2}{c}{ Coordinates (Angstroms) } \\
Number & Number & Type & X & Y & $Z$ \\
- \hdashline & 6 & 0 & 1.373881 & 0.485816 & -0.095962 \\
2 & 1 & 0 & 2.173376 & -0.169762 & 0.261379 \\
3 & 1 & 0 & 1.420854 & 0.502552 & -1.190336 \\
4 & 1 & 0 & 1.583683 & 1.498144 & 0.261587 \\
5 & 6 & 0 & 0.000004 & 0.000007 & 0.375648 \\
6 & 1 & 0 & -0.000007 & -0.000006 & 1.475537 \\
7 & 6 & 0 & -0.266202 & -1.432704 & -0.095962 \\
8 & 1 & 0 & -1.233624 & -1.797359 & 0.261515 \\
9 & 1 & 0 & -0.275363 & -1.481694 & -1.190336 \\
10 & 1 & 0 & 0.505684 & -2.120543 & 0.261441 \\
11 & 6 & 0 & -1.107677 & 0.946885 & -0.095962 \\
12 & 1 & 0 & -2.089316 & 0.622161 & 0.261259 \\
13 & 1 & 0 & -0.939915 & 1.966974 & 0.261723 \\
14 & 1 & 0 & -1.145409 & 0.979512 & -1.190340
\end{tabular}




$\begin{array}{lc}\text { Zero-point correction= } & 0.132717 \text { (Hartree/Particle) } \\ \text { Thermal correction to Energy= } & 0.138407 \\ \text { Thermal correction to Enthalpy= } & 0.139352 \\ \text { Thermal correction to Gibbs Free Energy= } & 0.105092 \\ \text { Sum of electronic and zero-point Energies }= & -158.030519 \\ \text { Sum of electronic and thermal Energies }= & -158.024829 \\ \text { Sum of electronic and thermal Enthalpies }= & -158.023884 \\ \text { Sum of electronic and thermal Free Energies }= & -158.058144 \\ \text { Pentane } & \end{array}$

Standard orientation:

\begin{tabular}{cccccc} 
Center & Atomic & \multicolumn{2}{c}{ Atomic } & \multicolumn{3}{c}{ Coordinates } \\
Number & Number & Type & X & $Y$ & $Z$ \\
---------- & Znstroms) \\
\hline 1 & 6 & 0 & 2.550260 & 0.326886 & -0.000008 \\
2 & 1 & 0 & 2.589849 & 0.972350 & -0.882851 \\
3 & 1 & 0 & 2.589845 & 0.972413 & 0.882789 \\
4 & 1 & 0 & 3.451632 & -0.292027 & 0.000016 \\
5 & 6 & 0 & 1.280245 & -0.525705 & 0.000019 \\
6 & 1 & 0 & 1.279933 & -1.183709 & -0.877921 \\
7 & 1 & 0 & 1.279936 & -1.183657 & 0.877998 \\
8 & 6 & 0 & 0.000000 & 0.312002 & 0.000000 \\
9 & 1 & 0 & -0.000014 & 0.971539 & 0.878396 \\
10 & 1 & 0 & 0.000014 & 0.971537 & -0.878397 \\
11 & 6 & 0 & -1.280245 & -0.525705 & -0.000019 \\
12 & 1 & 0 & -1.279933 & -1.183711 & 0.877920 \\
13 & 1 & 0 & -1.279937 & -1.183655 & -0.877998 \\
14 & 6 & 0 & -2.550260 & 0.326886 & 0.000009 \\
15 & 1 & 0 & -3.451632 & -0.292027 & -0.000075 \\
16 & 1 & 0 & -2.589876 & 0.972305 & 0.882882 \\
17 & 1 & 0 & -2.589819 & 0.972457 & -0.882759 \\
------------------------------------------------------------
\end{tabular}

Zero-point correction $=$

Thermal correction to Energy=

Thermal correction to Enthalpy=

0.161862 (Hartree/Particle)

0.168945

0.169889

Thermal correction to Gibbs Free Energy $=0.131493$

Sum of electronic and zero-point Energies $=\quad-197.242173$

Sum of electronic and thermal Energies $=\quad-197.235090$

Sum of electronic and thermal Enthalpies $=\quad-197.234146$

Sum of electronic and thermal Free Energies $=\quad-197.272542$

Neopentane

Standard orientation:

\begin{tabular}{|c|c|c|c|c|}
\hline Center & Atomic & Atomic & Coordinate & s (Angstroms) \\
\hline Number & Number & Type & $X$ & Z \\
\hline 1 & 6 & -0.329576 & 0.337213 & 1.461777 \\
\hline 2 & 1 & -1.311083 & -0.056447 & 1.744270 \\
\hline 3 & 1 & 0.412829 & -0.092985 & 2.141405 \\
\hline
\end{tabular}




$\begin{array}{cccrrr}4 & 1 & 0 & -0.343508 & 1.419943 & 1.621862 \\ 5 & 6 & 0 & 0.000001 & 0.000007 & -0.000006 \\ 6 & 6 & 0 & 0.010098 & -1.524865 & -0.183918 \\ 7 & 1 & 0 & 0.243106 & -1.794399 & -1.218900 \\ 8 & 1 & 0 & 0.759376 & -1.993696 & 0.461769 \\ 9 & 1 & 0 & -0.964430 & -1.957145 & 0.064185 \\ 10 & 6 & 0 & -1.062113 & 0.619679 & -0.920368 \\ 11 & 1 & 0 & -1.091290 & 1.708273 & -0.809725 \\ 12 & 1 & 0 & -0.851651 & 0.394644 & -1.970694 \\ 13 & 1 & 0 & -2.058788 & 0.231833 & -0.687238 \\ 14 & 6 & 0 & 1.381590 & 0.567974 & -0.357490 \\ 15 & 1 & 0 & 1.403067 & 1.655527 & -0.235446 \\ 16 & 1 & 0 & 2.159454 & 0.142793 & 0.284597 \\ 17 & 1 & 0 & 1.642916 & 0.341612 & -1.396048\end{array}$

Zero-point correction=

Thermal correction to Energy=

Thermal correction to Enthalpy=

Thermal correction to Gibbs Free Energy=

Sum of electronic and zero-point Energies=

Sum of electronic and thermal Energies=

Sum of electronic and thermal Enthalpies=

Sum of electronic and thermal Free Energies=

Hexane
0.160772 (Hartree/Particle)

$$
0.167664
$$

0.168609
0.131669

$-197.247634$

$-197.240742$

$-197.239797$

$-197.276737$

Standard orientation:

\begin{tabular}{|c|c|c|c|c|}
\hline \multirow{2}{*}{$\begin{array}{l}\text { Center } \\
\text { Number }\end{array}$} & Atomic & \multirow{2}{*}{$\begin{array}{r}\text { Atomic } \\
\text { Type }\end{array}$} & \multicolumn{2}{|c|}{ Coordinates (Angstrom } \\
\hline & Numbe & & $\mathrm{X}$ & $\mathrm{Z}$ \\
\hline 1 & 6 & -3.212594 & 0.209013 & 0.000051 \\
\hline 2 & 1 & -3.297523 & 0.850029 & 0.882975 \\
\hline 3 & 1 & -3.297521 & 0.850294 & -0.882682 \\
\hline 4 & 1 & -4.068193 & -0.471805 & -0.000054 \\
\hline 5 & 6 & -1.885643 & -0.551795 & -0.000064 \\
\hline 6 & 1 & -1.838681 & -1.208399 & 0.877777 \\
\hline 7 & 1 & -1.838722 & -1.208216 & -0.878044 \\
\hline 8 & 6 & -0.667267 & 0.373817 & -0.000003 \\
\hline 9 & 1 & -0.714245 & 1.031461 & -0.878417 \\
\hline 10 & 1 & -0.714289 & 1.031415 & 0.878443 \\
\hline 11 & 6 & 0.667267 & -0.373817 & 0.000018 \\
\hline 12 & 1 & 0.714255 & -1.031491 & -0.878374 \\
\hline 13 & 1 & 0.714279 & -1.031385 & 0.878486 \\
\hline 14 & 6 & 1.885643 & 0.551795 & -0.000054 \\
\hline 15 & 1 & 1.838689 & 1.208396 & 0.877790 \\
\hline 16 & 1 & 1.838715 & 1.208219 & -0.878031 \\
\hline 17 & 6 & 3.212594 & -0.209014 & 0.000041 \\
\hline 18 & 1 & 3.297529 & -0.850260 & -0.882716 \\
\hline 19 & 1 & 4.068193 & 0.471805 & -0.000028 \\
\hline 20 & 1 & 3.297516 & -0.850063 & 0.882942 \\
\hline
\end{tabular}


Zero-point correction=

Thermal correction to Energy=

Thermal correction to Enthalpy=

Thermal correction to Gibbs Free Energy=

Sum of electronic and zero-point Energies=

Sum of electronic and thermal Energies=

Sum of electronic and thermal Enthalpies=

Sum of electronic and thermal Free Energies=

22dimethylbutane
0.190527 (Hartree/Particle)

$$
0.198945
$$

0.199889

$$
\begin{gathered}
0.157865 \\
-236.456285 \\
-236.447867 \\
-236.446923 \\
-236.488947
\end{gathered}
$$

\begin{tabular}{|c|c|c|c|c|}
\hline \multirow{2}{*}{$\begin{array}{l}\text { Center } \\
\text { Number }\end{array}$} & Atomic & \multirow{2}{*}{$\begin{array}{r}\text { Atomic } \\
\text { Type }\end{array}$} & \multicolumn{2}{|c|}{ Coordinates (Angstroms } \\
\hline & Numbe & & $\mathrm{X}$ & Z \\
\hline 1 & 6 & -1.618921 & 0.979376 & 0.001602 \\
\hline 2 & 1 & -1.596233 & 1.622349 & 0.887212 \\
\hline 3 & 1 & -1.596525 & 1.624855 & -0.882192 \\
\hline 4 & 1 & -2.572643 & 0.442502 & 0.000995 \\
\hline 5 & 6 & -0.434460 & -0.000069 & 0.000013 \\
\hline 6 & 6 & 0.871012 & 0.828537 & 0.000629 \\
\hline 7 & 1 & 0.853763 & 1.488081 & -0.876602 \\
\hline 8 & 1 & 0.853866 & 1.486679 & 0.878919 \\
\hline 9 & 6 & 2.181490 & 0.036965 & -0.000005 \\
\hline 10 & 1 & 2.272652 & -0.599770 & -0.883739 \\
\hline 11 & 1 & 3.037612 & 0.717241 & -0.000098 \\
\hline 12 & 1 & 2.273155 & -0.600198 & 0.883361 \\
\hline 13 & 6 & -0.519195 & -0.884404 & 1.253881 \\
\hline 14 & 1 & 0.271514 & -1.639005 & 1.273141 \\
\hline 15 & 1 & -0.435065 & -0.283432 & 2.165448 \\
\hline 16 & 1 & -1.477523 & -1.412019 & 1.288551 \\
\hline 17 & 6 & -0.520000 & -0.881212 & -1.256057 \\
\hline 18 & 1 & -1.478123 & -1.409171 & -1.291177 \\
\hline 19 & 1 & -0.437012 & -0.277837 & -2.166136 \\
\hline 20 & 1 & 0.271005 & -1.635430 & -1.278059 \\
\hline
\end{tabular}

Standard orientation:

Zero-point correction $=$

0.189532 (Hartree/Particle)

Thermal correction to Energy=

0.197763

Thermal correction to Enthalpy=

0.198707

Thermal correction to Gibbs Free Energy=

0.158185

Sum of electronic and zero-point Energies=

$-236.459367$

Sum of electronic and thermal Energies $=$

$-236.451137$

Sum of electronic and thermal Enthalpies=

$-236.450193$

Sum of electronic and thermal Free Energies $=\quad-236.490715$

Heptane

Standard orientation:

\begin{tabular}{cccccc} 
Center & Atomic & \multicolumn{2}{c}{ Atomic } & \multicolumn{2}{c}{ Coordinates (Angstroms) } \\
Number & Number & Type & X & Y & Z \\
- \hdashline 1 & 6 & 0 & -3.828511 & -0.357430 & -0.000012
\end{tabular}




$\begin{array}{cccccc}2 & 1 & 0 & -3.867691 & -1.002929 & -0.882824 \\ 3 & 1 & 0 & -3.867680 & -1.003013 & 0.882740 \\ 4 & 1 & 0 & -4.730068 & 0.260994 & 0.000024 \\ 5 & 6 & 0 & -2.559059 & 0.495851 & 0.000021 \\ 6 & 1 & 0 & -2.558601 & 1.153928 & -0.877893 \\ 7 & 1 & 0 & -2.558603 & 1.153865 & 0.877983 \\ 8 & 6 & 0 & -1.278311 & -0.341163 & -0.000005 \\ 9 & 1 & 0 & -1.278390 & -1.000512 & 0.878384 \\ 10 & 1 & 0 & -1.278403 & -1.000482 & -0.878416 \\ 11 & 6 & 0 & 0.000000 & 0.498944 & -0.000003 \\ 12 & 1 & 0 & -0.000004 & 1.157773 & 0.878512 \\ 13 & 1 & 0 & 0.000004 & 1.157761 & -0.878526 \\ 14 & 6 & 0 & 1.278311 & -0.341163 & 0.000009 \\ 15 & 1 & 0 & 1.278392 & -1.000518 & -0.878375 \\ 16 & 1 & 0 & 1.278402 & -1.000476 & 0.878425 \\ 17 & 6 & 0 & 2.559059 & 0.495851 & -0.000019 \\ 18 & 1 & 0 & 2.558603 & 1.153925 & 0.877898 \\ 19 & 1 & 0 & 2.558601 & 1.153868 & -0.877978 \\ 20 & 6 & 0 & 3.828511 & -0.357430 & 0.000008 \\ 21 & 1 & 0 & 3.867683 & -1.003001 & -0.882752 \\ 22 & 1 & 0 & 4.730068 & 0.260994 & -0.000016 \\ 23 & 1 & 0 & 3.867687 & -1.002941 & 0.882811 \\ --------------------------------------------------------\end{array}$

Zero-point correction $=$

Thermal correction to Energy=

Thermal correction to Enthalpy=

0.219188 (Hartree/Particle)

0.228961

0.229905

Thermal correction to Gibbs Free Energy $=\quad 0.184220$

Sum of electronic and zero-point Energies $=\quad-275.670420$

Sum of electronic and thermal Energies $=\quad-275.660647$

Sum of electronic and thermal Enthalpies $=\quad-275.659703$

Sum of electronic and thermal Free Energies $=\quad-275.705388$

223trimetthylbutane

Standard orientation:

\begin{tabular}{|c|c|c|c|c|}
\hline \multirow{2}{*}{$\begin{array}{l}\text { Center } \\
\text { Number }\end{array}$} & Atomic & \multirow{2}{*}{$\begin{array}{l}\text { Atomic } \\
\text { Type }\end{array}$} & \multicolumn{2}{|c|}{ Coordinates (Angstrom } \\
\hline & Number & & $X$ & Z \\
\hline 1 & 6 & -1.328561 & -1.298336 & -0.462363 \\
\hline 2 & 1 & -1.183878 & -1.460996 & -1.535926 \\
\hline 3 & 1 & -0.947517 & -2.174731 & 0.066762 \\
\hline 4 & 1 & -2.405398 & -1.245625 & -0.275311 \\
\hline 5 & 6 & -0.642622 & -0.000083 & -0.000677 \\
\hline 6 & 6 & 0.852268 & -0.001067 & -0.453325 \\
\hline 7 & 1 & 0.825474 & -0.005358 & -1.554403 \\
\hline 8 & 6 & 1.629909 & 1.250448 & -0.020348 \\
\hline 9 & 1 & 1.760012 & 1.280646 & 1.065614 \\
\hline 10 & 1 & 2.628603 & 1.244619 & -0.466319 \\
\hline 11 & 1 & 1.141860 & 2.177673 & -0.327539 \\
\hline 12 & 6 & 1.633886 & -1.248715 & -0.016054 \\
\hline 13 & 1 & 1.667798 & -1.338446 & 1.074190 \\
\hline
\end{tabular}




$\begin{array}{rrrrrr}14 & 1 & 0 & 1.209373 & -2.170734 & -0.417555 \\ 15 & 1 & 0 & 2.667887 & -1.185298 & -0.367216 \\ 16 & 6 & 0 & -0.784837 & 0.119754 & 1.525788 \\ 17 & 1 & 0 & -0.387399 & 1.068008 & 1.897372 \\ 18 & 1 & 0 & -1.840999 & 0.073191 & 1.809471 \\ 19 & 1 & 0 & -0.270261 & -0.691924 & 2.047827 \\ 20 & 6 & 0 & -1.381514 & 1.178089 & -0.660761 \\ 21 & 1 & 0 & -1.288332 & 1.139112 & -1.751284 \\ 22 & 1 & 0 & -2.447422 & 1.144342 & -0.415374 \\ 23 & 1 & 0 & -1.000970 & 2.144973 & -0.323872\end{array}$

Zero-point correction=

Thermal correction to Energy=

Thermal correction to Enthalpy=

Thermal correction to Gibbs Free Energy=

Sum of electronic and zero-point Energies=

Sum of electronic and thermal Energies=

Sum of electronic and thermal Enthalpies=

Sum of electronic and thermal Free Energies=

Octane

Standard orientation:

\begin{tabular}{|c|c|c|c|c|}
\hline \multirow{2}{*}{$\begin{array}{l}\text { Center } \\
\text { Number }\end{array}$} & Atomic & \multirow{2}{*}{$\begin{array}{l}\text { Atomic } \\
\text { Type }\end{array}$} & \multicolumn{2}{|c|}{ Coordinates (Angstroms) } \\
\hline & Numbe & & $X$ & Z \\
\hline 1 & 6 & -4.482676 & 0.257963 & 0.000052 \\
\hline 2 & 1 & -4.547483 & 0.901361 & 0.883018 \\
\hline 3 & 1 & -4.547436 & 0.901724 & -0.882652 \\
\hline 4 & 1 & -5.359167 & -0.395742 & -0.000108 \\
\hline 5 & 6 & -3.180074 & -0.543765 & -0.000078 \\
\hline 6 & 1 & -3.153462 & -1.201622 & 0.877752 \\
\hline 7 & 1 & -3.153483 & -1.201370 & -0.878097 \\
\hline 8 & 6 & -1.933454 & 0.343382 & 0.000025 \\
\hline 9 & 1 & -1.959786 & 1.002456 & -0.878301 \\
\hline 10 & 1 & -1.959833 & 1.002333 & 0.878442 \\
\hline 11 & 6 & -0.622369 & -0.444776 & 0.000010 \\
\hline 12 & 1 & -0.596019 & -1.103508 & -0.878382 \\
\hline 13 & 1 & -0.595981 & -1.103457 & 0.878436 \\
\hline 14 & 6 & 0.622369 & 0.444776 & -0.000041 \\
\hline 15 & 1 & 0.595991 & 1.103548 & 0.878319 \\
\hline 16 & 1 & 0.596009 & 1.103417 & -0.878500 \\
\hline 17 & 6 & 1.933454 & -0.343382 & 0.000030 \\
\hline 18 & 1 & 1.959812 & -1.002469 & -0.878285 \\
\hline 19 & 1 & 1.959806 & -1.002319 & 0.878458 \\
\hline 20 & 6 & 3.180074 & 0.543765 & -0.000042 \\
\hline 21 & 1 & 3.153469 & 1.201572 & 0.877826 \\
\hline 22 & 1 & 3.153476 & 1.201420 & -0.878024 \\
\hline 23 & 6 & 4.482676 & -0.257963 & 0.000033 \\
\hline 24 & 1 & 4.547462 & -0.901620 & -0.882745 \\
\hline 25 & 1 & 5.359167 & 0.395742 & -0.000021 \\
\hline
\end{tabular}


0.882925

Zero-point correction=

Thermal correction to Energy=

Thermal correction to Enthalpy=

Thermal correction to Gibbs Free Energy=

Sum of electronic and zero-point Energies=

Sum of electronic and thermal Energies $=$

Sum of electronic and thermal Enthalpies=

Sum of electronic and thermal Free Energies= 2233tetramethylbutane

Standard orientation:

\begin{tabular}{|c|c|c|c|c|}
\hline \multirow{2}{*}{$\begin{array}{l}\text { Center } \\
\text { Number }\end{array}$} & Atomic & \multirow{2}{*}{$\begin{array}{r}\text { Atomic } \\
\text { Type }\end{array}$} & \multicolumn{2}{|c|}{ Coordinates (Angstroms } \\
\hline & Numbe & & $X$ & Z \\
\hline 1 & 6 & -1.350044 & -1.376624 & 0.409868 \\
\hline 2 & 1 & -1.147432 & -2.140675 & -0.344359 \\
\hline 3 & 1 & -0.939044 & -1.722660 & 1.362256 \\
\hline 4 & 1 & -2.436178 & -1.313280 & 0.527612 \\
\hline 5 & 6 & -0.791109 & 0.000007 & -0.000007 \\
\hline 6 & 6 & 0.791074 & 0.000003 & 0.000001 \\
\hline 7 & 6 & 1.350145 & 1.260497 & -0.688562 \\
\hline 8 & 1 & 1.147387 & 1.263419 & -1.762148 \\
\hline 9 & 1 & 0.939515 & 2.180393 & -0.263238 \\
\hline 10 & 1 & 2.436316 & 1.299184 & -0.560866 \\
\hline 11 & 6 & -1.350035 & 0.333347 & -1.397137 \\
\hline 12 & 1 & -1.147340 & 1.368520 & -1.681772 \\
\hline 13 & 1 & -0.939121 & -0.318481 & -2.173003 \\
\hline 14 & 1 & -2.436185 & 0.199802 & -1.401143 \\
\hline 15 & 6 & -1.350103 & 1.043300 & 0.987196 \\
\hline 16 & 1 & -2.436266 & 1.113402 & 0.873586 \\
\hline 17 & 1 & -1.147355 & 0.772321 & 2.026014 \\
\hline 18 & 1 & -0.939340 & 2.041168 & 0.810501 \\
\hline 19 & 6 & 1.350033 & -1.226609 & -0.747322 \\
\hline 20 & 1 & 2.436201 & -1.135450 & -0.844764 \\
\hline 21 & 1 & 1.147234 & -2.157768 & -0.212993 \\
\hline 22 & 1 & 0.939293 & -1.318236 & -1.756584 \\
\hline 23 & 6 & 1.349999 & -0.033926 & 1.435961 \\
\hline 24 & 1 & 2.436159 & -0.163985 & 1.405786 \\
\hline 25 & 1 & 1.147241 & 0.894443 & 1.975125 \\
\hline 26 & 1 & 0.939159 & -0.862078 & 2.020003 \\
\hline
\end{tabular}

Zero-point correction=

Thermal correction to Energy=

Thermal correction to Enthalpy=

Thermal correction to Gibbs Free Energy=

Sum of electronic and zero-point Energies=

Sum of electronic and thermal Energies $=$

Sum of electronic and thermal Enthalpies=
0.246593 (Hartree/Particle)

0.256968

0.257912

0.213321

$-314.883355$

$-314.872979$

$-314.872035$ 
Sum of electronic and thermal Free Energies=

PBE0DH/DHSVPD

Ethane

Standard orientation:

\begin{tabular}{crrrrr} 
Center & \multicolumn{2}{c}{ Atomic } & \multicolumn{2}{c}{ Atomic } & \multicolumn{2}{c}{ Coordinates (Angstroms) } \\
Number & Number & \multicolumn{1}{c}{ Type } & \multicolumn{1}{c}{ X } & \multicolumn{1}{c}{ Y } & Z \\
-1 & 6 & 0 & 0.000000 & -0.000000 & 0.759483 \\
2 & 1 & 0 & -0.508098 & 0.880090 & 1.158375 \\
3 & 1 & 0 & 1.016229 & -0.000019 & 1.158375 \\
4 & 1 & 0 & -0.508131 & -0.880071 & 1.158375 \\
5 & 6 & 0 & -0.000000 & -0.000000 & -0.759483 \\
6 & 1 & 0 & 0.508098 & 0.880090 & -1.158375 \\
7 & 1 & 0 & 0.508131 & -0.880071 & -1.158375 \\
8 & 1 & 0 & -1.016229 & -0.000019 & -1.158375
\end{tabular}

Zero-point correction $=$

0.076183 (Hartree/Particle)

Thermal correction to Energy=

0.079657

Thermal correction to Enthalpy=

0.080601

Thermal correction to Gibbs Free Energy=

0.054773

Sum of electronic and zero-point Energies $=$

$-79.596141$

Sum of electronic and thermal Energies=

$-79.592667$

Sum of electronic and thermal Enthalpies=

$-79.591723$

Sum of electronic and thermal Free Energies $=\quad-79.617551$

Methane

Standard orientation:

\begin{tabular}{|c|c|c|c|c|}
\hline \multirow{2}{*}{$\begin{array}{l}\text { Center } \\
\text { Number }\end{array}$} & \multirow{2}{*}{$\begin{array}{l}\text { Atomic } \\
\text { Number }\end{array}$} & \multirow{2}{*}{$\begin{array}{r}\text { Atomic } \\
\text { Type }\end{array}$} & \multicolumn{2}{|c|}{ Coordinates (Angstrom } \\
\hline & & & $\mathrm{X}$ & $\mathrm{Z}$ \\
\hline 1 & 6 & 0.000000 & 0.000000 & 0.000000 \\
\hline 2 & 1 & 0.628776 & 0.628776 & 0.628776 \\
\hline 3 & 1 & -0.628776 & -0.628776 & 0.628776 \\
\hline 4 & 1 & -0.628776 & 0.628776 & -0.628776 \\
\hline 5 & 1 & 0.628776 & -0.628776 & -0.628776 \\
\hline
\end{tabular}

Zero-point correction=

Thermal correction to Energy=

Thermal correction to Enthalpy=

Thermal correction to Gibbs Free Energy=

Sum of electronic and zero-point Energies=

Sum of electronic and thermal Energies $=$

Sum of electronic and thermal Enthalpies=

Sum of electronic and thermal Free Energies=

Propane
0.045648 (Hartree/Particle)

0.048515

$$
0.049459
$$

0.028335

$-40.388965$

$-40.386099$

$-40.385154$

$-40.406278$

Standard orientation:

\begin{tabular}{lcccc} 
Center & Atomic & Atomic & & \multicolumn{2}{c}{ Coordinates (Angstroms) } \\
Number & Number & Type & X & Y C Z
\end{tabular}




$\begin{array}{cccccc}1 & 6 & 0 & -1.263448 & -0.258495 & 0.000000 \\ 2 & 1 & 0 & -2.163701 & 0.359340 & -0.000028 \\ 3 & 1 & 0 & -1.304556 & -0.903994 & 0.880949 \\ 4 & 1 & 0 & -1.304532 & -0.904039 & -0.880918 \\ 5 & 6 & 0 & 0.000000 & 0.585482 & -0.000000 \\ 6 & 1 & 0 & 0.000000 & 1.243229 & 0.874891 \\ 7 & 1 & 0 & 0.000002 & 1.243214 & -0.874902 \\ 8 & 6 & 0 & 1.263447 & -0.258495 & 0.000000 \\ 9 & 1 & 0 & 1.304559 & -0.903987 & 0.880955 \\ 10 & 1 & 0 & 1.304525 & -0.904046 & -0.880913 \\ 11 & 1 & 0 & 2.163702 & 0.359337 & -0.000038 \\ --------------------------------------------------------\end{array}$

Zero-point correction=

Thermal correction to Energy=

Thermal correction to Enthalpy=

Thermal correction to Gibbs Free Energy=

Sum of electronic and zero-point Energies=

Sum of electronic and thermal Energies $=$

Sum of electronic and thermal Enthalpies $=$

Sum of electronic and thermal Free Energies=

Neopentane

Standard orientation:
0.105462 (Hartree/Particle)

0.109962

0.110906

0.079834

$-118.807501$

$-118.803001$

$-118.802057$

$-118.833130$

\begin{tabular}{|c|c|c|c|c|}
\hline \multirow{2}{*}{$\begin{array}{l}\text { Center } \\
\text { Number }\end{array}$} & \multirow{2}{*}{$\begin{array}{l}\text { Atomic } \\
\text { Number }\end{array}$} & \multirow{2}{*}{$\begin{array}{r}\text { Atomic } \\
\text { Type }\end{array}$} & \multicolumn{2}{|c|}{ Coordinates (Angstrom } \\
\hline & & & $X$ & $\mathrm{Z}$ \\
\hline 1 & 6 & 0.121858 & 1.111862 & -1.037720 \\
\hline 2 & 1 & -0.638852 & 1.008660 & -1.815895 \\
\hline 3 & 1 & -0.002525 & 2.095252 & -0.576893 \\
\hline 4 & 1 & 1.101241 & 1.091411 & -1.522774 \\
\hline 5 & 6 & -0.000015 & -0.000003 & 0.000023 \\
\hline 6 & 6 & -1.377738 & 0.062264 & 0.652707 \\
\hline 7 & 1 & -1.493623 & -0.724640 & 1.402532 \\
\hline 8 & 1 & -1.534447 & 1.023236 & 1.149555 \\
\hline 9 & 1 & -2.170427 & -0.063651 & -0.089364 \\
\hline 10 & 6 & 0.179332 & -1.353404 & -0.681252 \\
\hline 11 & 1 & 1.160083 & -1.426599 & -1.158386 \\
\hline 12 & 1 & 0.096531 & -2.170704 & 0.039890 \\
\hline 13 & 1 & -0.579934 & -1.509394 & -1.452011 \\
\hline 14 & 6 & 1.076545 & 0.179279 & 1.066263 \\
\hline 15 & 1 & 2.076385 & 0.138724 & 0.626302 \\
\hline 16 & 1 & 0.972609 & 1.142794 & 1.571944 \\
\hline 17 & 1 & 1.013068 & -0.605073 & 1.824974 \\
\hline
\end{tabular}

Zero-point correction $=$

Thermal correction to Energy=

0.162340 (Hartree/Particle)

Thermal correction to Enthalpy=

0.169194

0.170138

Thermal correction to Gibbs Free Energy=

0.133287

Sum of electronic and zero-point Energies=

$-197.235824$ 
Sum of electronic and thermal Energies $=$

Sum of electronic and thermal Enthalpies=

Sum of electronic and thermal Free Energies=

Butane

Standard orientation:

\begin{tabular}{|c|c|c|c|c|}
\hline \multirow{2}{*}{$\begin{array}{l}\text { Center } \\
\text { Number }\end{array}$} & Atomic & \multirow{2}{*}{$\begin{array}{r}\text { Atomic } \\
\text { Type }\end{array}$} & \multicolumn{2}{|c|}{ Coordinates (Angstrom } \\
\hline & Numbe & & $X$ & $\mathrm{Z}$ \\
\hline 1 & 6 & 1.942814 & -0.118982 & 0.000025 \\
\hline 2 & 1 & 2.087754 & -0.749142 & -0.880984 \\
\hline 3 & 1 & 2.087708 & -0.749120 & 0.881058 \\
\hline 4 & 1 & 2.732290 & 0.635134 & 0.000036 \\
\hline 5 & 6 & 0.561254 & 0.512595 & -0.000029 \\
\hline 6 & 1 & 0.452964 & 1.162734 & 0.875368 \\
\hline 7 & 1 & 0.453000 & 1.162697 & -0.875457 \\
\hline 8 & 6 & -0.561254 & -0.512595 & -0.000029 \\
\hline 9 & 1 & -0.453000 & -1.162695 & -0.875458 \\
\hline 10 & 1 & -0.452964 & -1.162736 & 0.875366 \\
\hline 11 & 6 & -1.942814 & 0.118982 & 0.000025 \\
\hline 12 & 1 & -2.087753 & 0.749145 & -0.880982 \\
\hline 13 & 1 & -2.732290 & -0.635134 & 0.000033 \\
\hline 14 & 1 & -2.087708 & 0.749117 & 0.881060 \\
\hline
\end{tabular}

Zero-point correction=

Thermal correction to Energy=

0.134519 (Hartree/Particle)

Thermal correction to Enthalpy=

0.140268

Thermal correction to Gibbs Free Energy=

0.141212

Sum of electronic and zero-point Energies $=$

0.106528

Sum of electronic and thermal Energies=

$-158.019049$

$-158.013301$

Sum of electronic and thermal Enthalpies=

$-158.012357$

Sum of electronic and thermal Free Energies= $-158.047040$

Isobutane

Standard orientation:

\begin{tabular}{cccccc} 
Center & \multicolumn{2}{c}{ Atomic } & \multicolumn{2}{c}{ Atomic } & \multicolumn{3}{c}{ Coordinates (Angstroms) } \\
Number & Number & Type & X & Y & $Z$ \\
\hline 1 & 6 & 0 & -0.682713 & -1.276384 & -0.095180 \\
2 & 1 & 0 & -1.713967 & -1.332234 & 0.260838 \\
3 & 1 & 0 & -0.706658 & -1.320840 & -1.188099 \\
4 & 1 & 0 & -0.156589 & -2.165186 & 0.260605 \\
5 & 6 & 0 & 0.000027 & 0.000014 & 0.373598 \\
6 & 1 & 0 & 0.000023 & 0.000020 & 1.471803 \\
7 & 6 & 0 & -0.764044 & 1.229427 & -0.095188 \\
8 & 1 & 0 & -0.296871 & 2.150471 & 0.260874 \\
9 & 1 & 0 & -0.790523 & 1.272386 & -1.188108 \\
10 & 1 & 0 & -1.796844 & 1.218094 & 0.260555 \\
11 & 6 & 0 & 1.446749 & 0.046952 & -0.095195 \\
12 & 1 & 0 & 1.953412 & 0.946956 & 0.260668
\end{tabular}




$\begin{array}{rrrrrr}13 & 1 & 0 & 2.010717 & -0.818255 & 0.260759 \\ 14 & 1 & 0 & 1.497182 & 0.048530 & -1.188109\end{array}$

Zero-point correction=

Thermal correction to Energy=

Thermal correction to Enthalpy=

Thermal correction to Gibbs Free Energy=

Sum of electronic and zero-point Energies=

Sum of electronic and thermal Energies=

Sum of electronic and thermal Enthalpies=

Sum of electronic and thermal Free Energies=

Pentane
0.134042 (Hartree/Particle)

$$
0.139702
$$

0.140646

0.106461

$-158.021330$

$-158.015670$

$-158.014726$

$-158.048911$

Standard orientation:

\begin{tabular}{|c|c|c|c|c|}
\hline \multirow{2}{*}{$\begin{array}{l}\text { Center } \\
\text { Number }\end{array}$} & Atomic & \multirow{2}{*}{$\begin{array}{l}\text { Atomic } \\
\text { Type }\end{array}$} & \multicolumn{2}{|c|}{ Coordinates (Angstroms } \\
\hline & Number & & $X$ & Z \\
\hline 1 & 6 & 2.532807 & 0.324274 & -0.000002 \\
\hline 2 & 1 & 2.573693 & 0.969592 & -0.880989 \\
\hline 3 & 1 & 2.573690 & 0.969608 & 0.880975 \\
\hline 4 & 1 & 3.433496 & -0.292622 & 0.000006 \\
\hline 5 & 6 & 1.271421 & -0.522103 & 0.000004 \\
\hline 6 & 1 & 1.270200 & -1.180699 & -0.875560 \\
\hline 7 & 1 & 1.270201 & -1.180689 & 0.875576 \\
\hline 8 & 6 & 0.000000 & 0.310402 & -0.000000 \\
\hline 9 & 1 & -0.000002 & 0.970576 & 0.875958 \\
\hline 10 & 1 & 0.000002 & 0.970575 & -0.875959 \\
\hline 11 & 6 & -1.271421 & -0.522103 & -0.000004 \\
\hline 12 & 1 & -1.270200 & -1.180698 & 0.875561 \\
\hline 13 & 1 & -1.270201 & -1.180689 & -0.875575 \\
\hline 14 & 6 & -2.532807 & 0.324274 & 0.000002 \\
\hline 15 & 1 & -3.433496 & -0.292622 & -0.000002 \\
\hline 16 & 1 & -2.573691 & 0.969595 & 0.880988 \\
\hline 17 & 1 & -2.573692 & 0.969606 & -0.880976 \\
\hline
\end{tabular}

Zero-point correction=

0.163566 (Hartree/Particle)

Thermal correction to Energy=

0.170612

Thermal correction to Enthalpy=

0.171556

Thermal correction to Gibbs Free Energy=

Sum of electronic and zero-point Energies=

0.133251

Sum of electronic and thermal Energies $=$

$-197.230577$

$-197.223532$

Sum of electronic and thermal Enthalpies=

$-197.222588$

Sum of electronic and thermal Free Energies=

$-197.260892$

Hexane

Standard orientation:

\begin{tabular}{cccccc} 
Center & Atomic & \multicolumn{2}{c}{ Atomic } & \multicolumn{2}{c}{ Coordinates (Angstroms) } \\
Number & Number & Type & X & Y & Z \\
- \hdashline 1 & 6 & 0 & -3.190539 & 0.207310 & 0.000009
\end{tabular}




$\begin{array}{cccccc}2 & 1 & 0 & -3.276679 & 0.848185 & 0.881012 \\ 3 & 1 & 0 & -3.276678 & 0.848233 & -0.880960 \\ 4 & 1 & 0 & -4.045672 & -0.471342 & -0.000010 \\ 5 & 6 & 0 & -1.872746 & -0.548154 & -0.000011 \\ 6 & 1 & 0 & -1.825024 & -1.205228 & 0.875485 \\ 7 & 1 & 0 & -1.825031 & -1.205193 & -0.875534 \\ 8 & 6 & 0 & -0.662608 & 0.371444 & 0.000001 \\ 9 & 1 & 0 & -0.709613 & 1.029702 & -0.876010 \\ 10 & 1 & 0 & -0.709619 & 1.029688 & 0.876023 \\ 11 & 6 & 0 & 0.662608 & -0.371444 & 0.000001 \\ 12 & 1 & 0 & 0.709613 & -1.029702 & -0.876010 \\ 13 & 1 & 0 & 0.709619 & -1.029688 & 0.876023 \\ 14 & 6 & 0 & 1.872746 & 0.548154 & -0.000011 \\ 15 & 1 & 0 & 1.825024 & 1.205228 & 0.875485 \\ 16 & 1 & 0 & 1.825031 & 1.205193 & -0.875534 \\ 17 & 6 & 0 & 3.190539 & -0.207310 & 0.000009 \\ 18 & 1 & 0 & 3.276679 & -0.848231 & -0.880961 \\ 19 & 1 & 0 & 4.045672 & 0.471342 & -0.000008 \\ 20 & 1 & 0 & 3.276678 & -0.848186 & 0.881012\end{array}$

Zero-point correction=

Thermal correction to Energy=

Thermal correction to Enthalpy=

Thermal correction to Gibbs Free Energy=

Sum of electronic and zero-point Energies=

Sum of electronic and thermal Energies $=$

Sum of electronic and thermal Enthalpies=

Sum of electronic and thermal Free Energies=

22dimethylbutane
0.192559 (Hartree/Particle)

$$
0.200930
$$$$
0.201874
$$

$$
\begin{aligned}
& 0.159964 \\
& -236.442185 \\
& -236.433815 \\
& -236.432871 \\
& -236.474780
\end{aligned}
$$

\begin{tabular}{|c|c|c|c|c|}
\hline \multirow{2}{*}{$\begin{array}{l}\text { Center } \\
\text { Number }\end{array}$} & Atomic & \multirow{2}{*}{$\begin{array}{l}\text { Atomic } \\
\text { Type }\end{array}$} & \multicolumn{2}{|c|}{ Coordinates (Angstroms) } \\
\hline & Numbe & & $\mathrm{X}$ & $\mathrm{Z}$ \\
\hline 1 & 6 & -1.609541 & 0.970775 & 0.001479 \\
\hline 2 & 1 & -1.588501 & 1.613374 & 0.885612 \\
\hline 3 & 1 & -1.588726 & 1.615754 & -0.880925 \\
\hline 4 & 1 & -2.561575 & 0.433930 & 0.000873 \\
\hline 5 & 6 & -0.431545 & -0.000198 & 0.000014 \\
\hline 6 & 6 & 0.863673 & 0.824847 & 0.000660 \\
\hline 7 & 1 & 0.844721 & 1.484706 & -0.874465 \\
\hline 8 & 1 & 0.844812 & 1.483238 & 0.876897 \\
\hline 9 & 6 & 2.164502 & 0.038439 & -0.000004 \\
\hline 10 & 1 & 2.255907 & -0.597866 & -0.882415 \\
\hline 11 & 1 & 3.021156 & 0.715404 & 0.000051 \\
\hline 12 & 1 & 2.256313 & -0.598552 & 0.881864 \\
\hline 13 & 6 & -0.513219 & -0.878816 & 1.245502 \\
\hline 14 & 1 & 0.279030 & -1.629886 & 1.265080 \\
\hline 15 & 1 & -0.431690 & -0.279095 & 2.156315 \\
\hline 16 & 1 & -1.468042 & -1.409600 & 1.280458 \\
\hline
\end{tabular}

Standard orientation: 


$\begin{array}{llllll}17 & 6 & 0 & -0.513892 & -0.875768 & -1.247585 \\ 18 & 1 & 0 & -1.468548 & -1.406817 & -1.283089 \\ 19 & 1 & 0 & -0.433319 & -0.273748 & -2.156961 \\ 20 & 1 & 0 & 0.278595 & -1.626517 & -1.269695\end{array}$

Zero-point correction=

Thermal correction to Energy=

Thermal correction to Enthalpy=

Thermal correction to Gibbs Free Energy=

Sum of electronic and zero-point Energies=

Sum of electronic and thermal Energies $=$

Sum of electronic and thermal Enthalpies=

Sum of electronic and thermal Free Energies=

Heptane
0.191416 (Hartree/Particle)

0.199602

0.200546

0.160125

$-236.445217$

$-236.437031$

$-236.436087$

$-236.476507$

Standard orientation:

\begin{tabular}{|c|c|c|c|c|}
\hline \multirow{3}{*}{$\begin{array}{c}\text { Center } \\
\text { Number } \\
-1\end{array}$} & \multirow{2}{*}{$\begin{array}{l}\text { Atomic } \\
\text { Number }\end{array}$} & \multirow{2}{*}{$\begin{array}{r}\text { Atomic } \\
\text { Type }\end{array}$} & \multicolumn{2}{|c|}{ Coordinates (Angstrom } \\
\hline & & & $\mathrm{X}$ & Z \\
\hline & 6 & -3.802997 & -0.354427 & -0.000009 \\
\hline 2 & 1 & -3.843381 & -0.999858 & -0.881020 \\
\hline 3 & 1 & -3.843375 & -0.999915 & 0.880961 \\
\hline 4 & 1 & -4.704113 & 0.261852 & 0.000014 \\
\hline 5 & 6 & -2.542031 & 0.492491 & 0.000015 \\
\hline 6 & 1 & -2.540909 & 1.151320 & -0.875485 \\
\hline 7 & 1 & -2.540912 & 1.151277 & 0.875547 \\
\hline 8 & 6 & -1.269891 & -0.339110 & -0.000002 \\
\hline 9 & 1 & -1.270169 & -0.999257 & 0.875940 \\
\hline 10 & 1 & -1.270177 & -0.999236 & -0.875961 \\
\hline 11 & 6 & 0.000000 & 0.495115 & -0.000000 \\
\hline 12 & 1 & -0.000003 & 1.154749 & 0.876101 \\
\hline 13 & 1 & 0.000003 & 1.154744 & -0.876106 \\
\hline 14 & 6 & 1.269891 & -0.339110 & 0.000006 \\
\hline 15 & 1 & 1.270168 & -0.999262 & -0.875933 \\
\hline 16 & 1 & 1.270177 & -0.999232 & 0.875968 \\
\hline 17 & 6 & 2.542031 & 0.492491 & -0.000015 \\
\hline 18 & 1 & 2.540911 & 1.151320 & 0.875485 \\
\hline 19 & 1 & 2.540910 & 1.151277 & -0.875547 \\
\hline 20 & 6 & 3.802997 & -0.354427 & 0.000006 \\
\hline 21 & 1 & 3.843377 & -0.999910 & -0.880968 \\
\hline 22 & 1 & 4.704113 & 0.261852 & -0.000013 \\
\hline 23 & 1 & 3.843380 & -0.999863 & 0.881013 \\
\hline
\end{tabular}

Zero-point correction=

Thermal correction to Energy=

Thermal correction to Enthalpy=

Thermal correction to Gibbs Free Energy=

Sum of electronic and zero-point Energies=

Sum of electronic and thermal Energies=
0.221550 (Hartree/Particle)

0.231252

0.232196

0.186700

$-275.653811$

$-275.644109$ 
Sum of electronic and thermal Enthalpies=

Sum of electronic and thermal Free Energies=

223trimethylbutane

Standard orientation:

\begin{tabular}{cccccc} 
Center & Atomic & \multicolumn{2}{c}{ Atomic } & \multicolumn{3}{c}{ Coordinates $($ Angstroms } \\
Number & Number & Type & X & Y & Z \\
-1 & 6 & 0 & -1.315888 & -1.294799 & -0.448312 \\
2 & 1 & 0 & -1.171396 & -1.466660 & -1.519000 \\
3 & 1 & 0 & -0.933247 & -2.164759 & 0.087716 \\
4 & 1 & 0 & -2.391471 & -1.243930 & -0.262205 \\
5 & 6 & 0 & -0.637749 & -0.000099 & -0.000652 \\
6 & 6 & 0 & 0.845599 & -0.001084 & -0.451745 \\
7 & 1 & 0 & 0.817216 & -0.005772 & -1.551141 \\
8 & 6 & 0 & 1.616048 & 1.242458 & -0.021527 \\
9 & 1 & 0 & 1.748029 & 1.271506 & 1.062917 \\
10 & 1 & 0 & 2.613127 & 1.240401 & -0.467279 \\
11 & 1 & 0 & 1.126662 & 2.168493 & -0.325742 \\
12 & 6 & 0 & 1.620415 & -1.240393 & -0.016824 \\
13 & 1 & 0 & 1.651351 & -1.331912 & 1.072078 \\
14 & 1 & 0 & 1.197928 & -2.161080 & -0.420034 \\
15 & 1 & 0 & 2.654447 & -1.177639 & -0.363068 \\
16 & 6 & 0 & -0.775459 & 0.130261 & 1.514785 \\
17 & 1 & 0 & -0.382725 & 1.082409 & 1.877356 \\
18 & 1 & 0 & -1.828780 & 0.080427 & 1.802392 \\
19 & 1 & 0 & -0.255820 & -0.673368 & 2.041487 \\
20 & 6 & 0 & -1.374309 & 1.163762 & -0.664006 \\
21 & 1 & 0 & -1.286817 & 1.115000 & -1.753092 \\
22 & 1 & 0 & -2.437717 & 1.133468 & -0.414051 \\
23 & 1 & 0 & -0.992735 & 2.132780 & -0.338655
\end{tabular}

Zero-point correction=

0.220131 (Hartree/Particle)

Thermal correction to Energy=

Thermal correction to Enthalpy=

0.229559

0.230504

Thermal correction to Gibbs Free Energy=

0.187068

Sum of electronic and zero-point Energies=

$-275.655049$

Sum of electronic and thermal Energies=

$-275.645620$

Sum of electronic and thermal Enthalpies=

$-275.644676$

Sum of electronic and thermal Free Energies $=\quad-275.688111$

Octane

Standard orientation:

\begin{tabular}{|c|c|c|c|c|}
\hline \multirow{2}{*}{$\begin{array}{l}\text { Center } \\
\text { Number }\end{array}$} & Atomic & \multirow{2}{*}{$\begin{array}{l}\text { Atomic } \\
\text { Type }\end{array}$} & \multicolumn{2}{|c|}{ Coordinates (Angstroms } \\
\hline & Number & & $\mathrm{X}$ & $\mathrm{Z}$ \\
\hline 1 & 6 & -4.451935 & 0.255844 & 0.000015 \\
\hline 2 & 1 & -4.518009 & 0.899163 & 0.881028 \\
\hline 3 & 1 & -4.518008 & 0.899232 & -0.880949 \\
\hline 4 & 1 & -5.327797 & -0.395832 & -0.000011 \\
\hline
\end{tabular}




$\begin{array}{cccccc}5 & 6 & 0 & -3.158214 & -0.540121 & -0.000017 \\ 6 & 1 & 0 & -3.130771 & -1.198460 & 0.875477 \\ 7 & 1 & 0 & -3.130779 & -1.198407 & -0.875551 \\ 8 & 6 & 0 & -1.920144 & 0.341403 & 0.000003 \\ 9 & 1 & 0 & -1.946644 & 1.001049 & -0.875942 \\ 10 & 1 & 0 & -1.946649 & 1.001020 & 0.875970 \\ 11 & 6 & 0 & -0.618185 & -0.441699 & -0.000003 \\ 12 & 1 & 0 & -0.591987 & -1.101015 & -0.876033 \\ 13 & 1 & 0 & -0.591980 & -1.101016 & 0.876025 \\ 14 & 6 & 0 & 0.618185 & 0.441699 & -0.000008 \\ 15 & 1 & 0 & 0.591979 & 1.101027 & 0.876012 \\ 16 & 1 & 0 & 0.591988 & 1.101003 & -0.876046 \\ 17 & 6 & 0 & 1.920144 & -0.341403 & 0.000009 \\ 18 & 1 & 0 & 1.946646 & -1.001054 & -0.875932 \\ 19 & 1 & 0 & 1.946647 & -1.001015 & 0.875979 \\ 20 & 6 & 0 & 3.158215 & 0.540121 & -0.000012 \\ 21 & 1 & 0 & 3.130774 & 1.198455 & 0.875486 \\ 22 & 1 & 0 & 3.130776 & 1.198411 & -0.875542 \\ 23 & 6 & 0 & 4.451935 & -0.255844 & 0.000010 \\ 24 & 1 & 0 & 4.518010 & -0.899220 & -0.880962 \\ 25 & 1 & 0 & 5.327797 & 0.395832 & -0.000006 \\ 26 & 1 & 0 & 4.518008 & -0.899175 & 0.881015\end{array}$

Zero-point correction $=$

Thermal correction to Energy=

Thermal correction to Enthalpy=

0.250528 (Hartree/Particle)

0.261571

0.262515

Thermal correction to Gibbs Free Energy $=0.213436$

Sum of electronic and zero-point Energies $=\quad-314.865448$

Sum of electronic and thermal Energies $=\quad-314.854405$

Sum of electronic and thermal Enthalpies $=\quad-314.853461$

Sum of electronic and thermal Free Energies $=\quad-314.902540$

2233tetramethylbutane

Standard orientation:

\begin{tabular}{|c|c|c|c|c|}
\hline \multirow{2}{*}{$\begin{array}{l}\text { Center } \\
\text { Number }\end{array}$} & Atomic & \multirow{2}{*}{$\begin{array}{r}\text { Atomic } \\
\text { Type }\end{array}$} & \multicolumn{2}{|c|}{ Coordinates (Angstrom } \\
\hline & Numbe & & $\mathrm{X}$ & Z \\
\hline 1 & 6 & -1.337937 & -1.390711 & -0.318703 \\
\hline 2 & 1 & -1.136081 & -1.683565 & -1.350413 \\
\hline 3 & 1 & -0.925784 & -2.159808 & 0.338334 \\
\hline 4 & 1 & -2.422442 & -1.395972 & -0.184293 \\
\hline 5 & 6 & -0.784494 & 0.000044 & -0.000044 \\
\hline 6 & 6 & 0.784546 & 0.000047 & -0.000002 \\
\hline 7 & 6 & 1.338094 & 1.426740 & 0.012353 \\
\hline 8 & 1 & 1.136333 & 1.950661 & -0.923451 \\
\hline 9 & 1 & 0.925949 & 2.022702 & 0.829681 \\
\hline 10 & 1 & 2.422595 & 1.400654 & 0.144367 \\
\hline 11 & 6 & -1.338074 & 0.971335 & -1.045077 \\
\hline 12 & 1 & -1.135963 & 2.011233 & -0.782943 \\
\hline 13 & 1 & -0.926235 & 0.786732 & -2.039746 \\
\hline
\end{tabular}




$\begin{array}{rrrrrr}14 & 1 & 0 & -2.422621 & 0.857761 & -1.116539 \\ 15 & 6 & 0 & -1.338066 & 0.419345 & 1.363687 \\ 16 & 1 & 0 & -2.422595 & 0.538162 & 1.301039 \\ 17 & 1 & 0 & -1.136047 & -0.327728 & 2.133118 \\ 18 & 1 & 0 & -0.926149 & 1.372980 & 1.701289 \\ 19 & 6 & 0 & 1.338073 & -0.702722 & -1.241694 \\ 20 & 1 & 0 & 2.422606 & -0.575635 & -1.285017 \\ 21 & 1 & 0 & 1.136000 & -1.775061 & -1.227580 \\ 22 & 1 & 0 & 0.926131 & -0.292717 & -2.166489 \\ 23 & 6 & 0 & 1.337902 & -0.724026 & 1.229441 \\ 24 & 1 & 0 & 2.422452 & -0.825073 & 1.141188 \\ 25 & 1 & 0 & 1.135659 & -0.175709 & 2.151058 \\ 26 & 1 & 0 & 0.925932 & -1.729932 & 1.336624 \\ -------------------------------------------------------------------\end{array}$

Zero-point correction $=$

Thermal correction to Energy=

0.249010 (Hartree/Particle)

Thermal correction to Enthalpy=

0.259304

0.260248

Thermal correction to Gibbs Free Energy=

Sum of electronic and zero-point Energies=

0.215842

Sum of electronic and thermal Energies $=$

$-314.864361$

$-314.854067$

Sum of electronic and thermal Enthalpies=

$-314.853123$

Sum of electronic and thermal Free Energies= $-314.897529$

PBEQIDH/DHSVPD

Ethane

Standard orientation:

\begin{tabular}{cccrcc} 
Center & \multicolumn{2}{c}{ Atomic } & \multicolumn{2}{c}{ Atomic } & \multicolumn{2}{c}{ Coordinates (Angstroms) } \\
Number & Number & \multicolumn{1}{c}{ Type } & X & Y & Z \\
-1 & 6 & 0 & -0.000000 & -0.000000 & 0.759733 \\
2 & 1 & 0 & -0.507964 & 0.879853 & 1.157410 \\
3 & 1 & 0 & 1.015957 & -0.000017 & 1.157410 \\
4 & 1 & 0 & -0.507993 & -0.879836 & 1.157410 \\
5 & 6 & 0 & 0.000000 & -0.000000 & -0.759733 \\
6 & 1 & 0 & 0.507964 & 0.879853 & -1.157410 \\
7 & 1 & 0 & 0.507993 & -0.879836 & -1.157410 \\
8 & 1 & 0 & -1.015957 & -0.000017 & -1.157410
\end{tabular}

Zero-point correction=

Thermal correction to Energy=

0.076572 (Hartree/Particle)

0.080039

Thermal correction to Enthalpy=

0.080983

Thermal correction to Gibbs Free Energy $=0.055167$

Sum of electronic and zero-point Energies=

$-79.568002$

Sum of electronic and thermal Energies $=$

$-79.564536$

Sum of electronic and thermal Enthalpies=

$-79.563591$

Sum of electronic and thermal Free Energies $=\quad-79.589407$

Methane

Standard orientation:

Center Atomic Atomic Coordinates (Angstroms)




$\begin{array}{cccccc}\text { Number } & \text { Number } & \text { Type } & X & Y & Z \\ -1 & 6 & 0 & 0.000000 & 0.000000 & 0.000000 \\ 2 & 1 & 0 & 0.628415 & 0.628415 & 0.628415 \\ 3 & 1 & 0 & -0.628415 & -0.628415 & 0.628415 \\ 4 & 1 & 0 & -0.628415 & 0.628415 & -0.628415 \\ 5 & 1 & 0 & 0.628415 & -0.628415 & -0.628415\end{array}$

Zero-point correction=

Thermal correction to Energy=

Thermal correction to Enthalpy=

Thermal correction to Gibbs Free Energy=

Sum of electronic and zero-point Energies=

Sum of electronic and thermal Energies $=$

Sum of electronic and thermal Enthalpies=

Sum of electronic and thermal Free Energies=

Propane

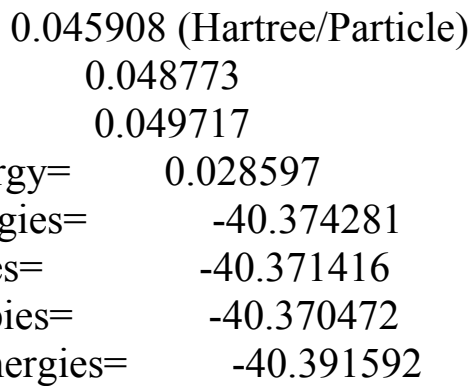

Standard orientation:

\begin{tabular}{|c|c|c|c|c|}
\hline \multirow{2}{*}{$\begin{array}{l}\text { Center } \\
\text { Number }\end{array}$} & \multirow{2}{*}{$\begin{array}{l}\text { Atomic } \\
\text { Number }\end{array}$} & \multirow{2}{*}{$\begin{array}{r}\text { Atomic } \\
\text { Type }\end{array}$} & \multicolumn{2}{|c|}{ Coordinates (Angstron } \\
\hline & & & $\mathrm{X}$ & $\mathrm{Z}$ \\
\hline 1 & 6 & -1.262105 & -0.259097 & 0.000000 \\
\hline 2 & 1 & -2.162979 & 0.356809 & -0.000034 \\
\hline 3 & 1 & -1.300482 & -0.903896 & 0.880821 \\
\hline 4 & 1 & -1.300452 & -0.903950 & -0.880783 \\
\hline 5 & 6 & 0.000000 & 0.587304 & 0.000000 \\
\hline 6 & 1 & 0.000001 & 1.243718 & 0.875062 \\
\hline 7 & 1 & 0.000002 & 1.243702 & -0.875073 \\
\hline 8 & 6 & 1.262105 & -0.259097 & 0.000000 \\
\hline 9 & 1 & 1.300483 & -0.903891 & 0.880825 \\
\hline 10 & 1 & 1.300446 & -0.903955 & -0.880779 \\
\hline 11 & 1 & 2.162980 & 0.356807 & -0.000042 \\
\hline
\end{tabular}

Zero-point correction $=$

Thermal correction to Energy=

Thermal correction to Enthalpy=

Thermal correction to Gibbs Free Energy=

Sum of electronic and zero-point Energies=

Sum of electronic and thermal Energies=

Sum of electronic and thermal Enthalpies=

Sum of electronic and thermal Free Energies=

Butane
0.105972 (Hartree/Particle)

$$
0.110457
$$

0.111401

0.080356

$-118.766342$

$-118.761858$

$-118.760913$

$-118.791959$

Standard orientation:

\begin{tabular}{|c|c|c|c|c|}
\hline Center & Atomic & Atomic & Coordinate & s (Angstroms) \\
\hline Number & Number & Type & $X$ & Z \\
\hline 1 & 6 & 1.94146 & -0.119006 & 0028 \\
\hline 2 & 1 & 2.084161 & -0.748787 & -0.880865 \\
\hline
\end{tabular}




$\begin{array}{cccccc}3 & 1 & 0 & 2.084102 & -0.748778 & 0.880938 \\ 4 & 1 & 0 & 2.731167 & 0.634085 & 0.000050 \\ 5 & 6 & 0 & 0.560147 & 0.513732 & -0.000032 \\ 6 & 1 & 0 & 0.451200 & 1.162435 & 0.875614 \\ 7 & 1 & 0 & 0.451240 & 1.162395 & -0.875714 \\ 8 & 6 & 0 & -0.560147 & -0.513732 & -0.000032 \\ 9 & 1 & 0 & -0.451240 & -1.162393 & -0.875716 \\ 10 & 1 & 0 & -0.451200 & -1.162438 & 0.875612 \\ 11 & 6 & 0 & -1.941462 & 0.119006 & 0.000029 \\ 12 & 1 & 0 & -2.084161 & 0.748790 & -0.880863 \\ 13 & 1 & 0 & -2.731167 & -0.634085 & 0.000048 \\ 14 & 1 & 0 & -2.084102 & 0.748775 & 0.880939\end{array}$

Zero-point correction=

Thermal correction to Energy=

Thermal correction to Enthalpy=

Thermal correction to Gibbs Free Energy=

Sum of electronic and zero-point Energies=

Sum of electronic and thermal Energies $=$

Sum of electronic and thermal Enthalpies=

Sum of electronic and thermal Free Energies=

Isobutane

Standard orientation:

\begin{tabular}{|c|c|c|c|c|}
\hline \multirow{2}{*}{$\begin{array}{l}\text { Center } \\
\text { Number }\end{array}$} & Atomic & \multirow{2}{*}{$\begin{array}{r}\text { Atomic } \\
\text { Type }\end{array}$} & \multicolumn{2}{|c|}{ Coordinates (Angstroms } \\
\hline & Numbe & & $X$ & Z \\
\hline 1 & 6 & 1.382016 & 0.426603 & -0.095582 \\
\hline 2 & 1 & 2.154203 & -0.259172 & 0.259011 \\
\hline 3 & 1 & 1.425745 & 0.440142 & -1.188205 \\
\hline 4 & 1 & 1.633344 & 1.428278 & 0.259073 \\
\hline 5 & 6 & 0.000001 & 0.000003 & 0.376183 \\
\hline 6 & 1 & -0.000003 & -0.000000 & 1.473754 \\
\hline 7 & 6 & -0.321556 & -1.410154 & -0.095582 \\
\hline 8 & 1 & -1.301549 & -1.736009 & 0.259005 \\
\hline 9 & 1 & -0.331688 & -1.454778 & -1.188208 \\
\hline 10 & 1 & 0.420254 & -2.128652 & 0.259072 \\
\hline 11 & 6 & -1.060461 & 0.983550 & -0.095582 \\
\hline 12 & 1 & -2.053639 & 0.700194 & 0.258823 \\
\hline 13 & 1 & -0.852829 & 1.995121 & 0.259256 \\
\hline 14 & 1 & -1.093840 & 1.014867 & -1.188206 \\
\hline
\end{tabular}

Zero-point correction=

Thermal correction to Energy=

Thermal correction to Enthalpy=

Thermal correction to Gibbs Free Energy=

Sum of electronic and zero-point Energies=

Sum of electronic and thermal Energies $=$

Sum of electronic and thermal Enthalpies=

Sum of electronic and thermal Free Energies=
0.140876
0.141820
0.107175
$-157.964944$
$-157.959214$
$-157.958270$
$-157.992915$ 
Pentane

Standard orientation:

\begin{tabular}{|c|c|c|c|c|c|}
\hline \multirow{2}{*}{$\begin{array}{l}\text { Center } \\
\text { Number }\end{array}$} & Atomic & \multirow{2}{*}{\multicolumn{2}{|c|}{$\begin{array}{l}\text { Atomic } \\
\text { Type }\end{array}$}} & \multicolumn{2}{|c|}{ Coordinates (Angstrom } \\
\hline & Numbe & & & $X$ & Z \\
\hline 1 & 6 & 0 & 2.530354 & 0.325596 & -0.000001 \\
\hline 2 & 1 & 0 & 2.568057 & 0.970254 & -0.880891 \\
\hline 3 & 1 & 0 & 2.568053 & 0.970269 & 0.880878 \\
\hline 4 & 1 & 0 & 3.432021 & -0.288938 & 0.000006 \\
\hline 5 & 6 & 0 & 1.270588 & -0.523659 & 0.000003 \\
\hline 6 & 1 & 0 & 1.269261 & -1.180905 & -0.875869 \\
\hline 7 & 1 & 0 & 1.269262 & -1.180896 & 0.875882 \\
\hline 8 & 6 & 0 & 0.000000 & 0.309931 & -0.000000 \\
\hline 9 & 1 & 0 & -0.000002 & 0.968795 & 0.876318 \\
\hline 10 & 1 & 0 & 0.000002 & 0.968794 & -0.876320 \\
\hline 11 & 6 & 0 & -1.270588 & -0.523659 & -0.000003 \\
\hline 12 & 1 & 0 & -1.269261 & -1.180904 & $4 \quad 0.875869$ \\
\hline 13 & 1 & 0 & -1.269262 & -1.180896 & $5 \quad-0.875881$ \\
\hline 14 & 6 & 0 & -2.530354 & 0.325596 & 0.000002 \\
\hline 15 & 1 & 0 & -3.432021 & -0.288938 & $\begin{array}{ll}3 & -0.000002\end{array}$ \\
\hline 16 & 1 & 0 & -2.568055 & 0.970257 & 0.880889 \\
\hline 17 & 1 & 0 & -2.568056 & 0.970266 & -0.880880 \\
\hline
\end{tabular}

Zero-point correction $=$

0.164311 (Hartree/Particle)

Thermal correction to Energy=

0.171332

Thermal correction to Enthalpy=

0.172276

Thermal correction to Gibbs Free Energy=

Sum of electronic and zero-point Energies=

0.134029

Sum of electronic and thermal Energies $=$

$-197.163566$

$-197.156545$

Sum of electronic and thermal Enthalpies=

$-197.155601$

Sum of electronic and thermal Free Energies=

$-197.193848$

Neopentane

Standard orientation:

\begin{tabular}{cccccc} 
Center & \multicolumn{2}{c}{ Atomic } & \multicolumn{2}{c}{ Atomic } & \multicolumn{2}{c}{ Coordinates (Angstroms } \\
Number & Number & Type & X & Y & $Z$ \\
- \hdashline 1 & 6 & 0 & 1.118484 & -0.927017 & 0.464130 \\
2 & 1 & 0 & 1.040344 & -1.906063 & -0.014900 \\
3 & 1 & 0 & 2.099034 & -0.511274 & 0.219631 \\
4 & 1 & 0 & 1.078602 & -1.078591 & 1.545575 \\
5 & 6 & 0 & -0.000000 & -0.000002 & 0.000013 \\
6 & 6 & 0 & 0.088488 & 0.186532 & -1.510996 \\
7 & 1 & 0 & -0.703078 & 0.848089 & -1.871376 \\
8 & 1 & 0 & 1.047882 & 0.624982 & -1.796337 \\
9 & 1 & 0 & -0.011089 & -0.769653 & -2.030505 \\
10 & 6 & 0 & -1.349756 & -0.612793 & 0.358377 \\
11 & 1 & 0 & -1.440403 & -0.757571 & 1.437694
\end{tabular}




$\begin{array}{rrrrrc}12 & 1 & 0 & -2.170996 & 0.032141 & 0.036318 \\ 13 & 1 & 0 & -1.478744 & -1.585518 & -0.122529 \\ 14 & 6 & 0 & 0.142783 & 1.353282 & 0.688486 \\ 15 & 1 & 0 & 0.082329 & 1.248918 & 1.774561 \\ 16 & 1 & 0 & 1.103503 & 1.815579 & 0.448899 \\ 17 & 1 & 0 & -0.647376 & 2.038959 & 0.372908\end{array}$

Zero-point correction=

Thermal correction to Energy=

Thermal correction to Enthalpy=

Thermal correction to Gibbs Free Energy=

Sum of electronic and zero-point Energies=

Sum of electronic and thermal Energies $=$

Sum of electronic and thermal Enthalpies=

Sum of electronic and thermal Free Energies=

Hexane
0.163078 (Hartree/Particle)

0.169896

0.170840

0.134061

$-197.170260$

$-197.163442$

$-197.162498$

$-197.199277$

Standard orientation:

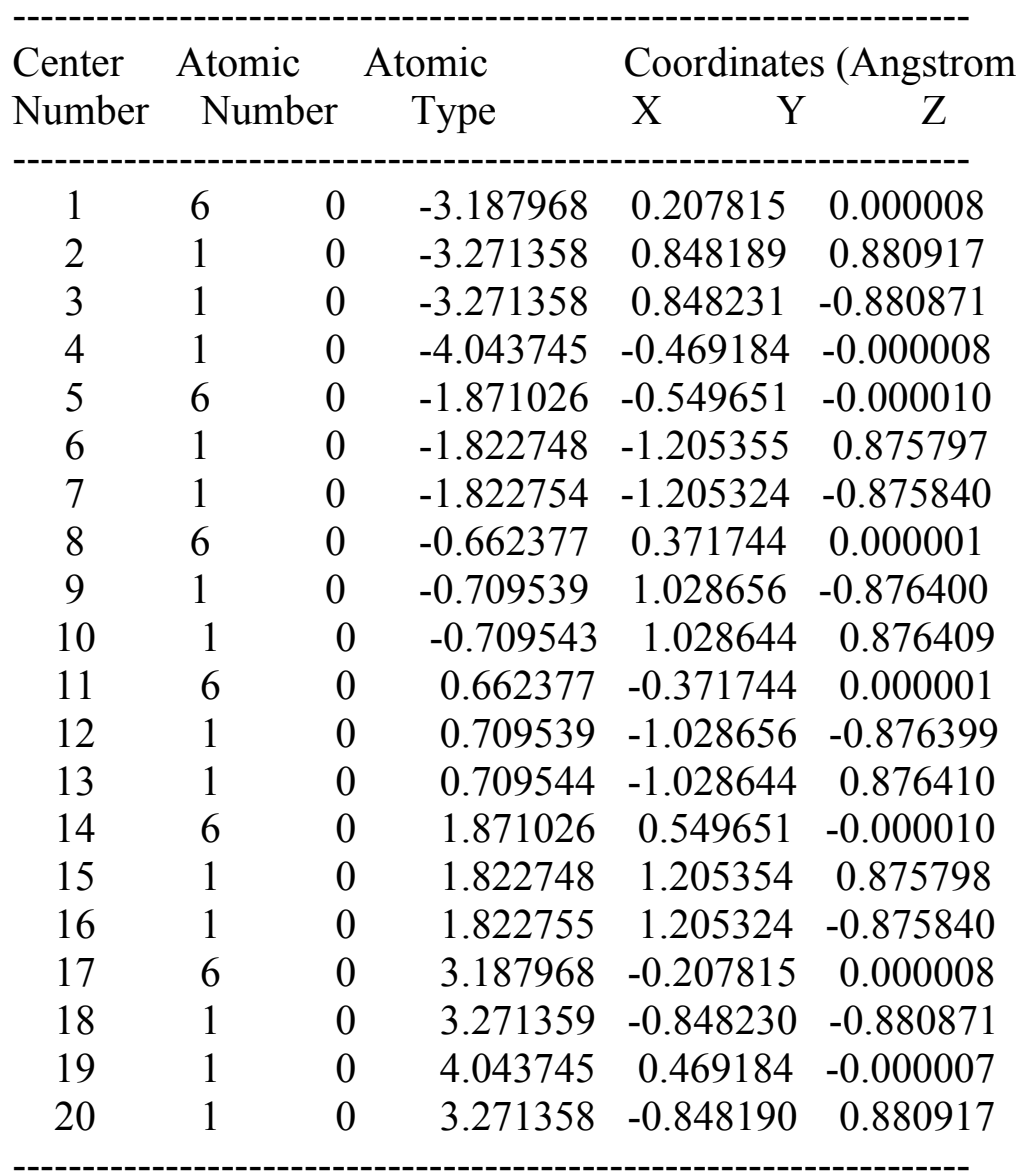

Zero-point correction $=$

Thermal correction to Energy=

0.193420 (Hartree/Particle)

Thermal correction to Enthalpy=

0.201761

0.202705

Thermal correction to Gibbs Free Energy=

0.160866

Sum of electronic and zero-point Energies $=$

$-236.362270$

Sum of electronic and thermal Energies=

$-236.353929$

Sum of electronic and thermal Enthalpies=

$-236.352985$

Sum of electronic and thermal Free Energies=

$-236.394824$ 
22dimethylbutane

Standard orientation:

\begin{tabular}{|c|c|c|c|c|c|}
\hline \multirow{2}{*}{$\begin{array}{l}\text { Center } \\
\text { Number }\end{array}$} & Atomic & \multirow{2}{*}{\multicolumn{2}{|c|}{$\begin{array}{l}\text { Atomic } \\
\text { Type }\end{array}$}} & \multicolumn{2}{|c|}{ Coordinates (Angstroms } \\
\hline & Number & & & $X$ & Z \\
\hline 1 & 6 & 0 & -1.610512 & 0.967876 & 0.001576 \\
\hline 2 & 1 & 0 & -1.589577 & 1.609744 & 0.885824 \\
\hline 3 & 1 & 0 & -1.589906 & 1.612177 & -0.880910 \\
\hline 4 & 1 & 0 & -2.560631 & 0.428314 & 0.001006 \\
\hline 5 & 6 & 0 & -0.430907 & -0.000080 & 0.000014 \\
\hline 6 & 6 & 0 & 0.861546 & 0.827080 & 0.000602 \\
\hline 7 & 1 & 0 & 0.841978 & 1.485745 & -0.875019 \\
\hline 8 & 1 & 0 & 0.842084 & 1.484388 & 0.877250 \\
\hline 9 & 6 & 0 & 2.161641 & 0.038949 & -0.000014 \\
\hline 10 & 1 & 0 & 2.250688 & -0.596920 & -0.882277 \\
\hline 11 & 1 & 0 & 3.018678 & 0.714680 & -0.000115 \\
\hline 12 & 1 & 0 & 2.251189 & -0.597323 & 0.881901 \\
\hline 13 & 6 & 0 & -0.510417 & -0.878529 & 1.244891 \\
\hline 14 & 1 & 0 & 0.281487 & -1.629442 & 1.261996 \\
\hline 15 & 1 & 0 & -0.426952 & -0.278218 & 2.154772 \\
\hline 16 & 1 & 0 & -1.465472 & -1.408235 & 1.280403 \\
\hline 17 & 6 & 0 & -0.511255 & -0.875419 & -1.247009 \\
\hline 18 & 1 & 0 & -1.466106 & -1.405469 & -1.282909 \\
\hline 19 & 1 & 0 & -0.428974 & -0.272753 & -2.155436 \\
\hline 20 & 1 & 0 & 0.280947 & -1.625951 & -1.266844 \\
\hline
\end{tabular}

Zero-point correction $=$

Thermal correction to Energy=

0.192262 (Hartree/Particle)

Thermal correction to Enthalpy=

0.200411

Thermal correction to Gibbs Free Energy=

Sum of electronic and zero-point Energies=

Sum of electronic and thermal Energies $=$

0.201355

0.160988

$-236.367060$

$-236.358912$

Sum of electronic and thermal Enthalpies=

$-236.357968$

Sum of electronic and thermal Free Energies=

$-236.398335$

Heptane

Standard orientation:

\begin{tabular}{|c|c|c|c|c|}
\hline \multirow{2}{*}{$\begin{array}{l}\text { Center } \\
\text { Number }\end{array}$} & Atomic & \multirow{2}{*}{$\begin{array}{l}\text { Atomic } \\
\text { Type }\end{array}$} & \multicolumn{2}{|c|}{ Coordinates (Angstroms) } \\
\hline & Number & & $\mathrm{X}$ & $\mathrm{Z}$ \\
\hline 1 & 6 & -3.799552 & -0.356257 & -0.000009 \\
\hline 2 & 1 & -3.836685 & -1.001026 & -0.880928 \\
\hline 3 & 1 & -3.836681 & -1.001077 & 0.880873 \\
\hline 4 & 1 & -4.701717 & 0.257559 & 0.000011 \\
\hline 5 & 6 & -2.540298 & 0.493673 & 0.000014 \\
\hline 6 & 1 & -2.539082 & 1.151163 & -0.875806 \\
\hline 7 & 1 & -2.539086 & 1.151123 & 0.875863 \\
\hline 8 & 6 & -1.268839 & -0.338783 & -0.000001 \\
\hline 9 & 1 & -1.268807 & -0.997629 & 0.876327 \\
\hline
\end{tabular}




$\begin{array}{rrrrrr}10 & 1 & 0 & -1.268814 & -0.997611 & -0.876343 \\ 11 & 6 & 0 & 0.000000 & 0.496854 & 0.000000 \\ 12 & 1 & 0 & -0.000002 & 1.155138 & 0.876513 \\ 13 & 1 & 0 & 0.000002 & 1.155133 & -0.876516 \\ 14 & 6 & 0 & 1.268839 & -0.338783 & 0.000006 \\ 15 & 1 & 0 & 1.268806 & -0.997634 & -0.876319 \\ 16 & 1 & 0 & 1.268815 & -0.997606 & 0.876351 \\ 17 & 6 & 0 & 2.540298 & 0.493673 & -0.000014 \\ 18 & 1 & 0 & 2.539084 & 1.151164 & 0.875805 \\ 19 & 1 & 0 & 2.539083 & 1.151123 & -0.875863 \\ 20 & 6 & 0 & 3.799552 & -0.356257 & 0.000005 \\ 21 & 1 & 0 & 3.836681 & -1.001074 & -0.880880 \\ 22 & 1 & 0 & 4.701717 & 0.257559 & -0.000013 \\ 23 & 1 & 0 & 3.836685 & -1.001029 & 0.880922\end{array}$

Zero-point correction=

0.222525 (Hartree/Particle)

Thermal correction to Energy=

0.232193

Thermal correction to Enthalpy=

0.233138

Thermal correction to Gibbs Free Energy=

Sum of electronic and zero-point Energies=

0.187724

Sum of electronic and thermal Energies $=$

$-275.560995$

$-275.551326$

Sum of electronic and thermal Enthalpies=

$-275.550382$

Sum of electronic and thermal Free Energies $=\quad-275.595795$

223trimethylbutane

Standard orientation:

\begin{tabular}{|c|c|c|c|c|}
\hline \multirow{2}{*}{$\begin{array}{l}\text { Center } \\
\text { Number }\end{array}$} & Atomic & \multirow{2}{*}{$\begin{array}{r}\text { Atomic } \\
\text { Type }\end{array}$} & \multicolumn{2}{|c|}{ Coordinates (Angstroms } \\
\hline & & & $\mathrm{X}$ & $\mathrm{Z}$ \\
\hline 1 & 6 & -1.308156 & -1.307146 & -0.419779 \\
\hline 2 & 1 & -1.160448 & -1.501559 & -1.485876 \\
\hline 3 & 1 & -0.921452 & -2.161783 & 0.136906 \\
\hline 4 & 1 & -2.383836 & -1.255894 & -0.236082 \\
\hline 5 & 6 & -0.636744 & -0.000236 & -0.000944 \\
\hline 6 & 6 & 0.843674 & -0.001342 & -0.455316 \\
\hline 7 & 1 & 0.814627 & -0.007332 & -1.554404 \\
\hline 8 & 6 & 1.612745 & 1.242186 & -0.023024 \\
\hline 9 & 1 & 1.755781 & 1.259460 & 1.059793 \\
\hline 10 & 1 & 2.604150 & 1.249106 & -0.480255 \\
\hline 11 & 1 & 1.114674 & 2.167971 & -0.312134 \\
\hline 12 & 6 & 1.618727 & -1.239191 & -0.017045 \\
\hline 13 & 1 & 1.637157 & -1.333125 & 1.071530 \\
\hline 14 & 1 & 1.203651 & -2.159185 & -0.428196 \\
\hline 15 & 1 & 2.655766 & -1.170447 & -0.351835 \\
\hline 16 & 6 & -0.770363 & 0.160469 & 1.511281 \\
\hline 17 & 1 & -0.391818 & 1.126479 & 1.850380 \\
\hline 18 & 1 & -1.821690 & 0.099479 & 1.802984 \\
\hline 19 & 1 & -0.235546 & -0.623152 & 2.052181 \\
\hline 20 & 6 & -1.381287 & 1.145336 & -0.685273 \\
\hline 21 & 1 & -1.303633 & 1.068325 & -1.773123 \\
\hline
\end{tabular}




$\begin{array}{llllll}22 & 1 & 0 & -2.441699 & 1.119314 & -0.423579 \\ 23 & 1 & 0 & -0.997258 & 2.121893 & -0.387685\end{array}$

$\begin{array}{lc}\text { Zero-point correction }= & 0.221130 \text { (Hartree/Particle) } \\ \text { Thermal correction to Energy= } & 0.230478 \\ \text { Thermal correction to Enthalpy= } & 0.231422 \\ \text { Thermal correction to Gibbs Free Energy= } & 0.188289 \\ \text { Sum of electronic and zero-point Energies }= & -275.564819 \\ \text { Sum of electronic and thermal Energies }= & -275.555471 \\ \text { Sum of electronic and thermal Enthalpies }= & -275.554527 \\ \text { Sum of electronic and thermal Free Energies }= & -275.597660\end{array}$

Octane

Standard orientation:

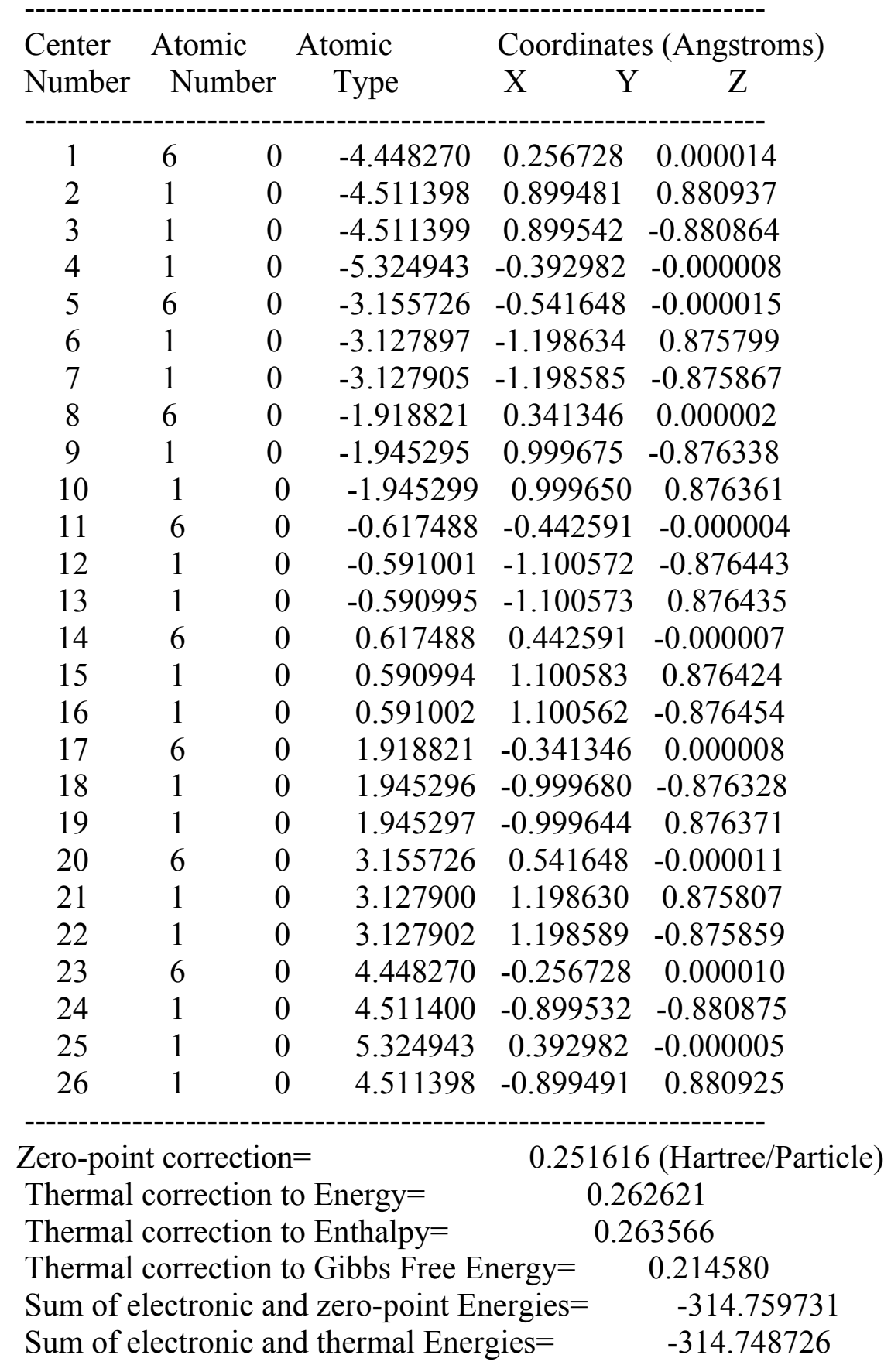


Sum of electronic and thermal Enthalpies=

$-314.747782$

Sum of electronic and thermal Free Energies=

2233tetramethylbutane

Standard orientation:

\begin{tabular}{|c|c|c|c|c|c|}
\hline \multirow{2}{*}{$\begin{array}{l}\text { Center } \\
\text { Number }\end{array}$} & \multicolumn{2}{|c|}{ Atomic } & \multirow{2}{*}{$\begin{array}{l}\text { Atomic } \\
\text { Type }\end{array}$} & \multicolumn{2}{|c|}{ Coordinates (Angstrom } \\
\hline & & & & $X$ & Z \\
\hline 1 & 6 & 0 & -1.335834 & 1.090175 & 0.919866 \\
\hline 2 & 1 & 0 & -1.136361 & 0.875141 & 1.970707 \\
\hline 3 & 1 & 0 & -0.920083 & 2.073291 & 0.689550 \\
\hline 4 & 1 & 0 & -2.419631 & 1.158458 & 0.799613 \\
\hline 5 & 6 & 0 & -0.783148 & 0.000031 & 0.000036 \\
\hline 6 & 6 & 0 & 0.783169 & -0.000034 & -0.000006 \\
\hline 7 & 6 & 0 & 1.335749 & -1.267562 & -0.654171 \\
\hline 8 & 1 & 0 & 1.136266 & -2.155569 & -0.052564 \\
\hline 9 & 1 & 0 & 0.919800 & -1.431828 & -1.650362 \\
\hline 10 & 1 & 0 & 2.419532 & -1.182732 & -0.763495 \\
\hline 11 & 6 & 0 & -1.335887 & -1.341668 & 0.484152 \\
\hline 12 & 1 & 0 & -1.136014 & -2.144197 & -0.227395 \\
\hline 13 & 1 & 0 & -0.920455 & -1.633710 & 1.450873 \\
\hline 14 & 1 & 0 & -2.419737 & -1.271807 & 0.602997 \\
\hline 15 & 6 & 0 & -1.335836 & 0.251597 & -1.403996 \\
\hline 16 & 1 & 0 & -2.419664 & 0.113577 & -1.402957 \\
\hline 17 & 1 & 0 & -1.136114 & 1.269109 & -1.743205 \\
\hline 18 & 1 & 0 & -0.920269 & -0.439521 & -2.140268 \\
\hline 19 & 6 & 0 & 1.335907 & 0.067190 & 1.424753 \\
\hline 20 & 1 & 0 & 2.419705 & -0.069766 & 1.405802 \\
\hline 21 & 1 & 0 & 1.136376 & 1.032153 & 1.893083 \\
\hline 22 & 1 & 0 & 0.920135 & -0.713503 & 2.065099 \\
\hline 23 & 6 & 0 & 1.335918 & 1.200268 & -0.770604 \\
\hline 24 & 1 & 0 & 2.419749 & 1.252205 & -0.642755 \\
\hline 25 & 1 & 0 & 1.136159 & 1.123519 & -1.840413 \\
\hline 26 & 1 & 0 & 0.920376 & 2.145203 & -0.414504 \\
\hline
\end{tabular}

Zero-point correction $=$

0.250080 (Hartree/Particle)

Thermal correction to Energy=

0.260295

Thermal correction to Enthalpy=

0.261239

Thermal correction to Gibbs Free Energy $=0.217009$

Sum of electronic and zero-point Energies $=\quad-314.762853$

Sum of electronic and thermal Energies $=$

$-314.752638$

Sum of electronic and thermal Enthalpies $=$

$-314.751694$

Sum of electronic and thermal Free Energies= $-314.795923$

B2PLYPD3/DHSVPD

Ethane

Standard orientation:

\begin{tabular}{lllll} 
Center & Atomic & Atomic & & \multicolumn{2}{c}{ Coordinates (Angstroms) } \\
Number & Number & Type & X & Y C Z
\end{tabular}




$\begin{array}{rrrrrr}1 & 6 & 0 & 0.000000 & 0.000000 & 0.765093 \\ 2 & 1 & 0 & -0.509212 & 0.882037 & 1.162361 \\ 3 & 1 & 0 & 1.018472 & -0.000028 & 1.162361 \\ 4 & 1 & 0 & -0.509261 & -0.882009 & 1.162361 \\ 5 & 6 & 0 & 0.000000 & 0.000000 & -0.765093 \\ 6 & 1 & 0 & 0.509212 & 0.882037 & -1.162361 \\ 7 & 1 & 0 & 0.509261 & -0.882009 & -1.162361 \\ 8 & 1 & 0 & -1.018472 & -0.000028 & -1.162361\end{array}$

Zero-point correction=

Thermal correction to Energy=

Thermal correction to Enthalpy=

Thermal correction to Gibbs Free Energy=

Sum of electronic and zero-point Energies=

Sum of electronic and thermal Energies $=$

Sum of electronic and thermal Enthalpies=

Sum of electronic and thermal Free Energies=

Methane
0.075491 (Hartree/Particle)

$$
0.078969
$$

0.079913

\subsection{9}

$-79.601258$

$-79.597780$

$-79.596836$

$-79.622680$

Standard orientation:

\begin{tabular}{|c|c|c|c|c|}
\hline \multirow{2}{*}{$\begin{array}{l}\text { Center } \\
\text { Number }\end{array}$} & \multirow{2}{*}{$\begin{array}{l}\text { Atomic } \\
\text { Number }\end{array}$} & \multirow{2}{*}{$\begin{array}{l}\text { Atomic } \\
\text { Type }\end{array}$} & \multicolumn{2}{|c|}{ Coordinates (Angstror } \\
\hline & & & $\mathrm{X}$ & $\mathrm{Z}$ \\
\hline 1 & 6 & 0.000000 & 0.000000 & 0.000000 \\
\hline 2 & 1 & 0.629541 & 0.629541 & 0.629541 \\
\hline 3 & 1 & -0.629541 & -0.629541 & 0.629541 \\
\hline 4 & 1 & -0.629541 & 0.629541 & -0.629541 \\
\hline 5 & 1 & 0.629541 & -0.629541 & -0.629541 \\
\hline
\end{tabular}

Zero-point correction=

Thermal correction to Energy=

Thermal correction to Enthalpy=

Thermal correction to Gibbs Free Energy=

Sum of electronic and zero-point Energies=

Sum of electronic and thermal Energies $=$

Sum of electronic and thermal Enthalpies=

Sum of electronic and thermal Free Energies=

Propane

$$
\begin{array}{cc}
0.045299 & \text { (Hartree/Particle) } \\
& 0.048165 \\
& 0.049110 \\
\text { rgy }= & 0.027983 \\
\text { gies }= & -40.390842 \\
\text { es }= & -40.387975 \\
\text { pies }= & -40.387031 \\
\text { Energies }= & -40.408158
\end{array}
$$

\begin{tabular}{|c|c|c|c|c|}
\hline \multirow{2}{*}{$\begin{array}{l}\text { Center } \\
\text { Number }\end{array}$} & Atomic & \multirow{2}{*}{$\begin{array}{r}\text { Atomic } \\
\text { Type }\end{array}$} & \multicolumn{2}{|c|}{ Coordinates (Angstroms) } \\
\hline & Number & & $\mathrm{X}$ & $\mathrm{Z}$ \\
\hline 1 & 6 & -1.270645 & -0.260851 & 0.000000 \\
\hline 2 & 1 & -2.172616 & 0.357275 & -0.000025 \\
\hline 3 & 1 & -1.308060 & -0.906543 & 0.882842 \\
\hline 4 & 1 & -1.308038 & -0.906583 & -0.882814 \\
\hline 5 & 6 & 0.000000 & 0.591093 & 0.000000 \\
\hline 6 & 1 & -0.000001 & 1.247685 & 0.877605 \\
\hline 7 & 1 & 0.000001 & 1.247668 & -0.877616 \\
\hline
\end{tabular}

Standard orientation: 


$\begin{array}{cccccc}8 & 6 & 0 & 1.270645 & -0.260851 & 0.000000 \\ 9 & 1 & 0 & 1.308087 & -0.906499 & 0.882873 \\ 10 & 1 & 0 & 1.308007 & -0.906626 & -0.882783 \\ 11 & 1 & 0 & 2.172618 & 0.357272 & -0.000086\end{array}$

Zero-point correction=

Thermal correction to Energy=

Thermal correction to Enthalpy=

Thermal correction to Gibbs Free Energy=

Sum of electronic and zero-point Energies=

Sum of electronic and thermal Energies $=$

Sum of electronic and thermal Enthalpies=

Sum of electronic and thermal Free Energies=

Butane
0.104516 (Hartree/Particle)

0.109008

0.109952

\subsection{6}

$-118.816402$

$-118.811910$

$-118.810966$

$-118.842032$

Standard orientation:

\begin{tabular}{|c|c|c|c|c|}
\hline Center & Atomic & Atomic & Coordina & stro \\
\hline Number & Numbe & Type & $X$ & Z \\
\hline 1 & 6 & 1.954297 & -0.119998 & 0.000045 \\
\hline 2 & 1 & 2.096009 & -0.750855 & -0.882849 \\
\hline 3 & 1 & 2.095923 & -0.750804 & 0.882988 \\
\hline 4 & 1 & 2.744777 & 0.635414 & 0.000061 \\
\hline 5 & 6 & 0.563798 & 0.516964 & -0.000050 \\
\hline 6 & 1 & 0.455251 & 1.165948 & 0.878040 \\
\hline 7 & 1 & 0.455321 & 1.165875 & -0.878204 \\
\hline 8 & 6 & -0.563798 & -0.516964 & -0.000051 \\
\hline 9 & 1 & -0.455321 & -1.165873 & -0.878205 \\
\hline 10 & 1 & -0.455251 & -1.165950 & 0.878039 \\
\hline 11 & 6 & -1.954297 & 0.119998 & 0.000045 \\
\hline 12 & 1 & -2.096005 & 0.750865 & -0.882842 \\
\hline 13 & 1 & -2.744777 & -0.635414 & 0.000049 \\
\hline 14 & 1 & -2.095926 & 0.750794 & 0.882995 \\
\hline
\end{tabular}

Zero-point correction=

Thermal correction to Energy=

Thermal correction to Enthalpy=

Thermal correction to Gibbs Free Energy=

Sum of electronic and zero-point Energies=

Sum of electronic and thermal Energies=

Sum of electronic and thermal Enthalpies=

Sum of electronic and thermal Free Energies=

Isobutane
0.133322 (Hartree/Particle)

$$
0.139055
$$$$
0.139999
$$

$$
\begin{gathered}
0.105345 \\
-158.031816 \\
-158.026082 \\
-158.025138 \\
-158.059793
\end{gathered}
$$

Standard orientation:

\begin{tabular}{cccccc} 
Center & \multicolumn{2}{c}{ Atomic } & \multicolumn{2}{c}{ Atomic } & \multicolumn{2}{c}{ Coordinates (Angstroms) } \\
Number & Number & Type & X & Y & Z \\
-1 & 6 & 0 & -0.833495 & -1.194158 & -0.096147 \\
2 & 1 & 0 & -1.865685 & -1.126833 & 0.259879
\end{tabular}




$\begin{array}{cccccc}3 & 1 & 0 & -0.859708 & -1.231685 & -1.190738 \\ 4 & 1 & 0 & -0.414363 & -2.139823 & 0.259869 \\ 5 & 6 & 0 & 0.000003 & 0.000005 & 0.377684 \\ 6 & 1 & 0 & 0.000008 & 0.000024 & 1.477543 \\ 7 & 6 & 0 & -0.617425 & 1.318903 & -0.096149 \\ 8 & 1 & 0 & -0.043004 & 2.179142 & 0.259844 \\ 9 & 1 & 0 & -0.636876 & 1.360365 & -1.190742 \\ 10 & 1 & 0 & -1.645946 & 1.428757 & 0.259909 \\ 11 & 6 & 0 & 1.450919 & -0.124748 & -0.096149 \\ 12 & 1 & 0 & 2.060260 & 0.711232 & 0.259582 \\ 13 & 1 & 0 & 1.908796 & -1.052163 & 0.260156 \\ 14 & 1 & 0 & 1.496503 & -0.129023 & -1.190738\end{array}$

Zero-point correction=

Thermal correction to Energy=

Thermal correction to Enthalpy=

Thermal correction to Gibbs Free Energy=

Sum of electronic and zero-point Energies=

Sum of electronic and thermal Energies $=$

Sum of electronic and thermal Enthalpies $=$

Sum of electronic and thermal Free Energies=

Pentane
0.132922 (Hartree/Particle)

$$
0.138550
$$

0.139494
0.105364

$-158.034595$

$-158.028967$

$-158.028023$

$-158.062153$

Standard orientation:

\begin{tabular}{|c|c|c|c|c|}
\hline \multirow{2}{*}{$\begin{array}{l}\text { Center } \\
\text { Number }\end{array}$} & Atomic & \multirow{2}{*}{$\begin{array}{l}\text { Atomic } \\
\text { Type }\end{array}$} & \multicolumn{2}{|c|}{ Coordinates (Angstrom } \\
\hline & Numbe & & $\mathrm{X}$ & Z \\
\hline 1 & 6 & 2.547628 & 0.327849 & -0.000011 \\
\hline 2 & 1 & 2.584132 & 0.973477 & -0.882922 \\
\hline 3 & 1 & 2.584134 & 0.973527 & 0.882864 \\
\hline 4 & 1 & 3.450758 & -0.288512 & 0.000006 \\
\hline 5 & 6 & 1.279245 & -0.526944 & 0.000016 \\
\hline 6 & 1 & 1.278395 & -1.184630 & -0.878156 \\
\hline 7 & 1 & 1.278407 & -1.184592 & 0.878215 \\
\hline 8 & 6 & 0.000000 & 0.311476 & 0.000009 \\
\hline 9 & 1 & -0.000012 & 0.970862 & 0.878535 \\
\hline 10 & 1 & 0.000012 & 0.970878 & -0.878505 \\
\hline 11 & 6 & -1.279245 & -0.526944 & -0.000016 \\
\hline 12 & 1 & -1.278404 & -1.184637 & 0.878152 \\
\hline 13 & 1 & -1.278398 & -1.184585 & -0.878220 \\
\hline 14 & 6 & -2.547628 & 0.327849 & 0.000003 \\
\hline 15 & 1 & -3.450758 & -0.288512 & -0.000072 \\
\hline 16 & 1 & -2.584160 & 0.973436 & 0.882942 \\
\hline 17 & 1 & -2.584106 & 0.973567 & -0.882845 \\
\hline
\end{tabular}

Zero-point correction=

0.162098 (Hartree/Particle)

Thermal correction to Energy=

0.169119

Thermal correction to Enthalpy=

0.170064

Thermal correction to Gibbs Free Energy=

0.131826

Sum of electronic and zero-point Energies=

$-197.247286$ 
Sum of electronic and thermal Energies $=$

Sum of electronic and thermal Enthalpies=

Sum of electronic and thermal Free Energies=

Neopentane

Standard orientation:

\begin{tabular}{cccccc} 
Center & Atomic & \multicolumn{2}{c}{ Atomic } & \multicolumn{3}{c}{ Coordinates } \\
Number & Number & Type & X & $Y$ & $Z$ \\
----------- & Y \\
1 & 6 & 0 & -0.312235 & -0.065472 & -1.501958 \\
2 & 1 & 0 & -1.391495 & -0.120295 & -1.676148 \\
3 & 1 & 0 & 0.067751 & 0.820450 & -2.020520 \\
4 & 1 & 0 & 0.147779 & -0.946739 & -1.960108 \\
5 & 6 & 0 & 0.000008 & -0.000002 & 0.000011 \\
6 & 6 & 0 & -0.660822 & 1.246378 & 0.606276 \\
7 & 1 & 0 & -0.453359 & 1.317955 & 1.678654 \\
8 & 1 & 0 & -0.288381 & 2.158764 & 0.129845 \\
9 & 1 & 0 & -1.747124 & 1.217478 & 0.474885 \\
10 & 6 & 0 & -0.546929 & -1.257019 & 0.691728 \\
11 & 1 & 0 & -0.091236 & -2.162106 & 0.277826 \\
12 & 1 & 0 & -0.337793 & -1.235980 & 1.765955 \\
13 & 1 & 0 & -1.630858 & -1.336183 & 0.561409 \\
14 & 6 & 0 & 1.519986 & 0.076114 & 0.203954 \\
15 & 1 & 0 & 2.016975 & -0.802503 & -0.219343 \\
16 & 1 & 0 & 1.936748 & 0.964627 & -0.280820 \\
17 & 1 & 0 & 1.770945 & 0.124542 & 1.268295
\end{tabular}

Zero-point correction $=$

0.161126 (Hartree/Particle)

Thermal correction to Energy=

0.167910

Thermal correction to Enthalpy=

0.168854

Thermal correction to Gibbs Free Energy=

Sum of electronic and zero-point Energies=

0.132133

Sum of electronic and thermal Energies=

$-197.254004$

$-197.247219$

Sum of electronic and thermal Enthalpies=

$-197.246275$

Sum of electronic and thermal Free Energies=

$-197.282996$

Hexane

Standard orientation:

\begin{tabular}{|c|c|c|c|c|}
\hline \multirow{2}{*}{$\begin{array}{l}\text { Center } \\
\text { Number }\end{array}$} & Atomic & \multirow{2}{*}{$\begin{array}{l}\text { Atomic } \\
\text { Type }\end{array}$} & \multicolumn{2}{|c|}{ Coordinates (Angstroms) } \\
\hline & Numbe & & $\mathrm{X}$ & Z \\
\hline 1 & 6 & -3.209617 & 0.209281 & 0.000046 \\
\hline 2 & 1 & -3.291842 & 0.850646 & 0.883029 \\
\hline 3 & 1 & -3.291842 & 0.850868 & -0.882776 \\
\hline 4 & 1 & -4.066758 & -0.469610 & -0.000040 \\
\hline 5 & 6 & -1.883703 & -0.553047 & -0.000054 \\
\hline 6 & 1 & -1.835804 & -1.209269 & 0.878022 \\
\hline 7 & 1 & -1.835841 & -1.209114 & -0.878248 \\
\hline 8 & 6 & -0.666902 & 0.373849 & -0.000004 \\
\hline 9 & 1 & -0.714072 & 1.031309 & -0.878544 \\
\hline
\end{tabular}




$\begin{array}{rrrrrr}10 & 1 & 0 & -0.714102 & 1.031269 & 0.878563 \\ 11 & 6 & 0 & 0.666902 & -0.373849 & 0.000010 \\ 12 & 1 & 0 & 0.714076 & -1.031331 & -0.878513 \\ 13 & 1 & 0 & 0.714098 & -1.031247 & 0.878594 \\ 14 & 6 & 0 & 1.883703 & 0.553047 & -0.000047 \\ 15 & 1 & 0 & 1.835808 & 1.209268 & 0.878030 \\ 16 & 1 & 0 & 1.835837 & 1.209115 & -0.878240 \\ 17 & 6 & 0 & 3.209617 & -0.209281 & 0.000039 \\ 18 & 1 & 0 & 3.291849 & -0.850845 & -0.882799 \\ 19 & 1 & 0 & 4.066759 & 0.469610 & -0.000022 \\ 20 & 1 & 0 & 3.291835 & -0.850669 & 0.883006\end{array}$

Zero-point correction=

0.190831 (Hartree/Particle)

Thermal correction to Energy=

Thermal correction to Enthalpy=

0.199173

0.200117

Thermal correction to Gibbs Free Energy=

0.158300

Sum of electronic and zero-point Energies=

$-236.462840$

Sum of electronic and thermal Energies=

$-236.454497$

$-236.453553$

Sum of electronic and thermal Enthalpies=

$-236.495370$

Sum of electronic and thermal Free Energies=

22dimethylbutane

Standard orientation:

\begin{tabular}{cccccc}
\hline Center & \multicolumn{2}{c}{ Atomic } & \multicolumn{2}{c}{ Atomic } & \multicolumn{3}{c}{ Coordinates } & Angstroms) \\
Number & Number & Type & X & Y & Z \\
\hline 1 & 6 & 0 & -1.620655 & 0.976558 & 0.000682 \\
2 & 1 & 0 & -1.599154 & 1.619822 & 0.886122 \\
3 & 1 & 0 & -1.599146 & 1.621037 & -0.883874 \\
4 & 1 & 0 & -2.572701 & 0.436696 & 0.000306 \\
5 & 6 & 0 & -0.433983 & 0.000256 & 0.000010 \\
6 & 6 & 0 & 0.869428 & 0.830807 & 0.000477 \\
7 & 1 & 0 & 0.851898 & 1.489659 & -0.877274 \\
8 & 1 & 0 & 0.851938 & 1.488624 & 0.879007 \\
9 & 6 & 0 & 2.178874 & 0.037862 & 0.000006 \\
10 & 1 & 0 & 2.267647 & -0.598852 & -0.883894 \\
11 & 1 & 0 & 3.036408 & 0.716308 & 0.000266 \\
12 & 1 & 0 & 2.267751 & -0.599668 & 0.883308 \\
13 & 6 & 0 & -0.516806 & -0.883503 & 1.253864 \\
14 & 1 & 0 & 0.273713 & -1.638259 & 1.270783 \\
15 & 1 & 0 & -0.430090 & -0.281526 & 2.164486 \\
16 & 1 & 0 & -1.475448 & -1.410428 & 1.289818 \\
17 & 6 & 0 & -0.516938 & -0.881872 & -1.254992 \\
18 & 1 & 0 & -1.475571 & -1.408775 & -1.291497 \\
19 & 1 & 0 & -0.430360 & -0.278698 & -2.164833 \\
20 & 1 & 0 & 0.273596 & -1.636587 & -1.273010
\end{tabular}

Zero-point correction=

Thermal correction to Energy=

Thermal correction to Enthalpy=
0.189934 (Hartree/Particle)

0.198057

0.199001 
Thermal correction to Gibbs Free Energy=

Sum of electronic and zero-point Energies=

Sum of electronic and thermal Energies $=$

Sum of electronic and thermal Enthalpies=

Sum of electronic and thermal Free Energies=

Heptane
0.158649

$-236.467737$

$-236.459614$

$-236.458669$

$-236.499022$

Standard orientation:

\begin{tabular}{|c|c|c|c|c|}
\hline \multirow{2}{*}{$\begin{array}{l}\text { Center } \\
\text { Number }\end{array}$} & Atomic & \multirow{2}{*}{$\begin{array}{l}\text { Atomic } \\
\text { Type }\end{array}$} & \multicolumn{2}{|c|}{ Coordinates (Angstroms } \\
\hline & Number & & $\mathrm{X}$ & Z \\
\hline 1 & 6 & -3.824584 & -0.358715 & -0.000010 \\
\hline 2 & 1 & -3.860628 & -1.004381 & -0.882878 \\
\hline 3 & 1 & -3.860621 & -1.004445 & 0.882813 \\
\hline 4 & 1 & -4.727939 & 0.257084 & 0.000017 \\
\hline 5 & 6 & -2.556801 & 0.496819 & 0.000017 \\
\hline 6 & 1 & -2.555743 & 1.154573 & -0.878127 \\
\hline 7 & 1 & -2.555745 & 1.154523 & 0.878198 \\
\hline 8 & 6 & -1.277058 & -0.340795 & -0.000003 \\
\hline 9 & 1 & -1.276919 & -0.999956 & 0.878518 \\
\hline 10 & 1 & -1.276927 & -0.999932 & -0.878543 \\
\hline 11 & 6 & 0.000000 & 0.500019 & -0.000001 \\
\hline 12 & 1 & -0.000003 & 1.158625 & 0.878649 \\
\hline 13 & 1 & 0.000003 & 1.158617 & -0.878657 \\
\hline 14 & 6 & 1.277058 & -0.340795 & 0.000007 \\
\hline 15 & 1 & 1.276918 & -0.999961 & -0.878511 \\
\hline 16 & 1 & 1.276928 & -0.999928 & 0.878550 \\
\hline 17 & 6 & 2.556801 & 0.496819 & -0.000015 \\
\hline 18 & 1 & 2.555744 & 1.154571 & 0.878130 \\
\hline 19 & 1 & 2.555744 & 1.154525 & -0.878195 \\
\hline 20 & 6 & 3.824584 & -0.358715 & 0.000006 \\
\hline 21 & 1 & 3.860622 & -1.004439 & -0.882821 \\
\hline 22 & 1 & 4.727939 & 0.257084 & -0.000014 \\
\hline 23 & 1 & 3.860627 & -1.004387 & 0.882870 \\
\hline
\end{tabular}

Zero-point correction $=$

Thermal correction to Energy=

Thermal correction to Enthalpy=

Thermal correction to Gibbs Free Energy=

Sum of electronic and zero-point Energies=

Sum of electronic and thermal Energies=

Sum of electronic and thermal Enthalpies=

Sum of electronic and thermal Free Energies=
0.219562 (Hartree/Particle)

0.229246

0.230190

0.184760

$-275.678416$

$-275.668733$

$-275.667789$

$-275.713218$

223trimethylbutane

Standard orientation:

\begin{tabular}{|c|c|c|c|}
\hline Center & Atomic & Atomic & Coordinates (Angstroms) \\
\hline Number & Number & Type & $X$ \\
\hline
\end{tabular}




$\begin{array}{cccccc}1 & 6 & 0 & -1.313261 & -1.325768 & -0.401210 \\ 2 & 1 & 0 & -1.158138 & -1.540789 & -1.463979 \\ 3 & 1 & 0 & -0.926955 & -2.169495 & 0.175207 \\ 4 & 1 & 0 & -2.391772 & -1.273611 & -0.224070 \\ 5 & 6 & 0 & -0.642033 & -0.000217 & -0.001328 \\ 6 & 6 & 0 & 0.850782 & -0.001610 & -0.457580 \\ 7 & 1 & 0 & 0.824045 & -0.008184 & -1.558565 \\ 8 & 6 & 0 & 1.625910 & 1.250570 & -0.022749 \\ 9 & 1 & 0 & 1.779794 & 1.260669 & 1.060345 \\ 10 & 1 & 0 & 2.613800 & 1.264018 & -0.491843 \\ 11 & 1 & 0 & 1.119762 & 2.177558 & -0.300084 \\ 12 & 6 & 0 & 1.632648 & -1.247560 & -0.016688 \\ 13 & 1 & 0 & 1.639566 & -1.348411 & 1.073083 \\ 14 & 1 & 0 & 1.224524 & -2.167783 & -0.438542 \\ 15 & 1 & 0 & 2.674321 & -1.171938 & -0.341701 \\ 16 & 6 & 0 & -0.778950 & 0.185950 & 1.518537 \\ 17 & 1 & 0 & -0.412910 & 1.164198 & 1.840940 \\ 18 & 1 & 0 & -1.830928 & 0.114879 & 1.812216 \\ 19 & 1 & 0 & -0.231184 & -0.581600 & 2.072654 \\ 20 & 6 & 0 & -1.396905 & 1.138744 & -0.709549 \\ 21 & 1 & 0 & -1.328519 & 1.036728 & -1.797685 \\ 22 & 1 & 0 & -2.456701 & 1.120614 & -0.437367 \\ 23 & 1 & 0 & -1.007859 & 2.122499 & -0.437203\end{array}$

Zero-point correction=

Thermal correction to Energy=

0.218503 (Hartree/Particle)

0.227802

0.228747

Thermal correction to Gibbs Free Energy=

0.185784

Sum of electronic and zero-point Energies=

$-275.682451$

Sum of electronic and thermal Energies $=$

$-275.673152$

Sum of electronic and thermal Enthalpies=

$-275.672208$

Sum of electronic and thermal Free Energies $=\quad-275.715170$

Octane

Standard orientation:

\begin{tabular}{|c|c|c|c|c|}
\hline \multirow{2}{*}{$\begin{array}{l}\text { Center } \\
\text { Number }\end{array}$} & Atomic & \multirow{2}{*}{$\begin{array}{l}\text { Atomic } \\
\text { Type }\end{array}$} & \multicolumn{2}{|c|}{ Coordinates (Angstroms) } \\
\hline & Number & & $\mathrm{X}$ & $\mathrm{Z}$ \\
\hline 1 & 6 & -4.477473 & 0.258533 & 0.000018 \\
\hline 2 & 1 & -4.539537 & 0.902229 & 0.882889 \\
\hline 3 & 1 & -4.539540 & 0.902302 & -0.882800 \\
\hline 4 & 1 & -5.355263 & -0.393194 & -0.000008 \\
\hline 5 & 6 & -3.176185 & -0.545106 & -0.000019 \\
\hline 6 & 1 & -3.148502 & -1.202362 & 0.878126 \\
\hline 7 & 1 & -3.148513 & -1.202303 & -0.878209 \\
\hline 8 & 6 & -1.931201 & 0.343359 & 0.000002 \\
\hline 9 & 1 & -1.957551 & 1.002006 & -0.878534 \\
\hline 10 & 1 & -1.957556 & 1.001978 & 0.878558 \\
\hline 11 & 6 & -0.621448 & -0.445378 & -0.000004 \\
\hline 12 & 1 & -0.594870 & -1.103670 & -0.878589 \\
\hline
\end{tabular}




$\begin{array}{rrrrrr}13 & 1 & 0 & -0.594865 & -1.103669 & 0.878582 \\ 14 & 6 & 0 & 0.621448 & 0.445378 & -0.000009 \\ 15 & 1 & 0 & 0.594863 & 1.103683 & 0.878567 \\ 16 & 1 & 0 & 0.594872 & 1.103657 & -0.878605 \\ 17 & 6 & 0 & 1.931201 & -0.343359 & 0.000009 \\ 18 & 1 & 0 & 1.957553 & -1.002013 & -0.878521 \\ 19 & 1 & 0 & 1.957553 & -1.001972 & 0.878570 \\ 20 & 6 & 0 & 3.176185 & 0.545106 & -0.000012 \\ 21 & 1 & 0 & 3.148506 & 1.202356 & 0.878138 \\ 22 & 1 & 0 & 3.148510 & 1.202309 & -0.878197 \\ 23 & 6 & 0 & 4.477473 & -0.258533 & 0.000012 \\ 24 & 1 & 0 & 4.539540 & -0.902290 & -0.882815 \\ 25 & 1 & 0 & 5.355263 & 0.393194 & -0.000004 \\ 26 & 1 & 0 & 4.539537 & -0.902242 & 0.882874\end{array}$

Zero-point correction=

Thermal correction to Energy=

Thermal correction to Enthalpy=

Thermal correction to Gibbs Free Energy=

Sum of electronic and zero-point Energies=

Sum of electronic and thermal Energies $=$

Sum of electronic and thermal Enthalpies=

Sum of electronic and thermal Free Energies=

2233tetramethylbutane

Standard orientation:

\begin{tabular}{|c|c|c|c|c|}
\hline \multirow{2}{*}{$\begin{array}{l}\text { Center } \\
\text { Number }\end{array}$} & Atomic & \multirow{2}{*}{$\begin{array}{l}\text { Atomic } \\
\text { Type }\end{array}$} & \multicolumn{2}{|c|}{ Coordinates (Angstroms } \\
\hline & Numbe & & $X$ & Z \\
\hline 1 & 6 & -1.347845 & -1.288962 & 0.633935 \\
\hline 2 & 1 & -1.154328 & -2.165360 & 0.010937 \\
\hline 3 & 1 & -0.925075 & -1.476457 & 1.624753 \\
\hline 4 & 1 & -2.432418 & -1.203546 & 0.751888 \\
\hline 5 & 6 & -0.790290 & -0.000003 & 0.000043 \\
\hline 6 & 6 & 0.790298 & -0.000061 & 0.000005 \\
\hline 7 & 6 & 1.347735 & 1.109580 & -0.912127 \\
\hline 8 & 1 & 1.154952 & 0.903845 & -1.967670 \\
\hline 9 & 1 & 0.924439 & 2.089301 & -0.674186 \\
\hline 10 & 1 & 2.432218 & 1.182240 & -0.785044 \\
\hline 11 & 6 & -1.347637 & 0.095567 & -1.433138 \\
\hline 12 & 1 & -1.154627 & 1.073514 & -1.880361 \\
\hline 13 & 1 & -0.924370 & -0.668373 & -2.091036 \\
\hline 14 & 1 & -2.432145 & -0.049882 & -1.418289 \\
\hline 15 & 6 & -1.347543 & 1.193625 & 0.799194 \\
\hline 16 & 1 & -2.431984 & 1.253790 & 0.665438 \\
\hline 17 & 1 & -1.154903 & 1.091932 & 1.869767 \\
\hline 18 & 1 & -0.923963 & 2.145291 & 0.466698 \\
\hline 19 & 6 & 1.347571 & -1.344908 & -0.504947 \\
\hline 20 & 1 & 2.432166 & -1.271690 & -0.630507 \\
\hline 21 & 1 & 1.153738 & -2.156179 & 0.200679 \\
\hline
\end{tabular}




$\begin{array}{llllrc}22 & 1 & 0 & 0.924845 & -1.628183 & -1.472754 \\ 23 & 6 & 0 & 1.347712 & 0.235097 & 1.417051 \\ 24 & 1 & 0 & 2.432199 & 0.088824 & 1.416505 \\ 25 & 1 & 0 & 1.154793 & 1.252094 & 1.766459 \\ 26 & 1 & 0 & 0.924458 & -0.460768 & 2.146628\end{array}$

Zero-point correction=

0.247266 (Hartree/Particle)

Thermal correction to Energy=

0.257410

Thermal correction to Enthalpy=

0.258355

Thermal correction to Gibbs Free Energy $=\quad 0.214285$

Sum of electronic and zero-point Energies=

Sum of electronic and thermal Energies $=$

$-314.897348$

$-314.887203$

Sum of electronic and thermal Enthalpies=

$-314.886259$

Sum of electronic and thermal Free Energies=

$-314.930328$

DSDPBEP86/DHSVPD

Ethane

Standard orientation:

\begin{tabular}{|c|c|c|c|c|}
\hline \multirow{2}{*}{$\begin{array}{l}\text { Center } \\
\text { Number }\end{array}$} & Atomic & \multirow{2}{*}{$\begin{array}{l}\text { Atomic } \\
\text { Type }\end{array}$} & \multicolumn{2}{|c|}{ Coordinates (Angstrom } \\
\hline & Numbe & & $X$ & Z \\
\hline 1 & 6 & 0.000000 & -0.000000 & 0.764607 \\
\hline 2 & 1 & -0.510418 & 0.884119 & 1.162591 \\
\hline 3 & 1 & 1.020879 & -0.000024 & 1.162591 \\
\hline 4 & 1 & -0.510460 & -0.884095 & 1.162591 \\
\hline 5 & 6 & 0.000000 & -0.000000 & -0.764607 \\
\hline 6 & 1 & 0.510418 & 0.884119 & -1.162591 \\
\hline 7 & 1 & 0.510460 & -0.884095 & -1.162591 \\
\hline 8 & 1 & -1.020879 & -0.000024 & -1.162591 \\
\hline
\end{tabular}

Zero-point correction=

Thermal correction to Energy=

0.075552 (Hartree/Particle)

Thermal correction to Enthalpy=

0.079035

0.079979

Thermal correction to Gibbs Free Energy=

Sum of electronic and zero-point Energies=

0.054123

$-79.508501$

Sum of electronic and thermal Energies $=$

$-79.505019$

Sum of electronic and thermal Enthalpies=

$-79.504075$

Sum of electronic and thermal Free Energies=

$-79.529930$

Methane

Standard orientation:

\begin{tabular}{cccccc} 
Center & \multicolumn{2}{c}{ Atomic } & \multicolumn{2}{c}{ Atomic } & \multicolumn{3}{c}{ Coordinates } \\
Number & Number & Type & X & Y & Z \\
- & \multicolumn{1}{c}{ Nustroms) } \\
\hline 1 & 6 & 0 & 0.000000 & 0.000000 & 0.000000 \\
2 & 1 & 0 & 0.631013 & 0.631013 & 0.631013 \\
3 & 1 & 0 & -0.631013 & -0.631013 & 0.631013 \\
4 & 1 & 0 & -0.631013 & 0.631013 & -0.631013 \\
5 & 1 & 0 & 0.631013 & -0.631013 & -0.631013
\end{tabular}


Zero-point correction $=$

Thermal correction to Energy=

Thermal correction to Enthalpy=

Thermal correction to Gibbs Free Energy=

Sum of electronic and zero-point Energies=

Sum of electronic and thermal Energies=

Sum of electronic and thermal Enthalpies=

Sum of electronic and thermal Free Energies=

Propane
0.045357 (Hartree/Particle)

0.048223

0.049167

0.028034

$-40.342963$

$-40.340097$

$-40.339153$

$-40.360286$

Standard orientation:

\begin{tabular}{cccccc} 
Center & \multicolumn{2}{c}{ Atomic } & \multicolumn{2}{c}{ Atomic } & \multicolumn{2}{c}{ Coordinates (Angstroms) } \\
Number & \multicolumn{2}{c}{ Number } & Type & X & $Y$ \\
- \hdashline 1 & 6 & 0 & -1.268726 & -0.261102 & 0.000000 \\
2 & 1 & 0 & -2.173785 & 0.357037 & -0.000030 \\
3 & 1 & 0 & -1.304818 & -0.908204 & 0.885071 \\
4 & 1 & 0 & -1.304791 & -0.908253 & -0.885037 \\
5 & 6 & 0 & 0.000000 & 0.592033 & -0.000000 \\
6 & 1 & 0 & -0.000000 & 1.249942 & 0.879815 \\
7 & 1 & 0 & 0.000001 & 1.249925 & -0.879827 \\
8 & 6 & 0 & 1.268726 & -0.261102 & 0.000000 \\
9 & 1 & 0 & 1.304829 & -0.908184 & 0.885085 \\
10 & 1 & 0 & 1.304775 & -0.908273 & -0.885022 \\
11 & 1 & 0 & 2.173786 & 0.357035 & -0.000059
\end{tabular}

Zero-point correction=

Thermal correction to Energy=

Thermal correction to Enthalpy=

Thermal correction to Gibbs Free Energy=

Sum of electronic and zero-point Energies=

Sum of electronic and thermal Energies $=$

Sum of electronic and thermal Enthalpies=

Sum of electronic and thermal Free Energies=

Butane
0.104542 (Hartree/Particle)

0.109055

0.109999

0.078891

$-118.679012$

$-118.674499$

$-118.673555$

$-118.704663$

Standard orientation:

\begin{tabular}{|c|c|c|c|c|}
\hline \multirow{2}{*}{$\begin{array}{l}\text { Center } \\
\text { Number }\end{array}$} & Atomic & \multirow{2}{*}{$\begin{array}{l}\text { Atomic } \\
\text { Type }\end{array}$} & \multicolumn{2}{|c|}{ Coordinates (Angstrom } \\
\hline & Numbe & & $X$ & Z \\
\hline 1 & 6 & 1.951880 & -0.119862 & 0.000038 \\
\hline 2 & 1 & 2.092955 & -0.752234 & -0.885107 \\
\hline 3 & 1 & 2.092885 & -0.752193 & 0.885222 \\
\hline 4 & 1 & 2.744950 & 0.636575 & 0.000051 \\
\hline 5 & 6 & 0.562585 & 0.517550 & -0.000042 \\
\hline 6 & 1 & 0.453018 & 1.167697 & 0.880356 \\
\hline 7 & 1 & 0.453075 & 1.167638 & -0.880492 \\
\hline 8 & 6 & -0.562585 & -0.517550 & -0.000043 \\
\hline 9 & 1 & -0.453075 & -1.167636 & -0.880494 \\
\hline 10 & 1 & -0.453019 & -1.167700 & 0.880355 \\
\hline
\end{tabular}




$\begin{array}{rrrrrr}11 & 6 & 0 & -1.951880 & 0.119862 & 0.000038 \\ 12 & 1 & 0 & -2.092953 & 0.752240 & -0.885103 \\ 13 & 1 & 0 & -2.744951 & -0.636575 & 0.000044 \\ 14 & 1 & 0 & -2.092887 & 0.752187 & 0.885226\end{array}$

Zero-point correction=

Thermal correction to Energy=

Thermal correction to Enthalpy=

Thermal correction to Gibbs Free Energy=

Sum of electronic and zero-point Energies=

Sum of electronic and thermal Energies $=$

Sum of electronic and thermal Enthalpies=

Sum of electronic and thermal Free Energies= Isobutane
0.133312 (Hartree/Particle)

0.139081

0.140025
0.105291

$-157.849814$

$-157.844046$

$-157.843101$

$-157.877835$

Standard orientation:

\begin{tabular}{|c|c|c|c|c|c|}
\hline \multirow{2}{*}{$\begin{array}{l}\text { Center } \\
\text { Number }\end{array}$} & \multicolumn{2}{|l|}{ Atomic } & & \multicolumn{2}{|c|}{ Coordinates (Angstrom } \\
\hline & Numbe & & & $\mathrm{X}$ & Z \\
\hline 1 & 6 & 0 & -1.438003 & -0.217135 & -0.096397 \\
\hline 2 & 1 & 0 & -2.101740 & 0.579969 & 0.259442 \\
\hline 3 & 1 & 0 & -1.480437 & -0.223672 & -1.193829 \\
\hline 4 & 1 & 0 & -1.836863 & -1.174575 & 0.259639 \\
\hline 5 & 6 & 0 & -0.000011 & -0.000006 & 0.379564 \\
\hline 6 & 1 & 0 & -0.000001 & -0.000002 & 1.481982 \\
\hline 7 & 6 & 0 & 0.530954 & 1.353896 & -0.096393 \\
\hline 8 & 1 & 0 & 1.553175 & 1.530116 & 0.259352 \\
\hline 9 & 1 & 0 & 0.546416 & 1.393918 & -1.193823 \\
\hline 10 & 1 & 0 & -0.098711 & 2.178068 & 0.259707 \\
\hline 11 & 6 & 0 & 0.907053 & -1.136757 & -0.096394 \\
\hline 12 & 1 & 0 & 1.935732 & -1.003252 & 0.259330 \\
\hline 13 & 1 & 0 & 0.548838 & -2.110096 & 0.259741 \\
\hline 14 & 1 & 0 & 0.933635 & -1.170458 & -1.193824 \\
\hline
\end{tabular}

Zero-point correction=

Thermal correction to Energy=

0.132868 (Hartree/Particle)

Thermal correction to Enthalpy=

0.138544

Thermal correction to Gibbs Free Energy=

0.139488

Sum of electronic and zero-point Energies=

0.105265

Sum of electronic and thermal Energies=

$-157.852890$

$-157.847214$

Sum of electronic and thermal Enthalpies=

$-157.846270$

Sum of electronic and thermal Free Energies= $-157.880494$

Pentane

Standard orientation:

\begin{tabular}{|c|c|c|c|c|}
\hline Center & Atomic & Atomic & Coordinate & tes (Angstroms) \\
\hline Number & Number & Type & $\mathrm{X}$ & $\mathrm{Z}$ \\
\hline 1 & 6 & 2. & & 001 \\
\hline 2 & 1 & 2.578575 & 0.975448 & -0.885113 \\
\hline
\end{tabular}




$\begin{array}{cccccc}3 & 1 & 0 & 2.578569 & 0.975472 & 0.885093 \\ 4 & 1 & 0 & 3.449558 & -0.287908 & 0.000011 \\ 5 & 6 & 0 & 1.277289 & -0.527838 & 0.000006 \\ 6 & 1 & 0 & 1.275995 & -1.186610 & -0.880575 \\ 7 & 1 & 0 & 1.275993 & -1.186592 & 0.880601 \\ 8 & 6 & 0 & 0.000000 & 0.311492 & -0.000004 \\ 9 & 1 & 0 & -0.000004 & 0.971935 & 0.881003 \\ 10 & 1 & 0 & 0.000004 & 0.971927 & -0.881017 \\ 11 & 6 & 0 & -1.277289 & -0.527838 & -0.000006 \\ 12 & 1 & 0 & -1.275992 & -1.186606 & 0.880578 \\ 13 & 1 & 0 & -1.275996 & -1.186595 & -0.880598 \\ 14 & 6 & 0 & -2.543469 & 0.328468 & 0.000005 \\ 15 & 1 & 0 & -3.449558 & -0.287908 & 0.000002 \\ 16 & 1 & 0 & -2.578569 & 0.975454 & 0.885112 \\ 17 & 1 & 0 & -2.578574 & 0.975466 & -0.885094\end{array}$

Zero-point correction=

Thermal correction to Energy=

Thermal correction to Enthalpy=

Thermal correction to Gibbs Free Energy=

Sum of electronic and zero-point Energies=

Sum of electronic and thermal Energies $=$

Sum of electronic and thermal Enthalpies=

Sum of electronic and thermal Free Energies=

Neopentane

Standard orientation:

\begin{tabular}{|c|c|c|c|c|c|}
\hline \multirow{3}{*}{$\begin{array}{l}\text { Center } \\
\text { Number } \\
------- \\
1\end{array}$} & \multirow{2}{*}{\multicolumn{2}{|c|}{$\begin{array}{l}\text { Atomic } \\
\text { Number }\end{array}$}} & \multirow{3}{*}{$\begin{array}{l}\text { Atomic } \\
\text { Type } \\
-0.416552\end{array}$} & \multicolumn{2}{|c|}{ Coordinates (Angstrom } \\
\hline & & & & $\mathrm{X}$ & $\mathrm{Z}$ \\
\hline & 6 & 0 & & 0.881414 & -1.183919 \\
\hline 2 & 1 & 0 & -1.407207 & 0.593588 & -1.558160 \\
\hline 3 & 1 & 0 & -0.459859 & 1.938009 & -0.890602 \\
\hline 4 & 1 & 0 & 0.297520 & 0.789487 & -2.012141 \\
\hline 5 & 6 & 0 & 0.000006 & -0.000000 & 0.000006 \\
\hline 6 & 6 & 0 & -1.019294 & 0.154039 & 1.135517 \\
\hline 7 & 1 & 0 & -0.744217 & -0.467633 & 1.997002 \\
\hline 8 & 1 & 0 & -1.074644 & 1.196263 & 1.474638 \\
\hline 9 & 1 & 0 & -2.021755 & -0.148212 & 0.806861 \\
\hline 10 & 6 & 0 & 0.049763 & -1.466262 & -0.446880 \\
\hline 11 & 1 & 0 & 0.773117 & -1.604358 & -1.260505 \\
\hline 12 & 1 & 0 & 0.346018 & -2.119840 & 0.383401 \\
\hline 13 & 1 & 0 & -0.931618 & -1.800543 & -0.806668 \\
\hline 14 & 6 & 0 & 1.386081 & 0.430805 & 0.495280 \\
\hline 15 & 1 & 0 & 2.135909 & 0.329661 & -0.299596 \\
\hline 16 & 1 & 0 & 1.378265 & 1.478639 & 0.821285 \\
\hline 17 & 1 & 0 & 1.708445 & -0.185036 & 1.344467 \\
\hline
\end{tabular}

Zero-point correction=

Thermal correction to Energy=
0.162067 (Hartree/Particle)

0.169141

0.170085

\subsection{3}

$-197.020680$

$-197.013605$

$-197.012661$

$-197.051034$ 
Thermal correction to Enthalpy=

Thermal correction to Gibbs Free Energy=

Sum of electronic and zero-point Energies=

Sum of electronic and thermal Energies $=$

Sum of electronic and thermal Enthalpies=

Sum of electronic and thermal Free Energies=

Hexane
0.168762

0.131872

$-197.028414$

$-197.021544$

$-197.020599$

$-197.057490$

Standard orientation:

\begin{tabular}{cccccc} 
Center & Atomic & \multicolumn{2}{c}{ Atomic } & \multicolumn{3}{c}{ Coordinates } \\
Number & Number & Type & X & Y & $Z$ \\
-1 & 6 & 0 & -3.205527 & 0.209429 & 0.000038 \\
2 & 1 & 0 & -3.286644 & 0.852320 & 0.885260 \\
3 & 1 & 0 & -3.286635 & 0.852521 & -0.885040 \\
4 & 1 & 0 & -4.065687 & -0.469817 & -0.000045 \\
5 & 6 & 0 & -1.881165 & -0.553804 & -0.000047 \\
6 & 1 & 0 & -1.832552 & -1.211305 & 0.880376 \\
7 & 1 & 0 & -1.832580 & -1.211171 & -0.880572 \\
8 & 6 & 0 & -0.666271 & 0.373958 & 0.000000 \\
9 & 1 & 0 & -0.713736 & 1.032685 & -0.880990 \\
10 & 1 & 0 & -0.713762 & 1.032641 & 0.881022 \\
11 & 6 & 0 & 0.666271 & -0.373958 & 0.000009 \\
12 & 1 & 0 & 0.713737 & -1.032699 & -0.880972 \\
13 & 1 & 0 & 0.713761 & -1.032628 & 0.881041 \\
14 & 6 & 0 & 1.881165 & 0.553804 & -0.000043 \\
15 & 1 & 0 & 1.832554 & 1.211307 & 0.880379 \\
16 & 1 & 0 & 1.832579 & 1.211169 & -0.880568 \\
17 & 6 & 0 & 3.205527 & -0.209429 & 0.000034 \\
18 & 1 & 0 & 3.286644 & -0.852502 & -0.885057 \\
19 & 1 & 0 & 4.065687 & 0.469817 & -0.000024 \\
20 & 1 & 0 & 3.286635 & -0.852339 & 0.885243
\end{tabular}

Zero-point correction=

0.190760 (Hartree/Particle)

Thermal correction to Energy=

Thermal correction to Enthalpy=

0.199158

0.200102

Thermal correction to Gibbs Free Energy=

0.158142

Sum of electronic and zero-point Energies=

$-236.191640$

Sum of electronic and thermal Energies=

$-236.183242$

$-236.182298$

Sum of electronic and thermal Enthalpies=

$-236.224258$

Sum of electronic and thermal Free Energies=

22dimethylbutane

Standard orientation:

\begin{tabular}{cccccc} 
Center & \multicolumn{2}{c}{ Atomic } & Atomic & \multicolumn{3}{c}{ Coordinates (Angstroms) } \\
Number & Number & Type & X & Y & Z \\
-1 & 6 & 0 & -1.620603 & 0.972010 & 0.000709 \\
2 & 1 & 0 & -1.599515 & 1.616679 & 0.888633 \\
3 & 1 & 0 & -1.599552 & 1.617895 & -0.886334
\end{tabular}




$\begin{array}{cccccc}4 & 1 & 0 & -2.573477 & 0.427921 & 0.000354 \\ 5 & 6 & 0 & -0.433033 & 0.000133 & 0.000010 \\ 6 & 6 & 0 & 0.865653 & 0.833154 & 0.000443 \\ 7 & 1 & 0 & 0.846373 & 1.493261 & -0.879917 \\ 8 & 1 & 0 & 0.846419 & 1.492294 & 0.881531 \\ 9 & 6 & 0 & 2.173016 & 0.039105 & 0.000002 \\ 10 & 1 & 0 & 2.260378 & -0.599196 & -0.886319 \\ 11 & 1 & 0 & 3.033812 & 0.717639 & 0.000180 \\ 12 & 1 & 0 & 2.260532 & -0.599865 & 0.885823 \\ 13 & 6 & 0 & -0.512455 & -0.882654 & 1.252375 \\ 14 & 1 & 0 & 0.281587 & -1.637893 & 1.267712 \\ 15 & 1 & 0 & -0.425545 & -0.278500 & 2.164991 \\ 16 & 1 & 0 & -1.472908 & -1.412130 & 1.288973 \\ 17 & 6 & 0 & -0.512655 & -0.881032 & -1.253492 \\ 18 & 1 & 0 & -1.473080 & -1.410529 & -1.290562 \\ 19 & 1 & 0 & -0.425994 & -0.275674 & -2.165333 \\ 20 & 1 & 0 & 0.281430 & -1.636200 & -1.270003\end{array}$

Zero-point correction=

Thermal correction to Energy=

Thermal correction to Enthalpy=

Thermal correction to Gibbs Free Energy=

Sum of electronic and zero-point Energies=

Sum of electronic and thermal Energies $=$

Sum of electronic and thermal Enthalpies=

Sum of electronic and thermal Free Energies=

Heptane
0.189746 (Hartree/Particle)

$$
0.197956
$$

0.198900
0.158380

$-236.197807$

$-236.189597$

$-236.188653$

$-236.229173$

Standard orientation:

\begin{tabular}{|c|c|c|c|}
\hline \multirow{2}{*}{$\begin{array}{l}\text { Center } \\
\text { Number }\end{array}$} & Atomic & \multirow{2}{*}{$\begin{array}{l}\text { Atomic } \\
\text { Type }\end{array}$} & Coordinates (Angstroms) \\
\hline & Numbe & & $X$ \\
\hline 1 & 6 & -3.819077 & $\begin{array}{ll}-0.359701 & -0.000009\end{array}$ \\
\hline 2 & 1 & -3.853492 & $\begin{array}{ll}-1.006791 & -0.885160\end{array}$ \\
\hline 3 & 1 & -3.853481 & $-1.006860 \quad 0.885092$ \\
\hline 4 & 1 & -4.725764 & $0.255810 \quad 0.000021$ \\
\hline 5 & 6 & -2.553531 & $0.497429 \quad 0.000017$ \\
\hline 6 & 1 & -2.552330 & $1.156458-0.880523$ \\
\hline 7 & 1 & -2.552331 & $1.156406 \quad 0.880595$ \\
\hline 8 & 6 & -1.275276 & $-0.340568 \quad-0.000004$ \\
\hline 9 & 1 & -1.274952 & $-1.001003 \quad 0.881007$ \\
\hline 10 & 1 & -1.274962 & $-1.000978 \quad-0.881034$ \\
\hline 11 & 6 & 0.000000 & $0.501043-0.000001$ \\
\hline 12 & 1 & -0.000003 & $1.160875 \quad 0.881188$ \\
\hline 13 & 1 & 0.000003 & $1.160868 \quad-0.881196$ \\
\hline 14 & 6 & 1.275276 & $-0.340568 \quad 0.000007$ \\
\hline 15 & 1 & 1.274952 & $-1.001007-0.881001$ \\
\hline 16 & 1 & 1.274962 & $-1.000974 \quad 0.881040$ \\
\hline 17 & 6 & 2.553531 & $0.497429-0.000015$ \\
\hline 18 & 1 & 2.552331 & $1.156455 \quad 0.880526$ \\
\hline
\end{tabular}




$\begin{array}{rrrrrr}19 & 1 & 0 & 2.552330 & 1.156408 & -0.880592 \\ 20 & 6 & 0 & 3.819077 & -0.359701 & 0.000006 \\ 21 & 1 & 0 & 3.853484 & -1.006852 & -0.885101 \\ 22 & 1 & 0 & 4.725764 & 0.255810 & -0.000014 \\ 23 & 1 & 0 & 3.853489 & -1.006800 & 0.885151\end{array}$

Zero-point correction=

Thermal correction to Energy=

Thermal correction to Enthalpy=

Thermal correction to Gibbs Free Energy=

Sum of electronic and zero-point Energies=

Sum of electronic and thermal Energies $=$

Sum of electronic and thermal Enthalpies=

Sum of electronic and thermal Free Energies=
0.219465 (Hartree/Particle)

0.229212

0.230156

0.184557

$-275.362610$

$-275.352863$

$-275.351919$

$-275.397518$

223trimethylbutane

Standard orientation:

\begin{tabular}{cccccc} 
Center & Atomic & \multicolumn{2}{c}{ Atomic } & \multicolumn{2}{c}{ Coordinates $($ Angstroms) } \\
Number & Number & Type & X & Y & $Z$ \\
\hline 1 & 6 & 0 & -1.313115 & -1.319395 & -0.410965 \\
2 & 1 & 0 & -1.161347 & -1.523923 & -1.479333 \\
3 & 1 & 0 & -0.924290 & -2.171002 & 0.157586 \\
4 & 1 & 0 & -2.393686 & -1.266378 & -0.228966 \\
5 & 6 & 0 & -0.640107 & -0.000264 & -0.001184 \\
6 & 6 & 0 & 0.847955 & -0.001451 & -0.459507 \\
7 & 1 & 0 & 0.819322 & -0.007856 & -1.563375 \\
8 & 6 & 0 & 1.621388 & 1.249160 & -0.023393 \\
9 & 1 & 0 & 1.768874 & 1.260980 & 1.063487 \\
10 & 1 & 0 & 2.614608 & 1.259089 & -0.487696 \\
11 & 1 & 0 & 1.117134 & 2.178512 & -0.307401 \\
12 & 6 & 0 & 1.627908 & -1.245940 & -0.017079 \\
13 & 1 & 0 & 1.638737 & -1.341789 & 1.076007 \\
14 & 1 & 0 & 1.214825 & -2.169153 & -0.434900 \\
15 & 1 & 0 & 2.671070 & -1.172852 & -0.347171 \\
16 & 6 & 0 & -0.773341 & 0.173220 & 1.518419 \\
17 & 1 & 0 & -0.399862 & 1.149139 & 1.849154 \\
18 & 1 & 0 & -1.828486 & 0.106025 & 1.812439 \\
19 & 1 & 0 & -0.228828 & -0.605089 & 2.066464 \\
20 & 6 & 0 & -1.392268 & 1.144766 & -0.697568 \\
21 & 1 & 0 & -1.316543 & 1.056395 & -1.789332 \\
22 & 1 & 0 & -2.456199 & 1.119339 & -0.430722 \\
23 & 1 & 0 & -1.005844 & 2.127984 & -0.408574
\end{tabular}

Zero-point correction=

Thermal correction to Energy=

Thermal correction to Enthalpy=

Thermal correction to Gibbs Free Energy=

Sum of electronic and zero-point Energies=
0.218285 (Hartree/Particle)

0.227683

0.228627

0.185447

$-275.368579$ 
Sum of electronic and thermal Energies $=$

$-275.359181$

Sum of electronic and thermal Enthalpies=

Sum of electronic and thermal Free Energies=

Octane

Standard orientation:

\begin{tabular}{|c|c|c|c|c|}
\hline \multirow{2}{*}{$\begin{array}{l}\text { Center } \\
\text { Number }\end{array}$} & Atomic & \multirow{2}{*}{$\begin{array}{l}\text { Atomic } \\
\text { Type }\end{array}$} & \multicolumn{2}{|c|}{ Coordinates (Angstrom } \\
\hline & Numbe & & $X$ & Z \\
\hline 1 & 6 & -4.471291 & 0.258928 & 0.000017 \\
\hline 2 & 1 & -4.532020 & 0.904092 & 0.885170 \\
\hline 3 & 1 & -4.532023 & 0.904163 & -0.885084 \\
\hline 4 & 1 & -5.352180 & -0.392974 & -0.000008 \\
\hline 5 & 6 & -3.171876 & -0.545917 & -0.000018 \\
\hline 6 & 1 & -3.143748 & -1.204434 & 0.880523 \\
\hline 7 & 1 & -3.143759 & -1.204377 & -0.880602 \\
\hline 8 & 6 & -1.928723 & 0.343333 & 0.000001 \\
\hline 9 & 1 & -1.955185 & 1.003241 & -0.881030 \\
\hline 10 & 1 & -1.955191 & 1.003214 & 0.881053 \\
\hline 11 & 6 & -0.620462 & -0.445857 & -0.000004 \\
\hline 12 & 1 & -0.593627 & -1.105394 & -0.881121 \\
\hline 13 & 1 & -0.593621 & -1.105393 & 0.881115 \\
\hline 14 & 6 & 0.620462 & 0.445857 & -0.000009 \\
\hline 15 & 1 & 0.593619 & 1.105406 & 0.881100 \\
\hline 16 & 1 & 0.593628 & 1.105381 & -0.881136 \\
\hline 17 & 6 & 1.928723 & -0.343333 & 0.000009 \\
\hline 18 & 1 & 1.955188 & -1.003248 & -0.881018 \\
\hline 19 & 1 & 1.955188 & -1.003207 & 0.881066 \\
\hline 20 & 6 & 3.171876 & 0.545917 & -0.000012 \\
\hline 21 & 1 & 3.143752 & 1.204428 & 0.880533 \\
\hline 22 & 1 & 3.143755 & 1.204382 & -0.880592 \\
\hline 23 & 6 & 4.471291 & -0.258928 & 0.000011 \\
\hline 24 & 1 & 4.532023 & -0.904151 & -0.885099 \\
\hline 25 & 1 & 5.352180 & 0.392974 & -0.000005 \\
\hline 26 & 1 & 4.532020 & -0.904104 & 0.885156 \\
\hline
\end{tabular}

Zero-point correction $=$

0.248150 (Hartree/Particle)

Thermal correction to Energy=

0.259244

Thermal correction to Enthalpy=

0.260189

Thermal correction to Gibbs Free Energy $=\quad 0.210988$

Sum of electronic and zero-point Energies $=\quad-314.533599$

Sum of electronic and thermal Energies $=\quad-314.522504$

Sum of electronic and thermal Enthalpies $=\quad-314.521560$

Sum of electronic and thermal Free Energies $=\quad-314.570760$

2233tetamethylbutane

Standard orientation:

\begin{tabular}{|c|c|c|c|}
\hline Center & Atomic & Atomic & Coordinates (Angstroms) \\
\hline Number & Number & Type & $\mathrm{X}$ \\
\hline
\end{tabular}




$\begin{array}{cccccc}1 & 6 & 0 & -1.343632 & -0.913916 & -1.105685 \\ 2 & 1 & 0 & -1.145728 & -0.511034 & -2.104974 \\ 3 & 1 & 0 & -0.922879 & -1.925142 & -1.055499 \\ 4 & 1 & 0 & -2.431563 & -1.004973 & -0.996249 \\ 5 & 6 & 0 & -0.787517 & 0.000008 & 0.000017 \\ 6 & 6 & 0 & 0.787495 & 0.000013 & 0.000012 \\ 7 & 6 & 0 & 1.343671 & 1.139051 & 0.872085 \\ 8 & 1 & 0 & 1.145468 & 2.122617 & 0.432342 \\ 9 & 1 & 0 & 0.923273 & 1.126457 & 1.884641 \\ 10 & 1 & 0 & 2.431646 & 1.033258 & 0.966773 \\ 11 & 6 & 0 & -1.343709 & 1.414534 & -0.238616 \\ 12 & 1 & 0 & -1.145169 & 2.078728 & 0.609615 \\ 13 & 1 & 0 & -0.923596 & 1.876365 & -1.139908 \\ 14 & 1 & 0 & -2.431728 & 1.365302 & -0.371414 \\ 15 & 6 & 0 & -1.343611 & -0.500651 & 1.344345 \\ 16 & 1 & 0 & -2.431493 & -0.360036 & 1.368646 \\ 17 & 1 & 0 & -1.145972 & -1.567584 & 1.494758 \\ 18 & 1 & 0 & -0.922552 & 0.048123 & 2.195042 \\ 19 & 6 & 0 & 1.343621 & 0.185775 & -1.422424 \\ 20 & 1 & 0 & 2.431540 & 0.321122 & -1.378125 \\ 21 & 1 & 0 & 1.145837 & -0.686957 & -2.054315 \\ 22 & 1 & 0 & 0.922896 & 1.068774 & -1.917854 \\ 23 & 6 & 0 & 1.343639 & -1.324793 & 0.550299 \\ 24 & 1 & 0 & 2.431617 & -1.353927 & 0.411390 \\ 25 & 1 & 0 & 1.145372 & -1.435875 & 1.621938 \\ 26 & 1 & 0 & 0.923280 & -2.195342 & 0.032996\end{array}$

Zero-point correction=

0.246901 (Hartree/Particle)

Thermal correction to Energy=

0.257188

Thermal correction to Enthalpy=

0.258133

Thermal correction to Gibbs Free Energy=

Sum of electronic and zero-point Energies=

Sum of electronic and thermal Energies $=$

0.213773

$-314.540044$

$-314.529757$

Sum of electronic and thermal Enthalpies=

$-314.528812$

Sum of electronic and thermal Free Energies=

$-314.573172$

PBE0DHD3BJ/DHSVPD

Ethane

Standard orientation:

\begin{tabular}{|c|c|c|c|c|}
\hline \multirow{2}{*}{$\begin{array}{l}\text { Center } \\
\text { Number }\end{array}$} & Atomic & \multirow{2}{*}{$\begin{array}{l}\text { Atomic } \\
\text { Type }\end{array}$} & \multicolumn{2}{|c|}{ Coordinates (Angstroms) } \\
\hline & Numbe & & $\mathrm{X}$ & $\mathrm{Z}$ \\
\hline 1 & 6 & -0.000000 & 0.000000 & 0.759379 \\
\hline 2 & 1 & -0.508108 & 0.880106 & 1.158088 \\
\hline 3 & 1 & 1.016248 & -0.000019 & 1.158088 \\
\hline 4 & 1 & -0.508140 & -0.880087 & 1.158088 \\
\hline 5 & 6 & 0.000000 & 0.000000 & -0.759379 \\
\hline 6 & 1 & 0.508108 & 0.880106 & -1.158088 \\
\hline 7 & 1 & 0.508140 & -0.880087 & -1.158088 \\
\hline 8 & 1 & -1.016248 & -0.000019 & -1.158088 \\
\hline
\end{tabular}


Zero-point correction=

Thermal correction to Energy=

Thermal correction to Enthalpy=

Thermal correction to Gibbs Free Energy=

Sum of electronic and zero-point Energies=

Sum of electronic and thermal Energies=

Sum of electronic and thermal Enthalpies=

Sum of electronic and thermal Free Energies=

Methane

Standard orientation:
0.076192 (Hartree/Particle)

0.079666

0.080610

0.054783

$-79.598580$

$-79.595107$

$-79.594162$

$-79.619989$

\begin{tabular}{cccccc}
\multicolumn{4}{c}{ Standard orientation: } \\
\hline $\begin{array}{c}\text { Center } \\
\text { Number }\end{array}$ & Atomic & Number & Atomic & \multicolumn{2}{c}{ Coordinates (Angstrom } \\
- & Type & X & Y & Z \\
\hline 1 & 6 & 0 & 0.000000 & 0.000000 & 0.000000 \\
2 & 1 & 0 & 0.628773 & 0.628773 & 0.628773 \\
3 & 1 & 0 & -0.628773 & -0.628773 & 0.628773 \\
4 & 1 & 0 & -0.628773 & 0.628773 & -0.628773 \\
5 & 1 & 0 & 0.628773 & -0.628773 & -0.628773
\end{tabular}

Zero-point correction $=$

Thermal correction to Energy=

Thermal correction to Enthalpy=

Thermal correction to Gibbs Free Energy=

Sum of electronic and zero-point Energies=

Sum of electronic and thermal Energies $=$

Sum of electronic and thermal Enthalpies=

Sum of electronic and thermal Free Energies=

Propane
0.045648 (Hartree/Particle)

0.048514

0.049459

0.028335

$-40.389783$

$-40.386917$

$-40.385973$

$-40.407096$

Standard orientation:

\begin{tabular}{cccccc} 
Center & Atomic & \multicolumn{2}{c}{ Atomic } & \multicolumn{2}{c}{ Coordinates } \\
Number & Number & Type & X & Y & Z \\
--------- & \\
\hline 1 & 6 & 0 & -1.262158 & -0.258687 & 0.000000 \\
2 & 1 & 0 & -2.163268 & 0.357709 & -0.000031 \\
3 & 1 & 0 & -1.301660 & -0.904217 & 0.880910 \\
4 & 1 & 0 & -1.301634 & -0.904266 & -0.880875 \\
5 & 6 & 0 & 0.000000 & 0.586362 & 0.000000 \\
6 & 1 & 0 & 0.000000 & 1.243821 & 0.874991 \\
7 & 1 & 0 & 0.000002 & 1.243805 & -0.875002 \\
8 & 6 & 0 & 1.262157 & -0.258688 & 0.000000 \\
9 & 1 & 0 & 1.301662 & -0.904211 & 0.880915 \\
10 & 1 & 0 & 1.301627 & -0.904272 & -0.880871 \\
11 & 1 & 0 & 2.163269 & 0.357707 & -0.000039
\end{tabular}

Zero-point correction=

Thermal correction to Energy=

Thermal correction to Enthalpy=
0.105491 (Hartree/Particle)

0.109987

0.110931 
Thermal correction to Gibbs Free Energy= Sum of electronic and zero-point Energies= Sum of electronic and thermal Energies $=$ Sum of electronic and thermal Enthalpies= Sum of electronic and thermal Free Energies= Butane
0.079866

$-118.812073$

$-118.807577$

$-118.806633$

$-118.837698$

Standard orientation:

\begin{tabular}{cccccc} 
Center & \multicolumn{2}{c}{ Atomic } & \multicolumn{2}{c}{ Atomic } & \multicolumn{3}{c}{ Coordinates } \\
Number & Number & Type & X & Y & $Z$ \\
-1 & 6 & 0 & 1.941001 & 0.118790 & -0.000027 \\
2 & 1 & 0 & 2.084616 & 0.749173 & 0.880940 \\
3 & 1 & 0 & 2.084565 & 0.749156 & -0.881014 \\
4 & 1 & 0 & 2.731174 & -0.634430 & -0.000043 \\
5 & 6 & 0 & 0.560266 & -0.513094 & 0.000030 \\
6 & 1 & 0 & 0.451158 & -1.162844 & -0.875411 \\
7 & 1 & 0 & 0.451196 & -1.162806 & 0.875506 \\
8 & 6 & 0 & -0.560266 & 0.513094 & 0.000031 \\
9 & 1 & 0 & -0.451196 & 1.162804 & 0.875508 \\
10 & 1 & 0 & -0.451158 & 1.162847 & -0.875410 \\
11 & 6 & 0 & -1.941001 & -0.118790 & -0.000027 \\
12 & 1 & 0 & -2.084616 & -0.749176 & 0.880937 \\
13 & 1 & 0 & -2.731174 & 0.634430 & -0.000039 \\
14 & 1 & 0 & -2.084565 & -0.749153 & -0.881016
\end{tabular}

Zero-point correction=

Thermal correction to Energy=

Thermal correction to Enthalpy=

Thermal correction to Gibbs Free Energy=

Sum of electronic and zero-point Energies=

Sum of electronic and thermal Energies=

Sum of electronic and thermal Enthalpies=

Sum of electronic and thermal Free Energies= Isobutane
0.134565 (Hartree/Particle)

0.140308

0.141253

\subsection{1}

$-158.025849$

$-158.020106$

$-158.019162$

$-158.053834$

Standard orientation:

\begin{tabular}{|c|c|c|c|c|}
\hline \multirow{2}{*}{$\begin{array}{l}\text { Center } \\
\text { Number }\end{array}$} & Atomic & \multirow{2}{*}{$\begin{array}{r}\text { Atomic } \\
\text { Type }\end{array}$} & \multicolumn{2}{|c|}{ Coordinates (Angstroms } \\
\hline & Numbe & & $\mathrm{X}$ & Z \\
\hline 1 & 6 & -0.663071 & 1.285284 & 0.095287 \\
\hline 2 & 1 & -1.693636 & 1.356408 & -0.259611 \\
\hline 3 & 1 & -0.684945 & 1.327929 & 1.188224 \\
\hline 4 & 1 & -0.123798 & 2.166301 & -0.259772 \\
\hline 5 & 6 & 0.000004 & -0.000005 & -0.374806 \\
\hline 6 & 1 & -0.000000 & -0.000021 & -1.472880 \\
\hline 7 & 6 & -0.781563 & -1.216871 & 0.095290 \\
\hline 8 & 1 & -0.327852 & -2.144938 & -0.259556 \\
\hline 9 & 1 & -0.807601 & -1.257107 & 1.188226 \\
\hline 10 & 1 & -1.814169 & -1.190370 & -0.259814 \\
\hline
\end{tabular}




$\begin{array}{rrrrrr}11 & 6 & 0 & 1.444630 & -0.068410 & 0.095289 \\ 12 & 1 & 0 & 1.937995 & -0.975920 & -0.259809 \\ 13 & 1 & 0 & 2.021476 & 0.788550 & -0.259595 \\ 14 & 1 & 0 & 1.492534 & -0.070816 & 1.188228\end{array}$

Zero-point correction=

Thermal correction to Energy=

Thermal correction to Enthalpy=

Thermal correction to Gibbs Free Energy=

Sum of electronic and zero-point Energies=

Sum of electronic and thermal Energies $=$

Sum of electronic and thermal Enthalpies=

Sum of electronic and thermal Free Energies=

Pentane
0.134100 (Hartree/Particle)

0.139751

0.140696

\subsection{0}

$-158.028577$

$-158.022925$

$-158.021981$

$-158.056147$

Standard orientation:

\begin{tabular}{cccccc} 
Center & \multicolumn{2}{c}{ Atomic } & \multicolumn{2}{c}{ Atomic } & \multicolumn{3}{c}{ Coordinates } \\
Number & Number & Type & X & $Y$ & $Z$ \\
---------------- \\
1 & 6 & 0 & -2.529874 & 0.324764 & 0.000002 \\
2 & 1 & 0 & -2.568811 & 0.970134 & 0.880941 \\
3 & 1 & 0 & -2.568808 & 0.970150 & -0.880926 \\
4 & 1 & 0 & -3.431645 & -0.290343 & -0.000006 \\
5 & 6 & 0 & -1.270059 & -0.522759 & -0.000004 \\
6 & 1 & 0 & -1.268357 & -1.181083 & 0.875612 \\
7 & 1 & 0 & -1.268358 & -1.181073 & -0.875627 \\
8 & 6 & 0 & 0.000000 & 0.310055 & 0.000000 \\
9 & 1 & 0 & 0.000002 & 0.970021 & -0.875950 \\
10 & 1 & 0 & -0.000002 & 0.970020 & 0.875950 \\
11 & 6 & 0 & 1.270059 & -0.522759 & 0.000004 \\
12 & 1 & 0 & 1.268357 & -1.181082 & -0.875613 \\
13 & 1 & 0 & 1.268358 & -1.181074 & 0.875626 \\
14 & 6 & 0 & 2.529874 & 0.324764 & -0.000002 \\
15 & 1 & 0 & 3.431645 & -0.290343 & 0.000002 \\
16 & 1 & 0 & 2.568809 & 0.970137 & -0.880939 \\
17 & 1 & 0 & 2.568810 & 0.970147 & 0.880928
\end{tabular}

Zero-point correction=

Thermal correction to Energy=

Thermal correction to Enthalpy=

Thermal correction to Gibbs Free Energy=

Sum of electronic and zero-point Energies=

Sum of electronic and thermal Energies $=$

Sum of electronic and thermal Enthalpies=

Sum of electronic and thermal Free Energies=

Neopentane

Standard orientation:

\begin{tabular}{lcccc} 
Center & Atomic & Atomic & & \multicolumn{2}{c}{ Coordinates (Angstroms) } \\
Number & Number & Type & X & Y
\end{tabular}




$\begin{array}{cccccc}1 & 6 & 0 & -0.059626 & -1.185807 & -0.956459 \\ 2 & 1 & 0 & 0.601939 & -1.032996 & -1.812923 \\ 3 & 1 & 0 & 0.246437 & -2.108621 & -0.457173 \\ 4 & 1 & 0 & -1.073360 & -1.331717 & -1.338037 \\ 5 & 6 & 0 & 0.000019 & 0.000005 & 0.000013 \\ 6 & 6 & 0 & 1.426872 & 0.170818 & 0.509381 \\ 7 & 1 & 0 & 1.499023 & 1.015680 & 1.198980 \\ 8 & 1 & 0 & 1.764329 & -0.723697 & 1.039013 \\ 9 & 1 & 0 & 2.119348 & 0.352383 & -0.316486 \\ 10 & 6 & 0 & -0.433319 & 1.265435 & -0.731733 \\ 11 & 1 & 0 & -1.454956 & 1.170740 & -1.108347 \\ 12 & 1 & 0 & -0.399933 & 2.133399 & -0.068356 \\ 13 & 1 & 0 & 0.220212 & 1.469573 & -1.583655 \\ 14 & 6 & 0 & -0.933929 & -0.250447 & 1.178812 \\ 15 & 1 & 0 & -1.965990 & -0.376714 & 0.841974 \\ 16 & 1 & 0 & -0.646117 & -1.153754 & 1.722637 \\ 17 & 1 & 0 & -0.911035 & 0.585701 & 1.882285\end{array}$

Zero-point correction=

Thermal correction to Energy=

Thermal correction to Enthalpy=

Thermal correction to Gibbs Free Energy=

Sum of electronic and zero-point Energies=

Sum of electronic and thermal Energies=

Sum of electronic and thermal Enthalpies=

Sum of electronic and thermal Free Energies=

Hexane
0.162433 (Hartree/Particle)

$$
0.169276
$$

0.170220

\subsection{3}

$-197.246303$

$-197.239460$

$-197.238516$

$-197.275343$

Standard orientation:

\begin{tabular}{ccccccc} 
Center & \multicolumn{2}{c}{ Atomic } & \multicolumn{2}{c}{ Atomic } & \multicolumn{3}{c}{ Coordinates } \\
Number & Number & Type & X & $Y$ & $Z$ \\
- & Nustroms) \\
\hline 1 & 6 & 0 & 3.186977 & -0.207362 & 0.000009 \\
2 & 1 & 0 & 3.271424 & -0.848394 & 0.880962 \\
3 & 1 & 0 & 3.271424 & -0.848440 & -0.880911 \\
4 & 1 & 0 & 4.043044 & 0.469923 & -0.000010 \\
5 & 6 & 0 & 1.870360 & 0.548797 & -0.000011 \\
6 & 1 & 0 & 1.821858 & 1.205553 & 0.875532 \\
7 & 1 & 0 & 1.821865 & 1.205518 & -0.875580 \\
8 & 6 & 0 & 0.661958 & -0.371425 & 0.000001 \\
9 & 1 & 0 & 0.709040 & -1.029447 & -0.876007 \\
10 & 1 & 0 & 0.709046 & -1.029433 & 0.876020 \\
11 & 6 & 0 & -0.661958 & 0.371425 & 0.000001 \\
12 & 1 & 0 & -0.709040 & 1.029447 & -0.876008 \\
13 & 1 & 0 & -0.709046 & 1.029433 & 0.876020 \\
14 & 6 & 0 & -1.870360 & -0.548797 & -0.000011 \\
15 & 1 & 0 & -1.821858 & -1.205552 & 0.875532 \\
16 & 1 & 0 & -1.821865 & -1.205518 & -0.875580 \\
17 & 6 & 0 & -3.186977 & 0.207362 & 0.000009
\end{tabular}




$\begin{array}{lllrrc}18 & 1 & 0 & -3.271425 & 0.848439 & -0.880912 \\ 19 & 1 & 0 & -4.043044 & -0.469923 & -0.000008 \\ 20 & 1 & 0 & -3.271424 & 0.848395 & 0.880962\end{array}$

Zero-point correction=

0.192642 (Hartree/Particle)

Thermal correction to Energy=

0.201004

Thermal correction to Enthalpy=

0.201948

Thermal correction to Gibbs Free Energy=

Sum of electronic and zero-point Energies=

0.160055

Sum of electronic and thermal Energies=

$-236.453514$

$-236.445151$

Sum of electronic and thermal Enthalpies=

$-236.444207$

Sum of electronic and thermal Free Energies $=\quad-236.486100$

22dimethylbutane

Standard orientation:

\begin{tabular}{|c|c|c|c|c|}
\hline \multirow{2}{*}{$\begin{array}{l}\text { Center } \\
\text { Number }\end{array}$} & Atomic & \multirow{2}{*}{$\begin{array}{r}\text { Atomic } \\
\text { Type }\end{array}$} & \multicolumn{2}{|c|}{ Coordinates (Angstrom } \\
\hline & Numbe & & $\mathrm{X}$ & $\mathrm{Z}$ \\
\hline 1 & 6 & 1.611203 & 0.966200 & -0.001481 \\
\hline 2 & 1 & 1.591440 & 1.608479 & -0.885681 \\
\hline 3 & 1 & 1.591709 & 1.610817 & 0.881022 \\
\hline 4 & 1 & 2.561101 & 0.425917 & -0.000907 \\
\hline 5 & 6 & 0.431007 & -0.000080 & -0.000014 \\
\hline 6 & 6 & -0.860507 & 0.827680 & -0.000609 \\
\hline 7 & 1 & -0.840424 & 1.486882 & 0.874756 \\
\hline 8 & 1 & -0.840520 & 1.485520 & -0.877005 \\
\hline 9 & 6 & -2.159551 & 0.040190 & 0.000009 \\
\hline 10 & 1 & -2.248521 & -0.596423 & 0.882258 \\
\hline 11 & 1 & -3.017640 & 0.715050 & 0.000041 \\
\hline 12 & 1 & -2.248967 & -0.596936 & $5-0.881819$ \\
\hline 13 & 6 & 0.508705 & -0.878341 & -1.244505 \\
\hline 14 & 1 & -0.285174 & -1.627433 & $3-1.261726$ \\
\hline 15 & 1 & 0.426326 & -0.278043 & -2.154663 \\
\hline 16 & 1 & 1.462420 & -1.410662 & -1.280645 \\
\hline 17 & 6 & 0.509449 & -0.875360 & 1.246539 \\
\hline 18 & 1 & 1.462986 & -1.407972 & 1.283129 \\
\hline 19 & 1 & 0.428115 & -0.272809 & 2.155298 \\
\hline 20 & 1 & -0.284687 & -1.624117 & $7 \quad 1.266313$ \\
\hline
\end{tabular}

Zero-point correction $=$

Thermal correction to Energy=

0.191543 (Hartree/Particle)

Thermal correction to Enthalpy=

0.199713

0.200657

Thermal correction to Gibbs Free Energy=

0.160260

Sum of electronic and zero-point Energies=

$-236.458628$

Sum of electronic and thermal Energies=

$-236.450459$

Sum of electronic and thermal Enthalpies= $-236.449514$

Sum of electronic and thermal Free Energies= $-236.489912$

Heptane

Standard orientation: 


\begin{tabular}{|c|c|c|c|c|}
\hline \multirow{2}{*}{$\begin{array}{l}\text { Center } \\
\text { Number }\end{array}$} & Atomic & \multirow{2}{*}{$\begin{array}{r}\text { Atomic } \\
\text { Type }\end{array}$} & \multicolumn{2}{|c|}{ Coordinates (Angstroms } \\
\hline & Numbe & & $\mathrm{X}$ & $\mathrm{Z}$ \\
\hline 1 & 6 & 3.798434 & -0.355127 & 0.000009 \\
\hline 2 & 1 & 3.836821 & -1.000614 & 0.880970 \\
\hline 3 & 1 & 3.836815 & -1.000670 & -0.880912 \\
\hline 4 & 1 & 4.700665 & 0.259310 & -0.000014 \\
\hline 5 & 6 & 2.539071 & 0.492962 & -0.000015 \\
\hline 6 & 1 & 2.537428 & 1.151528 & 0.875532 \\
\hline 7 & 1 & 2.537430 & 1.151486 & -0.875593 \\
\hline 8 & 6 & 1.268261 & -0.338814 & 0.000002 \\
\hline 9 & 1 & 1.268301 & -0.998742 & -0.875931 \\
\hline 10 & 1 & 1.268308 & -0.998721 & 0.875952 \\
\hline 11 & 6 & -0.000000 & 0.495730 & 0.000000 \\
\hline 12 & 1 & 0.000003 & 1.155109 & -0.876105 \\
\hline 13 & 1 & -0.000003 & 1.155104 & 0.876109 \\
\hline 14 & 6 & -1.268261 & -0.338814 & -0.000006 \\
\hline 15 & 1 & -1.268300 & -0.998746 & 0.875925 \\
\hline 16 & 1 & -1.268309 & -0.998717 & -0.875959 \\
\hline 17 & 6 & -2.539071 & 0.492962 & 0.000014 \\
\hline 18 & 1 & -2.537429 & 1.151528 & -0.875532 \\
\hline 19 & 1 & -2.537428 & 1.151486 & 0.875593 \\
\hline 20 & 6 & -3.798434 & -0.355127 & -0.000006 \\
\hline 21 & 1 & -3.836816 & -1.000665 & 0.880918 \\
\hline 22 & 1 & -4.700665 & 0.259310 & 0.000013 \\
\hline 23 & 1 & -3.836820 & -1.000619 & -0.880963 \\
\hline
\end{tabular}

Zero-point correction=

Thermal correction to Energy=

Thermal correction to Enthalpy=

Thermal correction to Gibbs Free Energy=

Sum of electronic and zero-point Energies=

Sum of electronic and thermal Energies $=$

Sum of electronic and thermal Enthalpies=

Sum of electronic and thermal Free Energies=

223trimethylbutane

\subsection{1 (Hartree/Particle)}

0.231344

$$
0.232288
$$

\subsection{2}

$-275.667411$

$-275.657718$

$-275.656774$

$-275.702250$

Standard orientation:

\begin{tabular}{|c|c|c|c|c|}
\hline \multirow{2}{*}{$\begin{array}{l}\text { Center } \\
\text { Number }\end{array}$} & Atomic & \multirow{2}{*}{$\begin{array}{l}\text { Atomic } \\
\text { Type }\end{array}$} & \multicolumn{2}{|c|}{ Coordinates (Angstroms) } \\
\hline & Number & & $\mathrm{X}$ & $\mathrm{Z}$ \\
\hline 1 & 6 & 1.311355 & 1.299687 & -0.434591 \\
\hline 2 & 1 & 1.165401 & 1.481833 & -1.503210 \\
\hline 3 & 1 & 0.926047 & 2.162267 & 0.110987 \\
\hline 4 & 1 & 2.386856 & 1.248916 & -0.249133 \\
\hline 5 & 6 & 0.636643 & 0.000145 & -0.000829 \\
\hline 6 & 6 & -0.843474 & 0.001178 & -0.454575 \\
\hline 7 & 1 & -0.814051 & 0.006244 & -1.553721 \\
\hline 8 & 6 & -1.611570 & -1.241746 & -0.022839 \\
\hline
\end{tabular}




$\begin{array}{cccccc}9 & 1 & 0 & -1.746808 & -1.265037 & 1.061148 \\ 10 & 1 & 0 & -2.606737 & -1.244851 & -0.472346 \\ 11 & 1 & 0 & -1.117194 & -2.167132 & -0.320301 \\ 12 & 6 & 0 & -1.616512 & 1.239436 & -0.017890 \\ 13 & 1 & 0 & -1.639769 & 1.331862 & 1.070969 \\ 14 & 1 & 0 & -1.196460 & 2.159256 & -0.425033 \\ 15 & 1 & 0 & -2.652515 & 1.175057 & -0.357283 \\ 16 & 6 & 0 & 0.768801 & -0.143254 & 1.512549 \\ 17 & 1 & 0 & 0.381637 & -1.101743 & 1.863472 \\ 18 & 1 & 0 & 1.820352 & -0.087758 & 1.804759 \\ 19 & 1 & 0 & 0.240144 & 0.651364 & 2.043543 \\ 20 & 6 & 0 & 1.376273 & -1.155546 & -0.671738 \\ 21 & 1 & 0 & 1.293437 & -1.094817 & -1.760388 \\ 22 & 1 & 0 & 2.438155 & -1.127570 & -0.415901 \\ 23 & 1 & 0 & 0.992409 & -2.127296 & -0.358083\end{array}$

Zero-point correction=

Thermal correction to Energy=

Thermal correction to Enthalpy=

Thermal correction to Gibbs Free Energy=

Sum of electronic and zero-point Energies=

Sum of electronic and thermal Energies $=$

Sum of electronic and thermal Enthalpies=

Sum of electronic and thermal Free Energies=

Octane
0.220319 (Hartree/Particle)
0.229711
0.230655
0.187360
$-275.671974$
$-275.662582$
$-275.661638$
$-275.704933$

Standard orientation:

\begin{tabular}{cccccc} 
Center & \multicolumn{2}{c}{ Atomic } & \multicolumn{2}{c}{ Atomic } & \multicolumn{3}{c}{ Coordinates } \\
Number & Number & Type & X & Y & $Z$ \\
---------- & Z \\
\hline 1 & 6 & 0 & -4.446683 & 0.256085 & 0.000015 \\
2 & 1 & 0 & -4.510934 & 0.899523 & 0.880978 \\
3 & 1 & 0 & -4.510933 & 0.899590 & -0.880900 \\
4 & 1 & 0 & -5.323560 & -0.394029 & -0.000012 \\
5 & 6 & 0 & -3.154303 & -0.540765 & -0.000016 \\
6 & 1 & 0 & -3.126168 & -1.198814 & 0.875524 \\
7 & 1 & 0 & -3.126176 & -1.198762 & -0.875596 \\
8 & 6 & 0 & -1.917803 & 0.341206 & 0.000003 \\
9 & 1 & 0 & -1.944217 & 1.000636 & -0.875932 \\
10 & 1 & 0 & -1.944222 & 1.000606 & 0.875961 \\
11 & 6 & 0 & -0.617267 & -0.441932 & -0.000004 \\
12 & 1 & 0 & -0.590867 & -1.100995 & -0.876031 \\
13 & 1 & 0 & -0.590860 & -1.100997 & 0.876023 \\
14 & 6 & 0 & 0.617267 & 0.441932 & -0.000008 \\
15 & 1 & 0 & 0.590859 & 1.101007 & 0.876011 \\
16 & 1 & 0 & 0.590868 & 1.100985 & -0.876044 \\
17 & 6 & 0 & 1.917803 & -0.341206 & 0.000008 \\
18 & 1 & 0 & 1.944219 & -1.000640 & -0.875924 \\
19 & 1 & 0 & 1.944220 & -1.000601 & 0.875969 \\
20 & 6 & 0 & 3.154303 & 0.540765 & -0.000012
\end{tabular}




$\begin{array}{rrrrrr}21 & 1 & 0 & 3.126171 & 1.198810 & 0.875532 \\ 22 & 1 & 0 & 3.126173 & 1.198767 & -0.875588 \\ 23 & 6 & 0 & 4.446683 & -0.256085 & 0.000010 \\ 24 & 1 & 0 & 4.510935 & -0.899579 & -0.880912 \\ 25 & 1 & 0 & 5.323560 & 0.394029 & -0.000005 \\ 26 & 1 & 0 & 4.510933 & -0.899534 & 0.880965\end{array}$

Zero-point correction=

Thermal correction to Energy=

Thermal correction to Enthalpy=

Thermal correction to Gibbs Free Energy=

Sum of electronic and zero-point Energies=

Sum of electronic and thermal Energies $=$

Sum of electronic and thermal Enthalpies=

Sum of electronic and thermal Free Energies=

2233tetramethylbutane
0.250647 (Hartree/Particle)

0.261679

0.262624

0.213566

$-314.881322$

$-314.870290$

$-314.869346$

$-314.918403$

Standard orientation:

\begin{tabular}{|c|c|c|c|c|}
\hline \multirow{2}{*}{$\begin{array}{l}\text { Center } \\
\text { Number }\end{array}$} & Atomic & \multirow{2}{*}{$\begin{array}{l}\text { Atomic } \\
\text { Type }\end{array}$} & \multicolumn{2}{|c|}{ Coordinates (Angstroms } \\
\hline & Numbe & & $X$ & Z \\
\hline 1 & 6 & -1.334461 & -1.384624 & -0.340759 \\
\hline 2 & 1 & -1.133227 & -1.658721 & -1.377492 \\
\hline 3 & 1 & -0.919025 & -2.163188 & 0.302574 \\
\hline 4 & 1 & -2.418480 & -1.393670 & -0.204451 \\
\hline 5 & 6 & -0.782936 & -0.000039 & -0.000020 \\
\hline 6 & 6 & 0.782966 & 0.000006 & -0.000004 \\
\hline 7 & 6 & 1.334404 & 1.425545 & 0.031527 \\
\hline 8 & 1 & 1.133406 & 1.959998 & -0.898192 \\
\hline 9 & 1 & 0.918541 & 2.009743 & 0.855152 \\
\hline 10 & 1 & 2.418377 & 1.398891 & 0.165915 \\
\hline 11 & 6 & -1.334516 & 0.987389 & -1.028651 \\
\hline 12 & 1 & -1.132967 & 2.022257 & -0.747781 \\
\hline 13 & 1 & -0.919415 & 0.819386 & -2.024685 \\
\hline 14 & 1 & -2.418582 & 0.874090 & -1.104318 \\
\hline 15 & 6 & -1.334442 & 0.397157 & 1.369452 \\
\hline 16 & 1 & -2.418442 & 0.519858 & 1.309106 \\
\hline 17 & 1 & -1.133282 & -0.363668 & 2.125167 \\
\hline 18 & 1 & -0.918878 & 1.343520 & 1.722072 \\
\hline 19 & 6 & 1.334513 & -0.685405 & -1.250323 \\
\hline 20 & 1 & 2.418564 & -0.556199 & -1.293952 \\
\hline 21 & 1 & 1.133010 & -1.757707 & -1.248697 \\
\hline 22 & 1 & 0.919295 & -0.263767 & -2.168145 \\
\hline 23 & 6 & 1.334522 & -0.740058 & 1.218767 \\
\hline 24 & 1 & 2.418518 & -0.842839 & 1.128509 \\
\hline 25 & 1 & 1.133349 & -0.202259 & 2.146525 \\
\hline 26 & 1 & 0.918943 & -1.745554 & 1.312766 \\
\hline
\end{tabular}

Zero-point correction=

Thermal correction to Energy=
0.249257 (Hartree/Particle)

0.259506 
Thermal correction to Enthalpy= 0.260450

Thermal correction to Gibbs Free Energy=

Sum of electronic and zero-point Energies= $-314.885417$

Sum of electronic and thermal Energies= $-314.875168$

Sum of electronic and thermal Enthalpies= $-314.874224$

Sum of electronic and thermal Free Energies= $-314.918523$

PBEQIDHD3BJ/DHSVPD

Ethane

Standard orientation:

\begin{tabular}{|c|c|c|c|c|}
\hline \multirow{2}{*}{$\begin{array}{l}\text { Center } \\
\text { Number }\end{array}$} & Atomic & \multirow{2}{*}{$\begin{array}{r}\text { Atomic } \\
\text { Type }\end{array}$} & \multicolumn{2}{|c|}{ Coordinates (Angstrom } \\
\hline & Numbe & & $X$ & Z \\
\hline 1 & 6 & -0.000000 & -0.000000 & 0.759729 \\
\hline 2 & 1 & -0.507965 & 0.879854 & 1.157398 \\
\hline 3 & 1 & 1.015959 & -0.000016 & 1.157398 \\
\hline 4 & 1 & -0.507994 & -0.879838 & 1.157398 \\
\hline 5 & 6 & 0.000000 & -0.000000 & -0.759729 \\
\hline 6 & 1 & 0.507965 & 0.879854 & -1.157398 \\
\hline 7 & 1 & 0.507994 & -0.879838 & -1.157398 \\
\hline 8 & 1 & -1.015959 & -0.000016 & -1.157398 \\
\hline
\end{tabular}

Zero-point correction=

Thermal correction to Energy=

Thermal correction to Enthalpy=

Thermal correction to Gibbs Free

Sum of electronic and zero-point Energies=

Sum of electronic and thermal Energies $=$

Sum of electronic and thermal Enthalpies=

Sum of electronic and thermal Free Energies=

Methane

\section{Standard orientation:}

\subsection{2 (Hartree/Particle) 0.080039} 0.080983

0.055167

$-79.568440$

$-79.564974$

$-79.564029$

$-79.589845$

\begin{tabular}{|c|c|c|c|c|}
\hline \multirow{2}{*}{$\begin{array}{l}\text { Center } \\
\text { Number }\end{array}$} & \multirow{2}{*}{$\begin{array}{l}\text { Atomic } \\
\text { Number }\end{array}$} & \multirow{2}{*}{$\begin{array}{r}\text { Atomic } \\
\text { Type }\end{array}$} & \multicolumn{2}{|c|}{ Coordinates (Angstrom } \\
\hline & & & $\mathrm{X}$ & $\mathrm{Z}$ \\
\hline 1 & 6 & 0.000000 & 0.000000 & 0.000000 \\
\hline 2 & 1 & 0.628415 & 0.628415 & 0.628415 \\
\hline 3 & 1 & -0.628415 & -0.628415 & 0.628415 \\
\hline 4 & 1 & -0.628415 & 0.628415 & -0.628415 \\
\hline 5 & 1 & 0.628415 & -0.628415 & -0.628415 \\
\hline
\end{tabular}

Zero-point correction=

Thermal correction to Energy=

Thermal correction to Enthalpy=

Thermal correction to Gibbs Free Energy=

Sum of electronic and zero-point Energies=

Sum of electronic and thermal Energies $=$

Sum of electronic and thermal Enthalpies=
0.045908 (Hartree/Particle)

0.048773

0.049717

0.028597

$-40.374421$

$-40.371556$

$-40.370612$ 
Sum of electronic and thermal Free Energies= $-40.391732$

Propane

Standard orientation:

\begin{tabular}{|c|c|c|c|c|c|}
\hline \multirow{2}{*}{$\begin{array}{l}\text { Center } \\
\text { Number }\end{array}$} & \multicolumn{2}{|l|}{ Atomic } & \multirow{2}{*}{$\begin{array}{l}\text { tomic } \\
\text { Type }\end{array}$} & \multicolumn{2}{|c|}{ Coordinates (Angstrom } \\
\hline & Numbe & & & $\mathrm{X}$ & Z \\
\hline 1 & 6 & 0 & -1.262014 & -0.259113 & 0.000000 \\
\hline 2 & 1 & 0 & -2.162939 & 0.356695 & -0.000034 \\
\hline 3 & 1 & 0 & -1.300277 & -0.903915 & 0.880819 \\
\hline 4 & 1 & 0 & -1.300247 & -0.903969 & -0.880781 \\
\hline 5 & 6 & 0 & -0.000000 & 0.587370 & 0.000000 \\
\hline 6 & 1 & 0 & 0.000001 & 1.243768 & 0.875070 \\
\hline 7 & 1 & 0 & 0.000002 & 1.243752 & -0.875081 \\
\hline 8 & 6 & 0 & 1.262014 & -0.259114 & 0.000000 \\
\hline 9 & 1 & 0 & 1.300279 & -0.903910 & 0.880823 \\
\hline 10 & 1 & 0 & 1.300241 & -0.903975 & -0.880777 \\
\hline 11 & 1 & 0 & 2.162941 & 0.356694 & -0.000042 \\
\hline
\end{tabular}

Zero-point correction=

Thermal correction to Energy=

0.105973 (Hartree/Particle)

Thermal correction to Enthalpy=

0.110458

Thermal correction to Gibbs Free Energy=

0.111402

0.080357

Sum of electronic and zero-point Energies=

$-118.767220$

Sum of electronic and thermal Energies=

$-118.762735$

Sum of electronic and thermal Enthalpies=

$-118.761791$

Sum of electronic and thermal Free Energies=

$-118.792836$

Butane

Standard orientation:

\begin{tabular}{|c|c|c|c|c|}
\hline \multirow{2}{*}{$\begin{array}{l}\text { Center } \\
\text { Number }\end{array}$} & Atomic & \multirow{2}{*}{$\begin{array}{l}\text { Atomic } \\
\text { Type }\end{array}$} & \multicolumn{2}{|c|}{ Coordinates (Angstrom } \\
\hline & Numbe & & $\mathrm{X}$ & $\mathrm{Z}$ \\
\hline 1 & 6 & 1.941236 & 0.118989 & -0.000028 \\
\hline 2 & 1 & 2.083763 & 0.748799 & 0.880862 \\
\hline 3 & 1 & 2.083705 & 0.748790 & -0.880936 \\
\hline 4 & 1 & 2.731026 & -0.633988 & -0.000051 \\
\hline 5 & 6 & 0.560033 & -0.513802 & 0.000032 \\
\hline 6 & 1 & 0.450998 & -1.162472 & -0.875622 \\
\hline 7 & 1 & 0.451039 & -1.162431 & 0.875722 \\
\hline 8 & 6 & -0.560033 & 0.513802 & 0.000032 \\
\hline 9 & 1 & -0.451038 & 1.162429 & 0.875723 \\
\hline 10 & 1 & -0.450998 & 1.162474 & -0.875620 \\
\hline 11 & 6 & -1.941236 & -0.118989 & -0.000029 \\
\hline 12 & 1 & -2.083763 & -0.748802 & 0.880861 \\
\hline 13 & 1 & -2.731026 & 0.633988 & -0.000048 \\
\hline 14 & 1 & -2.083705 & -0.748787 & $7 \quad-0.880937$ \\
\hline
\end{tabular}


Thermal correction to Enthalpy=

0.141823

Thermal correction to Gibbs Free Energy=

0.107179

Sum of electronic and zero-point Energies=

$-157.966322$

Sum of electronic and thermal Energies $=$

$-157.960592$

$-157.959648$

$-157.994293$

Sum of electronic and thermal Free Energies=

Isobutane

Standard orientation:

\begin{tabular}{|c|c|c|c|c|}
\hline \multirow{2}{*}{$\begin{array}{l}\text { Center } \\
\text { Number }\end{array}$} & Atomic & \multirow{2}{*}{$\begin{array}{l}\text { Atomic } \\
\text { Type }\end{array}$} & \multicolumn{2}{|c|}{ Coordinates (Angstroms } \\
\hline & Numbe & & $X$ & Z \\
\hline 1 & 6 & 1.301374 & -0.630965 & 0.095590 \\
\hline 2 & 1 & 2.168523 & -0.070051 & -0.258946 \\
\hline 3 & 1 & 1.342388 & -0.650887 & 1.188216 \\
\hline 4 & 1 & 1.398078 & -1.659159 & -0.258998 \\
\hline 5 & 6 & -0.000004 & -0.000003 & -0.376273 \\
\hline 6 & 1 & -0.000007 & 0.000001 & -1.473839 \\
\hline 7 & 6 & -0.104250 & 1.442499 & 0.095591 \\
\hline 8 & 1 & -1.023564 & 1.913043 & -0.258978 \\
\hline 9 & 1 & -0.107546 & 1.487956 & 1.188219 \\
\hline 10 & 1 & 0.737870 & 2.040327 & -0.258958 \\
\hline 11 & 6 & -1.197123 & -0.811533 & 0.095593 \\
\hline 12 & 1 & -2.135927 & -0.381002 & -0.258748 \\
\hline 13 & 1 & -1.145092 & -1.842884 & -0.259191 \\
\hline 14 & 1 & -1.234704 & -0.837333 & 1.188218 \\
\hline
\end{tabular}

Zero-point correction=

Thermal correction to Energy=

Thermal correction to Enthalpy=

Thermal correction to Gibbs Free Energy=

Sum of electronic and zero-point Energies=

Sum of electronic and thermal Energies $=$

Sum of electronic and thermal Enthalpies=

Sum of electronic and thermal Free Energies=
0.134673 (Hartree/Particle)

0.140307

0.141252

0.107118

$-157.969119$

$-157.963485$

$-157.962541$

$-157.996674$

Pentane

Standard orientation:

\begin{tabular}{|c|c|c|c|c|}
\hline \multirow{2}{*}{$\begin{array}{l}\text { Center } \\
\text { Number }\end{array}$} & \multirow{2}{*}{$\begin{array}{l}\text { Atomic } \\
\text { Number }\end{array}$} & \multirow{2}{*}{$\begin{array}{l}\text { Atomic } \\
\text { Type }\end{array}$} & \multicolumn{2}{|c|}{ Coordinates (Angstroms) } \\
\hline & & & $\mathrm{X}$ & Z \\
\hline 1 & 6 & -2.529884 & 0.325680 & 0.000001 \\
\hline 2 & 1 & -2.567270 & 0.970349 & 0.880886 \\
\hline 3 & 1 & -2.567266 & 0.970364 & -0.880873 \\
\hline 4 & 1 & -3.431731 & -0.288556 & -0.000006 \\
\hline 5 & 6 & -1.270374 & -0.523775 & -0.000003 \\
\hline 6 & 1 & -1.268966 & -1.180990 & 0.875880 \\
\hline 7 & 1 & -1.268967 & -1.180981 & -0.875894 \\
\hline
\end{tabular}




$\begin{array}{cccrrr}8 & 6 & 0 & -0.000000 & 0.309885 & 0.000000 \\ 9 & 1 & 0 & 0.000002 & 0.968732 & -0.876323 \\ 10 & 1 & 0 & -0.000002 & 0.968731 & 0.876324 \\ 11 & 6 & 0 & 1.270374 & -0.523775 & 0.000003 \\ 12 & 1 & 0 & 1.268966 & -1.180990 & -0.875881 \\ 13 & 1 & 0 & 1.268967 & -1.180982 & 0.875893 \\ 14 & 6 & 0 & 2.529884 & 0.325680 & -0.000002 \\ 15 & 1 & 0 & 3.431731 & -0.288556 & 0.000002 \\ 16 & 1 & 0 & 2.567268 & 0.970352 & -0.880884 \\ 17 & 1 & 0 & 2.567269 & 0.970361 & 0.880875\end{array}$

Zero-point correction=

Thermal correction to Energy=

Thermal correction to Enthalpy=

Thermal correction to Gibbs Free Energy=

Sum of electronic and zero-point Energies=

Sum of electronic and thermal Energies=

Sum of electronic and thermal Enthalpies=

Sum of electronic and thermal Free Energies=

Neopentane

Standard orientation:
0.164317 (Hartree/Particle)

0.171337

0.172282

$$
\begin{gathered}
0.134034 \\
-197.165469 \\
-197.158449 \\
-197.157505 \\
-197.195752
\end{gathered}
$$

\begin{tabular}{|c|c|c|c|c|}
\hline \multirow{3}{*}{$\begin{array}{l}\text { Center } \\
\text { Number } \\
1\end{array}$} & \multirow{2}{*}{$\begin{array}{l}\text { Atomic } \\
\text { Number }\end{array}$} & \multirow{2}{*}{$\begin{array}{r}\text { Atomic } \\
\text { Type }\end{array}$} & \multicolumn{2}{|c|}{ Coordinates (Angstroms } \\
\hline & & & $\mathrm{X}$ & $\mathrm{Z}$ \\
\hline & 6 & 1.042377 & 1.019631 & -0.446466 \\
\hline 2 & 1 & 0.852200 & 1.997196 & 0.003278 \\
\hline 3 & 1 & 2.047622 & 0.705943 & -0.154667 \\
\hline 4 & 1 & 1.031051 & 1.141959 & -1.532266 \\
\hline 5 & 6 & 0.000000 & 0.000003 & -0.000012 \\
\hline 6 & 6 & 0.045932 & -0.142697 & 1.517567 \\
\hline 7 & 1 & -0.692549 & -0.869097 & 1.865507 \\
\hline 8 & 1 & 1.030732 & -0.480026 & 1.849893 \\
\hline 9 & 1 & -0.164956 & 0.811025 & 2.007464 \\
\hline 10 & 6 & -1.386728 & 0.471526 & -0.424482 \\
\hline 11 & 1 & -1.447874 & 0.582272 & -1.509868 \\
\hline 12 & 1 & -2.154738 & -0.242079 & -0.116274 \\
\hline 13 & 1 & -1.626840 & 1.437970 & 0.025404 \\
\hline 14 & 6 & 0.298418 & -1.348463 & -0.646616 \\
\hline 15 & 1 & 0.271353 & -1.275087 & -1.736549 \\
\hline 16 & 1 & 1.288610 & -1.710392 & -0.359224 \\
\hline 17 & 1 & -0.434606 & -2.099686 & -0.342642 \\
\hline
\end{tabular}

Zero-point correction=

Thermal correction to Energy=

Thermal correction to Enthalpy=

Thermal correction to Gibbs Free Energy=

Sum of electronic and zero-point Energies=

Sum of electronic and thermal Energies $=$

Sum of electronic and thermal Enthalpies=
0.163085 (Hartree/Particle)

$$
0.169903
$$

0.170847

0.134069

$-197.172447$

$-197.165630$

$-197.164686$ 
Sum of electronic and thermal Free Energies=

Hexane

Standard orientation:

\begin{tabular}{|c|c|c|c|c|c|}
\hline \multirow{2}{*}{$\begin{array}{l}\text { Center } \\
\text { Number }\end{array}$} & \multicolumn{2}{|c|}{ Atomic } & \multirow{2}{*}{$\begin{array}{l}\text { tomic } \\
\text { Type }\end{array}$} & \multicolumn{2}{|c|}{ Coordinates (Angstrom } \\
\hline & & & & $X$ & Z \\
\hline 1 & 6 & 0 & -1.409320 & 2.866395 & 0.000008 \\
\hline 2 & 1 & 0 & -0.895678 & 3.257799 & 0.880912 \\
\hline 3 & 1 & 0 & -0.895641 & 3.257820 & -0.880865 \\
\hline 4 & 1 & 0 & -2.422777 & 3.270801 & -0.000008 \\
\hline 5 & 6 & 0 & -1.409320 & 1.347262 & -0.000010 \\
\hline 6 & 1 & 0 & -1.953482 & 0.978305 & 0.875806 \\
\hline 7 & 1 & 0 & -1.953459 & 0.978326 & -0.875850 \\
\hline 8 & 6 & 0 & -0.007983 & 0.759417 & 0.000001 \\
\hline 9 & 1 & 0 & 0.537899 & 1.127834 & -0.876408 \\
\hline 10 & 1 & 0 & 0.537887 & 1.127832 & 0.876418 \\
\hline 11 & 6 & 0 & 0.007983 & -0.759417 & 0.000001 \\
\hline 12 & 1 & 0 & -0.537899 & -1.127834 & -0.876408 \\
\hline 13 & 1 & 0 & -0.537887 & -1.127832 & 0.876418 \\
\hline 14 & 6 & 0 & 1.409320 & -1.347262 & -0.000010 \\
\hline 15 & 1 & 0 & 1.953482 & -0.978305 & 0.875806 \\
\hline 16 & 1 & 0 & 1.953459 & -0.978326 & -0.875850 \\
\hline 17 & 6 & 0 & 1.409320 & -2.866395 & 0.000008 \\
\hline 18 & 1 & 0 & 0.895641 & -3.257820 & -0.880865 \\
\hline 19 & 1 & 0 & 2.422777 & -3.270801 & -0.000008 \\
\hline 20 & 1 & 0 & 0.895678 & -3.257799 & 0.880912 \\
\hline
\end{tabular}

Zero-point correction=

Thermal correction to Energy=

Thermal correction to Enthalpy=

Thermal correction to Gibbs Free Energy=

Sum of electronic and zero-point Energies=

Sum of electronic and thermal Energies=

Sum of electronic and thermal Enthalpies=

Sum of electronic and thermal Free Energies=

22dimethylbutane
0.193424 (Hartree/Particle)

0.201766

0.202710

0.161521

$-236.364709$

$-236.356367$

$-236.355422$

$-236.396612$

Standard orientation:

\begin{tabular}{|c|c|c|c|c|}
\hline \multirow{2}{*}{$\begin{array}{l}\text { Center } \\
\text { Number }\end{array}$} & Atomic & \multirow{2}{*}{$\begin{array}{l}\text { Atomic } \\
\text { Type }\end{array}$} & \multicolumn{2}{|c|}{ Coordinates (Angstro } \\
\hline & Numbe & & $X$ & $\mathrm{Z}$ \\
\hline 1 & 6 & 0 & 417 & -0. \\
\hline 2 & 1 & 1 & 1.609250 & 5827 \\
\hline 3 & 1 & 1 & 1.611680 & 0.880917 \\
\hline 4 & 1 & 6 & 0.42 & -0.001008 \\
\hline 5 & 6 & 0.4308 & -0.000088 & -0.000014 \\
\hline 6 & 6 & -0.861182 & 0.827426 & -0.000598 \\
\hline 7 & 1 & -0.8414 & 1.486025 & 0.87504 \\
\hline 8 & 1 & -0.841590 & 1.484677 & -0.87726 \\
\hline
\end{tabular}




$\begin{array}{cccccc}9 & 6 & 0 & -2.161036 & 0.039147 & 0.000014 \\ 10 & 1 & 0 & -2.249776 & -0.596759 & 0.882259 \\ 11 & 1 & 0 & -3.018231 & 0.714620 & 0.000123 \\ 12 & 1 & 0 & -2.250281 & -0.597147 & -0.881893 \\ 13 & 6 & 0 & 0.509907 & -0.878512 & -1.244816 \\ 14 & 1 & 0 & -0.282128 & -1.629259 & -1.261641 \\ 15 & 1 & 0 & 0.426265 & -0.278144 & -2.154621 \\ 16 & 1 & 0 & 1.464856 & -1.408344 & -1.280512 \\ 17 & 6 & 0 & 0.510751 & -0.875409 & 1.246929 \\ 18 & 1 & 0 & 1.465495 & -1.405587 & 1.283006 \\ 19 & 1 & 0 & 0.428299 & -0.272691 & 2.155284 \\ 20 & 1 & 0 & -0.281584 & -1.625774 & 1.266486\end{array}$

Zero-point correction=

Thermal correction to Energy=

Thermal correction to Enthalpy=

Thermal correction to Gibbs Free Energy=

Sum of electronic and zero-point Energies=

Sum of electronic and thermal Energies $=$

Sum of electronic and thermal Enthalpies=

Sum of electronic and thermal Free Energies=

Heptane
0.192276 (Hartree/Particle)

0.200422

0.201366
0.161002

$-236.369987$

$-236.361841$

$-236.360896$

$-236.401261$

Standard orientation:

\begin{tabular}{|c|c|c|c|c|}
\hline \multirow{2}{*}{$\begin{array}{l}\text { Center } \\
\text { Number }\end{array}$} & Atomic & \multirow{2}{*}{$\begin{array}{r}\text { Atomic } \\
\text { Type }\end{array}$} & \multicolumn{2}{|c|}{ Coordinates (Angstroms } \\
\hline & & & $X$ & $\mathrm{Z}$ \\
\hline 1 & 6 & 3.798696 & -0.356414 & 0.000009 \\
\hline 2 & 1 & 3.835481 & -1.001197 & 0.880923 \\
\hline 3 & 1 & 3.835477 & -1.001249 & -0.880867 \\
\hline 4 & 1 & 4.701064 & 0.257069 & -0.000011 \\
\hline 5 & 6 & 2.539722 & 0.493736 & -0.000014 \\
\hline 6 & 1 & 2.538401 & 1.151198 & 0.875815 \\
\hline 7 & 1 & 2.538404 & 1.151157 & -0.875872 \\
\hline 8 & 6 & 1.268467 & -0.338719 & 0.000001 \\
\hline 9 & 1 & 1.268299 & -0.997540 & -0.876332 \\
\hline 10 & 1 & 1.268305 & -0.997522 & 0.876349 \\
\hline 11 & 6 & 0.000000 & 0.497058 & 0.000000 \\
\hline 12 & 1 & 0.000002 & 1.155299 & -0.876527 \\
\hline 13 & 1 & -0.000002 & 1.155294 & 0.876530 \\
\hline 14 & 6 & -1.268467 & -0.338719 & -0.000006 \\
\hline 15 & 1 & -1.268297 & -0.997545 & 0.876324 \\
\hline 16 & 1 & -1.268307 & -0.997517 & -0.876356 \\
\hline 17 & 6 & -2.539722 & 0.493736 & 0.000014 \\
\hline 18 & 1 & -2.538403 & 1.151198 & -0.875814 \\
\hline 19 & 1 & -2.538402 & 1.151157 & 0.875873 \\
\hline 20 & 6 & -3.798696 & -0.356414 & - -0.000005 \\
\hline 21 & 1 & -3.835477 & -1.001246 & 0.880874 \\
\hline 22 & 1 & -4.701064 & 0.257069 & 0.000013 \\
\hline 23 & 1 & -3.835481 & -1.001200 & -0.880916 \\
\hline
\end{tabular}




$\begin{array}{lc}\text { Zero-point correction= } & 0.222536 \text { (Hartree/Particle) } \\ \text { Thermal correction to Energy= } & 0.232204 \\ \text { Thermal correction to Enthalpy= } & 0.233149 \\ \text { Thermal correction to Gibbs Free Energy= } & 0.187733 \\ \text { Sum of electronic and zero-point Energies }= & -275.563964 \\ \text { Sum of electronic and thermal Energies }= & -275.554296 \\ \text { Sum of electronic and thermal Enthalpies }= & -275.553352 \\ \text { Sum of electronic and thermal Free Energies }= & -275.598767 \\ \text { 223trimethylbune } & \end{array}$

223trimethylbutane

\begin{tabular}{|c|c|c|c|c|}
\hline \multirow{2}{*}{$\begin{array}{l}\text { Center } \\
\text { Number }\end{array}$} & Atomic & \multirow{2}{*}{$\begin{array}{l}\text { Atomic } \\
\text { Type }\end{array}$} & \multicolumn{2}{|c|}{ Coordinates (Angstroms } \\
\hline & Numbe & & $\mathrm{X}$ & $\mathrm{Z}$ \\
\hline 1 & 6 & 1.307678 & 1.307520 & -0.418626 \\
\hline 2 & 1 & 1.159721 & 1.502753 & -1.484517 \\
\hline 3 & 1 & 0.920729 & 2.161482 & 0.138874 \\
\hline 4 & 1 & 2.383350 & 1.256324 & -0.235051 \\
\hline 5 & 6 & 0.636626 & 0.000234 & -0.000941 \\
\hline 6 & 6 & -0.843441 & 0.001349 & -0.455618 \\
\hline 7 & 1 & -0.814278 & 0.007362 & -1.554683 \\
\hline 8 & 6 & -1.612197 & -1.242123 & -0.023142 \\
\hline 9 & 1 & -1.755406 & -1.258882 & 1.059638 \\
\hline 10 & 1 & -2.603435 & -1.249561 & -0.480633 \\
\hline 11 & 1 & -1.113619 & -2.167784 & -0.311683 \\
\hline 12 & 6 & -1.618210 & 1.239124 & -0.017166 \\
\hline 13 & 1 & -1.635821 & 1.333162 & 1.071395 \\
\hline 14 & 1 & -1.203284 & 2.158967 & -0.428727 \\
\hline 15 & 1 & -2.655432 & 1.170297 & -0.351253 \\
\hline 16 & 6 & 0.769631 & -0.161519 & 1.511105 \\
\hline 17 & 1 & 0.391527 & -1.128022 & 1.849197 \\
\hline 18 & 1 & 1.820756 & -0.100036 & 1.803300 \\
\hline 19 & 1 & 0.233992 & 0.621335 & 2.052254 \\
\hline 20 & 6 & 1.381339 & -1.144664 & -0.685864 \\
\hline 21 & 1 & 1.303884 & -1.066691 & -1.773635 \\
\hline 22 & 1 & 2.441636 & -1.118846 & -0.423813 \\
\hline 23 & 1 & 0.997123 & -2.121385 & -0.389148 \\
\hline
\end{tabular}

Zero-point correction=

Thermal correction to Energy=

Thermal correction to Enthalpy=

Thermal correction to Gibbs Free Energy=

Sum of electronic and zero-point Energies=

Sum of electronic and thermal Energies $=$

Sum of electronic and thermal Enthalpies=

Sum of electronic and thermal Free Energies=

Octane

$$
\begin{array}{cc}
0.221151 & \text { (Hartree/Particle) } \\
& 0.230495 \\
& 0.231439 \\
\text { rgy }= & 0.188319 \\
\text { gies }= & -275.568631 \\
\text { s }= & -275.559287 \\
\text { pies }= & -275.558343 \\
\text { Energies }= & -275.601464
\end{array}
$$

Standard orientation:

Center Atomic Atomic Coordinates (Angstroms)




\begin{tabular}{|c|c|c|c|c|c|}
\hline Number & \multicolumn{2}{|c|}{ Number } & Type & $\mathrm{X}$ & $\mathrm{Z}$ \\
\hline 1 & 6 & 0 & -4.447257 & 0.256759 & 0.000014 \\
\hline 2 & 1 & 0 & -4.510086 & 0.899535 & 0.880931 \\
\hline 3 & 1 & 0 & -4.510086 & 0.899597 & -0.880858 \\
\hline 4 & 1 & 0 & -5.324096 & -0.392693 & -0.000009 \\
\hline 5 & 6 & 0 & -3.154922 & -0.541748 & -0.000016 \\
\hline 6 & 1 & 0 & -3.126935 & -1.198700 & 0.875808 \\
\hline 7 & 1 & 0 & -3.126943 & -1.198651 & -0.875876 \\
\hline 8 & 6 & 0 & -1.918288 & 0.341328 & 0.000002 \\
\hline 9 & 1 & 0 & -1.944665 & 0.999634 & -0.876343 \\
\hline 10 & 1 & 0 & -1.944669 & 0.999609 & 0.876367 \\
\hline 11 & 6 & 0 & -0.617274 & -0.442668 & -0.000004 \\
\hline 12 & 1 & 0 & -0.590717 & -1.100607 & -0.876454 \\
\hline 13 & 1 & 0 & -0.590711 & -1.100609 & 0.876445 \\
\hline 14 & 6 & 0 & 0.617274 & 0.442668 & -0.000007 \\
\hline 15 & 1 & 0 & 0.590710 & 1.100618 & 0.876434 \\
\hline 16 & 1 & 0 & 0.590718 & 1.100598 & -0.876464 \\
\hline 17 & 6 & 0 & 1.918288 & -0.341328 & 0.000008 \\
\hline 18 & 1 & 0 & 1.944666 & -0.999640 & -0.876334 \\
\hline 19 & 1 & 0 & 1.944667 & -0.999603 & 0.876377 \\
\hline 20 & 6 & 0 & 3.154922 & 0.541748 & -0.000011 \\
\hline 21 & 1 & 0 & 3.126938 & 1.198696 & 0.875816 \\
\hline 22 & 1 & 0 & 3.126940 & 1.198655 & -0.875868 \\
\hline 23 & 6 & 0 & 4.447257 & -0.256759 & 0.000010 \\
\hline 24 & 1 & 0 & 4.510087 & -0.899587 & -0.880869 \\
\hline 25 & 1 & 0 & 5.324096 & 0.392693 & -0.000005 \\
\hline 26 & 1 & 0 & 4.510085 & -0.899545 & 0.880920 \\
\hline
\end{tabular}

2233tetramethylbutane

Standard orientation:

\begin{tabular}{|c|c|c|c|c|}
\hline \multirow{2}{*}{$\begin{array}{l}\text { Center } \\
\text { Number }\end{array}$} & Atomic & \multirow{2}{*}{$\begin{array}{l}\text { Atomic } \\
\text { Type }\end{array}$} & \multicolumn{2}{|c|}{ Coordinates (Angstroms) } \\
\hline & Number & & $\mathrm{X}$ & $\mathrm{Z}$ \\
\hline 1 & 6 & 1.335516 & -0.590974 & 1.298053 \\
\hline 2 & 1 & 1.136224 & 0.053689 & 2.155310 \\
\hline 3 & 1 & 0.919314 & -1.577739 & 1.511175 \\
\hline 4 & 1 & 2.419229 & -0.704548 & 1.218588 \\
\hline 5 & 6 & 0.783006 & 0.000034 & 0.000001 \\
\hline 6 & 6 & -0.782953 & -0.000068 & 0.000010 \\
\hline 7 & 6 & -1.335539 & 0.864559 & -1.134227 \\
\hline 8 & 1 & -1.136209 & 1.924738 & -0.971372 \\
\hline 9 & 1 & -0.919480 & 0.585944 & -2.104601 \\
\hline 10 & 1 & -2.419262 & 0.740854 & -1.196590 \\
\hline 11 & 6 & 1.335257 & 1.419749 & -0.137213 \\
\hline 12 & 1 & 1.135752 & 1.839762 & -1.124119 \\
\hline 13 & 1 & 0.918913 & 2.097582 & 0.610794 \\
\hline 14 & 1 & 2.418987 & 1.407954 & 0.000816 \\
\hline 15 & 6 & 1.335514 & -0.828520 & -1.160889 \\
\hline
\end{tabular}




$\begin{array}{rrrrrr}16 & 1 & 0 & 2.419245 & -0.702997 & -1.219389 \\ 17 & 1 & 0 & 1.136137 & -1.893240 & -1.031278 \\ 18 & 1 & 0 & 0.919441 & -0.519605 & -2.122030 \\ 19 & 6 & 0 & -1.335444 & 0.549948 & 1.315947 \\ 20 & 1 & 0 & -2.419211 & 0.665589 & 1.240225 \\ 21 & 1 & 0 & -1.135805 & -0.121040 & 2.152680 \\ 22 & 1 & 0 & -0.919550 & 1.529738 & 1.559635 \\ 23 & 6 & 0 & -1.335313 & -1.414749 & -0.181669 \\ 24 & 1 & 0 & -2.419058 & -1.407154 & -0.043474 \\ 25 & 1 & 0 & -1.135762 & -1.803646 & -1.181244 \\ 26 & 1 & 0 & -0.919172 & -2.115754 & 0.544799\end{array}$

Zero-point correction=

Thermal correction to Energy=

Thermal correction to Enthalpy=

Thermal correction to Gibbs Free Energy=

Sum of electronic and zero-point Energies=

Sum of electronic and thermal Energies=

Sum of electronic and thermal Enthalpies=

Sum of electronic and thermal Free Energies=
0.250090 (Hartree/Particle)

0.260307

0.261251

0.217019

$-314.767718$

$-314.757501$

$-314.756557$

$-314.800789$

cc-PVTZ Basis set:

M06L/CCPVTZ

Ethane

Standard orientation:

\begin{tabular}{|c|c|c|c|c|}
\hline \multirow{2}{*}{$\begin{array}{l}\text { Center } \\
\text { Number }\end{array}$} & Atomic & \multirow{2}{*}{$\begin{array}{r}\text { Atomic } \\
\text { Type }\end{array}$} & \multicolumn{2}{|c|}{ Coordinates (Angstrom } \\
\hline & Numbe & & $\mathrm{X}$ & $\mathrm{Z}$ \\
\hline 1 & 6 & -0.000000 & -0.000000 & 0.757121 \\
\hline 2 & 1 & -0.505922 & 0.876367 & 1.159714 \\
\hline 3 & 1 & 1.011917 & -0.000043 & 1.159714 \\
\hline 4 & 1 & -0.505995 & -0.876325 & 1.159714 \\
\hline 5 & 6 & 0.000000 & 0.000000 & -0.757121 \\
\hline 6 & 1 & 0.505922 & 0.876367 & -1.159714 \\
\hline 7 & 1 & 0.505995 & -0.876325 & -1.159714 \\
\hline 8 & 1 & -1.011917 & -0.000043 & -1.159714 \\
\hline
\end{tabular}

Zero-point correction=

Thermal correction to Energy=

0.074849 (Hartree/Particle)

Thermal correction to Enthalpy=

0.078327

0.079271

Thermal correction to Gibbs Free Energy $=\quad 0.053449$

Sum of electronic and zero-point Energies=

$-79.767219$

Sum of electronic and thermal Energies $=$

$-79.763740$

Sum of electronic and thermal Enthalpies $=$

$-79.762796$

Sum of electronic and thermal Free Energies=

$-79.788619$

Methane

Standard orientation: 


\begin{tabular}{|c|c|c|c|c|}
\hline \multirow{2}{*}{$\begin{array}{l}\text { Center } \\
\text { Number }\end{array}$} & \multirow{2}{*}{$\begin{array}{l}\text { Atomic } \\
\text { Number }\end{array}$} & \multirow{2}{*}{$\begin{array}{l}\text { Atomic } \\
\text { Type }\end{array}$} & \multicolumn{2}{|c|}{ Coordinates (Angstrom } \\
\hline & & & $\mathrm{X}$ & $\mathrm{Z}$ \\
\hline 1 & 6 & 0.000000 & 0.000000 & 0.000000 \\
\hline 2 & 1 & 0.626173 & 0.626173 & 0.626173 \\
\hline 3 & 1 & -0.626173 & -0.626173 & 0.626173 \\
\hline 4 & 1 & -0.626173 & 0.626173 & -0.626173 \\
\hline 5 & 1 & 0.626173 & -0.626173 & -0.626173 \\
\hline
\end{tabular}

Zero-point correction=

Thermal correction to Energy=

Thermal correction to Enthalpy=

Thermal correction to Gibbs Free Energy=

Sum of electronic and zero-point Energies=

Sum of electronic and thermal Energies=

Sum of electronic and thermal Enthalpies=

Sum of electronic and thermal Free Energies=

Propane
0.044895 (Hartree/Particle)

0.047764

0.048709

$$
\begin{gathered}
0.027593 \\
-40.479697 \\
-40.476827 \\
-40.475883 \\
-40.496998
\end{gathered}
$$

\begin{tabular}{|c|c|c|c|c|}
\hline \multirow{2}{*}{$\begin{array}{l}\text { Center } \\
\text { Number }\end{array}$} & Atomic & \multirow{2}{*}{$\begin{array}{l}\text { Atomic } \\
\text { Type }\end{array}$} & \multicolumn{2}{|c|}{ Coordinates (Angstroms) } \\
\hline & Number & & $\mathrm{X}$ & $\mathrm{Z}$ \\
\hline 1 & 6 & -1.262649 & -0.257185 & 0.000000 \\
\hline 2 & 1 & -2.163459 & 0.354352 & -0.000025 \\
\hline 3 & 1 & -1.306272 & -0.904077 & 0.876842 \\
\hline 4 & 1 & -1.306251 & -0.904117 & -0.876813 \\
\hline 5 & 6 & 0.000000 & 0.583899 & 0.000000 \\
\hline 6 & 1 & -0.000000 & 1.245265 & 0.869286 \\
\hline 7 & 1 & 0.000001 & 1.245251 & -0.869296 \\
\hline 8 & 6 & 1.262649 & -0.257185 & 0.000000 \\
\hline 9 & 1 & 1.306268 & -0.904081 & 0.876840 \\
\hline 10 & 1 & 1.306251 & -0.904113 & -0.876817 \\
\hline 11 & 1 & 2.163461 & 0.354349 & -0.000020 \\
\hline
\end{tabular}

Standard orientation:

Zero-point correction=

Thermal correction to Energy=

Thermal correction to Enthalpy=

Thermal correction to Gibbs Free Energy=

Sum of electronic and zero-point Energies=

Sum of electronic and thermal Energies $=$

Sum of electronic and thermal Enthalpies=

Sum of electronic and thermal Free Energies=

Butane
0.103623 (Hartree/Particle)

0.108144

0.109088

0.077991

$-119.058072$

\begin{tabular}{|c|c|c|c|}
\hline Cent & Atomic & Atomic & Coordinates (Angstrom \\
\hline Number & Number & Type & $\mathrm{X}$ \\
\hline
\end{tabular}

$-119.053552$

$-119.052608$

$-119.083704$

Standard orientation: 


$\begin{array}{cccccc}1 & 6 & 0 & 1.941465 & -0.117724 & 0.000018 \\ 2 & 1 & 0 & 2.087971 & -0.749115 & -0.876937 \\ 3 & 1 & 0 & 2.087938 & -0.749115 & 0.876979 \\ 4 & 1 & 0 & 2.733498 & 0.629215 & 0.000033 \\ 5 & 6 & 0 & 0.562183 & 0.511582 & -0.000020 \\ 6 & 1 & 0 & 0.452089 & 1.165690 & 0.869751 \\ 7 & 1 & 0 & 0.452113 & 1.165669 & -0.869812 \\ 8 & 6 & 0 & -0.562183 & -0.511582 & -0.000021 \\ 9 & 1 & 0 & -0.452114 & -1.165667 & -0.869813 \\ 10 & 1 & 0 & -0.452089 & -1.165692 & 0.869750 \\ 11 & 6 & 0 & -1.941465 & 0.117724 & 0.000018 \\ 12 & 1 & 0 & -2.087972 & 0.749114 & -0.876938 \\ 13 & 1 & 0 & -2.733498 & -0.629215 & 0.000035 \\ 14 & 1 & 0 & -2.087937 & 0.749117 & 0.876978\end{array}$

Zero-point correction=

Thermal correction to Energy=

Thermal correction to Enthalpy=

Thermal correction to Gibbs Free Energy=

Sum of electronic and zero-point Energies=

Sum of electronic and thermal Energies=

Sum of electronic and thermal Enthalpies=

Sum of electronic and thermal Free Energies=

Isobutane
0.132227 (Hartree/Particle)
0.138002
0.138946

0.104242

$-158.349260$

$-158.343485$

$-158.342540$

$-158.377244$

Standard orientation:

\begin{tabular}{|c|c|c|c|c|}
\hline \multirow{2}{*}{$\begin{array}{l}\text { Center } \\
\text { Number }\end{array}$} & Atomic & \multirow{2}{*}{$\begin{array}{l}\text { Atomic } \\
\text { Type }\end{array}$} & \multicolumn{2}{|c|}{ Coordinates (Angstrom } \\
\hline & Numbe & & $X$ & Z \\
\hline 1 & 6 & -0.266676 & -1.422010 & -0.094539 \\
\hline 2 & 1 & -1.230982 & -1.789008 & 0.256072 \\
\hline 3 & 1 & -0.275996 & -1.472212 & -1.185305 \\
\hline 4 & 1 & 0.499110 & -2.113420 & 0.256209 \\
\hline 5 & 6 & -0.000002 & 0.000001 & 0.375125 \\
\hline 6 & 1 & -0.000021 & 0.000046 & 1.470024 \\
\hline 7 & 6 & -1.098177 & 0.941946 & -0.094545 \\
\hline 8 & 1 & -0.933749 & 1.960587 & 0.255941 \\
\hline 9 & 1 & -1.137165 & 0.975010 & -1.185314 \\
\hline 10 & 1 & -2.079821 & 0.624558 & 0.256364 \\
\hline 11 & 6 & 1.364846 & 0.480052 & -0.094544 \\
\hline 12 & 1 & 1.580648 & 1.489030 & 0.256017 \\
\hline 13 & 1 & 2.164854 & -0.171400 & 0.256320 \\
\hline 14 & 1 & 1.413178 & 0.496874 & -1.185313 \\
\hline
\end{tabular}

Zero-point correction=

Thermal correction to Energy=

Thermal correction to Enthalpy=

Thermal correction to Gibbs Free Energy=

Sum of electronic and zero-point Energies=
0.131704 (Hartree/Particle)
0.137390
0.138334

0.104128

$-158.351092$ 
Sum of electronic and thermal Energies=

Sum of electronic and thermal Enthalpies=

Sum of electronic and thermal Free Energies=

Pentane

Standard orientation:

\begin{tabular}{|c|c|c|c|c|}
\hline \multirow{2}{*}{$\begin{array}{l}\text { Center } \\
\text { Number }\end{array}$} & Atomic & \multirow{2}{*}{$\begin{array}{l}\text { Atomic } \\
\text { Type }\end{array}$} & \multicolumn{2}{|c|}{ Coordinates (Angstroms } \\
\hline & Numbe & & $\mathrm{X}$ & $\mathrm{Z}$ \\
\hline 1 & 6 & 2.533432 & 0.320845 & 0.000000 \\
\hline 2 & 1 & 2.578122 & 0.967399 & -0.876834 \\
\hline 3 & 1 & 2.578121 & 0.967400 & 0.876834 \\
\hline 4 & 1 & 3.433609 & -0.291267 & 0.000001 \\
\hline 5 & 6 & 1.271533 & -0.519394 & -0.000000 \\
\hline 6 & 1 & 1.267235 & -1.182282 & -0.869884 \\
\hline 7 & 1 & 1.267235 & -1.182282 & 0.869884 \\
\hline 8 & 6 & 0.000000 & 0.311832 & -0.000000 \\
\hline 9 & 1 & 0.000000 & 0.976825 & 0.870280 \\
\hline 10 & 1 & -0.000000 & 0.976826 & -0.870280 \\
\hline 11 & 6 & -1.271533 & -0.519394 & - -0.000000 \\
\hline 12 & 1 & -1.267235 & -1.182282 & 0.869884 \\
\hline 13 & 1 & -1.267235 & -1.182282 & -0.869884 \\
\hline 14 & 6 & -2.533432 & 0.320845 & 0.000000 \\
\hline 15 & 1 & -3.433609 & -0.291267 & 0.000005 \\
\hline 16 & 1 & -2.578119 & 0.967403 & 0.876831 \\
\hline 17 & 1 & -2.578124 & 0.967395 & -0.876837 \\
\hline
\end{tabular}

Zero-point correction=

0.160692 (Hartree/Particle)

Thermal correction to Energy=

0.167795

Thermal correction to Enthalpy=

0.168739

Thermal correction to Gibbs Free Energy=

Sum of electronic and zero-point Energies=

0.130328

Sum of electronic and thermal Energies $=$

$-197.640485$

$-197.633381$

Sum of electronic and thermal Enthalpies=

$-197.632437$

Sum of electronic and thermal Free Energies=

$-197.670848$

Neopentane

Standard orientation:

\begin{tabular}{|c|c|c|c|c|}
\hline \multirow{2}{*}{$\begin{array}{l}\text { Center } \\
\text { Number }\end{array}$} & Atomic & \multirow{2}{*}{$\begin{array}{l}\text { Atomic } \\
\text { Type }\end{array}$} & \multicolumn{2}{|c|}{ Coordinates (Angstroms) } \\
\hline & Numbe & & $X$ & Z \\
\hline 1 & 6 & 0.277644 & 0.096454 & -1.497719 \\
\hline 2 & 1 & 0.136536 & -0.867464 & -1.988602 \\
\hline 3 & 1 & -0.389934 & 0.813093 & -1.978135 \\
\hline 4 & 1 & 1.302055 & 0.418543 & -1.689729 \\
\hline 5 & 6 & -0.000053 & -0.000005 & 0.000017 \\
\hline 6 & 6 & -1.440668 & -0.452589 & 0.222279 \\
\hline 7 & 1 & -1.670510 & -0.531063 & 1.285780 \\
\hline 8 & 1 & -2.148373 & 0.251224 & -0.217991 \\
\hline 9 & 1 & -1.622063 & -1.429392 & -0.228256 \\
\hline
\end{tabular}




$\begin{array}{rrrrrr}10 & 6 & 0 & 0.953990 & -1.011171 & 0.630170 \\ 11 & 1 & 0 & 1.994217 & -0.715581 & 0.486691 \\ 12 & 1 & 0 & 0.780989 & -1.102060 & 1.703408 \\ 13 & 1 & 0 & 0.827728 & -2.001226 & 0.189853 \\ 14 & 6 & 0 & 0.209045 & 1.367315 & 0.645265 \\ 15 & 1 & 0 & 1.231528 & 1.719542 & 0.502112 \\ 16 & 1 & 0 & -0.460961 & 2.113511 & 0.215947 \\ 17 & 1 & 0 & 0.019047 & 1.330840 & 1.718855\end{array}$

Zero-point correction=

Thermal correction to Energy=

Thermal correction to Enthalpy=

Thermal correction to Gibbs Free Energy=

Sum of electronic and zero-point Energies=

Sum of electronic and thermal Energies=

Sum of electronic and thermal Enthalpies=

Sum of electronic and thermal Free Energies=

Hexane

\subsection{1 (Hartree/Particle)}

$$
0.166263
$$

0.167207

\subsection{7}

$-197.645270$

$-197.638379$

$-197.637435$

$-197.674314$

Standard orientation:

\begin{tabular}{|c|c|c|c|c|}
\hline \multirow{2}{*}{$\begin{array}{l}\text { Center } \\
\text { Number }\end{array}$} & Atomic & \multirow{2}{*}{$\begin{array}{l}\text { Atomic } \\
\text { Type }\end{array}$} & \multicolumn{2}{|c|}{ Coordinates (Angstrom } \\
\hline & Numbe & & $X$ & Z \\
\hline 1 & 6 & -3.191308 & 0.205642 & -0.000007 \\
\hline 2 & 1 & -3.280085 & 0.847718 & 0.876919 \\
\hline 3 & 1 & -3.280035 & 0.847787 & -0.876889 \\
\hline 4 & 1 & -4.047884 & -0.466398 & -0.000063 \\
\hline 5 & 6 & -1.874665 & -0.546132 & -0.000005 \\
\hline 6 & 1 & -1.825111 & -1.207315 & 0.869857 \\
\hline 7 & 1 & -1.825094 & -1.207292 & -0.869883 \\
\hline 8 & 6 & -0.662735 & 0.370292 & 0.000020 \\
\hline 9 & 1 & -0.709291 & 1.033664 & -0.870312 \\
\hline 10 & 1 & -0.709298 & 1.033625 & 0.870381 \\
\hline 11 & 6 & 0.662735 & -0.370292 & 0.000009 \\
\hline 12 & 1 & 0.709283 & -1.033650 & -0.870334 \\
\hline 13 & 1 & 0.709305 & -1.033639 & 0.870359 \\
\hline 14 & 6 & 1.874665 & 0.546132 & -0.000012 \\
\hline 15 & 1 & 1.825106 & 1.207321 & 0.869845 \\
\hline 16 & 1 & 1.825099 & 1.207286 & -0.869894 \\
\hline 17 & 6 & 3.191308 & -0.205642 & -0.000003 \\
\hline 18 & 1 & 3.280053 & -0.847774 & -0.876892 \\
\hline 19 & 1 & 4.047884 & 0.466398 & -0.000026 \\
\hline 20 & 1 & 3.280067 & -0.847732 & 0.876916 \\
\hline
\end{tabular}

Zero-point correction=

Thermal correction to Energy=

Thermal correction to Enthalpy=

Thermal correction to Gibbs Free Energy=

Sum of electronic and zero-point Energies=

Sum of electronic and thermal Energies=
0.189223 (Hartree/Particle)

$$
0.197650
$$

0.198594

\subsection{3}

$-236.931655$

$-236.923228$ 
Sum of electronic and thermal Enthalpies=

Sum of electronic and thermal Free Energies=

22dimethylbutane

Standard orientation:

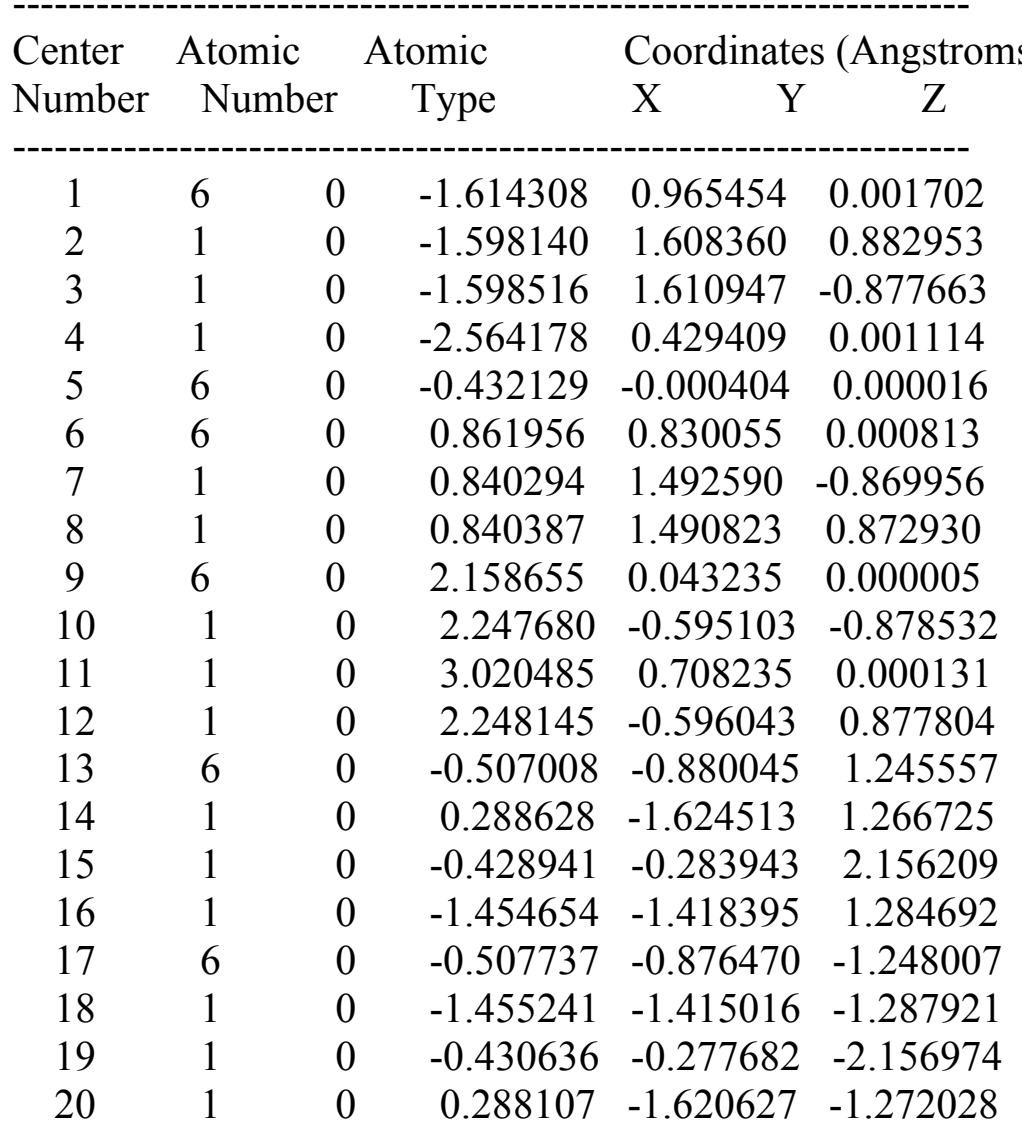

Zero-point correction=

Thermal correction to Energy=

Thermal correction to Enthalpy=

Thermal correction to Gibbs Free Energy=

Sum of electronic and zero-point Energies=

Sum of electronic and thermal Energies=

Sum of electronic and thermal Enthalpies=

Sum of electronic and thermal Free Energies=

Heptane
0.187883 (Hartree/Particle)

$$
\begin{gathered}
0.196196 \\
0.197140
\end{gathered}
$$

\subsection{7}

$-236.935531$

$-236.927218$

$-236.926274$

$-236.966997$

\begin{tabular}{|c|c|c|c|c|}
\hline \multirow{2}{*}{$\begin{array}{l}\text { Center } \\
\text { Number }\end{array}$} & Atomic & \multirow{2}{*}{$\begin{array}{l}\text { Atomic } \\
\text { Type }\end{array}$} & \multicolumn{2}{|c|}{ Coordinates (Angstroms } \\
\hline & Number & & $\mathrm{X}$ & $\mathrm{Z}$ \\
\hline 1 & 6 & -3.804539 & -0.349262 & -0.000004 \\
\hline 2 & 1 & -3.849266 & -0.995830 & -0.876844 \\
\hline 3 & 1 & -3.849273 & -0.995825 & 0.876839 \\
\hline 4 & 1 & -4.704727 & 0.262852 & -0.000009 \\
\hline 5 & 6 & -2.542639 & 0.490963 & 0.000001 \\
\hline 6 & 1 & -2.538350 & 1.153834 & -0.869874 \\
\hline 7 & 1 & -2.538356 & 1.153835 & 0.869874 \\
\hline
\end{tabular}

Standard orientation: 


$\begin{array}{cccccc}8 & 6 & 0 & -1.271009 & -0.340458 & 0.000004 \\ 9 & 1 & 0 & -1.271827 & -1.005241 & 0.870338 \\ 10 & 1 & 0 & -1.271825 & -1.005244 & -0.870328 \\ 11 & 6 & 0 & 0.000000 & 0.489889 & 0.000003 \\ 12 & 1 & 0 & -0.000000 & 1.154495 & 0.870431 \\ 13 & 1 & 0 & 0.000000 & 1.154495 & -0.870425 \\ 14 & 6 & 0 & 1.271009 & -0.340458 & 0.000002 \\ 15 & 1 & 0 & 1.271825 & -1.005245 & -0.870329 \\ 16 & 1 & 0 & 1.271828 & -1.005240 & 0.870337 \\ 17 & 6 & 0 & 2.542639 & 0.490963 & -0.000003 \\ 18 & 1 & 0 & 2.538354 & 1.153838 & 0.869868 \\ 19 & 1 & 0 & 2.538352 & 1.153831 & -0.869879 \\ 20 & 6 & 0 & 3.804539 & -0.349262 & -0.000002 \\ 21 & 1 & 0 & 3.849268 & -0.995831 & -0.876840 \\ 22 & 1 & 0 & 4.704727 & 0.262852 & -0.000005 \\ 23 & 1 & 0 & 3.849271 & -0.995824 & 0.876842\end{array}$

Zero-point correction=

Thermal correction to Energy=

Thermal correction to Enthalpy=

Thermal correction to Gibbs Free Energy=

Sum of electronic and zero-point Energies=

Sum of electronic and thermal Energies $=$

Sum of electronic and thermal Enthalpies=

Sum of electronic and thermal Free Energies=

223trimethylbutane

Standard orientation:

\begin{tabular}{|c|c|c|c|c|}
\hline \multirow{2}{*}{$\begin{array}{l}\text { Center } \\
\text { Number }\end{array}$} & Atomic & \multirow{2}{*}{$\begin{array}{l}\text { Atomic } \\
\text { Type }\end{array}$} & \multicolumn{2}{|c|}{ Coordinates (Angstroms } \\
\hline & Number & & $\mathrm{X}$ & Z \\
\hline 1 & 6 & -1.312945 & -1.303384 & -0.427995 \\
\hline 2 & 1 & -1.170818 & -1.492957 & -1.493629 \\
\hline 3 & 1 & -0.927618 & -2.163982 & 0.117122 \\
\hline 4 & 1 & -2.386603 & -1.258166 & -0.242788 \\
\hline 5 & 6 & -0.638548 & 0.000195 & -0.000557 \\
\hline 6 & 6 & 0.846142 & -0.001371 & -0.457920 \\
\hline 7 & 1 & 0.814731 & -0.006258 & -1.554611 \\
\hline 8 & 6 & 1.612069 & 1.242382 & -0.025128 \\
\hline 9 & 1 & 1.748621 & 1.267075 & 1.056951 \\
\hline 10 & 1 & 2.60 & 1.252967 & -0.469350 \\
\hline 11 & 1 & 1.118180 & 2.167291 & -0.316873 \\
\hline 12 & 6 & 1.615736 & -1.241385 & -0.020447 \\
\hline 13 & 1 & 1.631618 & -1.339692 & 1.066367 \\
\hline 14 & 1 & 1.200270 & -2.159862 & -0.429373 \\
\hline 15 & 1 & 2.653318 & -1.182137 & -0.347596 \\
\hline 16 & 6 & -0.764290 & 0.149543 & 1.514198 \\
\hline 17 & 1 & -0.378843 & 1.107711 & 1.862034 \\
\hline 18 & 1 & -1.810592 & 0.093394 & 1.816493 \\
\hline 19 & 1 & -0.231862 & -0.638387 & 2.047571 \\
\hline
\end{tabular}




$\begin{array}{llllll}20 & 6 & 0 & -1.379687 & 1.154354 & -0.675405 \\ 21 & 1 & 0 & -1.300810 & 1.092327 & -1.762081 \\ 22 & 1 & 0 & -2.439937 & 1.131606 & -0.421251 \\ 23 & 1 & 0 & -0.997262 & 2.127070 & -0.369473\end{array}$

$\begin{array}{lc}\text { Zero-point correction }= & 0.216396 \text { (Hartree/Particle) } \\ \text { Thermal correction to Energy= } & 0.225862 \\ \text { Thermal correction to Enthalpy= } & 0.226807 \\ \text { Thermal correction to Gibbs Free Energy= } & 0.183370 \\ \text { Sum of electronic and zero-point Energies }= & -276.225697 \\ \text { Sum of electronic and thermal Energies }= & -276.216230 \\ \text { Sum of electronic and thermal Enthalpies }= & -276.215286 \\ \text { Sum of electronic and thermal Free Energies }= & -276.258722\end{array}$

Octane

Standard orientation:

\begin{tabular}{|c|c|c|c|c|}
\hline \multirow{2}{*}{$\begin{array}{l}\text { Center } \\
\text { Number }\end{array}$} & Atomic & \multirow{2}{*}{$\begin{array}{l}\text { Atomic } \\
\text { Type }\end{array}$} & \multicolumn{2}{|c|}{ Coordinates (Angstroms } \\
\hline & Numbe & & $\mathrm{X}$ & Z \\
\hline 1 & 6 & -4.453085 & 0.253336 & 0.000007 \\
\hline 2 & 1 & -4.522601 & 0.897731 & 0.876836 \\
\hline 3 & 1 & -4.522610 & 0.897728 & -0.876823 \\
\hline 4 & 1 & -5.329119 & -0.392881 & 0.000012 \\
\hline 5 & 6 & -3.159872 & -0.537841 & -0.000001 \\
\hline 6 & 1 & -3.130130 & -1.200067 & 0.869866 \\
\hline 7 & 1 & -3.130138 & -1.200066 & -0.869869 \\
\hline 8 & 6 & -1.921061 & 0.341712 & -0.000005 \\
\hline 9 & 1 & -1.947399 & 1.005978 & -0.870344 \\
\hline 10 & 1 & -1.947396 & 1.005981 & 0.870330 \\
\hline 11 & 6 & -0.619171 & -0.439315 & -0.000004 \\
\hline 12 & 1 & -0.593666 & -1.103427 & -0.870402 \\
\hline 13 & 1 & -0.593665 & -1.103428 & 0.870393 \\
\hline 14 & 6 & 0.619171 & 0.439315 & -0.000003 \\
\hline 15 & 1 & 0.593664 & 1.103429 & 0.870393 \\
\hline 16 & 1 & 0.593667 & 1.103425 & -0.870402 \\
\hline 17 & 6 & 1.921061 & -0.341712 & 0.000001 \\
\hline 18 & 1 & 1.947399 & -1.005982 & -0.870334 \\
\hline 19 & 1 & 1.947396 & -1.005977 & 0.870340 \\
\hline 20 & 6 & 3.159872 & 0.537841 & 0.000001 \\
\hline 21 & 1 & 3.130133 & 1.200068 & 0.869867 \\
\hline 22 & 1 & 3.130135 & 1.200065 & -0.869868 \\
\hline 23 & 6 & 4.453085 & -0.253336 & 0.000003 \\
\hline 24 & 1 & 4.522607 & -0.897731 & -0.876826 \\
\hline 25 & 1 & 5.329119 & 0.392881 & 0.000004 \\
\hline 26 & 1 & 4.522604 & -0.897729 & 0.876833 \\
\hline
\end{tabular}

Zero-point correction=

Thermal correction to Energy=

Thermal correction to Enthalpy=
0.246134 (Hartree/Particle)

0.257300

0.258245 
Thermal correction to Gibbs Free Energy=

Sum of electronic and zero-point Energies=

Sum of electronic and thermal Energies $=$

Sum of electronic and thermal Enthalpies=

Sum of electronic and thermal Free Energies=

2233tetramethylbutane

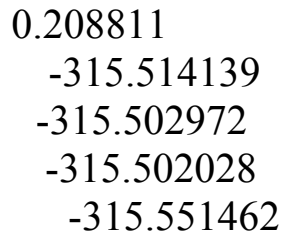

Standard orientation:

\begin{tabular}{|c|c|c|c|c|}
\hline \multirow{2}{*}{$\begin{array}{l}\text { Center } \\
\text { Number }\end{array}$} & Atomic & \multirow{2}{*}{$\begin{array}{l}\text { Atomic } \\
\text { Type }\end{array}$} & \multicolumn{2}{|c|}{ Coordinates (Angstroms } \\
\hline & Numbe & & $X$ & Z \\
\hline 1 & 6 & -1.335698 & 1.423606 & -0.115403 \\
\hline 2 & 1 & -0.919969 & 2.089820 & 0.640906 \\
\hline 3 & 1 & -1.134891 & 1.861726 & -1.092241 \\
\hline 4 & 1 & -2.417851 & 1.418615 & 0.019108 \\
\hline 5 & 6 & -0.786555 & -0.000017 & -0.000064 \\
\hline 6 & 6 & 0.786427 & -0.000038 & -0.000095 \\
\hline 7 & 6 & 1.335919 & -1.413911 & -0.201802 \\
\hline 8 & 1 & 0.920367 & -2.125040 & 0.512548 \\
\hline 9 & 1 & 1.135478 & -1.791965 & -1.203508 \\
\hline 10 & 1 & 2.418048 & -1.416731 & -0.067026 \\
\hline 11 & 6 & -1.335692 & -0.611747 & 1.290556 \\
\hline 12 & 1 & -0.919957 & -1.599759 & 1.489648 \\
\hline 13 & 1 & -1.134978 & 0.015347 & 2.158292 \\
\hline 14 & 1 & -2.417851 & -0.725830 & 1.218982 \\
\hline 15 & 6 & -1.335944 & -0.811878 & -1.175095 \\
\hline 16 & 1 & -2.418068 & -0.692597 & -1.238088 \\
\hline 17 & 1 & -0.920118 & -0.490508 & -2.130329 \\
\hline 18 & 1 & -1.135429 & -1.876936 & -1.065721 \\
\hline 19 & 6 & 1.335679 & 0.532118 & 1.325316 \\
\hline 20 & 1 & 2.417889 & 0.649812 & 1.260837 \\
\hline 21 & 1 & 0.920380 & 1.506477 & 1.583845 \\
\hline 22 & 1 & 1.134534 & -0.146310 & 2.153458 \\
\hline 23 & 6 & 1.335763 & 0.881831 & -1.123556 \\
\hline 24 & 1 & 2.417924 & 0.766877 & -1.193540 \\
\hline 25 & 1 & 0.920158 & 0.618998 & -2.096602 \\
\hline 26 & 1 & 1.134943 & 1.938221 & -0.949708 \\
\hline
\end{tabular}

Zero-point correction=

Thermal correction to Energy=

Thermal correction to Enthalpy=

Thermal correction to Gibbs Free Energy=

Sum of electronic and zero-point Energies=

Sum of electronic and thermal Energies $=$

Sum of electronic and thermal Enthalpies=

Sum of electronic and thermal Free Energies=

TPSSh/ccpVTZ

$$
\begin{array}{cc}
0.244532 & \text { (Hartree/Particle) } \\
& 0.254940 \\
& 0.255884 \\
\text { rgy }= & 0.211280 \\
\text { gies }= & -315.516344 \\
\text { s }= & -315.505937 \\
\text { pies }= & -315.504992 \\
\text { Energies }= & -315.549597
\end{array}
$$

Standard orientation:

Center Atomic Atomic Coordinates (Angstroms) 


\begin{tabular}{lllrrr} 
Number & Number & \multicolumn{1}{c}{ Type } & X & $Y$ & $Z$ \\
\hline 1 & 6 & 0 & 0.000000 & -0.000000 & 0.764091 \\
2 & 1 & 0 & -0.509123 & 0.881953 & 1.160283 \\
3 & 1 & 0 & 1.018355 & -0.000063 & 1.160283 \\
4 & 1 & 0 & -0.509233 & -0.881890 & 1.160283 \\
5 & 6 & 0 & 0.000000 & -0.000000 & -0.764091 \\
6 & 1 & 0 & 0.509123 & 0.881953 & -1.160283 \\
7 & 1 & 0 & 0.509233 & -0.881890 & -1.160283 \\
8 & 1 & 0 & -1.018355 & -0.000063 & -1.160283
\end{tabular}

Zero-point correction $=$

Thermal correction to Energy=

Thermal correction to Enthalpy=

Thermal correction to Gibbs Free Energy=

Sum of electronic and zero-point Energies=

Sum of electronic and thermal Energies=

Sum of electronic and thermal Enthalpies=

Sum of electronic and thermal Free Energies=
0.074420 (Hartree/Particle)

0.077910

0.078855

0.052995

$-79.791426$

$-79.787935$

$-79.786991$

$-79.812850$

Methane

Standard orientation:

\begin{tabular}{|c|c|c|c|c|}
\hline \multirow{2}{*}{$\begin{array}{l}\text { Center } \\
\text { Number }\end{array}$} & Atomic & \multirow{2}{*}{$\begin{array}{r}\text { Atomic } \\
\text { Type }\end{array}$} & \multicolumn{2}{|c|}{ Coordinates (Angstrom } \\
\hline & Numbe & & $\mathrm{X}$ & $\mathrm{Z}$ \\
\hline 1 & 6 & 0.000000 & 0.000000 & 0.000000 \\
\hline 2 & 1 & 0.629048 & 0.629048 & 0.629048 \\
\hline 3 & 1 & -0.629048 & -0.629048 & 0.629048 \\
\hline 4 & 1 & -0.629048 & 0.629048 & -0.629048 \\
\hline 5 & 1 & 0.629048 & -0.629048 & -0.629048 \\
\hline
\end{tabular}

Zero-point correction $=$

Thermal correction to Energy=

0.044721 (Hartree/Particle)

Thermal correction to Enthalpy=

0.047588

Thermal correction to Gibbs Free Energy=

0.048532

Sum of electronic and zero-point Energies $=$

0.027407

Sum of electronic and thermal Energies $=$

$-40.492811$

$-40.489944$

Sum of electronic and thermal Enthalpies=

$-40.489000$

Sum of electronic and thermal Free Energies $=\quad-40.510125$

Propane

Standard orientation:

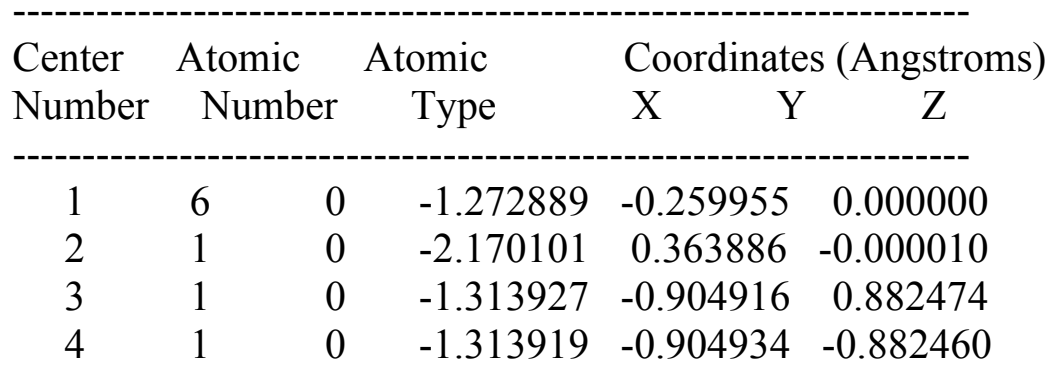




$\begin{array}{cccccc}5 & 6 & 0 & 0.000000 & 0.587136 & 0.000000 \\ 6 & 1 & 0 & -0.000000 & 1.244289 & 0.875712 \\ 7 & 1 & 0 & 0.000001 & 1.244277 & -0.875720 \\ 8 & 6 & 0 & 1.272889 & -0.259954 & 0.000000 \\ 9 & 1 & 0 & 1.313944 & -0.904888 & 0.882493 \\ 10 & 1 & 0 & 1.313898 & -0.904961 & -0.882442 \\ 11 & 1 & 0 & 2.170103 & 0.363883 & -0.000050\end{array}$

Zero-point correction=

Thermal correction to Energy=

Thermal correction to Enthalpy=

Thermal correction to Gibbs Free Energy=

Sum of electronic and zero-point Energies=

Sum of electronic and thermal Energies $=$

Sum of electronic and thermal Enthalpies=

Sum of electronic and thermal Free Energies=

Butane
0.102957 (Hartree/Particle)

0.107520

0.108464

0.077272

$-119.092904$

$-119.088341$

$-119.087397$

$-119.118589$

Standard orientation:

\begin{tabular}{|c|c|c|c|c|}
\hline \multirow{2}{*}{$\begin{array}{l}\text { Center } \\
\text { Number }\end{array}$} & Atomic & \multirow{2}{*}{$\begin{array}{r}\text { Atomic } \\
\text { Type }\end{array}$} & \multicolumn{2}{|c|}{ Coordinates (Angstrom } \\
\hline & Numbe & & $\mathrm{X}$ & Z \\
\hline 1 & 6 & 1.956149 & -0.120683 & 0.000029 \\
\hline 2 & 1 & 2.099632 & -0.750617 & -0.882487 \\
\hline 3 & 1 & 2.099582 & -0.750579 & 0.882579 \\
\hline 4 & 1 & 2.742932 & 0.637628 & 0.000034 \\
\hline 5 & 6 & 0.565766 & 0.515019 & -0.000032 \\
\hline 6 & 1 & 0.459782 & 1.164769 & 0.876156 \\
\hline 7 & 1 & 0.459825 & 1.164724 & -0.876260 \\
\hline 8 & 6 & -0.565766 & -0.515019 & -0.000033 \\
\hline 9 & 1 & -0.459825 & -1.164722 & -0.876262 \\
\hline 10 & 1 & -0.459782 & -1.164771 & 0.876155 \\
\hline 11 & 6 & -1.956149 & 0.120683 & 0.000029 \\
\hline 12 & 1 & -2.099628 & 0.750627 & -0.882480 \\
\hline 13 & 1 & -2.742932 & -0.637628 & 0.000022 \\
\hline 14 & 1 & -2.099586 & 0.750569 & 0.882585 \\
\hline
\end{tabular}

Zero-point correction=

Thermal correction to Energy=

Thermal correction to Enthalpy=

Thermal correction to Gibbs Free Energy=

Sum of electronic and zero-point Energies=

Sum of electronic and thermal Energies $=$

Sum of electronic and thermal Enthalpies=

Sum of electronic and thermal Free Energies=

Isobutane
0.131284 (Hartree/Particle)

0.137130

0.138074

0.103201

$-158.394513$

$-158.388667$

$-158.387723$

$-158.422597$

Standard orientation:

\begin{tabular}{lcccc} 
Center & Atomic & Atomic & & \multicolumn{2}{c}{ Coordinates (Angstroms) } \\
Number & Number & Type & X & Y C Z
\end{tabular}




$\begin{array}{cccccc}1 & 6 & 0 & -1.165564 & 0.874711 & -0.096078 \\ 2 & 1 & 0 & -1.061288 & 1.902071 & 0.262850 \\ 3 & 1 & 0 & -1.206718 & 0.905643 & -1.189587 \\ 4 & 1 & 0 & -2.122855 & 0.487456 & 0.262803 \\ 5 & 6 & 0 & 0.000027 & 0.000009 & 0.374956 \\ 6 & 1 & 0 & 0.000057 & 0.000003 & 1.471623 \\ 7 & 6 & 0 & 1.340309 & 0.572036 & -0.096096 \\ 8 & 1 & 0 & 2.177824 & -0.032145 & 0.262660 \\ 9 & 1 & 0 & 1.387569 & 0.592403 & -1.189599 \\ 10 & 1 & 0 & 1.483751 & 1.594609 & 0.262988 \\ 11 & 6 & 0 & -0.174759 & -1.446744 & -0.096083 \\ 12 & 1 & 0 & 0.639389 & -2.082147 & 0.262523 \\ 13 & 1 & 0 & -1.116492 & -1.870148 & 0.263131 \\ 14 & 1 & 0 & -0.181315 & -1.497813 & -1.189585\end{array}$

Zero-point correction=

Thermal correction to Energy=

Thermal correction to Enthalpy=

Thermal correction to Gibbs Free Energy=

Sum of electronic and zero-point Energies=

Sum of electronic and thermal Energies $=$

Sum of electronic and thermal Enthalpies=

Sum of electronic and thermal Free Energies=

Pentane
0.130799 (Hartree/Particle)

0.136595

0.137539

0.103094

$-158.395760$

$-158.389963$

$-158.389019$

$-158.423464$

Standard orientation:

\begin{tabular}{|c|c|c|c|c|}
\hline \multirow{2}{*}{$\begin{array}{l}\text { Center } \\
\text { Number }\end{array}$} & Atomic & \multirow{2}{*}{$\begin{array}{l}\text { Atomic } \\
\text { Type }\end{array}$} & \multicolumn{2}{|c|}{ Coordinates (Angstrom } \\
\hline & Numbe & & $X$ & Z \\
\hline 1 & 6 & 2.551822 & 0.324353 & -0.000000 \\
\hline 2 & 1 & 2.593983 & 0.968956 & -0.882370 \\
\hline 3 & 1 & 2.593981 & 0.968966 & 0.882362 \\
\hline 4 & 1 & 3.448328 & -0.300136 & 0.000004 \\
\hline 5 & 6 & 1.279612 & -0.523520 & 0.000003 \\
\hline 6 & 1 & 1.278588 & -1.181528 & -0.876139 \\
\hline 7 & 1 & 1.278587 & -1.181520 & 0.876151 \\
\hline 8 & 6 & 0.000000 & 0.315215 & -0.000002 \\
\hline 9 & 1 & -0.000001 & 0.974622 & 0.876572 \\
\hline 10 & 1 & 0.000001 & 0.974617 & -0.876580 \\
\hline 11 & 6 & -1.279612 & -0.523520 & -0.000003 \\
\hline 12 & 1 & -1.278586 & -1.181526 & 0.876141 \\
\hline 13 & 1 & -1.278589 & -1.181522 & -0.876149 \\
\hline 14 & 6 & -2.551822 & 0.324353 & 0.000002 \\
\hline 15 & 1 & -3.448328 & -0.300136 & 0.000003 \\
\hline 16 & 1 & -2.593980 & 0.968960 & 0.882369 \\
\hline 17 & 1 & -2.593984 & 0.968961 & -0.882364 \\
\hline
\end{tabular}

Zero-point correction=

Thermal correction to Energy=
0.159626 (Hartree/Particle)

0.166808 
Thermal correction to Enthalpy=

Thermal correction to Gibbs Free Energy=

Sum of electronic and zero-point Energies=

Sum of electronic and thermal Energies $=$

Sum of electronic and thermal Enthalpies=

Sum of electronic and thermal Free Energies=

Neopentane

Standard orientation:
0.167752

0.129167

$-197.696032$

$-197.688850$

$-197.687906$

$-197.726491$

\begin{tabular}{|c|c|c|c|c|}
\hline \multirow{3}{*}{\begin{tabular}{c} 
Center \\
Number \\
\hdashline 1
\end{tabular}} & \multirow{2}{*}{$\begin{array}{l}\text { Atomic } \\
\text { Number }\end{array}$} & \multirow{2}{*}{$\begin{array}{l}\text { Atomic } \\
\text { Type }\end{array}$} & \multicolumn{2}{|c|}{ Coordinates (Angstrom } \\
\hline & & & $\mathrm{X}$ & Z \\
\hline & 6 & -0.185606 & 1.420360 & -0.555510 \\
\hline 2 & 1 & -1.045272 & 1.466285 & -1.230326 \\
\hline 3 & 1 & -0.352429 & 2.137271 & 0.253636 \\
\hline 4 & 1 & 0.699358 & 1.740126 & -1.113271 \\
\hline 5 & 6 & 0.000022 & 0.000016 & 0.000015 \\
\hline 6 & 6 & -1.259453 & -0.415080 & 0.775868 \\
\hline 7 & 1 & -1.152452 & -1.424980 & 1.182281 \\
\hline 8 & 1 & -1.446363 & 0.266996 & 1.610342 \\
\hline 9 & 1 & -2.139544 & -0.403638 & 0.126388 \\
\hline 10 & 6 & 0.231303 & -0.978187 & -1.161936 \\
\hline 11 & 1 & 1.123959 & -0.703752 & -1.731463 \\
\hline 12 & 1 & 0.366893 & -1.998611 & -0.791996 \\
\hline 13 & 1 & -0.620682 & -0.977812 & -1.847955 \\
\hline 14 & 6 & 1.213757 & -0.027099 & 0.941574 \\
\hline 15 & 1 & 2.125086 & 0.265432 & 0.412040 \\
\hline 16 & 1 & 1.073488 & 0.662270 & 1.779182 \\
\hline 17 & 1 & 1.367817 & -1.029636 & 1.351070 \\
\hline
\end{tabular}

Zero-point correction=

Thermal correction to Energy=

Thermal correction to Enthalpy=

Thermal correction to Gibbs Free Energy=

Sum of electronic and zero-point Energies=

Sum of electronic and thermal Energies $=$

Sum of electronic and thermal Enthalpies=

Sum of electronic and thermal Free Energies=

Hexane
0.158244 (Hartree/Particle)

0.165313

0.166257
0.129010

$-197.698659$

$-197.691590$

$-197.690646$

$-197.727893$

Standard orientation:

\begin{tabular}{|c|c|c|c|c|}
\hline \multirow{2}{*}{$\begin{array}{l}\text { Center } \\
\text { Number }\end{array}$} & Atomic & \multirow{2}{*}{$\begin{array}{l}\text { Atomic } \\
\text { Type }\end{array}$} & \multicolumn{2}{|c|}{ Coordinates (Angstroms) } \\
\hline & Number & & $\mathrm{X}$ & $\mathrm{Z}$ \\
\hline 1 & 6 & -3.213695 & 0.208743 & 0.000032 \\
\hline 2 & 1 & -3.299673 & 0.848992 & 0.882586 \\
\hline 3 & 1 & -3.299683 & 0.849140 & -0.882413 \\
\hline 4 & 1 & -4.065773 & -0.475483 & -0.000023 \\
\hline 5 & 6 & -1.886329 & -0.550044 & -0.000040 \\
\hline 6 & 1 & -1.840427 & -1.206570 & 0.876115 \\
\hline
\end{tabular}




$\begin{array}{cccrrr}7 & 1 & 0 & -1.840456 & -1.206463 & -0.876276 \\ 8 & 6 & 0 & -0.667029 & 0.374529 & -0.000006 \\ 9 & 1 & 0 & -0.713221 & 1.032205 & -0.876666 \\ 10 & 1 & 0 & -0.713251 & 1.032187 & 0.876665 \\ 11 & 6 & 0 & 0.667029 & -0.374529 & 0.000013 \\ 12 & 1 & 0 & 0.713232 & -1.032230 & -0.876627 \\ 13 & 1 & 0 & 0.713240 & -1.032162 & 0.876703 \\ 14 & 6 & 0 & 1.886329 & 0.550044 & -0.000029 \\ 15 & 1 & 0 & 1.840436 & 1.206568 & 0.876127 \\ 16 & 1 & 0 & 1.840447 & 1.206465 & -0.876264 \\ 17 & 6 & 0 & 3.213695 & -0.208743 & 0.000023 \\ 18 & 1 & 0 & 3.299680 & -0.849124 & -0.882434 \\ 19 & 1 & 0 & 4.065773 & 0.475483 & -0.000019 \\ 20 & 1 & 0 & 3.299675 & -0.849009 & 0.882565\end{array}$

Zero-point correction=

Thermal correction to Energy=

Thermal correction to Enthalpy=

Thermal correction to Gibbs Free Energy=

Sum of electronic and zero-point Energies=

Sum of electronic and thermal Energies=

Sum of electronic and thermal Enthalpies=

Sum of electronic and thermal Free Energies=

22dimethylbutane

Standard orientation:

\begin{tabular}{|c|c|c|c|c|}
\hline \multirow{2}{*}{$\begin{array}{l}\text { Center } \\
\text { Number }\end{array}$} & Atomic & \multirow{2}{*}{$\begin{array}{r}\text { Atomic } \\
\text { Type }\end{array}$} & \multicolumn{2}{|c|}{ Coordinates (Angstroms } \\
\hline & Numbe & & $\mathrm{X}$ & Z \\
\hline 1 & 6 & -1.614273 & 0.983026 & 0.002358 \\
\hline 2 & 1 & -1.587645 & 1.624145 & 0.888204 \\
\hline 3 & 1 & -1.587831 & 1.628130 & -0.880598 \\
\hline 4 & 1 & -2.568892 & 0.449354 & 0.001249 \\
\hline 5 & 6 & -0.434210 & -0.000938 & 0.000016 \\
\hline 6 & 6 & 0.872678 & 0.825684 & 0.001488 \\
\hline 7 & 1 & 0.855652 & 1.485772 & -0.873456 \\
\hline 8 & 1 & 0.855729 & 1.482579 & 0.878836 \\
\hline 9 & 6 & 2.182242 & 0.034565 & 0.000047 \\
\hline 10 & 1 & 2.272531 & -0.600680 & -0.883898 \\
\hline 11 & 1 & 3.035955 & 0.716424 & 0.000816 \\
\hline 12 & 1 & 2.272898 & -0.603177 & 0.882147 \\
\hline 13 & 6 & -0.522794 & -0.885197 & 1.252914 \\
\hline 14 & 1 & 0.272362 & -1.633927 & 1.276482 \\
\hline 15 & 1 & -0.448361 & -0.283070 & 2.163223 \\
\hline 16 & 1 & -1.478139 & -1.416705 & 1.279907 \\
\hline 17 & 6 & -0.523326 & -0.879749 & -1.256669 \\
\hline 18 & 1 & -1.478568 & -1.411353 & -1.285452 \\
\hline 19 & 1 & -0.449559 & -0.273620 & -2.164373 \\
\hline 20 & 1 & 0.271965 & -1.628213 & -1.284012 \\
\hline
\end{tabular}


Zero-point correction=

Thermal correction to Energy=

Thermal correction to Enthalpy=

Thermal correction to Gibbs Free Energy=

Sum of electronic and zero-point Energies=

Sum of electronic and thermal Energies=

Sum of electronic and thermal Enthalpies=

Sum of electronic and thermal Free Energies= Heptane
0.186660 (Hartree/Particle)

0.195110

0.196054

0.155186
-236.997272
-236.988823
-236.987879
-237.028747

Standard orientation:

\begin{tabular}{|c|c|c|c|c|}
\hline \multirow{2}{*}{$\begin{array}{l}\text { Center } \\
\text { Number }\end{array}$} & Atomic & \multirow{2}{*}{$\begin{array}{r}\text { Atomic } \\
\text { Type }\end{array}$} & \multicolumn{2}{|c|}{ Coordinates (Angstroms } \\
\hline & Numbe & & $X$ & Z \\
\hline 1 & 6 & -3.831053 & -0.353057 & -0.000002 \\
\hline 2 & 1 & -3.873207 & -0.997669 & -0.882381 \\
\hline 3 & 1 & -3.873200 & -0.997700 & 0.882354 \\
\hline 4 & 1 & -4.727612 & 0.271382 & 0.000013 \\
\hline 5 & 6 & -2.558840 & 0.494834 & 0.000007 \\
\hline 6 & 1 & -2.557821 & 1.152846 & -0.876108 \\
\hline 7 & 1 & -2.557819 & 1.152825 & 0.876137 \\
\hline 8 & 6 & -1.279174 & -0.344201 & -0.000004 \\
\hline 9 & 1 & -1.280092 & -1.003385 & 0.876559 \\
\hline 10 & 1 & -1.280096 & -1.003371 & -0.876577 \\
\hline 11 & 6 & 0.000000 & 0.495162 & -0.000001 \\
\hline 12 & 1 & -0.000002 & 1.154127 & 0.876614 \\
\hline 13 & 1 & 0.000002 & 1.154125 & -0.876617 \\
\hline 14 & 6 & 1.279174 & -0.344201 & 0.000003 \\
\hline 15 & 1 & 1.280093 & -1.003385 & -0.876559 \\
\hline 16 & 1 & 1.280095 & -1.003371 & 0.876576 \\
\hline 17 & 6 & 2.558840 & 0.494834 & -0.000006 \\
\hline 18 & 1 & 2.557820 & 1.152844 & 0.876110 \\
\hline 19 & 1 & 2.557820 & 1.152826 & -0.876135 \\
\hline 20 & 6 & 3.831053 & -0.353057 & 0.000003 \\
\hline 21 & 1 & 3.873203 & -0.997694 & -0.882357 \\
\hline 22 & 1 & 4.727612 & 0.271382 & -0.000005 \\
\hline 23 & 1 & 3.873204 & -0.997675 & 0.882377 \\
\hline
\end{tabular}

Zero-point correction $=$

Thermal correction to Energy=

Thermal correction to Enthalpy=

Thermal correction to Gibbs Free Energy=

Sum of electronic and zero-point Energies=

Sum of electronic and thermal Energies $=$

Sum of electronic and thermal Enthalpies=

Sum of electronic and thermal Free Energies=

223trimethylbutane

Standard orientation:

Center Atomic Atomic Coordinates (Angstroms)




\begin{tabular}{cccccc} 
Number & Number & Type & $X$ & $Y$ & $Z$ \\
\hline 1 & 6 & 0 & -1.353625 & -1.241121 & -0.565775 \\
2 & 1 & 0 & -1.231246 & -1.302510 & -1.651382 \\
3 & 1 & 0 & -0.973824 & -2.166585 & -0.128744 \\
4 & 1 & 0 & -2.424908 & -1.192004 & -0.352225 \\
5 & 6 & 0 & -0.642015 & -0.000007 & 0.000367 \\
6 & 6 & 0 & 0.852739 & -0.000002 & -0.451599 \\
7 & 1 & 0 & 0.825108 & 0.000003 & -1.549280 \\
8 & 6 & 0 & 1.631616 & 1.249108 & -0.016839 \\
9 & 1 & 0 & 1.712257 & 1.310464 & 1.071814 \\
10 & 1 & 0 & 2.648518 & 1.210424 & -0.415688 \\
11 & 1 & 0 & 1.173744 & 2.172244 & -0.375000 \\
12 & 6 & 0 & 1.631645 & -1.249093 & -0.016827 \\
13 & 1 & 0 & 1.712539 & -1.310277 & 1.071813 \\
14 & 1 & 0 & 1.173643 & -2.172266 & -0.374732 \\
15 & 1 & 0 & 2.648460 & -1.210550 & -0.415915 \\
16 & 6 & 0 & -0.787767 & -0.000251 & 1.530206 \\
17 & 1 & 0 & -0.331213 & 0.884362 & 1.980231 \\
18 & 1 & 0 & -1.846315 & 0.000005 & 1.804831 \\
19 & 1 & 0 & -0.331730 & -0.885298 & 1.979894 \\
20 & 6 & 0 & -1.353507 & 1.241358 & -0.565346 \\
21 & 1 & 0 & -1.230988 & 1.303212 & -1.650911 \\
22 & 1 & 0 & -2.424820 & 1.192190 & -0.351954 \\
23 & 1 & 0 & -0.973735 & 2.166630 & -0.127873 \\
& & & & & \\
& 1 & & & &
\end{tabular}

Zero-point correction=

Thermal correction to Energy=

Thermal correction to Enthalpy=

Thermal correction to Gibbs Free Energy=

Sum of electronic and zero-point Energies=

Sum of electronic and thermal Energies $=$

Sum of electronic and thermal Enthalpies=

Sum of electronic and thermal Free Energies=

Octane
0.214604 (Hartree/Particle)

0.224432

0.225376

0.180887

$-276.295467$

$-276.285639$

$-276.284695$

$-276.329185$

Standard orientation:

\begin{tabular}{|c|c|c|c|c|}
\hline \multirow{2}{*}{$\begin{array}{l}\text { Center } \\
\text { Number }\end{array}$} & Atomic & \multirow{2}{*}{$\begin{array}{r}\text { Atomic } \\
\text { Type }\end{array}$} & \multicolumn{2}{|c|}{ Coordinates (Angstrom } \\
\hline & Numbe & & $\mathrm{X}$ & Z \\
\hline 1 & 6 & -4.484434 & 0.256723 & 0.000032 \\
\hline 2 & 1 & -4.550974 & 0.899276 & 0.882606 \\
\hline 3 & 1 & -4.550953 & 0.899501 & -0.882380 \\
\hline 4 & 1 & -5.356923 & -0.401308 & -0.000066 \\
\hline 5 & 6 & -3.180654 & -0.541894 & -0.000052 \\
\hline 6 & 1 & -3.154667 & -1.199534 & 0.876085 \\
\hline 7 & 1 & -3.154682 & -1.199374 & -0.876309 \\
\hline 8 & 6 & -1.933871 & 0.345349 & 0.000014 \\
\hline 9 & 1 & -1.960108 & 1.004148 & -0.876595 \\
\hline 10 & 1 & -1.960146 & 1.004077 & 0.876676 \\
\hline
\end{tabular}




$\begin{array}{rrrrrr}11 & 6 & 0 & -0.623092 & -0.444048 & 0.000012 \\ 12 & 1 & 0 & -0.597743 & -1.102639 & -0.876627 \\ 13 & 1 & 0 & -0.597724 & -1.102598 & 0.876680 \\ 14 & 6 & 0 & 0.623092 & 0.444048 & -0.000023 \\ 15 & 1 & 0 & 0.597731 & 1.102662 & 0.876598 \\ 16 & 1 & 0 & 0.597737 & 1.102576 & -0.876709 \\ 17 & 6 & 0 & 1.933871 & -0.345349 & 0.000020 \\ 18 & 1 & 0 & 1.960128 & -1.004159 & -0.876581 \\ 19 & 1 & 0 & 1.960126 & -1.004066 & 0.876690 \\ 20 & 6 & 0 & 3.180654 & 0.541893 & -0.000026 \\ 21 & 1 & 0 & 3.154674 & 1.199500 & 0.876137 \\ 22 & 1 & 0 & 3.154676 & 1.199408 & -0.876258 \\ 23 & 6 & 0 & 4.484434 & -0.256723 & 0.000018 \\ 24 & 1 & 0 & 4.550965 & -0.899435 & -0.882442 \\ 25 & 1 & 0 & 5.356923 & 0.401308 & -0.000016 \\ 26 & 1 & 0 & 4.550963 & -0.899342 & 0.882545\end{array}$

Zero-point correction=

Thermal correction to Energy=

0.244436 (Hartree/Particle)

0.255703

Thermal correction to Enthalpy=

0.256647

Thermal correction to Gibbs Free Energy=

Sum of electronic and zero-point Energies=

0.207111

Sum of electronic and thermal Energies $=$

$-315.600818$

$-315.589551$

Sum of electronic and thermal Enthalpies=

$-315.588607$

Sum of electronic and thermal Free Energies $=\quad-315.638143$

2233tetramethylbutane

Standard orientation:

\begin{tabular}{|c|c|c|c|c|}
\hline \multirow{2}{*}{$\begin{array}{l}\text { Center } \\
\text { Number }\end{array}$} & Atomic & \multirow{2}{*}{$\begin{array}{l}\text { Atomic } \\
\text { Type }\end{array}$} & \multicolumn{2}{|c|}{ Coordinates (Angstroms) } \\
\hline & Number & & $\mathrm{X}$ & $\mathrm{Z}$ \\
\hline 1 & 6 & -1.348555 & -0.081179 & -1.432960 \\
\hline 2 & 1 & -1.132660 & 0.823359 & -2.004875 \\
\hline 3 & 1 & -0.946932 & -0.935715 & -1.982624 \\
\hline 4 & 1 & -2.435414 & -0.195717 & -1.396342 \\
\hline 5 & 6 & -0.790251 & 0.000047 & -0.000083 \\
\hline 6 & 6 & 0.790232 & 0.000001 & -0.000046 \\
\hline 7 & 6 & 1.348203 & 0.368851 & 1.387056 \\
\hline 8 & 1 & 1.132522 & 1.406596 & 1.648680 \\
\hline 9 & 1 & 0.946070 & -0.271849 & 2.175408 \\
\hline 10 & 1 & 2.435017 & 0.248280 & 1.388299 \\
\hline 11 & 6 & -1.348525 & 1.281582 & 0.646066 \\
\hline 12 & 1 & -1.132803 & 1.324569 & 1.715419 \\
\hline 13 & 1 & -0.946776 & 2.184820 & 0.180917 \\
\hline 14 & 1 & -2.435359 & 1.307251 & 0.528447 \\
\hline 15 & 6 & -1.348384 & -1.200365 & 0.786700 \\
\hline 16 & 1 & -2.435110 & -1.110964 & 0.868658 \\
\hline 17 & 1 & -1.133331 & -2.147771 & 0.288663 \\
\hline 18 & 1 & -0.945751 & -1.249681 & 1.801156 \\
\hline 19 & 6 & 1.348831 & 1.016718 & -1.012851 \\
\hline
\end{tabular}




$\begin{array}{llllll}20 & 1 & 0 & 2.435660 & 1.077502 & -0.908870 \\ 21 & 1 & 0 & 1.133301 & 0.724834 & -2.042533 \\ 22 & 1 & 0 & 0.947300 & 2.019993 & -0.851975 \\ 23 & 6 & 0 & 1.348416 & -1.385653 & -0.373960 \\ 24 & 1 & 0 & 2.435260 & -1.326396 & -0.478629 \\ 25 & 1 & 0 & 1.132543 & -2.131162 & 0.393825 \\ 26 & 1 & 0 & 0.946664 & -1.747968 & -1.323156\end{array}$

Zero-point correction=

Thermal correction to Energy=

0.242575 (Hartree/Particle)

Thermal correction to Enthalpy=

0.253421

0.254365

Thermal correction to Gibbs Free Energy=

0.208757

Sum of electronic and zero-point Energies=

$-315.592539$

Sum of electronic and thermal Energies=

$-315.581693$

Sum of electronic and thermal Enthalpies=

$-315.580749$

Sum of electronic and thermal Free Energies=

$-315.626358$

B3LYP/ccpVTZ

Ethane

Standard orientation:

\begin{tabular}{|c|c|c|c|c|}
\hline \multirow{2}{*}{$\begin{array}{l}\text { Center } \\
\text { Number }\end{array}$} & Atomic & \multirow{2}{*}{$\begin{array}{l}\text { Atomic } \\
\text { Type }\end{array}$} & \multicolumn{2}{|c|}{ Coordinates (Angstroms } \\
\hline & Number & & $X$ & $\mathrm{Z}$ \\
\hline 1 & 6 & 0.000000 & -0.000000 & 0.763660 \\
\hline 2 & 1 & -0.508109 & 0.880134 & 1.161067 \\
\hline 3 & 1 & 1.016272 & -0.000032 & 1.161067 \\
\hline 4 & 1 & -0.508164 & -0.880102 & 1.161067 \\
\hline 5 & 6 & -0.000000 & -0.000000 & -0.763660 \\
\hline 6 & 1 & 0.508109 & 0.880134 & -1.161067 \\
\hline 7 & 1 & 0.508164 & -0.880102 & -1.161067 \\
\hline 8 & 1 & -1.016272 & -0.000032 & -1.161067 \\
\hline
\end{tabular}

Zero-point correction=

Thermal correction to Energy=

0.074372 (Hartree/Particle)

Thermal correction to Enthalpy=

0.077858

0.078803

Thermal correction to Gibbs Free Energy $=0.052951$

Sum of electronic and zero-point Energies $=\quad-79.789968$

Sum of electronic and thermal Energies $=\quad-79.786481$

Sum of electronic and thermal Enthalpies $=\quad-79.785537$

Sum of electronic and thermal Free Energies $=\quad-79.811389$

Mcc

Standard orientation:

\begin{tabular}{lrrrrr} 
Center & \multicolumn{2}{c}{ Atomic } & Atomic & \multicolumn{3}{c}{ Coordinates (Angstroms) } \\
Number & Number & Type & X & Y & Z \\
\hline 1 & 6 & 0 & 0.000000 & 0.000000 & 0.000000 \\
2 & 1 & 0 & 0.628170 & 0.628170 & 0.628170 \\
3 & 1 & 0 & -0.628170 & -0.628170 & 0.628170
\end{tabular}




$\begin{array}{rrrrrr}4 & 1 & 0 & -0.628170 & 0.628170 & -0.628170 \\ 5 & 1 & 0 & 0.628170 & -0.628170 & -0.628170\end{array}$

Zero-point correction $=$

Thermal correction to Energy=

0.044596 (Hartree/Particle)

Thermal correction to Enthalpy=

0.047465

0.048409

Thermal correction to Gibbs Free Energy $=0.027286$

Sum of electronic and zero-point Energies $=\quad-40.493641$

Sum of electronic and thermal Energies $=\quad-40.490773$

Sum of electronic and thermal Enthalpies $=\quad-40.489828$

Sum of electronic and thermal Free Energies $=\quad-40.510951$

Propane

Standard orientation:

\begin{tabular}{|c|c|c|c|c|c|}
\hline \multirow{2}{*}{$\begin{array}{l}\text { Center } \\
\text { Number }\end{array}$} & \multicolumn{2}{|l|}{ Atomic } & & \multicolumn{2}{|c|}{ Coordinates (Angstrom } \\
\hline & Numbe & & & $X$ & Z \\
\hline 1 & 6 & 0 & -1.274202 & -0.258973 & 0.000000 \\
\hline 2 & 1 & 0 & -2.169088 & 0.365510 & -0.000012 \\
\hline 3 & 1 & 0 & -1.319092 & -0.903468 & 0.880799 \\
\hline 4 & 1 & 0 & -1.319082 & -0.903489 & -0.880784 \\
\hline 5 & 6 & 0 & -0.000000 & 0.584380 & 0.000000 \\
\hline 6 & 1 & 0 & -0.000001 & 1.242155 & 0.873465 \\
\hline 7 & 1 & 0 & 0.000001 & 1.242142 & -0.873473 \\
\hline 8 & 6 & 0 & 1.274203 & -0.258973 & 0.000000 \\
\hline 9 & 1 & 0 & 1.319111 & -0.903438 & 0.880820 \\
\hline 10 & 1 & 0 & 1.319059 & -0.903518 & -0.880763 \\
\hline 11 & 1 & 0 & 2.169091 & 0.365507 & -0.000055 \\
\hline
\end{tabular}

Zero-point correction=

Thermal correction to Energy=

0.102999 (Hartree/Particle)

Thermal correction to Enthalpy=

0.107530

Thermal correction to Gibbs Free Energy=

0.108474

Sum of electronic and zero-point Energies=

0.077344

$-119.089333$

Sum of electronic and thermal Energies $=$

$-119.084803$

Sum of electronic and thermal Enthalpies=

$-119.083858$

Sum of electronic and thermal Free Energies $=\quad-119.114989$

Butane

Standard orientation:

\begin{tabular}{cccccc} 
Center & \multicolumn{2}{c}{ Atomic } & \multicolumn{2}{c}{ Atomic } & \multicolumn{2}{c}{ Coordinates } \\
Number & Number & Type & X & Y & Z \\
- & Numstroms) \\
\hline 1 & 6 & 0 & 1.957453 & -0.120435 & 0.000036 \\
2 & 1 & 0 & 2.104251 & -0.749396 & -0.880789 \\
3 & 1 & 0 & 2.104188 & -0.749346 & 0.880906 \\
4 & 1 & 0 & 2.742482 & 0.637434 & 0.000041 \\
5 & 6 & 0 & 0.567046 & 0.512859 & -0.000040 \\
6 & 1 & 0 & 0.462080 & 1.163303 & 0.873992
\end{tabular}




$\begin{array}{cccccc}7 & 1 & 0 & 0.462135 & 1.163244 & -0.874124 \\ 8 & 6 & 0 & -0.567046 & -0.512859 & -0.000040 \\ 9 & 1 & 0 & -0.462135 & -1.163243 & -0.874125 \\ 10 & 1 & 0 & -0.462080 & -1.163305 & 0.873991 \\ 11 & 6 & 0 & -1.957453 & 0.120435 & 0.000036 \\ 12 & 1 & 0 & -2.104246 & 0.749408 & -0.880782 \\ 13 & 1 & 0 & -2.742482 & -0.637434 & 0.000027 \\ 14 & 1 & 0 & -2.104193 & 0.749335 & 0.880913\end{array}$

Zero-point correction=

Thermal correction to Energy=

Thermal correction to Enthalpy=

Thermal correction to Gibbs Free Energy=

Sum of electronic and zero-point Energies=

Sum of electronic and thermal Energies $=$

Sum of electronic and thermal Enthalpies=

Sum of electronic and thermal Free Energies=

Isobutane
0.131430 (Hartree/Particle)

0.137219

0.138163

\subsection{5}

$-158.388778$

$-158.382989$

$-158.382045$

$-158.416793$

Standard orientation:

\begin{tabular}{|c|c|c|c|c|}
\hline \multirow{2}{*}{$\begin{array}{l}\text { Center } \\
\text { Number }\end{array}$} & Atomic & \multirow{2}{*}{$\begin{array}{l}\text { Atomic } \\
\text { Type }\end{array}$} & \multicolumn{2}{|c|}{ Coordinates (Angstroms } \\
\hline & Number & & $\mathrm{X}$ & $\mathrm{Z}$ \\
\hline 1 & 6 & -0.879375 & -1.163145 & -0.095403 \\
\hline 2 & 1 & -1.904475 & -1.056176 & 0.264287 \\
\hline 3 & 1 & -0.914127 & -1.209216 & -1.187132 \\
\hline 4 & 1 & -0.496980 & -2.120227 & 0.264348 \\
\hline 5 & 6 & 0.000004 & 0.000004 & 0.371063 \\
\hline 6 & 1 & 0.000009 & 0.000034 & 1.466429 \\
\hline 7 & 6 & -0.567635 & 1.343129 & -0.095406 \\
\hline 8 & 1 & 0.037582 & 2.177404 & 0.264245 \\
\hline 9 & 1 & -0.590160 & 1.396238 & -1.187134 \\
\hline 10 & 1 & -1.587683 & 1.490520 & 0.264358 \\
\hline 11 & 6 & 1.447002 & -0.179983 & -0.095406 \\
\hline 12 & 1 & 2.084632 & 0.629811 & 0.264194 \\
\hline 13 & 1 & 1.866937 & -1.121151 & 0.264465 \\
\hline 14 & 1 & 1.504287 & -0.187264 & -1.187139 \\
\hline
\end{tabular}

Zero-point correction=

Thermal correction to Energy=

Thermal correction to Enthalpy=

Thermal correction to Gibbs Free Energy=

Sum of electronic and zero-point Energies=

Sum of electronic and thermal Energies $=$

Sum of electronic and thermal Enthalpies=

Sum of electronic and thermal Free Energies=

Pentane
0.130982 (Hartree/Particle)

$$
0.136695
$$

0.137640

0.103354

$-158.390026$

$-158.384313$

$-158.383369$

$-158.417654$

Standard orientation:

Center Atomic Atomic Coordinates (Angstroms) 


\begin{tabular}{cccccc} 
Number & Number & Type & $X$ & $Y$ & $Z$ \\
\hline 1 & 6 & 0 & 2.554188 & 0.323299 & -0.000005 \\
2 & 1 & 0 & 2.599990 & 0.967506 & -0.880824 \\
3 & 1 & 0 & 2.599988 & 0.967543 & 0.880787 \\
4 & 1 & 0 & 3.448849 & -0.301437 & 0.000009 \\
5 & 6 & 0 & 1.280915 & -0.521363 & 0.000011 \\
6 & 1 & 0 & 1.280885 & -1.179961 & -0.874043 \\
7 & 1 & 0 & 1.280887 & -1.179930 & 0.874087 \\
8 & 6 & 0 & 0.000000 & 0.313686 & 0.000001 \\
9 & 1 & 0 & -0.000007 & 0.973605 & 0.874593 \\
10 & 1 & 0 & 0.000008 & 0.973605 & -0.874590 \\
11 & 6 & 0 & -1.280915 & -0.521363 & -0.000011 \\
12 & 1 & 0 & -1.280885 & -1.179963 & 0.874042 \\
13 & 1 & 0 & -1.280886 & -1.179928 & -0.874088 \\
14 & 6 & 0 & -2.554188 & 0.323299 & 0.000005 \\
15 & 1 & 0 & -3.448849 & -0.301437 & -0.000079 \\
16 & 1 & 0 & -2.600021 & 0.967456 & 0.880858 \\
17 & 1 & 0 & -2.599956 & 0.967593 & -0.880754
\end{tabular}

Zero-point correction=

Thermal correction to Energy=

Thermal correction to Enthalpy=

Thermal correction to Gibbs Free Energy=

Sum of electronic and zero-point Energies=

Sum of electronic and thermal Energies $=$

Sum of electronic and thermal Enthalpies=

Sum of electronic and thermal Free Energies=

Neopentane

Standard orientation:

\begin{tabular}{cccccc} 
Center & Atomic & \multicolumn{2}{c}{ Atomic } & \multicolumn{3}{c}{ Coordinates } \\
Number & Number & Type & X & Y & Z \\
------- & \\
\hline 1 & 6 & 0 & -0.935314 & -1.139074 & 0.432927 \\
2 & 1 & 0 & -1.930564 & -0.760313 & 0.674945 \\
3 & 1 & 0 & -1.044842 & -1.881211 & -0.360673 \\
4 & 1 & 0 & -0.549126 & -1.650765 & 1.317005 \\
5 & 6 & 0 & 0.000019 & -0.000033 & 0.000014 \\
6 & 6 & 0 & -0.577870 & 0.692365 & -1.243525 \\
7 & 1 & 0 & 0.067428 & 1.508703 & -1.574801 \\
8 & 1 & 0 & -0.679568 & -0.010150 & -2.073396 \\
9 & 1 & 0 & -1.565461 & 1.110502 & -1.037644 \\
10 & 6 & 0 & 0.126987 & 1.020109 & 1.141448 \\
11 & 1 & 0 & 0.535770 & 0.555071 & 2.040995 \\
12 & 1 & 0 & 0.787967 & 1.843157 & 0.861705 \\
13 & 1 & 0 & -0.845263 & 1.445783 & 1.398538 \\
14 & 6 & 0 & 1.386203 & -0.573394 & -0.330851 \\
15 & 1 & 0 & 1.822838 & -1.072484 & 0.536799 \\
16 & 1 & 0 & 1.326776 & -1.303581 & -1.140693
\end{tabular}


Zero-point correction=

Thermal correction to Energy=

Thermal correction to Enthalpy=

Thermal correction to Gibbs Free Energy=

Sum of electronic and zero-point Energies=

Sum of electronic and thermal Energies $=$

Sum of electronic and thermal Enthalpies=

Sum of electronic and thermal Free Energies=

Hexane

Standard orientation:
0.158620 (Hartree/Particle)

0.165557

0.166501
0.129500

$-197.690473$

$-197.683536$

$-197.682592$

$-197.719593$

\begin{tabular}{|c|c|c|c|c|c|}
\hline \multirow{2}{*}{$\begin{array}{l}\text { Center } \\
\text { Number }\end{array}$} & \multicolumn{2}{|c|}{ Atomic } & \multirow{2}{*}{$\begin{array}{l}\text { Atomic } \\
\text { Type }\end{array}$} & \multicolumn{2}{|c|}{ Coordinates (Angstroms } \\
\hline & & & & $\mathrm{X}$ & $\mathrm{Z}$ \\
\hline 1 & 6 & 0 & -3.216076 & 0.207973 & 0.000042 \\
\hline 2 & 1 & 0 & -3.305838 & 0.847483 & 0.880917 \\
\hline 3 & 1 & 0 & -3.305847 & 0.847674 & -0.880695 \\
\hline 4 & 1 & 0 & -4.065968 & -0.476450 & -0.000031 \\
\hline 5 & 6 & 0 & -1.888070 & -0.547680 & -0.000049 \\
\hline 6 & 1 & 0 & -1.843067 & -1.204795 & 0.873925 \\
\hline 7 & 1 & 0 & -1.843105 & -1.204656 & -0.874130 \\
\hline 8 & 6 & 0 & -0.667209 & 0.373191 & -0.000009 \\
\hline 9 & 1 & 0 & -0.713359 & 1.031279 & -0.874594 \\
\hline 10 & 1 & 0 & -0.713393 & 1.031260 & 0.874589 \\
\hline 11 & 6 & 0 & 0.667209 & -0.373191 & 0.000015 \\
\hline 12 & 1 & 0 & 0.713370 & -1.031311 & -0.874546 \\
\hline 13 & 1 & 0 & 0.713382 & -1.031227 & 0.874637 \\
\hline 14 & 6 & 0 & 1.888071 & 0.547680 & -0.000037 \\
\hline 15 & 1 & 0 & 1.843077 & 1.204790 & 0.873941 \\
\hline 16 & 1 & 0 & 1.843094 & 1.204661 & -0.874114 \\
\hline 17 & 6 & 0 & 3.216076 & -0.207973 & 0.000031 \\
\hline 18 & 1 & 0 & 3.305847 & -0.847649 & -0.880723 \\
\hline 19 & 1 & 0 & 4.065968 & 0.476450 & -0.000020 \\
\hline 20 & 1 & 0 & 3.305838 & -0.847507 & 0.880889 \\
\hline
\end{tabular}

Zero-point correction=

Thermal correction to Energy=

Thermal correction to Enthalpy=

Thermal correction to Gibbs Free Energy=

Sum of electronic and zero-point Energies=

Sum of electronic and thermal Energies $=$

Sum of electronic and thermal Enthalpies=

Sum of electronic and thermal Free Energies=
0.188211 (Hartree/Particle)

0.196637

0.197581

\subsection{2}

$-236.987575$

$-236.979148$

$-236.978204$

$-237.020173$

22dimethylbutane

Standard orientation:

Center Atomic Atomic Coordinates (Angstroms)




\begin{tabular}{ccccccc} 
Number & Number & Type & $X$ & $Y$ & $Z$ \\
\hline 1 & 6 & 0 & -1.611993 & 0.988557 & 0.002335 \\
2 & 1 & 0 & -1.585644 & 1.629744 & 0.885967 \\
3 & 1 & 0 & -1.585793 & 1.633729 & -0.878400 \\
4 & 1 & 0 & -2.568238 & 0.461509 & 0.001221 \\
5 & 6 & 0 & -0.435392 & -0.000699 & 0.000011 \\
6 & 6 & 0 & 0.876900 & 0.820034 & 0.001348 \\
7 & 1 & 0 & 0.862336 & 1.480717 & -0.870980 \\
8 & 1 & 0 & 0.862412 & 1.477811 & 0.875871 \\
9 & 6 & 0 & 2.188524 & 0.033145 & 0.000051 \\
10 & 1 & 0 & 2.284656 & -0.601164 & -0.881819 \\
11 & 1 & 0 & 3.038692 & 0.716983 & 0.000641 \\
12 & 1 & 0 & 2.285068 & -0.603252 & 0.880363 \\
13 & 6 & 0 & -0.528614 & -0.885093 & 1.253361 \\
14 & 1 & 0 & 0.258403 & -1.639519 & 1.278194 \\
15 & 1 & 0 & -0.448948 & -0.286899 & 2.163812 \\
16 & 1 & 0 & -1.485646 & -1.409780 & 1.283862 \\
17 & 6 & 0 & -0.529225 & -0.879849 & -1.256968 \\
18 & 1 & 0 & -1.486080 & -1.404775 & -1.289007 \\
19 & 1 & 0 & -0.450457 & -0.277783 & -2.164941 \\
20 & 1 & 0 & 0.258035 & -1.633892 & -1.285607
\end{tabular}

Zero-point correction $=$

0.187161 (Hartree/Particle)

Thermal correction to Energy=

0.195435

Thermal correction to Enthalpy=

0.196379

Thermal correction to Gibbs Free Energy=

Sum of electronic and zero-point Energies=

0.155885

$-236.986455$

Sum of electronic and thermal Energies $=$

$-236.978181$

Sum of electronic and thermal Enthalpies=

$-236.977237$

Sum of electronic and thermal Free Energies $=\quad-237.017732$

Heptane

Standard orientation:

\begin{tabular}{|c|c|c|c|c|}
\hline \multirow{2}{*}{$\begin{array}{l}\text { Center } \\
\text { Number }\end{array}$} & Atomic & \multirow{2}{*}{$\begin{array}{r}\text { Atomic } \\
\text { Type }\end{array}$} & \multicolumn{2}{|c|}{ Coordinates (Angstroms } \\
\hline & Numbe & & $X$ & Z \\
\hline 1 & 6 & -3.834493 & -0.351947 & -0.000032 \\
\hline 2 & 1 & -3.880339 & -0.996089 & -0.880918 \\
\hline 3 & 1 & -3.880305 & -0.996331 & 0.880680 \\
\hline 4 & 1 & -4.729218 & 0.272740 & 0.000074 \\
\hline 5 & 6 & -2.561235 & 0.492682 & 0.000060 \\
\hline 6 & 1 & -2.561239 & 1.151351 & -0.873905 \\
\hline 7 & 1 & -2.561249 & 1.151174 & 0.874159 \\
\hline 8 & 6 & -1.280307 & -0.342645 & -0.000009 \\
\hline 9 & 1 & -1.281382 & -1.002362 & 0.874526 \\
\hline 10 & 1 & -1.281430 & -1.002305 & -0.874587 \\
\hline 11 & 6 & 0.000000 & 0.493453 & -0.000020 \\
\hline 12 & 1 & -0.000009 & 1.152946 & 0.874553 \\
\hline 13 & 1 & 0.000009 & 1.152885 & -0.874638 \\
\hline
\end{tabular}




$\begin{array}{rrrrrr}14 & 6 & 0 & 1.280307 & -0.342645 & 0.000023 \\ 15 & 1 & 0 & 1.281400 & -1.002391 & -0.874490 \\ 16 & 1 & 0 & 1.281412 & -1.002275 & 0.874623 \\ 17 & 6 & 0 & 2.561235 & 0.492682 & -0.000041 \\ 18 & 1 & 0 & 2.561246 & 1.151327 & 0.873942 \\ 19 & 1 & 0 & 2.561243 & 1.151199 & -0.874121 \\ 20 & 6 & 0 & 3.834493 & -0.351947 & 0.000018 \\ 21 & 1 & 0 & 3.880320 & -0.996274 & -0.880734 \\ 22 & 1 & 0 & 4.729218 & 0.272740 & -0.000029 \\ 23 & 1 & 0 & 3.880323 & -0.996145 & 0.880865\end{array}$

Zero-point correction=

Thermal correction to Energy=

Thermal correction to Enthalpy=

Thermal correction to Gibbs Free Energy=

Sum of electronic and zero-point Energies=

Sum of electronic and thermal Energies=

Sum of electronic and thermal Enthalpies=

Sum of electronic and thermal Free Energies=

223trimethyibutane
0.216553 (Hartree/Particle)

0.226332

0.227276
0.181685

$-276.287022$

$-276.277242$

$-276.276298$

$-276.321889$

Standard orientation:

\begin{tabular}{|c|c|c|c|c|}
\hline \multirow{2}{*}{$\begin{array}{l}\text { Center } \\
\text { Number }\end{array}$} & Atomic & \multirow{2}{*}{$\begin{array}{l}\text { Atomic } \\
\text { Type }\end{array}$} & \multicolumn{2}{|c|}{ Coordinates (Angstroms } \\
\hline & Numbe & & $\mathrm{X}$ & $\mathrm{Z}$ \\
\hline 1 & 6 & -1.355461 & -1.241724 & -0.568371 \\
\hline 2 & 1 & -1.230463 & -1.306632 & -1.651709 \\
\hline 3 & 1 & -0.981998 & -2.167227 & -0.131219 \\
\hline 4 & 1 & -2.426133 & -1.193620 & -0.361346 \\
\hline 5 & 6 & -0.644457 & 0.000056 & 0.000220 \\
\hline 6 & 6 & 0.855962 & 0.000013 & -0.445826 \\
\hline 7 & 1 & 0.831096 & 0.000051 & -1.541709 \\
\hline 8 & 6 & 1.637677 & 1.249487 & -0.015756 \\
\hline 9 & 1 & 1.725076 & 1.315429 & 1.070354 \\
\hline 10 & 1 & 2.651482 & 1.210766 & -0.417721 \\
\hline 11 & 1 & 1.183208 & 2.172378 & -0.372653 \\
\hline 12 & 6 & 1.637551 & -1.249533 & -0.015870 \\
\hline 13 & 1 & 1.724801 & -1.315689 & 1.070257 \\
\hline 14 & 1 & 1.183120 & -2.172365 & -0.372963 \\
\hline 15 & 1 & 2.651414 & -1.210715 & -0.417677 \\
\hline 16 & 6 & -0.797237 & -0.000095 & 1.530402 \\
\hline 17 & 1 & -0.345186 & 0.882314 & . 1.984533 \\
\hline 18 & 1 & -1.854623 & -0.000445 & 1.802497 \\
\hline 19 & 1 & -0.344608 & -0.882288 & 1.984376 \\
\hline 20 & 6 & -1.355456 & 1.241832 & -0.568291 \\
\hline 21 & 1 & -1.230429 & 1.306799 & -1.651630 \\
\hline 22 & 1 & -2.426147 & 1.193689 & -0.361346 \\
\hline 23 & 1 & -0.982082 & 2.167339 & -0.131089 \\
\hline
\end{tabular}

Zero-point correction=

0.215298 (Hartree/Particle) 
Thermal correction to Energy=

Thermal correction to Enthalpy=

Thermal correction to Gibbs Free Energy=

Sum of electronic and zero-point Energies=

Sum of electronic and thermal Energies $=$

Sum of electronic and thermal Enthalpies=

Sum of electronic and thermal Free Energies=

Octane
0.224898

0.225842

0.182012

$-276.281644$

$-276.272044$

$-276.271100$

$-276.314930$

Standard orientation:

\begin{tabular}{|c|c|c|c|c|}
\hline \multirow{2}{*}{$\begin{array}{l}\text { Center } \\
\text { Number }\end{array}$} & Atomic & \multirow{2}{*}{$\begin{array}{l}\text { Atomic } \\
\text { Type }\end{array}$} & \multicolumn{2}{|c|}{ Coordinates (Angstrom } \\
\hline & & & $\mathrm{X}$ & Z \\
\hline 1 & 6 & -4.487771 & 0.255700 & 0.000039 \\
\hline 2 & 1 & -4.558269 & 0.897603 & 0.880938 \\
\hline 3 & 1 & -4.558233 & 0.897892 & -0.880654 \\
\hline 4 & 1 & -5.357940 & -0.402771 & -0.000092 \\
\hline 5 & 6 & -3.183130 & -0.539559 & -0.000064 \\
\hline 6 & 1 & -3.157962 & -1.197764 & 0.873885 \\
\hline 7 & 1 & -3.157977 & -1.197556 & -0.874171 \\
\hline 8 & 6 & -1.935073 & 0.344146 & 0.000021 \\
\hline 9 & 1 & -1.961391 & 1.003362 & -0.874500 \\
\hline 10 & 1 & -1.961436 & 1.003272 & 0.874608 \\
\hline 11 & 6 & -0.623706 & -0.442354 & 0.000015 \\
\hline 12 & 1 & -0.598500 & -1.101367 & -0.874517 \\
\hline 13 & 1 & -0.598472 & -1.101317 & 0.874583 \\
\hline 14 & 6 & 0.623706 & 0.442354 & -0.000030 \\
\hline 15 & 1 & 0.598481 & 1.101396 & 0.874481 \\
\hline 16 & 1 & 0.598490 & 1.101288 & -0.874620 \\
\hline 17 & 6 & 1.935073 & -0.344146 & 0.000025 \\
\hline 18 & 1 & 1.961414 & -1.003376 & -0.874484 \\
\hline 19 & 1 & 1.961412 & -1.003258 & 0.874623 \\
\hline 20 & 6 & 3.183130 & 0.539559 & -0.000033 \\
\hline 21 & 1 & 3.157969 & 1.197718 & 0.873951 \\
\hline 22 & 1 & 3.157971 & 1.197602 & -0.874105 \\
\hline 23 & 6 & 4.487771 & -0.255700 & 0.000021 \\
\hline 24 & 1 & 4.558252 & -0.897806 & -0.880732 \\
\hline 25 & 1 & 5.357940 & 0.402771 & -0.000021 \\
\hline 26 & 1 & 4.558250 & -0.897689 & 0.880860 \\
\hline
\end{tabular}

Zero-point correction=

Thermal correction to Energy=

Thermal correction to Enthalpy=

Thermal correction to Gibbs Free Energy=

Sum of electronic and zero-point Energies=

Sum of electronic and thermal Energies=

Sum of electronic and thermal Enthalpies=

Sum of electronic and thermal Free Energies=

2233tetramethylbutane

\subsection{3 (Hartree/Particle)}

0.256039

0.256983

0.207789

$-315.586462$

$-315.575326$

$-315.574382$

$-315.623576$

Standard orientation: 


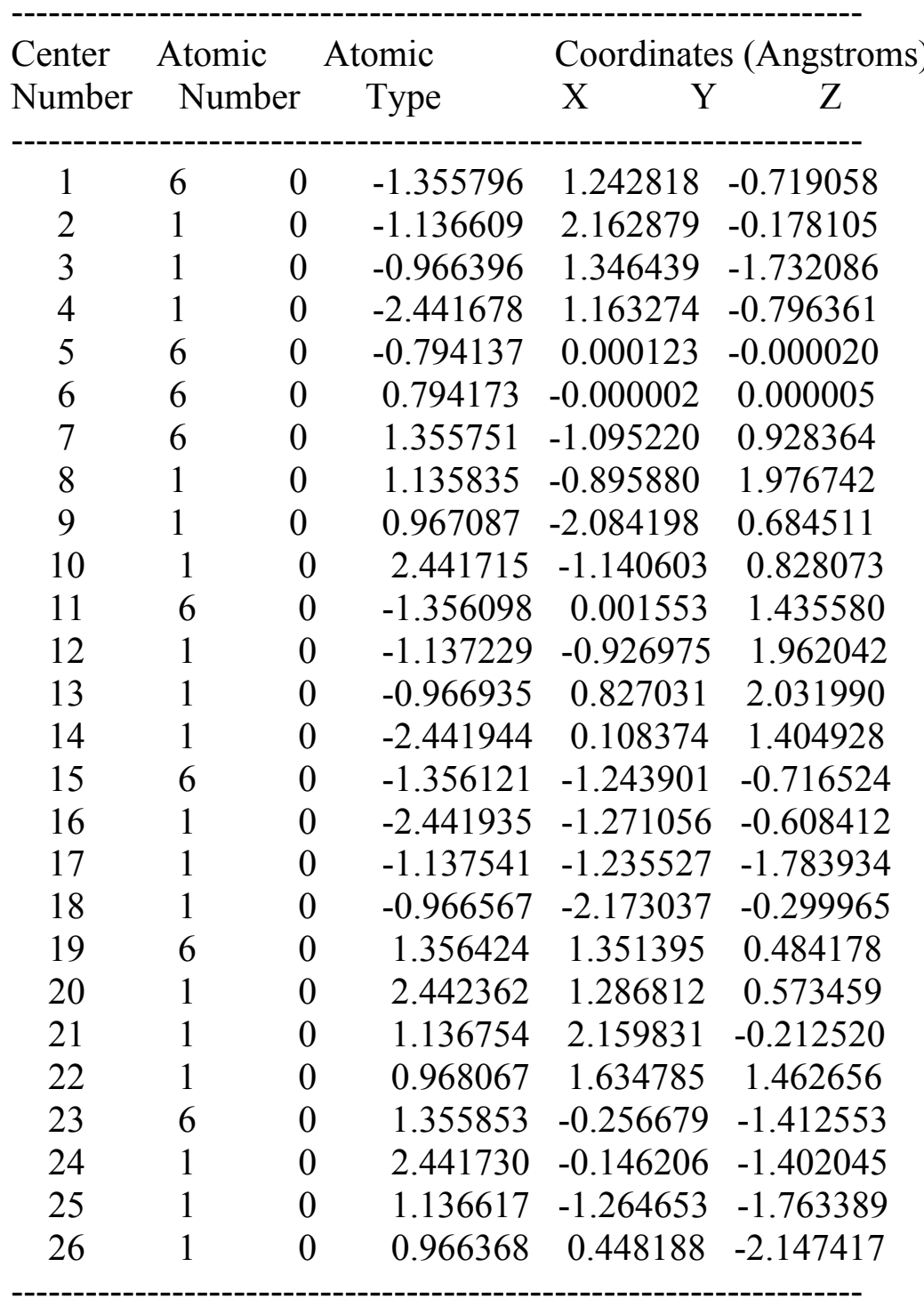

Zero-point correction=

Thermal correction to Energy=

Thermal correction to Enthalpy=

Thermal correction to Gibbs Free Energy=

Sum of electronic and zero-point Energies=

Sum of electronic and thermal Energies $=$

Sum of electronic and thermal Enthalpies=

Sum of electronic and thermal Free Energies=

PBE0/ccpvTZ

Ethane

Standard orientation: 


$\begin{array}{rrrrrr}5 & 6 & 0 & 0.000000 & -0.000000 & -0.759671 \\ 6 & 1 & 0 & 0.508352 & 0.880557 & -1.158407 \\ 7 & 1 & 0 & 0.508409 & -0.880525 & -1.158407 \\ 8 & 1 & 0 & -1.016761 & -0.000032 & -1.158407\end{array}$

Zero-point correction=

Thermal correction to Energy=

Thermal correction to Enthalpy=

Thermal correction to Gibbs Free Energy=

Sum of electronic and zero-point Energies=

Sum of electronic and thermal Energies $=$

Sum of electronic and thermal Enthalpies=

Sum of electronic and thermal Free Energies=

Methane

Standard orientation:
0.074634 (Hartree/Particle)

0.078120

0.079064

0.053222

$-79.675413$

$-79.671927$

$-79.670983$

$-79.696825$

\begin{tabular}{|c|c|c|c|c|}
\hline \multirow{2}{*}{$\begin{array}{l}\text { Center } \\
\text { Number }\end{array}$} & \multirow{2}{*}{$\begin{array}{l}\text { Atomic } \\
\text { Number }\end{array}$} & \multirow{2}{*}{$\begin{array}{r}\text { Atomic } \\
\text { Type }\end{array}$} & \multicolumn{2}{|c|}{ Coordinates (Angstron } \\
\hline & & & $\mathrm{X}$ & $\mathrm{Z}$ \\
\hline 1 & 6 & 0.000000 & 0.000000 & 0.000000 \\
\hline 2 & 1 & 0.628695 & 0.628695 & 0.628695 \\
\hline 3 & 1 & -0.628695 & -0.628695 & 0.628695 \\
\hline 4 & 1 & -0.628695 & 0.628695 & -0.628695 \\
\hline 5 & 1 & 0.628695 & -0.628695 & -0.628695 \\
\hline
\end{tabular}

Zero-point correction=

Thermal correction to Energy=

Thermal correction to Enthalpy=

Thermal correction to Gibbs Free Energy=

Sum of electronic and zero-point Energies=

Sum of electronic and thermal Energies=

Sum of electronic and thermal Enthalpies $=$

Sum of electronic and thermal Free Energies=

Propane

$$
\begin{array}{cc}
0.044713 \text { (Hartree/Particle) } & 0.047583 \\
& 0.048527 \\
\text { rgy }= & 0.027400 \\
\text { rgies }= & -40.429709 \\
\text { es }= & -40.426839 \\
\text { pies }= & -40.425895 \\
\text { Energies }= & -40.447022
\end{array}
$$

Standard orientation:

\begin{tabular}{cccccc} 
Center & \multicolumn{2}{c}{ Atomic } & \multicolumn{2}{c}{ Atomic } & \multicolumn{3}{c}{ Coordinates (Angstroms) } \\
Number & Number & Type & X & Y & $Z$ \\
- \hdashline 1 & 6 & 0 & -1.265580 & -0.258080 & 0.000000 \\
2 & 1 & 0 & -2.164373 & 0.362298 & -0.000024 \\
3 & 1 & 0 & -1.307529 & -0.903957 & 0.881209 \\
4 & 1 & 0 & -1.307510 & -0.903998 & -0.881181 \\
5 & 6 & 0 & 0.000000 & 0.583789 & 0.000000 \\
6 & 1 & 0 & -0.000000 & 1.242781 & 0.873995 \\
7 & 1 & 0 & 0.000001 & 1.242765 & -0.874006 \\
8 & 6 & 0 & 1.265580 & -0.258080 & 0.000000 \\
9 & 1 & 0 & 1.307536 & -0.903945 & 0.881218 \\
10 & 1 & 0 & 1.307498 & -0.904010 & -0.881173 \\
11 & 1 & 0 & 2.164375 & 0.362295 & -0.000042
\end{tabular}




$\begin{array}{lc}\text { Zero-point correction= } & 0.103365 \text { (Hartree/Particle) } \\ \text { Thermal correction to Energy= } & 0.107894 \\ \text { Thermal correction to Enthalpy= } & 0.108838 \\ \text { Thermal correction to Gibbs Free Energy= } & 0.077723 \\ \text { Sum of electronic and zero-point Energies }= & -118.924573 \\ \text { Sum of electronic and thermal Energies }= & -118.920043 \\ \text { Sum of electronic and thermal Enthalpies }= & -118.919099 \\ \text { Sum of electronic and thermal Free Energies }= & -118.950214 \\ \text { Butane } & \end{array}$

Standard orientation:

\begin{tabular}{cccccc} 
Center & \multicolumn{2}{c}{ Atomic } & \multicolumn{2}{c}{ Atomic } & \multicolumn{3}{c}{ Coordinates (Angstroms) } \\
Number & Number & Type & X & Y & $Z$ \\
-1 & 6 & 0 & 1.945358 & -0.119189 & 0.000030 \\
2 & 1 & 0 & 2.090522 & -0.749718 & -0.881229 \\
3 & 1 & 0 & 2.090462 & -0.749703 & 0.881311 \\
4 & 1 & 0 & 2.733734 & 0.636428 & 0.000051 \\
5 & 6 & 0 & 0.562774 & 0.511544 & -0.000034 \\
6 & 1 & 0 & 0.455094 & 1.163012 & 0.874507 \\
7 & 1 & 0 & 0.455139 & 1.162968 & -0.874615 \\
8 & 6 & 0 & -0.562774 & -0.511544 & -0.000035 \\
9 & 1 & 0 & -0.455139 & -1.162965 & -0.874617 \\
10 & 1 & 0 & -0.455094 & -1.163014 & 0.874506 \\
11 & 6 & 0 & -1.945358 & 0.119189 & 0.000031 \\
12 & 1 & 0 & -2.090520 & 0.749724 & -0.881225 \\
13 & 1 & 0 & -2.733734 & -0.636428 & 0.000043 \\
14 & 1 & 0 & -2.090464 & 0.749697 & 0.881315
\end{tabular}

Zero-point correction=

Thermal correction to Energy=

Thermal correction to Enthalpy=

Thermal correction to Gibbs Free Energy=

Sum of electronic and zero-point Energies=

Sum of electronic and thermal Energies $=$

Sum of electronic and thermal Enthalpies=

Sum of electronic and thermal Free Energies=
0.131888 (Hartree/Particle)

0.137676

0.138620
0.103888

$-158.173879$

$-158.168090$

$-158.167146$

$-158.201879$

Isobutane

Standard orientation:

\begin{tabular}{|c|c|c|c|}
\hline \multirow{2}{*}{$\begin{array}{l}\text { Center } \\
\text { Number }\end{array}$} & Atomic & \multirow{2}{*}{$\begin{array}{r}\text { Atomic } \\
\text { Type }\end{array}$} & Coordinates (Angstroms) \\
\hline & Numbe & & $\mathrm{X}$ \\
\hline 1 & 6 & 0.220551 & $-1.431996-0.095101$ \\
\hline 2 & 1 & -0.571100 & -2.095142 \\
\hline 3 & 1 & 0.228337 & $-1.483221-1.188326$ \\
\hline 4 & 1 & 1.175154 & $-1.826094 \quad 0.261720$ \\
\hline 5 & 6 & 0.000001 & $-0.000000 \quad 0.372727$ \\
\hline 6 & 1 & -0.000022 & $0.000025 \quad 1.469568$ \\
\hline
\end{tabular}




$\begin{array}{cccccc}7 & 6 & 0 & -1.350424 & 0.524994 & -0.095099 \\ 8 & 1 & 0 & -1.528924 & 1.542139 & 0.261917 \\ 9 & 1 & 0 & -1.398709 & 0.543930 & -1.188329 \\ 10 & 1 & 0 & -2.169000 & -0.104706 & 0.261701 \\ 11 & 6 & 0 & 1.129875 & 0.907001 & -0.095098 \\ 12 & 1 & 0 & 0.993847 & 1.930783 & 0.261642 \\ 13 & 1 & 0 & 2.099964 & 0.552989 & 0.261978 \\ 14 & 1 & 0 & 1.170434 & 0.939304 & -1.188325\end{array}$

Zero-point correction=

Thermal correction to Energy=

Thermal correction to Enthalpy=

Thermal correction to Gibbs Free Energy=

Sum of electronic and zero-point Energies=

Sum of electronic and thermal Energies $=$

Sum of electronic and thermal Enthalpies=

Sum of electronic and thermal Free Energies=
0.131417 (Hartree/Particle)

0.137131

0.138075

0.103806

$-158.175542$

$-158.169828$

$-158.168884$

$-158.203153$

Pentane

Standard orientation:

\begin{tabular}{cccccc} 
Center & Atomic & \multicolumn{2}{c}{ Atomic } & \multicolumn{3}{c}{ Coordinates } \\
Number & Number & Type & X & Y & Z \\
--------- & \\
\hline 1 & 6 & 0 & 2.537416 & 0.322505 & -0.000000 \\
2 & 1 & 0 & 2.580604 & 0.968019 & -0.881122 \\
3 & 1 & 0 & 2.580602 & 0.968028 & 0.881115 \\
4 & 1 & 0 & 3.435553 & -0.298423 & 0.000005 \\
5 & 6 & 0 & 1.272840 & -0.520186 & 0.000002 \\
6 & 1 & 0 & 1.271075 & -1.180097 & -0.874548 \\
7 & 1 & 0 & 1.271074 & -1.180090 & 0.874558 \\
8 & 6 & 0 & 0.000000 & 0.311800 & -0.000002 \\
9 & 1 & 0 & -0.000001 & 0.973250 & 0.875044 \\
10 & 1 & 0 & 0.000001 & 0.973246 & -0.875051 \\
11 & 6 & 0 & -1.272840 & -0.520186 & -0.000002 \\
12 & 1 & 0 & -1.271073 & -1.180095 & 0.874549 \\
13 & 1 & 0 & -1.271076 & -1.180092 & -0.874556 \\
14 & 6 & 0 & -2.537416 & 0.322505 & 0.000002 \\
15 & 1 & 0 & -3.435553 & -0.298423 & 0.000005 \\
16 & 1 & 0 & -2.580600 & 0.968025 & 0.881120 \\
17 & 1 & 0 & -2.580606 & 0.968022 & -0.881117
\end{tabular}

Zero-point correction=

0.160402 (Hartree/Particle)

Thermal correction to Energy=

Thermal correction to Enthalpy=

0.167505

0.168449

Thermal correction to Gibbs Free Energy=

0.130064

Sum of electronic and zero-point Energies $=$

$-197.423097$

Sum of electronic and thermal Energies=

$-197.415993$

Sum of electronic and thermal Enthalpies=

$-197.415049$

Sum of electronic and thermal Free Energies=

$-197.453434$ 
Neopentane

Standard orientation:

\begin{tabular}{cccccc} 
Center & \multicolumn{2}{c}{ Atomic } & \multicolumn{2}{c}{ Atomic } & \multicolumn{3}{c}{ Coordinates (Angstroms } \\
Number & Number & Type & X & Y & Z \\
- \hdashline 1 & 6 & 0 & -1.284667 & 0.607139 & 0.559349 \\
2 & 1 & 0 & -2.166705 & 0.139593 & 0.113703 \\
3 & 1 & 0 & -1.344695 & 0.470778 & 1.642399 \\
4 & 1 & 0 & -1.334436 & 1.679726 & 0.353727 \\
5 & 6 & 0 & -0.000019 & 0.000024 & 0.000024 \\
6 & 6 & 0 & 0.031677 & -1.495612 & 0.306526 \\
7 & 1 & 0 & 0.942898 & -1.957323 & -0.082964 \\
8 & 1 & 0 & -0.001041 & -1.676735 & 1.384177 \\
9 & 1 & 0 & -0.822296 & -2.007533 & -0.144996 \\
10 & 6 & 0 & 0.046493 & 0.210858 & -1.511670 \\
11 & 1 & 0 & 0.025113 & 1.275110 & -1.760882 \\
12 & 1 & 0 & 0.957770 & -0.214978 & -1.939964 \\
13 & 1 & 0 & -0.807466 & -0.264808 & -2.001255 \\
14 & 6 & 0 & 1.206496 & 0.677613 & 0.645792 \\
15 & 1 & 0 & 1.209563 & 1.751725 & 0.442153 \\
16 & 1 & 0 & 1.199084 & 0.542608 & 1.730651 \\
17 & 1 & 0 & 2.142330 & 0.261709 & 0.263118
\end{tabular}

Zero-point correction=

Thermal correction to Energy=

0.159109 (Hartree/Particle)

Thermal correction to Enthalpy=

0.166053

0.166997

Thermal correction to Gibbs Free Energy=

0.129999

Sum of electronic and zero-point Energies=

$-197.426769$

Sum of electronic and thermal Energies $=$

$-197.419825$

Sum of electronic and thermal Enthalpies=

$-197.418880$

Sum of electronic and thermal Free Energies=

$-197.455878$

Hexane

Standard orientation:

\begin{tabular}{|c|c|c|c|c|}
\hline \multirow{2}{*}{$\begin{array}{l}\text { Center } \\
\text { Number }\end{array}$} & Atomic & \multirow{2}{*}{$\begin{array}{r}\text { Atomic } \\
\text { Type }\end{array}$} & \multicolumn{2}{|c|}{ Coordinates (Angstroms } \\
\hline & Numbe & & $X$ & $\mathrm{Z}$ \\
\hline 1 & 6 & -3.196202 & 0.207018 & 0.000024 \\
\hline 2 & 1 & -3.283648 & 0.848073 & 0.881293 \\
\hline 3 & 1 & -3.283639 & 0.848208 & -0.881148 \\
\hline 4 & 1 & -4.049853 & -0.474075 & -0.000035 \\
\hline 5 & 6 & -1.876427 & -0.546545 & -0.000032 \\
\hline 6 & 1 & -1.829412 & -1.204944 & 0.874491 \\
\hline 7 & 1 & -1.829430 & -1.204853 & -0.874625 \\
\hline 8 & 6 & -0.663523 & 0.371123 & 0.000001 \\
\hline 9 & 1 & -0.710245 & 1.030827 & -0.875111 \\
\hline 10 & 1 & -0.710267 & 1.030798 & 0.875134 \\
\hline 11 & 6 & 0.663523 & -0.371123 & 0.000010 \\
\hline
\end{tabular}




$\begin{array}{rrrrrr}12 & 1 & 0 & 0.710247 & -1.030838 & -0.875094 \\ 13 & 1 & 0 & 0.710264 & -1.030787 & 0.875151 \\ 14 & 6 & 0 & 1.876427 & 0.546545 & -0.000028 \\ 15 & 1 & 0 & 1.829415 & 1.204945 & 0.874494 \\ 16 & 1 & 0 & 1.829427 & 1.204851 & -0.874621 \\ 17 & 6 & 0 & 3.196202 & -0.207018 & 0.000020 \\ 18 & 1 & 0 & 3.283645 & -0.848195 & -0.881160 \\ 19 & 1 & 0 & 4.049853 & 0.474075 & -0.000021 \\ 20 & 1 & 0 & 3.283642 & -0.848086 & 0.881280\end{array}$

Zero-point correction=

Thermal correction to Energy=

Thermal correction to Enthalpy=

Thermal correction to Gibbs Free Energy=

Sum of electronic and zero-point Energies=

Sum of electronic and thermal Energies=

Sum of electronic and thermal Enthalpies=

Sum of electronic and thermal Free Energies=

22dimethylbutane
0.188864 (Hartree/Particle)

0.197289

0.198233

Standard orientation:

\begin{tabular}{ccccccc} 
Center & Atomic & \multicolumn{2}{c}{ Atomic } & \multicolumn{3}{c}{ Coordinates } \\
Number & Number & Type & X & Y & $Z$ \\
--------- & Z \\
\hline 1 & 6 & 0 & -1.605809 & 0.977402 & 0.002283 \\
2 & 1 & 0 & -1.581045 & 1.619102 & 0.887158 \\
3 & 1 & 0 & -1.581247 & 1.622967 & -0.879785 \\
4 & 1 & 0 & -2.560631 & 0.445243 & 0.001222 \\
5 & 6 & 0 & -0.432257 & -0.000736 & 0.000016 \\
6 & 6 & 0 & 0.867995 & 0.820243 & 0.001307 \\
7 & 1 & 0 & 0.849910 & 1.481825 & -0.872226 \\
8 & 1 & 0 & 0.850001 & 1.478988 & 0.876987 \\
9 & 6 & 0 & 2.170663 & 0.035464 & 0.000043 \\
10 & 1 & 0 & 2.264128 & -0.600192 & -0.882722 \\
11 & 1 & 0 & 3.024903 & 0.715611 & 0.000558 \\
12 & 1 & 0 & 2.264584 & -0.602155 & 0.881332 \\
13 & 6 & 0 & -0.519728 & -0.880176 & 1.245912 \\
14 & 1 & 0 & 0.272310 & -1.631394 & 1.269399 \\
15 & 1 & 0 & -0.442595 & -0.280170 & 2.156856 \\
16 & 1 & 0 & -1.475247 & -1.410140 & 1.276099 \\
17 & 6 & 0 & -0.520377 & -0.875070 & -1.249427 \\
18 & 1 & 0 & -1.475758 & -1.405194 & -1.281133 \\
19 & 1 & 0 & -0.444083 & -0.271275 & -2.157931 \\
20 & 1 & 0 & 0.271846 & -1.625973 & -1.276621
\end{tabular}

Zero-point correction=

Thermal correction to Energy=

0.187734 (Hartree/Particle)

Thermal correction to Enthalpy=

0.196015

0.196959

Thermal correction to Gibbs Free Energy=

0.156443

Sum of electronic and zero-point Energies=

$-236.673129$ 
Sum of electronic and thermal Energies=

$-236.664848$

Sum of electronic and thermal Enthalpies=

Sum of electronic and thermal Free Energies=

Heptane

Standard orientation:

\begin{tabular}{|c|c|c|c|c|}
\hline \multirow{2}{*}{$\begin{array}{l}\text { Center } \\
\text { Number }\end{array}$} & Atomic & \multirow{2}{*}{$\begin{array}{l}\text { Atomic } \\
\text { Type }\end{array}$} & \multicolumn{2}{|c|}{ Coordinates (Angstroms } \\
\hline & Number & & $X$ & Z \\
\hline 1 & 6 & -3.809642 & -0.351208 & -0.000004 \\
\hline 2 & 1 & -3.852838 & -0.996732 & -0.881136 \\
\hline 3 & 1 & -3.852833 & -0.996762 & 0.881106 \\
\hline 4 & 1 & -4.707820 & 0.269685 & 0.000009 \\
\hline 5 & 6 & -2.545070 & 0.491488 & 0.000007 \\
\hline 6 & 1 & -2.543308 & 1.151407 & -0.874512 \\
\hline 7 & 1 & -2.543309 & 1.151384 & 0.874544 \\
\hline 8 & 6 & -1.272193 & -0.340799 & -0.000002 \\
\hline 9 & 1 & -1.273318 & -1.002005 & 0.875047 \\
\hline 10 & 1 & -1.273322 & -1.001991 & -0.875062 \\
\hline 11 & 6 & 0.000000 & 0.491793 & 0.000000 \\
\hline 12 & 1 & -0.000002 & 1.152751 & 0.875127 \\
\hline 13 & 1 & 0.000002 & 1.152750 & -0.875127 \\
\hline 14 & 6 & 1.272193 & -0.340799 & 0.000004 \\
\hline 15 & 1 & 1.273318 & -1.002006 & -0.875044 \\
\hline 16 & 1 & 1.273323 & -1.001989 & 0.875065 \\
\hline 17 & 6 & 2.545070 & 0.491488 & -0.000008 \\
\hline 18 & 1 & 2.543309 & 1.151408 & 0.874511 \\
\hline 19 & 1 & 2.543308 & 1.151384 & -0.874544 \\
\hline 20 & 6 & 3.809642 & -0.351208 & 0.000003 \\
\hline 21 & 1 & 3.852834 & -0.996760 & -0.881109 \\
\hline 22 & 1 & 4.707820 & 0.269685 & -0.000008 \\
\hline 23 & 1 & 3.852837 & -0.996734 & 0.881133 \\
\hline
\end{tabular}

Zero-point correction=

0.217318 (Hartree/Particle)

Thermal correction to Energy=

0.227111

Thermal correction to Enthalpy=

0.228055

Thermal correction to Gibbs Free Energy $=0.182417$

Sum of electronic and zero-point Energies $=\quad-275.921664$

Sum of electronic and thermal Energies $=\quad-275.911872$

Sum of electronic and thermal Enthalpies $=\quad-275.910927$

Sum of electronic and thermal Free Energies $=\quad-275.956566$

223trimethylbutane

Standard orientation:

\begin{tabular}{cccccc} 
Center & \multicolumn{2}{c}{ Atomic } & \multicolumn{2}{c}{ Atomic } & \multicolumn{2}{c}{ Coordinates } \\
Number & Number & Type & X & Y & Z \\
-1 & 6 & 0 & -1.346356 & -1.234344 & -0.563195 \\
2 & 1 & 0 & -1.221995 & -1.299325 & -1.647927 \\
3 & 1 & 0 & -0.971486 & -2.160269 & -0.124420
\end{tabular}




$\begin{array}{cccccc}4 & 1 & 0 & -2.417932 & -1.185431 & -0.354182 \\ 5 & 6 & 0 & -0.639114 & 0.000026 & 0.000012 \\ 6 & 6 & 0 & 0.848490 & 0.000017 & -0.447466 \\ 7 & 1 & 0 & 0.820262 & 0.000049 & -1.545285 \\ 8 & 6 & 0 & 1.623528 & 1.242053 & -0.016964 \\ 9 & 1 & 0 & 1.707709 & 1.304490 & 1.071015 \\ 10 & 1 & 0 & 2.639896 & 1.204850 & -0.415596 \\ 11 & 1 & 0 & 1.168478 & 2.166505 & -0.373364 \\ 12 & 6 & 0 & 1.623446 & -1.242082 & -0.017047 \\ 13 & 1 & 0 & 1.707901 & -1.304421 & 1.070920 \\ 14 & 1 & 0 & 1.168175 & -2.166515 & -0.373220 \\ 15 & 1 & 0 & 2.639715 & -1.205047 & -0.415943 \\ 16 & 6 & 0 & -0.784911 & -0.000603 & 1.521417 \\ 17 & 1 & 0 & -0.330449 & 0.883125 & 1.973971 \\ 18 & 1 & 0 & -1.842738 & -0.000534 & 1.796731 \\ 19 & 1 & 0 & -0.330749 & -0.884889 & 1.973181 \\ 20 & 6 & 0 & -1.346120 & 1.234951 & -0.562242 \\ 21 & 1 & 0 & -1.221501 & 1.300918 & -1.646888 \\ 22 & 1 & 0 & -2.417755 & 1.185930 & -0.353546 \\ 23 & 1 & 0 & -0.971305 & 2.160455 & -0.122535\end{array}$

Zero-point correction=

Thermal correction to Energy=

Thermal correction to Enthalpy=

Thermal correction to Gibbs Free Energy=

Sum of electronic and zero-point Energies=

Sum of electronic and thermal Energies $=$

Sum of electronic and thermal Enthalpies=

Sum of electronic and thermal Free Energies=

Octane

Standard orientation:

\begin{tabular}{|c|c|c|c|c|}
\hline \multirow{2}{*}{$\begin{array}{l}\text { Center } \\
\text { Number }\end{array}$} & Atomic & \multirow{2}{*}{$\begin{array}{l}\text { Atomic } \\
\text { Type }\end{array}$} & \multicolumn{2}{|c|}{ Coordinates (Angstroms } \\
\hline & Numbe & & $\mathrm{X}$ & Z \\
\hline 1 & 6 & -4.458923 & 0.254840 & 0.000010 \\
\hline 2 & 1 & -4.527007 & 0.898218 & 0.881145 \\
\hline 3 & 1 & -4.527008 & 0.898259 & -0.881095 \\
\hline 4 & 1 & -5.332486 & -0.400241 & -0.000005 \\
\hline 5 & 6 & -3.162782 & -0.538443 & -0.000010 \\
\hline 6 & 1 & -3.135582 & -1.197813 & 0.874503 \\
\hline 7 & 1 & -3.135587 & -1.197780 & -0.874548 \\
\hline 8 & 6 & -1.922929 & 0.342284 & 0.000002 \\
\hline 9 & 1 & -1.949549 & 1.002963 & -0.875052 \\
\hline 10 & 1 & -1.949550 & 1.002944 & 0.875069 \\
\hline 11 & 6 & -0.619596 & -0.440650 & -0.000003 \\
\hline 12 & 1 & -0.594126 & -1.101117 & -0.875095 \\
\hline 13 & 1 & -0.594120 & -1.101119 & 0.875086 \\
\hline 14 & 6 & 0.619597 & 0.440650 & -0.000006 \\
\hline 15 & 1 & 0.594120 & 1.101126 & 0.875078 \\
\hline
\end{tabular}




$\begin{array}{rrrrrr}16 & 1 & 0 & 0.594126 & 1.101110 & -0.875103 \\ 17 & 6 & 0 & 1.922929 & -0.342284 & 0.000005 \\ 18 & 1 & 0 & 1.949550 & -1.002966 & -0.875046 \\ 19 & 1 & 0 & 1.949549 & -1.002941 & 0.875074 \\ 20 & 6 & 0 & 3.162782 & 0.538443 & -0.000007 \\ 21 & 1 & 0 & 3.135583 & 1.197810 & 0.874508 \\ 22 & 1 & 0 & 3.135586 & 1.197783 & -0.874542 \\ 23 & 6 & 0 & 4.458923 & -0.254840 & 0.000007 \\ 24 & 1 & 0 & 4.527009 & -0.898252 & -0.881102 \\ 25 & 1 & 0 & 5.332486 & 0.400240 & -0.000002 \\ 26 & 1 & 0 & 4.527006 & -0.898225 & 0.881137\end{array}$

Zero-point correction $=$

Thermal correction to Energy=

Thermal correction to Enthalpy=

0.245764 (Hartree/Particle)

0.256915

0.257859

Thermal correction to Gibbs Free Energy=

Sum of electronic and zero-point Energies=

Sum of electronic and thermal Energies $=$

0.208601

$-315.170959$

$-315.159808$

Sum of electronic and thermal Enthalpies=

$-315.158864$

Sum of electronic and thermal Free Energies $=\quad-315.208122$

2233tetramethylbutane

Standard orientation:

\begin{tabular}{cccccc} 
Center & Atomic & \multicolumn{2}{c}{ Atomic } & \multicolumn{3}{c}{ Coordinates $($ Angstroms) } \\
Number & Number & Type & X & Y & Z \\
\hline 1 & 6 & 0 & -1.342450 & -1.234777 & -0.715962 \\
2 & 1 & 0 & -1.131488 & -1.215798 & -1.786351 \\
3 & 1 & 0 & -0.941634 & -2.165009 & -0.308076 \\
4 & 1 & 0 & -2.428420 & -1.269851 & -0.598471 \\
5 & 6 & 0 & -0.786712 & -0.000031 & -0.000047 \\
6 & 6 & 0 & 0.786710 & 0.000044 & 0.000053 \\
7 & 6 & 0 & 1.342473 & 1.355114 & 0.448209 \\
8 & 1 & 0 & 1.131515 & 2.142929 & -0.276654 \\
9 & 1 & 0 & 0.941504 & 1.667706 & 1.414557 \\
10 & 1 & 0 & 2.428444 & 1.290513 & 0.552532 \\
11 & 6 & 0 & -1.342676 & 1.237310 & -0.711319 \\
12 & 1 & 0 & -1.131473 & 2.154859 & -0.159865 \\
13 & 1 & 0 & -0.942275 & 1.349103 & -1.721035 \\
14 & 1 & 0 & -2.428684 & 1.153128 & -0.799988 \\
15 & 6 & 0 & -1.342659 & -0.002725 & 1.427164 \\
16 & 1 & 0 & -2.428604 & 0.116744 & 1.398638 \\
17 & 1 & 0 & -1.131924 & -0.939304 & 1.945800 \\
18 & 1 & 0 & -0.941787 & 0.815430 & 2.029067 \\
19 & 6 & 0 & 1.342707 & -0.289294 & -1.397476 \\
20 & 1 & 0 & 2.428743 & -0.167327 & -1.393246 \\
21 & 1 & 0 & 1.131242 & -1.310700 & -1.717832 \\
22 & 1 & 0 & 0.942527 & 0.391839 & -2.151289 \\
23 & 6 & 0 & 1.342632 & -1.065627 & 0.949399 \\
24 & 1 & 0 & 2.428598 & -1.123403 & 0.841253
\end{tabular}




$\begin{array}{lllll}1 & 0 & 1.131642 & -0.832097 & 1.994170 \\ 1 & 0 & 0.941923 & -2.058845 & 0.736653\end{array}$

Zero-point correction $=$

Thermal correction to Energy=

0.244204 (Hartree/Particle)

Thermal correction to Enthalpy=

0.254757

0.255701

Thermal correction to Gibbs Free Energy=

0.210713

Sum of electronic and zero-point Energies=

$-315.164334$

Sum of electronic and thermal Energies=

$-315.153780$

Sum of electronic and thermal Enthalpies=

$-315.152836$

Sum of electronic and thermal Free Energies=

M06/ccpVTZ

Ethane

Standard orientation:

\begin{tabular}{|c|c|c|c|c|}
\hline \multirow{2}{*}{$\begin{array}{l}\text { Center } \\
\text { Number }\end{array}$} & Atomic & \multirow{2}{*}{$\begin{array}{l}\text { Atomic } \\
\text { Type }\end{array}$} & \multicolumn{2}{|c|}{ Coordinates (Angstrol } \\
\hline & Numb & & $\mathrm{X}$ & $\mathrm{Z}$ \\
\hline 1 & 6 & 0.000000 & 0.000000 & 0.757216 \\
\hline 2 & 1 & -0.507251 & 0.878887 & 1.157438 \\
\hline 3 & 1 & 1.014764 & -0.000151 & 1.157438 \\
\hline 4 & 1 & -0.507513 & -0.878736 & 1.15743 \\
\hline 5 & 6 & 0.000000 & 0.000000 & -0.75721 \\
\hline 6 & 1 & 0.507251 & 0.878887 & -1.15743 \\
\hline 7 & 1 & 0.507513 & -0.878736 & -1.15743 \\
\hline 8 & 1 & -1.014764 & -0.000151 & -1.1574 \\
\hline
\end{tabular}

Zero-point correction=

Thermal correction to Energy=

Thermal correction to Enthalpy=

Thermal correction to Gibbs Free Energy=

Sum of electronic and zero-point Energies $=$

Sum of electronic and thermal Energies=

Sum of electronic and thermal Enthalpies=

Sum of electronic and thermal Free Energies=

Methane

\subsection{4 (Hartree/Particle)}

0.077546

0.078490

0.052628

$-79.709226$

$-79.705724$

$-79.704779$

$-79.730641$

Standard orientation:

\begin{tabular}{|c|c|c|c|c|}
\hline \multirow{2}{*}{$\begin{array}{l}\text { Center } \\
\text { Number }\end{array}$} & \multirow{2}{*}{$\begin{array}{l}\text { Atomic } \\
\text { Number }\end{array}$} & \multirow{2}{*}{$\begin{array}{r}\text { Atomic } \\
\text { Type }\end{array}$} & \multicolumn{2}{|c|}{ Coordinates (Angstror } \\
\hline & & & $\mathrm{X}$ & $\mathrm{Z}$ \\
\hline 1 & 6 & 0.000000 & 0.000000 & 0.000000 \\
\hline 2 & 1 & 0.627441 & 0.627441 & 0.627441 \\
\hline 3 & 1 & -0.627441 & -0.627441 & 0.627441 \\
\hline 4 & 1 & -0.627441 & 0.627441 & -0.627441 \\
\hline 5 & 1 & 0.627441 & -0.627441 & -0.627441 \\
\hline
\end{tabular}

Zero-point correction=

Thermal correction to Energy=
0.044429 (Hartree/Particle)

0.047301 
Thermal correction to Enthalpy=

0.048246

Thermal correction to Gibbs Free Energy=

0.027122

Sum of electronic and zero-point Energies=

$-40.448102$

Sum of electronic and thermal Energies=

$-40.445230$

Sum of electronic and thermal Enthalpies=

$-40.444286$

Sum of electronic and thermal Free Energies $=\quad-40.465410$

Propane

Standard orientation:

\begin{tabular}{|c|c|c|c|c|}
\hline \multirow{2}{*}{$\begin{array}{l}\text { Center } \\
\text { Number }\end{array}$} & Atomic & \multirow{2}{*}{$\begin{array}{l}\text { Atomic } \\
\text { Type }\end{array}$} & \multicolumn{2}{|c|}{ Coordinates (Angstrom } \\
\hline & Numbe & & $X$ & Z \\
\hline 1 & 6 & -1.255638 & -0.258719 & 0.000000 \\
\hline 2 & 1 & -2.162767 & 0.346570 & -0.000055 \\
\hline 3 & 1 & -1.287688 & -0.906257 & 0.879612 \\
\hline 4 & 1 & -1.287639 & -0.906342 & -0.879551 \\
\hline 5 & 6 & 0.000000 & 0.589760 & 0.000000 \\
\hline 6 & 1 & -0.000001 & 1.249074 & 0.872745 \\
\hline 7 & 1 & 0.000002 & 1.249049 & -0.872763 \\
\hline 8 & 6 & 1.255638 & -0.258719 & 0.000000 \\
\hline 9 & 1 & 1.287685 & -0.906258 & 0.879611 \\
\hline 10 & 1 & 1.287638 & -0.906340 & -0.879552 \\
\hline 11 & 1 & 2.162769 & 0.346567 & -0.000053 \\
\hline
\end{tabular}

Zero-point correction=

Thermal correction to Energy=

0.102594 (Hartree/Particle)

Thermal correction to Enthalpy=

0.107121

Thermal correction to Gibbs Free Energy=

0.108066

Sum of electronic and zero-point Energies $=$

0.076969

Sum of electronic and thermal Energies $=$

$-118.974251$

$-118.969723$

Sum of electronic and thermal Enthalpies=

$-118.968779$

Sum of electronic and thermal Free Energies $=\quad-118.999875$

Butane

Standard orientation:

\begin{tabular}{|c|c|c|c|c|}
\hline \multirow{2}{*}{$\begin{array}{l}\text { Center } \\
\text { Number }\end{array}$} & Atomic & \multirow{2}{*}{$\begin{array}{r}\text { Atomic } \\
\text { Type }\end{array}$} & \multicolumn{2}{|c|}{ Coordinates (Angstroms) } \\
\hline & Numbe & & $\mathrm{X}$ & $\mathrm{Z}$ \\
\hline 1 & 6 & 1.933878 & -0.117122 & 0.000058 \\
\hline 2 & 1 & 2.072471 & -0.750209 & -0.879636 \\
\hline 3 & 1 & 2.072346 & -0.750193 & 0.879783 \\
\hline 4 & 1 & 2.729553 & 0.628485 & 0.000107 \\
\hline 5 & 6 & 0.557339 & 0.514249 & -0.000065 \\
\hline 6 & 1 & 0.443063 & 1.165995 & 0.873387 \\
\hline 7 & 1 & 0.443155 & 1.165911 & -0.873595 \\
\hline 8 & 6 & -0.557339 & -0.514249 & -0.000066 \\
\hline 9 & 1 & -0.443155 & -1.165908 & -0.873597 \\
\hline 10 & 1 & -0.443063 & -1.165998 & 0.873385 \\
\hline 11 & 6 & -1.933878 & 0.117122 & 0.000058 \\
\hline 12 & 1 & -2.072470 & 0.750214 & -0.879633 \\
\hline
\end{tabular}




$\begin{array}{rrrrrr}13 & 1 & 0 & -2.729553 & -0.628485 & 0.000102 \\ 14 & 1 & 0 & -2.072347 & 0.750188 & 0.879786\end{array}$

Zero-point correction=

Thermal correction to Energy=

Thermal correction to Enthalpy=

Thermal correction to Gibbs Free Energy=

Sum of electronic and zero-point Energies=

Sum of electronic and thermal Energies=

Sum of electronic and thermal Enthalpies=

Sum of electronic and thermal Free Energies=

Isobutane

Standard orientation:
0.130824 (Hartree/Particle)

$$
0.136623
$$

0.137567

$$
\begin{gathered}
0.102829 \\
-158.239743 \\
-158.233945 \\
-158.233000 \\
-158.267738
\end{gathered}
$$

\begin{tabular}{cccccc}
\multicolumn{7}{c}{ Standard orientation: } \\
Center & Atomic & Atomic & \multicolumn{2}{c}{ Coordinates } \\
Number & Number & Type & X & Y & Z \\
- & Numstroms) \\
-1 & 6 & 0 & 0.410827 & -1.380477 & -0.095315 \\
2 & 1 & 0 & -0.282591 & -2.149230 & 0.251277 \\
3 & 1 & 0 & 0.421346 & -1.413289 & -1.189397 \\
4 & 1 & 0 & 1.411157 & -1.645656 & 0.252475 \\
5 & 6 & 0 & 0.000032 & 0.000013 & 0.382315 \\
6 & 1 & 0 & 0.000010 & 0.000006 & 1.478740 \\
7 & 6 & 0 & -1.400959 & 0.334450 & -0.095306 \\
8 & 1 & 0 & -1.720115 & 1.319226 & 0.251511 \\
9 & 1 & 0 & -1.434665 & 0.341999 & -1.189392 \\
10 & 1 & 0 & -2.130717 & -0.399439 & 0.252271 \\
11 & 6 & 0 & 0.990125 & 1.046025 & -0.095326 \\
12 & 1 & 0 & 0.719551 & 2.044933 & 0.252386 \\
13 & 1 & 0 & 2.002583 & 0.829943 & 0.251325 \\
14 & 1 & 0 & 1.013290 & 1.071442 & -1.189400
\end{tabular}

Zero-point correction=

0.130489 (Hartree/Particle)

Thermal correction to Energy=

Thermal correction to Enthalpy=

0.136154

0.137098

Thermal correction to Gibbs Free Energy=

Sum of electronic and zero-point Energies=

0.102948

$-158.242318$

Sum of electronic and thermal Energies $=$

$-158.236653$

\begin{tabular}{|c|c|c|c|c|}
\hline Center & Atomic & Atomic & Coordinat & tes (Angstrom \\
\hline Number & Number & Type & $X$ & Z \\
\hline 1 & 6 & 2.521065 & 0.324911 & 0.000004 \\
\hline 2 & 1 & 2.554142 & 0.972059 & -0.879552 \\
\hline 3 & 1 & 2.554125 & 0.972078 & 0.879547 \\
\hline 4 & 1 & 3.427743 & -0.280601 & 0.000020 \\
\hline
\end{tabular}

Sum of electronic and thermal Enthalpies=

$-158.235709$

Sum of electronic and thermal Free Energies $=\quad-158.269859$

Pentane

Standard orientation: 


$\begin{array}{cccccc}5 & 6 & 0 & 1.266720 & -0.523559 & 0.000002 \\ 6 & 1 & 0 & 1.261543 & -1.184741 & -0.873544 \\ 7 & 1 & 0 & 1.261533 & -1.184730 & 0.873555 \\ 8 & 6 & 0 & 0.000000 & 0.308504 & -0.000011 \\ 9 & 1 & 0 & -0.000001 & 0.972322 & 0.874189 \\ 10 & 1 & 0 & 0.000001 & 0.972302 & -0.874227 \\ 11 & 6 & 0 & -1.266720 & -0.523559 & -0.000002 \\ 12 & 1 & 0 & -1.261532 & -1.184733 & 0.873549 \\ 13 & 1 & 0 & -1.261544 & -1.184738 & -0.873550 \\ 14 & 6 & 0 & -2.521065 & 0.324911 & 0.000006 \\ 15 & 1 & 0 & -3.427743 & -0.280601 & 0.000022 \\ 16 & 1 & 0 & -2.554123 & 0.972077 & 0.879550 \\ 17 & 1 & 0 & -2.554144 & 0.972060 & -0.879549\end{array}$

Zero-point correction $=$

Thermal correction to Energy=

0.159158 (Hartree/Particle)

Thermal correction to Enthalpy=

$$
0.166254
$$

0.167198

Thermal correction to Gibbs Free Energy=

Sum of electronic and zero-point Energies=

0.128852

$-197.505038$

Sum of electronic and thermal Energies $=$

$-197.497942$

Sum of electronic and thermal Enthalpies=

$-197.496998$

Sum of electronic and thermal Free Energies=

$-197.535344$

Neopentane

Standard orientation:

\begin{tabular}{|c|c|c|c|c|}
\hline \multirow{2}{*}{$\begin{array}{l}\text { Center } \\
\text { Number }\end{array}$} & Atomic & \multirow{2}{*}{$\begin{array}{l}\text { Atomic } \\
\text { Type }\end{array}$} & \multicolumn{2}{|c|}{ Coordinates (Angstroms } \\
\hline & Number & & $\mathrm{X}$ & $\mathrm{Z}$ \\
\hline 1 & 6 & -1.301734 & 0.251301 & -0.746370 \\
\hline 2 & 1 & -1.930926 & 0.964959 & -0.207747 \\
\hline 3 & 1 & -1.110103 & 0.657993 & -1.742952 \\
\hline 4 & 1 & -1.870523 & -0.674802 & -0.865242 \\
\hline 5 & 6 & 0.000021 & 0.000015 & -0.000078 \\
\hline 6 & 6 & 0.761651 & 1.308894 & 0.146179 \\
\hline 7 & 1 & 1.703599 & 1.156777 & 0.679924 \\
\hline 8 & 1 & 0.995364 & 1.737843 & -0.831936 \\
\hline 9 & 1 & 0.174701 & 2.043783 & 0.703608 \\
\hline 10 & 6 & -0.307027 & -0.564807 & 1.378820 \\
\hline 11 & 1 & -0.855290 & -1.507667 & 1.302971 \\
\hline 12 & 1 & 0.612827 & -0.755286 & 1.938172 \\
\hline 13 & 1 & -0.916028 & 0.131858 & 1.961122 \\
\hline 14 & 6 & 0.847117 & -0.995387 & -0.778617 \\
\hline 15 & 1 & 0.322750 & -1.947248 & -0.898186 \\
\hline 16 & 1 & 1.082725 & -0.614122 & -1.775824 \\
\hline 17 & 1 & 1.790738 & -1.194187 & -0.263513 \\
\hline
\end{tabular}

Zero-point correction=

Thermal correction to Energy=

Thermal correction to Enthalpy=
0.158138 (Hartree/Particle)

0.164951

0.165895 
Thermal correction to Gibbs Free Energy=

Sum of electronic and zero-point Energies=

Sum of electronic and thermal Energies $=$

Sum of electronic and thermal Enthalpies=

Sum of electronic and thermal Free Energies=

Hexane
0.129171

$-197.512317$

$-197.505504$

$-197.504560$

$-197.541284$

Standard orientation:

\begin{tabular}{|c|c|c|c|c|c|}
\hline \multirow{2}{*}{$\begin{array}{l}\text { Center } \\
\text { Number }\end{array}$} & Atomic & \multirow{2}{*}{\multicolumn{2}{|c|}{$\begin{array}{l}\text { Atomic } \\
\text { Type }\end{array}$}} & \multicolumn{2}{|c|}{ Coordinates (Angstrom } \\
\hline & Numbe & & & $\mathrm{X}$ & Z \\
\hline 1 & 6 & 0 & -3.176730 & 0.206914 & 0.000002 \\
\hline 2 & 1 & 0 & -3.255795 & 0.850051 & 0.879564 \\
\hline 3 & 1 & 0 & -3.255781 & 0.850095 & -0.879530 \\
\hline 4 & 1 & 0 & -4.038102 & -0.461510 & -0.000022 \\
\hline 5 & 6 & 0 & -1.865283 & -0.550262 & -0.000007 \\
\hline 6 & 1 & 0 & -1.813201 & -1.209430 & 0.873473 \\
\hline 7 & 1 & 0 & -1.813201 & -1.209404 & -0.873506 \\
\hline 8 & 6 & 0 & -0.660773 & 0.369872 & 0.000007 \\
\hline 9 & 1 & 0 & -0.708692 & 1.031648 & -0.874262 \\
\hline 10 & 1 & 0 & -0.708698 & 1.031632 & 0.874287 \\
\hline 11 & 6 & 0 & 0.660773 & -0.369872 & 0.000003 \\
\hline 12 & 1 & 0 & 0.708690 & -1.031645 & -0.874268 \\
\hline 13 & 1 & 0 & 0.708700 & -1.031635 & 0.874281 \\
\hline 14 & 6 & 0 & 1.865283 & 0.550262 & -0.000009 \\
\hline 15 & 1 & 0 & 1.813200 & 1.209430 & 0.873472 \\
\hline 16 & 1 & 0 & 1.813203 & 1.209405 & -0.873508 \\
\hline 17 & 6 & 0 & 3.176730 & -0.206914 & 0.000003 \\
\hline 18 & 1 & 0 & 3.255785 & -0.850092 & -0.879530 \\
\hline 19 & 1 & 0 & 4.038102 & 0.461510 & -0.000014 \\
\hline 20 & 1 & 0 & 3.255791 & -0.850054 & 0.879564 \\
\hline
\end{tabular}

Zero-point correction=

0.187305 (Hartree/Particle)

Thermal correction to Energy=

Thermal correction to Enthalpy=

0.195763

0.196707

Thermal correction to Gibbs Free Energy=

0.154647

Sum of electronic and zero-point Energies=

$-236.770523$

Sum of electronic and thermal Energies=

$-236.762066$

Sum of electronic and thermal Enthalpies=

$-236.761121$

Sum of electronic and thermal Free Energies $=\quad-236.803182$

22dimethylbutane

Standard orientation:

\begin{tabular}{|c|c|c|c|c|}
\hline \multirow{2}{*}{$\begin{array}{l}\text { Center } \\
\text { Number }\end{array}$} & Atomic & \multirow{2}{*}{$\begin{array}{r}\text { Atomic } \\
\text { Type }\end{array}$} & \multicolumn{2}{|c|}{ Coordinates (Angstroms } \\
\hline & Numbe & & X & Z \\
\hline 1 & 6 & -1.602977 & 0.968916 & 0.002412 \\
\hline 2 & 1 & -1.580495 & 1.610358 & 0.887557 \\
\hline 3 & 1 & -1.580969 & 1.614068 & -0.880048 \\
\hline 4 & 1 & -2.554893 & 0.431673 & 0.001534 \\
\hline
\end{tabular}




$\begin{array}{cccccc}5 & 6 & 0 & -0.428771 & -0.000456 & 0.000027 \\ 6 & 6 & 0 & 0.863158 & 0.823121 & 0.001174 \\ 7 & 1 & 0 & 0.841409 & 1.485140 & -0.872966 \\ 8 & 1 & 0 & 0.841559 & 1.482509 & 0.877311 \\ 9 & 6 & 0 & 2.158695 & 0.036658 & -0.000026 \\ 10 & 1 & 0 & 2.246577 & -0.600640 & -0.881779 \\ 11 & 1 & 0 & 3.018941 & 0.706759 & 0.000206 \\ 12 & 1 & 0 & 2.247218 & -0.602098 & 0.880597 \\ 13 & 6 & 0 & -0.513236 & -0.877044 & 1.241792 \\ 14 & 1 & 0 & 0.270601 & -1.637013 & 1.258410 \\ 15 & 1 & 0 & -0.422045 & -0.277191 & 2.151819 \\ 16 & 1 & 0 & -1.474036 & -1.396977 & 1.279103 \\ 17 & 6 & 0 & -0.514267 & -0.871961 & -1.245257 \\ 18 & 1 & 0 & -1.474841 & -1.392248 & -1.283531 \\ 19 & 1 & 0 & -0.424553 & -0.268275 & -2.152883 \\ 20 & 1 & 0 & 0.269915 & -1.631472 & -1.266058\end{array}$

Zero-point correction= 0.186572 (Hartree/Particle)

Thermal correction to Energy=

0.194763

Thermal correction to Enthalpy=

0.195708

Thermal correction to Gibbs Free Energy=

Sum of electronic and zero-point Energies=

0.155198

Sum of electronic and thermal Energies=

$-236.776224$

$-236.768033$

Sum of electronic and thermal Enthalpies=

$-236.767089$

Sum of electronic and thermal Free Energies=

$-236.807598$

Heptane

Standard orientation:

\begin{tabular}{|c|c|c|c|c|}
\hline \multirow{2}{*}{$\begin{array}{l}\text { Center } \\
\text { Number }\end{array}$} & Atomic & \multirow{2}{*}{$\begin{array}{l}\text { Atomic } \\
\text { Type }\end{array}$} & \multicolumn{2}{|c|}{ Coordinates (Angstroms) } \\
\hline & Numbe & & $\mathrm{X}$ & $\mathrm{Z}$ \\
\hline 1 & 6 & -3.786848 & -0.354542 & 0.000003 \\
\hline 2 & 1 & -3.819901 & -1.001697 & -0.879566 \\
\hline 3 & 1 & -3.819886 & -1.001721 & 0.879555 \\
\hline 4 & 1 & -4.693580 & 0.250903 & 0.000019 \\
\hline 5 & 6 & -2.532579 & 0.494002 & 0.000005 \\
\hline 6 & 1 & -2.527430 & 1.155176 & -0.873526 \\
\hline 7 & 1 & -2.527424 & 1.155158 & 0.873549 \\
\hline 8 & 6 & -1.265751 & -0.338223 & -0.000008 \\
\hline 9 & 1 & -1.266574 & -1.001708 & 0.874265 \\
\hline 10 & 1 & -1.266579 & -1.001691 & -0.874293 \\
\hline 11 & 6 & 0.000000 & 0.493749 & -0.000003 \\
\hline 12 & 1 & -0.000003 & 1.156908 & 0.874400 \\
\hline 13 & 1 & 0.000003 & 1.156912 & -0.874403 \\
\hline 14 & 6 & 1.265751 & -0.338223 & -0.000002 \\
\hline 15 & 1 & 1.266576 & -1.001701 & -0.874280 \\
\hline 16 & 1 & 1.266577 & -1.001698 & 0.874278 \\
\hline 17 & 6 & 2.532579 & 0.494002 & -0.000003 \\
\hline 18 & 1 & 2.527424 & 1.155172 & 0.873531 \\
\hline
\end{tabular}




$\begin{array}{rrrrrr}19 & 1 & 0 & 2.527429 & 1.155162 & -0.873544 \\ 20 & 6 & 0 & 3.786848 & -0.354542 & 0.000006 \\ 21 & 1 & 0 & 3.819897 & -1.001714 & -0.879550 \\ 22 & 1 & 0 & 4.693580 & 0.250903 & 0.000006 \\ 23 & 1 & 0 & 3.819891 & -1.001704 & 0.879570\end{array}$

Zero-point correction=

Thermal correction to Energy=

Thermal correction to Enthalpy=

Thermal correction to Gibbs Free Energy=

Sum of electronic and zero-point Energies=

Sum of electronic and thermal Energies $=$

Sum of electronic and thermal Enthalpies=

Sum of electronic and thermal Free Energies=

223trimethylbutane

Standard orientation:

\begin{tabular}{|c|c|c|c|c|}
\hline \multirow{2}{*}{$\begin{array}{l}\text { Center } \\
\text { Number }\end{array}$} & Atomic & \multirow{2}{*}{$\begin{array}{l}\text { Atomic } \\
\text { Type }\end{array}$} & \multicolumn{2}{|c|}{ Coordinates (Angstrom } \\
\hline & Numbe & & $\mathrm{X}$ & Z \\
\hline 1 & 6 & -1.309413 & -1.301068 & -0.424225 \\
\hline 2 & 1 & -1.156629 & -1.494980 & -1.490121 \\
\hline 3 & 1 & -0.932960 & -2.160301 & 0.133340 \\
\hline 4 & 1 & -2.386247 & -1.244252 & -0.247188 \\
\hline 5 & 6 & -0.635657 & -0.000017 & -0.000543 \\
\hline 6 & 6 & 0.844099 & -0.001627 & -0.453490 \\
\hline 7 & 1 & 0.812413 & -0.007706 & -1.552651 \\
\hline 8 & 6 & 1.614570 & 1.237137 & -0.021728 \\
\hline 9 & 1 & 1.762493 & 1.249347 & 1.061388 \\
\hline 10 & 1 & 2.605622 & 1.242842 & -0.479137 \\
\hline 11 & 1 & 1.120074 & 2.166978 & -0.303539 \\
\hline 12 & 6 & 1.620759 & -1.234617 & -0.016054 \\
\hline 13 & 1 & 1.643718 & -1.324750 & 1.073490 \\
\hline 14 & 1 & 1.210036 & -2.158970 & -0.421366 \\
\hline 15 & 1 & 2.657198 & -1.163908 & -0.351773 \\
\hline 16 & 6 & -0.776802 & 0.155983 & 1.508506 \\
\hline 17 & 1 & -0.403318 & 1.121587 & 1.855222 \\
\hline 18 & 1 & -1.830451 & 0.092948 & 1.792660 \\
\hline 19 & 1 & -0.245698 & -0.628582 & 2.052235 \\
\hline 20 & 6 & -1.377765 & 1.144552 & -0.683033 \\
\hline 21 & 1 & -1.299337 & 1.068192 & -1.771211 \\
\hline 22 & 1 & -2.438960 & 1.117094 & -0.423161 \\
\hline 23 & 1 & -0.996700 & 2.122400 & -0.384785 \\
\hline
\end{tabular}

Zero-point correction=

Thermal correction to Energy=

Thermal correction to Enthalpy=

Thermal correction to Gibbs Free Energy=

Sum of electronic and zero-point Energies=
0.214794 (Hartree/Particle)

0.224165

0.225109

0.181940

$-276.040821$ 
Sum of electronic and thermal Energies $=$

$-276.031450$

Sum of electronic and thermal Enthalpies=

Sum of electronic and thermal Free Energies=

Octane

Standard orientation:

\begin{tabular}{cccccc} 
Center & Atomic & \multicolumn{2}{c}{ Atomic } & \multicolumn{3}{c}{ Coordinates } \\
Number & Number & Type & X & Y & Z \\
\hline-------- \\
\hline 1 & 6 & 0 & -4.433464 & 0.255879 & 0.000011 \\
2 & 1 & 0 & -4.492303 & 0.901198 & 0.879577 \\
3 & 1 & 0 & -4.492300 & 0.901242 & -0.879523 \\
4 & 1 & 0 & -5.315374 & -0.385208 & -0.000007 \\
5 & 6 & 0 & -3.146376 & -0.541998 & -0.000010 \\
6 & 1 & 0 & -3.114921 & -1.202464 & 0.873499 \\
7 & 1 & 0 & -3.114930 & -1.202431 & -0.873544 \\
8 & 6 & 0 & -1.913707 & 0.340015 & 0.000001 \\
9 & 1 & 0 & -1.940906 & 1.002946 & -0.874273 \\
10 & 1 & 0 & -1.940908 & 1.002927 & 0.874290 \\
11 & 6 & 0 & -0.615875 & -0.440927 & -0.000004 \\
12 & 1 & 0 & -0.589524 & -1.103565 & -0.874367 \\
13 & 1 & 0 & -0.589517 & -1.103567 & 0.874357 \\
14 & 6 & 0 & 0.615875 & 0.440927 & -0.000007 \\
15 & 1 & 0 & 0.589516 & 1.103574 & 0.874349 \\
16 & 1 & 0 & 0.589525 & 1.103558 & -0.874375 \\
17 & 6 & 0 & 1.913707 & -0.340015 & 0.000005 \\
18 & 1 & 0 & 1.940908 & -1.002949 & -0.874267 \\
19 & 1 & 0 & 1.940907 & -1.002923 & 0.874296 \\
20 & 6 & 0 & 3.146376 & 0.541998 & -0.000007 \\
21 & 1 & 0 & 3.114924 & 1.202461 & 0.873505 \\
22 & 1 & 0 & 3.114927 & 1.202434 & -0.873539 \\
23 & 6 & 0 & 4.433464 & -0.255879 & 0.000008 \\
24 & 1 & 0 & 4.492303 & -0.901233 & -0.879533 \\
25 & 1 & 0 & 5.315374 & 0.385207 & 0.000001 \\
26 & 1 & 0 & 4.492299 & -0.901208 & 0.879567
\end{tabular}

Zero-point correction=

0.243758 (Hartree/Particle)

Thermal correction to Energy=

0.254917

Thermal correction to Enthalpy=

0.255861

Thermal correction to Gibbs Free Energy=

Sum of electronic and zero-point Energies=

0.206545

Sum of electronic and thermal Energies=

$-315.301349$

$-315.290190$

Sum of electronic and thermal Enthalpies=

$-315.289246$

Sum of electronic and thermal Free Energies $=\quad-315.338561$

2233tetramethylbutane

Standard orientation:

\begin{tabular}{|c|c|c|c|}
\hline Center & Atomic & Atomic & Coordinates (Angstroms) \\
\hline Number & Number & Type & $\mathrm{X}$ \\
\hline
\end{tabular}




\begin{tabular}{|c|c|c|c|c|c|}
\hline 1 & 6 & 0 & -1.340696 & -0.404451 & -1.363713 \\
\hline 2 & 1 & 0 & -1.143507 & 0.349949 & -2.127169 \\
\hline 3 & 1 & 0 & -0.933853 & -1.355403 & -1.713885 \\
\hline 4 & 1 & 0 & -2.425186 & -0.521071 & -1.295957 \\
\hline 5 & 6 & 0 & -0.783392 & -0.000067 & 0.000012 \\
\hline 6 & 6 & 0 & 0.783355 & -0.000036 & -0.000013 \\
\hline 7 & 6 & 0 & 1.340502 & 0.681022 & 1.248848 \\
\hline 8 & 1 & 0 & 1.144198 & 1.754468 & 1.252267 \\
\hline 9 & 1 & 0 & 0.932649 & 0.258991 & 2.169726 \\
\hline 10 & 1 & 0 & 2.424855 & 0.549833 & 1.284419 \\
\hline 11 & 6 & 0 & -1.340656 & 1.383191 & 0.331618 \\
\hline 12 & 1 & 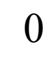 & -1.144060 & 1.666897 & 1.366857 \\
\hline 13 & 1 & 0 & -0.933260 & 2.162035 & -0.316398 \\
\hline 14 & 1 & 0 & -2.425059 & 1.382985 & 0.196063 \\
\hline 15 & 6 & 0 & -1.340614 & -0.978893 & 1.032086 \\
\hline 16 & 1 & 0 & -2.425004 & -0.861351 & 1.099902 \\
\hline 17 & 1 & 0 & -1.144081 & -2.017236 & 0.759921 \\
\hline 18 & 1 & 0 & -0.932992 & -0.807330 & 2.030525 \\
\hline 19 & 6 & 0 & 1.340609 & 0.741117 & -1.214114 \\
\hline 20 & 1 & 0 & 2.424990 & 0.837219 & -1.118181 \\
\hline 21 & 1 & 0 & 1.144242 & 0.207668 & -2.145650 \\
\hline 22 & 1 & 0 & 0.933010 & 1.749780 & -1.308807 \\
\hline 23 & 6 & 0 & 1.340861 & -1.421935 & -0.034717 \\
\hline 24 & 1 & 0 & 2.425315 & -1.386757 & -0.165218 \\
\hline 25 & 1 & 0 & 1.143982 & -1.962154 & 0.892793 \\
\hline 26 & 1 & 0 & 0.933952 & -2.008201 & -0.86125 \\
\hline
\end{tabular}

$\begin{array}{lc}\text { Zero-point correction= } & 0.243171 \text { (Hartree/Particle) } \\ \text { Thermal correction to Energy= } & 0.253407 \\ \text { Thermal correction to Enthalpy= } & 0.254351 \\ \text { Thermal correction to Gibbs Free Energy= } & 0.210100 \\ \text { Sum of electronic and zero-point Energies }= & -315.305854 \\ \text { Sum of electronic and thermal Energies }= & -315.295618 \\ \text { Sum of electronic and thermal Enthalpies }= & -315.294674 \\ \text { Sum of electronic and thermal Free Energies }= & -315.338925 \\ \text { CAM-B3LYP/ccpVTZ } & \end{array}$

Ethane

Standard orientation:

\begin{tabular}{|c|c|c|c|c|}
\hline \multirow{2}{*}{$\begin{array}{l}\text { Center } \\
\text { Number }\end{array}$} & Atomic & \multirow{2}{*}{$\begin{array}{l}\text { Atomic } \\
\text { Type }\end{array}$} & \multicolumn{2}{|c|}{ Coordinates (Angstroms) } \\
\hline & Numbe & & $\mathrm{X}$ & Z \\
\hline 1 & 6 & -0.000000 & 0.000000 & 0.760718 \\
\hline 2 & 1 & -0.507525 & 0.879093 & 1.157557 \\
\hline 3 & 1 & 1.015079 & -0.000017 & 1.157557 \\
\hline 4 & 1 & -0.507555 & -0.879076 & 1.157557 \\
\hline 5 & 6 & 0.000000 & 0.000000 & -0.760718 \\
\hline 6 & 1 & 0.507525 & 0.879093 & -1.157557 \\
\hline 7 & 1 & 0.507555 & -0.879076 & -1.157557 \\
\hline
\end{tabular}


$\begin{array}{llllll}8 & 1 & 0 & -1.015079 & -0.000017 & -1.157557\end{array}$

Zero-point correction $=$

0.074988 (Hartree/Particle)

Thermal correction to Energy=

0.078464

Thermal correction to Enthalpy=

0.079408

Thermal correction to Gibbs Free Energy $=\quad 0.053579$

Sum of electronic and zero-point Energies $=$

$-79.729644$

Sum of electronic and thermal Energies=

$-79.726168$

Sum of electronic and thermal Enthalpies=

$-79.725224$

Sum of electronic and thermal Free Energies=

$-79.751053$

Methane

Standard orientation:

\begin{tabular}{lrrrrr} 
Center & \multicolumn{2}{c}{ Atomic } & \multicolumn{2}{c}{ Atomic } & \multicolumn{2}{c}{ Coordinates (Angstroms) } \\
Number & Number & Type & X & Y & Z \\
-1 & 6 & 0 & 0.000000 & 0.000000 & 0.000000 \\
2 & 1 & 0 & 0.627616 & 0.627616 & 0.627616 \\
3 & 1 & 0 & -0.627616 & -0.627616 & 0.627616 \\
4 & 1 & 0 & -0.627616 & 0.627616 & -0.627616 \\
5 & 1 & 0 & 0.627616 & -0.627616 & -0.627616
\end{tabular}

Zero-point correction=

Thermal correction to Energy=

0.044877 (Hartree/Particle)

Thermal correction to Enthalpy=

0.047745

Thermal correction to Gibbs Free Energy=

0.048689

Sum of electronic and zero-point Energies=

0.027569

Sum of electronic and thermal Energies $=$

$-40.459108$

Sum of electronic and thermal Enthalpies=

$-40.456240$

Sum of electronic and thermal Free Energies=

$-40.455295$

Propane

$-40.476415$

Standard orientation:

\begin{tabular}{cccccc} 
Center & \multicolumn{2}{c}{ Atomic } & \multicolumn{2}{c}{ Atomic } & \multicolumn{3}{c}{ Coordinates (Angstroms) } \\
Number & Number & Type & X & Y & Z \\
\hline 1 & 6 & 0 & -1.266726 & -0.258504 & 0.000000 \\
2 & 1 & 0 & -2.163086 & 0.361426 & -0.000023 \\
3 & 1 & 0 & -1.308001 & -0.902541 & 0.879769 \\
4 & 1 & 0 & -1.307982 & -0.902578 & -0.879742 \\
5 & 6 & 0 & 0.000000 & 0.584526 & 0.000000 \\
6 & 1 & 0 & -0.000001 & 1.241146 & 0.872589 \\
7 & 1 & 0 & 0.000001 & 1.241131 & -0.872600 \\
8 & 6 & 0 & 1.266726 & -0.258504 & 0.000000 \\
9 & 1 & 0 & 1.308003 & -0.902537 & 0.879772 \\
10 & 1 & 0 & 1.307976 & -0.902582 & -0.879740 \\
11 & 1 & 0 & 2.163088 & 0.361424 & -0.000029
\end{tabular}

Zero-point correction $=$

0.103915 (Hartree/Particle)

Thermal correction to Energy=

0.108422 
Thermal correction to Enthalpy=

0.109366

Thermal correction to Gibbs Free Energy=

0.078285

Sum of electronic and zero-point Energies=

$-119.003726$

Sum of electronic and thermal Energies $=$

$-118.999219$

Sum of electronic and thermal Enthalpies=

$-118.998275$

Sum of electronic and thermal Free Energies=

$-119.029357$

Butane

Standard orientation:

\begin{tabular}{|c|c|c|c|c|}
\hline \multirow{2}{*}{$\begin{array}{l}\text { Center } \\
\text { Number }\end{array}$} & Atomic & \multirow{2}{*}{$\begin{array}{l}\text { Atomic } \\
\text { Type }\end{array}$} & \multicolumn{2}{|c|}{ Coordinates (Angstroms } \\
\hline & Numbe & & $\mathrm{X}$ & Z \\
\hline 1 & 6 & 1.946946 & -0.119520 & 0.000023 \\
\hline 2 & 1 & 2.091226 & -0.748338 & -0.879773 \\
\hline 3 & 1 & 2.091183 & -0.748324 & 0.879836 \\
\hline 4 & 1 & 2.732921 & 0.635344 & 0.000036 \\
\hline 5 & 6 & 0.563001 & 0.512153 & -0.000026 \\
\hline 6 & 1 & 0.456215 & 1.161284 & 0.873137 \\
\hline 7 & 1 & 0.456247 & 1.161253 & -0.873216 \\
\hline 8 & 6 & -0.563001 & -0.512153 & -0.000026 \\
\hline 9 & 1 & -0.456247 & -1.161251 & -0.873218 \\
\hline 10 & 1 & -0.456215 & -1.161286 & 0.873135 \\
\hline 11 & 6 & -1.946946 & 0.119520 & 0.000023 \\
\hline 12 & 1 & -2.091225 & 0.748340 & -0.879772 \\
\hline 13 & 1 & -2.732921 & -0.635344 & 0.000033 \\
\hline 14 & 1 & -2.091183 & 0.748322 & 0.879838 \\
\hline
\end{tabular}

Zero-point correction $=$

0.132642 (Hartree/Particle)

Thermal correction to Energy=

0.138394

Thermal correction to Enthalpy=

0.139338

Thermal correction to Gibbs Free Energy=

Sum of electronic and zero-point Energies=

0.104664

$-158.277918$

Sum of electronic and thermal Energies=

$-158.272165$

Sum of electronic and thermal Enthalpies=

$-158.271221$

Sum of electronic and thermal Free Energies=

$-158.305895$

Isobutane

Standard orientation:

\begin{tabular}{|c|c|c|c|c|}
\hline \multirow{2}{*}{$\begin{array}{l}\text { Center } \\
\text { Number }\end{array}$} & Atomic & \multirow{2}{*}{$\begin{array}{l}\text { Atomic } \\
\text { Type }\end{array}$} & \multicolumn{2}{|c|}{ Coordinates (Angstroms) } \\
\hline & Number & & $\mathrm{X}$ & Z \\
\hline 1 & 6 & -1.446880 & -0.098604 & -0.095258 \\
\hline 2 & 1 & -2.038801 & 0.744658 & 0.261734 \\
\hline 3 & 1 & -1.498678 & -0.102052 & -1.186168 \\
\hline 4 & 1 & -1.918835 & -1.014464 & 0.261617 \\
\hline 5 & 6 & -0.000001 & -0.000002 & 0.372742 \\
\hline 6 & 1 & 0.000013 & 0.000005 & 1.466726 \\
\hline 7 & 6 & 0.638047 & 1.302322 & -0.095260 \\
\hline 8 & 1 & 1.664381 & 1.393164 & 0.261513 \\
\hline 9 & 1 & 0.660699 & 1.348988 & -1.186167 \\
\hline
\end{tabular}




$\begin{array}{cccccc}10 & 1 & 0 & 0.081031 & 2.168997 & 0.261821 \\ 11 & 6 & 0 & 0.808836 & -1.203716 & -0.095262 \\ 12 & 1 & 0 & 1.838039 & -1.154418 & 0.261414 \\ 13 & 1 & 0 & 0.374634 & -2.137957 & 0.261902 \\ 14 & 1 & 0 & 0.837510 & -1.246921 & -1.186168\end{array}$

Zero-point correction=

Thermal correction to Energy=

Thermal correction to Enthalpy=

Thermal correction to Gibbs Free Energy=

Sum of electronic and zero-point Energies=

Sum of electronic and thermal Energies=

Sum of electronic and thermal Enthalpies=

Sum of electronic and thermal Free Energies=

Pentane
0.132182 (Hartree/Particle)

0.137853

0.138797

\subsection{5}

$-158.279665$

$-158.273994$

$-158.273050$

$-158.307252$

Standard orientation:

\begin{tabular}{cccccc} 
Center & Atomic & \multicolumn{2}{c}{ Atomic } & \multicolumn{3}{c}{ Coordinates } \\
Number & Number & Type & X & Y & Z \\
- & Nutroms \\
\hline 1 & 6 & 0 & 2.539763 & 0.323253 & -0.000007 \\
2 & 1 & 0 & 2.582033 & 0.967012 & -0.879785 \\
3 & 1 & 0 & 2.582033 & 0.967048 & 0.879746 \\
4 & 1 & 0 & 3.435927 & -0.296845 & 0.000006 \\
5 & 6 & 0 & 1.274038 & -0.520923 & 0.000011 \\
6 & 1 & 0 & 1.273177 & -1.178532 & -0.873164 \\
7 & 1 & 0 & 1.273183 & -1.178504 & 0.873206 \\
8 & 6 & 0 & 0.000000 & 0.311688 & 0.000005 \\
9 & 1 & 0 & -0.000008 & 0.970773 & 0.873727 \\
10 & 1 & 0 & 0.000008 & 0.970780 & -0.873712 \\
11 & 6 & 0 & -1.274038 & -0.520923 & -0.000011 \\
12 & 1 & 0 & -1.273180 & -1.178537 & 0.873161 \\
13 & 1 & 0 & -1.273180 & -1.178499 & -0.873209 \\
14 & 6 & 0 & -2.539763 & 0.323253 & 0.000003 \\
15 & 1 & 0 & -3.435927 & -0.296845 & -0.000035 \\
16 & 1 & 0 & -2.582044 & 0.966995 & 0.879792 \\
17 & 1 & 0 & -2.582021 & 0.967065 & -0.879739
\end{tabular}

Zero-point correction=

Thermal correction to Energy=

Thermal correction to Enthalpy=

Thermal correction to Gibbs Free Energy=

Sum of electronic and zero-point Energies=

Sum of electronic and thermal Energies $=$

Sum of electronic and thermal Enthalpies=

Sum of electronic and thermal Free Energies=

Neopentane

Standard orientation:
0.161338 (Hartree/Particle)

0.168384

0.169328

0.131061

$-197.552043$

$-197.544998$

$-197.544053$

$-197.582320$

Center Atomic Atomic Coordinates (Angstroms)




\begin{tabular}{cccccc} 
Number & Number & Type & $X$ & $Y$ & $Z$ \\
\hline 1 & 6 & 0 & 1.114640 & 0.978794 & -0.367746 \\
2 & 1 & 0 & 0.705408 & 1.900631 & -0.783443 \\
3 & 1 & 0 & 1.709605 & 1.242270 & 0.507844 \\
4 & 1 & 0 & 1.786514 & 0.546586 & -1.110603 \\
5 & 6 & 0 & 0.000019 & 0.000024 & -0.000007 \\
6 & 6 & 0 & -0.918178 & 0.640948 & 1.040094 \\
7 & 1 & 0 & -1.724840 & -0.037257 & 1.321876 \\
8 & 1 & 0 & -0.366037 & 0.897829 & 1.945200 \\
9 & 1 & 0 & -1.370193 & 1.555445 & 0.653511 \\
10 & 6 & 0 & -0.808690 & -0.344265 & -1.250261 \\
11 & 1 & 0 & -0.176982 & -0.804469 & -2.011327 \\
12 & 1 & 0 & -1.613245 & -1.042947 & -1.016588 \\
13 & 1 & 0 & -1.258128 & 0.549697 & -1.684877 \\
14 & 6 & 0 & 0.612226 & -1.275479 & 0.577914 \\
15 & 1 & 0 & 1.273606 & -1.755220 & -0.144921 \\
16 & 1 & 0 & 1.196642 & -1.058990 & 1.473269 \\
17 & 1 & 0 & -0.162457 & -1.993709 & 0.850093
\end{tabular}

Zero-point correction=

Thermal correction to Energy=

Thermal correction to Enthalpy=

Thermal correction to Gibbs Free Energy=

Sum of electronic and zero-point Energies=

Sum of electronic and thermal Energies $=$

Sum of electronic and thermal Enthalpies=

Sum of electronic and thermal Free Energies=

Hexane
0.160099 (Hartree/Particle)

$$
0.166974
$$

0.167918

$$
\begin{gathered}
0.131031 \\
-197.555867 \\
-197.548993 \\
-197.548049 \\
-197.584935
\end{gathered}
$$

\begin{tabular}{|c|c|c|c|c|c|}
\hline \multirow{2}{*}{$\begin{array}{l}\text { Center } \\
\text { Number }\end{array}$} & Atomic & \multirow{2}{*}{\multicolumn{2}{|c|}{$\begin{array}{l}\text { Atomic } \\
\text { Type }\end{array}$}} & \multicolumn{2}{|c|}{ Coordinates (Angstrom } \\
\hline & Numbe & & & $\mathrm{X}$ & $\mathrm{Z}$ \\
\hline 1 & 6 & 0 & -3.198418 & 0.207434 & 0.000021 \\
\hline 2 & 1 & 0 & -3.285128 & 0.846689 & 0.879842 \\
\hline 3 & 1 & 0 & -3.285104 & 0.846844 & -0.879690 \\
\hline 4 & 1 & 0 & -4.049604 & -0.473113 & -0.000053 \\
\hline 5 & 6 & 0 & -1.877404 & -0.547242 & -0.000032 \\
\hline 6 & 1 & 0 & -1.831152 & -1.203262 & 0.873081 \\
\hline 7 & 1 & 0 & -1.831163 & -1.203164 & -0.873219 \\
\hline 8 & 6 & 0 & -0.663926 & 0.371639 & 0.000008 \\
\hline 9 & 1 & 0 & -0.710570 & 1.028870 & -0.873702 \\
\hline 10 & 1 & 0 & -0.710591 & 1.028828 & 0.873747 \\
\hline 11 & 6 & 0 & 0.663926 & -0.371639 & 0.000010 \\
\hline 12 & 1 & 0 & 0.710569 & -1.028874 & -0.873696 \\
\hline 13 & 1 & 0 & 0.710593 & -1.028824 & 0.873753 \\
\hline 14 & 6 & 0 & 1.877404 & 0.547242 & -0.000032 \\
\hline 15 & 1 & 0 & 1.831152 & 1.203263 & 0.873081 \\
\hline 16 & 1 & 0 & 1.831163 & 1.203162 & -0.873220 \\
\hline
\end{tabular}

Standard orientation: 


$\begin{array}{rrrrrr}17 & 6 & 0 & 3.198418 & -0.207434 & 0.000020 \\ 18 & 1 & 0 & 3.285117 & -0.846825 & -0.879704 \\ 19 & 1 & 0 & 4.049604 & 0.473113 & -0.000024 \\ 20 & 1 & 0 & 3.285115 & -0.846708 & 0.879829\end{array}$

Zero-point correction=

Thermal correction to Energy=

Thermal correction to Enthalpy=

Thermal correction to Gibbs Free Energy=

Sum of electronic and zero-point Energies=

Sum of electronic and thermal Energies $=$

Sum of electronic and thermal Enthalpies=

Sum of electronic and thermal Free Energies=

22dimethylbutane

Standard orientation:

\begin{tabular}{|c|c|c|c|c|}
\hline Center & Atomic & Atomic & Coordinate & str \\
\hline Number & Numbe & Type & $X$ & Z \\
\hline 1 & 6 & -1.607521 & 0.978471 & 0.003005 \\
\hline 2 & 1 & -1.582989 & 1.618052 & 0.886461 \\
\hline 3 & 1 & -1.583215 & 1.623181 & -0.876725 \\
\hline 4 & 1 & -2.559978 & 0.446968 & 0.001577 \\
\hline 5 & 6 & -0.432584 & -0.000355 & 0.000016 \\
\hline 6 & 6 & 0.868917 & 0.820709 & 0.001592 \\
\hline 7 & 1 & 0.852557 & 1.480463 & -0.869945 \\
\hline 8 & 1 & 0.852665 & 1.477008 & 0.875739 \\
\hline 9 & 6 & 2.173555 & 0.035043 & 0.000049 \\
\hline 10 & 1 & 2.266603 & -0.598728 & -0.881085 \\
\hline 11 & 1 & 3.024919 & 0.715195 & 0.000623 \\
\hline 12 & 1 & 2.267208 & -0.601025 & 0.879453 \\
\hline 13 & 6 & -0.520505 & -0.881599 & 1.246459 \\
\hline 14 & 1 & 0.267049 & -1.633915 & 1.267500 \\
\hline 15 & 1 & -0.439230 & -0.283899 & 2.155783 \\
\hline 16 & 1 & -1.475950 & -1.406742 & 1.278069 \\
\hline 17 & 6 & -0.521491 & -0.875050 & -1.250964 \\
\hline 18 & 1 & -1.476669 & -1.400578 & -1.284254 \\
\hline 19 & 1 & -0.441640 & -0.272476 & -2.157188 \\
\hline 20 & 1 & 0.266446 & -1.626811 & -1.276956 \\
\hline
\end{tabular}

Zero-point correction=

Thermal correction to Energy=

Thermal correction to Enthalpy=

Thermal correction to Gibbs Free Energy=

Sum of electronic and zero-point Energies=

Sum of electronic and thermal Energies $=$

Sum of electronic and thermal Enthalpies=

Sum of electronic and thermal Free Energies=

Heptane
0.188966 (Hartree/Particle)

$$
0.197160
$$

0.198105

$$
\begin{gathered}
0.157734 \\
-236.826889 \\
-236.818695 \\
-236.817751 \\
-236.858122
\end{gathered}
$$

0.198372

.157466

$-236.826212$

$-236.816899$

$-236.858749$ 


\begin{tabular}{|c|c|c|c|c|}
\hline \multirow{2}{*}{$\begin{array}{l}\text { Center } \\
\text { Number }\end{array}$} & Atomic & \multirow{2}{*}{$\begin{array}{l}\text { Atomic } \\
\text { Type }\end{array}$} & \multicolumn{2}{|c|}{ Coordinates (Angstroms } \\
\hline & Numbe & & $\mathrm{X}$ & $\mathrm{Z}$ \\
\hline 1 & 6 & -3.812446 & -0.351893 & -0.000008 \\
\hline 2 & 1 & -3.854922 & -0.995597 & -0.879707 \\
\hline 3 & 1 & -3.854920 & -0.995636 & 0.879665 \\
\hline 4 & 1 & -4.708267 & 0.268484 & 0.000008 \\
\hline 5 & 6 & -2.546709 & 0.492137 & 0.000010 \\
\hline 6 & 1 & -2.545564 & 1.149639 & -0.873117 \\
\hline 7 & 1 & -2.545568 & 1.149609 & 0.873161 \\
\hline 8 & 6 & -1.273020 & -0.341148 & -0.000001 \\
\hline 9 & 1 & -1.274201 & -0.999866 & 0.873680 \\
\hline 10 & 1 & -1.274206 & -0.999853 & -0.873692 \\
\hline 11 & 6 & 0.000000 & 0.492538 & 0.000000 \\
\hline 12 & 1 & -0.000002 & 1.151030 & 0.873721 \\
\hline 13 & 1 & 0.000002 & 1.151026 & -0.873723 \\
\hline 14 & 6 & 1.273020 & -0.341148 & 0.000005 \\
\hline 15 & 1 & 1.274200 & -0.999871 & -0.873672 \\
\hline 16 & 1 & 1.274207 & -0.999848 & 0.873700 \\
\hline 17 & 6 & 2.546709 & 0.492137 & -0.000011 \\
\hline 18 & 1 & 2.545567 & 1.149640 & 0.873117 \\
\hline 19 & 1 & 2.545565 & 1.149608 & -0.873162 \\
\hline 20 & 6 & 3.812446 & -0.351893 & 0.000003 \\
\hline 21 & 1 & 3.854920 & -0.995634 & -0.879671 \\
\hline 22 & 1 & 4.708267 & 0.268484 & -0.000011 \\
\hline 23 & 1 & 3.854923 & -0.995600 & 0.879701 \\
\hline
\end{tabular}

Zero-point correction=

Thermal correction to Energy=

Thermal correction to Enthalpy=

Thermal correction to Gibbs Free Energy=

Sum of electronic and zero-point Energies=

Sum of electronic and thermal Energies $=$

Sum of electronic and thermal Enthalpies=

Sum of electronic and thermal Free Energies=

223trimethylbutane
0.218648 (Hartree/Particle) 0.228370

0.229314

0.183816

$-276.100397$

$-276.090676$

$-276.089732$

$-276.135229$

Standard orientation:

\begin{tabular}{|c|c|c|c|c|}
\hline \multirow{2}{*}{$\begin{array}{l}\text { Center } \\
\text { Number }\end{array}$} & Atomic & \multirow{2}{*}{$\begin{array}{l}\text { Atomic } \\
\text { Type }\end{array}$} & \multicolumn{2}{|c|}{ Coordinates (Angstroms) } \\
\hline & Number & & $\mathrm{X}$ & $\mathrm{Z}$ \\
\hline 1 & 6 & -1.348157 & -1.235825 & -0.563773 \\
\hline 2 & 1 & -1.221893 & -1.302455 & -1.645779 \\
\hline 3 & 1 & -0.975813 & -2.159225 & -0.124073 \\
\hline 4 & 1 & -2.417654 & -1.185483 & -0.357343 \\
\hline 5 & 6 & -0.639769 & 0.000015 & -0.000398 \\
\hline 6 & 6 & 0.849406 & 0.000009 & -0.447392 \\
\hline 7 & 1 & 0.823463 & 0.000030 & -1.542109 \\
\hline 8 & 6 & 1.626256 & 1.243287 & -0.016570 \\
\hline
\end{tabular}




$\begin{array}{cccccc}9 & 1 & 0 & 1.710715 & 1.305738 & 1.068876 \\ 10 & 1 & 0 & 2.639855 & 1.205239 & -0.415686 \\ 11 & 1 & 0 & 1.172227 & 2.165476 & -0.372229 \\ 12 & 6 & 0 & 1.626221 & -1.243295 & -0.016620 \\ 13 & 1 & 0 & 1.710901 & -1.305654 & 1.068816 \\ 14 & 1 & 0 & 1.172041 & -2.165486 & -0.372088 \\ 15 & 1 & 0 & 2.639744 & -1.205374 & -0.415942 \\ 16 & 6 & 0 & -0.787164 & -0.000372 & 1.522612 \\ 17 & 1 & 0 & -0.334079 & 0.881279 & 1.974480 \\ 18 & 1 & 0 & -1.843023 & -0.000287 & 1.796064 \\ 19 & 1 & 0 & -0.334332 & -0.882400 & 1.973997 \\ 20 & 6 & 0 & -1.348010 & 1.236189 & -0.563187 \\ 21 & 1 & 0 & -1.221639 & 1.303405 & -1.645148 \\ 22 & 1 & 0 & -2.417537 & 1.185816 & -0.356906 \\ 23 & 1 & 0 & -0.975668 & 2.159339 & -0.122956\end{array}$

Zero-point correction=

Thermal correction to Energy=

Thermal correction to Enthalpy=

Thermal correction to Gibbs Free Energy=

Sum of electronic and zero-point Energies=

Sum of electronic and thermal Energies $=$

Sum of electronic and thermal Enthalpies=

Sum of electronic and thermal Free Energies=

Octane
0.217404 (Hartree/Particle)
0.226919
0.227863
0.183894
$-276.097698$
$-276.088183$
$-276.087239$
$-276.131208$

Standard orientation:

\begin{tabular}{cccccc} 
Center & \multicolumn{2}{c}{ Atomic } & \multicolumn{2}{c}{ Atomic } & \multicolumn{3}{c}{ Coordinates } \\
Number & Number & Type & X & Y & $Z$ \\
- & Nustroms) \\
\hline 1 & 6 & 0 & -4.462188 & 0.255342 & 0.000015 \\
2 & 1 & 0 & -4.529509 & 0.896926 & 0.879718 \\
3 & 1 & 0 & -4.529513 & 0.896976 & -0.879651 \\
4 & 1 & 0 & -5.333383 & -0.399172 & -0.000002 \\
5 & 6 & 0 & -3.164790 & -0.539163 & -0.000013 \\
6 & 1 & 0 & -3.138260 & -1.196140 & 0.873108 \\
7 & 1 & 0 & -3.138270 & -1.196099 & -0.873165 \\
8 & 6 & 0 & -1.924216 & 0.342666 & -0.000000 \\
9 & 1 & 0 & -1.950835 & 1.000856 & -0.873684 \\
10 & 1 & 0 & -1.950837 & 1.000836 & 0.873698 \\
11 & 6 & 0 & -0.619969 & -0.441247 & -0.000004 \\
12 & 1 & 0 & -0.594569 & -1.099248 & -0.873693 \\
13 & 1 & 0 & -0.594563 & -1.099248 & 0.873684 \\
14 & 6 & 0 & 0.619969 & 0.441247 & -0.000008 \\
15 & 1 & 0 & 0.594562 & 1.099258 & 0.873672 \\
16 & 1 & 0 & 0.594570 & 1.099238 & -0.873704 \\
17 & 6 & 0 & 1.924216 & -0.342666 & 0.000006 \\
18 & 1 & 0 & 1.950837 & -1.000861 & -0.873673 \\
19 & 1 & 0 & 1.950835 & -1.000830 & 0.873709 \\
20 & 6 & 0 & 3.164790 & 0.539163 & -0.000008
\end{tabular}




$\begin{array}{rrrrrr}21 & 1 & 0 & 3.138264 & 1.196136 & 0.873116 \\ 22 & 1 & 0 & 3.138267 & 1.196103 & -0.873157 \\ 23 & 6 & 0 & 4.462188 & -0.255342 & 0.000010 \\ 24 & 1 & 0 & 4.529513 & -0.896968 & -0.879662 \\ 25 & 1 & 0 & 5.333383 & 0.399172 & -0.000001 \\ 26 & 1 & 0 & 4.529509 & -0.896934 & 0.879707\end{array}$

Zero-point correction=

Thermal correction to Energy=

Thermal correction to Enthalpy=

Thermal correction to Gibbs Free Energy=

Sum of electronic and zero-point Energies=

Sum of electronic and thermal Energies $=$

Sum of electronic and thermal Enthalpies=

Sum of electronic and thermal Free Energies=

2233tetramethylbutane

\begin{tabular}{|c|c|c|c|c|}
\hline \multirow{2}{*}{$\begin{array}{l}\text { Center } \\
\text { Number }\end{array}$} & Atomic & \multirow{2}{*}{$\begin{array}{l}\text { Atomic } \\
\text { Type }\end{array}$} & \multicolumn{2}{|c|}{ Coordinates (Angstroms } \\
\hline & Numbe & & $X$ & Z \\
\hline 1 & 6 & -1.345357 & -1.421833 & -0.139489 \\
\hline 2 & 1 & -0.944070 & -1.939145 & -1.010010 \\
\hline 3 & 1 & -1.137186 & -2.029219 & 0.739539 \\
\hline 4 & 1 & -2.428596 & -1.380700 & -0.257126 \\
\hline 5 & 6 & -0.787651 & 0.000097 & -0.000003 \\
\hline 6 & 6 & 0.787682 & 0.000078 & 0.000112 \\
\hline 7 & 6 & 1.345652 & 1.364012 & 0.425089 \\
\hline 8 & 1 & 0.945047 & 2.179492 & -0.175762 \\
\hline 9 & 1 & 1.137198 & 1.581728 & 1.471080 \\
\hline 10 & 1 & 2.428956 & 1.371551 & 0.301225 \\
\hline 11 & 6 & -1.345334 & 0.831646 & -1.161785 \\
\hline 12 & 1 & -0.944650 & 1.844436 & -1.174331 \\
\hline 13 & 1 & -1.136592 & 0.374222 & -2.127260 \\
\hline 14 & 1 & -2.428641 & 0.912433 & -1.067759 \\
\hline 15 & 6 & -1.345783 & 0.590216 & 1.300972 \\
\hline 16 & 1 & -2.429102 & 0.468409 & 1.323526 \\
\hline 17 & 1 & -0.945380 & 0.094475 & 2.184358 \\
\hline 18 & 1 & -1.137177 & 1.655052 & 1.387980 \\
\hline 19 & 6 & 1.345517 & -0.313968 & -1.393645 \\
\hline 20 & 1 & 2.428781 & -0.425325 & -1.338280 \\
\hline 21 & 1 & 0.944547 & -1.241909 & -1.799386 \\
\hline 22 & 1 & 1.137187 & 0.483134 & -2.105100 \\
\hline 23 & 6 & 1.345275 & -1.050155 & 0.968811 \\
\hline 24 & 1 & 2.428613 & -0.947072 & 1.037194 \\
\hline 25 & 1 & 0.944735 & -0.937363 & 1.975443 \\
\hline 26 & 1 & 1.136320 & -2.064755 & 0.634303 \\
\hline
\end{tabular}

Zero-point correction=

Thermal correction to Energy=
0.247289 (Hartree/Particle)

0.258358

0.259303

0.210210

$-315.374587$

$-315.363518$

$-315.362574$

$-315.411666$

Standard orientation: 
Thermal correction to Enthalpy=

0.257307

Thermal correction to Gibbs Free Energy $=$

0.212549

Sum of electronic and zero-point Energies=

$-315.367353$

Sum of electronic and thermal Energies $=$

$-315.356929$

$-315.355985$

$-315.400743$

Sum of electronic and thermal Free Energies=

wB97XD/ccpvTZ

ethane

Standard orientation:

\begin{tabular}{|c|c|c|c|c|}
\hline \multirow{2}{*}{$\begin{array}{l}\text { Center } \\
\text { Number }\end{array}$} & Atomic & \multirow{2}{*}{$\begin{array}{l}\text { Atomic } \\
\text { Type }\end{array}$} & \multicolumn{2}{|c|}{ Coordinates (Angstrom } \\
\hline & Numbe & & $X$ & $\mathrm{Z}$ \\
\hline 1 & 6 & 0.000000 & -0.000000 & 0.761693 \\
\hline 2 & 1 & 0.507974 & 0.880065 & 1.157297 \\
\hline 3 & 1 & 0.508171 & -0.879951 & 1.157297 \\
\hline 4 & 1 & -1.016146 & -0.000114 & - 1.157297 \\
\hline 5 & 6 & 0.000000 & -0.000000 & -0.761693 \\
\hline 6 & 1 & 1.016146 & -0.000114 & -1.157297 \\
\hline 7 & 1 & -0.508171 & -0.879951 & -1.157297 \\
\hline 8 & 1 & -0.507974 & 0.880065 & -1.157297 \\
\hline
\end{tabular}

Zero-point correction=

0.074985 (Hartree/Particle)

Thermal correction to Energy=

0.078462

Thermal correction to Enthalpy=

0.079406

Thermal correction to Gibbs Free Energy $=\quad 0.053575$

Sum of electronic and zero-point Energies $=\quad-79.757738$

Sum of electronic and thermal Energies $=\quad-79.754261$

Sum of electronic and thermal Enthalpies $=\quad-79.753317$

Sum of electronic and thermal Free Energies $=\quad-79.779148$

Methane

Standard orientation:

\begin{tabular}{|c|c|c|c|c|}
\hline \multirow{2}{*}{$\begin{array}{l}\text { Center } \\
\text { Number }\end{array}$} & \multirow{2}{*}{$\begin{array}{l}\text { Atomic } \\
\text { Numbe }\end{array}$} & \multirow{2}{*}{$\begin{array}{r}\text { Atomic } \\
\text { Type }\end{array}$} & \multicolumn{2}{|c|}{ Coordinates (Angstroms) } \\
\hline & & & $\mathrm{X}$ & $\mathrm{Z}$ \\
\hline 1 & 6 & 0.000000 & 0.000000 & 0.000000 \\
\hline 2 & 1 & 0.627970 & 0.627970 & 0.627970 \\
\hline 3 & 1 & -0.627970 & -0.627970 & 0.627970 \\
\hline 4 & 1 & -0.627970 & 0.627970 & -0.627970 \\
\hline 5 & 1 & 0.627970 & -0.627970 & -0.627970 \\
\hline \multicolumn{3}{|c|}{ Zero-point correction $=$} & \multicolumn{2}{|c|}{0.044890 (Hartree/Particle) } \\
\hline \multicolumn{3}{|c|}{ Thermal correction to Energy= } & \multicolumn{2}{|c|}{0.047758} \\
\hline \multicolumn{3}{|c|}{ Thermal correction to Enthalpy= } & \multicolumn{2}{|c|}{0.048702} \\
\hline \multicolumn{4}{|c|}{ Thermal correction to Gibbs Free Energy $=$} & 0.027580 \\
\hline \multicolumn{4}{|c|}{ Sum of electronic and zero-point Energies $=$} & -40.474930 \\
\hline \multicolumn{4}{|c|}{ Sum of electronic and thermal Energies $=$} & -40.472062 \\
\hline \multicolumn{4}{|c|}{ Sum of electronic and thermal Enthalpies= } & -40.471118 \\
\hline
\end{tabular}


Sum of electronic and thermal Free Energies=

$-40.492239$

Propane

Standard orientation:

\begin{tabular}{cccccc}
$\begin{array}{c}\text { Center } \\
\text { Number }\end{array}$ & \multicolumn{2}{c}{ Atomic } & \multicolumn{2}{c}{ Atomic } & \multicolumn{2}{c}{ Coordinates (Angstrom } \\
Number & Type & X & Y & $Z$ \\
\hline 1 & 6 & 0 & -1.267674 & -0.259211 & 0.000000 \\
2 & 1 & 0 & -2.164473 & 0.360981 & -0.000029 \\
3 & 1 & 0 & -1.306508 & -0.903347 & 0.880645 \\
4 & 1 & 0 & -1.306483 & -0.903392 & -0.880613 \\
5 & 6 & 0 & 0.000000 & 0.586284 & 0.000000 \\
6 & 1 & 0 & -0.000001 & 1.242181 & 0.873630 \\
7 & 1 & 0 & 0.000001 & 1.242164 & -0.873641 \\
8 & 6 & 0 & 1.267674 & -0.259211 & 0.000000 \\
9 & 1 & 0 & 1.306518 & -0.903331 & 0.880657 \\
10 & 1 & 0 & 1.306470 & -0.903408 & -0.880602 \\
11 & 1 & 0 & 2.164474 & 0.360979 & -0.000052
\end{tabular}

Zero-point correction=

Thermal correction to Energy=

0.103899 (Hartree/Particle)

Thermal correction to Enthalpy=

0.108396

Thermal correction to Gibbs Free Energy=

0.109340

Sum of electronic and zero-point Energies=

0.078281

Sum of electronic and thermal Energies=

$-119.044532$

$-119.040035$

$-119.039091$

Sum of electronic and thermal Enthalpies=

$-119.070151$

Butane

Standard orientation:

\begin{tabular}{cccccc} 
Center & \multicolumn{2}{c}{ Atomic } & \multicolumn{2}{c}{ Atomic } & \multicolumn{2}{c}{ Coordinates } \\
Number & Number & Type & X & $Y$ & $Z$ \\
- \hdashline & 6 & 0 & 1.948301 & -0.119880 & 0.000033 \\
2 & 1 & 0 & 2.089961 & -0.749241 & -0.880659 \\
3 & 1 & 0 & 2.089902 & -0.749207 & 0.880757 \\
4 & 1 & 0 & 2.734678 & 0.635303 & 0.000044 \\
5 & 6 & 0 & 0.563149 & 0.513678 & -0.000037 \\
6 & 1 & 0 & 0.455970 & 1.162331 & 0.874124 \\
7 & 1 & 0 & 0.456019 & 1.162283 & -0.874241 \\
8 & 6 & 0 & -0.563149 & -0.513678 & -0.000037 \\
9 & 1 & 0 & -0.456019 & -1.162281 & -0.874242 \\
10 & 1 & 0 & -0.455970 & -1.162333 & 0.874123 \\
11 & 6 & 0 & -1.948301 & 0.119880 & 0.000033 \\
12 & 1 & 0 & -2.089960 & 0.749245 & -0.880656 \\
13 & 1 & 0 & -2.734678 & -0.635303 & 0.000039 \\
14 & 1 & 0 & -2.089903 & 0.749203 & 0.880760
\end{tabular}

Zero-point correction=

0.132587 (Hartree/Particle)

Thermal correction to Energy=

0.138347 
Thermal correction to Enthalpy=

0.139291

Thermal correction to Gibbs Free Energy $=$

0.104593

Sum of electronic and zero-point Energies=

$-158.331671$

Sum of electronic and thermal Energies=

$-158.325911$

Sum of electronic and thermal Enthalpies=

$-158.324967$

Sum of electronic and thermal Free Energies= $-158.359664$

Isobutane

Standard orientation:

\begin{tabular}{|c|c|c|c|c|}
\hline \multirow{2}{*}{$\begin{array}{l}\text { Center } \\
\text { Number }\end{array}$} & Atomic & \multirow{2}{*}{$\begin{array}{l}\text { Atomic } \\
\text { Type }\end{array}$} & \multicolumn{2}{|c|}{ Coordinates (Angstroms } \\
\hline & Numbe & & $\mathrm{X}$ & Z \\
\hline 1 & 6 & -0.615208 & -1.315087 & -0.095588 \\
\hline 2 & 1 & -1.640442 & -1.423710 & 0.261498 \\
\hline 3 & 1 & -0.636060 & -1.359098 & -1.187517 \\
\hline 4 & 1 & -0.041280 & -2.171713 & 0.261072 \\
\hline 5 & 6 & 0.000005 & 0.000027 & 0.374475 \\
\hline 6 & 1 & 0.000024 & 0.000017 & 1.468700 \\
\hline 7 & 6 & -0.831308 & 1.190331 & -0.095594 \\
\hline 8 & 1 & -0.412494 & 2.132587 & 0.261009 \\
\hline 9 & 1 & -0.859415 & 1.230039 & -1.187519 \\
\hline 10 & 1 & -1.859987 & 1.121755 & 0.261514 \\
\hline 11 & 6 & 1.446519 & 0.124759 & -0.095589 \\
\hline 12 & 1 & 1.901643 & 1.049779 & 0.261643 \\
\hline 13 & 1 & 2.052988 & -0.709228 & 0.260890 \\
\hline 14 & 1 & 1.494971 & 0.129384 & -1.187517 \\
\hline
\end{tabular}

Zero-point correction $=$

0.132162 (Hartree/Particle)

Thermal correction to Energy=

0.137807

Thermal correction to Enthalpy=

0.138751

Thermal correction to Gibbs Free Energy=

Sum of electronic and zero-point Energies=

0.104605

$-158.333732$

$-158.328087$

Sum of electronic and thermal Energies=

$-158.327143$

Sum of electronic and thermal Enthalpies $=$

$-158.361289$

Pentane

Standard orientation:

\begin{tabular}{|c|c|c|c|c|}
\hline \multirow{2}{*}{$\begin{array}{l}\text { Center } \\
\text { Number }\end{array}$} & Atomic & \multirow{2}{*}{$\begin{array}{l}\text { Atomic } \\
\text { Type }\end{array}$} & \multicolumn{2}{|c|}{ Coordinates (Angstroms) } \\
\hline & Numbe & & $X$ & Z \\
\hline 1 & 6 & 2.540942 & 0.324187 & -0.000009 \\
\hline 2 & 1 & 2.580012 & 0.968087 & -0.880679 \\
\hline 3 & 1 & 2.580027 & 0.968086 & 0.880662 \\
\hline 4 & 1 & 3.437917 & -0.295622 & -0.000017 \\
\hline 5 & 6 & 1.274765 & -0.522579 & 0.000003 \\
\hline 6 & 1 & 1.273246 & -1.179680 & -0.874245 \\
\hline 7 & 1 & 1.273261 & -1.179679 & 0.874251 \\
\hline 8 & 6 & 0.000000 & 0.312561 & 0.000016 \\
\hline 9 & 1 & -0.000004 & 0.971460 & 0.874751 \\
\hline
\end{tabular}




$\begin{array}{rrrrrr}10 & 1 & 0 & 0.000004 & 0.971492 & -0.874694 \\ 11 & 6 & 0 & -1.274765 & -0.522579 & -0.000003 \\ 12 & 1 & 0 & -1.273261 & -1.179692 & 0.874237 \\ 13 & 1 & 0 & -1.273246 & -1.179666 & -0.874259 \\ 14 & 6 & 0 & -2.540942 & 0.324187 & -0.000006 \\ 15 & 1 & 0 & -3.437917 & -0.295622 & -0.000046 \\ 16 & 1 & 0 & -2.580037 & 0.968058 & 0.880684 \\ 17 & 1 & 0 & -2.580002 & 0.968115 & -0.880658\end{array}$

Zero-point correction=

Thermal correction to Energy=

Thermal correction to Enthalpy=

Thermal correction to Gibbs Free Energy=

Sum of electronic and zero-point Energies=

Sum of electronic and thermal Energies=

Sum of electronic and thermal Enthalpies=

Sum of electronic and thermal Free Energies=
0.161298 (Hartree/Particle)

0.168323

0.169267

\subsection{1}

$-197.618776$

$-197.611751$

$-197.610807$

$-197.649013$

Neopentane

Standard orientation:

\begin{tabular}{cccccc} 
Center & \multicolumn{2}{c}{ Atomic } & \multicolumn{2}{c}{ Atomic } & \multicolumn{3}{c}{ Coordinates $($ Angstroms) } \\
Number & Number & Type & X & Y & Z \\
- \hdashline 1 & 6 & 0 & 0.813370 & -1.006274 & -0.817357 \\
2 & 1 & 0 & 0.346248 & -1.191267 & -1.786698 \\
3 & 1 & 0 & 1.825066 & -0.637169 & -0.997023 \\
4 & 1 & 0 & 0.892302 & -1.961619 & -0.294739 \\
5 & 6 & 0 & -0.000010 & -0.000017 & -0.000014 \\
6 & 6 & 0 & -0.088755 & 1.323778 & -0.762857 \\
7 & 1 & 0 & -0.665013 & 2.061191 & -0.200532 \\
8 & 1 & 0 & 0.904895 & 1.739370 & -0.941719 \\
9 & 1 & 0 & -0.574197 & 1.185382 & -1.730976 \\
10 & 6 & 0 & -1.408990 & -0.550915 & 0.231374 \\
11 & 1 & 0 & -1.374524 & -1.497299 & 0.774712 \\
12 & 1 & 0 & -2.011619 & 0.148921 & 0.813709 \\
13 & 1 & 0 & -1.920767 & -0.726527 & -0.716943 \\
14 & 6 & 0 & 0.684371 & 0.233412 & 1.348831 \\
15 & 1 & 0 & 0.760806 & -0.697173 & 1.914706 \\
16 & 1 & 0 & 1.693454 & 0.627334 & 1.212331 \\
17 & 1 & 0 & 0.123434 & 0.948951 & 1.953317
\end{tabular}

Zero-point correction=

0.160053 (Hartree/Particle)

Thermal correction to Energy=

0.166876

Thermal correction to Enthalpy=

$$
0.167820
$$

Thermal correction to Gibbs Free Energy=

0.131041

Sum of electronic and zero-point Energies=

$-197.623921$

Sum of electronic and thermal Energies=

$-197.617097$

Sum of electronic and thermal Enthalpies=

$-197.616153$ 
Sum of electronic and thermal Free Energies= $-197.652933$

Hexane

Standard orientation:

\begin{tabular}{cccccc} 
Center & Atomic & \multicolumn{2}{c}{ Atomic } & \multicolumn{3}{c}{ Coordinates } \\
Number & Number & Type & X & Y & $Z$ \\
-1 & 6 & 0 & -3.199904 & 0.207998 & 0.000030 \\
2 & 1 & 0 & -3.283545 & 0.847600 & 0.880746 \\
3 & 1 & 0 & -3.283542 & 0.847733 & -0.880591 \\
4 & 1 & 0 & -4.051805 & -0.472455 & -0.000025 \\
5 & 6 & 0 & -1.878074 & -0.548864 & -0.000030 \\
6 & 1 & 0 & -1.830995 & -1.204340 & 0.874142 \\
7 & 1 & 0 & -1.831021 & -1.204256 & -0.874267 \\
8 & 6 & 0 & -0.664273 & 0.372752 & -0.000006 \\
9 & 1 & 0 & -0.710581 & 1.029796 & -0.874749 \\
10 & 1 & 0 & -0.710593 & 1.029773 & 0.874754 \\
11 & 6 & 0 & 0.664273 & -0.372752 & -0.000000 \\
12 & 1 & 0 & 0.710580 & -1.029805 & -0.874738 \\
13 & 1 & 0 & 0.710594 & -1.029764 & 0.874766 \\
14 & 6 & 0 & 1.878074 & 0.548864 & -0.000028 \\
15 & 1 & 0 & 1.830996 & 1.204342 & 0.874144 \\
16 & 1 & 0 & 1.831021 & 1.204255 & -0.874265 \\
17 & 6 & 0 & 3.199904 & -0.207998 & 0.000027 \\
18 & 1 & 0 & 3.283550 & -0.847719 & -0.880603 \\
19 & 1 & 0 & 4.051805 & 0.472455 & -0.000007 \\
20 & 1 & 0 & 3.283537 & -0.847614 & 0.880734
\end{tabular}

Zero-point correction=

0.189938 (Hartree/Particle)

Thermal correction to Energy=

0.198300

Thermal correction to Enthalpy=

0.199244

Thermal correction to Gibbs Free Energy=

Sum of electronic and zero-point Energies=

0.157404

Sum of electronic and thermal Energies=

$-236.905961$

$-236.897599$

Sum of electronic and thermal Enthalpies=

$-236.896655$

Sum of electronic and thermal Free Energies=

$-236.938495$

22dimethylbutane

Standard orientation:

\begin{tabular}{|c|c|c|c|c|}
\hline \multirow{2}{*}{$\begin{array}{l}\text { Center } \\
\text { Number }\end{array}$} & Atomic & \multirow{2}{*}{$\begin{array}{l}\text { Atomic } \\
\text { Type }\end{array}$} & \multicolumn{2}{|c|}{ Coordinates (Angstrom } \\
\hline & Numbe & & $\mathrm{X}$ & $\mathrm{Z}$ \\
\hline 1 & 6 & -1.611967 & 0.976966 & 0.000556 \\
\hline 2 & 1 & -1.588157 & 1.618570 & 0.883706 \\
\hline 3 & 1 & -1.588125 & 1.619575 & -0.881863 \\
\hline 4 & 1 & -2.563280 & 0.441543 & 0.000232 \\
\hline 5 & 6 & -0.432810 & -0.000186 & 0.000011 \\
\hline 6 & 6 & 0.868305 & 0.824875 & 0.000415 \\
\hline 7 & 1 & 0.851815 & 1.482961 & -0.873462 \\
\hline
\end{tabular}




$\begin{array}{cccccc}8 & 1 & 0 & 0.851849 & 1.482058 & 0.874973 \\ 9 & 6 & 0 & 2.172911 & 0.036398 & 0.000000 \\ 10 & 1 & 0 & 2.262308 & -0.598746 & -0.881620 \\ 11 & 1 & 0 & 3.025999 & 0.715051 & 0.000273 \\ 12 & 1 & 0 & 2.262371 & -0.599535 & 0.881044 \\ 13 & 6 & 0 & -0.518130 & -0.880474 & 1.249765 \\ 14 & 1 & 0 & 0.268747 & -1.635222 & 1.266964 \\ 15 & 1 & 0 & -0.430626 & -0.280001 & 2.157707 \\ 16 & 1 & 0 & -1.475608 & -1.403484 & 1.285272 \\ 17 & 6 & 0 & -0.518217 & -0.879113 & -1.250702 \\ 18 & 1 & 0 & -1.475718 & -1.402045 & -1.286723 \\ 19 & 1 & 0 & -0.430743 & -0.277646 & -2.157989 \\ 20 & 1 & 0 & 0.268623 & -1.633879 & -1.268781\end{array}$

Zero-point correction=

Thermal correction to Energy=

Thermal correction to Enthalpy=

Thermal correction to Gibbs Free Energy=

Sum of electronic and zero-point Energies=

Sum of electronic and thermal Energies $=$

Sum of electronic and thermal Enthalpies=

Sum of electronic and thermal Free Energies=
0.188744 (Hartree/Particle)

0.196951

0.197896
0.157413

$-236.909415$

$-236.901208$

$-236.900264$

$-236.940747$

Heptane

Standard orientation:

\begin{tabular}{cccccc} 
Center & Atomic & \multicolumn{2}{c}{ Atomic } & \multicolumn{3}{c}{ Coordinates } \\
Number & Number & Type & X & Y & $Z$ \\
\hline 1 & 6 & 0 & -3.813772 & -0.353251 & -0.000000 \\
2 & 1 & 0 & -3.852914 & -0.997092 & -0.880579 \\
3 & 1 & 0 & -3.852912 & -0.997100 & 0.880572 \\
4 & 1 & 0 & -4.710535 & 0.266612 & 0.000004 \\
5 & 6 & 0 & -2.547780 & 0.493639 & 0.000002 \\
6 & 1 & 0 & -2.546023 & 1.150624 & -0.874187 \\
7 & 1 & 0 & -2.546022 & 1.150617 & 0.874197 \\
8 & 6 & 0 & -1.273265 & -0.341851 & -0.000001 \\
9 & 1 & 0 & -1.273860 & -1.000345 & 0.874708 \\
10 & 1 & 0 & -1.273861 & -1.000341 & -0.874714 \\
11 & 6 & 0 & 0.000000 & 0.494395 & -0.000001 \\
12 & 1 & 0 & -0.000001 & 1.152617 & 0.874785 \\
13 & 1 & 0 & 0.000001 & 1.152616 & -0.874787 \\
14 & 6 & 0 & 1.273265 & -0.341851 & 0.000000 \\
15 & 1 & 0 & 1.273860 & -1.000344 & -0.874710 \\
16 & 1 & 0 & 1.273861 & -1.000342 & 0.874712 \\
17 & 6 & 0 & 2.547780 & 0.493639 & -0.000002 \\
18 & 1 & 0 & 2.546022 & 1.150623 & 0.874189 \\
19 & 1 & 0 & 2.546023 & 1.150618 & -0.874196 \\
20 & 6 & 0 & 3.813772 & -0.353251 & 0.000002 \\
21 & 1 & 0 & 3.852914 & -0.997098 & -0.880573
\end{tabular}




$\begin{array}{lllll}1 & 0 & 4.710535 & 0.266612 & 0.000001 \\ 1 & 0 & 3.852912 & -0.997094 & 0.880578\end{array}$

Zero-point correction $=$

Thermal correction to Energy=

Thermal correction to Enthalpy=

Thermal correction to Gibbs Free Energy=

Sum of electronic and zero-point Energies=

Sum of electronic and thermal Energies $=$

Sum of electronic and thermal Enthalpies=

Sum of electronic and thermal Free Energies= 223trimethylbutane

Standard orientation:

\begin{tabular}{|c|c|c|c|c|c|}
\hline \multirow{2}{*}{$\begin{array}{l}\text { Center } \\
\text { Number }\end{array}$} & Atomic & & \multirow{2}{*}{$\begin{array}{l}\text { Atomic } \\
\text { Type }\end{array}$} & \multicolumn{2}{|c|}{ Coordinates (Angstroms } \\
\hline & Numbe & & & $\mathrm{X}$ & $\mathrm{Z}$ \\
\hline 1 & 6 & 0 & -1.321260 & -1.297485 & -0.452885 \\
\hline 2 & 1 & 0 & -1.167171 & -1.471002 & -1.520308 \\
\hline 3 & 1 & 0 & -0.945706 & -2.165408 & 0.088548 \\
\hline 4 & 1 & 0 & -2.396605 & -1.242006 & -0.275563 \\
\hline 5 & 6 & 0 & -0.640277 & 0.000279 & -0.000877 \\
\hline 6 & 6 & 0 & 0.849497 & -0.000873 & -0.451581 \\
\hline 7 & 1 & 0 & 0.824077 & -0.005095 & -1.547074 \\
\hline 8 & 6 & 0 & 1.623946 & 1.245604 & -0.019840 \\
\hline 9 & 1 & 0 & 1.752888 & 1.275135 & 1.063563 \\
\hline 10 & 1 & 0 & 2.620119 & 1.238335 & -0.463739 \\
\hline 11 & 1 & 0 & 1.137093 & 2.169869 & -0.326910 \\
\hline 12 & 6 & 0 & 1.627053 & -1.244202 & -0.016054 \\
\hline 13 & 1 & 0 & 1.659504 & -1.334078 & 1.071479 \\
\hline 14 & 1 & 0 & 1.202980 & -2.162528 & -0.418414 \\
\hline 15 & 1 & 0 & 2.658266 & -1.180180 & -0.365565 \\
\hline 16 & 6 & 0 & -0.782440 & 0.127998 & 1.519091 \\
\hline 17 & 1 & 0 & -0.387978 & 1.076943 & 1.883405 \\
\hline 18 & 1 & 0 & -1.835711 & 0.080695 & 1.801612 \\
\hline 19 & 1 & 0 & -0.266760 & -0.677756 & 2.043005 \\
\hline 20 & 6 & 0 & -1.378111 & 1.169182 & -0.665598 \\
\hline 21 & 1 & 0 & -1.281024 & 1.125797 & -1.752510 \\
\hline 22 & 1 & 0 & -2.441799 & 1.132478 & -0.424004 \\
\hline 23 & 1 & 0 & -1.002614 & 2.135784 & -0.331060 \\
\hline
\end{tabular}

Zero-point correction=

Thermal correction to Energy=

Thermal correction to Enthalpy=

Thermal correction to Gibbs Free Energy=

Sum of electronic and zero-point Energies=

Sum of electronic and thermal Energies=

Sum of electronic and thermal Enthalpies=

Sum of electronic and thermal Free Energies=
0.218583 (Hartree/Particle)
0.228279
0.229223
0.183816
$-276.193155$
$-276.183460$
$-276.182515$
$-276.227923$


Octane

Standard orientation:

\begin{tabular}{|c|c|c|c|c|}
\hline \multirow{2}{*}{$\begin{array}{l}\text { Center } \\
\text { Number }\end{array}$} & Atomic & \multirow{2}{*}{$\begin{array}{l}\text { Atomic } \\
\text { Type }\end{array}$} & \multicolumn{2}{|c|}{ Coordinates (Angstroms } \\
\hline & Numbe & & $\mathrm{X}$ & $\mathrm{Z}$ \\
\hline 1 & 6 & -4.463775 & 0.256128 & 0.000008 \\
\hline 2 & 1 & -4.527907 & 0.897973 & 0.880585 \\
\hline 3 & 1 & -4.527913 & 0.897990 & -0.880556 \\
\hline 4 & 1 & -5.335788 & -0.398091 & 0.000004 \\
\hline 5 & 6 & -3.165788 & -0.540858 & -0.000005 \\
\hline 6 & 1 & -3.138501 & -1.197291 & 0.874176 \\
\hline 7 & 1 & -3.138508 & -1.197276 & -0.874197 \\
\hline 8 & 6 & -1.924739 & 0.343563 & -0.000003 \\
\hline 9 & 1 & -1.950904 & 1.001540 & -0.874717 \\
\hline 10 & 1 & -1.950904 & 1.001536 & 0.874714 \\
\hline 11 & 6 & -0.619965 & -0.442561 & -0.000003 \\
\hline 12 & 1 & -0.594389 & -1.100298 & -0.874749 \\
\hline 13 & 1 & -0.594388 & -1.100295 & 0.874746 \\
\hline 14 & 6 & 0.619965 & 0.442562 & -0.000004 \\
\hline 15 & 1 & 0.594386 & 1.100301 & 0.874740 \\
\hline 16 & 1 & 0.594391 & 1.100292 & -0.874755 \\
\hline 17 & 6 & 1.924739 & -0.343563 & 0.000003 \\
\hline 18 & 1 & 1.950905 & -1.001544 & -0.874708 \\
\hline 19 & 1 & 1.950903 & -1.001532 & 0.874723 \\
\hline 20 & 6 & 3.165788 & 0.540858 & -0.000002 \\
\hline 21 & 1 & 3.138504 & 1.197288 & 0.874181 \\
\hline 22 & 1 & 3.138505 & 1.197278 & -0.874193 \\
\hline 23 & 6 & 4.463775 & -0.256128 & 0.000004 \\
\hline 24 & 1 & 4.527911 & -0.897986 & -0.880563 \\
\hline 25 & 1 & 5.335788 & 0.398091 & 0.000002 \\
\hline 26 & 1 & 4.527908 & -0.897977 & 0.880578 \\
\hline
\end{tabular}

Zero-point correction=

0.247208 (Hartree/Particle)

Thermal correction to Energy=

0.258258

Thermal correction to Enthalpy=

0.259202

Thermal correction to Gibbs Free Energy $=\quad 0.210162$

Sum of electronic and zero-point Energies $=\quad-315.480365$

Sum of electronic and thermal Energies $=\quad-315.469315$

Sum of electronic and thermal Enthalpies $=\quad-315.468371$

Sum of electronic and thermal Free Energies $=\quad-315.517411$

2233tetramethylbutane

Standard orientation:

\begin{tabular}{cccccc}
$\begin{array}{c}\text { Center } \\
\text { Number }\end{array}$ & \multicolumn{2}{c}{ Atomic } & \multicolumn{2}{c}{ Atomic } & \multicolumn{2}{c}{ Coordinates } \\
Number & Type & X & Y & Z \\
\hline 1 & 6 & 0 & -1.344910 & 1.431007 & 0.019540 \\
2 & 1 & 0 & -0.931043 & 2.022542 & 0.836872 \\
3 & 1 & 0 & -1.144836 & 1.956708 & -0.913782
\end{tabular}




$\begin{array}{cccccc}4 & 1 & 0 & -2.427604 & 1.402624 & 0.153137 \\ 5 & 6 & 0 & -0.788301 & 0.000138 & -0.000144 \\ 6 & 6 & 0 & 0.788330 & 0.000022 & -0.000076 \\ 7 & 6 & 0 & 1.344846 & -1.388506 & -0.346365 \\ 8 & 1 & 0 & 0.929939 & -2.169334 & 0.291879 \\ 9 & 1 & 0 & 1.146132 & -1.657993 & -1.383368 \\ 10 & 1 & 0 & 2.427371 & -1.395630 & -0.208585 \\ 11 & 6 & 0 & -1.344865 & -0.732360 & 1.229192 \\ 12 & 1 & 0 & -0.929381 & -1.735174 & 1.333935 \\ 13 & 1 & 0 & -1.146868 & -0.185993 & 2.151015 \\ 14 & 1 & 0 & -2.427295 & -0.835751 & 1.136760 \\ 15 & 6 & 0 & -1.345113 & -0.698424 & -1.248991 \\ 16 & 1 & 0 & -2.427716 & -0.567826 & -1.291409 \\ 17 & 1 & 0 & -0.930892 & -0.287201 & -2.170135 \\ 18 & 1 & 0 & -1.145819 & -1.769694 & -1.237038 \\ 19 & 6 & 0 & 1.344578 & 0.394037 & 1.375746 \\ 20 & 1 & 0 & 2.427333 & 0.515241 & 1.313782 \\ 21 & 1 & 0 & 0.930718 & 1.337940 & 1.732129 \\ 22 & 1 & 0 & 1.143892 & -0.368770 & 2.127650 \\ 23 & 6 & 0 & 1.345470 & 0.994239 & -1.029042 \\ 24 & 1 & 0 & 2.428011 & 0.878313 & -1.103514 \\ 25 & 1 & 0 & 0.931098 & 0.832053 & -2.024625 \\ 26 & 1 & 0 & 1.146745 & 2.027019 & -0.743865\end{array}$

Zero-point correction=

Thermal correction to Energy=

0.245819 (Hartree/Particle)

0.256119

Thermal correction to Enthalpy=

0.257063

Thermal correction to Gibbs Free Energy=

Sum of electronic and zero-point Energies=

0.212641

Sum of electronic and thermal Energies $=$

$-315.480416$

$-315.470116$

Sum of electronic and thermal Enthalpies=

$-315.469172$

Sum of electronic and thermal Free Energies=

$-315.513593$

B3LYPD3/ccpVTZ

Ethane

Standard orientation:

\begin{tabular}{cccccc} 
Center & \multicolumn{2}{c}{ Atomic } & \multicolumn{2}{c}{ Atomic } & \multicolumn{2}{c}{ Coordinates (Angstroms) } \\
Number & Number & Type & X & Y & $Z$ \\
- \hdashline 1 & 6 & 0 & -0.000000 & -0.000000 & 0.764046 \\
2 & 1 & 0 & -0.508264 & 0.880412 & 1.160974 \\
3 & 1 & 0 & 1.016591 & -0.000036 & 1.160974 \\
4 & 1 & 0 & -0.508327 & -0.880376 & 1.160974 \\
5 & 6 & 0 & 0.000000 & -0.000000 & -0.764046 \\
6 & 1 & 0 & 0.508264 & 0.880412 & -1.160974 \\
7 & 1 & 0 & 0.508327 & -0.880376 & -1.160974 \\
8 & 1 & 0 & -1.016591 & -0.000036 & -1.160974
\end{tabular}

Zero-point correction=

0.074398 (Hartree/Particle)

Thermal correction to Energy=

0.077873 
Thermal correction to Enthalpy=

0.078817

Thermal correction to Gibbs Free Energy=

0.052986

Sum of electronic and zero-point Energies=

$-79.791739$

Sum of electronic and thermal Energies $=$

$-79.788264$

Sum of electronic and thermal Enthalpies=

$-79.787320$

Sum of electronic and thermal Free Energies=

$-79.813152$

Methane

Standard orientation:

\begin{tabular}{|c|c|c|c|c|}
\hline \multirow{2}{*}{$\begin{array}{l}\text { Center } \\
\text { Number }\end{array}$} & Atomic & \multirow{2}{*}{$\begin{array}{l}\text { Atomic } \\
\text { Type }\end{array}$} & \multicolumn{2}{|c|}{ Coordinates (Angstro } \\
\hline & Numbe & & $X$ & Z \\
\hline 1 & 6 & 0.000000 & 0.000000 & 0.000000 \\
\hline 2 & 1 & 0.628276 & 0.628276 & 0.628276 \\
\hline 3 & 1 & -0.628276 & -0.628276 & 0.62827 \\
\hline 4 & 1 & -0.628276 & 0.628276 & -0.62827 \\
\hline 5 & 1 & 0.628276 & -0.628276 & -0.628276 \\
\hline
\end{tabular}

Zero-point correction=

Thermal correction to Energy=

0.044561 (Hartree/Particle)

Thermal correction to Enthalpy=

0.047430

0.048374

Thermal correction to Gibbs Free Energy $=\quad 0.027250$

Sum of electronic and zero-point Energies=

$-40.493829$

Sum of electronic and thermal Energies=

$-40.490960$

Sum of electronic and thermal Enthalpies=

$-40.490016$

Sum of electronic and thermal Free Energies=

$-40.511139$

Propane

Standard orientation:

\begin{tabular}{|c|c|c|c|c|}
\hline \multirow{2}{*}{$\begin{array}{l}\text { Center } \\
\text { Number }\end{array}$} & Atomic & \multirow{2}{*}{$\begin{array}{l}\text { Atomic } \\
\text { Type }\end{array}$} & \multicolumn{2}{|c|}{ Coordinates (Angstrom } \\
\hline & Number & & $\mathrm{X}$ & $\mathrm{Z}$ \\
\hline 1 & 6 & -1.271960 & -0.259722 & 0.000000 \\
\hline 2 & 1 & -2.169625 & 0.360802 & -0.000019 \\
\hline 3 & 1 & -1.312282 & -0.904559 & 0.880890 \\
\hline 4 & 1 & -1.312266 & -0.904591 & -0.880867 \\
\hline 5 & 6 & -0.000000 & 0.587373 & 0.000000 \\
\hline 6 & 1 & -0.000001 & 1.244569 & 0.873967 \\
\hline 7 & 1 & 0.000001 & 1.244552 & -0.873979 \\
\hline 8 & 6 & 1.271960 & -0.259722 & 0.000000 \\
\hline 9 & 1 & 1.312312 & -0.904512 & 0.880923 \\
\hline 10 & 1 & 1.312232 & -0.904637 & -0.880835 \\
\hline 11 & 1 & 2.169628 & 0.360800 & -0.000085 \\
\hline
\end{tabular}

Zero-point correction=

Thermal correction to Energy=

0.103145 (Hartree/Particle)

Thermal correction to Enthalpy=

0.107631

Thermal correction to Gibbs Free Energy=

0.108575

Sum of electronic and zero-point Energies=

0.077536

Sum of electronic and thermal Energies=

$-119.093522$

$-119.089035$ 
Sum of electronic and thermal Enthalpies=

Sum of electronic and thermal Free Energies=

Butane
$-119.088091$

$-119.119130$

Standard orientation:

\begin{tabular}{cccccc} 
Center & \multicolumn{2}{c}{ Atomic } & \multicolumn{2}{c}{ Atomic } & \multicolumn{2}{c}{ Coordinates (Angstroms) } \\
Number & Number & Type & X & Y & $Z$ \\
-1 & 6 & 0 & 1.955003 & -0.120093 & 0.000053 \\
2 & 1 & 0 & 2.098308 & -0.749875 & -0.880867 \\
3 & 1 & 0 & 2.098193 & -0.749839 & 0.881017 \\
4 & 1 & 0 & 2.742108 & 0.635634 & 0.000088 \\
5 & 6 & 0 & 0.565275 & 0.514521 & -0.000060 \\
6 & 1 & 0 & 0.458363 & 1.164309 & 0.874291 \\
7 & 1 & 0 & 0.458448 & 1.164224 & -0.874485 \\
8 & 6 & 0 & -0.565275 & -0.514521 & -0.000060 \\
9 & 1 & 0 & -0.458447 & -1.164222 & -0.874487 \\
10 & 1 & 0 & -0.458364 & -1.164311 & 0.874290 \\
11 & 6 & 0 & -1.955003 & 0.120093 & 0.000053 \\
12 & 1 & 0 & -2.098302 & 0.749888 & -0.880859 \\
13 & 1 & 0 & -2.742108 & -0.635634 & 0.000071 \\
14 & 1 & 0 & -2.098198 & 0.749825 & 0.881026
\end{tabular}

Zero-point correction=

0.131691 (Hartree/Particle)

Thermal correction to Energy=

0.137407

Thermal correction to Enthalpy=

0.138351

Thermal correction to Gibbs Free Energy=

0.103773

Sum of electronic and zero-point Energies=

$-158.395535$

Sum of electronic and thermal Energies $=$

$-158.389819$

Sum of electronic and thermal Enthalpies=

$-158.388875$

Sum of electronic and thermal Free Energies= $-158.423452$

Isobutane

Standard orientation:

\begin{tabular}{|c|c|c|c|c|c|}
\hline \multirow{2}{*}{$\begin{array}{l}\text { Center } \\
\text { Number }\end{array}$} & Atomic & \multirow{2}{*}{\multicolumn{2}{|c|}{$\begin{array}{l}\text { Atomic } \\
\text { Type }\end{array}$}} & \multicolumn{2}{|c|}{ Coordinates (Angstroms } \\
\hline & Numbe & & & $\mathrm{X}$ & $\mathrm{Z}$ \\
\hline 1 & 6 & 0 & -0.396285 & -1.401682 & -0.095715 \\
\hline 2 & 1 & 0 & -1.391156 & -1.674727 & 0.261167 \\
\hline 3 & 1 & 0 & -0.409699 & -1.450832 & -1.187859 \\
\hline 4 & 1 & 0 & 0.307878 & -2.155291 & 0.261921 \\
\hline 5 & 6 & 0 & -0.000007 & 0.000030 & 0.374578 \\
\hline 6 & 1 & 0 & -0.000029 & 0.000062 & 1.469975 \\
\hline 7 & 6 & 0 & -1.015755 & 1.044030 & -0.095735 \\
\hline 8 & 1 & 0 & -0.754947 & 2.042070 & 0.261440 \\
\hline 9 & 1 & 0 & -1.051316 & 1.080430 & -1.187873 \\
\hline 10 & 1 & 0 & -2.020538 & 0.810818 & 0.261610 \\
\hline 11 & 6 & 0 & 1.412041 & 0.357642 & -0.095724 \\
\hline 12 & 1 & 0 & 1.712323 & 1.344627 & 0.261164 \\
\hline 13 & 1 & 0 & 2.146015 & -0.366973 & 0.261907 \\
\hline
\end{tabular}


Zero-point correction=

Thermal correction to Energy=

Thermal correction to Enthalpy=

Thermal correction to Gibbs Free Energy=

Sum of electronic and zero-point Energies=

Sum of electronic and thermal Energies=

Sum of electronic and thermal Enthalpies=

Sum of electronic and thermal Free Energies=
0.131308 (Hartree/Particle)

0.136921

0.137865

0.103788

$-158.397545$

$-158.391932$

$-158.390988$

$-158.425066$

Pentane

Standard orientation:

\begin{tabular}{cccccc} 
Center & \multicolumn{2}{c}{ Atomic } & \multicolumn{2}{c}{ Atomic } & \multicolumn{3}{c}{ Coordinates (Angstroms) } \\
Number & Number & Type & X & Y & Z \\
-1 & 6 & 0 & 2.549866 & 0.325029 & -0.000008 \\
2 & 1 & 0 & 2.590530 & 0.969620 & -0.880910 \\
3 & 1 & 0 & 2.590535 & 0.969636 & 0.880883 \\
4 & 1 & 0 & 3.447669 & -0.295198 & -0.000005 \\
5 & 6 & 0 & 1.279346 & -0.523536 & 0.000005 \\
6 & 1 & 0 & 1.278475 & -1.181726 & -0.874399 \\
7 & 1 & 0 & 1.278487 & -1.181718 & 0.874415 \\
8 & 6 & 0 & 0.000000 & 0.312655 & 0.000012 \\
9 & 1 & 0 & -0.000004 & 0.972453 & 0.874758 \\
10 & 1 & 0 & 0.000004 & 0.972475 & -0.874717 \\
11 & 6 & 0 & -1.279346 & -0.523536 & -0.000005 \\
12 & 1 & 0 & -1.278487 & -1.181735 & 0.874394 \\
13 & 1 & 0 & -1.278475 & -1.181709 & -0.874420 \\
14 & 6 & 0 & -2.549866 & 0.325029 & -0.000003 \\
15 & 1 & 0 & -3.447669 & -0.295198 & -0.000083 \\
16 & 1 & 0 & -2.590567 & 0.969568 & 0.880934 \\
17 & 1 & 0 & -2.590497 & 0.969687 & -0.880859
\end{tabular}

Zero-point correction $=$

0.160209 (Hartree/Particle)

Thermal correction to Energy=

0.167204

Thermal correction to Enthalpy=

0.168148

Thermal correction to Gibbs Free Energy=

Sum of electronic and zero-point Energies=

0.130034

Sum of electronic and thermal Energies $=$

$-197.697535$

$-197.690539$

Sum of electronic and thermal Enthalpies=

$-197.689595$

Sum of electronic and thermal Free Energies=

$-197.727710$

Neopentane

Standard orientation:

\begin{tabular}{|c|c|c|c|}
\hline Center & Atomic & Atomic & Coordinates (Angstroms) \\
\hline Number & Number & Type & $\mathrm{X}$ \\
\hline
\end{tabular}




$\begin{array}{rrrrrr}1 & 6 & 0 & -0.835080 & -1.231337 & 0.379611 \\ 2 & 1 & 0 & -1.828144 & -0.939359 & 0.727609 \\ 3 & 1 & 0 & -0.962591 & -1.897549 & -0.476216 \\ 4 & 1 & 0 & -0.354194 & -1.800223 & 1.178144 \\ 5 & 6 & 0 & 0.000018 & -0.000041 & -0.000003 \\ 6 & 6 & 0 & -0.707508 & 0.771759 & -1.123116 \\ 7 & 1 & 0 & -0.134306 & 1.654780 & -1.413379 \\ 8 & 1 & 0 & -0.832124 & 0.146915 & -2.010005 \\ 9 & 1 & 0 & -1.698057 & 1.104776 & -0.806172 \\ 10 & 6 & 0 & 0.156491 & 0.910263 & 1.226568 \\ 11 & 1 & 0 & 0.657630 & 0.385623 & 2.042748 \\ 12 & 1 & 0 & 0.747859 & 1.795855 & 0.984604 \\ 13 & 1 & 0 & -0.816155 & 1.246574 & 1.591809 \\ 14 & 6 & 0 & 1.386096 & -0.450679 & -0.483056 \\ 15 & 1 & 0 & 1.913159 & -1.002625 & 0.298030 \\ 16 & 1 & 0 & 1.304501 & -1.101510 & -1.356151 \\ 17 & 1 & 0 & 2.002315 & 0.406950 & -0.761043\end{array}$

Zero-point correction=

Thermal correction to Energy=

Thermal correction to Enthalpy=

Thermal correction to Gibbs Free Energy=

Sum of electronic and zero-point Energies=

Sum of electronic and thermal Energies $=$

Sum of electronic and thermal Enthalpies=

Sum of electronic and thermal Free Energies=

Hexane

Standard orientation:

\begin{tabular}{|c|c|c|c|c|}
\hline \multirow{2}{*}{$\begin{array}{l}\text { Center } \\
\text { Number }\end{array}$} & Atomic & \multirow{2}{*}{$\begin{array}{l}\text { Atomic } \\
\text { Type }\end{array}$} & \multicolumn{2}{|c|}{ Coordinates (Angstroms } \\
\hline & Numbe & & $\mathrm{X}$ & Z \\
\hline 1 & 6 & -3.211226 & 0.208428 & 0.000044 \\
\hline 2 & 1 & -3.296557 & 0.848601 & 0.880992 \\
\hline 3 & 1 & -3.296586 & 0.848732 & -0.880806 \\
\hline 4 & 1 & -4.063832 & -0.472625 & 0.000006 \\
\hline 5 & 6 & -1.884897 & -0.549852 & -0.000040 \\
\hline 6 & 1 & -1.838315 & -1.206456 & 0.874284 \\
\hline 7 & 1 & -1.838358 & -1.206351 & -0.874446 \\
\hline 8 & 6 & -0.666634 & 0.373207 & -0.000021 \\
\hline 9 & 1 & -0.713172 & 1.031101 & -0.874769 \\
\hline 10 & 1 & -0.713189 & 1.031101 & 0.874726 \\
\hline 11 & 6 & 0.666634 & -0.373207 & 0.000002 \\
\hline 12 & 1 & 0.713180 & -1.031131 & -0.874723 \\
\hline 13 & 1 & 0.713181 & -1.031071 & 0.874771 \\
\hline 14 & 6 & 1.884897 & 0.549852 & -0.000028 \\
\hline 15 & 1 & 1.838323 & 1.206453 & 0.874299 \\
\hline 16 & 1 & 1.838350 & 1.206354 & -0.874431 \\
\hline 17 & 6 & 3.211226 & -0.208428 & 0.000034 \\
\hline 18 & 1 & 3.296580 & -0.848723 & -0.880823 \\
\hline
\end{tabular}




$\begin{array}{rrrrrr}19 & 1 & 0 & 4.063832 & 0.472625 & -0.000002 \\ 20 & 1 & 0 & 3.296563 & -0.848609 & 0.880975\end{array}$

Zero-point correction=

0.188693 (Hartree/Particle)

Thermal correction to Energy=

0.197000

Thermal correction to Enthalpy=

0.197945

Thermal correction to Gibbs Free Energy=

0.156300

Sum of electronic and zero-point Energies=

$-236.999591$

Sum of electronic and thermal Energies=

$-236.991284$

Sum of electronic and thermal Enthalpies=

$-236.990340$

Sum of electronic and thermal Free Energies= $-237.031985$

22dimethylbutane

Standard orientation:

\begin{tabular}{|c|c|c|c|c|}
\hline \multirow{2}{*}{$\begin{array}{l}\text { Center } \\
\text { Number }\end{array}$} & Atomic & \multirow{2}{*}{$\begin{array}{r}\text { Atomic } \\
\text { Type }\end{array}$} & \multicolumn{2}{|c|}{ Coordinates (Angstroms } \\
\hline & Numbe & & $\mathrm{X}$ & Z \\
\hline 1 & 6 & -1.615920 & 0.982908 & 0.003056 \\
\hline 2 & 1 & -1.592030 & 1.622994 & 0.887562 \\
\hline 3 & 1 & -1.592253 & 1.628215 & -0.877657 \\
\hline 4 & 1 & -2.568932 & 0.450070 & 0.001599 \\
\hline 5 & 6 & -0.434681 & -0.000116 & 0.000013 \\
\hline 6 & 6 & 0.873751 & 0.824537 & 0.001687 \\
\hline 7 & 1 & 0.858387 & 1.484751 & -0.870969 \\
\hline 8 & 1 & 0.858512 & 1.481066 & 0.877122 \\
\hline 9 & 6 & 2.183668 & 0.035290 & 0.000049 \\
\hline 10 & 1 & 2.275335 & -0.599290 & -0.881953 \\
\hline 11 & 1 & 3.036292 & 0.715876 & 0.000617 \\
\hline 12 & 1 & 2.276005 & -0.601658 & 0.880264 \\
\hline 13 & 6 & -0.522920 & -0.885730 & 1.251996 \\
\hline 14 & 1 & 0.264538 & -1.639646 & 1.270859 \\
\hline 15 & 1 & -0.438670 & -0.287525 & 2.161938 \\
\hline 16 & 1 & -1.479873 & -1.410285 & 1.284734 \\
\hline 17 & 6 & -0.523855 & -0.878976 & -1.256636 \\
\hline 18 & 1 & -1.480585 & -1.403832 & -1.291216 \\
\hline 19 & 1 & -0.440902 & -0.275801 & -2.163411 \\
\hline 20 & 1 & 0.263920 & -1.632420 & -1.280485 \\
\hline
\end{tabular}

Zero-point correction=

0.187852 (Hartree/Particle)

Thermal correction to Energy=

0.195928

Thermal correction to Enthalpy=

0.196872

Thermal correction to Gibbs Free Energy=

0.156776

Sum of electronic and zero-point Energies=

$-237.001894$

Sum of electronic and thermal Energies $=$

$-236.993818$

Sum of electronic and thermal Enthalpies=

$-236.992874$

Sum of electronic and thermal Free Energies=

$-237.032970$

Heptane

Standard orientation:

Center Atomic Atomic Coordinates (Angstroms) 


\begin{tabular}{|c|c|c|c|c|c|}
\hline Number & Numbe & & Type & $X$ & Z \\
\hline 1 & 6 & 0 & -3.828114 & -0.354223 & -0.000028 \\
\hline 2 & 1 & 0 & -3.868766 & -0.998779 & -0.880983 \\
\hline 3 & 1 & 0 & -3.868744 & -0.998950 & 0.880803 \\
\hline 4 & 1 & 0 & -4.726016 & 0.265904 & 0.000046 \\
\hline 5 & 6 & 0 & -2.557666 & 0.494331 & 0.000042 \\
\hline 6 & 1 & 0 & -2.556779 & 1.152590 & -0.874282 \\
\hline 7 & 1 & 0 & -2.556789 & 1.152464 & 0.874462 \\
\hline 8 & 6 & 0 & -1.278281 & -0.341929 & -0.000002 \\
\hline 9 & 1 & 0 & -1.278978 & -1.001495 & 0.874690 \\
\hline 10 & 1 & 0 & -1.279002 & -1.001457 & -0.874722 \\
\hline 11 & 6 & 0 & -0.000000 & 0.495348 & -0.000007 \\
\hline 12 & 1 & 0 & -0.000007 & 1.154624 & 0.874741 \\
\hline 13 & 1 & 0 & 0.000007 & 1.154585 & -0.874784 \\
\hline 14 & 6 & 0 & 1.278281 & -0.341929 & 0.000020 \\
\hline 15 & 1 & 0 & 1.278981 & -1.001521 & -0.874652 \\
\hline 16 & 1 & 0 & 1.278999 & -1.001430 & 0.874760 \\
\hline 17 & 6 & 0 & 2.557666 & 0.494331 & -0.000035 \\
\hline 18 & 1 & 0 & 2.556787 & 1.152579 & 0.874298 \\
\hline 19 & 1 & 0 & 2.556781 & 1.152474 & -0.874446 \\
\hline 20 & 6 & 0 & 3.828114 & -0.354223 & 0.000011 \\
\hline 21 & 1 & 0 & 3.868752 & -0.998917 & -0.880844 \\
\hline 22 & 1 & 0 & 4.726016 & 0.265904 & -0.000030 \\
\hline 23 & 1 & 0 & 3.868759 & -0.998812 & 0.880942 \\
\hline
\end{tabular}

Zero-point correction=

Thermal correction to Energy=

Thermal correction to Enthalpy=

Thermal correction to Gibbs Free Energy=

Sum of electronic and zero-point Energies=

Sum of electronic and thermal Energies $=$

Sum of electronic and thermal Enthalpies=

Sum of electronic and thermal Free Energies= 223trimethylbutane
0.217148 (Hartree/Particle)

0.226784

0.227729

$-276.301678$

$-276.292042$

$-276.291098$

$-276.336282$

Standard orientation:

\begin{tabular}{|c|c|c|c|c|}
\hline \multirow{2}{*}{$\begin{array}{l}\text { Center } \\
\text { Number }\end{array}$} & Atomic & \multirow{2}{*}{$\begin{array}{r}\text { Atomic } \\
\text { Type }\end{array}$} & \multicolumn{2}{|c|}{ Coordinates (Angstrom } \\
\hline & Numbe & & $\mathrm{X}$ & Z \\
\hline 1 & 6 & -1.355561 & -1.241930 & -0.566301 \\
\hline 2 & 1 & -1.229725 & -1.308186 & -1.649360 \\
\hline 3 & 1 & -0.981243 & -2.165697 & -0.126526 \\
\hline 4 & 1 & -2.425935 & -1.193714 & -0.358163 \\
\hline 5 & 6 & -0.643519 & 0.000021 & -0.000586 \\
\hline 6 & 6 & 0.854305 & 0.000005 & -0.449824 \\
\hline 7 & 1 & 0.829245 & 0.000031 & -1.545725 \\
\hline 8 & 6 & 1.634734 & 1.248730 & -0.016711 \\
\hline 9 & 1 & 1.718640 & 1.309293 & 1.069829 \\
\hline 10 & 1 & 2.649306 & 1.212546 & -0.416725 \\
\hline
\end{tabular}




$\begin{array}{rrrrrr}11 & 1 & 0 & 1.179117 & 2.171794 & -0.371107 \\ 12 & 6 & 0 & 1.634656 & -1.248750 & -0.016746 \\ 13 & 1 & 0 & 1.718730 & -1.309241 & 1.069789 \\ 14 & 1 & 0 & 1.178888 & -2.171807 & -0.370980 \\ 15 & 1 & 0 & 2.649173 & -1.212718 & -0.416917 \\ 16 & 6 & 0 & -0.790873 & -0.000494 & 1.529350 \\ 17 & 1 & 0 & -0.336269 & 0.881856 & 1.980492 \\ 18 & 1 & 0 & -1.847394 & -0.000385 & 1.804391 \\ 19 & 1 & 0 & -0.336599 & -0.883332 & 1.979862 \\ 20 & 6 & 0 & -1.355363 & 1.242426 & -0.565489 \\ 21 & 1 & 0 & -1.229287 & 1.309562 & -1.648469 \\ 22 & 1 & 0 & -2.425795 & 1.194110 & -0.357649 \\ 23 & 1 & 0 & -0.981127 & 2.165842 & -0.124900\end{array}$

Zero-point correction=

Thermal correction to Energy=

Thermal correction to Enthalpy=

Thermal correction to Gibbs Free Energy=

Sum of electronic and zero-point Energies=

Sum of electronic and thermal Energies $=$

Sum of electronic and thermal Enthalpies=

Sum of electronic and thermal Free Energies=

Octane
0.216034 (Hartree/Particle)

0.225453

0.226397
0.182533

$-276.301752$

$-276.292333$

$-276.291389$

$-276.335252$

Standard orientation:

\begin{tabular}{cccccc} 
Center & \multicolumn{2}{c}{ Atomic } & \multicolumn{2}{c}{ Atomic } & \multicolumn{3}{c}{ Coordinates } \\
Number & Number & Type & X & Y & $Z$ \\
- & Nustroms) & Z \\
1 & 6 & 0 & -4.480681 & 0.256711 & 0.000026 \\
2 & 1 & 0 & -4.546434 & 0.899185 & 0.880993 \\
3 & 1 & 0 & -4.546393 & 0.899400 & -0.880788 \\
4 & 1 & 0 & -5.353770 & -0.397900 & -0.000077 \\
5 & 6 & 0 & -3.178177 & -0.541718 & -0.000043 \\
6 & 1 & 0 & -3.151703 & -1.199460 & 0.874268 \\
7 & 1 & 0 & -3.151705 & -1.199311 & -0.874468 \\
8 & 6 & 0 & -1.932288 & 0.343672 & 0.000020 \\
9 & 1 & 0 & -1.958640 & 1.002741 & -0.874656 \\
10 & 1 & 0 & -1.958659 & 1.002665 & 0.874753 \\
11 & 6 & 0 & -0.622412 & -0.443214 & 0.000005 \\
12 & 1 & 0 & -0.596733 & -1.102006 & -0.874696 \\
13 & 1 & 0 & -0.596706 & -1.101983 & 0.874722 \\
14 & 6 & 0 & 0.622412 & 0.443214 & -0.000024 \\
15 & 1 & 0 & 0.596714 & 1.102033 & 0.874657 \\
16 & 1 & 0 & 0.596725 & 1.101956 & -0.874761 \\
17 & 6 & 0 & 1.932288 & -0.343672 & 0.000019 \\
18 & 1 & 0 & 1.958650 & -1.002748 & -0.874651 \\
19 & 1 & 0 & 1.958648 & -1.002658 & 0.874757 \\
20 & 6 & 0 & 3.178177 & 0.541718 & -0.000025 \\
21 & 1 & 0 & 3.151703 & 1.199431 & 0.874309 \\
22 & 1 & 0 & 3.151705 & 1.199341 & -0.874427
\end{tabular}




$\begin{array}{rrrrrr}23 & 6 & 0 & 4.480681 & -0.256711 & 0.000018 \\ 24 & 1 & 0 & 4.546415 & -0.899338 & -0.880840 \\ 25 & 1 & 0 & 5.353770 & 0.397900 & -0.000015 \\ 26 & 1 & 0 & 4.546412 & -0.899248 & 0.880941\end{array}$

Zero-point correction=

0.245607 (Hartree/Particle)

Thermal correction to Energy=

0.256578

Thermal correction to Enthalpy=

0.257522

Thermal correction to Gibbs Free Energy=

Sum of electronic and zero-point Energies=

Sum of electronic and thermal Energies=

0.208809

$-315.603765$

$-315.592793$

Sum of electronic and thermal Enthalpies=

$-315.591849$

Sum of electronic and thermal Free Energies $=\quad-315.640562$

2233tetramethylbutane

Standard orientation:

\begin{tabular}{|c|c|c|c|c|}
\hline \multirow{2}{*}{$\begin{array}{l}\text { Center } \\
\text { Number }\end{array}$} & Atomic & \multirow{2}{*}{$\begin{array}{l}\text { Atomic } \\
\text { Type }\end{array}$} & \multicolumn{2}{|c|}{ Coordinates (Angstrom } \\
\hline & Numbe & & $X$ & Z \\
\hline 1 & 6 & -1.351620 & 0.909234 & 1.111231 \\
\hline 2 & 1 & -1.149171 & 0.509771 & 2.104409 \\
\hline 3 & 1 & -0.940972 & 1.917900 & 1.062739 \\
\hline 4 & 1 & -2.435020 & 0.993479 & 1.009302 \\
\hline 5 & 6 & -0.792714 & -0.000023 & 0.000006 \\
\hline 6 & 6 & 0.792833 & -0.000043 & -0.000099 \\
\hline 7 & 6 & 1.351627 & -1.124515 & -0.892932 \\
\hline 8 & 1 & 1.148991 & -2.111521 & -0.478586 \\
\hline 9 & 1 & 0.940686 & -1.090989 & -1.902039 \\
\hline 10 & 1 & 2.435007 & -1.023840 & -0.978865 \\
\hline 11 & 6 & -1.351832 & -1.416979 & 0.231816 \\
\hline 12 & 1 & -1.148686 & -2.077498 & -0.610443 \\
\hline 13 & 1 & -0.941875 & -1.879081 & 1.130020 \\
\hline 14 & 1 & -2.435313 & -1.370808 & 0.354903 \\
\hline 15 & 6 & -1.351711 & 0.507887 & -1.342967 \\
\hline 16 & 1 & -2.435151 & 0.377914 & -1.364787 \\
\hline 17 & 1 & -1.148763 & 1.567678 & -1.493404 \\
\hline 18 & 1 & -0.941334 & -0.038480 & -2.192373 \\
\hline 19 & 6 & 1.351804 & -0.211096 & 1.420122 \\
\hline 20 & 1 & 2.435240 & -0.335384 & 1.375855 \\
\hline 21 & 1 & 1.148893 & 0.640917 & 2.068101 \\
\hline 22 & 1 & 0.941311 & -1.102122 & 1.895377 \\
\hline 23 & 6 & 1.351753 & 1.335471 & -0.527278 \\
\hline 24 & 1 & 2.435106 & 1.359474 & -0.396915 \\
\hline 25 & 1 & 1.149428 & 1.470545 & -1.589239 \\
\hline 26 & 1 & 0.940780 & 2.192434 & 0.006546 \\
\hline
\end{tabular}

Zero-point correction=

0.244419 (Hartree/Particle)

Thermal correction to Energy=

0.254711

Thermal correction to Enthalpy=

0.255655

Thermal correction to Gibbs Free Energy= $\quad 0.211202$ 
Sum of electronic and zero-point Energies=

$-315.600870$

Sum of electronic and thermal Energies=

Sum of electronic and thermal Enthalpies=

Sum of electronic and thermal Free Energies=

B2PLYP/ccpVTZ

Ethane

Standard orientation:

\begin{tabular}{|c|c|c|c|c|}
\hline \multirow{2}{*}{$\begin{array}{l}\text { Center } \\
\text { Number }\end{array}$} & Atomic & \multirow{2}{*}{$\begin{array}{l}\text { Atomic } \\
\text { Type }\end{array}$} & \multicolumn{2}{|c|}{ Coordinates (Angstrom } \\
\hline & Numbe & & $\mathrm{X}$ & $\mathrm{Z}$ \\
\hline 1 & 6 & 0.000000 & 0.000000 & 0.762780 \\
\hline 2 & 1 & -0.507483 & 0.879024 & 1.158340 \\
\hline 3 & 1 & 1.014999 & -0.000019 & 1.158340 \\
\hline 4 & 1 & -0.507516 & -0.879005 & 1.158340 \\
\hline 5 & 6 & 0.000000 & 0.000000 & -0.762780 \\
\hline 6 & 1 & 0.507483 & 0.879024 & -1.158340 \\
\hline 7 & 1 & 0.507516 & -0.879005 & -1.158340 \\
\hline 8 & 1 & -1.014999 & -0.000019 & -1.158340 \\
\hline
\end{tabular}

Zero-point correction $=$

Thermal correction to Energy=

Thermal correction to Enthalpy=

Thermal correction to Gibbs Free Energy=

Sum of electronic and zero-point Energies=

Sum of electronic and thermal Energies $=$

Sum of electronic and thermal Enthalpies=

Sum of electronic and thermal Free Energies=

Methane

Standard orientation:
0.075079 (Hartree/Particle)

$$
0.078553
$$

0.079497

$$
\begin{gathered}
0.053670 \\
-79.696688 \\
-79.693214 \\
-79.692270 \\
-79.718097
\end{gathered}
$$

\begin{tabular}{|c|c|c|c|c|}
\hline \multirow{2}{*}{$\begin{array}{l}\text { Center } \\
\text { Number }\end{array}$} & \multirow{2}{*}{$\begin{array}{l}\text { Atomic } \\
\text { Number }\end{array}$} & \multirow{2}{*}{$\begin{array}{r}\text { Atomic } \\
\text { Type }\end{array}$} & \multicolumn{2}{|c|}{ Coordinates (Angstrom } \\
\hline & & & $\mathrm{X}$ & $\mathrm{Z}$ \\
\hline 1 & 6 & 0.000000 & 0.000000 & 0.000000 \\
\hline 2 & 1 & 0.627204 & 0.627204 & 0.627204 \\
\hline 3 & 1 & -0.627204 & -0.627204 & 0.627204 \\
\hline 4 & 1 & -0.627204 & 0.627204 & -0.627204 \\
\hline 5 & 1 & 0.627204 & -0.627204 & -0.627204 \\
\hline
\end{tabular}

Zero-point correction=

Thermal correction to Energy=

Thermal correction to Enthalpy=

Thermal correction to Gibbs Free Energy=

Sum of electronic and zero-point Energies=

Sum of electronic and thermal Energies $=$

Sum of electronic and thermal Enthalpies=

Sum of electronic and thermal Free Energies=

Propane

$$
\begin{array}{cc}
0.045020 & \text { (Hartree/Particle) } \\
& 0.047887 \\
& 0.048831 \\
\text { rgy }= & 0.027715 \\
\text { gies }= & -40.441365 \\
\text { s }= & -40.438498 \\
\text { pies }= & -40.437554 \\
\text { Energies }= & -40.458670
\end{array}
$$

Standard orientation: 


\begin{tabular}{|c|c|c|c|c|}
\hline \multirow{2}{*}{$\begin{array}{l}\text { Center } \\
\text { Number }\end{array}$} & Atomic & \multirow{2}{*}{$\begin{array}{l}\text { Atomic } \\
\text { Type }\end{array}$} & \multicolumn{2}{|c|}{ Coordinates (Angstroms) } \\
\hline & Number & & $\mathrm{X}$ & $\mathrm{Z}$ \\
\hline 1 & 6 & -1.269110 & -0.259616 & 0.000000 \\
\hline 2 & 1 & -2.165686 & 0.359417 & -0.000023 \\
\hline 3 & 1 & -1.308379 & -0.903003 & 0.879795 \\
\hline 4 & 1 & -1.308360 & -0.903039 & -0.879769 \\
\hline 5 & 6 & 0.000000 & 0.587226 & 0.000000 \\
\hline 6 & 1 & -0.000001 & 1.242651 & 0.873028 \\
\hline 7 & 1 & 0.000001 & 1.242637 & -0.873038 \\
\hline 8 & 6 & 1.269110 & -0.259616 & 0.000000 \\
\hline 9 & 1 & 1.308388 & -0.902988 & 0.879806 \\
\hline 10 & 1 & 1.308347 & -0.903054 & -0.879759 \\
\hline 11 & 1 & 2.165687 & 0.359414 & -0.000044 \\
\hline
\end{tabular}

$\begin{array}{lc}\text { Zero-point correction }= & 0.103924 \text { (Hartree/Particle) } \\ \text { Thermal correction to Energy= } & 0.108437 \\ \text { Thermal correction to Enthalpy= } & 0.109381 \\ \text { Thermal correction to Gibbs Free Energy= } & 0.078286 \\ \text { Sum of electronic and zero-point Energies }= & -118.955795 \\ \text { Sum of electronic and thermal Energies }= & -118.951282 \\ \text { Sum of electronic and thermal Enthalpies }= & -118.950337 \\ \text { Sum of electronic and thermal Free Energies }= & -118.981433\end{array}$

Butane

Standard orientation:

\begin{tabular}{|c|c|c|c|c|}
\hline \multirow{2}{*}{$\begin{array}{l}\text { Center } \\
\text { Number }\end{array}$} & Atomic & \multirow{2}{*}{$\begin{array}{r}\text { Atomic } \\
\text { Type }\end{array}$} & \multicolumn{2}{|c|}{ Coordinates (Angstroms } \\
\hline & Numbe & & $X$ & Z \\
\hline 1 & 6 & 1.951265 & -0.120006 & 0.000032 \\
\hline 2 & 1 & 2.093698 & -0.748414 & -0.879832 \\
\hline 3 & 1 & 2.093638 & -0.748387 & 0.879924 \\
\hline 4 & 1 & 2.737145 & 0.634566 & 0.000046 \\
\hline 5 & 6 & 0.563814 & 0.514278 & -0.000036 \\
\hline 6 & 1 & 0.456934 & 1.162214 & 0.873663 \\
\hline 7 & 1 & 0.456982 & 1.162167 & -0.873776 \\
\hline 8 & 6 & -0.563814 & -0.514278 & -0.000036 \\
\hline 9 & 1 & -0.456982 & -1.162165 & -0.873778 \\
\hline 10 & 1 & -0.456934 & -1.162216 & 0.873662 \\
\hline 11 & 6 & -1.951265 & 0.120006 & 0.000032 \\
\hline 12 & 1 & -2.093696 & 0.748421 & -0.879828 \\
\hline 13 & 1 & -2.737145 & -0.634566 & 0.000038 \\
\hline 14 & 1 & -2.093641 & 0.748381 & 0.879929 \\
\hline
\end{tabular}

Zero-point correction=

Thermal correction to Energy=

Thermal correction to Enthalpy=

Thermal correction to Gibbs Free Energy=
0.132572 (Hartree/Particle)
0.138338
0.139282

0.104577 
Sum of electronic and zero-point Energies=

$-158.215071$

Sum of electronic and thermal Energies=

$-158.209304$

Sum of electronic and thermal Enthalpies=

$-158.208360$

Sum of electronic and thermal Free Energies=

$-158.243066$

Isobutane

Standard orientation:

\begin{tabular}{|c|c|c|c|c|}
\hline \multirow{2}{*}{$\begin{array}{l}\text { Center } \\
\text { Number }\end{array}$} & Atomic & \multirow{2}{*}{$\begin{array}{l}\text { Atomic } \\
\text { Type }\end{array}$} & \multicolumn{2}{|c|}{ Coordinates (Angstrom } \\
\hline & Numbe & & $X$ & Z \\
\hline 1 & 6 & 1.162761 & 0.871814 & -0.095825 \\
\hline 2 & 1 & 2.118132 & 0.485976 & 0.260299 \\
\hline 3 & 1 & 1.201594 & 0.901193 & -1.186567 \\
\hline 4 & 1 & 1.060382 & 1.896933 & 0.260646 \\
\hline 5 & 6 & -0.000003 & 0.000004 & 0.375421 \\
\hline 6 & 1 & -0.000004 & -0.000007 & 1.469205 \\
\hline 7 & 6 & 0.173644 & -1.442875 & -0.095827 \\
\hline 8 & 1 & -0.638008 & -2.077429 & 0.260533 \\
\hline 9 & 1 & 0.179379 & -1.491199 & -1.186570 \\
\hline 10 & 1 & 1.112761 & -1.866642 & $2 \quad 0.260408$ \\
\hline 11 & 6 & -1.336405 & 0.571059 & -0.095825 \\
\hline 12 & 1 & -2.172949 & -0.030459 & $9 \quad 0.260258$ \\
\hline 13 & 1 & -1.480179 & 1.591179 & 9.260698 \\
\hline 14 & 1 & -1.381089 & 0.590439 & -1.186571 \\
\hline
\end{tabular}

Zero-point correction $=$

0.132080 (Hartree/Particle)

Thermal correction to Energy=

0.137771

Thermal correction to Enthalpy=

0.138715

Thermal correction to Gibbs Free Energy=

0.104473

Sum of electronic and zero-point Energies=

$-158.217117$

Sum of electronic and thermal Energies=

$-158.211426$

Sum of electronic and thermal Enthalpies $=$

$-158.210482$

Sum of electronic and thermal Free Energies=

$-158.244724$

Pentane

Standard orientation:

\begin{tabular}{|c|c|c|c|c|}
\hline \multirow{2}{*}{$\begin{array}{l}\text { Center } \\
\text { Number }\end{array}$} & Atomic & \multirow{2}{*}{$\begin{array}{r}\text { Atomic } \\
\text { Type }\end{array}$} & \multicolumn{2}{|c|}{ Coordinates (Angstroms } \\
\hline & Numbe & & $X$ & Z \\
\hline 1 & 6 & 2.545197 & 0.324952 & -0.000007 \\
\hline 2 & 1 & 2.585172 & 0.968026 & -0.879876 \\
\hline 3 & 1 & 2.585172 & 0.968062 & 0.879836 \\
\hline 4 & 1 & 3.441700 & -0.294213 & 0.000005 \\
\hline 5 & 6 & 1.277027 & -0.523327 & 0.000011 \\
\hline 6 & 1 & 1.276213 & -1.179723 & -0.873746 \\
\hline 7 & 1 & 1.276219 & -1.179696 & 0.873787 \\
\hline 8 & 6 & 0.000000 & 0.312461 & 0.000006 \\
\hline 9 & 1 & -0.000008 & 0.970402 & 0.874370 \\
\hline 10 & 1 & 0.000008 & 0.970412 & -0.874350 \\
\hline 11 & 6 & -1.277027 & -0.523327 & -0.000011 \\
\hline
\end{tabular}




$\begin{array}{cccccc}12 & 1 & 0 & -1.276217 & -1.179729 & 0.873742 \\ 13 & 1 & 0 & -1.276215 & -1.179690 & -0.873790 \\ 14 & 6 & 0 & -2.545197 & 0.324952 & 0.000002 \\ 15 & 1 & 0 & -3.441700 & -0.294213 & -0.000059 \\ 16 & 1 & 0 & -2.585195 & 0.967992 & 0.879894 \\ 17 & 1 & 0 & -2.585149 & 0.968095 & -0.879818\end{array}$

Zero-point correction=

Thermal correction to Energy=

Thermal correction to Enthalpy=

Thermal correction to Gibbs Free Energy=

Sum of electronic and zero-point Energies=

Sum of electronic and thermal Energies $=$

Sum of electronic and thermal Enthalpies=

Sum of electronic and thermal Free Energies=

Neopentane
0.161190 (Hartree/Particle)

0.168257

0.169202

0.130884

$-197.474307$

$-197.467240$

$-197.466295$

$-197.504613$

\begin{tabular}{|c|c|c|c|c|}
\hline \multirow{2}{*}{$\begin{array}{l}\text { Center } \\
\text { Number }\end{array}$} & Atomic & \multirow{2}{*}{$\begin{array}{r}\text { Atomic } \\
\text { Type }\end{array}$} & \multicolumn{2}{|c|}{ Coordinates (Angstrom } \\
\hline & Numbe & & X & Z \\
\hline 1 & 6 & -1.232175 & 0.104964 & 0.904227 \\
\hline 2 & 1 & -1.366006 & -0.805413 & 1.490071 \\
\hline 3 & 1 & -1.136973 & 0.940020 & 1.599592 \\
\hline 4 & 1 & -2.137145 & 0.260672 & 0.315416 \\
\hline 5 & 6 & -0.000008 & 0.000011 & 0.000013 \\
\hline 6 & 6 & 1.248222 & -0.217977 & 0.861007 \\
\hline 7 & 1 & 2.142249 & -0.296036 & 0.240889 \\
\hline 8 & 1 & 1.393568 & 0.610300 & 1.555806 \\
\hline 9 & 1 & 1.164730 & -1.135119 & 1.445640 \\
\hline 10 & 6 & -0.170540 & -1.181295 & -0.960378 \\
\hline 11 & 1 & -1.054061 & -1.051572 & -1.586863 \\
\hline 12 & 1 & 0.694729 & -1.279158 & -1.617377 \\
\hline 13 & 1 & -0.282853 & -2.117768 & -0.412359 \\
\hline 14 & 6 & 0.154492 & 1.294300 & -0.804856 \\
\hline 15 & 1 & -0.722277 & 1.474047 & -1.428461 \\
\hline 16 & 1 & 0.277537 & 2.153392 & -0.143999 \\
\hline 17 & 1 & 1.026553 & 1.246622 & -1.458426 \\
\hline
\end{tabular}

Zero-point correction=

Thermal correction to Energy=

Thermal correction to Enthalpy=

Thermal correction to Gibbs Free Energy=

Sum of electronic and zero-point Energies=

Sum of electronic and thermal Energies $=$

Sum of electronic and thermal Enthalpies=

Sum of electronic and thermal Free Energies=
0.159867 (Hartree/Particle)

$$
0.166777
$$

0.167722

\subsection{6}

$-197.479186$

$-197.472275$

$-197.471331$

$-197.508286$

Hexane

Standard orientation: 


\begin{tabular}{|c|c|c|c|c|c|}
\hline \multirow{2}{*}{$\begin{array}{l}\text { Center } \\
\text { Number }\end{array}$} & Atomic & \multirow{2}{*}{\multicolumn{2}{|c|}{$\begin{array}{l}\text { Atomic } \\
\text { Type }\end{array}$}} & \multicolumn{2}{|c|}{ Coordinates (Angstrom } \\
\hline & Numbe & & & $\mathrm{X}$ & Z \\
\hline 1 & 6 & 0 & -3.205656 & 0.208437 & 0.000037 \\
\hline 2 & 1 & 0 & -3.290224 & 0.847124 & 0.879961 \\
\hline 3 & 1 & 0 & -3.290224 & 0.847300 & -0.879761 \\
\hline 4 & 1 & 0 & -4.057016 & -0.471503 & -0.000034 \\
\hline 5 & 6 & 0 & -1.881641 & -0.549703 & -0.000044 \\
\hline 6 & 1 & 0 & -1.835230 & -1.204504 & 0.873654 \\
\hline 7 & 1 & 0 & -1.835262 & -1.204382 & -0.873836 \\
\hline 8 & 6 & 0 & -0.665695 & 0.372992 & -0.000004 \\
\hline 9 & 1 & 0 & -0.712296 & 1.029032 & -0.874397 \\
\hline 10 & 1 & 0 & -0.712326 & 1.029004 & 0.874407 \\
\hline 11 & 6 & 0 & 0.665695 & -0.372992 & 0.000011 \\
\hline 12 & 1 & 0 & 0.712302 & -1.029053 & -0.874366 \\
\hline 13 & 1 & 0 & 0.712320 & -1.028983 & 0.874438 \\
\hline 14 & 6 & 0 & 1.881641 & 0.549703 & -0.000036 \\
\hline 15 & 1 & 0 & 1.835237 & 1.204503 & 0.873663 \\
\hline 16 & 1 & 0 & 1.835255 & 1.204382 & -0.873827 \\
\hline 17 & 6 & 0 & 3.205656 & -0.208437 & 0.000029 \\
\hline 18 & 1 & 0 & 3.290228 & -0.847281 & -0.879781 \\
\hline 19 & 1 & 0 & 4.057016 & 0.471502 & -0.000020 \\
\hline 20 & 1 & 0 & 3.290220 & -0.847143 & 0.879941 \\
\hline
\end{tabular}

Zero-point correction=

Thermal correction to Energy=

0.189775 (Hartree/Particle)

Thermal correction to Enthalpy=

0.198174

0.199118

Thermal correction to Gibbs Free Energy=

0.157194

Sum of electronic and zero-point Energies=

$-236.733593$

Sum of electronic and thermal Energies=

$-236.725194$

Sum of electronic and thermal Enthalpies=

$-236.724250$

Sum of electronic and thermal Free Energies $=\quad-236.766174$

22dimethylbutane

Standard orientation:

\begin{tabular}{|c|c|c|c|c|}
\hline \multirow{2}{*}{$\begin{array}{l}\text { Center } \\
\text { Number }\end{array}$} & Atomic & \multirow{2}{*}{$\begin{array}{l}\text { Atomic } \\
\text { Type }\end{array}$} & \multicolumn{2}{|c|}{ Coordinates (Angstroms) } \\
\hline & Numbe & & $\mathrm{X}$ & Z \\
\hline 1 & 6 & -1.612375 & 0.979353 & 0.000834 \\
\hline 2 & 1 & -1.587696 & 1.620343 & 0.883214 \\
\hline 3 & 1 & -1.587813 & 1.621684 & -0.880574 \\
\hline 4 & 1 & -2.563716 & 0.445833 & 0.000487 \\
\hline 5 & 6 & -0.433251 & -0.000337 & 0.000008 \\
\hline 6 & 6 & 0.870186 & 0.824401 & 0.000411 \\
\hline 7 & 1 & 0.853641 & 1.482040 & -0.872896 \\
\hline 8 & 1 & 0.853690 & 1.481143 & 0.874397 \\
\hline 9 & 6 & 2.177717 & 0.035097 & 0.000006 \\
\hline 10 & 1 & 2.268867 & -0.599103 & -0.880600 \\
\hline 11 & 1 & 3.029235 & 0.714775 & 0.000130 \\
\hline 12 & 1 & 2.269032 & -0.599650 & 0.880197 \\
\hline
\end{tabular}




$\begin{array}{rrrrrr}13 & 6 & 0 & -0.520741 & -0.881285 & 1.251241 \\ 14 & 1 & 0 & 0.267396 & -1.632938 & 1.272911 \\ 15 & 1 & 0 & -0.439549 & -0.280875 & 2.158750 \\ 16 & 1 & 0 & -1.476296 & -1.406215 & 1.283108 \\ 17 & 6 & 0 & -0.521059 & -0.879511 & -1.252458 \\ 18 & 1 & 0 & -1.476509 & -1.404611 & -1.284692 \\ 19 & 1 & 0 & -0.440375 & -0.277768 & -2.159127 \\ 20 & 1 & 0 & 0.267227 & -1.630967 & -1.275552\end{array}$

Zero-point correction=

Thermal correction to Energy=

Thermal correction to Enthalpy=

Thermal correction to Gibbs Free Energy=

Sum of electronic and zero-point Energies=

Sum of electronic and thermal Energies $=$

Sum of electronic and thermal Enthalpies=

Sum of electronic and thermal Free Energies=

Heptane

Standard orientation:

\begin{tabular}{|c|c|c|c|c|}
\hline \multirow{2}{*}{$\begin{array}{l}\text { Center } \\
\text { Number }\end{array}$} & Atomic & \multirow{2}{*}{$\begin{array}{l}\text { Atomic } \\
\text { Type }\end{array}$} & \multicolumn{2}{|c|}{ Coordinates (Angstroms) } \\
\hline & Number & & $\mathrm{X}$ & Z \\
\hline 1 & 6 & -3.821226 & -0.353886 & -0.000006 \\
\hline 2 & 1 & -3.861445 & -0.996903 & -0.879786 \\
\hline 3 & 1 & -3.861437 & -0.996951 & 0.879740 \\
\hline 4 & 1 & -4.717406 & 0.265516 & 0.000016 \\
\hline 5 & 6 & -2.553011 & 0.494276 & 0.000011 \\
\hline 6 & 1 & -2.551878 & 1.150589 & -0.873680 \\
\hline 7 & 1 & -2.551878 & 1.150554 & 0.873730 \\
\hline 8 & 6 & -1.276178 & -0.342128 & -0.000004 \\
\hline 9 & 1 & -1.277120 & -0.999691 & 0.874332 \\
\hline 10 & 1 & -1.277127 & -0.999672 & -0.874354 \\
\hline 11 & 6 & 0.000000 & 0.494922 & -0.000001 \\
\hline 12 & 1 & -0.000003 & 1.152220 & 0.874398 \\
\hline 13 & 1 & 0.000003 & 1.152216 & -0.874403 \\
\hline 14 & 6 & 1.276178 & -0.342128 & 0.000005 \\
\hline 15 & 1 & 1.277120 & -0.999693 & -0.874328 \\
\hline 16 & 1 & 1.277126 & -0.999669 & 0.874357 \\
\hline 17 & 6 & 2.553011 & 0.494276 & -0.000010 \\
\hline 18 & 1 & 2.551878 & 1.150588 & 0.873682 \\
\hline 19 & 1 & 2.551877 & 1.150555 & -0.873727 \\
\hline 20 & 6 & 3.821226 & -0.353886 & 0.000004 \\
\hline 21 & 1 & 3.861439 & -0.996945 & -0.879746 \\
\hline 22 & 1 & 4.717406 & 0.265516 & -0.000010 \\
\hline 23 & 1 & 3.861442 & -0.996909 & 0.879780 \\
\hline
\end{tabular}

Zero-point correction=

Thermal correction to Energy=

Thermal correction to Enthalpy=
0.188643 (Hartree/Particle)

0.196879

0.197824

\subsection{6}

$-236.735641$

$-236.727404$

$-236.726460$

$-236.766918$ 
Thermal correction to Gibbs Free Energy=

Sum of electronic and zero-point Energies=

Sum of electronic and thermal Energies $=$

Sum of electronic and thermal Enthalpies=

Sum of electronic and thermal Free Energies=

223trimethylbutane
0.183449

$-275.992897$

$-275.983137$

$-275.982192$

$-276.0277$

Standard orientation:

\begin{tabular}{|c|c|c|c|c|}
\hline \multirow{2}{*}{$\begin{array}{l}\text { Center } \\
\text { Number }\end{array}$} & Atomic & \multirow{2}{*}{$\begin{array}{r}\text { Atomic } \\
\text { Type }\end{array}$} & \multicolumn{2}{|c|}{ Coordinates (Angstrom } \\
\hline & Number & & $X$ & Z \\
\hline 1 & 6 & -1.351817 & -1.238757 & -0.564481 \\
\hline 2 & 1 & -1.226607 & -1.303773 & -1.646726 \\
\hline 3 & 1 & -0.977881 & -2.161831 & -0.125336 \\
\hline 4 & 1 & -2.420944 & -1.187590 & -0.355832 \\
\hline 5 & 6 & -0.640864 & 0.000006 & -0.000375 \\
\hline 6 & 6 & 0.850848 & -0.000003 & -0.450616 \\
\hline 7 & 1 & 0.824337 & 0.000015 & -1.545708 \\
\hline 8 & 6 & 1.629568 & 1.246139 & -0.016780 \\
\hline 9 & 1 & 1.712748 & 1.305610 & 1.068750 \\
\hline 10 & 1 & 2.643112 & 1.207723 & -0.415950 \\
\hline 11 & 1 & 1.174933 & 2.168343 & -0.371395 \\
\hline 12 & 6 & 1.629570 & -1.246137 & -0.016814 \\
\hline 13 & 1 & 1.713148 & -1.305387 & 1.068697 \\
\hline 14 & 1 & 1.174715 & -2.168379 & -0.371055 \\
\hline 15 & 1 & 2.642977 & -1.207926 & -0.416356 \\
\hline 16 & 6 & -0.786824 & -0.000483 & 1.526513 \\
\hline 17 & 1 & -0.332911 & 0.881392 & 1.977166 \\
\hline 18 & 1 & -1.842841 & -0.000201 & 1.799692 \\
\hline 19 & 1 & -0.333532 & -0.882992 & 1.976537 \\
\hline 20 & 6 & -1.351588 & 1.239228 & -0.563699 \\
\hline 21 & 1 & -1.226174 & 1.305068 & -1.645873 \\
\hline 22 & 1 & -2.420767 & 1.188013 & -0.355306 \\
\hline 23 & 1 & -0.977670 & 2.161952 & -0.123792 \\
\hline
\end{tabular}

Zero-point correction $=$

Thermal correction to Energy=

Thermal correction to Enthalpy=

Thermal correction to Gibbs Free Energy=

Sum of electronic and zero-point Energies=

Sum of electronic and thermal Energies $=$

Sum of electronic and thermal Enthalpies=

0.216950 (Hartree/Particle)

0.226522

0.227466

0.183332

$-275.992343$

$-275.982771$

$-275.981827$

$-276.025961$

Sum of electronic and thermal Free Energies=

Octane

Standard orientation:

\begin{tabular}{cccccc} 
Center & Atomic & \multicolumn{2}{c}{ Atomic } & \multicolumn{2}{c}{ Coordinates } \\
Number & Number & Type & X & Y & Z \\
- \hdashline 1 & 6 & 0 & -4.473339 & 0.256704 & 0.000035
\end{tabular}




$\begin{array}{cccccc}2 & 1 & 0 & -4.538357 & 0.897649 & 0.879992 \\ 3 & 1 & 0 & -4.538308 & 0.897938 & -0.879716 \\ 4 & 1 & 0 & -5.345152 & -0.396827 & -0.000101 \\ 5 & 6 & 0 & -3.173137 & -0.541554 & -0.000062 \\ 6 & 1 & 0 & -3.146794 & -1.197502 & 0.873614 \\ 7 & 1 & 0 & -3.146804 & -1.197303 & -0.873888 \\ 8 & 6 & 0 & -1.929507 & 0.343539 & 0.000025 \\ 9 & 1 & 0 & -1.955956 & 1.000732 & -0.874323 \\ 10 & 1 & 0 & -1.956000 & 1.000631 & 0.874448 \\ 11 & 6 & 0 & -0.621481 & -0.443079 & 0.000013 \\ 12 & 1 & 0 & -0.595887 & -1.100007 & -0.874394 \\ 13 & 1 & 0 & -0.595853 & -1.099968 & 0.874447 \\ 14 & 6 & 0 & 0.621481 & 0.443079 & -0.000029 \\ 15 & 1 & 0 & 0.595864 & 1.100039 & 0.874352 \\ 16 & 1 & 0 & 0.595875 & 1.099935 & -0.874489 \\ 17 & 6 & 0 & 1.929507 & -0.343539 & 0.000026 \\ 18 & 1 & 0 & 1.955979 & -1.000741 & -0.874316 \\ 19 & 1 & 0 & 1.955978 & -1.000622 & 0.874456 \\ 20 & 6 & 0 & 3.173137 & 0.541554 & -0.000034 \\ 21 & 1 & 0 & 3.146799 & 1.197462 & 0.873672 \\ 22 & 1 & 0 & 3.146800 & 1.197343 & -0.873829 \\ 23 & 6 & 0 & 4.473339 & -0.256704 & 0.000021 \\ 24 & 1 & 0 & 4.538333 & -0.897853 & -0.879789 \\ 25 & 1 & 0 & 5.345152 & 0.396827 & -0.000022 \\ 26 & 1 & 0 & 4.538332 & -0.897734 & 0.879918\end{array}$

Zero-point correction=

0.246898 (Hartree/Particle)

Thermal correction to Energy=

0.257997

Thermal correction to Enthalpy=

0.258942

Thermal correction to Gibbs Free Energy $=0.209802$

Sum of electronic and zero-point Energies $=$

Sum of electronic and thermal Energies=

$-315.252212$

$-315.241112$

Sum of electronic and thermal Enthalpies=

$-315.240167$

Sum of electronic and thermal Free Energies= $-315.289307$

2233tetramethylbutane

Standard orientation:

\begin{tabular}{|c|c|c|c|c|}
\hline \multirow{2}{*}{$\begin{array}{l}\text { Center } \\
\text { Number }\end{array}$} & Atomic & \multirow{2}{*}{$\begin{array}{r}\text { Atomic } \\
\text { Type }\end{array}$} & \multicolumn{2}{|c|}{ Coordinates (Angstrom } \\
\hline & Numbe & & $\mathrm{X}$ & Z \\
\hline 1 & 6 & -1.348040 & -1.315627 & -0.566623 \\
\hline 2 & 1 & -1.139439 & -1.421555 & -1.629813 \\
\hline 3 & 1 & -0.946022 & -2.188601 & -0.053834 \\
\hline 4 & 1 & -2.431397 & -1.335476 & -0.443160 \\
\hline 5 & 6 & -0.789192 & -0.000070 & -0.000105 \\
\hline 6 & 6 & 0.789181 & 0.000030 & 0.000001 \\
\hline 7 & 6 & 1.347772 & 1.403212 & 0.288403 \\
\hline 8 & 1 & 1.138807 & 2.098485 & -0.522801 \\
\hline 9 & 1 & 0.945671 & 1.825476 & 1.208536 \\
\hline 10 & 1 & 2.431152 & 1.349146 & 0.400856 \\
\hline
\end{tabular}




$\begin{array}{rrrrrr}11 & 6 & 0 & -1.348268 & 1.148381 & -0.855971 \\ 12 & 1 & 0 & -1.139587 & 2.122056 & -0.416076 \\ 13 & 1 & 0 & -0.946487 & 1.140864 & -1.868473 \\ 14 & 1 & 0 & -2.431637 & 1.051323 & -0.934655 \\ 15 & 6 & 0 & -1.347995 & 0.166943 & 1.422531 \\ 16 & 1 & 0 & -2.431445 & 0.282983 & 1.378166 \\ 17 & 1 & 0 & -1.138602 & -0.700592 & 2.045961 \\ 18 & 1 & 0 & -0.946457 & 1.047849 & 1.921935 \\ 19 & 6 & 0 & 1.348413 & -0.451694 & -1.359128 \\ 20 & 1 & 0 & 2.431787 & -0.327061 & -1.367973 \\ 21 & 1 & 0 & 1.139824 & -1.501889 & -1.555836 \\ 22 & 1 & 0 & 0.946741 & 0.134008 & -2.185110 \\ 23 & 6 & 0 & 1.348115 & -0.951176 & 1.070846 \\ 24 & 1 & 0 & 2.431502 & -1.021187 & 0.967517 \\ 25 & 1 & 0 & 1.139322 & -0.596515 & 2.078675 \\ 26 & 1 & 0 & 0.946353 & -1.959303 & 0.976361\end{array}$

Zero-point correction=

Thermal correction to Energy=

Thermal correction to Enthalpy=

Thermal correction to Gibbs Free Energy=

Sum of electronic and zero-point Energies=

Sum of electronic and thermal Energies=

Sum of electronic and thermal Enthalpies=

Sum of electronic and thermal Free Energies=

PBE0DH/ccpVTZ

Ethane

Standard orientation:

\begin{tabular}{|c|c|c|c|c|}
\hline \multirow{2}{*}{$\begin{array}{l}\text { Center } \\
\text { Number }\end{array}$} & Atomic & \multirow{2}{*}{$\begin{array}{l}\text { Atomic } \\
\text { Type }\end{array}$} & \multicolumn{2}{|c|}{ Coordinates (Angstrom } \\
\hline & Number & & $\mathrm{X}$ & $\mathrm{Z}$ \\
\hline 1 & 6 & 0.000000 & 0.000000 & 0.757742 \\
\hline 2 & 1 & -0.506742 & 0.877737 & 1.154308 \\
\hline 3 & 1 & 1.013513 & -0.000018 & 1.154308 \\
\hline 4 & 1 & -0.506772 & -0.877720 & 1.154308 \\
\hline 5 & 6 & 0.000000 & 0.000000 & -0.757742 \\
\hline 6 & 1 & 0.506742 & 0.877737 & -1.154308 \\
\hline 7 & 1 & 0.506772 & -0.877720 & -1.154308 \\
\hline 8 & 1 & -1.013513 & -0.000018 & -1.154308 \\
\hline
\end{tabular}

Zero-point correction $=$

Thermal correction to Energy=

Thermal correction to Enthalpy=

Thermal correction to Gibbs Free Energy=

Sum of electronic and zero-point Energies=

Sum of electronic and thermal Energies $=$

Sum of electronic and thermal Enthalpies=

Sum of electronic and thermal Free Energies=

Methane
0.075754 (Hartree/Particle)

$$
0.079220
$$

0.080165

$$
0.054361
$$

$-79.682110$

$-79.678644$

$-79.677699$

$-79.703503$ 
Standard orientation:

\begin{tabular}{cccccc} 
Center & \multicolumn{2}{c}{ Atomic } & Atomic & \multicolumn{2}{c}{ Coordinates (Angstroms) } \\
Number & Number & Type & X & Y & Z \\
-1 & 6 & 0 & 0.000000 & 0.000000 & 0.000000 \\
2 & 1 & 0 & 0.626735 & 0.626735 & 0.626735 \\
3 & 1 & 0 & -0.626735 & -0.626735 & 0.626735 \\
4 & 1 & 0 & -0.626735 & 0.626735 & -0.626735 \\
5 & 1 & 0 & 0.626735 & -0.626735 & -0.626735
\end{tabular}

Zero-point correction=

Thermal correction to Energy=

Thermal correction to Enthalpy=

Thermal correction to Gibbs Free Energy=

Sum of electronic and zero-point Energies=

Sum of electronic and thermal Energies=

Sum of electronic and thermal Enthalpies=

Sum of electronic and thermal Free Energies=

Propane
0.045347 (Hartree/Particle)

0.048214

0.049158

$$
\begin{gathered}
0.028043 \\
-40.433830 \\
-40.430963 \\
-40.430019 \\
-40.451133
\end{gathered}
$$

Standard orientation:

\begin{tabular}{cccccc} 
Center & Atomic & \multicolumn{2}{c}{ Atomic } & \multicolumn{3}{c}{ Coordinates (Angstroms) } \\
Number & Number & Type & X & Y & $Z$ \\
- \hdashline 1 & 6 & 0 & -1.260404 & -0.257956 & 0.000000 \\
2 & 1 & 0 & -2.157617 & 0.358107 & -0.000029 \\
3 & 1 & 0 & -1.299364 & -0.901496 & 0.878410 \\
4 & 1 & 0 & -1.299339 & -0.901542 & -0.878377 \\
5 & 6 & 0 & 0.000000 & 0.584189 & 0.000000 \\
6 & 1 & 0 & 0.000000 & 1.240110 & 0.871479 \\
7 & 1 & 0 & 0.000002 & 1.240095 & -0.871489 \\
8 & 6 & 0 & 1.260404 & -0.257957 & 0.000000 \\
9 & 1 & 0 & 1.299359 & -0.901501 & 0.878407 \\
10 & 1 & 0 & 1.299339 & -0.901537 & -0.878381 \\
11 & 1 & 0 & 2.157619 & 0.358104 & -0.000023
\end{tabular}

Zero-point correction=

Thermal correction to Energy=

Thermal correction to Enthalpy=

Thermal correction to Gibbs Free Energy=

Sum of electronic and zero-point Energies=

Sum of electronic and thermal Energies=

Sum of electronic and thermal Enthalpies=

Sum of electronic and thermal Free Energies=

Butane
0.104913 (Hartree/Particle)

0.109409

0.110353

0.079300

$-118.934178$

$-118.929682$

$-118.928738$

$-118.959790$

Standard orientation:

\begin{tabular}{lcccc} 
Center & Atomic & Atomic & & \multicolumn{2}{c}{ Coordinates (Angstroms) } \\
Number & Number & Type & X & Y
\end{tabular}




$\begin{array}{cccccc}1 & 6 & 0 & 1.938153 & -0.118769 & 0.000020 \\ 2 & 1 & 0 & 2.080674 & -0.747305 & -0.878454 \\ 3 & 1 & 0 & 2.080630 & -0.747319 & 0.878491 \\ 4 & 1 & 0 & 2.724850 & 0.633209 & 0.000045 \\ 5 & 6 & 0 & 0.559866 & 0.511425 & -0.000022 \\ 6 & 1 & 0 & 0.451618 & 1.159781 & 0.872018 \\ 7 & 1 & 0 & 0.451644 & 1.159759 & -0.872082 \\ 8 & 6 & 0 & -0.559866 & -0.511425 & -0.000022 \\ 9 & 1 & 0 & -0.451644 & -1.159756 & -0.872084 \\ 10 & 1 & 0 & -0.451618 & -1.159783 & 0.872016 \\ 11 & 6 & 0 & -1.938153 & 0.118769 & 0.000020 \\ 12 & 1 & 0 & -2.080675 & 0.747305 & -0.878454 \\ 13 & 1 & 0 & -2.724850 & -0.633209 & 0.000046 \\ 14 & 1 & 0 & -2.080629 & 0.747320 & 0.878490\end{array}$

Zero-point correction=

Thermal correction to Energy=

Thermal correction to Enthalpy=

Thermal correction to Gibbs Free Energy=

Sum of electronic and zero-point Energies=

Sum of electronic and thermal Energies=

Sum of electronic and thermal Enthalpies=

Sum of electronic and thermal Free Energies=
0.133861 (Hartree/Particle)

0.139604

0.140548

0.105902

$-158.186449$

$-158.180707$

$-158.179762$

$-158.214408$

Isobutane

Standard orientation:

\begin{tabular}{|c|c|c|c|c|}
\hline \multirow{2}{*}{$\begin{array}{l}\text { Center } \\
\text { Number }\end{array}$} & Atomic & \multirow{2}{*}{$\begin{array}{r}\text { Atomic } \\
\text { Type }\end{array}$} & \multicolumn{2}{|c|}{ Coordinates (Angstroms } \\
\hline & Numbe & & $X$ & Z \\
\hline 1 & 6 & -1.416090 & -0.279852 & -0.095167 \\
\hline 2 & 1 & -2.110259 & 0.480859 & 0.259011 \\
\hline 3 & 1 & -1.462292 & -0.289071 & -1.184980 \\
\hline 4 & 1 & -1.768746 & -1.247366 & 0.259149 \\
\hline 5 & 6 & -0.000005 & -0.000008 & 0.374364 \\
\hline 6 & 1 & 0.000006 & 0.000005 & 1.467340 \\
\hline 7 & 6 & 0.465681 & 1.366274 & -0.095168 \\
\hline 8 & 1 & 1.471606 & 1.587040 & 0.258908 \\
\hline 9 & 1 & 0.480681 & 1.410897 & -1.184975 \\
\hline 10 & 1 & -0.195809 & 2.155467 & 0.259229 \\
\hline 11 & 6 & 0.950410 & -1.086420 & -0.095171 \\
\hline 12 & 1 & 1.964712 & -0.907807 & 0.258782 \\
\hline 13 & 1 & 0.638988 & -2.067906 & 0.259368 \\
\hline 14 & 1 & 0.981140 & -1.122085 & -1.184980 \\
\hline
\end{tabular}

Zero-point correction=

Thermal correction to Energy=

Thermal correction to Enthalpy=

Thermal correction to Gibbs Free Energy=

Sum of electronic and zero-point Energies=
0.133363 (Hartree/Particle)

$$
\begin{array}{r}
0.139027 \\
0.139971
\end{array}
$$

0.105794

$-158.188475$ 
Sum of electronic and thermal Energies $=$

$-158.182811$

Sum of electronic and thermal Enthalpies=

Sum of electronic and thermal Free Energies=

Pentane

Standard orientation:

\begin{tabular}{|c|c|c|c|c|}
\hline \multirow{2}{*}{$\begin{array}{l}\text { Center } \\
\text { Number }\end{array}$} & Atomic & \multirow{2}{*}{$\begin{array}{r}\text { Atomic } \\
\text { Type }\end{array}$} & \multicolumn{2}{|c|}{ Coordinates (Angstroms } \\
\hline & Numbe & & $X$ & Z \\
\hline 1 & 6 & 2.527404 & 0.322739 & -0.000001 \\
\hline 2 & 1 & 2.567364 & 0.965907 & -0.878426 \\
\hline 3 & 1 & 2.567362 & 0.965915 & 0.878419 \\
\hline 4 & 1 & 3.424106 & -0.293839 & 0.000003 \\
\hline 5 & 6 & 1.268027 & -0.520312 & 0.000001 \\
\hline 6 & 1 & 1.265985 & -1.177178 & -0.872159 \\
\hline 7 & 1 & 1.265986 & -1.177175 & 0.872164 \\
\hline 8 & 6 & 0.000000 & 0.310829 & -0.000000 \\
\hline 9 & 1 & -0.000001 & 0.969317 & 0.872675 \\
\hline 10 & 1 & 0.000001 & 0.969318 & -0.872674 \\
\hline 11 & 6 & -1.268027 & -0.520312 & -0.000001 \\
\hline 12 & 1 & -1.265985 & -1.177178 & 0.872159 \\
\hline 13 & 1 & -1.265986 & -1.177175 & -0.872164 \\
\hline 14 & 6 & -2.527404 & 0.322739 & 0.000001 \\
\hline 15 & 1 & -3.424106 & -0.293839 & 0.000000 \\
\hline 16 & 1 & -2.567363 & 0.965910 & 0.878423 \\
\hline 17 & 1 & -2.567364 & 0.965912 & -0.878421 \\
\hline
\end{tabular}

Zero-point correction $=$

0.162799 (Hartree/Particle)

Thermal correction to Energy=

0.169838

Thermal correction to Enthalpy=

0.170782

Thermal correction to Gibbs Free Energy=

Sum of electronic and zero-point Energies=

0.132526

Sum of electronic and thermal Energies=

$-197.438646$

$-197.431606$

Sum of electronic and thermal Enthalpies $=$

$-197.430662$

Sum of electronic and thermal Free Energies=

$-197.468919$

Neopentane:

Standard orientation:

\begin{tabular}{|c|c|c|c|c|}
\hline \multirow{2}{*}{$\begin{array}{l}\text { Center } \\
\text { Number }\end{array}$} & Atomic & \multirow{2}{*}{$\begin{array}{l}\text { Atomic } \\
\text { Type }\end{array}$} & \multicolumn{2}{|c|}{ Coordinates (Angstroms) } \\
\hline & Numbe & & $\mathrm{X}$ & $\mathrm{Z}$ \\
\hline 1 & 6 & -0.589792 & 0.995815 & 0.987998 \\
\hline 2 & 1 & -1.679020 & 0.971367 & 0.964323 \\
\hline 3 & 1 & -0.272071 & 0.771324 & 2.005954 \\
\hline 4 & 1 & -0.272731 & 2.012036 & 0.754881 \\
\hline 5 & 6 & 0.000012 & -0.000012 & 0.000008 \\
\hline 6 & 6 & -0.464426 & -1.404453 & 0.357052 \\
\hline 7 & 1 & -0.055683 & -2.139302 & -0.336135 \\
\hline 8 & 1 & -0.144354 & -1.678229 & 1.362129 \\
\hline
\end{tabular}




$\begin{array}{cccrrr}9 & 1 & 0 & -1.551085 & -1.477905 & 0.320253 \\ 10 & 6 & 0 & -0.464959 & 0.345609 & -1.407114 \\ 11 & 1 & 0 & -0.145115 & 1.348478 & -1.689130 \\ 12 & 1 & 0 & -0.056336 & -0.353440 & -2.136449 \\ 13 & 1 & 0 & -1.551637 & 0.308070 & -1.479911 \\ 14 & 6 & 0 & 1.519171 & 0.063031 & 0.062065 \\ 15 & 1 & 0 & 1.879588 & 1.060048 & -0.190194 \\ 16 & 1 & 0 & 1.879934 & -0.180353 & 1.061159 \\ 17 & 1 & 0 & 1.968475 & -0.642031 & -0.636929\end{array}$

Zero-point correction $=$

Thermal correction to Energy=

Thermal correction to Enthalpy=

Thermal correction to Gibbs Free Energy=

Sum of electronic and zero-point Energies=

Sum of electronic and thermal Energies=

Sum of electronic and thermal Enthalpies=

Sum of electronic and thermal Free Energies=

Hexane

Standard orientation:

\begin{tabular}{|c|c|c|c|c|}
\hline \multirow{3}{*}{$\begin{array}{l}\text { Center } \\
\text { Number } \\
-1\end{array}$} & \multirow{2}{*}{$\begin{array}{l}\text { Atomic } \\
\text { Number }\end{array}$} & \multirow{2}{*}{$\begin{array}{l}\text { Atomic } \\
\text { Type }\end{array}$} & \multicolumn{2}{|c|}{ Coordinates (Angstroms) } \\
\hline & & & $\mathrm{X}$ & Z \\
\hline & 6 & -3.183204 & 0.206878 & 0.000005 \\
\hline 2 & 1 & -3.267755 & 0.845718 & 0.878442 \\
\hline 3 & 1 & -3.267756 & 0.845739 & -0.878417 \\
\hline 4 & 1 & -4.034934 & -0.470488 & -0.000003 \\
\hline 5 & 6 & -1.868325 & -0.546681 & -0.000005 \\
\hline 6 & 1 & -1.820685 & -1.201831 & 0.872122 \\
\hline 7 & 1 & -1.820689 & -1.201815 & -0.872145 \\
\hline 8 & 6 & -0.660998 & 0.370742 & -0.000000 \\
\hline 9 & 1 & -0.707712 & 1.027327 & -0.872724 \\
\hline 10 & 1 & -0.707714 & 1.027321 & 0.872728 \\
\hline 11 & 6 & 0.660998 & -0.370742 & -0.000000 \\
\hline 12 & 1 & 0.707712 & -1.027327 & -0.872724 \\
\hline 13 & 1 & 0.707715 & -1.027321 & 0.872727 \\
\hline 14 & 6 & 1.868325 & 0.546681 & -0.000005 \\
\hline 15 & 1 & 1.820685 & 1.201831 & 0.872122 \\
\hline 16 & 1 & 1.820689 & 1.201815 & -0.872145 \\
\hline 17 & 6 & 3.183204 & -0.206878 & 0.000005 \\
\hline 18 & 1 & 3.267755 & -0.845740 & -0.878416 \\
\hline 19 & 1 & 4.034934 & 0.470488 & -0.000004 \\
\hline 20 & 1 & 3.267755 & -0.845717 & 0.878442 \\
\hline
\end{tabular}

Zero-point correction=

Thermal correction to Energy=

Thermal correction to Enthalpy=

Thermal correction to Gibbs Free Energy=
0.161437 (Hartree/Particle)

0.168310

0.169254
0.132386

$-197.443538$

$-197.436666$

$-197.435721$

$-197.472590$ 
Sum of electronic and zero-point Energies=

$-236.690905$

Sum of electronic and thermal Energies $=$

Sum of electronic and thermal Enthalpies=

Sum of electronic and thermal Free Energies=

22dimethylbutane

Standard orientation:

\begin{tabular}{cccccc} 
Center & \multicolumn{2}{c}{ Atomic } & \multicolumn{2}{c}{ Atomic } & \multicolumn{3}{c}{ Coordinates (Angstroms) } \\
Number & Number & Type & X & $Y$ & $Z$ \\
-------------- \\
1 & 6 & 0 & -1.602886 & 0.970480 & 0.000963 \\
2 & 1 & 0 & -1.579336 & 1.610884 & 0.882541 \\
3 & 1 & 0 & -1.579435 & 1.612482 & -0.879455 \\
4 & 1 & 0 & -2.552569 & 0.436186 & 0.000528 \\
5 & 6 & 0 & -0.430129 & -0.000428 & 0.000010 \\
6 & 6 & 0 & 0.862533 & 0.821113 & 0.000526 \\
7 & 1 & 0 & 0.843912 & 1.478991 & -0.871426 \\
8 & 1 & 0 & 0.843967 & 1.477839 & 0.873352 \\
9 & 6 & 0 & 2.160501 & 0.036405 & 0.000005 \\
10 & 1 & 0 & 2.251092 & -0.597638 & -0.879720 \\
11 & 1 & 0 & 3.013196 & 0.712704 & 0.000227 \\
12 & 1 & 0 & 2.251259 & -0.598446 & 0.879126 \\
13 & 6 & 0 & -0.514497 & -0.875870 & 1.242658 \\
14 & 1 & 0 & 0.275406 & -1.624350 & 1.264143 \\
15 & 1 & 0 & -0.435741 & -0.276157 & 2.149552 \\
16 & 1 & 0 & -1.467174 & -1.403805 & 1.274224 \\
17 & 6 & 0 & -0.514824 & -0.873757 & -1.244108 \\
18 & 1 & 0 & -1.467408 & -1.401830 & -1.276195 \\
19 & 1 & 0 & -0.436562 & -0.272458 & -2.149993 \\
20 & 1 & 0 & 0.275208 & -1.622053 & -1.267222
\end{tabular}

Zero-point correction $=$

0.190502 (Hartree/Particle)

Thermal correction to Energy=

0.198693

Thermal correction to Enthalpy=

0.199637

Thermal correction to Gibbs Free Energy=

0.159265

Sum of electronic and zero-point Energies=

$-236.693141$

Sum of electronic and thermal Energies=

$-236.684949$

Sum of electronic and thermal Enthalpies=

$-236.684005$

Sum of electronic and thermal Free Energies $=\quad-236.724377$

Heptane

Standard orientation:

\begin{tabular}{|c|c|c|c|c|}
\hline \multirow{2}{*}{$\begin{array}{l}\text { Center } \\
\text { Number }\end{array}$} & \multirow{2}{*}{$\begin{array}{l}\text { Atomic } \\
\text { Number }\end{array}$} & \multirow{2}{*}{$\begin{array}{l}\text { Atomic } \\
\text { Type }\end{array}$} & \multicolumn{2}{|c|}{ Coordinates (Angstroms) } \\
\hline & & & $\mathrm{X}$ & $\mathrm{Z}$ \\
\hline 1 & 6 & -3.794864 & -0.351616 & -0.000007 \\
\hline 2 & 1 & -3.834815 & -0.994793 & -0.878444 \\
\hline 3 & 1 & -3.834814 & -0.994824 & 0.878408 \\
\hline 4 & 1 & -4.691616 & 0.264918 & 0.000005 \\
\hline 5 & 6 & -2.535493 & 0.491446 & 0.000009 \\
\hline
\end{tabular}




$\begin{array}{cccccc}6 & 1 & 0 & -2.533437 & 1.148324 & -0.872127 \\ 7 & 1 & 0 & -2.533441 & 1.148299 & 0.872163 \\ 8 & 6 & 0 & -1.267392 & -0.339953 & -0.000000 \\ 9 & 1 & 0 & -1.268399 & -0.998193 & 0.872700 \\ 10 & 1 & 0 & -1.268402 & -0.998182 & -0.872709 \\ 11 & 6 & 0 & 0.000000 & 0.491801 & 0.000001 \\ 12 & 1 & 0 & -0.000002 & 1.149788 & 0.872784 \\ 13 & 1 & 0 & 0.000002 & 1.149786 & -0.872784 \\ 14 & 6 & 0 & 1.267392 & -0.339953 & 0.000005 \\ 15 & 1 & 0 & 1.268397 & -0.998198 & -0.872692 \\ 16 & 1 & 0 & 1.268404 & -0.998178 & 0.872717 \\ 17 & 6 & 0 & 2.535493 & 0.491446 & -0.000010 \\ 18 & 1 & 0 & 2.533440 & 1.148325 & 0.872125 \\ 19 & 1 & 0 & 2.533438 & 1.148297 & -0.872165 \\ 20 & 6 & 0 & 3.794864 & -0.351616 & 0.000002 \\ 21 & 1 & 0 & 3.834813 & -0.994824 & -0.878413 \\ 22 & 1 & 0 & 4.691616 & 0.264918 & -0.000010 \\ 23 & 1 & 0 & 3.834817 & -0.994793 & 0.878440\end{array}$

Zero-point correction=

Thermal correction to Energy=

Thermal correction to Enthalpy=

Thermal correction to Gibbs Free Energy=

Sum of electronic and zero-point Energies=

Sum of electronic and thermal Energies $=$

Sum of electronic and thermal Enthalpies=

Sum of electronic and thermal Free Energies=

223trimethylbutane

\subsection{1 (Hartree/Particle)}

0.230263

$$
0.231207
$$

\subsection{5}

$-275.943179$

$-275.933477$

$-275.932533$

$-275.977985$

Standard orientation:

\begin{tabular}{cccccc} 
Center & \multicolumn{2}{c}{ Atomic } & \multicolumn{2}{c}{ Atomic } & \multicolumn{3}{c}{ Coordinates } \\
Number & Number & Type & X & $Y$ & $Z$ \\
-1 & 6 & 0 & -1.341458 & -1.230765 & -0.559399 \\
2 & 1 & 0 & -1.216697 & -1.296943 & -1.640584 \\
3 & 1 & 0 & -0.967742 & -2.152541 & -0.119452 \\
4 & 1 & 0 & -2.409547 & -1.180102 & -0.350779 \\
5 & 6 & 0 & -0.635719 & 0.000004 & -0.000463 \\
6 & 6 & 0 & 0.843898 & 0.000000 & -0.449233 \\
7 & 1 & 0 & 0.815519 & 0.000002 & -1.543596 \\
8 & 6 & 0 & 1.615895 & 1.237770 & -0.017350 \\
9 & 1 & 0 & 1.697950 & 1.296350 & 1.067449 \\
10 & 1 & 0 & 2.629332 & 1.201053 & -0.413898 \\
11 & 1 & 0 & 1.161736 & 2.159433 & -0.371340 \\
12 & 6 & 0 & 1.615919 & -1.237753 & -0.017385 \\
13 & 1 & 0 & 1.698040 & -1.296332 & 1.067412 \\
14 & 1 & 0 & 1.161762 & -2.159428 & -0.371344 \\
15 & 1 & 0 & 2.629333 & -1.201005 & -0.413990 \\
16 & 6 & 0 & -0.777924 & -0.000112 & 1.516181 \\
17 & 1 & 0 & -0.324187 & 0.881435 & 1.965415
\end{tabular}




$\begin{array}{rrrrrr}18 & 1 & 0 & -1.832352 & 0.000002 & 1.791139 \\ 19 & 1 & 0 & -0.324408 & -0.881855 & 1.965251 \\ 20 & 6 & 0 & -1.341423 & 1.230854 & -0.559252 \\ 21 & 1 & 0 & -1.216561 & 1.297230 & -1.640416 \\ 22 & 1 & 0 & -2.409538 & 1.180145 & -0.350760 \\ 23 & 1 & 0 & -0.967775 & 2.152562 & -0.119108\end{array}$

Zero-point correction=

Thermal correction to Energy=

Thermal correction to Enthalpy=

Thermal correction to Gibbs Free Energy=

Sum of electronic and zero-point Energies=

Sum of electronic and thermal Energies $=$

Sum of electronic and thermal Enthalpies=

Sum of electronic and thermal Free Energies=

Octane
0.219068 (Hartree/Particle)

0.228608

0.229552

0.184890

$-275.943038$

$-275.933498$

$-275.932554$

$-275.977216$

Standard orientation:

\begin{tabular}{cccccc} 
Center & Atomic & \multicolumn{2}{c}{ Atomic } & \multicolumn{3}{c}{ Coordinates } \\
Number & Number & Type & X & Y & $Z$ \\
\hline 1 & 6 & 0 & -4.441893 & 0.254882 & 0.000013 \\
2 & 1 & 0 & -4.506855 & 0.896017 & 0.878453 \\
3 & 1 & 0 & -4.506857 & 0.896060 & -0.878397 \\
4 & 1 & 0 & -5.313984 & -0.396076 & -0.000003 \\
5 & 6 & 0 & -3.150672 & -0.538537 & -0.000011 \\
6 & 1 & 0 & -3.123073 & -1.194849 & 0.872118 \\
7 & 1 & 0 & -3.123081 & -1.194814 & -0.872167 \\
8 & 6 & 0 & -1.915848 & 0.341529 & 0.000001 \\
9 & 1 & 0 & -1.942441 & 0.999241 & -0.872703 \\
10 & 1 & 0 & -1.942443 & 0.999222 & 0.872719 \\
11 & 6 & 0 & -0.617084 & -0.440324 & -0.000004 \\
12 & 1 & 0 & -0.591498 & -1.097814 & -0.872761 \\
13 & 1 & 0 & -0.591492 & -1.097816 & 0.872750 \\
14 & 6 & 0 & 0.617084 & 0.440324 & -0.000007 \\
15 & 1 & 0 & 0.591491 & 1.097824 & 0.872742 \\
16 & 1 & 0 & 0.591498 & 1.097807 & -0.872769 \\
17 & 6 & 0 & 1.915848 & -0.341529 & 0.000006 \\
18 & 1 & 0 & 1.942442 & -0.999246 & -0.872695 \\
19 & 1 & 0 & 1.942441 & -0.999218 & 0.872727 \\
20 & 6 & 0 & 3.150672 & 0.538537 & -0.000007 \\
21 & 1 & 0 & 3.123076 & 1.194846 & 0.872124 \\
22 & 1 & 0 & 3.123079 & 1.194816 & -0.872161 \\
23 & 6 & 0 & 4.441893 & -0.254882 & 0.000008 \\
24 & 1 & 0 & 4.506857 & -0.896054 & -0.878405 \\
25 & 1 & 0 & 5.313984 & 0.396076 & -0.000001 \\
26 & 1 & 0 & 4.506855 & -0.896023 & 0.878444
\end{tabular}

Zero-point correction=

Thermal correction to Energy=
0.249427 (Hartree/Particle)

0.260476 
Thermal correction to Enthalpy=

Thermal correction to Gibbs Free Energy=

Sum of electronic and zero-point Energies=

Sum of electronic and thermal Energies $=$

Sum of electronic and thermal Enthalpies=

Sum of electronic and thermal Free Energies=

2233tetramethylbutane
0.261421

0.212373

$-315.195460$

$-315.184411$

$-315.183467$

$-315.232514$

Standard orientation:

\begin{tabular}{|c|c|c|c|c|}
\hline \multirow{2}{*}{$\begin{array}{l}\text { Center } \\
\text { Number }\end{array}$} & Atomic & \multirow{2}{*}{$\begin{array}{l}\text { Atomic } \\
\text { Type }\end{array}$} & \multicolumn{2}{|c|}{ Coordinates (Angstrom } \\
\hline & Numbe & & $X$ & Z \\
\hline 1 & 6 & -1.335572 & -0.271512 & -1.396435 \\
\hline 2 & 1 & -1.130588 & 0.548936 & -2.080655 \\
\hline 3 & 1 & -0.929237 & -1.183605 & -1.830083 \\
\hline 4 & 1 & -2.417360 & -0.390067 & -1.344164 \\
\hline 5 & 6 & -0.782182 & -0.000089 & 0.000051 \\
\hline 6 & 6 & 0.782177 & -0.000054 & -0.000016 \\
\hline 7 & 6 & 1.335365 & 0.569651 & 1.303643 \\
\hline 8 & 1 & 1.130303 & 1.633523 & 1.400690 \\
\hline 9 & 1 & 0.928687 & 0.065337 & 2.178472 \\
\hline 10 & 1 & 2.417141 & 0.442325 & 1.328274 \\
\hline 11 & 6 & -1.335370 & 1.345169 & 0.463183 \\
\hline 12 & 1 & -1.130545 & 1.527391 & 1.515872 \\
\hline 13 & 1 & -0.928580 & 2.176649 & -0.109728 \\
\hline 14 & 1 & -2.417108 & 1.359610 & 0.334076 \\
\hline 15 & 6 & -1.335385 & -1.073864 & 0.933394 \\
\hline 16 & 1 & -2.417151 & -0.969386 & 1.010285 \\
\hline 17 & 1 & -1.130395 & -2.076553 & 0.564753 \\
\hline 18 & 1 & -0.928697 & -0.993561 & 1.939995 \\
\hline 19 & 6 & 1.335227 & 0.844342 & -1.145084 \\
\hline 20 & 1 & 2.416996 & 0.929389 & -1.047122 \\
\hline 21 & 1 & 1.130192 & 0.396619 & -2.115039 \\
\hline 22 & 1 & 0.928516 & 1.854120 & -1.145543 \\
\hline 23 & 6 & 1.335747 & -1.413742 & -0.158694 \\
\hline 24 & 1 & 2.417450 & -1.370962 & -0.281701 \\
\hline 25 & 1 & 1.131342 & -2.029921 & 0.714148 \\
\hline 26 & 1 & 0.928990 & -1.919262 & -1.032785 \\
\hline
\end{tabular}

Zero-point correction $=$

Thermal correction to Energy=

Thermal correction to Enthalpy=

Thermal correction to Gibbs Free Energy=

Sum of electronic and zero-point Energies=

Sum of electronic and thermal Energies=

Sum of electronic and thermal Enthalpies=

Sum of electronic and thermal Free Energies=

PBEQIDH/DHSVPD

Ethane
0.247792 (Hartree/Particle)

0.258172

0.259116

0.214507

$-315.192439$

$-315.182059$

$-315.181114$

$-315.225723$

Standard orientation: 


\begin{tabular}{|c|c|c|c|c|}
\hline \multirow{2}{*}{$\begin{array}{l}\text { Center } \\
\text { Number }\end{array}$} & \multirow{2}{*}{$\begin{array}{l}\text { Atomic } \\
\text { Number }\end{array}$} & \multirow{2}{*}{$\begin{array}{r}\text { Atomic } \\
\text { Type }\end{array}$} & \multicolumn{2}{|c|}{ Coordinates (Angstroms) } \\
\hline & & & $X$ & $\mathrm{Z}$ \\
\hline 1 & 6 & 0.000000 & -0.000000 & 0.757775 \\
\hline 2 & 1 & -0.506314 & 0.876986 & 1.153161 \\
\hline 3 & 1 & 1.012649 & -0.000012 & 1.153161 \\
\hline 4 & 1 & -0.506335 & -0.876974 & 1.153161 \\
\hline 5 & 6 & 0.000000 & -0.000000 & -0.757775 \\
\hline 6 & 1 & 0.506314 & 0.876986 & -1.153161 \\
\hline 7 & 1 & 0.506335 & -0.876974 & -1.153161 \\
\hline 8 & 1 & -1.012649 & -0.000012 & -1.153161 \\
\hline \multicolumn{3}{|c|}{ Zero-point correction $=$} & \multicolumn{2}{|c|}{0.076114 (Hartree/Particle) } \\
\hline \multicolumn{3}{|c|}{ Thermal correction to Energy= } & \multicolumn{2}{|c|}{0.079573} \\
\hline \multicolumn{3}{|c|}{ Thermal correction to Enthalpy= } & \multicolumn{2}{|c|}{0.080517} \\
\hline \multicolumn{4}{|c|}{ Thermal correction to Gibbs Free Energy= } & 0.054726 \\
\hline \multicolumn{4}{|c|}{ Sum of electronic and zero-point Energies $=$} & -79.664992 \\
\hline & -79.661533 \\
\hline \multicolumn{4}{|c|}{$\begin{array}{l}\text { Sum of electronic and thermal Energies }= \\
\text { Sum of electronic and thermal Enthalpies }=\end{array}$} & -79.660589 \\
\hline \multicolumn{4}{|c|}{ Sum of electronic and thermal Free Energies $=$} & -79.686380 \\
\hline
\end{tabular}

Methane

Standard orientation:

\begin{tabular}{|c|c|c|c|c|}
\hline \multirow{2}{*}{$\begin{array}{l}\text { Center } \\
\text { Number }\end{array}$} & Atomic & \multirow{2}{*}{$\begin{array}{r}\text { Atomic } \\
\text { Type }\end{array}$} & \multicolumn{2}{|c|}{ Coordinates (Angstroms) } \\
\hline & Numbe & & $\mathrm{X}$ & $\mathrm{Z}$ \\
\hline 1 & 6 & 0.000000 & 0.000000 & 0.000000 \\
\hline 2 & 1 & 0.626051 & 0.626051 & 0.626051 \\
\hline 3 & 1 & -0.626051 & -0.626051 & 0.626051 \\
\hline 4 & 1 & -0.626051 & 0.626051 & -0.626051 \\
\hline 5 & 1 & 0.626051 & -0.626051 & -0.626051 \\
\hline \multicolumn{3}{|c|}{ Zero-point correction $=$} & \multicolumn{2}{|c|}{0.045575 (Hartree/Particle) } \\
\hline \multicolumn{3}{|c|}{ Thermal correction to Energy= } & \multicolumn{2}{|c|}{0.048440} \\
\hline \multicolumn{3}{|c|}{ Thermal correction to Enthalpy= } & \multicolumn{2}{|c|}{0.049385} \\
\hline \multicolumn{4}{|c|}{ Thermal correction to Gibbs Free Energy $=$} & 0.028274 \\
\hline \multicolumn{4}{|c|}{ Sum of electronic and zero-point Energies $=$} & -40.425008 \\
\hline \multicolumn{4}{|c|}{ Sum of electronic and thermal Energies $=$} & -40.422143 \\
\hline \multicolumn{4}{|c|}{ Sum of electronic and thermal Enthalpies= } & -40.421198 \\
\hline \multicolumn{4}{|c|}{ Sum of electronic and thermal Free Energies= } & -40.442309 \\
\hline
\end{tabular}

Propane

Standard orientation:

\begin{tabular}{cccccc} 
Center & \multicolumn{2}{c}{ Atomic } & Atomic & \multicolumn{2}{c}{ Coordinates } \\
Number & Number & Type & X & Y & Z \\
-1 & 6 & 0 & -1.258683 & -0.258433 & 0.000000 \\
2 & 1 & 0 & -2.156349 & 0.354964 & -0.000033 \\
3 & 1 & 0 & -1.294884 & -0.901142 & 0.877723
\end{tabular}




$\begin{array}{cccccc}4 & 1 & 0 & -1.294855 & -0.901193 & -0.877686 \\ 5 & 6 & 0 & 0.000000 & 0.585890 & 0.000000 \\ 6 & 1 & 0 & 0.000001 & 1.240309 & 0.871097 \\ 7 & 1 & 0 & 0.000002 & 1.240295 & -0.871107 \\ 8 & 6 & 0 & 1.258683 & -0.258433 & 0.000000 \\ 9 & 1 & 0 & 1.294877 & -0.901150 & 0.877718 \\ 10 & 1 & 0 & 1.294857 & -0.901186 & -0.877692 \\ 11 & 1 & 0 & 2.156351 & 0.354961 & -0.000023\end{array}$

Zero-point correction=

Thermal correction to Energy=

Thermal correction to Enthalpy=
0.105375 (Hartree/Particle)

0.109861

0.110805

0.079770

$-118.909156$

$-118.904670$

$-118.903726$

Sum of electronic and thermal Free Energies $=\quad-118.934760$

Butane

Standard orientation:

\begin{tabular}{|c|c|c|c|c|}
\hline \multirow{2}{*}{$\begin{array}{l}\text { Center } \\
\text { Number }\end{array}$} & Atomic & \multirow{2}{*}{$\begin{array}{r}\text { Atomic } \\
\text { Type }\end{array}$} & \multicolumn{2}{|c|}{ Coordinates (Angstroms } \\
\hline & Numbe & & $\mathrm{X}$ & $\mathrm{Z}$ \\
\hline 1 & 6 & 1.936333 & -0.118672 & 0.000021 \\
\hline 2 & 1 & 2.076650 & -0.746673 & -0.877785 \\
\hline 3 & 1 & 2.076602 & -0.746693 & 0.877820 \\
\hline 4 & 1 & 2.723105 & 0.631634 & 0.000050 \\
\hline 5 & 6 & 0.558629 & 0.512401 & -0.000023 \\
\hline 6 & 1 & 0.449626 & 1.159194 & 0.871689 \\
\hline 7 & 1 & 0.449653 & 1.159172 & -0.871756 \\
\hline 8 & 6 & -0.558629 & -0.512401 & -0.000023 \\
\hline 9 & 1 & -0.449653 & -1.159170 & -0.871758 \\
\hline 10 & 1 & -0.449626 & -1.159197 & 0.871687 \\
\hline 11 & 6 & -1.936333 & 0.118672 & 0.000021 \\
\hline 12 & 1 & -2.076652 & 0.746671 & -0.877786 \\
\hline 13 & 1 & -2.723105 & -0.631634 & 0.000053 \\
\hline 14 & 1 & -2.076601 & 0.746694 & 0.877819 \\
\hline
\end{tabular}

Zero-point correction=

Thermal correction to Energy=

Thermal correction to Enthalpy=

Thermal correction to Gibbs Free Energy=

Sum of electronic and zero-point Energies=

Sum of electronic and thermal Energies $=$

Sum of electronic and thermal Enthalpies=

Sum of electronic and thermal Free Energies=

Isobutane

\section{Standard orientation:}

0.134426 (Hartree/Particle)

0.140157

0.141101

0.106477

$-158.153569$

$-158.147839$

$-158.146895$

$-158.181519$

Center Atomic Atomic Coordinates (Angstroms) 


\begin{tabular}{cccccc} 
Number & Number & Type & $X$ & $Y$ & $Z$ \\
\hline 1 & 6 & 0 & -0.834670 & -1.175786 & -0.095456 \\
2 & 1 & 0 & -1.861287 & -1.101129 & 0.257048 \\
3 & 1 & 0 & -0.859285 & -1.210518 & -1.184438 \\
4 & 1 & 0 & -0.425657 & -2.120333 & 0.257123 \\
5 & 6 & 0 & 0.000005 & 0.000002 & 0.376750 \\
6 & 1 & 0 & 0.000006 & 0.000018 & 1.468546 \\
7 & 6 & 0 & -0.600929 & 1.310732 & -0.095459 \\
8 & 1 & 0 & -0.023028 & 2.162496 & 0.257109 \\
9 & 1 & 0 & -0.618654 & 1.349458 & -1.184442 \\
10 & 1 & 0 & -1.623462 & 1.428729 & 0.257070 \\
11 & 6 & 0 & 1.435599 & -0.134950 & -0.095458 \\
12 & 1 & 0 & 2.049039 & 0.691666 & 0.256924 \\
13 & 1 & 0 & 1.884330 & -1.061242 & 0.257241 \\
14 & 1 & 0 & 1.477968 & -0.139132 & -1.184439
\end{tabular}

Zero-point correction=

Thermal correction to Energy=

Thermal correction to Enthalpy=

Thermal correction to Gibbs Free Energy=

Sum of electronic and zero-point Energies=

Sum of electronic and thermal Energies $=$

Sum of electronic and thermal Enthalpies=

Sum of electronic and thermal Free Energies=

Pentane
0.133905 (Hartree/Particle)

0.139556

$$
0.140500
$$

0.106347

$-158.156020$

$-158.150368$

$-158.149424$

$-158.183577$

Standard orientation:

\begin{tabular}{|c|c|c|c|c|}
\hline \multirow{2}{*}{$\begin{array}{l}\text { Center } \\
\text { Number }\end{array}$} & Atomic & \multirow{2}{*}{$\begin{array}{l}\text { Atomic } \\
\text { Type }\end{array}$} & \multicolumn{2}{|c|}{ Coordinates (Angstroms } \\
\hline & Numbe & & $\mathrm{X}$ & Z \\
\hline 1 & 6 & 2.524554 & 0.323786 & -0.000001 \\
\hline 2 & 1 & 2.561591 & 0.966112 & -0.877787 \\
\hline 3 & 1 & 2.561589 & 0.966120 & 0.877781 \\
\hline 4 & 1 & 3.421834 & -0.290046 & 0.000003 \\
\hline 5 & 6 & 1.266919 & -0.521594 & 0.000001 \\
\hline 6 & 1 & 1.264633 & -1.177011 & -0.871873 \\
\hline 7 & 1 & 1.264633 & -1.177008 & 0.871878 \\
\hline 8 & 6 & 0.000000 & 0.310387 & 0.000000 \\
\hline 9 & 1 & -0.000001 & 0.967521 & 0.872433 \\
\hline 10 & 1 & 0.000001 & 0.967522 & -0.872432 \\
\hline 11 & 6 & -1.266919 & -0.521594 & -0.000001 \\
\hline 12 & 1 & -1.264633 & -1.177011 & 0.871873 \\
\hline 13 & 1 & -1.264633 & -1.177008 & -0.871877 \\
\hline 14 & 6 & -2.524554 & 0.323786 & 0.000001 \\
\hline 15 & 1 & -3.421834 & -0.290046 & -0.000001 \\
\hline 16 & 1 & -2.561590 & 0.966115 & 0.877785 \\
\hline 17 & 1 & -2.561590 & 0.966117 & -0.877783 \\
\hline
\end{tabular}


Thermal correction to Energy=

Thermal correction to Enthalpy=

Thermal correction to Gibbs Free Energy=

Sum of electronic and zero-point Energies=

Sum of electronic and thermal Energies=

Sum of electronic and thermal Enthalpies=

Sum of electronic and thermal Free Energies=

Neopentane

Standard orientation:
0.170488

0.171433

0.133203

$-197.397927$

$-197.390902$

$-197.389958$

$-197.428188$

\begin{tabular}{|c|c|c|c|c|}
\hline \multirow{3}{*}{$\begin{array}{l}\text { Center } \\
\text { Number } \\
-1 \\
-1\end{array}$} & \multirow{2}{*}{$\begin{array}{l}\text { Atomic } \\
\text { Number }\end{array}$} & \multirow{2}{*}{$\begin{array}{l}\text { Atomic } \\
\text { Type }\end{array}$} & \multicolumn{2}{|c|}{ Coordinates (Angstrom } \\
\hline & & & $\mathrm{X}$ & $\mathrm{Z}$ \\
\hline & 6 & -0.592632 & 0.215981 & 1.383976 \\
\hline 2 & 1 & -0.911775 & -0.728507 & 1.822341 \\
\hline 3 & 1 & 0.137699 & 0.667554 & 2.053873 \\
\hline 4 & 1 & -1.458704 & 0.874668 & 1.337929 \\
\hline 5 & 6 & 0.000006 & -0.000002 & 0.000001 \\
\hline 6 & 6 & 1.205023 & -0.922296 & 0.102775 \\
\hline 7 & 1 & 1.648612 & -1.093588 & -0.876999 \\
\hline 8 & 1 & 1.970230 & -0.492374 & 0.747520 \\
\hline 9 & 1 & 0.921112 & -1.888806 & 0.516690 \\
\hline 10 & 6 & -1.045645 & -0.631122 & -0.906404 \\
\hline 11 & 1 & -1.920777 & 0.010790 & -0.996760 \\
\hline 12 & 1 & -0.645604 & -0.796439 & -1.905758 \\
\hline 13 & 1 & -1.373120 & -1.592150 & -0.512381 \\
\hline 14 & 6 & 0.433255 & 1.337444 & -0.580344 \\
\hline 15 & 1 & -0.413041 & 2.017777 & -0.664016 \\
\hline 16 & 1 & 1.183772 & 1.810832 & 0.051084 \\
\hline 17 & 1 & 0.861555 & 1.210217 & -1.573542 \\
\hline
\end{tabular}

Zero-point correction $=$

0.162038 (Hartree/Particle)

Thermal correction to Energy=

0.168874

Thermal correction to Enthalpy=

0.169818

Thermal correction to Gibbs Free Energy=

Sum of electronic and zero-point Energies=

0.133026

$-197.404183$

Sum of electronic and thermal Energies $=$

$-197.397348$

Sum of electronic and thermal Enthalpies=

$-197.396404$

Sum of electronic and thermal Free Energies $=\quad-197.433196$

Hexane

Standard orientation:

\begin{tabular}{cccccc} 
Center & Atomic & Atomic & \multicolumn{2}{c}{ Coordinates (Angstroms) } \\
Number & Number & Type & X & Y & Z \\
- \hdashline 1 & 6 & 0 & -3.180085 & 0.207263 & 0.000006 \\
2 & 1 & 0 & -3.262014 & 0.845418 & 0.877808 \\
3 & 1 & 0 & -3.262016 & 0.845442 & -0.877778 \\
4 & 1 & 0 & -4.032200 & -0.467890 & -0.000003 \\
5 & 6 & 0 & -1.866341 & -0.547985 & -0.000007
\end{tabular}




$\begin{array}{cccccc}6 & 1 & 0 & -1.818154 & -1.201644 & 0.871842 \\ 7 & 1 & 0 & -1.818160 & -1.201624 & -0.871870 \\ 8 & 6 & 0 & -0.660674 & 0.370880 & -0.000000 \\ 9 & 1 & 0 & -0.707566 & 1.026073 & -0.872503 \\ 10 & 1 & 0 & -0.707569 & 1.026067 & 0.872506 \\ 11 & 6 & 0 & 0.660674 & -0.370880 & -0.000000 \\ 12 & 1 & 0 & 0.707566 & -1.026074 & -0.872502 \\ 13 & 1 & 0 & 0.707569 & -1.026067 & 0.872507 \\ 14 & 6 & 0 & 1.866341 & 0.547985 & -0.000006 \\ 15 & 1 & 0 & 1.818154 & 1.201644 & 0.871843 \\ 16 & 1 & 0 & 1.818159 & 1.201625 & -0.871870 \\ 17 & 6 & 0 & 3.180085 & -0.207263 & 0.000006 \\ 18 & 1 & 0 & 3.262015 & -0.845443 & -0.877778 \\ 19 & 1 & 0 & 4.032200 & 0.467890 & -0.000004 \\ 20 & 1 & 0 & 3.262014 & -0.845417 & 0.877809\end{array}$

Zero-point correction=

Thermal correction to Energy=

Thermal correction to Enthalpy=

Thermal correction to Gibbs Free Energy=

Sum of electronic and zero-point Energies=

Sum of electronic and thermal Energies $=$

Sum of electronic and thermal Enthalpies=

Sum of electronic and thermal Free Energies=
0.192452 (Hartree/Particle)

0.200800

0.201744

$-236.642351$

$-236.634003$

$-236.633059$

$-236.674883$

22dimethylbutane

Standard orientation:

\begin{tabular}{|c|c|c|c|c|}
\hline \multirow{2}{*}{$\begin{array}{l}\text { Center } \\
\text { Number }\end{array}$} & Atomic & \multirow{2}{*}{$\begin{array}{l}\text { Atomic } \\
\text { Type }\end{array}$} & \multicolumn{2}{|c|}{ Coordinates (Angstroms) } \\
\hline & Number & & $\mathrm{X}$ & Z \\
\hline 1 & 6 & -1.603907 & 0.966819 & 0.001262 \\
\hline 2 & 1 & -1.581062 & 1.606087 & 0.882614 \\
\hline 3 & 1 & -1.581224 & 1.608156 & -0.878589 \\
\hline 4 & 1 & -2.551032 & 0.429726 & 0.000714 \\
\hline 5 & 6 & -0.429360 & -0.000238 & 0.000011 \\
\hline 6 & 6 & 0.859998 & 0.823527 & 0.000652 \\
\hline 7 & 1 & 0.840664 & 1.480248 & -0.871113 \\
\hline 8 & 1 & 0.840731 & 1.478820 & 0.873496 \\
\hline 9 & 6 & 2.156837 & 0.037202 & 0.000005 \\
\hline 10 & 1 & 2.244892 & -0.596063 & -0.879210 \\
\hline 11 & 1 & 3.009792 & 0.711509 & 0.000243 \\
\hline 12 & 1 & 2.245135 & -0.597008 & 0.878512 \\
\hline 13 & 6 & -0.511123 & -0.875657 & 1.241572 \\
\hline 14 & 1 & 0.278348 & -1.623367 & 1.260426 \\
\hline 15 & 1 & -0.430606 & -0.275969 & 2.147329 \\
\hline 16 & 1 & -1.463329 & -1.402671 & 1.273809 \\
\hline 17 & 6 & -0.511597 & -0.872937 & -1.243438 \\
\hline 18 & 1 & -1.463661 & -1.400174 & -1.276270 \\
\hline 19 & 1 & -0.431814 & -0.271207 & -2.147902 \\
\hline
\end{tabular}


Zero-point correction=

Thermal correction to Energy=

Thermal correction to Enthalpy=

Thermal correction to Gibbs Free Energy=

Sum of electronic and zero-point Energies=

Sum of electronic and thermal Energies $=$

Sum of electronic and thermal Enthalpies=

Sum of electronic and thermal Free Energies=

Heptane
0.191220 (Hartree/Particle)

0.199392

0.200337
0.159976
-236.646260
-236.638087
-236.637143
-236.677503

Standard orientation:

\begin{tabular}{|c|c|c|c|c|}
\hline \multirow{2}{*}{$\begin{array}{l}\text { Center } \\
\text { Number }\end{array}$} & Atomic & \multirow{2}{*}{$\begin{array}{r}\text { Atomic } \\
\text { Type }\end{array}$} & \multicolumn{2}{|c|}{ Coordinates (Angstroms } \\
\hline & Numbe & & $X$ & Z \\
\hline 1 & 6 & -3.790942 & -0.352947 & -0.000008 \\
\hline 2 & 1 & -3.827947 & -0.995279 & -0.877812 \\
\hline 3 & 1 & -3.827947 & -0.995314 & 0.877770 \\
\hline 4 & 1 & -4.688287 & 0.260825 & 0.000005 \\
\hline 5 & 6 & -2.533321 & 0.492469 & 0.000010 \\
\hline 6 & 1 & -2.531001 & 1.147899 & -0.871848 \\
\hline 7 & 1 & -2.531006 & 1.147869 & 0.871891 \\
\hline 8 & 6 & -1.266278 & -0.339732 & 0.000000 \\
\hline 9 & 1 & -1.267149 & -0.996600 & 0.872487 \\
\hline 10 & 1 & -1.267154 & -0.996588 & -0.872495 \\
\hline 11 & 6 & 0.000000 & 0.492964 & 0.000001 \\
\hline 12 & 1 & -0.000002 & 1.149555 & 0.872582 \\
\hline 13 & 1 & 0.000002 & 1.149552 & -0.872582 \\
\hline 14 & 6 & 1.266278 & -0.339732 & 0.000006 \\
\hline 15 & 1 & 1.267148 & -0.996606 & -0.872477 \\
\hline 16 & 1 & 1.267156 & -0.996582 & 0.872505 \\
\hline 17 & 6 & 2.533321 & 0.492469 & -0.000011 \\
\hline 18 & 1 & 2.531005 & 1.147901 & 0.871846 \\
\hline 19 & 1 & 2.531003 & 1.147868 & -0.871893 \\
\hline 20 & 6 & 3.790942 & -0.352947 & 0.000003 \\
\hline 21 & 1 & 3.827944 & -0.995314 & -0.877775 \\
\hline 22 & 1 & 4.688287 & 0.260825 & -0.000012 \\
\hline 23 & 1 & 3.827949 & -0.995279 & 0.877807 \\
\hline
\end{tabular}

Zero-point correction=

Thermal correction to Energy=

Thermal correction to Enthalpy=

Thermal correction to Gibbs Free Energy=

Sum of electronic and zero-point Energies=

Sum of electronic and thermal Energies=

Sum of electronic and thermal Enthalpies=

Sum of electronic and thermal Free Energies=

223 trimethylbutane
0.221427 (Hartree/Particle)

0.231112

0.232056

0.186635

$-275.886790$

$-275.877105$

$-275.876161$

$-275.921582$ 
Standard orientation:

\begin{tabular}{|c|c|c|c|c|}
\hline \multirow{2}{*}{$\begin{array}{l}\text { Center } \\
\text { Number }\end{array}$} & Atomic & \multirow{2}{*}{$\begin{array}{l}\text { Atomic } \\
\text { Type }\end{array}$} & \multicolumn{2}{|c|}{ Coordinates (Angstroms } \\
\hline & Numbe & & $X$ & $\mathrm{Z}$ \\
\hline 1 & 6 & -1.315666 & -1.282391 & -0.462048 \\
\hline 2 & 1 & -1.171859 & -1.439301 & -1.530726 \\
\hline 3 & 1 & -0.936768 & -2.155892 & 0.062012 \\
\hline 4 & 1 & -2.386626 & -1.227166 & -0.274693 \\
\hline 5 & 6 & -0.634516 & 0.000183 & -0.000767 \\
\hline 6 & 6 & 0.841660 & -0.000801 & -0.453180 \\
\hline 7 & 1 & 0.812545 & -0.004765 & -1.546699 \\
\hline 8 & 6 & 1.611223 & 1.237550 & -0.020620 \\
\hline 9 & 1 & 1.733331 & 1.266266 & 1.060559 \\
\hline 10 & 1 & 2.607919 & 1.227045 & -0.456566 \\
\hline 11 & 1 & 1.129811 & 2.160418 & -0.330348 \\
\hline 12 & 6 & 1.614193 & -1.236008 & -0.016664 \\
\hline 13 & 1 & 1.655277 & -1.314839 & 1.068374 \\
\hline 14 & 1 & 1.185906 & -2.154590 & -0.406003 \\
\hline 15 & 1 & 2.640386 & -1.177118 & -0.373380 \\
\hline 16 & 6 & -0.771618 & 0.111624 & 1.511265 \\
\hline 17 & 1 & -0.369791 & 1.050793 & 1.884357 \\
\hline 18 & 1 & -1.822843 & 0.070873 & 1.792197 \\
\hline 19 & 1 & -0.265068 & -0.703018 & 2.024286 \\
\hline 20 & 6 & -1.366181 & 1.170268 & -0.646995 \\
\hline 21 & 1 & -1.272505 & 1.136870 & -1.731863 \\
\hline 22 & 1 & -2.426506 & 1.132194 & -0.403030 \\
\hline 23 & 1 & -0.987789 & 2.129687 & -0.304417 \\
\hline
\end{tabular}

Zero-point correction=

Thermal correction to Energy=

Thermal correction to Enthalpy=

Thermal correction to Gibbs Free Energy=

Sum of electronic and zero-point Energies=

Sum of electronic and thermal Energies=

Sum of electronic and thermal Enthalpies=

Sum of electronic and thermal Free Energies=

Octane

\subsection{5 (Hartree/Particle)}

0.229397

0.230342

0.186509

$-275.889109$

$-275.879647$

$-275.878702$

$-275.922535$

Standard orientation:

\begin{tabular}{cccccc} 
Center & \multicolumn{2}{c}{ Atomic } & \multicolumn{2}{c}{ Atomic } & \multicolumn{2}{c}{ Coordinates (Angstroms) } \\
Number & Number & Type & X & Y & Z \\
\hline 1 & 6 & 0 & -4.437609 & 0.255564 & 0.000015 \\
2 & 1 & 0 & -4.499810 & 0.895947 & 0.877823 \\
3 & 1 & 0 & -4.499814 & 0.895997 & -0.877758 \\
4 & 1 & 0 & -5.310175 & -0.392958 & -0.000003 \\
5 & 6 & 0 & -3.147766 & -0.539827 & -0.000013 \\
6 & 1 & 0 & -3.119727 & -1.194673 & 0.871839 \\
7 & 1 & 0 & -3.119738 & -1.194631 & -0.871897
\end{tabular}




$\begin{array}{cccrrr}8 & 6 & 0 & -1.914336 & 0.341430 & 0.000001 \\ 9 & 1 & 0 & -1.940952 & 0.997765 & -0.872492 \\ 10 & 1 & 0 & -1.940955 & 0.997743 & 0.872510 \\ 11 & 6 & 0 & -0.616378 & -0.440970 & -0.000004 \\ 12 & 1 & 0 & -0.590602 & -1.097055 & -0.872568 \\ 13 & 1 & 0 & -0.590595 & -1.097057 & 0.872557 \\ 14 & 6 & 0 & 0.616378 & 0.440970 & -0.000008 \\ 15 & 1 & 0 & 0.590595 & 1.097066 & 0.872547 \\ 16 & 1 & 0 & 0.590603 & 1.097046 & -0.872579 \\ 17 & 6 & 0 & 1.914336 & -0.341430 & 0.000007 \\ 18 & 1 & 0 & 1.940954 & -0.997770 & -0.872482 \\ 19 & 1 & 0 & 1.940953 & -0.997738 & 0.872520 \\ 20 & 6 & 0 & 3.147766 & 0.539827 & -0.000009 \\ 21 & 1 & 0 & 3.119731 & 1.194670 & 0.871846 \\ 22 & 1 & 0 & 3.119734 & 1.194635 & -0.871889 \\ 23 & 6 & 0 & 4.437609 & -0.255564 & 0.000010 \\ 24 & 1 & 0 & 4.499814 & -0.895989 & -0.877768 \\ 25 & 1 & 0 & 5.310175 & 0.392958 & -0.000002 \\ 26 & 1 & 0 & 4.499811 & -0.895954 & 0.877813\end{array}$

Zero-point correction=

Thermal correction to Energy=

Thermal correction to Enthalpy=

Thermal correction to Gibbs Free Energy=

Sum of electronic and zero-point Energies=

Sum of electronic and thermal Energies $=$

Sum of electronic and thermal Enthalpies=

Sum of electronic and thermal Free Energies=

2233tetramethylbutane
0.250394 (Hartree/Particle)

$$
0.261424
$$

0.262368

\subsection{2}

$-315.131237$

$-315.120208$

$-315.119263$

$-315.168279$

Standard orientation:

\begin{tabular}{|c|c|c|c|c|}
\hline \multirow{2}{*}{$\begin{array}{l}\text { Center } \\
\text { Number }\end{array}$} & Atomic & \multirow{2}{*}{$\begin{array}{l}\text { Atomic } \\
\text { Type }\end{array}$} & \multicolumn{2}{|c|}{ Coordinates (Angstroms) } \\
\hline & Number & & $\mathrm{X}$ & Z \\
\hline 1 & 6 & -1.333023 & -1.351305 & -0.442796 \\
\hline 2 & 1 & -1.131381 & -1.546041 & -1.493047 \\
\hline 3 & 1 & -0.922548 & -2.173266 & 0.139642 \\
\hline 4 & 1 & -2.413476 & -1.364503 & -0.309246 \\
\hline 5 & 6 & -0.780543 & -0.000091 & -0.000022 \\
\hline 6 & 6 & 0.780557 & 0.000053 & -0.000021 \\
\hline 7 & 6 & 1.332675 & 1.416270 & 0.128083 \\
\hline 8 & 1 & 1.130153 & 2.010756 & -0.759139 \\
\hline 9 & 1 & 0.922410 & 1.940280 & 0.988561 \\
\hline 10 & 1 & 2.413220 & 1.375942 & 0.255301 \\
\hline 11 & 6 & -1.333061 & 1.059039 & -0.948715 \\
\hline 12 & 1 & -1.130840 & 2.065904 & -0.592433 \\
\hline 13 & 1 & -0.923033 & 0.965343 & -1.951932 \\
\hline 14 & 1 & -2.413580 & 0.950374 & -1.026438 \\
\hline 15 & 6 & -1.332877 & 0.292029 & 1.391589 \\
\hline 16 & 1 & -2.413424 & 0.413569 & 1.336522 \\
\hline
\end{tabular}




$\begin{array}{rrrrrr}17 & 1 & 0 & -1.130459 & -0.519893 & 2.085426 \\ 18 & 1 & 0 & -0.922849 & 1.207756 & 1.811947 \\ 19 & 6 & 0 & 1.333148 & -0.597051 & -1.290441 \\ 20 & 1 & 0 & 2.413693 & -0.466613 & -1.318665 \\ 21 & 1 & 0 & 1.130822 & -1.662690 & -1.361844 \\ 22 & 1 & 0 & 0.923265 & -0.113841 & -2.174645 \\ 23 & 6 & 0 & 1.333159 & -0.818957 & 1.162294 \\ 24 & 1 & 0 & 2.413724 & -0.908440 & 1.063555 \\ 25 & 1 & 0 & 1.130655 & -0.348195 & 2.120926 \\ 26 & 1 & 0 & 0.923443 & -1.826368 & 1.185682\end{array}$

Zero-point correction=

Thermal correction to Energy=

Thermal correction to Enthalpy=

Thermal correction to Gibbs Free Energy=

Sum of electronic and zero-point Energies=

Sum of electronic and thermal Energies=

Sum of electronic and thermal Enthalpies=

Sum of electronic and thermal Free Energies=

B2PLYPD3BJ/ccpVTZ

Ethane

Standard orientation:

\begin{tabular}{cccccc}
$\begin{array}{c}\text { Center } \\
\text { Number }\end{array}$ & \multicolumn{2}{c}{ Atomic } & \multicolumn{2}{c}{ Atomic } & \multicolumn{2}{c}{ Coordinates (Angstroms) } \\
- & Number & \multicolumn{1}{c}{ Type } & X & Y & Z \\
\hline 1 & 6 & 0 & 0.000000 & -0.000000 & 0.762970 \\
2 & 1 & 0 & -0.507580 & 0.879197 & 1.158175 \\
3 & 1 & 0 & 1.015197 & -0.000021 & 1.158175 \\
4 & 1 & 0 & -0.507617 & -0.879176 & 1.158175 \\
5 & 6 & 0 & -0.000000 & -0.000000 & -0.762970 \\
6 & 1 & 0 & 0.507580 & 0.879197 & -1.158175 \\
7 & 1 & 0 & 0.507617 & -0.879176 & -1.158175 \\
8 & 1 & 0 & -1.015197 & -0.000021 & -1.158175
\end{tabular}

Zero-point correction=

Thermal correction to Energy=

Thermal correction to Enthalpy=

Thermal correction to Gibbs Free Energy=

Sum of electronic and zero-point Energies=

Sum of electronic and thermal Energies $=$

Sum of electronic and thermal Enthalpies=

Sum of electronic and thermal Free Energies=

Methane

Standard orientation:

\begin{tabular}{|c|c|c|c|c|}
\hline Center & Atomic & Atomic & Coordinate & es (Angstroms) \\
\hline Number & Number & Type & $\mathrm{X}$ & Z \\
\hline 1 & 6 & 0.000000 & 0.000000 & 0.000000 \\
\hline
\end{tabular}




$\begin{array}{rrrrrr}2 & 1 & 0 & 0.627266 & 0.627266 & 0.627266 \\ 3 & 1 & 0 & -0.627266 & -0.627266 & 0.627266 \\ 4 & 1 & 0 & -0.627266 & 0.627266 & -0.627266 \\ 5 & 1 & 0 & 0.627266 & -0.627266 & -0.627266\end{array}$

Zero-point correction=

Thermal correction to Energy=

Thermal correction to Enthalpy=

Thermal correction to Gibbs Free Energy=

Sum of electronic and zero-point Energies=

Sum of electronic and thermal Energies $=$

Sum of electronic and thermal Enthalpies=

Sum of electronic and thermal Free Energies=

Propane
0.045000 (Hartree/Particle)

0.047867

0.048811

0.027694

$-40.441473$

$-40.438606$

$-40.437662$

$-40.458779$

Standard orientation:

\begin{tabular}{|c|c|c|c|c|}
\hline \multirow{2}{*}{$\begin{array}{l}\text { Center } \\
\text { Number }\end{array}$} & Atomic & \multirow{2}{*}{$\begin{array}{l}\text { Atomic } \\
\text { Type }\end{array}$} & \multicolumn{2}{|c|}{ Coordinates (Angstrom } \\
\hline & Numbe & & $X$ & Z \\
\hline 1 & 6 & -1.267767 & -0.260044 & 0.000000 \\
\hline 2 & 1 & -2.165916 & 0.356730 & -0.000031 \\
\hline 3 & 1 & -1.304327 & -0.903580 & 0.879865 \\
\hline 4 & 1 & -1.304299 & -0.903629 & -0.879830 \\
\hline 5 & 6 & 0.000000 & 0.588934 & 0.000000 \\
\hline 6 & 1 & -0.000001 & 1.243950 & 0.873335 \\
\hline 7 & 1 & 0.000001 & 1.243933 & -0.873347 \\
\hline 8 & 6 & 1.267768 & -0.260044 & 0.000000 \\
\hline 9 & 1 & 1.304339 & -0.903560 & 0.879879 \\
\hline 10 & 1 & 1.304284 & -0.903648 & -0.879817 \\
\hline 11 & 1 & 2.165917 & 0.356727 & -0.000059 \\
\hline
\end{tabular}

Zero-point correction=

Thermal correction to Energy=

Thermal correction to Enthalpy=

Thermal correction to Gibbs Free Energy=

Sum of electronic and zero-point Energies=

Sum of electronic and thermal Energies=

Sum of electronic and thermal Enthalpies=

Sum of electronic and thermal Free Energies=

Butane

Standard orientation:
0.104023 (Hartree/Particle)

0.108507

0.109452

$$
0.078415
$$

$-118.958070$

$-118.953586$

$-118.952641$

$-118.983678$

\begin{tabular}{|c|c|c|c|c|}
\hline \multirow{2}{*}{$\begin{array}{l}\text { Center } \\
\text { Number }\end{array}$} & Atomic & \multirow{2}{*}{$\begin{array}{l}\text { Atomic } \\
\text { Type }\end{array}$} & \multicolumn{2}{|c|}{ Coordinates (Angstroms) } \\
\hline & Numbe & & $\mathrm{X}$ & $\mathrm{Z}$ \\
\hline 1 & 6 & 1.949784 & -0.119815 & 0.000041 \\
\hline 2 & 1 & 2.090107 & -0.748673 & -0.879892 \\
\hline 3 & 1 & 2.090015 & -0.748663 & 0.879996 \\
\hline 4 & 1 & 2.736843 & 0.633538 & 0.000077 \\
\hline 5 & 6 & 0.562765 & 0.515228 & -0.000046 \\
\hline
\end{tabular}




$\begin{array}{cccccc}6 & 1 & 0 & 0.454775 & 1.162715 & 0.873858 \\ 7 & 1 & 0 & 0.454838 & 1.162654 & -0.874005 \\ 8 & 6 & 0 & -0.562765 & -0.515228 & -0.000047 \\ 9 & 1 & 0 & -0.454838 & -1.162652 & -0.874006 \\ 10 & 1 & 0 & -0.454775 & -1.162718 & 0.873856 \\ 11 & 6 & 0 & -1.949784 & 0.119815 & 0.000041 \\ 12 & 1 & 0 & -2.090105 & 0.748680 & -0.879888 \\ 13 & 1 & 0 & -2.736843 & -0.633538 & 0.000069 \\ 14 & 1 & 0 & -2.090017 & 0.748656 & 0.880000\end{array}$

Zero-point correction $=$

Thermal correction to Energy=

Thermal correction to Enthalpy=

Thermal correction to Gibbs Free Energy=

Sum of electronic and zero-point Energies=

Sum of electronic and thermal Energies $=$

Sum of electronic and thermal Enthalpies=

Sum of electronic and thermal Free Energies=
0.132743 (Hartree/Particle)

$$
0.138464
$$

0.139408

$$
\begin{gathered}
0.104809 \\
-158.218748 \\
-158.213028 \\
-158.212083 \\
-158.246682
\end{gathered}
$$
Isobutane

Standard orientation:

\begin{tabular}{cccccc} 
Center & \multicolumn{2}{c}{ Atomic } & \multicolumn{2}{c}{ Atomic } & \multicolumn{3}{c}{ Coordinates } \\
Number & Number & Type & X & Y & Z \\
\hline-------- & \\
1 & 6 & 0 & 1.416676 & 0.320049 & -0.096005 \\
2 & 1 & 0 & 2.131285 & -0.422790 & 0.258949 \\
3 & 1 & 0 & 1.460336 & 0.329832 & -1.186965 \\
4 & 1 & 0 & 1.742474 & 1.298016 & 0.258826 \\
5 & 6 & 0 & 0.000007 & 0.000007 & 0.377402 \\
6 & 1 & 0 & -0.000005 & -0.000003 & 1.471162 \\
7 & 6 & 0 & -0.431166 & -1.386897 & -0.096000 \\
8 & 1 & 0 & -1.431811 & -1.634333 & 0.258902 \\
9 & 1 & 0 & -0.444476 & -1.429653 & -1.186964 \\
10 & 1 & 0 & 0.252852 & -2.158025 & 0.258891 \\
11 & 6 & 0 & -0.985513 & 1.066848 & -0.096002 \\
12 & 1 & 0 & -1.995385 & 0.859910 & 0.258684 \\
13 & 1 & 0 & -0.699593 & 2.057107 & 0.259107 \\
14 & 1 & 0 & -1.015701 & 1.099900 & -1.186964
\end{tabular}

Zero-point correction=

Thermal correction to Energy=

Thermal correction to Enthalpy=

Thermal correction to Gibbs Free Energy=

Sum of electronic and zero-point Energies=

Sum of electronic and thermal Energies=

Sum of electronic and thermal Enthalpies=

Sum of electronic and thermal Free Energies=

Pentane

Standard orientation:
0.132292 (Hartree/Particle)

$$
0.137919
$$

0.138864 


\begin{tabular}{cccccc} 
Center & \multicolumn{2}{c}{ Atomic } & \multicolumn{2}{c}{ Atomic } & \multicolumn{3}{c}{ Coordinates (Angstrom } \\
Number & Number & Type & X & $Y$ & $Z$ \\
-------------------- \\
1 & 6 & 0 & 2.542620 & 0.325941 & -0.000010 \\
2 & 1 & 0 & 2.579531 & 0.969192 & -0.879937 \\
3 & 1 & 0 & 2.579538 & 0.969215 & 0.879900 \\
4 & 1 & 0 & 3.440913 & -0.290635 & -0.000006 \\
5 & 6 & 0 & 1.276080 & -0.524573 & 0.000007 \\
6 & 1 & 0 & 1.274759 & -1.180668 & -0.873968 \\
7 & 1 & 0 & 1.274774 & -1.180655 & 0.873991 \\
8 & 6 & 0 & 0.000000 & 0.311880 & 0.000015 \\
9 & 1 & 0 & -0.000006 & 0.969688 & 0.874494 \\
10 & 1 & 0 & 0.000006 & 0.969717 & -0.874441 \\
11 & 6 & 0 & -1.276080 & -0.524573 & -0.000007 \\
12 & 1 & 0 & -1.274773 & -1.180679 & 0.873961 \\
13 & 1 & 0 & -1.274760 & -1.180643 & -0.873998 \\
14 & 6 & 0 & -2.542620 & 0.325941 & -0.000004 \\
15 & 1 & 0 & -3.440913 & -0.290635 & -0.000062 \\
16 & 1 & 0 & -2.579559 & 0.969158 & 0.879946 \\
17 & 1 & 0 & -2.579510 & 0.969248 & -0.879892 \\
----------------------------------------------------------
\end{tabular}

Zero-point correction $=$

Thermal correction to Energy=

0.161433 (Hartree/Particle)

Thermal correction to Enthalpy=

0.168438

0.169383

Thermal correction to Gibbs Free Energy=

0.131221

Sum of electronic and zero-point Energies=

$-197.479417$

Sum of electronic and thermal Energies=

$-197.472411$

Sum of electronic and thermal Enthalpies=

$-197.471467$

Sum of electronic and thermal Free Energies=

$-197.509629$

Neopentane

Standard orientation:

\begin{tabular}{|c|c|c|c|c|c|}
\hline \multirow{2}{*}{$\begin{array}{l}\text { Center } \\
\text { Number }\end{array}$} & \multicolumn{2}{|c|}{ Atomic } & \multirow{2}{*}{$\begin{array}{l}\text { Atomic } \\
\text { Type }\end{array}$} & \multicolumn{2}{|c|}{ Coordinates (Angstroms } \\
\hline & & & & $X$ & $\mathrm{Z}$ \\
\hline 1 & 6 & 0 & -0.405183 & 1.189432 & 0.875605 \\
\hline 2 & 1 & 0 & -1.099566 & 0.879610 & 1.657766 \\
\hline 3 & 1 & 0 & 0.466622 & 1.634369 & 1.357194 \\
\hline 4 & 1 & 0 & -0.892315 & 1.963554 & 0.281152 \\
\hline 5 & 6 & 0 & -0.000010 & 0.000003 & 0.000010 \\
\hline 6 & 6 & 0 & 0.679123 & -1.064289 & 0.866999 \\
\hline 7 & 1 & 0 & 0.978799 & -1.924037 & 0.266192 \\
\hline 8 & 1 & 0 & 1.571928 & -0.663573 & 1.348938 \\
\hline 9 & 1 & 0 & 0.005793 & -1.418833 & 1.648574 \\
\hline 10 & 6 & 0 & -1.247065 & -0.597471 & -0.658391 \\
\hline 11 & 1 & 0 & -1.750661 & 0.141546 & -1.283043 \\
\hline 12 & 1 & 0 & -0.985725 & -1.448352 & -1.288975 \\
\hline 13 & 1 & 0 & -1.958095 & -0.942349 & 0.093564 \\
\hline 14 & 6 & 0 & 0.973125 & 0.472330 & -1.084211 \\
\hline
\end{tabular}




\begin{tabular}{|c|c|c|c|c|c|}
\hline 15 & 1 & 0 & 0.512939 & 1.231924 & -1.717602 \\
\hline 16 & 1 & 0 & 1.871697 & 0.903724 & -0.641031 \\
\hline 17 & 1 & 0 & 1.278637 & -0.357615 & -1.722802 \\
\hline \multicolumn{4}{|c|}{ Zero-point correction $=$} & \multicolumn{2}{|c|}{0.160230 (Hartree/Particle) } \\
\hline \multicolumn{4}{|c|}{ Thermal correction to Energy= } & \multicolumn{2}{|c|}{0.167029} \\
\hline \multicolumn{4}{|c|}{ Thermal correction to Enthalpy= } & \multicolumn{2}{|c|}{0.167973} \\
\hline \multicolumn{5}{|c|}{ Thermal correction to Gibbs Free Energy= } & 0.131241 \\
\hline \multicolumn{5}{|c|}{ Sum of electronic and zero-point Energies $=$} & -197.485558 \\
\hline \multicolumn{5}{|c|}{ Sum of electronic and thermal Energies $=$} & -197.478759 \\
\hline \multicolumn{5}{|c|}{ Sum of electronic and thermal Enthalpies= } & -197.477815 \\
\hline \multicolumn{5}{|c|}{ Sum of electronic and thermal Free Energies $=$} & -197.514546 \\
\hline
\end{tabular}

Hexane

Standard orientation:

\begin{tabular}{|c|c|c|c|c|}
\hline \multirow{2}{*}{$\begin{array}{l}\text { Center } \\
\text { Number }\end{array}$} & Atomic & \multirow{2}{*}{$\begin{array}{r}\text { Atomic } \\
\text { Type }\end{array}$} & \multicolumn{2}{|c|}{ Coordinates (Angstroms } \\
\hline & Numbe & & $X$ & $\mathrm{Z}$ \\
\hline 1 & 6 & -3.202761 & 0.208699 & 0.000039 \\
\hline 2 & 1 & -3.284668 & 0.847739 & 0.880014 \\
\hline 3 & 1 & -3.284680 & 0.847878 & -0.879836 \\
\hline 4 & 1 & -4.055668 & -0.469306 & -0.000011 \\
\hline 5 & 6 & -1.879754 & -0.550944 & -0.000038 \\
\hline 6 & 1 & -1.832410 & -1.205383 & 0.873878 \\
\hline 7 & 1 & -1.832446 & -1.205281 & -0.874032 \\
\hline 8 & 6 & -0.665345 & 0.373010 & -0.000011 \\
\hline 9 & 1 & -0.712148 & 1.028882 & -0.874518 \\
\hline 10 & 1 & -0.712166 & 1.028864 & 0.874507 \\
\hline 11 & 6 & 0.665345 & -0.373010 & 0.000002 \\
\hline 12 & 1 & 0.712151 & -1.028899 & -0.874492 \\
\hline 13 & 1 & 0.712163 & -1.028847 & 0.874533 \\
\hline 14 & 6 & 1.879754 & 0.550944 & -0.000031 \\
\hline 15 & 1 & 1.832415 & 1.205384 & 0.873885 \\
\hline 16 & 1 & 1.832441 & 1.205280 & -0.874026 \\
\hline 17 & 6 & 3.202761 & -0.208699 & 0.000032 \\
\hline 18 & 1 & 3.284680 & -0.847870 & -0.879848 \\
\hline 19 & 1 & 4.055668 & 0.469306 & -0.000009 \\
\hline 20 & 1 & 3.284667 & -0.847747 & 0.880003 \\
\hline
\end{tabular}

Zero-point correction=

Thermal correction to Energy=

Thermal correction to Enthalpy=

Thermal correction to Gibbs Free Energy=

Sum of electronic and zero-point Energies=

Sum of electronic and thermal Energies $=$

Sum of electronic and thermal Enthalpies=

Sum of electronic and thermal Free Energies=

22dimethylbutane
0.190086 (Hartree/Particle)

0.198410

0.199354

0.157633

$-236.740146$

$-236.731823$

$-236.730879$

$-236.772599$

Standard orientation: 


\begin{tabular}{|c|c|c|c|c|c|}
\hline \multirow{2}{*}{$\begin{array}{l}\text { Center } \\
\text { Number }\end{array}$} & Atomic & & \multirow{2}{*}{$\begin{array}{l}\text { Atomic } \\
\text { Type }\end{array}$} & \multicolumn{2}{|c|}{ Coordinates (Angstrom } \\
\hline & Numbe & & & $\mathrm{X}$ & Z \\
\hline 1 & 6 & 0 & -1.614292 & 0.976496 & 0.000252 \\
\hline 2 & 1 & 0 & -1.590823 & 1.617655 & 0.882558 \\
\hline 3 & 1 & 0 & -1.590865 & 1.618029 & -0.881784 \\
\hline 4 & 1 & 0 & -2.563944 & 0.439955 & 0.000158 \\
\hline 5 & 6 & 0 & -0.432816 & 0.000004 & 0.000011 \\
\hline 6 & 6 & 0 & 0.868589 & 0.826738 & 0.000270 \\
\hline 7 & 1 & 0 & 0.851745 & 1.483727 & -0.873525 \\
\hline 8 & 1 & 0 & 0.851773 & 1.483182 & 0.874479 \\
\hline 9 & 6 & 0 & 2.175135 & 0.036103 & 0.000016 \\
\hline 10 & 1 & 0 & 2.263775 & -0.598394 & -0.880539 \\
\hline 11 & 1 & 0 & 3.028077 & 0.713937 & 0.000082 \\
\hline 12 & 1 & 0 & 2.263895 & -0.598719 & 0.880324 \\
\hline 13 & 6 & 0 & -0.518126 & -0.880698 & 1.251080 \\
\hline 14 & 1 & 0 & 0.269680 & -1.632679 & 1.269900 \\
\hline 15 & 1 & 0 & -0.433858 & -0.279618 & 2.157826 \\
\hline 16 & 1 & 0 & -1.474063 & -1.404800 & 1.284531 \\
\hline 17 & 6 & 0 & -0.518058 & -0.879966 & -1.251597 \\
\hline 18 & 1 & 0 & -1.474020 & -1.403996 & -1.285432 \\
\hline 19 & 1 & 0 & -0.433671 & -0.278353 & -2.157976 \\
\hline 20 & 1 & 0 & 0.269709 & -1.631982 & -1.270794 \\
\hline
\end{tabular}

Zero-point correction=

Thermal correction to Energy=

0.189053 (Hartree/Particle)

Thermal correction to Enthalpy=

0.197179

0.198124

Thermal correction to Gibbs Free Energy=

0.157855

Sum of electronic and zero-point Energies=

$-236.744012$

Sum of electronic and thermal Energies=

$-236.735886$

Sum of electronic and thermal Enthalpies=

$-236.734942$

Sum of electronic and thermal Free Energies $=\quad-236.775211$

Heptane

Standard orientation:

\begin{tabular}{|c|c|c|c|c|}
\hline \multirow{2}{*}{$\begin{array}{l}\text { Center } \\
\text { Number }\end{array}$} & Atomic & \multirow{2}{*}{$\begin{array}{l}\text { Atomic } \\
\text { Type }\end{array}$} & \multicolumn{2}{|c|}{ Coordinates (Angstrom } \\
\hline & Number & & $\mathrm{X}$ & Z \\
\hline 1 & 6 & -3.817873 & -0.355396 & -0.000026 \\
\hline 2 & 1 & -3.854764 & -0.998597 & -0.880017 \\
\hline 3 & 1 & -3.854725 & -0.998791 & 0.879825 \\
\hline 4 & 1 & -4.716270 & 0.261080 & 0.000063 \\
\hline 5 & 6 & -2.551394 & 0.495143 & 0.000046 \\
\hline 6 & 1 & -2.550038 & 1.151299 & -0.873868 \\
\hline 7 & 1 & -2.550044 & 1.151164 & 0.874062 \\
\hline 8 & 6 & -1.275216 & -0.341433 & -0.000009 \\
\hline 9 & 1 & -1.275848 & -0.998994 & 0.874458 \\
\hline 10 & 1 & -1.275878 & -0.998937 & -0.874519 \\
\hline 11 & 6 & 0.000000 & 0.496166 & -0.000008 \\
\hline 12 & 1 & -0.000011 & 1.153410 & 0.874541 \\
\hline
\end{tabular}




$\begin{array}{rrrrrr}13 & 1 & 0 & 0.000011 & 1.153376 & -0.874581 \\ 14 & 6 & 0 & 1.275216 & -0.341433 & 0.000022 \\ 15 & 1 & 0 & 1.275853 & -0.999015 & -0.874430 \\ 16 & 1 & 0 & 1.275873 & -0.998916 & 0.874547 \\ 17 & 6 & 0 & 2.551394 & 0.495143 & -0.000038 \\ 18 & 1 & 0 & 2.550044 & 1.151290 & 0.873883 \\ 19 & 1 & 0 & 2.550038 & 1.151173 & -0.874047 \\ 20 & 6 & 0 & 3.817873 & -0.355396 & 0.000014 \\ 21 & 1 & 0 & 3.854741 & -0.998753 & -0.879864 \\ 22 & 1 & 0 & 4.716270 & 0.261080 & -0.000030 \\ 23 & 1 & 0 & 3.854748 & -0.998635 & 0.879978\end{array}$

Zero-point correction=

Thermal correction to Energy=

Thermal correction to Enthalpy=

Thermal correction to Gibbs Free Energy=

Sum of electronic and zero-point Energies=

Sum of electronic and thermal Energies $=$

Sum of electronic and thermal Enthalpies=

Sum of electronic and thermal Free Energies=

223trimethylbutane

Standard orientation:

\begin{tabular}{|c|c|c|c|c|}
\hline \multirow{2}{*}{$\begin{array}{l}\text { Center } \\
\text { Number }\end{array}$} & Atomic & \multirow{2}{*}{$\begin{array}{l}\text { Atomic } \\
\text { Type }\end{array}$} & \multicolumn{2}{|c|}{ Coordinates (Angstroms) } \\
\hline & Number & & $X$ & Z \\
\hline 1 & 6 & -1.323457 & -1.298183 & -0.452922 \\
\hline 2 & 1 & -1.173691 & -1.468714 & -1.520486 \\
\hline 3 & 1 & -0.945383 & -2.166005 & 0.084488 \\
\hline 4 & 1 & -2.397198 & -1.242524 & -0.271015 \\
\hline 5 & 6 & -0.640386 & 0.000249 & -0.000934 \\
\hline 6 & 6 & 0.849562 & -0.000972 & -0.454315 \\
\hline 7 & 1 & 0.823091 & -0.005253 & -1.549336 \\
\hline 8 & 6 & 1.625662 & 1.246346 & -0.020035 \\
\hline 9 & 1 & 1.755922 & 1.271525 & 1.062026 \\
\hline 10 & 1 & 2.620445 & 1.239918 & -0.465343 \\
\hline 11 & 1 & 1.138420 & 2.170517 & -0.322696 \\
\hline 12 & 6 & 1.628901 & -1.244980 & -0.016130 \\
\hline 13 & 1 & 1.663567 & -1.329540 & 1.070418 \\
\hline 14 & 1 & 1.204150 & -2.163942 & -0.413187 \\
\hline 15 & 1 & 2.658562 & -1.181879 & -0.368230 \\
\hline 16 & 6 & -0.781603 & 0.127695 & 1.520655 \\
\hline 17 & 1 & -0.387456 & 1.075619 & 1.884521 \\
\hline 18 & 1 & -1.834348 & 0.080412 & 1.802056 \\
\hline 19 & 1 & -0.266597 & -0.677700 & 2.043320 \\
\hline 20 & 6 & -1.380118 & 1.170331 & -0.665013 \\
\hline 21 & 1 & -1.290771 & 1.121972 & -1.751491 \\
\hline 22 & 1 & -2.441278 & 1.137055 & -0.415688 \\
\hline 23 & 1 & -0.998801 & 2.135622 & -0.337192 \\
\hline
\end{tabular}




$\begin{array}{lc}\text { Zero-point correction= } & 0.217460 \text { (Hartree/Particle) } \\ \text { Thermal correction to Energy= } & 0.226870 \\ \text { Thermal correction to Enthalpy= } & 0.227814 \\ \text { Thermal correction to Gibbs Free Energy= } & 0.184214 \\ \text { Sum of electronic and zero-point Energies }= & -276.003170 \\ \text { Sum of electronic and thermal Energies }= & -275.993760 \\ \text { Sum of electronic and thermal Enthalpies }= & -275.992816 \\ \text { Sum of electronic and thermal Free Energies }= & -276.036416\end{array}$

Octane

Standard orientation:

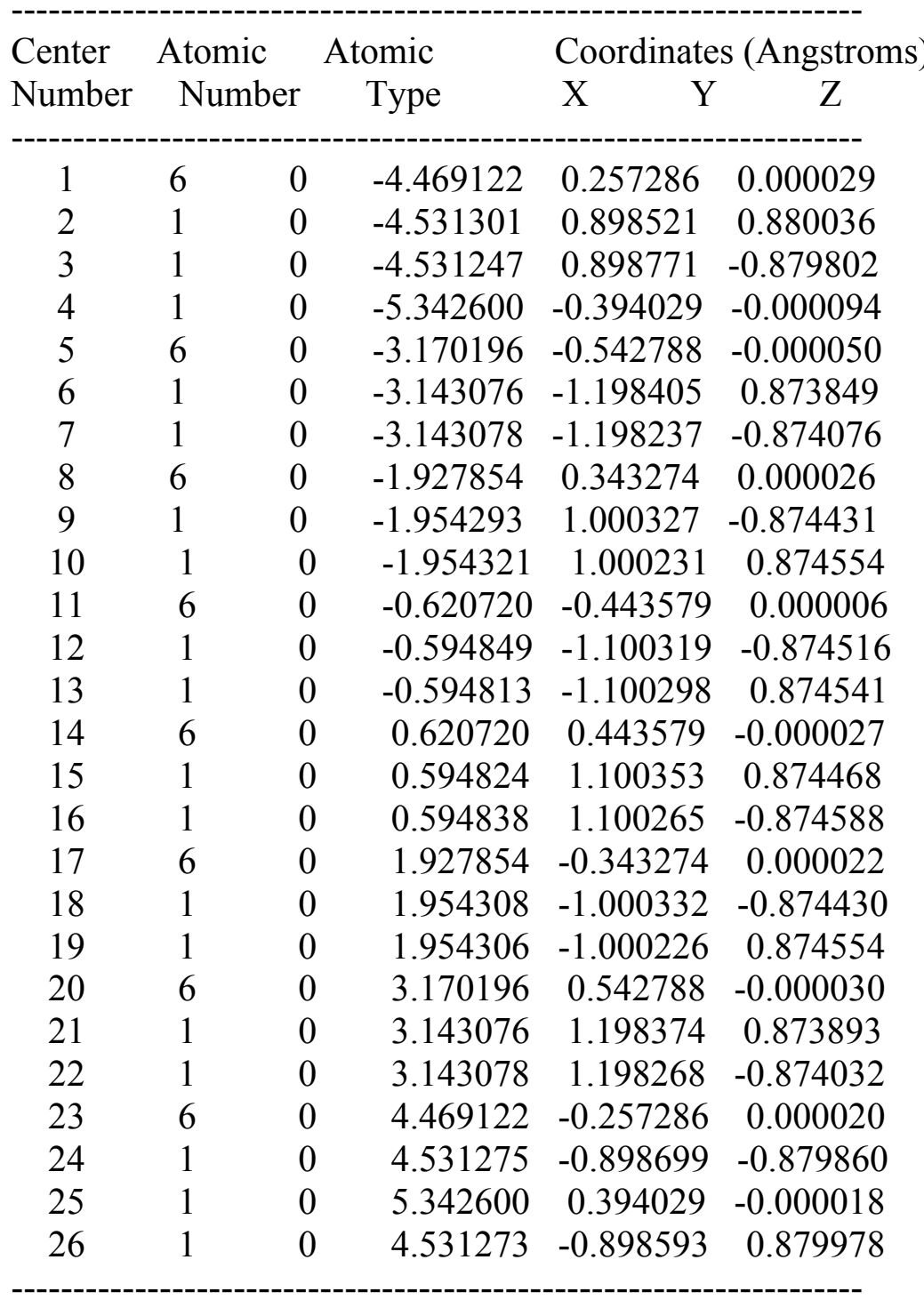

Zero-point correction $=$

Thermal correction to Energy=

Thermal correction to Enthalpy=

Thermal correction to Gibbs Free Energy=

Sum of electronic and zero-point Energies=

Sum of electronic and thermal Energies $=$

Sum of electronic and thermal Enthalpies=
0.247349 (Hartree/Particle)

0.258345

0.259289

0.210451

$-315.261658$

$-315.250661$

$-315.249717$ 
Sum of electronic and thermal Free Energies=

2233tetramethylbutane

Standard orientation:

\begin{tabular}{|c|c|c|c|c|c|}
\hline \multirow{2}{*}{$\begin{array}{l}\text { Center } \\
\text { Number }\end{array}$} & \multicolumn{2}{|c|}{ Atomic } & \multirow{2}{*}{$\begin{array}{l}\text { Atomic } \\
\text { Type }\end{array}$} & \multicolumn{2}{|c|}{ Coordinates (Angstrom } \\
\hline & & & & $\mathrm{X}$ & Z \\
\hline 1 & 6 & 0 & -1.345597 & 1.069611 & 0.952971 \\
\hline 2 & 1 & 0 & -0.929315 & 0.982311 & 1.955795 \\
\hline 3 & 1 & 0 & -1.148236 & 2.076982 & 0.590631 \\
\hline 4 & 1 & 0 & -2.427186 & 0.958146 & 1.036763 \\
\hline 5 & 6 & 0 & -0.788467 & 0.000009 & 0.000005 \\
\hline 6 & 6 & 0 & 0.788450 & -0.000030 & 0.000005 \\
\hline 7 & 6 & 0 & 1.345752 & -0.827252 & -1.169493 \\
\hline 8 & 1 & 0 & 0.930043 & -1.833800 & -1.194716 \\
\hline 9 & 1 & 0 & 1.148208 & -0.354776 & -2.130134 \\
\hline 10 & 1 & 0 & 2.427400 & -0.922463 & -1.068210 \\
\hline 11 & 6 & 0 & -1.345843 & -1.360031 & 0.449716 \\
\hline 12 & 1 & 0 & -0.929965 & -2.184815 & -0.127617 \\
\hline 13 & 1 & 0 & -1.148405 & -1.550292 & 1.503237 \\
\hline 14 & 1 & 0 & -2.427454 & -1.376510 & 0.311465 \\
\hline 15 & 6 & 0 & -1.345707 & 0.290566 & -1.402730 \\
\hline 16 & 1 & 0 & -2.427351 & 0.418405 & -1.348032 \\
\hline 17 & 1 & 0 & -0.929863 & 1.202973 & -1.828348 \\
\hline 18 & 1 & 0 & -1.148058 & -0.526665 & -2.094220 \\
\hline 19 & 6 & 0 & 1.345623 & -0.599297 & 1.301181 \\
\hline 20 & 1 & 0 & 2.427245 & -0.463844 & 1.333244 \\
\hline 21 & 1 & 0 & 0.929609 & -0.118124 & 2.185506 \\
\hline 22 & 1 & 0 & 1.148119 & -1.667496 & 1.372042 \\
\hline 23 & 6 & 0 & 1.345742 & 1.426425 & -0.131633 \\
\hline 24 & 1 & 0 & 2.427432 & 1.386373 & -0.264336 \\
\hline 25 & 1 & 0 & 0.930295 & 1.951408 & -0.990934 \\
\hline 26 & 1 & 0 & 1.147762 & 2.022184 & 0.757723 \\
\hline
\end{tabular}

Zero-point correction $=$

Thermal correction to Energy=

0.246073 (Hartree/Particle)

0.256314

Thermal correction to Enthalpy=

0.257258

Thermal correction to Gibbs Free Energy=

0.212973

Sum of electronic and zero-point Energies=

$-315.262395$

Sum of electronic and thermal Energies=

$-315.252155$

Sum of electronic and thermal Enthalpies=

$-315.251211$

Sum of electronic and thermal Free Energies=

$-315.295495$

DSDPBEP86/ccpVTZ

Ethane

Standard orientation:

\begin{tabular}{cccccc} 
Center & Atomic & \multicolumn{2}{c}{ Atomic } & \multicolumn{2}{c}{ Coordinates (Angstroms) } \\
Number & Number & Type & X & Y & Z \\
-1 & 6 & 0 & 0.000000 & -0.000000 & 0.762530
\end{tabular}




$\begin{array}{rrrrrr}2 & 1 & 0 & -0.508608 & 0.880966 & 1.158265 \\ 3 & 1 & 0 & 1.017243 & -0.000015 & 1.158265 \\ 4 & 1 & 0 & -0.508635 & -0.880951 & 1.158265 \\ 5 & 6 & 0 & 0.000000 & -0.000000 & -0.762530 \\ 6 & 1 & 0 & 0.508608 & 0.880966 & -1.158265 \\ 7 & 1 & 0 & 0.508635 & -0.880951 & -1.158265 \\ 8 & 1 & 0 & -1.017243 & -0.000015 & -1.158265\end{array}$

Zero-point correction=

Thermal correction to Energy=

Thermal correction to Enthalpy=

Sum of electronic and zero-point Energies=

Sum of electronic and thermal Energies $=$

Sum of electronic and thermal Enthalpies=

Sum of electronic and thermal Free Energies=

Methane

Standard orientation:

$$
\begin{array}{cc}
0.075091 & \text { (Hartree/Particle) } \\
0.078565 \\
& 0.079509 \\
\text { rgy }= & 0.053682 \\
\text { gies }= & -79.613394 \\
\text { s }= & -79.609920 \\
\text { pies }= & -79.608976 \\
\text { Energies }= & -79.634802
\end{array}
$$

\begin{tabular}{|c|c|c|c|c|}
\hline \multirow{2}{*}{$\begin{array}{l}\text { Center } \\
\text { Number }\end{array}$} & \multirow{2}{*}{$\begin{array}{l}\text { Atomic } \\
\text { Number }\end{array}$} & \multirow{2}{*}{$\begin{array}{r}\text { Atomic } \\
\text { Type }\end{array}$} & \multicolumn{2}{|c|}{ Coordinates (Angstroms) } \\
\hline & & & $X$ & $\mathrm{Z}$ \\
\hline 1 & 6 & 0.000000 & 0.000000 & 0.000000 \\
\hline 2 & 1 & 0.628452 & 0.628452 & 0.628452 \\
\hline 3 & 1 & -0.628452 & -0.628452 & 0.628452 \\
\hline 4 & 1 & -0.628452 & 0.628452 & -0.628452 \\
\hline 5 & 1 & 0.628452 & -0.628452 & -0.628452 \\
\hline \multicolumn{3}{|c|}{ Zero-point correction $=$} & \multicolumn{2}{|c|}{0.045012 (Hartree/Particle) } \\
\hline \multicolumn{3}{|c|}{ Thermal correction to Energy= } & \multicolumn{2}{|c|}{0.047880} \\
\hline \multicolumn{3}{|c|}{ Thermal correction to Enthalpy= } & \multicolumn{2}{|c|}{0.048824} \\
\hline \multicolumn{4}{|c|}{ Thermal correction to Gibbs Free Energy= } & 0.027701 \\
\hline \multicolumn{4}{|c|}{ Sum of electronic and zero-point Energies $=$} & -40.398110 \\
\hline \multicolumn{4}{|c|}{ Sum of electronic and thermal Energies $=$} & -40.395243 \\
\hline \multicolumn{4}{|c|}{ Sum of electronic and thermal Enthalpies $=$} & -40.394299 \\
\hline \multicolumn{4}{|c|}{ Sum of electronic and thermal Free Energies $=$} & -40.415422 \\
\hline
\end{tabular}

\begin{tabular}{|c|c|c|c|c|}
\hline \multirow{2}{*}{$\begin{array}{l}\text { Center } \\
\text { Number }\end{array}$} & Atomic & \multirow{2}{*}{$\begin{array}{l}\text { Atomic } \\
\text { Type }\end{array}$} & \multicolumn{2}{|c|}{ Coordinates (Angstroms } \\
\hline & Numbe & & $\mathrm{X}$ & $\mathrm{Z}$ \\
\hline 1 & 6 & -1.265491 & -0.260341 & 0.000000 \\
\hline 2 & 1 & -2.166812 & 0.355550 & -0.000032 \\
\hline 3 & 1 & -1.300046 & -0.905182 & 0.881706 \\
\hline 4 & 1 & -1.300019 & -0.905233 & -0.881669 \\
\hline 5 & 6 & 0.000000 & 0.590244 & 0.000000 \\
\hline 6 & 1 & -0.000000 & 1.246191 & 0.875365 \\
\hline 7 & 1 & 0.000002 & 1.246173 & -0.875377 \\
\hline 8 & 6 & 1.265491 & -0.260341 & 0.000000 \\
\hline
\end{tabular}

Propane

Standard orientation: 


$\begin{array}{cccccl}9 & 1 & 0 & 1.300056 & -0.905166 & 0.881717 \\ 10 & 1 & 0 & 1.300005 & -0.905249 & -0.881658 \\ 11 & 1 & 0 & 2.166814 & 0.355547 & -0.000055\end{array}$

Zero-point correction $=$

0.103948 (Hartree/Particle)

Thermal correction to Energy=

0.108459

Thermal correction to Enthalpy=

0.109404

Thermal correction to Gibbs Free Energy=

Sum of electronic and zero-point Energies=

0.078312

Sum of electronic and thermal Energies $=$

$-118.833137$

$-118.828625$

Sum of electronic and thermal Enthalpies=

$-118.827681$

Sum of electronic and thermal Free Energies $=\quad-118.858772$

Butane

Standard orientation:

\begin{tabular}{|c|c|c|c|c|c|}
\hline \multirow{2}{*}{$\begin{array}{l}\text { Center } \\
\text { Number }\end{array}$} & \multicolumn{2}{|c|}{ Atomic } & \multirow{2}{*}{$\begin{array}{l}\text { Atomic } \\
\text { Type }\end{array}$} & \multicolumn{2}{|c|}{ Coordinates (Angstrom } \\
\hline & & ber & & $\mathrm{X}$ & $\mathrm{Z}$ \\
\hline 1 & 6 & 0 & 1.946953 & -0.119573 & 0.000034 \\
\hline 2 & 1 & 0 & 2.086147 & -0.749909 & -0.881757 \\
\hline 3 & 1 & 0 & 2.086075 & -0.749901 & 0.881843 \\
\hline 4 & 1 & 0 & 2.736623 & 0.634144 & 0.000062 \\
\hline 5 & 6 & 0 & 0.561268 & 0.515991 & -0.000039 \\
\hline 6 & 1 & 0 & 0.451985 & 1.164291 & 0.875951 \\
\hline 7 & 1 & 0 & 0.452036 & 1.164242 & -0.876072 \\
\hline 8 & 6 & 0 & -0.561268 & -0.515991 & -0.000039 \\
\hline 9 & 1 & 0 & -0.452036 & -1.164240 & -0.876073 \\
\hline 10 & 1 & 0 & -0.451985 & -1.164294 & 0.875949 \\
\hline 11 & 6 & 0 & -1.946953 & 0.119573 & 0.000034 \\
\hline 12 & 1 & 0 & -2.086145 & 0.749914 & -0.881754 \\
\hline 13 & 1 & 0 & -2.736623 & -0.634144 & 0.000057 \\
\hline 14 & 1 & 0 & -2.086076 & 0.749896 & 0.881846 \\
\hline
\end{tabular}

Isobutane

Zero-point correction=

Thermal correction to Energy=

0.132602 (Hartree/Particle)

Thermal correction to Enthalpy=

0.138368

Thermal correction to Gibbs Free Energy=

0.139312

Sum of electronic and zero-point Energies=

0.104610

Sum of electronic and thermal Energies $=$

$-158.053150$

$-158.047385$

Sum of electronic and thermal Enthalpies=

$-158.046440$

Sum of electronic and thermal Free Energies $=\quad-158.081142$

Pentane

Standard orientation:

\begin{tabular}{|c|c|c|c|c|}
\hline Center & Atomic & Atomic & Coordinate & es (Angstroms) \\
\hline Number & Numbe & Type & $\mathrm{X}$ & Z \\
\hline 1 & 6 & 0 & 0.326402 & 0.000000 \\
\hline 2 & 1 & 2.573399 & 0.970867 & -0.881707 \\
\hline
\end{tabular}




$\begin{array}{cccccc}3 & 1 & 0 & 2.573393 & 0.970883 & 0.881697 \\ 4 & 1 & 0 & 3.439174 & -0.289689 & 0.000009 \\ 5 & 6 & 0 & 1.273795 & -0.525455 & 0.000003 \\ 6 & 1 & 0 & 1.271720 & -1.182435 & -0.876055 \\ 7 & 1 & 0 & 1.271718 & -1.182424 & 0.876070 \\ 8 & 6 & 0 & 0.000000 & 0.312097 & -0.000004 \\ 9 & 1 & 0 & -0.000002 & 0.970828 & 0.876611 \\ 10 & 1 & 0 & 0.000002 & 0.970820 & -0.876624 \\ 11 & 6 & 0 & -1.273795 & -0.525455 & -0.000004 \\ 12 & 1 & 0 & -1.271716 & -1.182431 & 0.876057 \\ 13 & 1 & 0 & -1.271721 & -1.182428 & -0.876067 \\ 14 & 6 & 0 & -2.538138 & 0.326402 & 0.000003 \\ 15 & 1 & 0 & -3.439174 & -0.289689 & 0.000007 \\ 16 & 1 & 0 & -2.573392 & 0.970876 & 0.881705 \\ 17 & 1 & 0 & -2.573400 & 0.970874 & -0.881699\end{array}$

Zero-point correction=

Thermal correction to Energy=

Thermal correction to Enthalpy=

Thermal correction to Gibbs Free Energy=

Sum of electronic and zero-point Energies=

Sum of electronic and thermal Energies $=$

Sum of electronic and thermal Enthalpies=

Sum of electronic and thermal Free Energies=

Neopentane

Standard orientation:

\begin{tabular}{|c|c|c|c|c|}
\hline \multirow{2}{*}{$\begin{array}{l}\text { Center } \\
\text { Number }\end{array}$} & Atomic & \multirow{2}{*}{$\begin{array}{l}\text { Atomic } \\
\text { Type }\end{array}$} & \multicolumn{2}{|c|}{ Coordinates (Angstroms } \\
\hline & Numbe & & $\mathrm{X}$ & Z \\
\hline 1 & 6 & -1.000135 & -0.523318 & 1.031896 \\
\hline 2 & 1 & -1.869462 & 0.135845 & 1.102148 \\
\hline 3 & 1 & -1.354376 & -1.521433 & 0.760903 \\
\hline 4 & 1 & -0.542399 & -0.585074 & 2.022764 \\
\hline 5 & 6 & 0.000018 & -0.000007 & -0.000008 \\
\hline 6 & 6 & -0.673442 & 0.073138 & -1.371154 \\
\hline 7 & 1 & 0.021924 & 0.445240 & -2.128228 \\
\hline 8 & 1 & -1.021414 & -0.913526 & -1.688276 \\
\hline 9 & 1 & -1.536509 & 0.743717 & -1.346832 \\
\hline 10 & 6 & 0.473196 & 1.395147 & 0.410613 \\
\hline 11 & 1 & 0.959353 & 1.370057 & 1.389478 \\
\hline 12 & 1 & 1.190431 & 1.792584 & -0.312417 \\
\hline 13 & 1 & -0.367898 & 2.091055 & 0.469195 \\
\hline 14 & 6 & 1.200381 & -0.944967 & -0.071354 \\
\hline 15 & 1 & 1.700535 & -1.014568 & 0.898262 \\
\hline 16 & 1 & 0.888205 & -1.951222 & -0.363159 \\
\hline 17 & 1 & 1.931506 & -0.592637 & -0.803796 \\
\hline
\end{tabular}


Thermal correction to Energy=

Thermal correction to Enthalpy=

Thermal correction to Gibbs Free Energy=

Sum of electronic and zero-point Energies=

Sum of electronic and thermal Energies $=$

Sum of electronic and thermal Enthalpies=

Sum of electronic and thermal Free Energies=

Hexane

Standard orientation:
0.166794

0.167738

0.130785

$-197.280353$

$-197.273442$

$-197.272498$

$-197.309451$

\begin{tabular}{|c|c|c|c|c|}
\hline \multirow{3}{*}{$\begin{array}{l}\text { Center } \\
\text { Number } \\
----- \\
1\end{array}$} & \multirow{2}{*}{$\begin{array}{l}\text { Atomic } \\
\text { Number }\end{array}$} & \multirow{2}{*}{$\begin{array}{l}\text { Atomic } \\
\text { Type }\end{array}$} & \multicolumn{2}{|c|}{ Coordinates (Angstron } \\
\hline & & & $\mathrm{X}$ & Z \\
\hline & 6 & -3.198060 & 0.208838 & 0.000035 \\
\hline 2 & 1 & -3.278411 & 0.849276 & 0.881881 \\
\hline 3 & 1 & -3.278410 & 0.849432 & -0.881698 \\
\hline 4 & 1 & -4.053955 & -0.468856 & -0.000027 \\
\hline 5 & 6 & -1.876787 & -0.551880 & -0.000040 \\
\hline 6 & 1 & -1.828598 & -1.207297 & 0.875977 \\
\hline 7 & 1 & -1.828629 & -1.207189 & -0.876139 \\
\hline 8 & 6 & -0.664619 & 0.373103 & -0.000005 \\
\hline 9 & 1 & -0.711762 & 1.030044 & -0.876679 \\
\hline 10 & 1 & -0.711787 & 1.030015 & 0.876690 \\
\hline 11 & 6 & 0.664619 & -0.373103 & 0.000006 \\
\hline 12 & 1 & 0.711765 & -1.030058 & -0.876658 \\
\hline 13 & 1 & 0.711785 & -1.030001 & 0.876712 \\
\hline 14 & 6 & 1.876787 & 0.551880 & -0.000034 \\
\hline 15 & 1 & 1.828603 & 1.207300 & 0.875981 \\
\hline 16 & 1 & 1.828624 & 1.207186 & -0.876135 \\
\hline 17 & 6 & 3.198060 & -0.208838 & 0.000030 \\
\hline 18 & 1 & 3.278415 & -0.849421 & -0.881711 \\
\hline 19 & 1 & 4.053955 & 0.468856 & -0.000017 \\
\hline 20 & 1 & 3.278406 & -0.849287 & 0.881868 \\
\hline
\end{tabular}

Zero-point correction=

0.189818 (Hartree/Particle)

Thermal correction to Energy=

Thermal correction to Enthalpy=

0.198216

0.199161

Thermal correction to Gibbs Free Energy=

0.157239

Sum of electronic and zero-point Energies=

$-236.493209$

Sum of electronic and thermal Energies=

$-236.484811$

Sum of electronic and thermal Enthalpies=

$-236.483866$

Sum of electronic and thermal Free Energies $=\quad-236.525788$

22dimethylbutane

Standard orientation:

\begin{tabular}{cccccc} 
Center & \multicolumn{2}{c}{ Atomic } & Atomic & \multicolumn{2}{c}{ Coordinates } \\
Number & Number & Type & X & Y & Z \\
-1 & 6 & 0 & -1.614250 & 0.971112 & 0.001346 \\
2 & 1 & 0 & -1.591420 & 1.612501 & 0.886481
\end{tabular}




$\begin{array}{cccccc}3 & 1 & 0 & -1.591640 & 1.614649 & -0.882234 \\ 4 & 1 & 0 & -2.563988 & 0.429869 & 0.000801 \\ 5 & 6 & 0 & -0.431593 & -0.000017 & 0.000012 \\ 6 & 6 & 0 & 0.864271 & 0.829497 & 0.000590 \\ 7 & 1 & 0 & 0.845461 & 1.487819 & -0.875298 \\ 8 & 1 & 0 & 0.845545 & 1.486514 & 0.877464 \\ 9 & 6 & 0 & 2.168469 & 0.037467 & -0.000002 \\ 10 & 1 & 0 & 2.255104 & -0.598260 & -0.882806 \\ 11 & 1 & 0 & 3.024641 & 0.714721 & 0.000088 \\ 12 & 1 & 0 & 2.255426 & -0.598925 & 0.882287 \\ 13 & 6 & 0 & -0.512847 & -0.880664 & 1.248661 \\ 14 & 1 & 0 & 0.278364 & -1.632705 & 1.264965 \\ 15 & 1 & 0 & -0.428287 & -0.278217 & 2.157430 \\ 16 & 1 & 0 & -1.470207 & -1.407195 & 1.282426 \\ 17 & 6 & 0 & -0.513467 & -0.877904 & -1.250549 \\ 18 & 1 & 0 & -1.470651 & -1.404727 & -1.284761 \\ 19 & 1 & 0 & -0.429843 & -0.273374 & -2.158017 \\ 20 & 1 & 0 & 0.278002 & -1.629620 & -1.269176\end{array}$

Zero-point correction=

Thermal correction to Energy=

Thermal correction to Enthalpy=

Thermal correction to Gibbs Free Energy=

Sum of electronic and zero-point Energies=

Sum of electronic and thermal Energies $=$

Sum of electronic and thermal Enthalpies=

Sum of electronic and thermal Free Energies= Heptane
0.188691 (Hartree/Particle)

$$
0.196925
$$

0.197869

$$
\begin{aligned}
& 0.157373 \\
& -236.498397 \\
& -236.490164 \\
& -236.489219 \\
& -236.529716
\end{aligned}
$$

Standard orientation:

\begin{tabular}{cccccc} 
Center & \multicolumn{2}{c}{ Atomic } & \multicolumn{2}{c}{ Atomic } & \multicolumn{3}{c}{ Coordinates } \\
Number & Number & Type & X & Y & Z \\
\hline 1 & 6 & 0 & -3.811299 & -0.355931 & -0.000007 \\
2 & 1 & 0 & -3.846502 & -1.000399 & -0.881733 \\
3 & 1 & 0 & -3.846495 & -1.000446 & 0.881687 \\
4 & 1 & 0 & -4.712417 & 0.260078 & 0.000014 \\
5 & 6 & 0 & -2.546985 & 0.495987 & 0.000012 \\
6 & 1 & 0 & -2.544854 & 1.152984 & -0.876033 \\
7 & 1 & 0 & -2.544856 & 1.152948 & 0.876083 \\
8 & 6 & 0 & -1.273026 & -0.341708 & -0.000003 \\
9 & 1 & 0 & -1.273721 & -1.000159 & 0.876658 \\
10 & 1 & 0 & -1.273728 & -1.000141 & -0.876679 \\
11 & 6 & 0 & 0.000000 & 0.496724 & -0.000000 \\
12 & 1 & 0 & -0.000003 & 1.154873 & 0.876748 \\
13 & 1 & 0 & 0.000003 & 1.154869 & -0.876752 \\
14 & 6 & 0 & 1.273026 & -0.341708 & 0.000006 \\
15 & 1 & 0 & 1.273721 & -1.000162 & -0.876654 \\
16 & 1 & 0 & 1.273728 & -1.000138 & 0.876683
\end{tabular}




$\begin{array}{rrrrrr}17 & 6 & 0 & 2.546985 & 0.495987 & -0.000011 \\ 18 & 1 & 0 & 2.544855 & 1.152983 & 0.876034 \\ 19 & 1 & 0 & 2.544854 & 1.152948 & -0.876083 \\ 20 & 6 & 0 & 3.811299 & -0.355931 & 0.000004 \\ 21 & 1 & 0 & 3.846496 & -1.000441 & -0.881692 \\ 22 & 1 & 0 & 4.712417 & 0.260078 & -0.000011 \\ 23 & 1 & 0 & 3.846500 & -1.000404 & 0.881728\end{array}$

Zero-point correction=

Thermal correction to Energy=

Thermal correction to Enthalpy=

Thermal correction to Gibbs Free Energy=

Sum of electronic and zero-point Energies=

Sum of electronic and thermal Energies $=$

Sum of electronic and thermal Enthalpies=

Sum of electronic and thermal Free Energies=

223trimethylbutane
0.218401 (Hartree/Particle)

0.228160

0.229104

0.183513

$-275.713279$

$-275.703520$

$-275.702576$

$-275.748167$

Standard orientation:

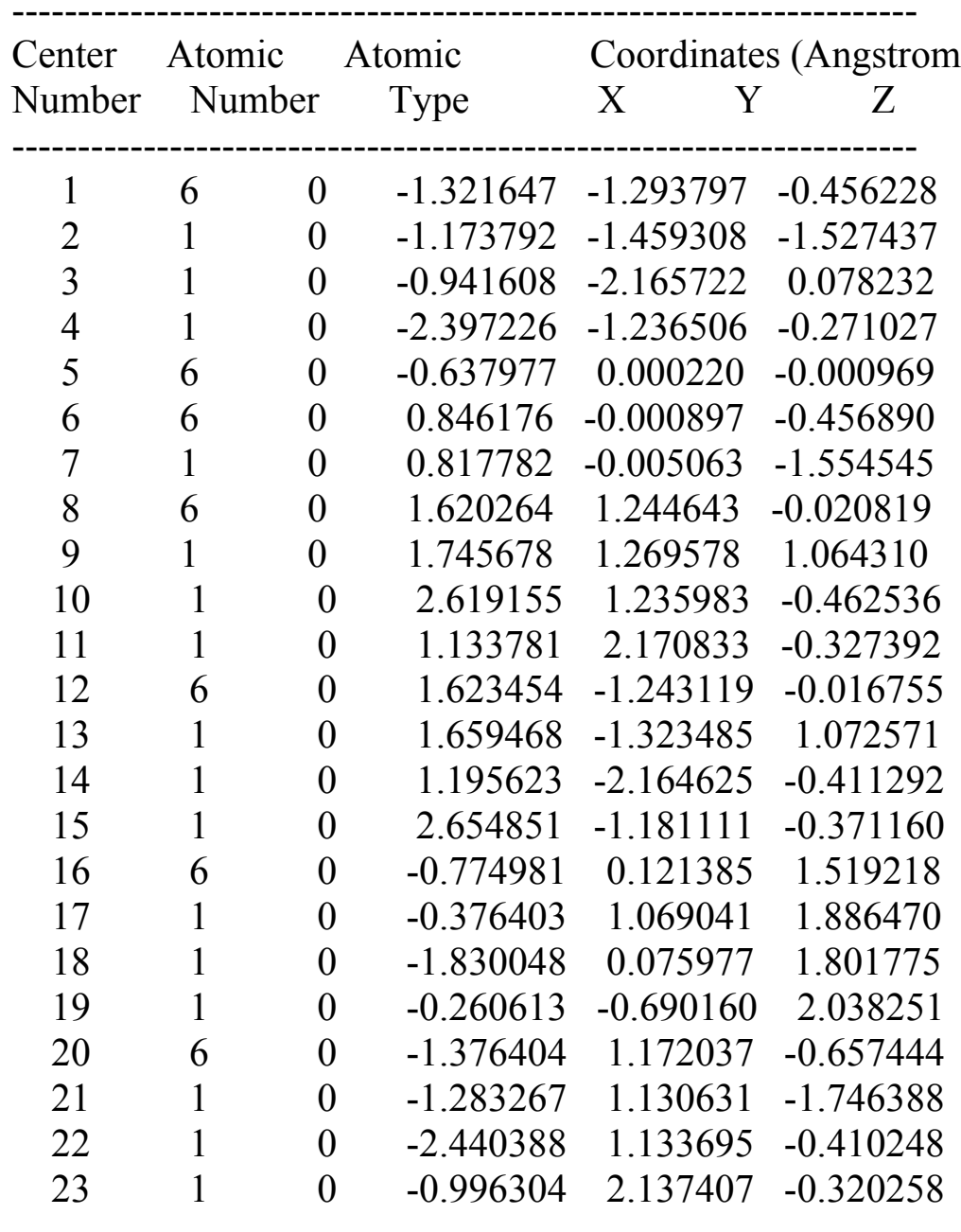

Zero-point correction=

Thermal correction to Energy=

Thermal correction to Enthalpy=
0.217068 (Hartree/Particle)

0.226592

0.227536 
Thermal correction to Gibbs Free Energy=

Sum of electronic and zero-point Energies=

Sum of electronic and thermal Energies $=$

Sum of electronic and thermal Enthalpies=

Sum of electronic and thermal Free Energies=
0.183656

$-275.717578$

$-275.708055$

$-275.707111$

$-275.750990$

Octane

\begin{tabular}{cccccc} 
Center & Atomic & \multicolumn{2}{c}{ Atomic } & \multicolumn{3}{c}{ Coordinates } \\
Number & Number & Type & X & Y & $Z$ \\
- & Nustroms) \\
\hline 1 & 6 & 0 & -4.461542 & 0.257589 & 0.000015 \\
2 & 1 & 0 & -4.522160 & 0.900164 & 0.881746 \\
3 & 1 & 0 & -4.522161 & 0.900223 & -0.881675 \\
4 & 1 & 0 & -5.337651 & -0.393507 & -0.000007 \\
5 & 6 & 0 & -3.164582 & -0.543758 & -0.000015 \\
6 & 1 & 0 & -3.136524 & -1.200171 & 0.876024 \\
7 & 1 & 0 & -3.136533 & -1.200123 & -0.876090 \\
8 & 6 & 0 & -1.924645 & 0.343524 & 0.000002 \\
9 & 1 & 0 & -1.951280 & 1.001440 & -0.876669 \\
10 & 1 & 0 & -1.951285 & 1.001415 & 0.876691 \\
11 & 6 & 0 & -0.619562 & -0.444066 & -0.000004 \\
12 & 1 & 0 & -0.593564 & -1.101705 & -0.876738 \\
13 & 1 & 0 & -0.593556 & -1.101705 & 0.876729 \\
14 & 6 & 0 & 0.619562 & 0.444066 & -0.000009 \\
15 & 1 & 0 & 0.593556 & 1.101716 & 0.876716 \\
16 & 1 & 0 & 0.593564 & 1.101694 & -0.876751 \\
17 & 6 & 0 & 1.924645 & -0.343524 & 0.000007 \\
18 & 1 & 0 & 1.951283 & -1.001445 & -0.876660 \\
19 & 1 & 0 & 1.951282 & -1.001411 & 0.876700 \\
20 & 6 & 0 & 3.164582 & 0.543758 & -0.000010 \\
21 & 1 & 0 & 3.136527 & 1.200165 & 0.876033 \\
22 & 1 & 0 & 3.136530 & 1.200128 & -0.876080 \\
23 & 6 & 0 & 4.461542 & -0.257589 & 0.000010 \\
24 & 1 & 0 & 4.522162 & -0.900212 & -0.881687 \\
25 & 1 & 0 & 5.337651 & 0.393507 & -0.000003 \\
26 & 1 & 0 & 4.522159 & -0.900175 & 0.881734
\end{tabular}

Zero-point correction=

Thermal correction to Energy=

Thermal correction to Enthalpy=

Thermal correction to Gibbs Free Energy=

Sum of electronic and zero-point Energies=

Sum of electronic and thermal Energies $=$

Sum of electronic and thermal Enthalpies=

Sum of electronic and thermal Free Energies=

2233tetramethylbutane
0.246969 (Hartree/Particle)

0.258084

0.259028

0.209816

$-314.933365$

$-314.922250$

$-314.921306$

$-314.970518$

Standard orientation:

Center Atomic Atomic Coordinates (Angstroms) 


\begin{tabular}{|c|c|c|c|c|c|}
\hline Number & Numbe & & Type & $X$ & Z \\
\hline 1 & 6 & 0 & -1.341087 & -0.892594 & -1.117493 \\
\hline 2 & 1 & 0 & -0.927752 & -0.633709 & -2.094449 \\
\hline 3 & 1 & 0 & -1.138983 & -1.948511 & -0.930795 \\
\hline 4 & 1 & 0 & -2.425651 & -0.770498 & -1.176636 \\
\hline 5 & 6 & 0 & -0.785006 & 0.000062 & 0.000007 \\
\hline 6 & 6 & 0 & 0.785022 & -0.000003 & -0.000033 \\
\hline 7 & 6 & 0 & 1.341152 & 0.619293 & 1.289138 \\
\hline 8 & 1 & 0 & 0.927083 & 1.611384 & 1.480362 \\
\hline 9 & 1 & 0 & 1.139949 & -0.007619 & 2.159284 \\
\hline 10 & 1 & 0 & 2.425593 & 0.726471 & 1.204346 \\
\hline 11 & 6 & 0 & -1.341066 & 1.414171 & -0.214208 \\
\hline 12 & 1 & 0 & -0.926922 & 2.130942 & 0.497855 \\
\hline 13 & 1 & 0 & -1.140000 & 1.780155 & -1.222325 \\
\hline 14 & 1 & 0 & -2.425496 & 1.404509 & -0.077786 \\
\hline 15 & 6 & 0 & -1.340894 & -0.521458 & 1.331836 \\
\hline 16 & 1 & 0 & -2.425333 & -0.634830 & 1.255428 \\
\hline 17 & 1 & 0 & -0.926616 & -1.496459 & 1.596582 \\
\hline 18 & 1 & 0 & -1.139685 & 0.168710 & 2.152711 \\
\hline 19 & 6 & 0 & 1.341098 & 0.806778 & -1.180998 \\
\hline 20 & 1 & 0 & 2.425561 & 0.679896 & -1.231399 \\
\hline 21 & 1 & 0 & 0.927075 & 0.476280 & -2.135796 \\
\hline 22 & 1 & 0 & 1.139739 & 1.873781 & -1.073207 \\
\hline 23 & 6 & 0 & 1.340819 & -1.426220 & -0.108265 \\
\hline 24 & 1 & 0 & 2.425225 & -1.406725 & 0.027314 \\
\hline 25 & 1 & 0 & 0.926228 & -2.087894 & 0.655017 \\
\hline 26 & 1 & 0 & 1.139759 & -1.866050 & -1.086398 \\
\hline
\end{tabular}

Zero-point correction=

Thermal correction to Energy=

Thermal correction to Enthalpy=

Thermal correction to Gibbs Free Energy=

Sum of electronic and zero-point Energies=

Sum of electronic and thermal Energies=

Sum of electronic and thermal Enthalpies=

Sum of electronic and thermal Free Energies=

PBE0DHD3BJ/ccpVTZ

Ethane

Standard orientation:
0.245566 (Hartree/Particle)

0.255952

0.256896

0.212320

$-314.937344$

$-314.926958$

$-314.926014$

$-314.970590$

\begin{tabular}{|c|c|c|c|c|}
\hline \multirow{2}{*}{$\begin{array}{l}\text { Center } \\
\text { Number }\end{array}$} & \multirow{2}{*}{$\begin{array}{l}\text { Atomic } \\
\text { Number }\end{array}$} & \multirow{2}{*}{$\begin{array}{r}\text { Atomic } \\
\text { Type }\end{array}$} & \multicolumn{2}{|c|}{ Coordinates (Angstrom } \\
\hline & & & $\mathrm{X}$ & $\mathrm{Z}$ \\
\hline 1 & 6 & 0.000000 & 0.000000 & 0.757637 \\
\hline 2 & 1 & -0.506750 & 0.877752 & 1.154023 \\
\hline 3 & 1 & 1.013531 & -0.000018 & 1.154023 \\
\hline 4 & 1 & -0.506781 & -0.877734 & 1.154023 \\
\hline 5 & 6 & 0.000000 & 0.000000 & -0.757637 \\
\hline 6 & 1 & 0.506750 & 0.877752 & -1.154023 \\
\hline
\end{tabular}




$\begin{array}{rrrrrr}7 & 1 & 0 & 0.506781 & -0.877734 & -1.154023 \\ 8 & 1 & 0 & -1.013531 & -0.000018 & -1.154023\end{array}$

Zero-point correction $=$

Thermal correction to Energy=

Thermal correction to Enthalpy=

Thermal correction to Gibbs Free Energy=

Sum of electronic and zero-point Energies=

Sum of electronic and thermal Energies=

Sum of electronic and thermal Enthalpies=

Sum of electronic and thermal Free Energies=

Methane

Standard orientation:

\begin{tabular}{|c|c|c|c|c|}
\hline Center & Atomic & Atomic & Coordinate & es (Angstrom \\
\hline Number & Numbe & Type & $\mathrm{X}$ & Z \\
\hline 1 & 6 & 0.000000 & 0.000000 & 0.000000 \\
\hline 2 & 1 & 0.626732 & 0.626732 & 0.626732 \\
\hline 3 & 1 & -0.626732 & -0.626732 & 0.626732 \\
\hline 4 & 1 & -0.626732 & 0.626732 & -0.626732 \\
\hline 5 & 1 & 0.626732 & -0.626732 & -0.626732 \\
\hline
\end{tabular}

Zero-point correction $=$

Thermal correction to Energy=

Thermal correction to Enthalpy=

0.045347 (Hartree/Particle)

0.048214

0.049158

Thermal correction to Gibbs Free Energy $=\quad 0.028043$

Sum of electronic and zero-point Energies=

Sum of electronic and thermal Energies=

$-40.434648$

$-40.431781$

$-40.430837$

Sum of electronic and thermal Enthalpies=

$-40.451952$

Propane

Standard orientation:

\begin{tabular}{|c|c|c|c|c|}
\hline \multirow{2}{*}{$\begin{array}{l}\text { Center } \\
\text { Number }\end{array}$} & Atomic & \multirow{2}{*}{$\begin{array}{l}\text { Atomic } \\
\text { Type }\end{array}$} & \multicolumn{2}{|c|}{ Coordinates (Angstroms } \\
\hline & Numbe & & $X$ & Z \\
\hline 1 & 6 & -1.259088 & -0.258152 & 0.000000 \\
\hline 2 & 1 & -2.157186 & 0.356428 & -0.000031 \\
\hline 3 & 1 & -1.296409 & -0.901726 & 0.878367 \\
\hline 4 & 1 & -1.296383 & -0.901775 & -0.878333 \\
\hline 5 & 6 & -0.000000 & 0.585092 & 0.000000 \\
\hline 6 & 1 & 0.000001 & 1.240721 & 0.871578 \\
\hline 7 & 1 & 0.000002 & 1.240706 & -0.871588 \\
\hline 8 & 6 & 1.259088 & -0.258153 & 0.000000 \\
\hline 9 & 1 & 1.296404 & -0.901731 & 0.878364 \\
\hline 10 & 1 & 1.296382 & -0.901770 & -0.878336 \\
\hline 11 & 1 & 2.157187 & 0.356425 & -0.000025 \\
\hline
\end{tabular}

Zero-point correction $=$

0.104942 (Hartree/Particle) 
Thermal correction to Energy=

Thermal correction to Enthalpy=

Thermal correction to Gibbs Free Energy=

Sum of electronic and zero-point Energies=

Sum of electronic and thermal Energies=

Sum of electronic and thermal Enthalpies=

Sum of electronic and thermal Free Energies=

Butane

Standard orientation:

\begin{tabular}{|c|c|c|c|c|}
\hline \multirow{2}{*}{$\begin{array}{l}\text { Center } \\
\text { Number }\end{array}$} & Atomic & \multirow{2}{*}{$\begin{array}{l}\text { Atomic } \\
\text { Type }\end{array}$} & \multicolumn{2}{|c|}{ Coordinates (Angstrom } \\
\hline & Number & & $\mathrm{X}$ & $\mathrm{Z}$ \\
\hline 1 & 6 & 1.936314 & 0.118570 & -0.000022 \\
\hline 2 & 1 & 2.077498 & 0.747337 & 0.878404 \\
\hline 3 & 1 & 2.077448 & 0.747354 & -0.878443 \\
\hline 4 & 1 & 2.723731 & -0.632483 & -0.000051 \\
\hline 5 & 6 & 0.558863 & -0.511931 & 0.000025 \\
\hline 6 & 1 & 0.449774 & -1.159896 & -0.872057 \\
\hline 7 & 1 & 0.449803 & -1.159871 & 0.872129 \\
\hline 8 & 6 & -0.558863 & 0.511931 & 0.000025 \\
\hline 9 & 1 & -0.449803 & 1.159869 & 0.872130 \\
\hline 10 & 1 & -0.449774 & 1.159899 & -0.872055 \\
\hline 11 & 6 & -1.936314 & -0.118570 & -0.000022 \\
\hline 12 & 1 & -2.077499 & -0.747337 & $7 \quad 0.878404$ \\
\hline 13 & 1 & -2.723731 & 0.632483 & -0.000052 \\
\hline 14 & 1 & -2.077447 & -0.747354 & $4-0.878443$ \\
\hline
\end{tabular}

Zero-point correction=

Thermal correction to Energy=

Thermal correction to Enthalpy=

Thermal correction to Gibbs Free Energy=

Sum of electronic and zero-point Energies=

Sum of electronic and thermal Energies=

Sum of electronic and thermal Enthalpies=

Sum of electronic and thermal Free Energies=

Isobutane

Standard orientation:

\begin{tabular}{|c|c|c|c|c|}
\hline \multirow{2}{*}{$\begin{array}{l}\text { Center } \\
\text { Number }\end{array}$} & Atomic & \multirow{2}{*}{$\begin{array}{r}\text { Atomic } \\
\text { Type }\end{array}$} & \multicolumn{2}{|c|}{ Coordinates (Angstroms } \\
\hline & Numbe & & $\mathrm{X}$ & $\mathrm{Z}$ \\
\hline 1 & 6 & -1.439454 & -0.088556 & 0.095281 \\
\hline 2 & 1 & -1.918006 & -1.000629 & -0.258016 \\
\hline 3 & 1 & -1.483823 & -0.091305 & 1.185116 \\
\hline 4 & 1 & -2.026189 & 0.757996 & -0.257993 \\
\hline 5 & 6 & -0.000006 & -0.000003 & -0.375658 \\
\hline 6 & 1 & 0.000006 & -0.000012 & -1.468496 \\
\hline 7 & 6 & 0.796423 & -1.202311 & 0.095281 \\
\hline
\end{tabular}

0.109434

0.110378

0.079333

$-118.938759$

$-118.934266$

$-118.933322$

$-118.964367$ 


$\begin{array}{cccccc}8 & 1 & 0 & 1.825608 & -1.160650 & -0.257918 \\ 9 & 1 & 0 & 0.820881 & -1.239361 & 1.185111 \\ 10 & 1 & 0 & 0.356734 & -2.133731 & -0.258048 \\ 11 & 6 & 0 & 0.643038 & 1.290866 & 0.095280 \\ 12 & 1 & 0 & 1.669629 & 1.375625 & -0.257761 \\ 13 & 1 & 0 & 0.092575 & 2.161349 & -0.258215 \\ 14 & 1 & 0 & 0.662576 & 1.330734 & 1.185111\end{array}$

Zero-point correction=

Thermal correction to Energy=

Thermal correction to Enthalpy=

Thermal correction to Gibbs Free Energy=

Sum of electronic and zero-point Energies=

Sum of electronic and thermal Energies=

Sum of electronic and thermal Enthalpies=

Sum of electronic and thermal Free Energies=

Pentane

\begin{tabular}{cccccc} 
Center & Atomic & \multicolumn{2}{c}{ Atomic } & \multicolumn{3}{c}{ Coordinates } \\
Number & Number & Type & X & Y & $Z$ \\
\hline 1 & 6 & 0 & -2.524427 & 0.323241 & 0.000001 \\
2 & 1 & 0 & -2.562414 & 0.966466 & 0.878374 \\
3 & 1 & 0 & -2.562412 & 0.966472 & -0.878369 \\
4 & 1 & 0 & -3.422240 & -0.291506 & -0.000003 \\
5 & 6 & 0 & -1.266646 & -0.520979 & -0.000001 \\
6 & 1 & 0 & -1.264122 & -1.177574 & 0.872210 \\
7 & 1 & 0 & -1.264122 & -1.177571 & -0.872214 \\
8 & 6 & 0 & 0.000000 & 0.310464 & 0.000000 \\
9 & 1 & 0 & 0.000001 & 0.968747 & -0.872666 \\
10 & 1 & 0 & -0.000001 & 0.968748 & 0.872665 \\
11 & 6 & 0 & 1.266646 & -0.520979 & 0.000001 \\
12 & 1 & 0 & 1.264122 & -1.177574 & -0.872210 \\
13 & 1 & 0 & 1.264122 & -1.177571 & 0.872214 \\
14 & 6 & 0 & 2.524427 & 0.323241 & -0.000001 \\
15 & 1 & 0 & 3.422240 & -0.291506 & 0.000000 \\
16 & 1 & 0 & 2.562412 & 0.966469 & -0.878372 \\
17 & 1 & 0 & 2.562413 & 0.966469 & 0.878371
\end{tabular}

Zero-point correction=

Thermal correction to Energy=

Thermal correction to Enthalpy=

Thermal correction to Gibbs Free Energy=

Sum of electronic and zero-point Energies=

Sum of electronic and thermal Energies $=$

Sum of electronic and thermal Enthalpies=

Sum of electronic and thermal Free Energies=

Neopentane

Standard orientation:
0.139075

0.140019

$$
\begin{gathered}
0.105861 \\
-158.195744 \\
-158.190089 \\
-158.189145 \\
-158.223303
\end{gathered}
$$

Standard orientation: 


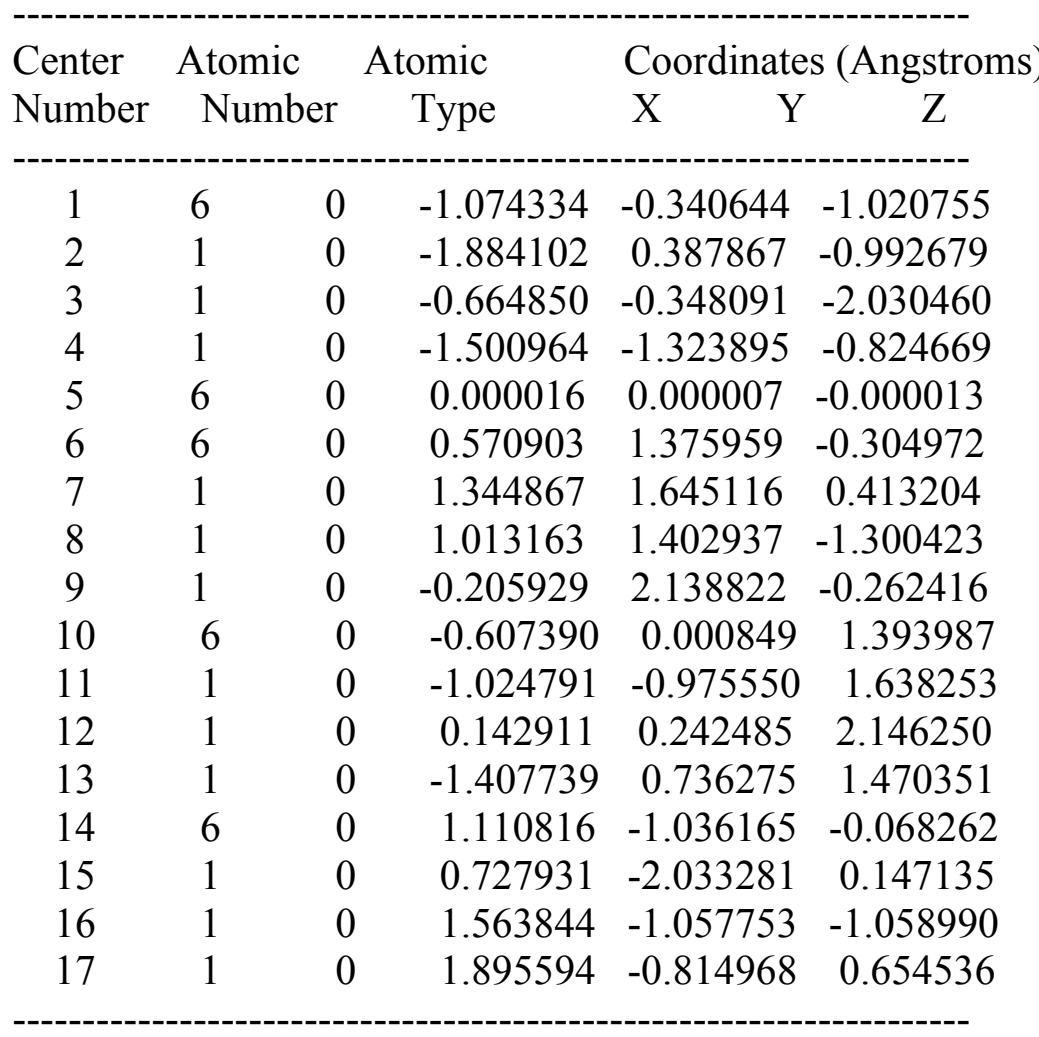

Zero-point correction=

Thermal correction to Energy=

Thermal correction to Enthalpy=

Thermal correction to Gibbs Free Energy=

Sum of electronic and zero-point Energies=

Sum of electronic and thermal Energies=

Sum of electronic and thermal Enthalpies=

Sum of electronic and thermal Free Energies=

Hexane

Standard orientation:

\begin{tabular}{|c|c|c|c|c|}
\hline \multirow{2}{*}{$\begin{array}{l}\text { Center } \\
\text { Number }\end{array}$} & Atomic & \multirow{2}{*}{$\begin{array}{l}\text { Atomic } \\
\text { Type }\end{array}$} & \multicolumn{2}{|c|}{ Coordinates (Angstroms) } \\
\hline & Numbe & & $\mathrm{X}$ & Z \\
\hline 1 & 6 & 3.179586 & -0.206932 & 0.000005 \\
\hline 2 & 1 & 3.262430 & -0.845933 & 0.878390 \\
\hline 3 & 1 & 3.262431 & -0.845953 & -0.878365 \\
\hline 4 & 1 & 4.032277 & 0.469033 & -0.000003 \\
\hline 5 & 6 & 1.865904 & 0.547337 & -0.000005 \\
\hline 6 & 1 & 1.817480 & 1.202166 & 0.872168 \\
\hline 7 & 1 & 1.817484 & 1.202150 & -0.872191 \\
\hline 8 & 6 & 0.660339 & -0.370713 & -0.000000 \\
\hline 9 & 1 & 0.707141 & -1.027065 & -0.872720 \\
\hline 10 & 1 & 0.707143 & -1.027059 & 0.872724 \\
\hline 11 & 6 & -0.660339 & 0.370713 & -0.000000 \\
\hline 12 & 1 & -0.707141 & 1.027065 & -0.872720 \\
\hline 13 & 1 & -0.707144 & 1.027059 & 0.872724 \\
\hline
\end{tabular}




$\begin{array}{rrrrrr}14 & 6 & 0 & -1.865904 & -0.547337 & -0.000005 \\ 15 & 1 & 0 & -1.817480 & -1.202166 & 0.872168 \\ 16 & 1 & 0 & -1.817484 & -1.202150 & -0.872191 \\ 17 & 6 & 0 & -3.179586 & 0.206932 & 0.000005 \\ 18 & 1 & 0 & -3.262431 & 0.845955 & -0.878364 \\ 19 & 1 & 0 & -4.032277 & -0.469033 & -0.000004 \\ 20 & 1 & 0 & -3.262430 & 0.845932 & 0.878391\end{array}$

Zero-point correction=

Thermal correction to Energy=

Thermal correction to Enthalpy=

Thermal correction to Gibbs Free Energy=

Sum of electronic and zero-point Energies=

Sum of electronic and thermal Energies $=$

Sum of electronic and thermal Enthalpies=

Sum of electronic and thermal Free Energies=

22dimethylbutane

Standard orientation:

\begin{tabular}{|c|c|c|c|c|}
\hline \multirow{2}{*}{$\begin{array}{l}\text { Center } \\
\text { Number }\end{array}$} & Atomic & \multirow{2}{*}{$\begin{array}{l}\text { Atomic } \\
\text { Type }\end{array}$} & \multicolumn{2}{|c|}{ Coordinates (Angstroms) } \\
\hline & Numbe & & $\mathrm{X}$ & Z \\
\hline 1 & 6 & 1.604608 & 0.965826 & -0.001040 \\
\hline 2 & 1 & 1.582388 & 1.605847 & -0.882738 \\
\hline 3 & 1 & 1.582513 & 1.607559 & 0.879417 \\
\hline 4 & 1 & 2.552132 & 0.428045 & -0.000580 \\
\hline 5 & 6 & 0.429585 & -0.000299 & -0.000011 \\
\hline 6 & 6 & -0.859308 & 0.824006 & -0.000561 \\
\hline 7 & 1 & -0.839541 & 1.481316 & 0.871561 \\
\hline 8 & 1 & -0.839600 & 1.480088 & -0.873613 \\
\hline 9 & 6 & -2.155462 & 0.038209 & -0.000004 \\
\hline 10 & 1 & -2.243624 & -0.596041 & 0.879628 \\
\hline 11 & 1 & -3.009608 & 0.712381 & -0.000224 \\
\hline 12 & 1 & -2.243818 & -0.596880 & -0.879009 \\
\hline 13 & 6 & 0.509902 & -0.875517 & -1.241567 \\
\hline 14 & 1 & -0.281643 & -1.622017 & -1.260561 \\
\hline 15 & 1 & 0.430254 & -0.275343 & -2.147876 \\
\hline 16 & 1 & 1.461466 & -1.405010 & -1.274317 \\
\hline 17 & 6 & 0.510272 & -0.873247 & 1.243126 \\
\hline 18 & 1 & 1.461734 & -1.402890 & 1.276418 \\
\hline 19 & 1 & 0.431177 & -0.271370 & 2.148351 \\
\hline 20 & 1 & -0.281416 & -1.619549 & 1.263883 \\
\hline \multicolumn{3}{|c|}{ Zero-point correction $=$} & \multicolumn{2}{|c|}{0.190634 (Hartree/Particle) } \\
\hline \multicolumn{3}{|c|}{ Thermal correction to Energy= } & \multicolumn{2}{|c|}{0.198809} \\
\hline \multicolumn{3}{|c|}{ Thermal correction to Enthalpy= } & \multicolumn{2}{|c|}{0.199753} \\
\hline \multicolumn{5}{|c|}{ Thermal correction to Gibbs Free Energy $=0.159408$} \\
\hline \multicolumn{4}{|c|}{ Sum of electronic and zero-point Energies $=$} & -236.706592 \\
\hline \multicolumn{4}{|c|}{ Sum of electronic and thermal Energies $=$} & -236.698417 \\
\hline \multicolumn{4}{|c|}{ Sum of electronic and thermal Enthalpies $=$} & -236.697473 \\
\hline
\end{tabular}


Sum of electronic and thermal Free Energies=

Heptane

Standard orientation:

\begin{tabular}{|c|c|c|c|c|c|}
\hline \multirow{2}{*}{$\begin{array}{l}\text { Center } \\
\text { Number }\end{array}$} & \multicolumn{2}{|c|}{ Atomic } & \multirow{2}{*}{$\begin{array}{l}\text { tomic } \\
\text { Type }\end{array}$} & \multicolumn{2}{|c|}{ Coordinates (Angstrom } \\
\hline & & & & $\mathrm{X}$ & Z \\
\hline 1 & 6 & 0 & -3.790237 & -0.352321 & -0.000007 \\
\hline 2 & 1 & 0 & -3.828176 & -0.995558 & -0.878392 \\
\hline 3 & 1 & 0 & -3.828176 & -0.995588 & 0.878357 \\
\hline 4 & 1 & 0 & -4.688130 & 0.262339 & 0.000005 \\
\hline 5 & 6 & 0 & -2.532487 & 0.491925 & 0.000009 \\
\hline 6 & 1 & 0 & -2.529906 & 1.148539 & -0.872173 \\
\hline 7 & 1 & 0 & -2.529910 & 1.148514 & 0.872209 \\
\hline 8 & 6 & 0 & -1.265741 & -0.339651 & 0.000000 \\
\hline 9 & 1 & 0 & -1.266520 & -0.997673 & 0.872691 \\
\hline 10 & 1 & 0 & -1.266524 & -0.997662 & -0.872700 \\
\hline 11 & 6 & 0 & 0.000000 & 0.492407 & 0.000001 \\
\hline 12 & 1 & 0 & -0.000002 & 1.150144 & 0.872787 \\
\hline 13 & 1 & 0 & 0.000002 & 1.150142 & -0.872786 \\
\hline 14 & 6 & 0 & 1.265741 & -0.339651 & 0.000005 \\
\hline 15 & 1 & 0 & 1.266518 & -0.997677 & -0.872684 \\
\hline 16 & 1 & 0 & 1.266525 & -0.997658 & 0.872708 \\
\hline 17 & 6 & 0 & 2.532487 & 0.491925 & -0.000010 \\
\hline 18 & 1 & 0 & 2.529909 & 1.148541 & 0.872171 \\
\hline 19 & 1 & 0 & 2.529907 & 1.148513 & -0.872211 \\
\hline 20 & 6 & 0 & 3.790237 & -0.352321 & 0.000002 \\
\hline 21 & 1 & 0 & 3.828174 & -0.995588 & -0.878361 \\
\hline 22 & 1 & 0 & 4.688130 & 0.262339 & -0.000010 \\
\hline 23 & 1 & 0 & 3.828178 & -0.995558 & 0.878388 \\
\hline
\end{tabular}

Zero-point correction $=$

0.220664 (Hartree/Particle)

Thermal correction to Energy=

0.230357

Thermal correction to Enthalpy=

0.231301

Thermal correction to Gibbs Free Energy=

0.185869

$-275.956814$

Sum of electronic and zero-point Energies= $-275.947121$

Sum of electronic and thermal Energies= $-275.946177$

Sum of electronic and thermal Enthalpies= $-275.991609$

Sum of electronic and thermal Free Energies=

223 trimethylbutane

Standard orientation:

\begin{tabular}{|c|c|c|c|c|}
\hline \multirow{2}{*}{$\begin{array}{l}\text { Center } \\
\text { Number }\end{array}$} & Atomic & \multirow{2}{*}{$\begin{array}{l}\text { Atomic } \\
\text { Type }\end{array}$} & \multicolumn{2}{|c|}{ Coordinates (Angstroms } \\
\hline & Numbe & & $X$ & Z \\
\hline 1 & 6 & 1.326541 & 1.259205 & -0.506660 \\
\hline 2 & 1 & 1.190038 & 1.375451 & -1.582080 \\
\hline 3 & 1 & 0.950049 & 2.155439 & -0.019305 \\
\hline 4 & 1 & 2.396661 & 1.205577 & -0.310583 \\
\hline 5 & 6 & 0.634609 & -0.000097 & -0.000587 \\
\hline
\end{tabular}




$\begin{array}{cccccc}6 & 6 & 0 & -0.841758 & 0.000383 & -0.452008 \\ 7 & 1 & 0 & -0.812268 & 0.002511 & -1.546124 \\ 8 & 6 & 0 & -1.610918 & -1.237355 & -0.019642 \\ 9 & 1 & 0 & -1.713280 & -1.279757 & 1.063943 \\ 10 & 1 & 0 & -2.616313 & -1.215627 & -0.436668 \\ 11 & 1 & 0 & -1.140842 & -2.159562 & -0.350318 \\ 12 & 6 & 0 & -1.612395 & 1.236531 & -0.017536 \\ 13 & 1 & 0 & -1.669957 & 1.306611 & 1.068053 \\ 14 & 1 & 0 & -1.171474 & 2.156190 & -0.392096 \\ 15 & 1 & 0 & -2.634018 & 1.188405 & -0.390733 \\ 16 & 6 & 0 & 0.771174 & -0.059953 & 1.513973 \\ 17 & 1 & 0 & 0.344378 & -0.974120 & 1.921742 \\ 18 & 1 & 0 & 1.823942 & -0.037452 & 1.793531 \\ 19 & 1 & 0 & 0.287092 & 0.786654 & 1.997149 \\ 20 & 6 & 0 & 1.353744 & -1.198965 & -0.605994 \\ 21 & 1 & 0 & 1.243792 & -1.213392 & -1.690465 \\ 22 & 1 & 0 & 2.418312 & -1.153717 & -0.379702 \\ 23 & 1 & 0 & 0.977907 & -2.141708 & -0.215618\end{array}$

Zero-point correction=

Thermal correction to Energy=

Thermal correction to Enthalpy=

Thermal correction to Gibbs Free Energy=

Sum of electronic and zero-point Energies=

Sum of electronic and thermal Energies=

Sum of electronic and thermal Enthalpies=

Sum of electronic and thermal Free Energies=

Octane

\subsection{0 (Hartree/Particle)}

0.228761

$$
0.229706
$$

0.185373

$-275.960017$

$-275.950515$

$-275.949571$

$-275.993904$

Standard orientation:

\begin{tabular}{|c|c|c|c|c|}
\hline \multirow{2}{*}{$\begin{array}{l}\text { Center } \\
\text { Number }\end{array}$} & Atomic & \multirow{2}{*}{$\begin{array}{l}\text { Atomic } \\
\text { Type }\end{array}$} & \multicolumn{2}{|c|}{ Coordinates (Angstrom } \\
\hline & Numbe & & $X$ & Z \\
\hline 1 & 6 & 1.326541 & 1.259205 & -0.506660 \\
\hline 2 & 1 & 1.190038 & 1.375451 & -1.582080 \\
\hline 3 & 1 & 0.950049 & 2.155439 & -0.019305 \\
\hline 4 & 1 & 2.396661 & 1.205577 & -0.310583 \\
\hline 5 & 6 & 0.634609 & -0.000097 & -0.000587 \\
\hline 6 & 6 & -0.841758 & 0.000383 & -0.452008 \\
\hline 7 & 1 & -0.812268 & 0.002511 & -1.546124 \\
\hline 8 & 6 & -1.610918 & -1.237355 & -0.019642 \\
\hline 9 & 1 & -1.713280 & -1.279757 & 1.063943 \\
\hline 10 & 1 & -2.616313 & -1.215627 & $7 \quad-0.436668$ \\
\hline 11 & 1 & -1.140842 & -2.159562 & -0.350318 \\
\hline 12 & 6 & -1.612395 & 1.236531 & -0.017536 \\
\hline 13 & 1 & -1.669957 & 1.306611 & 1.068053 \\
\hline 14 & 1 & -1.171474 & 2.156190 & -0.392096 \\
\hline 15 & 1 & -2.634018 & 1.188405 & -0.390733 \\
\hline 16 & 6 & 0.771174 & -0.059953 & $3 \quad 1.513973$ \\
\hline 17 & 1 & 0.344378 & -0.974120 & 1.921742 \\
\hline
\end{tabular}




$\begin{array}{llllrc}18 & 1 & 0 & 1.823942 & -0.037452 & 1.793531 \\ 19 & 1 & 0 & 0.287092 & 0.786654 & 1.997149 \\ 20 & 6 & 0 & 1.353744 & -1.198965 & -0.605994 \\ 21 & 1 & 0 & 1.243792 & -1.213392 & -1.690465 \\ 22 & 1 & 0 & 2.418312 & -1.153717 & -0.379702 \\ 23 & 1 & 0 & 0.977907 & -2.141708 & -0.215618\end{array}$

Zero-point correction=

Thermal correction to Energy=

Thermal correction to Enthalpy=

Thermal correction to Gibbs Free Energy=

Sum of electronic and zero-point Energies=

Sum of electronic and thermal Energies $=$

Sum of electronic and thermal Enthalpies=

Sum of electronic and thermal Free Energies=

2233tetramethylbutane

Standard orientation:
0.249549 (Hartree/Particle)

0.260587

$$
0.261532
$$

0.212506

$-315.211376$

$-315.200337$

$-315.199393$

$-315.248419$

\begin{tabular}{|c|c|c|c|c|}
\hline \multirow{2}{*}{$\begin{array}{l}\text { Center } \\
\text { Number }\end{array}$} & Atomic & \multirow{2}{*}{$\begin{array}{l}\text { Atomic } \\
\text { Type }\end{array}$} & \multicolumn{2}{|c|}{ Coordinates (Angstroms } \\
\hline & Number & & $\begin{array}{ll}X & Y\end{array}$ & $\mathrm{Z}$ \\
\hline 1 & 6 & 1.332015 & -1.264871 & -0.649210 \\
\hline 2 & 1 & 0.922236 & -2.168086 & -0.201097 \\
\hline 3 & 1 & 1.127723 & -1.293641 & -1.717036 \\
\hline 4 & 1 & 2.413307 & -1.299236 & -0.521959 \\
\hline 5 & 6 & 0.780604 & -0.000034 & -0.000045 \\
\hline 6 & 6 & -0.780555 & -0.000084 & -0.000001 \\
\hline 7 & 6 & -1.331767 & 1.376823 & 0.354506 \\
\hline 8 & 1 & -0.922016 & 1.754665 & 1.289371 \\
\hline 9 & 1 & -1.127240 & 2.107331 & -0.424827 \\
\hline 10 & 1 & -2.413084 & 1.318259 & 0.472332 \\
\hline 11 & 6 & 1.331857 & 0.070240 & 1.419977 \\
\hline 12 & 1 & 0.921579 & 0.909635 & 1.978241 \\
\hline 13 & 1 & 1.127917 & -0.840296 & 1.978642 \\
\hline 14 & 1 & 2.413099 & 0.198113 & 1.386222 \\
\hline 15 & 6 & 1.331672 & 1.194752 & -0.770800 \\
\hline 16 & 1 & 2.412903 & 1.101728 & -0.864892 \\
\hline 17 & 1 & 0.921171 & 1.258761 & -1.776750 \\
\hline 18 & 1 & 1.127667 & 2.133658 & -0.261264 \\
\hline 19 & 6 & -1.331866 & -0.995441 & 1.015189 \\
\hline 20 & 1 & -2.413196 & -1.068061 & 0.905514 \\
\hline 21 & 1 & -0.922274 & -1.994012 & 0.874935 \\
\hline 22 & 1 & -1.127403 & -0.685850 & 2.037530 \\
\hline 23 & 6 & -1.331881 & -0.381463 & -1.369657 \\
\hline 24 & 1 & -2.413138 & -0.249597 & -1.377861 \\
\hline 25 & 1 & -0.921820 & 0.238879 & -2.164427 \\
\hline 26 & 1 & -1.127915 & -1.421791 & -1.612427 \\
\hline
\end{tabular}

Zero-point correction=

Thermal correction to Energy=
0.248107 (Hartree/Particle) 0.258426 
Thermal correction to Enthalpy=

Thermal correction to Gibbs Free Energy=

Sum of electronic and zero-point Energies=

Sum of electronic and thermal Energies $=$

Sum of electronic and thermal Enthalpies=

Sum of electronic and thermal Free Energies=

PBEQIDHD3BJ/ccpVTZ

Ethane

Standard orientation:
0.259370

0.214911

$-315.213519$

$-315.203200$

$-315.202256$

$-315.246715$

\begin{tabular}{|c|c|c|c|c|}
\hline \multirow{2}{*}{$\begin{array}{l}\text { Center } \\
\text { Number }\end{array}$} & Atomic & \multirow{2}{*}{$\begin{array}{l}\text { Atomic } \\
\text { Type }\end{array}$} & \multicolumn{2}{|c|}{ Coordinates (Angstron } \\
\hline & Number & & $X$ & Z \\
\hline 1 & 6 & -0.000000 & -0.000000 & 0.757771 \\
\hline 2 & 1 & -0.506315 & 0.876988 & 1.153149 \\
\hline 3 & 1 & 1.012651 & -0.000012 & 1.153149 \\
\hline 4 & 1 & -0.506336 & -0.876975 & 1.153149 \\
\hline 5 & 6 & 0.000000 & 0.000000 & -0.757771 \\
\hline 6 & 1 & 0.506315 & 0.876988 & -1.153149 \\
\hline 7 & 1 & 0.506336 & -0.876975 & -1.153149 \\
\hline 8 & 1 & -1.012651 & -0.000012 & -1.153149 \\
\hline
\end{tabular}

Zero-point correction=

Thermal correction to Energy=

0.076114 (Hartree/Particle)

Thermal correction to Enthalpy=

0.079573

0.080517

Thermal correction to Gibbs Free Energy $=0.054726$

Sum of electronic and zero-point Energies $=\quad-79.665431$

Sum of electronic and thermal Energies $=\quad-79.661971$

Sum of electronic and thermal Enthalpies $=\quad-79.661027$

Sum of electronic and thermal Free Energies $=\quad-79.686818$

Methane

Standard orientation:

\begin{tabular}{|c|c|c|c|c|}
\hline \multirow{2}{*}{$\begin{array}{l}\text { Center } \\
\text { Number }\end{array}$} & Atomic & \multirow{2}{*}{$\begin{array}{r}\text { Atomic } \\
\text { Type }\end{array}$} & \multicolumn{2}{|c|}{ Coordinates (Angstroms) } \\
\hline & Numbe & & $\mathrm{X}$ & Z \\
\hline 1 & 6 & 0.000000 & 0.000000 & 0.000000 \\
\hline 2 & 1 & 0.626051 & 0.626051 & 0.626051 \\
\hline 3 & 1 & -0.626051 & -0.626051 & 0.626051 \\
\hline 4 & 1 & -0.626051 & 0.626051 & -0.626051 \\
\hline 5 & 1 & 0.626051 & -0.626051 & -0.626051 \\
\hline \multicolumn{3}{|c|}{ Zero-point correction $=$} & \multicolumn{2}{|c|}{0.045574 (Hartree/Particle) } \\
\hline \multicolumn{3}{|c|}{ Thermal correction to Energy= } & \multicolumn{2}{|c|}{0.048440} \\
\hline \multicolumn{3}{|c|}{ Thermal correction to Enthalpy= } & \multicolumn{2}{|c|}{0.049384} \\
\hline \multicolumn{4}{|c|}{ Thermal correction to Gibbs Free Energy= } & 0.028274 \\
\hline \multicolumn{4}{|c|}{ Sum of electronic and zero-point Energies $=$} & -40.425148 \\
\hline \multicolumn{4}{|c|}{ Sum of electronic and thermal Energies $=$} & -40.422282 \\
\hline \multicolumn{4}{|c|}{ Sum of electronic and thermal Enthalpies $=$} & -40.421338 \\
\hline
\end{tabular}


Sum of electronic and thermal Free Energies=

$-40.442448$

Propane

Standard orientation:

\begin{tabular}{|c|c|c|c|c|c|}
\hline \multirow{2}{*}{$\begin{array}{l}\text { Center } \\
\text { Number }\end{array}$} & \multicolumn{2}{|c|}{ Atomic } & \multirow{2}{*}{$\begin{array}{l}\text { tomic } \\
\text { Type }\end{array}$} & \multicolumn{2}{|c|}{ Coordinates (Angstrom } \\
\hline & & & & $\mathrm{X}$ & Z \\
\hline 1 & 6 & 0 & -1.258590 & -0.258449 & 0.000000 \\
\hline 2 & 1 & 0 & -2.156311 & 0.354847 & -0.000033 \\
\hline 3 & 1 & 0 & -1.294676 & -0.901162 & 0.877721 \\
\hline 4 & 1 & 0 & -1.294647 & -0.901213 & -0.877684 \\
\hline 5 & 6 & 0 & 0.000000 & 0.585958 & 0.000000 \\
\hline 6 & 1 & 0 & 0.000001 & 1.240360 & 0.871105 \\
\hline 7 & 1 & 0 & 0.000002 & 1.240346 & -0.871115 \\
\hline 8 & 6 & 0 & 1.258590 & -0.258450 & 0.000000 \\
\hline 9 & 1 & 0 & 1.294668 & -0.901170 & 0.877716 \\
\hline 10 & 1 & 0 & 1.294648 & -0.901205 & -0.877690 \\
\hline 11 & 1 & 0 & 2.156312 & 0.354844 & -0.000023 \\
\hline
\end{tabular}

Zero-point correction=

Thermal correction to Energy=

Thermal correction to Enthalpy=

Thermal correction to Gibbs Free Energy=

Sum of electronic and zero-point Energies=

Sum of electronic and thermal Energies=

Sum of electronic and thermal Enthalpies=

Sum of electronic and thermal Free Energies=

Butane

Standard orientation:
0.105376 (Hartree/Particle)

0.109862

0.110806

0.079772

$-118.910034$

$-118.905548$

$-118.904604$

$-118.935638$

\begin{tabular}{|c|c|c|c|c|}
\hline \multirow{3}{*}{$\begin{array}{l}\text { Center } \\
\text { Number } \\
------- \\
1\end{array}$} & \multirow{2}{*}{$\begin{array}{l}\text { Atomic } \\
\text { Number }\end{array}$} & \multirow{3}{*}{$\begin{array}{l}\text { Atomic } \\
\text { Type }\end{array}$} & \multicolumn{2}{|c|}{ Coordinates (Angstrom } \\
\hline & & & $\mathrm{X}$ & $\mathrm{Z}$ \\
\hline & 6 & & 0.118654 & -0.000021 \\
\hline 2 & 1 & 2.076248 & 0.746685 & 0.877783 \\
\hline 3 & 1 & 2.076199 & 0.746705 & -0.877817 \\
\hline 4 & 1 & 2.722963 & -0.631534 & -0.000051 \\
\hline 5 & 6 & 0.558514 & -0.512472 & 0.000023 \\
\hline 6 & 1 & 0.449420 & -1.159231 & -0.871697 \\
\hline 7 & 1 & 0.449447 & -1.159209 & 0.871764 \\
\hline 8 & 6 & -0.558514 & 0.512472 & 0.000023 \\
\hline 9 & 1 & -0.449447 & 1.159206 & 0.871766 \\
\hline 10 & 1 & -0.449420 & 1.159234 & -0.871695 \\
\hline 11 & 6 & -1.936104 & -0.118654 & -0.000021 \\
\hline 12 & 1 & -2.076249 & -0.746683 & 0.877783 \\
\hline 13 & 1 & -2.722963 & 0.631534 & -0.000053 \\
\hline 14 & 1 & -2.076198 & -0.746706 & -0.877816 \\
\hline
\end{tabular}

Zero-point correction=

Thermal correction to Energy=
0.134430 (Hartree/Particle)

0.140160 
Thermal correction to Enthalpy=

Thermal correction to Gibbs Free Energy=

Sum of electronic and zero-point Energies=

Sum of electronic and thermal Energies $=$

Sum of electronic and thermal Enthalpies=

Sum of electronic and thermal Free Energies=

Isobutane

Standard orientation:
0.141104

0.106481

$-158.154949$

$-158.149219$

$-158.148275$

$-158.182899$

\begin{tabular}{|c|c|c|c|c|}
\hline \multirow{2}{*}{$\begin{array}{l}\text { Center } \\
\text { Number }\end{array}$} & Atomic & \multirow{2}{*}{$\begin{array}{r}\text { Atomic } \\
\text { Type }\end{array}$} & \multicolumn{2}{|c|}{ Coordinates (Angstrom } \\
\hline & Numbe & & $\mathrm{X}$ & Z \\
\hline 1 & 6 & 0.794818 & -1.202974 & 0.095466 \\
\hline 2 & 1 & 1.823364 & -1.162695 & -0.256976 \\
\hline 3 & 1 & 0.818152 & -1.238353 & 1.184449 \\
\hline 4 & 1 & 0.354423 & -2.133311 & -0.257051 \\
\hline 5 & 6 & -0.000005 & 0.000002 & -0.376842 \\
\hline 6 & 1 & -0.000005 & 0.000018 & -1.468634 \\
\hline 7 & 6 & 0.644401 & 1.289813 & 0.095469 \\
\hline 8 & 1 & 0.095308 & 2.160433 & -0.257037 \\
\hline 9 & 1 & 0.663327 & 1.327756 & 1.184453 \\
\hline 10 & 1 & 1.670317 & 1.373527 & -0.256998 \\
\hline 11 & 6 & -1.439219 & -0.086843 & 0.095468 \\
\hline 12 & 1 & -2.024658 & 0.759844 & -0.256851 \\
\hline 13 & 1 & -1.918682 & -0.997614 & -0.257169 \\
\hline 14 & 1 & -1.481516 & -0.089594 & 1.184451 \\
\hline
\end{tabular}

Zero-point correction=

Thermal correction to Energy=

Thermal correction to Enthalpy=

Thermal correction to Gibbs Free Energy=

Sum of electronic and zero-point Energies=

Sum of electronic and thermal Energies $=$

Sum of electronic and thermal Enthalpies=

Sum of electronic and thermal Free Energies=

Pentane

Standard orientation:

\begin{tabular}{|c|c|c|c|c|}
\hline \multirow{2}{*}{$\begin{array}{l}\text { Center } \\
\text { Number }\end{array}$} & Atomic & \multirow{2}{*}{$\begin{array}{l}\text { Atomic } \\
\text { Type }\end{array}$} & \multicolumn{2}{|c|}{ Coordinates (Angstroms) } \\
\hline & Number & & $\mathrm{X}$ & $\mathrm{Z}$ \\
\hline 1 & 6 & -2.524077 & 0.323871 & 0.000001 \\
\hline 2 & 1 & -2.560790 & 0.966211 & 0.877782 \\
\hline 3 & 1 & -2.560788 & 0.966219 & -0.877775 \\
\hline 4 & 1 & -3.421542 & -0.289653 & -0.000003 \\
\hline 5 & 6 & -1.266702 & -0.521713 & -0.000001 \\
\hline 6 & 1 & -1.264334 & -1.177098 & 0.871884 \\
\hline 7 & 1 & -1.264335 & -1.177095 & -0.871889 \\
\hline 8 & 6 & -0.000000 & 0.310338 & -0.000000 \\
\hline 9 & 1 & 0.000001 & 0.967454 & -0.872438 \\
\hline
\end{tabular}




$\begin{array}{rrrrrr}10 & 1 & 0 & -0.000001 & 0.967455 & 0.872437 \\ 11 & 6 & 0 & 1.266702 & -0.521713 & 0.000001 \\ 12 & 1 & 0 & 1.264335 & -1.177098 & -0.871884 \\ 13 & 1 & 0 & 1.264335 & -1.177095 & 0.871889 \\ 14 & 6 & 0 & 2.524077 & 0.323871 & -0.000001 \\ 15 & 1 & 0 & 3.421542 & -0.289653 & 0.000001 \\ 16 & 1 & 0 & 2.560789 & 0.966214 & -0.877780 \\ 17 & 1 & 0 & 2.560789 & 0.966216 & 0.877777\end{array}$

Zero-point correction $=$

Thermal correction to Energy=

Thermal correction to Enthalpy=

Thermal correction to Gibbs Free Energy=

Sum of electronic and zero-point Energies=

Sum of electronic and thermal Energies=

Sum of electronic and thermal Enthalpies=

Sum of electronic and thermal Free Energies=
0.163470 (Hartree/Particle)

0.170494

0.171439

0.133209

$-197.399833$

$-197.392809$

$-197.391864$

$-197.430094$

Neopentane

Standard orientation:

\begin{tabular}{cccccc} 
Center & Atomic & \multicolumn{2}{c}{ Atomic } & \multicolumn{3}{c}{ Coordinates } \\
Number & Number & Type & X & Y & Z \\
--------- & \\
\hline 1 & 6 & 0 & 0.581898 & -0.205871 & 1.389974 \\
2 & 1 & 0 & 0.893791 & 0.742312 & 1.825533 \\
3 & 1 & 0 & -0.152291 & -0.656437 & 2.056297 \\
4 & 1 & 0 & 1.450789 & -0.861482 & 1.354789 \\
5 & 6 & 0 & -0.000006 & 0.000002 & 0.000001 \\
6 & 6 & 0 & -1.209252 & 0.918197 & 0.087537 \\
7 & 1 & 0 & -1.645283 & 1.082205 & -0.896848 \\
8 & 1 & 0 & -1.978110 & 0.488957 & 0.728356 \\
9 & 1 & 0 & -0.932392 & 1.888078 & 0.498286 \\
10 & 6 & 0 & 1.050691 & 0.629953 & -0.901238 \\
11 & 1 & 0 & 1.928924 & -0.009138 & -0.980651 \\
12 & 1 & 0 & 0.658294 & 0.788059 & -1.904763 \\
13 & 1 & 0 & 1.371203 & 1.594419 & -0.509944 \\
14 & 6 & 0 & -0.423338 & -1.342285 & -0.576270 \\
15 & 1 & 0 & 0.426216 & -2.019781 & -0.649036 \\
16 & 1 & 0 & -1.177263 & -1.814911 & 0.051630 \\
17 & 1 & 0 & -0.843837 & -1.222256 & -1.573671
\end{tabular}

Zero-point correction=

0.162047 (Hartree/Particle)

Thermal correction to Energy=

0.168881

Thermal correction to Enthalpy=

0.169825

Thermal correction to Gibbs Free Energy=

Sum of electronic and zero-point Energies $=$

0.133035

Sum of electronic and thermal Energies=

$-197.406373$

$-197.399539$

Sum of electronic and thermal Enthalpies=

$-197.398595$

Sum of electronic and thermal Free Energies=

$-197.435385$ 
Hexane

Standard orientation:

\begin{tabular}{|c|c|c|c|c|}
\hline \multirow{2}{*}{$\begin{array}{l}\text { Center } \\
\text { Number }\end{array}$} & Atomic & \multirow{2}{*}{$\begin{array}{l}\text { Atomic } \\
\text { Type }\end{array}$} & \multicolumn{2}{|c|}{ Coordinates (Angstroms } \\
\hline & Numbe & & $\mathrm{X}$ & $\mathrm{Z}$ \\
\hline 1 & 6 & -3.179459 & 0.207256 & 0.000006 \\
\hline 2 & 1 & -3.261120 & 0.845440 & 0.877802 \\
\hline 3 & 1 & -3.261122 & 0.845464 & -0.877772 \\
\hline 4 & 1 & -4.031720 & -0.467681 & -0.000003 \\
\hline 5 & 6 & -1.865891 & -0.548078 & -0.000007 \\
\hline 6 & 1 & -1.817530 & -1.201696 & 0.871851 \\
\hline 7 & 1 & -1.817536 & -1.201676 & -0.871880 \\
\hline 8 & 6 & -0.660531 & 0.370916 & -0.000000 \\
\hline 9 & 1 & -0.707386 & 1.026083 & -0.872511 \\
\hline 10 & 1 & -0.707389 & 1.026076 & 0.872515 \\
\hline 11 & 6 & 0.660531 & -0.370916 & -0.000000 \\
\hline 12 & 1 & 0.707386 & -1.026083 & -0.872511 \\
\hline 13 & 1 & 0.707389 & -1.026076 & 0.872516 \\
\hline 14 & 6 & 1.865891 & 0.548078 & -0.000006 \\
\hline 15 & 1 & 1.817530 & 1.201696 & 0.871852 \\
\hline 16 & 1 & 1.817536 & 1.201676 & -0.871879 \\
\hline 17 & 6 & 3.179459 & -0.207256 & 0.000006 \\
\hline 18 & 1 & 3.261122 & -0.845466 & -0.877771 \\
\hline 19 & 1 & 4.031720 & 0.467680 & -0.000004 \\
\hline 20 & 1 & 3.261121 & -0.845438 & 0.877803 \\
\hline
\end{tabular}

Zero-point correction=

Thermal correction to Energy=

Thermal correction to Enthalpy=

Thermal correction to Gibbs Free Energy=

Sum of electronic and zero-point Energies=

Sum of electronic and thermal Energies $=$

Sum of electronic and thermal Enthalpies=

Sum of electronic and thermal Free Energies=

22dimethylbutane

Standard orientation:

\begin{tabular}{|c|c|c|c|c|}
\hline \multirow{2}{*}{$\begin{array}{l}\text { Center } \\
\text { Number }\end{array}$} & Atomic & \multirow{2}{*}{$\begin{array}{l}\text { Atomic } \\
\text { Type }\end{array}$} & \multicolumn{2}{|c|}{ Coordinates (Angstrom } \\
\hline & Numbe & & $\mathrm{X}$ & Z \\
\hline 1 & 6 & 1.604052 & 0.966354 & -0.001267 \\
\hline 2 & 1 & 1.581274 & 1.605583 & -0.882625 \\
\hline 3 & 1 & 1.581437 & 1.607659 & 0.878587 \\
\hline 4 & 1 & 2.550992 & 0.428976 & -0.000716 \\
\hline 5 & 6 & 0.429306 & -0.000246 & -0.000011 \\
\hline 6 & 6 & -0.859628 & 0.823878 & -0.000653 \\
\hline 7 & 1 & -0.840161 & 1.480538 & 0.871133 \\
\hline 8 & 1 & -0.840228 & 1.479109 & -0.873519 \\
\hline 9 & 6 & -2.156223 & 0.037405 & -0.000005 \\
\hline
\end{tabular}




$\begin{array}{rrrrrr}10 & 1 & 0 & -2.243972 & -0.595891 & 0.879196 \\ 11 & 1 & 0 & -3.009337 & 0.711452 & -0.000240 \\ 12 & 1 & 0 & -2.244217 & -0.596833 & -0.878499 \\ 13 & 6 & 0 & 0.510605 & -0.875648 & -1.241492 \\ 14 & 1 & 0 & -0.279000 & -1.623191 & -1.260055 \\ 15 & 1 & 0 & 0.429905 & -0.275911 & -2.147178 \\ 16 & 1 & 0 & 1.462703 & -1.402792 & -1.273917 \\ 17 & 6 & 0 & 0.511083 & -0.872922 & 1.243363 \\ 18 & 1 & 0 & 1.463039 & -1.400288 & 1.276382 \\ 19 & 1 & 0 & 0.431122 & -0.271138 & 2.147753 \\ 20 & 1 & 0 & -0.278726 & -1.620193 & 1.264087\end{array}$

Zero-point correction=

Thermal correction to Energy=

Thermal correction to Enthalpy=

Thermal correction to Gibbs Free Energy=

Sum of electronic and zero-point Energies=

Sum of electronic and thermal Energies $=$

Sum of electronic and thermal Enthalpies=

Sum of electronic and thermal Free Energies=

Heptane

Standard orientation:

\begin{tabular}{|c|c|c|c|c|c|}
\hline \multirow{2}{*}{$\begin{array}{l}\text { Center } \\
\text { Number }\end{array}$} & \multicolumn{2}{|c|}{ Atomic } & \multirow{2}{*}{$\begin{array}{l}\text { Atomic } \\
\text { Type }\end{array}$} & \multicolumn{2}{|c|}{ Coordinates (Angstroms } \\
\hline & & & & $\mathrm{X}$ & Z \\
\hline 1 & 6 & 0 & -3.790074 & -0.353103 & -0.000008 \\
\hline 2 & 1 & 0 & -3.826728 & -0.995450 & -0.877806 \\
\hline 3 & 1 & 0 & -3.826728 & -0.995486 & 0.877764 \\
\hline 4 & 1 & 0 & -4.687625 & 0.260332 & 0.000005 \\
\hline 5 & 6 & 0 & -2.532734 & 0.492533 & 0.000011 \\
\hline 6 & 1 & 0 & -2.530307 & 1.147934 & -0.871857 \\
\hline 7 & 1 & 0 & -2.530312 & 1.147904 & 0.871901 \\
\hline 8 & 6 & 0 & -1.265900 & -0.339669 & -0.000000 \\
\hline 9 & 1 & 0 & -1.266636 & -0.996512 & 0.872492 \\
\hline 10 & 1 & 0 & -1.266641 & -0.996499 & -0.872502 \\
\hline 11 & 6 & 0 & 0.000000 & 0.493167 & 0.000001 \\
\hline 12 & 1 & 0 & -0.000002 & 1.149715 & 0.872595 \\
\hline 13 & 1 & 0 & 0.000002 & 1.149711 & -0.872596 \\
\hline 14 & 6 & 0 & 1.265900 & -0.339669 & 0.000006 \\
\hline 15 & 1 & 0 & 1.266634 & -0.996517 & -0.872483 \\
\hline 16 & 1 & 0 & 1.266643 & -0.996493 & 0.872511 \\
\hline 17 & 6 & 0 & 2.532734 & 0.492533 & -0.000011 \\
\hline 18 & 1 & 0 & 2.530310 & 1.147935 & 0.871855 \\
\hline 19 & 1 & 0 & 2.530308 & 1.147902 & -0.871903 \\
\hline 20 & 6 & 0 & 3.790074 & -0.353103 & 0.000003 \\
\hline 21 & 1 & 0 & 3.826726 & -0.995486 & -0.877769 \\
\hline 22 & 1 & 0 & 4.687625 & 0.260332 & -0.000012 \\
\hline 23 & 1 & 0 & 3.826730 & -0.995450 & 0.877801 \\
\hline
\end{tabular}


Zero-point correction=

Thermal correction to Energy=

Thermal correction to Enthalpy=

Thermal correction to Gibbs Free Energy=

Sum of electronic and zero-point Energies=

Sum of electronic and thermal Energies=

Sum of electronic and thermal Enthalpies=

Sum of electronic and thermal Free Energies=
0.221439 (Hartree/Particle)

0.231123

0.232067

0.186645

$-275.889765$

$-275.880081$

$-275.879137$

$-275.924559$

223trimethylbutane

Standard orientation:

\begin{tabular}{|c|c|c|c|c|c|}
\hline \multirow{2}{*}{$\begin{array}{l}\text { Center } \\
\text { Number }\end{array}$} & \multicolumn{2}{|l|}{ Atomic } & \multirow{2}{*}{$\begin{array}{l}\text { Atomic } \\
\text { Type }\end{array}$} & \multicolumn{2}{|c|}{ Coordinates (Angstroms } \\
\hline & Numbe & & & $\mathrm{X}$ & $\mathrm{Z}$ \\
\hline 1 & 6 & 0 & 1.314821 & 1.283523 & -0.459425 \\
\hline 2 & 1 & 0 & 1.170487 & 1.442616 & -1.527689 \\
\hline 3 & 1 & 0 & 0.935604 & 2.155621 & 0.066690 \\
\hline 4 & 1 & 0 & 2.385830 & 1.228323 & -0.272518 \\
\hline 5 & 6 & 0 & 0.634398 & -0.000189 & -0.000766 \\
\hline 6 & 6 & 0 & -0.841416 & 0.000823 & -0.453514 \\
\hline 7 & 1 & 0 & -0.812182 & 0.004871 & -1.547008 \\
\hline 8 & 6 & 0 & -1.610637 & -1.237493 & -0.020790 \\
\hline 9 & 1 & 0 & -1.733508 & -1.265312 & 1.060303 \\
\hline 10 & 1 & 0 & -2.606936 & -1.227883 & -0.457568 \\
\hline 11 & 1 & 0 & -1.128335 & -2.160268 & -0.329331 \\
\hline 12 & 6 & 0 & -1.613674 & 1.235930 & -0.016770 \\
\hline 13 & 1 & 0 & -1.653402 & 1.315186 & 1.068271 \\
\hline 14 & 1 & 0 & -1.185880 & 2.154321 & -0.407018 \\
\hline 15 & 1 & 0 & -2.640234 & 1.176667 & -0.372256 \\
\hline 16 & 6 & 0 & 0.770835 & -0.114364 & 1.510999 \\
\hline 17 & 1 & 0 & 0.370188 & -1.054858 & 1.881927 \\
\hline 18 & 1 & 0 & 1.821817 & -0.072542 & 1.792548 \\
\hline 19 & 1 & 0 & 0.262704 & 0.698492 & 2.025243 \\
\hline 20 & 6 & 0 & 1.366605 & -1.168665 & -0.648915 \\
\hline 21 & 1 & 0 & 1.273623 & -1.132772 & -1.733736 \\
\hline 22 & 1 & 0 & 2.426709 & -1.131059 & -0.404048 \\
\hline 23 & 1 & 0 & 0.987929 & -2.128796 & -0.308734 \\
\hline
\end{tabular}

Zero-point correction=

Thermal correction to Energy=

Thermal correction to Enthalpy=

Thermal correction to Gibbs Free Energy=

Sum of electronic and zero-point Energies=

Sum of electronic and thermal Energies $=$

Sum of electronic and thermal Enthalpies=

Sum of electronic and thermal Free Energies=

Octane
0.219958 (Hartree/Particle)

0.229416

0.230360

\subsection{1}

$-275.892927$

$-275.883470$

$-275.882526$

$-275.926325$

Standard orientation: 


\begin{tabular}{|c|c|c|c|c|}
\hline \multirow{2}{*}{$\begin{array}{l}\text { Center } \\
\text { Number }\end{array}$} & Atomic & \multirow{2}{*}{$\begin{array}{l}\text { Atomic } \\
\text { Type }\end{array}$} & \multicolumn{2}{|c|}{ Coordinates (Angstroms } \\
\hline & Numbe & & $\mathrm{X}$ & $\mathrm{Z}$ \\
\hline 1 & 6 & 4.436578 & -0.255595 & 0.000015 \\
\hline 2 & 1 & 4.498478 & -0.896002 & 0.877817 \\
\hline 3 & 1 & 4.498481 & -0.896053 & -0.877751 \\
\hline 4 & 1 & 5.309316 & 0.392663 & -0.000003 \\
\hline 5 & 6 & 3.146949 & 0.539929 & -0.000013 \\
\hline 6 & 1 & 3.118752 & 1.194740 & 0.871847 \\
\hline 7 & 1 & 3.118762 & 1.194698 & -0.871906 \\
\hline 8 & 6 & 1.913793 & -0.341409 & 0.000001 \\
\hline 9 & 1 & 1.940313 & -0.997722 & -0.872498 \\
\hline 10 & 1 & 1.940317 & -0.997700 & 0.872516 \\
\hline 11 & 6 & 0.616161 & 0.441048 & -0.000004 \\
\hline 12 & 1 & 0.590314 & 1.097091 & -0.872579 \\
\hline 13 & 1 & 0.590307 & 1.097093 & 0.872568 \\
\hline 14 & 6 & -0.616161 & -0.441048 & -0.000008 \\
\hline 15 & 1 & -0.590306 & -1.097102 & 0.872558 \\
\hline 16 & 1 & -0.590314 & -1.097081 & -0.872589 \\
\hline 17 & 6 & -1.913793 & 0.341409 & 0.000007 \\
\hline 18 & 1 & -1.940315 & 0.997728 & -0.872488 \\
\hline 19 & 1 & -1.940314 & 0.997695 & 0.872526 \\
\hline 20 & 6 & -3.146949 & -0.539929 & -0.000009 \\
\hline 21 & 1 & -3.118755 & -1.194736 & 0.871855 \\
\hline 22 & 1 & -3.118758 & -1.194701 & -0.871899 \\
\hline 23 & 6 & -4.436578 & 0.255595 & 0.000010 \\
\hline 24 & 1 & -4.498481 & 0.896045 & -0.877762 \\
\hline 25 & 1 & -5.309316 & -0.392663 & -0.000002 \\
\hline 26 & 1 & -4.498478 & 0.896010 & 0.877807 \\
\hline
\end{tabular}

Zero-point correction=

Thermal correction to Energy=

Thermal correction to Enthalpy=

Thermal correction to Gibbs Free Energy=

Sum of electronic and zero-point Energies=

Sum of electronic and thermal Energies $=$

Sum of electronic and thermal Enthalpies=

Sum of electronic and thermal Free Energies=

2233tertamethylbutane

Standard orientation:
0.250408 (Hartree/Particle)

$$
0.261438
$$$$
0.262382
$$

$$
\begin{gathered}
0.213363 \\
-315.134750 \\
-315.123720 \\
-315.122776 \\
-315.171795
\end{gathered}
$$

\begin{tabular}{|c|c|c|c|c|}
\hline \multirow{2}{*}{$\begin{array}{l}\text { Center } \\
\text { Number }\end{array}$} & \multirow{2}{*}{$\begin{array}{l}\text { Atomic } \\
\text { Number }\end{array}$} & \multirow{2}{*}{$\begin{array}{l}\text { Atomic } \\
\text { Type }\end{array}$} & \multicolumn{2}{|c|}{ Coordinates (Angstroms) } \\
\hline & & & $\mathrm{X}$ & $\mathrm{Z}$ \\
\hline 1 & 6 & -1.332405 & -1.218389 & -0.733068 \\
\hline 2 & 1 & -1.130622 & -1.173577 & -1.800224 \\
\hline 3 & 1 & -0.921328 & -2.149323 & -0.348788 \\
\hline 4 & 1 & -2.412792 & -1.261546 & -0.605798 \\
\hline 5 & 6 & -0.780405 & -0.000045 & -0.000007 \\
\hline
\end{tabular}




$\begin{array}{cccccc}6 & 6 & 0 & 0.780390 & 0.000040 & -0.000015 \\ 7 & 6 & 0 & 1.332317 & 1.352096 & 0.440126 \\ 8 & 1 & 0 & 1.130671 & 2.128860 & -0.293027 \\ 9 & 1 & 0 & 0.921015 & 1.671805 & 1.395070 \\ 10 & 1 & 0 & 2.412678 & 1.284232 & 0.556354 \\ 11 & 6 & 0 & -1.332508 & 1.243986 & -0.688564 \\ 12 & 1 & 0 & -1.130378 & 2.145817 & -0.116379 \\ 13 & 1 & 0 & -0.921781 & 1.376493 & -1.687073 \\ 14 & 1 & 0 & -2.412942 & 1.155503 & -0.789173 \\ 15 & 6 & 0 & -1.332387 & -0.025746 & 1.421683 \\ 16 & 1 & 0 & -2.412784 & 0.105952 & 1.395490 \\ 17 & 1 & 0 & -1.130502 & -0.972319 & 1.916410 \\ 18 & 1 & 0 & -0.921346 & 0.772521 & 2.035785 \\ 19 & 6 & 0 & 1.332444 & -0.294792 & -1.390961 \\ 20 & 1 & 0 & 2.412852 & -0.160590 & -1.390093 \\ 21 & 1 & 0 & 1.130517 & -1.317991 & -1.697307 \\ 22 & 1 & 0 & 0.921536 & 0.372620 & -2.145288 \\ 23 & 6 & 0 & 1.332541 & -1.057149 & 0.950788 \\ 24 & 1 & 0 & 2.412961 & -1.123352 & 0.834192 \\ 25 & 1 & 0 & 1.130533 & -0.810939 & 1.990068 \\ 26 & 1 & 0 & 0.921795 & -2.044174 & 0.749887\end{array}$

Zero-point correction=

Thermal correction to Energy=

0.248826 (Hartree/Particle)

Thermal correction to Enthalpy=

0.259117

0.260061

Thermal correction to Gibbs Free Energy=

Sum of electronic and zero-point Energies=

0.215667

Sum of electronic and thermal Energies $=$

$-315.137010$

$-315.126719$

$-315.125775$

Sum of electronic and thermal Enthalpies=

$-315.170169$

Sum of electronic and thermal Free Energies=

cc-pVQZ basis set:

M06L/ccpVQZ

Ethane

Standard orientation:

\begin{tabular}{|c|c|c|c|c|}
\hline \multirow{2}{*}{$\begin{array}{l}\text { Center } \\
\text { Number }\end{array}$} & Atomic & \multirow{2}{*}{$\begin{array}{l}\text { Atomic } \\
\text { Type }\end{array}$} & \multicolumn{2}{|c|}{ Coordinates (Angstroms) } \\
\hline & Numbe & & $\mathrm{X}$ & Z \\
\hline 1 & 6 & 0.000000 & -0.000000 & 0.757655 \\
\hline 2 & 1 & -0.505807 & 0.876126 & 1.158100 \\
\hline 3 & 1 & 1.011650 & -0.000021 & 1.158100 \\
\hline 4 & 1 & -0.505844 & -0.876104 & 1.158100 \\
\hline 5 & 6 & -0.000000 & -0.000000 & -0.757655 \\
\hline 6 & 1 & 0.505807 & 0.876126 & -1.158100 \\
\hline 7 & 1 & 0.505844 & -0.876104 & -1.158100 \\
\hline 8 & 1 & -1.011650 & -0.000021 & -1.158100 \\
\hline \multicolumn{3}{|c|}{ Zero-point correction $=$} & \multicolumn{2}{|c|}{0.075043 (Hartree/Particle) } \\
\hline \multicolumn{3}{|c|}{ Thermal correction to Energy= } & \multicolumn{2}{|c|}{0.078520} \\
\hline \multicolumn{3}{|c|}{ Thermal correction to Enthalpy= } & \multicolumn{2}{|c|}{0.079464} \\
\hline
\end{tabular}


Thermal correction to Gibbs Free Energy=

Sum of electronic and zero-point Energies=

Sum of electronic and thermal Energies $=$

Sum of electronic and thermal Enthalpies=

$-79.773218$

Sum of electronic and thermal Free Energies=

$-79.799035$

Methane

Standard orientation:

\begin{tabular}{cccccc} 
Center & Atomic & \multicolumn{2}{c}{ Atomic } & \multicolumn{2}{c}{ Coordinates (Angstrom } \\
Number & Number & Type & X & Y & Z \\
\hline 1 & 6 & 0 & 0.000000 & 0.000000 & 0.000000 \\
2 & 1 & 0 & 0.625937 & 0.625937 & 0.625937 \\
3 & 1 & 0 & -0.625937 & -0.625937 & 0.625937 \\
4 & 1 & 0 & -0.625937 & 0.625937 & -0.625937 \\
5 & 1 & 0 & 0.625937 & -0.625937 & -0.625937
\end{tabular}

Zero-point correction=

Thermal correction to Energy=

Thermal correction to Enthalpy=

Thermal correction to Gibbs Free Energy=

Sum of electronic and zero-point Energies=

Sum of electronic and thermal Energies $=$

Sum of electronic and thermal Enthalpies=

Sum of electronic and thermal Free Energies=

Propane
0.045019 (Hartree/Particle)

0.047888

0.048832

0.027718

$-40.485686$

$-40.482817$

$-40.481872$

$-40.502986$

Standard orientation:

\begin{tabular}{|c|c|c|c|c|}
\hline \multirow{2}{*}{$\begin{array}{l}\text { Center } \\
\text { Number }\end{array}$} & Atomic & \multirow{2}{*}{$\begin{array}{r}\text { Atomic } \\
\text { Type }\end{array}$} & \multicolumn{2}{|c|}{ Coordinates (Angstrom } \\
\hline & Number & & $\mathrm{X}$ & $\mathrm{Z}$ \\
\hline 1 & 6 & -1.263053 & -0.257468 & 0.000000 \\
\hline 2 & 1 & -2.161861 & 0.355262 & -0.000029 \\
\hline 3 & 1 & -1.305586 & -0.902834 & 0.876573 \\
\hline 4 & 1 & -1.305562 & -0.902879 & -0.876541 \\
\hline 5 & 6 & 0.000000 & 0.584042 & 0.000000 \\
\hline 6 & 1 & -0.000000 & 1.243143 & 0.869420 \\
\hline 7 & 1 & 0.000001 & 1.243127 & -0.869430 \\
\hline 8 & 6 & 1.263054 & -0.257468 & 0.000000 \\
\hline 9 & 1 & 1.305584 & -0.902836 & 0.876572 \\
\hline 10 & 1 & 1.305560 & -0.902876 & -0.876543 \\
\hline 11 & 1 & 2.161862 & 0.355259 & -0.000026 \\
\hline
\end{tabular}

Zero-point correction=

Thermal correction to Energy=

0.103961 (Hartree/Particle)

Thermal correction to Enthalpy=

0.108461

Thermal correction to Gibbs Free Energy=

0.109405

Sum of electronic and zero-point Energies=

0.078357

Sum of electronic and thermal Energies=

$-119.073009$

$-119.068509$

Sum of electronic and thermal Enthalpies=

$-119.067565$ 
Sum of electronic and thermal Free Energies $=\quad-119.098613$

Butane

Standard orientation:

\begin{tabular}{cccccc} 
Center & \multicolumn{2}{c}{ Atomic } & \multicolumn{2}{c}{ Atomic } & \multicolumn{3}{c}{ Coordinates } & Angstroms \\
Number & Number & Type & X & $Y$ & $Z$ \\
- \hdashline---------- \\
1 & 6 & 0 & 1.942165 & -0.118179 & 0.000020 \\
2 & 1 & 0 & 2.087533 & -0.748245 & -0.876574 \\
3 & 1 & 0 & 2.087494 & -0.748246 & 0.876620 \\
4 & 1 & 0 & 2.731838 & 0.629803 & 0.000038 \\
5 & 6 & 0 & 0.562279 & 0.511578 & -0.000023 \\
6 & 1 & 0 & 0.453228 & 1.163488 & 0.869771 \\
7 & 1 & 0 & 0.453255 & 1.163462 & -0.869840 \\
8 & 6 & 0 & -0.562279 & -0.511578 & -0.000023 \\
9 & 1 & 0 & -0.453255 & -1.163460 & -0.869841 \\
10 & 1 & 0 & -0.453227 & -1.163490 & 0.869770 \\
11 & 6 & 0 & -1.942165 & 0.118179 & 0.000020 \\
12 & 1 & 0 & -2.087534 & 0.748244 & -0.876575 \\
13 & 1 & 0 & -2.731838 & -0.629803 & 0.000040 \\
14 & 1 & 0 & -2.087493 & 0.748247 & 0.876620
\end{tabular}

Zero-point correction=

Thermal correction to Energy=

Thermal correction to Enthalpy=

Thermal correction to Gibbs Free Energy=

Sum of electronic and zero-point Energies=

Sum of electronic and thermal Energies=

Sum of electronic and thermal Enthalpies=

Sum of electronic and thermal Free Energies=

Isobutane
0.132741 (Hartree/Particle)

$$
\begin{aligned}
& 0.138491 \\
& 0.139435
\end{aligned}
$$

\begin{tabular}{|c|c|c|c|c|}
\hline \multirow{2}{*}{$\begin{array}{l}\text { Center } \\
\text { Number }\end{array}$} & Atomic & \multirow{2}{*}{$\begin{array}{l}\text { Atomic } \\
\text { Type }\end{array}$} & \multicolumn{2}{|c|}{ Coordinates (Angstroms } \\
\hline & Numbe & & $\mathrm{X}$ & Z \\
\hline 1 & 6 & -0.891227 & 1.140277 & -0.094742 \\
\hline 2 & 1 & -0.528719 & 2.104506 & 0.257214 \\
\hline 3 & 1 & -0.922212 & 1.180355 & -1.184208 \\
\hline 4 & 1 & -1.914595 & 1.021094 & 0.256756 \\
\hline 5 & 6 & 0.000019 & 0.000008 & 0.374624 \\
\hline 6 & 1 & -0.000022 & 0.000032 & 1.468258 \\
\hline 7 & 6 & 1.433140 & 0.201669 & -0.094738 \\
\hline 8 & 1 & 2.086851 & -0.594629 & 0.256843 \\
\hline 9 & 1 & 1.483244 & 0.208890 & -1.184205 \\
\hline 10 & 1 & 1.841742 & 1.147333 & 0.257112 \\
\hline 11 & 6 & -0.541918 & -1.341953 & -0.094728 \\
\hline 12 & 1 & 0.072574 & -2.168701 & 0.257301 \\
\hline 13 & 1 & -1.558485 & -1.509824 & 0.256631 \\
\hline 14 & 1 & -0.560464 & -1.389056 & -1.184199 \\
\hline
\end{tabular}

$$
\begin{gathered}
0.104797 \\
-158.368606 \\
-158.362856 \\
-158.361912 \\
-158.396550
\end{gathered}
$$


Zero-point correction=

Thermal correction to Energy=

Thermal correction to Enthalpy=

Thermal correction to Gibbs Free Energy=

Sum of electronic and zero-point Energies=

Sum of electronic and thermal Energies=

Sum of electronic and thermal Enthalpies=

Sum of electronic and thermal Free Energies=

Pentane
0.132041 (Hartree/Particle)

0.137761

0.138706

0.104413

$-158.370702$

$-158.364982$

$-158.364037$

$-158.398330$

Standard orientation:

\begin{tabular}{|c|c|c|c|c|}
\hline \multirow{2}{*}{$\begin{array}{l}\text { Center } \\
\text { Number }\end{array}$} & Atomic & \multirow{2}{*}{$\begin{array}{l}\text { Atomic } \\
\text { Type }\end{array}$} & \multicolumn{2}{|c|}{ Coordinates (Angstroms } \\
\hline & Numbe & & $X$ & Z \\
\hline 1 & 6 & 2.534106 & 0.321491 & -0.000000 \\
\hline 2 & 1 & 2.577670 & 0.966596 & -0.876477 \\
\hline 3 & 1 & 2.577668 & 0.966601 & 0.876474 \\
\hline 4 & 1 & 3.432405 & -0.291656 & 0.000002 \\
\hline 5 & 6 & 1.271927 & -0.519725 & 0.000001 \\
\hline 6 & 1 & 1.268570 & -1.180292 & -0.869847 \\
\hline 7 & 1 & 1.268570 & -1.180289 & 0.869851 \\
\hline 8 & 6 & 0.000000 & 0.311481 & -0.000000 \\
\hline 9 & 1 & -0.000000 & 0.973999 & 0.870264 \\
\hline 10 & 1 & 0.000000 & 0.973998 & -0.870265 \\
\hline 11 & 6 & -1.271927 & -0.519725 & -0.000001 \\
\hline 12 & 1 & -1.268569 & -1.180291 & 0.869848 \\
\hline 13 & 1 & -1.268570 & -1.180290 & -0.869850 \\
\hline 14 & 6 & -2.534106 & 0.321491 & 0.000001 \\
\hline 15 & 1 & -3.432405 & -0.291656 & 0.000004 \\
\hline 16 & 1 & -2.577667 & 0.966600 & 0.876475 \\
\hline 17 & 1 & -2.577671 & 0.966596 & -0.876476 \\
\hline
\end{tabular}

Zero-point correction=

Thermal correction to Energy=

0.161261 (Hartree/Particle)

Thermal correction to Enthalpy=

0.168322

0.169266

Thermal correction to Gibbs Free Energy=

Sum of electronic and zero-point Energies=

0.130994

Sum of electronic and thermal Energies=

$-197.664352$

$-197.657292$

Sum of electronic and thermal Enthalpies=

$-197.656347$

Sum of electronic and thermal Free Energies $=\quad-197.694620$

Neopentane

Standard orientation:

\begin{tabular}{|c|c|c|c|c|}
\hline Center & Atomic & Atomic & Coordinate & s (Angstrom \\
\hline Number & Numbe & Type & $X$ & Z \\
\hline 1 & 6 & -1.407077 & 0.591116 & 0.041757 \\
\hline 2 & 1 & -1.425681 & 1.594319 & -0.382725 \\
\hline 3 & 1 & -2.108445 & -0.020950 & -0.524292 \\
\hline 4 & 1 & -1.776338 & 0.657552 & 1.064597 \\
\hline
\end{tabular}




$\begin{array}{cccccc}5 & 6 & 0 & -0.000028 & -0.000053 & 0.000064 \\ 6 & 6 & 0 & 0.477665 & -0.075136 & -1.448109 \\ 7 & 1 & 0 & 1.481716 & -0.493394 & -1.510573 \\ 8 & 1 & 0 & -0.180387 & -0.702907 & -2.047966 \\ 9 & 1 & 0 & 0.501403 & 0.912801 & -1.906770 \\ 10 & 6 & 0 & 0.948505 & 0.886527 & 0.803340 \\ 11 & 1 & 0 & 0.632987 & 0.960149 & 1.843524 \\ 12 & 1 & 0 & 1.962954 & 0.489388 & 0.792424 \\ 13 & 1 & 0 & 0.983891 & 1.896307 & 0.395845 \\ 14 & 6 & 0 & -0.019082 & -1.402497 & 0.602998 \\ 15 & 1 & 0 & -0.356337 & -1.381413 & 1.638738 \\ 16 & 1 & 0 & -0.689033 & -2.059928 & 0.049926 \\ 17 & 1 & 0 & 0.973363 & -1.851664 & 0.586963\end{array}$

Zero-point correction $=$

Thermal correction to Energy=

0.160076 (Hartree/Particle)

Thermal correction to Enthalpy=

0.166959

Thermal correction to Gibbs Free Energy=

Sum of electronic and zero-point Energies $=$

0.167903

Sum of electronic and thermal Energies $=$

0.131033

$-197.669080$

$-197.662198$

Sum of electronic and thermal Enthalpies=

$-197.661254$

Sum of electronic and thermal Free Energies $=\quad-197.698124$

Hexane

Standard orientation:

\begin{tabular}{|c|c|c|c|c|}
\hline Center & Atomic & Atomic & Coordinate & str \\
\hline Number & Numbe & Type & $\mathrm{X}$ & Z \\
\hline 1 & 6 & -3.192194 & 0.206140 & 0.000003 \\
\hline 2 & 1 & -3.279822 & 0.846835 & 0.876552 \\
\hline 3 & 1 & -3.279777 & 0.846945 & -0.876472 \\
\hline 4 & 1 & -4.046686 & -0.466944 & -0.000066 \\
\hline 5 & 6 & -1.875119 & -0.546396 & -0.000016 \\
\hline 6 & 1 & -1.826538 & -1.205353 & 0.869774 \\
\hline 7 & 1 & -1.826531 & -1.205297 & -0.869848 \\
\hline 8 & 6 & -0.662993 & 0.370290 & 0.000018 \\
\hline 9 & 1 & -0.709641 & 1.031215 & -0.870219 \\
\hline 10 & 1 & -0.709656 & 1.031171 & 0.870287 \\
\hline 11 & 6 & 0.662993 & -0.370290 & 0.000011 \\
\hline 12 & 1 & 0.709636 & -1.031209 & -0.870231 \\
\hline 13 & 1 & 0.709661 & -1.031177 & 0.870276 \\
\hline 14 & 6 & 1.875119 & 0.546396 & -0.000020 \\
\hline 15 & 1 & 1.826535 & 1.205357 & 0.869767 \\
\hline 16 & 1 & 1.826535 & 1.205293 & -0.869855 \\
\hline 17 & 6 & 3.192194 & -0.206140 & 0.000005 \\
\hline 18 & 1 & 3.279795 & -0.846926 & -0.876481 \\
\hline 19 & 1 & 4.046686 & 0.466944 & -0.000028 \\
\hline 20 & 1 & 3.279804 & -0.846853 & 0.876543 \\
\hline
\end{tabular}


Thermal correction to Energy=

Thermal correction to Enthalpy=

Thermal correction to Gibbs Free Energy=

Sum of electronic and zero-point Energies=

Sum of electronic and thermal Energies=

Sum of electronic and thermal Enthalpies=

Sum of electronic and thermal Free Energies=

22dimethylbutane
0.198308

0.199252

0.157449

$-236.959962$

$-236.951588$

$-236.950644$

$-236.992447$

Standard orientation:

\begin{tabular}{|c|c|c|c|c|}
\hline \multirow{2}{*}{$\begin{array}{l}\text { Center } \\
\text { Number }\end{array}$} & Atomic & \multirow{2}{*}{$\begin{array}{l}\text { Atomic } \\
\text { Type }\end{array}$} & \multicolumn{2}{|c|}{ Coordinates (Angstrom } \\
\hline & Numbe & & $\mathrm{X}$ & $\mathrm{Z}$ \\
\hline 1 & 6 & -1.614023 & 0.966970 & 0.000673 \\
\hline 2 & 1 & -1.596486 & 1.609192 & 0.880704 \\
\hline 3 & 1 & -1.596517 & 1.610338 & -0.878520 \\
\hline 4 & 1 & -2.562361 & 0.430965 & 0.000341 \\
\hline 5 & 6 & -0.432163 & -0.000180 & 0.000012 \\
\hline 6 & 6 & 0.862607 & 0.829451 & 0.000466 \\
\hline 7 & 1 & 0.841994 & 1.489647 & -0.870115 \\
\hline 8 & 1 & 0.842033 & 1.488654 & 0.871803 \\
\hline 9 & 6 & 2.160120 & 0.042240 & 0.000006 \\
\hline 10 & 1 & 2.248544 & -0.594816 & -0.877938 \\
\hline 11 & 1 & 3.019380 & 0.708934 & 0.000258 \\
\hline 12 & 1 & 2.248653 & -0.595616 & 0.877357 \\
\hline 13 & 6 & -0.508561 & -0.879314 & 1.246513 \\
\hline 14 & 1 & 0.284874 & -1.624203 & 1.266202 \\
\hline 15 & 1 & -0.428306 & -0.282776 & 2.155016 \\
\hline 16 & 1 & -1.456732 & -1.414074 & 1.285000 \\
\hline 17 & 6 & -0.508704 & -0.877711 & -1.247621 \\
\hline 18 & 1 & -1.456860 & -1.412461 & -1.286643 \\
\hline 19 & 1 & -0.428625 & -0.279977 & -2.155351 \\
\hline 20 & 1 & 0.284752 & -1.622546 & -1.268413 \\
\hline
\end{tabular}

Zero-point correction=

Thermal correction to Energy=

0.188592 (Hartree/Particle)

Thermal correction to Enthalpy=

0.196876

Thermal correction to Gibbs Free Energy=

0.197820

Sum of electronic and zero-point Energies=

0.157225

Sum of electronic and thermal Energies=

$-236.963704$

$-236.955420$

Sum of electronic and thermal Enthalpies=

$-236.954476$

Sum of electronic and thermal Free Energies=

$-236.995071$

Heptane

Standard orientation:

\begin{tabular}{cccccc}
$\begin{array}{c}\text { Center } \\
\text { Number }\end{array}$ & \multicolumn{2}{c}{ Atomic } & Atomic & \multicolumn{2}{c}{ Coordinates (Angstroms) } \\
-1 & 6 & 0 & -3.805578 & -0.349984 & -0.000007 \\
2 & 1 & 0 & -3.849151 & -0.995105 & -0.876487
\end{tabular}




$\begin{array}{cccccc}3 & 1 & 0 & -3.849156 & -0.995121 & 0.876462 \\ 4 & 1 & 0 & -4.703905 & 0.263141 & -0.000003 \\ 5 & 6 & 0 & -2.543380 & 0.491198 & 0.000006 \\ 6 & 1 & 0 & -2.539993 & 1.151776 & -0.869809 \\ 7 & 1 & 0 & -2.539998 & 1.151760 & 0.869834 \\ 8 & 6 & 0 & -1.271416 & -0.340317 & 0.000002 \\ 9 & 1 & 0 & -1.272397 & -1.002666 & 0.870246 \\ 10 & 1 & 0 & -1.272399 & -1.002660 & -0.870247 \\ 11 & 6 & 0 & 0.000000 & 0.490327 & 0.000002 \\ 12 & 1 & 0 & -0.000001 & 1.152512 & 0.870289 \\ 13 & 1 & 0 & 0.000001 & 1.152510 & -0.870288 \\ 14 & 6 & 0 & 1.271416 & -0.340317 & 0.000004 \\ 15 & 1 & 0 & 1.272394 & -1.002671 & -0.870236 \\ 16 & 1 & 0 & 1.272401 & -1.002655 & 0.870257 \\ 17 & 6 & 0 & 2.543380 & 0.491198 & -0.000008 \\ 18 & 1 & 0 & 2.539997 & 1.151779 & 0.869805 \\ 19 & 1 & 0 & 2.539994 & 1.151757 & -0.869837 \\ 20 & 6 & 0 & 3.805578 & -0.349984 & 0.000001 \\ 21 & 1 & 0 & 3.849152 & -0.995124 & -0.876466 \\ 22 & 1 & 0 & 4.703905 & 0.263141 & -0.000008 \\ 23 & 1 & 0 & 3.849154 & -0.995103 & 0.876483\end{array}$

Zero-point correction=

Thermal correction to Energy=

Thermal correction to Enthalpy=

Thermal correction to Gibbs Free Energy=

Sum of electronic and zero-point Energies=

Sum of electronic and thermal Energies=

Sum of electronic and thermal Enthalpies=

Sum of electronic and thermal Free Energies=

223trimethylbutane

Standard orientation: 


$\begin{array}{rrrrrr}15 & 1 & 0 & 2.654990 & -1.176123 & -0.340169 \\ 16 & 6 & 0 & -0.765412 & 0.179473 & 1.511334 \\ 17 & 1 & 0 & -0.395401 & 1.150081 & 1.836355 \\ 18 & 1 & 0 & -1.809325 & 0.111945 & 1.814635 \\ 19 & 1 & 0 & -0.218995 & -0.587330 & 2.058495 \\ 20 & 6 & 0 & -1.386032 & 1.137242 & -0.698851 \\ 21 & 1 & 0 & -1.316323 & 1.046072 & -1.782627 \\ 22 & 1 & 0 & -2.442373 & 1.120631 & -0.433981 \\ 23 & 1 & 0 & -0.999799 & 2.115532 & -0.421399\end{array}$

Zero-point correction=

Thermal correction to Energy=

Thermal correction to Enthalpy=

Thermal correction to Gibbs Free Energy=

Sum of electronic and zero-point Energies=

Sum of electronic and thermal Energies=

Sum of electronic and thermal Enthalpies=

Sum of electronic and thermal Free Energies=

Octane

Standard orientation:

\begin{tabular}{cccccc} 
Center & \multicolumn{2}{c}{ Atomic } & \multicolumn{2}{l}{ Atomic } & \multicolumn{3}{c}{ Coordinates (Angstroms) } \\
Number & Number & Type & X & Y & Z \\
------------- \\
1 & 6 & 0 & -4.454361 & 0.253899 & 0.000012 \\
2 & 1 & 0 & -4.522697 & 0.896900 & 0.876473 \\
3 & 1 & 0 & -4.522707 & 0.896919 & -0.876435 \\
4 & 1 & 0 & -5.328460 & -0.393305 & 0.000009 \\
5 & 6 & 0 & -3.160762 & -0.538137 & -0.000007 \\
6 & 1 & 0 & -3.131937 & -1.198106 & 0.869799 \\
7 & 1 & 0 & -3.131948 & -1.198087 & -0.869827 \\
8 & 6 & 0 & -1.921682 & 0.341616 & -0.000004 \\
9 & 1 & 0 & -1.948161 & 1.003453 & -0.870243 \\
10 & 1 & 0 & -1.948160 & 1.003446 & 0.870241 \\
11 & 6 & 0 & -0.619308 & -0.439572 & -0.000005 \\
12 & 1 & 0 & -0.593821 & -1.101273 & -0.870255 \\
13 & 1 & 0 & -0.593817 & -1.101271 & 0.870246 \\
14 & 6 & 0 & 0.619308 & 0.439572 & -0.000007 \\
15 & 1 & 0 & 0.593816 & 1.101279 & 0.870239 \\
16 & 1 & 0 & 0.593822 & 1.101265 & -0.870263 \\
17 & 6 & 0 & 1.921682 & -0.341616 & 0.000003 \\
18 & 1 & 0 & 1.948162 & -1.003459 & -0.870232 \\
19 & 1 & 0 & 1.948158 & -1.003441 & 0.870252 \\
20 & 6 & 0 & 3.160762 & 0.538137 & -0.000002 \\
21 & 1 & 0 & 3.131941 & 1.198104 & 0.869805 \\
22 & 1 & 0 & 3.131945 & 1.198089 & -0.869821 \\
23 & 6 & 0 & 4.454361 & -0.253899 & 0.000007 \\
24 & 1 & 0 & 4.522705 & -0.896916 & -0.876442 \\
25 & 1 & 0 & 5.328460 & 0.393305 & 0.000004
\end{tabular}


Zero-point correction $=$

Thermal correction to Energy=

Thermal correction to Enthalpy=

Thermal correction to Gibbs Free Energy=

Sum of electronic and zero-point Energies=

Sum of electronic and thermal Energies=

Sum of electronic and thermal Enthalpies=

Sum of electronic and thermal Free Energies=

2233tetramethylbutane

Standard orientation:
0.247059 (Hartree/Particle)

0.258143

0.259087
0.210043

$-315.551387$

$-315.540303$

$-315.539359$

$-315.588403$

\begin{tabular}{|c|c|c|c|c|}
\hline \multirow{2}{*}{$\begin{array}{l}\text { Center } \\
\text { Number }\end{array}$} & Atomic & \multirow{2}{*}{$\begin{array}{l}\text { Atomic } \\
\text { Type }\end{array}$} & \multicolumn{2}{|c|}{ Coordinates (Angstroms } \\
\hline & & & $X$ & $\mathrm{Z}$ \\
\hline 1 & 6 & -1.336104 & -1.428881 & 0.012511 \\
\hline 2 & 1 & -1.141053 & -1.948257 & -0.923272 \\
\hline 3 & 1 & -0.914172 & -2.025181 & 0.820057 \\
\hline 4 & 1 & -2.415862 & -1.410877 & 0.153635 \\
\hline 5 & 6 & -0.786478 & -0.000003 & -0.000073 \\
\hline 6 & 6 & 0.786456 & -0.000100 & 0.000182 \\
\hline 7 & 6 & 1.336266 & 1.388438 & -0.336576 \\
\hline 8 & 1 & 1.140406 & 1.664195 & -1.370556 \\
\hline 9 & 1 & 0.915208 & 2.163586 & 0.301598 \\
\hline 10 & 1 & 2.416135 & 1.405026 & -0.196072 \\
\hline 11 & 6 & -1.335204 & 0.703621 & -1.244062 \\
\hline 12 & 1 & -1.139460 & 1.773602 & -1.225578 \\
\hline 13 & 1 & -0.912795 & 0.302370 & -2.164065 \\
\hline 14 & 1 & -2.415012 & 0.572906 & -1.299700 \\
\hline 15 & 6 & -1.336529 & 0.725571 & 1.230748 \\
\hline 16 & 1 & -2.416459 & 0.837300 & 1.144673 \\
\hline 17 & 1 & -1.140576 & 0.176206 & 2.149145 \\
\hline 18 & 1 & -0.916021 & 1.723825 & 1.342153 \\
\hline 19 & 6 & 1.335936 & -0.986173 & -1.033933 \\
\hline 20 & 1 & 2.415530 & -0.871539 & -1.120358 \\
\hline 21 & 1 & 1.141672 & -2.019346 & -0.754223 \\
\hline 22 & 1 & 0.912821 & -0.822593 & -2.023780 \\
\hline 23 & 6 & 1.335710 & -0.402612 & 1.371251 \\
\hline 24 & 1 & 2.415443 & -0.533967 & 1.315547 \\
\hline 25 & 1 & 1.140629 & 0.355671 & 2.126547 \\
\hline 26 & 1 & 0.913249 & -1.342094 & 1.723967 \\
\hline
\end{tabular}

Zero-point correction=

Thermal correction to Energy=

0.245457 (Hartree/Particle)

Thermal correction to Enthalpy=

0.255841

0.256785

Thermal correction to Gibbs Free Energy=

0.212274

Sum of electronic and zero-point Energies=

$-315.553223$

Sum of electronic and thermal Energies $=$

$-315.542839$

Sum of electronic and thermal Enthalpies=

$-315.541895$ 
Sum of electronic and thermal Free Energies=

TPSSh/ccpVQZ

Ethane

Standard orientation:

\begin{tabular}{cccccc} 
Center & \multicolumn{2}{c}{ Atomic } & \multicolumn{2}{c}{ Atomic } & \multicolumn{3}{c}{ Coordinates (Angstroms) } \\
Number & Number & \multicolumn{1}{c}{ Type } & X & Y & Z \\
\hline 1 & 6 & 0 & -0.000000 & 0.000000 & 0.763946 \\
2 & 1 & 0 & -0.508795 & 0.881392 & 1.159812 \\
3 & 1 & 0 & 1.017706 & -0.000067 & 1.159812 \\
4 & 1 & 0 & -0.508911 & -0.881326 & 1.159812 \\
5 & 6 & 0 & 0.000000 & 0.000000 & -0.763946 \\
6 & 1 & 0 & 0.508795 & 0.881392 & -1.159812 \\
7 & 1 & 0 & 0.508911 & -0.881326 & -1.159812 \\
8 & 1 & 0 & -1.017706 & -0.000067 & -1.159812
\end{tabular}

Zero-point correction $=$

Thermal correction to Energy=

0.074385 (Hartree/Particle)

Thermal correction to Enthalpy=

0.077876

Thermal correction to Gibbs Free Energy $=\quad 0.052961$

Sum of electronic and zero-point Energies $=$

$-79.797241$

Sum of electronic and thermal Energies $=$

$-79.793750$

Sum of electronic and thermal Enthalpies=

$-79.792806$

Sum of electronic and thermal Free Energies $=\quad-79.818665$

Methane

Standard orientation:

\begin{tabular}{cccccc} 
Center & \multicolumn{2}{c}{ Atomic } & \multicolumn{2}{c}{ Atomic } & \multicolumn{2}{c}{ Coordinates } \\
Number & Number & Type & X & Y & Z \\
- & Numstroms) \\
\hline 1 & 6 & 0 & 0.000000 & 0.000000 & 0.000000 \\
2 & 1 & 0 & 0.628602 & 0.628602 & 0.628602 \\
3 & 1 & 0 & -0.628602 & -0.628602 & 0.628602 \\
4 & 1 & 0 & -0.628602 & 0.628602 & -0.628602 \\
5 & 1 & 0 & 0.628602 & -0.628602 & -0.628602
\end{tabular}

Zero-point correction=

0.044700 (Hartree/Particle)

Thermal correction to Energy=

0.047567

Thermal correction to Enthalpy=

0.048511

Thermal correction to Gibbs Free Energy $=0.027388$

Sum of electronic and zero-point Energies $=$

$-40.495884$

Sum of electronic and thermal Energies=

$-40.493017$

Sum of electronic and thermal Enthalpies=

$-40.492072$

Sum of electronic and thermal Free Energies=

$-40.513196$

Propane

Standard orientation:

\begin{tabular}{|c|c|c|c|}
\hline Center & Atomic & Atomic & Coordinates (Angstroms) \\
\hline Number & Number & Type & $\mathrm{X}$ \\
\hline
\end{tabular}




$\begin{array}{cccccc}1 & 6 & 0 & -1.272342 & -0.259973 & 0.000000 \\ 2 & 1 & 0 & -2.169210 & 0.363129 & -0.000012 \\ 3 & 1 & 0 & -1.313005 & -0.904461 & 0.881972 \\ 4 & 1 & 0 & -1.312996 & -0.904483 & -0.881957 \\ 5 & 6 & 0 & 0.000000 & 0.587250 & 0.000000 \\ 6 & 1 & 0 & -0.000000 & 1.243911 & 0.875223 \\ 7 & 1 & 0 & 0.000001 & 1.243899 & -0.875231 \\ 8 & 6 & 0 & 1.272342 & -0.259973 & 0.000000 \\ 9 & 1 & 0 & 1.313020 & -0.904438 & 0.881989 \\ 10 & 1 & 0 & 1.312977 & -0.904506 & -0.881941 \\ 11 & 1 & 0 & 2.169212 & 0.363126 & -0.000046 \\ ------------------------------------------------------\end{array}$

Zero-point correction=

Thermal correction to Energy=

Thermal correction to Enthalpy=

Thermal correction to Gibbs Free Energy=

Sum of electronic and zero-point Energies=

Sum of electronic and thermal Energies=

Sum of electronic and thermal Enthalpies=

Sum of electronic and thermal Free Energies=

Butane
0.102916 (Hartree/Particle)

$$
\begin{array}{r}
0.107476 \\
0.108421
\end{array}
$$

$$
\begin{gathered}
0.077234 \\
-119.101511 \\
-119.096951 \\
-119.096006 \\
-119.127193
\end{gathered}
$$

\begin{tabular}{|c|c|c|c|c|c|}
\hline \multirow{2}{*}{$\begin{array}{l}\text { Center } \\
\text { Number }\end{array}$} & \multicolumn{2}{|c|}{ Atomic } & \multirow{2}{*}{$\begin{array}{l}\text { Atomic } \\
\text { Type }\end{array}$} & \multicolumn{2}{|c|}{ Coordinates (Angstroms) } \\
\hline & & & & $\mathrm{X}$ & $\mathrm{Z}$ \\
\hline 1 & 6 & 0 & 1.955477 & -0.120638 & 0.000029 \\
\hline 2 & 1 & 0 & 2.098637 & -0.750127 & -0.881987 \\
\hline 3 & 1 & 0 & 2.098588 & -0.750089 & 0.882080 \\
\hline 4 & 1 & 0 & 2.741863 & 0.637071 & 0.000033 \\
\hline 5 & 6 & 0 & 0.565444 & 0.515007 & -0.000032 \\
\hline 6 & 1 & 0 & 0.459429 & 1.164229 & 0.875719 \\
\hline 7 & 1 & 0 & 0.459472 & 1.164183 & -0.875823 \\
\hline 8 & 6 & 0 & -0.565444 & -0.515007 & -0.000032 \\
\hline 9 & 1 & 0 & -0.459472 & -1.164181 & -0.875824 \\
\hline 10 & 1 & 0 & -0.459429 & -1.164231 & 0.875718 \\
\hline 11 & 6 & 0 & -1.955476 & 0.120638 & 0.000029 \\
\hline 12 & 1 & 0 & -2.098633 & 0.750137 & -0.881981 \\
\hline 13 & 1 & 0 & -2.741863 & -0.637071 & 0.000022 \\
\hline 14 & 1 & 0 & -2.098592 & 0.750079 & 0.882086 \\
\hline \multicolumn{4}{|c|}{ Zero-point correction $=$} & \multicolumn{2}{|c|}{0.131235 (Hartree/Particle) } \\
\hline \multicolumn{4}{|c|}{ Thermal correction to Energy= } & \multicolumn{2}{|c|}{0.137077} \\
\hline \multicolumn{4}{|c|}{ Thermal correction to Enthalpy= } & \multicolumn{2}{|c|}{0.138021} \\
\hline \multicolumn{5}{|c|}{ Thermal correction to Gibbs Free Energy= } & 0103156 \\
\hline \multirow{2}{*}{\multicolumn{5}{|c|}{$\begin{array}{l}\text { Sum of electronic and zero-point Energies }= \\
\text { Sum of electronic and thermal Energies }=\end{array}$}} & -158.405911 \\
\hline & & & & & -158.400069 \\
\hline \multicolumn{5}{|c|}{ Sum of electronic and thermal Enthalpies $=$} & -158.399125 \\
\hline
\end{tabular}

Standard orientation: 
Sum of electronic and thermal Free Energies=

Isobutane

Standard orientation:

\begin{tabular}{cccccc} 
Center & \multicolumn{2}{c}{ Atomic } & \multicolumn{2}{c}{ Atomic } & \multicolumn{3}{c}{ Coordinates (Angstroms) } \\
Number & Number & Type & X & Y & Z \\
\hline 1 & 6 & 0 & -1.318470 & -0.619603 & -0.096108 \\
2 & 1 & 0 & -2.176701 & -0.046435 & 0.262500 \\
3 & 1 & 0 & -1.364596 & -0.641274 & -1.188968 \\
4 & 1 & 0 & -1.424939 & -1.646127 & 0.262504 \\
5 & 6 & 0 & -0.000023 & -0.000038 & 0.375073 \\
6 & 1 & 0 & -0.000005 & 0.000002 & 1.471142 \\
7 & 6 & 0 & 0.122632 & 1.451609 & -0.096085 \\
8 & 1 & 0 & 1.048027 & 1.908378 & 0.262658 \\
9 & 1 & 0 & 0.127127 & 1.502435 & -1.188957 \\
10 & 1 & 0 & -0.713238 & 2.057027 & 0.262358 \\
11 & 6 & 0 & 1.195845 & -0.831998 & -0.096095 \\
12 & 1 & 0 & 2.138106 & -0.410422 & 0.261826 \\
13 & 1 & 0 & 1.129067 & -1.861627 & 0.263191 \\
14 & 1 & 0 & 1.237249 & -0.861785 & -1.188963
\end{tabular}

Zero-point correction=

0.130761 (Hartree/Particle)

Thermal correction to Energy=

0.136548

Thermal correction to Enthalpy=

0.137492

Thermal correction to Gibbs Free Energy=

0.103065

Sum of electronic and zero-point Energies=

$-158.407214$

Sum of electronic and thermal Energies $=$

$-158.401426$

Sum of electronic and thermal Enthalpies=

$-158.400482$

Sum of electronic and thermal Free Energies=

$-158.434909$

Pentane

Standard orientation:

\begin{tabular}{|c|c|c|c|c|}
\hline \multirow{2}{*}{$\begin{array}{l}\text { Center } \\
\text { Number }\end{array}$} & Atomic & \multirow{2}{*}{$\begin{array}{l}\text { Atomic } \\
\text { Type }\end{array}$} & \multicolumn{2}{|c|}{ Coordinates (Angstroms } \\
\hline & Numbe & & $X$ & Z \\
\hline 1 & 6 & 2.551110 & 0.324688 & -0.000004 \\
\hline 2 & 1 & 2.592486 & 0.968897 & -0.882010 \\
\hline 3 & 1 & 2.592483 & 0.968926 & 0.881980 \\
\hline 4 & 1 & 3.447775 & -0.298697 & 0.000008 \\
\hline 5 & 6 & 1.279516 & -0.523655 & 0.000008 \\
\hline 6 & 1 & 1.278801 & -1.181223 & -0.875770 \\
\hline 7 & 1 & 1.278802 & -1.181199 & 0.875804 \\
\hline 8 & 6 & 0.000000 & 0.314534 & 0.000001 \\
\hline 9 & 1 & -0.000006 & 0.973494 & 0.876266 \\
\hline 10 & 1 & 0.000006 & 0.973495 & -0.876263 \\
\hline 11 & 6 & -1.279516 & -0.523655 & -0.000009 \\
\hline 12 & 1 & -1.278801 & -1.181226 & 0.875768 \\
\hline 13 & 1 & -1.278802 & -1.181196 & -0.875806 \\
\hline 14 & 6 & -2.551110 & 0.324688 & 0.000004 \\
\hline
\end{tabular}




$\begin{array}{rrrrrr}15 & 1 & 0 & -3.447775 & -0.298697 & -0.000063 \\ 16 & 1 & 0 & -2.592510 & 0.968857 & 0.882036 \\ 17 & 1 & 0 & -2.592458 & 0.968966 & -0.881954\end{array}$

Zero-point correction=

Thermal correction to Energy=

Thermal correction to Enthalpy=

Thermal correction to Gibbs Free Energy=

Sum of electronic and zero-point Energies=

Sum of electronic and thermal Energies $=$

Sum of electronic and thermal Enthalpies=

Sum of electronic and thermal Free Energies=

Neopentane

Standard orientation:
0.159563 (Hartree/Particle)

$$
\begin{gathered}
0.166726 \\
0.167670
\end{gathered}
$$

\begin{tabular}{|c|c|c|c|c|}
\hline \multirow{2}{*}{$\begin{array}{l}\text { Center } \\
\text { Number }\end{array}$} & Atomic & \multirow{2}{*}{$\begin{array}{l}\text { Atomic } \\
\text { Type }\end{array}$} & \multicolumn{2}{|c|}{ Coordinates (Angstron } \\
\hline & Numbe & & $\mathrm{X}$ & Z \\
\hline 1 & 6 & -0.974003 & 0.024782 & -1.186528 \\
\hline 2 & 1 & -1.470576 & -0.941721 & -1.305410 \\
\hline 3 & 1 & -1.746629 & 0.784518 & -1.042644 \\
\hline 4 & 1 & -0.449667 & 0.250566 & -2.118699 \\
\hline 5 & 6 & 0.000021 & -0.000016 & 0.000010 \\
\hline 6 & 6 & -0.771289 & -0.319278 & 1.288526 \\
\hline 7 & 1 & -0.099883 & -0.342983 & 2.150777 \\
\hline 8 & 1 & -1.539941 & 0.433639 & 1.481247 \\
\hline 9 & 1 & -1.263777 & -1.292626 & 1.218673 \\
\hline 10 & 6 & 1.068340 & -1.076859 & -0.237183 \\
\hline 11 & 1 & 1.633132 & -0.873122 & -1.150592 \\
\hline 12 & 1 & 1.776443 & -1.115482 & 0.594649 \\
\hline 13 & 1 & 0.612335 & -2.065268 & -0.337022 \\
\hline 14 & 6 & 0.676953 & 1.371355 & 0.135189 \\
\hline 15 & 1 & 1.234762 & 1.623402 & -0.770450 \\
\hline 16 & 1 & -0.062948 & 2.157955 & 0.304425 \\
\hline 17 & 1 & 1.376616 & 1.381220 & 0.974961 \\
\hline
\end{tabular}

$$
\begin{gathered}
0.129141 \\
-197.710221 \\
-197.703058 \\
-197.702113 \\
-197.740643
\end{gathered}
$$

Zero-point correction=

Thermal correction to Energy=

Thermal correction to Enthalpy=

Thermal correction to Gibbs Free Energy=

Sum of electronic and zero-point Energies=

Sum of electronic and thermal Energies=

Sum of electronic and thermal Enthalpies=

Sum of electronic and thermal Free Energies=

Hexane
0.158221 (Hartree/Particle)

$$
\begin{gathered}
0.165295 \\
0.166239
\end{gathered}
$$

\begin{tabular}{|c|c|c|c|}
\hline Cent & Atomic & Atomic & Coordinates (Angstrom \\
\hline Number & Number & Type & $\mathrm{X}$ \\
\hline
\end{tabular}

\subsection{6}

$-197.713021$

$-197.705948$

$-197.705003$

$-197.742267$

Standard orientation: 


$\begin{array}{cccccc}1 & 6 & 0 & -3.212614 & 0.208768 & 0.000035 \\ 2 & 1 & 0 & -3.298249 & 0.848549 & 0.882093 \\ 3 & 1 & 0 & -3.298256 & 0.848716 & -0.881901 \\ 4 & 1 & 0 & -4.064303 & -0.474822 & -0.000028 \\ 5 & 6 & 0 & -1.885683 & -0.550086 & -0.000043 \\ 6 & 1 & 0 & -1.839768 & -1.206101 & 0.875665 \\ 7 & 1 & 0 & -1.839799 & -1.205982 & -0.875842 \\ 8 & 6 & 0 & -0.666869 & 0.374384 & -0.000006 \\ 9 & 1 & 0 & -0.713076 & 1.031517 & -0.876275 \\ 10 & 1 & 0 & -0.713107 & 1.031496 & 0.876277 \\ 11 & 6 & 0 & 0.666869 & -0.374383 & 0.000014 \\ 12 & 1 & 0 & 0.713086 & -1.031544 & -0.876235 \\ 13 & 1 & 0 & 0.713097 & -1.031470 & 0.876317 \\ 14 & 6 & 0 & 1.885683 & 0.550086 & -0.000033 \\ 15 & 1 & 0 & 1.839778 & 1.206099 & 0.875678 \\ 16 & 1 & 0 & 1.839790 & 1.205985 & -0.875829 \\ 17 & 6 & 0 & 3.212614 & -0.208768 & 0.000026 \\ 18 & 1 & 0 & 3.298256 & -0.848696 & -0.881925 \\ 19 & 1 & 0 & 4.064303 & 0.474822 & -0.000020 \\ 20 & 1 & 0 & 3.298250 & -0.848569 & 0.882069\end{array}$

Zero-point correction=

Thermal correction to Energy=

Thermal correction to Enthalpy=

Thermal correction to Gibbs Free Energy=

Sum of electronic and zero-point Energies=

Sum of electronic and thermal Energies $=$

Sum of electronic and thermal Enthalpies=

Sum of electronic and thermal Free Energies=

22dimethylbutane

Standard orientation:

\begin{tabular}{|c|c|c|c|c|}
\hline \multirow{2}{*}{$\begin{array}{l}\text { Center } \\
\text { Number }\end{array}$} & Atomic & \multirow{2}{*}{$\begin{array}{l}\text { Atomic } \\
\text { Type }\end{array}$} & \multicolumn{2}{|c|}{ Coordinates (Angstroms } \\
\hline & Numbe & & $\mathrm{X}$ & Z \\
\hline 1 & 6 & -1.614557 & 0.982091 & 0.002575 \\
\hline 2 & 1 & -1.588401 & 1.622659 & 0.888031 \\
\hline 3 & 1 & -1.588612 & 1.627013 & -0.879724 \\
\hline 4 & 1 & -2.568218 & 0.448038 & 0.001366 \\
\hline 5 & 6 & -0.434070 & -0.000829 & 0.000015 \\
\hline 6 & 6 & 0.872121 & 0.826000 & 0.001558 \\
\hline 7 & 1 & 0.855109 & 1.485747 & -0.872882 \\
\hline 8 & 1 & 0.855193 & 1.482395 & 0.878522 \\
\hline 9 & 6 & 2.181276 & 0.034836 & 0.000048 \\
\hline 10 & 1 & 2.271493 & -0.599830 & -0.883436 \\
\hline 11 & 1 & 3.034462 & 0.716247 & 0.000809 \\
\hline 12 & 1 & 2.271915 & -0.602375 & 0.881647 \\
\hline 13 & 6 & -0.521923 & -0.885208 & 1.252438 \\
\hline 14 & 1 & 0.272390 & -1.633887 & 1.275104 \\
\hline 15 & 1 & -0.446542 & -0.283690 & 2.162296 \\
\hline
\end{tabular}




$\begin{array}{rrrrrr}16 & 1 & 0 & -1.476892 & -1.416032 & 1.279967 \\ 17 & 6 & 0 & -0.522577 & -0.879347 & -1.256475 \\ 18 & 1 & 0 & -1.477414 & -1.410313 & -1.285840 \\ 19 & 1 & 0 & -0.448022 & -0.273522 & -2.163540 \\ 20 & 1 & 0 & 0.271912 & -1.627707 & -1.283276\end{array}$

Zero-point correction=

Thermal correction to Energy=

Thermal correction to Enthalpy=

Thermal correction to Gibbs Free Energy=

Sum of electronic and zero-point Energies=

Sum of electronic and thermal Energies=

Sum of electronic and thermal Enthalpies=

Sum of electronic and thermal Free Energies=

Heptane

Standard orientation:

\begin{tabular}{|c|c|c|c|c|}
\hline \multirow{2}{*}{$\begin{array}{l}\text { Center } \\
\text { Number }\end{array}$} & Atomic & \multirow{2}{*}{$\begin{array}{l}\text { Atomic } \\
\text { Type }\end{array}$} & \multicolumn{2}{|c|}{ Coordinates (Angstrom } \\
\hline & Number & & $\mathrm{X}$ & Z \\
\hline 1 & 6 & -3.829771 & -0.353193 & -0.000003 \\
\hline 2 & 1 & -3.871548 & -0.997330 & -0.881878 \\
\hline 3 & 1 & -3.871540 & -0.997369 & 0.881844 \\
\hline 4 & 1 & -4.725974 & 0.270534 & 0.000015 \\
\hline 5 & 6 & -2.558064 & 0.494845 & 0.000009 \\
\hline 6 & 1 & -2.557049 & 1.152352 & -0.875656 \\
\hline 7 & 1 & -2.557047 & 1.152324 & 0.875694 \\
\hline 8 & 6 & -1.278823 & -0.344012 & -0.000004 \\
\hline 9 & 1 & -1.279753 & -1.002663 & 0.876161 \\
\hline 10 & 1 & -1.279758 & -1.002647 & -0.876182 \\
\hline 11 & 6 & 0.000000 & 0.495131 & -0.000001 \\
\hline 12 & 1 & -0.000002 & 1.153567 & 0.876209 \\
\hline 13 & 1 & 0.000002 & 1.153564 & -0.876215 \\
\hline 14 & 6 & 1.278823 & -0.344011 & 0.000004 \\
\hline 15 & 1 & 1.279754 & -1.002664 & -0.876160 \\
\hline 16 & 1 & 1.279757 & -1.002646 & 0.876182 \\
\hline 17 & 6 & 2.558064 & 0.494845 & -0.000007 \\
\hline 18 & 1 & 2.557049 & 1.152350 & 0.875659 \\
\hline 19 & 1 & 2.557048 & 1.152326 & -0.875691 \\
\hline 20 & 6 & 3.829771 & -0.353193 & 0.000004 \\
\hline 21 & 1 & 3.871543 & -0.997362 & -0.881849 \\
\hline 22 & 1 & 4.725974 & 0.270534 & -0.000006 \\
\hline 23 & 1 & 3.871545 & -0.997337 & 0.881874 \\
\hline
\end{tabular}

Zero-point correction $=$

Thermal correction to Energy=

Thermal correction to Enthalpy=

Thermal correction to Gibbs Free Energy=

Sum of electronic and zero-point Energies=

Sum of electronic and thermal Energies=
0.216100 (Hartree/Particle)

0.226005

0.226949

0.181006

$-276.318973$

$-276.309068$ 
Sum of electronic and thermal Enthalpies=

Sum of electronic and thermal Free Energies=

223trimethylbutane

Standard orientation:

\begin{tabular}{|c|c|c|c|c|}
\hline \multirow{2}{*}{$\begin{array}{l}\text { Center } \\
\text { Number }\end{array}$} & Atomic & \multirow{2}{*}{$\begin{array}{l}\text { Atomic } \\
\text { Type }\end{array}$} & \multicolumn{2}{|c|}{ Coordinates (Angstrom } \\
\hline & Number & & $X$ & Z \\
\hline 1 & 6 & -1.353460 & -1.240762 & -0.565806 \\
\hline 2 & 1 & -1.230872 & -1.302350 & -1.650733 \\
\hline 3 & 1 & -0.974311 & -2.165681 & -0.128708 \\
\hline 4 & 1 & -2.424146 & -1.191472 & -0.352627 \\
\hline 5 & 6 & -0.641814 & -0.000001 & 0.000203 \\
\hline 6 & 6 & 0.852389 & 0.000003 & -0.451784 \\
\hline 7 & 1 & 0.824987 & 0.000025 & -1.548953 \\
\hline 8 & 6 & 1.630974 & 1.248832 & -0.016898 \\
\hline 9 & 1 & 1.711817 & 1.309734 & 1.071083 \\
\hline 10 & 1 & 2.647159 & 1.210382 & -0.415765 \\
\hline 11 & 1 & 1.173435 & 2.171490 & -0.374617 \\
\hline 12 & 6 & 1.630974 & -1.248828 & -0.016911 \\
\hline 13 & 1 & 1.712421 & -1.309348 & 1.071042 \\
\hline 14 & 1 & 1.173068 & -2.171538 & -0.374035 \\
\hline 15 & 1 & 2.646946 & -1.210732 & -0.416359 \\
\hline 16 & 6 & -0.786922 & -0.000749 & 1.529762 \\
\hline 17 & 1 & -0.330388 & 0.883090 & 1.979715 \\
\hline 18 & 1 & -1.844778 & -0.000333 & 1.804511 \\
\hline 19 & 1 & -0.331333 & -0.885562 & 1.978748 \\
\hline 20 & 6 & -1.353127 & 1.241499 & -0.564555 \\
\hline 21 & 1 & -1.230181 & 1.304399 & -1.649368 \\
\hline 22 & 1 & -2.423890 & 1.192077 & -0.351788 \\
\hline 23 & 1 & -0.974023 & 2.165853 & -0.126214 \\
\hline
\end{tabular}

Zero-point correction=

0.214567 (Hartree/Particle)

Thermal correction to Energy=

0.224380

Thermal correction to Enthalpy=

0.225324

Thermal correction to Gibbs Free Energy=

0.180778

Sum of electronic and zero-point Energies=

$-276.315451$

Sum of electronic and thermal Energies=

$-276.305638$

Sum of electronic and thermal Enthalpies=

$-276.304693$

Sum of electronic and thermal Free Energies=

$-276.349240 \mathrm{PBE} 0$

Octane

Standard orientation:

\begin{tabular}{cccccc} 
Center & \multicolumn{2}{c}{ Atomic } & \multicolumn{2}{c}{ Atomic } & \multicolumn{2}{c}{ Coordinates (Angstroms) } \\
Number & Number & Type & X & Y & Z \\
\hline 1 & 6 & 0 & -4.482949 & 0.256780 & 0.000036 \\
2 & 1 & 0 & -4.549140 & 0.898854 & 0.882117 \\
3 & 1 & 0 & -4.549115 & 0.899106 & -0.881863 \\
4 & 1 & 0 & -5.355053 & -0.400594 & -0.000074
\end{tabular}




$\begin{array}{cccccc}5 & 6 & 0 & -3.179634 & -0.541934 & -0.000058 \\ 6 & 1 & 0 & -3.153646 & -1.199068 & 0.875631 \\ 7 & 1 & 0 & -3.153662 & -1.198888 & -0.875881 \\ 8 & 6 & 0 & -1.933327 & 0.345187 & 0.000016 \\ 9 & 1 & 0 & -1.959578 & 1.003448 & -0.876202 \\ 10 & 1 & 0 & -1.959619 & 1.003370 & 0.876291 \\ 11 & 6 & 0 & -0.622895 & -0.443968 & 0.000013 \\ 12 & 1 & 0 & -0.597542 & -1.102025 & -0.876228 \\ 13 & 1 & 0 & -0.597520 & -1.101980 & 0.876286 \\ 14 & 6 & 0 & 0.622895 & 0.443968 & -0.000027 \\ 15 & 1 & 0 & 0.597527 & 1.102050 & 0.876195 \\ 16 & 1 & 0 & 0.597534 & 1.101955 & -0.876319 \\ 17 & 6 & 0 & 1.933327 & -0.345187 & 0.000022 \\ 18 & 1 & 0 & 1.959600 & -1.003461 & -0.876186 \\ 19 & 1 & 0 & 1.959597 & -1.003357 & 0.876308 \\ 20 & 6 & 0 & 3.179634 & 0.541934 & -0.000029 \\ 21 & 1 & 0 & 3.153653 & 1.199029 & 0.875689 \\ 22 & 1 & 0 & 3.153655 & 1.198926 & -0.875824 \\ 23 & 6 & 0 & 4.482949 & -0.256781 & 0.000020 \\ 24 & 1 & 0 & 4.549129 & -0.899031 & -0.881932 \\ 25 & 1 & 0 & 5.355053 & 0.400594 & -0.000017 \\ 26 & 1 & 0 & 4.549126 & -0.898928 & 0.882048\end{array}$

Zero-point correction $=$

Thermal correction to Energy=

Thermal correction to Enthalpy=

0.244345 (Hartree/Particle)

0.255606

0.256550

Thermal correction to Gibbs Free Energy=

Sum of electronic and zero-point Energies=

0.207027

Sum of electronic and thermal Energies $=$

$-315.623371$

$-315.612110$

Sum of electronic and thermal Enthalpies=

$-315.611166$

Sum of electronic and thermal Free Energies $=\quad-315.660689$

2233tetramethylbutane

Standard orientation:

\begin{tabular}{|c|c|c|c|c|}
\hline \multirow{2}{*}{$\begin{array}{l}\text { Center } \\
\text { Number }\end{array}$} & Atomic & \multirow{2}{*}{$\begin{array}{l}\text { Atomic } \\
\text { Type }\end{array}$} & \multicolumn{2}{|c|}{ Coordinates (Angstroms } \\
\hline & Numbe & & $\mathrm{X}$ & Z \\
\hline 1 & 6 & -1.347532 & -0.641879 & -1.283556 \\
\hline 2 & 1 & -1.134718 & -0.036143 & -2.165736 \\
\hline 3 & 1 & -0.942900 & -1.642035 & -1.451624 \\
\hline 4 & 1 & -2.433367 & -0.736247 & -1.203735 \\
\hline 5 & 6 & -0.789951 & 0.000034 & -0.000003 \\
\hline 6 & 6 & 0.789979 & -0.000026 & 0.000088 \\
\hline 7 & 6 & 1.347780 & 0.891795 & 1.124317 \\
\hline 8 & 1 & 1.135160 & 1.947396 & 0.948298 \\
\hline 9 & 1 & 0.943387 & 0.621396 & 2.101874 \\
\hline 10 & 1 & 2.433616 & 0.779442 & 1.175860 \\
\hline 11 & 6 & -1.347694 & 1.432520 & 0.085707 \\
\hline 12 & 1 & -1.134863 & 1.893841 & 1.051298 \\
\hline 13 & 1 & -0.943273 & 2.078130 & -0.696551 \\
\hline
\end{tabular}




$\begin{array}{rrrrrr}14 & 1 & 0 & -2.433551 & 1.410432 & -0.035785 \\ 15 & 6 & 0 & -1.347977 & -0.790458 & 1.197561 \\ 16 & 1 & 0 & -2.433809 & -0.674011 & 1.238919 \\ 17 & 1 & 0 & -1.135302 & -1.857391 & 1.114326 \\ 18 & 1 & 0 & -0.943741 & -0.435829 & 2.147884 \\ 19 & 6 & 0 & 1.347866 & 0.527641 & -1.334415 \\ 20 & 1 & 0 & 2.433683 & 0.628546 & -1.262850 \\ 21 & 1 & 0 & 1.135268 & -0.152614 & -2.160573 \\ 22 & 1 & 0 & 0.943385 & 1.509386 & -1.589066 \\ 23 & 6 & 0 & 1.347539 & -1.419636 & 0.210344 \\ 24 & 1 & 0 & 2.433401 & -1.408387 & 0.087431 \\ 25 & 1 & 0 & 1.134580 & -1.794981 & 1.212460 \\ 26 & 1 & 0 & 0.942987 & -2.130878 & -0.512685\end{array}$

Zero-point correction $=$

Thermal correction to Energy=

0.242532 (Hartree/Particle)

Thermal correction to Enthalpy=

0.253349

0.254293

Thermal correction to Gibbs Free Energy=

Sum of electronic and zero-point Energies=

0.208744

Sum of electronic and thermal Energies $=$

$-315.615456$

$-315.604639$

Sum of electronic and thermal Enthalpies=

$-315.603695$

Sum of electronic and thermal Free Energies $=\quad-315.649244$

B3LYP/ccpVQZ

Ethane

Standard orientation:

\begin{tabular}{cccrrr} 
Center & \multicolumn{2}{c}{ Atomic } & \multicolumn{2}{c}{ Atomic } & \multicolumn{2}{c}{ Coordinates (Angstroms) } \\
Number & Number & \multicolumn{1}{c}{ Type } & \multicolumn{1}{c}{ X } & Y & Z \\
\hline 1 & 6 & 0 & -0.000000 & -0.000000 & 0.763553 \\
2 & 1 & 0 & -0.507792 & 0.879565 & 1.160727 \\
3 & 1 & 0 & 1.015622 & -0.000022 & 1.160727 \\
4 & 1 & 0 & -0.507830 & -0.879543 & 1.160727 \\
5 & 6 & 0 & 0.000000 & -0.000000 & -0.763553 \\
6 & 1 & 0 & 0.507792 & 0.879565 & -1.160727 \\
7 & 1 & 0 & 0.507830 & -0.879543 & -1.160727 \\
8 & 1 & 0 & -1.015622 & -0.000022 & -1.160727
\end{tabular}

Zero-point correction=

Thermal correction to Energy=

0.074333 (Hartree/Particle)

0.077822

Thermal correction to Enthalpy= $\quad 0.078766$

Thermal correction to Gibbs Free Energy $=0.052912$

Sum of electronic and zero-point Energies $=\quad-79.796205$

Sum of electronic and thermal Energies $=\quad-79.792716$

Sum of electronic and thermal Enthalpies $=\quad-79.791772$

Sum of electronic and thermal Free Energies $=\quad-79.817626$

Methane

Standard orientation:

Center Atomic Atomic Coordinates (Angstroms) 


\begin{tabular}{lllrrr} 
Number & \multicolumn{1}{c}{ Number } & \multicolumn{1}{c}{ Type } & X & $Y$ & $Z$ \\
- \hdashline 1 & 6 & 0 & 0.000000 & 0.000000 & 0.000000 \\
2 & 1 & 0 & 0.627740 & 0.627740 & 0.627740 \\
3 & 1 & 0 & -0.627740 & -0.627740 & 0.627740 \\
4 & 1 & 0 & -0.627740 & 0.627740 & -0.627740 \\
5 & 1 & 0 & 0.627740 & -0.627740 & -0.627740
\end{tabular}

Zero-point correction=

Thermal correction to Energy=

Thermal correction to Enthalpy=

Thermal correction to Gibbs Free Energy=
Sum of electronic and zero-point Energies

Sum of electronic and thermal Energies $=$

Sum of electronic and thermal Enthalpies=

Sum of electronic and thermal Free Energies=

Propane

Standard orientation:

\subsection{4 (Hartree/Particle)}

0.047443

0.048387

0.027265

$-40.496930$

$-40.494061$

$-40.493117$

$-40.514239$

\begin{tabular}{cccccc} 
Center & \multicolumn{2}{c}{ Atomic } & \multicolumn{2}{c}{ Atomic } & \multicolumn{3}{c}{ Coordinates } \\
Number & Number & Type & X & Y & Z \\
- & Numbon \\
\hline 1 & 6 & 0 & -1.273815 & -0.258987 & 0.000000 \\
2 & 1 & 0 & -2.168314 & 0.364871 & -0.000012 \\
3 & 1 & 0 & -1.318451 & -0.903061 & 0.880279 \\
4 & 1 & 0 & -1.318441 & -0.903082 & -0.880264 \\
5 & 6 & 0 & -0.000000 & 0.584461 & 0.000000 \\
6 & 1 & 0 & -0.000001 & 1.241814 & 0.872988 \\
7 & 1 & 0 & 0.000001 & 1.241801 & -0.872997 \\
8 & 6 & 0 & 1.273815 & -0.258986 & 0.000000 \\
9 & 1 & 0 & 1.318467 & -0.903035 & 0.880297 \\
10 & 1 & 0 & 1.318421 & -0.903107 & -0.880246 \\
11 & 1 & 0 & 2.168316 & 0.364869 & -0.000049
\end{tabular}

Zero-point correction=

Thermal correction to Energy=

0.102949 (Hartree/Particle)

Thermal correction to Enthalpy=

0.107481

Thermal correction to Gibbs Free Energy=

Sum of electronic and zero-point Energies=

0.108425

Sum of electronic and thermal Energies $=$

0.077293

$-119.098558$

$-119.094026$

Sum of electronic and thermal Enthalpies=

$-119.093082$

Sum of electronic and thermal Free Energies= $-119.124214$

Butane

Standard orientation:

\begin{tabular}{cccccc} 
Center & \multicolumn{2}{c}{ Atomic } & Atomic & \multicolumn{2}{c}{ Coordinates (Angstroms) } \\
Number & Number & Type & X & Y & Z \\
-1 & 6 & 0 & 1.956968 & -0.120393 & 0.000035 \\
2 & 1 & 0 & 2.103527 & -0.748958 & -0.880269
\end{tabular}




$\begin{array}{cccccc}3 & 1 & 0 & 2.103468 & -0.748907 & 0.880384 \\ 4 & 1 & 0 & 2.741586 & 0.636927 & 0.000038 \\ 5 & 6 & 0 & 0.566815 & 0.512853 & -0.000039 \\ 6 & 1 & 0 & 0.461805 & 1.162836 & 0.873558 \\ 7 & 1 & 0 & 0.461858 & 1.162779 & -0.873685 \\ 8 & 6 & 0 & -0.566815 & -0.512853 & -0.000039 \\ 9 & 1 & 0 & -0.461858 & -1.162778 & -0.873686 \\ 10 & 1 & 0 & -0.461806 & -1.162838 & 0.873557 \\ 11 & 6 & 0 & -1.956968 & 0.120393 & 0.000035 \\ 12 & 1 & 0 & -2.103523 & 0.748969 & -0.880262 \\ 13 & 1 & 0 & -2.741586 & -0.636927 & 0.000025 \\ 14 & 1 & 0 & -2.103472 & 0.748897 & 0.880391\end{array}$

Zero-point correction=

Thermal correction to Energy=

Thermal correction to Enthalpy=

Thermal correction to Gibbs Free Energy=

Sum of electronic and zero-point Energies=

Sum of electronic and thermal Energies=

Sum of electronic and thermal Enthalpies=

Sum of electronic and thermal Free Energies=

Isobutane

Standard orientation:

\begin{tabular}{|c|c|c|c|c|}
\hline \multirow{2}{*}{$\begin{array}{l}\text { Center } \\
\text { Number }\end{array}$} & Atomic & \multirow{2}{*}{$\begin{array}{r}\text { Atomic } \\
\text { Type }\end{array}$} & \multicolumn{2}{|c|}{ Coordinates (Angstroms } \\
\hline & Numbe & & $\mathrm{X}$ & Z \\
\hline 1 & 6 & -1.456721 & 0.056604 & -0.095418 \\
\hline 2 & 1 & -1.954584 & 0.958384 & 0.263918 \\
\hline 3 & 1 & -1.514009 & 0.058695 & -1.186514 \\
\hline 4 & 1 & -2.023110 & -0.803701 & 0.264135 \\
\hline 5 & 6 & -0.000012 & 0.000001 & 0.371148 \\
\hline 6 & 1 & -0.000001 & 0.000018 & 1.466009 \\
\hline 7 & 6 & 0.777384 & 1.233238 & -0.095416 \\
\hline 8 & 1 & 1.807207 & 1.213603 & 0.264133 \\
\hline 9 & 1 & 0.808061 & 1.281648 & -1.186515 \\
\hline 10 & 1 & 0.315416 & 2.153930 & 0.263909 \\
\hline 11 & 6 & 0.679340 & -1.289841 & -0.095414 \\
\hline 12 & 1 & 1.707663 & -1.350085 & 0.263928 \\
\hline 13 & 1 & 0.147464 & -2.171912 & 0.264114 \\
\hline 14 & 1 & 0.705944 & -1.340589 & -1.186519 \\
\hline
\end{tabular}

Zero-point correction=

Thermal correction to Energy=

Thermal correction to Enthalpy=

Thermal correction to Gibbs Free Energy=

Sum of electronic and zero-point Energies=

Sum of electronic and thermal Energies $=$

Sum of electronic and thermal Enthalpies=

Sum of electronic and thermal Free Energies=
0.137159
0.138103
0.103353
$-158.400991$
$-158.395201$
$-158.394257$
$-158.429008$ 
Pentane

Standard orientation:

\begin{tabular}{cccccc}
\hline Center & \multicolumn{2}{c}{ Atomic } & \multicolumn{2}{c}{ Atomic } & \multicolumn{3}{c}{ Coordinates } \\
Number & Number & Type & X & Y & $Z$ \\
-1 & 6 & 0 & 2.553536 & 0.323351 & -0.000005 \\
1 & 1 & 0 & 2.599094 & 0.967138 & -0.880300 \\
2 & 1 & 0 & 2.599091 & 0.967178 & 0.880260 \\
3 & 1 & 0 & 3.447763 & -0.300826 & 0.000010 \\
4 & 1 & 0 & 1.280599 & -0.521370 & 0.000012 \\
5 & 6 & 0 & 1.280525 & -1.179527 & -0.873596 \\
6 & 1 & 0 & 1.280527 & -1.179494 & 0.873644 \\
7 & 1 & 0 & 0.000000 & 0.313551 & 0.000001 \\
8 & 6 & 0 & -0.000008 & 0.972996 & 0.874194 \\
9 & 1 & 0 & 0.000008 & 0.972995 & -0.874193 \\
10 & 1 & 0 & -1.280599 & -0.521370 & -0.000012 \\
11 & 6 & 0 & -1.280525 & -1.179528 & 0.873596 \\
12 & 1 & 0 & -1.280527 & -1.179493 & -0.873644 \\
13 & 1 & 0 & -2.553536 & 0.323351 & 0.000005 \\
14 & 6 & 0 & -3.447763 & -0.300826 & -0.000079 \\
15 & 1 & 0 & -2.599125 & 0.967088 & 0.880334 \\
16 & 1 & 0 & -2.599059 & 0.967228 & -0.880226
\end{tabular}

Zero-point correction=

Thermal correction to Energy=

0.159764 (Hartree/Particle)

Thermal correction to Enthalpy=

0.166859

Thermal correction to Gibbs Free Energy=

0.129434

Sum of electronic and zero-point Energies $=$

$-197.703353$

Sum of electronic and thermal Energies $=$

$-197.696258$

Sum of electronic and thermal Enthalpies=

$-197.695314$

Sum of electronic and thermal Free Energies $=\quad-197.733683$

Neopentane

Standard orientation:

\begin{tabular}{|c|c|c|c|c|c|}
\hline \multirow{2}{*}{$\begin{array}{l}\text { Center } \\
\text { Number }\end{array}$} & \multicolumn{2}{|l|}{ Atomic } & \multirow{2}{*}{$\begin{array}{l}\text { Atomic } \\
\text { Type }\end{array}$} & \multicolumn{2}{|c|}{ Coordinates (Angstrom } \\
\hline & Numbe & & & $X$ & Z \\
\hline 1 & 6 & 0 & -0.982557 & -0.985007 & 0.651878 \\
\hline 2 & 1 & 0 & -1.900982 & -1.066662 & 0.067660 \\
\hline 3 & 1 & 0 & -0.544845 & -1.982006 & 0.728700 \\
\hline 4 & 1 & 0 & -1.253551 & -0.659863 & 1.658015 \\
\hline 5 & 6 & 0 & -0.000005 & -0.000016 & 0.000015 \\
\hline 6 & 6 & 0 & 0.356679 & -0.491052 & -1.411463 \\
\hline 7 & 1 & 0 & 1.054794 & 0.191808 & -1.899150 \\
\hline 8 & 1 & 0 & 0.822315 & -1.477697 & -1.376966 \\
\hline 9 & 1 & 0 & -0.534206 & -0.562991 & -2.038067 \\
\hline 10 & 6 & 0 & -0.651174 & 1.388681 & -0.090198 \\
\hline 11 & 1 & 0 & -0.915541 & 1.762610 & 0.900655 \\
\hline 12 & 1 & 0 & 0.026536 & 2.110025 & -0.550466 \\
\hline
\end{tabular}




$\begin{array}{rrrrrr}13 & 1 & 0 & -1.562681 & 1.355800 & -0.689837 \\ 14 & 6 & 0 & 1.277054 & 0.087386 & 0.849775 \\ 15 & 1 & 0 & 1.052453 & 0.434254 & 1.860084 \\ 16 & 1 & 0 & 1.761497 & -0.887448 & 0.930412 \\ 17 & 1 & 0 & 1.994229 & 0.782219 & 0.408918\end{array}$

Zero-point correction=

Thermal correction to Energy=

Thermal correction to Enthalpy=

Thermal correction to Gibbs Free Energy=

Sum of electronic and zero-point Energies=

Sum of electronic and thermal Energies=

Sum of electronic and thermal Enthalpies=

Sum of electronic and thermal Free Energies=

Hexane

Standard orientation:

\begin{tabular}{cccccc} 
Center & Atomic & \multicolumn{2}{c}{ Atomic } & \multicolumn{3}{c}{ Coordinates } \\
Number & Number & Type & X & Y & Z \\
- & Nutroms \\
\hline 1 & 6 & 0 & -3.215287 & 0.207986 & 0.000043 \\
2 & 1 & 0 & -3.304792 & 0.847090 & 0.880395 \\
3 & 1 & 0 & -3.304800 & 0.847290 & -0.880163 \\
4 & 1 & 0 & -4.064765 & -0.475872 & -0.000033 \\
5 & 6 & 0 & -1.887596 & -0.547713 & -0.000051 \\
6 & 1 & 0 & -1.842549 & -1.204384 & 0.873476 \\
7 & 1 & 0 & -1.842588 & -1.204239 & -0.873690 \\
8 & 6 & 0 & -0.667090 & 0.373076 & -0.000009 \\
9 & 1 & 0 & -0.713241 & 1.030695 & -0.874189 \\
10 & 1 & 0 & -0.713277 & 1.030674 & 0.874185 \\
11 & 6 & 0 & 0.667090 & -0.373076 & 0.000015 \\
12 & 1 & 0 & 0.713253 & -1.030728 & -0.874140 \\
13 & 1 & 0 & 0.713266 & -1.030640 & 0.874235 \\
14 & 6 & 0 & 1.887596 & 0.547713 & -0.000039 \\
15 & 1 & 0 & 1.842560 & 1.204379 & 0.873493 \\
16 & 1 & 0 & 1.842577 & 1.204245 & -0.873673 \\
17 & 6 & 0 & 3.215287 & -0.207986 & 0.000032 \\
18 & 1 & 0 & 3.304801 & -0.847264 & -0.880193 \\
19 & 1 & 0 & 4.064765 & 0.475872 & -0.000021 \\
20 & 1 & 0 & 3.304791 & -0.847116 & 0.880366
\end{tabular}

Zero-point correction=

Thermal correction to Energy=

Thermal correction to Enthalpy=

Thermal correction to Gibbs Free Energy=

Sum of electronic and zero-point Energies=

Sum of electronic and thermal Energies=

Sum of electronic and thermal Enthalpies=

Sum of electronic and thermal Free Energies=

22dimethylbutane
0.188126 (Hartree/Particle)

$$
0.196555
$$

0.197499

$$
\begin{aligned}
& 0.155522 \\
& -237.005758 \\
& -236.997330 \\
& -236.996385 \\
& -237.038362
\end{aligned}
$$


Standard orientation:

\begin{tabular}{|c|c|c|c|c|}
\hline Center & Atomic & Atomic & Coordinat & igstron \\
\hline Number & Number & Type & $X$ & Z \\
\hline 1 & 6 & -1.612200 & 0.987806 & 0.002321 \\
\hline 2 & 1 & -1.586083 & 1.628583 & 0.885463 \\
\hline 3 & 1 & -1.586229 & 1.632553 & -0.877935 \\
\hline 4 & 1 & -2.567537 & 0.460440 & 0.001208 \\
\hline 5 & 6 & -0.435270 & -0.000660 & 0.000009 \\
\hline 6 & 6 & 0.876457 & 0.820321 & 0.001344 \\
\hline 7 & 1 & 0.861686 & 1.480571 & -0.870583 \\
\hline 8 & 1 & 0.861757 & 1.477678 & 0.875456 \\
\hline 9 & 6 & 2.187744 & 0.033372 & 0.000054 \\
\hline 10 & 1 & 2.283636 & -0.600527 & -0.881296 \\
\hline 11 & 1 & 3.037493 & 0.716651 & 0.000640 \\
\hline 12 & 1 & 2.284047 & -0.602603 & 0.879854 \\
\hline 13 & 6 & -0.527976 & -0.884933 & 1.253168 \\
\hline 14 & 1 & 0.259074 & -1.638387 & 1.277915 \\
\hline 15 & 1 & -0.448697 & -0.286863 & 2.162965 \\
\hline 16 & 1 & -1.484166 & -1.409824 & 1.283500 \\
\hline 17 & 6 & -0.528576 & -0.879715 & -1.256759 \\
\hline 18 & 1 & -1.484584 & -1.404852 & -1.288626 \\
\hline 19 & 1 & -0.450196 & -0.277797 & -2.164092 \\
\hline 20 & 1 & 0.258722 & -1.632780 & -1.285287 \\
\hline
\end{tabular}

Zero-point correction $=$

0.187110 (Hartree/Particle)

Thermal correction to Energy=

0.195377

Thermal correction to Enthalpy=

0.196321

Thermal correction to Gibbs Free Energy=

Sum of electronic and zero-point Energies=

0.155842

Sum of electronic and thermal Energies=

$-237.004764$

$-236.996497$

Sum of electronic and thermal Enthalpies $=$

$-236.995553$

Sum of electronic and thermal Free Energies=

$-237.036032$

\section{Heptane}

Standard orientation:

\begin{tabular}{|c|c|c|c|c|}
\hline \multirow{2}{*}{$\begin{array}{l}\text { Center } \\
\text { Number }\end{array}$} & Atomic & \multirow{2}{*}{$\begin{array}{l}\text { Atomic } \\
\text { Type }\end{array}$} & \multicolumn{2}{|c|}{ Coordinates (Angstrom } \\
\hline & Numbe & & $\mathrm{X}$ & Z \\
\hline 1 & 6 & -3.833541 & -0.352007 & -0.000034 \\
\hline 2 & 1 & -3.879133 & -0.995728 & -0.880396 \\
\hline 3 & 1 & -3.879098 & -0.995981 & 0.880147 \\
\hline 4 & 1 & -4.727838 & 0.272111 & 0.000077 \\
\hline 5 & 6 & -2.560633 & 0.492693 & 0.000063 \\
\hline 6 & 1 & -2.560588 & 1.150923 & -0.873454 \\
\hline 7 & 1 & -2.560599 & 1.150739 & 0.873719 \\
\hline 8 & 6 & -1.280043 & -0.342534 & -0.000009 \\
\hline 9 & 1 & -1.281124 & -1.001785 & 0.874119 \\
\hline
\end{tabular}




$\begin{array}{rrrrrr}10 & 1 & 0 & -1.281174 & -1.001726 & -0.874181 \\ 11 & 6 & 0 & 0.000000 & 0.493384 & -0.000020 \\ 12 & 1 & 0 & -0.000010 & 1.152418 & 0.874138 \\ 13 & 1 & 0 & 0.000010 & 1.152354 & -0.874227 \\ 14 & 6 & 0 & 1.280043 & -0.342534 & 0.000024 \\ 15 & 1 & 0 & 1.281143 & -1.001816 & -0.874080 \\ 16 & 1 & 0 & 1.281156 & -1.001695 & 0.874220 \\ 17 & 6 & 0 & 2.560633 & 0.492693 & -0.000043 \\ 18 & 1 & 0 & 2.560596 & 1.150898 & 0.873493 \\ 19 & 1 & 0 & 2.560591 & 1.150764 & -0.873680 \\ 20 & 6 & 0 & 3.833541 & -0.352007 & 0.000019 \\ 21 & 1 & 0 & 3.879113 & -0.995922 & -0.880203 \\ 22 & 1 & 0 & 4.727838 & 0.272111 & -0.000031 \\ 23 & 1 & 0 & 3.879118 & -0.995787 & 0.880339\end{array}$

Zero-point correction=

Thermal correction to Energy=

Thermal correction to Enthalpy=

Thermal correction to Gibbs Free Energy=

Sum of electronic and zero-point Energies=

Sum of electronic and thermal Energies $=$

Sum of electronic and thermal Enthalpies=

Sum of electronic and thermal Free Energies= 223trimethylbutane

Standard orientation:
0.216460 (Hartree/Particle)

0.226241

0.227186

0.181588

$-276.308189$

$-276.298408$

$-276.297463$

$-276.343061$

\begin{tabular}{|c|c|c|c|c|}
\hline \multirow{2}{*}{$\begin{array}{l}\text { Center } \\
\text { Number }\end{array}$} & Atomic & \multirow{2}{*}{$\begin{array}{l}\text { Atomic } \\
\text { Type }\end{array}$} & \multicolumn{2}{|c|}{ Coordinates (Angstrom } \\
\hline & Number & & $\mathrm{X}$ & Z \\
\hline 1 & 6 & -1.355234 & -1.241590 & -0.568068 \\
\hline 2 & 1 & -1.230463 & -1.306377 & -1.650796 \\
\hline 3 & 1 & -0.981709 & -2.166418 & -0.131157 \\
\hline 4 & 1 & -2.425205 & -1.193489 & -0.360798 \\
\hline 5 & 6 & -0.644259 & 0.000058 & 0.000170 \\
\hline 6 & 6 & 0.855650 & 0.000010 & -0.446046 \\
\hline 7 & 1 & 0.830658 & 0.000050 & -1.541476 \\
\hline 8 & 6 & 1.637064 & 1.249294 & -0.015863 \\
\hline 9 & 1 & 1.724158 & 1.314921 & 1.069626 \\
\hline 10 & 1 & 2.650285 & 1.210648 & -0.417512 \\
\hline 11 & 1 & 1.182837 & 2.171602 & -0.372552 \\
\hline 12 & 6 & 1.636939 & -1.249343 & -0.015982 \\
\hline 13 & 1 & 1.723905 & -1.315179 & 1.069524 \\
\hline 14 & 1 & 1.182743 & -2.171596 & -0.372850 \\
\hline 15 & 1 & 2.650210 & -1.210601 & -0.417491 \\
\hline 16 & 6 & -0.796399 & -0.000100 & 1.530172 \\
\hline 17 & 1 & -0.344409 & 0.881847 & 1.983705 \\
\hline 18 & 1 & -1.853094 & -0.000477 & 1.802369 \\
\hline 19 & 1 & -0.343786 & -0.881811 & 1.983541 \\
\hline 20 & 6 & -1.355216 & 1.241707 & -0.567983 \\
\hline 21 & 1 & -1.230434 & 1.306538 & -1.650714 \\
\hline
\end{tabular}




$\begin{array}{llllll}22 & 1 & 0 & -2.425204 & 1.193586 & -0.360776 \\ 23 & 1 & 0 & -0.981761 & 2.166537 & -0.131037\end{array}$

Zero-point correction=

Thermal correction to Energy=

Thermal correction to Enthalpy=

Thermal correction to Gibbs Free Energy=

Sum of electronic and zero-point Energies=

Sum of electronic and thermal Energies $=$

Sum of electronic and thermal Enthalpies=

Sum of electronic and thermal Free Energies=

Octane

Standard orientation:

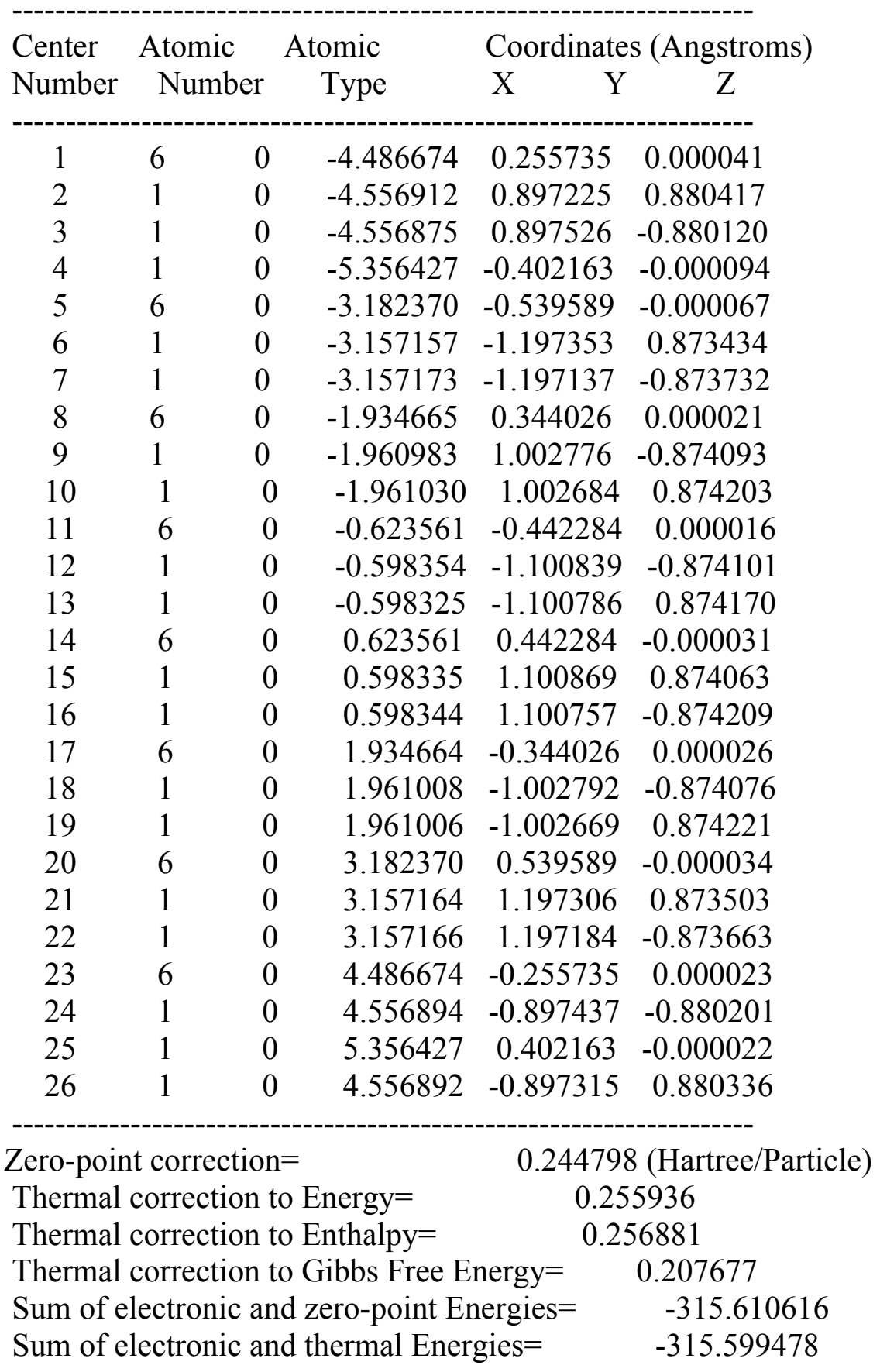


Sum of electronic and thermal Enthalpies=

Sum of electronic and thermal Free Energies=

2233tetramethylbutane

Standard orientation:

\begin{tabular}{|c|c|c|c|c|}
\hline \multirow{2}{*}{$\begin{array}{l}\text { Center } \\
\text { Number }\end{array}$} & Atomic & \multirow{2}{*}{$\begin{array}{l}\text { Atomic } \\
\text { Type }\end{array}$} & \multicolumn{2}{|c|}{ Coordinates (Angstrom } \\
\hline & Number & & $X$ & Z \\
\hline 1 & 6 & -1.355635 & 0.174466 & 1.424748 \\
\hline 2 & 1 & -1.135674 & -0.682851 & 2.059107 \\
\hline 3 & 1 & -0.967209 & 1.065960 & 1.916221 \\
\hline 4 & 1 & -2.440978 & 0.275684 & 1.381735 \\
\hline 5 & 6 & -0.793978 & 0.000012 & -0.000007 \\
\hline 6 & 6 & 0.793798 & 0.000023 & 0.000052 \\
\hline 7 & 6 & 1.355382 & -0.425599 & -1.370873 \\
\hline 8 & 1 & 1.137000 & -1.468264 & -1.596620 \\
\hline 9 & 1 & 0.965508 & 0.184406 & -2.185211 \\
\hline 10 & 1 & 2.440554 & -0.313994 & -1.373888 \\
\hline 11 & 6 & -1.355368 & -1.321154 & -0.561370 \\
\hline 12 & 1 & -1.135727 & -1.441449 & -1.621113 \\
\hline 13 & 1 & -0.966260 & -2.192451 & -0.035411 \\
\hline 14 & 1 & -2.440661 & -1.335019 & -0.451749 \\
\hline 15 & 6 & -1.355380 & 1.146741 & -0.863498 \\
\hline 16 & 1 & -2.440687 & 1.058754 & -0.930193 \\
\hline 17 & 1 & -1.135701 & 2.124658 & -0.437817 \\
\hline 18 & 1 & -0.966370 & 1.126911 & -1.881090 \\
\hline 19 & 6 & 1.355426 & -0.974467 & 1.054050 \\
\hline 20 & 1 & 2.440627 & -1.032589 & 0.958987 \\
\hline 21 & 1 & 1.136890 & -0.648854 & 2.069936 \\
\hline 22 & 1 & 0.965847 & -1.984815 & 0.932759 \\
\hline 23 & 6 & 1.355564 & 1.399992 & 0.316913 \\
\hline 24 & 1 & 2.440785 & 1.346676 & 0.414501 \\
\hline 25 & 1 & 1.136916 & 2.117080 & -0.472906 \\
\hline 26 & 1 & 0.966281 & 1.800078 & 1.252667 \\
\hline
\end{tabular}

Zero-point correction $=$

Thermal correction to Energy=

0.243374 (Hartree/Particle)

Thermal correction to Enthalpy=

0.253989

0.254933

Thermal correction to Gibbs Free Energy=

0.209702

Sum of electronic and zero-point Energies=

$-315.599531$

Sum of electronic and thermal Energies $=$

$-315.588916$

Sum of electronic and thermal Enthalpies=

$-315.587972$

Sum of electronic and thermal Free Energies= $-315.633203$

PBE0/ccpVQZ

Ethane

Standard orientation:

Center Atomic Atomic Coordinates (Angstroms)

Number Number Type X $\quad$ Y Z 


$\begin{array}{rrrrrr}1 & 6 & 0 & 0.000000 & -0.000000 & 0.759507 \\ 2 & 1 & 0 & -0.508084 & 0.880082 & 1.158115 \\ 3 & 1 & 0 & 1.016215 & -0.000027 & 1.158115 \\ 4 & 1 & 0 & -0.508131 & -0.880054 & 1.158115 \\ 5 & 6 & 0 & -0.000000 & 0.000000 & -0.759507 \\ 6 & 1 & 0 & 0.508084 & 0.880082 & -1.158115 \\ 7 & 1 & 0 & 0.508131 & -0.880054 & -1.158115 \\ 8 & 1 & 0 & -1.016215 & -0.000027 & -1.158115\end{array}$

Zero-point correction=

Thermal correction to Energy=

Thermal correction to Enthalpy=

Thermal correction to Gibbs Free Energy=

Sum of electronic and zero-point Energies=

Sum of electronic and thermal Energies=

Sum of electronic and thermal Enthalpies=

Sum of electronic and thermal Free Energies=

Methane

Standard orientation:

\begin{tabular}{|c|c|c|c|c|}
\hline \multirow{2}{*}{$\begin{array}{l}\text { Center } \\
\text { Number }\end{array}$} & \multirow{2}{*}{$\begin{array}{l}\text { Atomic } \\
\text { Number }\end{array}$} & \multirow{2}{*}{$\begin{array}{l}\text { Atomic } \\
\text { Type }\end{array}$} & \multicolumn{2}{|c|}{ Coordinates (Angstro } \\
\hline & & & $\mathrm{X}$ & Z \\
\hline 1 & 6 & 0.000000 & 0.000000 & 0.000000 \\
\hline 2 & 1 & 0.628332 & 0.628332 & 0.62833 \\
\hline 3 & 1 & -0.628332 & -0.628332 & 0.62833 \\
\hline 4 & 1 & -0.628332 & 0.628332 & $-0.6283 ?$ \\
\hline 5 & 1 & 0.628332 & -0.628332 & -0.62833 \\
\hline
\end{tabular}

Zero-point correction=

Thermal correction to Energy=

Thermal correction to Enthalpy=

Thermal correction to Gibbs Free Energy=

Sum of electronic and zero-point Energies=

Sum of electronic and thermal Energies=

Sum of electronic and thermal Enthalpies=

Sum of electronic and thermal Free Energies=

Propane

Standard orientation:

\begin{tabular}{cccrrr} 
Center & \multicolumn{2}{c}{ Atomic } & \multicolumn{2}{c}{ Atomic } & \multicolumn{2}{c}{ Coordinates (Angstroms) } \\
Number & Number & Type & X & Y & Z \\
\hline 1 & 6 & 0 & -1.265166 & -0.258052 & 0.000000 \\
2 & 1 & 0 & -2.163683 & 0.361784 & -0.000025 \\
3 & 1 & 0 & -1.306980 & -0.903609 & 0.880788 \\
4 & 1 & 0 & -1.306959 & -0.903651 & -0.880758 \\
5 & 6 & 0 & 0.000000 & 0.583768 & 0.000000 \\
6 & 1 & 0 & -0.000000 & 1.242497 & 0.873581 \\
7 & 1 & 0 & 0.000001 & 1.242480 & -0.873592
\end{tabular}




$\begin{array}{cccccc}8 & 6 & 0 & 1.265166 & -0.258052 & 0.000000 \\ 9 & 1 & 0 & 1.306985 & -0.903601 & 0.880794 \\ 10 & 1 & 0 & 1.306950 & -0.903659 & -0.880752 \\ 11 & 1 & 0 & 2.163685 & 0.361781 & -0.000038\end{array}$

Zero-point correction=

Thermal correction to Energy=

Thermal correction to Enthalpy=

Thermal correction to Gibbs Free Energy=

Sum of electronic and zero-point Energies=

Sum of electronic and thermal Energies $=$

Sum of electronic and thermal Enthalpies=

Sum of electronic and thermal Free Energies=

Butane

Standard orientation:
0.103317 (Hartree/Particle)

0.107847

0.108791

$-118.933259$

$-118.928730$

$-118.927786$

$-118.958900$

\begin{tabular}{|c|c|c|c|c|}
\hline \multirow{2}{*}{$\begin{array}{l}\text { Center } \\
\text { Number }\end{array}$} & Atomic & \multirow{2}{*}{$\begin{array}{r}\text { Atomic } \\
\text { Type }\end{array}$} & \multicolumn{2}{|c|}{ Coordinates (Angstron } \\
\hline & Numbe & & $X$ & Z \\
\hline 1 & 6 & 1.944833 & -0.119132 & 0.000030 \\
\hline 2 & 1 & 2.089900 & -0.749348 & -0.880806 \\
\hline 3 & 1 & 2.089841 & -0.749333 & 0.880885 \\
\hline 4 & 1 & 2.732914 & 0.636018 & 0.000049 \\
\hline 5 & 6 & 0.562567 & 0.511454 & -0.000033 \\
\hline 6 & 1 & 0.454840 & 1.162633 & 0.874136 \\
\hline 7 & 1 & 0.454883 & 1.162591 & -0.874240 \\
\hline 8 & 6 & -0.562567 & -0.511454 & -0.000034 \\
\hline 9 & 1 & -0.454883 & -1.162589 & -0.874242 \\
\hline 10 & 1 & -0.454840 & -1.162636 & 0.874134 \\
\hline 11 & 6 & -1.944833 & 0.119132 & 0.000030 \\
\hline 12 & 1 & -2.089898 & 0.749353 & -0.880802 \\
\hline 13 & 1 & -2.732914 & -0.636018 & 0.000043 \\
\hline 14 & 1 & -2.089843 & 0.749328 & 0.880888 \\
\hline
\end{tabular}

Zero-point correction=

Thermal correction to Energy=

Thermal correction to Enthalpy=

Thermal correction to Gibbs Free Energy=

Sum of electronic and zero-point Energies=

Sum of electronic and thermal Energies $=$

Sum of electronic and thermal Enthalpies=

Sum of electronic and thermal Free Energies=

Isobutane

Standard orientation:
0.131831 (Hartree/Particle)

$$
0.137619
$$

0.138563

0.103832

$-158.185394$

$-158.179606$

$-158.178662$

$-158.213393$

\begin{tabular}{cccccc} 
Center & \multicolumn{2}{c}{ Atomic } & \multicolumn{2}{c}{ Atomic } & \multicolumn{2}{c}{ Coordinates (Angstroms) } \\
Number & Number & Type & X & Y & Z \\
- \hdashline 1 & 6 & 0 & 1.447142 & 0.063886 & -0.095085
\end{tabular}




$\begin{array}{cccccc}2 & 1 & 0 & 2.020219 & -0.794658 & 0.261635 \\ 3 & 1 & 0 & 1.498790 & 0.066192 & -1.187811 \\ 4 & 1 & 0 & 1.942276 & 0.969616 & 0.261653 \\ 5 & 6 & 0 & 0.000024 & 0.000007 & 0.372656 \\ 6 & 1 & 0 & -0.000016 & -0.000007 & 1.469127 \\ 7 & 6 & 0 & -0.668244 & -1.285177 & -0.095076 \\ 8 & 1 & 0 & -1.698427 & -1.352018 & 0.261326 \\ 9 & 1 & 0 & -0.691754 & -1.331175 & -1.187806 \\ 10 & 1 & 0 & -0.131666 & -2.166888 & 0.261912 \\ 11 & 6 & 0 & -0.778906 & 1.221285 & -0.095079 \\ 12 & 1 & 0 & -1.810957 & 1.197044 & 0.261362 \\ 13 & 1 & 0 & -0.322130 & 2.146856 & 0.261904 \\ 14 & 1 & 0 & -0.806435 & 1.265032 & -1.187803\end{array}$

Zero-point correction $=$

Thermal correction to Energy=

Thermal correction to Enthalpy=

Thermal correction to Gibbs Free Energy=

Sum of electronic and zero-point Energies=

Sum of electronic and thermal Energies=

Sum of electronic and thermal Enthalpies=

Sum of electronic and thermal Free Energies=

Pentane
0.131364 (Hartree/Particle)

$$
0.137075
$$

0.138020

$$
\begin{gathered}
0.103756 \\
-158.187108 \\
-158.181397 \\
-158.180453 \\
-158.214716
\end{gathered}
$$

\begin{tabular}{|c|c|c|c|c|}
\hline \multirow{2}{*}{$\begin{array}{l}\text { Center } \\
\text { Number }\end{array}$} & Atomic & \multirow{2}{*}{$\begin{array}{r}\text { Atomic } \\
\text { Type }\end{array}$} & \multicolumn{2}{|c|}{ Coordinates (Angstroms) } \\
\hline & Numbe & & $\mathrm{X}$ & $\mathrm{Z}$ \\
\hline 1 & 6 & 2.536738 & 0.322512 & -0.000000 \\
\hline 2 & 1 & 2.579811 & 0.967715 & -0.880691 \\
\hline 3 & 1 & 2.579808 & 0.967725 & 0.880684 \\
\hline 4 & 1 & 3.434574 & -0.297917 & 0.000005 \\
\hline 5 & 6 & 1.272529 & -0.520111 & 0.000002 \\
\hline 6 & 1 & 1.270740 & -1.179753 & -0.874163 \\
\hline 7 & 1 & 1.270739 & -1.179746 & 0.874173 \\
\hline 8 & 6 & 0.000000 & 0.311604 & -0.000002 \\
\hline 9 & 1 & -0.000001 & 0.972756 & 0.874711 \\
\hline 10 & 1 & 0.000001 & 0.972752 & -0.874718 \\
\hline 11 & 6 & -1.272529 & -0.520111 & -0.000003 \\
\hline 12 & 1 & -1.270738 & -1.179751 & 0.874164 \\
\hline 13 & 1 & -1.270741 & -1.179748 & -0.874171 \\
\hline 14 & 6 & -2.536738 & 0.322512 & 0.000002 \\
\hline 15 & 1 & -3.434574 & -0.297917 & $7 \quad 0.000005$ \\
\hline 16 & 1 & -2.579807 & 0.967721 & 0.880689 \\
\hline 17 & 1 & -2.579812 & 0.967720 & -0.880686 \\
\hline Zero-poi & tt correct & & 0.16033 & 33 (Hartree/Particle) \\
\hline Thermal & correctior & to Energy= & 0.16 & 67436 \\
\hline Thermal & correctior & to Enthalpy= & 0.1 & 68380 \\
\hline Thermal & correctior & to Gibbs Free E & nergy $=$ & 0.129997 \\
\hline
\end{tabular}

Standard orientation: 
Sum of electronic and zero-point Energies=

$-197.437437$

Sum of electronic and thermal Energies=

$-197.430335$

Sum of electronic and thermal Enthalpies=

$-197.429391$

Sum of electronic and thermal Free Energies=

$-197.467774$

Neopentane

Standard orientation:

\begin{tabular}{|c|c|c|c|c|}
\hline \multirow{2}{*}{$\begin{array}{l}\text { Center } \\
\text { Number }\end{array}$} & Atomic & \multirow{2}{*}{$\begin{array}{l}\text { Atomic } \\
\text { Type }\end{array}$} & \multicolumn{2}{|c|}{ Coordinates (Angstrom } \\
\hline & Number & & $X$ & Z \\
\hline 1 & 6 & 1.193139 & 0.122087 & 0.944691 \\
\hline 2 & 1 & 1.643393 & 1.115811 & 0.882353 \\
\hline 3 & 1 & 0.891741 & -0.044218 & 1.981803 \\
\hline 4 & 1 & 1.965255 & -0.611106 & 0.698963 \\
\hline 5 & 6 & -0.000025 & 0.000039 & 0.000042 \\
\hline 6 & 6 & -1.054315 & 1.038730 & 0.374775 \\
\hline 7 & 1 & -1.921853 & 0.973959 & -0.286497 \\
\hline 8 & 1 & -1.403260 & 0.892088 & 1.399879 \\
\hline 9 & 1 & -0.651509 & 2.051781 & 0.300095 \\
\hline 10 & 6 & 0.461280 & 0.238208 & -1.435714 \\
\hline 11 & 1 & 1.217660 & -0.492850 & -1.731611 \\
\hline 12 & 1 & -0.374235 & 0.157076 & -2.135305 \\
\hline 13 & 1 & 0.896499 & 1.234201 & -1.548326 \\
\hline 14 & 6 & -0.600109 & -1.399029 & 0.116239 \\
\hline 15 & 1 & 0.134025 & -2.164443 & -0.147020 \\
\hline 16 & 1 & -0.939374 & -1.597440 & 1.135865 \\
\hline 17 & 1 & -1.458162 & -1.515067 & -0.550399 \\
\hline
\end{tabular}

Zero-point correction=

Thermal correction to Energy=

0.159073 (Hartree/Particle)

Thermal correction to Enthalpy=

0.166006

Thermal correction to Gibbs Free Energy=

0.166950

Sum of electronic and zero-point Energies=

0.129975

Sum of electronic and thermal Energies $=$

$-197.441242$

$-197.434309$

Sum of electronic and thermal Enthalpies=

$-197.433365$

Sum of electronic and thermal Free Energies=

$-197.470340$

Hexane

Standard orientation:

\begin{tabular}{|c|c|c|c|c|}
\hline \multirow{2}{*}{$\begin{array}{l}\text { Center } \\
\text { Number }\end{array}$} & \multirow{2}{*}{$\begin{array}{l}\text { Atomic } \\
\text { Number }\end{array}$} & \multirow{2}{*}{$\begin{array}{l}\text { Atomic } \\
\text { Type }\end{array}$} & \multicolumn{2}{|c|}{ Coordinates (Angstroms } \\
\hline & & & $\mathrm{X}$ & $\mathrm{Z}$ \\
\hline 1 & 6 & -3.195393 & 0.206998 & 0.000024 \\
\hline 2 & 1 & -3.282732 & 0.847743 & 0.880865 \\
\hline 3 & 1 & -3.282719 & 0.847888 & -0.880713 \\
\hline 4 & 1 & -4.048742 & -0.473616 & -0.000040 \\
\hline 5 & 6 & -1.875976 & -0.546484 & -0.000033 \\
\hline 6 & 1 & -1.828933 & -1.204619 & 0.874103 \\
\hline 7 & 1 & -1.828950 & -1.204522 & -0.874243 \\
\hline 8 & 6 & -0.663398 & 0.370953 & 0.000003 \\
\hline
\end{tabular}




$\begin{array}{ccrrrr}9 & 1 & 0 & -0.710139 & 1.030371 & -0.874767 \\ 10 & 1 & 0 & -0.710162 & 1.030338 & 0.874797 \\ 11 & 6 & 0 & 0.663398 & -0.370953 & 0.000010 \\ 12 & 1 & 0 & 0.710141 & -1.030381 & -0.874753 \\ 13 & 1 & 0 & 0.710161 & -1.030328 & 0.874811 \\ 14 & 6 & 0 & 1.875976 & 0.546484 & -0.000030 \\ 15 & 1 & 0 & 1.828936 & 1.204620 & 0.874105 \\ 16 & 1 & 0 & 1.828947 & 1.204521 & -0.874240 \\ 17 & 6 & 0 & 3.195393 & -0.206998 & 0.000021 \\ 18 & 1 & 0 & 3.282727 & -0.847873 & -0.880726 \\ 19 & 1 & 0 & 4.048742 & 0.473616 & -0.000022 \\ 20 & 1 & 0 & 3.282724 & -0.847758 & 0.880852\end{array}$

Zero-point correction $=$

Thermal correction to Energy=

0.188783 (Hartree/Particle)

Thermal correction to Enthalpy=

0.197208

0.198152

Thermal correction to Gibbs Free Energy=

Sum of electronic and zero-point Energies=

0.156202

$-236.689545$

Sum of electronic and thermal Energies $=$

$-236.681121$

Sum of electronic and thermal Enthalpies=

$-236.680176$

Sum of electronic and thermal Free Energies $=\quad-236.722126$

Heptane

Standard orientation:

\begin{tabular}{|c|c|c|c|c|}
\hline \multirow{2}{*}{$\begin{array}{l}\text { Center } \\
\text { Number }\end{array}$} & Atomic & \multirow{2}{*}{$\begin{array}{r}\text { Atomic } \\
\text { Type }\end{array}$} & \multicolumn{2}{|c|}{ Coordinates (Angstroms } \\
\hline & Numbe & & $\mathrm{X}$ & $\mathrm{Z}$ \\
\hline 1 & 6 & -3.808685 & -0.351228 & -0.000005 \\
\hline 2 & 1 & -3.851757 & -0.996442 & -0.880706 \\
\hline 3 & 1 & -3.851752 & -0.996477 & 0.880670 \\
\hline 4 & 1 & -4.706569 & 0.269156 & 0.000010 \\
\hline 5 & 6 & -2.544492 & 0.491413 & 0.000009 \\
\hline 6 & 1 & -2.542704 & 1.151068 & -0.874122 \\
\hline 7 & 1 & -2.542705 & 1.151041 & 0.874160 \\
\hline 8 & 6 & -1.271937 & -0.340621 & -0.000002 \\
\hline 9 & 1 & -1.273084 & -1.001542 & 0.874702 \\
\hline 10 & 1 & -1.273089 & -1.001526 & -0.874718 \\
\hline 11 & 6 & 0.000000 & 0.491664 & 0.000000 \\
\hline 12 & 1 & -0.000002 & 1.152346 & 0.874773 \\
\hline 13 & 1 & 0.000002 & 1.152345 & -0.874774 \\
\hline 14 & 6 & 1.271937 & -0.340621 & 0.000005 \\
\hline 15 & 1 & 1.273084 & -1.001544 & -0.874698 \\
\hline 16 & 1 & 1.273089 & -1.001524 & 0.874722 \\
\hline 17 & 6 & 2.544492 & 0.491413 & -0.000009 \\
\hline 18 & 1 & 2.542705 & 1.151068 & 0.874122 \\
\hline 19 & 1 & 2.542703 & 1.151041 & -0.874160 \\
\hline 20 & 6 & 3.808685 & -0.351228 & 0.000003 \\
\hline 21 & 1 & 3.851753 & -0.996474 & -0.880674 \\
\hline 22 & 1 & 4.706569 & 0.269156 & -0.000009 \\
\hline 23 & 1 & 3.851756 & -0.996444 & 0.880702 \\
\hline
\end{tabular}


Zero-point correction=

Thermal correction to Energy=

Thermal correction to Enthalpy=

Thermal correction to Gibbs Free Energy=

Sum of electronic and zero-point Energies=

Sum of electronic and thermal Energies=

Sum of electronic and thermal Enthalpies=

Sum of electronic and thermal Free Energies=

223trimethylbutane

Standard orientation:
0.217227 (Hartree/Particle)

0.227018

0.227963

0.182327

$-275.941657$

$-275.931865$

$-275.930921$

$-275.976557$

\begin{tabular}{cccccc} 
Center & \multicolumn{2}{c}{ Atomic } & \multicolumn{2}{c}{ Atomic } & \multicolumn{3}{c}{ Coordinates } \\
Number & Number & Type & X & Y & $Z$ \\
\hline 1 & 6 & 0 & -1.346112 & -1.234235 & -0.562891 \\
2 & 1 & 0 & -1.221872 & -1.299416 & -1.647131 \\
3 & 1 & 0 & -0.971409 & -2.159634 & -0.124106 \\
4 & 1 & 0 & -2.417185 & -1.185372 & -0.353864 \\
5 & 6 & 0 & -0.638989 & 0.000029 & -0.000061 \\
6 & 6 & 0 & 0.848245 & 0.000017 & -0.447560 \\
7 & 1 & 0 & 0.819975 & 0.000051 & -1.545068 \\
8 & 6 & 0 & 1.622947 & 1.241842 & -0.017066 \\
9 & 1 & 0 & 1.707378 & 1.303966 & 1.070394 \\
10 & 1 & 0 & 2.638744 & 1.204939 & -0.415776 \\
11 & 1 & 0 & 1.168036 & 2.165921 & -0.373033 \\
12 & 6 & 0 & 1.622863 & -1.241870 & -0.017155 \\
13 & 1 & 0 & 1.707551 & -1.303918 & 1.070294 \\
14 & 1 & 0 & 1.167746 & -2.165928 & -0.372920 \\
15 & 1 & 0 & 2.638568 & -1.205116 & -0.416110 \\
16 & 6 & 0 & -0.784163 & -0.000592 & 1.521117 \\
17 & 1 & 0 & -0.329816 & 0.882768 & 1.973294 \\
18 & 1 & 0 & -1.841430 & -0.000549 & 1.796631 \\
19 & 1 & 0 & -0.330068 & -0.884479 & 1.972516 \\
20 & 6 & 0 & -1.345885 & 1.234829 & -0.561963 \\
21 & 1 & 0 & -1.221395 & 1.300968 & -1.646120 \\
22 & 1 & 0 & -2.417016 & 1.185858 & -0.353248 \\
23 & 1 & 0 & -0.971242 & 2.159821 & -0.122274
\end{tabular}

Zero-point correction=

Thermal correction to Energy=

Thermal correction to Enthalpy=

Thermal correction to Gibbs Free Energy=

Sum of electronic and zero-point Energies=

Sum of electronic and thermal Energies $=$

Sum of electronic and thermal Enthalpies=

Sum of electronic and thermal Free Energies=

Octane

Standard orientation:

\subsection{4 (Hartree/Particle)} 0.225467

0.226411

0.182291

$-275.939470$

$-275.929858$

$-275.928914$

$-275.973033$ 


\begin{tabular}{|c|c|c|c|c|c|}
\hline \multirow{2}{*}{$\begin{array}{l}\text { Center } \\
\text { Number }\end{array}$} & Atomic & \multirow{2}{*}{\multicolumn{2}{|c|}{$\begin{array}{l}\text { Atomic } \\
\text { Type }\end{array}$}} & \multicolumn{2}{|c|}{ Coordinates (Angstroms } \\
\hline & Numbe & & & X & Z \\
\hline 1 & 6 & 0 & -4.457831 & 0.254836 & 0.000012 \\
\hline 2 & 1 & 0 & -4.525791 & 0.897907 & 0.880716 \\
\hline 3 & 1 & 0 & -4.525792 & 0.897955 & -0.880658 \\
\hline 4 & 1 & 0 & -5.331111 & -0.399738 & -0.000006 \\
\hline 5 & 6 & 0 & -3.162060 & -0.538384 & -0.000012 \\
\hline 6 & 1 & 0 & -3.134828 & -1.197488 & 0.874113 \\
\hline 7 & 1 & 0 & -3.134835 & -1.197451 & -0.874165 \\
\hline 8 & 6 & 0 & -1.922537 & 0.342102 & 0.000002 \\
\hline 9 & 1 & 0 & -1.949182 & 1.002496 & -0.874706 \\
\hline 10 & 1 & 0 & -1.949184 & 1.002474 & 0.874725 \\
\hline 11 & 6 & 0 & -0.619463 & -0.440512 & -0.000004 \\
\hline 12 & 1 & 0 & -0.593985 & -1.100705 & -0.874740 \\
\hline 13 & 1 & 0 & -0.593978 & -1.100707 & 0.874731 \\
\hline 14 & 6 & 0 & 0.619463 & 0.440512 & -0.000007 \\
\hline 15 & 1 & 0 & 0.593978 & 1.100716 & 0.874721 \\
\hline 16 & 1 & 0 & 0.593985 & 1.100697 & -0.874750 \\
\hline 17 & 6 & 0 & 1.922537 & -0.342102 & 0.000006 \\
\hline 18 & 1 & 0 & 1.949183 & -1.002499 & -0.874699 \\
\hline 19 & 1 & 0 & 1.949182 & -1.002471 & 0.874732 \\
\hline 20 & 6 & 0 & 3.162060 & 0.538384 & -0.000008 \\
\hline 21 & 1 & 0 & 3.134830 & 1.197485 & 0.874119 \\
\hline 22 & 1 & 0 & 3.134833 & 1.197454 & -0.874158 \\
\hline 23 & 6 & 0 & 4.457831 & -0.254836 & 0.000008 \\
\hline 24 & 1 & 0 & 4.525793 & -0.897947 & -0.880667 \\
\hline 25 & 1 & 0 & 5.331111 & 0.399738 & -0.000002 \\
\hline 26 & 1 & 0 & 4.525790 & -0.897915 & 0.880707 \\
\hline
\end{tabular}

Zero-point correction=

Thermal correction to Energy=

Thermal correction to Enthalpy=

Thermal correction to Gibbs Free Energy=

Sum of electronic and zero-point Energies=

Sum of electronic and thermal Energies $=$

Sum of electronic and thermal Enthalpies=

Sum of electronic and thermal Free Energies=

2233tetramethylbutane

Standard orientation:
0.245662 (Hartree/Particle)

0.256811

$$
0.257756
$$

0.208500

$-315.193778$

$-315.182628$

$-315.181684$

$-315.230940$

\begin{tabular}{|c|c|c|c|c|}
\hline \multirow{2}{*}{$\begin{array}{l}\text { Center } \\
\text { Number }\end{array}$} & \multirow{2}{*}{$\begin{array}{l}\text { Atomic } \\
\text { Number }\end{array}$} & \multirow{2}{*}{$\begin{array}{l}\text { Atomic } \\
\text { Type }\end{array}$} & \multicolumn{2}{|c|}{ Coordinates (Angstroms } \\
\hline & & & $\mathrm{X}$ & $\mathrm{Z}$ \\
\hline 1 & 6 & -1.342151 & -1.389428 & 0.325817 \\
\hline 2 & 1 & -1.131357 & -2.109363 & -0.465886 \\
\hline 3 & 1 & -0.941371 & -1.786421 & 1.260123 \\
\hline 4 & 1 & -2.427622 & -1.334483 & 0.435510 \\
\hline 5 & 6 & -0.786707 & -0.000023 & 0.000074 \\
\hline
\end{tabular}




$\begin{array}{cccccc}6 & 6 & 0 & 0.786544 & -0.000002 & -0.000090 \\ 7 & 6 & 0 & 1.342032 & 1.294464 & -0.601016 \\ 8 & 1 & 0 & 1.130898 & 1.372685 & -1.668172 \\ 9 & 1 & 0 & 0.941743 & 2.183483 & -0.110397 \\ 10 & 1 & 0 & 2.427570 & 1.318730 & -0.481335 \\ 11 & 6 & 0 & -1.342364 & 0.412597 & -1.365953 \\ 12 & 1 & 0 & -1.130444 & 1.457842 & -1.594084 \\ 13 & 1 & 0 & -0.942744 & -0.198812 & -2.176902 \\ 14 & 1 & 0 & -2.427988 & 0.291388 & -1.372682 \\ 15 & 6 & 0 & -1.341990 & 0.976824 & 1.040496 \\ 16 & 1 & 0 & -2.427583 & 1.043873 & 0.938903 \\ 17 & 1 & 0 & -1.130189 & 0.651644 & 2.059759 \\ 18 & 1 & 0 & -0.941759 & 1.984559 & 0.916339 \\ 19 & 6 & 0 & 1.342061 & -1.167617 & -0.820615 \\ 20 & 1 & 0 & 2.427609 & -1.076067 & -0.901293 \\ 21 & 1 & 0 & 1.130897 & -2.130933 & -0.354747 \\ 22 & 1 & 0 & 0.941928 & -1.187426 & -1.835896 \\ 23 & 6 & 0 & 1.342370 & -0.126835 & 1.421268 \\ 24 & 1 & 0 & 2.427779 & -0.243565 & 1.382046 \\ 25 & 1 & 0 & 1.132349 & 0.758615 & 2.022436 \\ 26 & 1 & 0 & 0.941507 & -0.995630 & 1.946399\end{array}$

Zero-point correction=

Thermal correction to Energy=

0.244122 (Hartree/Particle)

Thermal correction to Enthalpy=

0.254668

0.255612

Thermal correction to Gibbs Free Energy=

Sum of electronic and zero-point Energies=

0.210636

Sum of electronic and thermal Energies=

$-315.187470$

$-315.176923$

$-315.175979$

Sum of electronic and thermal Enthalpies=

$-315.220956$

Sum of electronic and thermal Free Energies=

M06/ccpVQZ

Ethane

Standard orientation:

\begin{tabular}{|c|c|c|c|c|}
\hline \multirow{2}{*}{$\begin{array}{l}\text { Center } \\
\text { Number }\end{array}$} & Atomic & \multirow{2}{*}{$\begin{array}{l}\text { Atomic } \\
\text { Type }\end{array}$} & \multicolumn{2}{|c|}{ Coordinates (Angstrom } \\
\hline & Number & & $\mathrm{X}$ & Z \\
\hline 1 & 6 & -0.000000 & 0.000000 & 0.757853 \\
\hline 2 & 1 & -0.506831 & 0.878165 & 1.156051 \\
\hline 3 & 1 & 1.013928 & -0.000154 & 1.156051 \\
\hline 4 & 1 & -0.507098 & -0.878011 & 1.156051 \\
\hline 5 & 6 & 0.000000 & 0.000000 & -0.757853 \\
\hline 6 & 1 & 0.506831 & 0.878165 & -1.156051 \\
\hline 7 & 1 & 0.507098 & -0.878011 & -1.156051 \\
\hline 8 & 1 & -1.013928 & -0.000154 & -1.156051 \\
\hline
\end{tabular}

Zero-point correction=

0.074266 (Hartree/Particle)

Thermal correction to Energy=

0.077771

Thermal correction to Enthalpy=

0.078716

Thermal correction to Gibbs Free Energy $=\quad 0.052849$ 
Sum of electronic and zero-point Energies=

$-79.719286$

Sum of electronic and thermal Energies=

Sum of electronic and thermal Enthalpies=

Sum of electronic and thermal Free Energies=

$-79.740703$

Methane

Standard orientation:

\begin{tabular}{cccccc} 
Center & \multicolumn{2}{c}{ Atomic } & Atomic & \multicolumn{2}{c}{ Coordinates (Angstrom } \\
Number & Number & Type & X & Y & Z \\
-1 & 6 & 0 & 0.000000 & 0.000000 & 0.000000 \\
2 & 1 & 0 & 0.626823 & 0.626823 & 0.626823 \\
3 & 1 & 0 & -0.626823 & -0.626823 & 0.626823 \\
4 & 1 & 0 & -0.626823 & 0.626823 & -0.626823 \\
5 & 1 & 0 & 0.626823 & -0.626823 & -0.626823
\end{tabular}

Zero-point correction=

Thermal correction to Energy=

Thermal correction to Enthalpy=

Thermal correction to Gibbs Free Energy=

Sum of electronic and zero-point Energies=

Sum of electronic and thermal Energies=

Sum of electronic and thermal Enthalpies=

Sum of electronic and thermal Free Energies=

Propane

Standard orientation:
0.044577 (Hartree/Particle)

0.047449

0.048393

0.027272

$-40.453793$

$-40.450921$

$-40.449977$

$-40.471098$

\begin{tabular}{|c|c|c|c|c|}
\hline \multirow{2}{*}{$\begin{array}{l}\text { Center } \\
\text { Number }\end{array}$} & Atomic & \multirow{2}{*}{$\begin{array}{l}\text { Atomic } \\
\text { Type }\end{array}$} & \multicolumn{2}{|c|}{ Coordinates (Angstrom } \\
\hline & Numbe & & $\mathrm{X}$ & $\mathrm{Z}$ \\
\hline 1 & 6 & -1.257058 & -0.258885 & 0.000000 \\
\hline 2 & 1 & -2.160920 & 0.348794 & -0.000051 \\
\hline 3 & 1 & -1.289756 & -0.904536 & 0.878895 \\
\hline 4 & 1 & -1.289710 & -0.904616 & -0.878838 \\
\hline 5 & 6 & 0.000000 & 0.589115 & 0.000000 \\
\hline 6 & 1 & -0.000000 & 1.246338 & 0.872256 \\
\hline 7 & 1 & 0.000001 & 1.246314 & -0.872273 \\
\hline 8 & 6 & 1.257058 & -0.258885 & 0.000000 \\
\hline 9 & 1 & 1.289753 & -0.904537 & 0.878895 \\
\hline 10 & 1 & 1.289708 & -0.904614 & -0.878839 \\
\hline 11 & 1 & 2.160922 & 0.348791 & -0.000050 \\
\hline
\end{tabular}

Zero-point correction $=$

Thermal correction to Energy=

0.102945 (Hartree/Particle)

Thermal correction to Enthalpy=

0.107462

0.108406

Thermal correction to Gibbs Free Energy=

Sum of electronic and zero-point Energies=

0.077336

Sum of electronic and thermal Energies=

$-118.988768$

Sum of electronic and thermal Enthalpies=

$-118.984251$

$-118.983307$

Sum of electronic and thermal Free Energies $=\quad-119.014378$ 
Butane

Standard orientation:

\begin{tabular}{|c|c|c|c|c|}
\hline \multirow{2}{*}{$\begin{array}{l}\text { Center } \\
\text { Number }\end{array}$} & Atomic & \multirow{2}{*}{$\begin{array}{l}\text { Atomic } \\
\text { Type }\end{array}$} & \multicolumn{2}{|c|}{ Coordinates (Angstrom } \\
\hline & Numbe & & $\mathrm{X}$ & $\mathrm{Z}$ \\
\hline 1 & 6 & 1.935597 & -0.117742 & 0.000056 \\
\hline 2 & 1 & 2.074242 & -0.748998 & -0.878841 \\
\hline 3 & 1 & 2.074116 & -0.748990 & 0.878978 \\
\hline 4 & 1 & 2.728008 & 0.629294 & 0.000109 \\
\hline 5 & 6 & 0.558008 & 0.513987 & -0.000063 \\
\hline 6 & 1 & 0.445496 & 1.163724 & 0.872830 \\
\hline 7 & 1 & 0.445584 & 1.163643 & -0.873030 \\
\hline 8 & 6 & -0.558008 & -0.513987 & -0.000063 \\
\hline 9 & 1 & -0.445584 & -1.163640 & -0.873032 \\
\hline 10 & 1 & -0.445496 & -1.163727 & 0.872828 \\
\hline 11 & 6 & -1.935597 & 0.117742 & 0.000056 \\
\hline 12 & 1 & -2.074241 & 0.749002 & -0.878838 \\
\hline 13 & 1 & -2.728008 & -0.629294 & 0.000105 \\
\hline 14 & 1 & -2.074116 & 0.748986 & 0.878981 \\
\hline
\end{tabular}

Zero-point correction=

0.131375 (Hartree/Particle)

Thermal correction to Energy=

0.137147

Thermal correction to Enthalpy=

0.138091

Thermal correction to Gibbs Free Energy=

0.103427

Sum of electronic and zero-point Energies=

$-158.258563$

Sum of electronic and thermal Energies $=$

$-158.252791$

Sum of electronic and thermal Enthalpies=

$-158.251847$

Sum of electronic and thermal Free Energies $=\quad-158.286511$

Isobutane

Standard orientation:

\begin{tabular}{|c|c|c|c|c|c|}
\hline \multirow{2}{*}{$\begin{array}{l}\text { Center } \\
\text { Number }\end{array}$} & \multicolumn{2}{|c|}{ Atomic } & \multirow{2}{*}{$\begin{array}{l}\text { Atomic } \\
\text { Type }\end{array}$} & \multicolumn{2}{|c|}{ Coordinates (Angstroms } \\
\hline & & & & $\mathrm{X}$ & $\mathrm{Z}$ \\
\hline 1 & 6 & 0 & -0.634455 & -1.294629 & -0.095479 \\
\hline 2 & 1 & 0 & -1.663030 & -1.388579 & 0.253667 \\
\hline 3 & 1 & 0 & -0.650548 & -1.326519 & -1.187740 \\
\hline 4 & 1 & 0 & -0.078139 & -2.165122 & 0.253018 \\
\hline 5 & 6 & 0 & 0.000024 & 0.000004 & 0.380998 \\
\hline 6 & 1 & 0 & 0.000030 & 0.000009 & 1.475874 \\
\hline 7 & 6 & 0 & -0.803980 & 1.196758 & -0.095472 \\
\hline 8 & 1 & 0 & -0.370912 & 2.134580 & 0.253297 \\
\hline 9 & 1 & 0 & -0.823893 & 1.226358 & -1.187740 \\
\hline 10 & 1 & 0 & -1.835908 & 1.150357 & 0.253345 \\
\hline 11 & 6 & 0 & 1.438429 & 0.097875 & -0.095489 \\
\hline 12 & 1 & 0 & 1.914285 & 1.014715 & 0.253323 \\
\hline 13 & 1 & 0 & 2.033967 & -0.746119 & 0.253359 \\
\hline 14 & 1 & 0 & 1.474040 & 0.100277 & -1.187751 \\
\hline
\end{tabular}


Zero-point correction $=$

Thermal correction to Energy=

Thermal correction to Enthalpy=

Thermal correction to Gibbs Free Energy=

Sum of electronic and zero-point Energies=

Sum of electronic and thermal Energies=

Sum of electronic and thermal Enthalpies=

Sum of electronic and thermal Free Energies=

Pentane

Standard orientation:
0.136564

0.137508

$$
\begin{gathered}
0.103319 \\
-158.261333 \\
-158.255653 \\
-158.254709 \\
-158.288898
\end{gathered}
$$

\begin{tabular}{cccccc} 
Center & \multicolumn{2}{c}{ Atomic } & \multicolumn{2}{c}{ Atomic } & \multicolumn{3}{c}{ Coordinates $($ Angstroms) } \\
Number & Number & Type & X & Y & $Z$ \\
- \hdashline 1 & 6 & 0 & 2.523384 & 0.324947 & 0.000003 \\
2 & 1 & 0 & 2.557175 & 0.970311 & -0.878749 \\
3 & 1 & 0 & 2.557161 & 0.970330 & 0.878742 \\
4 & 1 & 0 & 3.426830 & -0.282929 & 0.000017 \\
5 & 6 & 0 & 1.267543 & -0.523355 & 0.000002 \\
6 & 1 & 0 & 1.263461 & -1.182295 & -0.872934 \\
7 & 1 & 0 & 1.263454 & -1.182284 & 0.872947 \\
8 & 6 & 0 & 0.000000 & 0.309005 & -0.000009 \\
9 & 1 & 0 & -0.000001 & 0.970305 & 0.873617 \\
10 & 1 & 0 & 0.000002 & 0.970289 & -0.873648 \\
11 & 6 & 0 & -1.267543 & -0.523355 & -0.000003 \\
12 & 1 & 0 & -1.263453 & -1.182288 & 0.872938 \\
13 & 1 & 0 & -1.263462 & -1.182290 & -0.872942 \\
14 & 6 & 0 & -2.523384 & 0.324947 & 0.000005 \\
15 & 1 & 0 & -3.426830 & -0.282929 & 0.000017 \\
16 & 1 & 0 & -2.557160 & 0.970326 & 0.878747 \\
17 & 1 & 0 & -2.557176 & 0.970315 & -0.878744
\end{tabular}

Zero-point correction=

0.159731 (Hartree/Particle)

Thermal correction to Energy=

Thermal correction to Enthalpy=

0.166803

0.167747

Thermal correction to Gibbs Free Energy=

0.129489

Sum of electronic and zero-point Energies=

$-197.528317$

Sum of electronic and thermal Energies=

$-197.521246$

Sum of electronic and thermal Enthalpies=

$-197.520302$

\begin{tabular}{|c|c|c|c|c|}
\hline Center & Atomic & Atomic & Coordinate & (Angstron \\
\hline Number & Numbe & Type & $\mathrm{X}$ & Z \\
\hline 1 & 6 & -1.056296 & -0.782185 & 0.768120 \\
\hline 2 & 1 & -0.593527 & -1.465915 & 1.482039 \\
\hline 3 & 1 & -1.712983 & -0.110063 & 1.323417 \\
\hline 4 & 1 & -1.675086 & -1.372473 & 0.089897 \\
\hline
\end{tabular}

Sum of electronic and thermal Free Energies=

$-197.558560$

Neopentane

Standard orientation: 


$\begin{array}{cccccl}5 & 6 & 0 & -0.000014 & 0.000003 & 0.000007 \\ 6 & 6 & 0 & 0.842281 & 0.809155 & 0.976404 \\ 7 & 1 & 0 & 1.609328 & 1.380169 & 0.450182 \\ 8 & 1 & 0 & 0.223419 & 1.513159 & 1.535615 \\ 9 & 1 & 0 & 1.342213 & 0.156794 & 1.694612 \\ 10 & 6 & 0 & 0.895074 & -0.968268 & -0.760828 \\ 11 & 1 & 0 & 0.314769 & -1.561676 & -1.469656 \\ 12 & 1 & 0 & 1.663175 & -0.431939 & -1.320918 \\ 13 & 1 & 0 & 1.396065 & -1.656150 & -0.077333 \\ 14 & 6 & 0 & -0.681066 & 0.941294 & -0.983690 \\ 15 & 1 & 0 & -1.293102 & 0.385338 & -1.696186 \\ 16 & 1 & 0 & -1.329712 & 1.647807 & -0.462714 \\ 17 & 1 & 0 & 0.055563 & 1.514964 & -1.549033\end{array}$

Zero-point correction $=$

Thermal correction to Energy=

0.158775 (Hartree/Particle)

Thermal correction to Enthalpy=

0.165569

0.166513

Thermal correction to Gibbs Free Energy=

Sum of electronic and zero-point Energies $=$

0.129833

$-197.535408$

$-197.528614$

Sum of electronic and thermal Energies $=$

$-197.527670$

Sum of electronic and thermal Enthalpies=

$-197.564350$

Sum of electronic and thermal Free Energies=

Hexane

Standard orientation:

\begin{tabular}{|c|c|c|c|c|}
\hline \multirow{2}{*}{$\begin{array}{l}\text { Center } \\
\text { Number }\end{array}$} & Atomic & \multirow{2}{*}{$\begin{array}{l}\text { Atomic } \\
\text { Type }\end{array}$} & \multicolumn{2}{|c|}{ Coordinates (Angstrom } \\
\hline & Numbe & & $\mathrm{X}$ & $\mathrm{Z}$ \\
\hline 1 & 6 & -3.179442 & 0.207256 & 0.000004 \\
\hline 2 & 1 & -3.258891 & 0.848616 & 0.878745 \\
\hline 3 & 1 & -3.258880 & 0.848662 & -0.878705 \\
\hline 4 & 1 & -4.037592 & -0.463071 & -0.000019 \\
\hline 5 & 6 & -1.866716 & -0.549981 & -0.000008 \\
\hline 6 & 1 & -1.815998 & -1.207029 & 0.872842 \\
\hline 7 & 1 & -1.816001 & -1.206999 & -0.872880 \\
\hline 8 & 6 & -0.661177 & 0.370226 & 0.000005 \\
\hline 9 & 1 & -0.708958 & 1.029561 & -0.873621 \\
\hline 10 & 1 & -0.708963 & 1.029545 & 0.873644 \\
\hline 11 & 6 & 0.661177 & -0.370226 & 0.000002 \\
\hline 12 & 1 & 0.708956 & -1.029559 & -0.873626 \\
\hline 13 & 1 & 0.708965 & -1.029547 & 0.873639 \\
\hline 14 & 6 & 1.866716 & 0.549981 & -0.000010 \\
\hline 15 & 1 & 1.815997 & 1.207028 & 0.872840 \\
\hline 16 & 1 & 1.816002 & 1.207000 & -0.872881 \\
\hline 17 & 6 & 3.179442 & -0.207256 & 0.000005 \\
\hline 18 & 1 & 3.258884 & -0.848659 & -0.878705 \\
\hline 19 & 1 & 4.037592 & 0.463071 & -0.000012 \\
\hline 20 & 1 & 3.258888 & -0.848619 & 0.878744 \\
\hline
\end{tabular}


Thermal correction to Energy=

Thermal correction to Enthalpy=

Thermal correction to Gibbs Free Energy=

Sum of electronic and zero-point Energies=

Sum of electronic and thermal Energies=

Sum of electronic and thermal Enthalpies=

Sum of electronic and thermal Free Energies=

22dimethylbutane

Standard orientation:
0.196461

0.197405

0.155526

$-236.798121$

$-236.789707$

$-236.788763$

$-236.830643$

\begin{tabular}{|c|c|c|c|c|}
\hline \multirow{2}{*}{$\begin{array}{l}\text { Center } \\
\text { Number }\end{array}$} & Atomic & \multirow{2}{*}{$\begin{array}{r}\text { Atomic } \\
\text { Type }\end{array}$} & \multicolumn{2}{|c|}{ Coordinates (Angstrom } \\
\hline & Numbe & & $\mathrm{X}$ & $\mathrm{Z}$ \\
\hline 1 & 6 & -1.603464 & 0.970456 & 0.001800 \\
\hline 2 & 1 & -1.579795 & 1.610898 & 0.885427 \\
\hline 3 & 1 & -1.580284 & 1.613498 & -0.879950 \\
\hline 4 & 1 & -2.553570 & 0.433601 & 0.001272 \\
\hline 5 & 6 & -0.429041 & -0.000345 & 0.000020 \\
\hline 6 & 6 & 0.864002 & 0.822944 & 0.000726 \\
\hline 7 & 1 & 0.843231 & 1.482467 & -0.872817 \\
\hline 8 & 1 & 0.843359 & 1.480802 & 0.875533 \\
\hline 9 & 6 & 2.160531 & 0.035924 & -0.000030 \\
\hline 10 & 1 & 2.248306 & -0.600156 & -0.880562 \\
\hline 11 & 1 & 3.017887 & 0.707434 & -0.000064 \\
\hline 12 & 1 & 2.248848 & -0.600782 & 0.879987 \\
\hline 13 & 6 & -0.514402 & -0.876841 & 1.243069 \\
\hline 14 & 1 & 0.268185 & -1.635641 & 1.258986 \\
\hline 15 & 1 & -0.423002 & -0.276875 & 2.150808 \\
\hline 16 & 1 & -1.474289 & -1.394843 & 1.279287 \\
\hline 17 & 6 & -0.515326 & -0.873241 & -1.245513 \\
\hline 18 & 1 & -1.474976 & -1.391655 & -1.282159 \\
\hline 19 & 1 & -0.425319 & -0.270535 & -2.151566 \\
\hline 20 & 1 & 0.267622 & -1.631596 & -1.264615 \\
\hline
\end{tabular}

Zero-point correction=

Thermal correction to Energy=

Thermal correction to Enthalpy=

Thermal correction to Gibbs Free Energy=

Sum of electronic and zero-point Energies=

Sum of electronic and thermal Energies $=$

Sum of electronic and thermal Enthalpies=

Sum of electronic and thermal Free Energies=

Heptane

Standard orientation:
0.187237 (Hartree/Particle)

$$
\begin{gathered}
0.195411 \\
0.196355 \\
0.155969 \\
-236.803578 \\
-236.795404 \\
\quad-236.794460 \\
\\
\quad-236.834846
\end{gathered}
$$

\begin{tabular}{|c|c|c|c|}
\hline Center & Atomic & Atomic & Coordinates (Angstroms) \\
\hline Number & Number & Type & $\mathrm{X}$ \\
\hline 1 & 6 & -3.790125 & $-0.354454-0.000003$ \\
\hline 2 & 1 & -3.823864 & $-0.999829-0.878764$ \\
\hline
\end{tabular}




$\begin{array}{cccccc}3 & 1 & 0 & -3.823851 & -0.999873 & 0.878728 \\ 4 & 1 & 0 & -4.693640 & 0.253335 & 0.000019 \\ 5 & 6 & 0 & -2.534342 & 0.493892 & 0.000011 \\ 6 & 1 & 0 & -2.530235 & 1.152861 & -0.872879 \\ 7 & 1 & 0 & -2.530232 & 1.152827 & 0.872926 \\ 8 & 6 & 0 & -1.266727 & -0.338695 & -0.000007 \\ 9 & 1 & 0 & -1.267728 & -0.999756 & 0.873604 \\ 10 & 1 & 0 & -1.267736 & -0.999733 & -0.873635 \\ 11 & 6 & 0 & 0.000000 & 0.493732 & -0.000002 \\ 12 & 1 & 0 & -0.000003 & 1.154512 & 0.873693 \\ 13 & 1 & 0 & 0.000003 & 1.154512 & -0.873697 \\ 14 & 6 & 0 & 1.266727 & -0.338695 & 0.000003 \\ 15 & 1 & 0 & 1.267729 & -0.999754 & -0.873611 \\ 16 & 1 & 0 & 1.267735 & -0.999736 & 0.873629 \\ 17 & 6 & 0 & 2.534342 & 0.493892 & -0.000009 \\ 18 & 1 & 0 & 2.530232 & 1.152859 & 0.872883 \\ 19 & 1 & 0 & 2.530235 & 1.152830 & -0.872922 \\ 20 & 6 & 0 & 3.790125 & -0.354454 & 0.000006 \\ 21 & 1 & 0 & 3.823858 & -0.999866 & -0.878729 \\ 22 & 1 & 0 & 4.693640 & 0.253335 & -0.000003 \\ 23 & 1 & 0 & 3.823857 & -0.999836 & 0.878764\end{array}$

Zero-point correction $=$

Thermal correction to Energy=

Thermal correction to Enthalpy=

Thermal correction to Gibbs Free Energy=

Sum of electronic and zero-point Energies=

Sum of electronic and thermal Energies $=$

Sum of electronic and thermal Enthalpies=

Sum of electronic and thermal Free Energies=
0.216385 (Hartree/Particle)

0.226129

0.227074

0.181655

$-276.067905$

$-276.058160$

$-276.057216$

$-276.102635$

223 trimethylbutane

Standard orientation:

\begin{tabular}{|c|c|c|c|c|}
\hline \multirow{2}{*}{$\begin{array}{l}\text { Center } \\
\text { Number }\end{array}$} & Atomic & \multirow{2}{*}{$\begin{array}{r}\text { Atomic } \\
\text { Type }\end{array}$} & \multicolumn{2}{|c|}{ Coordinates (Angstroms } \\
\hline & Numbe & & $X$ & Z \\
\hline 1 & 6 & -1.310851 & -1.300324 & -0.428793 \\
\hline 2 & 1 & -1.158683 & -1.489471 & -1.493731 \\
\hline 3 & 1 & -0.933880 & -2.159431 & 0.125182 \\
\hline 4 & 1 & -2.385654 & -1.242834 & -0.250551 \\
\hline 5 & 6 & -0.636028 & -0.000012 & -0.000530 \\
\hline 6 & 6 & 0.844794 & -0.001540 & -0.452573 \\
\hline 7 & 1 & 0.814066 & -0.007450 & -1.549698 \\
\hline 8 & 6 & 1.615953 & 1.238059 & -0.021086 \\
\hline 9 & 1 & 1.761024 & 1.252474 & 1.060507 \\
\hline 10 & 1 & 2.605981 & 1.240452 & -0.476899 \\
\hline 11 & 1 & 1.123024 & 2.165599 & -0.306869 \\
\hline 12 & 6 & 1.621749 & -1.235687 & -0.015509 \\
\hline 13 & 1 & 1.647791 & -1.324356 & 1.072218 \\
\hline
\end{tabular}




$\begin{array}{rrrrrr}14 & 1 & 0 & 1.208382 & -2.157747 & -0.419042 \\ 15 & 1 & 0 & 2.655191 & -1.165438 & -0.355237 \\ 16 & 6 & 0 & -0.778670 & 0.151937 & 1.509860 \\ 17 & 1 & 0 & -0.402620 & 1.114053 & 1.858002 \\ 18 & 1 & 0 & -1.831402 & 0.091375 & 1.791106 \\ 19 & 1 & 0 & -0.251056 & -0.635077 & 2.049874 \\ 20 & 6 & 0 & -1.377288 & 1.147875 & -0.680844 \\ 21 & 1 & 0 & -1.294629 & 1.076118 & -1.767186 \\ 22 & 1 & 0 & -2.437420 & 1.117288 & -0.424497 \\ 23 & 1 & 0 & -0.998066 & 2.122591 & -0.376335\end{array}$

Zero-point correction=

Thermal correction to Energy=

Thermal correction to Enthalpy=

Thermal correction to Gibbs Free Energy=

Sum of electronic and zero-point Energies=

Sum of electronic and thermal Energies=

Sum of electronic and thermal Enthalpies=

Sum of electronic and thermal Free Energies=

Octane

Standard orientation:

\begin{tabular}{cccccc} 
Center & Atomic & \multicolumn{2}{l}{ Atomic } & \multicolumn{3}{c}{ Coordinates (Angstroms) } \\
Number & Number & Type & X & Y & Z \\
------------- \\
1 & 6 & 0 & -4.437169 & 0.256072 & 0.000020 \\
2 & 1 & 0 & -4.496528 & 0.899601 & 0.878775 \\
3 & 1 & 0 & -4.496530 & 0.899672 & -0.878685 \\
4 & 1 & 0 & -5.315865 & -0.387096 & -0.000006 \\
5 & 6 & 0 & -3.148701 & -0.541741 & -0.000017 \\
6 & 1 & 0 & -3.118444 & -1.200053 & 0.872849 \\
7 & 1 & 0 & -3.118458 & -1.199997 & -0.872925 \\
8 & 6 & 0 & -1.915109 & 0.340463 & 0.000002 \\
9 & 1 & 0 & -1.942306 & 1.000981 & -0.873601 \\
10 & 1 & 0 & -1.942308 & 1.000949 & 0.873629 \\
11 & 6 & 0 & -0.616416 & -0.441098 & -0.000008 \\
12 & 1 & 0 & -0.590233 & -1.101382 & -0.873648 \\
13 & 1 & 0 & -0.590222 & -1.101386 & 0.873630 \\
14 & 6 & 0 & 0.616416 & 0.441098 & -0.000013 \\
15 & 1 & 0 & 0.590221 & 1.101397 & 0.873617 \\
16 & 1 & 0 & 0.590233 & 1.101371 & -0.873661 \\
17 & 6 & 0 & 1.915109 & -0.340463 & 0.000007 \\
18 & 1 & 0 & 1.942308 & -1.000986 & -0.873592 \\
19 & 1 & 0 & 1.942306 & -1.000945 & 0.873638 \\
20 & 6 & 0 & 3.148701 & 0.541741 & -0.000012 \\
21 & 1 & 0 & 3.118447 & 1.200048 & 0.872858 \\
22 & 1 & 0 & 3.118455 & 1.200002 & -0.872916 \\
23 & 6 & 0 & 4.437169 & -0.256072 & 0.000015 \\
24 & 1 & 0 & 4.496533 & -0.899660 & -0.878698 \\
25 & 1 & 0 & 5.315865 & 0.387096 & 0.000002
\end{tabular}


Zero-point correction $=$

Thermal correction to Energy=

Thermal correction to Enthalpy=

Thermal correction to Gibbs Free Energy=

Sum of electronic and zero-point Energies=

Sum of electronic and thermal Energies=

Sum of electronic and thermal Enthalpies=

Sum of electronic and thermal Free Energies=

2233tetramethylbutane

Standard orientation:
0.244684 (Hartree/Particle)

0.255787

0.256731
0.207710
-315.337725
-315.326623
-315.325678
-315.374700

\begin{tabular}{|c|c|c|c|c|}
\hline \multirow{2}{*}{$\begin{array}{l}\text { Center } \\
\text { Number }\end{array}$} & Atomic & \multirow{2}{*}{$\begin{array}{l}\text { Atomic } \\
\text { Type }\end{array}$} & \multicolumn{2}{|c|}{ Coordinates (Angstroms } \\
\hline & Numbe & & $X$ & Z \\
\hline 1 & 6 & -1.341382 & 0.842945 & 1.147084 \\
\hline 2 & 1 & -1.142969 & 0.391689 & 2.118677 \\
\hline 3 & 1 & -0.934916 & 1.854539 & 1.153254 \\
\hline 4 & 1 & -2.424133 & 0.928513 & 1.043499 \\
\hline 5 & 6 & -0.783891 & 0.000022 & 0.000031 \\
\hline 6 & 6 & 0.783807 & -0.000044 & -0.000036 \\
\hline 7 & 6 & 1.341190 & -1.064492 & -0.945244 \\
\hline 8 & 1 & 1.142683 & -2.073533 & -0.585454 \\
\hline 9 & 1 & 0.934729 & -0.977464 & -1.953128 \\
\hline 10 & 1 & 2.423957 & -0.953651 & -1.020934 \\
\hline 11 & 6 & -1.341496 & -1.414765 & 0.156565 \\
\hline 12 & 1 & -1.141230 & -2.031437 & -0.718997 \\
\hline 13 & 1 & -0.936901 & -1.925058 & 1.030910 \\
\hline 14 & 1 & -2.424492 & -1.367788 & 0.280228 \\
\hline 15 & 6 & -1.341358 & 0.571987 & -1.303500 \\
\hline 16 & 1 & -2.424222 & 0.440375 & -1.325433 \\
\hline 17 & 1 & -1.142124 & 1.638839 & -1.398891 \\
\hline 18 & 1 & -0.935676 & 0.070988 & -2.182685 \\
\hline 19 & 6 & 1.341592 & -0.286434 & 1.394216 \\
\hline 20 & 1 & 2.424388 & -0.406915 & 1.335680 \\
\hline 21 & 1 & 1.143072 & 0.529452 & 2.088432 \\
\hline 22 & 1 & 0.935741 & -1.203106 & 1.822714 \\
\hline 23 & 6 & 1.341456 & 1.350757 & -0.449144 \\
\hline 24 & 1 & 2.424182 & 1.360650 & -0.315032 \\
\hline 25 & 1 & 1.143312 & 1.543933 & -1.502891 \\
\hline 26 & 1 & 0.935091 & 2.180118 & 0.130221 \\
\hline
\end{tabular}

Zero-point correction=

Thermal correction to Energy=

0.243596 (Hartree/Particle)

Thermal correction to Enthalpy=

0.253952

0.254896

Thermal correction to Gibbs Free Energy=

Sum of electronic and zero-point Energies=

0.210369

Sum of electronic and thermal Energies $=$

$-315.341941$

$-315.331585$

Sum of electronic and thermal Enthalpies=

$-315.330641$ 


\section{CAMB3LYP/ccpVQZ}

Ethane

Standard orientation:

\begin{tabular}{|c|c|c|c|c|}
\hline \multirow{2}{*}{$\begin{array}{l}\text { Center } \\
\text { Number }\end{array}$} & Atomic & \multirow{2}{*}{$\begin{array}{l}\text { Atomic } \\
\text { Type }\end{array}$} & \multicolumn{2}{|c|}{ Coordinates (Angstrom } \\
\hline & Numbe & & $X$ & Z \\
\hline 1 & 6 & -0.000000 & -0.000000 & 0.760639 \\
\hline 2 & 1 & -0.507192 & 0.878501 & 1.157275 \\
\hline 3 & 1 & 1.014400 & -0.000010 & 1.157275 \\
\hline 4 & 1 & -0.507209 & -0.878491 & 1.157275 \\
\hline 5 & 6 & 0.000000 & 0.000000 & -0.760639 \\
\hline 6 & 1 & 0.507192 & 0.878501 & -1.157275 \\
\hline 7 & 1 & 0.507209 & -0.878491 & -1.157275 \\
\hline 8 & 1 & -1.014400 & -0.000010 & -1.157275 \\
\hline
\end{tabular}

Zero-point correction=

Thermal correction to Energy=

Thermal correction to Enthalpy=

Thermal correction to Gibbs Free Energy=

Sum of electronic and zero-point Energies=

Sum of electronic and thermal Energies=

Sum of electronic and thermal Enthalpies=

Sum of electronic and thermal Free Energies=

Methane

Standard orientation:

\begin{tabular}{|c|c|c|c|c|}
\hline \multirow{2}{*}{$\begin{array}{l}\text { Center } \\
\text { Number }\end{array}$} & \multirow{2}{*}{$\begin{array}{l}\text { Atomic } \\
\text { Number }\end{array}$} & \multirow{2}{*}{$\begin{array}{r}\text { Atomic } \\
\text { Type }\end{array}$} & \multicolumn{2}{|c|}{ Coordinates (Angstro } \\
\hline & & & $X$ & $\mathrm{Z}$ \\
\hline 1 & 6 & 0 & 0.000000 & 0.000000 \\
\hline 2 & 1 & 0.6271 & 0.627177 & 0.627177 \\
\hline 3 & 1 & -0.627177 & -0.627177 & 0.627177 \\
\hline 4 & 1 & -0.627177 & 0.627177 & -0.62717 \\
\hline 5 & 1 & 0.627177 & -0.627177 & -0.627177 \\
\hline
\end{tabular}

Zero-point correction=

Thermal correction to Energy=

0.044857 (Hartree/Particle)

Thermal correction to Enthalpy=

0.047725

0.048669

Thermal correction to Gibbs Free Energy=

Sum of electronic and zero-point Energies=

0.027551

Sum of electronic and thermal Energies $=$

$-40.462435$

$-40.459567$

$-40.458623$

$-40.479741$

Sum of electronic and thermal Free Energies=

Propane

Standard orientation: 


\begin{tabular}{|c|c|c|c|c|}
\hline \multirow{2}{*}{$\begin{array}{l}\text { Center } \\
\text { Number }\end{array}$} & Atomic & \multirow{2}{*}{$\begin{array}{l}\text { Atomic } \\
\text { Type }\end{array}$} & \multicolumn{2}{|c|}{ Coordinates (Angstroms } \\
\hline & Numbe & & $\mathrm{X}$ & Z \\
\hline 1 & 6 & -1.266485 & -0.258514 & 0.000000 \\
\hline 2 & 1 & -2.162357 & 0.360929 & -0.000023 \\
\hline 3 & 1 & -1.307676 & -0.902117 & 0.879232 \\
\hline 4 & 1 & -1.307656 & -0.902153 & -0.879206 \\
\hline 5 & 6 & 0.000000 & 0.584550 & 0.000000 \\
\hline 6 & 1 & -0.000001 & 1.240782 & 0.872064 \\
\hline 7 & 1 & 0.000001 & 1.240767 & -0.872074 \\
\hline 8 & 6 & 1.266485 & -0.258514 & 0.000000 \\
\hline 9 & 1 & 1.307675 & -0.902116 & 0.879232 \\
\hline 10 & 1 & 1.307654 & -0.902153 & -0.879206 \\
\hline 11 & 1 & 2.162358 & 0.360926 & -0.000024 \\
\hline
\end{tabular}

Zero-point correction=

Thermal correction to Energy=

Thermal correction to Enthalpy=

Thermal correction to Gibbs Free Energy=

Sum of electronic and zero-point Energies=

Sum of electronic and thermal Energies=

Sum of electronic and thermal Enthalpies=

Sum of electronic and thermal Free Energies=

Butane

Standard orientation:

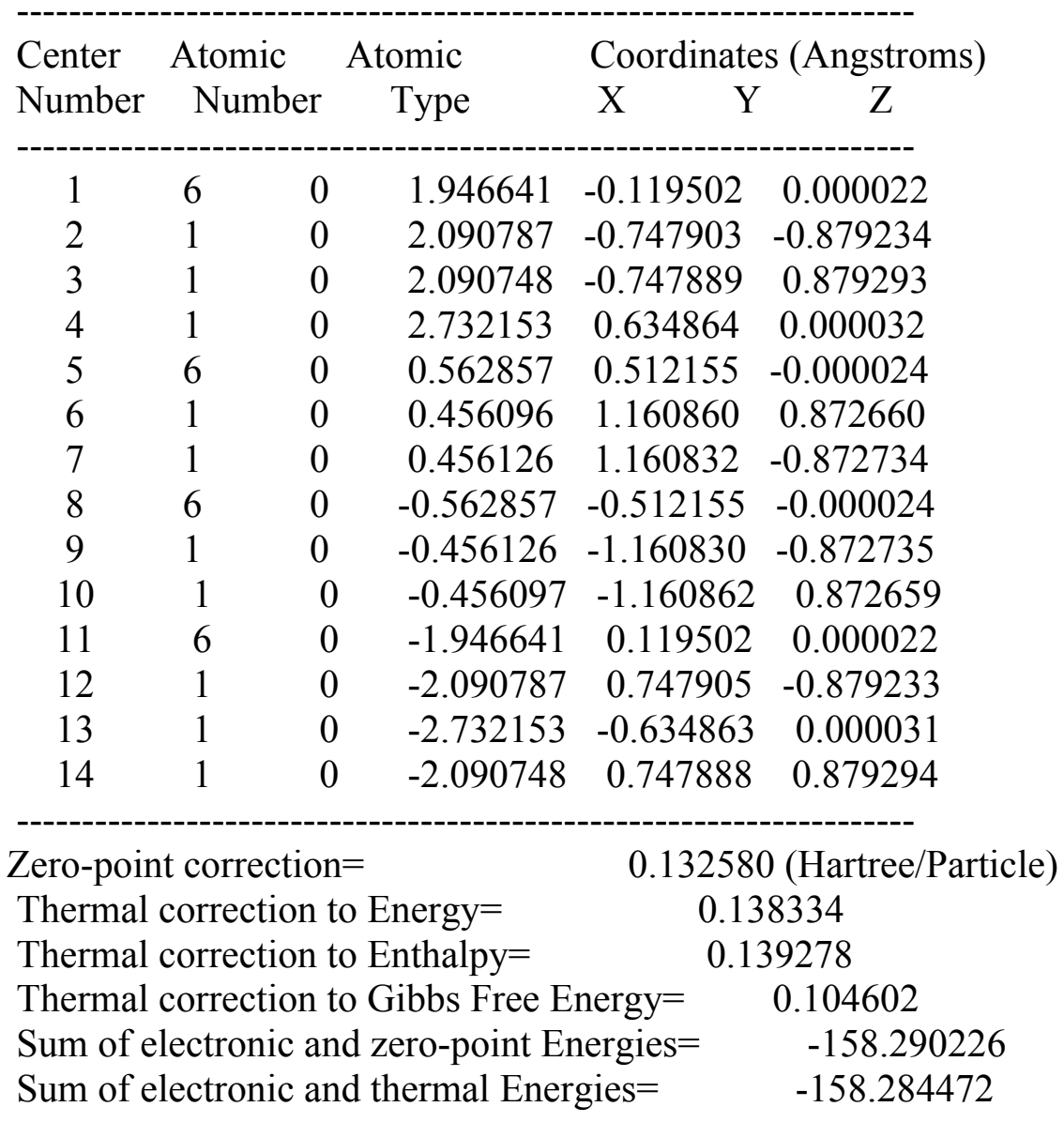


Sum of electronic and thermal Enthalpies=

Sum of electronic and thermal Free Energies=

Isobutane

Standard orientation:
$-158.283528$

$-158.318205$

\begin{tabular}{|c|c|c|c|c|}
\hline \multirow{2}{*}{$\begin{array}{l}\text { Center } \\
\text { Number }\end{array}$} & Atomic & \multirow{2}{*}{$\begin{array}{l}\text { Atomic } \\
\text { Type }\end{array}$} & \multicolumn{2}{|c|}{ Coordinates (Angstroms } \\
\hline & Numbe & & $\mathrm{X}$ & Z \\
\hline 1 & 6 & -1.442774 & 0.145022 & -0.095285 \\
\hline 2 & 1 & -1.884798 & 1.074971 & 0.261437 \\
\hline 3 & 1 & -1.494184 & 0.150176 & -1.185529 \\
\hline 4 & 1 & -2.061028 & -0.678347 & 0.261451 \\
\hline 5 & 6 & -0.000026 & 0.000016 & 0.372776 \\
\hline 6 & 1 & -0.000027 & 0.000014 & 1.466227 \\
\hline 7 & 6 & 0.846988 & 1.176944 & -0.095277 \\
\hline 8 & 1 & 1.873417 & 1.094617 & 0.261252 \\
\hline 9 & 1 & 0.876983 & 1.219015 & -1.185526 \\
\hline 10 & 1 & 0.443247 & 2.124067 & 0.261641 \\
\hline 11 & 6 & 0.595794 & -1.321971 & -0.095270 \\
\hline 12 & 1 & 1.617968 & -1.445736 & $5 \quad 0.261495$ \\
\hline 13 & 1 & 0.011440 & -2.169767 & 0.261407 \\
\hline 14 & 1 & 0.617090 & -1.369079 & -1.185519 \\
\hline
\end{tabular}

Zero-point correction $=$

0.132123 (Hartree/Particle)

Thermal correction to Energy=

0.137793

Thermal correction to Enthalpy=

0.138737

Thermal correction to Gibbs Free Energy=

0.104539

Sum of electronic and zero-point Energies=

$-158.292004$

Sum of electronic and thermal Energies $=$

$-158.286334$

Sum of electronic and thermal Enthalpies=

$-158.285390$

Sum of electronic and thermal Free Energies= $-158.319588$

Pentane

Standard orientation:

\begin{tabular}{|c|c|c|c|c|}
\hline \multirow{2}{*}{$\begin{array}{l}\text { Center } \\
\text { Number }\end{array}$} & Atomic & \multirow{2}{*}{$\begin{array}{r}\text { Atomic } \\
\text { Type }\end{array}$} & \multicolumn{2}{|c|}{ Coordinates (Angstroms) } \\
\hline & Numbe & & $X$ & $\mathrm{Z}$ \\
\hline 1 & 6 & -1.266485 & -0.258514 & 0.000000 \\
\hline 2 & 1 & -2.162357 & 0.360929 & -0.000023 \\
\hline 3 & 1 & -1.307676 & -0.902117 & 0.879232 \\
\hline 4 & 1 & -1.307656 & -0.902153 & -0.879206 \\
\hline 5 & 6 & 0.000000 & 0.584550 & 0.000000 \\
\hline 6 & 1 & -0.000001 & 1.240782 & 0.872064 \\
\hline 7 & 1 & 0.000001 & 1.240767 & -0.872074 \\
\hline 8 & 6 & 1.266485 & -0.258514 & 0.000000 \\
\hline 9 & 1 & 1.307675 & -0.902116 & 0.879232 \\
\hline 10 & 1 & 1.307654 & -0.902153 & -0.879206 \\
\hline 11 & 1 & 2.162358 & 0.360926 & -0.000024 \\
\hline
\end{tabular}


Zero-point correction $=$

Thermal correction to Energy=

Thermal correction to Enthalpy=

Thermal correction to Gibbs Free Energy=

Sum of electronic and zero-point Energies=

Sum of electronic and thermal Energies=

Sum of electronic and thermal Enthalpies=

Sum of electronic and thermal Free Energies=

Neopentane

Standard orientation:

\begin{tabular}{cccccc} 
Center & Atomic & \multicolumn{2}{c}{ Atomic } & \multicolumn{3}{c}{ Coordinates } \\
Number & Number & Type & X & Y & Z \\
-------- & \\
1 & 6 & 0 & -0.974956 & -1.119200 & -0.363493 \\
2 & 1 & 0 & -0.957121 & -1.321621 & -1.434635 \\
3 & 1 & 0 & -0.720969 & -2.043729 & 0.155518 \\
4 & 1 & 0 & -1.996357 & -0.852721 & -0.090792 \\
5 & 6 & 0 & 0.000008 & -0.000009 & -0.000002 \\
6 & 6 & 0 & 1.417462 & -0.410199 & -0.397287 \\
7 & 1 & 0 & 2.135588 & 0.371544 & -0.148679 \\
8 & 1 & 0 & 1.721494 & -1.320130 & 0.120615 \\
9 & 1 & 0 & 1.485089 & -0.597393 & -1.469212 \\
10 & 6 & 0 & -0.388743 & 1.276198 & -0.745288 \\
11 & 1 & 0 & -1.397905 & 1.592471 & -0.480333 \\
12 & 1 & 0 & 0.291605 & 2.093258 & -0.504089 \\
13 & 1 & 0 & -0.358815 & 1.124023 & -1.824446 \\
14 & 6 & 0 & -0.053765 & 0.253207 & 1.506067 \\
15 & 1 & 0 & -1.055764 & 0.548744 & 1.817975 \\
16 & 1 & 0 & 0.219086 & -0.642941 & 2.063842 \\
17 & 1 & 0 & 0.634033 & 1.048514 & 1.794249
\end{tabular}

Zero-point correction=

Thermal correction to Energy=

Thermal correction to Enthalpy=

Thermal correction to Gibbs Free Energy=

Sum of electronic and zero-point Energies=

Sum of electronic and thermal Energies=

Sum of electronic and thermal Enthalpies=

Sum of electronic and thermal Free Energies=

Hexane

Standard orientation:
0.108374

0.109318

0.078234

$-119.013030$

$-119.008522$

$-119.007578$

$-119.038662$ 


$\begin{array}{cccccc}5 & 6 & 0 & -1.877132 & -0.547251 & -0.000031 \\ 6 & 1 & 0 & -1.830865 & -1.202859 & 0.872590 \\ 7 & 1 & 0 & -1.830875 & -1.202762 & -0.872726 \\ 8 & 6 & 0 & -0.663858 & 0.371599 & 0.000009 \\ 9 & 1 & 0 & -0.710472 & 1.028387 & -0.873253 \\ 10 & 1 & 0 & -0.710492 & 1.028344 & 0.873303 \\ 11 & 6 & 0 & 0.663858 & -0.371598 & 0.000010 \\ 12 & 1 & 0 & 0.710469 & -1.028390 & -0.873250 \\ 13 & 1 & 0 & 0.710494 & -1.028341 & 0.873306 \\ 14 & 6 & 0 & 1.877132 & 0.547251 & -0.000032 \\ 15 & 1 & 0 & 1.830864 & 1.202861 & 0.872589 \\ 16 & 1 & 0 & 1.830875 & 1.202760 & -0.872728 \\ 17 & 6 & 0 & 3.197961 & -0.207440 & 0.000019 \\ 18 & 1 & 0 & 3.284537 & -0.846418 & -0.879156 \\ 19 & 1 & 0 & 4.048655 & 0.472639 & -0.000025 \\ 20 & 1 & 0 & 3.284536 & -0.846301 & 0.879279\end{array}$

Zero-point correction=

0.189918 (Hartree/Particle)

Thermal correction to Energy=

0.198289

Thermal correction to Enthalpy=

0.199233

Thermal correction to Gibbs Free Energy=

Sum of electronic and zero-point Energies=

0.157380

Sum of electronic and thermal Energies=

$-236.844524$

$-236.836153$

Sum of electronic and thermal Enthalpies=

$-236.835209$

Sum of electronic and thermal Free Energies=

$-236.877063$

22dimethylbutane

Standard orientation:

\begin{tabular}{|c|c|c|c|c|}
\hline \multirow{2}{*}{$\begin{array}{l}\text { Center } \\
\text { Number }\end{array}$} & Atomic & \multirow{2}{*}{$\begin{array}{r}\text { Atomic } \\
\text { Type }\end{array}$} & \multicolumn{2}{|c|}{ Coordinates (Angstroms } \\
\hline & Number & & $\mathrm{X}$ & $\mathrm{Z}$ \\
\hline 1 & 6 & -1.607773 & 0.977931 & 0.002886 \\
\hline 2 & 1 & -1.583415 & 1.617190 & 0.885775 \\
\hline 3 & 1 & -1.583631 & 1.622119 & -0.876421 \\
\hline 4 & 1 & -2.559385 & 0.446242 & 0.001512 \\
\hline 5 & 6 & -0.432530 & -0.000340 & 0.000015 \\
\hline 6 & 6 & 0.868638 & 0.820966 & 0.001538 \\
\hline 7 & 1 & 0.852107 & 1.480238 & -0.869608 \\
\hline 8 & 1 & 0.852210 & 1.476903 & 0.875202 \\
\hline 9 & 6 & 2.173041 & 0.035225 & 0.000048 \\
\hline 10 & 1 & 2.265934 & -0.598175 & -0.880514 \\
\hline 11 & 1 & 3.023950 & 0.714855 & 0.000604 \\
\hline 12 & 1 & 2.266514 & -0.600393 & 0.878939 \\
\hline 13 & 6 & -0.520051 & -0.881397 & 1.246455 \\
\hline 14 & 1 & 0.267621 & -1.632632 & 1.267643 \\
\hline 15 & 1 & -0.439399 & -0.283737 & 2.155080 \\
\hline 16 & 1 & -1.474586 & -1.406861 & 1.277809 \\
\hline 17 & 6 & -0.520991 & -0.875096 & -1.250790 \\
\hline 18 & 1 & -1.475271 & -1.400926 & -1.283776 \\
\hline 19 & 1 & -0.441694 & -0.272751 & -2.156432 \\
\hline
\end{tabular}


Zero-point correction $=$

Thermal correction to Energy=

Thermal correction to Enthalpy=

Thermal correction to Gibbs Free Energy=

Sum of electronic and zero-point Energies=

Sum of electronic and thermal Energies=

Sum of electronic and thermal Enthalpies=

Sum of electronic and thermal Free Energies=

Heptane

Standard orientation:
0.188903 (Hartree/Particle)

0.197091

0.198035

$$
\begin{gathered}
0.157680 \\
-236.845283 \\
-236.837095 \\
-236.836151 \\
-236.876506
\end{gathered}
$$

\begin{tabular}{|c|c|c|c|c|}
\hline \multirow{2}{*}{$\begin{array}{l}\text { Center } \\
\text { Number }\end{array}$} & Atomic & \multirow{2}{*}{$\begin{array}{r}\text { Atomic } \\
\text { Type }\end{array}$} & \multicolumn{2}{|c|}{ Coordinates (Angstroms } \\
\hline & Numbe & & $X$ & $\mathrm{Z}$ \\
\hline 1 & 6 & -3.811949 & -0.351886 & -0.000010 \\
\hline 2 & 1 & -3.854331 & -0.995171 & -0.879161 \\
\hline 3 & 1 & -3.854329 & -0.995219 & 0.879107 \\
\hline 4 & 1 & -4.707263 & 0.268042 & 0.000009 \\
\hline 5 & 6 & -2.546385 & 0.492135 & 0.000013 \\
\hline 6 & 1 & -2.545186 & 1.149237 & -0.872613 \\
\hline 7 & 1 & -2.545191 & 1.149199 & 0.872668 \\
\hline 8 & 6 & -1.272899 & -0.341122 & -0.000000 \\
\hline 9 & 1 & -1.274079 & -0.999404 & 0.873224 \\
\hline 10 & 1 & -1.274085 & -0.999388 & -0.873236 \\
\hline 11 & 6 & 0.000000 & 0.492469 & 0.000000 \\
\hline 12 & 1 & -0.000002 & 1.150535 & 0.873255 \\
\hline 13 & 1 & 0.000002 & 1.150529 & -0.873259 \\
\hline 14 & 6 & 1.272899 & -0.341122 & 0.000007 \\
\hline 15 & 1 & 1.274078 & -0.999411 & -0.873213 \\
\hline 16 & 1 & 1.274086 & -0.999382 & 0.873247 \\
\hline 17 & 6 & 2.546385 & 0.492135 & -0.000013 \\
\hline 18 & 1 & 2.545190 & 1.149238 & 0.872613 \\
\hline 19 & 1 & 2.545187 & 1.149199 & -0.872668 \\
\hline 20 & 6 & 3.811949 & -0.351886 & 0.000004 \\
\hline 21 & 1 & 3.854328 & -0.995217 & -0.879115 \\
\hline 22 & 1 & 4.707263 & 0.268042 & -0.000014 \\
\hline 23 & 1 & 3.854333 & -0.995174 & 0.879153 \\
\hline
\end{tabular}

Zero-point correction $=$

Thermal correction to Energy=

Thermal correction to Enthalpy=

Thermal correction to Gibbs Free Energy=

Sum of electronic and zero-point Energies=

Sum of electronic and thermal Energies $=$

Sum of electronic and thermal Enthalpies=

Sum of electronic and thermal Free Energies=

223trimethylbutane

Standard orientation:
0.218554 (Hartree/Particle)

0.228277

0.229221

0.183721

$-276.121710$

$-276.111987$

$-276.111043$

$-276.156543$ 


\begin{tabular}{|c|c|c|c|c|}
\hline \multirow{2}{*}{$\begin{array}{l}\text { Center } \\
\text { Number }\end{array}$} & Atomic & \multirow{2}{*}{$\begin{array}{l}\text { Atomic } \\
\text { Type }\end{array}$} & \multicolumn{2}{|c|}{ Coordinates (Angstroms } \\
\hline & Numbe & & $\mathrm{X}$ & $\mathrm{Z}$ \\
\hline 1 & 6 & -1.348066 & -1.235750 & -0.563620 \\
\hline 2 & 1 & -1.222328 & -1.301969 & -1.645059 \\
\hline 3 & 1 & -0.975410 & -2.158540 & -0.124526 \\
\hline 4 & 1 & -2.416812 & -1.185618 & -0.356658 \\
\hline 5 & 6 & -0.639691 & 0.000012 & -0.000408 \\
\hline 6 & 6 & 0.849250 & 0.000008 & -0.447554 \\
\hline 7 & 1 & 0.823145 & 0.000031 & -1.541783 \\
\hline 8 & 6 & 1.625846 & 1.243216 & -0.016674 \\
\hline 9 & 1 & 1.710062 & 1.305517 & 1.068127 \\
\hline 10 & 1 & 2.638843 & 1.205243 & -0.415509 \\
\hline 11 & 1 & 1.172018 & 2.164785 & -0.372161 \\
\hline 12 & 6 & 1.625816 & -1.243222 & -0.016721 \\
\hline 13 & 1 & 1.710310 & -1.305389 & 1.068068 \\
\hline 14 & 1 & 1.171804 & -2.164800 & -0.371957 \\
\hline 15 & 1 & 2.638717 & -1.205410 & -0.415818 \\
\hline 16 & 6 & -0.786521 & -0.000435 & 1.522524 \\
\hline 17 & 1 & -0.333431 & 0.880686 & 1.973846 \\
\hline 18 & 1 & -1.841687 & -0.000309 & 1.796067 \\
\hline 19 & 1 & -0.333774 & -0.882016 & 1.973287 \\
\hline 20 & 6 & -1.347890 & 1.236176 & -0.562927 \\
\hline 21 & 1 & -1.222006 & 1.303098 & -1.644309 \\
\hline 22 & 1 & -2.416675 & 1.185996 & -0.356155 \\
\hline 23 & 1 & -0.975242 & 2.158662 & -0.123188 \\
\hline
\end{tabular}

Zero-point correction=

Thermal correction to Energy=

Thermal correction to Enthalpy=

Thermal correction to Gibbs Free Energy=

Sum of electronic and zero-point Energies=

Sum of electronic and thermal Energies $=$

Sum of electronic and thermal Enthalpies=

Sum of electronic and thermal Free Energies=

Octane

Standard orientation:

\begin{tabular}{|c|c|c|c|c|}
\hline \multirow{2}{*}{$\begin{array}{l}\text { Center } \\
\text { Number }\end{array}$} & Atomic & \multirow{2}{*}{$\begin{array}{l}\text { Atomic } \\
\text { Type }\end{array}$} & \multicolumn{2}{|c|}{ Coordinates (Angstroms) } \\
\hline & Number & & $X$ & Z \\
\hline 1 & 6 & -4.461616 & 0.255347 & 0.000019 \\
\hline 2 & 1 & -4.528821 & 0.896513 & 0.879175 \\
\hline 3 & 1 & -4.528827 & 0.896579 & -0.879090 \\
\hline 4 & 1 & -5.332326 & -0.398693 & -0.000003 \\
\hline 5 & 6 & -3.164396 & -0.539163 & -0.000017 \\
\hline 6 & 1 & -3.137831 & -1.195742 & 0.872601 \\
\hline 7 & 1 & -3.137844 & -1.195688 & -0.872675 \\
\hline 8 & 6 & -1.924020 & 0.342623 & 0.000000 \\
\hline
\end{tabular}




$\begin{array}{cccccc}9 & 1 & 0 & -1.950618 & 1.000381 & -0.873225 \\ 10 & 1 & 0 & -1.950622 & 1.000355 & 0.873244 \\ 11 & 6 & 0 & -0.619906 & -0.441203 & -0.000005 \\ 12 & 1 & 0 & -0.594515 & -1.098782 & -0.873227 \\ 13 & 1 & 0 & -0.594509 & -1.098782 & 0.873216 \\ 14 & 6 & 0 & 0.619906 & 0.441203 & -0.000010 \\ 15 & 1 & 0 & 0.594507 & 1.098794 & 0.873202 \\ 16 & 1 & 0 & 0.594517 & 1.098769 & -0.873241 \\ 17 & 6 & 0 & 1.924020 & -0.342623 & 0.000008 \\ 18 & 1 & 0 & 1.950621 & -1.000387 & -0.873212 \\ 19 & 1 & 0 & 1.950620 & -1.000348 & 0.873257 \\ 20 & 6 & 0 & 3.164396 & 0.539163 & -0.000011 \\ 21 & 1 & 0 & 3.137835 & 1.195736 & 0.872611 \\ 22 & 1 & 0 & 3.137840 & 1.195693 & -0.872665 \\ 23 & 6 & 0 & 4.461616 & -0.255348 & 0.000012 \\ 24 & 1 & 0 & 4.528826 & -0.896569 & -0.879104 \\ 25 & 1 & 0 & 5.332326 & 0.398693 & -0.000002 \\ 26 & 1 & 0 & 4.528822 & -0.896524 & 0.879162\end{array}$

Zero-point correction=

Thermal correction to Energy=

Thermal correction to Enthalpy=

Thermal correction to Gibbs Free Energy=

Sum of electronic and zero-point Energies=

Sum of electronic and thermal Energies $=$

Sum of electronic and thermal Enthalpies=

Sum of electronic and thermal Free Energies=
0.247183 (Hartree/Particle)

0.258254

0.259198

0.210103

$-315.398903$

$-315.387833$

$-315.386888$

$-315.435983$

2233tetramethylbutane

Standard orientation:

\begin{tabular}{cccccc} 
Center & \multicolumn{2}{c}{ Atomic } & \multicolumn{2}{c}{ Atomic } & \multicolumn{3}{c}{ Coordinates } \\
Number & Number & Type & X & Y & Z \\
- & Nums & \\
\hline 1 & 6 & 0 & -1.345111 & -1.212669 & 0.755303 \\
2 & 1 & 0 & -1.136382 & -2.146207 & 0.237192 \\
3 & 1 & 0 & -0.944406 & -1.289906 & 1.764510 \\
4 & 1 & 0 & -2.427788 & -1.124120 & 0.841907 \\
5 & 6 & 0 & -0.787622 & -0.000005 & -0.000015 \\
6 & 6 & 0 & 0.787559 & -0.000029 & -0.000000 \\
7 & 6 & 0 & 1.345109 & 1.034453 & -0.985351 \\
8 & 1 & 0 & 1.136458 & 0.766155 & -2.018789 \\
9 & 1 & 0 & 0.944464 & 2.030985 & -0.807915 \\
10 & 1 & 0 & 2.427782 & 1.096123 & -0.877910 \\
11 & 6 & 0 & -1.345051 & -0.047773 & -1.427883 \\
12 & 1 & 0 & -1.135974 & 0.867576 & -1.977360 \\
13 & 1 & 0 & -0.944599 & -0.883350 & -1.999263 \\
14 & 1 & 0 & -2.427764 & -0.166706 & -1.394560 \\
15 & 6 & 0 & -1.345045 & 1.260479 & 0.672522 \\
16 & 1 & 0 & -2.427722 & 1.291265 & 0.552557 \\
17 & 1 & 0 & -1.136267 & 1.278534 & 1.740035
\end{tabular}




$\begin{array}{rrrrrr}18 & 1 & 0 & -0.944302 & 2.173071 & 0.234787 \\ 19 & 6 & 0 & 1.345083 & -1.370606 & -0.403163 \\ 20 & 1 & 0 & 2.427786 & -1.308509 & -0.510044 \\ 21 & 1 & 0 & 1.136193 & -2.131462 & 0.345831 \\ 22 & 1 & 0 & 0.944634 & -1.715126 & -1.355001 \\ 23 & 6 & 0 & 1.345012 & 0.336130 & 1.388572 \\ 24 & 1 & 0 & 2.427669 & 0.212124 & 1.388439 \\ 25 & 1 & 0 & 1.136437 & 1.365324 & 1.672777 \\ 26 & 1 & 0 & 0.944177 & -0.315646 & 2.162895\end{array}$

Zero-point correction=

Thermal correction to Energy=

Thermal correction to Enthalpy=

Thermal correction to Gibbs Free Energy=

Sum of electronic and zero-point Energies=

Sum of electronic and thermal Energies=

Sum of electronic and thermal Enthalpies=

Sum of electronic and thermal Free Energies=

wB97XD/ccpVQZ

Ethane

Standard orientation:
0.245919 (Hartree/Particle)

$$
0.256324
$$

0.257268

$$
\begin{aligned}
& 0.212555 \\
& -315.391814 \\
& -315.381409 \\
& -315.380465 \\
& -315.425178
\end{aligned}
$$

\begin{tabular}{cccccc} 
Center & \multicolumn{2}{c}{ Atomic } & \multicolumn{2}{c}{ Atomic } & \multicolumn{2}{c}{ Coordinates (Angstrom } \\
Number & Number & Type & \multicolumn{1}{c}{ X } & Y & Z \\
- \hdashline 1 & 6 & 0 & -0.000000 & 0.000000 & 0.761514 \\
2 & 1 & 0 & 0.507730 & 0.879706 & 1.157053 \\
3 & 1 & 0 & 0.507983 & -0.879560 & 1.157053 \\
4 & 1 & 0 & -1.015713 & -0.000146 & 1.157053 \\
5 & 6 & 0 & 0.000000 & 0.000000 & -0.761514 \\
6 & 1 & 0 & 1.015713 & -0.000146 & -1.157053 \\
7 & 1 & 0 & -0.507983 & -0.879560 & -1.157053 \\
8 & 1 & 0 & -0.507730 & 0.879706 & -1.157053
\end{tabular}

Zero-point correction=

Thermal correction to Energy=

0.074927 (Hartree/Particle)

Thermal correction to Enthalpy=

0.078406

0.079351

Thermal correction to Gibbs Free Energy $=0.053516$

Sum of electronic and zero-point Energies=

$-79.764297$

Sum of electronic and thermal Energies $=$

$-79.760818$

\begin{tabular}{|c|c|c|c|c|}
\hline Center & Atomic & Atomic & Coordinat & es (Angstron \\
\hline Number & Number & Type & $\mathrm{X}$ & $\mathrm{Z}$ \\
\hline 1 & 6 & 0.000000 & 0.000000 & 0.000000 \\
\hline 2 & 1 & 0.627706 & 0.627706 & 0.627706 \\
\hline
\end{tabular}

Sum of electronic and thermal Enthalpies=

$-79.759874$

Sum of electronic and thermal Free Energies $=\quad-79.785708$

Methane

Standard orientation: 


$\begin{array}{rrrrrr}3 & 1 & 0 & -0.627706 & -0.627706 & 0.627706 \\ 4 & 1 & 0 & -0.627706 & 0.627706 & -0.627706 \\ 5 & 1 & 0 & 0.627706 & -0.627706 & -0.627706\end{array}$

Zero-point correction $=$

Thermal correction to Energy=

Thermal correction to Enthalpy=

Thermal correction to Gibbs Free Energy=

Sum of electronic and zero-point Energies=

Sum of electronic and thermal Energies=

Sum of electronic and thermal Enthalpies=

Sum of electronic and thermal Free Energies=

Propane

Standard orientation:

\begin{tabular}{cccccc}
$\begin{array}{c}\text { Center } \\
\text { Number }\end{array}$ & Atomic & Number & Atomic & \multicolumn{2}{c}{ Coordinates (Angstrom } \\
Nype & X & $Y$ & $Z$ \\
\hline 1 & 6 & 0 & -1.267374 & -0.259136 & 0.000000 \\
2 & 1 & 0 & -2.163719 & 0.360982 & -0.000031 \\
3 & 1 & 0 & -1.306508 & -0.902961 & 0.880308 \\
4 & 1 & 0 & -1.306481 & -0.903010 & -0.880273 \\
5 & 6 & 0 & 0.000000 & 0.586028 & 0.000000 \\
6 & 1 & 0 & -0.000001 & 1.241729 & 0.873279 \\
7 & 1 & 0 & 0.000001 & 1.241711 & -0.873292 \\
8 & 6 & 0 & 1.267375 & -0.259136 & 0.000000 \\
9 & 1 & 0 & 1.306516 & -0.902948 & 0.880317 \\
10 & 1 & 0 & 1.306469 & -0.903023 & -0.880264 \\
11 & 1 & 0 & 2.163721 & 0.360979 & -0.000050
\end{tabular}

Zero-point correction $=$

Thermal correction to Energy=

0.103857 (Hartree/Particle)

Thermal correction to Enthalpy=

0.108344

0.109288

Thermal correction to Gibbs Free Energy=

Sum of electronic and zero-point Energies=

0.078252

$-119.054220$

$-119.049733$

$-119.048789$

$-119.079824$

Sum of electronic and thermal Free Energies=

$-40.478359$

$-40.475491$

$-40.495667$

Sum of electronic and thermal Energies=

Butane

Standard orientation:

\begin{tabular}{|c|c|c|c|c|}
\hline \multirow{2}{*}{$\begin{array}{l}\text { Center } \\
\text { Number }\end{array}$} & \multirow{2}{*}{$\begin{array}{l}\text { Atomic } \\
\text { Number }\end{array}$} & \multirow{2}{*}{$\begin{array}{r}\text { Atomic } \\
\text { Type }\end{array}$} & \multicolumn{2}{|c|}{ Coordinates (Angstroms } \\
\hline & & & $\mathrm{X}$ & $\mathrm{Z}$ \\
\hline 1 & 6 & 1.947875 & -0.119867 & 0.000031 \\
\hline 2 & 1 & 2.089866 & -0.748879 & -0.880310 \\
\hline 3 & 1 & 2.089811 & -0.748844 & 0.880407 \\
\hline 4 & 1 & 2.733786 & 0.635212 & 0.000040 \\
\hline 5 & 6 & 0.563035 & 0.513421 & -0.000035 \\
\hline 6 & 1 & 0.456112 & 1.161895 & 0.873798 \\
\hline
\end{tabular}




$\begin{array}{cccccc}7 & 1 & 0 & 0.456158 & 1.161849 & -0.873910 \\ 8 & 6 & 0 & -0.563035 & -0.513421 & -0.000036 \\ 9 & 1 & 0 & -0.456158 & -1.161848 & -0.873911 \\ 10 & 1 & 0 & -0.456112 & -1.161897 & 0.873797 \\ 11 & 6 & 0 & -1.947875 & 0.119868 & 0.000032 \\ 12 & 1 & 0 & -2.089865 & 0.748881 & -0.880309 \\ 13 & 1 & 0 & -2.733786 & -0.635211 & 0.000037 \\ 14 & 1 & 0 & -2.089812 & 0.748841 & 0.880409\end{array}$

Zero-point correction=

Thermal correction to Energy=

Thermal correction to Enthalpy=

Thermal correction to Gibbs Free Energy=

Sum of electronic and zero-point Energies=

Sum of electronic and thermal Energies=

Sum of electronic and thermal Enthalpies=

Sum of electronic and thermal Free Energies=

Isobutane

Standard orientation:

\begin{tabular}{|c|c|c|c|c|}
\hline \multirow{2}{*}{$\begin{array}{l}\text { Center } \\
\text { Number }\end{array}$} & Atomic & \multirow{2}{*}{$\begin{array}{l}\text { Atomic } \\
\text { Type }\end{array}$} & \multicolumn{2}{|c|}{ Coordinates (Angstroms } \\
\hline & Numbe & & $X$ & Z \\
\hline 1 & 6 & 1.000778 & -1.051333 & -0.095570 \\
\hline 2 & 1 & 0.729006 & -2.045398 & 0.261446 \\
\hline 3 & 1 & 1.034562 & -1.086985 & -1.187031 \\
\hline 4 & 1 & 2.007047 & -0.828679 & 0.261233 \\
\hline 5 & 6 & -0.000011 & 0.000002 & 0.374207 \\
\hline 6 & 1 & 0.000011 & 0.000011 & 1.468156 \\
\hline 7 & 6 & -1.410879 & -0.341030 & -0.095567 \\
\hline 8 & 1 & -2.135847 & 0.391485 & 0.261272 \\
\hline 9 & 1 & -1.458559 & -0.352599 & -1.187026 \\
\hline 10 & 1 & -1.721295 & -1.323754 & 0.261332 \\
\hline 11 & 6 & 0.410100 & 1.392361 & -0.095572 \\
\hline 12 & 1 & -0.285615 & 2.152582 & 0.261556 \\
\hline 13 & 1 & 1.407043 & 1.653797 & 0.261103 \\
\hline 14 & 1 & 0.423717 & 1.439535 & -1.187031 \\
\hline
\end{tabular}

Zero-point correction=

Thermal correction to Energy=

Thermal correction to Enthalpy=

Thermal correction to Gibbs Free Energy=

Sum of electronic and zero-point Energies $=$

Sum of electronic and thermal Energies $=$

Sum of electronic and thermal Enthalpies=

Sum of electronic and thermal Free Energies=

Pentane

Standard orientation:
0.138273

0.139217

$-158.344513$

$-158.338757$

$-158.337812$

$-158.372499$ 


\begin{tabular}{cccccc} 
Number & Number & Type & $X$ & $Y$ & $Z$ \\
\hline 1 & 6 & 0 & 2.540417 & 0.324105 & -0.000010 \\
2 & 1 & 0 & 2.579845 & 0.967727 & -0.880314 \\
3 & 1 & 0 & 2.579866 & 0.967716 & 0.880301 \\
4 & 1 & 0 & 3.436903 & -0.295679 & -0.000024 \\
5 & 6 & 0 & 1.274491 & -0.522338 & 0.000002 \\
6 & 1 & 0 & 1.273212 & -1.179222 & -0.873903 \\
7 & 1 & 0 & 1.273231 & -1.179227 & 0.873902 \\
8 & 6 & 0 & 0.000000 & 0.312359 & 0.000020 \\
9 & 1 & 0 & -0.000003 & 0.970986 & 0.874449 \\
10 & 1 & 0 & 0.000003 & 0.971024 & -0.874380 \\
11 & 6 & 0 & -1.274491 & -0.522338 & -0.000001 \\
12 & 1 & 0 & -1.273231 & -1.179236 & 0.873894 \\
13 & 1 & 0 & -1.273212 & -1.179212 & -0.873911 \\
14 & 6 & 0 & -2.540417 & 0.324105 & -0.000008 \\
15 & 1 & 0 & -3.436903 & -0.295679 & -0.000043 \\
16 & 1 & 0 & -2.579872 & 0.967699 & 0.880314 \\
17 & 1 & 0 & -2.579839 & 0.967744 & -0.880301
\end{tabular}

Zero-point correction=

Thermal correction to Energy=

Thermal correction to Enthalpy=

Thermal correction to Gibbs Free Energy=

Sum of electronic and zero-point Energies=

Sum of electronic and thermal Energies $=$

Sum of electronic and thermal Enthalpies=

Sum of electronic and thermal Free Energies=

Neopentane
0.161255 (Hartree/Particle)

0.168259

0.169204

0.131064

$-197.634721$

$-197.627717$

$-197.626772$

$-197.664912$

Standard orientation:

\begin{tabular}{|c|c|c|c|c|}
\hline \multirow{2}{*}{$\begin{array}{l}\text { Center } \\
\text { Number }\end{array}$} & Atomic & \multirow{2}{*}{$\begin{array}{l}\text { Atomic } \\
\text { Type }\end{array}$} & \multicolumn{2}{|c|}{ Coordinates (Angstroms } \\
\hline & Numbe & & $\mathrm{X}$ & $\mathrm{Z}$ \\
\hline 1 & 6 & -0.714074 & -1.330463 & -0.246844 \\
\hline 2 & 1 & -0.304866 & -2.117585 & 0.388826 \\
\hline 3 & 1 & -1.781012 & -1.247078 & -0.032892 \\
\hline 4 & 1 & -0.603776 & -1.646643 & -1.285568 \\
\hline 5 & 6 & 0.000031 & -0.000017 & -0.000032 \\
\hline 6 & 6 & -0.176783 & 0.409863 & 1.463425 \\
\hline 7 & 1 & 0.323322 & 1.358311 & 1.666973 \\
\hline 8 & 1 & -1.233025 & 0.527380 & 1.711685 \\
\hline 9 & 1 & 0.243739 & -0.341918 & 2.133565 \\
\hline 10 & 6 & 1.490294 & -0.155876 & -0.309515 \\
\hline 11 & 1 & 1.645138 & -0.449071 & -1.349302 \\
\hline 12 & 1 & 2.024482 & 0.780936 & -0.141853 \\
\hline 13 & 1 & 1.943695 & -0.919020 & 0.325407 \\
\hline 14 & 6 & -0.599431 & 1.076492 & -0.907086 \\
\hline 15 & 1 & -0.486369 & 0.808774 & -1.959054 \\
\hline 16 & 1 & -1.664187 & 1.207922 & -0.706788 \\
\hline
\end{tabular}


Zero-point correction $=$

0.159995 (Hartree/Particle)

Thermal correction to Energy=

0.166812

Thermal correction to Enthalpy=

0.167756

Thermal correction to Gibbs Free Energy=

0.130989

Sum of electronic and zero-point Energies=

$-197.639938$

Sum of electronic and thermal Energies $=$

$-197.633121$

Sum of electronic and thermal Enthalpies=

$-197.632177$

Sum of electronic and thermal Free Energies= $-197.668944$

Hexane

Standard orientation:

\begin{tabular}{|c|c|c|c|c|}
\hline \multirow{2}{*}{$\begin{array}{l}\text { Center } \\
\text { Number }\end{array}$} & Atomic & \multirow{2}{*}{$\begin{array}{l}\text { Atomic } \\
\text { Type }\end{array}$} & \multicolumn{2}{|c|}{ Coordinates (Angstroms } \\
\hline & Numbe & & $\mathrm{X}$ & $\mathrm{Z}$ \\
\hline 1 & 6 & -3.199234 & 0.207963 & 0.000029 \\
\hline 2 & 1 & -3.283181 & 0.847276 & 0.880378 \\
\hline 3 & 1 & -3.283177 & 0.847402 & -0.880231 \\
\hline 4 & 1 & -4.050696 & -0.472374 & -0.000024 \\
\hline 5 & 6 & -1.877704 & -0.548639 & -0.000028 \\
\hline 6 & 1 & -1.830903 & -1.203927 & 0.873798 \\
\hline 7 & 1 & -1.830928 & -1.203849 & -0.873914 \\
\hline 8 & 6 & -0.664149 & 0.372525 & -0.000006 \\
\hline 9 & 1 & -0.710481 & 1.029320 & -0.874427 \\
\hline 10 & 1 & -0.710490 & 1.029297 & 0.874432 \\
\hline 11 & 6 & 0.664149 & -0.372525 & -0.000002 \\
\hline 12 & 1 & 0.710479 & -1.029327 & -0.874419 \\
\hline 13 & 1 & 0.710492 & -1.029291 & 0.874440 \\
\hline 14 & 6 & 1.877704 & 0.548639 & -0.000027 \\
\hline 15 & 1 & 1.830903 & 1.203929 & 0.873798 \\
\hline 16 & 1 & 1.830928 & 1.203847 & -0.873913 \\
\hline 17 & 6 & 3.199234 & -0.207964 & 0.000027 \\
\hline 18 & 1 & 3.283186 & -0.847389 & -0.880241 \\
\hline 19 & 1 & 4.050696 & 0.472374 & -0.000005 \\
\hline 20 & 1 & 3.283171 & -0.847289 & 0.880368 \\
\hline
\end{tabular}

Zero-point correction=

Thermal correction to Energy=

Thermal correction to Enthalpy=

Thermal correction to Gibbs Free Energy=

Sum of electronic and zero-point Energies=

Sum of electronic and thermal Energies $=$

Sum of electronic and thermal Enthalpies=

Sum of electronic and thermal Free Energies=

22dimethylbutane

Standard orientation:
0.189878 (Hartree/Particle)

$$
0.198222
$$

0.199166

$$
\begin{gathered}
0.157391 \\
-236.925050 \\
-236.916706 \\
-236.915762 \\
-236.957537
\end{gathered}
$$

Center Atomic Atomic Coordinates (Angstroms) 


\begin{tabular}{cccccc} 
Number & Number & Type & $X$ & $Y$ & $Z$ \\
\hline 1 & 6 & 0 & -1.611879 & 0.976421 & 0.000688 \\
2 & 1 & 0 & -1.588459 & 1.617744 & 0.883501 \\
3 & 1 & 0 & -1.588471 & 1.618921 & -0.881268 \\
4 & 1 & 0 & -2.562691 & 0.440997 & 0.000335 \\
5 & 6 & 0 & -0.432725 & -0.000089 & 0.000014 \\
6 & 6 & 0 & 0.867936 & 0.824757 & 0.000474 \\
7 & 1 & 0 & 0.851584 & 1.482747 & -0.872977 \\
8 & 1 & 0 & 0.851629 & 1.481713 & 0.874705 \\
9 & 6 & 0 & 2.172230 & 0.036553 & -0.000005 \\
10 & 1 & 0 & 2.261992 & -0.598190 & -0.881321 \\
11 & 1 & 0 & 3.024849 & 0.715128 & 0.000287 \\
12 & 1 & 0 & 2.262083 & -0.599064 & 0.880670 \\
13 & 6 & 0 & -0.517702 & -0.880371 & 1.249294 \\
14 & 1 & 0 & 0.269049 & -1.634627 & 1.266373 \\
15 & 1 & 0 & -0.430436 & -0.280427 & 2.157074 \\
16 & 1 & 0 & -1.474559 & -1.403613 & 1.284757 \\
17 & 6 & 0 & -0.517859 & -0.878740 & -1.250413 \\
18 & 1 & 0 & -1.474734 & -1.401911 & -1.286435 \\
19 & 1 & 0 & -0.430701 & -0.277599 & -2.157410 \\
20 & 1 & 0 & 0.268860 & -1.633004 & -1.268602 \\
------------------------------------------------------------
\end{tabular}

Zero-point correction=

Thermal correction to Energy=

Thermal correction to Enthalpy=

Thermal correction to Gibbs Free Energy=

Sum of electronic and zero-point Energies=

Sum of electronic and thermal Energies=

Sum of electronic and thermal Enthalpies=

Sum of electronic and thermal Free Energies=

Heptane

\subsection{9 (Hartree/Particle)}

0.196868

0.197812

0.157361

$-236.928527$

$-236.920329$

$-236.919385$

$-236.959835$

Standard orientation:

\begin{tabular}{|c|c|c|c|c|}
\hline \multirow{2}{*}{$\begin{array}{l}\text { Center } \\
\text { Number }\end{array}$} & Atomic & \multirow{2}{*}{$\begin{array}{l}\text { Atomic } \\
\text { Type }\end{array}$} & \multicolumn{2}{|c|}{ Coordinates (Angstroms) } \\
\hline & Numbe & & $\mathrm{X}$ & $\mathrm{Z}$ \\
\hline 1 & 6 & -3.812996 & -0.353181 & 0.000001 \\
\hline 2 & 1 & -3.852464 & -0.996751 & -0.880209 \\
\hline 3 & 1 & -3.852461 & -0.996755 & 0.880209 \\
\hline 4 & 1 & -4.709306 & 0.266610 & 0.000004 \\
\hline 5 & 6 & -2.547275 & 0.493414 & 0.000001 \\
\hline 6 & 1 & -2.545762 & 1.150190 & -0.873837 \\
\hline 7 & 1 & -2.545760 & 1.150186 & 0.873843 \\
\hline 8 & 6 & -1.273039 & -0.341628 & -0.000002 \\
\hline 9 & 1 & -1.273684 & -0.999879 & 0.874377 \\
\hline 10 & 1 & -1.273685 & -0.999875 & -0.874383 \\
\hline 11 & 6 & 0.000000 & 0.494164 & -0.000001 \\
\hline 12 & 1 & -0.000000 & 1.152153 & 0.874443 \\
\hline
\end{tabular}




$\begin{array}{rrrrrr}13 & 1 & 0 & 0.000000 & 1.152154 & -0.874445 \\ 14 & 6 & 0 & 1.273039 & -0.341628 & -0.000002 \\ 15 & 1 & 0 & 1.273684 & -0.999876 & -0.874382 \\ 16 & 1 & 0 & 1.273685 & -0.999878 & 0.874378 \\ 17 & 6 & 0 & 2.547275 & 0.493414 & -0.000000 \\ 18 & 1 & 0 & 2.545760 & 1.150188 & 0.873840 \\ 19 & 1 & 0 & 2.545762 & 1.150188 & -0.873840 \\ 20 & 6 & 0 & 3.812996 & -0.353181 & 0.000002 \\ 21 & 1 & 0 & 3.852465 & -0.996752 & -0.880207 \\ 22 & 1 & 0 & 4.709306 & 0.266610 & 0.000005 \\ 23 & 1 & 0 & 3.852461 & -0.996754 & 0.880210\end{array}$

$\begin{array}{lc}\text { Zero-point correction }= & 0.218541 \text { (Hartree/Particle) } \\ \text { Thermal correction to Energy= } & 0.228204 \\ \text { Thermal correction to Enthalpy= } & 0.229148 \\ \text { Thermal correction to Gibbs Free Energy= } & 0.183873 \\ \text { Sum of electronic and zero-point Energies }= & -276.215356 \\ \text { Sum of electronic and thermal Energies }= & -276.205694 \\ \text { Sum of electronic and thermal Enthalpies }= & -276.204749 \\ \text { Sum of electronic and thermal Free Energies }= & -276.250025 \\ \text { 223trimethylbutane } & \end{array}$

Standard orientation:

\begin{tabular}{|c|c|c|c|c|}
\hline \multirow{2}{*}{$\begin{array}{l}\text { Center } \\
\text { Number }\end{array}$} & Atomic & \multirow{2}{*}{$\begin{array}{l}\text { Atomic } \\
\text { Type }\end{array}$} & \multicolumn{2}{|c|}{ Coordinates (Angstroms) } \\
\hline & Number & & $\mathrm{X}$ & $\mathrm{Z}$ \\
\hline 1 & 6 & -1.319190 & -1.300204 & -0.446592 \\
\hline 2 & 1 & -1.164705 & -1.478992 & -1.512637 \\
\hline 3 & 1 & -0.943019 & -2.164641 & 0.099106 \\
\hline 4 & 1 & -2.394246 & -1.245317 & -0.269989 \\
\hline 5 & 6 & -0.640113 & 0.000224 & -0.000943 \\
\hline 6 & 6 & 0.849186 & -0.000895 & -0.451444 \\
\hline 7 & 1 & 0.823880 & -0.005491 & -1.546654 \\
\hline 8 & 6 & 1.623201 & 1.245384 & -0.020114 \\
\hline 9 & 1 & 1.755453 & 1.273385 & 1.062479 \\
\hline 10 & 1 & 2.617662 & 1.240106 & -0.466830 \\
\hline 11 & 1 & 1.134867 & 2.169403 & -0.324045 \\
\hline 12 & 6 & 1.626587 & -1.243762 & -0.015973 \\
\hline 13 & 1 & 1.657712 & -1.334899 & 1.071046 \\
\hline 14 & 1 & 1.204115 & -2.161644 & -0.419832 \\
\hline 15 & 1 & 2.657872 & -1.178940 & -0.363799 \\
\hline 16 & 6 & -0.781991 & 0.134917 & 1.518034 \\
\hline 17 & 1 & -0.390597 & 1.086511 & 1.877364 \\
\hline 18 & 1 & -1.834540 & 0.085740 & 1.801241 \\
\hline 19 & 1 & -0.263841 & -0.666221 & 2.045670 \\
\hline 20 & 6 & -1.379356 & 1.164835 & -0.670714 \\
\hline 21 & 1 & -1.284491 & 1.115492 & -1.757104 \\
\hline 22 & 1 & -2.442155 & 1.129369 & -0.427013 \\
\hline 23 & 1 & -1.003909 & 2.133144 & -0.342527 \\
\hline
\end{tabular}


Zero-point correction=

Thermal correction to Energy=

Thermal correction to Enthalpy=

Thermal correction to Gibbs Free Energy=

Sum of electronic and zero-point Energies=

Sum of electronic and thermal Energies $=$

Sum of electronic and thermal Enthalpies=

Sum of electronic and thermal Free Energies=

Octane

Standard orientation:
0.217455 (Hartree/Particle)

0.226813

0.227757

0.184385

$-276.216962$

$-276.207604$

$-276.206660$

$-276.250032$

\begin{tabular}{|c|c|c|c|c|}
\hline \multirow{2}{*}{$\begin{array}{l}\text { Center } \\
\text { Number }\end{array}$} & Atomic & \multirow{2}{*}{$\begin{array}{l}\text { Atomic } \\
\text { Type }\end{array}$} & \multicolumn{2}{|c|}{ Coordinates (Angstroms } \\
\hline & Numbe & & $\mathrm{X}$ & Z \\
\hline 1 & 6 & -4.462863 & 0.256088 & 0.000006 \\
\hline 2 & 1 & -4.527288 & 0.897656 & 0.880213 \\
\hline 3 & 1 & -4.527294 & 0.897666 & -0.880194 \\
\hline 4 & 1 & -5.334453 & -0.398008 & 0.000005 \\
\hline 5 & 6 & -3.165173 & -0.540636 & -0.000004 \\
\hline 6 & 1 & -3.138150 & -1.196871 & 0.873827 \\
\hline 7 & 1 & -3.138157 & -1.196862 & -0.873842 \\
\hline 8 & 6 & -1.924385 & 0.343324 & -0.000003 \\
\hline 9 & 1 & -1.950585 & 1.001057 & -0.874388 \\
\hline 10 & 1 & -1.950584 & 1.001057 & 0.874381 \\
\hline 11 & 6 & -0.619853 & -0.442344 & -0.000002 \\
\hline 12 & 1 & -0.594286 & -1.099857 & -0.874401 \\
\hline 13 & 1 & -0.594286 & -1.099854 & 0.874401 \\
\hline 14 & 6 & 0.619853 & 0.442344 & -0.000003 \\
\hline 15 & 1 & 0.594284 & 1.099859 & 0.874396 \\
\hline 16 & 1 & 0.594288 & 1.099852 & -0.874406 \\
\hline 17 & 6 & 1.924385 & -0.343324 & 0.000003 \\
\hline 18 & 1 & 1.950585 & -1.001062 & -0.874378 \\
\hline 19 & 1 & 1.950584 & -1.001052 & 0.874390 \\
\hline 20 & 6 & 3.165173 & 0.540636 & -0.000001 \\
\hline 21 & 1 & 3.138153 & 1.196870 & 0.873832 \\
\hline 22 & 1 & 3.138154 & 1.196863 & -0.873838 \\
\hline 23 & 6 & 4.462863 & -0.256088 & 0.000002 \\
\hline 24 & 1 & 4.527292 & -0.897664 & -0.880200 \\
\hline 25 & 1 & 5.334453 & 0.398008 & 0.000001 \\
\hline 26 & 1 & 4.527291 & -0.897659 & 0.880208 \\
\hline
\end{tabular}

Zero-point correction=

Thermal correction to Energy=

Thermal correction to Enthalpy=

Thermal correction to Gibbs Free Energy=

Sum of electronic and zero-point Energies=

Sum of electronic and thermal Energies=

Sum of electronic and thermal Enthalpies=

Sum of electronic and thermal Free Energies=

2233tetramethylbutane
0.247158 (Hartree/Particle)

$$
0.258174
$$

0.259118

$$
0.210229
$$

$-315.505702$

$-315.494687$

$-315.493742$

$-315.542631$ 
Standard orientation:

\begin{tabular}{|c|c|c|c|c|c|}
\hline \multirow{2}{*}{$\begin{array}{l}\text { Center } \\
\text { Number }\end{array}$} & \multicolumn{2}{|c|}{ Atomic } & \multirow{2}{*}{$\begin{array}{l}\text { Atomic } \\
\text { Type }\end{array}$} & \multicolumn{2}{|c|}{ Coordinates (Angstroms } \\
\hline & & & & $X$ & Z \\
\hline 1 & 6 & 0 & -1.344853 & -0.672330 & 1.262759 \\
\hline 2 & 1 & 0 & -0.934007 & -1.670749 & 1.412826 \\
\hline 3 & 1 & 0 & -1.142357 & -0.085122 & 2.157572 \\
\hline 4 & 1 & 0 & -2.427512 & -0.775047 & 1.177677 \\
\hline 5 & 6 & 0 & -0.788135 & -0.000026 & 0.000007 \\
\hline 6 & 6 & 0 & 0.788028 & -0.000115 & -0.000044 \\
\hline 7 & 6 & 0 & 1.344688 & 0.939729 & -1.078607 \\
\hline 8 & 1 & 0 & 0.932949 & 0.724512 & -2.064671 \\
\hline 9 & 1 & 0 & 1.143240 & 1.985213 & -0.848714 \\
\hline 10 & 1 & 0 & 2.427207 & 0.822814 & -1.145059 \\
\hline 11 & 6 & 0 & -1.345130 & -0.757247 & -1.213584 \\
\hline 12 & 1 & 0 & -0.932442 & -0.389782 & -2.153184 \\
\hline 13 & 1 & 0 & -1.145326 & -1.826209 & -1.151296 \\
\hline 14 & 1 & 0 & -2.427441 & -0.629741 & -1.261183 \\
\hline 15 & 6 & 0 & -1.344427 & 1.429885 & -0.048956 \\
\hline 16 & 1 & 0 & -2.427047 & 1.408034 & 0.082857 \\
\hline 17 & 1 & 0 & -0.932986 & 2.058833 & 0.740535 \\
\hline 18 & 1 & 0 & -1.141918 & 1.911120 & -1.004940 \\
\hline 19 & 6 & 0 & 1.344808 & -1.404098 & -0.274693 \\
\hline 20 & 1 & 0 & 2.427345 & -1.402937 & -0.140426 \\
\hline 21 & 1 & 0 & 0.933374 & -2.150390 & 0.405001 \\
\hline 22 & 1 & 0 & 1.143294 & -1.727873 & -1.295024 \\
\hline 23 & 6 & 0 & 1.344884 & 0.464091 & 1.353093 \\
\hline 24 & 1 & 0 & 2.427321 & 0.580472 & 1.284575 \\
\hline 25 & 1 & 0 & 0.932948 & 1.425477 & 1.660037 \\
\hline 26 & 1 & 0 & 1.144188 & -0.257958 & 2.143566 \\
\hline
\end{tabular}

Zero-point correction=

Thermal correction to Energy=

Thermal correction to Enthalpy=

Thermal correction to Gibbs Free Energy=

Sum of electronic and zero-point Energies=

Sum of electronic and thermal Energies=

Sum of electronic and thermal Enthalpies=

Sum of electronic and thermal Free Energies=

B3LYPD3/ccpVQZ

Ethane

Standard orientation:

\begin{tabular}{cccrrr} 
Center & \multicolumn{2}{c}{ Atomic } & \multicolumn{2}{c}{ Atomic } & \multicolumn{2}{c}{ Coordinates (Angstroms) } \\
Number & Number & Type & \multicolumn{1}{c}{ X } & Y & Z \\
-1 & 6 & 0 & 0.000000 & -0.000000 & 0.763939 \\
2 & 1 & 0 & -0.507947 & 0.879842 & 1.160638 \\
3 & 1 & 0 & 1.015939 & -0.000026 & 1.160638
\end{tabular}




$\begin{array}{rrrrrr}4 & 1 & 0 & -0.507992 & -0.879816 & 1.160638 \\ 5 & 6 & 0 & -0.000000 & 0.000000 & -0.763939 \\ 6 & 1 & 0 & 0.507947 & 0.879842 & -1.160638 \\ 7 & 1 & 0 & 0.507992 & -0.879816 & -1.160638 \\ 8 & 1 & 0 & -1.015939 & -0.000026 & -1.160638\end{array}$

Zero-point correction=

Thermal correction to Energy=

Thermal correction to Enthalpy=

Thermal correction to Gibbs Free Energy=

Sum of electronic and zero-point Energies=

Sum of electronic and thermal Energies=

Sum of electronic and thermal Enthalpies=

Sum of electronic and thermal Free Energies=

Methane

Standard orientation:
0.074360 (Hartree/Particle)

0.077837

0.078781

$$
0.052948
$$

$-79.797975$

$-79.794498$

$-79.793553$

$-79.819387$

\begin{tabular}{|c|c|c|c|c|}
\hline \multirow{2}{*}{$\begin{array}{l}\text { Center } \\
\text { Number }\end{array}$} & Atomic & \multirow{2}{*}{$\begin{array}{l}\text { Atomic } \\
\text { Type }\end{array}$} & \multicolumn{2}{|c|}{ Coordinates (Angstrom } \\
\hline & Numbe & & $X$ & Z \\
\hline 1 & 6 & 0.000000 & 0.000000 & 0.000000 \\
\hline 2 & 1 & 0.627846 & 0.627846 & 0.627846 \\
\hline 3 & 1 & -0.627846 & -0.627846 & 0.627846 \\
\hline 4 & 1 & -0.627846 & 0.627846 & -0.627846 \\
\hline 5 & 1 & 0.627846 & -0.627846 & -0.627846 \\
\hline
\end{tabular}

Zero-point correction=

Thermal correction to Energy=

Thermal correction to Enthalpy=

Thermal correction to Gibbs Free Energy=

Sum of electronic and zero-point Energies=

Sum of electronic and thermal Energies=

Sum of electronic and thermal Enthalpies=

Sum of electronic and thermal Free Energies=

Propane

Standard orientation:
0.044539 (Hartree/Particle)

0.047408

$$
0.048352
$$

0.027230

$-40.497117$

$-40.494248$

$-40.493304$

\begin{tabular}{|c|c|c|c|c|}
\hline \multirow{2}{*}{$\begin{array}{l}\text { Center } \\
\text { Number }\end{array}$} & Atomic & \multirow{2}{*}{$\begin{array}{l}\text { Atomic } \\
\text { Type }\end{array}$} & \multicolumn{2}{|c|}{ Coordinates (Angstrom } \\
\hline & Numbe & & $\mathrm{X}$ & $\mathrm{Z}$ \\
\hline 1 & 6 & -1.271593 & -0.259731 & 0.000000 \\
\hline 2 & 1 & -2.168853 & 0.360193 & -0.000020 \\
\hline 3 & 1 & -1.311690 & -0.904148 & 0.880369 \\
\hline 4 & 1 & -1.311674 & -0.904180 & -0.880346 \\
\hline 5 & 6 & -0.000000 & 0.587437 & 0.000000 \\
\hline 6 & 1 & -0.000001 & 1.244218 & 0.873488 \\
\hline 7 & 1 & 0.000001 & 1.244202 & -0.873499 \\
\hline 8 & 6 & 1.271593 & -0.259731 & 0.000000 \\
\hline 9 & 1 & 1.311717 & -0.904107 & 0.880398 \\
\hline
\end{tabular}

$-40.514426$ 


$\begin{array}{rrrrrr}10 & 1 & 0 & 1.311644 & -0.904220 & -0.880318 \\ 11 & 1 & 0 & 2.168855 & 0.360190 & -0.000077\end{array}$

Zero-point correction $=$

Thermal correction to Energy=

0.103097 (Hartree/Particle)

Thermal correction to Enthalpy=

0.107584

0.108528

Thermal correction to Gibbs Free Energy=

0.077487

Sum of electronic and zero-point Energies $=$

$-119.102745$

Sum of electronic and thermal Energies $=$

$-119.098257$

Sum of electronic and thermal Enthalpies=

$-119.097313$

Sum of electronic and thermal Free Energies $=\quad-119.128354$

Butane

Standard orientation:

\begin{tabular}{|c|c|c|c|c|c|}
\hline \multirow{2}{*}{$\begin{array}{l}\text { Center } \\
\text { Number }\end{array}$} & \multirow{2}{*}{\multicolumn{2}{|c|}{$\begin{array}{l}\text { Atomic } \\
\text { Number }\end{array}$}} & \multirow{2}{*}{$\begin{array}{r}\text { Atomic } \\
\text { Type }\end{array}$} & \multicolumn{2}{|c|}{ Coordinates (Angstrom } \\
\hline & & & & $X$ & Z \\
\hline 1 & 6 & 0 & 1.954541 & -0.120054 & 0.000051 \\
\hline 2 & 1 & 0 & 2.097626 & -0.749436 & -0.880348 \\
\hline 3 & 1 & 0 & 2.097516 & -0.749401 & 0.880492 \\
\hline 4 & 1 & 0 & 2.741223 & 0.635138 & 0.000084 \\
\hline 5 & 6 & 0 & 0.565058 & 0.514507 & -0.000058 \\
\hline 6 & 1 & 0 & 0.458115 & 1.163841 & 0.873855 \\
\hline 7 & 1 & 0 & 0.458196 & 1.163760 & -0.874042 \\
\hline 8 & 6 & 0 & -0.565058 & -0.514507 & -0.000058 \\
\hline 9 & 1 & 0 & -0.458196 & -1.163758 & -0.874043 \\
\hline 10 & 1 & 0 & -0.458115 & -1.163843 & 0.873854 \\
\hline 11 & 6 & 0 & -1.954541 & 0.120054 & 0.000051 \\
\hline 12 & 1 & 0 & -2.097621 & 0.749448 & -0.880340 \\
\hline 13 & 1 & 0 & -2.741224 & -0.635138 & 0.000069 \\
\hline 14 & 1 & 0 & -2.097521 & 0.749388 & 0.880500 \\
\hline
\end{tabular}

Zero-point correction $=$

Thermal correction to Energy=

0.131632 (Hartree/Particle)

Thermal correction to Enthalpy=

0.137349

0.138293

Thermal correction to Gibbs Free Energy=

0.103714

Sum of electronic and zero-point Energies=

$-158.407747$

Sum of electronic and thermal Energies=

$-158.402030$

Sum of electronic and thermal Enthalpies=

$-158.401086$

Sum of electronic and thermal Free Energies $=\quad-158.435665$

Isobutane

Standard orientation:

\begin{tabular}{cccccc}
$\begin{array}{c}\text { Center } \\
\text { Number }\end{array}$ & \multicolumn{2}{c}{ Atomic } & \multicolumn{2}{c}{ Atomic } & \multicolumn{2}{c}{ Coordinates (Angstroms) } \\
- & Number & Type & X & Y & Z \\
\hline 1 & 6 & 0 & -1.456073 & 0.027308 & -0.095714 \\
2 & 1 & 0 & -1.972612 & 0.919428 & 0.261423 \\
3 & 1 & 0 & -1.506799 & 0.028401 & -1.187226
\end{tabular}




$\begin{array}{cccccc}4 & 1 & 0 & -2.005671 & -0.844932 & 0.261199 \\ 5 & 6 & 0 & -0.000011 & 0.000005 & 0.374548 \\ 6 & 1 & 0 & 0.000016 & 0.000002 & 1.469454 \\ 7 & 6 & 0 & 0.751687 & 1.247311 & -0.095720 \\ 8 & 1 & 0 & 1.782687 & 1.248318 & 0.261044 \\ 9 & 1 & 0 & 0.777584 & 1.290887 & -1.187229 \\ 10 & 1 & 0 & 0.271415 & 2.159419 & 0.261562 \\ 11 & 6 & 0 & 0.704384 & -1.274619 & -0.095716 \\ 12 & 1 & 0 & 1.734569 & -1.314434 & 0.261190 \\ 13 & 1 & 0 & 0.190096 & -2.168043 & 0.261413 \\ 14 & 1 & 0 & 0.728793 & -1.319075 & -1.187221\end{array}$

Zero-point correction=

0.131251 (Hartree/Particle)

Thermal correction to Energy=

Thermal correction to Enthalpy=

0.136863

0.137807

Thermal correction to Gibbs Free Energy=

Sum of electronic and zero-point Energies=

0.103732

$-158.409803$

$-158.404191$

Sum of electronic and thermal Energies=

$-158.403247$

Sum of electronic and thermal Enthalpies $=$

$-158.437323$

Sum of electronic and thermal Free Energies=

Pentane

Standard orientation:

\begin{tabular}{|c|c|c|c|c|c|}
\hline \multirow{2}{*}{$\begin{array}{l}\text { Center } \\
\text { Number }\end{array}$} & Atomic & \multicolumn{2}{|c|}{ Atomic } & \multicolumn{2}{|c|}{ Coordinates (Angstroms) } \\
\hline & \multicolumn{2}{|c|}{ Number } & Type & $\mathrm{X}$ & Z \\
\hline 1 & 6 & 0 & 2.549244 & 0.325075 & -0.000008 \\
\hline 2 & 1 & 0 & 2.589685 & 0.969246 & -0.880384 \\
\hline 3 & 1 & 0 & 2.589691 & 0.969265 & 0.880354 \\
\hline 4 & 1 & 0 & 3.446599 & -0.294611 & -0.000004 \\
\hline 5 & 6 & 0 & 1.279046 & -0.523536 & 0.000006 \\
\hline 6 & 1 & 0 & 1.278135 & -1.181291 & -0.873950 \\
\hline 7 & 1 & 0 & 1.278147 & -1.181280 & 0.873969 \\
\hline 8 & 6 & 0 & 0.000000 & 0.312523 & 0.000012 \\
\hline 9 & 1 & 0 & -0.000005 & 0.971854 & 0.874357 \\
\hline 10 & 1 & 0 & 0.000005 & 0.971875 & -0.874317 \\
\hline 11 & 6 & 0 & -1.279046 & -0.523536 & -0.000005 \\
\hline 12 & 1 & 0 & -1.278147 & -1.181299 & 0.873945 \\
\hline 13 & 1 & 0 & -1.278135 & -1.181271 & -0.873974 \\
\hline 14 & 6 & 0 & -2.549244 & 0.325075 & -0.000003 \\
\hline 15 & 1 & 0 & -3.446599 & -0.294611 & -0.000082 \\
\hline 16 & 1 & 0 & -2.589722 & 0.969196 & 0.880407 \\
\hline 17 & 1 & 0 & -2.589654 & 0.969314 & -0.880331 \\
\hline Zero-po & t correcti & Unt- & & 0.16014 & 0 (Hartree/Particle) \\
\hline Thermal & correctio & $n$ to & Energy= & 0.1 & 67137 \\
\hline Thermal & correctio & $n$ to & Enthalpy= & 0.1 & 68081 \\
\hline Thermal & correctio & $n$ to & Gibbs Free E & nergy $=$ & 0.129963 \\
\hline Sum of e & lectronic & and & zero-point E & nergies $=$ & -197.712730 \\
\hline Sum of e & lectronic & and & hermal En & gies $=$ & -197.705734 \\
\hline
\end{tabular}


Sum of electronic and thermal Enthalpies=

Sum of electronic and thermal Free Energies=

Neopentane

Standard orientation:

\begin{tabular}{|c|c|c|c|c|}
\hline \multirow{2}{*}{$\begin{array}{l}\text { Center } \\
\text { Number }\end{array}$} & Atomic & \multirow{2}{*}{$\begin{array}{r}\text { Atomic } \\
\text { Type }\end{array}$} & \multicolumn{2}{|c|}{ Coordinates (Angstrom } \\
\hline & Numbe & & $\mathrm{X}$ & $\mathrm{Z}$ \\
\hline 1 & 6 & -1.437741 & -0.537858 & 0.023650 \\
\hline 2 & 1 & -1.851579 & -0.593877 & -0.984672 \\
\hline 3 & 1 & -1.474344 & -1.538975 & 0.456720 \\
\hline 4 & 1 & -2.087693 & 0.107644 & 0.616959 \\
\hline 5 & 6 & -0.000048 & -0.000018 & 0.000002 \\
\hline 6 & 6 & 0.881444 & -0.932473 & -0.842841 \\
\hline 7 & 1 & 1.911334 & -0.572904 & -0.876937 \\
\hline 8 & 1 & 0.892737 & -1.941805 & -0.427820 \\
\hline 9 & 1 & 0.515017 & -0.996437 & -1.868884 \\
\hline 10 & 6 & 0.009966 & 1.406987 & -0.614084 \\
\hline 11 & 1 & -0.610076 & 2.092208 & -0.033438 \\
\hline 12 & 1 & 1.022057 & 1.814348 & -0.643401 \\
\hline 13 & 1 & -0.374392 & 1.391339 & -1.635432 \\
\hline 14 & 6 & 0.546334 & 0.063346 & 1.433282 \\
\hline 15 & 1 & -0.062433 & 0.721368 & 2.055791 \\
\hline 16 & 1 & 0.550032 & -0.925501 & 1.895119 \\
\hline 17 & 1 & 1.569602 & 0.442684 & 1.445942 \\
\hline
\end{tabular}

Zero-point correction=

Thermal correction to Energy=

Thermal correction to Enthalpy=

Thermal correction to Gibbs Free Energy=

Sum of electronic and zero-point Energies=

Sum of electronic and thermal Energies=

Sum of electronic and thermal Enthalpies=

Sum of electronic and thermal Free Energies=

Hexane

Standard orientation:

\begin{tabular}{|c|c|c|c|c|}
\hline \multirow{2}{*}{$\begin{array}{l}\text { Center } \\
\text { Number }\end{array}$} & Atomic & \multirow{2}{*}{$\begin{array}{r}\text { Atomic } \\
\text { Type }\end{array}$} & \multicolumn{2}{|c|}{ Coordinates (Angstroms } \\
\hline & Numbe & & $\mathrm{X}$ & $\mathrm{Z}$ \\
\hline 1 & 6 & -3.210472 & 0.208438 & 0.000045 \\
\hline 2 & 1 & -3.295568 & 0.848205 & 0.880467 \\
\hline 3 & 1 & -3.295596 & 0.848344 & -0.880274 \\
\hline 4 & 1 & -4.062650 & -0.472067 & 0.000002 \\
\hline 5 & 6 & -1.884446 & -0.549874 & -0.000041 \\
\hline 6 & 1 & -1.837824 & -1.206041 & 0.873833 \\
\hline 7 & 1 & -1.837868 & -1.205932 & -0.874000 \\
\hline 8 & 6 & -0.666520 & 0.373094 & -0.000020 \\
\hline 9 & 1 & -0.713054 & 1.030526 & -0.874360 \\
\hline 10 & 1 & -0.713072 & 1.030524 & 0.874320 \\
\hline
\end{tabular}




$\begin{array}{rrrrrr}11 & 6 & 0 & 0.666520 & -0.373094 & 0.000002 \\ 12 & 1 & 0 & 0.713062 & -1.030555 & -0.874315 \\ 13 & 1 & 0 & 0.713064 & -1.030494 & 0.874365 \\ 14 & 6 & 0 & 1.884446 & 0.549874 & -0.000030 \\ 15 & 1 & 0 & 1.837832 & 1.206038 & 0.873848 \\ 16 & 1 & 0 & 1.837860 & 1.205935 & -0.873986 \\ 17 & 6 & 0 & 3.210472 & -0.208438 & 0.000034 \\ 18 & 1 & 0 & 3.295590 & -0.848333 & -0.880292 \\ 19 & 1 & 0 & 4.062650 & 0.472067 & -0.000003 \\ 20 & 1 & 0 & 3.295573 & -0.848215 & 0.880449\end{array}$

Zero-point correction=

Thermal correction to Energy=

Thermal correction to Enthalpy=

Thermal correction to Gibbs Free Energy=

Sum of electronic and zero-point Energies=

Sum of electronic and thermal Energies $=$

Sum of electronic and thermal Enthalpies=

Sum of electronic and thermal Free Energies=
0.188612 (Hartree/Particle)

0.196921

0.197865

22dimethylbutane

Standard orientation:

\begin{tabular}{|c|c|c|c|c|}
\hline \multirow{2}{*}{$\begin{array}{l}\text { Center } \\
\text { Number }\end{array}$} & Atomic & \multirow{2}{*}{$\begin{array}{r}\text { Atomic } \\
\text { Type }\end{array}$} & \multicolumn{2}{|c|}{ Coordinates (Angstroms } \\
\hline & Numbe & & $X$ & $\mathrm{Z}$ \\
\hline 1 & 6 & -1.616080 & 0.982226 & 0.003075 \\
\hline 2 & 1 & -1.592400 & 1.621853 & 0.887123 \\
\hline 3 & 1 & -1.592589 & 1.627153 & -0.877122 \\
\hline 4 & 1 & -2.568219 & 0.449139 & 0.001574 \\
\hline 5 & 6 & -0.434566 & -0.000079 & 0.000013 \\
\hline 6 & 6 & 0.873346 & 0.824783 & 0.001696 \\
\hline 7 & 1 & 0.857779 & 1.484588 & -0.870541 \\
\hline 8 & 1 & 0.857904 & 1.480882 & 0.876726 \\
\hline 9 & 6 & 2.182936 & 0.035488 & 0.000049 \\
\hline 10 & 1 & 2.274391 & -0.598670 & -0.881437 \\
\hline 11 & 1 & 3.035121 & 0.715540 & 0.000614 \\
\hline 12 & 1 & 2.275068 & -0.601041 & 0.879745 \\
\hline 13 & 6 & -0.522332 & -0.885607 & 1.251787 \\
\hline 14 & 1 & 0.265080 & -1.638634 & 1.270527 \\
\hline 15 & 1 & -0.438390 & -0.287569 & 2.161097 \\
\hline 16 & 1 & -1.478490 & -1.410279 & 1.284369 \\
\hline 17 & 6 & -0.523279 & -0.878814 & -1.256454 \\
\hline 18 & 1 & -1.479215 & -1.403784 & -1.290888 \\
\hline 19 & 1 & -0.440638 & -0.275783 & -2.162579 \\
\hline 20 & 1 & 0.264446 & -1.631372 & -1.280203 \\
\hline
\end{tabular}

Zero-point correction=

Thermal correction to Energy=

Thermal correction to Enthalpy=
0.187802 (Hartree/Particle)

0.195871

0.196815 
Thermal correction to Gibbs Free Energy=

Sum of electronic and zero-point Energies=

Sum of electronic and thermal Energies $=$

Sum of electronic and thermal Enthalpies=

Sum of electronic and thermal Free Energies=

Heptane
0.156731

$-237.020205$

$-237.012136$

$-237.011192$

$-237.051276$

Standard orientation:

\begin{tabular}{|c|c|c|c|c|c|}
\hline \multirow{2}{*}{$\begin{array}{l}\text { Center } \\
\text { Number }\end{array}$} & \multicolumn{2}{|l|}{ Atomic } & \multirow{2}{*}{$\begin{array}{l}\text { Atomic } \\
\text { Type }\end{array}$} & \multicolumn{2}{|c|}{ Coordinates (Angstroms } \\
\hline & Numbe & & & $\mathrm{X}$ & $\mathrm{Z}$ \\
\hline 1 & 6 & 0 & -3.827204 & -0.354278 & -0.000030 \\
\hline 2 & 1 & 0 & -3.867625 & -0.998414 & -0.880458 \\
\hline 3 & 1 & 0 & -3.867602 & -0.998593 & 0.880269 \\
\hline 4 & 1 & 0 & -4.724663 & 0.265300 & 0.000048 \\
\hline 5 & 6 & 0 & -2.557091 & 0.494333 & 0.000044 \\
\hline 6 & 1 & 0 & -2.556159 & 1.152159 & -0.873830 \\
\hline 7 & 1 & 0 & -2.556169 & 1.152027 & 0.874018 \\
\hline 8 & 6 & 0 & -1.278027 & -0.341819 & -0.000003 \\
\hline 9 & 1 & 0 & -1.278726 & -1.000925 & 0.874279 \\
\hline 10 & 1 & 0 & -1.278751 & -1.000885 & -0.874315 \\
\hline 11 & 6 & 0 & -0.000000 & 0.495279 & -0.000008 \\
\hline 12 & 1 & 0 & -0.000008 & 1.154102 & 0.874323 \\
\hline 13 & 1 & 0 & 0.000008 & 1.154060 & -0.874369 \\
\hline 14 & 6 & 0 & 1.278027 & -0.341819 & 0.000021 \\
\hline 15 & 1 & 0 & 1.278729 & -1.000953 & -0.874240 \\
\hline 16 & 1 & 0 & 1.278748 & -1.000858 & 0.874354 \\
\hline 17 & 6 & 0 & 2.557091 & 0.494333 & -0.000036 \\
\hline 18 & 1 & 0 & 2.556168 & 1.152147 & 0.873846 \\
\hline 19 & 1 & 0 & 2.556161 & 1.152038 & -0.874001 \\
\hline 20 & 6 & 0 & 3.827204 & -0.354278 & 0.000011 \\
\hline 21 & 1 & 0 & 3.867610 & -0.998558 & -0.880312 \\
\hline 22 & 1 & 0 & 4.724663 & 0.265300 & -0.000031 \\
\hline 23 & 1 & 0 & 3.867617 & -0.998448 & 0.880415 \\
\hline
\end{tabular}

Zero-point correction $=$

Thermal correction to Energy=

Thermal correction to Enthalpy=

Thermal correction to Gibbs Free Energy=

Sum of electronic and zero-point Energies=

Sum of electronic and thermal Energies $=$

Sum of electronic and thermal Enthalpies=

0.217058 (Hartree/Particle)

0.226696

0.227640

0.182450

$-276.322845$

$-276.313207$

$-276.312263$

Sum of electronic and thermal Free Energies $=\quad-276.357453$

223trimethylbutane

Standard orientation:

\begin{tabular}{cccccc} 
Center & Atomic & Atomic & & \multicolumn{2}{c}{ Coordinates } \\
Number & Number & Type & X & Y & Z \\
-1 & 6 & 0 & -1.355308 & -1.241855 & -0.565949
\end{tabular}




$\begin{array}{cccccc}2 & 1 & 0 & -1.229697 & -1.308030 & -1.648395 \\ 3 & 1 & 0 & -0.980931 & -2.164939 & -0.126400 \\ 4 & 1 & 0 & -2.424988 & -1.193676 & -0.357587 \\ 5 & 6 & 0 & -0.643348 & 0.000003 & -0.000621 \\ 6 & 6 & 0 & 0.854038 & -0.000001 & -0.449990 \\ 7 & 1 & 0 & 0.828832 & 0.000015 & -1.545437 \\ 8 & 6 & 0 & 1.634124 & 1.248555 & -0.016818 \\ 9 & 1 & 0 & 1.717732 & 1.308905 & 1.069098 \\ 10 & 1 & 0 & 2.648129 & 1.212445 & -0.416483 \\ 11 & 1 & 0 & 1.178755 & 2.171023 & -0.371056 \\ 12 & 6 & 0 & 1.634097 & -1.248568 & -0.016827 \\ 13 & 1 & 0 & 1.717972 & -1.308758 & 1.069076 \\ 14 & 1 & 0 & 1.178549 & -2.171050 & -0.370804 \\ 15 & 1 & 0 & 2.648009 & -1.212637 & -0.416748 \\ 16 & 6 & 0 & -0.790131 & -0.000382 & 1.529127 \\ 17 & 1 & 0 & -0.335599 & 0.881529 & 1.979635 \\ 18 & 1 & 0 & -1.845972 & -0.000258 & 1.804226 \\ 19 & 1 & 0 & -0.335930 & -0.882707 & 1.979155 \\ 20 & 6 & 0 & -1.355126 & 1.242245 & -0.565306 \\ 21 & 1 & 0 & -1.229312 & 1.309109 & -1.647687 \\ 22 & 1 & 0 & -2.424850 & 1.194009 & -0.357176 \\ 23 & 1 & 0 & -0.980778 & 2.165035 & -0.125108 \\ ----------------------------------------------------------\end{array}$

Zero-point correction $=$

Thermal correction to Energy=

0.215985 (Hartree/Particle) 0.225395

Thermal correction to Enthalpy= 0.226339

Thermal correction to Gibbs Free Energy=

Sum of electronic and zero-point Energies=

0.182482

Sum of electronic and thermal Energies $=$

$-276.323094$

$-276.313684$

Sum of electronic and thermal Enthalpies=

$-276.312740$

Sum of electronic and thermal Free Energies $=\quad-276.356597$

Octane

Standard orientation:

\begin{tabular}{|c|c|c|c|c|}
\hline \multirow{2}{*}{$\begin{array}{l}\text { Center } \\
\text { Number }\end{array}$} & Atomic & \multirow{2}{*}{$\begin{array}{r}\text { Atomic } \\
\text { Type }\end{array}$} & \multicolumn{2}{|c|}{ Coordinates (Angstroms } \\
\hline & Numbe & & $\mathrm{X}$ & $\mathrm{Z}$ \\
\hline 1 & 6 & -4.479632 & 0.256742 & 0.000028 \\
\hline 2 & 1 & -4.545146 & 0.898803 & 0.880470 \\
\hline 3 & 1 & -4.545104 & 0.899028 & -0.880252 \\
\hline 4 & 1 & -5.352290 & -0.397315 & -0.000079 \\
\hline 5 & 6 & -3.177450 & -0.541739 & -0.000046 \\
\hline 6 & 1 & -3.150936 & -1.199046 & 0.873815 \\
\hline 7 & 1 & -3.150939 & -1.198888 & -0.874026 \\
\hline 8 & 6 & -1.931895 & 0.343555 & 0.000021 \\
\hline 9 & 1 & -1.958243 & 1.002164 & -0.874246 \\
\hline 10 & 1 & -1.958264 & 1.002085 & 0.874346 \\
\hline 11 & 6 & -0.622273 & -0.443143 & 0.000005 \\
\hline 12 & 1 & -0.596593 & -1.101483 & -0.874278 \\
\hline
\end{tabular}




$\begin{array}{rrrrrr}13 & 1 & 0 & -0.596565 & -1.101458 & 0.874306 \\ 14 & 6 & 0 & 0.622273 & 0.443143 & -0.000025 \\ 15 & 1 & 0 & 0.596573 & 1.101511 & 0.874237 \\ 16 & 1 & 0 & 0.596585 & 1.101429 & -0.874347 \\ 17 & 6 & 0 & 1.931895 & -0.343555 & 0.000020 \\ 18 & 1 & 0 & 1.958255 & -1.002172 & -0.874240 \\ 19 & 1 & 0 & 1.958253 & -1.002077 & 0.874351 \\ 20 & 6 & 0 & 3.177450 & 0.541739 & -0.000026 \\ 21 & 1 & 0 & 3.150936 & 1.199014 & 0.873859 \\ 22 & 1 & 0 & 3.150939 & 1.198920 & -0.873982 \\ 23 & 6 & 0 & 4.479632 & -0.256742 & 0.000019 \\ 24 & 1 & 0 & 4.545127 & -0.898963 & -0.880308 \\ 25 & 1 & 0 & 5.352290 & 0.397315 & -0.000015 \\ 26 & 1 & 0 & 4.545124 & -0.898868 & 0.880414\end{array}$

Zero-point correction=

Thermal correction to Energy=

Thermal correction to Enthalpy=

Thermal correction to Gibbs Free Energy=

Sum of electronic and zero-point Energies=

Sum of electronic and thermal Energies $=$

Sum of electronic and thermal Enthalpies=

Sum of electronic and thermal Free Energies=

2233tetramethylbutane

Standard orientation:

\begin{tabular}{|c|c|c|c|c|}
\hline \multirow{2}{*}{$\begin{array}{l}\text { Center } \\
\text { Number }\end{array}$} & Atomic & \multirow{2}{*}{$\begin{array}{l}\text { Atomic } \\
\text { Type }\end{array}$} & \multicolumn{2}{|c|}{ Coordinates (Angstroms } \\
\hline & Numbe & & $X$ & Z \\
\hline 1 & 6 & -1.351134 & -1.099520 & 0.923188 \\
\hline 2 & 1 & -1.149084 & -2.096870 & 0.535893 \\
\hline 3 & 1 & -0.939910 & -1.038628 & 1.930225 \\
\hline 4 & 1 & -2.433806 & -0.996259 & 1.006684 \\
\hline 5 & 6 & -0.792534 & -0.000026 & 0.000020 \\
\hline 6 & 6 & 0.792511 & -0.000036 & -0.000017 \\
\hline 7 & 6 & 1.351087 & 0.877621 & -1.136200 \\
\hline 8 & 1 & 1.148528 & 0.450516 & -2.117082 \\
\hline 9 & 1 & 0.940320 & 1.886472 & -1.115893 \\
\hline 10 & 1 & 2.433835 & 0.964786 & -1.036799 \\
\hline 11 & 6 & -1.351290 & -0.249639 & -1.413738 \\
\hline 12 & 1 & -1.149292 & 0.584405 & -2.083905 \\
\hline 13 & 1 & -0.940208 & -1.152240 & -1.864578 \\
\hline 14 & 1 & -2.433975 & -0.373499 & -1.365966 \\
\hline 15 & 6 & -1.351004 & 1.349252 & 0.490721 \\
\hline 16 & 1 & -2.433731 & 1.369939 & 0.359988 \\
\hline 17 & 1 & -1.148479 & 1.512742 & 1.547989 \\
\hline 18 & 1 & -0.939983 & 2.190818 & -0.065819 \\
\hline 19 & 6 & 1.351143 & -1.422821 & -0.192029 \\
\hline 20 & 1 & 2.433834 & -1.380287 & -0.317710 \\
\hline 21 & 1 & 1.148961 & -2.058523 & 0.668524 \\
\hline
\end{tabular}




$\begin{array}{rrrrrr}22 & 1 & 0 & 0.939989 & -1.909844 & -1.075585 \\ 23 & 6 & 0 & 1.351208 & 0.545123 & 1.328074 \\ 24 & 1 & 0 & 2.433919 & 0.415148 & 1.353859 \\ 25 & 1 & 0 & 1.148924 & 1.608218 & 1.448361 \\ 26 & 1 & 0 & 0.940243 & 0.023378 & 2.191693\end{array}$

$\begin{array}{lc}\text { Zero-point correction }= & 0.244395 \text { (Hartree/Particle) } \\ \text { Thermal correction to Energy= } & 0.254670 \\ \text { Thermal correction to Enthalpy= } & 0.255614 \\ \text { Thermal correction to Gibbs Free Energy= } & 0.211200 \\ \text { Sum of electronic and zero-point Energies= } & -315.625290 \\ \text { Sum of electronic and thermal Energies= } & -315.615016 \\ \text { Sum of electronic and thermal Enthalpies }= & -315.614071 \\ \text { Sum of electronic and thermal Free Energies }= & -315.658485 \\ \text { B2PLYP/ccpVQZ } & \\ \text { Ethane } & \\ \text { Standard orientation: }\end{array}$

\begin{tabular}{|c|c|c|c|c|}
\hline \multirow{2}{*}{$\begin{array}{l}\text { Center } \\
\text { Number }\end{array}$} & Atomic & \multirow{2}{*}{$\begin{array}{l}\text { Atomic } \\
\text { Type }\end{array}$} & \multicolumn{2}{|c|}{ Coordinates (Angstrom } \\
\hline & Number & & $\mathrm{X}$ & Z \\
\hline 1 & 6 & -0.000000 & -0.000000 & 0.762374 \\
\hline 2 & 1 & -0.507110 & 0.878381 & 1.157708 \\
\hline 3 & 1 & 1.014255 & -0.000021 & 1.157708 \\
\hline 4 & 1 & -0.507146 & -0.878361 & 1.157708 \\
\hline 5 & 6 & 0.000000 & 0.000000 & -0.762374 \\
\hline 6 & 1 & 0.507110 & 0.878381 & -1.157708 \\
\hline 7 & 1 & 0.507146 & -0.878361 & -1.157708 \\
\hline 8 & 1 & -1.014255 & -0.000021 & -1.157708 \\
\hline
\end{tabular}

Zero-point correction=

Thermal correction to Energy=

Thermal correction to Enthalpy=

Thermal correction to Gibbs Free Energy=

Sum of electronic and zero-point Energies=

Sum of electronic and thermal Energies $=$

Sum of electronic and thermal Enthalpies=

Sum of electronic and thermal Free Energies=

Methane

Standard orientation:

\begin{tabular}{|c|c|c|c|}
\hline \multirow{2}{*}{$\begin{array}{l}\text { Center } \\
\text { Number }\end{array}$} & \multirow{2}{*}{$\begin{array}{l}\text { Atomic } \\
\text { Number }\end{array}$} & \multirow{2}{*}{$\begin{array}{l}\text { Atomic } \\
\text { Type }\end{array}$} & Coordinates (Angstroms) \\
\hline & & & $\mathrm{Y} \quad \mathrm{Z}$ \\
\hline 1 & 6 & 0.000000 & $0.000000 \quad 0.000000$ \\
\hline 2 & 1 & 0.626719 & $0.626719 \quad 0.626719$ \\
\hline 3 & 1 & -0.626719 & $-0.626719 \quad 0.626719$ \\
\hline 4 & 1 & -0.626719 & $0.626719-0.626719$ \\
\hline 5 & 1 & 0.626719 & $-0.626719 \quad-0.626719$ \\
\hline
\end{tabular}


Zero-point correction $=$

Thermal correction to Energy=

Thermal correction to Enthalpy=

Thermal correction to Gibbs Free Energy=

Sum of electronic and zero-point Energies=

Sum of electronic and thermal Energies=

Sum of electronic and thermal Enthalpies=

Sum of electronic and thermal Free Energies=

Propane

Standard orientation:
0.045013 (Hartree/Particle)

0.047879

0.048824

0.027709

$-40.447848$

$-40.444981$

$-40.444037$

$-40.465152$

\begin{tabular}{|c|c|c|c|c|}
\hline \multirow{2}{*}{$\begin{array}{l}\text { Center } \\
\text { Number }\end{array}$} & Atomic & \multirow{2}{*}{$\begin{array}{l}\text { Atomic } \\
\text { Type }\end{array}$} & \multicolumn{2}{|c|}{ Coordinates (Angstrom } \\
\hline & Numbe & & $X$ & Z \\
\hline 1 & 6 & -1.268311 & -0.259508 & 0.000000 \\
\hline 2 & 1 & -2.164374 & 0.358939 & -0.000022 \\
\hline 3 & 1 & -1.307465 & -0.902448 & 0.879189 \\
\hline 4 & 1 & -1.307446 & -0.902484 & -0.879163 \\
\hline 5 & 6 & 0.000000 & 0.586999 & 0.000000 \\
\hline 6 & 1 & -0.000001 & 1.242049 & 0.872451 \\
\hline 7 & 1 & 0.000001 & 1.242034 & -0.872461 \\
\hline 8 & 6 & 1.268311 & -0.259508 & 0.000000 \\
\hline 9 & 1 & 1.307471 & -0.902437 & 0.879196 \\
\hline 10 & 1 & 1.307436 & -0.902494 & -0.879156 \\
\hline 11 & 1 & 2.164376 & 0.358936 & -0.000038 \\
\hline
\end{tabular}

Zero-point correction=

Thermal correction to Energy=

Thermal correction to Enthalpy=

Thermal correction to Gibbs Free Energy=

Sum of electronic and zero-point Energies=

Sum of electronic and thermal Energies=

Sum of electronic and thermal Enthalpies=

Sum of electronic and thermal Free Energies=

Butane

Standard orientation:

\begin{tabular}{cccccc} 
Center & \multicolumn{2}{c}{ Atomic } & \multicolumn{2}{c}{ Atomic } & \multicolumn{3}{c}{ Coordinates } \\
Number & Number & Type & X & Y & Z \\
- & Nustroms) \\
\hline 1 & 6 & 0 & 1.950105 & -0.119923 & 0.000030 \\
2 & 1 & 0 & 2.092402 & -0.747889 & -0.879225 \\
3 & 1 & 0 & 2.092343 & -0.747867 & 0.879310 \\
4 & 1 & 0 & 2.735479 & 0.634080 & 0.000046 \\
5 & 6 & 0 & 0.563421 & 0.514035 & -0.000034 \\
6 & 1 & 0 & 0.456535 & 1.161573 & 0.873130 \\
7 & 1 & 0 & 0.456579 & 1.161529 & -0.873237 \\
8 & 6 & 0 & -0.563421 & -0.514035 & -0.000034 \\
9 & 1 & 0 & -0.456579 & -1.161527 & -0.873239
\end{tabular}




$\begin{array}{rrrrrr}10 & 1 & 0 & -0.456535 & -1.161575 & 0.873129 \\ 11 & 6 & 0 & -1.950105 & 0.119923 & 0.000030 \\ 12 & 1 & 0 & -2.092400 & 0.747895 & -0.879221 \\ 13 & 1 & 0 & -2.735479 & -0.634080 & 0.000040 \\ 14 & 1 & 0 & -2.092345 & 0.747862 & 0.879314\end{array}$

Zero-point correction=

Thermal correction to Energy=

Thermal correction to Enthalpy=

Thermal correction to Gibbs Free Energy=

Sum of electronic and zero-point Energies=

Sum of electronic and thermal Energies=

Sum of electronic and thermal Enthalpies=

Sum of electronic and thermal Free Energies=

Isobutane
0.132537 (Hartree/Particle)

0.138301

0.139245

0.104549

$-158.238833$

$-158.233070$

$-158.232125$

$-158.266821$

Standard orientation:

\begin{tabular}{|c|c|c|c|c|}
\hline \multirow{2}{*}{$\begin{array}{l}\text { Center } \\
\text { Number }\end{array}$} & Atomic & \multirow{2}{*}{$\begin{array}{r}\text { Atomic } \\
\text { Type }\end{array}$} & \multicolumn{2}{|c|}{ Coordinates (Angstrom } \\
\hline & Numbe & & $\mathrm{X}$ & $\mathrm{Z}$ \\
\hline 1 & 6 & -0.207563 & -1.437578 & -0.095781 \\
\hline 2 & 1 & -1.155868 & -1.838703 & 0.260095 \\
\hline 3 & 1 & -0.214243 & -1.485755 & -1.185799 \\
\hline 4 & 1 & 0.588270 & -2.090660 & 0.260534 \\
\hline 5 & 6 & -0.000009 & 0.000026 & 0.375214 \\
\hline 6 & 1 & -0.000021 & 0.000036 & 1.468490 \\
\hline 7 & 6 & -1.141203 & 0.898542 & -0.095797 \\
\hline 8 & 1 & -1.014532 & 1.920314 & 0.260244 \\
\hline 9 & 1 & -1.179416 & 0.928538 & -1.185809 \\
\hline 10 & 1 & -2.104715 & 0.535744 & 0.260355 \\
\hline 11 & 6 & 1.348768 & 0.539029 & -0.095789 \\
\hline 12 & 1 & 1.516345 & 1.554887 & 0.260270 \\
\hline 13 & 1 & 2.170293 & -0.081531 & 0.260349 \\
\hline 14 & 1 & 1.393927 & 0.557016 & -1.185807 \\
\hline
\end{tabular}

Zero-point correction=

Thermal correction to Energy=

0.132060 (Hartree/Particle)

Thermal correction to Enthalpy=

0.137742

Thermal correction to Gibbs Free Energy=

Sum of electronic and zero-point Energies=

0.138687

Sum of electronic and thermal Energies $=$

0.104465

$-158.240910$

$-158.235227$

Sum of electronic and thermal Enthalpies=

$-158.234283$

Sum of electronic and thermal Free Energies= $-158.268504$

Pentane

Standard orientation:

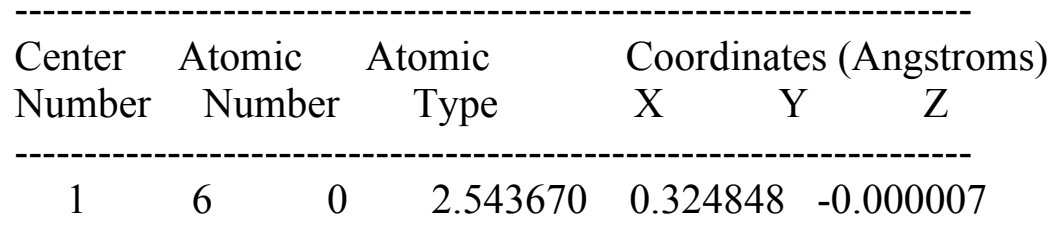




$\begin{array}{cccccc}2 & 1 & 0 & 2.583529 & 0.967477 & -0.879257 \\ 3 & 1 & 0 & 2.583529 & 0.967509 & 0.879219 \\ 4 & 1 & 0 & 3.439615 & -0.293789 & 0.000004 \\ 5 & 6 & 0 & 1.276275 & -0.523088 & 0.000010 \\ 6 & 1 & 0 & 1.275436 & -1.179093 & -0.873204 \\ 7 & 1 & 0 & 1.275442 & -1.179068 & 0.873241 \\ 8 & 6 & 0 & 0.000000 & 0.312215 & 0.000006 \\ 9 & 1 & 0 & -0.000007 & 0.969753 & 0.873872 \\ 10 & 1 & 0 & 0.000007 & 0.969763 & -0.873852 \\ 11 & 6 & 0 & -1.276275 & -0.523088 & -0.000010 \\ 12 & 1 & 0 & -1.275441 & -1.179099 & 0.873200 \\ 13 & 1 & 0 & -1.275438 & -1.179062 & -0.873244 \\ 14 & 6 & 0 & -2.543670 & 0.324848 & 0.000002 \\ 15 & 1 & 0 & -3.439615 & -0.293789 & -0.000054 \\ 16 & 1 & 0 & -2.583550 & 0.967447 & 0.879272 \\ 17 & 1 & 0 & -2.583509 & 0.967540 & -0.879204\end{array}$

Zero-point correction=

Thermal correction to Energy=

Thermal correction to Enthalpy=

Thermal correction to Gibbs Free Energy=

Sum of electronic and zero-point Energies=

Sum of electronic and thermal Energies $=$

Sum of electronic and thermal Enthalpies=

Sum of electronic and thermal Free Energies=

Neopentane

Standard orientation:

\begin{tabular}{|c|c|c|c|c|}
\hline \multirow{2}{*}{$\begin{array}{l}\text { Center } \\
\text { Number }\end{array}$} & Atomic & \multirow{2}{*}{$\begin{array}{l}\text { Atomic } \\
\text { Type }\end{array}$} & \multicolumn{2}{|c|}{ Coordinates (Angstroms } \\
\hline & Number & & $\mathrm{X}$ & $\mathrm{Z}$ \\
\hline 1 & 6 & -0.593945 & 0.464909 & 1.332480 \\
\hline 2 & 1 & -1.428004 & -0.170223 & 1.631295 \\
\hline 3 & 1 & 0.152980 & 0.432238 & 2.125803 \\
\hline 4 & 1 & -0.961510 & 1.488645 & 1.260412 \\
\hline 5 & 6 & 0.000016 & -0.000004 & -0.000009 \\
\hline 6 & 6 & 0.504232 & -1.438932 & 0.140308 \\
\hline 7 & 1 & 0.933309 & -1.795698 & -0.796146 \\
\hline 8 & 1 & 1.273162 & -1.510187 & 0.909727 \\
\hline 9 & 1 & -0.307773 & -2.112452 & 0.414813 \\
\hline 10 & 6 & -1.076521 & 0.059863 & -1.087186 \\
\hline 11 & 1 & -1.453627 & 1.075488 & -1.208178 \\
\hline 12 & 1 & -0.679666 & -0.266873 & -2.048479 \\
\hline 13 & 1 & -1.920448 & -0.583249 & -0.837132 \\
\hline 14 & 6 & 1.166232 & 0.914164 & -0.385605 \\
\hline 15 & 1 & 0.834295 & 1.946992 & -0.492455 \\
\hline 16 & 1 & 1.948638 & 0.890537 & 0.373094 \\
\hline 17 & 1 & 1.608558 & 0.604788 & -1.332683 \\
\hline
\end{tabular}


Thermal correction to Energy=

Thermal correction to Enthalpy=

Thermal correction to Gibbs Free Energy=

Sum of electronic and zero-point Energies=

Sum of electronic and thermal Energies=

Sum of electronic and thermal Enthalpies=

Sum of electronic and thermal Free Energies=

Hexane

Standard orientation:
0.166762

0.167706

0.130784

$-197.508794$

$-197.501898$

$-197.500954$

$-197.537875$

\begin{tabular}{|c|c|c|c|c|}
\hline \multirow{2}{*}{$\begin{array}{l}\text { Center } \\
\text { Number }\end{array}$} & Atomic & \multirow{2}{*}{$\begin{array}{l}\text { Atomic } \\
\text { Type }\end{array}$} & \multicolumn{2}{|c|}{ Coordinates (Angstrom } \\
\hline & Numbe & & $\mathrm{X}$ & $\mathrm{Z}$ \\
\hline 1 & 6 & -3.203741 & 0.208364 & 0.000033 \\
\hline 2 & 1 & -3.288162 & 0.846622 & 0.879332 \\
\hline 3 & 1 & -3.288160 & 0.846783 & -0.879151 \\
\hline 4 & 1 & -4.054580 & -0.471010 & -0.000033 \\
\hline 5 & 6 & -1.880528 & -0.549489 & -0.000039 \\
\hline 6 & 1 & -1.834106 & -1.203889 & 0.873119 \\
\hline 7 & 1 & -1.834133 & -1.203778 & -0.873283 \\
\hline 8 & 6 & -0.665340 & 0.372724 & -0.000003 \\
\hline 9 & 1 & -0.711955 & 1.028361 & -0.873891 \\
\hline 10 & 1 & -0.711980 & 1.028334 & 0.873903 \\
\hline 11 & 6 & 0.665340 & -0.372724 & 0.000009 \\
\hline 12 & 1 & 0.711959 & -1.028378 & -0.873865 \\
\hline 13 & 1 & 0.711976 & -1.028316 & 0.873929 \\
\hline 14 & 6 & 1.880528 & 0.549489 & -0.000033 \\
\hline 15 & 1 & 1.834111 & 1.203889 & 0.873126 \\
\hline 16 & 1 & 1.834128 & 1.203778 & -0.873276 \\
\hline 17 & 6 & 3.203741 & -0.208364 & 0.000027 \\
\hline 18 & 1 & 3.288165 & -0.846766 & -0.879169 \\
\hline 19 & 1 & 4.054580 & 0.471010 & -0.000019 \\
\hline 20 & 1 & 3.288158 & -0.846639 & 0.879315 \\
\hline
\end{tabular}

Zero-point correction=

Thermal correction to Energy=

0.189730 (Hartree/Particle)

Thermal correction to Enthalpy=

0.198124

0.199068

Thermal correction to Gibbs Free Energy=

0.157167

Sum of electronic and zero-point Energies=

$-236.768902$

Sum of electronic and thermal Energies=

$-236.760508$

Sum of electronic and thermal Enthalpies=

$-236.759564$

Sum of electronic and thermal Free Energies $=\quad-236.801465$

22dimethylbutane

Standard orientation:

\begin{tabular}{cccccc}
$\begin{array}{l}\text { Center } \\
\text { Number }\end{array}$ & \multicolumn{2}{c}{ Atomic } & Atomic & \multicolumn{2}{c}{ Coordinates (Angstroms) } \\
- & Number & Type & X & Y & Z \\
\hline 1 & 6 & 0 & -1.611749 & 0.978587 & 0.002979 \\
2 & 1 & 0 & -1.587174 & 1.617299 & 0.886125
\end{tabular}




$\begin{array}{cccccc}3 & 1 & 0 & -1.587414 & 1.622373 & -0.876485 \\ 4 & 1 & 0 & -2.562286 & 0.445106 & 0.001568 \\ 5 & 6 & 0 & -0.433032 & -0.000325 & 0.000010 \\ 6 & 6 & 0 & 0.869664 & 0.824121 & 0.001568 \\ 7 & 1 & 0 & 0.852953 & 1.482613 & -0.870293 \\ 8 & 1 & 0 & 0.853052 & 1.479210 & 0.875992 \\ 9 & 6 & 0 & 2.176415 & 0.035167 & 0.000048 \\ 10 & 1 & 0 & 2.267309 & -0.597679 & -0.880635 \\ 11 & 1 & 0 & 3.027331 & 0.714387 & 0.000639 \\ 12 & 1 & 0 & 2.267876 & -0.599974 & 0.879008 \\ 13 & 6 & 0 & -0.519895 & -0.883165 & 1.248964 \\ 14 & 1 & 0 & 0.268191 & -1.633871 & 1.269275 \\ 15 & 1 & 0 & -0.439149 & -0.284683 & 2.156921 \\ 16 & 1 & 0 & -1.474511 & -1.408387 & 1.279697 \\ 17 & 6 & 0 & -0.520893 & -0.876690 & -1.253416 \\ 18 & 1 & 0 & -1.475243 & -1.402300 & -1.285794 \\ 19 & 1 & 0 & -0.441567 & -0.273414 & -2.158318 \\ 20 & 1 & 0 & 0.267570 & -1.626853 & -1.278620\end{array}$

Zero-point correction=

Thermal correction to Energy=

Thermal correction to Enthalpy=

Thermal correction to Gibbs Free Energy=

Sum of electronic and zero-point Energies=

Sum of electronic and thermal Energies $=$

Sum of electronic and thermal Enthalpies=

Sum of electronic and thermal Free Energies=

Heptane

Standard orientation:
0.188633 (Hartree/Particle)

$$
\begin{gathered}
0.196854 \\
0.197798
\end{gathered}
$$

\subsection{5}

$-236.770998$

$-236.762777$

$-236.761833$

\begin{tabular}{|c|c|c|c|c|}
\hline \multirow{2}{*}{$\begin{array}{l}\text { Center } \\
\text { Number }\end{array}$} & Atomic & \multirow{2}{*}{$\begin{array}{l}\text { Atomic } \\
\text { Type }\end{array}$} & \multicolumn{2}{|c|}{ Coordinates (Angstrom } \\
\hline & Numbe & & $X$ & $\mathrm{Z}$ \\
\hline 1 & 6 & -3.819387 & -0.353953 & -0.000024 \\
\hline 2 & 1 & -3.859261 & -0.996523 & -0.879338 \\
\hline 3 & 1 & -3.859216 & -0.996740 & 0.879134 \\
\hline 4 & 1 & -4.715412 & 0.264620 & 0.000078 \\
\hline 5 & 6 & -2.552022 & 0.493991 & 0.000051 \\
\hline 6 & 1 & -2.551167 & 1.150048 & -0.873101 \\
\hline 7 & 1 & -2.551169 & 1.149897 & 0.873317 \\
\hline 8 & 6 & -1.275717 & -0.341622 & -0.000014 \\
\hline 9 & 1 & -1.276628 & -0.998926 & 0.873836 \\
\hline 10 & 1 & -1.276667 & -0.998861 & -0.873914 \\
\hline 11 & 6 & 0.000000 & 0.494745 & -0.000015 \\
\hline 12 & 1 & -0.000011 & 1.151776 & 0.873903 \\
\hline 13 & 1 & 0.000011 & 1.151735 & -0.873963 \\
\hline 14 & 6 & 1.275717 & -0.341622 & 0.000021 \\
\hline 15 & 1 & 1.276641 & -0.998944 & -0.873816 \\
\hline 16 & 1 & 1.276654 & -0.998843 & 0.873933 \\
\hline 17 & 6 & 2.552022 & 0.493991 & -0.000037 \\
\hline
\end{tabular}

$-236.802246$ 


$\begin{array}{rrrrrr}18 & 1 & 0 & 2.551169 & 1.150031 & 0.873128 \\ 19 & 1 & 0 & 2.551167 & 1.149914 & -0.873290 \\ 20 & 6 & 0 & 3.819387 & -0.353953 & 0.000018 \\ 21 & 1 & 0 & 3.859237 & -0.996691 & -0.879175 \\ 22 & 1 & 0 & 4.715412 & 0.264620 & -0.000025 \\ 23 & 1 & 0 & 3.859240 & -0.996573 & 0.879297\end{array}$

Zero-point correction=

Thermal correction to Energy=

Thermal correction to Enthalpy=

Thermal correction to Gibbs Free Energy=

Sum of electronic and zero-point Energies=

Sum of electronic and thermal Energies $=$

Sum of electronic and thermal Enthalpies=

Sum of electronic and thermal Free Energies=

223trimethylbutane

Standard orientation:

\begin{tabular}{|c|c|c|c|c|}
\hline \multirow{2}{*}{$\begin{array}{l}\text { Center } \\
\text { Number }\end{array}$} & Atomic & \multirow{2}{*}{$\begin{array}{l}\text { Atomic } \\
\text { Type }\end{array}$} & \multicolumn{2}{|c|}{ Coordinates (Angstroms } \\
\hline & Number & & $\mathrm{X}$ & Z \\
\hline 1 & 6 & -1.351151 & -1.238152 & -0.564108 \\
\hline 2 & 1 & -1.226663 & -1.302757 & -1.645728 \\
\hline 3 & 1 & -0.976907 & -2.160618 & -0.125731 \\
\hline 4 & 1 & -2.419434 & -1.187331 & -0.354793 \\
\hline 5 & 6 & -0.640602 & 0.000016 & -0.000381 \\
\hline 6 & 6 & 0.850398 & 0.000007 & -0.450445 \\
\hline 7 & 1 & 0.823714 & 0.000032 & -1.545039 \\
\hline 8 & 6 & 1.628609 & 1.245521 & -0.016843 \\
\hline 9 & 1 & 1.711806 & 1.304885 & 1.067959 \\
\hline 10 & 1 & 2.641408 & 1.207176 & -0.415924 \\
\hline 11 & 1 & 1.174222 & 2.167091 & -0.371214 \\
\hline 12 & 6 & 1.628573 & -1.245528 & -0.016893 \\
\hline 13 & 1 & 1.712053 & -1.304759 & 1.067896 \\
\hline 14 & 1 & 1.174000 & -2.167108 & -0.371008 \\
\hline 15 & 1 & 2.641275 & -1.207351 & -0.416239 \\
\hline 16 & 6 & -0.786016 & -0.000497 & 1.525777 \\
\hline 17 & 1 & -0.332353 & 0.880863 & 1.975966 \\
\hline 18 & 1 & -1.841268 & -0.000346 & 1.799050 \\
\hline 19 & 1 & -0.332757 & -0.882388 & 1.975325 \\
\hline 20 & 6 & -1.350946 & 1.238638 & -0.563312 \\
\hline 21 & 1 & -1.226265 & 1.304071 & -1.644863 \\
\hline 22 & 1 & -2.419279 & 1.187747 & -0.354244 \\
\hline 23 & 1 & -0.976737 & 2.160759 & -0.124173 \\
\hline
\end{tabular}

Zero-point correction=

Thermal correction to Energy=

Thermal correction to Enthalpy=

Thermal correction to Gibbs Free Energy=

Sum of electronic and zero-point Energies=
0.216946 (Hartree/Particle)

0.226496

0.227440

0.183413

$-276.033482$ 
Sum of electronic and thermal Energies=

$-276.023931$

Sum of electronic and thermal Enthalpies=

Sum of electronic and thermal Free Energies=

Octane

Standard orientation:

\begin{tabular}{cccccc} 
Center & Atomic & \multicolumn{2}{c}{ Atomic } & \multicolumn{3}{c}{ Coordinates } & Angstroms \\
Number & Number & Type & X & Y & $Z$ \\
-------------- \\
1 & 6 & 0 & -4.470668 & 0.256625 & 0.000029 \\
2 & 1 & 0 & -4.535546 & 0.897140 & 0.879358 \\
3 & 1 & 0 & -4.535494 & 0.897409 & -0.879109 \\
4 & 1 & 0 & -5.341953 & -0.396344 & -0.000100 \\
5 & 6 & 0 & -3.171273 & -0.541340 & -0.000057 \\
6 & 1 & 0 & -3.144915 & -1.196883 & 0.873083 \\
7 & 1 & 0 & -3.144920 & -1.196699 & -0.873334 \\
8 & 6 & 0 & -1.928421 & 0.343290 & 0.000027 \\
9 & 1 & 0 & -1.954892 & 1.000080 & -0.873816 \\
10 & 1 & 0 & -1.954930 & 0.999982 & 0.873941 \\
11 & 6 & 0 & -0.621125 & -0.442820 & 0.000012 \\
12 & 1 & 0 & -0.595535 & -1.099351 & -0.873880 \\
13 & 1 & 0 & -0.595502 & -1.099318 & 0.873927 \\
14 & 6 & 0 & 0.621125 & 0.442820 & -0.000026 \\
15 & 1 & 0 & 0.595514 & 1.099383 & 0.873841 \\
16 & 1 & 0 & 0.595523 & 1.099287 & -0.873965 \\
17 & 6 & 0 & 1.928421 & -0.343290 & 0.000024 \\
18 & 1 & 0 & 1.954911 & -1.000086 & -0.873813 \\
19 & 1 & 0 & 1.954911 & -0.999976 & 0.873943 \\
20 & 6 & 0 & 3.171273 & 0.541340 & -0.000032 \\
21 & 1 & 0 & 3.144917 & 1.196846 & 0.873135 \\
22 & 1 & 0 & 3.144918 & 1.196736 & -0.873282 \\
23 & 6 & 0 & 4.470668 & -0.256625 & 0.000019 \\
24 & 1 & 0 & 4.535520 & -0.897330 & -0.879175 \\
25 & 1 & 0 & 5.341953 & 0.396344 & -0.000022 \\
26 & 1 & 0 & 4.535520 & -0.897219 & 0.879293
\end{tabular}

Zero-point correction $=$

0.246846 (Hartree/Particle)

Thermal correction to Energy=

0.257936

Thermal correction to Enthalpy=

0.258881

Thermal correction to Gibbs Free Energy=

Sum of electronic and zero-point Energies=

0.209779

Sum of electronic and thermal Energies=

$-315.299066$

$-315.287976$

Sum of electronic and thermal Enthalpies=

$-315.287032$

Sum of electronic and thermal Free Energies $=\quad-315.336133$

2233tetramethylbutane

Standard orientation:

\begin{tabular}{|c|c|c|c|}
\hline Center & Atomic & Atomic & Coordinates (Angstroms) \\
\hline Number & Number & Type & $\mathrm{X}$ \\
\hline
\end{tabular}




$\begin{array}{cccccc}1 & 6 & 0 & -1.347216 & 0.853083 & -1.149826 \\ 2 & 1 & 0 & -1.137892 & 1.911344 & -1.009137 \\ 3 & 1 & 0 & -0.945733 & 0.554114 & -2.116548 \\ 4 & 1 & 0 & -2.429956 & 0.738231 & -1.197164 \\ 5 & 6 & 0 & -0.788927 & -0.000006 & 0.000001 \\ 6 & 6 & 0 & 0.788877 & -0.000003 & 0.000012 \\ 7 & 6 & 0 & 1.347334 & -0.600919 & 1.299487 \\ 8 & 1 & 0 & 1.138064 & 0.029232 & 2.161267 \\ 9 & 1 & 0 & 0.946149 & -1.592894 & 1.499970 \\ 10 & 1 & 0 & 2.430092 & -0.697250 & 1.221246 \\ 11 & 6 & 0 & -1.347329 & 0.569283 & 1.313655 \\ 12 & 1 & 0 & -1.137690 & -0.081365 & 2.159975 \\ 13 & 1 & 0 & -0.946322 & 1.556223 & 1.537844 \\ 14 & 1 & 0 & -2.430119 & 0.667221 & 1.237930 \\ 15 & 6 & 0 & -1.347329 & -1.422291 & -0.163831 \\ 16 & 1 & 0 & -2.430082 & -1.405737 & -0.040842 \\ 17 & 1 & 0 & -1.137940 & -1.829771 & -1.150573 \\ 18 & 1 & 0 & -0.946056 & -2.109943 & 0.578607 \\ 19 & 6 & 0 & 1.347322 & 1.425827 & -0.129353 \\ 20 & 1 & 0 & 2.430099 & 1.406240 & -0.007019 \\ 21 & 1 & 0 & 1.137832 & 1.857149 & -1.105894 \\ 22 & 1 & 0 & 0.946276 & 2.095354 & 0.629621 \\ 23 & 6 & 0 & 1.347204 & -0.824982 & -1.170153 \\ 24 & 1 & 0 & 2.429944 & -0.709026 & -1.214673 \\ 25 & 1 & 0 & 1.137928 & -1.886360 & -1.055159 \\ 26 & 1 & 0 & 0.945787 & -0.502718 & -2.129411\end{array}$

Zero-point correction=

Thermal correction to Energy=

Thermal correction to Enthalpy=

Thermal correction to Gibbs Free Energy=

Sum of electronic and zero-point Energies=

Sum of electronic and thermal Energies $=$

Sum of electronic and thermal Enthalpies=

Sum of electronic and thermal Free Energies=

PBE0DH/ccpVQZ

Ethane

Standard orientation:

\begin{tabular}{|c|c|c|c|c|}
\hline \multirow{2}{*}{$\begin{array}{l}\text { Center } \\
\text { Number }\end{array}$} & Atomic & \multirow{2}{*}{$\begin{array}{l}\text { Atomic } \\
\text { Type }\end{array}$} & \multicolumn{2}{|c|}{ Coordinates (Angstroms } \\
\hline & Numbe & & X & Z \\
\hline 1 & 6 & 0.000000 & 0.000000 & 0.757448 \\
\hline 2 & 1 & -0.506439 & 0.877209 & 1.153860 \\
\hline 3 & 1 & 1.012905 & -0.000015 & 1.153860 \\
\hline 4 & 1 & -0.506466 & -0.877194 & 1.153860 \\
\hline 5 & 6 & 0.000000 & 0.000000 & -0.757448 \\
\hline 6 & 1 & 0.506439 & 0.877209 & -1.153860 \\
\hline 7 & 1 & 0.506466 & -0.877194 & -1.153860 \\
\hline 8 & 1 & -1.012905 & -0.000015 & -1.153860 \\
\hline
\end{tabular}


Zero-point correction=

Thermal correction to Energy=

Thermal correction to Enthalpy=

Thermal correction to Gibbs Free Energy=

Sum of electronic and zero-point Energies=

Sum of electronic and thermal Energies=

Sum of electronic and thermal Enthalpies=

Sum of electronic and thermal Free Energies=

Methane

Standard orientation:
0.075731 (Hartree/Particle)

0.079199

0.080143

0.054337

$-79.690642$

$-79.687174$

$-79.686230$

$-79.712036$

\begin{tabular}{|c|c|c|c|c|}
\hline \multirow{2}{*}{$\begin{array}{l}\text { Center } \\
\text { Number }\end{array}$} & Atomic & \multirow{2}{*}{$\begin{array}{l}\text { Atomic } \\
\text { Type }\end{array}$} & \multicolumn{2}{|c|}{ Coordinates (Angstrom } \\
\hline & Number & & $\mathrm{X}$ & Z \\
\hline 1 & 6 & 0.000000 & 0.000000 & 0.000000 \\
\hline 2 & 1 & 0.626337 & 0.626337 & 0.626337 \\
\hline 3 & 1 & -0.626337 & -0.626337 & 0.626337 \\
\hline 4 & 1 & -0.626337 & 0.626337 & -0.626337 \\
\hline 5 & 1 & 0.626337 & -0.626337 & -0.626337 \\
\hline
\end{tabular}

Zero-point correction=

Thermal correction to Energy=

Thermal correction to Enthalpy=

Thermal correction to Gibbs Free Energy=

Sum of electronic and zero-point Energies=

Sum of electronic and thermal Energies $=$

Sum of electronic and thermal Enthalpies=

Sum of electronic and thermal Free Energies=

Propane

Standard orientation:
0.045339 (Hartree/Particle)

0.048206

0.049150

$$
\begin{gathered}
0.028037 \\
-40.438355 \\
-40.435488 \\
-40.434544 \\
-40.455657
\end{gathered}
$$

\begin{tabular}{|c|c|c|c|c|}
\hline \multirow{2}{*}{$\begin{array}{l}\text { Center } \\
\text { Number }\end{array}$} & Atomic & \multirow{2}{*}{$\begin{array}{l}\text { Atomic } \\
\text { Type }\end{array}$} & \multicolumn{2}{|c|}{ Coordinates (Angstrom } \\
\hline & Number & & $\mathrm{X}$ & Z \\
\hline 1 & 6 & -1.259828 & -0.257876 & 0.000000 \\
\hline 2 & 1 & -2.156655 & 0.357699 & -0.000029 \\
\hline 3 & 1 & -1.298733 & -0.901058 & 0.877930 \\
\hline 4 & 1 & -1.298709 & -0.901103 & -0.877898 \\
\hline 5 & 6 & 0.000000 & 0.584021 & 0.000000 \\
\hline 6 & 1 & 0.000001 & 1.239666 & 0.870999 \\
\hline 7 & 1 & 0.000002 & 1.239652 & -0.871009 \\
\hline 8 & 6 & 1.259828 & -0.257877 & 0.000000 \\
\hline 9 & 1 & 1.298727 & -0.901065 & 0.877926 \\
\hline 10 & 1 & 1.298710 & -0.901096 & -0.877903 \\
\hline 11 & 1 & 2.156656 & 0.357696 & -0.000020 \\
\hline
\end{tabular}

Zero-point correction=

Thermal correction to Energy=

Thermal correction to Enthalpy=
0.104885 (Hartree/Particle)

0.109380

0.110324 
Thermal correction to Gibbs Free Energy=

Sum of electronic and zero-point Energies=

Sum of electronic and thermal Energies $=$

Sum of electronic and thermal Enthalpies=

Sum of electronic and thermal Free Energies=

Butane

Standard orientation:
0.079276

$-118.946765$

$-118.942270$

$-118.941326$

$-118.972374$

\begin{tabular}{|c|c|c|c|c|}
\hline \multirow{2}{*}{$\begin{array}{l}\text { Center } \\
\text { Number }\end{array}$} & Atomic & \multirow{2}{*}{$\begin{array}{l}\text { Atomic } \\
\text { Type }\end{array}$} & \multicolumn{2}{|c|}{ Coordinates (Angstrom } \\
\hline & Number & & $\mathrm{X}$ & $\mathrm{Z}$ \\
\hline 1 & 6 & 1.937347 & -0.118703 & 0.000019 \\
\hline 2 & 1 & 2.079816 & -0.746885 & -0.877967 \\
\hline 3 & 1 & 2.079772 & -0.746900 & 0.878002 \\
\hline 4 & 1 & 2.723665 & 0.632807 & 0.000046 \\
\hline 5 & 6 & 0.559599 & 0.511235 & -0.000022 \\
\hline 6 & 1 & 0.451344 & 1.159303 & 0.871576 \\
\hline 7 & 1 & 0.451369 & 1.159283 & -0.871640 \\
\hline 8 & 6 & -0.559599 & -0.511235 & -0.000022 \\
\hline 9 & 1 & -0.451369 & -1.159280 & -0.871641 \\
\hline 10 & 1 & -0.451344 & -1.159306 & 0.871575 \\
\hline 11 & 6 & -1.937347 & 0.118703 & 0.000020 \\
\hline 12 & 1 & -2.079817 & 0.746884 & -0.877968 \\
\hline 13 & 1 & -2.723665 & -0.632807 & 0.000048 \\
\hline 14 & 1 & -2.079770 & 0.746902 & 0.878001 \\
\hline
\end{tabular}

Zero-point correction=

Thermal correction to Energy=

Thermal correction to Enthalpy=

Thermal correction to Gibbs Free Energy=

Sum of electronic and zero-point Energies=

Sum of electronic and thermal Energies $=$

Sum of electronic and thermal Enthalpies=

Sum of electronic and thermal Free Energies=

Isobutane

Standard orientation:
0.133828 (Hartree/Particle)

0.139567

0.140512

0.105876

$-158.203094$

$-158.197355$

$-158.196410$

$-158.231046$

\begin{tabular}{|c|c|c|c|c|}
\hline \multirow{2}{*}{$\begin{array}{l}\text { Center } \\
\text { Number }\end{array}$} & Atomic & \multirow{2}{*}{$\begin{array}{l}\text { Atomic } \\
\text { Type }\end{array}$} & \multicolumn{2}{|c|}{ Coordinates (Angstrom } \\
\hline & Numbe & & $\mathrm{X}$ & Z \\
\hline 1 & 6 & -0.978483 & -1.060454 & -0.095130 \\
\hline 2 & 1 & -1.987113 & -0.855353 & 0.258908 \\
\hline 3 & 1 & -1.010368 & -1.095118 & -1.184362 \\
\hline 4 & 1 & -0.693131 & -2.049340 & 0.259014 \\
\hline 5 & 6 & -0.000001 & 0.000004 & 0.374160 \\
\hline 6 & 1 & -0.000004 & 0.000005 & 1.466721 \\
\hline 7 & 6 & -0.429143 & 1.377614 & -0.095134 \\
\hline 8 & 1 & 0.252704 & 2.148601 & 0.258997 \\
\hline 9 & 1 & -0.443108 & 1.422567 & -1.184368 \\
\hline 10 & 1 & -1.428270 & 1.624852 & 0.258926 \\
\hline
\end{tabular}




$\begin{array}{cccccc}11 & 6 & 0 & 1.407630 & -0.317160 & -0.095128 \\ 12 & 1 & 0 & 2.121335 & 0.424463 & 0.258935 \\ 13 & 1 & 0 & 1.734365 & -1.293167 & 0.258985 \\ 14 & 1 & 0 & 1.453565 & -0.327534 & -1.184365\end{array}$

Zero-point correction=

Thermal correction to Energy=

Thermal correction to Enthalpy=

Thermal correction to Gibbs Free Energy=

Sum of electronic and zero-point Energies=

Sum of electronic and thermal Energies $=$

Sum of electronic and thermal Enthalpies=

Sum of electronic and thermal Free Energies=

Pentane

Standard orientation:
0.133339 (Hartree/Particle)

0.138996

0.139940

\subsection{0}

$-158.205157$

$-158.199500$

$-158.198556$

$-158.232716$

\begin{tabular}{cccccc} 
Center & \multicolumn{2}{c}{ Atomic } & \multicolumn{2}{c}{ Atomic } & \multicolumn{3}{c}{ Coordinates } \\
Number & Number & Type & X & $Y$ & $Z$ \\
--------------- \\
1 & 6 & 0 & 2.526348 & 0.322674 & -0.000001 \\
2 & 1 & 0 & 2.566248 & 0.965498 & -0.877934 \\
3 & 1 & 0 & 2.566245 & 0.965505 & 0.877927 \\
4 & 1 & 0 & 3.422651 & -0.293440 & 0.000003 \\
5 & 6 & 0 & 1.267524 & -0.520132 & 0.000001 \\
6 & 1 & 0 & 1.265478 & -1.176721 & -0.871710 \\
7 & 1 & 0 & 1.265479 & -1.176718 & 0.871715 \\
8 & 6 & 0 & 0.000000 & 0.310608 & 0.000000 \\
9 & 1 & 0 & -0.000001 & 0.968798 & 0.872276 \\
10 & 1 & 0 & 0.000001 & 0.968798 & -0.872276 \\
11 & 6 & 0 & -1.267524 & -0.520132 & -0.000001 \\
12 & 1 & 0 & -1.265478 & -1.176721 & 0.871710 \\
13 & 1 & 0 & -1.265478 & -1.176718 & -0.871715 \\
14 & 6 & 0 & -2.526349 & 0.322674 & 0.000001 \\
15 & 1 & 0 & -3.422651 & -0.293440 & -0.000000 \\
16 & 1 & 0 & -2.566246 & 0.965501 & 0.877932 \\
17 & 1 & 0 & -2.566247 & 0.965502 & -0.877929
\end{tabular}

Zero-point correction=

Thermal correction to Energy=

Thermal correction to Enthalpy=

Thermal correction to Gibbs Free Energy=

Sum of electronic and zero-point Energies=

Sum of electronic and thermal Energies $=$

Sum of electronic and thermal Enthalpies=

Sum of electronic and thermal Free Energies=

Neopentane

Standard orientation:

\begin{tabular}{lcccc} 
Center & Atomic & Atomic & & \multicolumn{2}{c}{ Coordinates (Angstroms) } \\
Number & Number & Type & X & Y C Z
\end{tabular}




$\begin{array}{cccccc}1 & 6 & 0 & -1.057827 & 0.635215 & 0.890157 \\ 2 & 1 & 0 & -1.306191 & 1.638480 & 0.545827 \\ 3 & 1 & 0 & -1.973284 & 0.044685 & 0.890341 \\ 4 & 1 & 0 & -0.707700 & 0.711021 & 1.918966 \\ 5 & 6 & 0 & 0.000010 & 0.000007 & 0.000016 \\ 6 & 6 & 0 & -0.524026 & -0.089637 & -1.425552 \\ 7 & 1 & 0 & 0.215624 & -0.541733 & -2.085327 \\ 8 & 1 & 0 & -1.428560 & -0.695035 & -1.471326 \\ 9 & 1 & 0 & -0.762198 & 0.898917 & -1.816479 \\ 10 & 6 & 0 & 1.260952 & 0.851076 & 0.024259 \\ 11 & 1 & 0 & 1.657557 & 0.931939 & 1.035663 \\ 12 & 1 & 0 & 2.036439 & 0.417533 & -0.606178 \\ 13 & 1 & 0 & 1.058719 & 1.858372 & -0.338014 \\ 14 & 6 & 0 & 0.320903 & -1.396656 & 0.511128 \\ 15 & 1 & 0 & 0.698298 & -1.361142 & 1.532450 \\ 16 & 1 & 0 & -0.566656 & -2.028294 & 0.503526 \\ 17 & 1 & 0 & 1.077881 & -1.874771 & -0.109497\end{array}$

Zero-point correction=

Thermal correction to Energy=

Thermal correction to Enthalpy=

Thermal correction to Gibbs Free Energy=

Sum of electronic and zero-point Energies=

Sum of electronic and thermal Energies=

Sum of electronic and thermal Enthalpies=

Sum of electronic and thermal Free Energies=

Hexane

Standard orientation:

\begin{tabular}{|c|c|c|c|c|}
\hline \multirow{2}{*}{$\begin{array}{l}\text { Center } \\
\text { Number }\end{array}$} & Atomic & \multirow{2}{*}{$\begin{array}{l}\text { Atomic } \\
\text { Type }\end{array}$} & \multicolumn{2}{|c|}{ Coordinates (Angstrom } \\
\hline & Number & & $X$ & Z \\
\hline 1 & 6 & -3.181903 & 0.206821 & 0.000006 \\
\hline 2 & 1 & -3.266378 & 0.845320 & 0.877951 \\
\hline 3 & 1 & -3.266379 & 0.845345 & -0.877921 \\
\hline 4 & 1 & -4.033260 & -0.470065 & -0.000004 \\
\hline 5 & 6 & -1.867588 & -0.546515 & -0.000006 \\
\hline 6 & 1 & -1.819940 & -1.201389 & 0.871669 \\
\hline 7 & 1 & -1.819945 & -1.201370 & -0.871696 \\
\hline 8 & 6 & -0.660767 & 0.370520 & -0.000000 \\
\hline 9 & 1 & -0.707508 & 1.026814 & -0.872314 \\
\hline 10 & 1 & -0.707510 & 1.026808 & 0.872318 \\
\hline 11 & 6 & 0.660767 & -0.370520 & 0.000000 \\
\hline 12 & 1 & 0.707507 & -1.026815 & -0.872313 \\
\hline 13 & 1 & 0.707511 & -1.026807 & 0.872319 \\
\hline 14 & 6 & 1.867588 & 0.546515 & -0.000006 \\
\hline 15 & 1 & 1.819940 & 1.201389 & 0.871669 \\
\hline 16 & 1 & 1.819945 & 1.201370 & -0.871696 \\
\hline 17 & 6 & 3.181903 & -0.206821 & 0.000006 \\
\hline
\end{tabular}




$\begin{array}{rrrrrr}18 & 1 & 0 & 3.266379 & -0.845346 & -0.877921 \\ 19 & 1 & 0 & 4.033260 & 0.470065 & -0.000005 \\ 20 & 1 & 0 & 3.266378 & -0.845319 & 0.877951\end{array}$

$\begin{array}{lc}\text { Zero-point correction }= & 0.191643 \text { (Hartree/Particle) } \\ \text { Thermal correction to Energy= } & 0.200001 \\ \text { Thermal correction to Enthalpy= } & 0.200945 \\ \text { Thermal correction to Gibbs Free Energy= } & 0.159114 \\ \text { Sum of electronic and zero-point Energies }= & -236.715658 \\ \text { Sum of electronic and thermal Energies }= & -236.707300 \\ \text { Sum of electronic and thermal Enthalpies }= & -236.706356 \\ \text { Sum of electronic and thermal Free Energies }= & -236.748187 \\ \text { 22dimethylbutane } & \\ \text { Standard orientation: }\end{array}$

\begin{tabular}{|c|c|c|c|c|}
\hline \multirow{2}{*}{$\begin{array}{l}\text { Center } \\
\text { Number }\end{array}$} & Atomic & \multirow{2}{*}{$\begin{array}{l}\text { Atomic } \\
\text { Type }\end{array}$} & \multicolumn{2}{|c|}{ Coordinates (Angstroms } \\
\hline & Numbe & & $\mathrm{X}$ & Z \\
\hline 1 & 6 & -1.602700 & 0.969745 & 0.000919 \\
\hline 2 & 1 & -1.579437 & 1.609867 & 0.882013 \\
\hline 3 & 1 & -1.579539 & 1.611384 & -0.879074 \\
\hline 4 & 1 & -2.551676 & 0.435332 & 0.000510 \\
\hline 5 & 6 & -0.430008 & -0.000390 & 0.000010 \\
\hline 6 & 6 & 0.862073 & 0.821071 & 0.000500 \\
\hline 7 & 1 & 0.843358 & 1.478651 & -0.871064 \\
\hline 8 & 1 & 0.843412 & 1.477556 & 0.872894 \\
\hline 9 & 6 & 2.159462 & 0.036585 & 0.000004 \\
\hline 10 & 1 & 2.250118 & -0.597094 & -0.879238 \\
\hline 11 & 1 & 3.011727 & 0.712479 & 0.000210 \\
\hline 12 & 1 & 2.250280 & -0.597852 & 0.878679 \\
\hline 13 & 6 & -0.513930 & -0.875511 & 1.242254 \\
\hline 14 & 1 & 0.275861 & -1.623295 & 1.263733 \\
\hline 15 & 1 & -0.435454 & -0.276044 & 2.148659 \\
\hline 16 & 1 & -1.465873 & -1.403626 & 1.273770 \\
\hline 17 & 6 & -0.514246 & -0.873498 & -1.243636 \\
\hline 18 & 1 & -1.466102 & -1.401740 & -1.275648 \\
\hline 19 & 1 & -0.436240 & -0.272520 & -2.149079 \\
\hline 20 & 1 & 0.275665 & -1.621109 & -1.266668 \\
\hline
\end{tabular}

Zero-point correction=

Thermal correction to Energy=

Thermal correction to Enthalpy=

Thermal correction to Gibbs Free Energy=

Sum of electronic and zero-point Energies=

Sum of electronic and thermal Energies $=$

Sum of electronic and thermal Enthalpies=

Sum of electronic and thermal Free Energies=

Heptane

Standard orientation:
0.190483 (Hartree/Particle)

$$
0.198661
$$$$
0.199606
$$

0.159265

$-236.717967$

$-236.709789$

$-236.708845$

$-236.749186$ 


\begin{tabular}{|c|c|c|c|c|}
\hline \multirow{2}{*}{$\begin{array}{l}\text { Center } \\
\text { Number }\end{array}$} & Atomic & \multirow{2}{*}{$\begin{array}{l}\text { Atomic } \\
\text { Type }\end{array}$} & \multicolumn{2}{|c|}{ Coordinates (Angstroms } \\
\hline & Numbe & & $\mathrm{X}$ & $\mathrm{Z}$ \\
\hline 1 & 6 & -3.793323 & -0.351545 & -0.000008 \\
\hline 2 & 1 & -3.833211 & -0.994377 & -0.877954 \\
\hline 3 & 1 & -3.833210 & -0.994413 & 0.877912 \\
\hline 4 & 1 & -4.689680 & 0.264520 & 0.000005 \\
\hline 5 & 6 & -2.534515 & 0.491278 & 0.000010 \\
\hline 6 & 1 & -2.532446 & 1.147881 & -0.871673 \\
\hline 7 & 1 & -2.532451 & 1.147852 & 0.871715 \\
\hline 8 & 6 & -1.266937 & -0.339747 & 0.000000 \\
\hline 9 & 1 & -1.267971 & -0.997702 & 0.872287 \\
\hline 10 & 1 & -1.267975 & -0.997689 & -0.872296 \\
\hline 11 & 6 & 0.000000 & 0.491576 & 0.000001 \\
\hline 12 & 1 & -0.000002 & 1.149288 & 0.872362 \\
\hline 13 & 1 & 0.000002 & 1.149284 & -0.872362 \\
\hline 14 & 6 & 1.266937 & -0.339747 & 0.000005 \\
\hline 15 & 1 & 1.267969 & -0.997707 & -0.872278 \\
\hline 16 & 1 & 1.267977 & -0.997684 & 0.872306 \\
\hline 17 & 6 & 2.534515 & 0.491278 & -0.000011 \\
\hline 18 & 1 & 2.532450 & 1.147882 & 0.871671 \\
\hline 19 & 1 & 2.532447 & 1.147850 & -0.871717 \\
\hline 20 & 6 & 3.793323 & -0.351545 & 0.000003 \\
\hline 21 & 1 & 3.833208 & -0.994412 & -0.877917 \\
\hline 22 & 1 & 4.689680 & 0.264520 & -0.000012 \\
\hline 23 & 1 & 3.833213 & -0.994377 & 0.877949 \\
\hline
\end{tabular}

Zero-point correction=

Thermal correction to Energy=

Thermal correction to Enthalpy=

Thermal correction to Gibbs Free Energy=

Sum of electronic and zero-point Energies=

Sum of electronic and thermal Energies $=$

Sum of electronic and thermal Enthalpies=

Sum of electronic and thermal Free Energies=

223trimethylbutane

Standard orientation:

\begin{tabular}{|c|c|c|c|c|}
\hline \multirow{2}{*}{$\begin{array}{l}\text { Center } \\
\text { Number }\end{array}$} & Atomic & \multirow{2}{*}{$\begin{array}{l}\text { Atomic } \\
\text { Type }\end{array}$} & \multicolumn{2}{|c|}{ Coordinates (Angstroms) } \\
\hline & Number & & $X$ & Z \\
\hline 1 & 6 & -1.341078 & -1.230309 & -0.559266 \\
\hline 2 & 1 & -1.216854 & -1.296212 & -1.639962 \\
\hline 3 & 1 & -0.967287 & -2.151668 & -0.119893 \\
\hline 4 & 1 & -2.408533 & -1.179927 & -0.350216 \\
\hline 5 & 6 & -0.635568 & 0.000003 & -0.000500 \\
\hline 6 & 6 & 0.843590 & 0.000003 & -0.449148 \\
\hline 7 & 1 & 0.815123 & 0.000014 & -1.543134 \\
\hline 8 & 6 & 1.615147 & 1.237378 & -0.017438 \\
\hline
\end{tabular}




$\begin{array}{cccccc}9 & 1 & 0 & 1.697410 & 1.295861 & 1.066772 \\ 10 & 1 & 0 & 2.627976 & 1.200872 & -0.414004 \\ 11 & 1 & 0 & 1.161179 & 2.158573 & -0.371146 \\ 12 & 6 & 0 & 1.615169 & -1.237361 & -0.017482 \\ 13 & 1 & 0 & 1.697675 & -1.295734 & 1.066717 \\ 14 & 1 & 0 & 1.161088 & -2.158580 & -0.370986 \\ 15 & 1 & 0 & 2.627911 & -1.200929 & -0.414279 \\ 16 & 6 & 0 & -0.777179 & -0.000339 & 1.515666 \\ 17 & 1 & 0 & -0.323445 & 0.880631 & 1.964679 \\ 18 & 1 & 0 & -1.830985 & -0.000137 & 1.790821 \\ 19 & 1 & 0 & -0.323887 & -0.881762 & 1.964231 \\ 20 & 6 & 0 & -1.340945 & 1.230624 & -0.558745 \\ 21 & 1 & 0 & -1.216533 & 1.297099 & -1.639387 \\ 22 & 1 & 0 & -2.408443 & 1.180171 & -0.349921 \\ 23 & 1 & 0 & -0.967207 & 2.151741 & -0.118822\end{array}$

Zero-point correction=

Thermal correction to Energy=

Thermal correction to Enthalpy=

Thermal correction to Gibbs Free Energy=

Sum of electronic and zero-point Energies=

Sum of electronic and thermal Energies $=$

Sum of electronic and thermal Enthalpies=

Sum of electronic and thermal Free Energies=

Octane

Standard orientation:

\begin{tabular}{|c|c|c|c|c|}
\hline \multirow{2}{*}{$\begin{array}{l}\text { Center } \\
\text { Number }\end{array}$} & Atomic & \multirow{2}{*}{$\begin{array}{l}\text { Atomic } \\
\text { Type }\end{array}$} & \multicolumn{2}{|c|}{ Coordinates (Angstroms } \\
\hline & Numbe & & $\mathrm{X}$ & $\mathrm{Z}$ \\
\hline 1 & 6 & -4.440107 & 0.254827 & 0.000015 \\
\hline 2 & 1 & -4.504993 & 0.895618 & 0.877965 \\
\hline 3 & 1 & -4.504996 & 0.895670 & -0.877898 \\
\hline 4 & 1 & -5.311822 & -0.395646 & -0.000004 \\
\hline 5 & 6 & -3.149457 & -0.538372 & -0.000013 \\
\hline 6 & 1 & -3.121853 & -1.194409 & 0.871662 \\
\hline 7 & 1 & -3.121862 & -1.194366 & -0.871721 \\
\hline 8 & 6 & -1.915150 & 0.341310 & 0.000001 \\
\hline 9 & 1 & -1.941767 & 0.998736 & -0.872290 \\
\hline 10 & 1 & -1.941770 & 0.998713 & 0.872308 \\
\hline 11 & 6 & -0.616855 & -0.440119 & -0.000005 \\
\hline 12 & 1 & -0.591263 & -1.097334 & -0.872337 \\
\hline 13 & 1 & -0.591257 & -1.097337 & 0.872325 \\
\hline 14 & 6 & 0.616855 & 0.440119 & -0.000008 \\
\hline 15 & 1 & 0.591256 & 1.097346 & 0.872315 \\
\hline 16 & 1 & 0.591264 & 1.097325 & -0.872346 \\
\hline 17 & 6 & 1.915149 & -0.341310 & 0.000007 \\
\hline 18 & 1 & 1.941769 & -0.998741 & -0.872280 \\
\hline 19 & 1 & 1.941768 & -0.998708 & 0.872318 \\
\hline 20 & 6 & 3.149457 & 0.538372 & -0.000009 \\
\hline
\end{tabular}




$\begin{array}{rrrrrr}21 & 1 & 0 & 3.121856 & 1.194406 & 0.871669 \\ 22 & 1 & 0 & 3.121859 & 1.194370 & -0.871714 \\ 23 & 6 & 0 & 4.440107 & -0.254827 & 0.000010 \\ 24 & 1 & 0 & 4.504996 & -0.895663 & -0.877907 \\ 25 & 1 & 0 & 5.311822 & 0.395645 & -0.000003 \\ 26 & 1 & 0 & 4.504993 & -0.895626 & 0.877955\end{array}$

Zero-point correction=

Thermal correction to Energy=

Thermal correction to Enthalpy=

Thermal correction to Gibbs Free Energy=

Sum of electronic and zero-point Energies=

Sum of electronic and thermal Energies $=$

Sum of electronic and thermal Enthalpies=

Sum of electronic and thermal Free Energies=
0.249371 (Hartree/Particle)

0.260412

0.261356

0.212339

$-315.228323$

$-315.217282$

$-315.216338$

$-315.265355$

2233tetramethylbutane

Standard orientation:

\begin{tabular}{|c|c|c|c|c|c|}
\hline \multirow{2}{*}{$\begin{array}{l}\text { Center } \\
\text { Number }\end{array}$} & \multicolumn{2}{|l|}{ Atomic } & & \multicolumn{2}{|c|}{ Coordinates (Angstroms) } \\
\hline & Numbe & & & $\mathrm{X}$ & Z \\
\hline 1 & 6 & 0 & -1.334941 & -1.418992 & 0.098173 \\
\hline 2 & 1 & 0 & -1.129967 & -1.997037 & -0.799586 \\
\hline 3 & 1 & 0 & -0.928666 & -1.960916 & 0.949710 \\
\hline 4 & 1 & 0 & -2.416175 & -1.381996 & 0.222259 \\
\hline 5 & 6 & 0 & -0.782029 & -0.000107 & 0.000008 \\
\hline 6 & 6 & 0 & 0.782020 & -0.000040 & 0.000001 \\
\hline 7 & 6 & 0 & 1.334590 & 1.363353 & -0.405219 \\
\hline 8 & 1 & 0 & 1.129399 & 1.590261 & -1.448551 \\
\hline 9 & 1 & 0 & 0.928172 & 2.169091 & 0.202608 \\
\hline 10 & 1 & 0 & 2.415839 & 1.372395 & -0.276164 \\
\hline 11 & 6 & 0 & -1.334765 & 0.624451 & -1.277844 \\
\hline 12 & 1 & 0 & -1.129434 & 1.690893 & -1.329617 \\
\hline 13 & 1 & 0 & -0.928655 & 0.157778 & -2.172907 \\
\hline 14 & 1 & 0 & -2.416041 & 0.498849 & -1.307798 \\
\hline 15 & 6 & 0 & -1.334789 & 0.794448 & 1.179675 \\
\hline 16 & 1 & 0 & -2.415953 & 0.883975 & 1.085340 \\
\hline 17 & 1 & 0 & -1.130297 & 0.305774 & 2.129142 \\
\hline 18 & 1 & 0 & -0.927993 & 1.802652 & 1.223393 \\
\hline 19 & 6 & 0 & 1.335003 & -1.032564 & -0.978065 \\
\hline 20 & 1 & 0 & 2.416216 & -0.924933 & -1.050426 \\
\hline 21 & 1 & 0 & 1.130220 & -2.049603 & -0.652794 \\
\hline 22 & 1 & 0 & 0.928583 & -0.909303 & -1.979790 \\
\hline 23 & 6 & 0 & 1.334920 & -0.330650 & 1.383283 \\
\hline 24 & 1 & 0 & 2.416114 & -0.447328 & 1.326276 \\
\hline 25 & 1 & 0 & 1.130245 & 0.459725 & 2.101290 \\
\hline 26 & 1 & 0 & 0.928337 & -1.259673 & 1.777549 \\
\hline
\end{tabular}


Zero-point correction=

Thermal correction to Energy=

Thermal correction to Enthalpy=

Thermal correction to Gibbs Free Energy=

Sum of electronic and zero-point Energies=

Sum of electronic and thermal Energies=

Sum of electronic and thermal Enthalpies=

Sum of electronic and thermal Free Energies=

PBEQIDH/ccpVQZ

Ethane

Standard orientation:
0.247816 (Hartree/Particle)

0.258168

0.259112

0.214567

$-315.225413$

$-315.215061$

$-315.214116$

$-315.258662$

\begin{tabular}{|c|c|c|c|c|}
\hline \multirow{2}{*}{$\begin{array}{l}\text { Center } \\
\text { Number }\end{array}$} & Atomic & \multirow{2}{*}{$\begin{array}{r}\text { Atomic } \\
\text { Type }\end{array}$} & \multicolumn{2}{|c|}{ Coordinates (Angstro } \\
\hline & Numbe & & $\mathrm{X}$ & Z \\
\hline 1 & 6 & 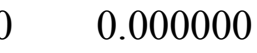 & -0 . & 0.7 \\
\hline 2 & 1 & -0.5 & 368 & 24 \\
\hline 3 & 1 & 6 & -0.0 & 1.15245 \\
\hline 4 & 1 & -0.505977 & -0.876357 & 1.1524 \\
\hline 5 & 6 & 0.0 & 0.0 & -0 . \\
\hline 6 & 1 & 0.505959 & 0.876368 & -1.1524 \\
\hline 7 & 1 & 0.505977 & -0.876357 & -1.1524 \\
\hline 8 & 1 & -1.011936 & -0.000011 & -1.1524 \\
\hline
\end{tabular}

Zero-point correction=

Thermal correction to Energy=

0.076101 (Hartree/Particle)

Thermal correction to Enthalpy=

0.079563

0.080507

Thermal correction to Gibbs Free Energy $=0.054713$

Sum of electronic and zero-point Energies=

$-79.678171$

Sum of electronic and thermal Energies $=$

$-79.674709$

Sum of electronic and thermal Enthalpies=

$-79.673765$

Sum of electronic and thermal Free Energies=

$-79.699559$

Methane

Standard orientation:

\begin{tabular}{|c|c|c|c|c|}
\hline \multirow{2}{*}{$\begin{array}{l}\text { Center } \\
\text { Number }\end{array}$} & \multirow{2}{*}{$\begin{array}{l}\text { Atomic } \\
\text { Number }\end{array}$} & \multirow{2}{*}{$\begin{array}{r}\text { Atomic } \\
\text { Type }\end{array}$} & \multicolumn{2}{|c|}{ Coordinates (Angstroms) } \\
\hline & & & $\mathrm{X}$ & $\mathrm{Z}$ \\
\hline 1 & 6 & 0.000000 & 0.000000 & 0.000000 \\
\hline 2 & 1 & 0.625582 & 0.625582 & 0.625582 \\
\hline 3 & 1 & -0.625582 & -0.625582 & 0.625582 \\
\hline 4 & 1 & -0.625582 & 0.625582 & -0.625582 \\
\hline 5 & 1 & 0.625582 & -0.625582 & -0.625582 \\
\hline \multicolumn{3}{|c|}{ Zero-point correction $=$} & \multicolumn{2}{|c|}{0.045578 (Hartree/Particle) } \\
\hline \multicolumn{3}{|c|}{ Thermal correction to Energy= } & \multicolumn{2}{|c|}{0.048444} \\
\hline \multicolumn{3}{|c|}{ Thermal correction to Enthalpy= } & \multicolumn{2}{|c|}{0.049388} \\
\hline \multicolumn{4}{|c|}{ Thermal correction to Gibbs Free Energy= } & 0.028280 \\
\hline \multicolumn{4}{|c|}{ Sum of electronic and zero-point Energies $=$} & -40.432009 \\
\hline \multicolumn{4}{|c|}{ Sum of electronic and thermal Energies $=$} & -40.429143 \\
\hline
\end{tabular}


Sum of electronic and thermal Enthalpies=

$-40.428199$

Sum of electronic and thermal Free Energies=

Propane

Standard orientation:

\begin{tabular}{|c|c|c|c|c|}
\hline \multirow{2}{*}{$\begin{array}{l}\text { Center } \\
\text { Number }\end{array}$} & Atomic & \multirow{2}{*}{$\begin{array}{l}\text { Atomic } \\
\text { Type }\end{array}$} & \multicolumn{2}{|c|}{ Coordinates (Angstrom } \\
\hline & Numbe & & $X$ & Z \\
\hline 1 & 6 & -1.257800 & -0.258278 & 0.000000 \\
\hline 2 & 1 & -2.154944 & 0.354632 & -0.000030 \\
\hline 3 & 1 & -1.293978 & -0.900565 & 0.877149 \\
\hline 4 & 1 & -1.293951 & -0.900613 & -0.877115 \\
\hline 5 & 6 & 0.000000 & 0.585527 & 0.000000 \\
\hline 6 & 1 & 0.000001 & 1.239642 & 0.870527 \\
\hline 7 & 1 & 0.000002 & 1.239628 & -0.870537 \\
\hline 8 & 6 & 1.257799 & -0.258278 & 0.000000 \\
\hline 9 & 1 & 1.293971 & -0.900574 & 0.877144 \\
\hline 10 & 1 & 1.293953 & -0.900605 & -0.877121 \\
\hline 11 & 1 & 2.154945 & 0.354629 & -0.000020 \\
\hline
\end{tabular}

Zero-point correction $=$

Thermal correction to Energy=

Thermal correction to Enthalpy=

Thermal correction to Gibbs Free Energy=

Sum of electronic and zero-point Energies=

Sum of electronic and thermal Energies=

Sum of electronic and thermal Enthalpies=

Sum of electronic and thermal Free Energies=
0.105364 (Hartree/Particle)

0.109847

0.110792

\subsection{5}

$-118.928563$

$-118.924080$

$-118.923135$

$-118.954162$

\section{Butane}

Standard orientation:

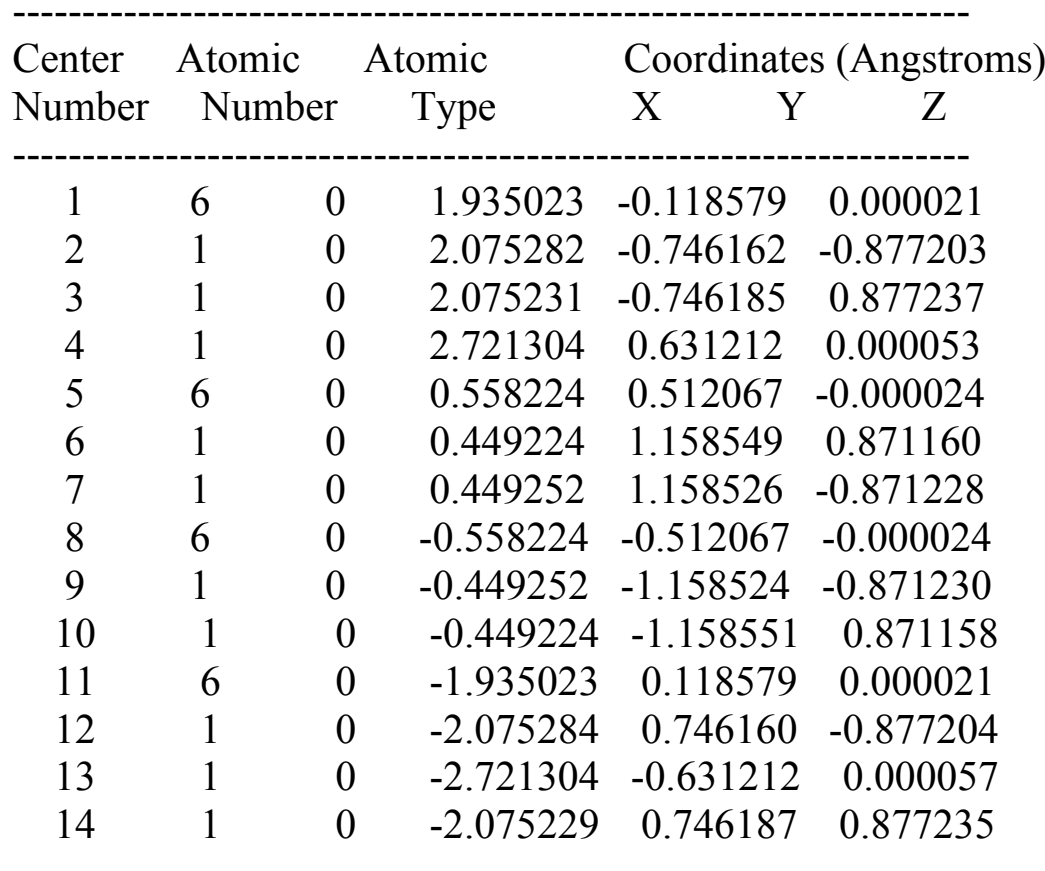


Zero-point correction $=$

Thermal correction to Energy=

Thermal correction to Enthalpy=

Thermal correction to Gibbs Free Energy=

Sum of electronic and zero-point Energies=

Sum of electronic and thermal Energies=

Sum of electronic and thermal Enthalpies=

Sum of electronic and thermal Free Energies=

Isobutane

Standard orientation:
0.134415 (Hartree/Particle)

0.140140

0.141084

$$
\begin{gathered}
0.106477 \\
-158.179211 \\
-158.173486 \\
-158.172541 \\
-158.207148
\end{gathered}
$$

\begin{tabular}{|c|c|c|c|c|}
\hline \multirow{2}{*}{$\begin{array}{l}\text { Center } \\
\text { Number }\end{array}$} & Atomic & \multirow{2}{*}{$\begin{array}{l}\text { Atomic } \\
\text { Type }\end{array}$} & \multicolumn{2}{|c|}{ Coordinates (Angstroms } \\
\hline & Numbe & & $X$ & Z \\
\hline 1 & 6 & 1.418488 & 0.253936 & -0.095396 \\
\hline 2 & 1 & 2.098167 & -0.518171 & 0.257000 \\
\hline 3 & 1 & 1.460556 & 0.261530 & -1.183685 \\
\hline 4 & 1 & 1.788164 & 1.213819 & 0.257112 \\
\hline 5 & 6 & 0.000016 & 0.000003 & 0.376351 \\
\hline 6 & 1 & 0.000010 & -0.000006 & 1.467690 \\
\hline 7 & 6 & -0.489328 & -1.355393 & -0.095391 \\
\hline 8 & 1 & -1.497850 & -1.557938 & 0.256968 \\
\hline 9 & 1 & -0.503750 & -1.395614 & -1.183679 \\
\hline 10 & 1 & 0.157084 & -2.155503 & 0.257131 \\
\hline 11 & 6 & -0.929166 & 1.101457 & -0.095389 \\
\hline 12 & 1 & -1.945349 & 0.941490 & 0.256865 \\
\hline 13 & 1 & -0.600540 & 2.076118 & 0.257230 \\
\hline 14 & 1 & -0.956546 & 1.134258 & -1.183679 \\
\hline
\end{tabular}

Zero-point correction=

Thermal correction to Energy=

0.133910 (Hartree/Particle)

Thermal correction to Enthalpy=

0.139549

Thermal correction to Gibbs Free Energy=

0.140494

Sum of electronic and zero-point Energies=

0.106368

Sum of electronic and thermal Energies $=$

$-158.181688$

$-158.176048$

$-158.175104$

\begin{tabular}{|c|c|c|c|c|}
\hline \multirow{2}{*}{$\begin{array}{l}\text { Center } \\
\text { Number }\end{array}$} & Atomic & \multirow{2}{*}{$\begin{array}{l}\text { Atomic } \\
\text { Type }\end{array}$} & \multicolumn{2}{|c|}{ Coordinates (Angstrom } \\
\hline & Number & & $\mathrm{X}$ & $\mathrm{Z}$ \\
\hline 1 & 6 & 2.522819 & 0.323626 & -0.000001 \\
\hline 2 & 1 & 2.559797 & 0.965544 & -0.877200 \\
\hline 3 & 1 & 2.559794 & 0.965551 & 0.877194 \\
\hline 4 & 1 & 3.419574 & -0.289724 & 0.000003 \\
\hline 5 & 6 & 1.266070 & -0.521268 & 0.000001 \\
\hline 6 & 1 & 1.263771 & -1.176375 & -0.871339 \\
\hline 7 & 1 & 1.263772 & -1.176373 & 0.871343 \\
\hline
\end{tabular}

$-158.209229$

Sum of electronic and thermal Free Energies=

Pentane

Standard orientation: 


$\begin{array}{cccccc}8 & 6 & 0 & 0.000000 & 0.310105 & 0.000000 \\ 9 & 1 & 0 & -0.000001 & 0.966919 & 0.871949 \\ 10 & 1 & 0 & 0.000001 & 0.966920 & -0.871949 \\ 11 & 6 & 0 & -1.266070 & -0.521268 & -0.000001 \\ 12 & 1 & 0 & -1.263772 & -1.176375 & 0.871339 \\ 13 & 1 & 0 & -1.263772 & -1.176372 & -0.871343 \\ 14 & 6 & 0 & -2.522819 & 0.323626 & 0.000000 \\ 15 & 1 & 0 & -3.419574 & -0.289724 & -0.000001 \\ 16 & 1 & 0 & -2.559796 & 0.965546 & 0.877199 \\ 17 & 1 & 0 & -2.559795 & 0.965549 & -0.877196\end{array}$

Zero-point correction=

Thermal correction to Energy=

Thermal correction to Enthalpy=

Thermal correction to Gibbs Free Energy=

Sum of electronic and zero-point Energies=

Sum of electronic and thermal Energies=

Sum of electronic and thermal Enthalpies=

Sum of electronic and thermal Free Energies=

Neopentane

Standard orientation:
0.163452 (Hartree/Particle)

0.170468

0.171413

0.133212

$-197.429797$

$-197.422780$

$-197.421836$

$-197.460037$

\begin{tabular}{|c|c|c|c|c|}
\hline \multirow{2}{*}{$\begin{array}{l}\text { Center } \\
\text { Number }\end{array}$} & Atomic & \multirow{2}{*}{$\begin{array}{l}\text { Atomic } \\
\text { Type }\end{array}$} & \multicolumn{2}{|c|}{ Coordinates (Angstrom } \\
\hline & Numbe & & $X$ & Z \\
\hline 1 & 6 & -1.472597 & 0.048399 & -0.373606 \\
\hline 2 & 1 & -1.912058 & -0.947116 & -0.352703 \\
\hline 3 & 1 & -2.029354 & 0.675828 & 0.319907 \\
\hline 4 & 1 & -1.606684 & 0.453648 & -1.374804 \\
\hline 5 & 6 & -0.000014 & 0.000008 & 0.000005 \\
\hline 6 & 6 & 0.148744 & -0.569815 & 1.401291 \\
\hline 7 & 1 & 1.196227 & -0.615583 & 1.693330 \\
\hline 8 & 1 & -0.376179 & 0.045932 & 2.129245 \\
\hline 9 & 1 & -0.259643 & -1.577159 & 1.456863 \\
\hline 10 & 6 & 0.744577 & -0.883328 & -0.987812 \\
\hline 11 & 1 & 0.653419 & -0.496386 & -2.001012 \\
\hline 12 & 1 & 1.803626 & -0.934691 & -0.742070 \\
\hline 13 & 1 & 0.348221 & -1.896948 & -0.978574 \\
\hline 14 & 6 & 0.579279 & 1.404742 & -0.039871 \\
\hline 15 & 1 & 0.484581 & 1.836378 & -1.034524 \\
\hline 16 & 1 & 0.062702 & 2.058471 & 0.660410 \\
\hline 17 & 1 & 1.635209 & 1.397588 & 0.223892 \\
\hline
\end{tabular}

Zero-point correction=

0.162068 (Hartree/Particle)

Thermal correction to Energy=

0.168885

Thermal correction to Enthalpy=

0.169829

Thermal correction to Gibbs Free Energy=

0.133078

Sum of electronic and zero-point Energies=

$-197.436095$

Sum of electronic and thermal Energies $=$

$-197.429278$

Sum of electronic and thermal Enthalpies=

$-197.428334$ 
Sum of electronic and thermal Free Energies $=\quad-197.465084$

Hexane

Standard orientation:

\begin{tabular}{cccccc} 
Center & \multicolumn{2}{c}{ Atomic } & \multicolumn{2}{c}{ Atomic } & \multicolumn{3}{c}{ Coordinates } \\
Number & Number & Type & X & Y & $Z$ \\
\hline 1 & 6 & 0 & -3.177909 & 0.207153 & 0.000006 \\
2 & 1 & 0 & -3.259748 & 0.844906 & 0.877220 \\
3 & 1 & 0 & -3.259750 & 0.844931 & -0.877190 \\
4 & 1 & 0 & -4.029537 & -0.467479 & -0.000003 \\
5 & 6 & 0 & -1.865092 & -0.547674 & -0.000006 \\
6 & 1 & 0 & -1.816894 & -1.201020 & 0.871306 \\
7 & 1 & 0 & -1.816900 & -1.201001 & -0.871334 \\
8 & 6 & 0 & -0.660262 & 0.370569 & -0.000001 \\
9 & 1 & 0 & -0.707180 & 1.025448 & -0.872008 \\
10 & 1 & 0 & -0.707183 & 1.025442 & 0.872011 \\
11 & 6 & 0 & 0.660262 & -0.370569 & 0.000000 \\
12 & 1 & 0 & 0.707180 & -1.025449 & -0.872007 \\
13 & 1 & 0 & 0.707183 & -1.025441 & 0.872012 \\
14 & 6 & 0 & 1.865092 & 0.547674 & -0.000006 \\
15 & 1 & 0 & 1.816894 & 1.201020 & 0.871307 \\
16 & 1 & 0 & 1.816899 & 1.201001 & -0.871333 \\
17 & 6 & 0 & 3.177909 & -0.207154 & 0.000006 \\
18 & 1 & 0 & 3.259749 & -0.844932 & -0.877189 \\
19 & 1 & 0 & 4.029537 & 0.467479 & -0.000005 \\
20 & 1 & 0 & 3.259749 & -0.844905 & 0.877220 \\
& & & & &
\end{tabular}

Zero-point correction=

Thermal correction to Energy=

Thermal correction to Enthalpy=

Thermal correction to Gibbs Free Energy=

Sum of electronic and zero-point Energies=

Sum of electronic and thermal Energies $=$

Sum of electronic and thermal Enthalpies=

Sum of electronic and thermal Free Energies=

22dimethylbutane

Standard orientation:

\begin{tabular}{|c|c|c|c|c|}
\hline \multirow{2}{*}{$\begin{array}{l}\text { Center } \\
\text { Number }\end{array}$} & Atomic & \multirow{2}{*}{$\begin{array}{r}\text { Atomic } \\
\text { Type }\end{array}$} & \multicolumn{2}{|c|}{ Coordinates (Angstroms } \\
\hline & Numbe & & $\mathrm{X}$ & $\mathrm{Z}$ \\
\hline 1 & 6 & -1.603199 & 0.965965 & 0.001122 \\
\hline 2 & 1 & -1.580591 & 1.604961 & 0.881841 \\
\hline 3 & 1 & -1.580743 & 1.606790 & -0.878272 \\
\hline 4 & 1 & -2.549558 & 0.428874 & 0.000641 \\
\hline 5 & 6 & -0.429152 & -0.000208 & 0.000010 \\
\hline 6 & 6 & 0.859400 & 0.823180 & 0.000596 \\
\hline 7 & 1 & 0.839994 & 1.479534 & -0.870720 \\
\hline 8 & 1 & 0.840055 & 1.478232 & 0.872896 \\
\hline
\end{tabular}




$\begin{array}{cccccc}9 & 6 & 0 & 2.155323 & 0.037301 & 0.000006 \\ 10 & 1 & 0 & 2.243470 & -0.595546 & -0.878637 \\ 11 & 1 & 0 & 3.007718 & 0.711192 & 0.000233 \\ 12 & 1 & 0 & 2.243680 & -0.596423 & 0.877992 \\ 13 & 6 & 0 & -0.510577 & -0.874973 & 1.240918 \\ 14 & 1 & 0 & 0.278943 & -1.621664 & 1.260110 \\ 15 & 1 & 0 & -0.430834 & -0.275493 & 2.146069 \\ 16 & 1 & 0 & -1.461788 & -1.402431 & 1.272828 \\ 17 & 6 & 0 & -0.510981 & -0.872536 & -1.242592 \\ 18 & 1 & 0 & -1.462076 & -1.400169 & -1.275067 \\ 19 & 1 & 0 & -0.431852 & -0.271229 & -2.146582 \\ 20 & 1 & 0 & 0.278703 & -1.619003 & -1.263687\end{array}$

Zero-point correction=

Thermal correction to Energy=

Thermal correction to Enthalpy=

Thermal correction to Gibbs Free Energy=

Sum of electronic and zero-point Energies=

Sum of electronic and thermal Energies $=$

Sum of electronic and thermal Enthalpies=

Sum of electronic and thermal Free Energies=

Heptane

Standard orientation:

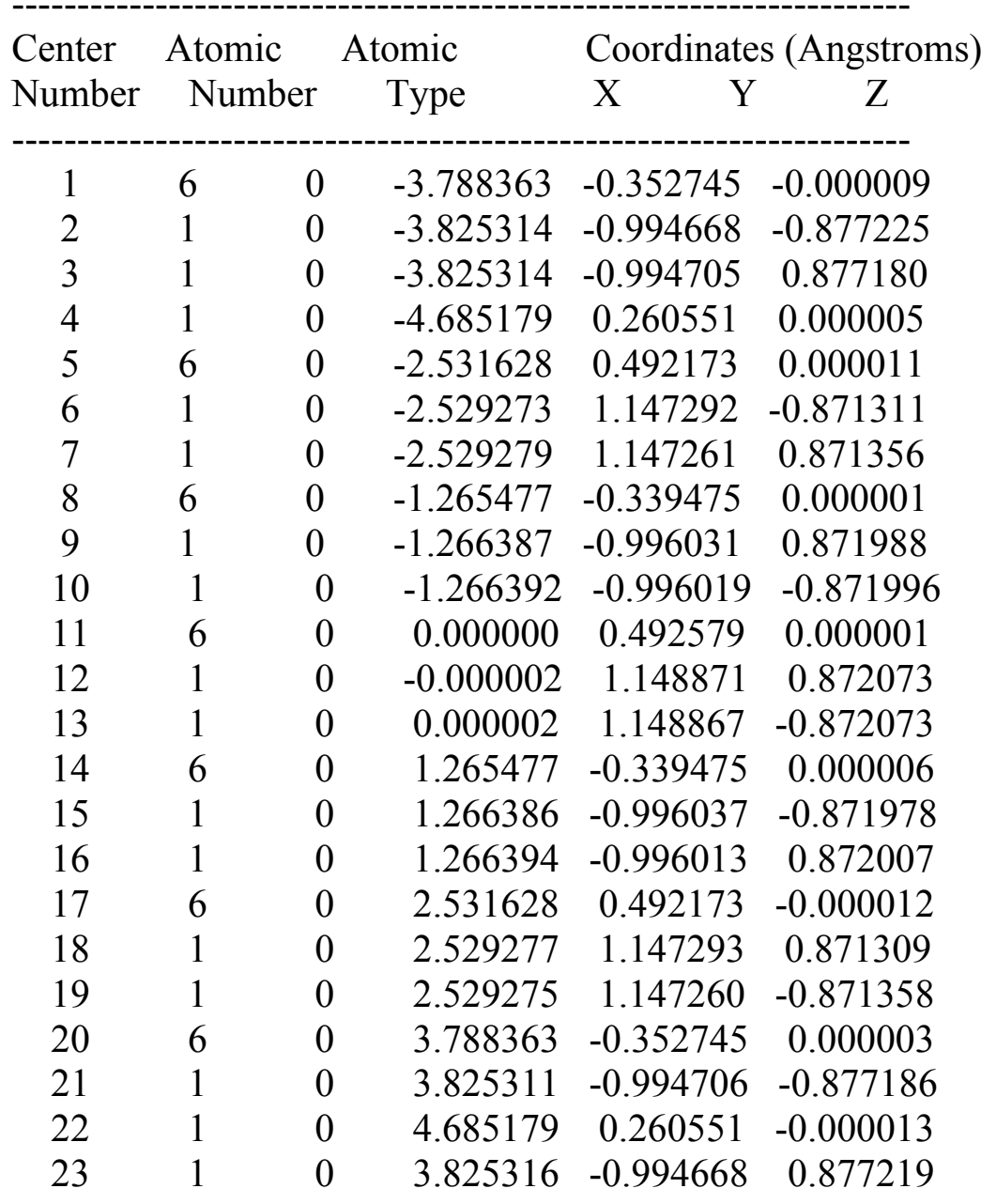


Zero-point correction=

Thermal correction to Energy=

Thermal correction to Enthalpy=

Thermal correction to Gibbs Free Energy=

Sum of electronic and zero-point Energies=

Sum of electronic and thermal Energies=

Sum of electronic and thermal Enthalpies=

Sum of electronic and thermal Free Energies=

223tributane

Standard orientation:
0.221416 (Hartree/Particle)

0.231087

0.232031

0.186660

$-275.931119$

$-275.921448$

$-275.920503$

$-275.965874$

\begin{tabular}{|c|c|c|c|c|}
\hline \multirow{2}{*}{$\begin{array}{l}\text { Center } \\
\text { Number }\end{array}$} & Atomic & \multirow{2}{*}{$\begin{array}{r}\text { Atomic } \\
\text { Type }\end{array}$} & \multicolumn{2}{|c|}{ Coordinates (Angstroms } \\
\hline & Number & & $\mathrm{X}$ & $\mathrm{Z}$ \\
\hline 1 & 6 & -1.317332 & -1.276916 & -0.471451 \\
\hline 2 & 1 & -1.175702 & -1.424799 & -1.541009 \\
\hline 3 & 1 & -0.938920 & -2.154810 & 0.044154 \\
\hline 4 & 1 & -2.387227 & -1.222108 & -0.281744 \\
\hline 5 & 6 & -0.634269 & 0.000127 & -0.000775 \\
\hline 6 & 6 & 0.841278 & -0.000720 & -0.452619 \\
\hline 7 & 1 & 0.812062 & -0.004431 & -1.545705 \\
\hline 8 & 6 & 1.610363 & 1.236823 & -0.020373 \\
\hline 9 & 1 & 1.728168 & 1.268426 & 1.060524 \\
\hline 10 & 1 & 2.608159 & 1.223541 & -0.452047 \\
\hline 11 & 1 & 1.132091 & 2.158995 & -0.334610 \\
\hline 12 & 6 & 1.613096 & -1.235361 & -0.016712 \\
\hline 13 & 1 & 1.658449 & -1.311788 & 1.067629 \\
\hline 14 & 1 & 1.182287 & -2.153634 & -0.402136 \\
\hline 15 & 1 & 2.637279 & -1.178878 & -0.377505 \\
\hline 16 & 6 & -0.771203 & 0.100538 & 1.511119 \\
\hline 17 & 1 & -0.363938 & 1.033656 & 1.891531 \\
\hline 18 & 1 & -1.822166 & 0.064346 & 1.791099 \\
\hline 19 & 1 & -0.270463 & -0.720775 & 2.017716 \\
\hline 20 & 6 & -1.362844 & 1.175872 & -0.637980 \\
\hline 21 & 1 & -1.265935 & 1.152826 & -1.722162 \\
\hline 22 & 1 & -2.423262 & 1.136024 & -0.397717 \\
\hline 23 & 1 & -0.985412 & 2.131232 & -0.285274 \\
\hline
\end{tabular}

Zero-point correction=

Thermal correction to Energy=

Thermal correction to Enthalpy=
0.219935 (Hartree/Particle)

0.229389

0.230333

0.186429

$-275.933489$

$-275.924035$

$-275.923091$

$-275.966995$

Sum of electronic and thermal Free Energies=

Octane

Standard orientation: 


\begin{tabular}{|c|c|c|c|c|c|}
\hline \multirow{2}{*}{$\begin{array}{l}\text { Center } \\
\text { Number }\end{array}$} & \multicolumn{2}{|c|}{ Atomic } & \multirow{2}{*}{$\begin{array}{l}\text { Atomic } \\
\text { Type }\end{array}$} & \multicolumn{2}{|c|}{ Coordinates (Angstrom } \\
\hline & & & & $X$ & Z \\
\hline 1 & 6 & 0 & -4.434590 & 0.255440 & 0.000015 \\
\hline 2 & 1 & 0 & -4.496708 & 0.895418 & 0.877235 \\
\hline 3 & 1 & 0 & -4.496712 & 0.895469 & -0.877168 \\
\hline 4 & 1 & 0 & -5.306663 & -0.392565 & -0.000003 \\
\hline 5 & 6 & 0 & -3.145672 & -0.539517 & -0.000014 \\
\hline 6 & 1 & 0 & -3.117621 & -1.194050 & 0.871302 \\
\hline 7 & 1 & 0 & -3.117631 & -1.194006 & -0.871362 \\
\hline 8 & 6 & 0 & -1.913101 & 0.341140 & 0.000001 \\
\hline 9 & 1 & 0 & -1.939747 & 0.997162 & -0.871993 \\
\hline 10 & 1 & 0 & -1.939750 & 0.997139 & 0.872011 \\
\hline 11 & 6 & 0 & -0.615978 & -0.440655 & -0.000005 \\
\hline 12 & 1 & 0 & -0.590200 & -1.096440 & -0.872056 \\
\hline 13 & 1 & 0 & -0.590194 & -1.096442 & 0.872045 \\
\hline 14 & 6 & 0 & 0.615978 & 0.440655 & -0.000008 \\
\hline 15 & 1 & 0 & 0.590193 & 1.096451 & 0.872036 \\
\hline 16 & 1 & 0 & 0.590201 & 1.096431 & -0.872066 \\
\hline 17 & 6 & 0 & 1.913101 & -0.341140 & 0.000007 \\
\hline 18 & 1 & 0 & 1.939749 & -0.997167 & -0.871982 \\
\hline 19 & 1 & 0 & 1.939749 & -0.997134 & 0.872021 \\
\hline 20 & 6 & 0 & 3.145672 & 0.539517 & -0.000009 \\
\hline 21 & 1 & 0 & 3.117625 & 1.194046 & 0.871309 \\
\hline 22 & 1 & 0 & 3.117628 & 1.194010 & -0.871355 \\
\hline 23 & 6 & 0 & 4.434590 & -0.255440 & 0.000010 \\
\hline 24 & 1 & 0 & 4.496711 & -0.895463 & -0.877178 \\
\hline 25 & 1 & 0 & 5.306663 & 0.392565 & -0.000003 \\
\hline 26 & 1 & 0 & 4.496709 & -0.895425 & 0.877225 \\
\hline
\end{tabular}

Zero-point correction=

Thermal correction to Energy=

Thermal correction to Enthalpy=

Thermal correction to Gibbs Free Energy=

Sum of electronic and zero-point Energies=

Sum of electronic and thermal Energies=

Sum of electronic and thermal Enthalpies=

Sum of electronic and thermal Free Energies=

2233tetramethylbutane

Standard orientation:

\begin{tabular}{|c|c|c|c|c|}
\hline \multirow{2}{*}{$\begin{array}{l}\text { Center } \\
\text { Number }\end{array}$} & Atomic & \multirow{2}{*}{$\begin{array}{l}\text { Atomic } \\
\text { Type }\end{array}$} & \multicolumn{2}{|c|}{ Coordinates (Angstroms } \\
\hline & Number & & $\mathrm{X}$ & Z \\
\hline 1 & 6 & -1.331980 & -0.728864 & 1.220058 \\
\hline 2 & 1 & -1.129990 & -1.795507 & 1.179064 \\
\hline 3 & 1 & -0.921664 & -0.341620 & 2.149373 \\
\hline 4 & 1 & -2.411817 & -0.602059 & 1.262588 \\
\hline 5 & 6 & -0.780302 & 0.000024 & -0.000002 \\
\hline 6 & 6 & 0.780299 & -0.000063 & 0.000006 \\
\hline
\end{tabular}




$\begin{array}{cccccc}7 & 6 & 0 & 1.331931 & 0.434744 & -1.353090 \\ 8 & 1 & 0 & 1.130015 & -0.300578 & -2.126862 \\ 9 & 1 & 0 & 0.921464 & 1.388216 & -1.676162 \\ 10 & 1 & 0 & 2.411749 & 0.550569 & -1.285970 \\ 11 & 6 & 0 & -1.332080 & -0.692092 & -1.241259 \\ 12 & 1 & 0 & -1.130191 & -0.123235 & -2.144498 \\ 13 & 1 & 0 & -0.921777 & -1.690512 & -1.370616 \\ 14 & 1 & 0 & -2.411901 & -0.792370 & -1.152579 \\ 15 & 6 & 0 & -1.331756 & 1.421152 & 0.021258 \\ 16 & 1 & 0 & -2.411562 & 1.394796 & -0.110143 \\ 17 & 1 & 0 & -1.129909 & 1.918704 & 0.965648 \\ 18 & 1 & 0 & -0.921017 & 2.032404 & -0.778501 \\ 19 & 6 & 0 & 1.331901 & -1.389283 & 0.299989 \\ 20 & 1 & 0 & 2.411739 & -1.389029 & 0.166209 \\ 21 & 1 & 0 & 1.129915 & -1.691873 & 1.323626 \\ 22 & 1 & 0 & 0.921559 & -2.145723 & -0.364361 \\ 23 & 6 & 0 & 1.331977 & 0.954372 & 1.053039 \\ 24 & 1 & 0 & 2.411843 & 0.838554 & 1.119496 \\ 25 & 1 & 0 & 1.129777 & 1.992133 & 0.803303 \\ 26 & 1 & 0 & 0.921837 & 0.757192 & 2.040392\end{array}$

Zero-point correction=

Thermal correction to Energy=

Thermal correction to Enthalpy=

Thermal correction to Gibbs Free Energy=

Sum of electronic and zero-point Energies=

Sum of electronic and thermal Energies $=$

Sum of electronic and thermal Enthalpies=

Sum of electronic and thermal Free Energies=
0.248791 (Hartree/Particle)

0.259073

0.260017

0.215641

$-315.182745$

$-315.172464$

$-315.171519$

$-315.215895$

\section{B2PLYPD3/ccpVQZ}

Ethane

Standard orientation:

\begin{tabular}{crrrrr} 
Center & \multicolumn{2}{c}{ Atomic } & \multicolumn{2}{c}{ Atomic } & \multicolumn{2}{c}{ Coordinates (Angstroms) } \\
Number & Number & \multicolumn{1}{c}{ Type } & \multicolumn{1}{c}{ X } & \multicolumn{1}{c}{ Y } & Z \\
\hline 1 & 6 & 0 & 0.000000 & 0.000000 & 0.762565 \\
2 & 1 & 0 & -0.507207 & 0.878553 & 1.157547 \\
3 & 1 & 0 & 1.014453 & -0.000022 & 1.157547 \\
4 & 1 & 0 & -0.507246 & -0.878531 & 1.157547 \\
5 & 6 & 0 & 0.000000 & 0.000000 & -0.762565 \\
6 & 1 & 0 & 0.507207 & 0.878553 & -1.157547 \\
7 & 1 & 0 & 0.507246 & -0.878531 & -1.157547 \\
8 & 1 & 0 & -1.014453 & -0.000022 & -1.157547
\end{tabular}

Zero-point correction=

Thermal correction to Energy=

Thermal correction to Enthalpy=

Thermal correction to Gibbs Free Energy=
0.075075 (Hartree/Particle)

0.078544

0.079489

0.053672 
Sum of electronic and zero-point Energies=

$-79.709863$

Sum of electronic and thermal Energies=

$-79.706394$

Sum of electronic and thermal Enthalpies=

Sum of electronic and thermal Free Energies=

$-79.731266$

Methane

Standard orientation:

\begin{tabular}{|c|c|c|c|c|}
\hline \multirow{2}{*}{$\begin{array}{l}\text { Center } \\
\text { Number }\end{array}$} & \multirow{2}{*}{$\begin{array}{l}\text { Atomic } \\
\text { Number }\end{array}$} & \multirow{2}{*}{$\begin{array}{r}\text { Atomic } \\
\text { Type }\end{array}$} & \multicolumn{2}{|c|}{ Coordinates (Angstro } \\
\hline & & & $\mathrm{X}$ & $\mathrm{Z}$ \\
\hline 1 & 6 & 0.000000 & 0.000000 & 0.000000 \\
\hline 2 & 1 & 0.626781 & 0.626781 & 0.626781 \\
\hline 3 & 1 & -0.626781 & -0.626781 & 0.62678 \\
\hline 4 & 1 & -0.626781 & 0.626781 & -0.626781 \\
\hline 5 & 1 & 0.626781 & -0.626781 & -0.626781 \\
\hline
\end{tabular}

Zero-point correction=

Thermal correction to Energy=

Thermal correction to Enthalpy=

Thermal correction to Gibbs Free Energy=
Sum of electronic and zero-point Energies

Sum of electronic and thermal Energies $=$

Sum of electronic and thermal Enthalpies=

Sum of electronic and thermal Free Energies=

Propane

Standard orientation:
0.044992 (Hartree/Particle)

0.047859

0.048803

0.027688

$-40.447956$

$-40.445089$

$-40.444145$

$-40.465260$

\begin{tabular}{|c|c|c|c|c|}
\hline \multirow{2}{*}{$\begin{array}{l}\text { Center } \\
\text { Number }\end{array}$} & Atomic & \multirow{2}{*}{$\begin{array}{l}\text { Atomic } \\
\text { Type }\end{array}$} & \multicolumn{2}{|c|}{ Coordinates (Angstrom } \\
\hline & Number & & $\mathrm{X}$ & Z \\
\hline 1 & 6 & -1.266990 & -0.259931 & 0.000000 \\
\hline 2 & 1 & -2.164609 & 0.356283 & -0.000031 \\
\hline 3 & 1 & -1.303468 & -0.903020 & 0.879258 \\
\hline 4 & 1 & -1.303440 & -0.903069 & -0.879223 \\
\hline 5 & 6 & 0.000000 & 0.588688 & 0.000000 \\
\hline 6 & 1 & -0.000001 & 1.243336 & 0.872755 \\
\hline 7 & 1 & 0.000001 & 1.243318 & -0.872767 \\
\hline 8 & 6 & 1.266990 & -0.259931 & 0.000000 \\
\hline 9 & 1 & 1.303476 & -0.903005 & 0.879268 \\
\hline 10 & 1 & 1.303428 & -0.903083 & -0.879213 \\
\hline 11 & 1 & 2.164611 & 0.356280 & -0.000052 \\
\hline
\end{tabular}

Zero-point correction=

0.103994 (Hartree/Particle)

Thermal correction to Energy=

Thermal correction to Enthalpy=

0.108477

0.109422

Thermal correction to Gibbs Free Energy $=\quad 0.078389$

Sum of electronic and zero-point Energies $=\quad-118.976053$

Sum of electronic and thermal Energies $=\quad-118.971569$

Sum of electronic and thermal Enthalpies $=\quad-118.970625$ 
Sum of electronic and thermal Free Energies $=\quad-119.001658$

Butane

Standard orientation:

\begin{tabular}{|c|c|c|c|c|}
\hline \multirow{2}{*}{$\begin{array}{l}\text { Center } \\
\text { Number }\end{array}$} & Atomic & \multirow{2}{*}{$\begin{array}{r}\text { Atomic } \\
\text { Type }\end{array}$} & \multicolumn{2}{|c|}{ Coordinates (Angstrom } \\
\hline & Numbe & & $\mathrm{X}$ & Z \\
\hline 1 & 6 & 1.948650 & -0.119734 & 0.000040 \\
\hline 2 & 1 & 2.088860 & -0.748147 & -0.879283 \\
\hline 3 & 1 & 2.088769 & -0.748142 & 0.879381 \\
\hline 4 & 1 & 2.735191 & 0.633064 & 0.000078 \\
\hline 5 & 6 & 0.562387 & 0.514974 & -0.000045 \\
\hline 6 & 1 & 0.454403 & 1.162073 & 0.873321 \\
\hline 7 & 1 & 0.454464 & 1.162015 & -0.873462 \\
\hline 8 & 6 & -0.562387 & -0.514974 & -0.000045 \\
\hline 9 & 1 & -0.454464 & -1.162013 & -0.873464 \\
\hline 10 & 1 & -0.454403 & -1.162076 & 0.873319 \\
\hline 11 & 6 & -1.948650 & 0.119734 & 0.000040 \\
\hline 12 & 1 & -2.088858 & 0.748153 & -0.879279 \\
\hline 13 & 1 & -2.735191 & -0.633064 & 0.000071 \\
\hline 14 & 1 & -2.088771 & 0.748136 & 0.879384 \\
\hline
\end{tabular}

Zero-point correction=

0.132710 (Hartree/Particle)

Thermal correction to Energy=

0.138427

Thermal correction to Enthalpy=

0.139371

Thermal correction to Gibbs Free Energy=

0.104784

Sum of electronic and zero-point Energies=

$-158.242510$

Sum of electronic and thermal Energies $=$

$-158.236792$

Sum of electronic and thermal Enthalpies=

$-158.235848$

Sum of electronic and thermal Free Energies $=\quad-158.270436$

Isobutane

Standard orientation:

\begin{tabular}{cccccc} 
Center & \multicolumn{2}{c}{ Atomic } & \multicolumn{2}{c}{ Atomic } & \multicolumn{2}{c}{ Coordinates } \\
Number & Number & Type & X & Y & $Z$ \\
-1 & 6 & 0 & 1.308306 & -0.628855 & 0.095963 \\
2 & 1 & 0 & 2.170482 & -0.065157 & -0.258668 \\
3 & 1 & 0 & 1.348575 & -0.648295 & 1.186194 \\
4 & 1 & 0 & 1.406803 & -1.654185 & -0.258810 \\
5 & 6 & 0 & -0.000001 & -0.000004 & -0.377181 \\
6 & 1 & 0 & -0.000012 & 0.000013 & -1.470435 \\
7 & 6 & 0 & -0.109544 & 1.447438 & 0.095966 \\
8 & 1 & 0 & -1.028813 & 1.912270 & -0.258641 \\
9 & 1 & 0 & -0.112800 & 1.492047 & 1.186199 \\
10 & 1 & 0 & 0.729158 & 2.045399 & -0.258846 \\
11 & 6 & 0 & -1.198760 & -0.818587 & 0.095966 \\
12 & 1 & 0 & -2.135983 & -0.390982 & -0.258492 \\
13 & 1 & 0 & -1.141901 & -1.847005 & -0.258987 \\
14 & 1 & 0 & -1.235518 & -0.844056 & 1.186198
\end{tabular}


Zero-point correction=

Thermal correction to Energy=

Thermal correction to Enthalpy=

Thermal correction to Gibbs Free Energy=

Sum of electronic and zero-point Energies=

Sum of electronic and thermal Energies=

Sum of electronic and thermal Enthalpies=

Sum of electronic and thermal Free Energies=

Pentane

Standard orientation:
0.132273 (Hartree/Particle)

0.137892

0.138836

0.104747

$-158.244983$

$-158.239365$

$-158.238421$

$-158.272510$

\begin{tabular}{|c|c|c|c|c|}
\hline \multirow{2}{*}{$\begin{array}{l}\text { Center } \\
\text { Number }\end{array}$} & Atomic & \multirow{2}{*}{$\begin{array}{l}\text { Atomic } \\
\text { Type }\end{array}$} & \multicolumn{2}{|c|}{ Coordinates (Angstrom } \\
\hline & Number & & $\mathrm{X}$ & $\mathrm{Z}$ \\
\hline 1 & 6 & -1.266990 & -0.259931 & 0.000000 \\
\hline 2 & 1 & -2.164609 & 0.356283 & -0.000031 \\
\hline 3 & 1 & -1.303468 & -0.903020 & 0.879258 \\
\hline 4 & 1 & -1.303440 & -0.903069 & -0.879223 \\
\hline 5 & 6 & 0.000000 & 0.588688 & 0.000000 \\
\hline 6 & 1 & -0.000001 & 1.243336 & 0.872755 \\
\hline 7 & 1 & 0.000001 & 1.243318 & -0.872767 \\
\hline 8 & 6 & 1.266990 & -0.259931 & 0.000000 \\
\hline 9 & 1 & 1.303476 & -0.903005 & 0.879268 \\
\hline 10 & 1 & 1.303428 & -0.903083 & -0.879213 \\
\hline 11 & 1 & 2.164611 & 0.356280 & -0.000052 \\
\hline
\end{tabular}

\begin{tabular}{|c|c|c|c|c|c|}
\hline \multicolumn{6}{|c|}{ Zero-point correction $=$} \\
\hline \multicolumn{6}{|c|}{ Thermal correction to Energy= } \\
\hline \multicolumn{6}{|c|}{ Thermal correction to Enthalpy= } \\
\hline \multicolumn{5}{|c|}{ Thermal correction to Gibbs Free Energy= } & 0.078389 \\
\hline \multicolumn{5}{|c|}{ Sum of electronic and zero-point Energies $=$} & -118.976 \\
\hline \multicolumn{5}{|c|}{ Sum of electronic and thermal Energies $=$} & \\
\hline \multicolumn{5}{|c|}{ Sum of electronic and thermal Enthalpies= } & -1 \\
\hline \multirow{2}{*}{\multicolumn{6}{|c|}{$\begin{array}{l}\text { Sum of electronic and thermal Free Energies= } \\
\text { Neopentane }\end{array}$}} \\
\hline \multicolumn{4}{|c|}{ Neopentane } & & \\
\hline \multicolumn{6}{|c|}{ Standard orientation: } \\
\hline \multirow{2}{*}{$\begin{array}{l}\text { Center } \\
\text { Number }\end{array}$} & \multirow{2}{*}{\multicolumn{2}{|c|}{$\begin{array}{l}\text { Atomic } \\
\text { Number }\end{array}$}} & \multirow{2}{*}{$\begin{array}{l}\text { Atomic } \\
\text { Type }\end{array}$} & \multicolumn{2}{|c|}{ Coordinates (Angstrom } \\
\hline & & & & $\mathrm{X}$ & Z \\
\hline 1 & 6 & & 1.050176 & 0.245808 & -1.086248 \\
\hline 2 & 1 & 0 & 1.713240 & 1.065512 & -0.809151 \\
\hline 3 & 1 & 0 & 1.662178 & -0.642233 & -1.244991 \\
\hline 4 & 1 & 0 & 0.577639 & 0.501988 & -2.034631 \\
\hline 5 & 6 & 0 & 0.000002 & 0.000010 & -0.000011 \\
\hline 6 & 6 & 0 & 0.699146 & -0.356311 & 1.314313 \\
\hline 7 & 1 & 0 & -0.027892 & -0.537198 & 2.106178 \\
\hline 8 & 1 & 0 & 1.304700 & -1.255842 & 1.202459 \\
\hline
\end{tabular}




$\begin{array}{cccccc}9 & 1 & 0 & 1.354936 & 0.451830 & 1.638677 \\ 10 & 6 & 0 & -0.838472 & 1.265780 & 0.194885 \\ 11 & 1 & 0 & -1.348117 & 1.541871 & -0.728370 \\ 12 & 1 & 0 & -1.595616 & 1.117183 & 0.964955 \\ 13 & 1 & 0 & -0.212440 & 2.105524 & 0.496998 \\ 14 & 6 & 0 & -0.910851 & -1.155278 & -0.422954 \\ 15 & 1 & 0 & -1.422058 & -0.926549 & -1.358212 \\ 16 & 1 & 0 & -0.337248 & -2.070721 & -0.568902 \\ 17 & 1 & 0 & -1.669328 & -1.351425 & 0.335074\end{array}$

Zero-point correction=

Thermal correction to Energy=

Thermal correction to Enthalpy=

Thermal correction to Gibbs Free Energy=

Sum of electronic and zero-point Energies=

Sum of electronic and thermal Energies $=$

Sum of electronic and thermal Enthalpies=

Sum of electronic and thermal Free Energies=

Hexane

Standard orientation:

\begin{tabular}{|c|c|c|c|c|c|}
\hline \multirow{2}{*}{$\begin{array}{l}\text { Center } \\
\text { Number }\end{array}$} & \multicolumn{2}{|l|}{ Atomic } & & \multicolumn{2}{|c|}{ Coordinates (Angstroms) } \\
\hline & Numbe & & & $\mathrm{X}$ & $\mathrm{Z}$ \\
\hline 1 & 6 & 0 & -3.200888 & 0.208623 & 0.000035 \\
\hline 2 & 1 & 0 & -3.282675 & 0.847232 & 0.879384 \\
\hline 3 & 1 & 0 & -3.282685 & 0.847356 & -0.879224 \\
\hline 4 & 1 & 0 & -4.053259 & -0.468837 & -0.000010 \\
\hline 5 & 6 & 0 & -1.878668 & -0.550717 & -0.000033 \\
\hline 6 & 1 & 0 & -1.831319 & -1.204762 & 0.873339 \\
\hline 7 & 1 & 0 & -1.831350 & -1.204671 & -0.873475 \\
\hline 8 & 6 & 0 & -0.664996 & 0.372742 & -0.000010 \\
\hline 9 & 1 & 0 & -0.711808 & 1.028219 & -0.874008 \\
\hline 10 & 1 & 0 & -0.711821 & 1.028202 & 0.873998 \\
\hline 11 & 6 & 0 & 0.664996 & -0.372742 & -0.000000 \\
\hline 12 & 1 & 0 & 0.711809 & -1.028233 & -0.873987 \\
\hline 13 & 1 & 0 & 0.711819 & -1.028188 & 0.874020 \\
\hline 14 & 6 & 0 & 1.878668 & 0.550717 & -0.000028 \\
\hline 15 & 1 & 0 & 1.831322 & 1.204763 & 0.873343 \\
\hline 16 & 1 & 0 & 1.831347 & 1.204670 & -0.873470 \\
\hline 17 & 6 & 0 & 3.200888 & -0.208623 & 0.000030 \\
\hline 18 & 1 & 0 & 3.282686 & -0.847350 & -0.879233 \\
\hline 19 & 1 & 0 & 4.053259 & 0.468837 & -0.000007 \\
\hline 20 & 1 & 0 & 3.282674 & -0.847238 & 0.879375 \\
\hline
\end{tabular}

Zero-point correction=

Thermal correction to Energy=

Thermal correction to Enthalpy=

Thermal correction to Gibbs Free Energy=

Sum of electronic and zero-point Energies=
0.190045 (Hartree/Particle)

$$
0.198362
$$

0.199306

$$
\begin{aligned}
& 0.157608 \\
& -236.775455
\end{aligned}
$$


Sum of electronic and thermal Energies $=$

$-236.767138$

Sum of electronic and thermal Enthalpies=

Sum of electronic and thermal Free Energies=

22dimethylbutane

Standard orientation:

\begin{tabular}{cccccc} 
Center & Atomic & \multicolumn{2}{c}{ Atomic } & \multicolumn{3}{c}{ Coordinates } \\
Number & Number & Type & X & Y & $Z$ \\
- & Nustroms \\
\hline 1 & 6 & 0 & 1.613748 & 0.975650 & -0.000858 \\
2 & 1 & 0 & 1.590333 & 1.615988 & -0.882863 \\
3 & 1 & 0 & 1.590639 & 1.617137 & 0.880321 \\
4 & 1 & 0 & 2.562553 & 0.439068 & -0.000669 \\
5 & 6 & 0 & 0.432609 & 0.000023 & -0.000010 \\
6 & 6 & 0 & -0.868017 & 0.826541 & -0.000215 \\
7 & 1 & 0 & -0.851007 & 1.483116 & 0.873090 \\
8 & 1 & 0 & -0.851087 & 1.482602 & -0.873914 \\
9 & 6 & 0 & -2.173781 & 0.036204 & 0.000018 \\
10 & 1 & 0 & -2.262254 & -0.598034 & 0.879852 \\
11 & 1 & 0 & -3.026153 & 0.713547 & 0.000192 \\
12 & 1 & 0 & -2.262535 & -0.597956 & -0.879841 \\
13 & 6 & 0 & 0.517226 & -0.880635 & -1.250205 \\
14 & 1 & 0 & -0.270592 & -1.631576 & -1.268717 \\
15 & 1 & 0 & 0.433344 & -0.280116 & -2.156488 \\
16 & 1 & 0 & 1.472180 & -1.405050 & -1.283420 \\
17 & 6 & 0 & 0.517795 & -0.879085 & 1.251250 \\
18 & 1 & 0 & 1.472582 & -1.403806 & 1.284454 \\
19 & 1 & 0 & 0.434787 & -0.277374 & 2.156819 \\
20 & 1 & 0 & -0.270265 & -1.629736 & 1.271311
\end{tabular}

Zero-point correction=

Thermal correction to Energy=

Thermal correction to Enthalpy=

Thermal correction to Gibbs Free Energy=

Sum of electronic and zero-point Energies=

Sum of electronic and thermal Energies=

Sum of electronic and thermal Enthalpies=

Sum of electronic and thermal Free Energies=

Heptane

Standard orientation:

\begin{tabular}{|c|c|c|c|c|}
\hline \multirow{2}{*}{$\begin{array}{l}\text { Center } \\
\text { Number }\end{array}$} & Atomic & \multirow{2}{*}{$\begin{array}{l}\text { Atomic } \\
\text { Type }\end{array}$} & \multicolumn{2}{|c|}{ Coordinates (Angstroms) } \\
\hline & Number & & $\mathrm{X}$ & Z \\
\hline 1 & 6 & -3.814871 & -0.355125 & -0.000013 \\
\hline 2 & 1 & -3.851884 & -0.997853 & -0.879242 \\
\hline 3 & 1 & -3.851867 & -0.997954 & 0.879145 \\
\hline 4 & 1 & -4.712316 & 0.261087 & 0.000032 \\
\hline 5 & 6 & -2.549199 & 0.495025 & 0.000023 \\
\hline 6 & 1 & -2.547491 & 1.150674 & -0.873314 \\
\hline
\end{tabular}




$\begin{array}{cccccc}7 & 1 & 0 & -2.547494 & 1.150604 & 0.873413 \\ 8 & 6 & 0 & -1.274153 & -0.341475 & -0.000006 \\ 9 & 1 & 0 & -1.274868 & -0.998493 & 0.873922 \\ 10 & 1 & 0 & -1.274884 & -0.998460 & -0.873959 \\ 11 & 6 & 0 & 0.000000 & 0.495782 & -0.000003 \\ 12 & 1 & 0 & -0.000005 & 1.152505 & 0.873989 \\ 13 & 1 & 0 & 0.000005 & 1.152492 & -0.874006 \\ 14 & 6 & 0 & 1.274153 & -0.341475 & 0.000011 \\ 15 & 1 & 0 & 1.274870 & -0.998500 & -0.873911 \\ 16 & 1 & 0 & 1.274882 & -0.998452 & 0.873969 \\ 17 & 6 & 0 & 2.549199 & 0.495025 & -0.000020 \\ 18 & 1 & 0 & 2.547494 & 1.150669 & 0.873320 \\ 19 & 1 & 0 & 2.547491 & 1.150608 & -0.873406 \\ 20 & 6 & 0 & 3.814871 & -0.355125 & 0.000008 \\ 21 & 1 & 0 & 3.851873 & -0.997935 & -0.879162 \\ 22 & 1 & 0 & 4.712316 & 0.261087 & -0.000017 \\ 23 & 1 & 0 & 3.851878 & -0.997872 & 0.879224\end{array}$

Zero-point correction=

Thermal correction to Energy=

Thermal correction to Enthalpy=
0.218683 (Hartree/Particle) 0.228347 0.229291

Thermal correction to Gibbs Free Energy=

Sum of electronic and zero-point Energies=

Sum of electronic and thermal Energies $=$

0.183975

$-276.041972$

$-276.032309$

$-276.031365$

$-276.076681$

223trimethylbutane

Standard orientation:

\begin{tabular}{|c|c|c|c|c|}
\hline \multirow{2}{*}{$\begin{array}{l}\text { Center } \\
\text { Number }\end{array}$} & Atomic & \multirow{2}{*}{$\begin{array}{l}\text { Atomic } \\
\text { Type }\end{array}$} & \multicolumn{2}{|c|}{ Coordinates (Angstroms } \\
\hline & Numbe & & $X$ & Z \\
\hline 1 & 6 & 1.325549 & 1.292132 & -0.464070 \\
\hline 2 & 1 & 1.178316 & 1.452040 & -1.532888 \\
\hline 3 & 1 & 0.947823 & 2.165219 & 0.063476 \\
\hline 4 & 1 & 2.398108 & 1.236902 & -0.279362 \\
\hline 5 & 6 & 0.640125 & -0.000209 & -0.000923 \\
\hline 6 & 6 & -0.849227 & 0.000845 & -0.453821 \\
\hline 7 & 1 & -0.822619 & 0.004825 & -1.548362 \\
\hline 8 & 6 & -1.625005 & -1.245726 & -0.019763 \\
\hline 9 & 1 & -1.749827 & -1.274331 & 1.062123 \\
\hline 10 & 1 & -2.621307 & -1.235845 & $5-0.459819$ \\
\hline 11 & 1 & -1.141496 & -2.169152 & -0.327970 \\
\hline 12 & 6 & -1.627892 & 1.244423 & -0.016157 \\
\hline 13 & 1 & -1.667366 & 1.326014 & 1.069708 \\
\hline 14 & 1 & -1.200092 & 2.163100 & -0.408598 \\
\hline 15 & 1 & -2.655328 & 1.184100 & -0.372951 \\
\hline 16 & 6 & 0.781273 & -0.114770 & 1.520919 \\
\hline 17 & 1 & 0.380638 & -1.055798 & 1.893389 \\
\hline 18 & 1 & 1.833863 & -0.072831 & 1.800989 \\
\hline
\end{tabular}




$\begin{array}{llllll}19 & 1 & 0 & 0.273032 & 0.698649 & 2.036196 \\ 20 & 6 & 0 & 1.376595 & -1.177143 & -0.654647 \\ 21 & 1 & 0 & 1.283072 & -1.140937 & -1.740530 \\ 22 & 1 & 0 & 2.437989 & -1.141288 & -0.409889 \\ 23 & 1 & 0 & 0.996686 & -2.137980 & -0.314737\end{array}$

$\begin{array}{lc}\text { Zero-point correction= } & 0.217432 \text { (Hartree/Particle) } \\ \text { Thermal correction to Energy= } & 0.226839 \\ \text { Thermal correction to Enthalpy= } & 0.227784 \\ \text { Thermal correction to Gibbs Free Energy= } & 0.184089 \\ \text { Sum of electronic and zero-point Energies }= & -276.044333 \\ \text { Sum of electronic and thermal Energies }= & -276.034926 \\ \text { Sum of electronic and thermal Enthalpies }= & -276.033981 \\ \text { Sum of electronic and thermal Free Energies }= & -276.077676 \\ \text { Octane } & \\ \text { Standard orientation: } & \end{array}$

\begin{tabular}{|c|c|c|c|c|}
\hline \multirow{2}{*}{$\begin{array}{l}\text { Center } \\
\text { Number }\end{array}$} & Atomic & \multirow{2}{*}{$\begin{array}{l}\text { Atomic } \\
\text { Type }\end{array}$} & \multicolumn{2}{|c|}{ Coordinates (Angstroms) } \\
\hline & Number & & $\mathrm{X}$ & Z \\
\hline 1 & 6 & -4.466509 & 0.257201 & 0.000023 \\
\hline 2 & 1 & -4.528576 & 0.898006 & 0.879402 \\
\hline 3 & 1 & -4.528520 & 0.898236 & -0.879192 \\
\hline 4 & 1 & -5.339443 & -0.393573 & -0.000092 \\
\hline 5 & 6 & -3.168372 & -0.542562 & -0.000045 \\
\hline 6 & 1 & -3.141244 & -1.197781 & 0.873313 \\
\hline 7 & 1 & -3.141241 & -1.197628 & -0.873517 \\
\hline 8 & 6 & -1.926790 & 0.343027 & 0.000027 \\
\hline 9 & 1 & -1.953245 & 0.999684 & -0.873919 \\
\hline 10 & 1 & -1.953267 & 0.999591 & 0.874042 \\
\hline 11 & 6 & -0.620372 & -0.443317 & 0.000005 \\
\hline 12 & 1 & -0.594505 & -1.099669 & -0.873997 \\
\hline 13 & 1 & -0.594471 & -1.099653 & 0.874016 \\
\hline 14 & 6 & 0.620372 & 0.443317 & -0.000024 \\
\hline 15 & 1 & 0.594481 & 1.099701 & 0.873953 \\
\hline 16 & 1 & 0.594494 & 1.099621 & -0.874060 \\
\hline 17 & 6 & 1.926790 & -0.343027 & 0.000021 \\
\hline 18 & 1 & 1.953256 & -0.999686 & -0.873923 \\
\hline 19 & 1 & 1.953256 & -0.999589 & 0.874037 \\
\hline 20 & 6 & 3.168372 & 0.542562 & -0.000028 \\
\hline 21 & 1 & 3.141242 & 1.197753 & 0.873351 \\
\hline 22 & 1 & 3.141244 & 1.197656 & -0.873480 \\
\hline 23 & 6 & 4.466509 & -0.257201 & 0.000018 \\
\hline 24 & 1 & 4.528548 & -0.898170 & -0.879244 \\
\hline 25 & 1 & 5.339443 & 0.393572 & -0.000018 \\
\hline 26 & 1 & 4.528547 & -0.898072 & 0.879350 \\
\hline
\end{tabular}

Zero-point correction=

Thermal correction to Energy=

Thermal correction to Enthalpy=
0.247300 (Hartree/Particle)

0.258287

0.259231 
Thermal correction to Gibbs Free Energy=

Sum of electronic and zero-point Energies=

Sum of electronic and thermal Energies=

Sum of electronic and thermal Enthalpies=

Sum of electronic and thermal Free Energies=

2233tetramethylbutane

Standard orientation:

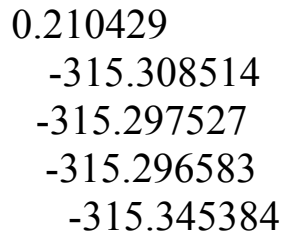

\begin{tabular}{|c|c|c|c|c|}
\hline \multirow{2}{*}{$\begin{array}{l}\text { Center } \\
\text { Number }\end{array}$} & Atomic & \multirow{2}{*}{$\begin{array}{l}\text { Atomic } \\
\text { Type }\end{array}$} & \multicolumn{2}{|c|}{ Coordinates (Angstroms } \\
\hline & Numbe & & $X$ & Z \\
\hline 1 & 6 & -1.344907 & 1.409361 & -0.253201 \\
\hline 2 & 1 & -1.146974 & 2.078555 & 0.581368 \\
\hline 3 & 1 & -0.929365 & 1.858412 & -1.153603 \\
\hline 4 & 1 & -2.425887 & 1.358340 & -0.382094 \\
\hline 5 & 6 & -0.788222 & 0.000059 & 0.000011 \\
\hline 6 & 6 & 0.788195 & 0.000012 & 0.000030 \\
\hline 7 & 6 & 1.344805 & -1.315699 & 0.565052 \\
\hline 8 & 1 & 1.146693 & -1.414597 & 1.630182 \\
\hline 9 & 1 & 0.929457 & -2.186498 & 0.060792 \\
\hline 10 & 1 & 2.425813 & -1.344036 & 0.429600 \\
\hline 11 & 6 & -1.344895 & -0.485388 & 1.347076 \\
\hline 12 & 1 & -1.146437 & -1.542626 & 1.509550 \\
\hline 13 & 1 & -0.929841 & 0.070211 & 2.186166 \\
\hline 14 & 1 & -2.425951 & -0.348821 & 1.367125 \\
\hline 15 & 6 & -1.344861 & -0.923924 & -1.093884 \\
\hline 16 & 1 & -2.425851 & -1.009986 & -0.985352 \\
\hline 17 & 1 & -1.146825 & -0.535851 & -2.090724 \\
\hline 18 & 1 & -0.929407 & -1.928250 & -1.032476 \\
\hline 19 & 6 & 1.344967 & 1.147187 & 0.856871 \\
\hline 20 & 1 & 2.426033 & 1.044266 & 0.948691 \\
\hline 21 & 1 & 1.146474 & 2.119079 & 0.410140 \\
\hline 22 & 1 & 0.930060 & 1.145690 & 1.863302 \\
\hline 23 & 6 & 1.344883 & 0.168443 & -1.421930 \\
\hline 24 & 1 & 2.425886 & 0.299943 & -1.378741 \\
\hline 25 & 1 & 1.146795 & -0.704614 & -2.040029 \\
\hline 26 & 1 & 0.929537 & 1.040479 & -1.924043 \\
\hline
\end{tabular}

Zero-point correction=

Thermal correction to Energy=

Thermal correction to Enthalpy=

Thermal correction to Gibbs Free Energy=

Sum of electronic and zero-point Energies=

Sum of electronic and thermal Energies=

Sum of electronic and thermal Enthalpies=

Sum of electronic and thermal Free Energies=

DSDPBEP86/ccpVQZ

Ethane

Standard orientation:
0.246033 (Hartree/Particle)

0.256267

0.257211

0.212936

$-315.309351$

$-315.299117$

$-315.298173$

$-315.342448$ 


\begin{tabular}{cccrrr}
$\begin{array}{c}\text { Center } \\
\text { Number }\end{array}$ & Atomic & \multicolumn{2}{c}{ Atomic } & \multicolumn{2}{c}{ Coordinates (Angstrom } \\
Number & \multicolumn{1}{c}{ Type } & X & Y & Z \\
\hline 1 & 6 & 0 & -0.000000 & -0.000000 & 0.761928 \\
2 & 1 & 0 & -0.508207 & 0.880263 & 1.157416 \\
3 & 1 & 0 & 1.016433 & -0.000011 & 1.157416 \\
4 & 1 & 0 & -0.508226 & -0.880252 & 1.157416 \\
5 & 6 & 0 & 0.000000 & 0.000000 & -0.761928 \\
6 & 1 & 0 & 0.508207 & 0.880263 & -1.157416 \\
7 & 1 & 0 & 0.508226 & -0.880252 & -1.157416 \\
8 & 1 & 0 & -1.016433 & -0.000011 & -1.157416
\end{tabular}

Zero-point correction=

Thermal correction to Energy=

Thermal correction to Enthalpy=

Thermal correction to Gibbs Free Energy=

Sum of electronic and zero-point Energies=

Sum of electronic and thermal Energies=

Sum of electronic and thermal Enthalpies=

Sum of electronic and thermal Free Energies=

Methane

Standard orientation:
0.075083 (Hartree/Particle)

$$
0.078559
$$

0.079504

0.053673

$-79.630172$

$-79.626695$

$-79.625751$

$-79.651581$

\begin{tabular}{|c|c|c|c|c|}
\hline \multirow{2}{*}{$\begin{array}{l}\text { Center } \\
\text { Number }\end{array}$} & \multirow{2}{*}{$\begin{array}{l}\text { Atomic } \\
\text { Number }\end{array}$} & \multirow{2}{*}{$\begin{array}{r}\text { Atomic } \\
\text { Type }\end{array}$} & \multicolumn{2}{|c|}{ Coordinates (Angstrom } \\
\hline & & & $\mathrm{X}$ & $\mathrm{Z}$ \\
\hline 1 & 6 & 0.0 & 0.000000 & 0.000000 \\
\hline 2 & 1 & 0.627919 & 0.627919 & 0.627919 \\
\hline 3 & 1 & -0.627919 & -0.627919 & 0.627919 \\
\hline 4 & 1 & -0.627919 & 0.627919 & -0.627919 \\
\hline 5 & 1 & 0.627919 & -0.627919 & -0.627919 \\
\hline
\end{tabular}

Zero-point correction $=$

Thermal correction to Energy=

0.045020 (Hartree/Particle)

Thermal correction to Enthalpy=

0.047887

0.048831

Thermal correction to Gibbs Free Energy $=0.027711$

Sum of electronic and zero-point Energies $=\quad-40.407057$

Sum of electronic and thermal Energies $=\quad-40.404189$

Sum of electronic and thermal Enthalpies $=\quad-40.403245$

Sum of electronic and thermal Free Energies $=\quad-40.424366$

Propane

Standard orientation:

\begin{tabular}{|c|c|c|c|c|}
\hline Center & Atomic & Atomic & Coordinate & s (Angstrom \\
\hline Number & Number & Type & $X$ & Z \\
\hline 1 & 6 & -1.264455 & -0.260140 & 0.000000 \\
\hline 2 & 1 & -2.165128 & 0.355256 & -0.000033 \\
\hline 3 & 1 & -1.299019 & -0.904497 & 0.881040 \\
\hline 4 & 1 & -1.298991 & -0.904549 & -0.881003 \\
\hline
\end{tabular}




$\begin{array}{cccccc}5 & 6 & 0 & 0.000000 & 0.589755 & 0.000000 \\ 6 & 1 & 0 & -0.000000 & 1.245377 & 0.874699 \\ 7 & 1 & 0 & 0.000001 & 1.245359 & -0.874711 \\ 8 & 6 & 0 & 1.264455 & -0.260140 & 0.000000 \\ 9 & 1 & 0 & 1.299019 & -0.904495 & 0.881042 \\ 10 & 1 & 0 & 1.298986 & -0.904551 & -0.881002 \\ 11 & 1 & 0 & 2.165130 & 0.355253 & -0.000037\end{array}$

Zero-point correction=

Thermal correction to Energy=

Thermal correction to Enthalpy=

Thermal correction to Gibbs Free Energy=

Sum of electronic and zero-point Energies=

Sum of electronic and thermal Energies $=$

Sum of electronic and thermal Enthalpies=

Sum of electronic and thermal Free Energies=

Butane

Standard orientation:

\begin{tabular}{cccccc} 
Center & Atomic & \multicolumn{2}{c}{ Atomic } & \multicolumn{3}{c}{ Coordinates } & (Angstroms) \\
Number & Number & Type & X & Y & Z \\
\hline 1 & 6 & 0 & 1.945364 & -0.119465 & 0.000034 \\
2 & 1 & 0 & 2.084478 & -0.749316 & -0.881085 \\
3 & 1 & 0 & 2.084403 & -0.749314 & 0.881167 \\
4 & 1 & 0 & 2.734438 & 0.633684 & 0.000067 \\
5 & 6 & 0 & 0.560785 & 0.515568 & -0.000039 \\
6 & 1 & 0 & 0.451510 & 1.163518 & 0.875334 \\
7 & 1 & 0 & 0.451560 & 1.163470 & -0.875454 \\
8 & 6 & 0 & -0.560785 & -0.515568 & -0.000039 \\
9 & 1 & 0 & -0.451560 & -1.163467 & -0.875456 \\
10 & 1 & 0 & -0.451510 & -1.163520 & 0.875332 \\
11 & 6 & 0 & -1.945364 & 0.119465 & 0.000034 \\
12 & 1 & 0 & -2.084477 & 0.749320 & -0.881082 \\
13 & 1 & 0 & -2.734438 & -0.633684 & 0.000062 \\
14 & 1 & 0 & -2.084404 & 0.749309 & 0.881170
\end{tabular}

Zero-point correction=

Thermal correction to Energy=

Thermal correction to Enthalpy=

Thermal correction to Gibbs Free Energy=

Sum of electronic and zero-point Energies=

Sum of electronic and thermal Energies=

Sum of electronic and thermal Enthalpies=

Sum of electronic and thermal Free Energies=

Isobutane

Standard orientation:
0.103945 (Hartree/Particle)

0.108454

0.109398

0.078315

$-118.857795$

$-118.853286$

$-118.852342$

$-118.883424$

Center Atomic Atomic

Number Number Type

Coordinates (Angstroms)

X $\quad \mathrm{Y} \quad \mathrm{Z}$ 


$\begin{array}{cccccc}1 & 6 & 0 & 1.445775 & -0.095979 & -0.096164 \\ 2 & 1 & 0 & 1.920573 & -1.012947 & 0.257896 \\ 3 & 1 & 0 & 1.486834 & -0.098688 & -1.188785 \\ 4 & 1 & 0 & 2.037578 & 0.750197 & 0.257918 \\ 5 & 6 & 0 & 0.000006 & -0.000001 & 0.379188 \\ 6 & 1 & 0 & -0.000005 & -0.000001 & 1.474705 \\ 7 & 6 & 0 & -0.806009 & -1.204081 & -0.096160 \\ 8 & 1 & 0 & -1.837562 & -1.156697 & 0.257778 \\ 9 & 1 & 0 & -0.828768 & -1.238353 & -1.188782 \\ 10 & 1 & 0 & -0.369202 & -2.139692 & 0.258026 \\ 11 & 6 & 0 & -0.639769 & 1.300057 & -0.096161 \\ 12 & 1 & 0 & -1.668571 & 1.389386 & 0.257683 \\ 13 & 1 & 0 & -0.083190 & 2.169725 & 0.258122 \\ 14 & 1 & 0 & -0.657702 & 1.337090 & -1.188780\end{array}$

Zero-point correction=

Thermal correction to Energy=

Thermal correction to Enthalpy=

Thermal correction to Gibbs Free Energy=

Sum of electronic and zero-point Energies=

Sum of electronic and thermal Energies $=$

Sum of electronic and thermal Enthalpies=

Sum of electronic and thermal Free Energies=

Pentane

Standard orientation:
0.132121 (Hartree/Particle)

0.137798

0.138742

0.104537

$-158.088479$

$-158.082802$

$-158.081857$

$-158.116063$

\begin{tabular}{ccccccc} 
Center & Atomic & \multicolumn{2}{c}{ Atomic } & \multicolumn{3}{c}{ Coordinates } \\
Number & Number & Type & X & Y & Z \\
- & Nustroms) \\
\hline 1 & 6 & 0 & 2.536053 & 0.326161 & 0.000000 \\
2 & 1 & 0 & 2.571279 & 0.970152 & -0.881023 \\
3 & 1 & 0 & 2.571274 & 0.970164 & 0.881015 \\
4 & 1 & 0 & 3.436426 & -0.289450 & 0.000007 \\
5 & 6 & 0 & 1.272742 & -0.525020 & 0.000002 \\
6 & 1 & 0 & 1.270616 & -1.181650 & -0.875430 \\
7 & 1 & 0 & 1.270614 & -1.181643 & 0.875440 \\
8 & 6 & 0 & 0.000000 & 0.311802 & -0.000003 \\
9 & 1 & 0 & -0.000001 & 0.970175 & 0.876033 \\
10 & 1 & 0 & 0.000001 & 0.970170 & -0.876043 \\
11 & 6 & 0 & -1.272742 & -0.525020 & -0.000003 \\
12 & 1 & 0 & -1.270613 & -1.181648 & 0.875432 \\
13 & 1 & 0 & -1.270617 & -1.181646 & -0.875438 \\
14 & 6 & 0 & -2.536053 & 0.326161 & 0.000003 \\
15 & 1 & 0 & -3.436426 & -0.289450 & 0.000006 \\
16 & 1 & 0 & -2.571273 & 0.970160 & 0.881021 \\
17 & 1 & 0 & -2.571280 & 0.970157 & -0.881017
\end{tabular}

Zero-point correction=

0.161240 (Hartree/Particle)

Thermal correction to Energy=

0.168307 


$\begin{array}{lc}\text { Thermal correction to Enthalpy= } & 0.169252 \\ \text { Thermal correction to Gibbs Free Energy= } & 0.130936 \\ \text { Sum of electronic and zero-point Energies= } & -197.313569 \\ \text { Sum of electronic and thermal Energies }= & -197.306502 \\ \text { Sum of electronic and thermal Enthalpies }= & -197.305558 \\ \text { Sum of electronic and thermal Free Energies }= & -197.343873 \\ \text { Neopentane } & \\ \text { Standard orientation: }\end{array}$

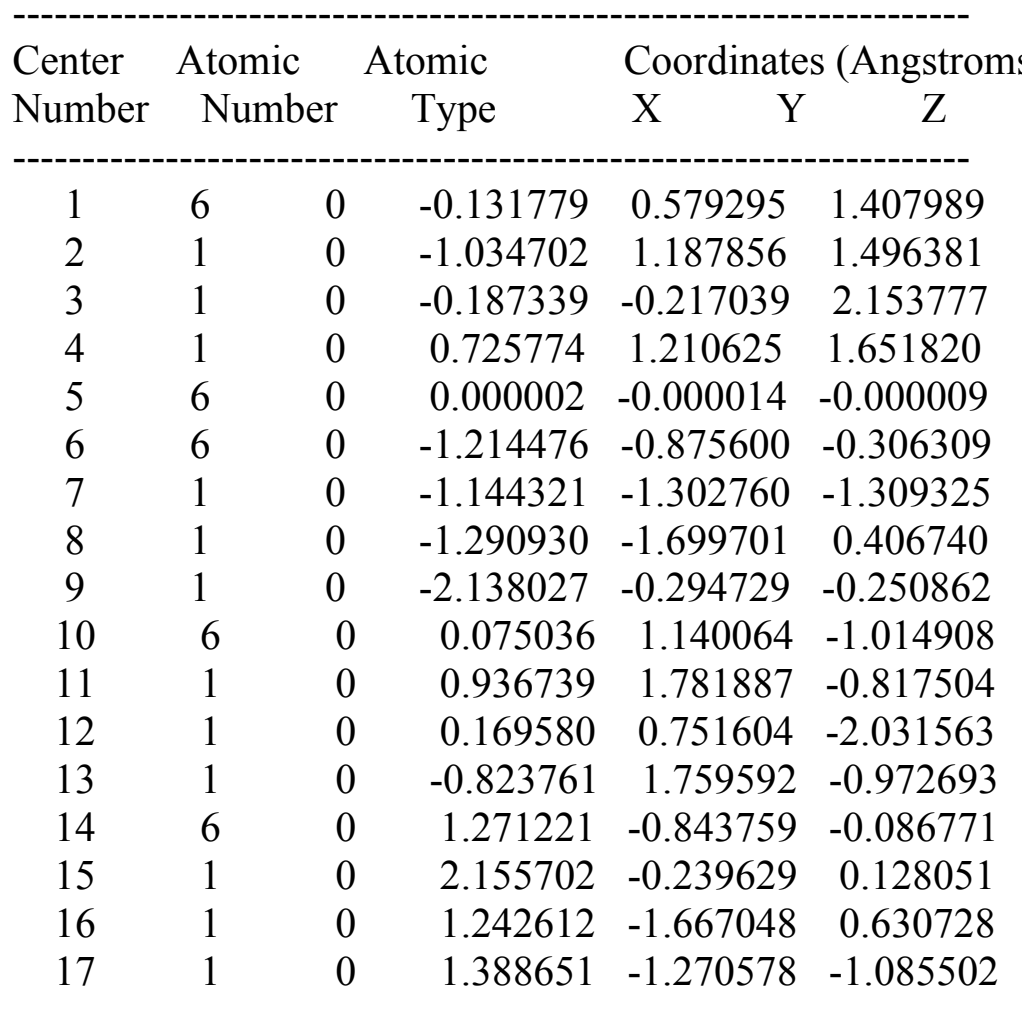

Zero-point correction=

Thermal correction to Energy=

Thermal correction to Enthalpy=

Thermal correction to Gibbs Free Energy=

Sum of electronic and zero-point Energies=

Sum of electronic and thermal Energies $=$

Sum of electronic and thermal Enthalpies=

Sum of electronic and thermal Free Energies=

Hexane

Standard orientation:

\begin{tabular}{cccccc} 
Center & \multicolumn{2}{c}{ Atomic } & \multicolumn{2}{c}{ Atomic } & \multicolumn{2}{c}{ Coordinates } \\
Number & Number & Type & X & Y & Z \\
-------- & \\
\hline 1 & 6 & 0 & -3.195422 & 0.208693 & 0.000032 \\
2 & 1 & 0 & -3.275694 & 0.848669 & 0.881183 \\
3 & 1 & 0 & -3.275695 & 0.848802 & -0.881024 \\
4 & 1 & 0 & -4.050692 & -0.468459 & -0.000021 \\
5 & 6 & 0 & -1.875255 & -0.551466 & -0.000034 \\
6 & 1 & 0 & -1.827046 & -1.206520 & 0.875361
\end{tabular}




$\begin{array}{ccccrr}7 & 1 & 0 & -1.827073 & -1.206427 & -0.875501 \\ 8 & 6 & 0 & -0.664099 & 0.372743 & -0.000006 \\ 9 & 1 & 0 & -0.711249 & 1.029326 & -0.876095 \\ 10 & 1 & 0 & -0.711267 & 1.029302 & 0.876100 \\ 11 & 6 & 0 & 0.664099 & -0.372743 & 0.000004 \\ 12 & 1 & 0 & 0.711250 & -1.029338 & -0.876076 \\ 13 & 1 & 0 & 0.711266 & -1.029290 & 0.876119 \\ 14 & 6 & 0 & 1.875255 & 0.551466 & -0.000030 \\ 15 & 1 & 0 & 1.827049 & 1.206522 & 0.875364 \\ 16 & 1 & 0 & 1.827069 & 1.206424 & -0.875497 \\ 17 & 6 & 0 & 3.195422 & -0.208693 & 0.000027 \\ 18 & 1 & 0 & 3.275698 & -0.848794 & -0.881033 \\ 19 & 1 & 0 & 4.050692 & 0.468459 & -0.000014 \\ 20 & 1 & 0 & 3.275690 & -0.848677 & 0.881173\end{array}$

Zero-point correction=

Thermal correction to Energy=

Thermal correction to Enthalpy=

Thermal correction to Gibbs Free Energy=

Sum of electronic and zero-point Energies=

Sum of electronic and thermal Energies=

Sum of electronic and thermal Enthalpies=

Sum of electronic and thermal Free Energies=
0.189824 (Hartree/Particle)

0.198211

0.199156

$$
\begin{aligned}
& 0.157274 \\
& -236.541520 \\
& -236.533132 \\
& -236.532188 \\
& -236.574069
\end{aligned}
$$

\begin{tabular}{|c|c|c|c|c|}
\hline \multirow{2}{*}{$\begin{array}{l}\text { Center } \\
\text { Number }\end{array}$} & Atomic & \multirow{2}{*}{$\begin{array}{l}\text { Atomic } \\
\text { Type }\end{array}$} & \multicolumn{2}{|c|}{ Coordinates (Angstroms } \\
\hline & Numbe & & $\mathrm{X}$ & $\mathrm{Z}$ \\
\hline 1 & 6 & -1.613220 & 0.970205 & 0.001088 \\
\hline 2 & 1 & -1.590576 & 1.611346 & 0.885425 \\
\hline 3 & 1 & -1.590763 & 1.613068 & -0.882003 \\
\hline 4 & 1 & -2.562122 & 0.429082 & 0.000656 \\
\hline 5 & 6 & -0.431333 & -0.000001 & 0.000011 \\
\hline 6 & 6 & 0.863597 & 0.828952 & 0.000484 \\
\hline 7 & 1 & 0.844701 & 1.486809 & -0.874899 \\
\hline 8 & 1 & 0.844774 & 1.485735 & 0.876679 \\
\hline 9 & 6 & 2.166718 & 0.037515 & -0.000002 \\
\hline 10 & 1 & 2.253416 & -0.597771 & -0.882121 \\
\hline 11 & 1 & 3.022222 & 0.714305 & 0.000071 \\
\hline 12 & 1 & 2.253676 & -0.598313 & 0.881696 \\
\hline 13 & 6 & -0.512351 & -0.879727 & 1.247927 \\
\hline 14 & 1 & 0.279038 & -1.630445 & 1.264883 \\
\hline 15 & 1 & -0.428956 & -0.277399 & 2.155930 \\
\hline 16 & 1 & -1.468494 & -1.406874 & 1.281187 \\
\hline 17 & 6 & -0.512846 & -0.877487 & -1.249459 \\
\hline 18 & 1 & -1.468857 & -1.404853 & -1.283102 \\
\hline 19 & 1 & -0.430182 & -0.273471 & -2.156404 \\
\hline 20 & 1 & 0.278734 & -1.627960 & -1.268286 \\
\hline
\end{tabular}

22dimethylbutane

Standard orientation: 


$\begin{array}{lc}\text { Zero-point correction }= & 0.188728 \text { (Hartree/Particle) } \\ \text { Thermal correction to Energy= } & 0.196942 \\ \text { Thermal correction to Enthalpy= } & 0.197886 \\ \text { Thermal correction to Gibbs Free Energy= } & 0.157449 \\ \text { Sum of electronic and zero-point Energies }= & -236.546691 \\ \text { Sum of electronic and thermal Energies }= & -236.538477 \\ \text { Sum of electronic and thermal Enthalpies }= & -236.537533 \\ \text { Sum of electronic and thermal Free Energies }= & -236.577970\end{array}$

Heptane

Standard orientation:

\begin{tabular}{|c|c|c|c|c|}
\hline \multirow{2}{*}{$\begin{array}{l}\text { Center } \\
\text { Number }\end{array}$} & Atomic & \multirow{2}{*}{$\begin{array}{r}\text { Atomic } \\
\text { Type }\end{array}$} & \multicolumn{2}{|c|}{ Coordinates (Angstroms } \\
\hline & Number & & $\mathrm{X}$ & $\mathrm{Z}$ \\
\hline 1 & 6 & -3.808160 & -0.355612 & -0.000007 \\
\hline 2 & 1 & -3.843335 & -0.999609 & -0.881046 \\
\hline 3 & 1 & -3.843331 & -0.999649 & 0.881004 \\
\hline 4 & 1 & -4.708607 & 0.259928 & 0.000010 \\
\hline 5 & 6 & -2.544872 & 0.495605 & 0.000011 \\
\hline 6 & 1 & -2.542657 & 1.152248 & -0.875406 \\
\hline 7 & 1 & -2.542660 & 1.152216 & 0.875451 \\
\hline 8 & 6 & -1.272028 & -0.341441 & -0.000002 \\
\hline 9 & 1 & -1.272773 & -0.999537 & 0.876068 \\
\hline 10 & 1 & -1.272779 & -0.999522 & -0.876083 \\
\hline 11 & 6 & 0.000000 & 0.496203 & 0.000000 \\
\hline 12 & 1 & -0.000002 & 1.154010 & 0.876147 \\
\hline 13 & 1 & 0.000002 & 1.154007 & -0.876148 \\
\hline 14 & 6 & 1.272028 & -0.341441 & 0.000005 \\
\hline 15 & 1 & 1.272772 & -0.999541 & -0.876062 \\
\hline 16 & 1 & 1.272780 & -0.999518 & 0.876089 \\
\hline 17 & 6 & 2.544872 & 0.495605 & -0.000011 \\
\hline 18 & 1 & 2.542659 & 1.152248 & 0.875406 \\
\hline 19 & 1 & 2.542658 & 1.152216 & -0.875451 \\
\hline 20 & 6 & 3.808160 & -0.355612 & 0.000004 \\
\hline 21 & 1 & 3.843331 & -0.999648 & -0.881008 \\
\hline 22 & 1 & 4.708607 & 0.259928 & -0.000011 \\
\hline 23 & 1 & 3.843335 & -0.999611 & 0.881042 \\
\hline
\end{tabular}

Zero-point correction=

Thermal correction to Energy=

Thermal correction to Enthalpy=

Thermal correction to Gibbs Free Energy=

Sum of electronic and zero-point Energies=

Sum of electronic and thermal Energies=

Sum of electronic and thermal Enthalpies=

Sum of electronic and thermal Free Energies=

223trimethylbutane

\subsection{1 (Hartree/Particle)}

0.228156

0.229100

0.183562

$-275.769470$

$-275.759726$

$-275.758782$

$-275.804320$

Standard orientation: 


\begin{tabular}{|c|c|c|c|c|c|}
\hline \multirow{2}{*}{$\begin{array}{l}\text { Center } \\
\text { Number }\end{array}$} & Atomic & \multirow{2}{*}{\multicolumn{2}{|c|}{$\begin{array}{l}\text { Atomic } \\
\text { Type }\end{array}$}} & \multicolumn{2}{|c|}{ Coordinates (Angstroms } \\
\hline & Numbe & & & $X$ & Z \\
\hline 1 & 6 & 0 & -1.324950 & -1.284556 & -0.473041 \\
\hline 2 & 1 & 0 & -1.180493 & -1.434345 & -1.546202 \\
\hline 3 & 1 & 0 & -0.945856 & -2.164602 & 0.046950 \\
\hline 4 & 1 & 0 & -2.399108 & -1.227739 & -0.284080 \\
\hline 5 & 6 & 0 & -0.637663 & 0.000147 & -0.000950 \\
\hline 6 & 6 & 0 & 0.845750 & -0.000739 & -0.455990 \\
\hline 7 & 1 & 0 & 0.817237 & -0.004396 & -1.553140 \\
\hline 8 & 6 & 0 & 1.619397 & 1.243724 & -0.020325 \\
\hline 9 & 1 & 0 & 1.736903 & 1.273911 & 1.064764 \\
\hline 10 & 1 & 0 & 2.620736 & 1.229939 & -0.454387 \\
\hline 11 & 1 & 0 & 1.138320 & 2.168998 & -0.335217 \\
\hline 12 & 6 & 0 & 1.622125 & -1.242343 & -0.016793 \\
\hline 13 & 1 & 0 & 1.665314 & -1.318606 & 1.071745 \\
\hline 14 & 1 & 0 & 1.189763 & -2.163614 & -0.404766 \\
\hline 15 & 1 & 0 & 2.650481 & -1.184320 & -0.378194 \\
\hline 16 & 6 & 0 & -0.774798 & 0.101890 & 1.519507 \\
\hline 17 & 1 & 0 & -0.366481 & 1.039280 & 1.899920 \\
\hline 18 & 1 & 0 & -1.829874 & 0.064474 & 1.800200 \\
\hline 19 & 1 & 0 & -0.270547 & -0.721794 & 2.027524 \\
\hline 20 & 6 & 0 & -1.370950 & 1.182260 & -0.641891 \\
\hline 21 & 1 & 0 & -1.271559 & 1.159175 & -1.730047 \\
\hline 22 & 1 & 0 & -2.435586 & 1.140418 & -0.401676 \\
\hline 23 & 1 & 0 & -0.992713 & 2.140922 & -0.286504 \\
\hline
\end{tabular}

Zero-point correction=

Thermal correction to Energy=

Thermal correction to Enthalpy=

Thermal correction to Gibbs Free Energy=

Sum of electronic and zero-point Energies=

Sum of electronic and thermal Energies $=$

Sum of electronic and thermal Enthalpies=

Sum of electronic and thermal Free Energies=

Octane

Standard orientation:

\begin{tabular}{|c|c|c|c|c|}
\hline \multirow{2}{*}{$\begin{array}{l}\text { Center } \\
\text { Number }\end{array}$} & Atomic & \multirow{2}{*}{$\begin{array}{l}\text { Atomic } \\
\text { Type }\end{array}$} & \multicolumn{2}{|c|}{ Coordinates (Angstrom } \\
\hline & Numbe & & $\mathrm{X}$ & Z \\
\hline 1 & 6 & -4.457841 & 0.257406 & 0.000014 \\
\hline 2 & 1 & -4.518382 & 0.899514 & 0.881057 \\
\hline 3 & 1 & -4.518384 & 0.899569 & -0.880991 \\
\hline 4 & 1 & -5.333332 & -0.393148 & -0.000006 \\
\hline 5 & 6 & -3.161974 & -0.543345 & -0.000014 \\
\hline 6 & 1 & -3.133875 & -1.199399 & 0.875398 \\
\hline 7 & 1 & -3.133884 & -1.199355 & -0.875459 \\
\hline 8 & 6 & -1.923098 & 0.343204 & 0.000002 \\
\hline 9 & 1 & -1.949757 & 1.000764 & -0.876076 \\
\hline
\end{tabular}




$\begin{array}{rrrrrr}10 & 1 & 0 & -1.949761 & 1.000741 & 0.876097 \\ 11 & 6 & 0 & -0.619069 & -0.443670 & -0.000004 \\ 12 & 1 & 0 & -0.593080 & -1.100966 & -0.876132 \\ 13 & 1 & 0 & -0.593074 & -1.100968 & 0.876123 \\ 14 & 6 & 0 & 0.619069 & 0.443670 & -0.000007 \\ 15 & 1 & 0 & 0.593073 & 1.100977 & 0.876113 \\ 16 & 1 & 0 & 0.593081 & 1.100957 & -0.876142 \\ 17 & 6 & 0 & 1.923098 & -0.343204 & 0.000007 \\ 18 & 1 & 0 & 1.949759 & -1.000769 & -0.876067 \\ 19 & 1 & 0 & 1.949759 & -1.000737 & 0.876105 \\ 20 & 6 & 0 & 3.161974 & 0.543345 & -0.000009 \\ 21 & 1 & 0 & 3.133878 & 1.199395 & 0.875405 \\ 22 & 1 & 0 & 3.133881 & 1.199359 & -0.875451 \\ 23 & 6 & 0 & 4.457841 & -0.257406 & 0.000009 \\ 24 & 1 & 0 & 4.518384 & -0.899560 & -0.881001 \\ 25 & 1 & 0 & 5.333332 & 0.393148 & -0.000004 \\ 26 & 1 & 0 & 4.518382 & -0.899523 & 0.881047\end{array}$

Zero-point correction=

Thermal correction to Energy=

Thermal correction to Enthalpy=
0.246983 (Hartree/Particle)

0.258081

0.259025

0.209877

$-314.997439$

$-314.986341$

$-314.985397$

$-315.034544$

Sum of electronic and thermal Free Energies=

PBE0DHD3BJ/ccpVQZ

Ethane

Standard orientation:

\begin{tabular}{|c|c|c|c|c|}
\hline \multirow{2}{*}{$\begin{array}{l}\text { Center } \\
\text { Number }\end{array}$} & Atomic & \multirow{2}{*}{$\begin{array}{l}\text { Atomic } \\
\text { Type }\end{array}$} & \multicolumn{2}{|c|}{ Coordinates (Angstroms) } \\
\hline & Number & & $\mathrm{X}$ & $\mathrm{Z}$ \\
\hline 1 & 6 & 0.000000 & 0.000000 & 0.757343 \\
\hline 2 & 1 & -0.506448 & 0.877224 & 1.153575 \\
\hline 3 & 1 & 1.012922 & -0.000015 & 1.153575 \\
\hline 4 & 1 & -0.506474 & -0.877209 & 1.153575 \\
\hline 5 & 6 & 0.000000 & 0.000000 & -0.757343 \\
\hline 6 & 1 & 0.506448 & 0.877224 & -1.153575 \\
\hline 7 & 1 & 0.506474 & -0.877209 & -1.153575 \\
\hline 8 & 1 & -1.012922 & -0.000015 & -1.153575 \\
\hline \multicolumn{3}{|c|}{ Zero-point correction $=$} & \multicolumn{2}{|c|}{0.075741 (Hartree/Particle) } \\
\hline \multicolumn{3}{|c|}{ Thermal correction to Energy= } & \multicolumn{2}{|c|}{0.079208} \\
\hline \multicolumn{3}{|c|}{ Thermal correction to Enthalpy= } & \multicolumn{2}{|c|}{0.080152} \\
\hline \multicolumn{4}{|c|}{ Thermal correction to Gibbs Free Energy= } & 0.054348 \\
\hline \multicolumn{4}{|c|}{ Sum of electronic and zero-point Energies $=$} & -79.693084 \\
\hline \multicolumn{4}{|c|}{ Sum of electronic and thermal Energies $=$} & -79.689616 \\
\hline \multicolumn{4}{|c|}{ Sum of electronic and thermal Enthalpies $=$} & -79.688672 \\
\hline \multicolumn{4}{|c|}{ Sum of electronic and thermal Free Energies $=$} & -79.714477 \\
\hline
\end{tabular}


Methane

Standard orientation:

\begin{tabular}{|c|c|c|c|c|}
\hline Center & Atomic & Atomic & Coordinate & tron \\
\hline Number & Numbe & Type & $X$ & Z \\
\hline 1 & 6 & 0.000000 & 0.000000 & 0.000000 \\
\hline 2 & 1 & 0.626334 & 0.626334 & 0.626334 \\
\hline 3 & 1 & -0.626334 & -0.626334 & 0.626334 \\
\hline 4 & 1 & -0.626334 & 0.626334 & -0.626334 \\
\hline 5 & 1 & 0.626334 & -0.626334 & -0.626334 \\
\hline
\end{tabular}

Zero-point correction= 0.045339 (Hartree/Particle)

Thermal correction to Energy= 0.048206

Thermal correction to Enthalpy= 0.049150

Thermal correction to Gibbs Free Energy $=\quad 0.028037$

Sum of electronic and zero-point Energies $=\quad-40.439174$

Sum of electronic and thermal Energies $=\quad-40.436306$

Sum of electronic and thermal Enthalpies $=\quad-40.435362$

Sum of electronic and thermal Free Energies $=\quad-40.456475$

Propane

Standard orientation:

\begin{tabular}{cccccc} 
Center & \multicolumn{2}{c}{ Atomic } & \multicolumn{2}{c}{ Atomic } & \multicolumn{3}{c}{ Coordinates } \\
Number & Number & Type & X & Y & Z \\
\hline 1 & 6 & 0 & -1.258520 & -0.258070 & 0.000000 \\
2 & 1 & 0 & -2.156223 & 0.356033 & -0.000030 \\
3 & 1 & 0 & -1.295796 & -0.901286 & 0.877887 \\
4 & 1 & 0 & -1.295770 & -0.901333 & -0.877853 \\
5 & 6 & 0 & -0.000000 & 0.584916 & 0.000000 \\
6 & 1 & 0 & 0.000001 & 1.240271 & 0.871097 \\
7 & 1 & 0 & 0.000002 & 1.240256 & -0.871107 \\
8 & 6 & 0 & 1.258520 & -0.258071 & 0.000000 \\
9 & 1 & 0 & 1.295790 & -0.901292 & 0.877883 \\
10 & 1 & 0 & 1.295770 & -0.901327 & -0.877858 \\
11 & 1 & 0 & 2.156225 & 0.356030 & -0.000022
\end{tabular}

Zero-point correction=

Thermal correction to Energy=

Thermal correction to Enthalpy=

Thermal correction to Gibbs Free Energy=

Sum of electronic and zero-point Energies=

Sum of electronic and thermal Energies $=$

Sum of electronic and thermal Enthalpies=

Sum of electronic and thermal Free Energies=

Butane

Standard orientation:
0.104914 (Hartree/Particle)

$$
0.109405
$$

0.110349

0.079309

$-118.951347$

$-118.946856$

$-118.945912$

$-118.976953$

Center Atomic Atomic Coordinates (Angstroms) 


\begin{tabular}{|c|c|c|c|c|c|}
\hline Number & Numbe & & Type & $X$ & Z \\
\hline 1 & 6 & 0 & 1.935516 & 0.118504 & -0.000022 \\
\hline 2 & 1 & 0 & 2.076654 & 0.746917 & 0.877916 \\
\hline 3 & 1 & 0 & 2.076603 & 0.746935 & -0.877955 \\
\hline 4 & 1 & 0 & 2.722549 & -0.632086 & -0.000052 \\
\hline 5 & 6 & 0 & 0.558601 & -0.511736 & 0.000025 \\
\hline 6 & 1 & 0 & 0.449508 & -1.159416 & -0.871615 \\
\hline 7 & 1 & 0 & 0.449537 & -1.159392 & 0.871686 \\
\hline 8 & 6 & 0 & -0.558601 & 0.511736 & 0.000025 \\
\hline 9 & 1 & 0 & -0.449537 & 1.159390 & 0.871688 \\
\hline 10 & 1 & 0 & -0.449508 & 1.159419 & -0.871613 \\
\hline 11 & 6 & 0 & -1.935516 & -0.118504 & -0.000022 \\
\hline 12 & 1 & 0 & -2.076655 & -0.746916 & 0.877917 \\
\hline 13 & 1 & 0 & -2.722549 & 0.632086 & -0.000054 \\
\hline 14 & 1 & 0 & -2.076602 & -0.746936 & -0.877954 \\
\hline
\end{tabular}

Zero-point correction=

Thermal correction to Energy=

Thermal correction to Enthalpy=

Thermal correction to Gibbs Free Energy=

Sum of electronic and zero-point Energies=

Sum of electronic and thermal Energies $=$

Sum of electronic and thermal Enthalpies=

Sum of electronic and thermal Free Energies=

Isobutane

Standard orientation:

\begin{tabular}{cccccc}
\multicolumn{7}{c}{ Standard orientation: } \\
Center & Atomic & \multicolumn{2}{c}{ Atomic } & \multicolumn{3}{c}{ Coordinates (Angstroms) } \\
Number & Number & Type & X & Y & Z \\
------------- \\
1 & 6 & 0 & 0.901194 & -1.125225 & 0.095238 \\
2 & 1 & 0 & 1.921878 & -0.991096 & -0.257855 \\
3 & 1 & 0 & 0.928945 & -1.159979 & 1.184490 \\
4 & 1 & 0 & 0.547389 & -2.091940 & -0.257948 \\
5 & 6 & 0 & 0.000002 & 0.000005 & -0.375423 \\
6 & 1 & 0 & 0.000005 & 0.000007 & -1.467846 \\
7 & 6 & 0 & 0.523879 & 1.343068 & 0.095243 \\
8 & 1 & 0 & -0.102550 & 2.159968 & -0.257919 \\
9 & 1 & 0 & 0.540020 & 1.384485 & 1.184496 \\
10 & 1 & 0 & 1.538015 & 1.519951 & -0.257884 \\
11 & 6 & 0 & -1.425079 & -0.217843 & 0.095236 \\
12 & 1 & 0 & -2.085358 & 0.571970 & -0.257869 \\
13 & 1 & 0 & -1.819295 & -1.168803 & -0.257929 \\
14 & 1 & 0 & -1.469029 & -0.224591 & 1.184492
\end{tabular}

Zero-point correction=

Thermal correction to Energy=

Thermal correction to Enthalpy=
0.133875 (Hartree/Particle)

0.139609

0.140554

0.105929

$-158.209911$

$-158.204177$

$-158.203233$

$-158.237857$ 
Thermal correction to Gibbs Free Energy=

Sum of electronic and zero-point Energies=

Sum of electronic and thermal Energies $=$

Sum of electronic and thermal Enthalpies=

Sum of electronic and thermal Free Energies=

Pentane

Standard orientation:
0.105848

$-158.212426$

$-158.206778$

$-158.205834$

$-158.239975$

\begin{tabular}{|c|c|c|c|c|}
\hline \multirow{2}{*}{$\begin{array}{l}\text { Center } \\
\text { Number }\end{array}$} & Atomic & \multirow{2}{*}{$\begin{array}{l}\text { Atomic } \\
\text { Type }\end{array}$} & \multicolumn{2}{|c|}{ Coordinates (Angstrom } \\
\hline & Number & & $\mathrm{X}$ & $\mathrm{Z}$ \\
\hline 1 & 6 & -2.523380 & 0.323173 & 0.000001 \\
\hline 2 & 1 & -2.561313 & 0.966052 & 0.877883 \\
\hline 3 & 1 & -2.561311 & 0.966059 & -0.877877 \\
\hline 4 & 1 & -3.420788 & -0.291119 & -0.000003 \\
\hline 5 & 6 & -1.266146 & -0.520796 & -0.000001 \\
\hline 6 & 1 & -1.263617 & -1.177114 & 0.871761 \\
\hline 7 & 1 & -1.263618 & -1.177111 & -0.871765 \\
\hline 8 & 6 & 0.000000 & 0.310246 & 0.000000 \\
\hline 9 & 1 & 0.000001 & 0.968231 & -0.872267 \\
\hline 10 & 1 & -0.000001 & 0.968232 & 0.872266 \\
\hline 11 & 6 & 1.266146 & -0.520796 & 0.000001 \\
\hline 12 & 1 & 1.263617 & -1.177114 & -0.871761 \\
\hline 13 & 1 & 1.263617 & -1.177111 & 0.871765 \\
\hline 14 & 6 & 2.523380 & 0.323173 & -0.000001 \\
\hline 15 & 1 & 3.420788 & -0.291119 & 0.000000 \\
\hline 16 & 1 & 2.561312 & 0.966055 & -0.877881 \\
\hline 17 & 1 & 2.561312 & 0.966057 & 0.877879 \\
\hline
\end{tabular}

Zero-point correction=

Thermal correction to Energy=

Thermal correction to Enthalpy=

Thermal correction to Gibbs Free Energy=

Sum of electronic and zero-point Energies=

Sum of electronic and thermal Energies=

Sum of electronic and thermal Enthalpies=

Sum of electronic and thermal Free Energies=

Neopentane

Standard orientation:

\begin{tabular}{|c|c|c|c|c|}
\hline \multirow{2}{*}{$\begin{array}{l}\text { Center } \\
\text { Number }\end{array}$} & Atomic & \multirow{2}{*}{$\begin{array}{r}\text { Atomic } \\
\text { Type }\end{array}$} & \multicolumn{2}{|c|}{ Coordinates (Angstroms } \\
\hline & Numbe & & $\mathrm{X}$ & $\mathrm{Z}$ \\
\hline 1 & 6 & -0.176431 & -1.192070 & -0.926463 \\
\hline 2 & 1 & -0.855096 & -1.925428 & -0.493315 \\
\hline 3 & 1 & -0.586246 & -0.882275 & -1.886722 \\
\hline 4 & 1 & 0.776314 & -1.685795 & -1.112275 \\
\hline 5 & 6 & -0.000010 & 0.000008 & 0.000012 \\
\hline 6 & 6 & -1.347823 & 0.661876 & 0.236333 \\
\hline 7 & 1 & -1.249632 & 1.520810 & 0.898607 \\
\hline
\end{tabular}




$\begin{array}{cccrcc}8 & 1 & 0 & -1.781351 & 1.008842 & -0.700512 \\ 9 & 1 & 0 & -2.049599 & -0.034727 & 0.692701 \\ 10 & 6 & 0 & 0.573268 & -0.470096 & 1.326979 \\ 11 & 1 & 0 & 1.541173 & -0.948897 & 1.185805 \\ 12 & 1 & 0 & 0.709692 & 0.366302 & 2.010966 \\ 13 & 1 & 0 & -0.089953 & -1.189413 & 1.805235 \\ 14 & 6 & 0 & 0.950984 & 1.000287 & -0.636848 \\ 15 & 1 & 0 & 1.926455 & 0.550735 & -0.816785 \\ 16 & 1 & 0 & 0.563510 & 1.353491 & -1.591381 \\ 17 & 1 & 0 & 1.094804 & 1.866324 & 0.007597\end{array}$

Zero-point correction $=$

Thermal correction to Energy=

Thermal correction to Enthalpy=

Thermal correction to Gibbs Free Energy=

Sum of electronic and zero-point Energies=

Sum of electronic and thermal Energies=

Sum of electronic and thermal Enthalpies=

Sum of electronic and thermal Free Energies=

Hexane

Standard orientation:
0.161524 (Hartree/Particle)

0.168371

0.169315
0.132500

$-197.474836$

$-197.467989$

$-197.467044$

$-197.503859$

\begin{tabular}{|c|c|c|c|c|}
\hline \multirow{2}{*}{$\begin{array}{l}\text { Center } \\
\text { Number }\end{array}$} & Atomic & \multirow{2}{*}{$\begin{array}{r}\text { Atomic } \\
\text { Type }\end{array}$} & \multicolumn{2}{|c|}{ Coordinates (Angstroms } \\
\hline & Numbe & & $X$ & $\mathrm{Z}$ \\
\hline 1 & 6 & 3.178296 & -0.206873 & 0.000006 \\
\hline 2 & 1 & 3.261069 & -0.845532 & 0.877899 \\
\hline 3 & 1 & 3.261070 & -0.845557 & -0.877870 \\
\hline 4 & 1 & 4.030606 & 0.468619 & -0.000003 \\
\hline 5 & 6 & 1.865173 & 0.547166 & -0.000006 \\
\hline 6 & 1 & 1.816741 & 1.201720 & 0.871714 \\
\hline 7 & 1 & 1.816746 & 1.201701 & -0.871741 \\
\hline 8 & 6 & 0.660109 & -0.370491 & -0.000000 \\
\hline 9 & 1 & 0.706934 & -1.026554 & -0.872310 \\
\hline 10 & 1 & 0.706937 & -1.026547 & 0.872314 \\
\hline 11 & 6 & -0.660109 & 0.370491 & -0.000000 \\
\hline 12 & 1 & -0.706934 & 1.026554 & -0.872309 \\
\hline 13 & 1 & -0.706937 & 1.026547 & 0.872315 \\
\hline 14 & 6 & -1.865173 & -0.547166 & -0.000006 \\
\hline 15 & 1 & -1.816741 & -1.201720 & 0.871714 \\
\hline 16 & 1 & -1.816746 & -1.201701 & -0.871741 \\
\hline 17 & 6 & -3.178296 & 0.206873 & 0.000006 \\
\hline 18 & 1 & -3.261070 & 0.845558 & -0.877869 \\
\hline 19 & 1 & -4.030606 & -0.468619 & -0.000005 \\
\hline 20 & 1 & -3.261070 & 0.845532 & 0.877900 \\
\hline
\end{tabular}

Zero-point correction=

Thermal correction to Energy=

Thermal correction to Enthalpy=
0.191727 (Hartree/Particle)

0.200078

0.201022 
Thermal correction to Gibbs Free Energy=

Sum of electronic and zero-point Energies=

Sum of electronic and thermal Energies $=$

Sum of electronic and thermal Enthalpies=

Sum of electronic and thermal Free Energies=

22dimethylbutane

Standard orientation:
0.159207

$-236.727019$

$-236.718669$

$-236.717725$

$-236.759539$

\begin{tabular}{|c|c|c|c|c|c|}
\hline \multirow{2}{*}{$\begin{array}{l}\text { Center } \\
\text { Number }\end{array}$} & \multicolumn{2}{|l|}{ Atomic } & & \multicolumn{2}{|c|}{ Coordinates (Angstroms } \\
\hline & Numbe & & & $\mathrm{X}$ & Z \\
\hline 1 & 6 & 0 & 1.604400 & 0.965119 & -0.001007 \\
\hline 2 & 1 & 0 & 1.582451 & 1.604847 & -0.882229 \\
\hline 3 & 1 & 0 & 1.582578 & 1.606499 & 0.879016 \\
\hline 4 & 1 & 0 & 2.551228 & 0.427244 & -0.000568 \\
\hline 5 & 6 & 0 & 0.429466 & -0.000261 & -0.000010 \\
\hline 6 & 6 & 0 & -0.858864 & 0.823945 & -0.000546 \\
\hline 7 & 1 & 0 & -0.839006 & 1.480971 & 0.871179 \\
\hline 8 & 1 & 0 & -0.839064 & 1.479777 & -0.873173 \\
\hline 9 & 6 & 0 & -2.154445 & 0.038374 & -0.000004 \\
\hline 10 & 1 & 0 & -2.242680 & -0.595499 & 0.879154 \\
\hline 11 & 1 & 0 & -3.008152 & 0.712153 & -0.000216 \\
\hline 12 & 1 & 0 & -2.242869 & -0.596311 & -0.878556 \\
\hline 13 & 6 & 0 & 0.509364 & -0.875175 & -1.241152 \\
\hline 14 & 1 & 0 & -0.282052 & -1.620996 & -1.260137 \\
\hline 15 & 1 & 0 & 0.429992 & -0.275262 & -2.146983 \\
\hline 16 & 1 & 0 & 1.460204 & -1.404828 & -1.273833 \\
\hline 17 & 6 & 0 & 0.509721 & -0.872972 & 1.242665 \\
\hline 18 & 1 & 0 & 1.460467 & -1.402764 & 1.275878 \\
\hline 19 & 1 & 0 & 0.430876 & -0.271409 & 2.147445 \\
\hline 20 & 1 & 0 & -0.281828 & -1.618608 & 1.263355 \\
\hline
\end{tabular}

Zero-point correction=

Thermal correction to Energy=

0.190615 (Hartree/Particle)

Thermal correction to Enthalpy=

0.198777

0.199721

Thermal correction to Gibbs Free Energy=

0.159408

Sum of electronic and zero-point Energies=

$-236.731425$

Sum of electronic and thermal Energies=

$-236.723264$

$-236.722320$

Sum of electronic and thermal Enthalpies=

$-236.762633$

Sum of electronic and thermal Free Energies=

Heptane

Standard orientation:

\begin{tabular}{cccccc} 
Center & \multicolumn{2}{c}{ Atomic } & \multicolumn{2}{c}{ Atomic } & \multicolumn{2}{c}{ Coordinates (Angstroms) } \\
Number & Number & Type & X & Y & Z \\
- \hdashline 1 & 6 & 0 & -3.788709 & -0.352244 & -0.000008 \\
2 & 1 & 0 & -3.826593 & -0.995136 & -0.877902 \\
3 & 1 & 0 & -3.826592 & -0.995172 & 0.877860 \\
4 & 1 & 0 & -4.686200 & 0.261956 & 0.000006
\end{tabular}




$\begin{array}{cccccc}5 & 6 & 0 & -2.531515 & 0.491754 & 0.000010 \\ 6 & 1 & 0 & -2.528920 & 1.148094 & -0.871718 \\ 7 & 1 & 0 & -2.528924 & 1.148064 & 0.871761 \\ 8 & 6 & 0 & -1.265290 & -0.339447 & 0.000000 \\ 9 & 1 & 0 & -1.266094 & -0.997185 & 0.872278 \\ 10 & 1 & 0 & -1.266098 & -0.997172 & -0.872288 \\ 11 & 6 & 0 & 0.000000 & 0.492178 & 0.000001 \\ 12 & 1 & 0 & -0.000002 & 1.149641 & 0.872364 \\ 13 & 1 & 0 & 0.000002 & 1.149638 & -0.872364 \\ 14 & 6 & 0 & 1.265290 & -0.339447 & 0.000005 \\ 15 & 1 & 0 & 1.266092 & -0.997190 & -0.872269 \\ 16 & 1 & 0 & 1.266100 & -0.997167 & 0.872297 \\ 17 & 6 & 0 & 2.531515 & 0.491754 & -0.000011 \\ 18 & 1 & 0 & 2.528923 & 1.148095 & 0.871717 \\ 19 & 1 & 0 & 2.528921 & 1.148063 & -0.871763 \\ 20 & 6 & 0 & 3.788709 & -0.352244 & 0.000003 \\ 21 & 1 & 0 & 3.826590 & -0.995171 & -0.877865 \\ 22 & 1 & 0 & 4.686200 & 0.261956 & -0.000012 \\ 23 & 1 & 0 & 3.826594 & -0.995136 & 0.877897\end{array}$

Zero-point correction=

Thermal correction to Energy=

0.220614 (Hartree/Particle)

Thermal correction to Enthalpy=

0.230300

0.231244

Thermal correction to Gibbs Free Energy=

0.185837

$-275.985628$

$-275.975941$

Sum of electronic and thermal Energies=

$-275.974997$

Sum of electronic and thermal Enthalpies=

$-276.020404$

Sum of electronic and thermal Free Energies=

223trimethylbutane

Standard orientation:

\begin{tabular}{|c|c|c|c|c|}
\hline \multirow{2}{*}{$\begin{array}{l}\text { Center } \\
\text { Number }\end{array}$} & Atomic & \multirow{2}{*}{$\begin{array}{r}\text { Atomic } \\
\text { Type }\end{array}$} & \multicolumn{2}{|c|}{ Coordinates (Angstroms } \\
\hline & Numbe & & $\mathrm{X}$ & $\mathrm{Z}$ \\
\hline 1 & 6 & 1.326062 & 1.258945 & -0.506225 \\
\hline 2 & 1 & 1.189953 & 1.375236 & -1.581121 \\
\hline 3 & 1 & 0.949481 & 2.154602 & -0.019123 \\
\hline 4 & 1 & 2.395577 & 1.205565 & -0.309847 \\
\hline 5 & 6 & 0.634462 & -0.000087 & -0.000625 \\
\hline 6 & 6 & -0.841460 & 0.000384 & -0.451915 \\
\hline 7 & 1 & -0.811886 & 0.002566 & -1.545654 \\
\hline 8 & 6 & -1.610173 & -1.236973 & -0.019737 \\
\hline 9 & 1 & -1.712820 & -1.279218 & 1.063249 \\
\hline 10 & 1 & -2.614923 & -1.215492 & -0.436855 \\
\hline 11 & 1 & -1.140247 & -2.158724 & -0.350034 \\
\hline 12 & 6 & -1.611669 & 1.236129 & -0.017607 \\
\hline 13 & 1 & -1.669478 & 1.306102 & 1.067399 \\
\hline 14 & 1 & -1.170902 & 2.155316 & -0.391860 \\
\hline 15 & 1 & -2.632676 & 1.188237 & -0.390865 \\
\hline
\end{tabular}




$\begin{array}{llllll}16 & 6 & 0 & 0.770468 & -0.060109 & 1.513447 \\ 17 & 1 & 0 & 0.343774 & -0.973862 & 1.920758 \\ 18 & 1 & 0 & 1.822622 & -0.037701 & 1.793172 \\ 19 & 1 & 0 & 0.286542 & 0.786008 & 1.996376 \\ 20 & 6 & 0 & 1.353358 & -1.198535 & -0.605802 \\ 21 & 1 & 0 & 1.243941 & -1.212758 & -1.689762 \\ 22 & 1 & 0 & 2.417285 & -1.153573 & -0.379105 \\ 23 & 1 & 0 & 0.977468 & -2.140824 & -0.215945\end{array}$

Zero-point correction=

Thermal correction to Energy=

Thermal correction to Enthalpy=

Thermal correction to Gibbs Free Energy=

Sum of electronic and zero-point Energies=

Sum of electronic and thermal Energies=

Sum of electronic and thermal Enthalpies=

Sum of electronic and thermal Free Energies=
0.219238 (Hartree/Particle)

0.228726

0.229670

0.185383

$-275.988939$

$-275.979451$

$-275.978507$

$-276.022794$

Octane

Standard orientation:

\begin{tabular}{|c|c|c|c|c|c|}
\hline \multirow{2}{*}{$\begin{array}{l}\text { Center } \\
\text { Number }\end{array}$} & \multicolumn{2}{|c|}{ Atomic } & \multirow{2}{*}{$\begin{array}{l}\text { Atomic } \\
\text { Type }\end{array}$} & \multicolumn{2}{|c|}{ Coordinates (Angstroms } \\
\hline & & & & $\mathrm{X}$ & $\mathrm{Z}$ \\
\hline 1 & 6 & 0 & 4.434790 & -0.255071 & 0.000014 \\
\hline 2 & 1 & 0 & 4.497844 & -0.895983 & 0.877913 \\
\hline 3 & 1 & 0 & 4.497847 & -0.896036 & -0.877846 \\
\hline 4 & 1 & 0 & 5.307541 & 0.393812 & -0.000005 \\
\hline 5 & 6 & 0 & 3.145497 & 0.539023 & -0.000013 \\
\hline 6 & 1 & 0 & 3.117200 & 1.194770 & 0.871708 \\
\hline 7 & 1 & 0 & 3.117210 & 1.194727 & -0.871767 \\
\hline 8 & 6 & 0 & 1.912779 & -0.341103 & 0.000001 \\
\hline 9 & 1 & 0 & 1.939319 & -0.998315 & -0.872280 \\
\hline 10 & 1 & 0 & 1.939322 & -0.998291 & 0.872300 \\
\hline 11 & 6 & 0 & 0.615926 & 0.440346 & -0.000005 \\
\hline 12 & 1 & 0 & 0.590132 & 1.097313 & -0.872334 \\
\hline 13 & 1 & 0 & 0.590125 & 1.097316 & 0.872322 \\
\hline 14 & 6 & 0 & -0.615926 & -0.440346 & -0.000008 \\
\hline 15 & 1 & 0 & -0.590124 & -1.097324 & 0.872312 \\
\hline 16 & 1 & 0 & -0.590133 & -1.097304 & -0.872343 \\
\hline 17 & 6 & 0 & -1.912779 & 0.341103 & 0.000007 \\
\hline 18 & 1 & 0 & -1.939320 & 0.998319 & -0.872271 \\
\hline 19 & 1 & 0 & -1.939320 & 0.998287 & 0.872309 \\
\hline 20 & 6 & 0 & -3.145497 & -0.539023 & -0.000009 \\
\hline 21 & 1 & 0 & -3.117203 & -1.194766 & 0.871714 \\
\hline 22 & 1 & 0 & -3.117207 & -1.194730 & -0.871760 \\
\hline 23 & 6 & 0 & -4.434790 & 0.255071 & 0.000010 \\
\hline 24 & 1 & 0 & -4.497847 & 0.896028 & -0.877856 \\
\hline 25 & 1 & 0 & -5.307541 & -0.393812 & -0.000003 \\
\hline
\end{tabular}


Zero-point correction $=$

Thermal correction to Energy=

Thermal correction to Enthalpy=

Thermal correction to Gibbs Free Energy=

Sum of electronic and zero-point Energies=

Sum of electronic and thermal Energies=

Sum of electronic and thermal Enthalpies=

Sum of electronic and thermal Free Energies=
0.249493 (Hartree/Particle)

0.260524

0.261468

0.212473

$-315.244246$

$-315.233215$

$-315.232271$

$-315.281266$

2233tetramethylbutane

Standard orientation:

\begin{tabular}{|c|c|c|c|c|c|}
\hline \multirow{2}{*}{$\begin{array}{l}\text { Center } \\
\text { Number }\end{array}$} & Atomic & \multirow{2}{*}{\multicolumn{2}{|c|}{$\begin{array}{r}\text { Atomic } \\
\text { Type }\end{array}$}} & \multicolumn{2}{|c|}{ Coordinates (Angstroms) } \\
\hline & Numbe & & & $\mathrm{X}$ & Z \\
\hline 1 & 6 & 0 & -1.331392 & -0.831223 & -1.152983 \\
\hline 2 & 1 & 0 & -0.921816 & -0.523860 & -2.112760 \\
\hline 3 & 1 & 0 & -1.127127 & -1.890747 & -1.021281 \\
\hline 4 & 1 & 0 & -2.412161 & -0.710851 & -1.206154 \\
\hline 5 & 6 & 0 & -0.780420 & -0.000001 & -0.000075 \\
\hline 6 & 6 & 0 & 0.780477 & -0.000023 & -0.000094 \\
\hline 7 & 6 & 0 & 1.331049 & 0.556298 & 1.307963 \\
\hline 8 & 1 & 0 & 0.920459 & 1.536090 & 1.542011 \\
\hline 9 & 1 & 0 & 1.127361 & -0.105203 & 2.146134 \\
\hline 10 & 1 & 0 & 2.411711 & 0.665160 & 1.232456 \\
\hline 11 & 6 & 0 & -1.331081 & 1.414202 & -0.143262 \\
\hline 12 & 1 & 0 & -0.920353 & 2.091784 & 0.602147 \\
\hline 13 & 1 & 0 & -1.127800 & 1.829580 & -1.127025 \\
\hline 14 & 1 & 0 & -2.411705 & 1.400483 & -0.011198 \\
\hline 15 & 6 & 0 & -1.331003 & -0.582945 & 1.296369 \\
\hline 16 & 1 & 0 & -2.411675 & -0.690129 & 1.218724 \\
\hline 17 & 1 & 0 & -0.920514 & -1.567411 & 1.510286 \\
\hline 18 & 1 & 0 & -1.127291 & 0.061228 & 2.147927 \\
\hline 19 & 6 & 0 & 1.331202 & 0.854660 & -1.135800 \\
\hline 20 & 1 & 0 & 2.411958 & 0.735430 & -1.191669 \\
\hline 21 & 1 & 0 & 0.921371 & 0.566918 & -2.101500 \\
\hline 22 & 1 & 0 & 1.126841 & 1.911227 & -0.982387 \\
\hline 23 & 6 & 0 & 1.331243 & -1.410978 & -0.172242 \\
\hline 24 & 1 & 0 & 2.411837 & -1.399942 & -0.039651 \\
\hline 25 & 1 & 0 & 0.920297 & -2.103625 & 0.559015 \\
\hline 26 & 1 & 0 & 1.128157 & -1.806068 & -1.164331 \\
\hline Zero-poir & it correcti & $\mathrm{n}=$ & & 0.24803 & 1 (Hartree/Particle) \\
\hline Thermal & correctior & to & Energy= & 0.25 & 58348 \\
\hline Thermal & correctior & to & Enthalpy= & 0.2 & 59293 \\
\hline Thermal & correctior & to & Gibbs Free E & Energy= & 0.214831 \\
\hline Sum of e & lectronic & and & zero-point El & nergies $=$ & -315.246593 \\
\hline Sum of e & lectronic & and & thermal Ener & rgies $=$ & -315.236276 \\
\hline
\end{tabular}


Sum of electronic and thermal Enthalpies=

PBEQIDHD3BJ/ccpVQZ

Ethane

Standard orientation:

\begin{tabular}{cccccc} 
Center & \multicolumn{2}{c}{ Atomic } & \multicolumn{2}{c}{ Atomic } & \multicolumn{2}{c}{ Coordinates (Angstroms) } \\
Number & Number & Type & X & Y & Z \\
- \hdashline 1 & 6 & 0 & 0.000000 & 0.000000 & 0.757269 \\
2 & 1 & 0 & -0.505959 & 0.876369 & 1.152446 \\
3 & 1 & 0 & 1.011937 & -0.000011 & 1.152446 \\
4 & 1 & 0 & -0.505978 & -0.876358 & 1.152446 \\
5 & 6 & 0 & 0.000000 & 0.000000 & -0.757269 \\
6 & 1 & 0 & 0.505959 & 0.876369 & -1.152446 \\
7 & 1 & 0 & 0.505978 & -0.876358 & -1.152446 \\
8 & 1 & 0 & -1.011937 & -0.000011 & -1.152446
\end{tabular}

Zero-point correction=

Thermal correction to Energy=

Thermal correction to Enthalpy=

Thermal correction to Gibbs Free Energy=

Sum of electronic and zero-point Energies=

Sum of electronic and thermal Energies $=$

Sum of electronic and thermal Enthalpies=

Sum of electronic and thermal Free Energies=

Methane

Standard orientation:

\subsection{1 (Hartree/Particle)} 0.079563

0.080507

0.054713

$-79.678609$

$-79.675147$

$-79.674203$

$-79.699997$

\begin{tabular}{|c|c|c|c|c|}
\hline \multirow{2}{*}{$\begin{array}{l}\text { Center } \\
\text { Number }\end{array}$} & \multirow{2}{*}{$\begin{array}{l}\text { Atomic } \\
\text { Number }\end{array}$} & \multirow{2}{*}{$\begin{array}{l}\text { Atomic } \\
\text { Type }\end{array}$} & \multicolumn{2}{|c|}{ Coordinates (Angstro } \\
\hline & & & $X$ & $\mathrm{Z}$ \\
\hline 1 & 6 & 0.000000 & 0.000000 & 0.000000 \\
\hline 2 & 1 & 0.625582 & 0.625582 & 0.62558 \\
\hline 3 & 1 & -0.625582 & -0.625582 & 0.62558 \\
\hline 4 & 1 & -0.625582 & 0.625582 & -0.62558 \\
\hline 5 & 1 & 0.625582 & -0.625582 & -0.62558 \\
\hline
\end{tabular}

Zero-point correction=

Thermal correction to Energy=

Thermal correction to Enthalpy=

Thermal correction to Gibbs Free Energy=

Sum of electronic and zero-point Energies=

Sum of electronic and thermal Energies=

Sum of electronic and thermal Enthalpies=

Sum of electronic and thermal Free Energies=

Propane

Standard orientation:

$$
\begin{array}{cc}
0.045578 & \text { (Hartree/Particle) } \\
0.048444 \\
0.049388 \\
\text { rgy }= & 0.028280 \\
\text { gies }= & -40.432149 \\
\text { s }= & -40.429283 \\
\text { pies }= & -40.428338 \\
\text { Energies }= & -40.449447
\end{array}
$$

Center Atomic Atomic Coordinates (Angstroms) 


\begin{tabular}{|c|c|c|c|c|c|}
\hline Number & Number & & Type & $X$ & Z \\
\hline 1 & 6 & 0 & -1.257708 & -0.258294 & 0.000000 \\
\hline 2 & 1 & 0 & -2.154905 & 0.354516 & -0.000030 \\
\hline 3 & 1 & 0 & -1.293771 & -0.900585 & 0.877147 \\
\hline 4 & 1 & 0 & -1.293745 & -0.900632 & -0.877113 \\
\hline 5 & 6 & 0 & 0.000000 & 0.585594 & 0.000000 \\
\hline 6 & 1 & 0 & 0.000001 & 1.239692 & 0.870535 \\
\hline 7 & 1 & 0 & 0.000002 & 1.239679 & -0.870544 \\
\hline 8 & 6 & 0 & 1.257708 & -0.258294 & 0.000000 \\
\hline 9 & 1 & 0 & 1.293764 & -0.900593 & 0.877142 \\
\hline 10 & 1 & 0 & 1.293747 & -0.900624 & -0.877119 \\
\hline 11 & 1 & 0 & 2.154907 & 0.354513 & -0.000020 \\
\hline
\end{tabular}

Zero-point correction=

Thermal correction to Energy=

Thermal correction to Enthalpy=

Thermal correction to Gibbs Free Energy=

Sum of electronic and zero-point Energies=

Sum of electronic and thermal Energies $=$

Sum of electronic and thermal Enthalpies=

Sum of electronic and thermal Free Energies=

Butane

Standard orientation:

\begin{tabular}{|c|c|c|c|c|}
\hline \multirow{2}{*}{$\begin{array}{l}\text { Center } \\
\text { Number }\end{array}$} & Atomic & Atomic & \multicolumn{2}{|c|}{ Coordinates (Angstroms) } \\
\hline & Numbe & Type & $X$ & Z \\
\hline 1 & 6 & -1.934795 & -0.118562 & -0.000021 \\
\hline 2 & 1 & -2.074881 & -0.746174 & 0.877200 \\
\hline 3 & 1 & -2.074830 & -0.746197 & -0.877234 \\
\hline 4 & 1 & -2.721162 & 0.631113 & -0.000054 \\
\hline 5 & 6 & -0.558110 & 0.512137 & 0.000024 \\
\hline 6 & 1 & -0.449019 & 1.158585 & -0.871167 \\
\hline 7 & 1 & -0.449046 & 1.158562 & 0.871236 \\
\hline 8 & 6 & 0.558110 & -0.512137 & 0.000024 \\
\hline 9 & 1 & 0.449046 & -1.158559 & 0.871238 \\
\hline 10 & 1 & 0.449019 & -1.158587 & -0.871166 \\
\hline 11 & 6 & 1.934795 & 0.118562 & -0.000021 \\
\hline 12 & 1 & 2.074883 & 0.746172 & 0.877202 \\
\hline 13 & 1 & 2.721162 & -0.631113 & -0.000057 \\
\hline 14 & 1 & 2.074828 & 0.746199 & -0.877233 \\
\hline \multicolumn{3}{|c|}{ Zero-point correction= } & \multicolumn{2}{|c|}{0.134418 (Hartree/Particle) } \\
\hline \multicolumn{3}{|c|}{ Thermal correction to Energy= } & \multicolumn{2}{|c|}{0.140143} \\
\hline \multicolumn{3}{|c|}{ Thermal correction to Enthalpy= } & \multicolumn{2}{|c|}{0.141087} \\
\hline \multicolumn{5}{|c|}{ Thermal correction to Gibbs Free Energy= } \\
\hline \multicolumn{4}{|c|}{ Sum of electronic and zero-point Energies= } & -158.180591 \\
\hline \multicolumn{4}{|c|}{ Sum of electronic and thermal Energies $=$} & -158.174866 \\
\hline \multicolumn{4}{|c|}{ Sum of electronic and thermal Enthalpies $=$} & -158.173922 \\
\hline
\end{tabular}


Sum of electronic and thermal Free Energies=

Isobutane

Standard orientation:

\begin{tabular}{|c|c|c|c|c|}
\hline \multirow{2}{*}{$\begin{array}{l}\text { Center } \\
\text { Number }\end{array}$} & Atomic & \multirow{2}{*}{$\begin{array}{l}\text { Atomic } \\
\text { Type }\end{array}$} & \multicolumn{2}{|c|}{ Coordinates (Angstrom } \\
\hline & Numbe & & $\mathrm{X}$ & Z \\
\hline 1 & 6 & 1.413716 & -0.278811 & 0.095406 \\
\hline 2 & 1 & 2.106855 & 0.481249 & -0.256920 \\
\hline 3 & 1 & 1.455457 & -0.287117 & 1.183696 \\
\hline 4 & 1 & 1.766463 & -1.245048 & -0.257049 \\
\hline 5 & 6 & 0.000015 & -0.000003 & -0.376443 \\
\hline 6 & 1 & 0.000010 & 0.000006 & -1.467777 \\
\hline 7 & 6 & -0.465400 & 1.363698 & 0.095401 \\
\hline 8 & 1 & -1.470212 & 1.583933 & -0.256900 \\
\hline 9 & 1 & -0.479057 & 1.403987 & 1.183691 \\
\hline 10 & 1 & 0.194991 & 2.152320 & -0.257055 \\
\hline 11 & 6 & -0.948322 & -1.084887 & 0.095399 \\
\hline 12 & 1 & -1.961540 & -0.907075 & -0.256796 \\
\hline 13 & 1 & -0.636864 & -2.065176 & -0.257156 \\
\hline 14 & 1 & -0.976154 & -1.117060 & 1.183691 \\
\hline
\end{tabular}

Zero-point correction=

0.133914 (Hartree/Particle)

Thermal correction to Energy=

0.139553

Thermal correction to Enthalpy=

0.140497

Thermal correction to Gibbs Free Energy=

0.106374

Sum of electronic and zero-point Energies=

$-158.183150$

Sum of electronic and thermal Energies $=$

$-158.177511$

Sum of electronic and thermal Enthalpies=

$-158.176567$

Sum of electronic and thermal Free Energies= $-158.210691$

Pentane

Standard orientation:

\begin{tabular}{|c|c|c|c|c|}
\hline Center & Atomic & Atomic & Coordinate & (Angstro \\
\hline Number & Numbe & Type & $X$ & Z \\
\hline 1 & 6 & -2.522343 & 0.323711 & 0.000001 \\
\hline 2 & 1 & -2.558999 & 0.965642 & 0.877195 \\
\hline 3 & 1 & -2.558996 & 0.965649 & -0.877189 \\
\hline 4 & 1 & -3.419282 & -0.289333 & -0.000003 \\
\hline 5 & 6 & -1.265854 & -0.521387 & -0.000001 \\
\hline 6 & 1 & -1.263473 & -1.176462 & 0.871351 \\
\hline 7 & 1 & -1.263474 & -1.176460 & -0.871355 \\
\hline 8 & 6 & -0.000000 & 0.310055 & -0.000000 \\
\hline 9 & 1 & 0.000001 & 0.966852 & -0.871954 \\
\hline 10 & 1 & -0.000001 & 0.966854 & 0.871953 \\
\hline 11 & 6 & 1.265854 & -0.521387 & 0.000001 \\
\hline 12 & 1 & 1.263474 & -1.176463 & -0.871351 \\
\hline 13 & 1 & 1.263474 & -1.176460 & 0.871355 \\
\hline 14 & 6 & 2.522343 & 0.323711 & -0.000000 \\
\hline
\end{tabular}




$\begin{array}{rrrrrr}15 & 1 & 0 & 3.419282 & -0.289333 & 0.000001 \\ 16 & 1 & 0 & 2.558998 & 0.965644 & -0.877193 \\ 17 & 1 & 0 & 2.558997 & 0.965647 & 0.877190\end{array}$

Zero-point correction=

Thermal correction to Energy=

Thermal correction to Enthalpy=

Thermal correction to Gibbs Free Energy=

Sum of electronic and zero-point Energies=

Sum of electronic and thermal Energies $=$

Sum of electronic and thermal Enthalpies=

Sum of electronic and thermal Free Energies=

Neopentane

Standard orientation:

\begin{tabular}{|c|c|c|c|c|}
\hline \multirow{2}{*}{$\begin{array}{l}\text { Center } \\
\text { Number }\end{array}$} & Atomic & \multirow{2}{*}{$\begin{array}{r}\text { Atomic } \\
\text { Type }\end{array}$} & \multicolumn{2}{|c|}{ Coordinates (Angstroms } \\
\hline & Number & & $X$ & Z \\
\hline 1 & 6 & -1.471267 & 0.032237 & -0.380223 \\
\hline 2 & 1 & -1.904142 & -0.965714 & -0.344261 \\
\hline 3 & 1 & -2.034949 & 0.667522 & 0.300400 \\
\hline 4 & 1 & -1.603865 & 0.419656 & -1.388634 \\
\hline 5 & 6 & -0.000014 & 0.000008 & 0.000004 \\
\hline 6 & 6 & 0.146759 & -0.545089 & 1.411219 \\
\hline 7 & 1 & 1.193317 & -0.578889 & 1.708107 \\
\hline 8 & 1 & -0.385226 & 0.079337 & 2.126521 \\
\hline 9 & 1 & -0.255175 & -1.554049 & 1.482090 \\
\hline 10 & 6 & 0.754422 & -0.894796 & -0.969737 \\
\hline 11 & 1 & 0.664823 & -0.525558 & -1.989644 \\
\hline 12 & 1 & 1.812746 & -0.934881 & -0.718898 \\
\hline 13 & 1 & 0.364721 & -1.910717 & -0.944933 \\
\hline 14 & 6 & 0.570089 & 1.407646 & -0.061256 \\
\hline 15 & 1 & 0.476590 & 1.821760 & -1.063427 \\
\hline 16 & 1 & 0.046292 & 2.069552 & 0.625832 \\
\hline 17 & 1 & 1.624934 & 1.411944 & 0.206808 \\
\hline
\end{tabular}

Zero-point correction=

Thermal correction to Energy=

Thermal correction to Enthalpy=

Thermal correction to Gibbs Free Energy=

Sum of electronic and zero-point Energies=

Sum of electronic and thermal Energies=

Sum of electronic and thermal Enthalpies=

Sum of electronic and thermal Free Energies=

Hexane

Standard orientation:
0.170474

0.171419

0.133218

$-197.431704$

$-197.424688$

$-197.423744$

$-197.461944$ 


$\begin{array}{rrrrrr}1 & 6 & 0 & -3.177285 & 0.207146 & 0.000006 \\ 2 & 1 & 0 & -3.258857 & 0.844928 & 0.877214 \\ 3 & 1 & 0 & -3.258859 & 0.844952 & -0.877184 \\ 4 & 1 & 0 & -4.029058 & -0.467271 & -0.000003 \\ 5 & 6 & 0 & -1.864643 & -0.547766 & -0.000007 \\ 6 & 1 & 0 & -1.816271 & -1.201072 & 0.871315 \\ 7 & 1 & 0 & -1.816277 & -1.201052 & -0.871343 \\ 8 & 6 & 0 & -0.660118 & 0.370605 & -0.000001 \\ 9 & 1 & 0 & -0.707000 & 1.025457 & -0.872017 \\ 10 & 1 & 0 & -0.707003 & 1.025451 & 0.872019 \\ 11 & 6 & 0 & 0.660118 & -0.370605 & 0.000000 \\ 12 & 1 & 0 & 0.707000 & -1.025458 & -0.872015 \\ 13 & 1 & 0 & 0.707003 & -1.025450 & 0.872021 \\ 14 & 6 & 0 & 1.864643 & 0.547766 & -0.000006 \\ 15 & 1 & 0 & 1.816272 & 1.201072 & 0.871316 \\ 16 & 1 & 0 & 1.816277 & 1.201053 & -0.871342 \\ 17 & 6 & 0 & 3.177285 & -0.207146 & 0.000006 \\ 18 & 1 & 0 & 3.258858 & -0.844954 & -0.877183 \\ 19 & 1 & 0 & 4.029058 & 0.467271 & -0.000005 \\ 20 & 1 & 0 & 3.258858 & -0.844927 & 0.877214\end{array}$

Zero-point correction=

Thermal correction to Energy=

Thermal correction to Enthalpy=

Thermal correction to Gibbs Free Energy=

Sum of electronic and zero-point Energies=

Sum of electronic and thermal Energies $=$

Sum of electronic and thermal Enthalpies=

Sum of electronic and thermal Free Energies=

22dimethylbutane

Standard orientation:

\begin{tabular}{|c|c|c|c|c|}
\hline \multirow{2}{*}{$\begin{array}{l}\text { Center } \\
\text { Number }\end{array}$} & Atomic & \multirow{2}{*}{$\begin{array}{l}\text { Atomic } \\
\text { Type }\end{array}$} & \multicolumn{2}{|c|}{ Coordinates (Angstroms } \\
\hline & Numbe & & $\mathrm{X}$ & Z \\
\hline 1 & 6 & 1.603341 & 0.965504 & -0.001108 \\
\hline 2 & 1 & 1.580800 & 1.604474 & -0.881825 \\
\hline 3 & 1 & 1.580948 & 1.606285 & 0.878296 \\
\hline 4 & 1 & 2.549515 & 0.428132 & -0.000631 \\
\hline 5 & 6 & 0.429099 & -0.000215 & -0.000010 \\
\hline 6 & 6 & -0.859033 & 0.823529 & -0.000583 \\
\hline 7 & 1 & -0.839495 & 1.479809 & 0.870762 \\
\hline 8 & 1 & -0.839556 & 1.478531 & -0.872895 \\
\hline 9 & 6 & -2.154713 & 0.037501 & -0.000005 \\
\hline 10 & 1 & -2.242557 & -0.595388 & 0.878616 \\
\hline 11 & 1 & -3.007265 & 0.711134 & -0.000223 \\
\hline 12 & 1 & -2.242766 & -0.596240 & o -0.877987 \\
\hline 13 & 6 & 0.510064 & -0.874944 & +-1.240853 \\
\hline 14 & 1 & -0.279587 & -1.621470 & o -1.259768 \\
\hline 15 & 1 & 0.430138 & -0.275400 & -2.145923 \\
\hline
\end{tabular}




$\begin{array}{rrrrrr}16 & 1 & 0 & 1.461170 & -1.402526 & -1.272957 \\ 17 & 6 & 0 & 0.510471 & -0.872542 & 1.242502 \\ 18 & 1 & 0 & 1.461458 & -1.400306 & 1.275151 \\ 19 & 1 & 0 & 0.431166 & -0.271197 & 2.146429 \\ 20 & 1 & 0 & -0.279348 & -1.618842 & 1.263302\end{array}$

Zero-point correction=

Thermal correction to Energy=

Thermal correction to Enthalpy=

Thermal correction to Gibbs Free Energy=

Sum of electronic and zero-point Energies=

Sum of electronic and thermal Energies $=$

Sum of electronic and thermal Enthalpies=

Sum of electronic and thermal Free Energies=

Heptane

Standard orientation:

\begin{tabular}{|c|c|c|c|c|}
\hline \multirow{2}{*}{$\begin{array}{l}\text { Center } \\
\text { Number }\end{array}$} & Atomic & \multirow{2}{*}{$\begin{array}{r}\text { Atomic } \\
\text { Type }\end{array}$} & \multicolumn{2}{|c|}{ Coordinates (Angstrom } \\
\hline & Number & & $X$ & $\mathrm{Z}$ \\
\hline 1 & 6 & -3.787497 & -0.352900 & -0.000009 \\
\hline 2 & 1 & -3.824098 & -0.994839 & -0.877219 \\
\hline 3 & 1 & -3.824099 & -0.994876 & 0.877174 \\
\hline 4 & 1 & -4.684518 & 0.260060 & 0.000005 \\
\hline 5 & 6 & -2.531043 & 0.492236 & 0.000011 \\
\hline 6 & 1 & -2.528579 & 1.147327 & -0.871320 \\
\hline 7 & 1 & -2.528585 & 1.147295 & 0.871365 \\
\hline 8 & 6 & -1.265100 & -0.339413 & 0.000000 \\
\hline 9 & 1 & -1.265875 & -0.995943 & 0.871994 \\
\hline 10 & 1 & -1.265880 & -0.995931 & -0.872003 \\
\hline 11 & 6 & 0.000000 & 0.492781 & 0.000001 \\
\hline 12 & 1 & -0.000002 & 1.149030 & 0.872086 \\
\hline 13 & 1 & 0.000002 & 1.149026 & -0.872087 \\
\hline 14 & 6 & 1.265100 & -0.339413 & 0.000006 \\
\hline 15 & 1 & 1.265873 & -0.995949 & -0.871984 \\
\hline 16 & 1 & 1.265882 & -0.995925 & 0.872013 \\
\hline 17 & 6 & 2.531043 & 0.492236 & -0.000012 \\
\hline 18 & 1 & 2.528583 & 1.147328 & 0.871318 \\
\hline 19 & 1 & 2.528581 & 1.147294 & -0.871367 \\
\hline 20 & 6 & 3.787497 & -0.352900 & 0.000003 \\
\hline 21 & 1 & 3.824096 & -0.994876 & -0.877180 \\
\hline 22 & 1 & 4.684518 & 0.260060 & -0.000013 \\
\hline 23 & 1 & 3.824101 & -0.994838 & 0.877213 \\
\hline
\end{tabular}

Zero-point correction=

Thermal correction to Energy=

Thermal correction to Enthalpy=

Thermal correction to Gibbs Free Energy=

Sum of electronic and zero-point Energies=
0.221428 (Hartree/Particle)

0.231099

0.232043

0.186670

$-275.934096$ 
Sum of electronic and thermal Energies $=$

$-275.924425$

Sum of electronic and thermal Enthalpies=

Sum of electronic and thermal Free Energies=

223trimethylbutane

Standard orientation:

\begin{tabular}{cccccc} 
Center & \multicolumn{1}{c}{ Atomic } & \multicolumn{2}{c}{ Atomic } & \multicolumn{3}{c}{ Coordinates (Angstroms) } \\
Number & Number & Type & X & Y & Z \\
------------- \\
1 & 6 & 0 & 1.316400 & 1.278243 & -0.468494 \\
2 & 1 & 0 & 1.174160 & 1.428635 & -1.537602 \\
3 & 1 & 0 & 0.937661 & 2.154577 & 0.049472 \\
4 & 1 & 0 & 2.386360 & 1.223440 & -0.279330 \\
5 & 6 & 0 & 0.634153 & -0.000133 & -0.000772 \\
6 & 6 & 0 & -0.841035 & 0.000745 & -0.452950 \\
7 & 1 & 0 & -0.811699 & 0.004559 & -1.546013 \\
8 & 6 & 0 & -1.609774 & -1.236770 & -0.020555 \\
9 & 1 & 0 & -1.728465 & -1.267409 & 1.060250 \\
10 & 1 & 0 & -2.607128 & -1.224449 & -0.453181 \\
11 & 1 & 0 & -1.130536 & -2.158858 & -0.333491 \\
12 & 6 & 0 & -1.612582 & 1.235282 & -0.016808 \\
13 & 1 & 0 & -1.656463 & 1.312207 & 1.067543 \\
14 & 1 & 0 & -1.182340 & 2.153356 & -0.403255 \\
15 & 1 & 0 & -2.637176 & 1.178361 & -0.376254 \\
16 & 6 & 0 & 0.770422 & -0.103681 & 1.510848 \\
17 & 1 & 0 & 0.364506 & -1.038335 & 1.888833 \\
18 & 1 & 0 & 1.821142 & -0.066291 & 1.791448 \\
19 & 1 & 0 & 0.267932 & 0.715624 & 2.018919 \\
20 & 6 & 0 & 1.363356 & -1.174060 & -0.640227 \\
21 & 1 & 0 & 1.267242 & -1.148166 & -1.724389 \\
22 & 1 & 0 & 2.423533 & -1.134722 & -0.398945 \\
23 & 1 & 0 & 0.985628 & -2.130281 & -0.290263 \\
--------------------------------------------------------
\end{tabular}

Zero-point correction=

Thermal correction to Energy=

0.219958 (Hartree/Particle)

0.229407

Thermal correction to Enthalpy=

0.230351

Thermal correction to Gibbs Free Energy=

0.186486

Sum of electronic and zero-point Energies=

$-275.937309$

Sum of electronic and thermal Energies $=$

$-275.927860$

Sum of electronic and thermal Enthalpies=

$-275.926916$

Sum of electronic and thermal Free Energies $=\quad-275.970781$

Octane

Standard orientation:

\begin{tabular}{cccccc} 
Center & \multicolumn{2}{c}{ Atomic } & Atomic & \multicolumn{3}{c}{ Coordinates (Angstroms) } \\
Number & Number & Type & X & Y & Z \\
-1 & 6 & 0 & -4.433562 & 0.255470 & 0.000015 \\
2 & 1 & 0 & -4.495378 & 0.895473 & 0.877229
\end{tabular}




\begin{tabular}{|c|c|c|c|c|c|}
\hline 3 & 1 & 0 & -4.495382 & 0.895525 & -0.877161 \\
\hline 4 & 1 & 0 & -5.305805 & -0.392271 & -0.000004 \\
\hline 5 & 6 & 0 & -3.144856 & -0.539618 & -0.000014 \\
\hline 6 & 1 & 0 & -3.116647 & -1.194116 & 0.871311 \\
\hline 7 & 1 & 0 & -3.116656 & -1.194072 & -0.871372 \\
\hline 8 & 6 & 0 & -1.912560 & 0.341119 & 0.000001 \\
\hline 9 & 1 & 0 & -1.939109 & 0.997119 & -0.871999 \\
\hline 10 & 1 & 0 & -1.939112 & 0.997097 & 0.872017 \\
\hline 11 & 6 & 0 & -0.615761 & -0.440732 & -0.000004 \\
\hline 12 & 1 & 0 & -0.589912 & -1.096475 & -0.872067 \\
\hline 13 & 1 & 0 & -0.589906 & -1.096478 & 0.872056 \\
\hline 14 & 6 & 0 & 0.615761 & 0.440732 & -0.000008 \\
\hline 15 & 1 & 0 & 0.589905 & 1.096487 & 0.872046 \\
\hline 16 & 1 & 0 & 0.589913 & 1.096467 & -0.872076 \\
\hline 17 & 6 & 0 & 1.912560 & -0.341119 & 0.000007 \\
\hline 18 & 1 & 0 & 1.939110 & -0.997125 & -0.871988 \\
\hline 19 & 1 & 0 & 1.939110 & -0.997091 & 0.872028 \\
\hline 20 & 6 & 0 & 3.144856 & 0.539618 & -0.000009 \\
\hline 21 & 1 & 0 & 3.116650 & 1.194113 & 0.871318 \\
\hline 22 & 1 & 0 & 3.116653 & 1.194076 & -0.871365 \\
\hline 23 & 6 & 0 & 4.433561 & -0.255470 & 0.000010 \\
\hline 24 & 1 & 0 & 4.495381 & -0.895518 & -0.877171 \\
\hline 25 & 1 & 0 & 5.305805 & 0.392271 & -0.000003 \\
\hline 26 & 1 & 0 & 4.495379 & -0.895480 & 0.877219 \\
\hline
\end{tabular}

Zero-point correction=

Thermal correction to Energy=

0.250397 (Hartree/Particle)

0.261411

Thermal correction to Enthalpy=

0.262355

Thermal correction to Gibbs Free Energy $=0.213397$

Sum of electronic and zero-point Energies=

$-315.185311$

Sum of electronic and thermal Energies=

$-315.174297$

Sum of electronic and thermal Enthalpies=

$-315.173353$

Sum of electronic and thermal Free Energies $=\quad-315.222311$ 2233tetramethylbutane

Standard orientation:

\begin{tabular}{|c|c|c|c|c|}
\hline \multirow{2}{*}{$\begin{array}{l}\text { Center } \\
\text { Number }\end{array}$} & Atomic & \multirow{2}{*}{$\begin{array}{l}\text { Atomic } \\
\text { Type }\end{array}$} & \multicolumn{2}{|c|}{ Coordinates (Angstroms } \\
\hline & Number & & $\mathrm{X}$ & Z \\
\hline 1 & 6 & 1.331323 & -1.116347 & 0.879637 \\
\hline 2 & 1 & 1.129804 & -0.938037 & 1.932139 \\
\hline 3 & 1 & 0.919965 & -2.087350 & 0.615437 \\
\hline 4 & 1 & 2.411017 & -1.175758 & 0.758894 \\
\hline 5 & 6 & 0.780162 & 0.000007 & 0.000086 \\
\hline 6 & 6 & -0.780134 & 0.000071 & 0.000032 \\
\hline 7 & 6 & -1.331206 & 1.286151 & -0.604871 \\
\hline 8 & 1 & -1.129993 & 2.147445 & 0.025860 \\
\hline 9 & 1 & -0.919340 & 1.484613 & -1.591185 \\
\hline 10 & 1 & -2.410850 & 1.204701 & -0.712544 \\
\hline 11 & 6 & 1.331640 & 1.319830 & 0.526871 \\
\hline
\end{tabular}




$\begin{array}{rrrrrr}12 & 1 & 0 & 1.129781 & 2.142202 & -0.153653 \\ 13 & 1 & 0 & 0.920907 & 1.576528 & 1.500156 \\ 14 & 1 & 0 & 2.411403 & 1.244864 & 0.638054 \\ 15 & 6 & 0 & 1.331299 & -0.203778 & -1.406466 \\ 16 & 1 & 0 & 2.411035 & -0.069823 & -1.397496 \\ 17 & 1 & 0 & 1.129475 & -1.204428 & -1.778110 \\ 18 & 1 & 0 & 0.920141 & 0.510475 & -2.115459 \\ 19 & 6 & 0 & -1.331619 & -0.119128 & 1.416087 \\ 20 & 1 & 0 & -2.411352 & 0.014112 & 1.398756 \\ 21 & 1 & 0 & -1.130006 & -1.095676 & 1.847208 \\ 22 & 1 & 0 & -0.920720 & 0.636442 & 2.081026 \\ 23 & 6 & 0 & -1.331450 & -1.166754 & -0.811318 \\ 24 & 1 & 0 & -2.411224 & -1.218291 & -0.687671 \\ 25 & 1 & 0 & -1.129445 & -1.051972 & -1.872524 \\ 26 & 1 & 0 & -0.920685 & -2.120359 & -0.489234\end{array}$

Zero-point correction=

0.248785 (Hartree/Particle)

Thermal correction to Energy=

Thermal correction to Enthalpy= $\quad 0.260016$

0.259072

Thermal correction to Gibbs Free Energy=

Sum of electronic and zero-point Energies $=$

Sum of electronic and thermal Energies=

0.215629

$-315.187638$

$-315.177351$

Sum of electronic and thermal Enthalpies=

$-315.176407$

Sum of electronic and thermal Free Energies=

$-315.220793$

S2: For the alkane system absolute energies:

DHSVPD basis set:

M06L/DHSVPD

2233tetra.out: SCF Done: $\mathrm{E}(\mathrm{RM} 06 \mathrm{~L})=-315.458510161$

223tri.out: SCF Done: E(RM06L $)=-276.176682279$

22dime.out: SCF Done: $\mathrm{E}(\mathrm{RM} 06 \mathrm{~L})=-236.894755637$

butane.out: SCF Done: $\mathrm{E}(\mathrm{RM} 06 \mathrm{~L})=-158.326972972$

ethane.out: SCF Done: $\mathrm{E}(\mathrm{RM} 06 \mathrm{~L})=-79.7621683825$

heptane.out: SCF Done: $\mathrm{E}(\mathrm{RM} 06 \mathrm{~L})=-276.174083742$

hexane.out: SCF Done: $\mathrm{E}(\mathrm{RM} 06 \mathrm{~L})=-236.891702210$

isobutane.out: SCF Done: $\mathrm{E}(\mathrm{RM} 06 \mathrm{~L})=-158.328562452$

Methane.out: SCF Done: $\mathrm{E}(\mathrm{RM} 06 \mathrm{~L})=-40.4823102112$

neopentane.out: SCF Done: $\mathrm{E}(\mathrm{RM} 06 \mathrm{~L})=-197.613093781$

octane.out: SCF Done: $\mathrm{E}(\mathrm{RM} 06 \mathrm{~L})=-315.456476281$

pentane.out: SCF Done: $\mathrm{E}(\mathrm{RM} 06 \mathrm{~L})=-197.609318901$

Propane.out: SCF Done: E(RM06L $)=-119.044530403$

TPSSh/DHSVPD

2233tetra.out: SCF Done: E(RTPSSh $)=-315.533338193$ 
223tri.out: SCF Done: E(RTPSSh) $=-276.245174274$

22dime.out: SCF Done: E(RTPSSh) $=-236.955922117$

butane.out: SCF Done: E(RTPSSh $)=-158.371902939$

ethane.out: SCF Done: E(RTPSSh $)=-79.7868868981$

heptane.out: SCF Done: E(RTPSSh $)=-276.249154154$

hexane.out: SCF Done: E(RTPSSh) $=-236.956720359$

isobutane.out: SCF Done: E(RTPSSh) $=-158.372962349$

Methane.out: SCF Done: E(RTPSSh $)=-40.4963862851$

neopentane.out: SCF Done: E(RTPSSh) $=-197.666056752$

octane.out: SCF Done: E(RTPSSh) $=-315.541586830$

pentane.out: SCF Done: E(RTPSSh $)=-197.664300789$

Propane.out: SCF Done: E(RTPSSh) $=-119.079463254$

\section{B3LYP/DHSVPD}

2233tetra.out: SCF Done: E(RB3LYP) $=-315.508149351$

223tri.out: SCF Done: $\mathrm{E}(\mathrm{RB} 3 \mathrm{LYP})=-276.224077654$

22dime.out: SCF Done: $E($ RB3LYP $)=-236.938456781$

butane.out: SCF Done: $\mathrm{E}(\mathrm{RB} 3 \mathrm{LYP})=-158.360931014$

ethane.out: SCF Done: $\mathrm{E}(\mathrm{RB} 3 \mathrm{LYP})=-79.7822892981$

heptane.out: SCF Done: $\mathrm{E}(\mathrm{RB} 3 \mathrm{LYP})=-276.228559176$

hexane.out: SCF Done: $\mathrm{E}(\mathrm{RB} 3 \mathrm{LYP})=-236.939328186$

isobutane.out: SCF Done: $\mathrm{E}(\mathrm{RB} 3 \mathrm{LYP})=-158.362160293$

Methane.out: SCF Done: E(RB3LYP $)=-40.4952526147$

neopentane.out: SCF Done: $\mathrm{E}(\mathrm{RB} 3 \mathrm{LYP})=-197.652107672$

octane.out: SCF Done: $\mathrm{E}(\mathrm{RB} 3 \mathrm{LYP})=-315.517791462$

pentane.out: SCF Done: $\mathrm{E}(\mathrm{RB} 3 \mathrm{LYP})=-197.650113685$

Propane.out: SCF Done: E(RB3LYP) $=-119.071686550$

\section{PBE0/DHSVPD}

2233tetra.out: SCF Done: E(RPBE1PBE) $=-315.108576685$

223tri.out: SCF Done: $\mathrm{E}(\mathrm{RPBE} 1 \mathrm{PBE})=-275.871858599$

22dime.out: SCF Done: $\mathrm{E}(\mathrm{RPBE} 1 \mathrm{PBE})=-236.634129818$

butane.out: SCF Done: $\mathrm{E}(\mathrm{RPBE} 1 \mathrm{PBE})=-158.152705985$

ethane.out: SCF Done: $\mathrm{E}(\mathrm{RPBE} 1 \mathrm{PBE})=-79.6715632187$

heptane.out: SCF Done: E(RPBE1PBE $)=-275.874142839$

hexane.out: SCF Done: $\mathrm{E}(\mathrm{RPBE} 1 \mathrm{PBE})=-236.633643601$

isobutane.out: SCF Done: $\mathrm{E}(\mathrm{RPBE} 1 \mathrm{PBE})=-158.154192003$

Methane.out: SCF Done: $\mathrm{E}(\mathrm{RPBE} 1 \mathrm{PBE})=-40.4335679107$

neopentane.out: SCF Done: $\mathrm{E}(\mathrm{RPBE} 1 \mathrm{PBE})=-197.396045905$

octane.out: SCF Done: $\mathrm{E}(\mathrm{RPBE} 1 \mathrm{PBE})=-315.114641845$

pentane.out: SCF Done: $\mathrm{E}(\mathrm{RPBE} 1 \mathrm{PBE})=-197.393160376$

Propane.out: SCF Done: E(RPBE1PBE $)=-118.912188297$

\section{M06/DHSVPD}

2233tetra.out: SCF Done: $\mathrm{E}(\mathrm{RM} 06)=-315.251922086$

223tri.out: SCF Done: $\mathrm{E}(\mathrm{RM} 06)=-275.994920611$

22dime.out: SCF Done: $\mathrm{E}(\mathrm{RM} 06)=-236.738385111$

butane.out: SCF Done: $\mathrm{E}(\mathrm{RM} 06)=-158.219242395$

ethane.out: SCF Done: $\mathrm{E}(\mathrm{RM} 06)=-79.7055583015$

heptane.out: SCF Done: $\mathrm{E}(\mathrm{RM} 06)=-275.989614562$ 
hexane.out: SCF Done: $\mathrm{E}(\mathrm{RM} 06)=-236.732799231$

isobutane.out: SCF Done: $\mathrm{E}(\mathrm{RM} 06)=-158.221772093$

Methane.out: SCF Done: E(RM06) $=-40.4518741408$

neopentane.out: SCF Done: $\mathrm{E}(\mathrm{RM} 06)=-197.482655905$

octane.out: SCF Done: $\mathrm{E}(\mathrm{RM} 06)=-315.246435331$

pentane.out: SCF Done: $\mathrm{E}(\mathrm{RM} 06)=-197.476001305$

Propane.out: SCF Done: E(RM06) $=-118.962395928$

\section{CAM-B3LYP/DHSVPD}

2233tetra.out: SCF Done: E(RCAM-B3LYP $)=-315.303109588$

223tri.out: SCF Done: E(RCAM-B3LYP $)=-276.042473969$

22dime.out: SCF Done: $\mathrm{E}(\mathrm{RCAM}-\mathrm{B} 3 \mathrm{LYP})=-236.780937381$

butane.out: SCF Done: E(RCAM-B3LYP) $=-158.251536721$

ethane.out: SCF Done: E(RCAM-B3LYP) $=-79.7227524405$

heptane.out: SCF Done: E(RCAM-B3LYP $)=-276.044409378$

hexane.out: SCF Done: $E($ RCAM-B3LYP $)=-236.780094820$

isobutane.out: SCF Done: $\mathrm{E}(\mathrm{RCAM}-\mathrm{B} 3 \mathrm{LYP})=-158.253209026$

Methane.out: SCF Done: E(RCAM-B3LYP $)=-40.4610911216$

neopentane.out: SCF Done: E(RCAM-B3LYP) $=-197.519177772$

octane.out: SCF Done: E(RCAM-B3LYP $)=-315.308723990$

pentane.out: SCF Done: $\mathrm{E}(\mathrm{RCAM}-\mathrm{B} 3 \mathrm{LYP})=-197.515799682$

Propane.out: SCF Done: E(RCAM-B3LYP $)=-118.987212096$

WB97XD/DH-SVPD:

2233tetra.out: SCF Done: $\mathrm{E}(\mathrm{RwB} 97 \mathrm{XD})=-315.425212651$

223tri.out: SCF Done: $\mathrm{E}(\mathrm{RwB} 97 \mathrm{XD})=-276.147916741$

22dime.out: SCF Done: $\mathrm{E}(\mathrm{RwB} 97 \mathrm{XD})=-236.870457798$

butane.out: SCF Done: $\mathrm{E}(\mathrm{RwB} 97 \mathrm{XD})=-158.310333251$

ethane.out: SCF Done: $\mathrm{E}(\mathrm{RwB} 97 \mathrm{XD})=-79.7535308766$

heptane.out: SCF Done: $E($ RwB97XD $)=-276.145750974$

hexane.out: SCF Done: $E(R w B 97 X D)=-236.867247052$

isobutane.out: SCF Done: $\mathrm{E}(\mathrm{RwB} 97 \mathrm{XD})=-158.312324330$

Methane.out: SCF Done: $\mathrm{E}(\mathrm{RwB} 97 \mathrm{XD})=-40.4783463973$

neopentane.out: SCF Done: $\mathrm{E}(\mathrm{RwB} 97 \mathrm{XD})=-197.593327500$

octane.out: SCF Done: $\mathrm{E}(\mathrm{RwB} 97 \mathrm{XD})=-315.424252967$

pentane.out: SCF Done: $E($ RwB97XD $)=-197.588774394$

Propane.out: SCF Done: E(RwB97XD) $=-119.031908531$

B3LYPD3/DHSVPD

2233tetra.out: SCF Done: E(RB3LYP $)=-315.534921796$

223tri.out: SCF Done: E(RB3LYP $)=-276.244915206$

22dime.out: SCF Done: $E($ RB3LYP $)=-236.954571931$

butane.out: SCF Done: $\mathrm{E}(\mathrm{RB} 3 \mathrm{LYP})=-158.367947807$

ethane.out: SCF Done: $\mathrm{E}(\mathrm{RB} 3 \mathrm{LYP})=-79.7840920181$

heptane.out: SCF Done: $\mathrm{E}(\mathrm{RB} 3 \mathrm{LYP})=-276.243799374$

hexane.out: SCF Done: $E(R B 3 L Y P)=-236.951819524$

isobutane.out: SCF Done: $\mathrm{E}(\mathrm{RB} 3 \mathrm{LYP})=-158.369996696$

Methane.out: SCF Done: E(RB3LYP $)=-40.4954081689$

neopentane.out: SCF Done: $\mathrm{E}(\mathrm{RB} 3 \mathrm{LYP})=-197.664449924$

octane.out: SCF Done: $\mathrm{E}(\mathrm{RB} 3 \mathrm{LYP})=-315.535781794$

pentane.out: SCF Done: $\mathrm{E}(\mathrm{RB} 3 \mathrm{LYP})=-197.659861643$

Propane.out: SCF Done: E(RB3LYP) $=-119.076023972$ 
B2PLYP/DHSVPD

2,2,3,3-tetramethylbutane.out: E2(B2PLYP) $=-0.4156483837 \mathrm{D}+00 \mathrm{E}(\mathrm{B} 2 \mathrm{PLYP})=$

$0.31512994776395 \mathrm{D}+03$

2,2,3-trimethylbutane.out: E2(B2PLYP $)=-0.3620866485 \mathrm{D}+00 \mathrm{E}(\mathrm{B} 2 \mathrm{PLYP})=-$

$0.27588959583474 \mathrm{D}+03$

2,2-dimethylbutane.out: E2(B2PLYP $)=-0.3098157221 \mathrm{D}+00 \mathrm{E}(\mathrm{B} 2 \mathrm{PLYP})=$

$0.23664889952164 \mathrm{D}+03$

butane.out: $\mathrm{E} 2(\mathrm{~B} 2 \mathrm{PLYP})=-0.2057823258 \mathrm{D}+00 \mathrm{E}(\mathrm{B} 2 \mathrm{PLYP})=-0.15816129107760 \mathrm{D}+03$

Ethane.out: E2(B2PLYP $)=-0.1056356664 \mathrm{D}+00 \mathrm{E}(\mathrm{B} 2 \mathrm{PLYP})=-0.79675758012117 \mathrm{D}+02$

heptane.out: E2(B2PLYP) $=-0.3563092175 \mathrm{D}+00 \mathrm{E}(\mathrm{B} 2 \mathrm{PLYP})=$

$0.27588960814815 \mathrm{D}+03$

hexane.out: E2(B2PLYP $)=-0.3061319179 \mathrm{D}+00 \mathrm{E}(\mathrm{B} 2 \mathrm{PLYP})=-0.23664681237314 \mathrm{D}+03$

isobutane.out: $\mathrm{E} 2(\mathrm{~B} 2 \mathrm{PLYP})=-0.2066777135 \mathrm{D}+00 \mathrm{E}(\mathrm{B} 2 \mathrm{PLYP})=\quad-$

$0.15816323587746 \mathrm{D}+03$

Methane.out: E2(B2PLYP) $=-0.5650899078 \mathrm{D}-01 \mathrm{E}(\mathrm{B} 2 \mathrm{PLYP})=\quad-$

$0.40436050651881 \mathrm{D}+02$

neopentane.out: $\mathrm{E} 2(\mathrm{~B} 2 \mathrm{PLYP})=-0.2587744064 \mathrm{D}+00 \mathrm{E}(\mathrm{B} 2 \mathrm{PLYP})=\quad-$

$0.19740840610188 \mathrm{D}+03$

octane.out: E2(B2PLYP) $=-0.4065000730 \mathrm{D}+00 \mathrm{E}(\mathrm{B} 2 \mathrm{PLYP})=-0.31513240327070 \mathrm{D}+03$

pentane.out: $\mathrm{E} 2(\mathrm{~B} 2 \mathrm{PLYP})=-0.2559517557 \mathrm{D}+00 \mathrm{E}(\mathrm{B} 2 \mathrm{PLYP})=-$

$0.19740403506752 \mathrm{D}+03$

Propane.out: E2(B2PLYP $)=-0.1556604770 \mathrm{D}+00 \mathrm{E}(\mathrm{B} 2 \mathrm{PLYP})=$

$0.11891854276324 \mathrm{D}+03$

PBE0DH/DHSVPD

2233tetra.out: $\mathrm{E} 2(\mathrm{PBE} 0 \mathrm{DH})=-0.1947696123 \mathrm{D}+00 \mathrm{E}(\mathrm{PBE} 0 \mathrm{DH})=\quad-$

$0.31511337095679 \mathrm{D}+03$

223tri.out: $\mathrm{E} 2(\mathrm{PBE0DH})=-0.1696051902 \mathrm{D}+00 \mathrm{E}(\mathrm{PBE} 0 \mathrm{DH})=-0.27587517917659 \mathrm{D}+03$

22dime.out: $\mathrm{E} 2(\mathrm{PBE} 0 \mathrm{DH})=-0.1450712819 \mathrm{D}+00 \mathrm{E}(\mathrm{PBE} 0 \mathrm{DH})=$

$0.23663663283481 \mathrm{D}+03$

butane.out: E2(PBE0DH $)=-0.9627319287 \mathrm{D}-01 \mathrm{E}(\mathrm{PBE} 0 \mathrm{DH})=-0.15815356834479 \mathrm{D}+03$

ethane.out: $\mathrm{E} 2(\mathrm{PBE} 0 \mathrm{DH})=-0.4938645655 \mathrm{D}-01 \mathrm{E}(\mathrm{PBE} 0 \mathrm{DH})=-0.79672324238464 \mathrm{D}+02$

heptane.out: $\mathrm{E} 2(\mathrm{PBE} 0 \mathrm{DH})=-0.1667575264 \mathrm{D}+00 \mathrm{E}(\mathrm{PBE} 0 \mathrm{DH})=\quad-$

$0.27587536067815 \mathrm{D}+03$

hexane.out: $\mathrm{E} 2(\mathrm{PBE} 0 \mathrm{DH})=-0.1432588784 \mathrm{D}+00 \mathrm{E}(\mathrm{PBE} 0 \mathrm{DH})=-$

$0.23663474450712 \mathrm{D}+03$

isobutane.out: $\mathrm{E} 2(\mathrm{PBE} 0 \mathrm{DH})=-0.9671131836 \mathrm{D}-01 \mathrm{E}(\mathrm{PBE} 0 \mathrm{DH})=\quad$

$0.15815537210651 \mathrm{D}+03$

Methane.out: E2(PBE0DH $)=-0.2641110113 \mathrm{D}-01 \mathrm{E}(\mathrm{PBE0DH})=\quad-$

$0.40434613067416 \mathrm{D}+02$

neopentane.out: $\mathrm{E} 2(\mathrm{PBE} 0 \mathrm{DH})=-0.1211383714 \mathrm{D}+00 \mathrm{E}(\mathrm{PBE} 0 \mathrm{DH})=-$

$0.19739816324525 \mathrm{D}+03$

octane.out: $\mathrm{E} 2(\mathrm{PBE} 0 \mathrm{DH})=-0.1902565301 \mathrm{D}+00 \mathrm{E}(\mathrm{PBE} 0 \mathrm{DH})=-$

$0.31511597600144 \mathrm{D}+03$

pentane.out: $\mathrm{E} 2(\mathrm{PBE} 0 \mathrm{DH})=-0.1197623712 \mathrm{D}+00 \mathrm{E}(\mathrm{PBE} 0 \mathrm{DH})=$

$0.19739414337713 \mathrm{D}+03$

Propane.out: E2(PBE0DH $)=-0.7280530639 \mathrm{D}-01 \mathrm{E}(\mathrm{PBE} 0 \mathrm{DH})=\quad$

$0.11891296322881 \mathrm{D}+03$

PBEQIDH/DHSVPD

2233tetra.out: E2(PBEQIDH) $=-0.4778045972 \mathrm{D}+00 \mathrm{E}(\mathrm{PBEQIDH})=\quad-$

$0.31501293288746 \mathrm{D}+03$ 


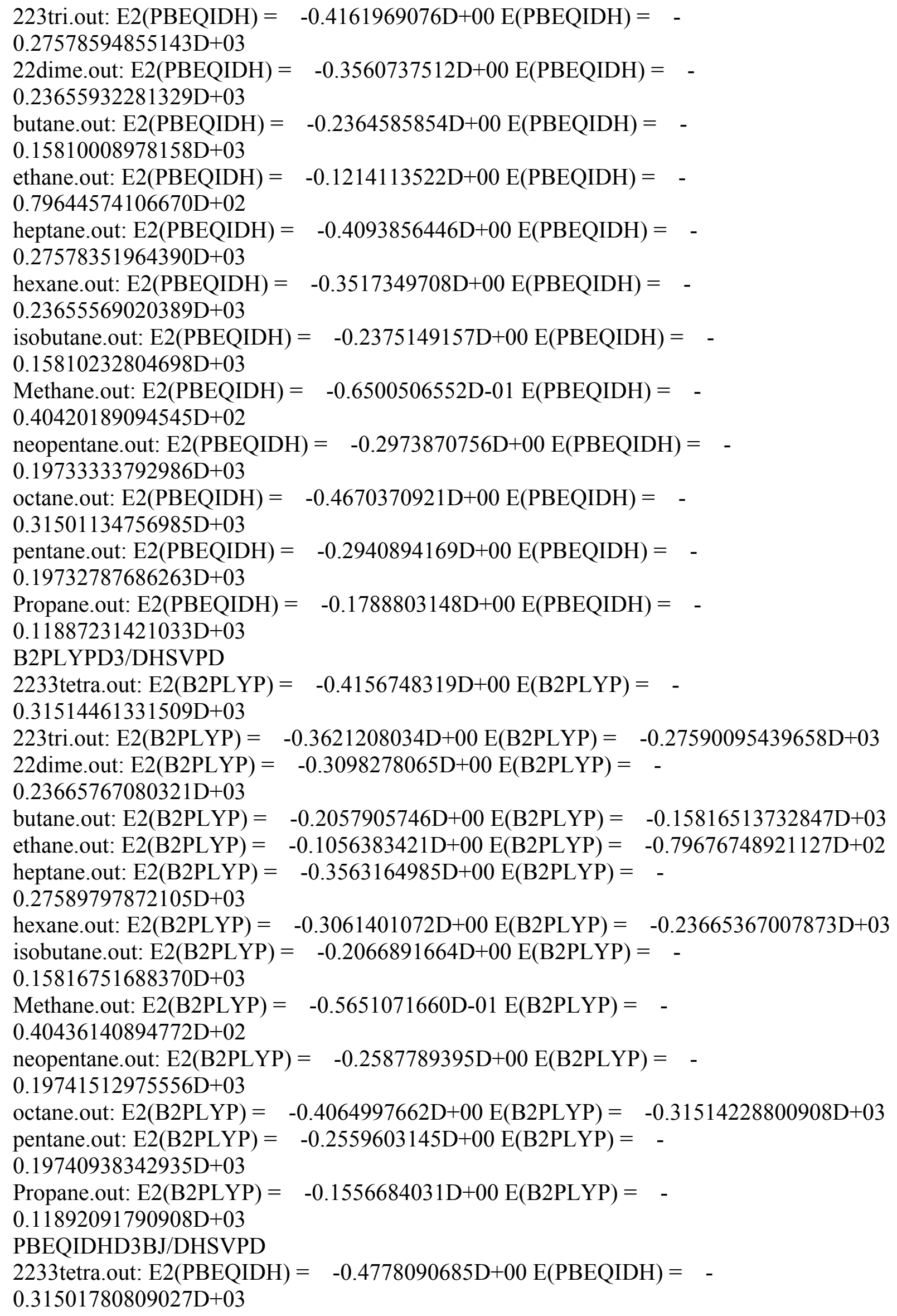




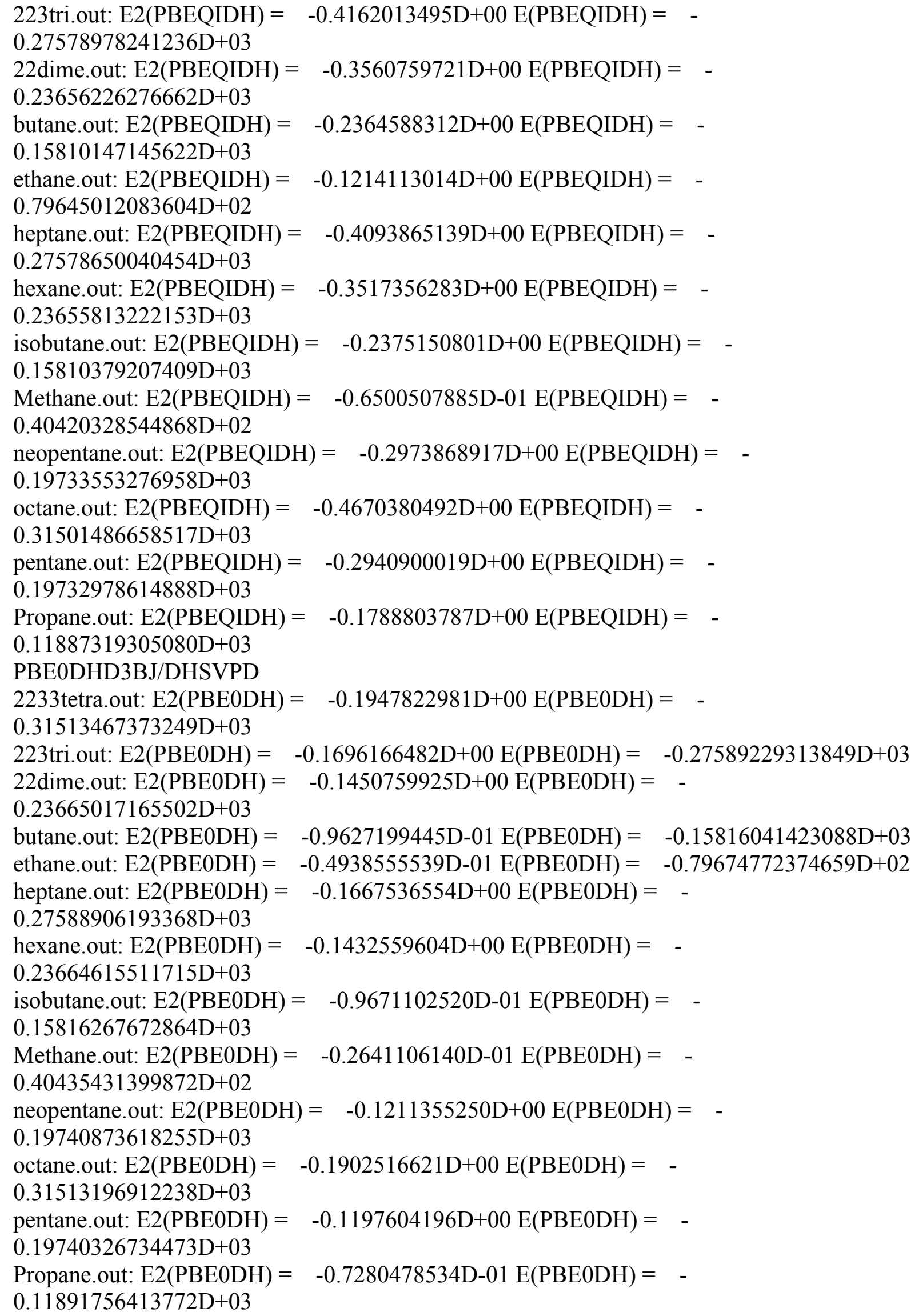


2233tetra.out: E2(DSDPBEP86) $=-0.6453792597 \mathrm{D}+00 \mathrm{E}(\mathrm{DSDPBEP} 86)=-0.3147869$ 4496493D+03

223tri.out: $\mathrm{E} 2(\mathrm{DSDPBEP} 86)=-0.5630800376 \mathrm{D}+00 \mathrm{E}(\mathrm{DSDPBEP} 86)=-0.2755868639$ $6707 \mathrm{D}+03$

22dime.out: E2(DSDPBEP86) $=-0.4824864294 \mathrm{D}+00 \mathrm{E}(\mathrm{DSDPBEP} 86)=-0.2363875529$ $5142 \mathrm{D}+03$

butane.out: $\mathrm{E} 2(\mathrm{DSDPBEP} 86)=-0.3220704935 \mathrm{D}+00 \mathrm{E}(\mathrm{DSDPBEP} 86)=-0.1579831265$ $2572 \mathrm{D}+03$

ethane.out: E2(DSDPBEP86 $)=-0.1668009585 \mathrm{D}+00 \mathrm{E}(\mathrm{DSDPBEP} 86)=-0.7958405343$ $9855 \mathrm{D}+02$

heptane.out: $\mathrm{E} 2(\mathrm{DSDPBEP} 86)=-0.5553860518 \mathrm{D}+00 \mathrm{E}(\mathrm{DSDPBEP} 86)=-0.275582075$ 14983D+03

hexane.out: E2(DSDPBEP86) $=-0.4776126677 \mathrm{D}+00 \mathrm{E}(\mathrm{DSDPBEP} 86)=-0.2363823996$ $4458 \mathrm{D}+03$

isobutane.out: $\mathrm{E} 2(\mathrm{DSDPBEP} 86)=-0.3232455612 \mathrm{D}+00 \mathrm{E}(\mathrm{DSDPBEP} 86)=-0.1579857$ $5808117 \mathrm{D}+03$

Methane.out: E2(DSDPBEP86) $=-0.9038647750 \mathrm{D}-01 \mathrm{E}(\mathrm{DSDPBEP} 86)=-0.403883194$ $24062 \mathrm{D}+02$

neopentane.out: E2(DSDPBEP86) $=-0.4035325870 \mathrm{D}+00 \mathrm{E}(\mathrm{DSDPBEP} 86)=-0.197189$ $36117287 \mathrm{D}+03$

octane.out: E2 $($ DSDPBEP86 $)=-0.6331686850 \mathrm{D}+00 \mathrm{E}(\mathrm{DSDPBEP} 86)=-0.3147817485$ $5841 \mathrm{D}+03$

pentane.out: $\mathrm{E} 2(\mathrm{DSDPBEP} 86)=-0.3998276494 \mathrm{D}+00 \mathrm{E}(\mathrm{DSDPBEP} 86)=-0.197182746$ 36214D+03

Propane.out: E2 $($ DSDPBEP86 $)=-0.2443758323 \mathrm{D}+00 \mathrm{E}(\mathrm{DSDPBEP} 86)=-0.118783554$ 41387D+03

CCPVTZ Basis Set

/ccpvtz/M06L

2233tetra 2.out

SCF Done: $\mathrm{E}(\mathrm{RM} 06 \mathrm{~L})=-315.760876779$ A.U. after 1 cycles

/ccpvtz/M06L

223tri.out

SCF Done: $\mathrm{E}(\mathrm{RM} 06 \mathrm{~L})=-276.442092556 \quad$ A.U. after 1 cycles

/ccpvtz/M06L

22dime.out

SCF Done: $\mathrm{E}(\mathrm{RM} 06 \mathrm{~L})=-237.123413978 \quad$ A.U. after 1 cycles

/ccpvtz/M06L

butane.out

SCF Done: $\mathrm{E}(\mathrm{RM} 06 \mathrm{~L})=-158.481486867 \quad$ A.U. after 1 cycles

/ccpvtz/M06L

ethane.out

SCF Done: $\mathrm{E}(\mathrm{RM} 06 \mathrm{~L})=-79.8420676038 \quad$ A.U. after 1 cycles

/ccpvtz/M06L

heptane.out 
SCF Done: $\mathrm{E}(\mathrm{RM} 06 \mathrm{~L})=-276.440573633$ A.U. after 1 cycles /ccpvtz/M06L

hexane.out

SCF Done: $\mathrm{E}(\mathrm{RM} 06 \mathrm{~L})=-237.120877452$ A.U. after 1 cycles

/ccpvtz/M06L

isobutane.out

SCF Done: $\mathrm{E}(\mathrm{RM} 06 \mathrm{~L})=-158.482795524 \quad$ A.U. after 1 cycles

/ccpvtz/M06L

Methane.out

SCF Done: $\mathrm{E}(\mathrm{RM} 06 \mathrm{~L})=-40.5245915273$ A.U. after 1 cycles

/ccpvtz/M06L

neopentane.out

SCF Done: $\mathrm{E}(\mathrm{RM} 06 \mathrm{~L})=-197.804641809$ A.U. after 1 cycles

/ccpvtz/M06L

octane.out

SCF Done: $\mathrm{E}(\mathrm{RM} 06 \mathrm{~L})=-315.760272684$ A.U. after 1 cycles

/ccpvtz/M06L

pentane.out

SCF Done: $\mathrm{E}(\mathrm{RM} 06 \mathrm{~L})=-197.801176437$ A.U. after 1 cycles

/ccpvtz/M06L

Propane.out

SCF Done: $\mathrm{E}(\mathrm{RM} 06 \mathrm{~L})=-119.161695846 \quad$ A.U. after 1 cycles

/ccpvtz/TPSSH

2233tetra.out

SCF Done: $\mathrm{E}(\mathrm{RTPSSh})=-315.835114386$ A.U. after 1 cycles

/ccpvtz/TPSSH

223tri.out

SCF Done: $\mathrm{E}(\mathrm{RTPSSh})=-276.510071512$ A.U. after 1 cycles

/ccpvtz/TPSSH

22dime.out

SCF Done: $\mathrm{E}(\mathrm{RTPSSh})=-237.183932861 \quad$ A.U. after 1 cycles

/ccpvtz/TPSSH

butane.out

SCF Done: $\mathrm{E}(\mathrm{RTPSSh})=-158.525797326 \quad$ A.U. after 1 cycles

/ccpvtz/TPSSH

ethane.out

SCF Done: $\mathrm{E}(\mathrm{RTPSSh})=-79.8658451374$ A.U. after 1 cycles 
/ccpvtz/TPSSH

heptane.out

SCF Done: $\mathrm{E}(\mathrm{RTPSSh})=-276.515389898$

A.U. after 1 cycles

/ccpvtz/TPSSH

hexane.out

SCF Done: E(RTPSSh $)=-237.185524509$

A.U. after 1 cycles

/ccpvtz/TPSSH

isobutane.out

SCF Done: $\mathrm{E}(\mathrm{RTPSSh})=-158.526558087 \quad$ A.U. after 1 cycles

/ccpvtz/TPSSH

Methane.out

SCF Done: $\mathrm{E}(\mathrm{RTPSSh})=-40.5375325514 \quad$ A.U. after 1 cycles

/ccpvtz/TPSSH

neopentane.out

SCF Done: $\mathrm{E}(\mathrm{RTPSSh})=-197.856902934 \quad$ A.U. after 1 cycles

/ccpvtz/TPSSH

octane.out

SCF Done: $\mathrm{E}(\mathrm{RTPSSh})=-315.845253954 \quad$ A.U. after 1 cycles

/ccpvtz/TPSSH

pentane.out

SCF Done: $\mathrm{E}(\mathrm{RTPSSh})=-197.855658033 \quad$ A.U. after 1 cycles

/ccpvtz/TPSSH

Propane.out

SCF Done: $\mathrm{E}(\mathrm{RTPSSh})=-119.195861232 \quad$ A.U. after 1 cycles

/ccpvtz/B3LYP

2,2,3,3-tetramethylbutane.log

SCF Done: $\mathrm{E}(\mathrm{RB} 3 \mathrm{LYP})=-315.818524035$ A.U. after 1 cycles

/ccpvtz/B3LYP

2,2,3-trimethylbutane.log

SCF Done: $E(R B 3 L Y P)=-276.496942335 \quad$ A.U. after 1 cycles

/ccpvtz/B3LYP

2,2-dimethylbutane.log

SCF Done: $\mathrm{E}(\mathrm{RB} 3 \mathrm{LYP})=-237.173616291 \quad$ A.U. after 1 cycles

/ccpvtz/B3LYP

butane.log

SCF Done: $E($ RB3LYP $)=-158.520208052 \quad$ A.U. after 1 cycles

/ccpvtz/B3LYP

Ethane.log 
SCF Done: $\mathrm{E}(\mathrm{RB} 3 \mathrm{LYP})=-79.8643398357 \quad$ A.U. after 1 cycles /ccpvtz/B3LYP

heptane.log

SCF Done: $\mathrm{E}(\mathrm{RB} 3 \mathrm{LYP})=-276.503574454 \quad$ A.U. after 1 cycles

/ccpvtz/B3LYP

hexane.log

SCF Done: $\mathrm{E}(\mathrm{RB} 3 \mathrm{LYP})=-237.175785137 \quad$ A.U. after 1 cycles

/ccpvtz/B3LYP

isobutane.log

SCF Done: $E($ RB3LYP $)=-158.521008258$ A.U. after 1 cycles

/ccpvtz/B3LYP

Methane.log

SCF Done: $E($ RB3LYP $)=-40.5382374155$ A.U. after 1 cycles

/ccpvtz/B3LYP

neopentane.log

SCF Done: $\mathrm{E}(\mathrm{RB} 3 \mathrm{LYP})=-197.849092971 \quad$ A.U. after 1 cycles

/ccpvtz/B3LYP

octane.log

SCF Done: $E(R B 3 L Y P)=-315.831365072 \quad$ A.U. after 1 cycles

/ccpvtz/B3LYP

pentane.log

SCF Done: $\mathrm{E}(\mathrm{RB} 3 \mathrm{LYP})=-197.847991525 \quad$ A.U. after 1 cycles

/ccpvtz/B3LYP

Propane.log

SCF Done: $E($ RB3LYP $)=-119.192332163 \quad$ A.U. after 1 cycles

/ccpvtz/PBE0

2233-tetramethylbutane.log

SCF Done: $\mathrm{E}(\mathrm{RPBE} 1 \mathrm{PBE})=-315.408537388 \quad$ A.U. after 1 cycles

/ccpvtz/PBE0

223-trimethylbutane.log

SCF Done: E(RPBE1PBE) $=-276.135202581 \quad$ A.U. after 1 cycles

/ccpvtz/PBE0

22-dimethylbutane.log

SCF Done: $\mathrm{E}(\mathrm{RPBE} 1 \mathrm{PBE})=-236.860863039$ A.U. after 1 cycles

/ccpvtz/PBE0

butane.log

SCF Done: $\mathrm{E}(\mathrm{RPBE} 1 \mathrm{PBE})=-158.305766527$ A.U. after 1 cycles 
/ccpvtz/PBE0

Ethane.log

SCF Done: $\mathrm{E}(\mathrm{RPBE} 1 \mathrm{PBE})=-79.7500469905 \quad$ A.U. after 1 cycles

/ccpvtz/PBE0

heptane.log

SCF Done: $\mathrm{E}(\mathrm{RPBE} 1 \mathrm{PBE})=-276.138982159 \quad$ A.U. after 1 cycles

/ccpvtz/PBE0

hexane.log

SCF Done: $\mathrm{E}(\mathrm{RPBE} 1 \mathrm{PBE})=-236.861241402 \quad$ A.U. after 1 cycles

/ccpvtz/PBE0

isobutane.log

SCF Done: $\mathrm{E}(\mathrm{RPBE} 1 \mathrm{PBE})=-158.306958706 \quad$ A.U. after 1 cycles

/ccpvtz/PBE0

Methane.log

SCF Done: $\mathrm{E}(\mathrm{RPBE} 1 \mathrm{PBE})=-40.4744219638 \quad$ A.U. after 1 cycles

/ccpvtz/PBE0

neopentane.log

SCF Done: $\mathrm{E}(\mathrm{RPBE} 1 \mathrm{PBE})=-197.585877406 \quad$ A.U. after 1 cycles

/ccpvtz/PBE0

octane.log

SCF Done: $\mathrm{E}(\mathrm{RPBE} 1 \mathrm{PBE})=-315.416723065 \quad$ A.U. after 1 cycles

/ccpvtz/PBE0

pentane.log

SCF Done: $\mathrm{E}(\mathrm{RPBE} 1 \mathrm{PBE})=-197.583498388 \quad$ A.U. after 1 cycles

/ccpvtz/PBE0

Propane.log

SCF Done: $\mathrm{E}(\mathrm{RPBE} 1 \mathrm{PBE})=-119.027937256 \quad$ A.U. after 1 cycles

/ccpvtz/M06

2233tetra.out

SCF Done: $\mathrm{E}(\mathrm{RM} 06)=-315.549025116 \quad$ A.U. after 1 cycles

/ccpvtz/M06

223tri.out

SCF Done: $\mathrm{E}(\mathrm{RM} 06)=-276.255614458 \quad$ A.U. after 1 cycles

/ccpvtz/M06

22dime.out

SCF Done: $\mathrm{E}(\mathrm{RM} 06)=-236.962796165 \quad$ A.U. after 1 cycles

/ccpvtz/M06

butane.out 
SCF Done: $\mathrm{E}(\mathrm{RM} 06)=-158.370567233 \quad$ A.U. after 1 cycles

/ccpvtz/M06

ethane.out

SCF Done: $\mathrm{E}(\mathrm{RM} 06)=-79.7832696740 \quad$ A.U. after 1 cycles

/ccpvtz/M06

heptane.out

SCF Done: $\mathrm{E}(\mathrm{RM} 06)=-276.251470841 \quad$ A.U. after 1 cycles

/ccpvtz/M06

hexane.out

SCF Done: $E(R M 06)=-236.957828534$ A.U. after 1 cycles

/ccpvtz/M06

isobutane.out

SCF Done: $\mathrm{E}(\mathrm{RM} 06)=-158.372807051 \quad$ A.U. after 1 cycles

/ccpvtz/M06

Methane.out

SCF Done: $\mathrm{E}(\mathrm{RM} 06)=-40.4925311611$ A.U. after 1 cycles

/ccpvtz/M06

neopentane.out

SCF Done: $\mathrm{E}(\mathrm{RM} 06)=-197.670455034$ A.U. after 1 cycles

/ccpvtz/M06

octane.out

SCF Done: $\mathrm{E}(\mathrm{RM} 06)=-315.545106604$ A.U. after 1 cycles

/ccpvtz/M06

pentane.out

SCF Done: $\mathrm{E}(\mathrm{RM} 06)=-197.664195851 \quad$ A.U. after 1 cycles

/ccpvtz/M06

Propane.out

SCF Done: $\mathrm{E}(\mathrm{RM} 06)=-119.076844423$ A.U. after 1 cycles

/ccpvtz/CAMB3LYP

2233tetra.out

SCF Done: E(RCAM-B3LYP) $=-315.613291723$ A.U. after 1 cycles

/ccpvtz/CAMB3LYP

223tri.out

SCF Done: E(RCAM-B3LYP $)=-276.315101854$ A.U. after 1 cycles

/ccpvtz/CAMB3LYP

22dime.out

SCF Done: E(RCAM-B3LYP $)=-237.015855713$ A.U. after 1 cycles 
/ccpvtz/CAMB3LYP

butane.out

SCF Done: $\mathrm{E}(\mathrm{RCAM}-\mathrm{B} 3 \mathrm{LYP})=-158.410559491 \quad$ A.U. after 1 cycles

/ccpvtz/CAMB3LYP

ethane.out

SCF Done: E(RCAM-B3LYP $)=-79.8046320094$ A.U. after 1 cycles

/ccpvtz/CAMB3LYP

heptane.out

SCF Done: $\mathrm{E}(\mathrm{RCAM}-\mathrm{B} 3 \mathrm{LYP})=-276.319045536$ A.U. after 1 cycles

/ccpvtz/CAMB3LYP

hexane.out

SCF Done: E(RCAM-B3LYP $)=-237.016214807$

A.U. after 1 cycles

/ccpvtz/CAMB3LYP

isobutane.out

SCF Done: E(RCAM-B3LYP) $=-158.411846909$ A.U. after 1 cycles

/ccpvtz/CAMB3LYP

Methane.out

SCF Done: E(RCAM-B3LYP $)=-40.5039846088$ A.U. after 1 cycles

/ccpvtz/CAMB3LYP

neopentane.out

SCF Done: E(RCAM-B3LYP $)=-197.715966539$ A.U. after 1 cycles

/ccpvtz/CAMB3LYP

octane.out

SCF Done: $\mathrm{E}(\mathrm{RCAM}-\mathrm{B} 3 \mathrm{LYP})=-315.621876407$ A.U. after 1 cycles

/ccpvtz/CAMB3LYP

pentane.out

SCF Done: E(RCAM-B3LYP $)=-197.713381352 \quad$ A.U. after 1 cycles

/ccpvtz/CAMB3LYP

Propane.out

SCF Done: E(RCAM-B3LYP) $=-119.107641390$ A.U. after 1 cycles

/ccpvtz/WB97XD

2233tetra.out

SCF Done: $\mathrm{E}(\mathrm{RwB} 97 \mathrm{XD})=-315.726234699 \quad$ A.U. after 1 cycles

/ccpvtz/WB97XD

223tri.out

SCF Done: $\mathrm{E}(\mathrm{RwB} 97 \mathrm{XD})=-276.412274211$ A.U. after 1 cycles

/ccpvtz/WB97XD

22dime.out 
SCF Done: $\mathrm{E}(\mathrm{RwB} 97 \mathrm{XD})=-237.098159836$

A.U. after 1 cycles

/ccpvtz/WB97XD

butane.out

SCF Done: E(RwB97XD) $=-158.464257343$

A.U. after 1 cycles

/ccpvtz/WB97XD

ethane.out

SCF Done: $\mathrm{E}(\mathrm{RwB} 97 \mathrm{XD})=-79.8327227545$

A.U. after 1 cycles

/ccpvtz/WB97XD

heptane.out

SCF Done: E(RwB97XD) = -276.411738659

A.U. after 1 cycles

/ccpvtz/WB97XD

hexane.out

SCF Done: E(RwB97XD) $=-237.095899622$

A.U. after 1 cycles

/ccpvtz/WB97XD

isobutane.out

SCF Done: $\mathrm{E}(\mathrm{RwB} 97 \mathrm{XD})=-158.465893903 \quad$ A.U. after 1 cycles

/ccpvtz/WB97XD

Methane.out

SCF Done: $\mathrm{E}(\mathrm{RwB} 97 \mathrm{XD})=-40.5198197157 \quad$ A.U. after 1 cycles

/ccpvtz/WB97XD

neopentane.out

SCF Done: $\mathrm{E}(\mathrm{RwB} 97 \mathrm{XD})=-197.783973575 \quad$ A.U. after 1 cycles

/ccpvtz/WB97XD

octane.out

SCF Done: $\mathrm{E}(\mathrm{RwB} 97 \mathrm{XD})=-315.727573512 \quad$ A.U. after 1 cycles

/ccpvtz/WB97XD

pentane.out

SCF Done: $\mathrm{E}(\mathrm{RwB} 97 \mathrm{XD})=-197.780073948$ A.U. after 1 cycles

/ccpvtz/WB97XD

Propane.out

SCF Done: $\mathrm{E}(\mathrm{RwB} 97 \mathrm{XD})=-119.148431411 \quad$ A.U. after 1 cycles

/ccpvtz/WB97XD

$* \log$

/ccpvtz/B3LYPD3

2,2,3,3-tetramethylbutane.log

SCF Done: $\mathrm{E}(\mathrm{RB} 3 \mathrm{LYP})=-315.845288277 \quad$ A.U. after 1 cycles

/ccpvtz/B3LYPD3 
2,2,3-trimethylbutane.log

SCF Done: $E(R B 3 L Y P)=-276.517785783 \quad$ A.U. after 1 cycles

/ccpvtz/B3LYPD3

2,2-dimethylbutane.log

SCF Done: $E($ RB3LYP $)=-237.189745996$ A.U. after 1 cycles

/ccpvtz/B3LYPD3

butane.log

SCF Done: $\mathrm{E}(\mathrm{RB} 3 \mathrm{LYP})=-158.527225709$ A.U. after 1 cycles

/ccpvtz/B3LYPD3

Ethane.log

SCF Done: $E(R B 3 L Y P)=-79.8661371985 \quad$ A.U. after 1 cycles

/ccpvtz/B3LYPD3

heptane.log

SCF Done: $E(R B 3 L Y P)=-276.518826631 \quad$ A.U. after 1 cycles

/ccpvtz/B3LYPD3

hexane.log

SCF Done: $\mathrm{E}(\mathrm{RB} 3 \mathrm{LYP})=-237.188284591 \quad$ A.U. after 1 cycles

/ccpvtz/B3LYPD3

isobutane.log

SCF Done: $\mathrm{E}(\mathrm{RB} 3 \mathrm{LYP})=-158.528853583$ A.U. after 1 cycles

/ccpvtz/B3LYPD3

Methane.log

SCF Done: $\mathrm{E}(\mathrm{RB} 3 \mathrm{LYP})=-40.5383897403 \quad$ A.U. after 1 cycles

/ccpvtz/B3LYPD3

neopentane.log

SCF Done: $E(R B 3 L Y P)=-197.861451938 \quad$ A.U. after 1 cycles

/ccpvtz/B3LYPD3

octane.log

SCF Done: $\mathrm{E}(\mathrm{RB} 3 \mathrm{LYP})=-315.849371570 \quad$ A.U. after 1 cycles

/ccpvtz/B3LYPD3

pentane.log

SCF Done: $\mathrm{E}(\mathrm{RB} 3 \mathrm{LYP})=-197.857743546 \quad$ A.U. after 1 cycles

/ccpvtz/B3LYPD3

Propane.log

SCF Done: $\mathrm{E}(\mathrm{RB} 3 \mathrm{LYP})=-119.196666453 \quad$ A.U. after 1 cycles

/ccpvtz/B2PLYP

2,2,3,3-tetramethylbutane.log: E2(B2PLYP) $=-0.4686252840 \mathrm{D}+00 \mathrm{E}(\mathrm{B} 2 \mathrm{PLYP})=$

$0.31549379221193 \mathrm{D}+03$ 
2,2,3-trimethylbutane.log: E2(B2PLYP $)=-0.4087402699 \mathrm{D}+00 \mathrm{E}(\mathrm{B} 2 \mathrm{PLYP})=-$ $0.27620929331978 \mathrm{D}+03$

2,2-dimethylbutane.log: E2(B2PLYP $)=-0.3499977516 \mathrm{D}+00 \mathrm{E}(\mathrm{B} 2 \mathrm{PLYP})=\quad-$ $0.23692428377684 \mathrm{D}+03$

butane.log: E2(B2PLYP $)=-0.2331813366 \mathrm{D}+00 \mathrm{E}(\mathrm{B} 2 \mathrm{PLYP})=-0.15834764272825 \mathrm{D}+03$

Ethane.log: E2(B2PLYP $)=-0.1198722449 \mathrm{D}+00 \mathrm{E}(\mathrm{B} 2 \mathrm{PLYP})=-0.79771766939028 \mathrm{D}+02$ heptane_2.log: E2(B2PLYP) $=-0.4033406200 \mathrm{D}+00 \mathrm{E}(\mathrm{B} 2 \mathrm{PLYP})=\quad-$ $0.27621123814764 \mathrm{D}+03$

hexane.log: E2(B2PLYP $)=-0.3466210456 \mathrm{D}+00 \mathrm{E}(\mathrm{B} 2 \mathrm{PLYP})=-0.23692336769092 \mathrm{D}+03$ isobutane.log: $\mathrm{E} 2(\mathrm{~B} 2 \mathrm{PLYP})=-0.2339743997 \mathrm{D}+00 \mathrm{E}(\mathrm{B} 2 \mathrm{PLYP})=\quad-$

$0.15834919681997 \mathrm{D}+03$

Methane.log: E2(B2PLYP $)=-0.6404642813 \mathrm{D}-01 \mathrm{E}(\mathrm{B} 2 \mathrm{PLYP})=\quad-$

$0.40486384825442 \mathrm{D}+02$

neopentane.log: E2(B2PLYP $)=-0.2923793537 \mathrm{D}+00 \mathrm{E}(\mathrm{B} 2 \mathrm{PLYP})=\quad-$

$0.19763905245557 \mathrm{D}+03$

octane.log: E2(B2PLYP $)=-0.4600750784 \mathrm{D}+00 \mathrm{E}(\mathrm{B} 2 \mathrm{PLYP})=-0.31549910909815 \mathrm{D}+03$

pentane.log: E2(B2PLYP $)=-0.2898969317 \mathrm{D}+00 \mathrm{E}(\mathrm{B} 2 \mathrm{PLYP})=\quad-$

$0.19763549693819 \mathrm{D}+03$

Propane.log: E2(B2PLYP) $=-0.1764851273 \mathrm{D}+00 \mathrm{E}(\mathrm{B} 2 \mathrm{PLYP})=\quad-$

$0.11905971854758 \mathrm{D}+03$

Ccpvtz/PBE0DH

2233tetramethylbutane.log: $\mathrm{E} 2(\mathrm{PBE} 0 \mathrm{DH})=-0.2193884004 \mathrm{D}+00 \mathrm{E}(\mathrm{PBE} 0 \mathrm{DH})=-$

$0.31544023059612 \mathrm{D}+03$

223trimethylbutane.log: $\mathrm{E} 2(\mathrm{PBE0DH})=-0.1912726169 \mathrm{D}+00 \mathrm{E}(\mathrm{PBE} 0 \mathrm{DH})=$

$0.27616210622640 \mathrm{D}+03$

22dimethylbutane.log: $\mathrm{E} 2(\mathrm{PBE} 0 \mathrm{DH})=-0.1637312776 \mathrm{D}+00 \mathrm{E}(\mathrm{PBE} 0 \mathrm{DH})=$

$0.23688364220272 \mathrm{D}+03$

butane.log: $\mathrm{E} 2(\mathrm{PBE} 0 \mathrm{DH})=-0.1089888783 \mathrm{D}+00 \mathrm{E}(\mathrm{PBE} 0 \mathrm{DH})=\quad-$

$0.15832031010394 \mathrm{D}+03$

Ethane.log: E2(PBE0DH $)=-0.5599504175 \mathrm{D}-01 \mathrm{E}(\mathrm{PBE} 0 \mathrm{DH})=\quad$

$0.79757863980627 \mathrm{D}+02$

heptane.log: $\mathrm{E} 2(\mathrm{PBE} 0 \mathrm{DH})=-0.1885796870 \mathrm{D}+00 \mathrm{E}(\mathrm{PBE} 0 \mathrm{DH})=$

$0.27616373989334 \mathrm{D}+03$

hexane.log: $\mathrm{E} 2(\mathrm{PBE0DH})=-0.1620465824 \mathrm{D}+00 \mathrm{E}(\mathrm{PBE} 0 \mathrm{DH})=\quad-$

$0.23688259277080 \mathrm{D}+03$

isobutane.log: $\mathrm{E} 2(\mathrm{PBE} 0 \mathrm{DH})=-0.1093827041 \mathrm{D}+00 \mathrm{E}(\mathrm{PBE} 0 \mathrm{DH})=\quad-$

$0.15832183777796 \mathrm{D}+03$

Methane.log: E2(PBE0DH $)=-0.2991462890 \mathrm{D}-01 \mathrm{E}(\mathrm{PBE0DH})=\quad-$

$0.40479176869682 \mathrm{D}+02$

neopentane.log: $\mathrm{E} 2(\mathrm{PBE} 0 \mathrm{DH})=-0.1367423060 \mathrm{D}+00 \mathrm{E}(\mathrm{PBE} 0 \mathrm{DH})=\quad-$

$0.19760497521259 \mathrm{D}+03$

octane.log: $\mathrm{E} 2(\mathrm{PBE} 0 \mathrm{DH})=-0.2151130659 \mathrm{D}+00 \mathrm{E}(\mathrm{PBE} 0 \mathrm{DH})=-$

$0.31544488754092 \mathrm{D}+03$

pentane.log: $\mathrm{E} 2(\mathrm{PBE} 0 \mathrm{DH})=-0.1355149081 \mathrm{D}+00 \mathrm{E}(\mathrm{PBE} 0 \mathrm{DH})=$

$0.19760144430543 \mathrm{D}+03$

Propane.log: E2 $(\mathrm{PBE0DH})=-0.8247073384 \mathrm{D}-01 \mathrm{E}(\mathrm{PBE0DH})=\quad-$

$0.11903909049199 \mathrm{D}+03$

Ccpvtz/PBEQIDH

2233tetramethylbutane.log: E2(PBEQIDH $)=-0.5403807915 \mathrm{D}+00 \mathrm{E}(\mathrm{PBEQIDH})=\quad-$

$0.31538094516467 \mathrm{D}+03$ 
223trimethylbutane.log: E2(PBEQIDH $)=-0.4712663478 \mathrm{D}+00 \mathrm{E}(\mathrm{PBEQIDH})=-$ $0.27610904398168 \mathrm{D}+03$

22dimethylbutane.log: E2(PBEQIDH $)=-0.4034982451 \mathrm{D}+00 \mathrm{E}(\mathrm{PBEQIDH})=\quad-$ $0.23683747937743 \mathrm{D}+03$

butane.log: $\mathrm{E} 2(\mathrm{PBEQIDH})=\quad-0.2687673254 \mathrm{D}+00 \mathrm{E}(\mathrm{PBEQIDH})=\quad-$ $0.15828799547164 \mathrm{D}+03$

Ethane.log: E2(PBEQIDH $)=-0.1382109984 \mathrm{D}+00 \mathrm{E}(\mathrm{PBEQIDH})=\quad-$ $0.79741106063982 \mathrm{D}+02$ heptane.log: $\mathrm{E} 2(\mathrm{PBEQIDH})=\quad-0.4648257359 \mathrm{D}+00 \mathrm{E}(\mathrm{PBEQIDH})=\quad-$ $0.27610821690953 \mathrm{D}+03$ hexane.log: $\mathrm{E} 2(\mathrm{PBEQIDH})=-0.3994669910 \mathrm{D}+00 \mathrm{E}(\mathrm{PBEQIDH})=\quad-$ $0.23683480325865 \mathrm{D}+03$ isobutane.log: $\mathrm{E} 2(\mathrm{PBEQIDH})=-0.2697136905 \mathrm{D}+00 \mathrm{E}(\mathrm{PBEQIDH})=\quad-$ $0.15828992429940 \mathrm{D}+03$

Methane.log: E2(PBEQIDH) $=-0.7392392040 \mathrm{D}-01 \mathrm{E}(\mathrm{PBEQIDH})=\quad-$ $0.40470583087129 \mathrm{D}+02$ neopentane.log: $\mathrm{E} 2(\mathrm{PBEQIDH})=-0.3370576748 \mathrm{D}+00 \mathrm{E}(\mathrm{PBEQIDH})=$ $0.19756622141521 \mathrm{D}+03$ octane.log: $\mathrm{E} 2(\mathrm{PBEQIDH})=-0.5301851710 \mathrm{D}+00 \mathrm{E}(\mathrm{PBEQIDH})=\quad$ $0.31538163121965 \mathrm{D}+03$ pentane.log: $\mathrm{E} 2(\mathrm{PBEQIDH})=-0.3341116147 \mathrm{D}+00 \mathrm{E}(\mathrm{PBEQIDH})=\quad-$ $0.19756139039201 \mathrm{D}+03$ Propane.log: E2(PBEQIDH $)=-0.2034425489 \mathrm{D}+00 \mathrm{E}(\mathrm{PBEQIDH})=\quad-$ $0.11901453069550 \mathrm{D}+03$ Ccpvtz/B2PLYPD3 2233tetramethylbutane.log: E2(B2PLYP $)=-0.4686459078 \mathrm{D}+00 \mathrm{E}(\mathrm{B} 2 \mathrm{PLYP})=-$ $0.31550846874703 \mathrm{D}+03$

223trimethylbutane.log: E2(B2PLYP $)=-0.4087667138 \mathrm{D}+00 \mathrm{E}(\mathrm{B} 2 \mathrm{PLYP})=-$ $0.27622062995549 \mathrm{D}+03$ 22dimethylbutane.log: E2(B2PLYP) $=-0.3500120456 \mathrm{D}+00 \mathrm{E}(\mathrm{B} 2 \mathrm{PLYP})=\quad-$ $0.23693306574091 \mathrm{D}+03$

Ethane.log: E2(B2PLYP $)=-0.1198744968 \mathrm{D}+00 \mathrm{E}(\mathrm{B} 2 \mathrm{PLYP})=-0.79772755066729 \mathrm{D}+02$ heptane.log: E2(B2PLYP $)=-0.4033565652 \mathrm{D}+00 \mathrm{E}(\mathrm{B} 2 \mathrm{PLYP})=\quad-$

$0.27621961835764 \mathrm{D}+03$

hexane.log: E2(B2PLYP $)=-0.3466301106 \mathrm{D}+00 \mathrm{E}(\mathrm{B} 2 \mathrm{PLYP})=-0.23693023269717 \mathrm{D}+03$ isobutane.log: $\mathrm{E} 2(\mathrm{~B} 2 \mathrm{PLYP})=-0.2339865313 \mathrm{D}+00 \mathrm{E}(\mathrm{B} 2 \mathrm{PLYP})=\quad$ $0.15835348314723 \mathrm{D}+03$

Methane.log: E2(B2PLYP $)=-0.6404786291 \mathrm{D}-01 \mathrm{E}(\mathrm{B} 2 \mathrm{PLYP})=\quad-$ $0.40486472698423 \mathrm{D}+02$ neopentane.log: E2(B2PLYP) $=-0.2923851634 \mathrm{D}+00 \mathrm{E}(\mathrm{B} 2 \mathrm{PLYP})=\quad-$ $0.19764578777526 \mathrm{D}+03$ pentane.log: E2(B2PLYP) $=-0.2899062698 \mathrm{D}+00 \mathrm{E}(\mathrm{B} 2 \mathrm{PLYP})=-$ $0.19764084969569 \mathrm{D}+03$ Propane.log: E2(B2PLYP $)=-0.1764932729 \mathrm{D}+00 \mathrm{E}(\mathrm{B} 2 \mathrm{PLYP})=-$ $0.11906209305433 \mathrm{D}+03$ Ccpvtz/PBEQIDHD3BJ 2233tetra.out: $\mathrm{E} 2(\mathrm{PBEQIDH})=-0.5403900009 \mathrm{D}+00 \mathrm{E}(\mathrm{PBEQIDH})=-$ $0.31538583596224 \mathrm{D}+03$ 223tri.out: $\mathrm{E} 2(\mathrm{PBEQIDH})=-0.4712726277 \mathrm{D}+00 \mathrm{E}(\mathrm{PBEQIDH})=$ $0.27611288541098 \mathrm{D}+03$ 


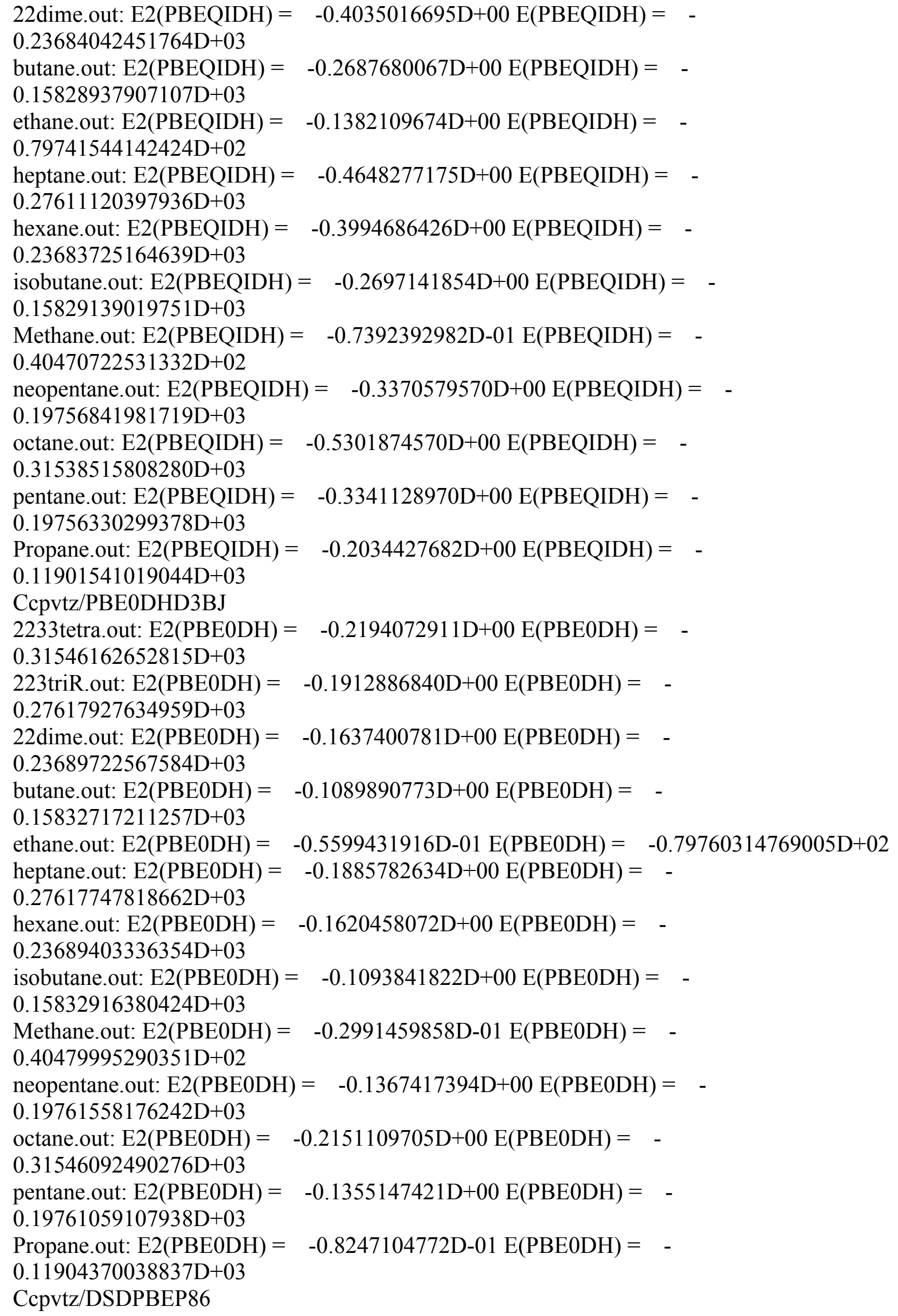


2233tetra.out: E2(DSDPBEP86 $)=-0.7330938322 \mathrm{D}+00 \mathrm{E}(\mathrm{DSDPBEP} 86)=-$ $0.31518291003674 \mathrm{D}+03$

223tri.out: $\mathrm{E} 2(\mathrm{DSDPBEP} 86)=-0.6403196826 \mathrm{D}+00 \mathrm{E}(\mathrm{DSDPBEP} 86)=$

$0.27593464638626 \mathrm{D}+03$

22dime.out: $\mathrm{E} 2(\mathrm{DSDPBEP} 86)=-0.5490657734 \mathrm{D}+00 \mathrm{E}(\mathrm{DSDPBEP} 86)=$

$0.23668708861821 \mathrm{D}+03$

butane.out: $\mathrm{E} 2(\mathrm{DSDPBEP} 86)=-0.3675263133 \mathrm{D}+00 \mathrm{E}(\mathrm{DSDPBEP} 86)=$

$0.15818575237433 \mathrm{D}+03$

ethane.out: E2(DSDPBEP86) $=-0.1905676731 \mathrm{D}+00 \mathrm{E}(\mathrm{DSDPBEP} 86)=$

$0.79688484654139 \mathrm{D}+02$

heptane.out: E2(DSDPBEP86) $=-0.6332089436 \mathrm{D}+00 \mathrm{E}(\mathrm{DSDPBEP} 86)=$

$0.27593168027755 \mathrm{D}+03$

hexane.out: $\mathrm{E} 2(\mathrm{DSDPBEP} 86)=-0.5446491507 \mathrm{D}+00 \mathrm{E}(\mathrm{DSDPBEP} 86)=-$

$0.23668302709461 \mathrm{D}+03$

isobutane.out: $\mathrm{E} 2(\mathrm{DSDPBEP} 86)=-0.3685385330 \mathrm{D}+00 \mathrm{E}(\mathrm{DSDPBEP} 86)=$

$0.15818801723245 \mathrm{D}+03$

Methane.out: E2 $(\mathrm{DSDPBEP} 86)=-0.1031130885 \mathrm{D}+00 \mathrm{E}(\mathrm{DSDPBEP} 86)=$

$0.40443122769949 \mathrm{D}+02$

neopentane.out: E2(DSDPBEP86) $=-0.4592669891 \mathrm{D}+00 \mathrm{E}(\mathrm{DSDPBEP} 86)=$

$0.19744023642966 \mathrm{D}+03$

octane.out: $\mathrm{E} 2(\mathrm{DSDPBEP} 86)=-0.7217785109 \mathrm{D}+00 \mathrm{E}(\mathrm{DSDPBEP} 86)=$

$0.31518033389029 \mathrm{D}+03$

pentane.out: E2 $(\mathrm{DSDPBEP} 86)=-0.4560749104 \mathrm{D}+00 \mathrm{E}(\mathrm{DSDPBEP} 86)=$

$0.19743437921122 \mathrm{D}+03$

Propane.out: E2 $(D S D P B E P 86)=-0.2789974297 D+00$ E $(D S D P B E P 86)=-$

$0.11893708460934 \mathrm{D}+03$

CCPVQZ Basis Set

/ccpvqz/M06L

2233tetra.out

SCF Done: $\mathrm{E}(\mathrm{RM} 06 \mathrm{~L})=-315.798679833$ A.U. after 1 cycles

/ccpvqz/M06L

223tri.out

SCF Done: $\mathrm{E}(\mathrm{RM} 06 \mathrm{~L})=-276.475419060 \quad$ A.U. after 1 cycles

/ccpvqz/M06L

22dime.out

SCF Done: $\mathrm{E}(\mathrm{RM} 06 \mathrm{~L})=-237.152296297 \quad$ A.U. after 1 cycles

/ccpvqz/M06L

butane.out

SCF Done: $\mathrm{E}(\mathrm{RM} 06 \mathrm{~L})=-158.501347028$ A.U. after 1 cycles

/ccpvqz/M06L

ethane.out

SCF Done: $\mathrm{E}(\mathrm{RM} 06 \mathrm{~L})=-79.8526821800 \quad$ A.U. after 1 cycles

/ccpvqz/M06L 
heptane.out

SCF Done: $\mathrm{E}(\mathrm{RM} 06 \mathrm{~L})=-276.474167202 \quad$ A.U. after 1 cycles

/ccpvqz/M06L

hexane.out

SCF Done: $\mathrm{E}(\mathrm{RM} 06 \mathrm{~L})=-237.149895459$ A.U. after 1 cycles

/ccpvqz/M06L

isobutane.out

SCF Done: $\mathrm{E}(\mathrm{RM} 06 \mathrm{~L})=-158.502743083$ A.U. after 1 cycles

/ccpvqz/M06L

Methane.out

SCF Done: $\mathrm{E}(\mathrm{RM} 06 \mathrm{~L})=-40.5307048147 \quad$ A.U. after 1 cycles

/ccpvqz/M06L

neopentane.out

SCF Done: $\mathrm{E}(\mathrm{RM} 06 \mathrm{~L})=-197.829156964$ A.U. after 1 cycles

/ccpvqz/M06L

octane.out

SCF Done: $\mathrm{E}(\mathrm{RM} 06 \mathrm{~L})=-315.798445546 \quad$ A.U. after 1 cycles

/ccpvqz/M06L

pentane.out

SCF Done: $\mathrm{E}(\mathrm{RM} 06 \mathrm{~L})=-197.825613609$ A.U. after 2 cycles

/ccpvqz/M06L

Propane.out

SCF Done: $\mathrm{E}(\mathrm{RM} 06 \mathrm{~L})=-119.176970018 \quad$ A.U. after 1 cycles

/ccpvqz/TPSSH

2233tetra.out

SCF Done: $\mathrm{E}(\mathrm{RTPSSh})=-315.857987997$ A.U. after 1 cycles

/ccpvqz/TPSSH

22dime.out

SCF Done: $\mathrm{E}(\mathrm{RTPSSh})=-237.201038005$ A.U. after 1 cycles

/ccpvqz/TPSSH

butane.out

SCF Done: $\mathrm{E}(\mathrm{RTPSSh})=-158.537146260 \quad$ A.U. after 1 cycles

/ccpvqz/TPSSH

ethane.out

SCF Done: E(RTPSSh $)=-79.8716261102$ A.U. after 1 cycles

/ccpvqz/TPSSH

heptane.out

SCF Done: $\mathrm{E}(\mathrm{RTPSSh})=-276.535072880 \quad$ A.U. after 1 cycles 
/ccpvqz/TPSSH

hexane.out

SCF Done: $\mathrm{E}(\mathrm{RTPSSh})=-237.202428867$ A.U. after 1 cycles

/ccpvqz/TPSSH

isobutane.out

SCF Done: E(RTPSSh) $=-158.537974706$ A.U. after 1 cycles

/ccpvqz/TPSSH

Methane.out

SCF Done: $\mathrm{E}(\mathrm{RTPSSh})=-40.5405833685$ A.U. after 1 cycles

/ccpvqz/TPSSH

neopentane.out

SCF Done: $\mathrm{E}(\mathrm{RTPSSh})=-197.871242421$ A.U. after 1 cycles

/ccpvqz/TPSSH

octane.out

SCF Done: $\mathrm{E}(\mathrm{RTPSSh})=-315.867715781 \quad$ A.U. after 1 cycles

/ccpvqz/TPSSH

pentane.out

SCF Done: $\mathrm{E}(\mathrm{RTPSSh})=-197.869783912$ A.U. after 1 cycles

/ccpvqz/TPSSH

Propane.out

SCF Done: E(RTPSSh) $=-119.204427028$ A.U. after 1 cycles

/ccpvqz/TPSSH

TP223trime.out

SCF Done: $\mathrm{E}(\mathrm{RTPSSh})=-276.530017841 \quad$ A.U. after 1 cycles

/ccpvqz/PBE0

2233tetrametbut.log

SCF Done: $\mathrm{E}(\mathrm{RPBE} 1 \mathrm{PBE})=-315.431591654$ A.U. after 1 cycles

/ccpvqz/PBE0

223trimethbut.log

SCF Done: E(RPBE1PBE $)=-276.155324776 \quad$ A.U. after 1 cycles

/ccpvqz/PBE0

22dimethbut.log

SCF Done: $\mathrm{E}(\mathrm{RPBE} 1 \mathrm{PBE})=-236.878111194$ A.U. after 1 cycles

/ccpvqz/PBE0

butane.log

SCF Done: E(RPBE1PBE $)=-158.317225044$ A.U. after 1 cycles

/ccpvqz/PBE0 
ethane.log

SCF Done: $\mathrm{E}(\mathrm{RPBE} 1 \mathrm{PBE})=-79.7558680512 \quad$ A.U. after 1 cycles

/ccpvqz/PBE0

heptane.log

SCF Done: $\mathrm{E}(\mathrm{RPBE} 1 \mathrm{PBE})=-276.158883883 \quad$ A.U. after 1 cycles

/ccpvqz/PBE0

hexane.log

SCF Done: $\mathrm{E}(\mathrm{RPBE} 1 \mathrm{PBE})=-236.878328103 \quad$ A.U. after 1 cycles

/ccpvqz/PBE0

isobutane.log

SCF Done: $\mathrm{E}(\mathrm{RPBE} 1 \mathrm{PBE})=-158.318472399$ A.U. after 1 cycles

/ccpvqz/PBE0

methane.log

SCF Done: $\mathrm{E}(\mathrm{RPBE} 1 \mathrm{PBE})=-40.4774838324$ A.U. after 1 cycles

/ccpvqz/PBE0

neopentane.log

SCF Done: E(RPBE1PBE $)=-197.600315294$ A.U. after 1 cycles

/ccpvqz/PBE0

octane.log

SCF Done: $\mathrm{E}(\mathrm{RPBE} 1 \mathrm{PBE})=-315.439439951 \quad$ A.U. after 1 cycles

/ccpvqz/PBE0

pentane.log

SCF Done: $\mathrm{E}(\mathrm{RPBE} 1 \mathrm{PBE})=-197.597770538 \quad$ A.U. after 1 cycles

/ccpvqz/PBE0

propane.log

SCF Done: $\mathrm{E}(\mathrm{RPBE} 1 \mathrm{PBE})=-119.036576412 \quad$ A.U. after 1 cycles

/ccpvqz/M06

2233tetra.out

SCF Done: $\mathrm{E}(\mathrm{RM} 06)=-315.585537345 \quad$ A.U. after 1 cycles

/ccpvqz/M06

22dime.out

SCF Done: $\mathrm{E}(\mathrm{RM} 06)=-236.990815065 \quad$ A.U. after 1 cycles

/ccpvqz/M06

butane.out

SCF Done: $\mathrm{E}(\mathrm{RM} 06)=-158.389937905$ A.U. after 1 cycles

/ccpvqz/M06

ethane.out

SCF Done: $\mathrm{E}(\mathrm{RM} 06)=-79.7935515961$ A.U. after 1 cycles 
/ccpvqz/M06

heptane.out

SCF Done: $E(R M 06)=-276.284289680 \quad$ A.U. after 1 cycles

/ccpvqz/M06

hexane.out

SCF Done: $\mathrm{E}(\mathrm{RM} 06)=-236.986168384$ A.U. after 1 cycles

/ccpvqz/M06

isobutane.out

SCF Done: $\mathrm{E}(\mathrm{RM} 06)=-158.392217222 \quad$ A.U. after 1 cycles

/ccpvqz/M06

M06223tri.out

SCF Done: $\mathrm{E}(\mathrm{RM} 06)=-276.287951371 \quad$ A.U. after 1 cycles

/ccpvqz/M06

Methane.out

SCF Done: $E(R M 06)=-40.4983701687$ A.U. after 1 cycles

/ccpvqz/M06

neopentane.out

SCF Done: $\mathrm{E}(\mathrm{RM} 06)=-197.694182697$ A.U. after 1 cycles

/ccpvqz/M06

octane.out

SCF Done: $\mathrm{E}(\mathrm{RM} 06)=-315.582409438$ A.U. after 1 cycles

/ccpvqz/M06

pentane.out

SCF Done: $\mathrm{E}(\mathrm{RM} 06)=-197.688048923$ A.U. after 1 cycles

/ccpvqz/M06

Propane.out

SCF Done: $\mathrm{E}(\mathrm{RM} 06)=-119.091713178 \quad$ A.U. after 1 cycles

/ccpvqz/B3LYPD3

2233tetra.out

SCF Done: $\mathrm{E}(\mathrm{RB} 3 \mathrm{LYP})=-315.869685265$ A.U. after 1 cycles

/ccpvqz/B3LYPD3

223tri.out

SCF Done: $E(R B 3 L Y P)=-276.539078987 \quad$ A.U. after 1 cycles

/ccpvqz/B3LYPD3

22dime.out

SCF Done: $E(R B 3 L Y P)=-237.208006972 \quad$ A.U. after 1 cycles

/ccpvqz/B3LYPD3 
butane.out

SCF Done: $\mathrm{E}(\mathrm{RB} 3 \mathrm{LYP})=-158.539378626$ A.U. after 1 cycles

/ccpvqz/B3LYPD3

ethane.out

SCF Done: $\mathrm{E}(\mathrm{RB} 3 \mathrm{LYP})=-79.8723348567$ A.U. after 1 cycles

/ccpvqz/B3LYPD3

heptane.out

SCF Done: $E($ RB3LYP $)=-276.539903062$ A.U. after 1 cycles

/ccpvqz/B3LYPD3

hexane.out

SCF Done: $E(R B 3 L Y P)=-237.206385741 \quad$ A.U. after 1 cycles

/ccpvqz/B3LYPD3

isobutane.out

SCF Done: $\mathrm{E}(\mathrm{RB} 3 \mathrm{LYP})=-158.541054624$ A.U. after 1 cycles

/ccpvqz/B3LYPD3

Methane.out

SCF Done: $\mathrm{E}(\mathrm{RB} 3 \mathrm{LYP})=-40.5416554705$ A.U. after 1 cycles

/ccpvqz/B3LYPD3

neopentane.out

SCF Done: $\mathrm{E}(\mathrm{RB} 3 \mathrm{LYP})=-197.876744309$ A.U. after 1 cycles

/ccpvqz/B3LYPD3

octane.out

SCF Done: $\mathrm{E}(\mathrm{RB} 3 \mathrm{LYP})=-315.873423367$ A.U. after 1 cycles

/ccpvqz/B3LYPD3

pentane.out

SCF Done: $E($ RB3LYP $)=-197.872870226$ A.U. after 1 cycles

/ccpvqz/B3LYPD3

Propane.out

SCF Done: $E($ RB3LYP $)=-119.205841182 \quad$ A.U. after 1 cycles

/ccpvqz/CAMB3LYP

2233tetra.out

SCF Done: $\mathrm{E}(\mathrm{RCAM}-\mathrm{B} 3 \mathrm{LYP})=-315.637733100 \quad$ A.U. after 1 cycles

/ccpvqz/CAMB3LYP

22dime.out

SCF Done: E(RCAM-B3LYP $)=-237.034185761 \quad$ A.U. after 1 cycles

/ccpvqz/CAMB3LYP

butane.out

SCF Done: E(RCAM-B3LYP $)=-158.422806413$ A.U. after 1 cycles 
/ccpvqz/CAMB3LYP

CAM223trime.out

SCF Done: E(RCAM-B3LYP $)=-276.336465770 \quad$ A.U. after 1 cycles

/ccpvqz/CAMB3LYP

ethane.out

SCF Done: E(RCAM-B3LYP $)=-79.8108969134$ A.U. after 1 cycles

/ccpvqz/CAMB3LYP

heptane.out

SCF Done: E(RCAM-B3LYP) $=-276.340264309$ A.U. after 1 cycles

/ccpvqz/CAMB3LYP

hexane.out

SCF Done: E(RCAM-B3LYP $)=-237.034442157$ A.U. after 1 cycles

/ccpvqz/CAMB3LYP

isobutane.out

SCF Done: $\mathrm{E}(\mathrm{RCAM}-\mathrm{B} 3 \mathrm{LYP})=-158.424126891 \quad$ A.U. after 1 cycles

/ccpvqz/CAMB3LYP

Methane.out

SCF Done: E(RCAM-B3LYP $)=-40.5072923705$ A.U. after 1 cycles

/ccpvqz/CAMB3LYP

neopentane.out

SCF Done: E(RCAM-B3LYP $)=-197.731311946$ A.U. after 1 cycles

/ccpvqz/CAMB3LYP

octane.out

SCF Done: E(RCAM-B3LYP $)=-315.646086699$ A.U. after 1 cycles

/ccpvqz/CAMB3LYP

pentane.out

SCF Done: E(RCAM-B3LYP $)=-197.728617956$ A.U. after 1 cycles

/ccpvqz/CAMB3LYP

Propane.out

SCF Done: E(RCAM-B3LYP $)=-119.116895430 \quad$ A.U. after 1 cycles

/ccpvqz/WB97XD/

2233tetra.out

SCF Done: $\mathrm{E}(\mathrm{RwB} 97 \mathrm{XD})=-315.751521669$ A.U. after 1 cycles

/ccpvqz/WB97XD/

223tri.out

SCF Done: $\mathrm{E}(\mathrm{RwB} 97 \mathrm{XD})=-276.434417302 \quad$ A.U. after 1 cycles

/ccpvqz/WB97XD/ 
22dime.out

SCF Done: $\mathrm{E}(\mathrm{RwB} 97 \mathrm{XD})=-237.117196732 \quad$ A.U. after 1 cycles

/ccpvqz/WB97XD/

butane.out

SCF Done: $\mathrm{E}(\mathrm{RwB} 97 \mathrm{XD})=-158.477029347 \quad$ A.U. after 1 cycles

/ccpvqz/WB97XD/

ethane.out

SCF Done: $\mathrm{E}(\mathrm{RwB} 97 \mathrm{XD})=-79.8392243501 \quad$ A.U. after 1 cycles

/ccpvqz/WB97XD/

heptane.out

SCF Done: $\mathrm{E}(\mathrm{RwB} 97 \mathrm{XD})=-276.433897761$

A.U. after 1 cycles

/ccpvqz/WB97XD/

hexane.out

SCF Done: $\mathrm{E}(\mathrm{RwB} 97 \mathrm{XD})=-237.114927977$

A.U. after 1 cycles

/ccpvqz/WB97XD/

isobutane.out

SCF Done: $\mathrm{E}(\mathrm{RwB} 97 \mathrm{XD})=-158.478696005 \quad$ A.U. after 1 cycles

/ccpvqz/WB97XD/

Methane.out

SCF Done: $\mathrm{E}(\mathrm{RwB} 97 \mathrm{XD})=-40.5232167777 \quad$ A.U. after 1 cycles

/ccpvqz/WB97XD/

neopentane.out

SCF Done: $\mathrm{E}(\mathrm{RwB} 97 \mathrm{XD})=-197.799933213 \quad$ A.U. after 1 cycles

/ccpvqz/WB97XD/

octane.out

SCF Done: E(RwB97XD) = -315.752860426

A.U. after 1 cycles

/ccpvqz/WB97XD/

pentane.out

SCF Done: $\mathrm{E}(\mathrm{RwB} 97 \mathrm{XD})=-197.795975934$ A.U. after 1 cycles

/ccpvqz/WB97XD/

Propane.out

SCF Done: $\mathrm{E}(\mathrm{RwB} 97 \mathrm{XD})=-119.158076885 \quad$ A.U. after 1 cycles

/ccpvqz/B3LYP

2233tetra.out

SCF Done: $\mathrm{E}(\mathrm{RB} 3 \mathrm{LYP})=-315.508149351 \quad$ A.U. after 1 cycles

/ccpvqz/B3LYP

223tri.out

SCF Done: $E(R B 3 L Y P)=-276.224077654$ A.U. after 1 cycles 
/ccpvqz/B3LYP

22dime.out

SCF Done: $E(R B 3 L Y P)=-236.938456781 \quad$ A.U. after 1 cycles

/ccpvqz/B3LYP

butane.out

SCF Done: $\mathrm{E}(\mathrm{RB} 3 \mathrm{LYP})=-158.360931014$ A.U. after 1 cycles

/ccpvqz/B3LYP

ethane.out

SCF Done: $\mathrm{E}(\mathrm{RB} 3 \mathrm{LYP})=-79.7822892981 \quad$ A.U. after 1 cycles

/ccpvqz/B3LYP

heptane.out

SCF Done: $E(R B 3 L Y P)=-276.228559176 \quad$ A.U. after 1 cycles

/ccpvqz/B3LYP

hexane.out

SCF Done: $\mathrm{E}(\mathrm{RB} 3 \mathrm{LYP})=-236.939328186 \quad$ A.U. after 1 cycles

/ccpvqz/B3LYP

isobutane.out

SCF Done: $E(R B 3 L Y P)=-158.362160293$ A.U. after 1 cycles

/ccpvqz/B3LYP

Methane.out

SCF Done: $E(R B 3 L Y P)=-40.4952526147 \quad$ A.U. after 1 cycles

/ccpvqz/B3LYP

neopentane.out

SCF Done: $E($ RB3LYP $)=-197.652107672 \quad$ A.U. after 1 cycles

/ccpvqz/B3LYP

octane.out

SCF Done: $\mathrm{E}(\mathrm{RB} 3 \mathrm{LYP})=-315.517791462 \quad$ A.U. after 1 cycles

/ccpvqz/B3LYP

pentane.out

SCF Done: $\mathrm{E}(\mathrm{RB} 3 \mathrm{LYP})=-197.650113685 \quad$ A.U. after 1 cycles

/ccpvqz/B3LYP

Propane.out

SCF Done: $E($ RB3LYP $)=-119.071686550 \quad$ A.U. after 1 cycles

/ccpvqz/B2PLYP

2233tetrametbut.log: E2(B2PLYP $)=-0.4935417239 \mathrm{D}+00 \mathrm{E}(\mathrm{B} 2 \mathrm{PLYP})=$

$0.31554070729453 \mathrm{D}+03$

223trimethbut.log: E2(B2PLYP) $=-0.4306352163 \mathrm{D}+00 \mathrm{E}(\mathrm{B} 2 \mathrm{PLYP})=-$

$0.27625042740379 \mathrm{D}+03$ 
22dimethbut.log: E2(B2PLYP) $=-0.3688251829 \mathrm{D}+00 \mathrm{E}(\mathrm{B} 2 \mathrm{PLYP})=\quad-$ $0.23695963137824 \mathrm{D}+03$

butane.log: $\mathrm{E} 2(\mathrm{~B} 2 \mathrm{PLYP})=-0.2458409859 \mathrm{D}+00 \mathrm{E}(\mathrm{B} 2 \mathrm{PLYP})=-0.15837137011382 \mathrm{D}+03$

Ethane.log: E2(B2PLYP $)=-0.1263672274 \mathrm{D}+00 \mathrm{E}(\mathrm{B} 2 \mathrm{PLYP})=-0.79783950561283 \mathrm{D}+02$ heptane.log: $\mathrm{E} 2(\mathrm{~B} 2 \mathrm{PLYP})=-0.4252459679 \mathrm{D}+00 \mathrm{E}(\mathrm{B} 2 \mathrm{PLYP})=\quad-$

$0.27625227141594 \mathrm{D}+03$

hexane.log: E2(B2PLYP $)=-0.3654398838 \mathrm{D}+00 \mathrm{E}(\mathrm{B} 2 \mathrm{PLYP})=-0.23695863207428 \mathrm{D}+03$

isobutane.log: $\mathrm{E} 2(\mathrm{~B} 2 \mathrm{PLYP})=-0.2466527390 \mathrm{D}+00 \mathrm{E}(\mathrm{B} 2 \mathrm{PLYP})=-$

$0.15837296935543 \mathrm{D}+03$

Methane.log: E2(B2PLYP) $=-0.6750081049 \mathrm{D}-01 \mathrm{E}(\mathrm{B} 2 \mathrm{PLYP})=\quad-$

$0.40492860683458 \mathrm{D}+02$

neopentane.log: E2(B2PLYP $)=-0.3081433130 \mathrm{D}+00 \mathrm{E}(\mathrm{B} 2 \mathrm{PLYP})=\quad-$

$0.19766865941352 \mathrm{D}+03$

octane.log: E2(B2PLYP) $=-0.4850528526 \mathrm{D}+00 \mathrm{E}(\mathrm{B} 2 \mathrm{PLYP})=-0.31554591221842 \mathrm{D}+03$

pentane.log: E2(B2PLYP $)=-0.3056361750 \mathrm{D}+00 \mathrm{E}(\mathrm{B} 2 \mathrm{PLYP})=\quad-$

$0.19766499255104 \mathrm{D}+03$

propane.log: E2(B2PLYP) $=-0.1860617786 \mathrm{D}+00 \mathrm{E}(\mathrm{B} 2 \mathrm{PLYP})=\quad-$

$0.11907767237074 \mathrm{D}+03$

/ccpvqz/PBE0DH

2233tetrametbut.log: E2(PBE0DH $)=-0.2310702745 \mathrm{D}+00 \mathrm{E}(\mathrm{PBE} 0 \mathrm{DH})=\quad-$

$0.31547322873018 \mathrm{D}+03$

223trimethbut.log: E2(PBE0DH $)=-0.2015398400 \mathrm{D}+00 \mathrm{E}(\mathrm{PBE} 0 \mathrm{DH})=$

$0.27619099789100 \mathrm{D}+03$

22dimethbut.log: $\mathrm{E} 2(\mathrm{PBE} 0 \mathrm{DH})=-0.1725610171 \mathrm{D}+00 \mathrm{E}(\mathrm{PBE} 0 \mathrm{DH})=\quad-$

$0.23690845041445 \mathrm{D}+03$

butane.log: $\mathrm{E} 2(\mathrm{PBE} 0 \mathrm{DH})=-0.1149288892 \mathrm{D}+00 \mathrm{E}(\mathrm{PBE0DH})=-$

$0.15833692195089 \mathrm{D}+03$

ethane.log: $\mathrm{E} 2(\mathrm{PBE} 0 \mathrm{DH})=-0.5904306981 \mathrm{D}-01 \mathrm{E}(\mathrm{PBE} 0 \mathrm{DH})=-0.79766373345657 \mathrm{D}+02$

heptane.log: $\mathrm{E} 2(\mathrm{PBE0DH})=-0.1988548143 \mathrm{D}+00 \mathrm{E}(\mathrm{PBE} 0 \mathrm{DH})=\quad$

$0.27619249715931 \mathrm{D}+03$

hexane.log: $\mathrm{E} 2(\mathrm{PBE} 0 \mathrm{DH})=-0.1708768683 \mathrm{D}+00 \mathrm{E}(\mathrm{PBE} 0 \mathrm{DH})=-$

$0.23690730102353 \mathrm{D}+03$

isobutane.log: $\mathrm{E} 2(\mathrm{PBE} 0 \mathrm{DH})=-0.1153297143 \mathrm{D}+00 \mathrm{E}(\mathrm{PBE} 0 \mathrm{DH})=-$

$0.15833849651071 \mathrm{D}+03$

methane.log: $\mathrm{E} 2(\mathrm{PBE} 0 \mathrm{DH})=-0.3153536098 \mathrm{D}-01 \mathrm{E}(\mathrm{PBE} 0 \mathrm{DH})=-$

$0.40483694306088 \mathrm{D}+02$

neopentane.log: $\mathrm{E} 2(\mathrm{PBE} 0 \mathrm{DH})=-0.1441417608 \mathrm{D}+00 \mathrm{E}(\mathrm{PBE} 0 \mathrm{DH})=\quad-$

$0.19762574591058 \mathrm{D}+03$

octane.log: $\mathrm{E} 2(\mathrm{PBE} 0 \mathrm{DH})=-0.2268331694 \mathrm{D}+00 \mathrm{E}(\mathrm{PBE} 0 \mathrm{DH})=\quad-$

$0.31547769404359 \mathrm{D}+03$

pentane.log: $\mathrm{E} 2(\mathrm{PBE} 0 \mathrm{DH})=-0.1429002232 \mathrm{D}+00 \mathrm{E}(\mathrm{PBE} 0 \mathrm{DH})=\quad-$

$0.19762210388314 \mathrm{D}+03$

propane.log: $\mathrm{E} 2(\mathrm{PBE} 0 \mathrm{DH})=-0.8696421655 \mathrm{D}-01 \mathrm{E}(\mathrm{PBE} 0 \mathrm{DH})=\quad$

$0.11905164999095 \mathrm{D}+03$

/ccpvqz/PBEQIDH

223trimethbut.log: E2(PBEQIDH) $=-0.4977729614 \mathrm{D}+00 \mathrm{E}(\mathrm{PBEQIDH})=$

$0.27615342388360 \mathrm{D}+03$ 


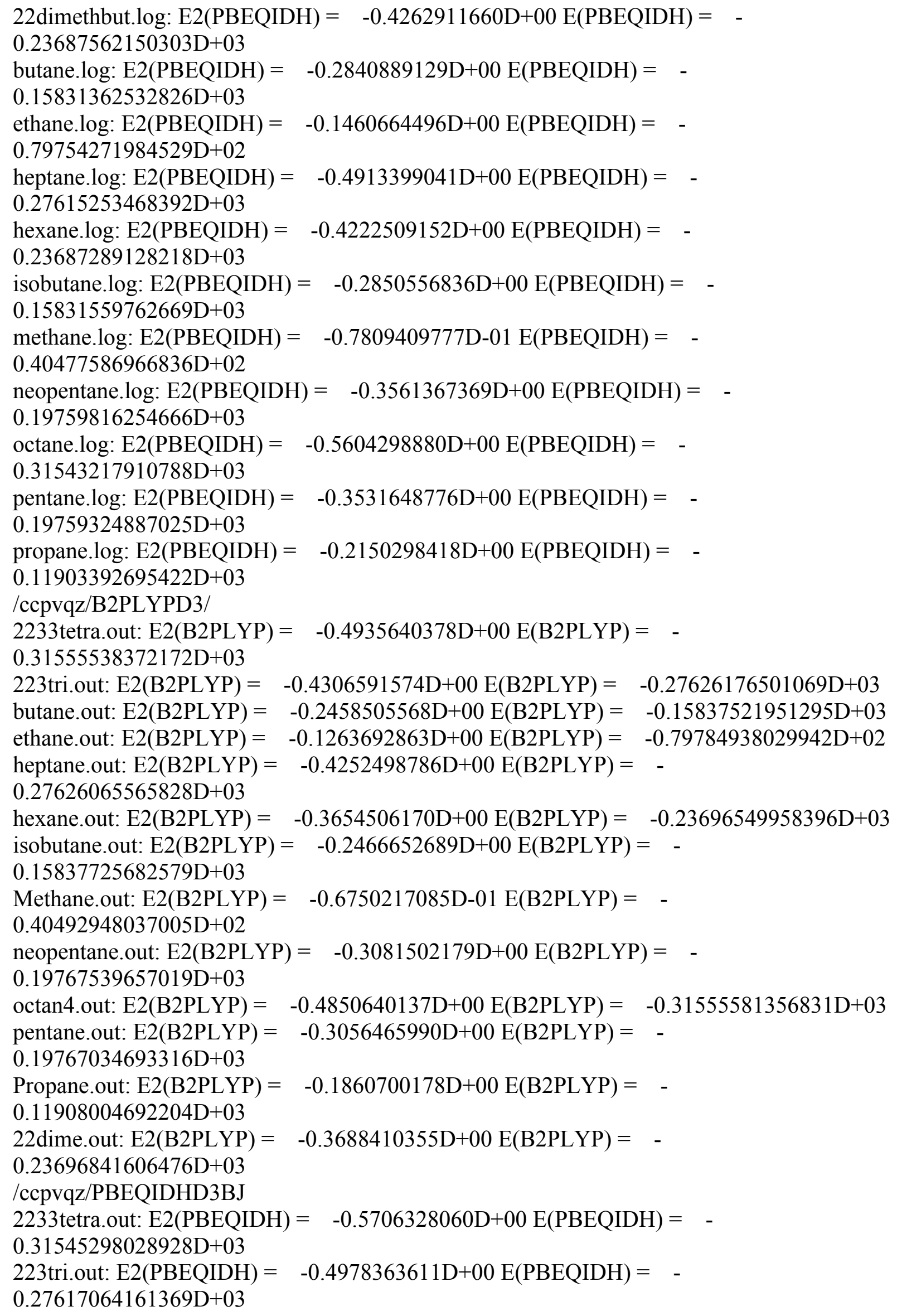




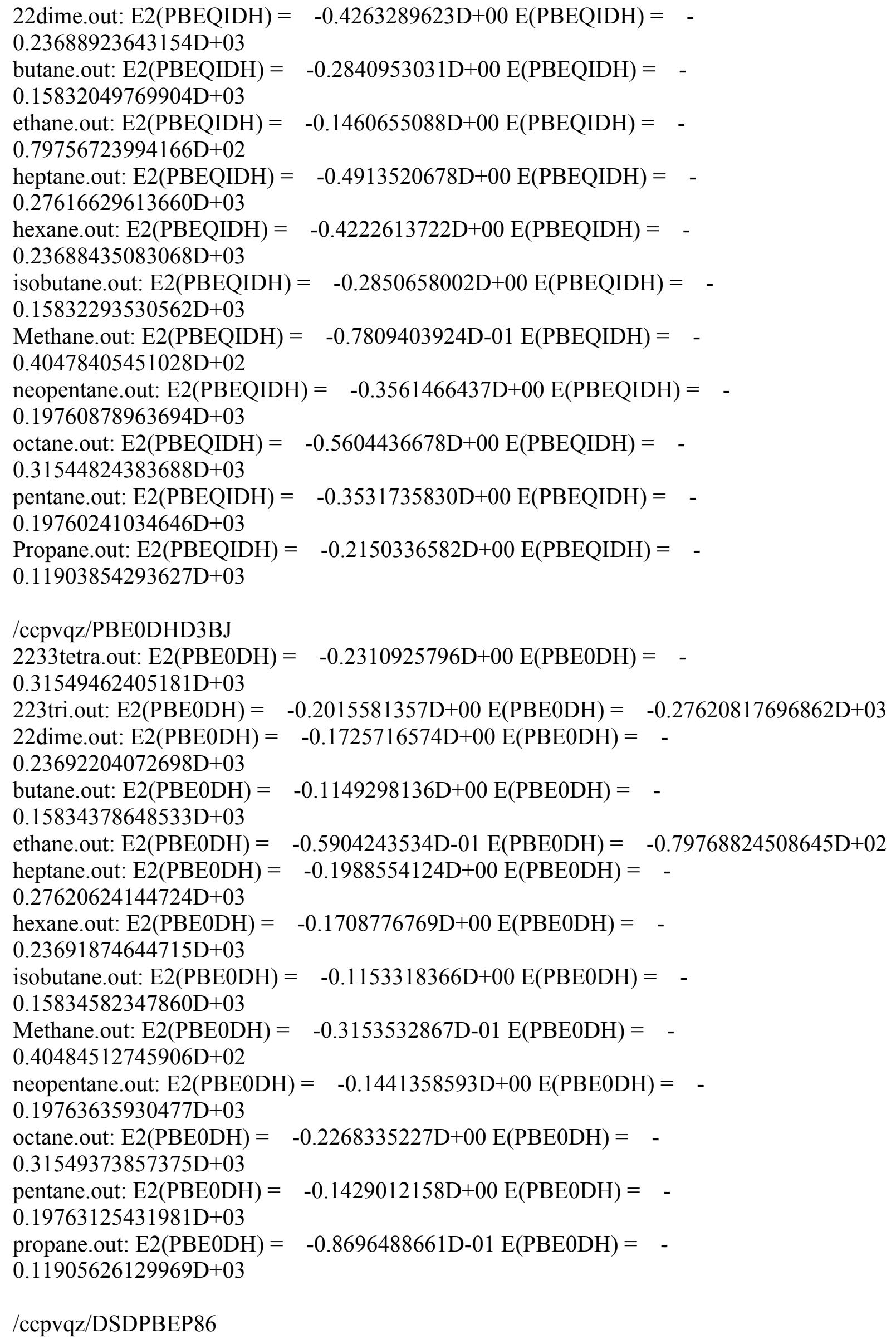




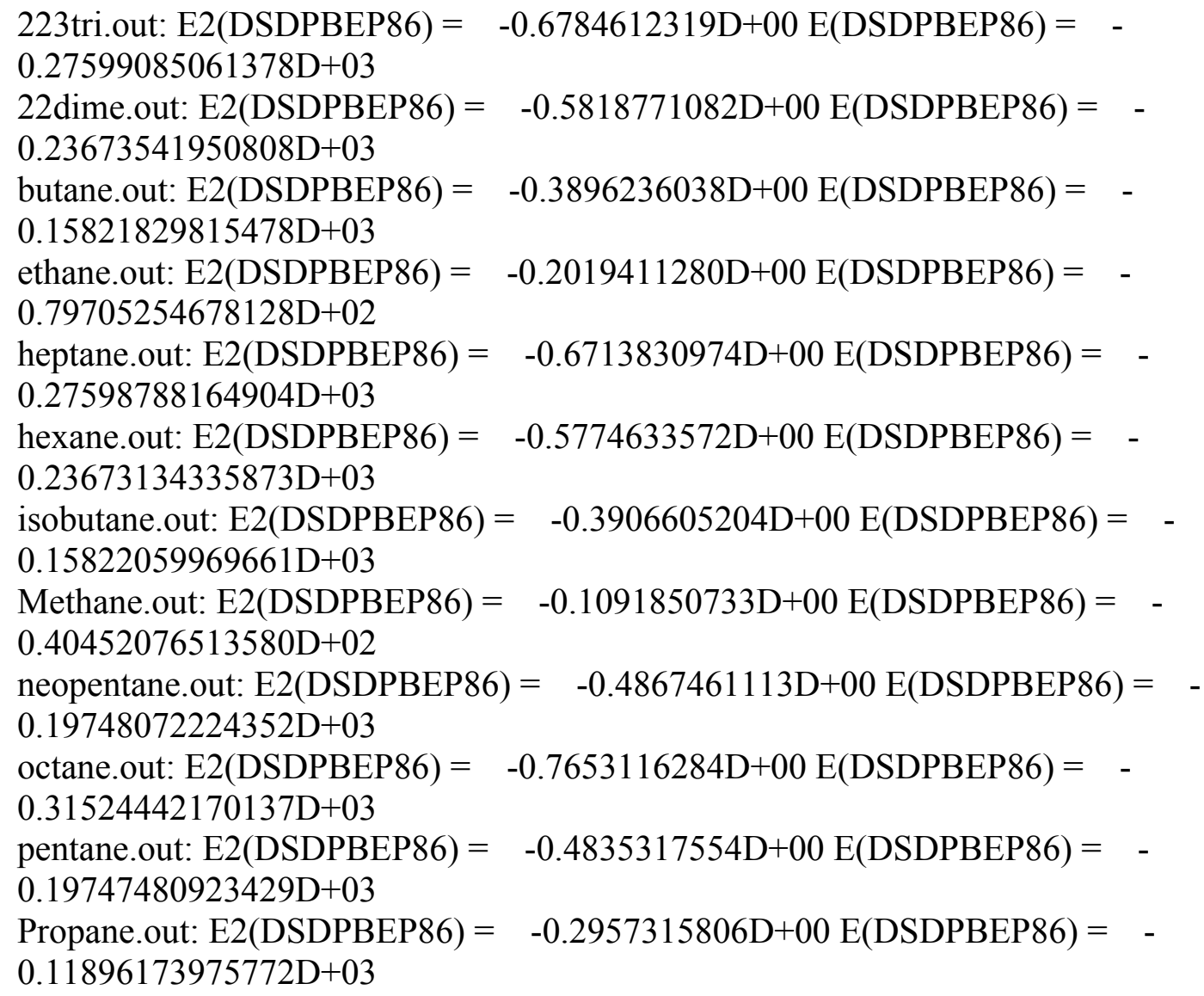

S3: For BSR36 dataset

The geometris from http://www.thch.uni-

bonn.de/tc.old/downloads/GMTKN/GMTKN55/BSR36.html

The energies of the main text considered as follows:

Basis set: DH-SVPD

Functional: M06

C1.out: SCF Done: $\mathrm{E}(\mathrm{RM} 06)=-390.177183841 \quad$ A.U.

C2H6.out: SCF Done: $\mathrm{E}(\mathrm{RM} 06)=-79.7054290106$ A.U

C2.out: SCF Done: $\mathrm{E}(\mathrm{RM} 06)=-468.702581452$ A.U. 
C3.out: SCF Done: $\mathrm{E}(\mathrm{RM} 06)=-468.691044276$ C4.out: SCF Done: $\mathrm{E}(\mathrm{RM} 06)=-547.227719967$ C5.out: SCF Done: $\mathrm{E}(\mathrm{RM} 06)=-544.820445723$ CH4.out: SCF Done: $E(R M 06)=-40.4518198303$ H10.out: SCF Done: $E($ RM06 $)=-275.991883805$ H11.out: SCF Done: $E(R M 06)=-275.994541755$ H12.out: SCF Done: $E(R M 06)=-275.994331260$ H13.out: SCF Done: $E(R M 06)=-275.990656723$ H14.out: SCF Done: $E(R M 06)=-275.988980207$ H15.out: SCF Done: $E(R M 06)=-315.251569205$ H1.out: SCF Done: $\mathrm{E}(\mathrm{RM} 06)=-236.735767421$ H2.out: SCF Done: $\mathrm{E}(\mathrm{RM} 06)=-236.734551239$ H3.out: SCF Done: $\mathrm{E}(\mathrm{RM} 06)=-236.738138051$ H4.out: SCF Done: $E(R M 06)=-236.734044225$ H5.out: SCF Done: $E(R M 06)=-236.732459884$ H6.out: SCF Done: E(RM06) $=-275.989212321$ H7.out: SCF Done: $\mathrm{E}(\mathrm{RM} 06)=-275.993888279$ H8.out: SCF Done: $E(R M 06)=-275.991362286$ H9.out: SCF Done: $E(R M 06)=-275.994654025$ r10.out: SCF Done: $\mathrm{E}(\mathrm{RM} 06)=-391.364735327$ r11.out: SCF Done: $\mathrm{E}(\mathrm{RM} 06)=-391.350810587$ r12.out: SCF Done: $E(R M 06)=-391.358175174$ r13.out: SCF Done: $E(R M 06)=-391.376336663$ r14.out: SCF Done: $\mathrm{E}(\mathrm{RM} 06)=-391.372281587$ r15.out: SCF Done: $E(R M 06)=-469.886513762$ r16.out: SCF Done: $E(R M 06)=-471.080627534$ r1.out: SCF Done: $\mathrm{E}(\mathrm{RM} 06)=-196.269517500$ r2.out: SCF Done: $E(R M 06)=-235.537567743$ r3.out: SCF Done: $E(R M 06)=-235.529182443$ r4.out: SCF Done: $\mathrm{E}(\mathrm{RM} 06)=-274.797483485$ r5.out: SCF Done: $\mathrm{E}(\mathrm{RM} 06)=-274.789677469$ r6.out: SCF Done: $\mathrm{E}(\mathrm{RM} 06)=-274.788368536$ r7.out: SCF Done: $\mathrm{E}(\mathrm{RM} 06)=-274.787574849$ r8.out: SCF Done: $\mathrm{E}(\mathrm{RM} 06)=-274.789600403$ r9.out: SCF Done: $E($ RM06 $)=-274.787800537$

\section{M06D3/DHSVPD}

C1.out: SCF Done: $E(R M 06)=-390.180915970$ C2H6.out: SCF Done: E(RM06) $=-79.7055432331$ C2.out: SCF Done: $E(R M 06)=-468.708056010$ C3.out: SCF Done: $E(R M 06)=-468.696733815$ C4.out: SCF Done: $E(R M 06)=-547.235090408$ C5.out: SCF Done: $\mathrm{E}(\mathrm{RM} 06)=-544.826794552$ CH4.out: SCF Done: $\mathrm{E}(\mathrm{RM} 06)=-40.4518221602$ H10.out: SCF Done: $E(R M 06)=-275.994497722$ H11.out: SCF Done: $\mathrm{E}(\mathrm{RM} 06)=-275.997372551$ H12.out: SCF Done: $E(R M 06)=-275.997117530$ H13.out: SCF Done: $E(R M 06)=-275.992935310$ H14.out: SCF Done: E(RM06) $=-275.991513822$ H15.out: SCF Done: $E(R M 06)=-315.255320717$
A.U.

A.U.

A.U.

A.U.

A.U.

A.U.

A.U.

A.U.

A.U.

A.U.

A.U.

A.U.

A.U.

A.U.

A.U.

A.U.

A.U.

A.U.

A.U.

A.U.

A.U.

A.U.

A.U.

A.U.

A.U.

A.U.

A.U.

A.U.

A.U.

A.U.

A.U.

A.U.

A.U.

A.U.

A.U.

A.U

A.U

A.U

A.U

A.U

A.U 
H1.out: SCF Done: $\mathrm{E}(\mathrm{RM} 06)=-236.737806294$ A.U. H2.out: SCF Done: $E(R M 06)=-236.736291578$ A.U. H3.out: SCF Done: $E(R M 06)=-236.740185380$ A.U. H4.out: SCF Done: $E(R M 06)=-236.735875184$ A.U. H5.out: SCF Done: $\mathrm{E}(\mathrm{RM} 06)=-236.733948676$ A.U. H6.out: SCF Done: $\mathrm{E}(\mathrm{RM} 06)=-275.991091374$ A.U. H7.out: SCF Done: $\mathrm{E}(\mathrm{RM} 06)=-275.996374548$ A.U. H8.out: SCF Done: $\mathrm{E}(\mathrm{RM} 06)=-275.993524214$ A.U. H9.out: SCF Done: $E(R M 06)=-275.997195845$ A.U. r10.out: SCF Done: $\mathrm{E}(\mathrm{RM} 06)=-391.368694977 \quad$ A.U r11.out: SCF Done: $\mathrm{E}(\mathrm{RM} 06)=-391.355278048$ A.U r12.out: SCF Done: $E(R M 06)=-391.362076522 \quad$ A.U r13.out: SCF Done: $E(R M 06)=-391.380082499$ A.U r14.out: SCF Done: $\mathrm{E}(\mathrm{RM} 06)=-391.376281267 \quad$ A.U r15.out: SCF Done: $\mathrm{E}(\mathrm{RM} 06)=-469.891638512 \quad$ A.U r16.out: SCF Done: $E(R M 06)=-471.085000188$ A.U r1.out: SCF Done: $\mathrm{E}(\mathrm{RM} 06)=-196.270421864$ A.U. r2.out: SCF Done: $E(R M 06)=-235.539069295$ A.U. r3.out: SCF Done: $E(R M 06)=-235.530626471$ A.U. r4.out: SCF Done: $\mathrm{E}(\mathrm{RM} 06)=-274.799570073$ A.U. r5.out: SCF Done: $E(R M 06)=-274.791910764$ A.U. r6.out: SCF Done: $\mathrm{E}(\mathrm{RM} 06)=-274.790403553$ A.U. r7.out: SCF Done: $E(R M 06)=-274.789782431$ A.U. r8.out: SCF Done: $E(R M 06)=-274.791725654$ A.U. r9.out: SCF Done: $E(R M 06)=-274.789859603$ A.U. WB97XD/DHSVPD

C1.out: SCF Done: $\mathrm{E}(\mathrm{RwB} 97 \mathrm{XD})=-390.380521176$ C2H6.out: SCF Done: $E($ RwB97XD $)=-79.7533876590$ C2.out: SCF Done: $E($ RwB97XD $)=-468.947271848$ C3.out: SCF Done: $E($ RwB97XD $)=-468.936748782$ C4.out: SCF Done: $\mathrm{E}(\mathrm{RwB} 97 \mathrm{XD})=-547.514311254$ C5.out: SCF Done: $\mathrm{E}(\mathrm{RwB} 97 \mathrm{XD})=-545.099721782$ CH4.out: SCF Done: $E($ RwB97XD $)=-40.4782499393$ H10.out: SCF Done: $E(R w B 97 X D)=-276.146396249$ H11.out: SCF Done: $E($ RwB97XD $)=-276.147361392$ H12.out: SCF Done: $E(R w B 97 X D)=-276.147861826$ H13.out: SCF Done: $E($ RwB97XD $)=-276.145907106$ H14.out: SCF Done: $E($ RwB97XD $)=-276.144278133$ H15.out: SCF Done: $E(R w B 97 X D)=-315.424606481$ H1.out: SCF Done: $E(R w B 97 X D)=-236.868653877$ H2.out: SCF Done: $E(R w B 97 X D)=-236.868226044$ H3.out: SCF Done: $E(R w B 97 X D)=-236.870060297$ H4.out: SCF Done: $E(R w B 97 X D)=-236.867506830$ H5.out: SCF Done: $E(R w B 97 X D)=-236.866897935$ H6.out: SCF Done: $E($ RwB97XD $)=-276.145359916$ H7.out: SCF Done: E(RwB97XD) $=-276.148463108$ H8.out: SCF Done: $E($ RwB97XD $)=-276.146753610$ H9.out: SCF Done: $E(R w B 97 X D)=-276.148397072$ r10.out: SCF Done: $E(R w B 97 X D)=-391.573483037$ r11.out: SCF Done: $E($ RwB97XD $)=-391.560874961$ 
r12.out: SCF Done: $E($ RwB97XD $)=-391.568010846$ r13.out: SCF Done: $E($ RwB97XD $)=-391.584986436$ r14.out: SCF Done: $E(R w B 97 X D)=-391.581681376$ r15.out: SCF Done: $E($ RwB97XD) $=-470.138554549$ r16.out: SCF Done: $E($ RwB97XD $)=-471.338771885$ r1.out: SCF Done: $E(R w B 97 X D)=-196.376627445$ r2.out: SCF Done: $E(R w B 97 X D)=-235.665858680$ r3.out: SCF Done: $E(R w B 97 X D)=-235.657778366$ r4.out: SCF Done: $E(R w B 97 X D)=-274.947022970$ r5.out: SCF Done: $\mathrm{E}(\mathrm{RwB} 97 \mathrm{XD})=-274.938675930$ r6.out: SCF Done: $E($ RwB97XD $)=-274.938621132$ r7.out: SCF Done: $\mathrm{E}(\mathrm{RwB} 97 \mathrm{XD})=-274.937321050$ r8.out: SCF Done: $E(R w B 97 X D)=-274.939442324$ r9.out: SCF Done: $E($ RwB97XD $)=-274.938138146$ B2PLYP/DHSVPD

C1.out: E2(B2PLYP) $=-0.4915492906 \mathrm{D}+00 \mathrm{E}(\mathrm{B} 2 \mathrm{PLYP})=$ C2H6.out: E2(B2PLYP $)=-0.1054852799 \mathrm{D}+00 \mathrm{E}(\mathrm{B} 2 \mathrm{PLYP})=$ C2.out: $\mathrm{E} 2(\mathrm{~B} 2 \mathrm{PLYP})=-0.5965694853 \mathrm{D}+00 \mathrm{E}(\mathrm{B} 2 \mathrm{PLYP})=$ C3.out: E2(B2PLYP) $=$ C4.out: E2(B2PLYP) $=$ C5.out: E2(B2PLYP) $=$ CH4.out: E2(B2PLYP) $=$ H10.out: E2(B2PLYP) = H11.out: E2(B2PLYP) $=$ H12.out: E2(B2PLYP) $=$ H13.out: E2(B2PLYP) $=$ H14.out: E2(B2PLYP) $=$ H15.out: E2(B2PLYP) $=$ H1.out: E2(B2PLYP) = H2.out: E2(B2PLYP) $=$ H3.out: E2(B2PLYP) = H4.out: E2(B2PLYP) = H5.out: E2(B2PLYP) $=$ H6.out: E2(B2PLYP) = H7.out: E2(B2PLYP) $=$ H8.out: E2(B2PLYP) = H9.out: E2(B2PLYP) $=$ r10.out: E2(B2PLYP) = r11.out: E2(B2PLYP) = r12.out: E2(B2PLYP) = r13.out: E2(B2PLYP) = r14.out: E2(B2PLYP) = r15.out: E2(B2PLYP) = r16.out: E2(B2PLYP) = r1.out: E2(B2PLYP) = r2.out: E2(B2PLYP) = r3.out: E2(B2PLYP) = r4.out: E2(B2PLYP) = r5.out: E2(B2PLYP) $=$ r6.out: E2(B2PLYP) =
$-0.5965694853 \mathrm{D}+00 \mathrm{E}(\mathrm{B} 2 \mathrm{PLYP})=$ $-0.5977033488 \mathrm{D}+00 \mathrm{E}(\mathrm{B} 2 \mathrm{PLYP})=$ $-0.7017729962 \mathrm{D}+00 \mathrm{E}(\mathrm{B} 2 \mathrm{PLYP})=$ $-0.6842646410 \mathrm{D}+00 \mathrm{E}(\mathrm{B} 2 \mathrm{PLYP})=$ $-0.5642992934 \mathrm{D}-01 \mathrm{E}(\mathrm{B} 2 \mathrm{PLYP})=$ $-0.3595254739 \mathrm{D}+00 \mathrm{E}(\mathrm{B} 2 \mathrm{PLYP})=$ $-0.3617980704 \mathrm{D}+00 \mathrm{E}(\mathrm{B} 2 \mathrm{PLYP})=$ $-0.3609043114 \mathrm{D}+00 \mathrm{E}(\mathrm{B} 2 \mathrm{PLYP})=$ $-0.3578637159 \mathrm{D}+00 \mathrm{E}(\mathrm{B} 2 \mathrm{PLYP})=$ $-0.3583130163 \mathrm{D}+00 \mathrm{E}(\mathrm{B} 2 \mathrm{PLYP})=$ $-0.4154010686 \mathrm{D}+00 \mathrm{E}(\mathrm{B} 2 \mathrm{PLYP})=$ $-0.3088081164 \mathrm{D}+00 \mathrm{E}(\mathrm{B} 2 \mathrm{PLYP})=$ $-0.3072216295 \mathrm{D}+00 \mathrm{E}(\mathrm{B} 2 \mathrm{PLYP})=$ $-0.3095584235 \mathrm{D}+00 \mathrm{E}(\mathrm{B} 2 \mathrm{PLYP})=$ $-0.3076457117 \mathrm{D}+00 \mathrm{E}(\mathrm{B} 2 \mathrm{PLYP})=$ $-0.3057569701 \mathrm{D}+00 \mathrm{E}(\mathrm{B} 2 \mathrm{PLYP})=$ $-0.3558784437 \mathrm{D}+00 \mathrm{E}(\mathrm{B} 2 \mathrm{PLYP})=$ $-0.3590757441 \mathrm{D}+00 \mathrm{E}(\mathrm{B} 2 \mathrm{PLYP})=$ $-0.3573933784 \mathrm{D}+00 \mathrm{E}(\mathrm{B} 2 \mathrm{PLYP})=$ $-0.3598707440 \mathrm{D}+00 \mathrm{E}(\mathrm{B} 2 \mathrm{PLYP})=$ $-0.4981460305 \mathrm{D}+00 \mathrm{E}(\mathrm{B} 2 \mathrm{PLYP})=$ $-0.4987643594 \mathrm{D}+00 \mathrm{E}(\mathrm{B} 2 \mathrm{PLYP})=$ $-0.4965274166 \mathrm{D}+00 \mathrm{E}(\mathrm{B} 2 \mathrm{PLYP})=$ $-0.4963967015 \mathrm{D}+00 \mathrm{E}(\mathrm{B} 2 \mathrm{PLYP})=$ $-0.4972134308 \mathrm{D}+00 \mathrm{E}(\mathrm{B} 2 \mathrm{PLYP})=$ $-0.5981760602 \mathrm{D}+00 \mathrm{E}(\mathrm{B} 2 \mathrm{PLYP})=$ $-0.6021023205 \mathrm{D}+00 \mathrm{E}(\mathrm{B} 2 \mathrm{PLYP})=$ $-0.2491120848 \mathrm{D}+00 \mathrm{E}(\mathrm{B} 2 \mathrm{PLYP})=$ $-0.2995417049 \mathrm{D}+00 \mathrm{E}(\mathrm{B} 2 \mathrm{PLYP})=$ $-0.2999898762 \mathrm{D}+00 \mathrm{E}(\mathrm{B} 2 \mathrm{PLYP})=$ $-0.3507580272 \mathrm{D}+00 \mathrm{E}(\mathrm{B} 2 \mathrm{PLYP})=$ $-0.3527044170 \mathrm{D}+00 \mathrm{E}(\mathrm{B} 2 \mathrm{PLYP})=$ $-0.3509055944 \mathrm{D}+00 \mathrm{E}(\mathrm{B} 2 \mathrm{PLYP})=$
$-0.39004136578654 \mathrm{D}+03$

$-0.79675644987819 \mathrm{D}+02$

$-0.46853594997038 \mathrm{D}+03$

$-0.46852416745872 \mathrm{D}+03$ $-0.54703064964877 \mathrm{D}+03$ $-0.54463375045951 \mathrm{D}+03$ $-0.40435994581415 \mathrm{D}+02$ $-0.27588816821801 \mathrm{D}+03$ $-0.27588901582102 \mathrm{D}+03$ $-0.27588915727717 \mathrm{D}+03$ $-0.27588875296174 \mathrm{D}+03$ $-0.27588669243007 \mathrm{D}+03$ $-0.31512922123920 \mathrm{D}+03$ $-0.23664663961289 \mathrm{D}+03$ $-0.23664723738919 \mathrm{D}+03$ $-0.23664845615889 \mathrm{D}+03$ $-0.23664607680511 \mathrm{D}+03$ $-0.23664645065030 \mathrm{D}+03$ $-0.27588918799077 \mathrm{D}+03$ $-0.27589090522861 \mathrm{D}+03$ $-0.27589003825743 \mathrm{D}+03$ $-0.27589101820111 \mathrm{D}+03$ $-0.39122425469289 \mathrm{D}+03$ $-0.39120962717902 \mathrm{D}+03$ $-0.39121872789914 \mathrm{D}+03$ $-0.39123622645738 \mathrm{D}+03$ $-0.39123176147406 \mathrm{D}+03$ $-0.46971691874093 \mathrm{D}+03$ $-0.47091087097243 \mathrm{D}+03$ $-0.19620046781391 \mathrm{D}+03$ $-0.23545304202344 \mathrm{D}+03$ $-0.23544567578224 \mathrm{D}+03$ $-0.27469829411463 \mathrm{D}+03$ $-0.27469012101316 \mathrm{D}+03$ $-0.27469054135188 \mathrm{D}+03$ 
r7.out: E2(B2PLYP) = r8.out: E2(B2PLYP) = r9.out: E2(B2PLYP) = PBEQIDH/DHSVPD

C1.out: E2(PBEQIDH) $=-0.5650107530 \mathrm{D}+00 \mathrm{E}(\mathrm{PBEQIDH})=-0.38989639912972 \mathrm{D}+03$ C2H6.out: $\mathrm{E} 2(\mathrm{PBEQIDH})=-0.1213521165 \mathrm{D}+00 \mathrm{E}(\mathrm{PBEQIDH})=-$

$0.79644539529983 \mathrm{D}+02$

C2.out: $\mathrm{E} 2(\mathrm{PBEQIDH})=$ C3.out: E2(PBEQIDH) $=$ C4.out: E2(PBEQIDH) $=$ C5.out: E2(PBEQIDH) $=$ CH4.out: E2(PBEQIDH) = 0.40420173944222D+02

H10.out: E2(PBEQIDH) = $0.27578394875207 \mathrm{D}+03$

H11.out: E2(PBEQIDH) $=$ $0.27578583475633 \mathrm{D}+03$

H12.out: E2(PBEQIDH) = $0.27578575902903 \mathrm{D}+03$

H13.out: E2(PBEQIDH) = $0.27578377623705 \mathrm{D}+03$

H14.out: E2(PBEQIDH) = $0.27578186049185 \mathrm{D}+03$

H15.out: E2(PBEQIDH) = $0.31501285941913 \mathrm{D}+03$

H1.out: E2(PBEQIDH) = H2.out: E2(PBEQIDH) $=$ H3.out: E2(PBEQIDH) $=$ H4.out: E2(PBEQIDH) $=$ H5.out: E2(PBEQIDH) $=$ H6.out: E2(PBEQIDH) = H7.out: E2(PBEQIDH) = H8.out: E2(PBEQIDH) = H9.out: E2(PBEQIDH) = r10.out: E2(PBEQIDH) = r11.out: E2(PBEQIDH) = r12.out: E2(PBEQIDH) = r13.out: E2(PBEQIDH) $=$ r14.out: E2(PBEQIDH) $=$ r15.out: E2(PBEQIDH) = r16.out: E2(PBEQIDH) = r1.out: $\mathrm{E} 2(\mathrm{PBEQIDH})=$ r2.out: $\mathrm{E} 2(\mathrm{PBEQIDH})=$ r3.out: $\mathrm{E} 2(\mathrm{PBEQIDH})=$ r4.out: $\mathrm{E} 2(\mathrm{PBEQIDH})=$ r5.out: E2(PBEQIDH) = r6.out: $\mathrm{E} 2(\mathrm{PBEQIDH})=$ r7.out: $\mathrm{E} 2(\mathrm{PBEQIDH})=$ r8.out: E2(PBEQIDH) = r9.out: E2(PBEQIDH) =
$-0.6857487255 \mathrm{D}+00 \mathrm{E}(\mathrm{PBEQIDH})=$ $-0.6870319061 \mathrm{D}+00 \mathrm{E}(\mathrm{PBEQIDH})=$ $-0.8067097258 \mathrm{D}+00 \mathrm{E}(\mathrm{PBEQIDH})=$ $-0.7864745997 \mathrm{D}+00 \mathrm{E}(\mathrm{PBEQIDH})=$ $-0.6495898791 \mathrm{D}-01 \mathrm{E}(\mathrm{PBEQIDH})=$ $-0.4134092240 \mathrm{D}+00 \mathrm{E}(\mathrm{PBEQIDH})=$ $-0.4160139046 \mathrm{D}+00 \mathrm{E}(\mathrm{PBEQIDH})=$ $-0.4150012171 \mathrm{D}+00 \mathrm{E}(\mathrm{PBEQIDH})=$ $-0.4114983480 \mathrm{D}+00 \mathrm{E}(\mathrm{PBEQIDH})=$ $-0.4119929580 \mathrm{D}+00 \mathrm{E}(\mathrm{PBEQIDH})=$ $-0.4776334549 \mathrm{D}+00 \mathrm{E}(\mathrm{PBEQIDH})=$ $-0.3551067517 \mathrm{D}+00 \mathrm{E}(\mathrm{PBEQIDH})=$ $-0.3532791923 \mathrm{D}+00 \mathrm{E}(\mathrm{PBEQIDH})=$ $-0.3559596717 \mathrm{D}+00 \mathrm{E}(\mathrm{PBEQIDH})=$ $-0.3537676898 \mathrm{D}+00 \mathrm{E}(\mathrm{PBEQIDH})=$ $-0.3516044351 \mathrm{D}+00 \mathrm{E}(\mathrm{PBEQIDH})=$ $-0.4092304328 \mathrm{D}+00 \mathrm{E}(\mathrm{PBEQIDH})=$ $-0.4128917422 \mathrm{D}+00 \mathrm{E}(\mathrm{PBEQIDH})=$ $-0.4109626030 \mathrm{D}+00 \mathrm{E}(\mathrm{PBEQIDH})=$ $-0.4137971124 \mathrm{D}+00 \mathrm{E}(\mathrm{PBEQIDH})=$ $-0.5725567953 \mathrm{D}+00 \mathrm{E}(\mathrm{PBEQIDH})=$ $-0.5732524097 \mathrm{D}+00 \mathrm{E}(\mathrm{PBEQIDH})=$ $-0.5706679817 \mathrm{D}+00 \mathrm{E}(\mathrm{PBEQIDH})=$ $-0.5706821528 \mathrm{D}+00 \mathrm{E}(\mathrm{PBEQIDH})=$ $-0.5716014538 \mathrm{D}+00 \mathrm{E}(\mathrm{PBEQIDH})=$ $-0.6876649074 \mathrm{D}+00 \mathrm{E}(\mathrm{PBEQIDH})=$ $-0.6922513710 \mathrm{D}+00 \mathrm{E}(\mathrm{PBEQIDH})=$ $-0.2862918339 \mathrm{D}+00 \mathrm{E}(\mathrm{PBEQIDH})=$ $-0.3443929020 \mathrm{D}+00 \mathrm{E}(\mathrm{PBEQIDH})=$ $-0.3448071392 \mathrm{D}+00 \mathrm{E}(\mathrm{PBEQIDH})=$ $-0.4032841474 \mathrm{D}+00 \mathrm{E}(\mathrm{PBEQIDH})=$ $-0.4054033327 \mathrm{D}+00 \mathrm{E}(\mathrm{PBEQIDH})=$ $-0.4033548806 \mathrm{D}+00 \mathrm{E}(\mathrm{PBEQIDH})=$ $-0.4046641538 \mathrm{D}+00 \mathrm{E}(\mathrm{PBEQIDH})=$ $-0.4038435004 \mathrm{D}+00 \mathrm{E}(\mathrm{PBEQIDH})=$ $-0.4033789757 \mathrm{D}+00 \mathrm{E}(\mathrm{PBEQIDH})=$
$-0.46836309122345 \mathrm{D}+03$ $-0.46835184715116 \mathrm{D}+03$ $-0.54682990112413 \mathrm{D}+03$ $-0.54443346068883 \mathrm{D}+03$ $-$

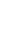

$-$ $-$

$-0.23655708920623 \mathrm{D}+03$ $-0.23655695580655 \mathrm{D}+03$ $-0.23655926343701 \mathrm{D}+03$ $-0.23655601784673 \mathrm{D}+03$ $-0.23655560082843 \mathrm{D}+03$ $-0.27578341526664 \mathrm{D}+03$ $-0.27578645229717 \mathrm{D}+03$ $-0.27578484473605 \mathrm{D}+03$ $-0.27578695753586 \mathrm{D}+03$ $-0.39107757212090 \mathrm{D}+03$ $-0.39106229717368 \mathrm{D}+03$ $-0.39107059906865 \mathrm{D}+03$ $-0.39108924858769 \mathrm{D}+03$ $-0.39108515925931 \mathrm{D}+03$ $-0.46954074014532 \mathrm{D}+03$ $-0.47073245027891 \mathrm{D}+03$ $-0.19612499685513 \mathrm{D}+03$ $-0.23536345643054 \mathrm{D}+03$ $-0.23535560040615 \mathrm{D}+03$ $-0.27459421014526 \mathrm{D}+03$ $-0.27458636344908 \mathrm{D}+03$ $-0.27458579788029 \mathrm{D}+03$ $-0.27458422561658 \mathrm{D}+03$ $-0.27458650499416 \mathrm{D}+03$ $-0.27458524498151 \mathrm{D}+03$ 
PBE0DH/DHSVPD

C1.out: E2(PBE0DH $)=-0.2304932121 \mathrm{D}+00 \mathrm{E}(\mathrm{PBE} 0 \mathrm{DH})=-0.39001905097813 \mathrm{D}+03$

C2H6.out: $\mathrm{E} 2(\mathrm{PBE} 0 \mathrm{DH})=-0.4935036186 \mathrm{D}-01 \mathrm{E}(\mathrm{PBE} 0 \mathrm{DH})=-0.79672270589238 \mathrm{D}+02$

C2.out: $\mathrm{E} 2(\mathrm{PBE} 0 \mathrm{DH})=$

C3.out: $\mathrm{E} 2(\mathrm{PBE} 0 \mathrm{DH})=$

C4.out: $\mathrm{E} 2(\mathrm{PBE} 0 \mathrm{DH})=$

C5.out: $\mathrm{E} 2(\mathrm{PBE} 0 \mathrm{DH})=$

CH4.out: E2(PBE0DH) $=$

H10.out: $\mathrm{E} 2(\mathrm{PBE} 0 \mathrm{DH})=$

H11.out: E2(PBE0DH) $=$

H12.out: E2(PBE0DH) $=$

H13.out: $\mathrm{E} 2(\mathrm{PBE} 0 \mathrm{DH})=$

H14.out: E2(PBE0DH) =

H15.out: $\mathrm{E} 2(\mathrm{PBE} 0 \mathrm{DH})=$

H1.out: E2(PBE0DH) =

H2.out: E2(PBE0DH) $=$

H3.out: E2(PBE0DH) =

H4.out: E2(PBE0DH) $=$

H5.out: E2(PBE0DH) =

H6.out: E2(PBE0DH) =

H7.out: E2(PBE0DH) $=$

H8.out: E2(PBE0DH) $=$

H9.out: E2(PBE0DH) =

r10.out: E2 $(\mathrm{PBE} 0 \mathrm{DH})=$

r11.out: E2(PBE0DH $)=$

r12.out: E2 $(\mathrm{PBE} 0 \mathrm{DH})=$

r13.out: E2(PBE0DH) =

r14.out: E2(PBE0DH $)=$

r15.out: E2(PBE0DH $)=$

r16.out: E2(PBE0DH $)=$ r1.out: E2 $(\mathrm{PBE} 0 \mathrm{DH})=$ r2.out: E2(PBE0DH) $=$ r3.out: E2(PBE0DH $)=$ r4.out: E2(PBE0DH $)=$ r5.out: E2(PBE0DH) = r6.out: E2(PBE0DH) = r7.out: E2(PBE0DH) $=$ r8.out: E2 $(\mathrm{PBE} 0 \mathrm{DH})=$ r9. out: E2(PBE0DH) = B2PLYPD3BJ/DHSVPD

C1.out: E2(B2PLYP) = C2H6.out: E2(B2PLYP) $=$ C2.out: E2(B2PLYP) = C3.out: E2(B2PLYP) = C4.out: E2(B2PLYP) $=$ C5.out: E2(B2PLYP) $=$ CH4.out: E2(B2PLYP) $=$ H10.out: E2(B2PLYP) = H11.out: E2(B2PLYP) = H12.out: E2(B2PLYP) $=$
$-0.2797765689 \mathrm{D}+00 \mathrm{E}(\mathrm{PBE} 0 \mathrm{DH})=$ $-0.2803255754 \mathrm{D}+00 \mathrm{E}(\mathrm{PBE} 0 \mathrm{DH})=$ $-0.3291511578 \mathrm{D}+00 \mathrm{E}(\mathrm{PBE} 0 \mathrm{DH})=$ $-0.3210102079 \mathrm{D}+00 \mathrm{E}(\mathrm{PBE} 0 \mathrm{DH})=$ $-0.2638366822 \mathrm{D}-01 \mathrm{E}(\mathrm{PBE} 0 \mathrm{DH})=$ $-0.1684375955 \mathrm{D}+00 \mathrm{E}(\mathrm{PBE} 0 \mathrm{DH})=$ $-0.1695277184 \mathrm{D}+00 \mathrm{E}(\mathrm{PBE} 0 \mathrm{DH})=$ $-0.1691008585 \mathrm{D}+00 \mathrm{E}(\mathrm{PBE} 0 \mathrm{DH})=$ $-0.1676343402 \mathrm{D}+00 \mathrm{E}(\mathrm{PBE} 0 \mathrm{DH})=$ $-0.1678485773 \mathrm{D}+00 \mathrm{E}(\mathrm{PBE} 0 \mathrm{DH})=$ $-0.1946960717 \mathrm{D}+00 \mathrm{E}(\mathrm{PBE} 0 \mathrm{DH})=$ $-0.1446622546 \mathrm{D}+00 \mathrm{E}(\mathrm{PBE} 0 \mathrm{DH})=$ $-0.1438948783 \mathrm{D}+00 \mathrm{E}(\mathrm{PBE} 0 \mathrm{DH})=$ $-0.1450156881 \mathrm{D}+00 \mathrm{E}(\mathrm{PBE} 0 \mathrm{DH})=$ $-0.1441015393 \mathrm{D}+00 \mathrm{E}(\mathrm{PBE} 0 \mathrm{DH})=$ $-0.1431903249 \mathrm{D}+00 \mathrm{E}(\mathrm{PBE} 0 \mathrm{DH})=$ $-0.1666773218 \mathrm{D}+00 \mathrm{E}(\mathrm{PBE} 0 \mathrm{DH})=$ $-0.1682167679 \mathrm{D}+00 \mathrm{E}(\mathrm{PBE} 0 \mathrm{DH})=$ $-0.1674062290 \mathrm{D}+00 \mathrm{E}(\mathrm{PBE} 0 \mathrm{DH})=$ $-0.1685937663 \mathrm{D}+00 \mathrm{E}(\mathrm{PBE} 0 \mathrm{DH})=$ $-0.2335038526 \mathrm{D}+00 \mathrm{E}(\mathrm{PBE} 0 \mathrm{DH})=$ $-0.2338165459 \mathrm{D}+00 \mathrm{E}(\mathrm{PBE} 0 \mathrm{DH})=$ $-0.2327245112 \mathrm{D}+00 \mathrm{E}(\mathrm{PBE} 0 \mathrm{DH})=$ $-0.2327099896 \mathrm{D}+00 \mathrm{E}(\mathrm{PBE} 0 \mathrm{DH})=$ $-0.2330980293 \mathrm{D}+00 \mathrm{E}(\mathrm{PBE} 0 \mathrm{DH})=$ $-0.2804224412 \mathrm{D}+00 \mathrm{E}(\mathrm{PBE} 0 \mathrm{DH})=$ $-0.2821557747 \mathrm{D}+00 \mathrm{E}(\mathrm{PBE} 0 \mathrm{DH})=$ $-0.1166669604 \mathrm{D}+00 \mathrm{E}(\mathrm{PBE} 0 \mathrm{DH})=$ $-0.1403446684 \mathrm{D}+00 \mathrm{E}(\mathrm{PBE} 0 \mathrm{DH})=$ $-0.1405242517 \mathrm{D}+00 \mathrm{E}(\mathrm{PBE} 0 \mathrm{DH})=$ $-0.1643632880 \mathrm{D}+00 \mathrm{E}(\mathrm{PBE} 0 \mathrm{DH})=$ $-0.1652543106 \mathrm{D}+00 \mathrm{E}(\mathrm{PBE} 0 \mathrm{DH})=$ $-0.1643991889 \mathrm{D}+00 \mathrm{E}(\mathrm{PBE} 0 \mathrm{DH})=$ $-0.1649477203 \mathrm{D}+00 \mathrm{E}(\mathrm{PBE} 0 \mathrm{DH})=$ $-0.1646024844 \mathrm{D}+00 \mathrm{E}(\mathrm{PBE} 0 \mathrm{DH})=$ $-0.1644126319 \mathrm{D}+00 \mathrm{E}(\mathrm{PBE} 0 \mathrm{DH})=$

$-0.4915492906 \mathrm{D}+00 \mathrm{E}(\mathrm{B} 2 \mathrm{PLYP})=$ $=-0.1054852799 \mathrm{D}+00 \mathrm{E}(\mathrm{B} 2 \mathrm{PLYP})=$ $-0.5965694853 \mathrm{D}+00 \mathrm{E}(\mathrm{B} 2 \mathrm{PLYP})=$ $-0.5977033488 \mathrm{D}+00 \mathrm{E}(\mathrm{B} 2 \mathrm{PLYP})=$ $-0.7017729962 \mathrm{D}+00 \mathrm{E}(\mathrm{B} 2 \mathrm{PLYP})=$ $-0.6842646410 \mathrm{D}+00 \mathrm{E}(\mathrm{B} 2 \mathrm{PLYP})=$ $-0.5642992934 \mathrm{D}-01 \mathrm{E}(\mathrm{B} 2 \mathrm{PLYP})=$ $-0.3595254739 \mathrm{D}+00 \mathrm{E}(\mathrm{B} 2 \mathrm{PLYP})=$ $-0.3617980704 \mathrm{D}+00 \mathrm{E}(\mathrm{B} 2 \mathrm{PLYP})=$ $-0.3609043114 \mathrm{D}+00 \mathrm{E}(\mathrm{B} 2 \mathrm{PLYP})=$
$-0.46850881004653 \mathrm{D}+03$ $-0.46849741571377 \mathrm{D}+03$ $-0.54699863660538 \mathrm{D}+03$ $-0.54460104709746 \mathrm{D}+03$ $-0.40434587225736 \mathrm{D}+02$ $-0.27587424802732 \mathrm{D}+03$ $-0.27587504902780 \mathrm{D}+03$ $-0.27587534132243 \mathrm{D}+03$ $-0.27587478239843 \mathrm{D}+03$ $-0.27587278363520 \mathrm{D}+03$ $-0.31511325632950 \mathrm{D}+03$ $-0.23663483017629 \mathrm{D}+03$ $-0.23663534324384 \mathrm{D}+03$ $-0.23663654380543 \mathrm{D}+03$ $-0.23663429852871 \mathrm{D}+03$ $-0.23663461465222 \mathrm{D}+03$ $-0.27587521210002 \mathrm{D}+03$ $-0.27587684746494 \mathrm{D}+03$ $-0.27587598607922 \mathrm{D}+03$ $-0.27587689879651 \mathrm{D}+03$ $-0.39120227707766 \mathrm{D}+03$ $-0.39118725078702 \mathrm{D}+03$ $-0.39119631983205 \mathrm{D}+03$ $-0.39121450813106 \mathrm{D}+03$ $-0.39121012606452 \mathrm{D}+03$ $-0.46969098147063 \mathrm{D}+03$ $-0.47088553108563 D+03$ $-0.19618988709868 \mathrm{D}+03$ $-0.23544071151534 \mathrm{D}+03$ $-0.23543289950431 \mathrm{D}+03$ $-0.27468371469860 \mathrm{D}+03$ $-0.27467514715305 \mathrm{D}+03$ $-0.27467550535512 \mathrm{D}+03$ $-0.27467341226371 \mathrm{D}+03$ $-0.27467601591555 \mathrm{D}+03$ $-0.27467495577305 \mathrm{D}+03$

$-0.39006554506906 \mathrm{D}+03$ $=-0.79677956222471 \mathrm{D}+02$ $-0.46856700861750 \mathrm{D}+03$ $-0.46855603845389 \mathrm{D}+03$ $-0.54706871673548 \mathrm{D}+03$ $-0.54467098174374 \mathrm{D}+03$ $-0.40436785791046 \mathrm{D}+02$ $-0.27590335433638 \mathrm{D}+03$ $-0.27590523323550 \mathrm{D}+03$ $-0.27590509240207 \mathrm{D}+03$ 
H13.out: E2(B2PLYP) = H14.out: E2(B2PLYP) = H15.out: E2(B2PLYP) = H1.out: E2(B2PLYP) = H2.out: E2(B2PLYP) = H3.out: E2(B2PLYP) $=$ H4.out: E2(B2PLYP) $=$ H5.out: E2(B2PLYP) $=$ H6.out: E2(B2PLYP) = H7.out: E2(B2PLYP) = H8.out: E2(B2PLYP) $=$ H9.out: E2(B2PLYP) = r10.out: E2(B2PLYP) = r11.out: E2(B2PLYP) = r12.out: E2(B2PLYP) = r13.out: E2(B2PLYP) = r14.out: E2(B2PLYP) = r15.out: E2(B2PLYP) = r16.out: E2(B2PLYP) = r1.out: E2(B2PLYP) = r2.out: E2(B2PLYP) $=$ r3.out: E2(B2PLYP) = r4.out: E2(B2PLYP) = r5.out: E2(B2PLYP) = r6.out: E2(B2PLYP) = r7.out: E2(B2PLYP) = r8.out: E2(B2PLYP) = r9.out: E2(B2PLYP) = DSDPBEP86/DHSVPD
$-0.3578637159 \mathrm{D}+00 \mathrm{E}(\mathrm{B} 2 \mathrm{PLYP})=$ $-0.3583130163 \mathrm{D}+00 \mathrm{E}(\mathrm{B} 2 \mathrm{PLYP})=$ $-0.4154010686 \mathrm{D}+00 \mathrm{E}(\mathrm{B} 2 \mathrm{PLYP})=$ $-0.3088081164 \mathrm{D}+00 \mathrm{E}(\mathrm{B} 2 \mathrm{PLYP})=$ $-0.3072216295 \mathrm{D}+00 \mathrm{E}(\mathrm{B} 2 \mathrm{PLYP})=$ $-0.3095584235 \mathrm{D}+00 \mathrm{E}(\mathrm{B} 2 \mathrm{PLYP})=$ $-0.3076457117 \mathrm{D}+00 \mathrm{E}(\mathrm{B} 2 \mathrm{PLYP})=$ $-0.3057569701 \mathrm{D}+00 \mathrm{E}(\mathrm{B} 2 \mathrm{PLYP})=$ $-0.3558784437 \mathrm{D}+00 \mathrm{E}(\mathrm{B} 2 \mathrm{PLYP})=$ $-0.3590757441 \mathrm{D}+00 \mathrm{E}(\mathrm{B} 2 \mathrm{PLYP})=$ $-0.3573933784 \mathrm{D}+00 \mathrm{E}(\mathrm{B} 2 \mathrm{PLYP})=$ $-0.3598707440 \mathrm{D}+00 \mathrm{E}(\mathrm{B} 2 \mathrm{PLYP})=$ $-0.4981460305 \mathrm{D}+00 \mathrm{E}(\mathrm{B} 2 \mathrm{PLYP})=$ $-0.4987643594 \mathrm{D}+00 \mathrm{E}(\mathrm{B} 2 \mathrm{PLYP})=$ $-0.4965274166 \mathrm{D}+00 \mathrm{E}(\mathrm{B} 2 \mathrm{PLYP})=$ $-0.4963967015 \mathrm{D}+00 \mathrm{E}(\mathrm{B} 2 \mathrm{PLYP})=$ $-0.4972134308 \mathrm{D}+00 \mathrm{E}(\mathrm{B} 2 \mathrm{PLYP})=$ $-0.5981760602 \mathrm{D}+00 \mathrm{E}(\mathrm{B} 2 \mathrm{PLYP})=$ $-0.6021023205 \mathrm{D}+00 \mathrm{E}(\mathrm{B} 2 \mathrm{PLYP})=$ $-0.2491120848 \mathrm{D}+00 \mathrm{E}(\mathrm{B} 2 \mathrm{PLYP})=$ $-0.2995417049 \mathrm{D}+00 \mathrm{E}(\mathrm{B} 2 \mathrm{PLYP})=$ $-0.2999898762 \mathrm{D}+00 \mathrm{E}(\mathrm{B} 2 \mathrm{PLYP})=$ $-0.3507580272 \mathrm{D}+00 \mathrm{E}(\mathrm{B} 2 \mathrm{PLYP})=$ $-0.3527044170 \mathrm{D}+00 \mathrm{E}(\mathrm{B} 2 \mathrm{PLYP})=$ $-0.3509055944 \mathrm{D}+00 \mathrm{E}(\mathrm{B} 2 \mathrm{PLYP})=$ $-0.3520430997 \mathrm{D}+00 \mathrm{E}(\mathrm{B} 2 \mathrm{PLYP})=$ $-0.3513249535 \mathrm{D}+00 \mathrm{E}(\mathrm{B} 2 \mathrm{PLYP})=$ $-0.3509366697 \mathrm{D}+00 \mathrm{E}(\mathrm{B} 2 \mathrm{PLYP})=$
$-0.27590284328693 \mathrm{D}+03$ $-0.27590115847852 \mathrm{D}+03$ $-0.31514943474768 \mathrm{D}+03$ $-0.23665924541335 \mathrm{D}+03$ $-0.23665880657441 \mathrm{D}+03$ $-0.23666126185546 \mathrm{D}+03$ $-0.23665795759210 \mathrm{D}+03$ $-0.23665719341695 \mathrm{D}+03$ $-0.27590208505228 \mathrm{D}+03$ $-0.27590567785097 \mathrm{D}+03$ $-0.27590379087708 \mathrm{D}+03$ $-0.27590610094031 \mathrm{D}+03$ $-0.39124769390653 \mathrm{D}+03$ $-0.39123421362639 D+03$ $-0.39124141996829 \mathrm{D}+03$ $-0.39125910069965 \mathrm{D}+03$ $-0.39125540089428 \mathrm{D}+03$ $-0.46974524024189 \mathrm{D}+03$ $-0.47093665781197 \mathrm{D}+03$ $-0.19620913992253 \mathrm{D}+03$ $-0.23546476292627 \mathrm{D}+03$ $-0.23545697661357 \mathrm{D}+03$ $-0.27471276591113 \mathrm{D}+03$ $-0.27470505041101 \mathrm{D}+03$ $-0.27470451298184 \mathrm{D}+03$ $-0.27470298624468 \mathrm{D}+03$ $-0.27470524886962 \mathrm{D}+03$ $-0.27470404743085 \mathrm{D}+03$

C1.out: E2(DSDPBEP86 $)=-0.7550703949 \mathrm{D}+00 \mathrm{E}(\mathrm{DSDPBEP} 86)=-$ $0.38961971654171 \mathrm{D}+03$ C2H6.out: E2(DSDPBEP86 $)=-0.1664805960 \mathrm{D}+00 \mathrm{E}(\mathrm{DSDPBEP} 86)=-$ $0.79583828648891 \mathrm{D}+02$

C2.out: $\mathrm{E} 2(\mathrm{DSDPBEP} 86)=-0.9165435027 \mathrm{D}+00 \mathrm{E}(\mathrm{DSDPBEP} 86)=-$ $0.46803179478970 \mathrm{D}+03$

C3.out: E2(DSDPBEP86) $=-0.9181378312 \mathrm{D}+00 \mathrm{E}(\mathrm{DSDPBEP} 86)=\quad-$ $0.46802092105295 \mathrm{D}+03$

C4.out: E2(DSDPBEP86 $)=-0.1078300297 \mathrm{D}+01 \mathrm{E}(\mathrm{DSDPBEP} 86)=-$ $0.54644410246044 \mathrm{D}+03$

C5.out: E2(DSDPBEP86 $)=-0.1046745114 \mathrm{D}+01 \mathrm{E}(\mathrm{DSDPBEP} 86)=-$ $0.54405125317008 \mathrm{D}+03$

CH4.out: E2(DSDPBEP86) $=-0.9020319233 \mathrm{D}-01 \mathrm{E}(\mathrm{DSDPBEP} 86)=\quad-$ $0.40388184430664 \mathrm{D}+02$ H10.out: E2(DSDPBEP86 $)=-0.5592091174 \mathrm{D}+00 \mathrm{E}(\mathrm{DSDPBEP} 86)=-$ $0.27558352599010 \mathrm{D}+03$ H11.out: E2(DSDPBEP86 $)=-0.5621909016 \mathrm{D}+00 \mathrm{E}(\mathrm{DSDPBEP} 86)=-$ $0.27558617887698 \mathrm{D}+03$ H12.out: $22(D S D P B E P 86)=-0.5610258824 \mathrm{D}+00 \mathrm{E}(\mathrm{DSDPBEP} 86)=-$ $0.27558580220576 \mathrm{D}+03$ 


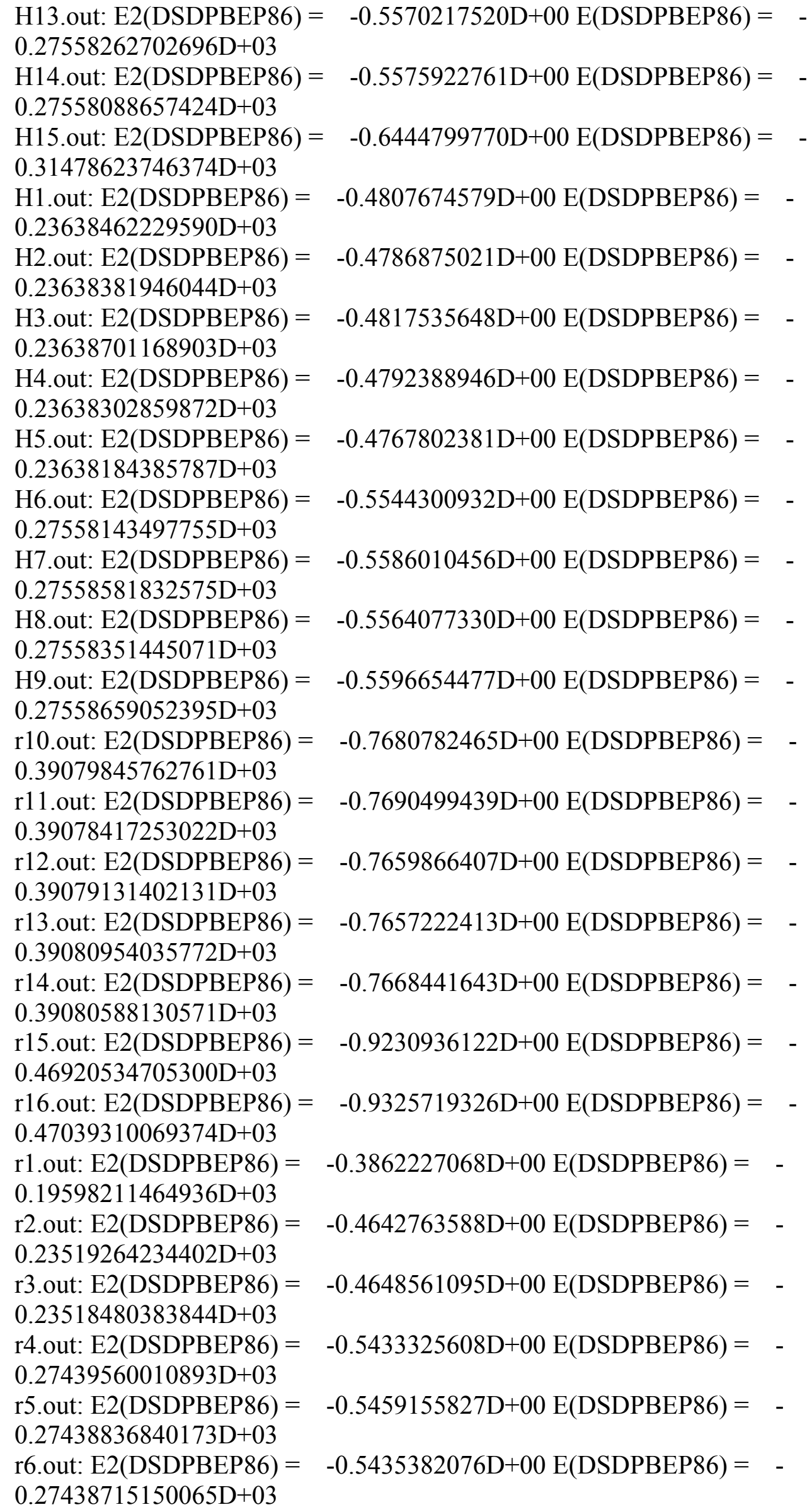


r7.out: E2(DSDPBEP86) = $0.27438601968558 \mathrm{D}+03$

r8.out: E2(DSDPBEP86) = $0.27438801287303 \mathrm{D}+03$

r9.out: E2(DSDPBEP86) = $0.27438665621955 \mathrm{D}+03$

PBEQIDHD3BJ/DHSVPD

C1.out: E2(PBEQIDH) $=-0.5650107530 \mathrm{D}+00 \mathrm{E}(\mathrm{PBEQIDH})=-0.38990223903710 \mathrm{D}+03$ C2H6.out: E2(PBEQIDH $)=-0.1213521165 \mathrm{D}+00 \mathrm{E}(\mathrm{PBEQIDH})=$

$0.79644977526968 \mathrm{D}+02$

C2.out: E2(PBEQIDH) $=$ C3.out: $\mathrm{E} 2(\mathrm{PBEQIDH})=$ C4.out: $\mathrm{E} 2(\mathrm{PBEQIDH})=$ C5.out: E2(PBEQIDH) $=$ CH4.out: E2(PBEQIDH) = 0.40420313390984D+02

H10.out: E2(PBEQIDH) = $0.27578754995456 \mathrm{D}+03$

H11.out: E2(PBEQIDH) = $0.27578966805339 \mathrm{D}+03$

H12.out: E2(PBEQIDH) $=$ $0.27578954443402 \mathrm{D}+03$

H13.out: E2(PBEQIDH) = $0.27578709073532 \mathrm{D}+03$

H14.out: E2(PBEQIDH) = $0.27578533582368 \mathrm{D}+03$

H15.out: $\mathrm{E} 2(\mathrm{PBEQIDH})=$ $0.31501773462230 \mathrm{D}+03$

H1.out: E2(PBEQIDH) = H2.out: E2(PBEQIDH) = H3.out: E2(PBEQIDH) = H4.out: E2(PBEQIDH) $=$ H5.out: E2(PBEQIDH) = H6.out: E2(PBEQIDH) $=$ H7.out: E2(PBEQIDH) = H8.out: E2(PBEQIDH) = H9.out: E2(PBEQIDH) $=$ r10.out: E2(PBEQIDH) = r11.out: E2(PBEQIDH) = r12.out: E2(PBEQIDH) = r13.out: E2(PBEQIDH) = r14.out: E2(PBEQIDH) $=$ r15.out: E2(PBEQIDH) = r16.out: E2(PBEQIDH) $=$ r1.out: E2(PBEQIDH) = r2.out: E2(PBEQIDH) = r3.out: $\mathrm{E} 2(\mathrm{PBEQIDH})=$ r4.out: $\mathrm{E} 2(\mathrm{PBEQIDH})=$ r5.out: $\mathrm{E} 2(\mathrm{PBEQIDH})=$ r6.out: E2(PBEQIDH) $=$
$-0.5450591461 \mathrm{D}+00 \mathrm{E}(\mathrm{DSDPBEP} 86)=-$

$-0.5440788394 \mathrm{D}+00 \mathrm{E}(\mathrm{DSDPBEP} 86)=-$

$-0.5435838828 \mathrm{D}+00 \mathrm{E}(\mathrm{DSDPBEP} 86)=-$
$-0.6857487255 \mathrm{D}+00 \mathrm{E}(\mathrm{PBEQIDH})=$ $-0.6870319061 \mathrm{D}+00 \mathrm{E}(\mathrm{PBEQIDH})=$ $-0.8067097258 \mathrm{D}+00 \mathrm{E}(\mathrm{PBEQIDH})=$ $-0.7864745997 \mathrm{D}+00 \mathrm{E}(\mathrm{PBEQIDH})=-0.54444297624022 \mathrm{D}+03$ $-0.6495898791 \mathrm{D}-01 \mathrm{E}(\mathrm{PBEQIDH})=-$

$-0.4134092240 \mathrm{D}+00 \mathrm{E}(\mathrm{PBEQIDH})=-$

$-0.4160139046 \mathrm{D}+00 \mathrm{E}(\mathrm{PBEQIDH})=-$

$-0.4150012171 \mathrm{D}+00 \mathrm{E}(\mathrm{PBEQIDH})=\quad-$

$-0.4114983480 \mathrm{D}+00 \mathrm{E}(\mathrm{PBEQIDH})=$

$-0.4119929580 \mathrm{D}+00 \mathrm{E}(\mathrm{PBEQIDH})=$ $-0.4776334549 \mathrm{D}+00 \mathrm{E}(\mathrm{PBEQIDH})=$ $-0.3551067517 \mathrm{D}+00 \mathrm{E}(\mathrm{PBEQIDH})=$ $-0.3532791923 \mathrm{D}+00 \mathrm{E}(\mathrm{PBEQIDH})=$ $-0.3559596717 \mathrm{D}+00 \mathrm{E}(\mathrm{PBEQIDH})=$ $-0.3537676898 \mathrm{D}+00 \mathrm{E}(\mathrm{PBEQIDH})=$ $-0.3516044351 \mathrm{D}+00 \mathrm{E}(\mathrm{PBEQIDH})=$ $-0.4092304328 \mathrm{D}+00 \mathrm{E}(\mathrm{PBEQIDH})=$ $-0.4128917422 \mathrm{D}+00 \mathrm{E}(\mathrm{PBEQIDH})=$ $-0.4109626030 \mathrm{D}+00 \mathrm{E}(\mathrm{PBEQIDH})=$ $-0.4137971124 \mathrm{D}+00 \mathrm{E}(\mathrm{PBEQIDH})=$ $-0.5725567953 \mathrm{D}+00 \mathrm{E}(\mathrm{PBEQIDH})=$ $-0.5732524097 \mathrm{D}+00 \mathrm{E}(\mathrm{PBEQIDH})=$ $-0.5706679817 \mathrm{D}+00 \mathrm{E}(\mathrm{PBEQIDH})=$ $-0.5706821528 \mathrm{D}+00 \mathrm{E}(\mathrm{PBEQIDH})=$ $-0.5716014538 \mathrm{D}+00 \mathrm{E}(\mathrm{PBEQIDH})=$ $-0.6876649074 \mathrm{D}+00 \mathrm{E}(\mathrm{PBEQIDH})=$ $-0.6922513710 \mathrm{D}+00 \mathrm{E}(\mathrm{PBEQIDH})=$ $-0.2862918339 \mathrm{D}+00 \mathrm{E}(\mathrm{PBEQIDH})=$ $-0.3443929020 \mathrm{D}+00 \mathrm{E}(\mathrm{PBEQIDH})=$ $-0.3448071392 \mathrm{D}+00 \mathrm{E}(\mathrm{PBEQIDH})=$ $-0.4032841474 \mathrm{D}+00 \mathrm{E}(\mathrm{PBEQIDH})=$ $-0.4054033327 \mathrm{D}+00 \mathrm{E}(\mathrm{PBEQIDH})=$ $-0.4033548806 \mathrm{D}+00 \mathrm{E}(\mathrm{PBEQIDH})=$
$-0.46837091828082 \mathrm{D}+03$ $-0.46835987441567 \mathrm{D}+03$ $-0.54683981839431 \mathrm{D}+03$ $0.54444297624022 \mathrm{D}+03$ 
r7.out: $\mathrm{E} 2(\mathrm{PBEQIDH})=$ r8.out: $\mathrm{E} 2(\mathrm{PBEQIDH})=$ r9.out: E2(PBEQIDH) $=$ PBE0DHD3BJ/DHSVPD

C1.out: E2(PBE0DH) $=$ C2H6 out: $\mathrm{E} 2(\mathrm{PBEODH})=-0.4935036186 \mathrm{D}-01 \mathrm{E}(\mathrm{PBE} 0 \mathrm{DH})$ C2.out: $\mathrm{E} 2(\mathrm{PBE} 0 \mathrm{DH})=$ C3.out: E2(PBE0DH) $=$ C4.out: $\mathrm{E} 2(\mathrm{PBE} 0 \mathrm{DH})=$ C5.out: E2(PBE0DH $)=$ CH4.out: E2(PBE0DH $)=$ H10.out: E2(PBE0DH) $=$ H11.out: E2(PBE0DH) $=$ H12.out: E2(PBE0DH) $=$ H13.out: E2(PBE0DH) $=$ H14.out: E2(PBE0DH) $=$ H15.out: E2(PBE0DH) $=$ H1.out: E2(PBE0DH) = H2.out: E2(PBE0DH) = H3.out: E2(PBE0DH) $=$ H4.out: E2(PBE0DH) $=$ H5.out: E2(PBE0DH) $=$ H6.out: E2(PBE0DH) = H7.out: E2(PBE0DH) $=$ H8.out: E2(PBE0DH) $=$ H9.out: E2(PBE0DH $)=$ r10.out: E2 $(\mathrm{PBE} 0 \mathrm{DH})=$ r11.out: E2(PBE0DH $)=$ r12.out: E2 $(\mathrm{PBE} 0 \mathrm{DH})=$ r13.out: E2(PBE0DH $)=$ r14.out: E2(PBE0DH) = r15.out: E2(PBE0DH) $=$ r16.out: E2(PBE0DH) $=$ r1.out: $\mathrm{E} 2(\mathrm{PBE} 0 \mathrm{DH})=$ r2.out: $\mathrm{E} 2(\mathrm{PBE} 0 \mathrm{DH})=$ r3.out: $\mathrm{E} 2(\mathrm{PBE} 0 \mathrm{DH})=$ r4.out: E2(PBE0DH) $=$ r5.out: E2(PBE0DH) $=$ r6.out: E2(PBE0DH) $=$ r7.out: $\mathrm{E} 2(\mathrm{PBE} 0 \mathrm{DH})=$ r8.out: E2(PBE0DH $)=$ r9.out: E2(PBE0DH) = M06/Def2QZPD

C1.out: SCF Done: $\mathrm{E}(\mathrm{RM} 06)=-390.591846655$ A.U. C2H6.out: SCF Done: $E(R M 06)=-79.7958940011 \quad$ A.U C2.out: SCF Done: $\mathrm{E}(\mathrm{RM} 06)=-469.201931821 \quad$ A.U. C3.out: SCF Done: $E(R M 06)=-469.189329297$ A.U. C4.out: SCF Done: $\mathrm{E}(\mathrm{RM} 06)=-547.811685244$ A.U. C5.out: SCF Done: $\mathrm{E}(\mathrm{RM} 06)=-545.394168590$ A.U. CH4.out: SCF Done: $E(R M 06)=-40.4995338051 \quad$ A.U.
$-0.27458766231649 \mathrm{D}+03$

$-0.27458984398860 \mathrm{D}+03$

$-0.27458850495611 \mathrm{D}+03$

$-0.39004512894566 \mathrm{D}+03$

$-0.79674719680086 \mathrm{D}+02$

$-0.46854211134997 \mathrm{D}+03$

$-0.46853150639649 \mathrm{D}+03$

$-0.54703929499043 \mathrm{D}+03$

$-0.54464117243532 \mathrm{D}+03$

$-0.40435405646961 \mathrm{D}+02$

$-0.27589026768448 \mathrm{D}+03$

$-0.27589216459343 \mathrm{D}+03$

$-0.27589213397929 \mathrm{D}+03$

$-0.27588969175674 \mathrm{D}+03$

$-0.27588805769481 D+03$

$-0.31513456528777 \mathrm{D}+03$

$-0.23664814185950 \mathrm{D}+03$

$-0.23664760329186 \mathrm{D}+03$

$-0.23665009266026 \mathrm{D}+03$

$-0.23664686574728 \mathrm{D}+03$

$-0.23664602452078 \mathrm{D}+03$

$-0.27588890961089 D+03$

$-0.27589246009840 \mathrm{D}+03$

$-0.27589056424301 \mathrm{D}+03$

$-0.27589285700429 \mathrm{D}+03$

$-0.39122731249304 \mathrm{D}+03$

$-0.39121331383307 \mathrm{D}+03$

$-0.39122052107755 \mathrm{D}+03$

$-0.39123897584284 \mathrm{D}+03$

$-0.39123533570176 \mathrm{D}+03$

$-0.46972116108862 \mathrm{D}+03$

$-0.47091298891599 \mathrm{D}+03$

$-0.19619927330720 \mathrm{D}+03$

$-0.23545328545838 D+03$

$-0.23544505919624 \mathrm{D}+03$

$-0.27469919152179 \mathrm{D}+03$

$-0.27469112429754 \mathrm{D}+03$

$-0.27469048025741 \mathrm{D}+03$

$-0.27468918893497 \mathrm{D}+03$

$-0.27469129360827 \mathrm{D}+03$

$-0.27468995567415 \mathrm{D}+03$ 
H10.out: SCF Done: $\mathrm{E}(\mathrm{RM} 06)=-276.293592007$

A.U. H11.out: SCF Done: $E(R M 06)=-276.296059154$ A.U. H12.out: SCF Done: $E(R M 06)=-276.295996417$ H13.out: SCF Done: $E(R M 06)=-276.292965040$ H14.out: SCF Done: $\mathrm{E}(\mathrm{RM} 06)=-276.291194975$ H15.out: SCF Done: $E(R M 06)=-315.594794350$ H1.out: SCF Done: $\mathrm{E}(\mathrm{RM} 06)=-236.995221518$ H2.out: SCF Done: $\mathrm{E}(\mathrm{RM} 06)=-236.994624521$ H3.out: SCF Done: $E(R M 06)=-236.997805428$ H4.out: SCF Done: $E(R M 06)=-236.993857072$ H5.out: SCF Done: $E(R M 06)=-236.992989351$ H6.out: SCF Done: E(RM06) $=-276.292226431$ H7.out: SCF Done: $E(R M 06)=-276.295990600$ H8.out: SCF Done: $E(R M 06)=-276.293887379$ H9.out: SCF Done: $\mathrm{E}(\mathrm{RM} 06)=-276.296820591$ r10.out: SCF Done: $\mathrm{E}(\mathrm{RM} 06)=-391.784836774$ r11.out: SCF Done: $\mathrm{E}(\mathrm{RM} 06)=-391.768893190$ r12.out: SCF Done: $\mathrm{E}(\mathrm{RM} 06)=-391.778301323$ r13.out: SCF Done: $\mathrm{E}(\mathrm{RM} 06)=-391.796044551$ r14.out: SCF Done: $\mathrm{E}(\mathrm{RM} 06)=-391.791554822$ r15.out: SCF Done: $E(\mathrm{RM} 06)=-470.390642300$ r16.out: SCF Done: $\mathrm{E}(\mathrm{RM} 06)=-471.590368809$ r1.out: SCF Done: $\mathrm{E}(\mathrm{RM} 06)=-196.483270806$ r2.out: SCF Done: $E(R M 06)=-235.792836986$ r3.out: SCF Done: $E(R M 06)=-235.785186001$ r4.out: SCF Done: $E(R M 06)=-275.095104390$ r5.out: SCF Done: $\mathrm{E}(\mathrm{RM} 06)=-275.087659908$ r6.out: SCF Done: $\mathrm{E}(\mathrm{RM} 06)=-275.086538188$ r7.out: SCF Done: $E(R M 06)=-275.085396180$ r8.out: SCF Done: $\mathrm{E}(\mathrm{RM} 06)=-275.087602982$ r9.out: SCF Done: $\mathrm{E}(\mathrm{RM} 06)=-275.085896971$ M06D3/Def2QZVP

C1.out: SCF Done: $\mathrm{E}(\mathrm{RM} 06)=-390.595578783$ A.U. C2H6.out: SCF Done: $\mathrm{E}(\mathrm{RM} 06)=-79.7960082237$ A.U C2.out: SCF Done: $E(R M 06)=-469.207406379$ A.U. C3.out: SCF Done: $E(R M 06)=-469.195018836$ A.U. C4.out: SCF Done: $\mathrm{E}(\mathrm{RM} 06)=-547.819055685$ A.U. C5.out: SCF Done: $E(R M 06)=-545.400517419$ A.U. CH4.out: SCF Done: $\mathrm{E}(\mathrm{RM} 06)=-40.4995361350$ A.U. H10.out: SCF Done: $E(R M 06)=-276.296205924$ A.U. H11.out: SCF Done: $\mathrm{E}(\mathrm{RM} 06)=-276.298889950$ A.U. H12.out: SCF Done: $E(R M 06)=-276.298782688$ A.U. H13.out: SCF Done: $E(R M 06)=-276.295243628$ A.U. H14.out: SCF Done: $E(R M 06)=-276.293728590$ A.U. H15.out: SCF Done: $E(R M 06)=-315.598545862$ A.U. H1.out: SCF Done: $\mathrm{E}(\mathrm{RM} 06)=-236.997260391 \quad$ A.U. H2.out: SCF Done: $\mathrm{E}(\mathrm{RM} 06)=-236.996364860$ A.U. H3.out: SCF Done: $E(R M 06)=-236.999852757$ A.U. H4.out: SCF Done: $\mathrm{E}(\mathrm{RM} 06)=-236.995688031$ A.U. H5.out: SCF Done: $E(R M 06)=-236.994478144$ A.U. 
H6.out: SCF Done: $\mathrm{E}(\mathrm{RM} 06)=-276.294105484$ H7.out: SCF Done: $\mathrm{E}(\mathrm{RM} 06)=-276.298476869$ H8.out: SCF Done: $\mathrm{E}(\mathrm{RM} 06)=-276.296049307$ H9.out: SCF Done: $\mathrm{E}(\mathrm{RM} 06)=-276.299362410$ r10.out: SCF Done: $\mathrm{E}(\mathrm{RM} 06)=-391.788796424$ r11.out: SCF Done: $\mathrm{E}(\mathrm{RM} 06)=-391.773360651$ r12.out: SCF Done: $E(R M 06)=-391.782202672$ r13.out: SCF Done: $E(R M 06)=-391.799790388$ r14.out: SCF Done: $\mathrm{E}(\mathrm{RM} 06)=-391.795554502$ r15.out: SCF Done: $\mathrm{E}(\mathrm{RM} 06)=-470.395767049$ r16.out: SCF Done: $\mathrm{E}(\mathrm{RM} 06)=-471.594741462$ r1.out: SCF Done: $\mathrm{E}(\mathrm{RM} 06)=-196.484175169$ r2.out: SCF Done: $\mathrm{E}(\mathrm{RM} 06)=-235.794338537$ r3.out: SCF Done: $E(R M 06)=-235.786630030$ r4.out: SCF Done: $E(R M 06)=-275.097190978$ r5.out: SCF Done: $E(R M 06)=-275.089893203$ r6.out: SCF Done: $\mathrm{E}(\mathrm{RM} 06)=-275.088573206$ r7.out: SCF Done: $\mathrm{E}(\mathrm{RM} 06)=-275.087603762$ r8.out: SCF Done: $\mathrm{E}(\mathrm{RM} 06)=-275.089728234$ r9.out: SCF Done: E(RM06) = -275.087956038 A.U. A.U. A.U. A.U. A.U. A.U. A.U. A.U. A.U. A.U. A.U. A.U. A.U. A.U. A.U. A.U. A.U. A.U. A.U. A.U. wB97XD/Def2QZVP

C1.out: SCF Done: $E(R w B 97 X D)=-390.781712910 \quad$ A.U. C2H6.out: SCF Done: $\mathrm{E}(\mathrm{RwB} 97 \mathrm{XD})=-79.8403313653$ A.U C2.out: SCF Done: $\mathrm{E}(\mathrm{RwB} 97 \mathrm{XD})=-469.430273046 \quad$ A.U. C3.out: SCF Done: $E(R w B 97 X D)=-469.418692282 \quad$ A.U. C4.out: SCF Done: $E(R w B 97 X D)=-548.079009753$ A.U. C5.out: SCF Done: $\mathrm{E}(\mathrm{RwB} 97 \mathrm{XD})=-545.655524158$ A.U. CH4.out: SCF Done: $E(R w B 97 X D)=-40.5238323257$ A.U. H10.out: SCF Done: $E(R w B 97 X D)=-276.437093001 \quad$ A.U. H11.out: SCF Done: $E(R w B 97 X D)=-276.437854538$ A.U. H12.out: SCF Done: $E(R w B 97 X D)=-276.438554596 \quad$ A.U. H13.out: SCF Done: $E(R w B 97 X D)=-276.437250959$ A.U. H14.out: SCF Done: $E(R w B 97 X D)=-276.435533599 \quad$ A.U. H15.out: SCF Done: $E(R w B 97 X D)=-315.755380220$ A.U. H1.out: SCF Done: $E($ RwB97XD $)=-237.118572128$ A.U. H2.out: SCF Done: $E(R w B 97 X D)=-237.118809462 \quad$ A.U. H3.out: SCF Done: $E(R w B 97 X D)=-237.120208314$ A.U. H4.out: SCF Done: $E(R w B 97 X D)=-237.117832408$ A.U. H5.out: SCF Done: $E(R w B 97 X D)=-237.117957848$ A.U. H6.out: SCF Done: $E(R w B 97 X D)=-276.437423141 \quad$ A.U. H7.out: SCF Done: $E(R w B 97 X D)=-276.439588429$ A.U. H8.out: SCF Done: $E(R w B 97 X D)=-276.438312221 \quad$ A.U. H9.out: SCF Done: $E(R w B 97 X D)=-276.439588181 \quad$ A.U. r10.out: SCF Done: $\mathrm{E}(\mathrm{RwB} 97 \mathrm{XD})=-391.979405060 \quad$ A.U. r11.out: SCF Done: $\mathrm{E}(\mathrm{RwB} 97 \mathrm{XD})=-391.965436955$ A.U. r12.out: SCF Done: $\mathrm{E}(\mathrm{RwB} 97 \mathrm{XD})=-391.974123480 \quad$ A.U. r13.out: SCF Done: $\mathrm{E}(\mathrm{RwB} 97 \mathrm{XD})=-391.990746580 \quad$ A.U. r14.out: SCF Done: $\mathrm{E}(\mathrm{RwB} 97 \mathrm{XD})=-391.986965371 \quad$ A.U. r15.out: SCF Done: $\mathrm{E}(\mathrm{RwB} 97 \mathrm{XD})=-470.625763521 \quad$ A.U. 
r16.out: SCF Done: $\mathrm{E}(\mathrm{RwB} 97 \mathrm{XD})=-471.830909290$ r1.out: SCF Done: $\mathrm{E}(\mathrm{RwB} 97 \mathrm{XD})=-196.582881565$ r2.out: SCF Done: $E(R w B 97 X D)=-235.912379910$ r3.out: SCF Done: $E(R w B 97 X D)=-235.904823203$ r4.out: SCF Done: $E(R w B 97 X D)=-275.234392539$ r5.out: SCF Done: $\mathrm{E}(\mathrm{RwB} 97 \mathrm{XD})=-275.226235483$ r6.out: SCF Done: $\mathrm{E}(\mathrm{RwB} 97 \mathrm{XD})=-275.226411394$ r7.out: SCF Done: $E(R w B 97 X D)=-275.224699140$ r8.out: SCF Done: $E(R w B 97 X D)=-275.227049523$ r9.out: SCF Done: $E(R w B 97 X D)=-275.225904265$

A.U.

A.U.

A.U.

A.U.

A.U.

A.U.

A.U.

A.U.

A.U.

A.U.

\section{B2PLYP/Def2QZVP}

C1.out: E2(B2PLYP $)=-0.5844809578 \mathrm{D}+00 \mathrm{E}(\mathrm{B} 2 \mathrm{PLYP})=-0.39054250252006 \mathrm{D}+03$

C2H6.out: E2(B2PLYP) $=-0.1261742243 \mathrm{D}+00 \mathrm{E}(\mathrm{B} 2 \mathrm{PLYP})=-0.79784825980402 \mathrm{D}+02$

C2.out: E2(B2PLYP $)=-0.7081590780 \mathrm{D}+00 \mathrm{E}(\mathrm{B} 2 \mathrm{PLYP})=-0.46913901284519 \mathrm{D}+03$

C3.out: E2(B2PLYP $)=-0.7094412058 \mathrm{D}+00 \mathrm{E}(\mathrm{B} 2 \mathrm{PLYP})=-0.46912623299109 \mathrm{D}+03$

C4.out: E2(B2PLYP $)=-0.8318855855 \mathrm{D}+00 \mathrm{E}(\mathrm{B} 2 \mathrm{PLYP})=-0.54773543761439 \mathrm{D}+03$

C5.out: E2(B2PLYP) $=-0.8120649390 \mathrm{D}+00 \mathrm{E}(\mathrm{B} 2 \mathrm{PLYP})=-0.54532715822186 \mathrm{D}+03$

CH4.out: E2(B2PLYP $)=-0.6739250537 \mathrm{D}-01 \mathrm{E}(\mathrm{B} 2 \mathrm{PLYP})=-0.40493357908707 \mathrm{D}+02$

H10.out: E2(B2PLYP) $=-0.4282898737 \mathrm{D}+00 \mathrm{E}(\mathrm{B} 2 \mathrm{PLYP})=$

H11.out: E2(B2PLYP) $=-0.4303600071 \mathrm{D}+00 \mathrm{E}(\mathrm{B} 2 \mathrm{PLYP})=$

H12.out: E2(B2PLYP) $=$

H13.out: E2(B2PLYP) $=$

$-0.4295231880 \mathrm{D}+00 \mathrm{E}(\mathrm{B} 2 \mathrm{PLYP})=$

$-0.4266804140 \mathrm{D}+00 \mathrm{E}(\mathrm{B} 2 \mathrm{PLYP})=$

H14.out: E2(B2PLYP) $=$

$-0.4272367689 \mathrm{D}+00 \mathrm{E}(\mathrm{B} 2 \mathrm{PLYP})=$

$-0.4932902424 \mathrm{D}+00 \mathrm{E}(\mathrm{B} 2 \mathrm{PLYP})=$

H15.out: E2(B2PLYP) $=$

H1.out: E2(B2PLYP) =

$-0.3679731632 \mathrm{D}+00 \mathrm{E}(\mathrm{B} 2 \mathrm{PLYP})=$

$-0.3664111457 \mathrm{D}+00 \mathrm{E}(\mathrm{B} 2 \mathrm{PLYP})=$

$-0.3685494749 \mathrm{D}+00 \mathrm{E}(\mathrm{B} 2 \mathrm{PLYP})=$

H3.out: E2(B2PLYP) $=$

H4.out: E2(B2PLYP) $=$

H5.out: E2(B2PLYP) $=$

H6.out: E2(B2PLYP) =

H7.out: E2(B2PLYP) =

H8.out: E2(B2PLYP) =

H9.out: E2(B2PLYP) $=$

r10.out: E2(B2PLYP) =

$-0.3668738701 \mathrm{D}+00 \mathrm{E}(\mathrm{B} 2 \mathrm{PLYP})=$

$-0.3650224223 \mathrm{D}+00 \mathrm{E}(\mathrm{B} 2 \mathrm{PLYP})=$

$-0.4247647093 \mathrm{D}+00 \mathrm{E}(\mathrm{B} 2 \mathrm{PLYP})=$

$-0.4277514178 \mathrm{D}+00 \mathrm{E}(\mathrm{B} 2 \mathrm{PLYP})=$

$-0.4261824525 \mathrm{D}+00 \mathrm{E}(\mathrm{B} 2 \mathrm{PLYP})=$ $-0.4284227070 \mathrm{D}+00 \mathrm{E}(\mathrm{B} 2 \mathrm{PLYP})=$

$-0.5926353708 \mathrm{D}+00 \mathrm{E}(\mathrm{B} 2 \mathrm{PLYP})=$

$-0.5934653622 \mathrm{D}+00 \mathrm{E}(\mathrm{B} 2 \mathrm{PLYP})=$

$-0.27625251273084 \mathrm{D}+03$

$-0.27625305149225 \mathrm{D}+03$

$-0.27625342586229 \mathrm{D}+03$

$-0.27625377668068 \mathrm{D}+03$

$-0.27625168748941 \mathrm{D}+03$

$-0.31554359711594 \mathrm{D}+03$

$-0.23695997384139 \mathrm{D}+03$

$-0.23696124664242 \mathrm{D}+03$

$-0.23696193071540 \mathrm{D}+03$

$-0.23695984914142 \mathrm{D}+03$

$-0.23696098300287 \mathrm{D}+03$

$-0.27625499421632 \mathrm{D}+03$

$-0.27625567251994 \mathrm{D}+03$

$-0.27625527610150 \mathrm{D}+03$

$-0.27625575403130 \mathrm{D}+03$

$-0.39173149577788 \mathrm{D}+03$

r11.out: E2(B2PLYP) =

r12.out: E2(B2PLYP) =

r13.out: E2(B2PLYP) =

r14.out: E2(B2PLYP) =

r15.out: E2(B2PLYP) =

r16.out: E2(B2PLYP) =

r1.out: E2(B2PLYP) =

r2.out: E2(B2PLYP) $=$

r3.out: E2(B2PLYP) =

r4.out: E2(B2PLYP) =

r5.out: E2(B2PLYP) =

r6.out: E2(B2PLYP) =

$-0.5912942419 \mathrm{D}+00 \mathrm{E}(\mathrm{B} 2 \mathrm{PLYP})=$

$-0.5910541640 \mathrm{D}+00 \mathrm{E}(\mathrm{B} 2 \mathrm{PLYP})=$

$-0.5918627339 \mathrm{D}+00 \mathrm{E}(\mathrm{B} 2 \mathrm{PLYP})=$

$-0.7119818327 \mathrm{D}+00 \mathrm{E}(\mathrm{B} 2 \mathrm{PLYP})=$ $-0.7175152643 \mathrm{D}+00 \mathrm{E}(\mathrm{B} 2 \mathrm{PLYP})=$ $-0.2973593399 \mathrm{D}+00 \mathrm{E}(\mathrm{B} 2 \mathrm{PLYP})=$ $-0.3574080233 \mathrm{D}+00 \mathrm{E}(\mathrm{B} 2 \mathrm{PLYP})=$ $-0.3577695312 \mathrm{D}+00 \mathrm{E}(\mathrm{B} 2 \mathrm{PLYP})=$ $-0.4181603756 \mathrm{D}+00 \mathrm{E}(\mathrm{B} 2 \mathrm{PLYP})=$ $-0.4198692062 \mathrm{D}+00 \mathrm{E}(\mathrm{B} 2 \mathrm{PLYP})=$ $-0.4182270095 \mathrm{D}+00 \mathrm{E}(\mathrm{B} 2 \mathrm{PLYP})=$ $-0.39171595281641 \mathrm{D}+03$

$-0.39172645663925 \mathrm{D}+03$

$-0.39174359626076 \mathrm{D}+03$

$-0.39173863433626 \mathrm{D}+03$

$-0.47032617395711 \mathrm{D}+03$

$-0.47152662732147 \mathrm{D}+03$

$-0.19645839051688 \mathrm{D}+03$

$-0.23576169346253 \mathrm{D}+03$

$-0.23575463779796 \mathrm{D}+03$

$-0.27505800852508 \mathrm{D}+03$

$-0.27504973898448 \mathrm{D}+03$

$-0.27505052545161 \mathrm{D}+03$

r7.out: E2(B2PLYP) = $-0.4193482498 \mathrm{D}+00 \mathrm{E}(\mathrm{B} 2 \mathrm{PLYP})=$ $-0.27504773433463 \mathrm{D}+03$ r8.out: E2(B2PLYP) = $-0.4186277794 \mathrm{D}+00 \mathrm{E}(\mathrm{B} 2 \mathrm{PLYP})=$ $-0.27505071172266 \mathrm{D}+03$ 
r9.out: E2(B2PLYP) $=-0.4182643209 \mathrm{D}+00 \mathrm{E}(\mathrm{B} 2 \mathrm{PLYP})=-0.27505004722881 \mathrm{D}+03$ PBEQIDH/Def2QZVP C1.out: E2(PBEQIDH $)=-0.6750510549 \mathrm{D}+00 \mathrm{E}(\mathrm{PBEQIDH})=-0.39040618782747 \mathrm{D}+03$ C2H6.out: E2(PBEQIDH) $=-0.1458632164 \mathrm{D}+00 \mathrm{E}(\mathrm{PBEQIDH})=\quad-$ $0.79754842024397 \mathrm{D}+0$ C2.out: E2(PBEQIDH) $=$ C3.out: E2(PBEQIDH) $=$ C4.out: E2(PBEQIDH) $=$ C5.out: $\mathrm{E} 2(\mathrm{PBEQIDH})=$ CH4.out: E2(PBEQIDH) $=$ $0.40477943358113 \mathrm{D}+02$ H10.out: E2(PBEQIDH) = $0.27615348904804 \mathrm{D}+03$ H11.out: E2(PBEQIDH) $=$ $0.27615516305317 \mathrm{D}+03$ H12.out: E2(PBEQIDH) $=$ $0.27615523495027 \mathrm{D}+03$ H13.out: E2(PBEQIDH) = $0.27615390488583 \mathrm{D}+03$ H14.out: E2(PBEQIDH) $=$ $0.27615197732013 \mathrm{D}+03$ H15.out: E2(PBEQIDH) = $0.31543350453022 \mathrm{D}+03$

H1.out: E2(PBEQIDH) = H2.out: E2(PBEQIDH) = H3.out: E2(PBEQIDH) = H4.out: E2(PBEQIDH) = H5.out: E2(PBEQIDH) $=$ H6.out: E2(PBEQIDH) $=$ H7.out: E2(PBEQIDH) = H8.out: E2(PBEQIDH) = H9.out: E2(PBEQIDH) = r10.out: E2(PBEQIDH) $=$ r11.out: E2(PBEQIDH) = r12.out: E2(PBEQIDH) = r13.out: E2(PBEQIDH) = r14.out: E2(PBEQIDH) = r15.out: E2(PBEQIDH) = r16.out: E2(PBEQIDH) = r1.out: E2(PBEQIDH) = r2.out: $\mathrm{E} 2(\mathrm{PBEQIDH})=$ r3.out: E2(PBEQIDH) = r4.out: $\mathrm{E} 2(\mathrm{PBEQIDH})=$ r5.out: E2(PBEQIDH) $=$ r6.out: $\mathrm{E} 2(\mathrm{PBEQIDH})=$ r7.out: $\mathrm{E} 2(\mathrm{PBEQIDH})=$ r8.out: E2(PBEQIDH) $=$ r9. out: E2(PBEQIDH) $=$ PBE0DH/Def2QZVP $-0.8179752809 \mathrm{D}+00 \mathrm{E}(\mathrm{PBEQIDH})=-0.46897641865017 \mathrm{D}+03$ $-0.8194254192 \mathrm{D}+00 \mathrm{E}(\mathrm{PBEQIDH})=-0.46896432022387 \mathrm{D}+03$ $-0.9609608171 \mathrm{D}+00 \mathrm{E}(\mathrm{PBEQIDH})=-0.54754658062952 \mathrm{D}+03$ $-0.9378508992 \mathrm{D}+00 \mathrm{E}(\mathrm{PBEQIDH})=-0.54513954088711 \mathrm{D}+03$ $-0.7797355538 \mathrm{D}-01 \mathrm{E}(\mathrm{PBEQIDH})=-$ $-0.4948548708 \mathrm{D}+00 \mathrm{E}(\mathrm{PBEQIDH})=$ $-0.4972580450 \mathrm{D}+00 \mathrm{E}(\mathrm{PBEQIDH})=$ $-0.4963028773 \mathrm{D}+00 \mathrm{E}(\mathrm{PBEQIDH})=$ $-0.4929897264 \mathrm{D}+00 \mathrm{E}(\mathrm{PBEQIDH})=$ $-0.4936154129 \mathrm{D}+00 \mathrm{E}(\mathrm{PBEQIDH})=$ $-0.5699778326 \mathrm{D}+00 \mathrm{E}(\mathrm{PBEQIDH})=$ $-0.4251806297 \mathrm{D}+00 \mathrm{E}(\mathrm{PBEQIDH})=$ $-0.4233705077 \mathrm{D}+00 \mathrm{E}(\mathrm{PBEQIDH})=$ $-0.4258536472 \mathrm{D}+00 \mathrm{E}(\mathrm{PBEQIDH})=$ $-0.4239052957 \mathrm{D}+00 \mathrm{E}(\mathrm{PBEQIDH})=$ $-0.4217692614 \mathrm{D}+00 \mathrm{E}(\mathrm{PBEQIDH})=$ $-0.4907832510 \mathrm{D}+00 \mathrm{E}(\mathrm{PBEQIDH})=$ $-0.4942355031 \mathrm{D}+00 \mathrm{E}(\mathrm{PBEQIDH})=$ $-0.4924183393 \mathrm{D}+00 \mathrm{E}(\mathrm{PBEQIDH})=$ $-0.4950162114 \mathrm{D}+00 \mathrm{E}(\mathrm{PBEQIDH})=$ $-0.6844453456 \mathrm{D}+00 \mathrm{E}(\mathrm{PBEQIDH})=$ $-0.6853499972 \mathrm{D}+00 \mathrm{E}(\mathrm{PBEQIDH})=$ $-0.6828290333 \mathrm{D}+00 \mathrm{E}(\mathrm{PBEQIDH})=$ $-0.6827317599 \mathrm{D}+00 \mathrm{E}(\mathrm{PBEQIDH})=$ $-0.6836512215 \mathrm{D}+00 \mathrm{E}(\mathrm{PBEQIDH})=$ $-0.8224034205 \mathrm{D}+00 \mathrm{E}(\mathrm{PBEQIDH})=$ $-0.8288797344 \mathrm{D}+00 \mathrm{E}(\mathrm{PBEQIDH})=$ $-0.3433835071 \mathrm{D}+00 \mathrm{E}(\mathrm{PBEQIDH})=$ $-0.4128752964 \mathrm{D}+00 \mathrm{E}(\mathrm{PBEQIDH})=$ $-0.4131905455 \mathrm{D}+00 \mathrm{E}(\mathrm{PBEQIDH})=$ $-0.4830684998 \mathrm{D}+00 \mathrm{E}(\mathrm{PBEQIDH})=$ $-0.4849493279 \mathrm{D}+00 \mathrm{E}(\mathrm{PBEQIDH})=$ $-0.4830451913 \mathrm{D}+00 \mathrm{E}(\mathrm{PBEQIDH})=$ $-0.4843491230 \mathrm{D}+00 \mathrm{E}(\mathrm{PBEQIDH})=$ $-0.4835145525 \mathrm{D}+00 \mathrm{E}(\mathrm{PBEQIDH})=$ $-0.4830781363 \mathrm{D}+00 \mathrm{E}(\mathrm{PBEQIDH})=$ $-0.23687483323109 \mathrm{D}+03$ $-0.23687527145510 \mathrm{D}+03$ $-0.23687713338412 \mathrm{D}+03$ $-0.23687412855190 \mathrm{D}+03$ $-0.23687435131045 \mathrm{D}+03$ $-0.27615421673096 \mathrm{D}+03$ $-0.27615636971115 \mathrm{D}+03$ $-0.27615516927854 \mathrm{D}+03$ $-0.27615683921045 \mathrm{D}+03$ $-0.39159300639989 \mathrm{D}+03$ $-0.39157688143128 \mathrm{D}+03$ $-0.39158654288285 \mathrm{D}+03$ $-0.39160478629047 \mathrm{D}+03$ $-0.39160021446689 \mathrm{D}+03$ $-0.47015973528725 \mathrm{D}+03$ $-0.47135753552781 \mathrm{D}+03$ $-0.19638679393208 \mathrm{D}+03$ $-0.23567671696723 \mathrm{D}+03$ $-0.23566925542235 \mathrm{D}+03$ $-0.27495936968715 \mathrm{D}+03$ $-0.27495149870719 \mathrm{D}+03$ $-0.27495132756415 \mathrm{D}+03$ $-0.27494929017582 \mathrm{D}+03$ $-0.27495177830019 \mathrm{D}+03$ $-0.27495081095295 \mathrm{D}+03$ C1.out: $\mathrm{E} 2(\mathrm{PBEODH})=-0.2736341513 \mathrm{D}+00 \mathrm{E}(\mathrm{PBE} 0 \mathrm{DH})=-0.39045946941629 \mathrm{D}+03$ 
C2H6.out: E2(PBE0DH) = C2.out: $\mathrm{E} 2(\mathrm{PBE} 0 \mathrm{DH})=$ C3.out: $\mathrm{E} 2(\mathrm{PBE} 0 \mathrm{DH})=$ C4.out: E2(PBE0DH $)=$ C5.out: E2(PBE0DH $)=$ CH4.out: E2(PBE0DH $)=$ H10.out: E2(PBE0DH) $=$ H11.out: E2(PBE0DH) $=$ H12.out: $\mathrm{E} 2(\mathrm{PBE} 0 \mathrm{DH})=$ H13.out: E2(PBE0DH) = H14.out: E2(PBE0DH) $=$ H15.out: E2(PBE0DH) $=$ H1.out: E2(PBE0DH) $=$ H2.out: E2(PBE0DH) $=$ H3.out: E2(PBE0DH $)=$ H4.out: E2(PBE0DH) $=$ H5.out: E2(PBE0DH) $=$ H6.out: E2(PBE0DH) = H7.out: E2(PBE0DH) $=$ H8.out: E2(PBE0DH) $=$ H9.out: E2(PBE0DH) $=$ r10.out: E2 $(\mathrm{PBE} 0 \mathrm{DH})=$ r11.out: E2(PBE0DH) $=$ r12.out: E2 $(\mathrm{PBE} 0 \mathrm{DH})=$ r13.out: E2(PBE0DH $)=$ r14.out: E2(PBE0DH) = r15.out: E2 $(\mathrm{PBE} 0 \mathrm{DH})=$ r16.out: E2(PBE0DH) = r1.out: $\mathrm{E} 2(\mathrm{PBE} 0 \mathrm{DH})=$ r2.out: $\mathrm{E} 2(\mathrm{PBE} 0 \mathrm{DH})=$ r3.out: $\mathrm{E} 2(\mathrm{PBE} 0 \mathrm{DH})=$ r4.out: $\mathrm{E} 2(\mathrm{PBE} 0 \mathrm{DH})=$ r5.out: $\mathrm{E} 2(\mathrm{PBE} 0 \mathrm{DH})=$ r6.out: $\mathrm{E} 2(\mathrm{PBE} 0 \mathrm{DH})=$ r7.out: $\mathrm{E} 2(\mathrm{PBE} 0 \mathrm{DH})=$ r8.out: $\mathrm{E} 2(\mathrm{PBE} 0 \mathrm{DH})=$ r9.out: $\mathrm{E} 2(\mathrm{PBE} 0 \mathrm{DH})=$ B2PLYPD3BJ/Def2QZVP

C1.out: E2(B2PLYP) $=-0.5844809578 \mathrm{D}+00 \mathrm{E}(\mathrm{B} 2 \mathrm{PLYP})=$ C2H6.out: E2(B2PLYP $)=-0.1261742243 \mathrm{D}+00 \mathrm{E}(\mathrm{B} 2 \mathrm{PLYP})=$ C2.out: E2(B2PLYP) $=$ C3.out: E2(B2PLYP) = C4.out: E2(B2PLYP) = C5.out: E2(B2PLYP) = CH4.out: E2(B2PLYP) $=$ H10.out: E2(B2PLYP) $=$ H11.out: E2(B2PLYP) = H12.out: E2(B2PLYP) = H13.out: E2(B2PLYP) = H14.out: E2(B2PLYP) =
$-0.5896553903 \mathrm{D}-01 \mathrm{E}(\mathrm{PBE} 0 \mathrm{DH})=$ $-0.3316064743 \mathrm{D}+00 \mathrm{E}(\mathrm{PBE} 0 \mathrm{DH})=$ $-0.3322233318 \mathrm{D}+00 \mathrm{E}(\mathrm{PBE} 0 \mathrm{DH})=$ $-0.3896053444 \mathrm{D}+00 \mathrm{E}(\mathrm{PBE} 0 \mathrm{DH})=$ $-0.3803439247 \mathrm{D}+00 \mathrm{E}(\mathrm{PBE} 0 \mathrm{DH})=$ $-0.3148680111 \mathrm{D}-01 \mathrm{E}(\mathrm{PBE} 0 \mathrm{DH})=$ $-0.2003837580 \mathrm{D}+00 \mathrm{E}(\mathrm{PBE} 0 \mathrm{DH})=$ $-0.2013895619 \mathrm{D}+00 \mathrm{E}(\mathrm{PBE} 0 \mathrm{DH})=$ $-0.2009864110 \mathrm{D}+00 \mathrm{E}(\mathrm{PBE} 0 \mathrm{DH})=$ $-0.1996007329 \mathrm{D}+00 \mathrm{E}(\mathrm{PBE} 0 \mathrm{DH})=$ $-0.1998679324 \mathrm{D}+00 \mathrm{E}(\mathrm{PBE} 0 \mathrm{DH})=$ $-0.2309036935 \mathrm{D}+00 \mathrm{E}(\mathrm{PBE} 0 \mathrm{DH})=$ $-0.1721487811 \mathrm{D}+00 \mathrm{E}(\mathrm{PBE} 0 \mathrm{DH})=$ $-0.1713894982 \mathrm{D}+00 \mathrm{E}(\mathrm{PBE} 0 \mathrm{DH})=$ $-0.1724274568 \mathrm{D}+00 \mathrm{E}(\mathrm{PBE} 0 \mathrm{DH})=$ $-0.1716150239 \mathrm{D}+00 \mathrm{E}(\mathrm{PBE} 0 \mathrm{DH})=$ $-0.1707163578 \mathrm{D}+00 \mathrm{E}(\mathrm{PBE} 0 \mathrm{DH})=$ $-0.2001209385 \mathrm{D}+00 \mathrm{E}(\mathrm{PBE} 0 \mathrm{DH})=$ $-0.1993581992 \mathrm{D}+00 \mathrm{E}(\mathrm{PBE} 0 \mathrm{DH})=$ $-0.2004463658 \mathrm{D}+00 \mathrm{E}(\mathrm{PBE} 0 \mathrm{DH})=$ $-0.2773781152 \mathrm{D}+00 \mathrm{E}(\mathrm{PBE} 0 \mathrm{DH})=$ $-0.2777852660 \mathrm{D}+00 \mathrm{E}(\mathrm{PBE} 0 \mathrm{DH})=$ $-0.2767164038 \mathrm{D}+00 \mathrm{E}(\mathrm{PBE} 0 \mathrm{DH})=$ $-0.2766535915 \mathrm{D}+00 \mathrm{E}(\mathrm{PBE} 0 \mathrm{DH})=$ $-0.2770398603 \mathrm{D}+00 \mathrm{E}(\mathrm{PBE} 0 \mathrm{DH})=$ $-0.3332617609 \mathrm{D}+00 \mathrm{E}(\mathrm{PBE} 0 \mathrm{DH})=$ $-0.3357449996 \mathrm{D}+00 \mathrm{E}(\mathrm{PBE} 0 \mathrm{DH})=$ $-0.1390613161 \mathrm{D}+00 \mathrm{E}(\mathrm{PBE} 0 \mathrm{DH})=$ $-0.1672066886 \mathrm{D}+00 \mathrm{E}(\mathrm{PBE} 0 \mathrm{DH})=$ $-0.1673472142 \mathrm{D}+00 \mathrm{E}(\mathrm{PBE} 0 \mathrm{DH})=$ $-0.1956573624 \mathrm{D}+00 \mathrm{E}(\mathrm{PBE} 0 \mathrm{DH})=$ $-0.1964484422 \mathrm{D}+00 \mathrm{E}(\mathrm{PBE} 0 \mathrm{DH})=$ $-0.1956558684 \mathrm{D}+00 \mathrm{E}(\mathrm{PBE} 0 \mathrm{DH})=$ $-0.1962004370 \mathrm{D}+00 \mathrm{E}(\mathrm{PBE} 0 \mathrm{DH})=$ $-0.1958512345 \mathrm{D}+00 \mathrm{E}(\mathrm{PBE} 0 \mathrm{DH})=$ $-0.1956722682 \mathrm{D}+00 \mathrm{E}(\mathrm{PBE} 0 \mathrm{DH})=$ $-0.5844809578 \mathrm{D}+00 \mathrm{E}(\mathrm{B} 2 \mathrm{PLYP})=$
$=-0.1261742243 \mathrm{D}+00 \mathrm{E}(\mathrm{B} 2 \mathrm{PLYP})$ $-0.7081590780 \mathrm{D}+00 \mathrm{E}(\mathrm{B} 2 \mathrm{PLYP})=$ $-0.7094412058 \mathrm{D}+00 \mathrm{E}(\mathrm{B} 2 \mathrm{PLYP})=$ $-0.8318855855 \mathrm{D}+00 \mathrm{E}(\mathrm{B} 2 \mathrm{PLYP})=$ $-0.8120649390 \mathrm{D}+00 \mathrm{E}(\mathrm{B} 2 \mathrm{PLYP})=$ $-0.6739250537 \mathrm{D}-01 \mathrm{E}(\mathrm{B} 2 \mathrm{PLYP})=$ $-0.4282898737 \mathrm{D}+00 \mathrm{E}(\mathrm{B} 2 \mathrm{PLYP})=$ $-0.4303600071 \mathrm{D}+00 \mathrm{E}(\mathrm{B} 2 \mathrm{PLYP})=$ $-0.4295231880 \mathrm{D}+00 \mathrm{E}(\mathrm{B} 2 \mathrm{PLYP})=$ $-0.4266804140 \mathrm{D}+00 \mathrm{E}(\mathrm{B} 2 \mathrm{PLYP})=$ $-0.4272367689 \mathrm{D}+00 \mathrm{E}(\mathrm{B} 2 \mathrm{PLYP})=$ $-0.1986704919 \mathrm{D}+00 \mathrm{E}(\mathrm{PBE} 0 \mathrm{DH})=$
$-0.79767160626152 \mathrm{D}+02$ $-0.46903889064146 \mathrm{D}+03$ $-0.46902652632709 \mathrm{D}+03$ $-0.54761826910917 \mathrm{D}+03$ $-0.54521162270356 \mathrm{D}+03$ $-0.40484175744216 \mathrm{D}+02$ $-0.27619266676155 \mathrm{D}+03$ $-0.27619336669925 \mathrm{D}+03$ $-0.27619377668667 \mathrm{D}+03$ $-0.27619376799516 \mathrm{D}+03$ $-0.27619169862314 \mathrm{D}+03$ $-0.31547590274370 \mathrm{D}+03$ $-0.23690856735221 \mathrm{D}+03$ $-0.23690965317751 \mathrm{D}+03$ $-0.23691050976409 \mathrm{D}+03$ $-0.23690837968747 \mathrm{D}+03$ $-0.23690932006623 \mathrm{D}+03$ $-0.27619482590083 \mathrm{D}+03$ $-0.27619569143505 \mathrm{D}+03$ $-0.27619517741283 \mathrm{D}+03$ $-0.27619578614544 \mathrm{D}+03$ $-0.39164734601160 \mathrm{D}+03$ $-0.39163130171890 \mathrm{D}+03$ $-0.39164170202836 \mathrm{D}+03$ $-0.39165958226016 \mathrm{D}+03$ $-0.39165469365437 \mathrm{D}+03$ $-0.47022528747839 D+03$ $-0.47142480736089 \mathrm{D}+03$ $-0.19641574629792 \mathrm{D}+03$ $-0.23571088568865 \mathrm{D}+03$ $-0.23570351624800 \mathrm{D}+03$ $-0.27499870244878 \mathrm{D}+03$ $-0.27499024775248 \mathrm{D}+03$ $-0.27499087251545 \mathrm{D}+03$ $-0.27498833623435 \mathrm{D}+03$ $-0.27499115969556 \mathrm{D}+03$ $-0.27499033861859 \mathrm{D}+03$

$-0.39056668180270 \mathrm{D}+03$

$-0.79787137215055 \mathrm{D}+02$

$-0.46917007149229 \mathrm{D}+03$ $-0.46915810398640 \mathrm{D}+03$ $-0.54777350470116 \mathrm{D}+03$ $-0.54536438950599 \mathrm{D}+03$ $-0.40494149118337 \mathrm{D}+02$ $-0.27626769884921 \mathrm{D}+03$ $-0.27626926890674 \mathrm{D}+03$ $-0.27626936098721 \mathrm{D}+03$ $-0.27626786700586 \mathrm{D}+03$ $-0.27626615353785 \mathrm{D}+03$ 


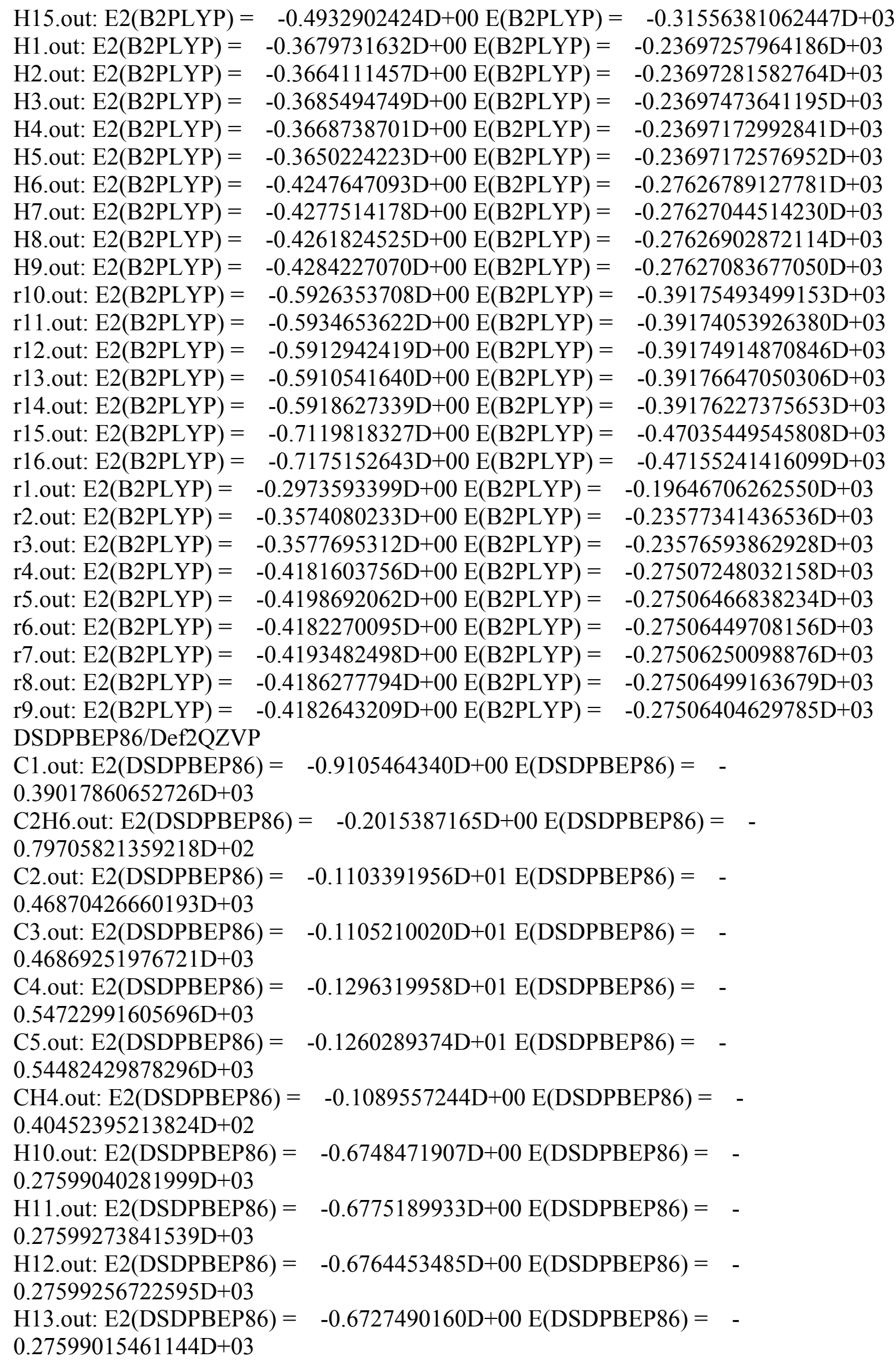


H14.out: E2(DSDPBEP86 $)=-0.6734799859 D+00 \mathrm{E}(\mathrm{DSDPBEP} 86)=-$ $0.27598842972889 \mathrm{D}+03$

H15.out: E2(DSDPBEP86 $)=-0.7754930002 \mathrm{D}+00 \mathrm{E}(\mathrm{DSDPBEP} 86)=-$ $0.31524907575015 \mathrm{D}+03$

H1.out: E2(DSDPBEP86 $)=-0.5803080489 \mathrm{D}+00 \mathrm{E}(\mathrm{DSDPBEP} 86)=$ $0.23673457493344 \mathrm{D}+03$

H2.out: E2(DSDPBEP86) $=-0.5782778818 \mathrm{D}+00 \mathrm{E}(\mathrm{DSDPBEP} 86)=-$ $0.23673440244427 \mathrm{D}+03$

H3.out: E2(DSDPBEP86 $)=-0.5810413811 \mathrm{D}+00 \mathrm{E}(\mathrm{DSDPBEP} 86)=-$ $0.23673705300728 \mathrm{D}+03$

H4.out: E2(DSDPBEP86 $)=-0.5788822122 \mathrm{D}+00 \mathrm{E}(\mathrm{DSDPBEP} 86)=-$ $0.23673339708040 \mathrm{D}+03$

H5.out: E2(DSDPBEP86 $)=-0.5764970877 \mathrm{D}+00 \mathrm{E}(\mathrm{DSDPBEP} 86)=-$ $0.23673293615492 \mathrm{D}+03$

H6.out: E2(DSDPBEP86 $)=-0.6702784478 D+00 \mathrm{E}(\mathrm{DSDPBEP} 86)=-$ $0.27598972917467 \mathrm{D}+03$

H7.out: E2(DSDPBEP86 $)=-0.6741108438 \mathrm{D}+00 \mathrm{E}(\mathrm{DSDPBEP} 86)=-$ $0.27599307091359 \mathrm{D}+03$

H8.out: E2(DSDPBEP86 $)=-0.6720966947 \mathrm{D}+00 \mathrm{E}(\mathrm{DSDPBEP} 86)=-$ $0.27599124480484 \mathrm{D}+03$

H9.out: E2(DSDPBEP86 $)=-0.6749974624 \mathrm{D}+00 \mathrm{E}(\mathrm{DSDPBEP} 86)=-$ $0.27599378055365 \mathrm{D}+03$

r10.out: E2(DSDPBEP86) $=-0.9263668850 \mathrm{D}+00 \mathrm{E}(\mathrm{DSDPBEP} 86)=-$ $0.39136422266410 \mathrm{D}+03$

r11.out: E2(DSDPBEP86 $)=-0.9276203959 \mathrm{D}+00 \mathrm{E}(\mathrm{DSDPBEP} 86)=-$ $0.39134914267711 \mathrm{D}+03$

r12.out: $\mathrm{E} 2(\mathrm{DSDPBEP} 86)=-0.9246592819 \mathrm{D}+00 \mathrm{E}(\mathrm{DSDPBEP} 86)=-$ $0.39135767424738 \mathrm{D}+03$

r13.out: E2(DSDPBEP86) $=-0.9242435822 \mathrm{D}+00 \mathrm{E}(\mathrm{DSDPBEP} 86)=$ $0.39137545606973 \mathrm{D}+03$

r14.out: $\mathrm{E} 2(\mathrm{DSDPBEP} 86)=-0.9253712713 \mathrm{D}+00 \mathrm{E}(\mathrm{DSDPBEP} 86)=-$ $0.39137133150421 \mathrm{D}+03$

r15.out: $\mathrm{E} 2(\mathrm{DSDPBEP} 86)=-0.1113746383 \mathrm{D}+01 \mathrm{E}(\mathrm{DSDPBEP} 86)=-$ $0.46988498139847 \mathrm{D}+03$

r16.out: E2(DSDPBEP86 $)=-0.1126169716 \mathrm{D}+01 \mathrm{E}(\mathrm{DSDPBEP} 86)=-$ $0.47108012412278 \mathrm{D}+03$

r1.out: E2(DSDPBEP86 $)=-0.4671852709 \mathrm{D}+00 \mathrm{E}(\mathrm{DSDPBEP} 86)=-$

$0.19626988223122 \mathrm{D}+03$

r2.out: E2(DSDPBEP86) $=-0.5613666900 \mathrm{D}+00 \mathrm{E}(\mathrm{DSDPBEP} 86)=\quad-$

$0.23553704757104 \mathrm{D}+03$

r3.out: E2(DSDPBEP86) $=-0.5618070822 \mathrm{D}+00 \mathrm{E}(\mathrm{DSDPBEP} 86)=-$

$0.23552953243471 \mathrm{D}+03$

r4.out: E2(DSDPBEP86 $)=-0.6564119590 \mathrm{D}+00 \mathrm{E}(\mathrm{DSDPBEP} 86)=-$

$0.27479698819766 \mathrm{D}+03$

r5.out: E2(DSDPBEP86 $)=-0.6586493084 \mathrm{D}+00 \mathrm{E}(\mathrm{DSDPBEP} 86)=-$

$0.27478963538567 \mathrm{D}+03$

r6.out: E2(DSDPBEP86 $)=-0.6564976558 \mathrm{D}+00 \mathrm{E}(\mathrm{DSDPBEP} 86)=$

$0.27478885949986 \mathrm{D}+03$

r7.out: E2(DSDPBEP86) $=-0.6579951049 \mathrm{D}+00 \mathrm{E}(\mathrm{DSDPBEP} 86)=$

$0.27478724512339 \mathrm{D}+03$ 
r8.out: E2(DSDPBEP86) $=$ $0.27478944343660 \mathrm{D}+03$

r9.out: E2(DSDPBEP86) = $0.27478840496454 \mathrm{D}+03$

PBEQIDHD3BJ/Def2QZVP

C1.out: E2(PBEQIDH $)=-0.6750510549 \mathrm{D}+00 \mathrm{E}(\mathrm{PBEQIDH})=-0.39041202773473 \mathrm{D}+03$

C2H6.out: E2(PBEQIDH) $=-0.1458632164 \mathrm{D}+00 \mathrm{E}(\mathrm{PBEQIDH})=-$

$0.79755280021383 \mathrm{D}+02$

C2.out: E2(PBEQIDH) $=$ C3.out: E2(PBEQIDH) $=$ C4.out: E2(PBEQIDH) $=$ C5.out: E2(PBEQIDH) $=$ CH4.out: E2(PBEQIDH) $=$ $0.40478082804875 \mathrm{D}+02$

H10.out: E2(PBEQIDH) $=$ $0.27615709025053 \mathrm{D}+03$

H11.out: E2(PBEQIDH) = $0.27615899635019 \mathrm{D}+03$

H12.out: E2(PBEQIDH) = $0.27615902035523 \mathrm{D}+03$

H13.out: E2(PBEQIDH) = $0.27615721938409 \mathrm{D}+03$

H14.out: E2(PBEQIDH) = $0.27615545265195 \mathrm{D}+03$

H15.out: E2(PBEQIDH) = $0.31543837973340 \mathrm{D}+03$

H1.out: E2(PBEQIDH) = H2.out: E2(PBEQIDH) $=$ H3.out: E2(PBEQIDH) $=$ H4.out: E2(PBEQIDH) $=$ H5.out: E2(PBEQIDH) = H6.out: E2(PBEQIDH) = H7.out: E2(PBEQIDH) = H8.out: E2(PBEQIDH) = H9.out: E2(PBEQIDH) = r10.out: E2(PBEQIDH) = r11.out: E2(PBEQIDH) = r12.out: E2(PBEQIDH) = r13.out: E2(PBEQIDH) $=$ r14.out: E2(PBEQIDH) = r15.out: E2(PBEQIDH) = r16.out: E2(PBEQIDH) = r1.out: E2(PBEQIDH) = r2.out: E2(PBEQIDH) = r3.out: E2(PBEQIDH) = r4.out: E2(PBEQIDH) = r5.out: E2(PBEQIDH) = r6.out: $\mathrm{E} 2(\mathrm{PBEQIDH})=$ r7.out: E2(PBEQIDH) $=$ r8.out: E2(PBEQIDH) =

$-0.6570021233 \mathrm{D}+00 \mathrm{E}(\mathrm{DSDPBEP} 86)=-$

$-0.6565561456 \mathrm{D}+00 \mathrm{E}(\mathrm{DSDPBEP} 86)=-$
$-0.8179752809 \mathrm{D}+00 \mathrm{E}(\mathrm{PBEQIDH})=$ $-0.8194254192 \mathrm{D}+00 \mathrm{E}(\mathrm{PBEQIDH})=$ $-0.9609608171 \mathrm{D}+00 \mathrm{E}(\mathrm{PBEQIDH})=$ $-0.9378508992 \mathrm{D}+00 \mathrm{E}(\mathrm{PBEQIDH})=$ $-0.7797355538 \mathrm{D}-01 \mathrm{E}(\mathrm{PBEQIDH})=$ $-0.4948548708 \mathrm{D}+00 \mathrm{E}(\mathrm{PBEQIDH})=$ $-0.4972580450 \mathrm{D}+00 \mathrm{E}(\mathrm{PBEQIDH})=$ $-0.4963028773 \mathrm{D}+00 \mathrm{E}(\mathrm{PBEQIDH})=$ $-0.4929897264 \mathrm{D}+00 \mathrm{E}(\mathrm{PBEQIDH})=$ $-0.4936154129 \mathrm{D}+00 \mathrm{E}(\mathrm{PBEQIDH})=$ $-0.5699778326 \mathrm{D}+00 \mathrm{E}(\mathrm{PBEQIDH})=$ $-0.4251806297 \mathrm{D}+00 \mathrm{E}(\mathrm{PBEQIDH})=$ $-0.4233705077 \mathrm{D}+00 \mathrm{E}(\mathrm{PBEQIDH})=$ $-0.4258536472 \mathrm{D}+00 \mathrm{E}(\mathrm{PBEQIDH})=$ $-0.4239052957 \mathrm{D}+00 \mathrm{E}(\mathrm{PBEQIDH})=$ $-0.4217692614 \mathrm{D}+00 \mathrm{E}(\mathrm{PBEQIDH})=$ $-0.4907832510 \mathrm{D}+00 \mathrm{E}(\mathrm{PBEQIDH})=$ $-0.4942355031 \mathrm{D}+00 \mathrm{E}(\mathrm{PBEQIDH})=$ $-0.4924183393 \mathrm{D}+00 \mathrm{E}(\mathrm{PBEQIDH})=$ $-0.4950162114 \mathrm{D}+00 \mathrm{E}(\mathrm{PBEQIDH})=$ $-0.6844453456 \mathrm{D}+00 \mathrm{E}(\mathrm{PBEQIDH})=$ $-0.6853499972 \mathrm{D}+00 \mathrm{E}(\mathrm{PBEQIDH})=$ $-0.6828290333 \mathrm{D}+00 \mathrm{E}(\mathrm{PBEQIDH})=$ $-0.6827317599 \mathrm{D}+00 \mathrm{E}(\mathrm{PBEQIDH})=$ $-0.6836512215 \mathrm{D}+00 \mathrm{E}(\mathrm{PBEQIDH})=$ $-0.8224034205 \mathrm{D}+00 \mathrm{E}(\mathrm{PBEQIDH})=$ $-0.8288797344 \mathrm{D}+00 \mathrm{E}(\mathrm{PBEQIDH})=$ $-0.3433835071 \mathrm{D}+00 \mathrm{E}(\mathrm{PBEQIDH})=$ $-0.4128752964 \mathrm{D}+00 \mathrm{E}(\mathrm{PBEQIDH})=$ $-0.4131905455 \mathrm{D}+00 \mathrm{E}(\mathrm{PBEQIDH})=$ $-0.4830684998 \mathrm{D}+00 \mathrm{E}(\mathrm{PBEQIDH})=$ $-0.4849493279 \mathrm{D}+00 \mathrm{E}(\mathrm{PBEQIDH})=$ $-0.4830451913 \mathrm{D}+00 \mathrm{E}(\mathrm{PBEQIDH})=$ $-0.4843491230 \mathrm{D}+00 \mathrm{E}(\mathrm{PBEQIDH})=$ $-0.4835145525 \mathrm{D}+00 \mathrm{E}(\mathrm{PBEQIDH})=$
$-0.46898424570760 \mathrm{D}+03$ $-0.46897234748831 \mathrm{D}+03$ $-0.54755649789996 \mathrm{D}+03$ $-0.54514905643858 \mathrm{D}+03$

$-0.23687774568467 \mathrm{D}+03$ $-0.23687793043433 \mathrm{D}+03$ $-0.23688007393553 \mathrm{D}+03$ $-0.23687686588922 \mathrm{D}+03$ $-0.23687679370976 \mathrm{D}+03$ $-0.27615719544158 \mathrm{D}+03$ $-0.27615987272973 \mathrm{D}+03$ $-0.27615838401319 \mathrm{D}+03$ $-0.27616039659006 \mathrm{D}+03$ $-0.39159876525190 \mathrm{D}+03$ $-0.39158301926646 \mathrm{D}+03$ $-0.39159214999356 \mathrm{D}+03$ $-0.39161038965231 \mathrm{D}+03$ $-0.39160603254761 \mathrm{D}+03$ $-0.47016682037079 \mathrm{D}+03$ $-0.47136382560374 \mathrm{D}+03$ $-0.19638865920651 \mathrm{D}+03$ $-0.23567934299780 \mathrm{D}+03$ $-0.23567179818935 \mathrm{D}+03$ $-0.27496272549757 \mathrm{D}+03$ $-0.27495495888951 \mathrm{D}+03$ $-0.27495457773301 \mathrm{D}+03$ $-0.27495272687573 \mathrm{D}+03$ $-0.27495511729463 \mathrm{D}+03$ 
r9.out: E2(PBEQIDH $)=-0.4830781363 \mathrm{D}+00 \mathrm{E}(\mathrm{PBEQIDH})=-0.27495407092755 \mathrm{D}+03$ PBE0DHD3BJ/Def2QZVP

C1.out: $\mathrm{E} 2(\mathrm{PBE} 0 \mathrm{DH})=-0.2736341513 \mathrm{D}+00 \mathrm{E}(\mathrm{PBE} 0 \mathrm{DH})=-0.39048554738367 \mathrm{D}+03$

C2H6.out: E2 $(\mathrm{PBE} 0 \mathrm{DH})=-0.5896553903 \mathrm{D}-01 \mathrm{E}(\mathrm{PBE} 0 \mathrm{DH})=-0.79769609717001 \mathrm{D}+0$

C2.out: $\mathrm{E} 2(\mathrm{PBE} 0 \mathrm{DH})=-0.3316064743 \mathrm{D}+00 \mathrm{E}(\mathrm{PBE} 0 \mathrm{DH})=-0.46907219194485 \mathrm{D}+03$

C3.out: $\mathrm{E} 2(\mathrm{PBE} 0 \mathrm{DH})=-0.3322233318 \mathrm{D}+00 \mathrm{E}(\mathrm{PBE} 0 \mathrm{DH})=-0.46906061700993 \mathrm{D}+03$

C4.out: E2 $(\mathrm{PBE} 0 \mathrm{DH})=-0.3896053444 \mathrm{D}+00 \mathrm{E}(\mathrm{PBE} 0 \mathrm{DH})=-0.54765892749445 \mathrm{D}+03$

C5.out: $\mathrm{E} 2(\mathrm{PBE} 0 \mathrm{DH})=-0.3803439247 \mathrm{D}+00 \mathrm{E}(\mathrm{PBE} 0 \mathrm{DH})=-0.54525174804157 \mathrm{D}+03$

CH4.out: E2(PBE0DH $)=-0.3148680111 \mathrm{D}-01 \mathrm{E}(\mathrm{PBE} 0 \mathrm{DH})=-0.40484994165441 \mathrm{D}+02$

H10.out: E2(PBE0DH $)=-0.2003837580 \mathrm{D}+00 \mathrm{E}(\mathrm{PBE} 0 \mathrm{DH})=-0.27620868641871 \mathrm{D}+03$

H11.out: E2(PBE0DH) $=$

H12.out: $\mathrm{E} 2(\mathrm{PBE} 0 \mathrm{DH})=$

H13.out: E2(PBE0DH) $=$

H14.out: E2(PBE0DH) $=$

H15.out: E2(PBE0DH) $=$

H1.out: $\mathrm{E} 2(\mathrm{PBE} 0 \mathrm{DH})=$

H2.out: E2(PBE0DH) $=$

H3.out: E2(PBE0DH) $=$

H4.out: E2(PBE0DH) $=$

H5.out: E2(PBE0DH $)=$

H6.out: E2(PBE0DH) $=$

H7.out: E2(PBE0DH) $=$

H8.out: E2(PBE0DH) $=$

H9.out: E2(PBE0DH) $=$

r10.out: $\mathrm{E} 2(\mathrm{PBE} 0 \mathrm{DH})=$

r11.out: E2(PBE0DH $)=$

r12.out: $\mathrm{E} 2(\mathrm{PBE} 0 \mathrm{DH})=$

r13.out: $\mathrm{E} 2(\mathrm{PBE} 0 \mathrm{DH})=$

r14.out: $\mathrm{E} 2(\mathrm{PBE} 0 \mathrm{DH})=$

r15.out: E2(PBE0DH $)=$

r16.out: E2(PBE0DH) =

r1.out: $\mathrm{E} 2(\mathrm{PBE} 0 \mathrm{DH})=$

r2.out: $\mathrm{E} 2(\mathrm{PBE} 0 \mathrm{DH})=$

r3.out: $\mathrm{E} 2(\mathrm{PBE} 0 \mathrm{DH})=$

r4.out: E2(PBE0DH) $=$

r5.out: E2(PBE0DH) $=$

$-0.2013895619 \mathrm{D}+00 \mathrm{E}(\mathrm{PBE} 0 \mathrm{DH})=$

$-0.2009864110 \mathrm{D}+00 \mathrm{E}(\mathrm{PBE} 0 \mathrm{DH})=$

$-0.27621048226490 \mathrm{D}+03$

$-0.1996007329 \mathrm{D}+00 \mathrm{E}(\mathrm{PBE} 0 \mathrm{DH})=$

$-0.1998679324 \mathrm{D}+00 \mathrm{E}(\mathrm{PBE} 0 \mathrm{DH})=$

$-0.27621056934351 \mathrm{D}+03$

$-0.2309036935 \mathrm{D}+00 \mathrm{E}(\mathrm{PBE} 0 \mathrm{DH})=$

$-0.27620867735348 \mathrm{D}+03$

$-0.27620697268274 \mathrm{D}+03$

$-0.1721487811 \mathrm{D}+00 \mathrm{E}(\mathrm{PBE} 0 \mathrm{DH})=$

$-0.1713894982 \mathrm{D}+00 \mathrm{E}(\mathrm{PBE} 0 \mathrm{DH})=$

$-0.1724274568 \mathrm{D}+00 \mathrm{E}(\mathrm{PBE} 0 \mathrm{DH})=$

$-0.1716150239 \mathrm{D}+00 \mathrm{E}(\mathrm{PBE} 0 \mathrm{DH})=$

$-0.1707163578 \mathrm{D}+00 \mathrm{E}(\mathrm{PBE} 0 \mathrm{DH})=$

$-0.1986704919 \mathrm{D}+00 \mathrm{E}(\mathrm{PBE} 0 \mathrm{DH})=$

$-0.2001209385 \mathrm{D}+00 \mathrm{E}(\mathrm{PBE} 0 \mathrm{DH})=$

$-0.1993581992 \mathrm{D}+00 \mathrm{E}(\mathrm{PBE} 0 \mathrm{DH})=$

$-0.2004463658 \mathrm{D}+00 \mathrm{E}(\mathrm{PBE} 0 \mathrm{DH})=$

$-0.31549721170197 \mathrm{D}+03$

$-0.23692187903542 \mathrm{D}+03$

$-0.23692191322554 \mathrm{D}+03$

$-0.23692405861892 \mathrm{D}+03$

$-0.23692094690605 \mathrm{D}+03$

$-0.23692072993478 \mathrm{D}+03$

$-0.27620852341170 \mathrm{D}+03$

$-0.27621130406849 \mathrm{D}+03$

$-0.27620975557662 \mathrm{D}+03$

$-0.2773781152 \mathrm{D}+00 \mathrm{E}(\mathrm{PBE} 0 \mathrm{DH})=$

$-0.2777852660 \mathrm{D}+00 \mathrm{E}(\mathrm{PBE} 0 \mathrm{DH})=$

$-0.2767164038 \mathrm{D}+00 \mathrm{E}(\mathrm{PBE} 0 \mathrm{DH})=$

$-0.2766535915 \mathrm{D}+00 \mathrm{E}(\mathrm{PBE} 0 \mathrm{DH})=$

$-0.2770398603 \mathrm{D}+00 \mathrm{E}(\mathrm{PBE} 0 \mathrm{DH})=$

$-0.3332617609 \mathrm{D}+00 \mathrm{E}(\mathrm{PBE} 0 \mathrm{DH})=$

$-0.3357449996 \mathrm{D}+00 \mathrm{E}(\mathrm{PBE} 0 \mathrm{DH})=$

$-0.1390613161 \mathrm{D}+00 \mathrm{E}(\mathrm{PBE} 0 \mathrm{DH})=$

$-0.27621174435322 \mathrm{D}+03$

$-0.39167238142695 \mathrm{D}+03$

$-0.39165736476497 \mathrm{D}+03$

$-0.39166590327386 \mathrm{D}+03$

$-0.39168404997197 \mathrm{D}+03$

$-0.39167990329165 \mathrm{D}+03$

$-0.47025546709642 \mathrm{D}+03$

$-0.47145226519126 \mathrm{D}+03$

$-0.1672066886 \mathrm{D}+00 \mathrm{E}(\mathrm{PBE} 0 \mathrm{DH})=$ $-0.1673472142 \mathrm{D}+00 \mathrm{E}(\mathrm{PBE} 0 \mathrm{DH})=$ $-0.1956573624 \mathrm{D}+00 \mathrm{E}(\mathrm{PBE} 0 \mathrm{DH})=$ $-0.1964484422 \mathrm{D}+00 \mathrm{E}(\mathrm{PBE} 0 \mathrm{DH})=$ $-0.1956558684 \mathrm{D}+00 \mathrm{E}(\mathrm{PBE} 0 \mathrm{DH})=$ $-0.1962004370 \mathrm{D}+00 \mathrm{E}(\mathrm{PBE} 0 \mathrm{DH})=$ $-0.1958512345 \mathrm{D}+00 \mathrm{E}(\mathrm{PBE} 0 \mathrm{DH})=$ $-0.19642513250644 \mathrm{D}+03$

$-0.23572345963167 \mathrm{D}+03$

$-0.23571567593993 \mathrm{D}+03$

$-0.27501417927197 \mathrm{D}+03$

$-0.27500622489695 \mathrm{D}+03$

$-0.27500584741774 \mathrm{D}+03$

$-0.27500411290560 \mathrm{D}+03$

r7.out: $\mathrm{E} 2(\mathrm{PBE} 0 \mathrm{DH})=$

r8.out: E2(PBE0DH $)=$ r9.out: E2(PBE0DH $)=$ $-0.1956722682 \mathrm{D}+00 \mathrm{E}(\mathrm{PBE} 0 \mathrm{DH})=$

$-0.27500643738829 D+03$

$-0.27500533851970 \mathrm{D}+03$ 